Quality of

Surface Waters of the

United States

1964

Parts 7 and 8. Lower Mississippi River Basin and Western Gulf of Mexico Basins

GEOLOGICAL SURVEY WATER-SUPPLY PAPER 1957

Prepared in cooperation with the States of Arkansas, Colorado, Kansas, Louisiana, Mississippi, Missouri, New Mexico, Oklahoma, Tennessee, Texas, and with other agencies

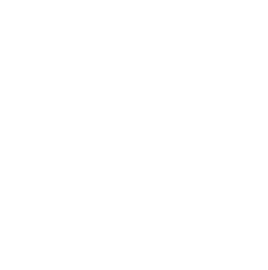




\section{UNITED STATES DEPARTMENT OF THE INTERIOR}

WALTER J. HICKEL, Secretary

\section{GEOLOGICAL SURVEY}

William T. Pecora, Director

Library of Congress catalog card No. GS 43-68 


\section{PREFACE}

This report was prepared by the Geological Survey in cooperation with the States of Arkansas, Colorado, Kansas, Louisiana, Mississippi, Missouri, New Mexico, Oklahoma, Tennessee, Texas, and with other agencies, by personnel of the Water Resources Division, E. L. Hendricks, chief hydrologist, G. W. Whetstone, assistant chief for Reports and Data Processing, under the general direction of S. M. Lang, chief, Reports Section, and B. A. Anderson, chief, Data Reports Unit.

The data were collected under supervision of district chiefs, district chemists, or engineers of the Water Resources Division, as follows:

J. S. Cragwell, Jr...........................Nashville, Tenn.

D. M. Culbertson...........................Lincoln, Nebr.

C. H. Hembree and Trigg Twichell.......Austin, Tex.

J. H. Hubble............................... Little Rock, Ark.

S. F. Kapustka succeeded by

R. L. McAvoy........................Baton Rouge, La.

R. H. Langford........................... Salt Lake City, Utah

R. P. Orth............................. Oklahoma City, Okla.

J. M. Stow.........................Albuquerque, N. Mex. 


\section{CONTENTS}

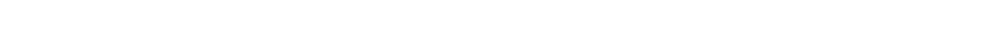

List of Water-Quality stations, in downstream

order, for which records are published.....

Introduction ..........................

Collection and examination of samples..........

Chemical quality.......................

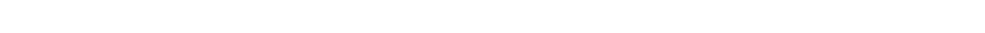

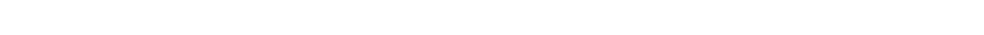

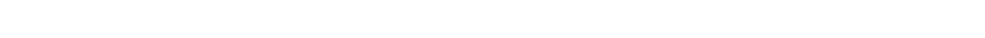

Composition of surface waters...............

Mineral constituents in solution............

Silica..........................

Aluminum.........................

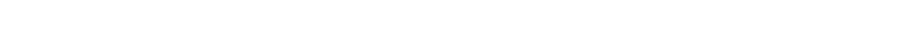

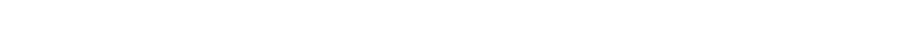

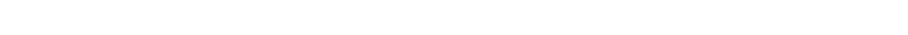

Magnesium........................

Strontium.........................

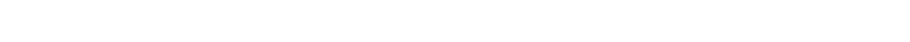

Lithium............................

Bicarbonate, carbonate and hydroxide........

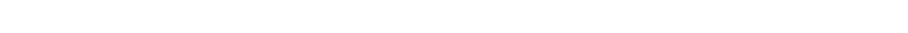

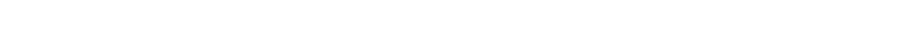

Fluoride..........................

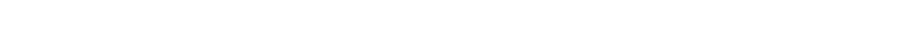

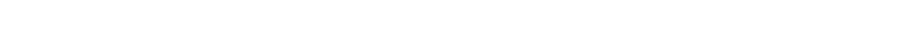

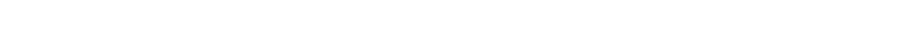

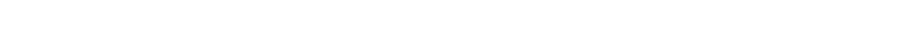

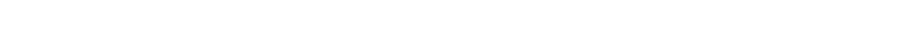

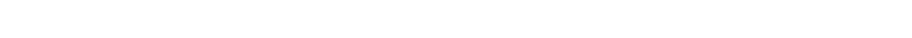

Copper.............................

Lead .............................

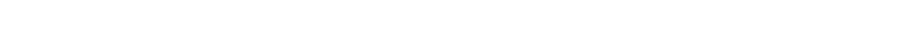

Barium............................

Bromide............................

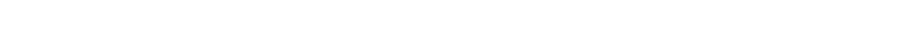

Hardness..........................

Acidity .............................

Sodium adsorption ratio................

Specific conductance..................

Hydrogen-ion concentration.............. 
Composition of surface waters--Continued

Properties and characteristics of water--Continued

Biochemical oxygen demand....

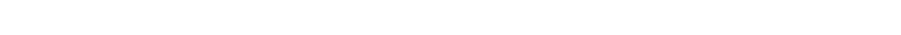

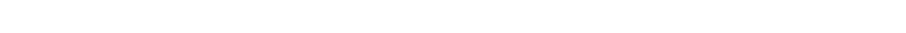

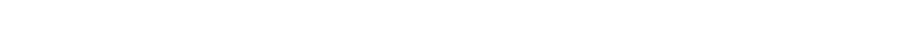

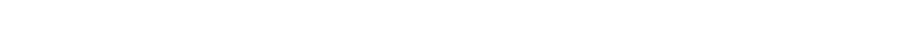

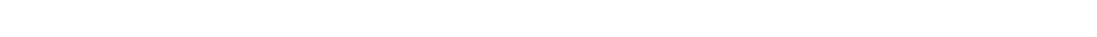

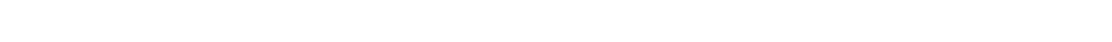

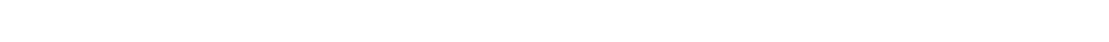

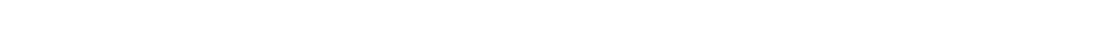

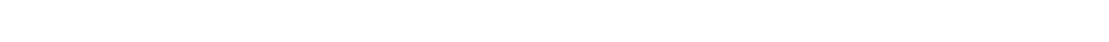

Index.................................. 599

\section{$\overline{\text { ILLUSTRATION }}$}

Figure 1. Map of the United States showing basins

Page covered by the six water-supply papers on quality of surface waters in 1964 


\section{WATER-QUALITY STATIONS, IN DOWNSTREAM ORDER, FOR WHICH RECORDS ARE PUBLISHED}

[Symbols after station name designate type of data: c, chemical;

$t$, water temperature; $s$, sediment]

PART 7. LOWER MISSISSIPPI RIVER BASIN.......... Mississippi River at St. Louis, Mo. (main stem)

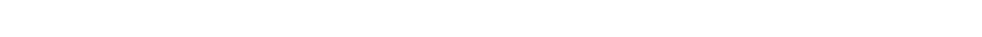

Meramec River basin...................... 41

Meramec River near Suliivan, Mo. c.......... 41

Bourbeuse River above Union, Mo. c.......... 43

Big River near DeSoto, Mo. c.............. 45

Meramec River at Paulina Hills, Mo. c....... 47

White River basin......................... 49

White River at Bull Shoals Dam, Ark. ct..... 49

White River at Buffalo City, Ark. t......... 51

Buffalo River near Rush, Ark. t........... 52

White River at Shipps Ferry, Ark. t........ 53

North Fork River at Norfork Dam, near

Norfork, Ark. c...................... 54

Little Red River near Heber Springs, Ark. c.. 55

White River at DeValls Bluff, Ark. t....... 56

White River at Clarendon, Ark. ct......... 58

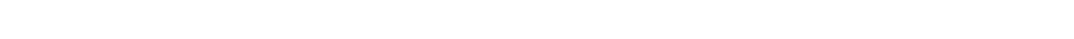

Arkansas River at Canon City, Colo. c....... 60

Arkansas River near Pueblo, Colo. c......... 61

Fountain Creek at Pueblo, Colo. c........... 62

Arkansas River near Nepesta, Colo. c........ 63

Apishapa River near Fowler, Colo. c......... 64

Arkansas River near La Junta, Colo. c........ 65

Arkansas River at Las Animas, Colo. c....... 66

Purgatoire River near Las Animas, Colo。c.... 67

Arkansas River below John Martin Reservoir,

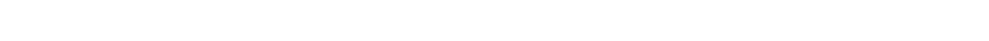

Arkansas River at Lamar, Colo. c........... 71

Arkansas River near Coolidge, Kans. cs...... 72

Arkansas River at Dodge City, Kans. cs...... 74

Arkansas River near Kinsley, Kans, ts....... 76

Pawnee River near Larned, Kans. cs.......... 81

Arkansas River at Great Bend, Kans. cs....... 83

Walnut Creek at Albert, Kans. cts........... 85

Rattlesnake Creek near Raymond, Kans. cs.... 9 90

Cow Creek near Lyons, Kans. cs............. 91

Arkansas River near Hutchinson, Kans. cts.... 93

Little Arkansas River at Valley Center,

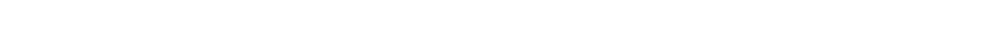

Arkansas River below Wichita, Kans. c....... 99 
LOWER MISSISSIPPI RIVER BASIN--Continued

Arkansas River basin--Continued

North Fork Ninnescah River near Cheney,

Kans. c.........................

Page

South Fork Ninnescah River near Pratt,

Kans. cs........................

South Fork Ninnescah River near Calista,

Kans. cs........................

South Fork Ninnescah River near Kingman,

Kans. cs........................

South Fork Ninnescah River near Murdock,

Kans. cs.......................

Ninnescah River near Peck, Kans. cs.........

Arkansas River at Arkansas City, Kans. cts...

East Branch Walnut River near El Dorado,

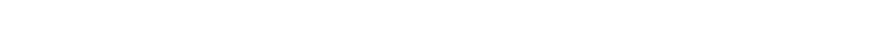

West Branch Walnut River near El Dorado,

Kans. c..........................

Walnut River near El Dorado, Kans. c........

Walnut River near Augusta, Kans. cs..........

Whitewater River at Towanda, Kans. cs........

Whitewater River at Augusta, Kans. cs........

Walnut River near Douglass, Kans. cs........

Walnut River at Winfield, Kans. cts.........

Chikaskia River near Corbin, Kans. cts.......

Chikaskia River near Blackwell, Okla. ct.....

Black Bear Creek at Pawnee, Okla. c.........

Cimarron River near Guy, $N$. Mex. s..........

Arkansas River at Sand Springs Bridge, near

Tulsa, okla. ct....................

Verdigris River near Coyville, Kans. c.......

Fall River near Eureka, Kans. c............

Fall River at Fredonia, Kans. c............

Elk River near Elk City, Kans. c...........

Verdigris River at Independence, Kans. ct....

Verdigris River near Lenapah, okla. ct.......

Verdigris River near Inola, Okla. ct.........

Neosho River near Americus, Kans. cs.........

Cottonwood River near Marion, Kans, cs.......

Mud Creek near Marion, Kans. cs.............

Cedar Creek near Cedar Point, Kans. c........

Cottonwood River at Cottonwood Falls, Kans.

cs...............................

Cottonwood River near Plymouth, Kans. cs.....

Neosho River at Burlington, Kans. c.........

Neosho River near Parsons, Kans. ct..........

Neosho River near Commerce, Okla. c.........

Center Creek near Cartervilile, Mo. c.........

Turkey Creek

Spring Creek near Joplin, Mo. c........... 182

Spring River near Baxter Springs, Kans. c.... 183

Big Cabin Creek near Big Cabin, Okla. c...... 184

Neosho River below Fort Gibson Reservoir,

near Fort Gibson, Okla. c.............. 185

rllinois River near Gore, Okla. c........... 186

Vermejo River near Dawson, N. Mex. c........ 187 
LOWER MISSISSIPPI RIVER BASIN--Continued

Arkansas River basin--Continued

Conchas Canal below Conchas Dam, N. Mex. c... 188

Ute Creek near Logan, N. Mex。c............ 189

Revuelto Creek near Logan, N. Mex. ct....... 190

Canadian River near Glenrio, N。Mex。c...... 193

Canadian River near Amarillo, Tex. ct....... 194

Little River below Hog Creek, near Norman,

Okla.c......................... 196

Little River near Sasakwa, Okla. ct......... 197

North Canadian River near Wetumka, Okla. ct.. 200

Deep Fork near Beggs, Okla。ct............. 204

Canadian River near Whitefield, Okla. ct..... 207

Arkansas River at Van Buren, Ark. ct......... 210

Arkansas River at Little Rock, Ark. ct....... 215

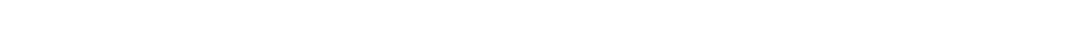

Little Wichita River near Henrietta, Tex。ct. 219

Foss Reservoir near Foss, Okla.c......... 222

Washita River at Carnegie, Okla.ct........ 223

Rush Creek near Maysville, Okla。c......... 226

Washita River near Durwood, Okla. ct........ 227

Red River at Denison Dam, near Denison, Tex.

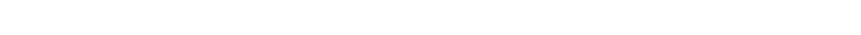

Clear Boggy Creek near Caney, Okla。c.......

South Sulphur River near Cooper, Tex。ct....

Red River near Hosston, La. ct.............

Ouachita River at Arkadelphia, Ark. ct......

Smackover Creek near Norphlet, Ark。ct......

Ouachita River near Felsenthal, Ark. ct......

Bayou Bartholomew near McGehee, Ark. c......

Bayou $D^{\prime A}$ Arbonne at Homer, La. c...........

Ouachita River at Monroe, La. c............

Little River near Pollock, La. c...........

Black River at Jonesville, La. c..........

Little Cypress Creek base-flow

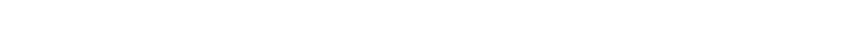

Mississippi River near St. Francisville, La.

(main stem) ct....................

Mississippi River at Luling Ferry, La.

(main stem) ct....................

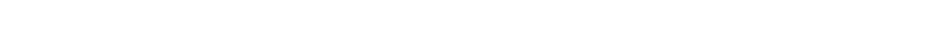

Tchefuncta River south of Covington, La. c...

Tangipahoa River near Ponchatoula, La. ct.... Miscellaneous analyses of lakes and streams in

lower Mississippi River basin..........

230

232

234

236

239

241

245

247

248

249

250

251

254

258

260

262

262

264

267

PART 8. WESTERN GULF OF MEXICO BASINS..........

Mermentau River basin................. Mermentau River at Lake Arthur, La. ct......

Sabine River basin...................... Sabine River near Tatum, Tex, ct........... Sabine River near Ruliff, Tex, ct..........

Neches River basin......................

Neches River near Alto, Tex. ct........... Angelina River near Lufkin, Tex. ct........ Angelina River below Sam Rayburn Dam, near Jasper, Tex. ct................... 
WESTERN GULF OF MEXICO BASINS--Continued

Neches River basin--Continued

Neches River at Evadale, Tex. ct..........

Trinity River basin...................

Elm Fork Trinity River near Muenster, Tex. cs

Trinity River near Rosser, Tex。cts........

Pin Oak Creek near Hubbard, Tex. s.........

Chambers Creek near Corsicana, Tex. ct......

Richland Creek near Fairfield, Tex. ct.......

Trinity River near Crockett, Tex. ct.........

Long King Creek near Livingston, Tex. ct..... Trinity River at Romayor, Tex. ct......... Trinity River near Moss Bluff, Tex. c....... old River near Cove, Tex. c............ Trinity River at Anahuac, Tex. c........... Trinity Bay at mouth of Trinity River, near

Anahuac, Tex. c...................

San Jacinto River basin...................

West Fork San Jacinto River near Conroe, Mex.

ct.............................

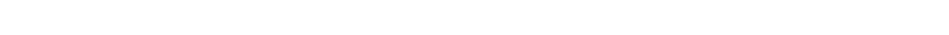

Double Moutain Fork Brazos River near Asper-

mont, Tex. ct...................

Croton Creek near Jayton, Tex。ct..........

Salt Croton Creek at weir $D$, near Aspermont,

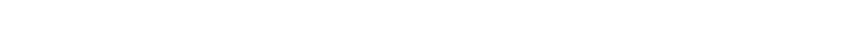

Haystack Creek near Aspermont, Tex. c.......

Salt Croton Creek near Aspermont, Tex. c....

Salt Fork Brazos River near Aspermont, Tex。

ct............................

Brazos River at Seymour, Tex. ct...........

California Creek near Stamford, Tex. ct.....

Hubbard Creek near Sedwick, Tex. ct.........

Deep Creek at Moran, Tex. ct.............

Hubbard Creek near Albany, Tex. ct..........

Salt Prong Hubbard Creek at U.S. Highway 380 , near Albany, Tex. ct.................. North Fork Hubbard Creek near Albany, Tex. ct Snailum Creek near Albany, Tex. ct......... Big Sandy Creek near Breckenridge, Tex. cts.. Hubbard Creek near Breckenridge, Tex。ct.... Clear Fork Brazos River at Eliasville, Tex。

ct..............................

Page

296

298

298

302

305

307

310

312

314

316

319

321

323

326

329

329

332

332

334

336

337

338

339

342

344

346

348

350

352

354

356

358

361

363

Brazos River at Possum Kingdom Dam, near

Graford, Tex $c \ldots \ldots \ldots \ldots \ldots \ldots \ldots \ldots$

Brazos River at Whitney Dam, near Whitney,

Tex。ct..........................

North Bosque River near Clifton, Tex, c..... Leon River near Belton, Tex. ct........... Lampasas River at Youngsport, Tex。ct........ Lampasas River near Belton, Tex。c......... San Gabriel River at Georgetown, Tex. c..... Little River at Cameron, Tex. ct........... Brazos River at State Highway 21 , near Bryan,

Tex.ct......................... 
WESTERN GULF OF MEXICO BASINS--Continued

Brazos River basin--Continued

Yegua Creek near Somerville, Tex.ct........

Navasota River near Bryan, Tex. ct........... Brazos River at Richmond, Tex. ct.......... Brazos River at Harris Reservoir, near

Angleton, Tex. c................... Brazos River at Brazoria Reservoir, near

Brazoria, Tex.c................... San Gabriel River base-flow study in Texas c.

Colorado River basin................... Colorado River near lra, Tex. ct........... Colorado River at Colorado City, Tex. ct..... Beals Creek near Westbrook, Tex。ct......... Colorado River near Silver, Tex. ct.......... Colorado River at Ballinger, Tex. ct........ San Saba River at San Saba, Tex. cts........ Colorado River near San Saba, Tex. ct....... Colorado River at Austin, Tex. ct.......... Colorado River at Columbus, Tex. ts......... Colorado River at Wharton, Tex. ct.........

Lavaca River basin..................... Navidad River near Ganado, Tex. ct..........

Guadalupe River basin.................... Guadalupe River at Victoria, Tex. ct........ Coleto Creek near Shroeder, Tex.c.......... San Antonio River at Goliad, Tex. ct........

Mission River basin................... Mission River near Refugio, Tex. ct.........

Nueces River basin...................... Nueces River near Mathis, Tex. ct.......... Rio Grande basin........................ Rio Grande above Culebra Creek, near Lobatos,

Colo.ct........................... Red River below Zwergle damsite, near Red

River, N. Mex. s.................... Rio Hondo at damsite, at Valdez, N . Mex. S... Rio Grande de Ranchos near Talpa, N. Mex. s.. Willow Creek near Park View, N. Mex. S...... Rio Chama above Abiquiu Reservoir, N. Mex。 ts Rio Chama below Abiquiu Dam, N. Mex. ts...... Rio Chama near Chamita, $N$. Mex. cts......... Rio Nambe at Nambe Falls, near Nambe,

N. Mex. s......................... Rio Grande at Otowi Bridge, near San

Ildefonso, N. Mex.cts................ Galisteo Creek at Domingo, N. Mex. ts....... Jemez River below East Fork, near Jemez

Springs, N. Mex. c.................. Bernalillo floodwater retarding reservoir

No. 1 (Piedra Lisa Arroyo), near Berna-

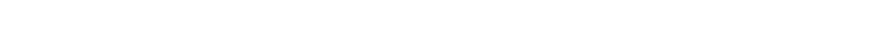
Rio Grande near Bernalilio, $N$. Mex. ts....... Rio Grande near Bernardo, N. Mex. cts........ Rio Puerco near Bernardo, N. Mex. cs........

390

393

393

396

398

400

403

406

408

411

413

417

419

419

422

422

424

425

428

428

431

431

433

433

435

436

437

438

439

443

447

453

454

460

464 
WESTERN GULF OF MEXICO BASINS--Continued

Rio Grande basin--Continued

Rio Salado near San Acacia, No Mex。 S.......

Rio Grande conveyance channel at San Acacia,

$\mathrm{N}$. Mex. ts...........................

Rio Grande floodway at San Acacia, N. Mex。ts

Rio Grande conveyance channel at San Marcial,

N. Mex. cts........................

Rio Grande floodway at San Marcial, $N$. Mex。

cts............................

Tortugas Arroyo near Las Cruces, N. Mex. S...

Rio Grande at El Paso, Tex. c............

Rio Grande at Fort Quitman, Tex.c.........

Rio Grande above Rio Conchos, near Presidio,

Tex.c.........................

Rio Grande at Johnson Ranch, Tex.c........

Rio Grande at Langtry, Tex.c............

Gallinas River near Montezuma, N。Mex。c....

Gallinas River at Montezuma, No Mex。c......

Pecos River at Santa Rosa, N。Mex。ts......

Pecos River below Alamogordo Dam, N. Mex。ct。

Pecos River near Acme, $N$. Mex。 ct..........

Rio Ruidoso at Hollywood, N. Mex. c........

Pecos River near Artesia, $N_{0}$ Mex。 cts.......

Rio Penasco at Dayton, $N$. Mex. s...........

Pecos River below McMillan Dam, N.Mex。c...

Pecos River at ford crossing, above Major

Johnson Springs, near Lakewood, N. Mex....

Pecos River at damsite 3, near Carlsbad,

N. Mex. c.........................

Pecos River at Carlsbad, NoMex. ct........

Black River at Harkey Crossing, near Malaga,

N. Mex。c ..........................

Pecos River near Malaga, No Mex. ct..........

Pecos River at Pierce Canyon Crossing, near

Malaga, $N$ 。 Mex。ct..................

Pecos River at Red Bluff, NoMex。ct........

Pecos River below Red Bluff Dam, near Orla,

Tex.ct........................

Pecos River near Girvin, Tex。ct..........

Pecos River near Shumla, Tex,c.........

Rio Grande at Laredo, Tex.c.............

Rio Grande below Falcon, Dam, Tex.c.......

Rio Grande at Fort Ringgold, Rio Grande City,

Tex.c............................

Rio Grande at Anzalduas Dam, Tex.c........

Pecos River seepage investigations $c \ldots \ldots \ldots$

Tularosa Valley basin..................

Rio Tularosa near Bent, $\mathrm{N}$. Mex。 $\ldots \ldots \ldots$ Miscellaneous analyses of streams in western

Gulf of Mexico basins................ 


\title{
QUALITY OF SURFACE WATERS OF THE UNITED STATES, 1964
}

\author{
PARTS 7 and 8
}

\section{IN'TRODUCTION}

The quality-of-water investigations of the United States Geological Survey are concerned with chemical and physical characteristics of the surface and ground water supplies of the Nation. Most of the investigations carried on in cooperation with State and Federal agencies deal with the amounts of matter in solution and in suspension in streams.

The record of chemical analysis, suspended sediment, and temperature of surface waters given in this volume serve as a basis for determining the suitability of waters for various uses. The flow and water quality of a stream are related to variations in rainfall and other forms of precipitation. In general, lower concentrations of dissolved solids may be expectedduring periods of high flow than during periods of low flow. Conversely, the suspended solids in some streams may change materially with relatively small variations in flow, whereas for other streams the quality of the water may remain relatively uniform throughout large ranges in discharge.

The Geological Survey has published annual records of chemical quality, suspended sediment, and water temperature since 1941. The records prior to 1948 were published each yea $r$ in a single volume for the entire country, and in two volumes in 1948 and 1949. From 1950 to 1958, the records were published in four volumes and from 1959 to 1963 in five volumes. Beginning with the 1964 water year, water quality records obtained by the Geological Survey were published in a new series of annual releases on a state-boundary basis. These records are then published in six volumes in the Geological Survey water-supply paper sories. The drainage basins covered in the six volumes are shown in Figure 1. The data given in this report were collected during the water year October 1, 1963 to September 30,1964. The records are 


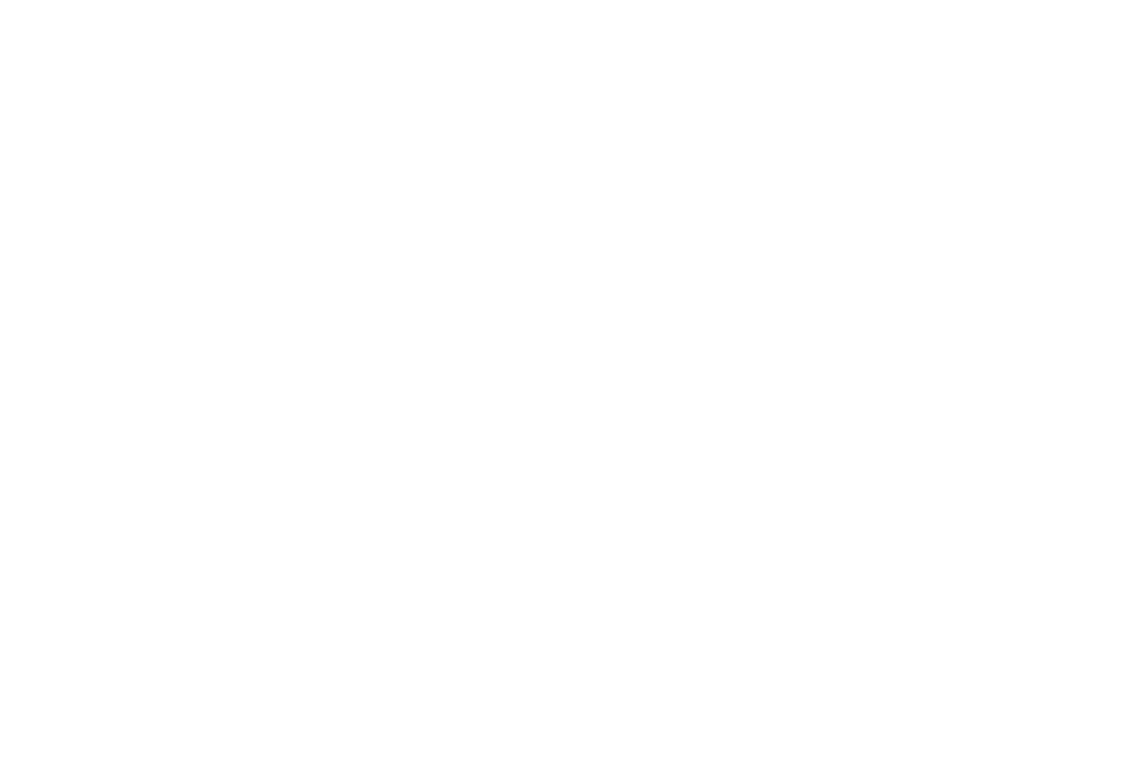

Figure 1.--Map of the United States showing basins covered by the six water-supply papers on quality of surface waters in 1964. The shaded part represents the section of the country covered by this volume; the unshaded part represents the soction of the country covered by other water-supply papers.

arranged by drainage basins in downstream order according to the Geological Survey method of reporting streamflow. St ation s on tributary streams are listed between stations on the main stem in the order in which those tributaries enter the main stem.

A station number has been assigned as an added means of identification for each stream location where regular measurements of water quantity or quality have been made. The numbers have been assigned to conform with the standard downstream order of listing gaging stations. The numbering system consists of 2 digits followed by a hyphen and a 6-digit number. The notation to the left of the hyphen identifies the Part or hydrologic region used by the Geological Survey for reporting hydrologic date. The number to the right of the hyphen represents the location of the station in the standard downstream order within each of the 15 parts (Fig. 1). The assigned numbers are in numerical order but are 
not consecutive. They are so selected from the complete 6-digitnumber scale that intervening numbers will be available for future assignments to new locations. The identification number for each station in this report is printed to the left of the station name and contains only the essential digits. For example, the number is printed as 4-100 for a station whose complete identification number is $04-0100.00$.

Descriptive statements are given for each sampling station where chemical analyses, temperature measurements, or sediment determinations have been made. These statements include location of the station, drainage area, periods of records available, extremes of dissolved solids, hardness, specific conductance, temperature, sediment loads, and other pertinent data. Records of discharge of the streams at or near the sampling station are included in most tables of analyses.

During the water year ending September 30, 1964, the Geological Survey maintained 228 stations on 114 streams for the study of chemical and physical characteristics of surface water. Samples were collected daily and monthly at 206 of these locations for chemical-quality studies. Samples also were collected less frequently at many other points. Water temperatures were measured continuously at 7 and daily at 107 stations. All surface water samples collected and analyzed during the year have not been included. Single analyses made of daily samples before compositing have not been reported. The specific conductance of almost all daily samples was determined, and as noted in the table headings this information is available for reference at the district offices listed under Division of Work, on page 28 .

Quantities of suspended sediment are reported for 48 stations during the year ending September 30, 1964. Sediment samples were collected one or more times daily at most stations, depending on the rate of flow and changes in stage of the stream. Particlesize distributions of sediments were determined at 32 of the stations.

\section{COLLECTION AND EXAMINATION OF SAMPIES}

Quality of water stations usually are located at or near points on streams where streamflow is measured by the U.S. Geological Survey. The concentration of solutes and sediments at different 
locations in the stream-cross section may vary widely with different rates of water discharge depending on the so'urce of the material and the turbulence and mixing of the stream. In general, the distribution of sediment in a stream section is much more variable than the distribution of solutes. It is necessary to sample some streams at several verticals across the chanrel and especially for sediment, to uniformly traverse the derth of flow. These measurements require special sampling equipment to adequately integrate the vertical and lateral variability of the concentration in the section. These procedures yield a velocityweighted mean concentration for the section.

The near uniformly dispersed ions of the solute load move with the velocity of the transporting water. Accordingly, the mean section concentration of solutes determined from samples is a precise measure of the total solute load. The mean section concentration obtained from suspended sediment samples is a less precise measure of the total sediment load, because the sediment samplers do not traverse the bottom 0.3 foot of the sampling vertical where the concentration of suspended sediment is greatest and because a significant part of the coarser particles in many streams move in essentially continuous contact with the bed and are not represented in the suspended sediment sample. Hence, the computed sediment loads presented in this report are usually less than the total sediment loads. For most streams the difference between the computed and total sediment loads will be small, in the order of a few percent.

\section{CHEMICAL QUALITY}

The methods of collecting and compositing water samples for chemical analysis are described by Rainwater and Thatcher (1960, 301 p.). No single method of compositing samples is applicable to all problems related to the study of water quality. Although the method of 10-day periods or the equivalent of three composite samples per month generally is practiced, modifications usually are made on the basis of dissolved-solids content as indicated by measurements of conductivity of daily samples, supplemented by other information such as chloride content, river stage, weather conditions and other background information of the stream. 


\section{TEMPERATURE}

Daily water temperatures were measured at most of the stations at the time samples were collected for chemical quality or sediment content. So far as practicable, the water temperatures were taken at about the same time each day in order that the data would be relatively unaffected by diurnal variations in temperature. Most large streams have a small diurnal variation in water temperature; small, shallow streams may have a daily range of several degrees and may follow closely the changes in air temperature. The thermometers used for determining water temperature were accurate to plus or minus $0.5^{\circ} \mathrm{F}$.

At stations where thermographs are located, the records consist of maximum and minimum temperatures for each day, and the monthly averages of maximum daily and minimum daily temperatures.

\section{SEDIMENT}

In general, suspended-sediment samples were collected daily with depth-integrating cable-suspended samples (U.S. InterAgency, 1963, and 1952.) from a fixed sampling point at one vertical in the cross section. A hand sampler was used at many stations during periods of low flow. Depth-integrated samples were collected periodically at three or more verticals in the cross section to determine the cross-sectional distribution of the concentration of suspended sediment with respect to that at the daily sampling vertical. In streams where transverse distribution of sediment concentration ranges widely, samples were taken at two or more verticals to define more accurately the average concentration of the cross section. During periods of high or rapidly changing flow, samples were taken two or more times a day at most sampling stations.

Sediment concentrations were determined by filtrationevaporation method. At many stations the daily mean concentration for some days was obtained by plotting the velocity-wejghted instantaneous concentrations on the gage-height chart. The plotted concentrations, adjusted if necessary, for cross-sectional distribution were connected or averaged by continuous curres to obtain a concentration graph. This graph represented the estimated velocity-weighted concentration at any time, and for most periods daily mean concentrations were determined from the 
graph. The days were divided into shorter intervals when the concentration and water discharge were changing rapidly. During some periods of minor variation in concentration, the average concentration of the samples was used as the daily mean concentration. During extended periods of relatively uniform concentration and flow, samples for a number of days were composited to obtain average concentrations and average daily loads for each period.

For some periods when no samples were collected, daily loads of suspended sediment were estimated on the basis of water discharge, sediment concentrations observed immediately before and after the periods, and suspended-sediment loads for other periods of similar discharge. The estimates were further guided by weather conditions and sediment discharge for other stations.

In many instances where there were no observations for several days, the suspended-sediment loads for individual days are not estimated, because numerous factors influencing the quantities of transported sediment made it very difficult to male accurate estimates for individual days. However, estimated loads of suspended sediment for missing days in an otherwise continuous period of sampling have been included in monthly and annual totals in order to provide a complete record. For some streams, samples were collected weekly, monthly, or less frequently, and only rates of sediment discharge at the time of sampling are shown.

In addition to the records of quantities of suspender sediment transported, records of the particle sizes of sediment are included. The particle sizes of the suspended sediment for many of the stations, and the particle sizes of the bed materic.I for some of the stations were determined periodically.

The size of particles in stream sediments commonly range from colloidal clay (finer than $0.001 \mathrm{~mm}$ ) to coarse sand or gravel (coarser than $1.0 \mathrm{~mm}$ ). The common methods of particle-size analyses cannot accommodate such a wide range in particle size. Hence, it was necessary to separate most samples into two parts, one coarser than $0.062 \mathrm{~mm}$ and one finer than $0.062 \mathrm{~mm}$. The separations were made by sieve or by a tube containing a settling medium of water. The coarse fractions were classified by sieve separation or by the visual accumulation tube (U.S. Inter-Agency, 1957). The fine fractions were classified by the pipet method (Kilmer and Alexander, 1949) or the bottom withdrawal tube method (U.S. Inter-Agency, 1943). 


\section{EXPRESSION OF RESULTS}

The quantities of solute concentrations analyzed in the laboratory are measured by weight-volume units (milligrams per liter) and for reporting, are converted to weight-weight units (parts per million). For most waters, this conversion is made by assuming that the liter of water sample weighs 1 kilogram; and thus milligrams per liter are equivalent to parts per million (ppm).

Equivalents per million are not reported, but they can be calculated easily from the parts per million data. An equivalent per million (epm) is a unit chemical combining weight of a constituent in a million unit weights of water. Chemical equivalence in equivalents per million can be obtained by (a) dividing the concentration in parts per million by the combining weight of that ion, or (b) multiplying the concentration (in ppm) by the reciprocals of the combining weights. The following table lists the reciprocals of the combining weights of cations and anions generally reported in water analyses.

The conversion factors are computed from atomic weights based on carbon-12 (International Union of Pure and Applied Chemistry, 1961).

Conversion factors: Parts per million to equivalents per million

\begin{tabular}{|c|c|c|c|}
\hline Ion & $\begin{array}{l}\text { Multi- } \\
\text { ply by }\end{array}$ & Ion & $\begin{array}{l}\text { Multi- } \\
\text { ply by }\end{array}$ \\
\hline Aluminum $(\mathrm{Al}+3) \ldots . .$. & 0.11119 & Iron $(\mathrm{Fe}+3)$ & 0.05372 \\
\hline Barium $\left(\mathrm{Ba}^{+2}\right) \ldots . . . \ldots$ & .01456 & Lead $(\mathrm{Pb}+2) .$. & .07965 \\
\hline Bicarbonate $\left(\mathrm{HCO}_{3}{ }^{-1}\right)$. & .01639 & Lithium $\left(\mathrm{Li}^{+1}\right) \ldots$ & .14411 \\
\hline Bromide $\left(\mathrm{Br}^{-1}\right) \ldots . . .$. & .01251 & Magnesium $\left(\mathrm{Mg}^{+2}\right) \ldots$ & .08226 \\
\hline Calcium $(\mathrm{Ca}+2) \ldots \ldots \ldots$. & .04990 & Manganese $(\mathrm{Mn}+2) \ldots$ & .03640 \\
\hline Carbonate $\left(\mathrm{CO}_{3}^{-2}\right) \ldots .$. & .03333 & Nickel $(\mathrm{Ni}+2) \ldots \ldots \ldots \ldots$ & .03406 \\
\hline Chloride $\left(\mathrm{Cl}^{-1}\right) \ldots . . . .$. & .02821 & Nitrate $\left(\mathrm{NO}_{3}^{-1}\right)$. & .01613 \\
\hline Chromium $(\mathrm{Cr}+6) \ldots . .$. & .11539 & Nitrite $\left(\mathrm{NO}_{2}{ }^{-1}\right) .$. & .02174 \\
\hline Cobalt $(\mathrm{Co}+2) \ldots . . . . . .$. & .03394 & Phosphate $\left(\mathrm{PO}_{4}{ }^{-3}\right) \ldots$ &.$C 3159$ \\
\hline Copper $(\mathrm{Cu}+2) \ldots \ldots \ldots \ldots$ & .03148 & Potassium $(K+1)$ &.$C 2557$ \\
\hline Fluoride $\left(F^{-1}\right) \ldots \ldots \ldots$ & .05264 & Sodium $(\mathrm{Na}+1)$. & .C4350 \\
\hline Hydrogen $(\mathrm{H}+1) \ldots$ & .99209 & Strontium $(\mathrm{Sr}+2)$ & .C2283 \\
\hline Hydroxide $\left(\mathrm{OH}^{-1}\right)$. & .05880 & Sulfate $\left(\mathrm{SO}_{4}{ }^{-2}\right)$ & .C 2082 \\
\hline Iodide $\left(\mathrm{I}^{-1}\right) \ldots \ldots \ldots \ldots$ & .00788 & Zinc $(Z n+2) \ldots$ & (C3060 \\
\hline
\end{tabular}


Results given in parts per million can be converted to grains per United States gallon by dividing by 17.12 .

The hardness of water is conventionally expressed in all water analyses in terms of an equivalent quantity of calcium carbonate. Such a procedure is required because hardness is caused by several different cations, present in variable pronortions. It should be remembered that hardness is an expressior in conventional terms of a property of water. The actual presence of calcium carbonate in the concentration given is not to ke assumed. The hardness caused by calcium and magnesium (and other cations if significant) equivalent to the carbonate and bicarbonate is called carbonate hardness; the hardness in excess of this quantity is called noncarbonate hardness. Hardness or alkalinity values expressed in parts per million as calcium carbonate may be converted to equivalents per million by dividing by 50 .

The value usually reported as dissolved solids is the residue on evaporation after drying at $180^{\circ} \mathrm{C}$ for 1 hour. For some waters, particularly those containing moderately large quantities of soluble salts, the value reported is calculated from the quantities of the various determined constituents using the carbonate equivalent of the reported bicarbonate. The calculated sum of the constituents may be given instead of or in addition to the residue. In the analyses of most waters used for irrigation, the quantity of dissolved solids is given in tons per acre-foot as well as in parts per million.

Specific conductance is given for most analyses $\varepsilon$ nd was determined by means of a conductance bridge and using a standard potassium chloride solution as reference. Specific conductance values are expressed in micromhos per centimeter at $25^{\circ} \mathrm{C}$. Specific conductance in micromhos is 1 million times the reciprocal of specific resistance at $25^{\circ} \mathrm{C}$. Specific resistance is the resistance in ohms of a column of water 1 centimeter long and 1 square centimeter in cross section.

The discharge of the streams is reported in cubic feet per second (see Streamflow, p. 25) and the temperature in degrees Fahrenheit. Color is expressed in units of the platinum-cobalt scale proposed by Hazen (1892). A unit of color is Froduced by one milligram per liter of platinum in the form of the chloroplatinate ion. Hydrogen-ion concentration is expressed in terms of $\mathrm{pH}$ units. By definition the $\mathrm{pH}$ value of a solution is the negative logarithm of the concentration of gram ions of hydrogen. 
An average of analyses for the water year is given for most daily sampling stations. Most of these averages are arithmetical, time-weighted, or discharge-weighted; when analyses during a year are all on 10-day composites of daily samples with no missing days, the arithmetical and time-weighted averages are equivalent. A time-weighted average represents the composition of water that would be contained in a vessel or reservoir that had received equal quantities of water from the river each day for the water year. A discharge-weighted average approximates the composition of water that would be found in a reservoir containing all of the water passing a given station during the year. A dischargeweighted average is computed by multiplying the discharge for the sampling period by the concentrations of individual constituents for the corresponding period and dividing the sum of the products by the sum of the discharges. For most streams, discrargeweighted averages are lower than arithmetical averages because at times of high discharge the rivers generally have low ccncentrations of dissolved solids.

A program for computing these averages on an electronic digital computer was instituted in the 1962 water year. This program extended computations to include averages for $\mathrm{pH}$ values expressed in terms of hydrogen ion and averages for the ccncentration of individual constituents expressed in tons per day. Concentrations in tons per day are computed the same as daily sediment loads.

The concentration of sediment in parts per million is computed as 1,000,000 times the ratio of the weight of sediment to the weight of water-sediment mixture. Daily sediment loads are expressed in tons per day and except for subdivided days, are usually obtained by multiplying daily mean sediment concentrations in parts per million by the daily mean discharge in cubic feat per second, and the conversion factor, normally 0.0027 .

Particle size analyses are expressed in percentages of material finer than classified sizes (in millimeters). The size classification used in this report agrees closely with recommendations made by the American Geophysical Union Subcommittee on sediment terminology (Lane and others, 1947). The particle size distributions given in this report are not necessarily representative of the particle sizes of sediment in transport in the natural stream. Most of the organic matter is removed and the sample is subjected to mechanical and chemical dispersion rofore analysis of the silt and clay. 


\section{COMPOSITION OF SURFACE WATERS}

All natural waters contain dissolved mineral matter. The quantity of dissolved mineral matter in a natural weter depends primarily on the type of rocks or soils with which the water has been in contact and the length of time of contact. Ground water is generally more highly mineralized than surface runoff because it remains in contact with the rocks and soils for much longer periods. Some streams are fed by both surface runoff and ground water from springs or seeps. Such streams reflect the chemical character of their concentrated underground sources during dry periods and are more dilute during periods of heavy rainfall. The dissolved-solids content in a river is frequently increased by drainage from mines or oil fields, by the addition of industrial or municipal wastes, or--in irrigated regions--by drainage from irrigated lands.

The mineral constituents and physical properties of natural waters reported in the tables of analyses include those that have a practical bearing on water use. The results of analyses generally include silica, iron, calcium, magnesium, sodium, potassium (or sodium and potassium together calculated as sodium), lithium, carbonate, bicarbonate, sulfate, chloride, fluoride, nitrate, boron, $\mathrm{pH}$, dissolved solids, and specific conductance. Aluminum, manganese, color, acidity, dissolved oxygen, and other dis solved constituents and physical properties are reported for certain streams. Phenolic material and minor elements including strontium, chromium, nickel, copper, lead, zinc, cobalt, and other trace elements are determined occasionally for a few streams in connection with specific problems and the results are reported. The source and significance of the different constituents and properties of natural waters are discussed in the following paragraphs. The constituents are arranged in the order that they appear in the tables.

\section{MINERAL CONSTITUENTS IN SOLUTION}

Silica $\left(\mathrm{SiO}_{2}\right)$

Silica is dissolved from practically all rocks. Some natural surface waters contain less than 5 parts per million $c^{f}$ silica and few contain more than 50 parts, but the more common range is from 10 to 30 parts per million. Silica affects the usefulness of 
a water because it contributes to the formation of boiler scale; it usually is removed from feed water for high-pressure bcilers. Silica also forms troublesome deposits on the blades of stearn turbines.

\section{Aluminum (Al)}

Aluminum is usually present only in negligible quantities in natural waters except in areas where the waters have been in contact with the more soluble rocks of high aluminum content such as bauxite and certain shales. Acid waters often contain large amounts of aluminum. It may be troublesome in feed waters where it tends to be deposited as a scale on boiler tubes.

$\operatorname{Iron}(\mathrm{Fe})$

Iron is dissolved from many rocks and soils. On exposire to the air, normal basic waters that contain more than 1 part per million of iron soon become turbid with the insoluble reddish ferric oxide produced by oxidation. Surface waters, therefore, seldom contain as much as 1 part per million of dissolved iron, although some acid waters carry large quantities of iron in solution. Iron causes reddish-brown stains on porcelain or enameled ware and fixtures and on fabrics washed in the water.

Manganese $(\mathrm{Mn})$

Manganese is dissolved in appreciable quantities from rocks in some sections of the country. It resemblesiron in its chemical behavior and in its occurrence in natural waters. However, manganese in rocks is less abundant than iron. As a result the concentration of manganese is much less than that of iron and is not regularly determined in many areas. It is especially objectionable in water used in laundry work and in textile processing. Concentrations as low as 0.2 part permillion may cause a dark-brcwn or black stain on fabrics and porcelain fixtures. Appreciable quantities of manganese are often found in waters containing objectionable quantities of iron.

\section{Calcium (Ca)}

Calcium is dissolved from almost all rocks and soils, r't the highest concentrations are usually found in waters that have been in contact with limestone, dolomite, and gypsum. Calcium and magnesium make water hard and are largely responsible for the formation of boiler scale. Most waters associated with granite or silicious sands contain less than 10 parts per million of calcium; 
waters in areas where rocks are composed of dolomite and limestone contain from 30 to 100 parts per million; and waters that have come in contact with deposits of gypsum may contain several hundred parts per million.

Magnesium (Mg)

Magnesium is dissolved from many rocks, particularly from dolomitic rocks. Its effect in water is similar to that of calcium. The magnesium in soft waters may amount to only 1 or 2 parts per million, but water in areas that contain large quantities of dolomite or other magnesium-bearing rocks may contain from 20 to 100 parts per million or more of magnesium.

Strontium (Sr)

Strontium is a typical alkaline-earth element and is similar chemically to calcium. Strontium may be present in natural water in amounts up to a few parts per million much more frequently than the available data indicate. In most surface water the amount of strontium is small in proportion to calcium. However, in sea water the ratio of strontium to calcium is $1: 30$.

Sodium and potassium ( $\mathrm{Na}$ and $\mathrm{K}$ )

Sodium and potassium are dissolved from practically all rocks. Sodium is the predominant cation in some of the more highly mineralized waters found in the western United States. Nat'iral waters that contain only 3 or 4 parts per million of the two together are likely to carry almost as much potassium as sodium. As the total quantity of these constituents increases, the proportion of sodium becomes much greater. Moderate quantities of sodium and potassium have little effect on the usefulness of the water for most purposes, but waters that carry more than 50 or 107 parts per million of the two may require careful operation of steam boilers to prevent foaming. More highly mineralized waters that contain a large proportion of sodium salts may be unsatisfactory for irrigation.

In this report, sodium and potassium values that are calculated and reported as sodium are indicated by footnote.

Lithium (Li)

Data concerning the quantity of lithium in water are scarce. It is usually found in small amounts in thermal springs and saline 
waters. Lithium also occurs in streams where some industries dump their waste water. The scarcity of lithium in rocks is responsible more than other factors for relatively small amounts present in water.

Bicarbonate, carbonate and hydroxide $\left(\mathrm{HCO}_{3}, \mathrm{CO}_{3}, \mathrm{OH}\right)$

Bicarbonate, carbonate, or hydroxide is sometimes reported as alkalinity. The alkalinity of a water is defined as its capacity to consume a strong acid to $\mathrm{pH}$ 4.5. Since the major causes of alkalinity in most natural waters are carbonate and bicarkonate ions dissolved from carbonate rocks, the results are usually reported in terms of these constituents. Although alkalinity may suggest the presence of definite amounts of carbonate, bicarbonate or hydroxide, it may not be true due to other ions that contribute to alkalinity such as silicates, phosphates, borates, possibly fluoride, and certain organic anions which may occur in colored waters. The significance of alkalinity to the domestic, agricultural, and industrial user is usually dependent upon the nature of the cations $(\mathrm{Ca}, \mathrm{Mg}, \mathrm{Na}, \mathrm{K})$ associated with it. However, alkalinity in moderate amounts does not adversely affect most users.

Hydroxide may occur in water that has been softened ry the lime process. Its presence in streams usually can be taken as an indication of contamination and does not represent the natural chemical character of the water.

Sulfate $\left(\mathrm{SO}_{4}\right)$

Sulfate is dissolved from many rocks and soils--in especially large quantities from gypsum and from beds of shale. It is formed also by the oxidation of sulfides of iron and is therefore present in considerable quantities in waters from mines. Sulfate in waters that contain much calcium and magnesium causes the formation of hard scale in steam boilers and may increase the cost of softening the water.

\section{Chloride $(\mathrm{Cl})$}

Chloride is dissolved from rock materials in all parts of the country. Surface waters in the humid regions are usually low in chloride, whereas streams in arid or semiarid regions may contain several hundred parts per million of chloride leached from soils and rocks, especially where the streams receive return drainage from irrigated lands or are affected by ground-waterinflow carrying appreciable quantities of chloride. Large quan- 
tities of chloride in water that contains a high content of calcium and magnesium increases the water's corrosiveness.

\section{Fluoride (F)}

Fluoride has been reported as being present in some rocks to about the same extent as chloride. However, the quantity of fluoride in natural surface waters is ordinarily very small compared to that of chloride. Investigations have proved that fluoride concentrations of about 0.6 to $1.7 \mathrm{ppm}$ reduced the incidence of dental caries and that concentrations greater than $1.7 \mathrm{ppm}$ also protect the teeth from cavities but cause an undesirable black stain (Durfor and Becker, 1964, p. 20). Public Health Service, 1962 (p. 8), states, "When fluoride is naturally present in drinking water, the concentration should not average more than the appropriate upper control limit (0.6 to $1.7 \mathrm{ppm})$. Presence of fluoride in average concentration greater than two times the optimum values shall constitute grounds for rejection of the supply." Concentration higher than the stated limits may cause mottled enamel in teeth, endemic cumulative fluorosis, and skeletal effects.

Nitrate $\left(\mathrm{NO}_{3}\right)$

Nitrate in water is considered a final oxidation product of nitrogenous material and may indicate contamination ly sewage or other organic matter. The quantities of nitrate presert in surface waters are generally less than 5 parts per million (as $\mathrm{NO}_{3}$ ) and have no effect on the value of the water for orcinary uses.

It has been reported that as much as 2 parts per million of nitrate in boiler water tends to decrease intercrystalline cracking of boiler steel. Studies made in Illinois indicate that nitrates in excess of 70 parts per million (as $\mathrm{NO}_{3}$ ) may contribute to methemoglobinemia ("blue babies") (Faucett and Miller, 1946), and more recent investigations conducted in Ohio show that drinking water containing nitrates in the range of 44 to $88 \mathrm{ppm}$ (as $\mathrm{NO}_{3}$ ) may cause methemoglobinemia (Waring, 1949). A report fublished by the National Research Council, Maxcy (1950) concludes that a nitrate content in excess of 44 parts per million (as $\mathrm{NO}_{3}$ ) should be regarded as unsafe for infant feeding. U.S. Public Health Service (1962) sets $45 \mathrm{ppm}$ as the upper limit.

Phosphate $\left(\mathrm{PO}_{4}\right)$

Phosphorus is an essential element in the growth of plants and animals. Some sources that contribute nitrate, such as organic 
wastes are also important sources of phosphate. The addition of phosphates in water treatment constitutes a possible so:irce, although the dosage is usually small. In some areas, phosnhate fertilizers may yield some phosphate to water. A moreimpcrtant source is the increasing use of phosphates in detergents. Domestic and industrial sewage effluents often contain considerable amounts of phosphate.

\section{Boron (B)}

Boron in small quantities has been found essential for plant growth, but irrigation water containing more than 1 part per million boron is detrimental to citrus and other boron-sensitive crops. Boron is reported in Survey analyses of surface waters in arid and semiarid regions of the Southwest and West where irrigation is practiced or contemplated, but few of the surface waters analyzed have harmful concentrations of boron.

\section{Dissolved solids}

The reported quantity of dissolved solids--the residue on evaporation--consists mainly of the dissolved mineral constituents in the water. It may also contain some organic matter and water of crystallization. Waters with less than 500 parts per million of dissolved solids are usually satisfactory for domestic and some industrial uses. Water containing several thousand parts permillion of dissolved solids are sometimes successfully used for irrigation where practices permit the removal of soluble salts through the application of large volumes of water on well-drained lands, but generally water containing more than about $2,000 \mathrm{ppm}$ is considered to be unsuitable for long-term irrigation under average conditions.

\section{Chromium (Cr)}

Few if any waters contain chromium from natural sources. Natural waters can probably contain only traces of chromium as a cation unless the $\mathrm{pH}$ is very low. When chromium is present in water, it is usually the result of pollution by industrial wastes. Fairly high concentrations of chromate anions are possible in waters having normal $\mathrm{pH}$ levels. Concentrations of more than $0.05 \mathrm{ppm}$ of chromium in the hexavalent form constitute gro' inds for rejection of a water for domestic use on the basis of the standards of the U.S. Public Health Service (1962). 
Nickel and Cobalt (Ni, Co)

Nickel and cobalt are very similar in chemical behavior and also closely related to iron. Both are present in ignexus rocks in small amounts and are more prevalent in silicic rocks. Any nickel in water is likely to be in small amounts and could be in a colloidal state. Cobalt may be taken into solution more readily than nickel. It may be taken into solution in small amounts through bacteriological activity similar to that causing solution of manganese. However, few data on the occurrence of either nickel or cobalt in natural water are available.

\section{Copper $(\mathrm{Cu})$}

Copper is a fairly common trace constituent of natural water. Small amounts may be introduced into water by solution of copper and brass water pipes and other copper-bearing equipment in contact with the water, or from copper salts added to control algae in open reservoirs. Copper salts such as the sulfate and chloride are highly soluble in waters with a low pH but in water of normal alkalinity these salts hydrolyze and the copper may be precipitated. In the normal $\mathrm{pH}$ range of natural water containing carbon dioxide, the copper might be precipitated as carbonate. The oxidized portions of sulfide-copper ore bodies contain other copper compounds. The presence of copper in mine water is common.

Copper imparts a disagreeable metallic taste to water. As little as $1.5 \mathrm{ppm}$ can usually be detected, and $5 \mathrm{ppm}$ can render the water unpalatable. Copper is not considered to be a cumulative systemic poison like lead and mercury; most copper ingested is excreted by the body and very little is retained. T'e pathological effects of copper are controversial, but it is generally believed very unlikely that humans could unknowingly ingest toxic quantities from palatable drinking water. The U.S. Public Health Service (1962) recommends that copper should not exceed $1.0 \mathrm{ppm}$ in drinking and culinary water.

Lead $(\mathrm{Pb})$

Lead is only a minor element in most natural waters, but industrial or mine and smelter effluents may contain relatively large amounts of lead. Many of the commonly used lead salts are water soluble. 
Traces of lead in water usually are the result of solution of lead pipe through which the water has passed. Amounts of lead of the order of $0.05 \mathrm{ppm}$ are significant, as this concentration is the upper limit for drinking water in the standards adopted by the U.S. Public Health Service (1962). Higher concentrations m ay be added to water through industrial and mine-waste disposal. Lead in the form of sulfate is reported to be soluble in water to the extent of $31 \mathrm{ppm}$ (Seidell, 1940) at $25^{\circ} \mathrm{C}$. In natural water this concentration would not be approached, however, since a $\mathrm{pH}$ of less than 4.5 would probably be required to prevent formation of lead hydroxide and carbonate. It is reported (Pleissner, 1907) that at $18^{\circ} \mathrm{C}$ water free of carbon dioxide will dissolve the equivalent of $1.4 \mathrm{ppm}$ of lead and the solubility is increased nearly four fold by the presence of $2.8 \mathrm{ppm}$ of carbon dioxide in the solution. Presence of other ions may increase the solubility of lead.

\section{Zinc $(\mathrm{Zn})$}

Zinc is abundant in rocks and ores but is only a minor constituent in natural water because the free metal and its oxides are only sparingly soluble. In most alkaline surface waters it is present only in trace quantities, but more may be present in acid water. Chlorides and sulfates of zinc are highly soluble. Zinc is used in many commercial products, and industrial wastes may contain large amounts.

Zinc in water does not cause serious effects on health, but produces undesirable esthetic effects. The U.S. Public Health Service (1962, p. 55) recommends that the zinc content not exceed $5 \mathrm{ppm}$ in drinking and culinary water.

Barium (Ba)

Barium may replace potassium in some of the igneous rock minerals, especially feldspar and barium sulfate (barite) is a common barium mineral of secondary origin. Only traces of barium are present in surface water and sea water. Berause natural water contains sulfate, barium will dissolve only in trace amounts. Barium sometimes occurs in brines from oil-well wastes.

The U.S. Public Health Service (1962) states that water containing concentrations of barium in excess of $1 \mathrm{ppm}$ is not suitable for drinking and culinary use because of the serious toxic effects of barium on heart, blood vessels, and nerves. 
Bromide (Br)

Bromine is a very minor element in the earth's crust and is normally present in surface waters in only minute quantities. Measurable amounts may be found in some streams that receive industrial wastes, and some natural brines may contain rather high concentrations. It resembles chloride in that it tends to be concentrated in sea water.

Iodide (I)

Iodide is considerably less abundant both in rocks and water than bromine. Measurable amounts may be found in sc me streams that receive industrial wastes, and some natural brines may contain rather high concentrations. It occurs in sea water to the extent of less than $1 \mathrm{ppm}$. Rankama and Sahama (1950) report iodide present in rainwater to the extent of 0.001 to $0.003 \mathrm{ppm}$ and in river water in about the same amount. Few waters will contain over $2.0 \mathrm{ppm}$.

\section{PROPERTIES AND CHARACTERISTICS OF WATER}

Hardness

Hardness is the characteristic of water that receires the most attention in industrial and domestic use. It is commonly recognized by the increased quantity of soap required to produce lather. The use of hard water is also objectionable because it contributes to the formation of scale in boilers, water heaters, radiators, and pipes, with the resultant decrease in rate of heat trensfer, possibility of boiler failure, and loss of flow.

Hardness is caused almost entirely by compounds of calcium and magnesium. Other constituents--such as iron, manganese, aluminum, barium, strontium, and free acid--also cause hariness, although they usually are not present in quantities large enough to have any appreciable effect.

Generally, bicarbonate and carbonate determine the proportions of "carbonate" hardness of water. Carbonate rardness is the amount of hardness chemically equivalent to the amount of bicarbonate and carbonate in solution. Carbonate hardness is approximately equal to the amount of hardness that is removed from water by boiling. 
Noncarbonate hardness is the difference between the hardness calculated from the total amount of calcium and magnesium in solution and the carbonate hardness. If the carbonate hardness (expressed as calcium carbonate) equal the amount of calcium and magnesium hardness (also expressed as calcium carbonate) there is no noncarbonate hardness. Noncarbonate hardness is about equal to the amount of hardness remaining after water is boiled. The scale formed at high temperatures by the evaporation of water containing noncarbonate hardness commonly is tough, heat resistant, and difficult to remove.

Although many people talk about soft water and hard water, there has been no firm line of demarcation. Water that seems hard to an easterner may seem soft to a westerner. In this report hardness of water is classified as follows:

\begin{tabular}{|c|c|}
\hline $\begin{array}{c}\text { Hardness range } \\
\text { (calcium carbonate } \\
\text { in ppm) }\end{array}$ & Hardness description \\
\hline $0-60$ & $\begin{array}{c}\text { Soft } \\
61-120 \\
121-180\end{array}$ \\
more than 180 & $\begin{array}{c}\text { Moderately hard } \\
\text { Hard } \\
\text { Very hard }\end{array}$ \\
\hline
\end{tabular}

For public use, water with hardness above 200 parts per million generally requires softening treatment (Durfor and Becker, 1964 , p. 23-27).

Acidity $\left(\mathrm{H}^{+1}\right)$

The use of the terms acidity and alkalinity is widespread in the literature of water analysis and is a cause of confusion to those who are more accustomed to seeing a $\mathrm{pH}$ of 7.0 used as neutral point. Acidity of a natural water represents the content of free carbon dioxide and other uncombined gases, organic acids and salts of strong acids and weak bases that hydrolyze to give hydrogen ions. Sulfates of iron and aluminum in mine and industrial wastes are common sources of acidity. The presence of acidity is reported in those waters which have a $\mathrm{pH}$ below 4.5.

Sodium adsorption ratio (SAR)

The term "sodium adsorption ratio (SAR)" was introd'ıced by the U.S. Salinity Laboratory Staff (1954). It is a ratio expressing the relative activity of sodium ions in exchange reaction with 
soil and is an index of the sodium or alkali hazard to the soil. Sodium adsorption ratio is expressed by the equation:

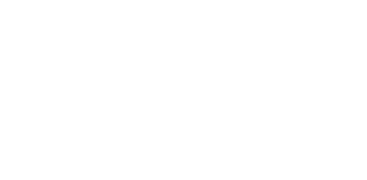

where the concentrations of the ions are expressed in milliequivalents per liter (or equivalents per million for most irrigation waters).

Waters are divided into four classes with respect to sodium or alkali hazard: low, medium, high, and very high, depending upon the SAR and the specific conductance. At a conductance of 100 micromhos per centimeter the dividing points are at SAR values of 10,18 , and 26 , but at 5,000 micromhos the corresponding dividing points are SAR values of approximately $2.5,6.5$, and 11 . Waters range in respect to sodium hazard from those which can be used for irrigation on almost all soils to those which are generally unsatisfactory for irrigation.

Specific conductance (micromhos per centimeter at $25^{\circ} \mathrm{C}$ )

Specific conductance is a convenient, rapid determination used to estimate the amount of dissolved solids in water. It is a measure of the ability of water to transmit a small electrical current (see p. 8). The more dissolved solids in water that can transmit electricity the greater the specific conductance of the vater. Commonly, the amount of dissolved solids (in parts per million) is about 65 percent of the specific conductance(in micrcmhos). This relation is not constant from stream to stream or from well to well and it may even vary in the same source with clanges in the composition of the water (Durfor and Becker, 196:4 p. 27-29).

Specific conductance of most waters in the eastern United States is less than 1,000 micromhos, but in the arid western parts of the country, a specific conductance of more than 1,000 micromhos is common.

Hydrogen-ion concentration $(\mathrm{pH})$

Hydrogen-ion concentration is expressed in terms of $\mathrm{pH}$ units (see p. 8). The values of $\mathrm{pH}$ often are used as a measure of the solvent power of water or as anindicator of the chemical behavior certain solutions may have toward rock minerals. 
The degree of acidity or alkalinity of water, as indicated by the hydrogen-ion concentration, expressed as $\mathrm{pH}$, is related to the corrosive properties of water and is useful in determining the proper treatment for coagulation that may be necessary at watertreatment plants. A pH of 7.0 indicates that the water is neither acid nor alkaline. $\mathrm{pH}$ readings progressively lower thar 7.0 denote increasing acidity and those progressively higher than 7.0 denote increa sing alkalinity. The $\mathrm{pH}$ of most natural surface waters ranges between 6 and 8 . Some alkaline surface waters have $\mathrm{pH}$ values greater than 8.0 and waters containing free mineral acid or organic matter usually have $\mathrm{pH}$ values less than 4.5.

The investigator who utilizes $\mathrm{pH}$ data in his interpretations of water analyses should be careful to place pH values in their proper perspective.

\section{Color}

In water analysis the term "color" refers to the appearance of water that is free from suspended solids. Many turbid waters that appear yellow, red, or brown when viewed in the stream show very little color after the suspended matter has been removed. The yellow-to-brown color of some waters is usually caused by organic matter extracted from leaves, roots, and other organic substances in the ground. In some areas objectionable color in water results from industrial wastes and sewage. Clear deep water may appear blue as the result of a scattering of sunlight by the water molecules. Water for domestic use and some industrial uses should be free from any perceptible color. A color less than 15 units generally passes unnoticed (U.S. Public Health Service, 1962). Some swamp waters have natural color in excess of 300 units.

The extent to which a water is colored by material in solution is commonly reported as a part of a water analysis because a significant color in water may indicate the presence of organic material that may have some bearing on the dissolved solids content. Color in water is expressed in terms of units between 0 and 500 or more based on the above standard (see p. 8).

\section{Oxygen consumed}

Oxygen consumed is a measure of the amount of oxygen required to oxidize unstable materials in water and may be correlated with natural-water color or with some carbonaceous organic pollution from sewage or industrial wastes. 
Tolerances for oxygen consumed in feed water for low- and high-pressure boilers are 15 and $3 \mathrm{ppm}$, respectively (Northeast Water Works Association, 1940). Wash water containing more than $8 \mathrm{ppm}$ has been reported to import a bad odor to textiles; concentrations for water used in beverages and brewing range from 0.5 to $5.0 \mathrm{ppm}$ (California State Water Pollution Control Board, 1952, 1954).

Dissolved oxygen (DO)

Adequate dissolved oxygen is necessary for the life of fish and other aquatic organisms and is an indicator for corrosivity of water, photosynthetic activity, and septicity. It js one of the most important indicators of the condition of a water supply for biological, chemical and sanitary investigations (Rose, 1965).

Biochemical oxygen demand (BOD)

Biochemical oxygen demand is a measure of the oxygen required to oxidize the carbonaceous organic material usable as a source of food by aerobic organisms.

Chemical oxygen demand (COD)

Chemical oxygen demand indicates the quantity of oxidizable compounds present in a water and will vary witl water compositions, concentration of reagent, temperature, period of contact, and other factors.

\section{Organics}

Phenols.--Phenolic material in water resources is invariably the result of pollution. Phenols are widely used as disinfectants and in the synthesis of many organic compounds. Weste products from oil refineries, coke areas, and chemical plants may contain high concentrations. Fortunately, phenols decompose in the presence of oxygen and organic material, and their persistence downstream from point of entry is relatively short lived. The rate of decomposition is dependent on the environment.

Very low concentrations impart such a disagreeable taste to water that it is highly improbable that harmful amounts could be consumed unknowingly. Reported thresholds of detection of taste and odor range from 0.001 to $0.01 \mathrm{ppm}$. 
Most probable number (MPN).--An index for determinin extent of pollution in water is the most probable number which is a direct count of coliform colonies per 100 milliliters of water.

Detergents (MBAS).--Anionic surfactants (methylene blue active substance, MBAS) in detergents resist chemical oxidation and biological breakdown. Their persistence in water over long periods of time contributes to pollution of both ground water and surface water. Some of the effects produced from detergent pollution are unpleasant taste, odor, and foaming (Wayman, and others, 1962). Although the physiological implicatiors of MBAS to human beings is unknown, prolonged ingestion of this material by rats is believed to be nontoxic (Paynter, 1960). The U.S. Public Health Service (1962) recommends that MBAS should not exceed $0.5 \mathrm{ppm}$ in drinking and culinary waters.

\section{Temperature}

Temperature is an important factor in property determining the quality of water. This is very evident for such a direct use as an industrial coolant. Temperature is also important, but perhaps not so evident, for its indirect influence upon aquatic riota, concentrations of dissolved gases, and distribution of chernical solutes in lakes and reservoirs as a consequence of thermal stratification and variation.

Surface water temperatures tend to change seasonally and daily with air temperatures, except for the outflow of large springs. Superimposed upon the annual temperature cycle is a daily fluctuation of temperature which is greater in warm seasons than in cold and greater in sunny periods than with a cloud cover. Natural warming is due mainly to absorption of a solar radiation by the water and secondarily to transfer of heat from the air. Condensation of water vapor at the water surface is reported to furnish measurable quantities of heat. Heat loss takes place largely through radiation, with further losses through evaporation and conduction to the air and to the stream bed. Thus the temperature of a small stream generally reaches a maximum in mid- to late afternoon due to solar heating and reaches a minimum from early to mid-morning after nocturnal radiation.

Temperature variations which commonly occur during summer in lakes and reservoirs of temperate regions result in a separation of the water volume into a circulating upper portion and a noncirculating lower portion. Separating the two is a stratum of water of variable vertical thickness in which the temperature 
decreases rapidly with increasing depth. This physical division of the water mass into a circulating and a stagnant portion is the result of density differences in the water column associated with the temperature distribution. Knowledge of the stratification in a body of water may result in increased utility by locating strata of more suitable characteristics. For example, the elevation of an intake pipe may be changed to obtain water of lower temperature, higher $\mathrm{pH}$, less dissolved iron, or other desirable properties.

Temperature is a major factor in determining the effect of pollution on aquatic organisms. The resistance of fish to certain toxin substances has been shown to vary widely with temperature. The quantity of dissolved oxygen which the water can contain is also temperature dependent. Oxygen is more soluble in cold water than in warm water, hence the reduction of oxygen concentrations by pollution is especially serious during periods of high temperature when oxygen levels are already low. Increased temperatures also accelerate biological activity including that of the oxygenutilizing bacteria which decompose organic wastes. These pollutional effects may be especially serious when low flow conditions coincide with high temperatures. Summary temperature data of water are essential for planning multiple uses of water.

\section{Turbidity}

Turbidity is the optical property of a suspension with reference to the extent to which the penetration of light is inhibited by the presence of insoluble material. Turbidity is a function on both the concentration and particle size of the suspended material. Although it is reported in terms of parts per million of silica, it is only partly synonymous with the weight of sediment per unit volume of water.

Turbid water is abrasive in pipes, pumps, and turbine blades. In process water, turbidities much more than $1 \mathrm{ppm}$ are not tolerated by several industries, but others permit up to $50 \mathrm{ppm}$ or higher (Rainwater, Thatcher, 1960, p. 289). Although turbidity does not directly measure the safety of drinking water, it is related to the consumers acceptance of the water. A level of 5 units of turbidity becomes objectionable to a considerable number of people (U.S. Public Health, 1962).

Sediment

Fluvial sediment is generally regarded as that sediment which is transported by, suspended in, or deposited by water. Suspended 
sediment is that part which remains in suspension in water owing to the upward components of turbulent currents or by colloidal suspension. Much fluvial sediment results from the natural process of erosion, which in turn is part of the geslogic cycle of rock transformation. This natural process may be accelerated by agricultural practices. Sediment is also contributed by a number of industrial and construction activities. In certain sections, waste materials from mining, logging, oil-field, and other industrial operations introduce large quantities of suspended as well as dissolved material.

The quantity of sediment, transported or available for transportation, is affected by climatic conditions, form or nature of precipitation, character of the solid mantle, plant cover, topography, and land use. The mode and rate of sediment erosion, transport, and deposition is determined largely by the size distribution of the particles or more precisely by the fall velorities of the particles in water. Sediment particles in the sandsize (larger than $0.062 \mathrm{~mm}$ ) range do not appear to be affected by flocculation or dispersion resulting from the mineral constituents in solution. In contrast, the sedimentation diameter of clay and silt particles in suspension may vary considerably from point to point in a stream or reservoir, depending on the mineral matter in solution and in suspension and the degree of turbulence present. The size of sediment particles in transport at any point depends on the type of erodible and soluble material in the drainage area, the degree of flocculation present, time in transport, and charecteristics of the transporting flow. The flow characteristics include velocity of water, turbulence, and the depth, width, and roughness of the channel. As a result of these variable characteristics, the size of particles transported, as well as the total sediment load, is in constant adjustment with the characteristics and physical features of the stream and drainage area.

\section{STREAMFLOW}

Most of the records of stream discharge, used in conjunction with the chemical analyses and in the computation of sediment loads in this volume, are published in The Geological Sirvey water-supply paper series, "Surface Water Supply of the United States, 1961-65." The discharge reported for a composite sample is usually the average of daily mean discharges for the composite period. The discharges reported in the tables of single anclyses 
are either daily mean discharges or discharges obtained at the time samples were collected and computed from a stage-discharge relation or from a discharge measurement.

The water-supply papers and numbers which contain more complete records of stream discharge for this report are listed below:

\begin{tabular}{|c|c||c|c|}
\hline Part 7 & WSP & Part 8 & W $\subseteq$ P \\
\hline Volume 1 & 1920 & Volume 1 & 1922 \\
Volume 2 & 1921 & Volume 2 & 1923 \\
\hline
\end{tabular}

\section{PUBLICATIONS}

Reports giving records of chemical quality and temperatures of surface waters and suspended-sediment loads of streams in the area covered by this volume for the water years 1941-64, are listed below:

Numbers of water-supply papers containing reccrds for Parts 7 and 8, 1941-64

\begin{tabular}{l|r||l|l||l|l||l|l}
\hline Year & \multicolumn{1}{|c||}{ WSP } & Year & WSP & Year & WSP & Year & WSP \\
\hline 1941 & 942 & 1947 & 1102 & 1953 & 1292 & 1959 & 1644 \\
1942 & 950 & 1948 & 1133 & 1954 & 1352 & 1960 & 1744 \\
1943 & 970 & 1949 & 1163 & 1955 & 1402 & 1961 & 1884 \\
1944 & 1022 & 1950 & 1188 & 1956 & 1452 & 1962 & 1944 \\
1945 & 1030 & 1951 & 1199 & 1957 & 1522 & 1963 & 1950 \\
1946 & 1050 & 1952 & 1252 & 1958 & 1573 & 1964 & 1957 \\
\hline
\end{tabular}

Geological Survey reports containing chemical quality, temperature, and sediment data obtained before 1941 are listed below. Publications dealing largely with the quality of groundwater supplies and only incidentally covering the chemical composition of surface waters are not included. Publications that are out of print are preceded by an asterisk.

PROFESSIONAL PAPER

*135. Composition of river and lake waters of the United States, 1924. 


\section{BULLETINS}

*479. The geochemical interpretation of water analyses, 1911.

770. The data of geochemistry, 1924 .

\section{WATER-SUPPLY PAPERS}

*108. Quality of water in the Susquehanna River drainage basin, with an introductory chapter on physiographic features. 1904.

*161. Quality of water in the upper Ohio River basin and at Erie, Pa., 1906.

*193. The quality of surface waters in Minnesota, 1907.

*236. The quality of surface waters in the United States, Part 1, Analyses of waters east of the one hundredth meridian, 1909.

*237. The quality of the surface waters of California, 1910.

*239. The quality of surface waters of I1linois, 1910.

*273. Quality of the water supplies of Kansas, with a preliminary report on stream pollution by mine waters in southeastern Kansas, 1911.

*274. Some stream waters of the western United States, with chapters on sediment carried by the Rio Grande and the industrial application of water analyses, 1911.

*339. Quality of the surface waters of Washington, 1914.

*363. Quality of the surface waters of Oregon, 1914.

*418. Mineral springs of Alaska, with a chapter on the chemical character of some surface waters of Alaska, 1917. *596-B. Quality of water of Colorado River in 1925-26, 1928. *596-D. Quality of water of Pecos River in Texas, 1928. *596-E. Quality of the surface waters of New Jersey, 1928. *636-A. Quality of water of the Colorado River in 1926-28, 1930.

*636-B. Suspended matter in the Colorado River in 1925-28, 1930.

*638-D. Quality of water of the Colorado River in 1928-30, 1932.

*839 Quality of water of the Rio Grande basin above. Fort Quitman, Tex., 1938.

*889-E. Chemical character of surface water of Georgia, 1944. *998. Suspended sediment in the Colorado River, 1925-41, 1947.

1048. Discharge and sediment loads in the Boise River drainage basin, Idaho, 1939-40, 1948.

1110-C. Quality of water of Conchas Reservoir, New Mexico, 1939-49, 1952. 
Many of the reports listed are available for consultation in the larger public and institutional libraries. Copies of Geological Survey publications still in print may be purchased at a nominal cost from the Superintendent of Documents, Government Printing Office, Washingtorı D.C. 20402, who will, upon request, furnish lists giving prices.

\section{COOPERATION}

Many Municipal, State, and Federal agencies assisted in collecting records for these quality-of-water investigations. Many of the investigations were supported by funds appropriated directly to the Geological Survey. Studies of suspended-sediment loads in the middle Rio Grande in New Mexico were started in 1948 as a Federal project.

Financial assistance was furnished by the Bureau of Reclamation, U.S. Department of the Interior for some investigations in the Arkansas River basin in Kansas and Oklahoma, and the Rio Grande basin in New Mexico. The Soil Conservat:on Service, U.S. Department of Agriculture assisted with special sediment studies in the Arkansas River basin in Arkansas, and the Rio Grande basin in New Mexico. The Corps of Engineers, U.S. Army provided funds and determined the sediment concentration and particle size of bed material in connection with the sediment investigations of the Mississippi River at St. Louis, Mo. The Corps also assisted in investigations in Texas and for the operation of some stations in the Rio Grande basin in New Mexico.

Records of 10 stations in the Rio Grande basin were furnished by the U.S. Department of Agriculture, in cooperation with the International Boundary and Water Commission.

\section{DIVISION OF WORK}

The quality-of-water program was conducted by the Water Resources Division of the Geological Survey, E. L. Hendricks, chief hydrologist, and G. W. Whetstone, assistant chief for Reports and Data Processing, under the general direction of S. M. Lang, chief, Reports Section, and B. A. Anderson, chief, Data Reports Unit. The data were collected and prepared for publication under the supervision of district chiefs, district chemists, or engineers as follows: In Arkansas and Missouri, J. H. Hubble; Colorado, R. H. Langford; Arkansas River basin in Kansas, D. M. Culbertson; 


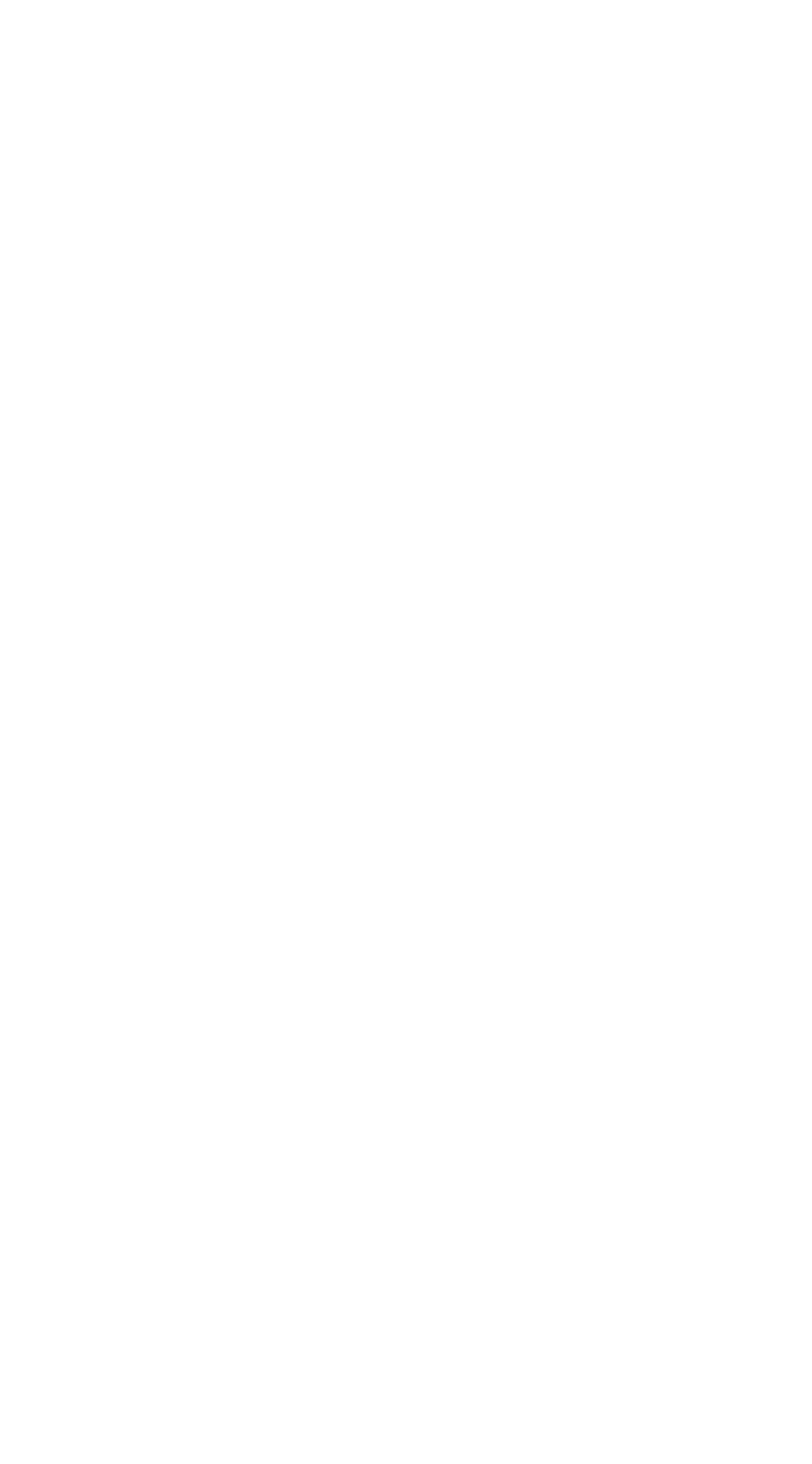




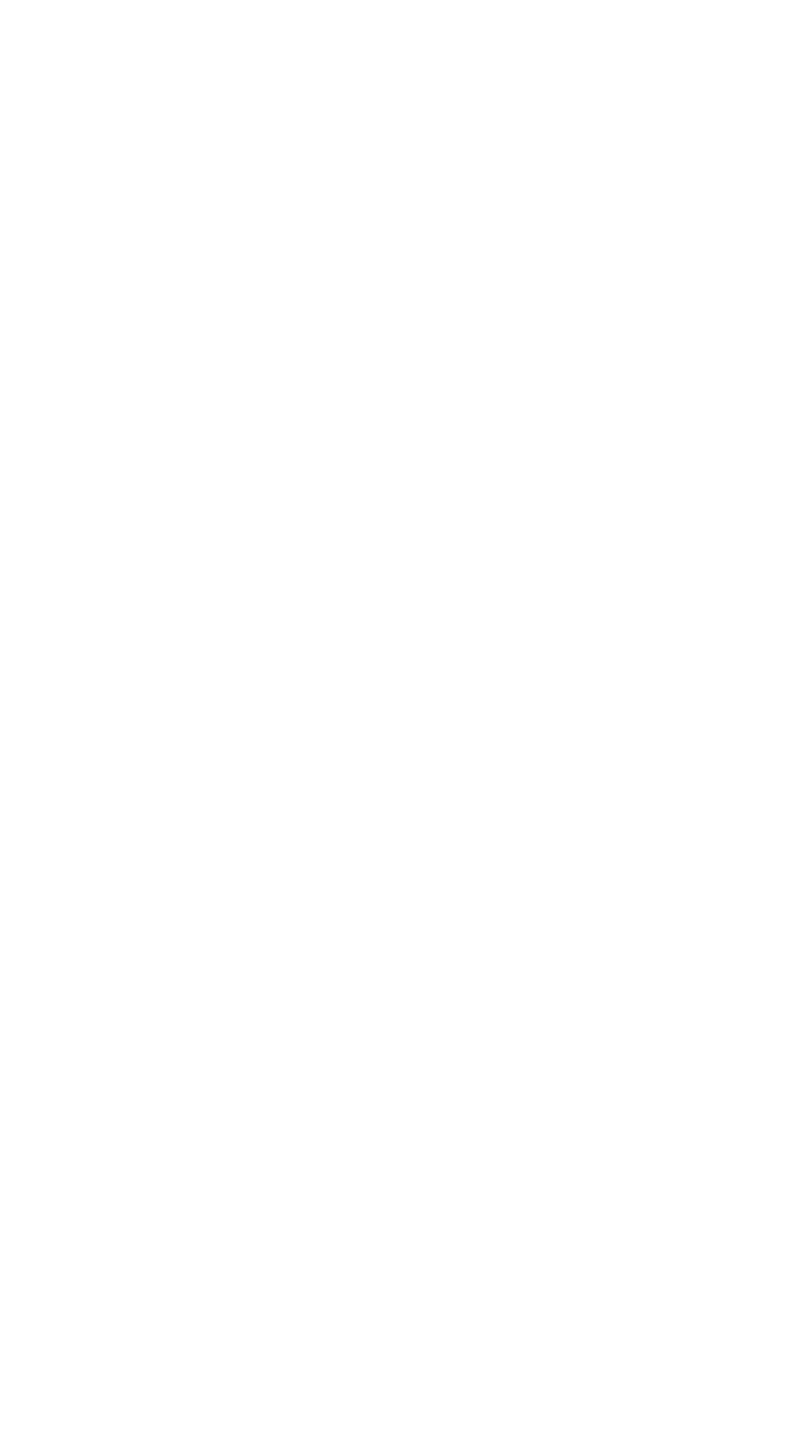




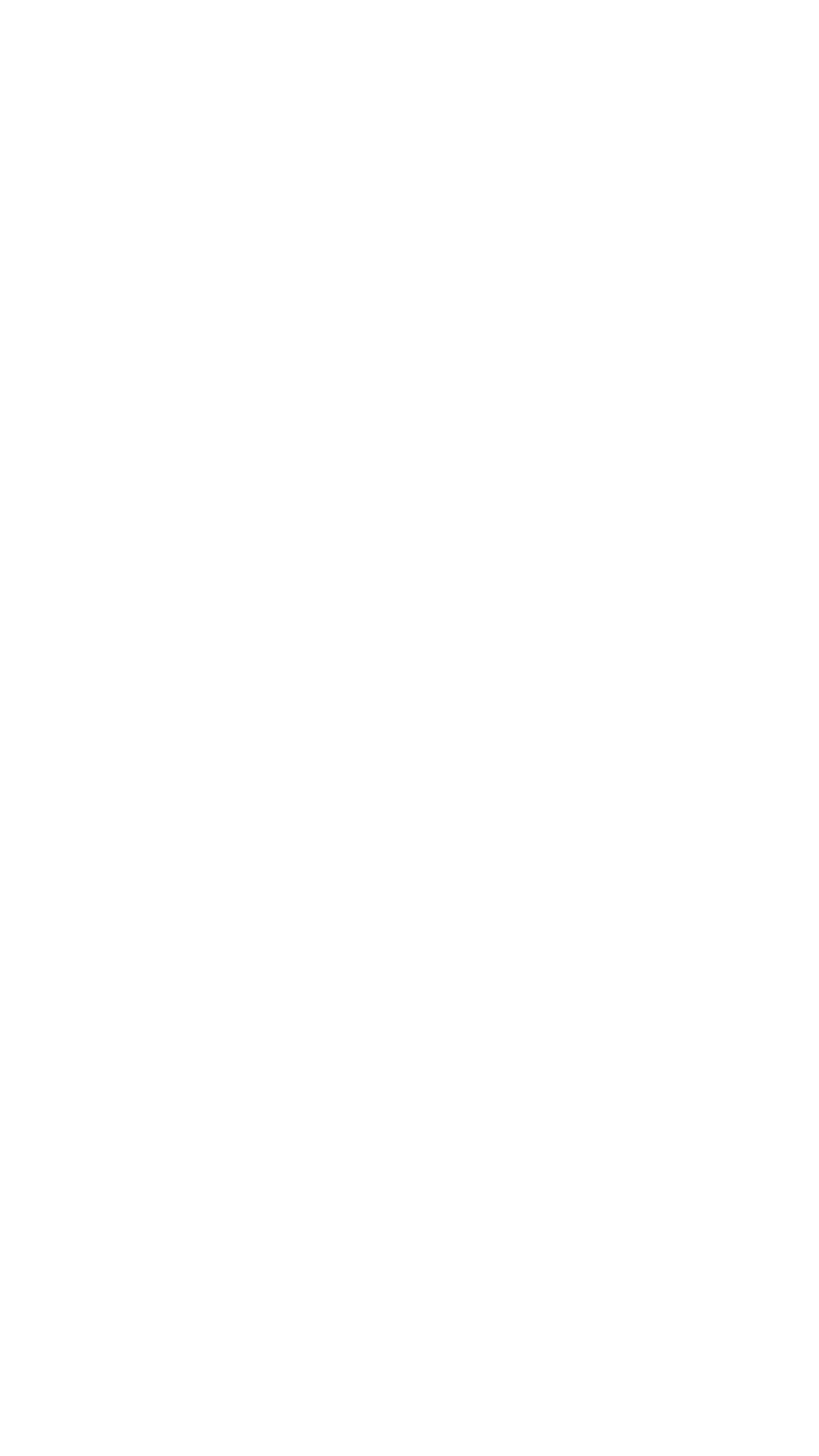




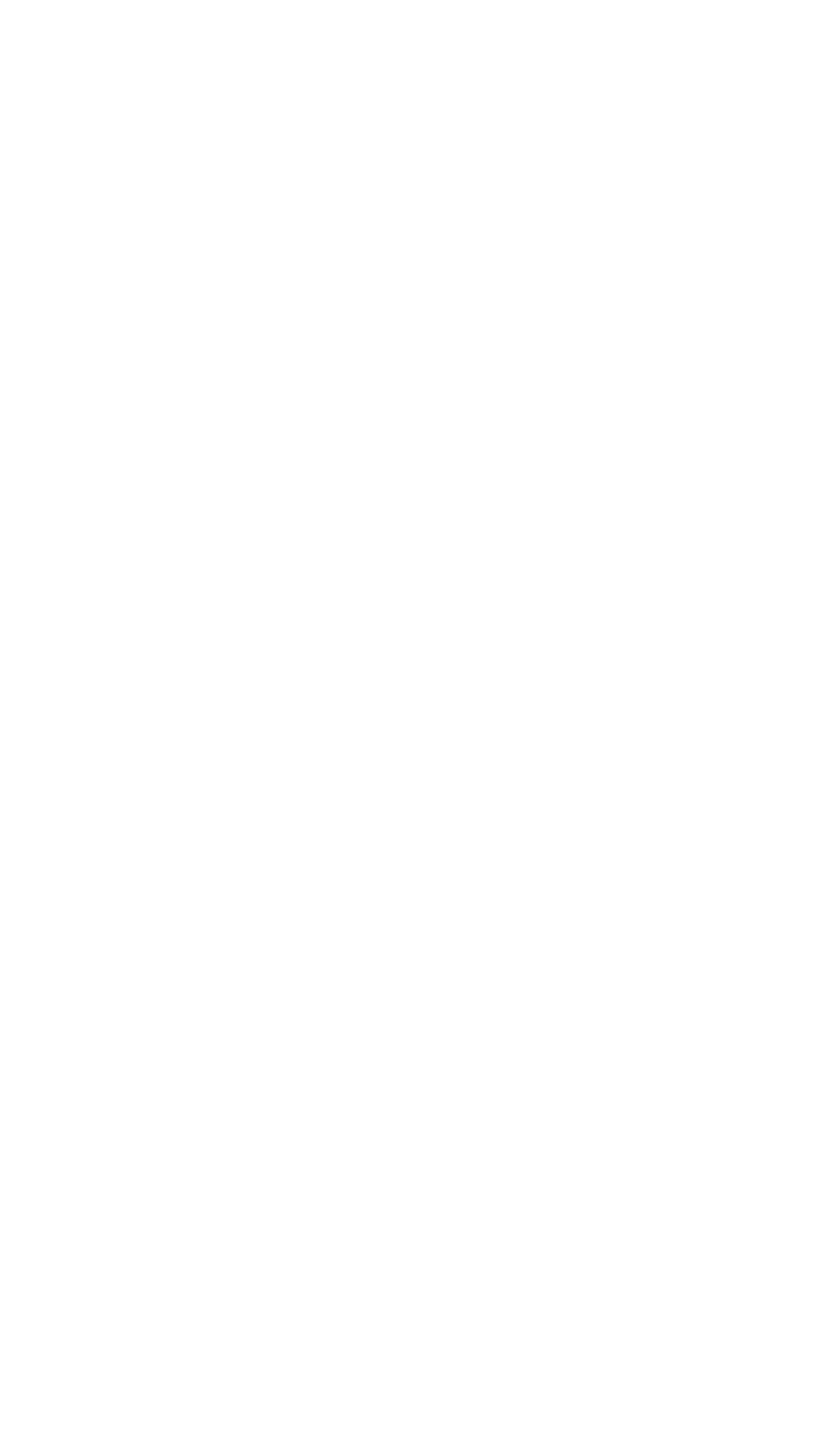




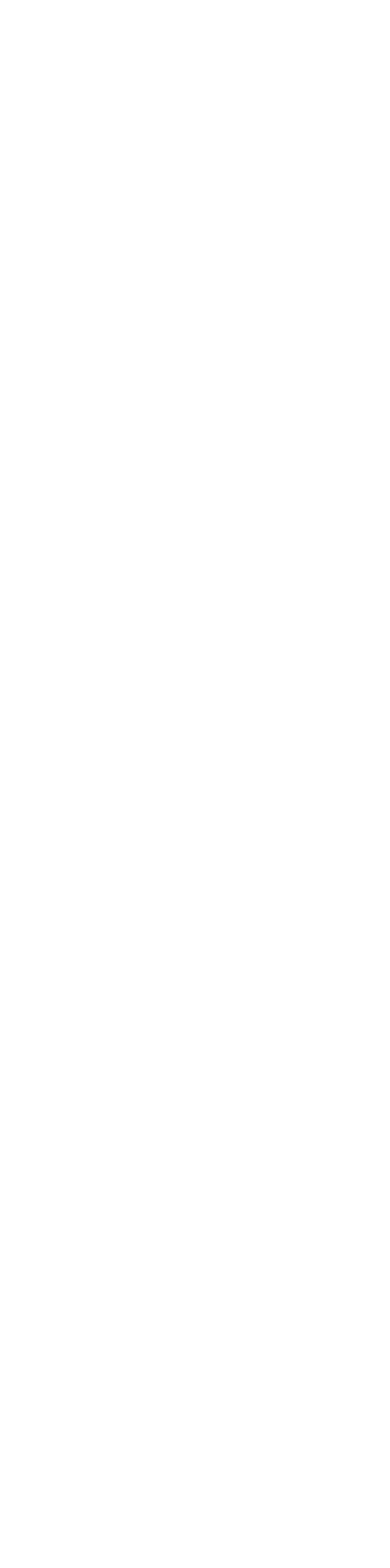


Louisiana and Mississippi, F. K. Kapustka succeeded by R. L. McAvoy; New Mexico, J. M. Stow; Oklahoma, R. P. Orth; Tennessee, J. S. Cragwell, Jr.; and in Texas, C. H. Hembree and Trigg Twichell.

Correspondence regarding the records on this report or any additional information should be directed to the district chief of the appropriate Geological Survey--Water Resources Division offices indicated in the table on page 35 . Because of reorganization in recent years, the offices now administering water-quality programs in most of the States differ from those that were administering the programs in 1964.

\section{LITERATURE CITED}

American Society for Testing Materials, 1954, Manual on industrial water: Am. Soc. for Testing Mat., Philadelphia, Pa., p. 356.

Durfor, C. N. and Becker, E., 1964, Public water supplies of the 100 largest cities in the United States; 1962: U.S. Geol. Survey Water-Supply Paper 1812, p. 20.

California State Water Pollution Control Board, 1952, Waterquality criteria: California State Water Pollution Control Board, pub. 3., p. 291-292, 377-378.

1954, Water-quality criteria: California State Water Pollution Control Board, pub. 3, Addendum no. 1., p. 291-292.

Faucett, R. L. and Miller, H. C., 1946, Methemoglobinemia occurring in infants fed milk diluted with well waters of high nitrate content: Jour. Pediatrics, v. 29, p. 593.

Hazen, Allen, 1892, A new color standard for natural waters: Am. Chem. Jour., v. 12, p. 427-428.

International Union of Pure and Applied Chemistry, 1961, Table of Atomic weights based on carbon-12: Chem. and Eng. News, v. 39, no. 42, Nov. 20, 1961, p. 43.

Kilmer, V. J. and Alexander, L. T., 1949, Methods of making mechanical analyses of soils: Soil Sci., v. 68, p. 15-24.

Lane, E. W., and others, 1947, Report of the Subcommittee on sediment terminology: Am. Geophys. Union Trans., v. 28, no. 6, p. 936-938.

Magistad, O. C., and Christiansen, J. E., 1944, Saline Soils, their nature and management: U. S. Dept., Agriculture Circ. 707, p. 8-9. 
Maxcy, K. F., 1950, Report on the relation of nitrate concentrations in well waters to the occurrence of methemoglobinemia: Natl. Research Council, Bull. Sanitary Eng. and Environment, App. D., p. 271.

Northeastern Water Works Association, 1940, Progress report, Committee on quality Tolerances of Water for Industrial Uses: Northeast Water Works Assoc. Jour., v. 54.

Paynter, O. E., 1960, The chronic toxicity of dodecylbenzene sodium sulfonate: U.S. Public Health Conference on Fhysiological Aspects of Water Quality Proc., Washington, D.C., Sept. 8-9, 1960, p. 175-179.

Pleissner, M., 1907, Uber die Löslichkeit eimiger Bleiverbindungen in wasser: Arb. Kais. Gesundeitsamt. v. 26. p.

Rainwater, F. H., and Thatcher, L. L., 1960, Methods for collection and analysis of water samples: U.S. Geol. Survey Water-Supply Paper 1454, 301 p.

Rankama, K., and Sahama, T. G., 1950, Geochemistry: Chicago Univ. Press, Chicago, Ill., p. 767.

Riffenburg, H. B., 1925, Chemical character of ground waters of the northern Great Plains: U.S. Geol. Survey Water-Supply Paper 560-B, p. 31-52.

Rose, Arthur and Elizabeth, 1965, The condensed chemical dictionary: Reinhold Pub. Corp., New York, 5th ed., p. 412.

Seidell, Atherton, 1940, Solubilities of inorganic and metal organic compounds, 3d ed., v. 1, D. van Nostrand, New York. p. 1409.

U.S. Inter-Agency Committee on Water Resources, Subcommittee on Sedimentation, A study of methods used in measurement and analysis of sediment loads in streams. Published b:r the St. Anthony Falls Hydraulic Laboratory, Minneapolis, Minn. 1943, A study of new methods of size analysis of suspended-sediment samplers, Rept. 7.

1952, The design of improved types of suspended-sediment samplers: Rept. 6,

1957, The development and calibration of visual accumulation tube: Rept 11.

1957, Some fundamentals of particle size analysis: Rept. 12.

1959, Federal Inter-agency sedimentation instruments and reports: Rept. AA.

1963, Determinations of fluvial sediment discharge: Rept. 14.

1963, A summary of the work of the Inter-agency sedimentation project: Rept. S.

U.S. Public Health Service, 1962, Drinking water standards: U.S. Dept. Health, Education, and Welfare, Public Health Service: Pub. no. 956. 
U.S. Salinity Laboratory Staff, 1954, Diagnosis and improvement of saline and alkali soils: U.S. Dept. Agriculture, Agriculture Handb. 60, p. 1-160.

Waring, F. H., 1949, Significance of nitrates in water supplies: Am. Water Works Assoc. Jour., v. 41, no. 2., p. 147-150.

Wayman, C. H., 1962, Limitations of the methylene blue method for ABS determinations: U.S. Geol. Survey, Prof. Paper 450-B, art. 49, p. B117-B120.

Wayman, C. H., Robertson, J. B., and Page, H. G., 1962, Foaming characteristics of synthetic-detergent solutions: U.S. Geol. Survey, Prof. Paper 450D, art. 178, p. D198. 


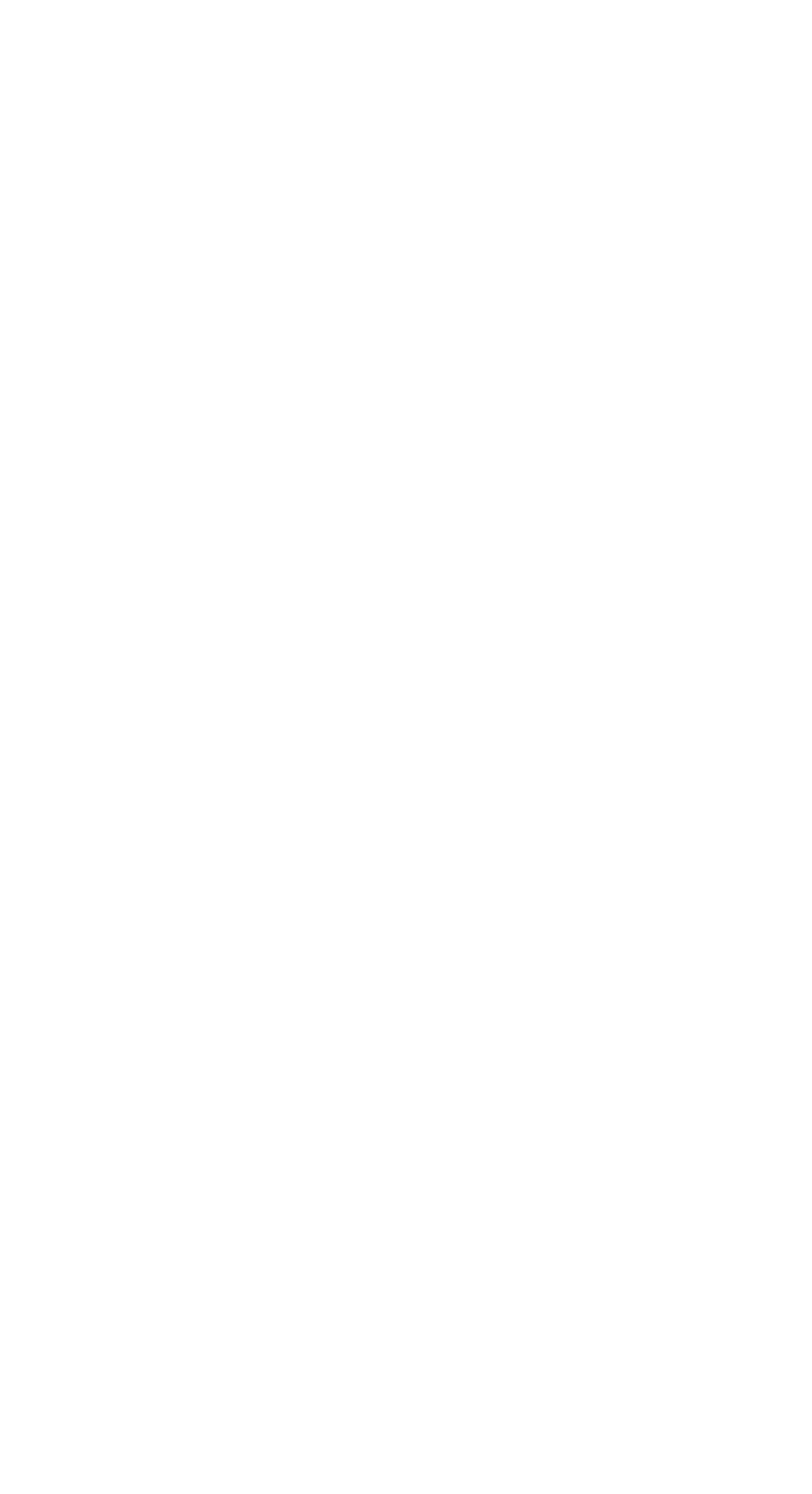


MISSISSIPPI RIVER MAIN STEM--COntinued

7-100. MISSISSIPPI RIVER AT ST. LOUIS, MO.--Continued Suspended sediment, water year October 1963 to September 1964

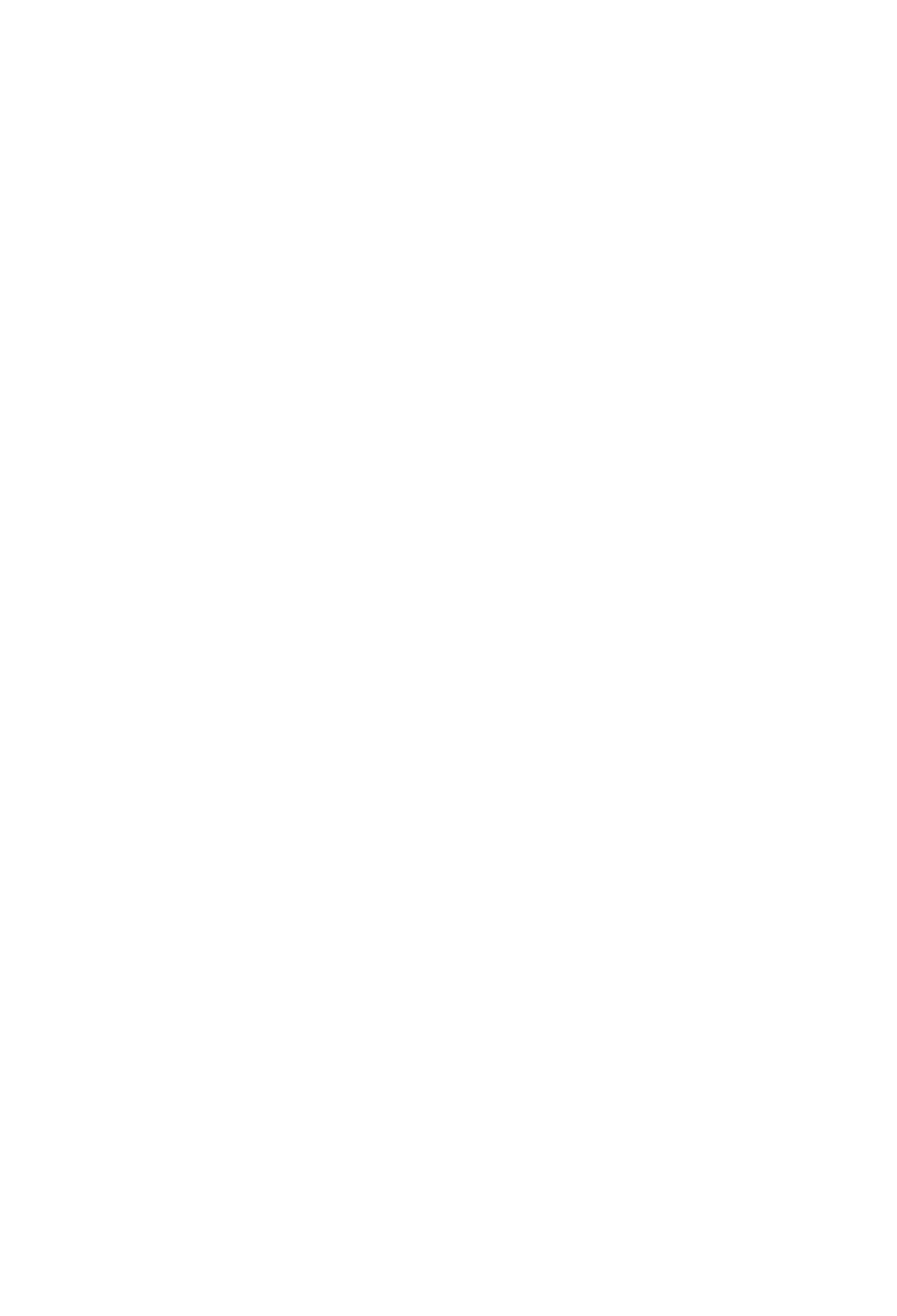


MISSISSIPPI RIVER MAIN STEM--Continued

7-100. MISSISSIPPI RIVER AT ST. LOUIS, MO.--Continued

Suspended sediment, water year October 1963 to September 1964--Continued

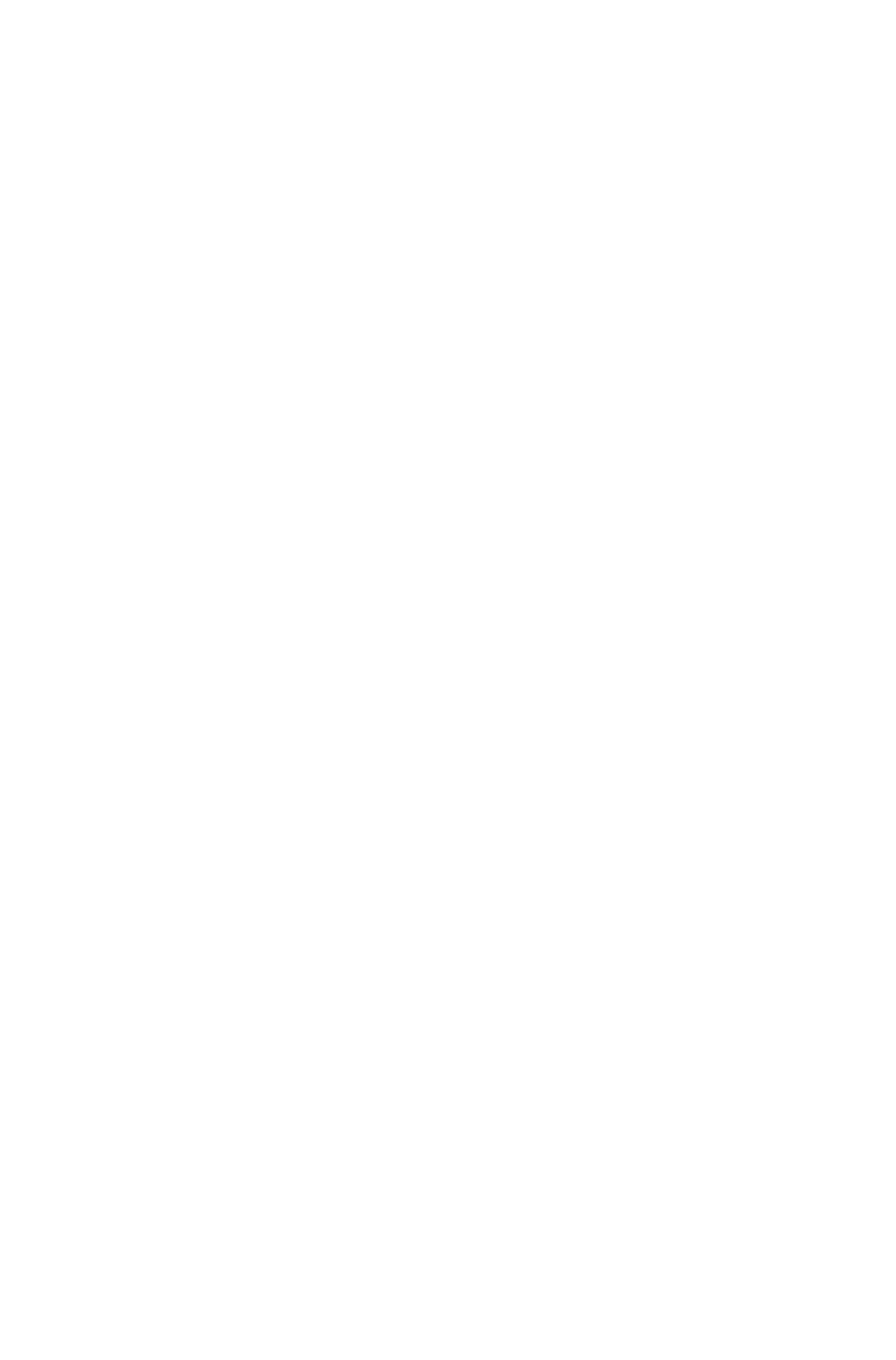



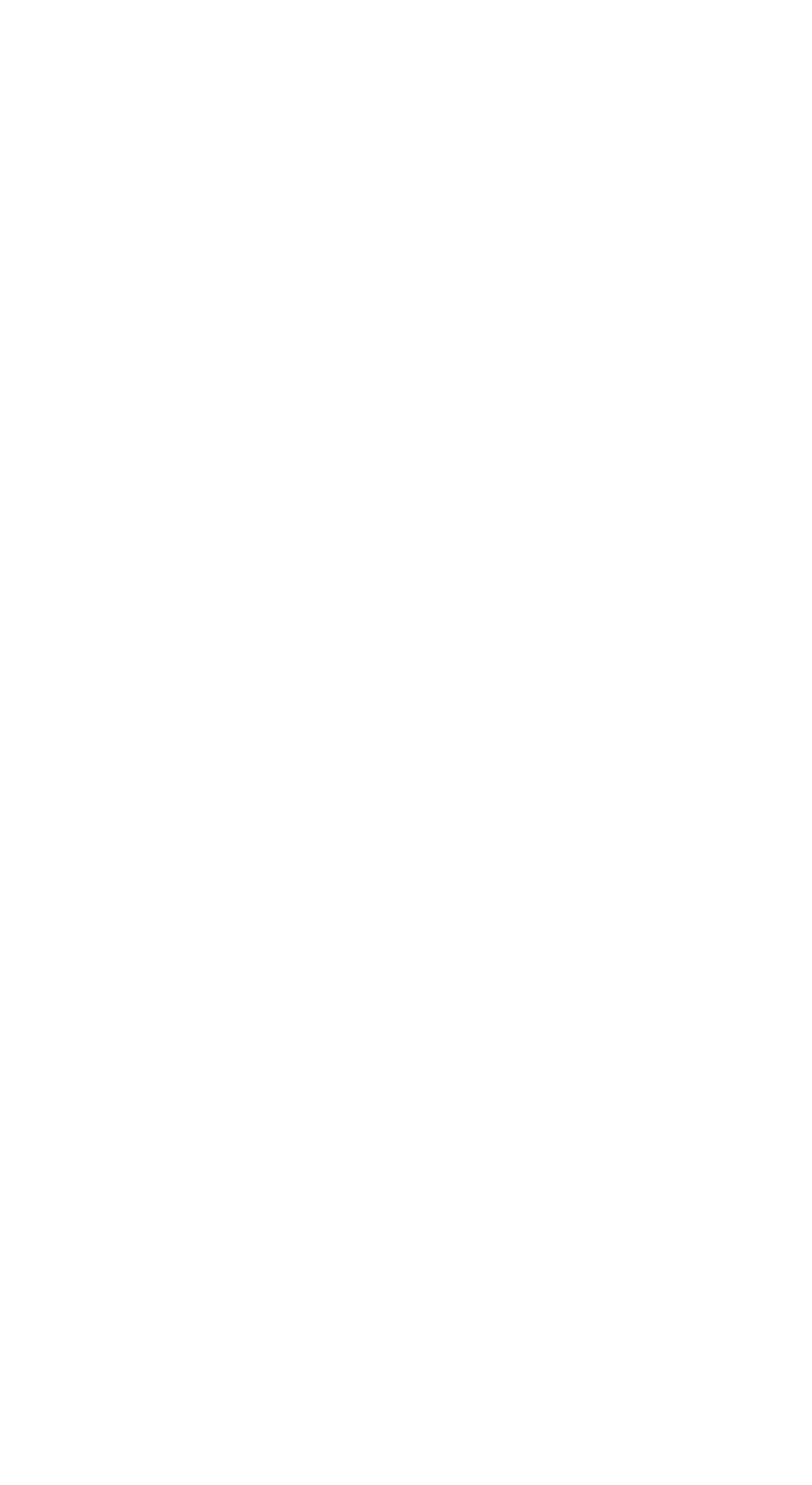


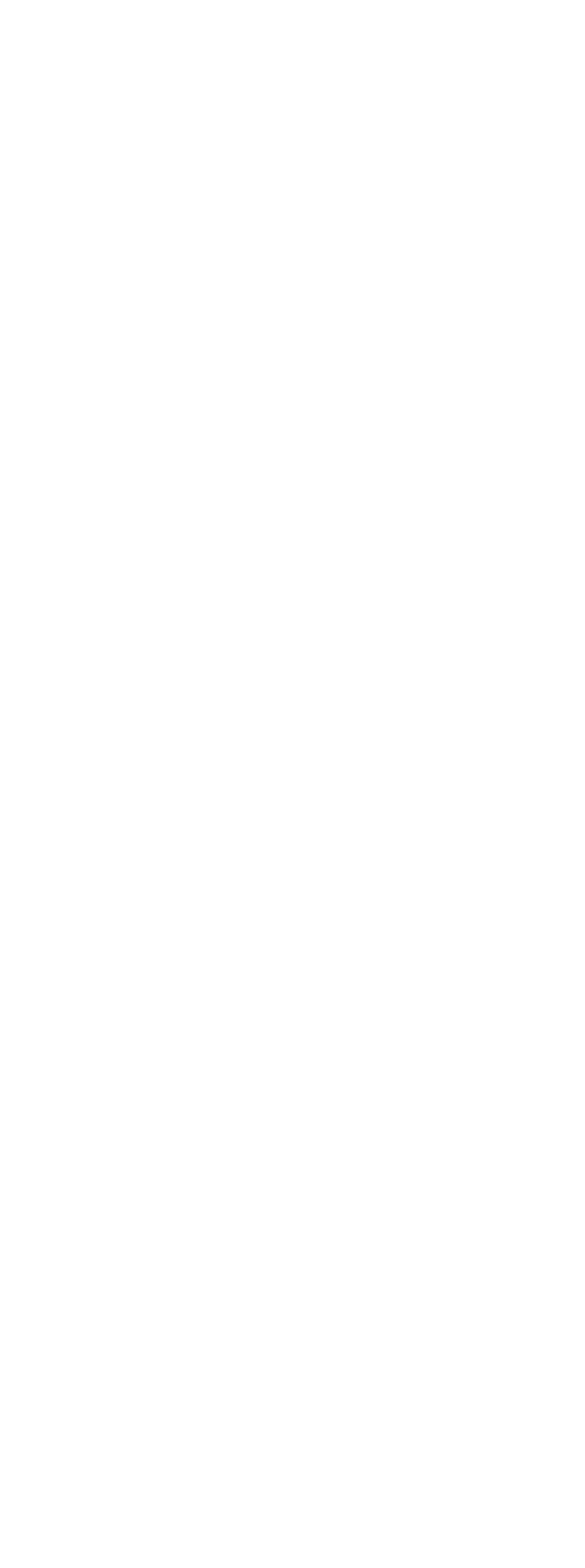



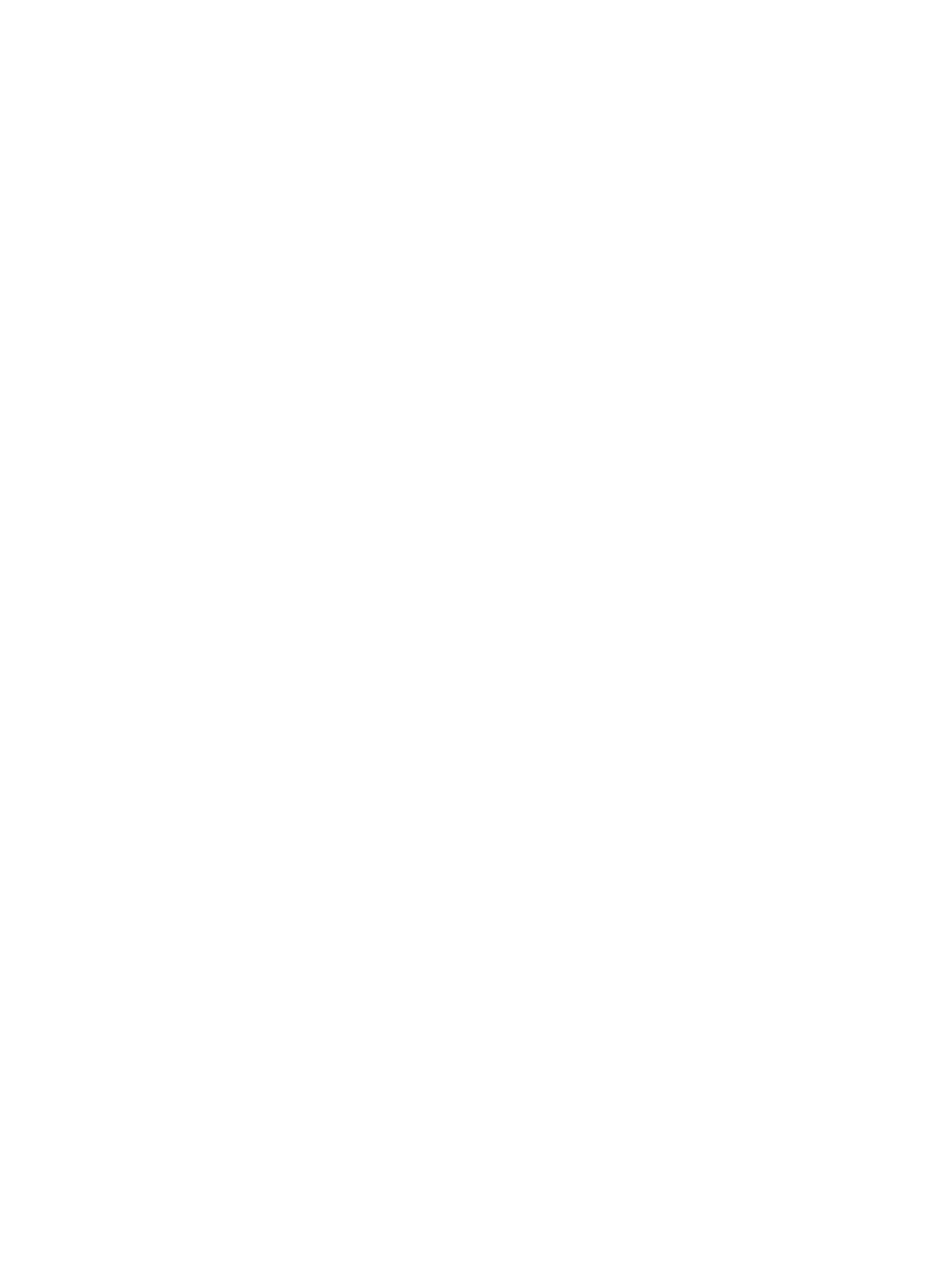


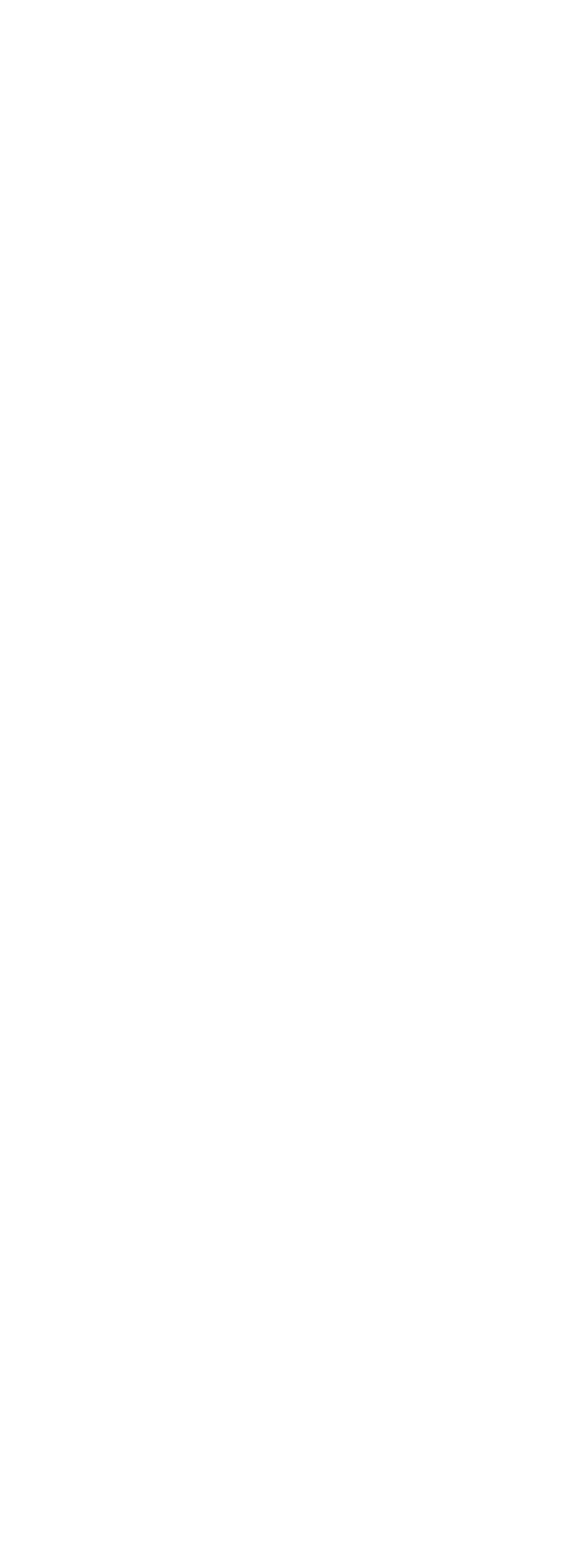




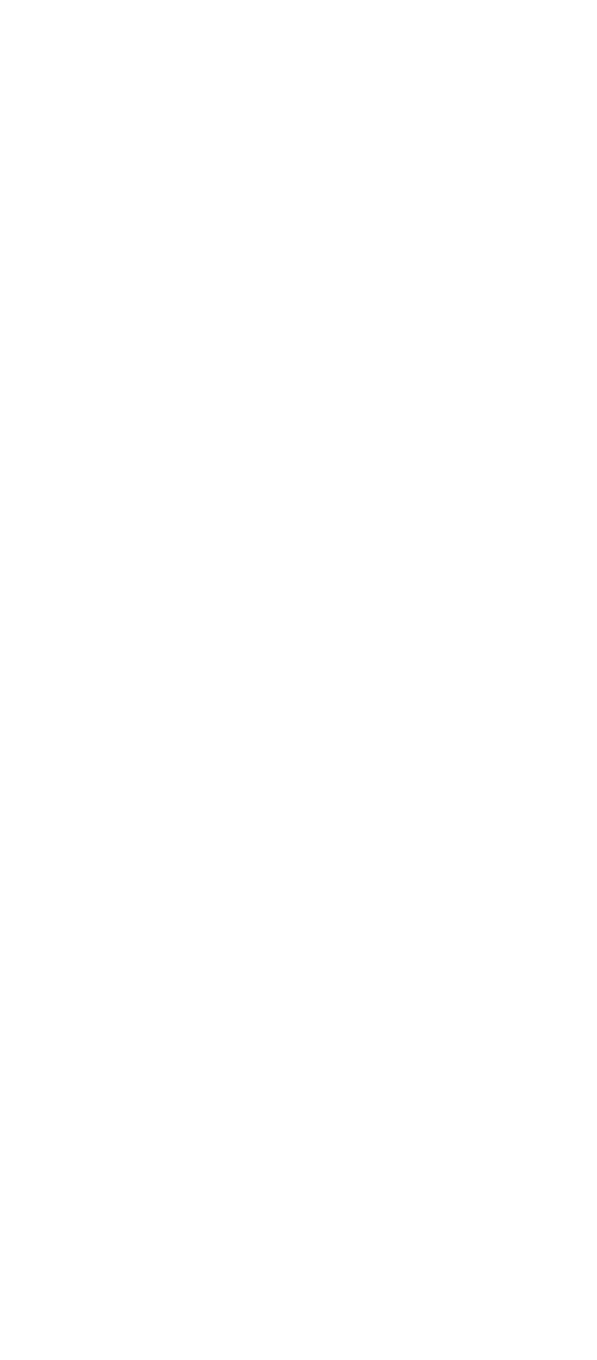




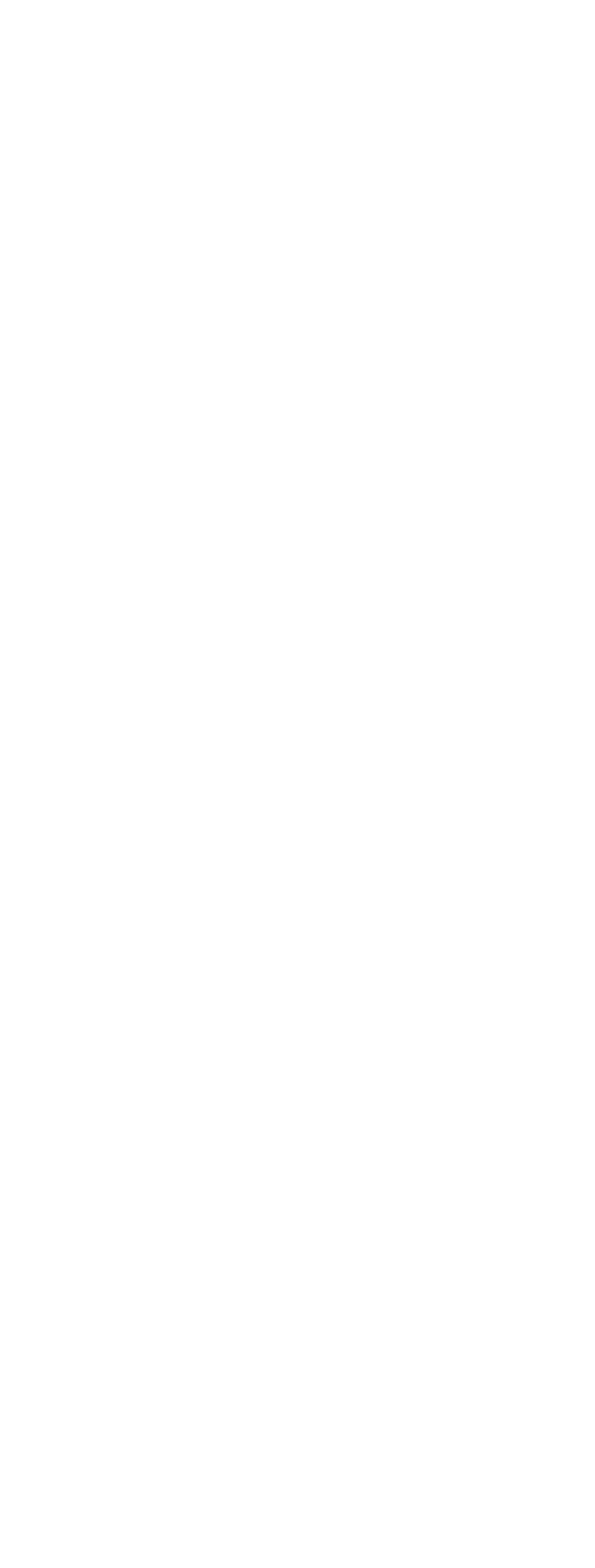




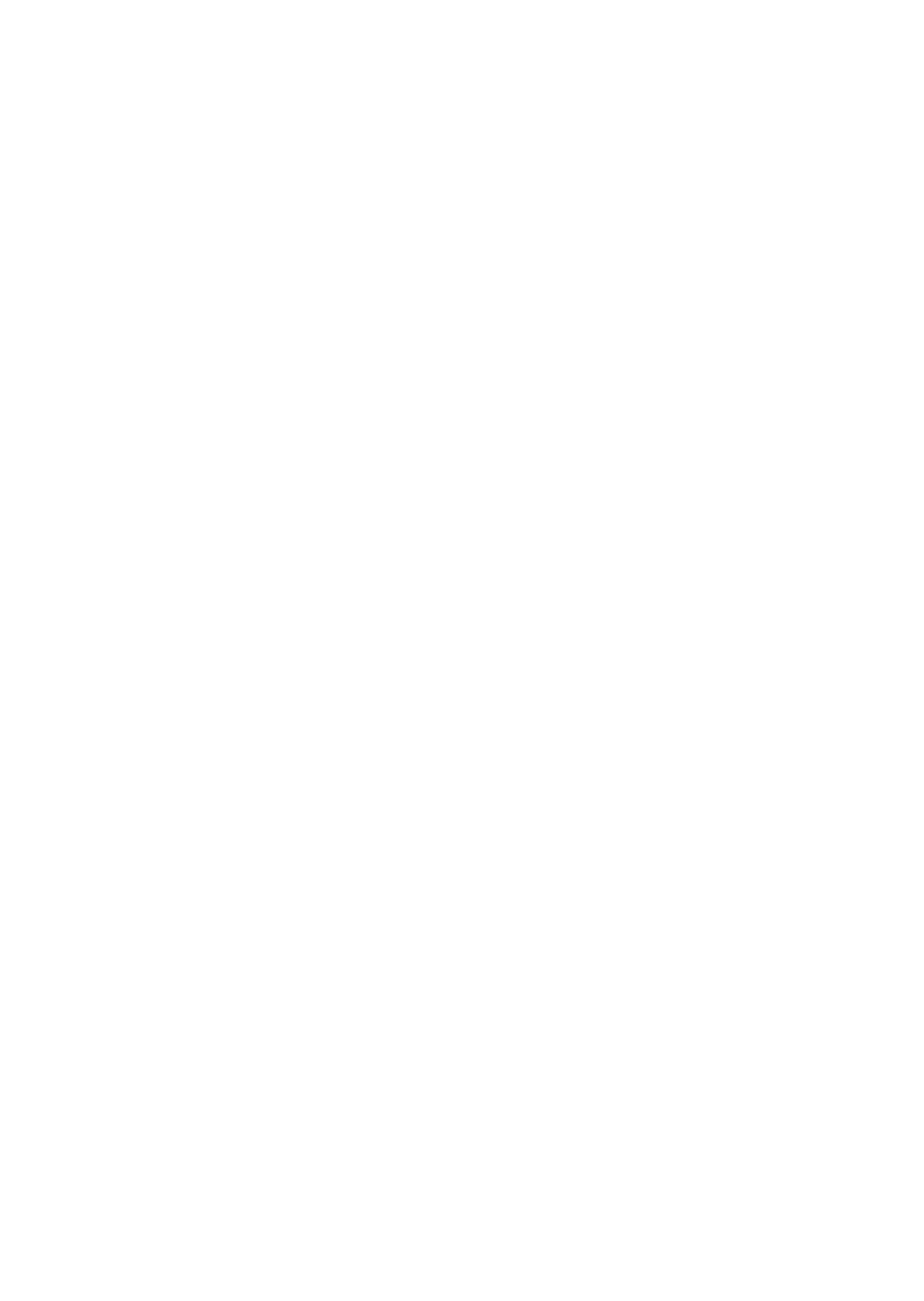




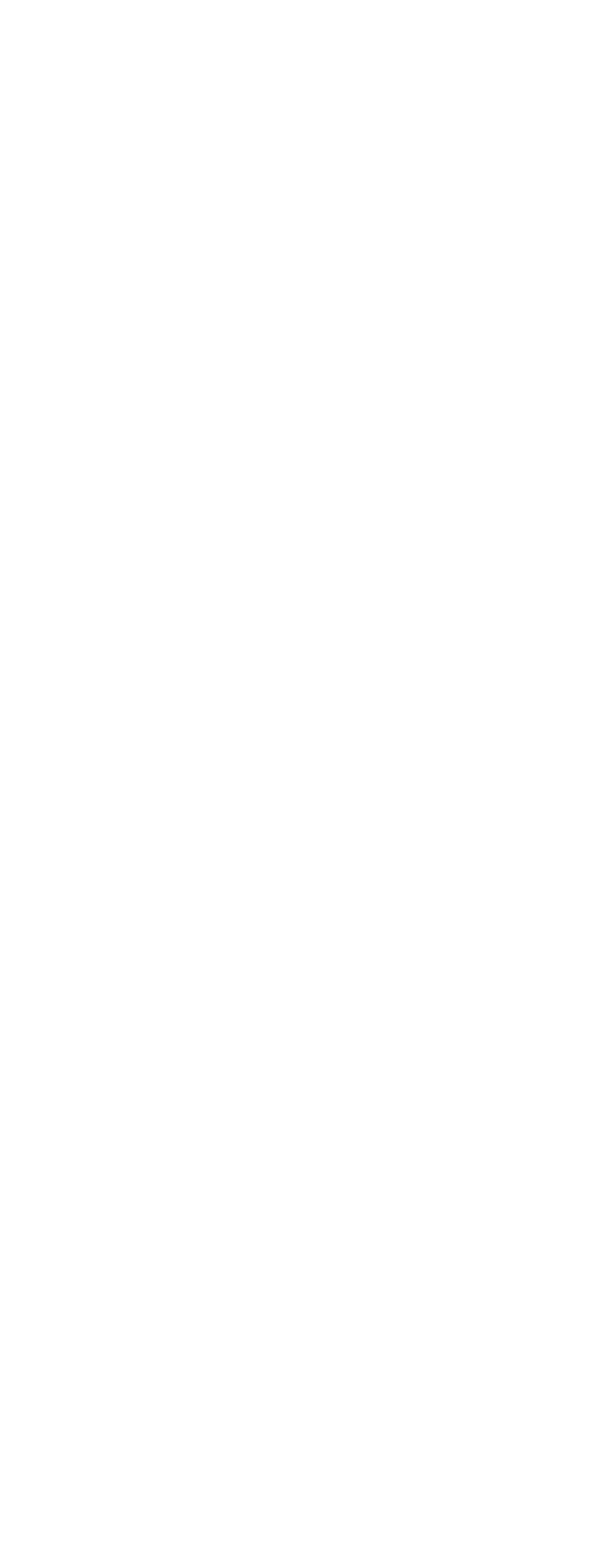




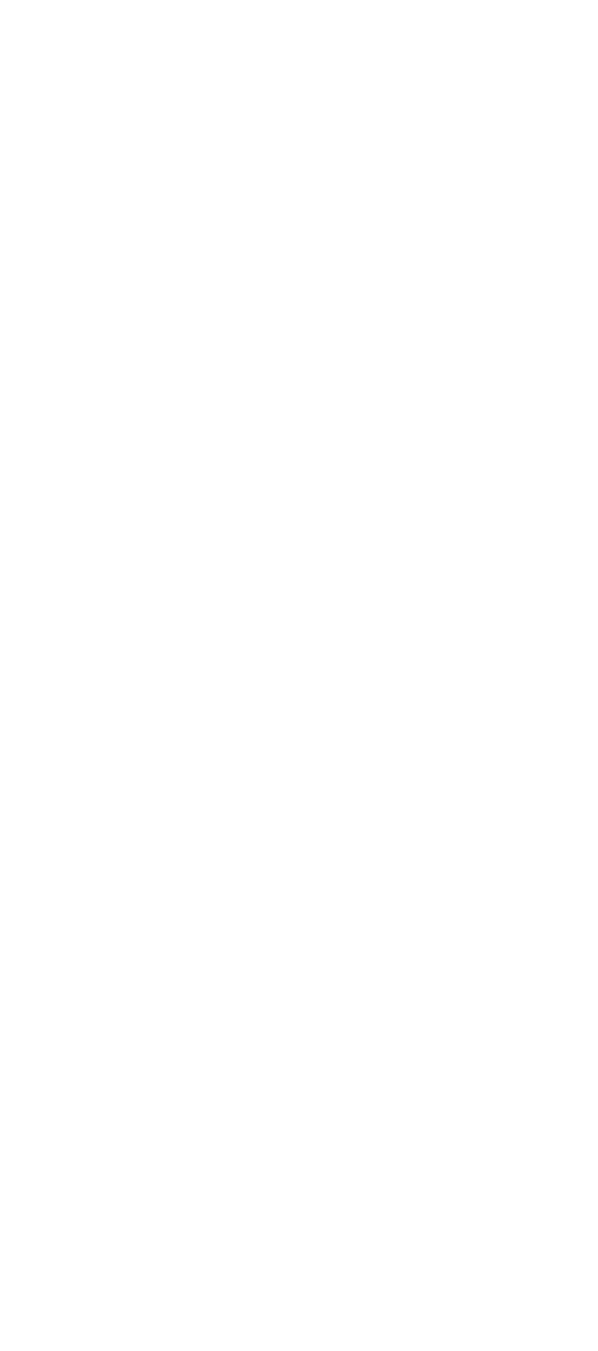




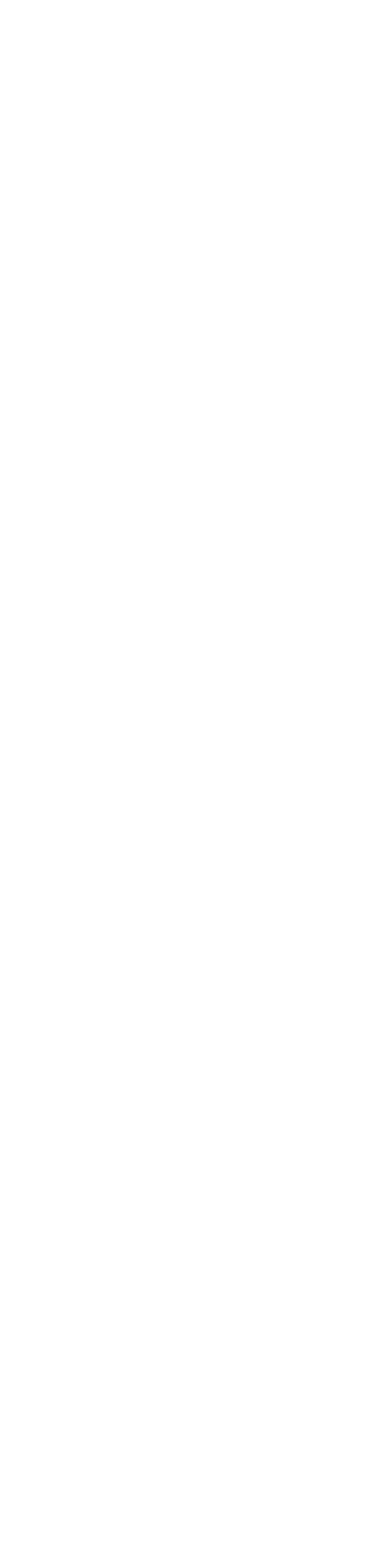

\begin{tabular}{|c|c|c|c|}
\hline 0 & & & \\
\hline$\frac{\pi}{2}$ & 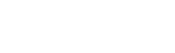 & 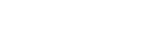 & 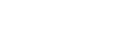 \\
\hline 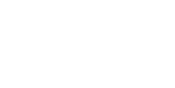 & 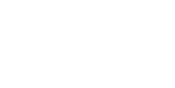 & 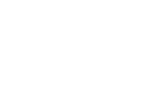 & 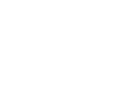 \\
\hline 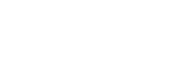 & & & \\
\hline 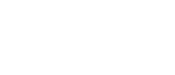 & N OA & nis & \\
\hline 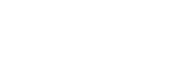 & 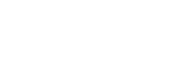 & 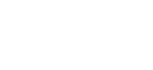 & 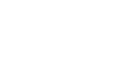 \\
\hline 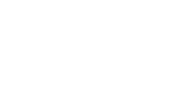 & 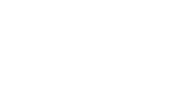 & 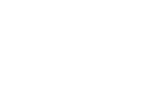 & 9 \\
\hline 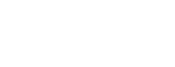 & & & \\
\hline 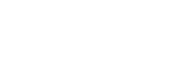 & 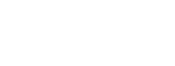 & & $\left|\begin{array}{cc|} & 9 \\
& 0\end{array}\right|$ \\
\hline 㝘害可 & $\because 0 \div: 90$ & 00 & $\begin{array}{|ll|} & \overrightarrow{0} \\
0 & 0\end{array}$ \\
\hline 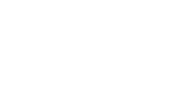 & 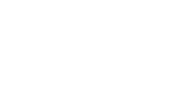 & 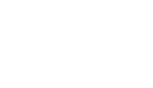 & 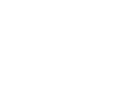 \\
\hline 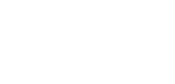 & 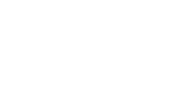 & & $\begin{array}{|cc|}\bullet & \vdots \\
\end{array}$ \\
\hline 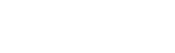 & $0 \infty$ & काण & ल \\
\hline 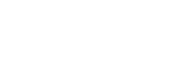 & 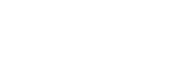 & 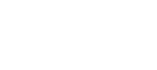 & 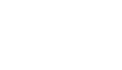 \\
\hline 产是会 & & & \\
\hline 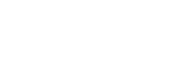 & تُ تُ & 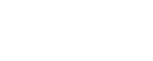 & $\because$ \\
\hline 熟要 & 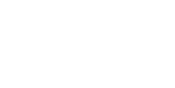 & 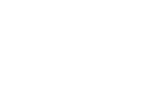 & $\tilde{\alpha}$ \\
\hline 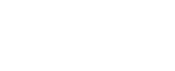 & ᄋㅋㄱㄱ 쿠 & 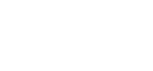 & $\Rightarrow=$ \\
\hline 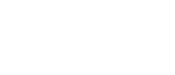 & 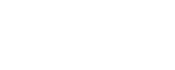 & 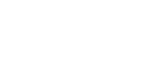 & \begin{tabular}{|c|c|}
0 & 0 \\
\hdashline & 0
\end{tabular} \\
\hline 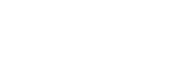 & 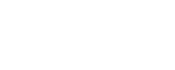 & 8.888 .89 & \begin{tabular}{|l|l|} 
& 0 \\
& 0 \\
& 0 \\
\end{tabular} \\
\hline 莡㐍 & 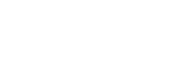 & $\begin{array}{llllll}1 & 1 & 1 & 1 & 1 & 1 \\
\end{array}$ & $i$ \\
\hline 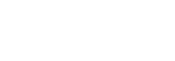 & & & \\
\hline 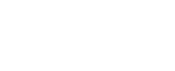 & 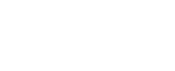 & 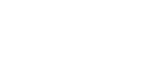 & 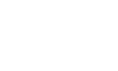 \\
\hline 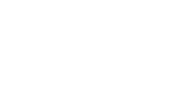 & 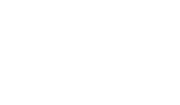 & 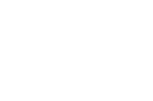 & 常 \\
\hline \&: & 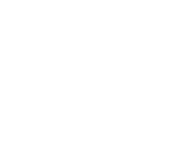 & 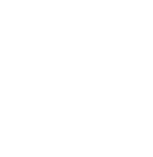 & 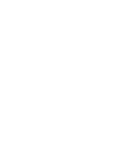 \\
\hline
\end{tabular}




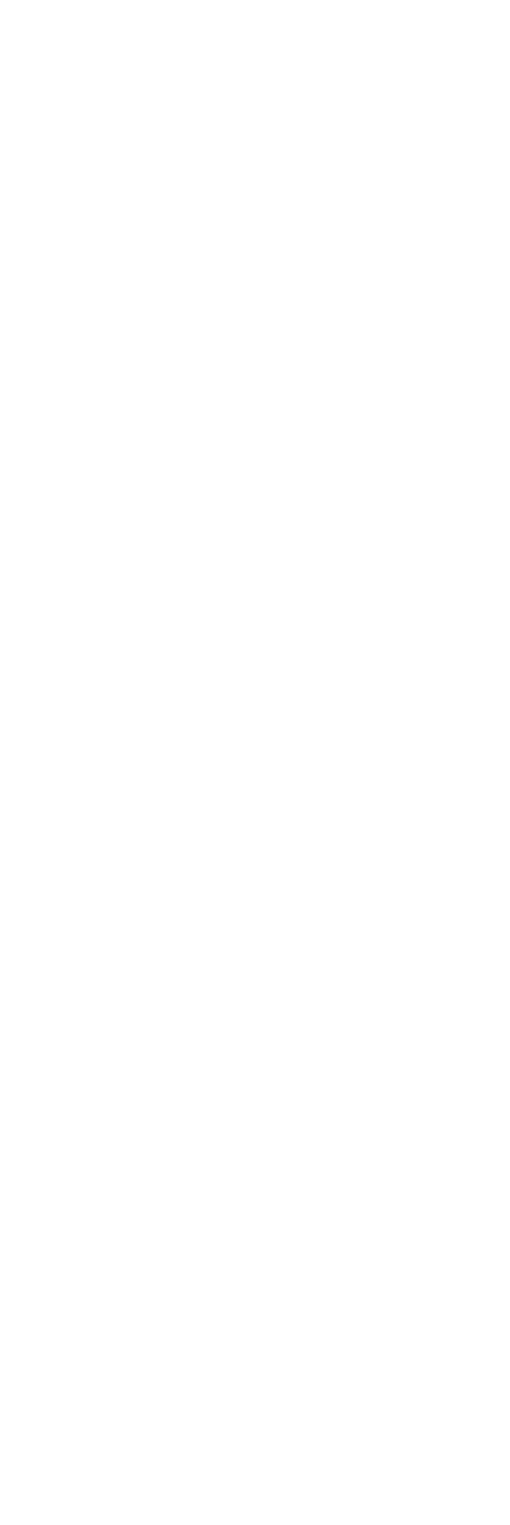




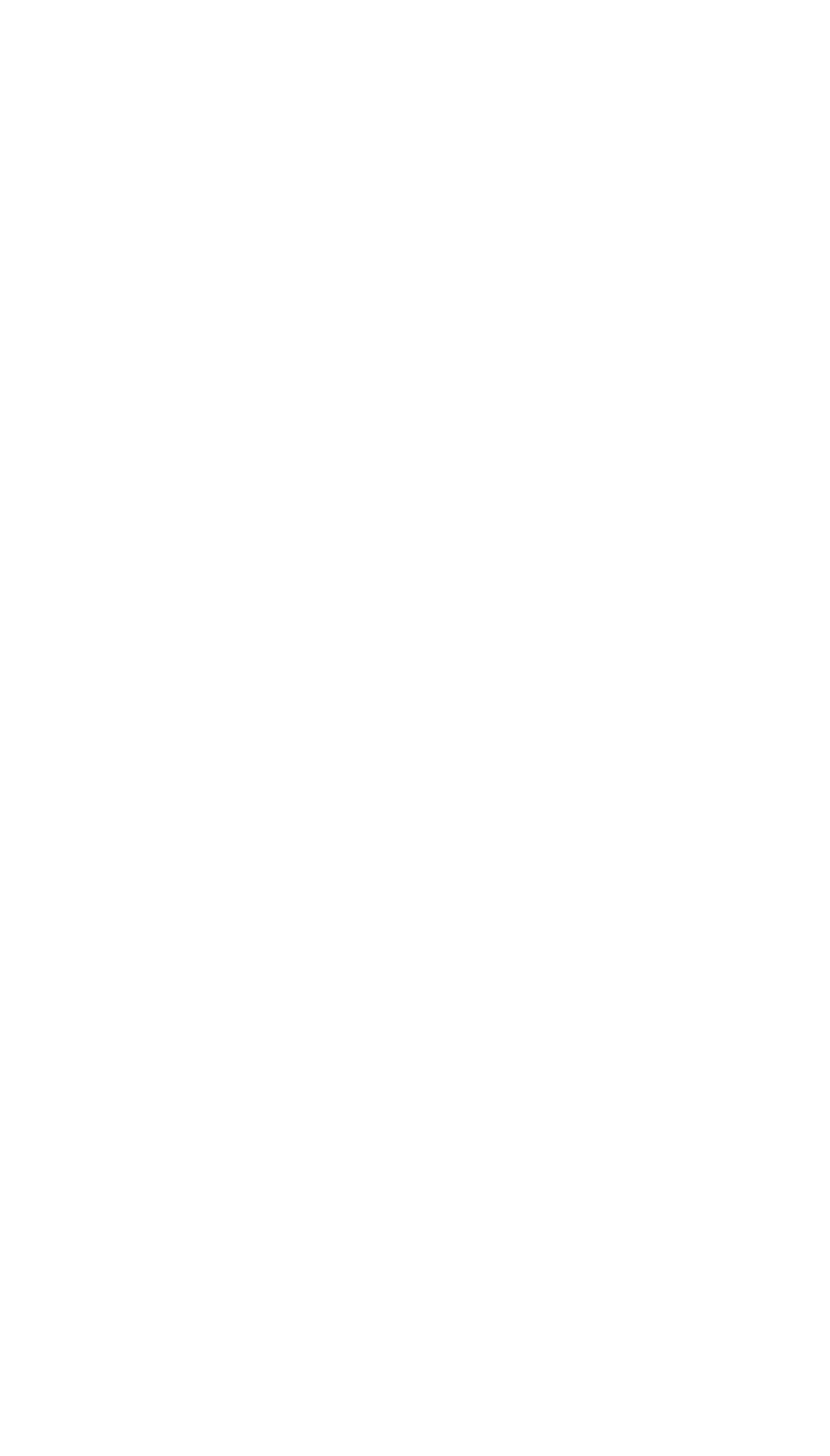




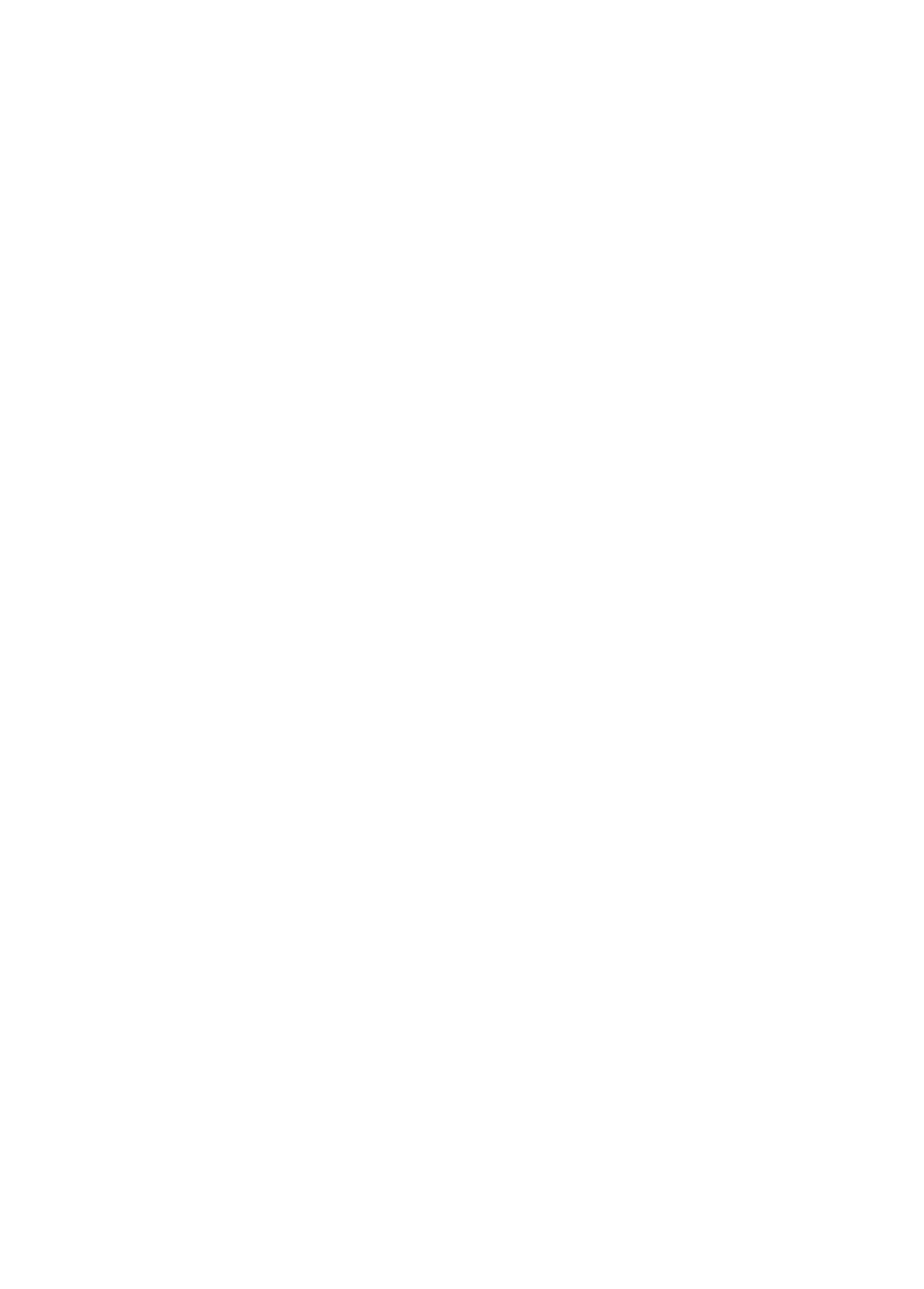




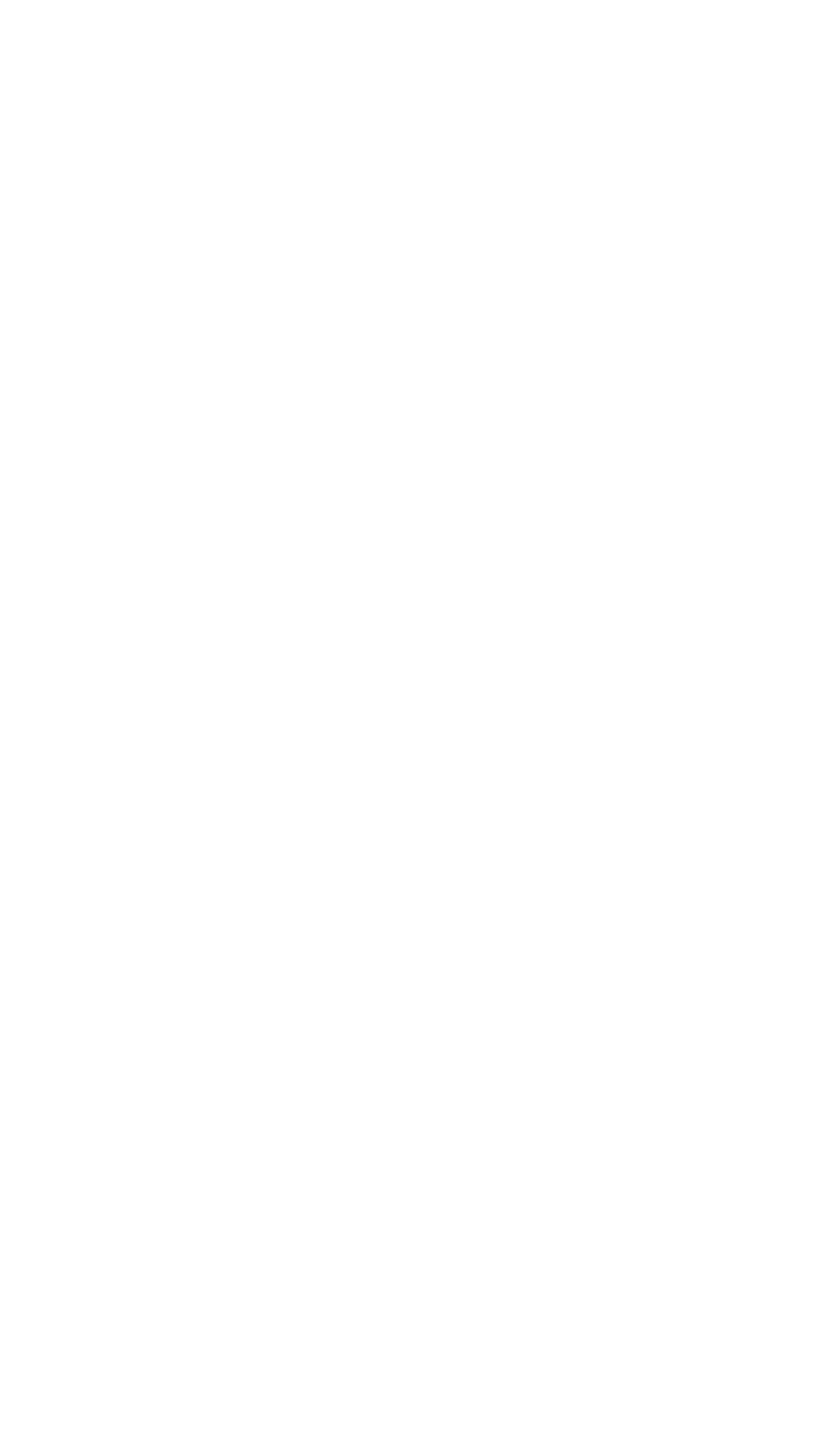




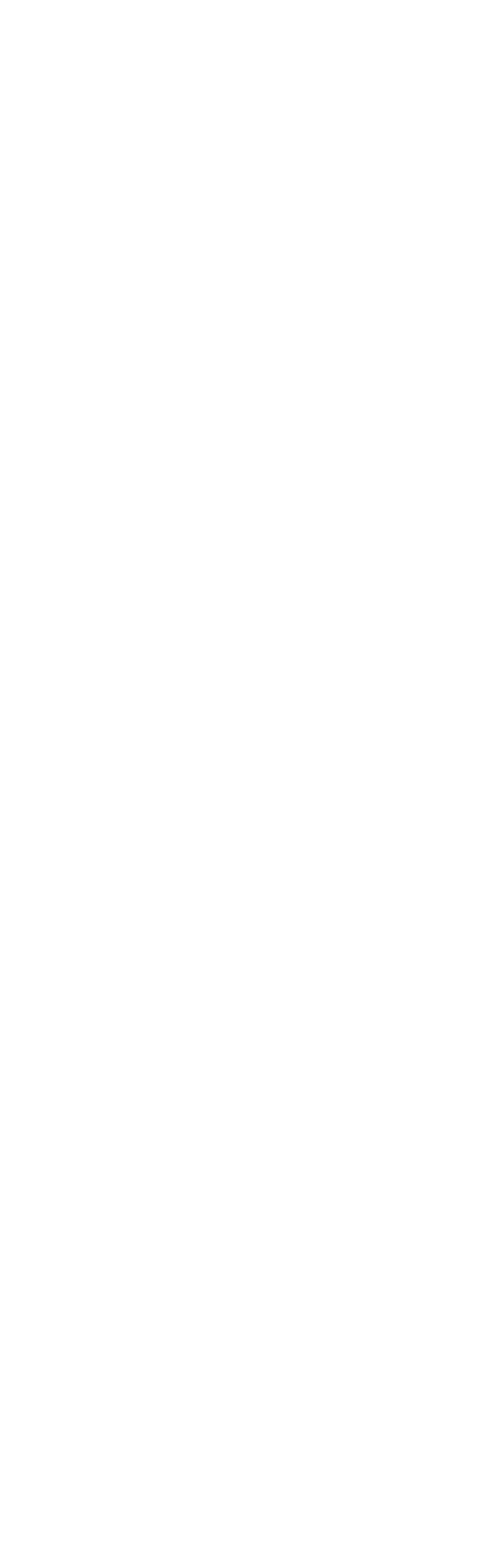




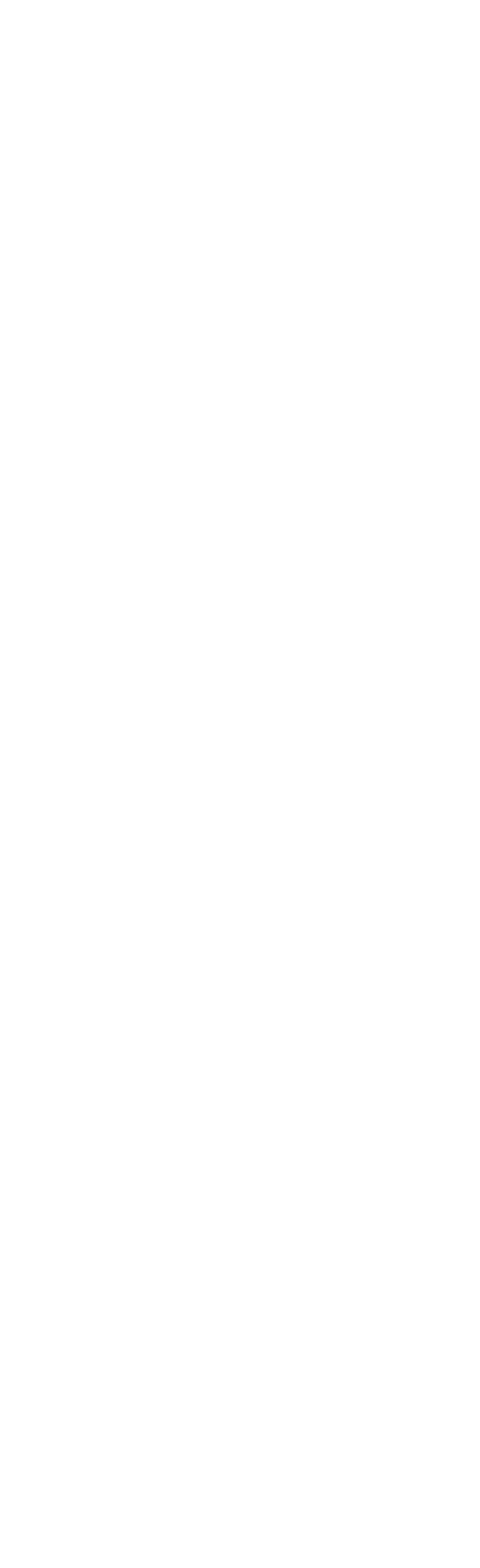




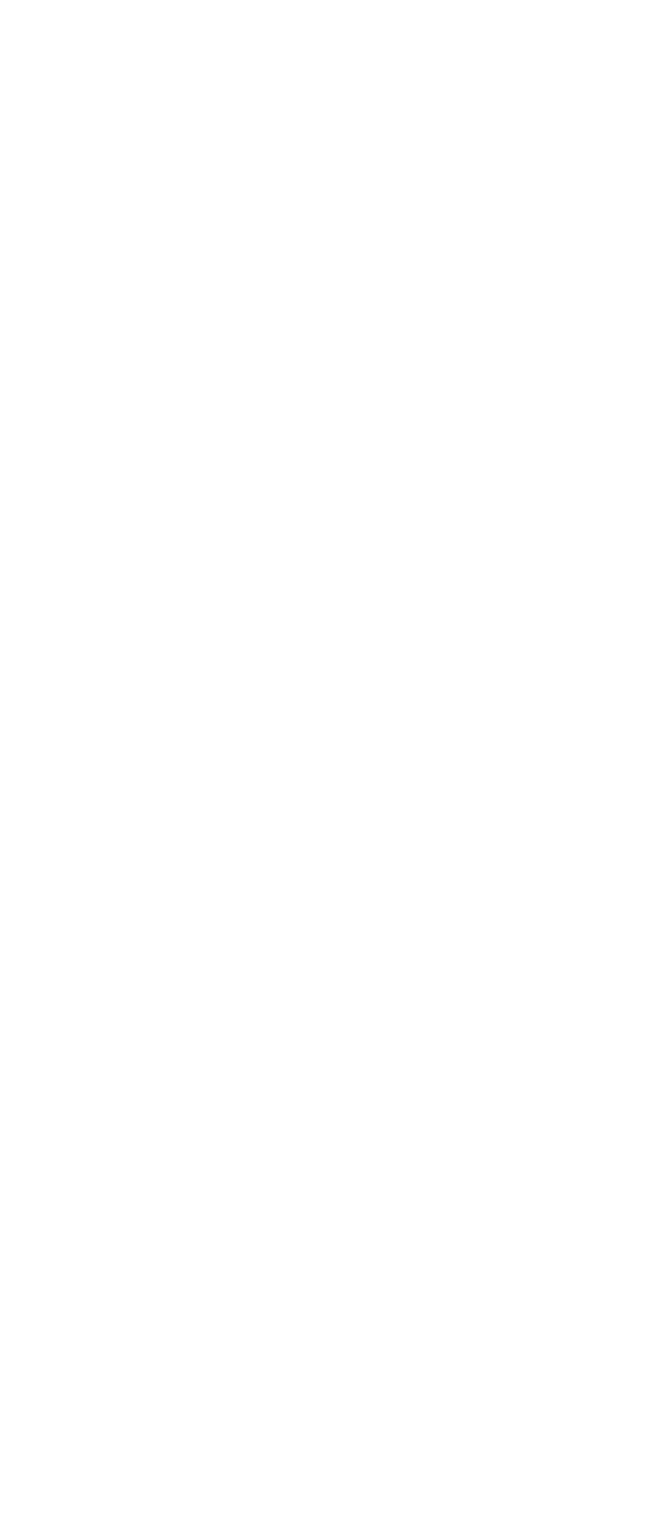




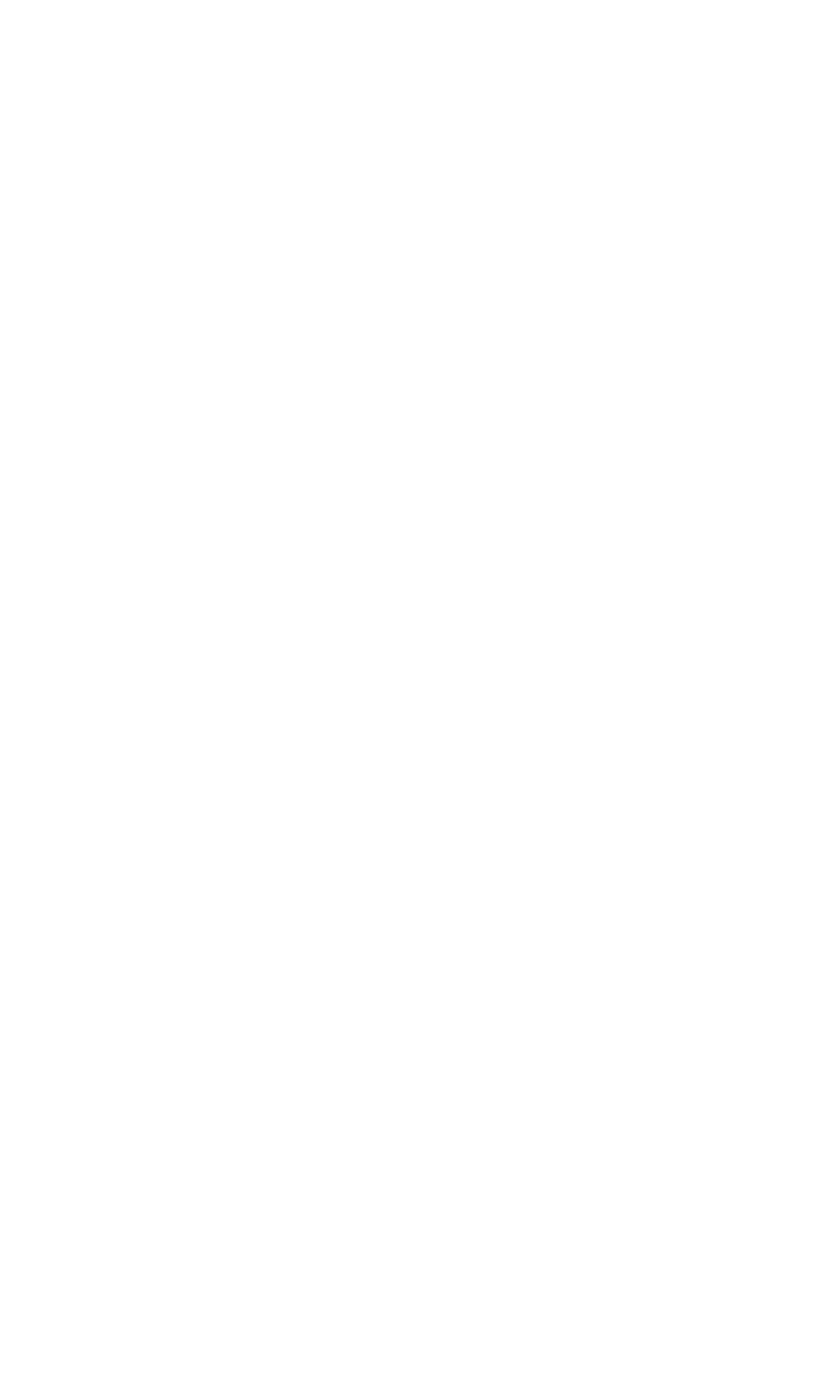




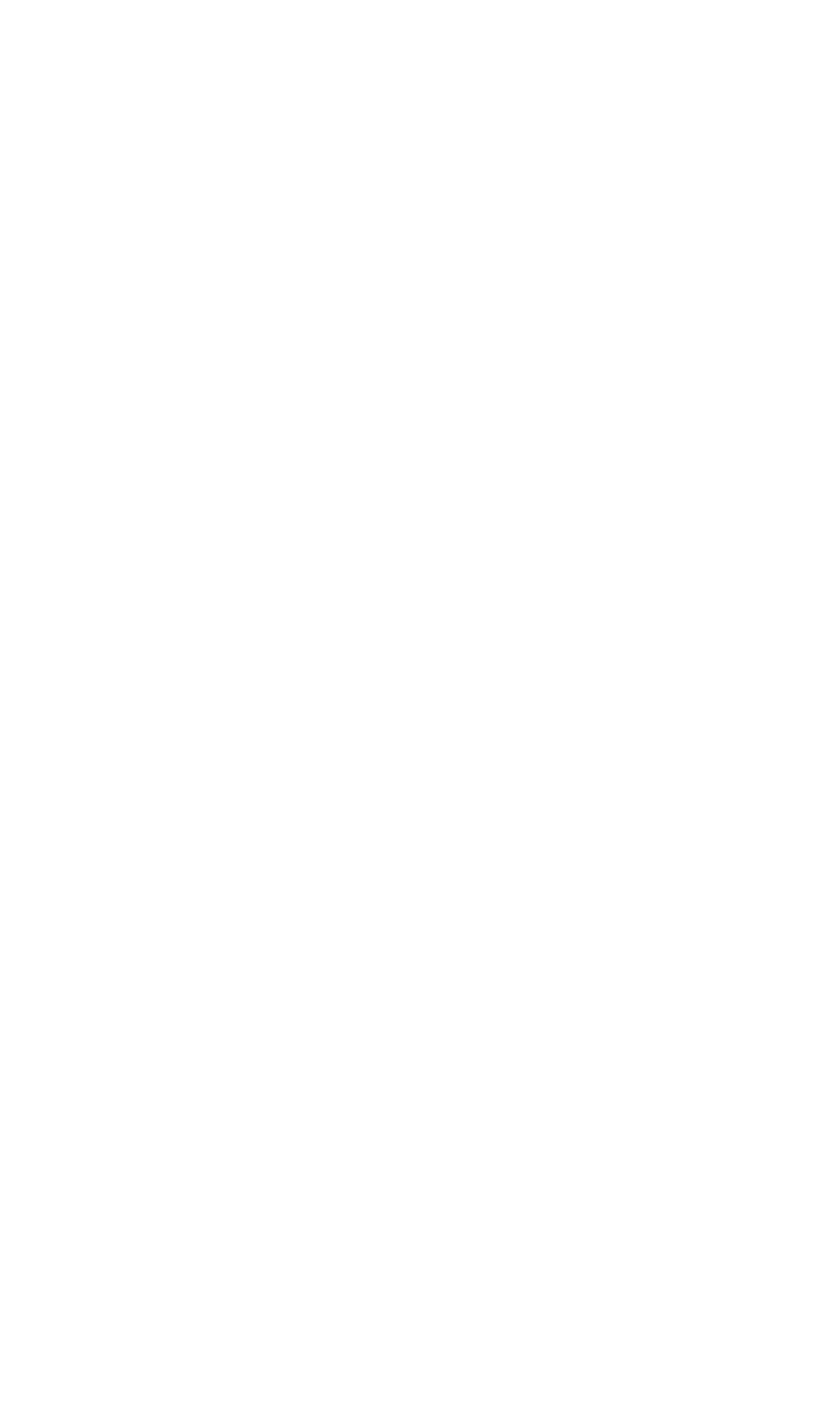




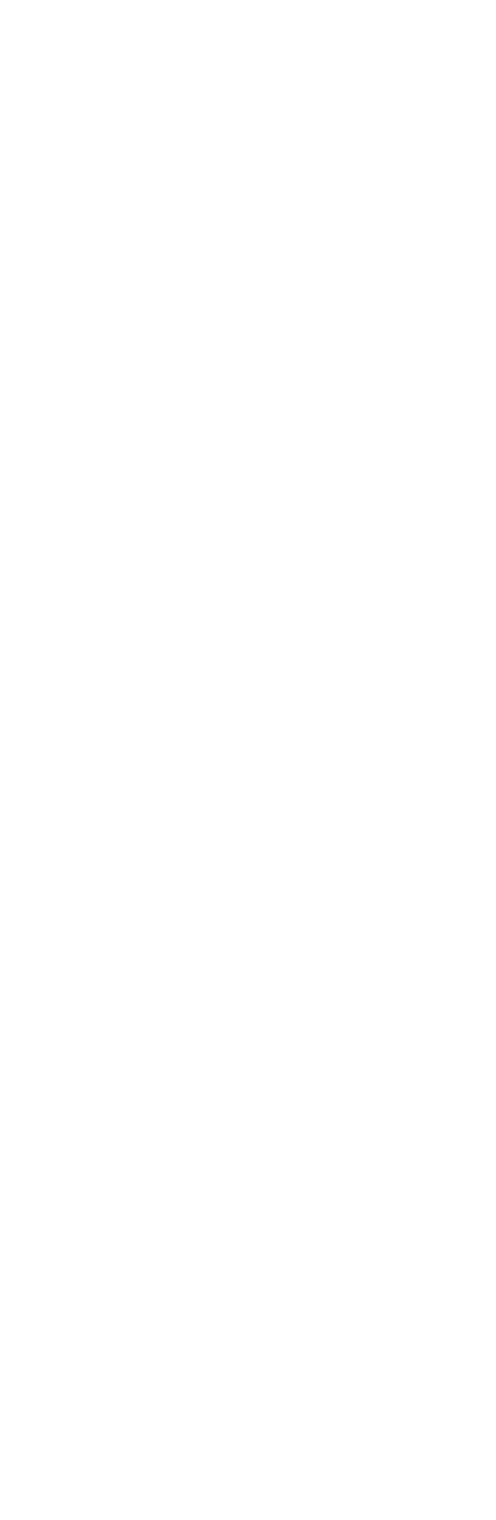




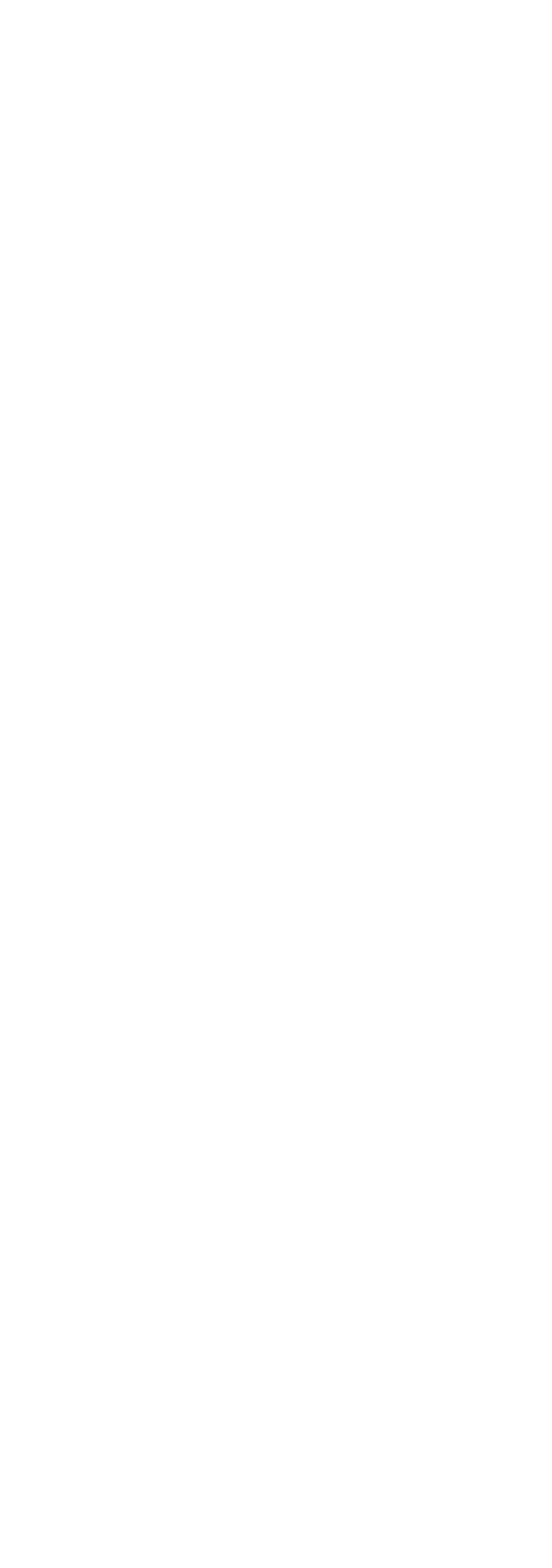




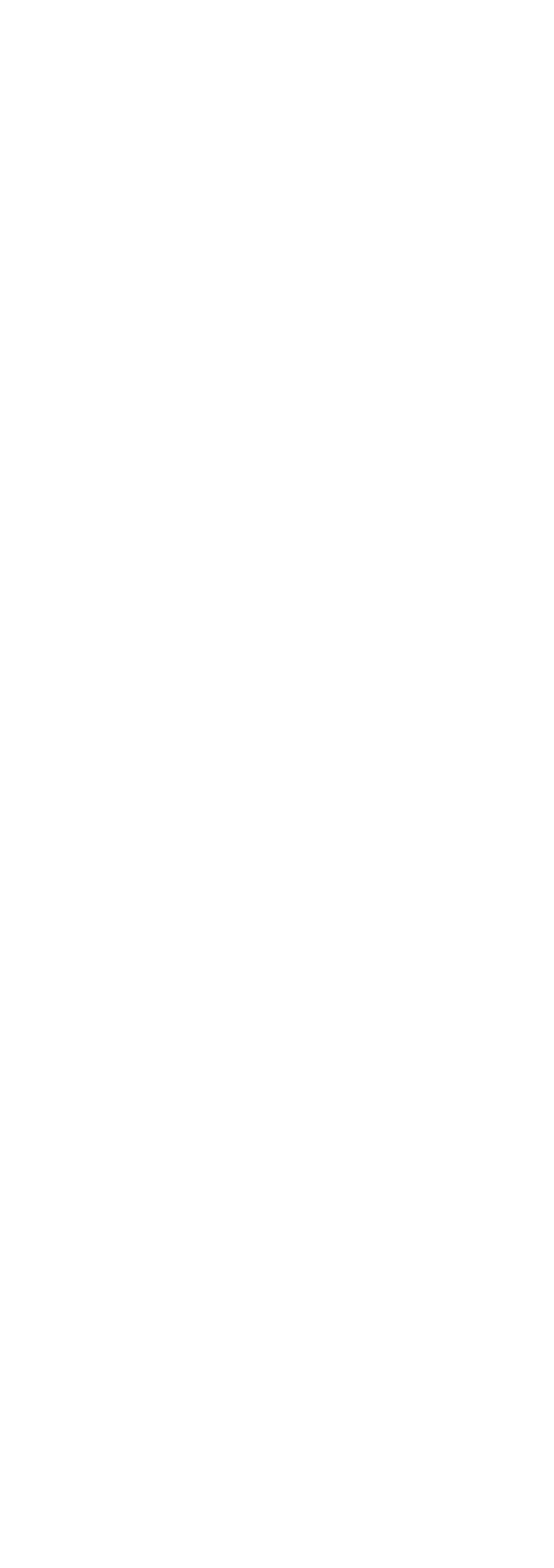




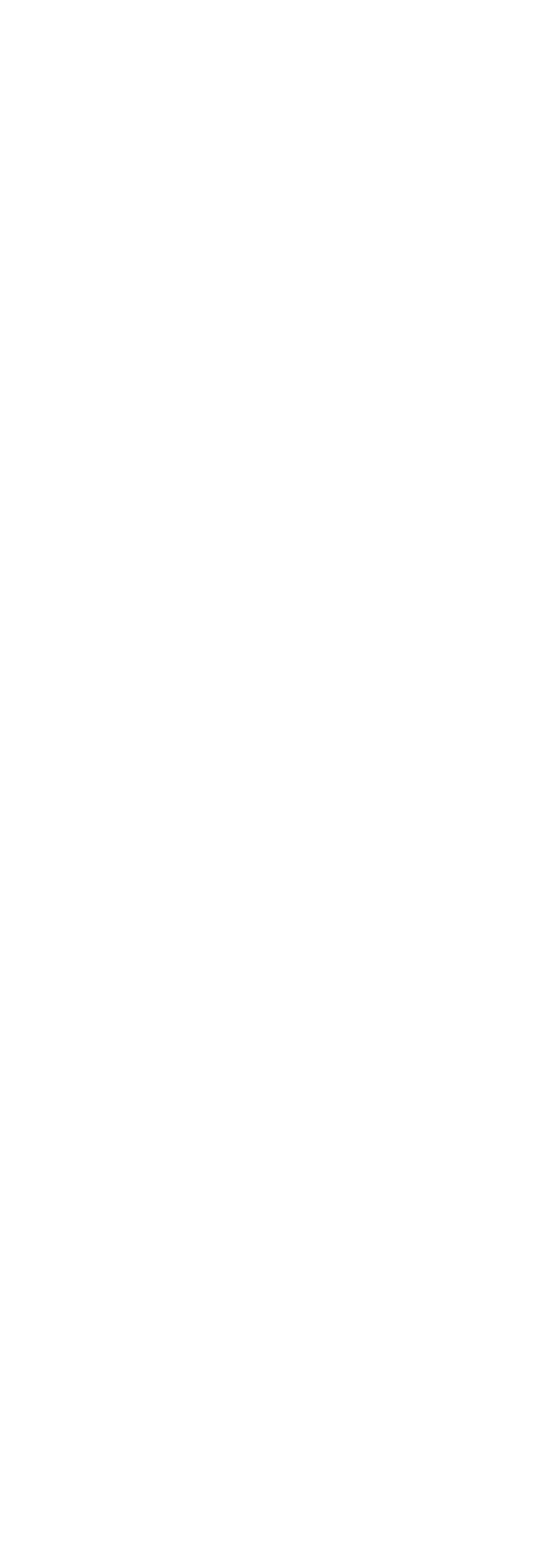




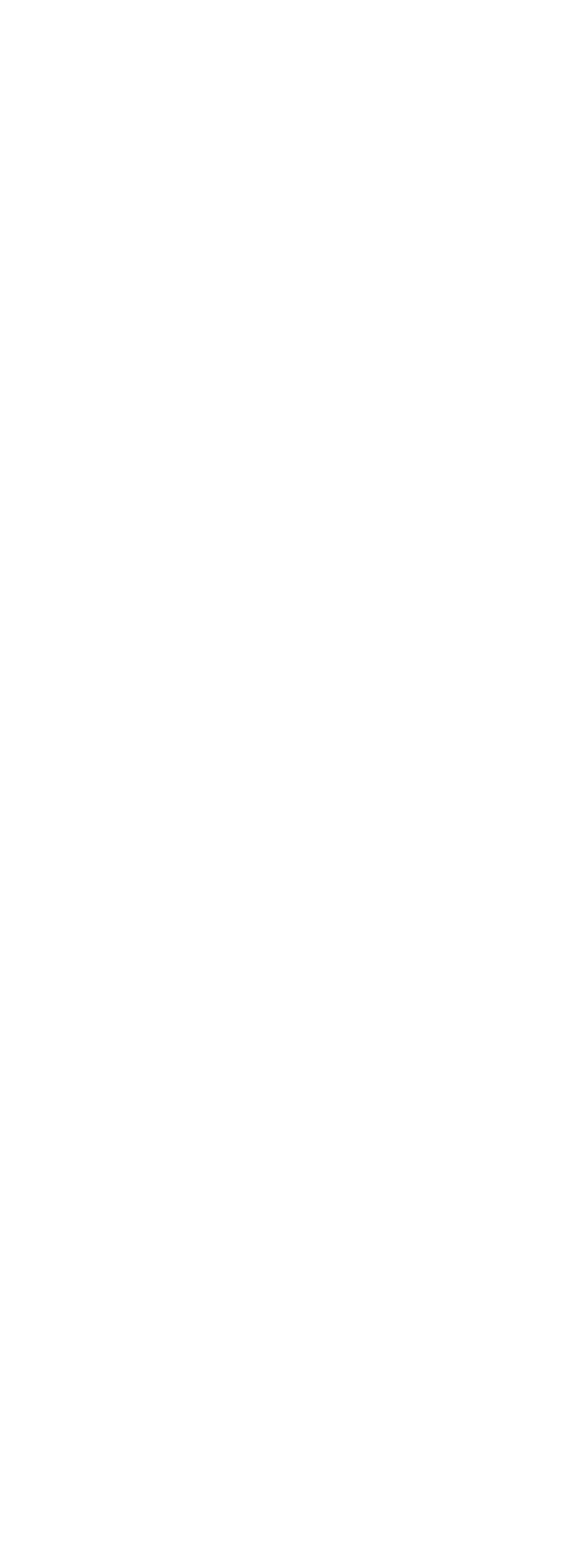




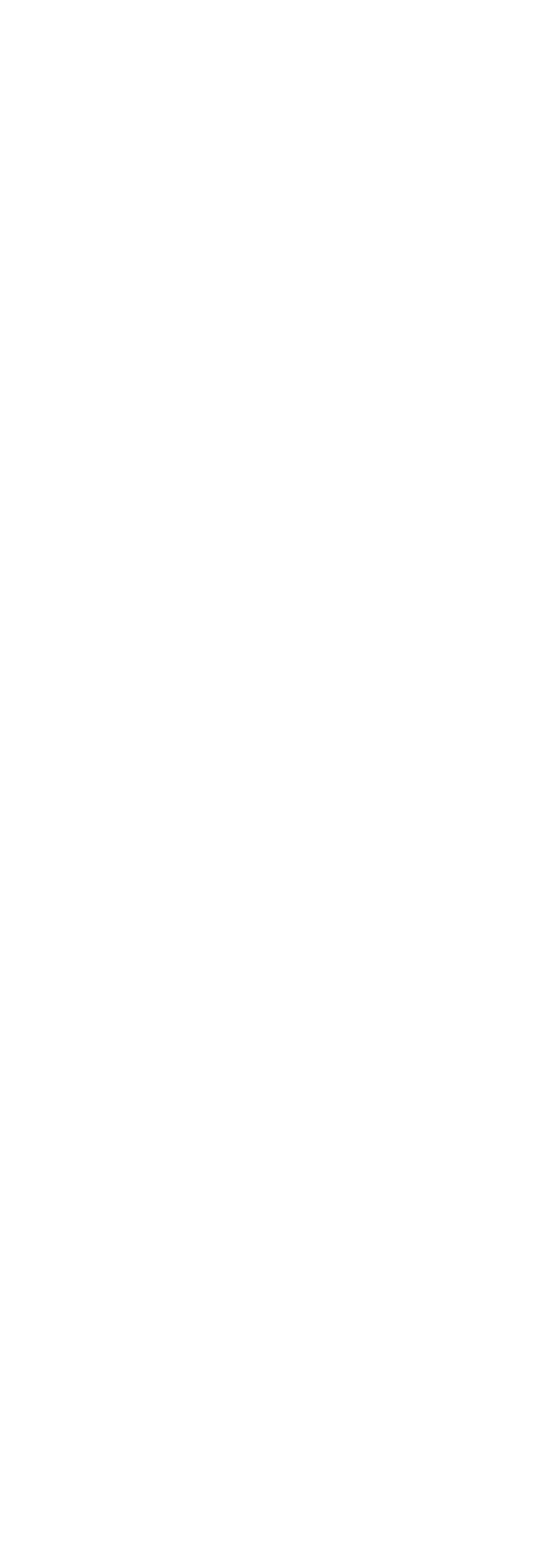




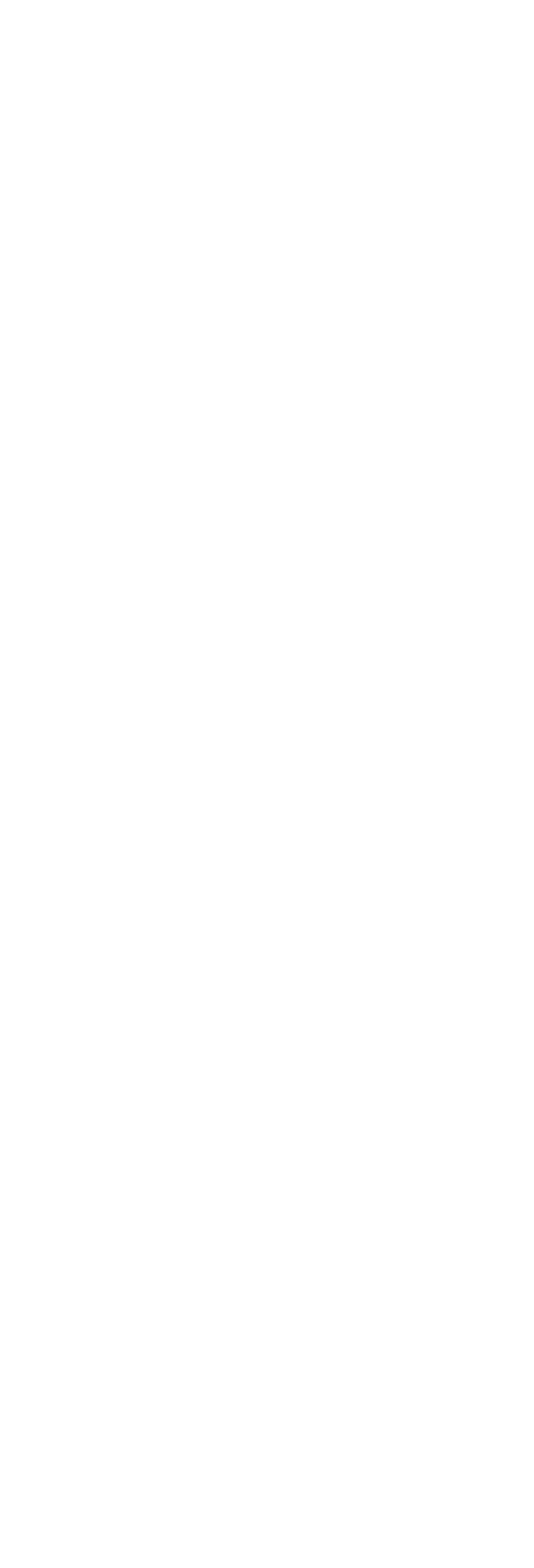




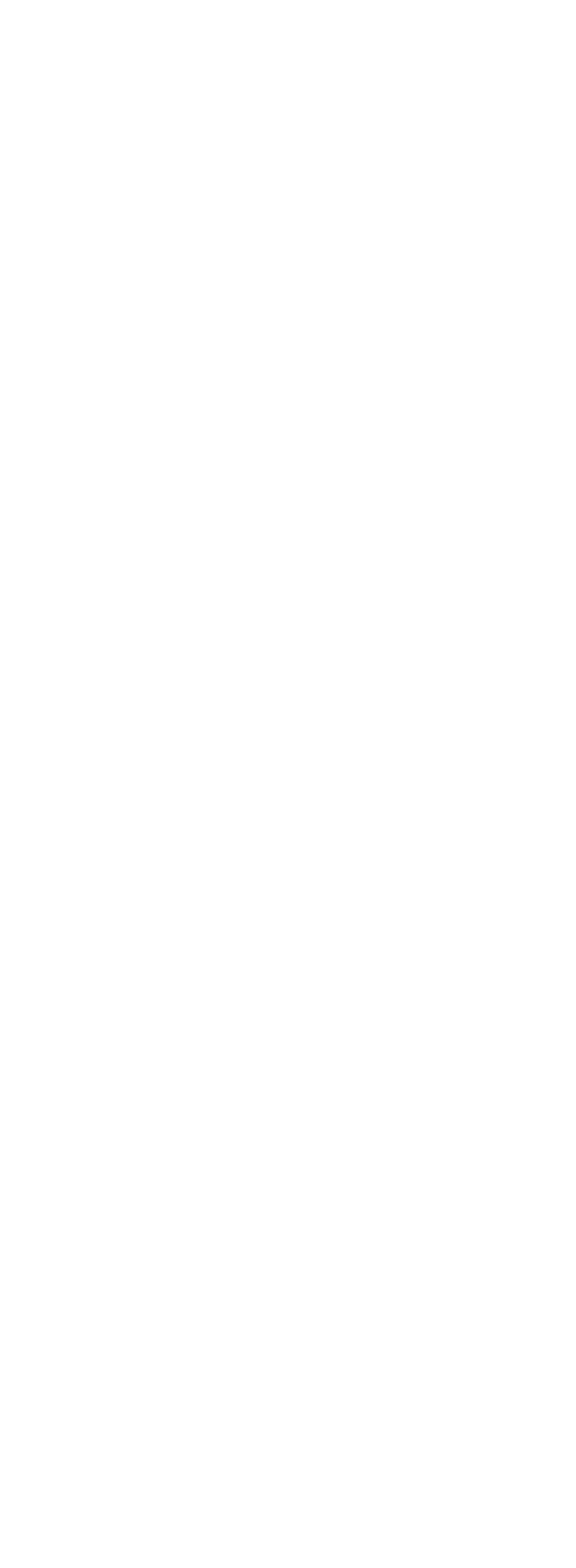




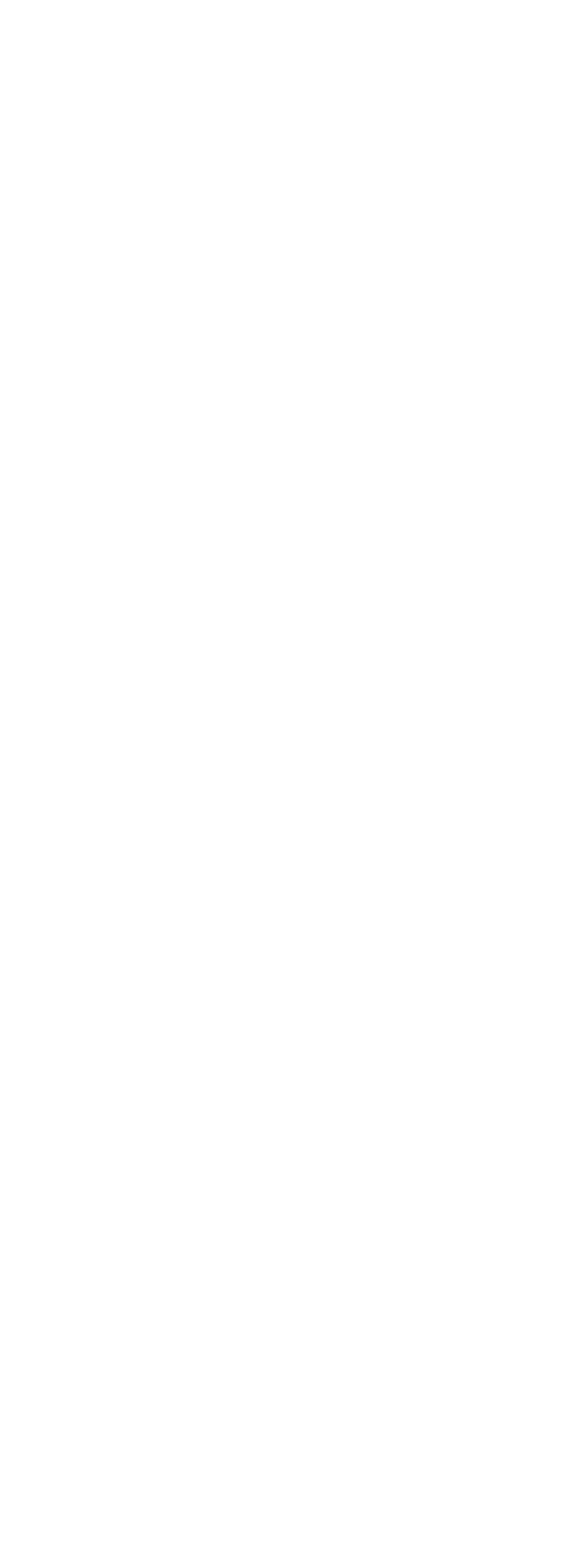




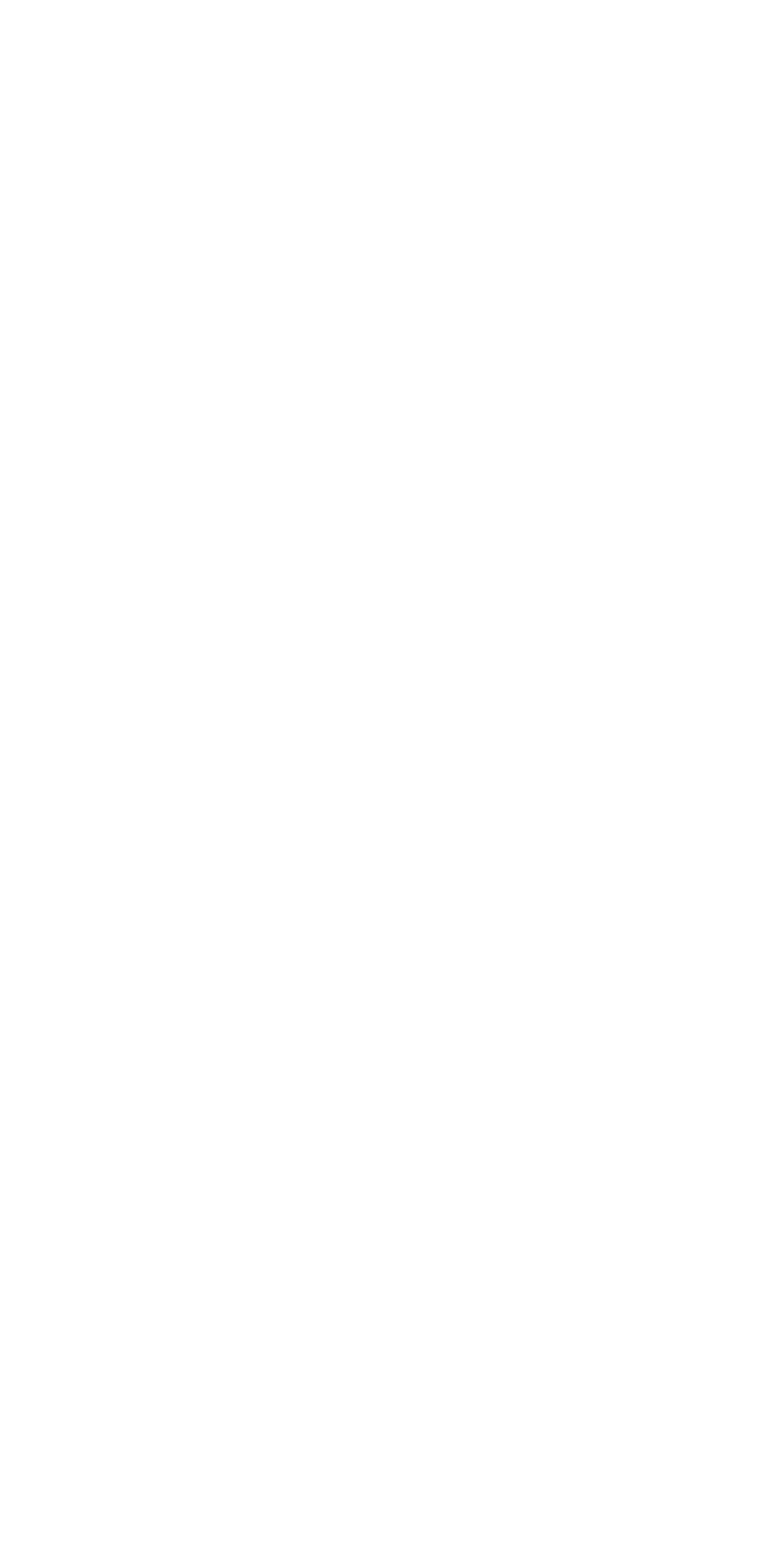




\begin{tabular}{|c|c|c|c|}
\hline 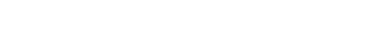 & $\left|\begin{array}{l}\pi \\
\hdashline\end{array}\right|$ & $\stackrel{\varphi}{:}$ & \\
\hline 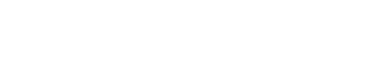 & 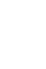 & : & 1 \\
\hline 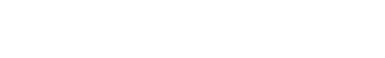 & $\dot{m}$ & i & $T$ \\
\hline 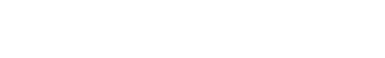 & $\begin{array}{l}\infty \\
\infty\end{array}$ & : & 1 \\
\hline 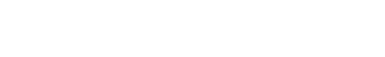 & 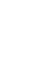 & 䙳 & $i$ \\
\hline 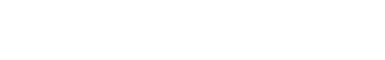 & 离 & 1 & $i$ \\
\hline 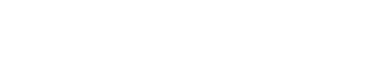 & $\begin{array}{c}0 \\
0 \\
\dot{m}\end{array}$ & 1 & $i$ \\
\hline 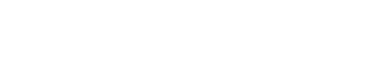 & ฟิ & 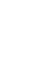 & i \\
\hline 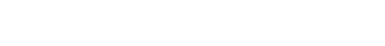 & 1 & $i$ & 1 \\
\hline 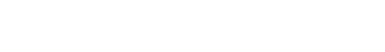 & $i$ & $i$ & I \\
\hline 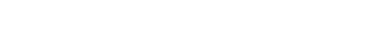 & I & สี & $\stackrel{\oplus}{\sim}$ \\
\hline 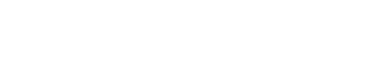 & 总 & 융 & 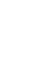 \\
\hline 000000000000000000 & $i$ & $i$ & $i$ \\
\hline 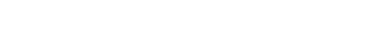 & $\underset{N}{\infty}$ & 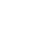 & 9 \\
\hline 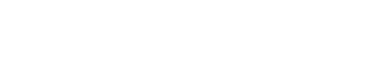 & 1 & $\mathrm{i}$ & $i$ \\
\hline 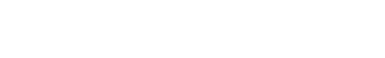 & $\stackrel{\leftrightarrow}{\circ}$ & $\hat{\Im}$ & is \\
\hline 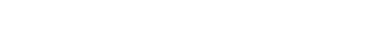 & 1 & $i$ & 1 \\
\hline 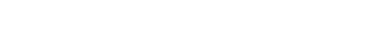 & $\mathrm{i}$ & $i$ & 1 \\
\hline 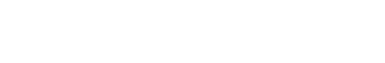 & 1 & 1 & $i$ \\
\hline $\begin{array}{l}0 \\
\dot{\infty} \\
\infty \\
\infty\end{array}$ & $i$ & $\begin{array}{l}0 \\
\dot{\infty} \\
\infty\end{array}$ & $!$ \\
\hline 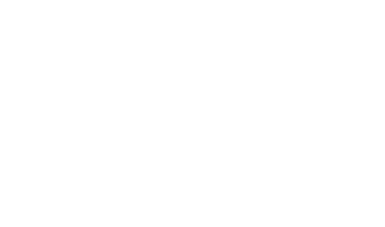 & 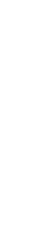 & 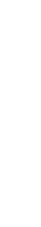 & 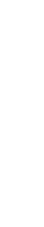 \\
\hline
\end{tabular}




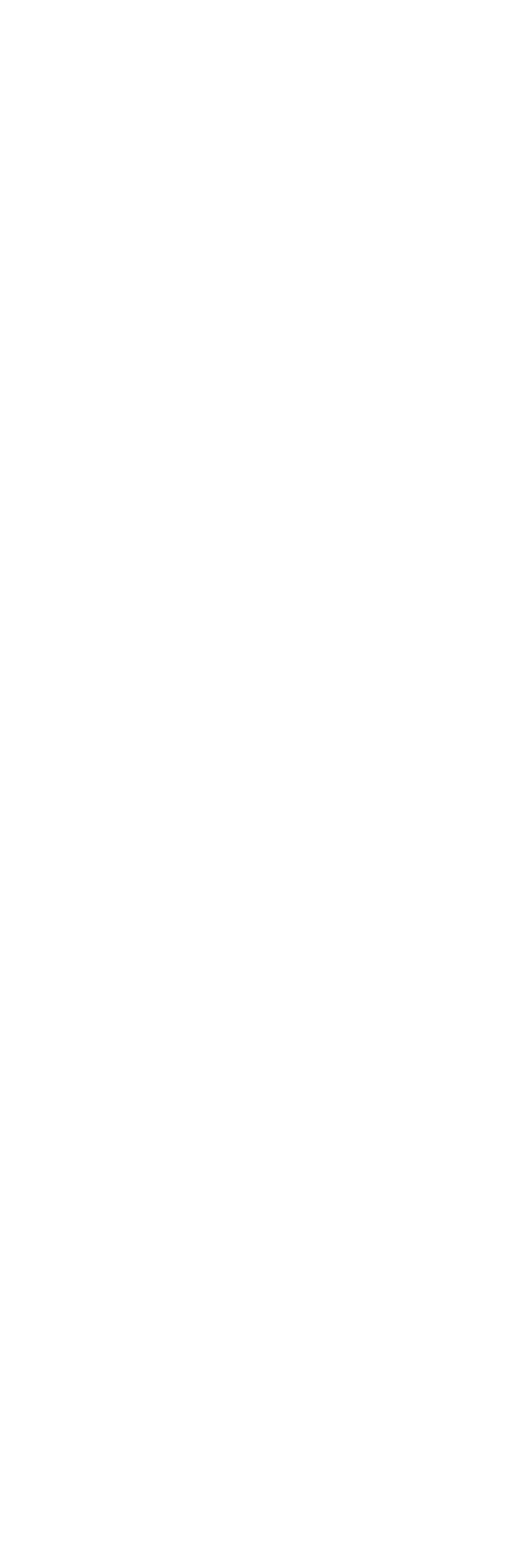

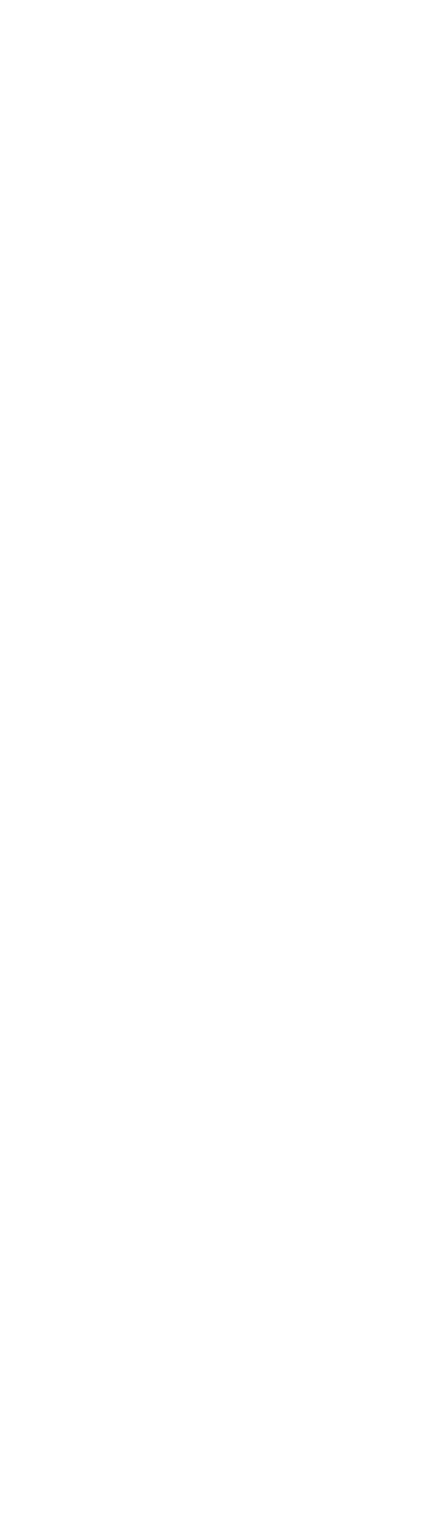




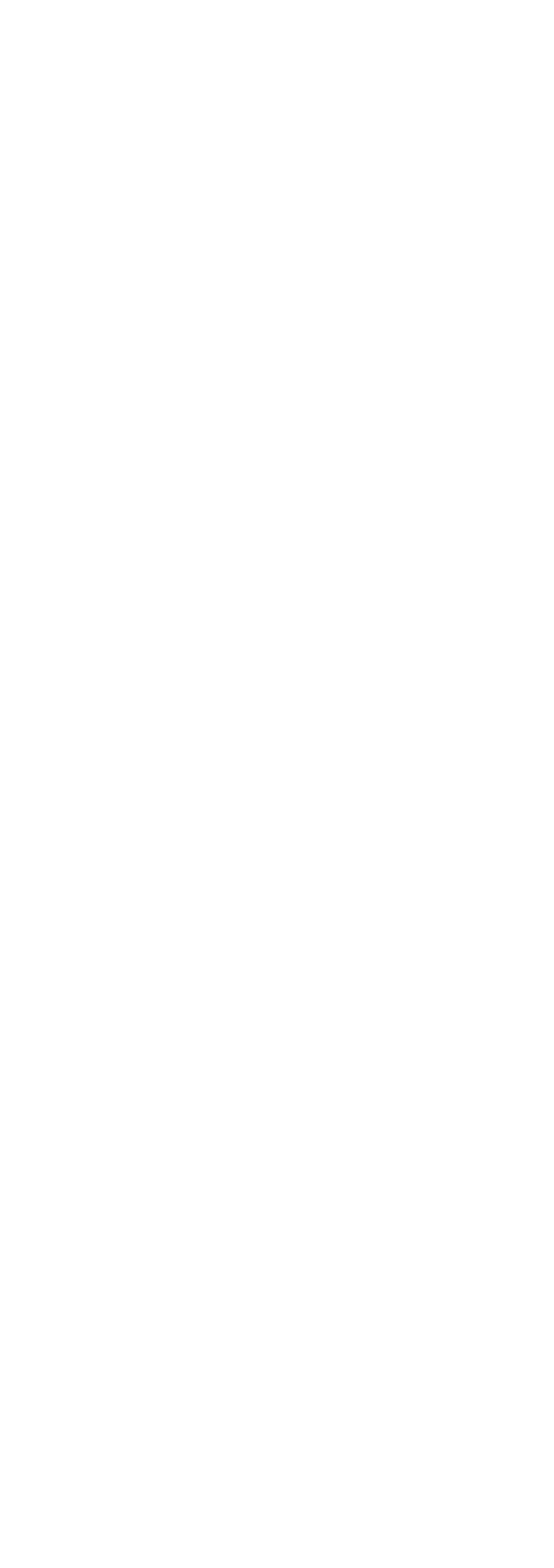




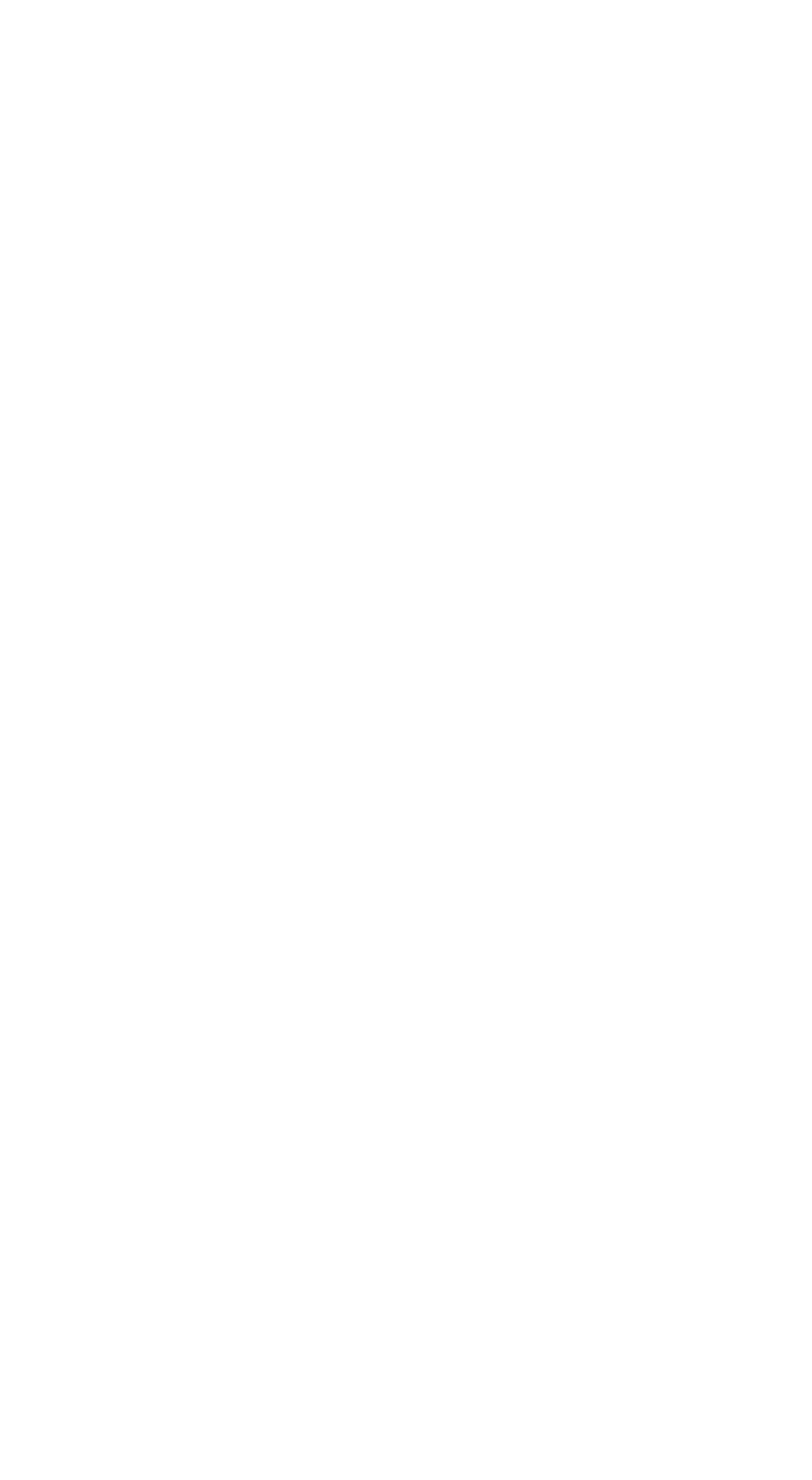



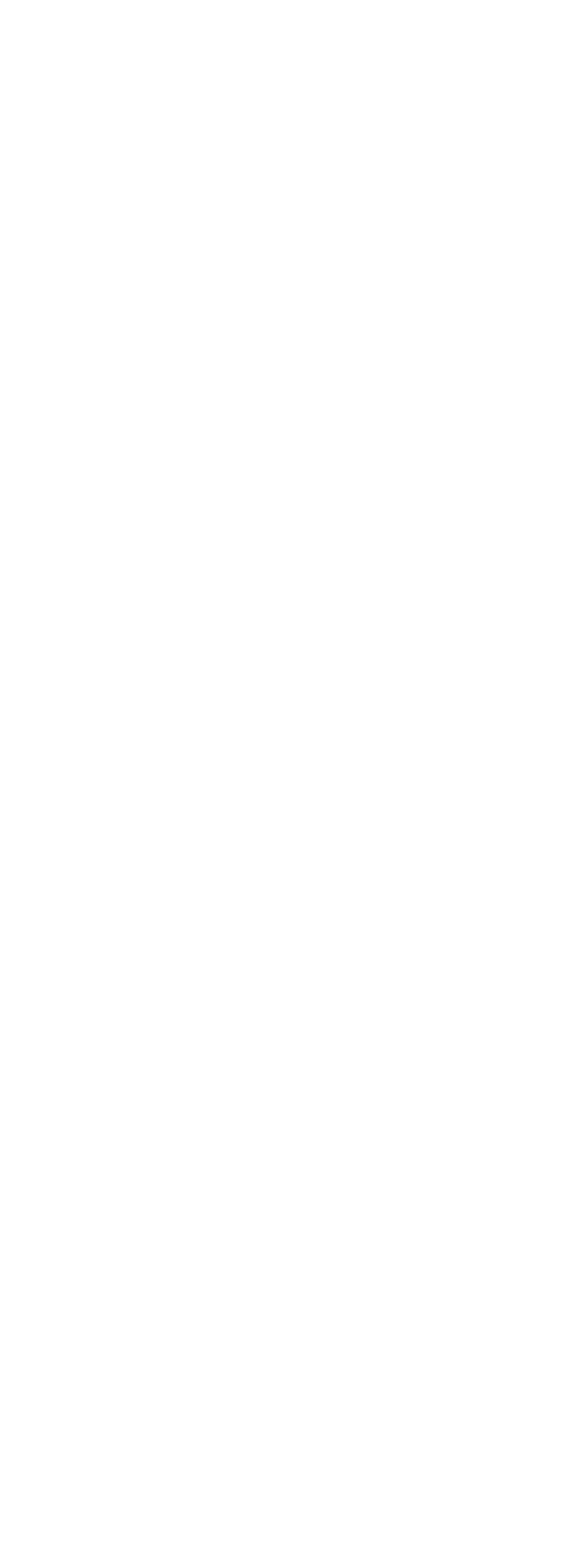


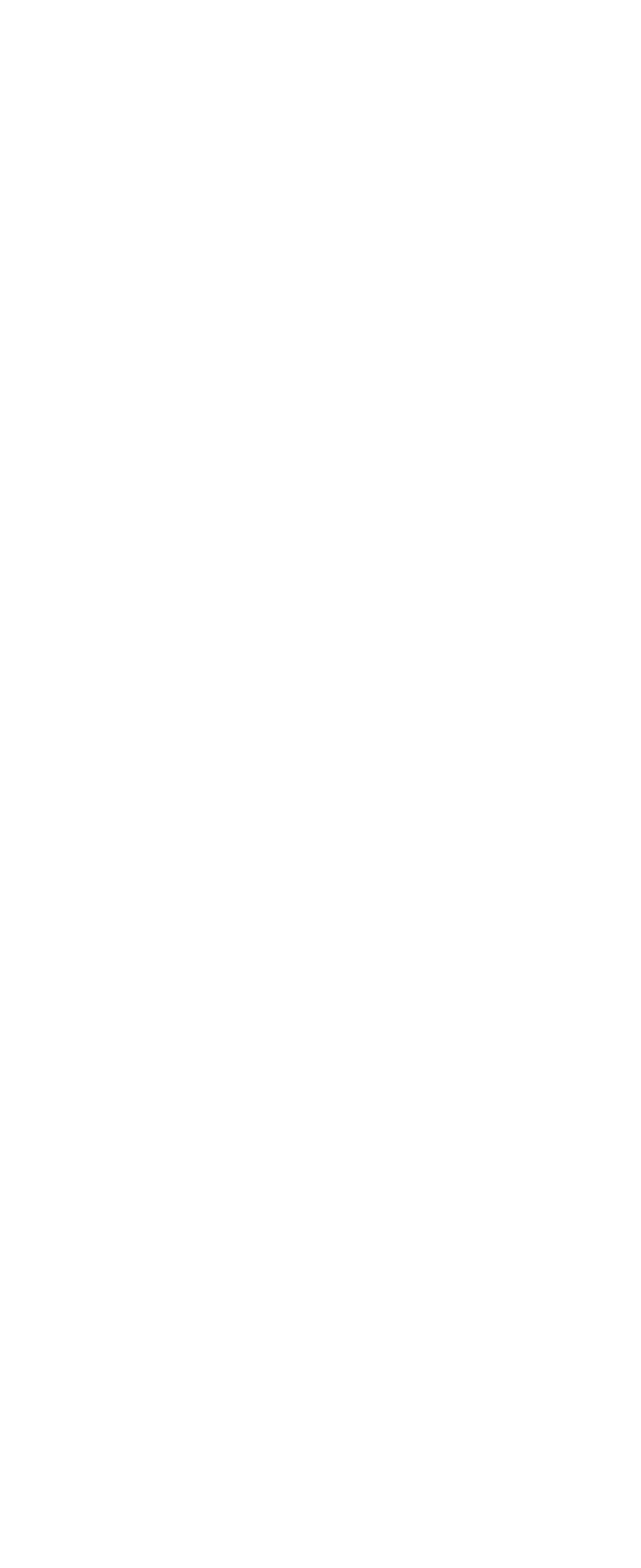



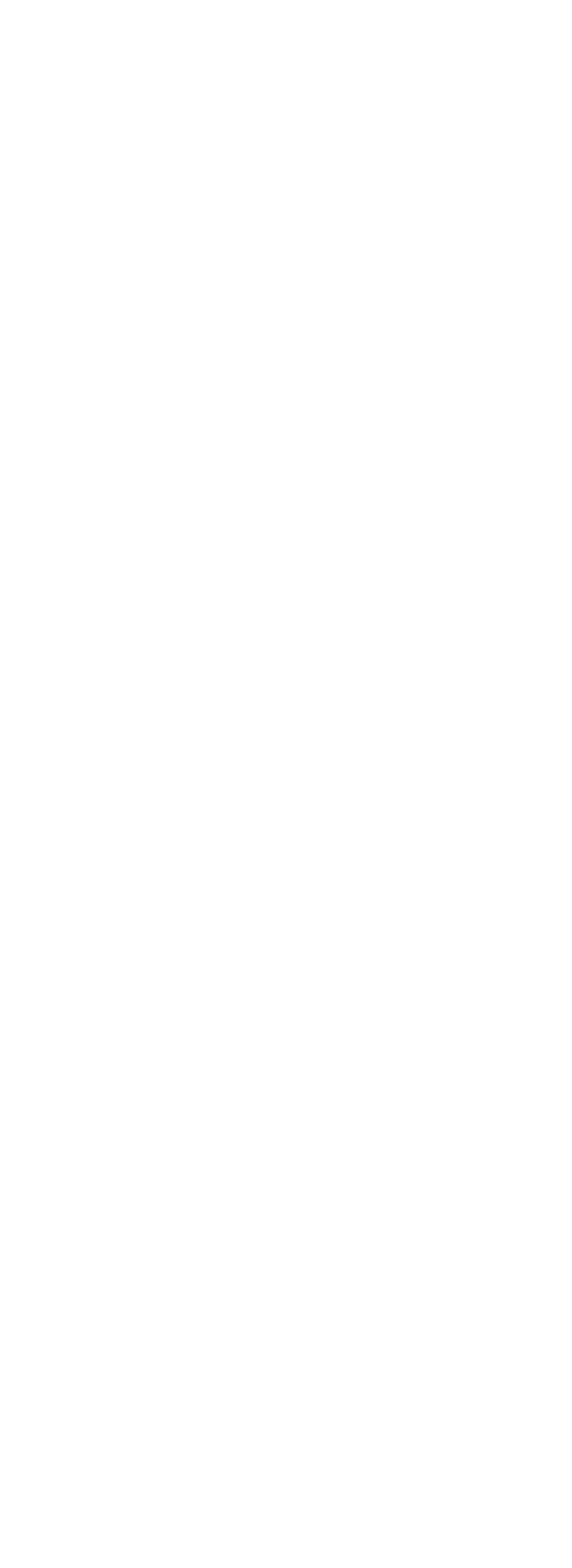


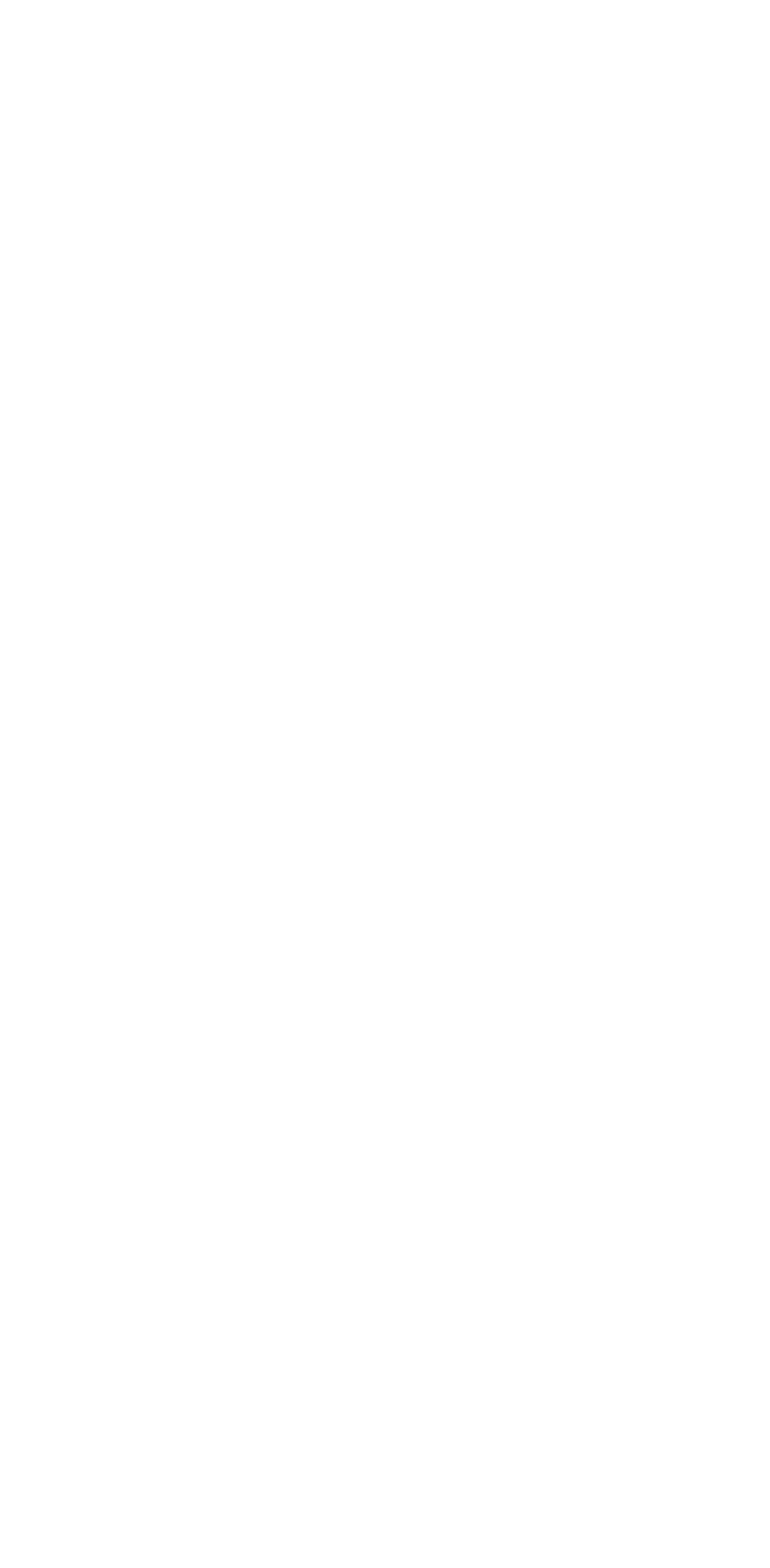




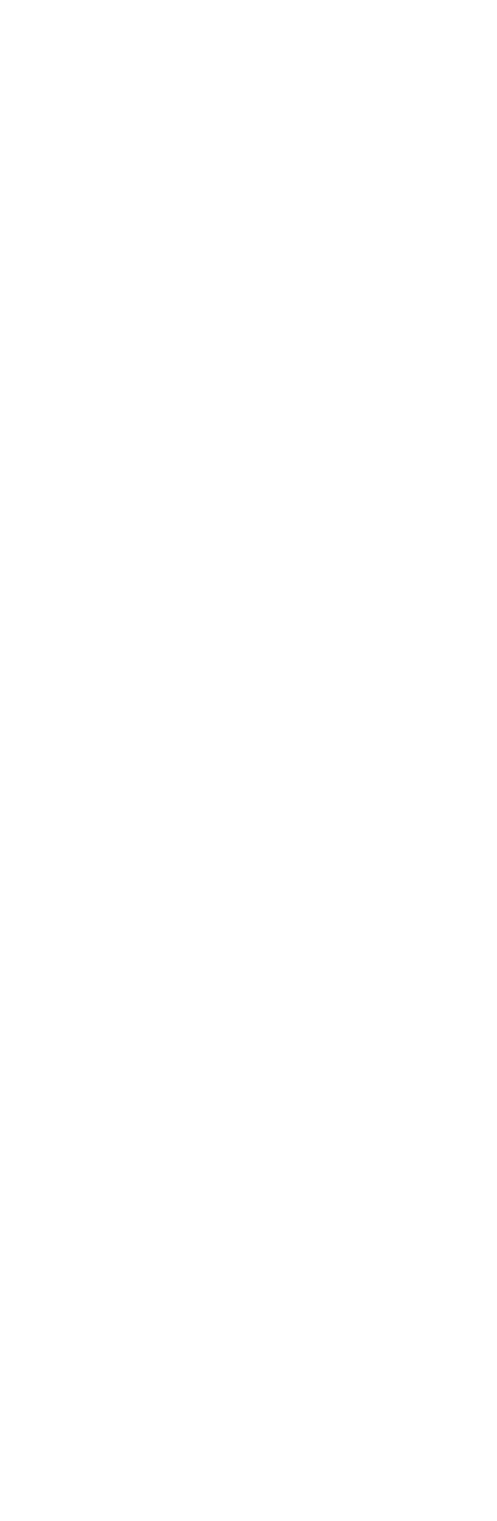


ARKANSAS RIVER BASIN--Continued

7-1400. ARKANSAS RIVER NEAR KINSLEY, KANS,--Continued

Suspended sediment, water year October 1963 to September 1964 (Where no daily concentrations are reported, loads are estimated)

\begin{tabular}{|c|c|c|c|c|c|c|c|c|c|}
\hline \multirow[b]{3}{*}{ Day } & \multicolumn{3}{|c|}{ OCTOBER } & \multicolumn{3}{|c|}{ NOVEMBER } & \multicolumn{3}{|c|}{ DECEMBER } \\
\hline & \multirow[b]{2}{*}{$\begin{array}{c}\text { Mean } \\
\text { dis- } \\
\text { charge } \\
(c f s)\end{array}$} & \multicolumn{2}{|c|}{ Suspended sediment } & \multirow[b]{2}{*}{$\begin{array}{c}\text { Mean } \\
\text { dis- } \\
\text { charge } \\
\text { (cfs) }\end{array}$} & \multicolumn{2}{|c|}{ Suspended sediment } & \multirow[b]{2}{*}{$\begin{array}{c}\text { Mean } \\
\text { dis- } \\
\text { charge } \\
\text { (cfs) }\end{array}$} & \multicolumn{2}{|c|}{ Suspended sediment } \\
\hline & & $\begin{array}{c}\text { Mean } \\
\text { concen- } \\
\text { tration } \\
(\text { ppm })\end{array}$ & $\begin{array}{l}\text { Tons } \\
\text { per } \\
\text { day }\end{array}$ & & $\begin{array}{c}\text { Mean } \\
\text { concen- } \\
\text { tration } \\
(\mathrm{ppm})\end{array}$ & $\begin{array}{l}\text { Tons } \\
\text { per } \\
\text { day }\end{array}$ & & $\begin{array}{c}\text { Mean } \\
\text { concen- } \\
\text { tration } \\
(\text { ppm })\end{array}$ & $\begin{array}{l}\text { Tons } \\
\text { per } \\
\text { day }\end{array}$ \\
\hline $\begin{array}{l}1 \ldots \\
2 \ldots \\
3 \ldots \\
4 \ldots \\
5 \ldots\end{array}$ & $\begin{array}{l}33 \\
32 \\
30 \\
31 \\
30\end{array}$ & $\begin{array}{l}42 \\
47 \\
60 \\
62 \\
58\end{array}$ & $\begin{array}{l}4 \\
4 \\
5 \\
5 \\
5\end{array}$ & $\begin{array}{l}40 \\
42 \\
42 \\
42 \\
41\end{array}$ & $\begin{array}{l}11 \\
10 \\
11 \\
11 \\
13\end{array}$ & $\begin{array}{l}1 \\
1 \\
1 \\
1 \\
1\end{array}$ & $\begin{array}{l}51 \\
50 \\
50 \\
50 \\
50\end{array}$ & $\begin{array}{l}55 \\
58 \\
57 \\
32 \\
55\end{array}$ & $\begin{array}{l}8 \\
8 \\
8 \\
4 \\
7\end{array}$ \\
\hline $\begin{array}{r}6 \ldots \\
7 \ldots \\
8 \ldots \\
9 \ldots \\
10 \ldots\end{array}$ & $\begin{array}{l}30 \\
30 \\
30 \\
29 \\
29\end{array}$ & $\begin{array}{l}50 \\
48 \\
22 \\
23 \\
19\end{array}$ & $\begin{array}{l}4 \\
4 \\
2 \\
2 \\
1\end{array}$ & $\begin{array}{l}40 \\
40 \\
40 \\
40 \\
40\end{array}$ & $\begin{array}{l}18 \\
10 \\
19 \\
12 \\
12\end{array}$ & $\begin{array}{l}2 \\
1 \\
2 \\
1 \\
1\end{array}$ & $\begin{array}{l}51 \\
51 \\
51 \\
50 \\
42\end{array}$ & $\begin{array}{l}52 \\
61 \\
65 \\
65 \\
40\end{array}$ & $\begin{array}{l}7 \\
8 \\
9 \\
9 \\
5\end{array}$ \\
\hline $\begin{array}{l}11 \ldots \\
12 \ldots \\
13 \ldots\end{array}$ & $\begin{array}{l}28 \\
28 \\
28\end{array}$ & $\begin{array}{l}68 \\
42 \\
43\end{array}$ & $\begin{array}{l}5 \\
3 \\
3\end{array}$ & $\begin{array}{l}41 \\
41 \\
40\end{array}$ & $\begin{array}{r}12 \\
9 \\
8\end{array}$ & $\begin{array}{l}1 \\
1 \\
1\end{array}$ & $\begin{array}{l}35 \\
40 \\
30\end{array}$ & $\begin{array}{l}31 \\
36 \\
27\end{array}$ & $\begin{array}{l}3 \\
4 \\
2\end{array}$ \\
\hline $\begin{array}{l}14 \ldots \\
15 \ldots\end{array}$ & $\begin{array}{l}27 \\
26\end{array}$ & $\begin{array}{l}24 \\
27\end{array}$ & $\begin{array}{l}2 \\
2\end{array}$ & $\begin{array}{l}40 \\
40\end{array}$ & $\begin{array}{l}9 \\
7\end{array}$ & $\begin{array}{l}1 \\
1\end{array}$ & $\begin{array}{l}25 \\
35\end{array}$ & $\begin{array}{l}18 \\
18\end{array}$ & $\begin{array}{l}1 \\
2\end{array}$ \\
\hline $\begin{array}{l}16 \ldots \\
17 \ldots \\
18 \ldots \\
19 \ldots \\
20 \ldots\end{array}$ & $\begin{array}{l}31 \\
29 \\
31 \\
34 \\
38\end{array}$ & $\begin{array}{l}28 \\
15 \\
13 \\
20 \\
54\end{array}$ & $\begin{array}{l}2 \\
1 \\
1 \\
2 \\
6\end{array}$ & $\begin{array}{l}40 \\
40 \\
40 \\
42 \\
43\end{array}$ & $\begin{array}{r}10 \\
11 \\
9 \\
14 \\
18\end{array}$ & $\begin{array}{l}1 \\
1 \\
1 \\
2 \\
2\end{array}$ & $\begin{array}{l}45 \\
50 \\
45 \\
47 \\
47\end{array}$ & $\begin{array}{l}19 \\
20 \\
24 \\
22 \\
18\end{array}$ & $\begin{array}{l}2 \\
3 \\
3 \\
3 \\
2\end{array}$ \\
\hline $\begin{array}{l}21 \ldots \\
22 \ldots \\
23 \ldots \\
24 \ldots \\
25 \ldots\end{array}$ & $\begin{array}{l}40 \\
40 \\
44 \\
44 \\
43\end{array}$ & $\begin{array}{l}58 \\
50 \\
53 \\
59 \\
52\end{array}$ & $\begin{array}{l}6 \\
5 \\
6 \\
7 \\
6\end{array}$ & $\begin{array}{l}45 \\
47 \\
45 \\
46 \\
48\end{array}$ & $\begin{array}{l}18 \\
20 \\
19 \\
27 \\
18\end{array}$ & $\begin{array}{l}2 \\
3 \\
2 \\
3 \\
2\end{array}$ & $\begin{array}{l}44 \\
37 \\
40 \\
46 \\
36\end{array}$ & $\begin{array}{l}18 \\
18 \\
23 \\
25 \\
24\end{array}$ & $\begin{array}{l}2 \\
2 \\
2 \\
3 \\
2\end{array}$ \\
\hline $\begin{array}{l}26 \ldots \\
27 \ldots \\
28 \ldots \\
29 \ldots \\
30 \ldots \\
31 \ldots\end{array}$ & $\begin{array}{l}42 \\
41 \\
41 \\
40 \\
40 \\
40\end{array}$ & \begin{tabular}{l|}
13 \\
15 \\
15
\end{tabular} & $\begin{array}{l}1 \\
2 \\
2 \\
2 \\
2 \\
1\end{array}$ & $\begin{array}{l}48 \\
49 \\
49 \\
50 \\
50 \\
--\end{array}$ & $\begin{array}{l}29 \\
27 \\
31 \\
31 \\
29 \\
--\end{array}$ & $\begin{array}{r}4 \\
4 \\
4 \\
4 \\
4 \\
-\end{array}$ & $\begin{array}{l}39 \\
43 \\
49 \\
50 \\
54 \\
57\end{array}$ & $\begin{array}{l}26 \\
28 \\
40 \\
48 \\
46 \\
47\end{array}$ & $\begin{array}{l}3 \\
3 \\
5 \\
6 \\
7 \\
7\end{array}$ \\
\hline \multirow[t]{2}{*}{ Total } & 1059 & -- & 103 & 1291 & -- & 56 & 1390 & -- & 140 \\
\hline & \multicolumn{3}{|c|}{ JANUARY } & \multicolumn{3}{|c|}{ FEBRUARY } & \multicolumn{3}{|c|}{ MARCH } \\
\hline $\begin{array}{l}1 \ldots \\
2 \ldots \\
3 \ldots \\
4 \ldots \\
5 \ldots\end{array}$ & $\begin{array}{l}61 \\
66 \\
76 \\
75 \\
77\end{array}$ & $\begin{array}{l}95 \\
87 \\
92 \\
96 \\
97\end{array}$ & $\begin{array}{l}16 \\
16 \\
19 \\
19 \\
20\end{array}$ & $\begin{array}{l}72 \\
72 \\
73 \\
75 \\
77\end{array}$ & $\begin{array}{l}45 \\
47 \\
40 \\
40 \\
37\end{array}$ & $\begin{array}{l}9 \\
9 \\
8 \\
8 \\
8\end{array}$ & $\begin{array}{l}82 \\
84 \\
84 \\
82 \\
81\end{array}$ & $\begin{array}{l}35 \\
22 \\
31 \\
29 \\
35\end{array}$ & $\begin{array}{l}8 \\
5 \\
7 \\
6 \\
8\end{array}$ \\
\hline $\begin{array}{r}6 \ldots \\
7 \ldots \\
9 \ldots \\
10 \ldots\end{array}$ & $\begin{array}{l}73 \\
75 \\
73 \\
72 \\
67\end{array}$ & $\begin{array}{l}93 \\
73 \\
72 \\
81 \\
19\end{array}$ & $\begin{array}{r}18 \\
15 \\
14 \\
16 \\
3\end{array}$ & $\begin{array}{l}77 \\
75 \\
73 \\
76 \\
76\end{array}$ & $\begin{array}{l}27 \\
22 \\
24 \\
24 \\
24\end{array}$ & $\begin{array}{l}6 \\
4 \\
5 \\
5 \\
5\end{array}$ & $\begin{array}{l}81 \\
78 \\
77 \\
77 \\
75\end{array}$ & $\begin{array}{l}32 \\
32 \\
31 \\
29 \\
34\end{array}$ & $\begin{array}{l}7 \\
7 \\
6 \\
6 \\
7\end{array}$ \\
\hline $\begin{array}{l}11 \ldots \\
12 \ldots \\
13 \ldots \\
14 \ldots \\
15 \ldots\end{array}$ & $\begin{array}{l}60 \\
50 \\
47 \\
54 \\
60\end{array}$ & $\begin{array}{l}26 \\
22 \\
19 \\
19 \\
19\end{array}$ & $\begin{array}{l}4 \\
3 \\
2 \\
3 \\
3\end{array}$ & $\begin{array}{l}76 \\
77 \\
76 \\
77 \\
82\end{array}$ & $\begin{array}{l}30 \\
32 \\
33 \\
33 \\
41\end{array}$ & $\begin{array}{l}6 \\
7 \\
7 \\
7 \\
9\end{array}$ & $\begin{array}{l}73 \\
71 \\
71 \\
72 \\
71\end{array}$ & $\begin{array}{l}35 \\
37 \\
30 \\
25 \\
26\end{array}$ & $\begin{array}{l}7 \\
7 \\
6 \\
5 \\
5\end{array}$ \\
\hline $\begin{array}{l}16 \ldots \\
17 \ldots \\
18 \ldots \\
19 \ldots \\
20 \ldots\end{array}$ & $\begin{array}{l}70 \\
75 \\
70 \\
76 \\
76\end{array}$ & $\begin{array}{r}57 \\
58 \\
58 \\
100 \\
97\end{array}$ & $\begin{array}{l}11 \\
12 \\
11 \\
20 \\
20\end{array}$ & $\begin{array}{l}81 \\
82 \\
82 \\
81 \\
81\end{array}$ & $\begin{array}{l}36 \\
52 \\
37 \\
37 \\
42\end{array}$ & $\begin{array}{r}8 \\
12 \\
8 \\
8 \\
9\end{array}$ & $\begin{array}{l}71 \\
70 \\
69 \\
72 \\
72\end{array}$ & $\begin{array}{l}20 \\
23 \\
24 \\
24 \\
25\end{array}$ & $\begin{array}{l}4 \\
4 \\
4 \\
5 \\
5\end{array}$ \\
\hline $\begin{array}{l}21 \ldots \\
22 \ldots \\
23 \ldots \\
24 \ldots \\
25 \ldots\end{array}$ & $\begin{array}{l}80 \\
93 \\
81 \\
73 \\
72\end{array}$ & $\begin{array}{l}96 \\
67 \\
66 \\
37 \\
68\end{array}$ & $\begin{array}{r}21 \\
17 \\
14 \\
7 \\
13\end{array}$ & $\begin{array}{l}82 \\
84 \\
82 \\
82 \\
82\end{array}$ & $\begin{array}{l}41 \\
40 \\
33 \\
26 \\
29\end{array}$ & $\begin{array}{l}9 \\
9 \\
7 \\
6 \\
6\end{array}$ & $\begin{array}{l}69 \\
67 \\
65 \\
64 \\
62\end{array}$ & $\begin{array}{l}22 \\
22 \\
21 \\
23 \\
20\end{array}$ & $\begin{array}{l}4 \\
4 \\
4 \\
4 \\
3\end{array}$ \\
\hline $\begin{array}{l}26 \ldots \\
27 \ldots \\
28 \ldots \\
29 \ldots \\
30 \ldots \\
31 \ldots\end{array}$ & $\begin{array}{l}73 \\
72 \\
71 \\
70 \\
70 \\
72\end{array}$ & $\begin{array}{l}68 \\
61 \\
61 \\
52 \\
45 \\
60\end{array}$ & $\begin{array}{r}13 \\
12 \\
12 \\
10 \\
8 \\
12\end{array}$ & $\begin{array}{l}82 \\
82 \\
82 \\
82 \\
-- \\
--\end{array}$ & $\begin{array}{l}34 \\
33 \\
34 \\
37 \\
-- \\
--\end{array}$ & $\begin{array}{r}8 \\
7 \\
8 \\
8 \\
-- \\
--\end{array}$ & $\begin{array}{l}61 \\
61 \\
61 \\
60 \\
58 \\
57\end{array}$ & $\begin{array}{l}21 \\
19 \\
22 \\
20 \\
23 \\
22\end{array}$ & $\begin{array}{l}3 \\
3 \\
4 \\
3 \\
4 \\
3\end{array}$ \\
\hline Total & 2180 & -- & 384 & 2281 & -- & 216 & 2198 & - & 158 \\
\hline
\end{tabular}


ARKANSAS RIVER BASIN--Continued

7-1400. ARKANSAS RIVER NEAR KINSLEY, KANS,--Continued

Suspended sediment water year October 1963 to September 1964--Continued (Where no daily concentrations are reported, loads are estimated)

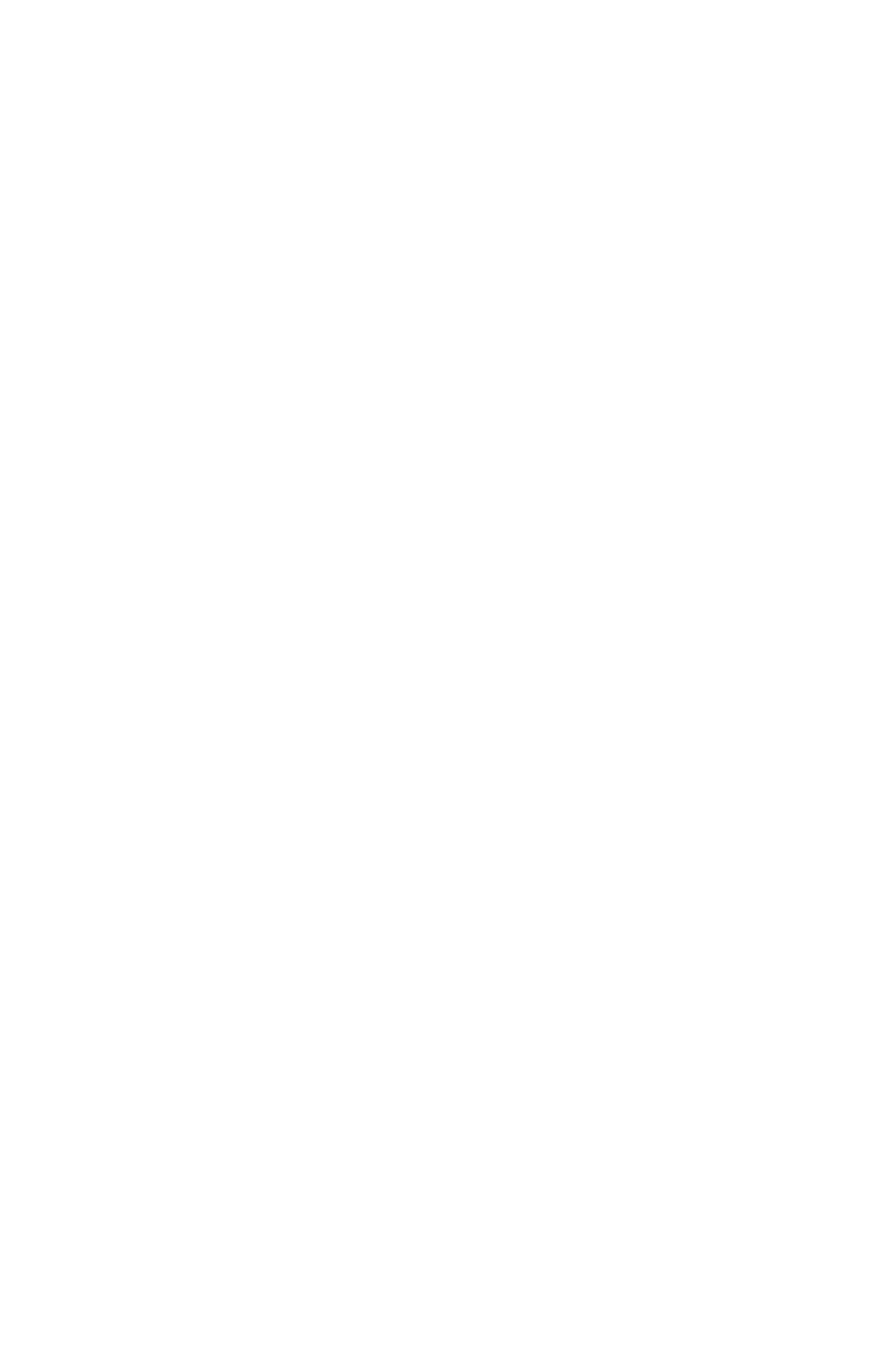

s Computed by subdividing day. 

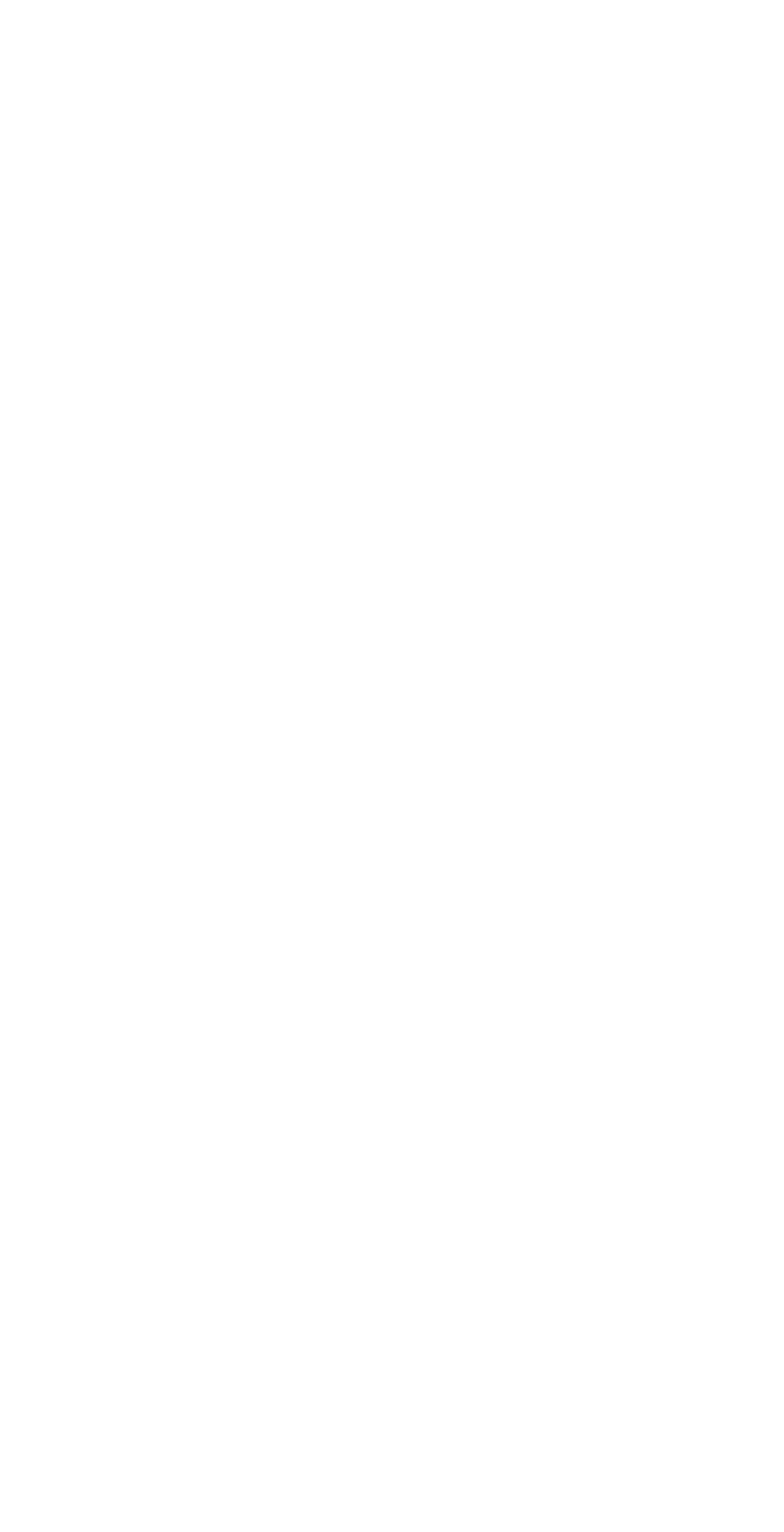


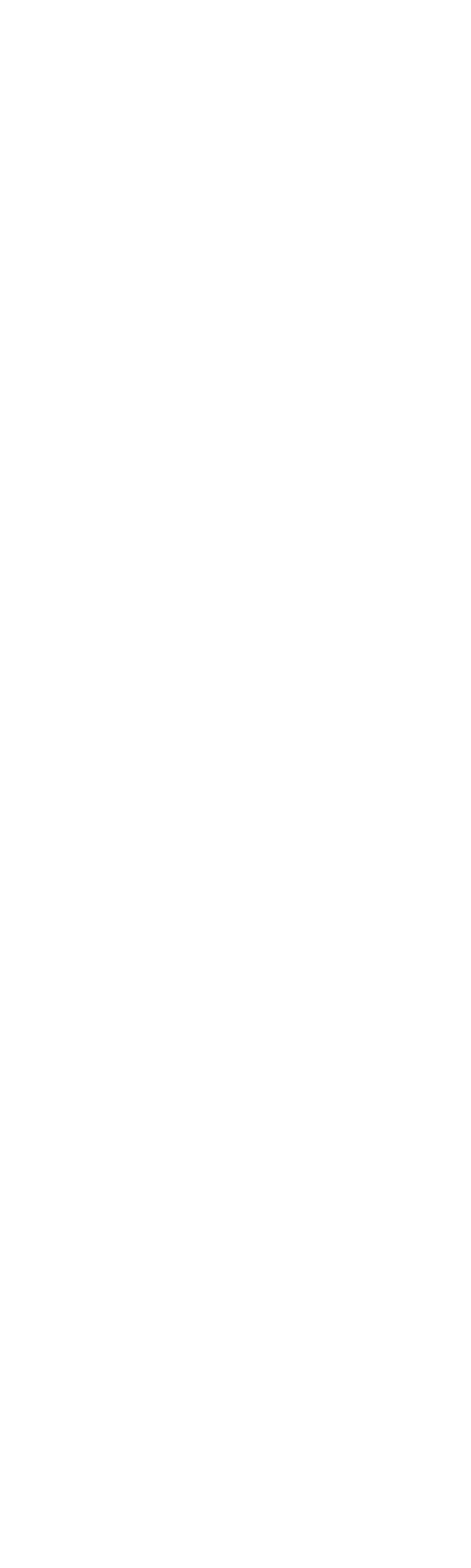




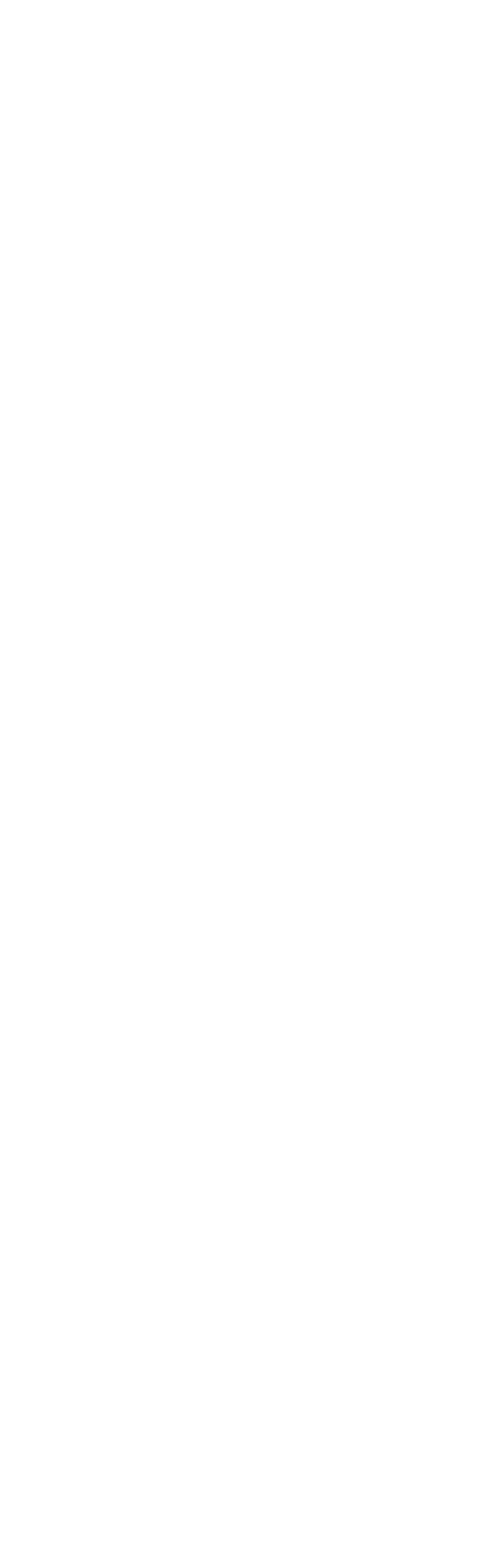




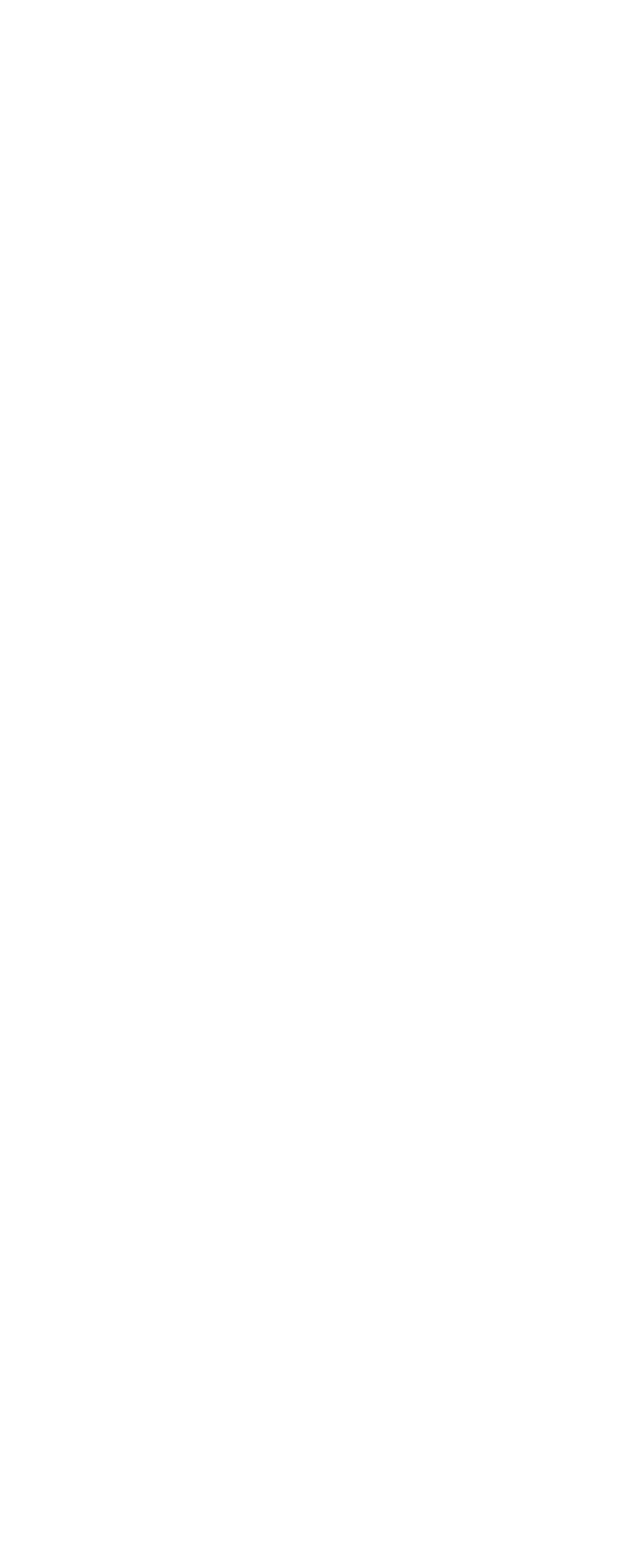



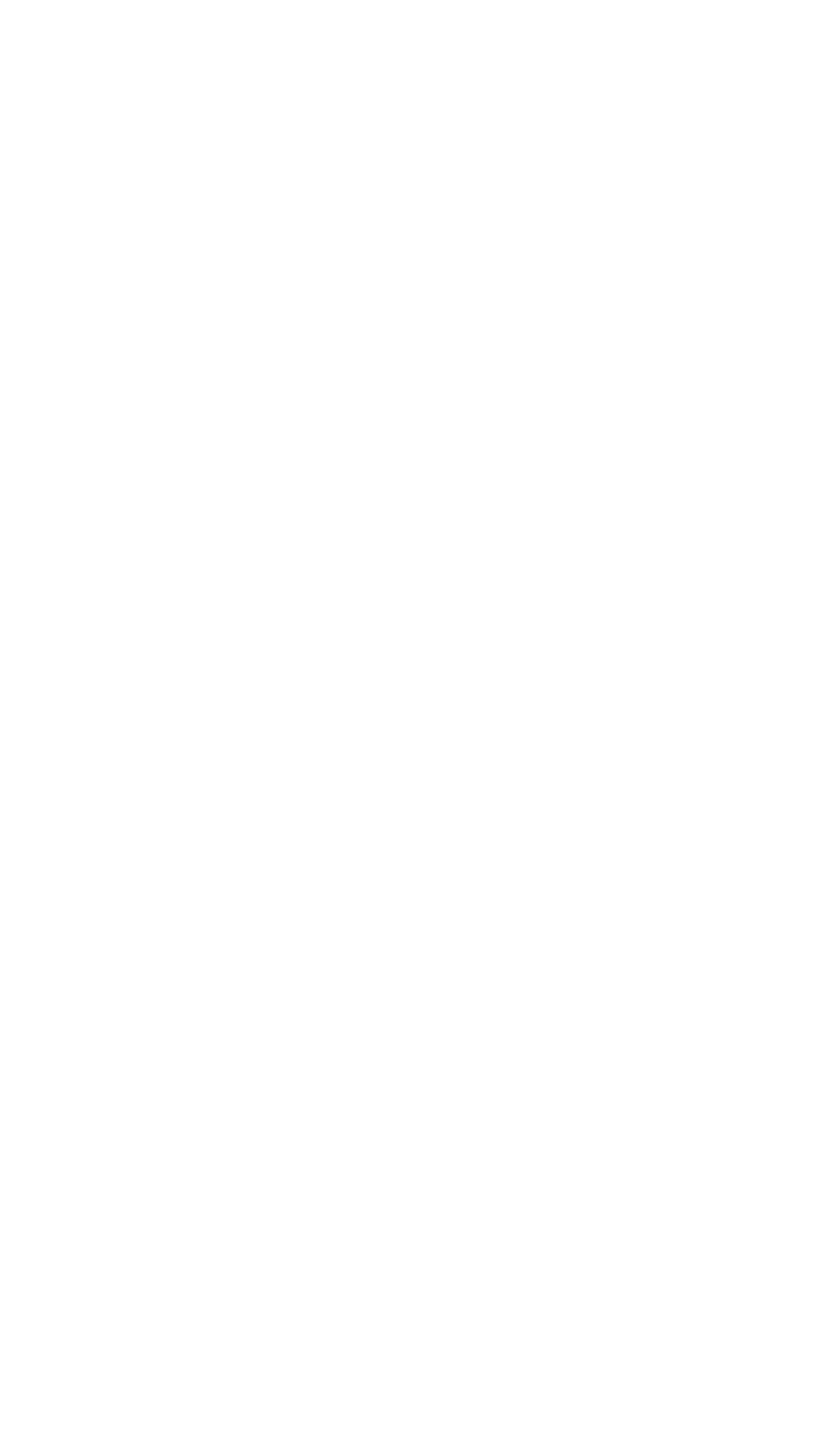


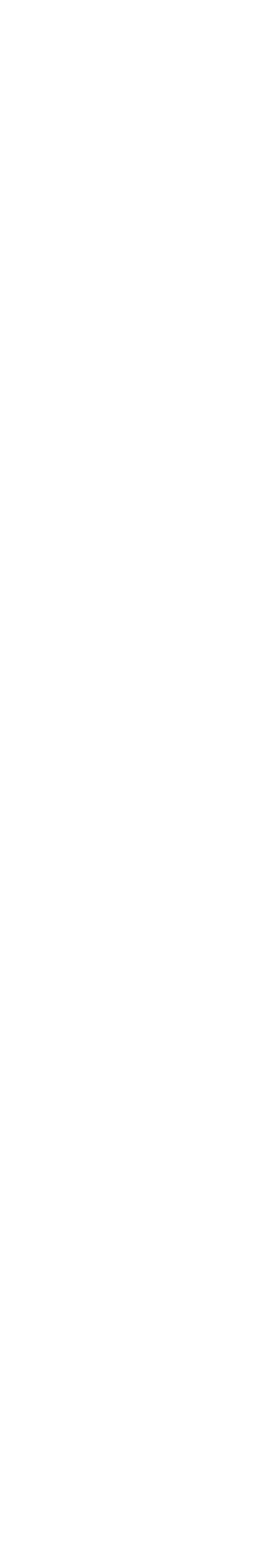

\begin{tabular}{|c|c|}
\hline $\bar{z}$ & \\
\hline 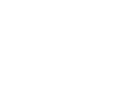 & 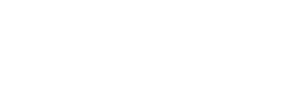 \\
\hline 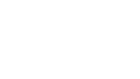 & 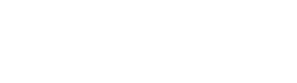 \\
\hline 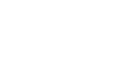 & 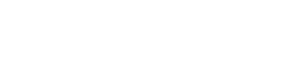 \\
\hline 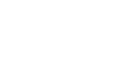 & 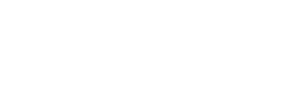 \\
\hline 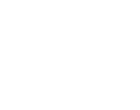 & 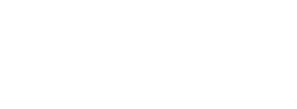 \\
\hline 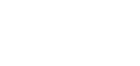 & क्ष \\
\hline 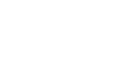 & 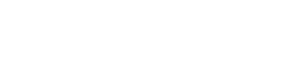 \\
\hline 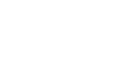 & 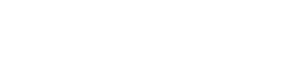 \\
\hline 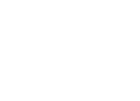 & 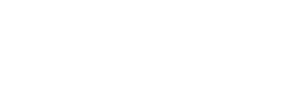 \\
\hline 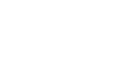 & 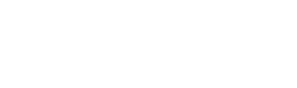 \\
\hline 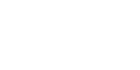 & $00000 \quad 00000$ \\
\hline$\frac{1}{1+\frac{8}{4}}$ & 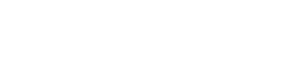 \\
\hline 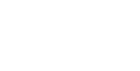 & 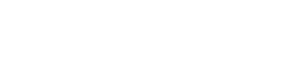 \\
\hline 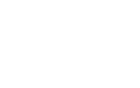 & 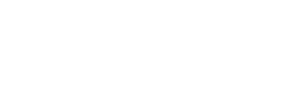 \\
\hline 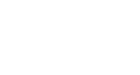 & 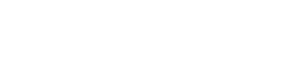 \\
\hline 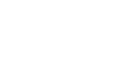 & 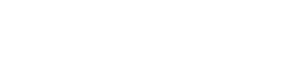 \\
\hline 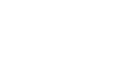 & \\
\hline 㿣䍃 & $\stackrel{\overrightarrow{0}}{\dot{0}}$ \\
\hline 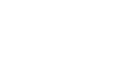 & \\
\hline 童产 & 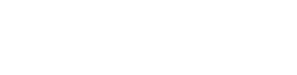 \\
\hline 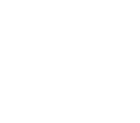 & 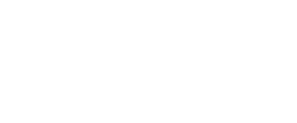 \\
\hline 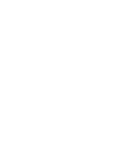 & 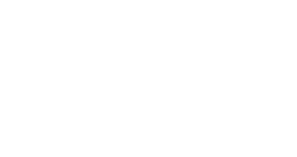 \\
\hline
\end{tabular}




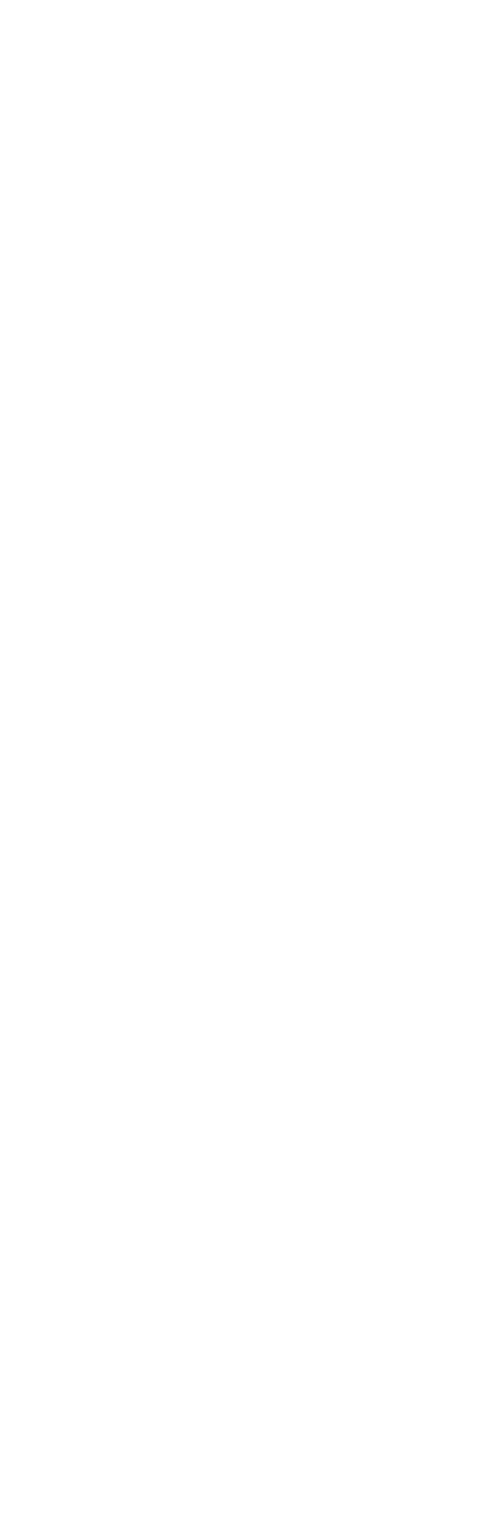


ARKANSAS RIVER BASIN--Continued

7-1419. WALNUT CREEK AT ALBERT, KANS.--Continued

Suspended sediment, water year October 1963 to September 1964

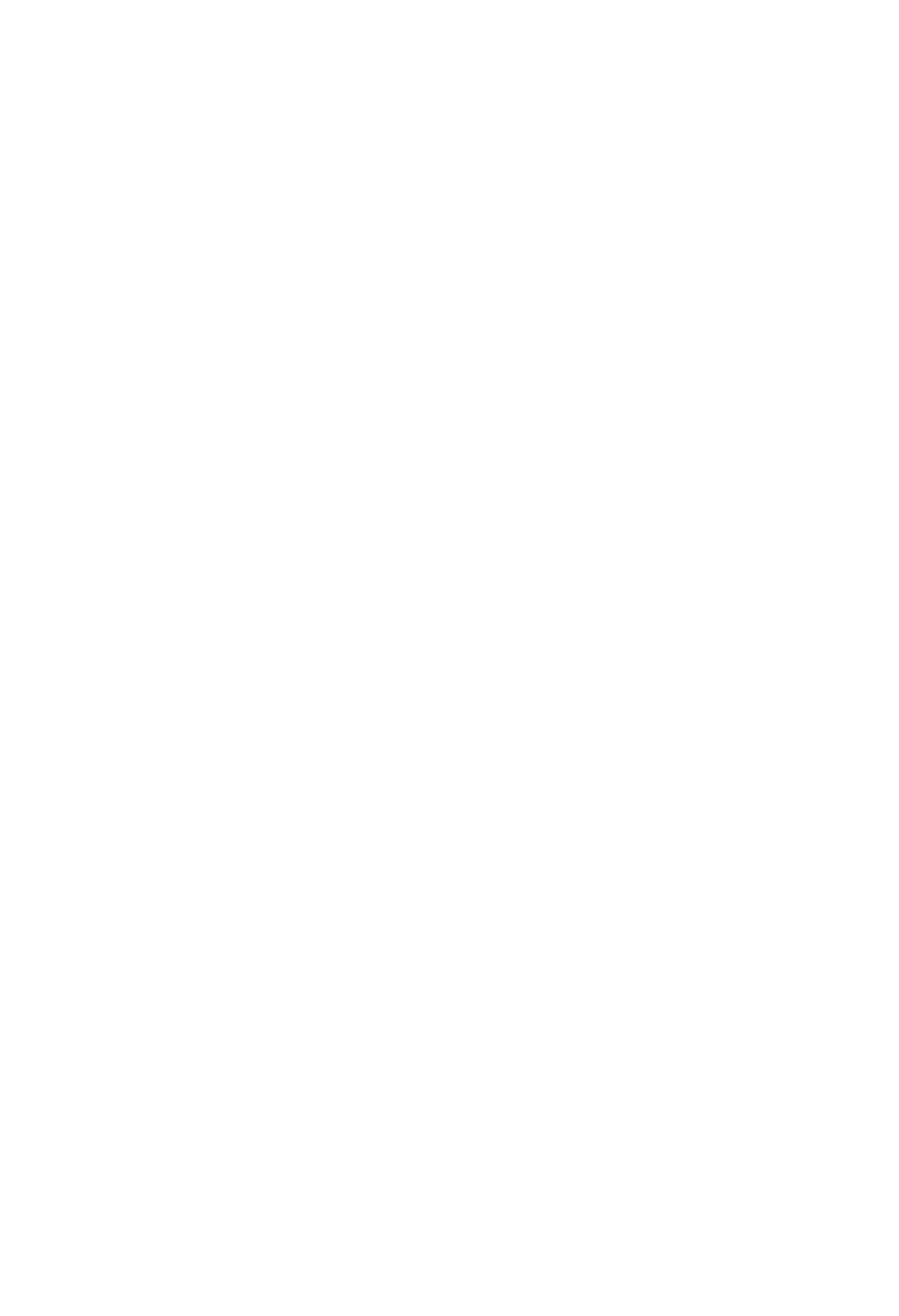

I) Estimated.

Computed by subdividing day.

Less than 0.50 ton. 
ARKANSAS RIVER BASIN--Continued

7-1419. WALNUT CREEK AT ALBERT, KANS.--Continued

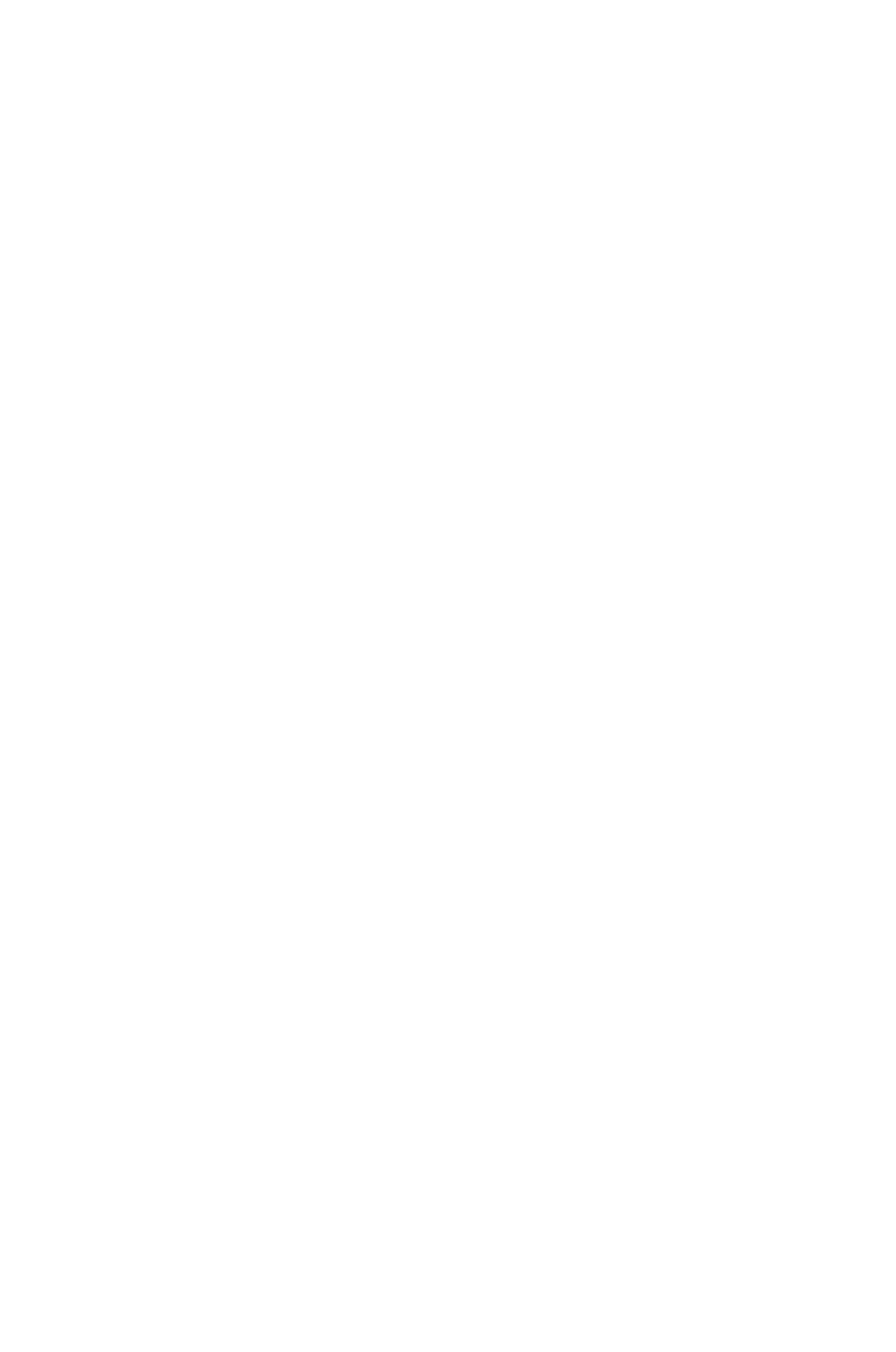

$S$ Computed by subdividing day.

$T$ Less than 0.50 ton. 


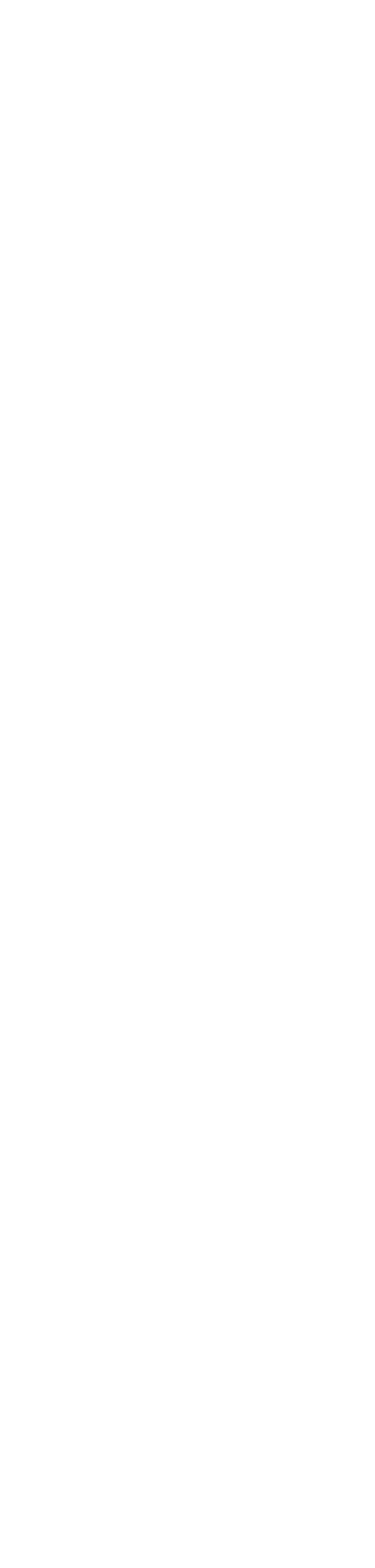




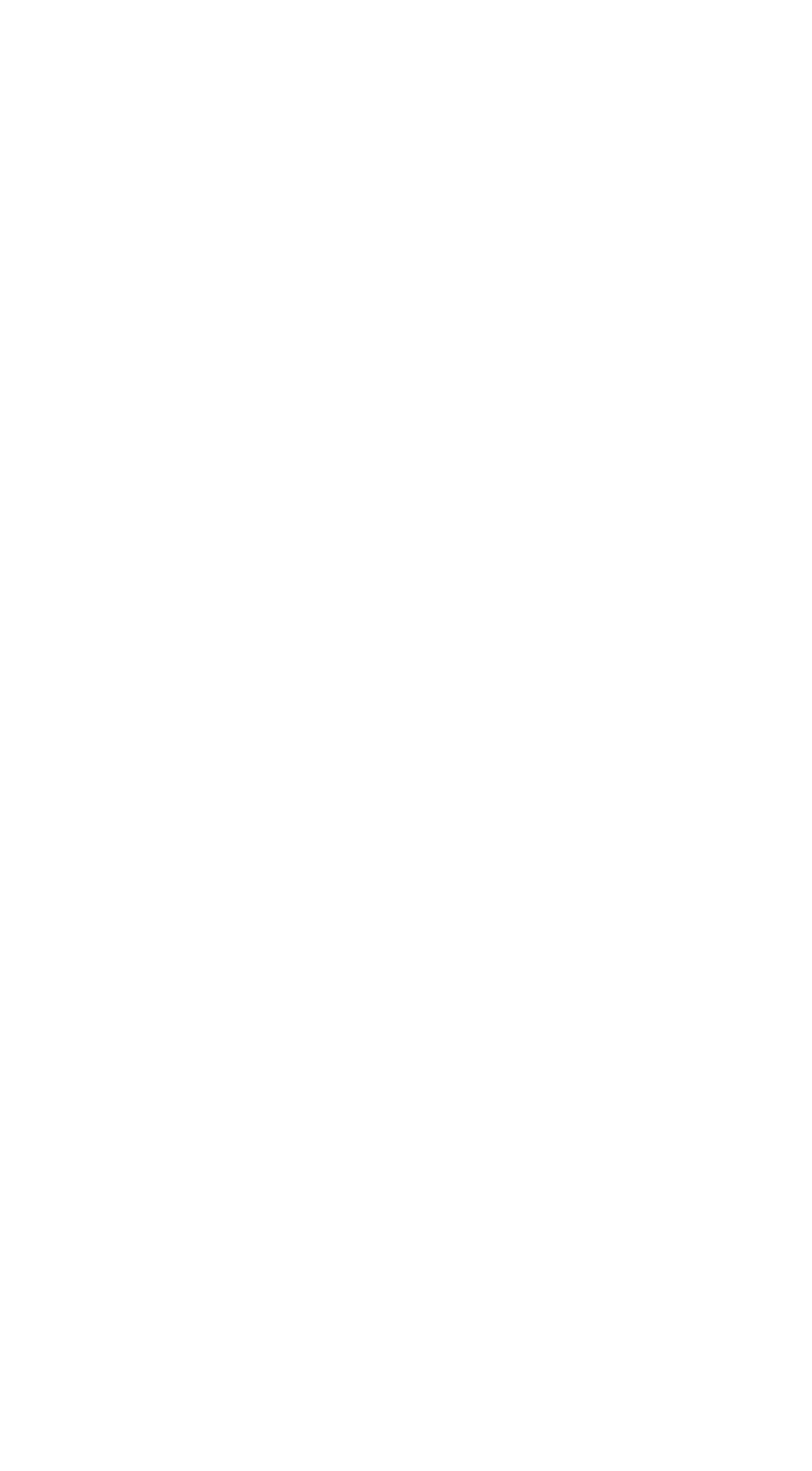




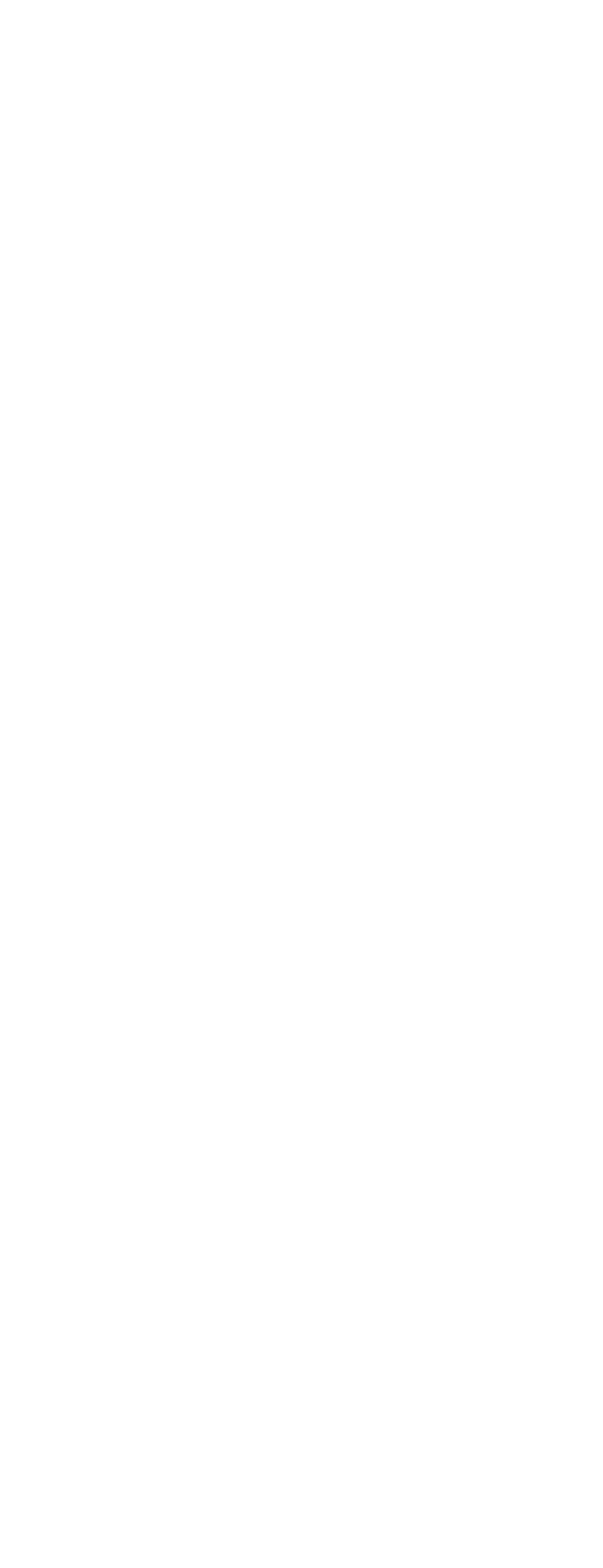




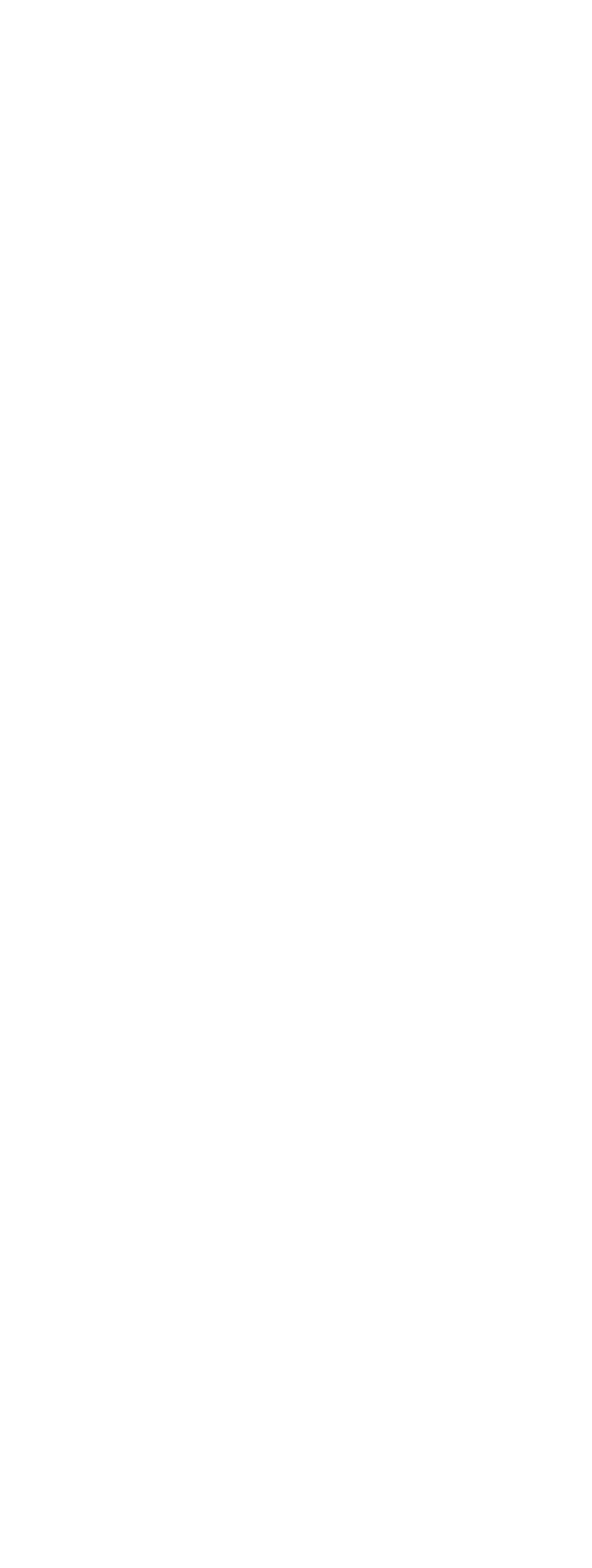




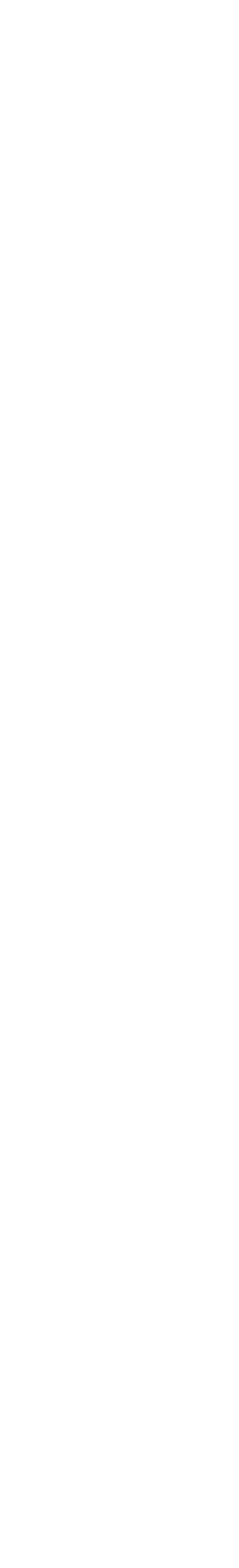

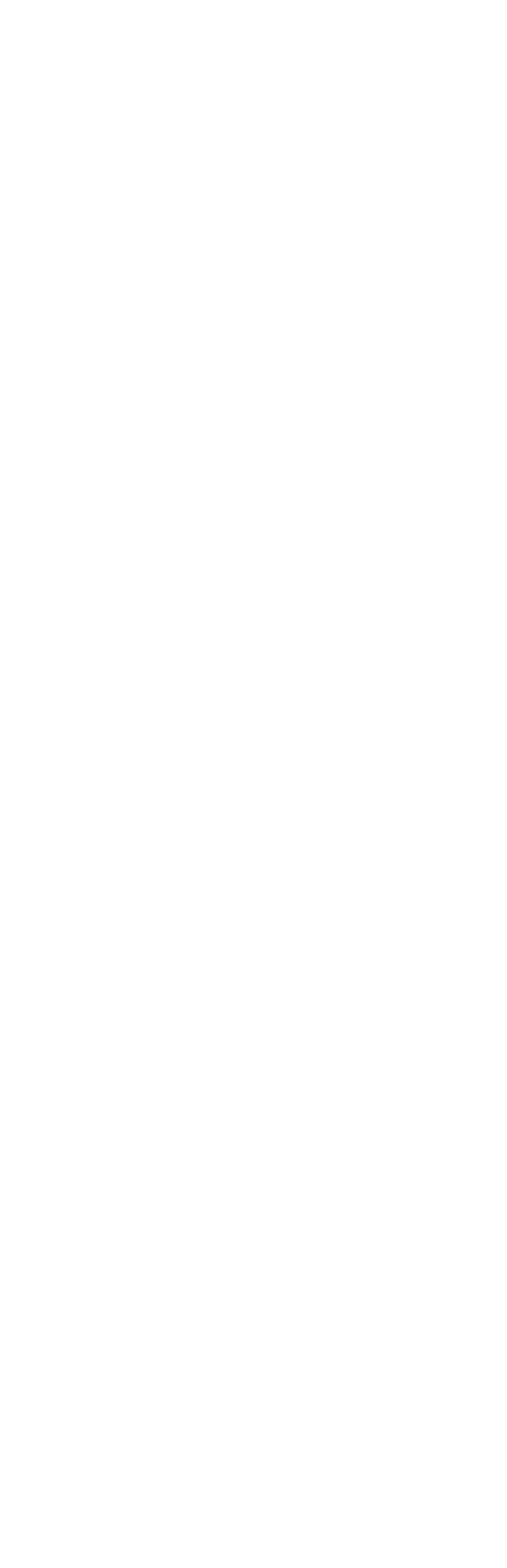




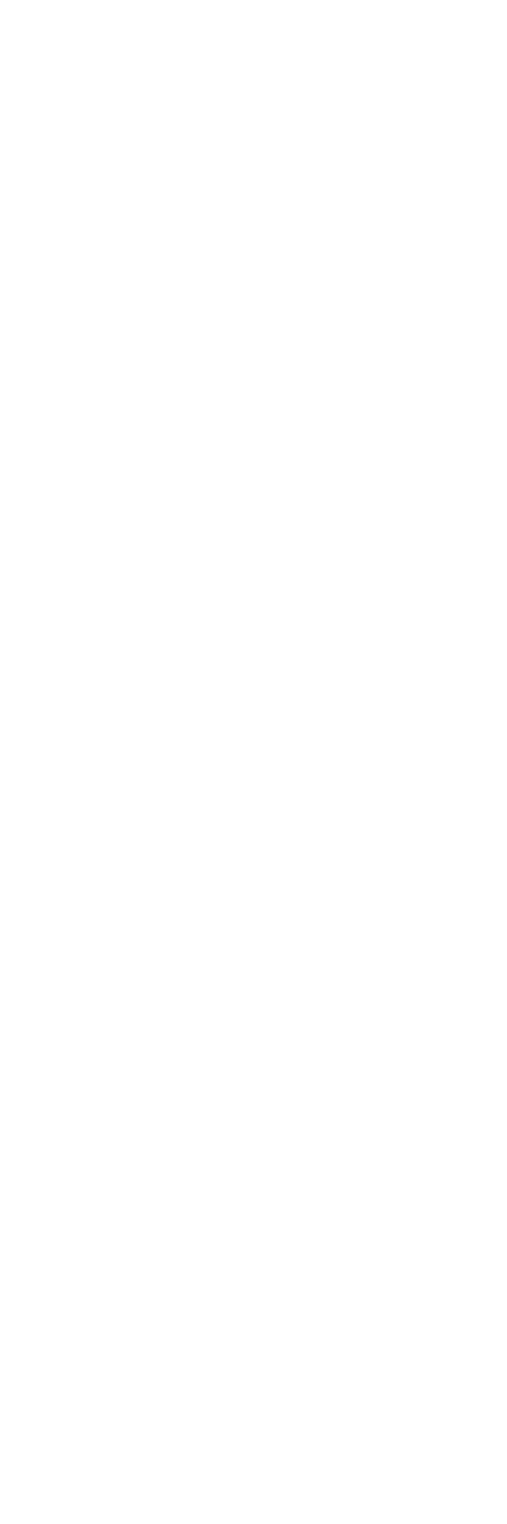


ARKANSAS RIVER BASIN--Continued

7-1433.3. ARKANSAS RIVER NEAR HUTCHINSON, KANS.--Continued

Suspended sediment, water year October 1963 to September 1964

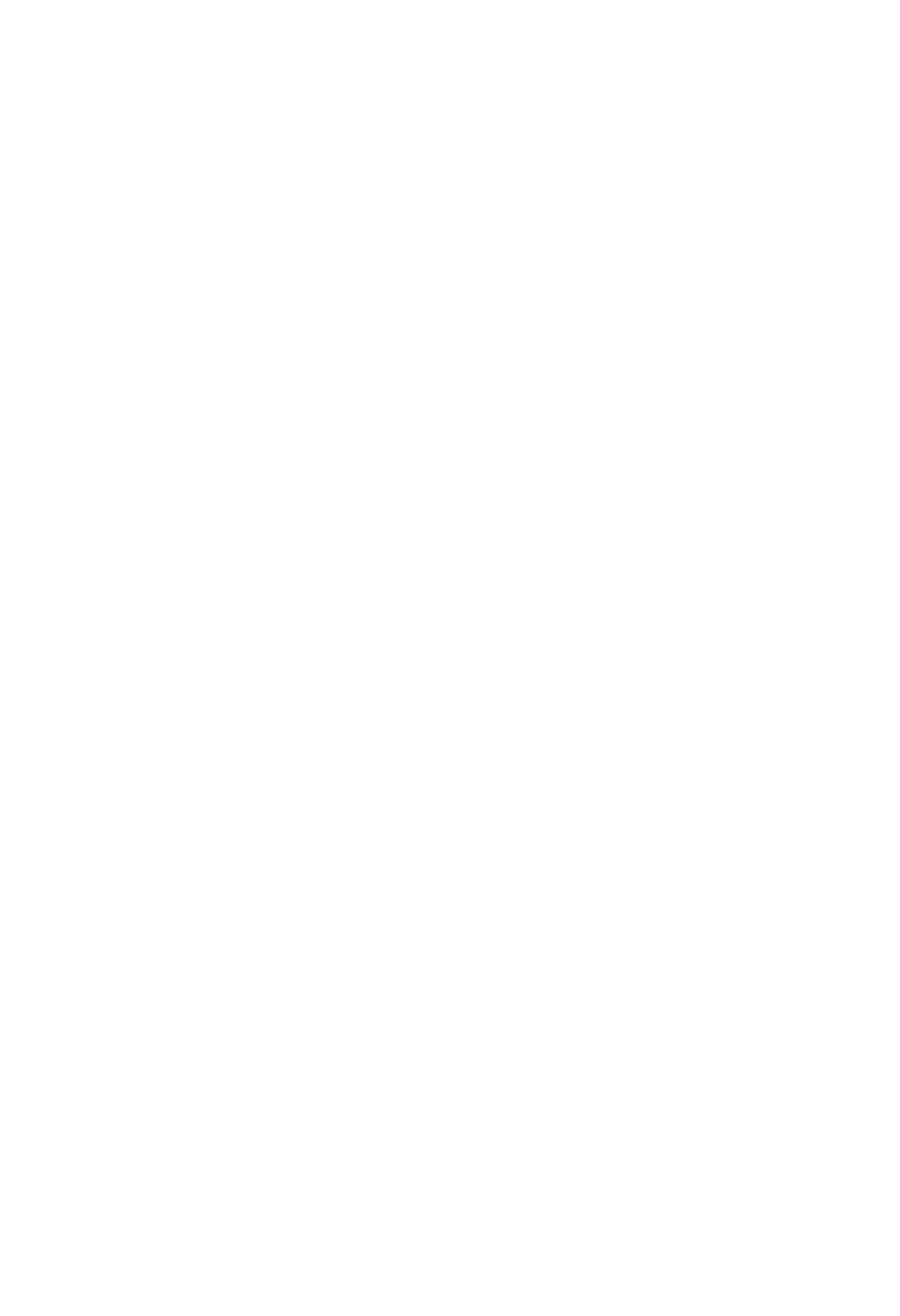

E Estimated.

$T$ Less than 0.50 ton. 
ARKANSAS RIVER BASIN--Continued

7-1433.3. ARKANSAS RIVER NEAR HUTCHINSON, KANS,--COntinued

Suspended sediment, water year October 1963 to September 1964--Continued

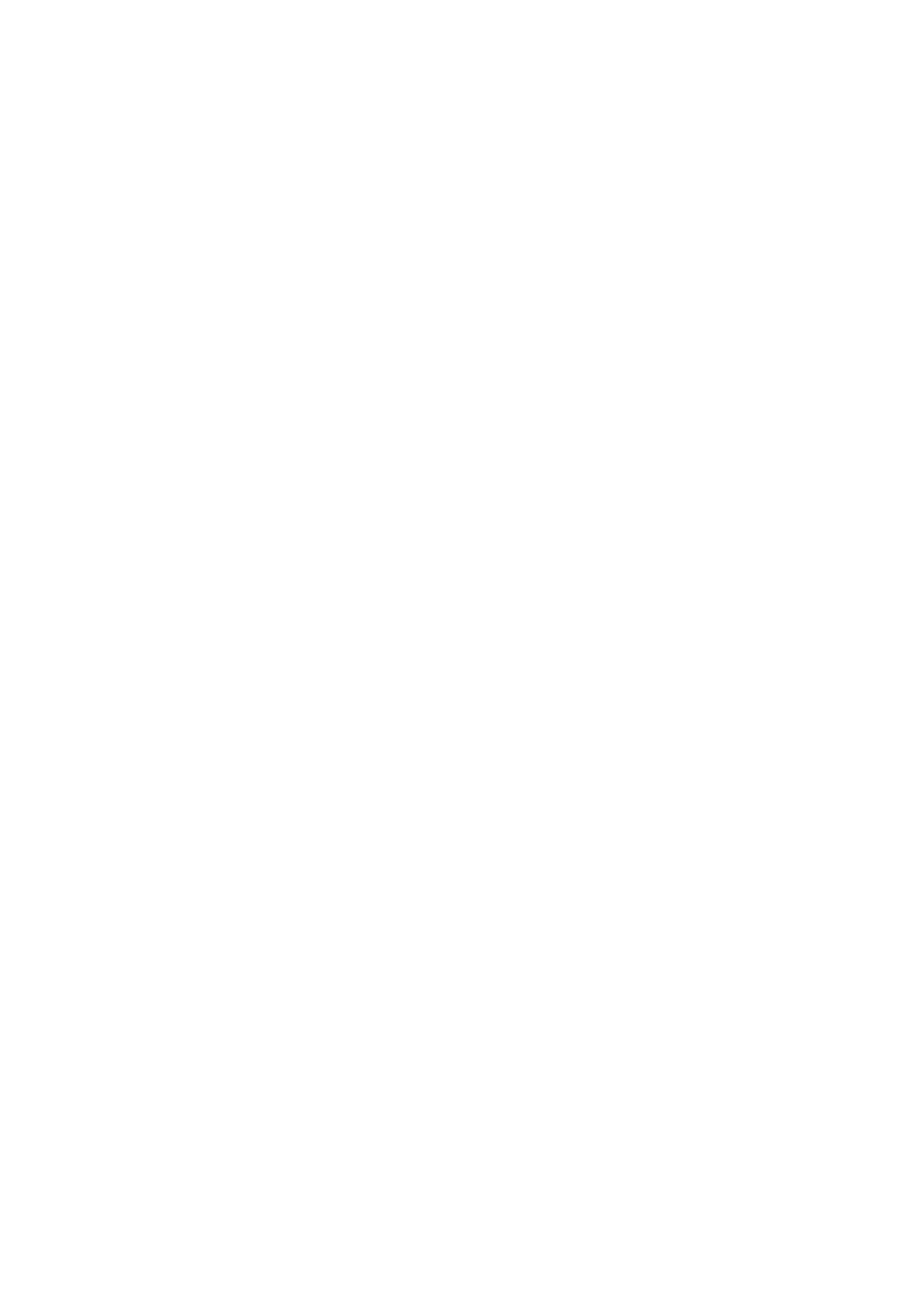

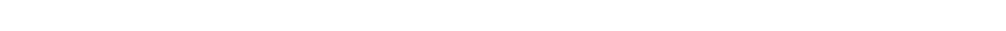



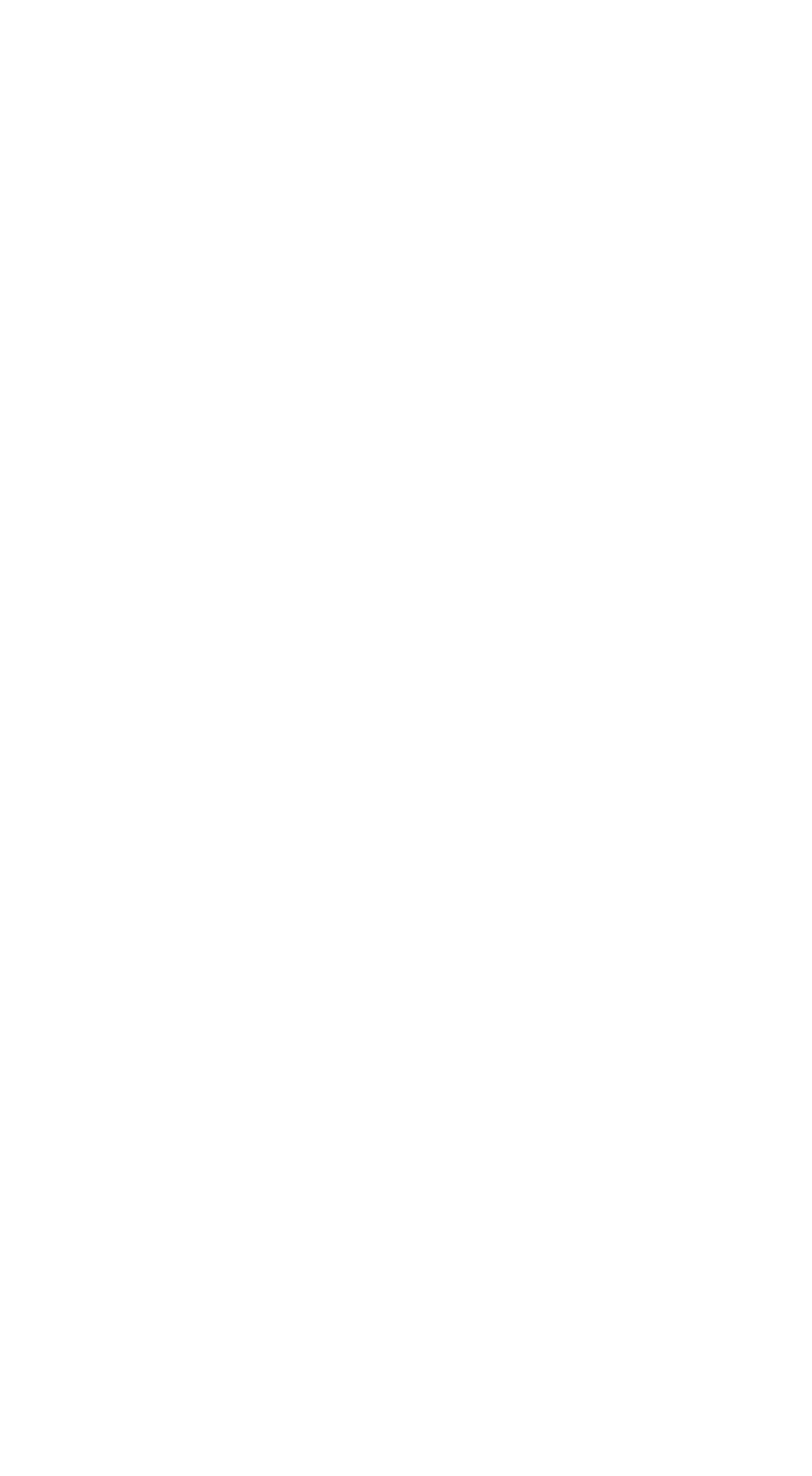


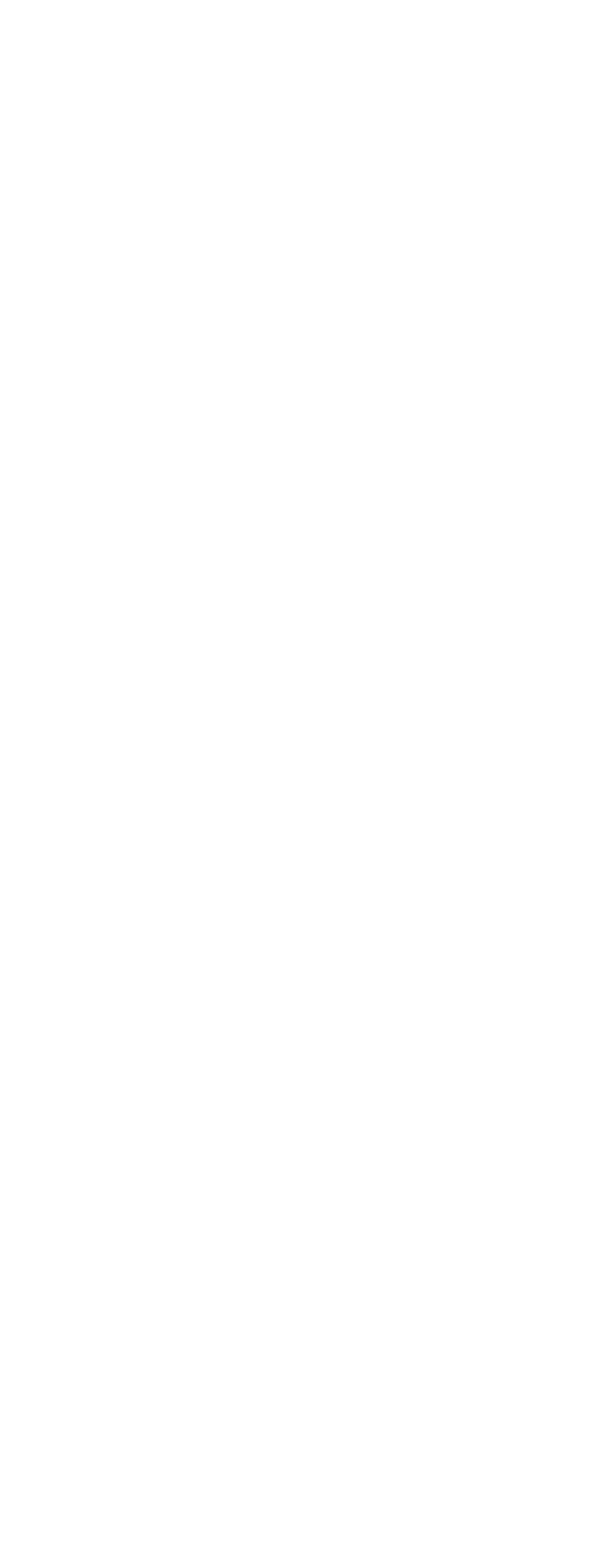




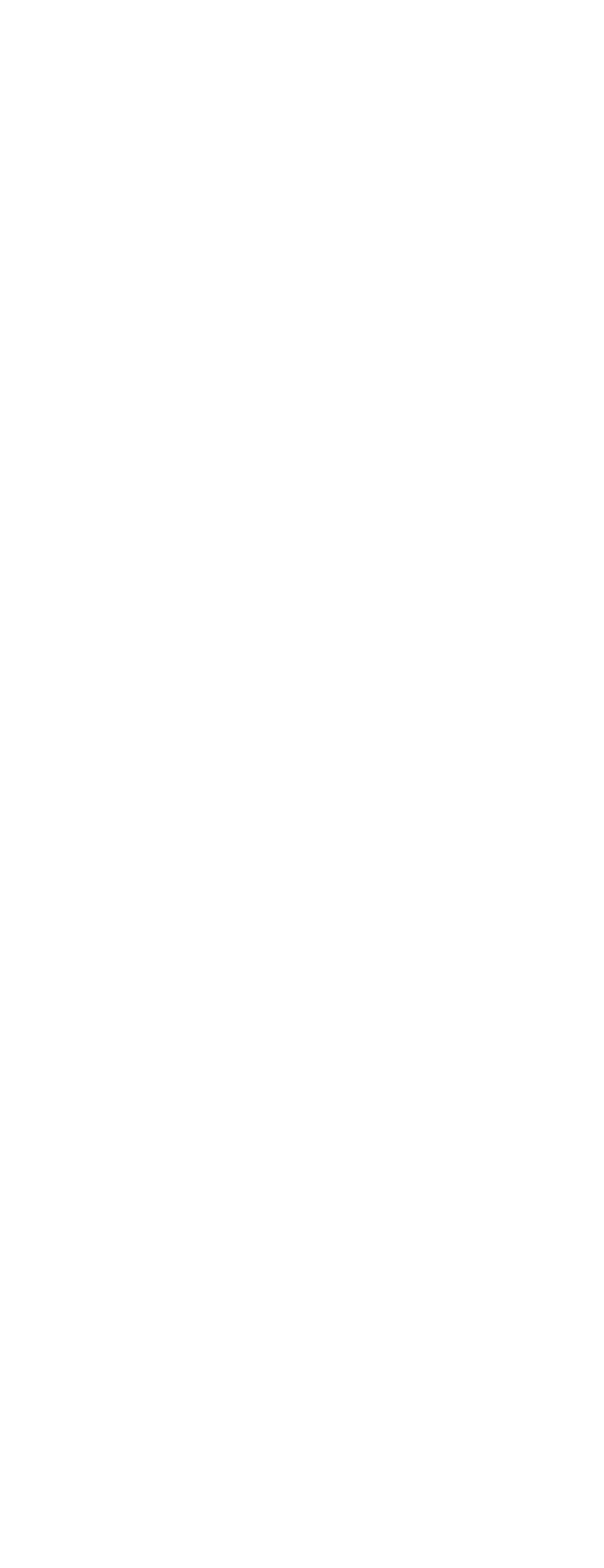




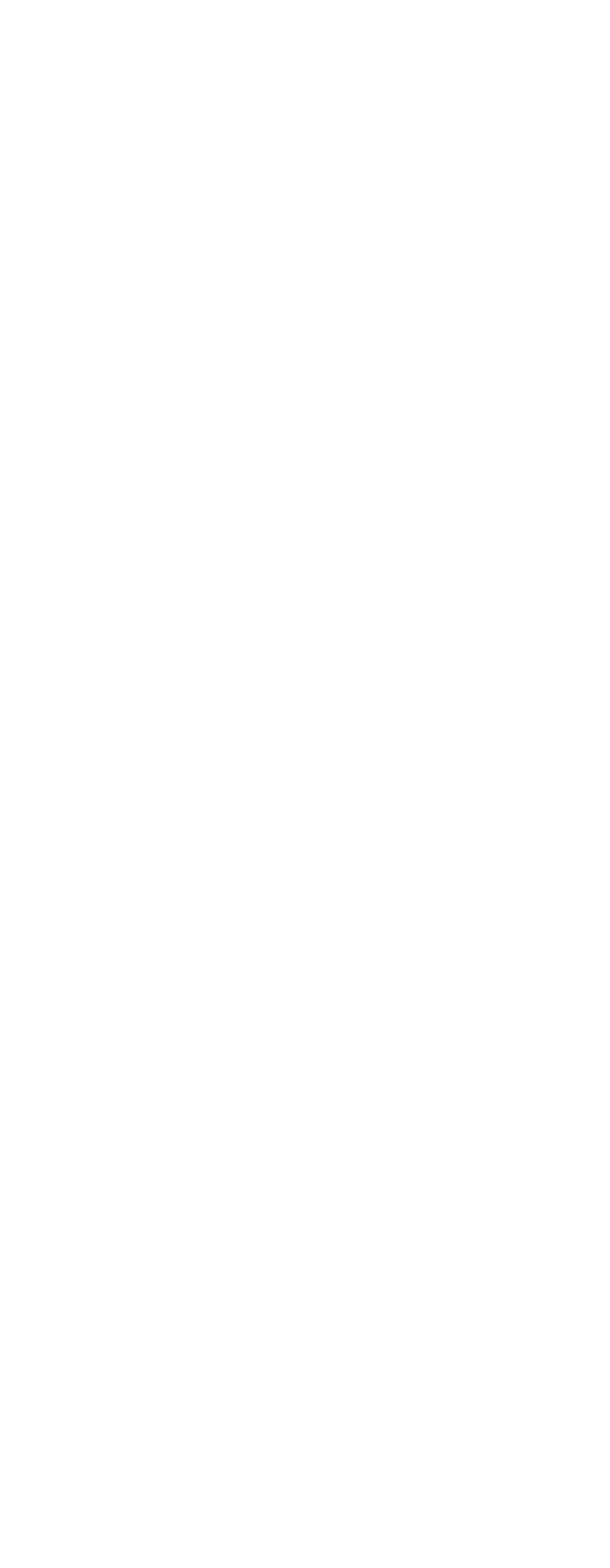




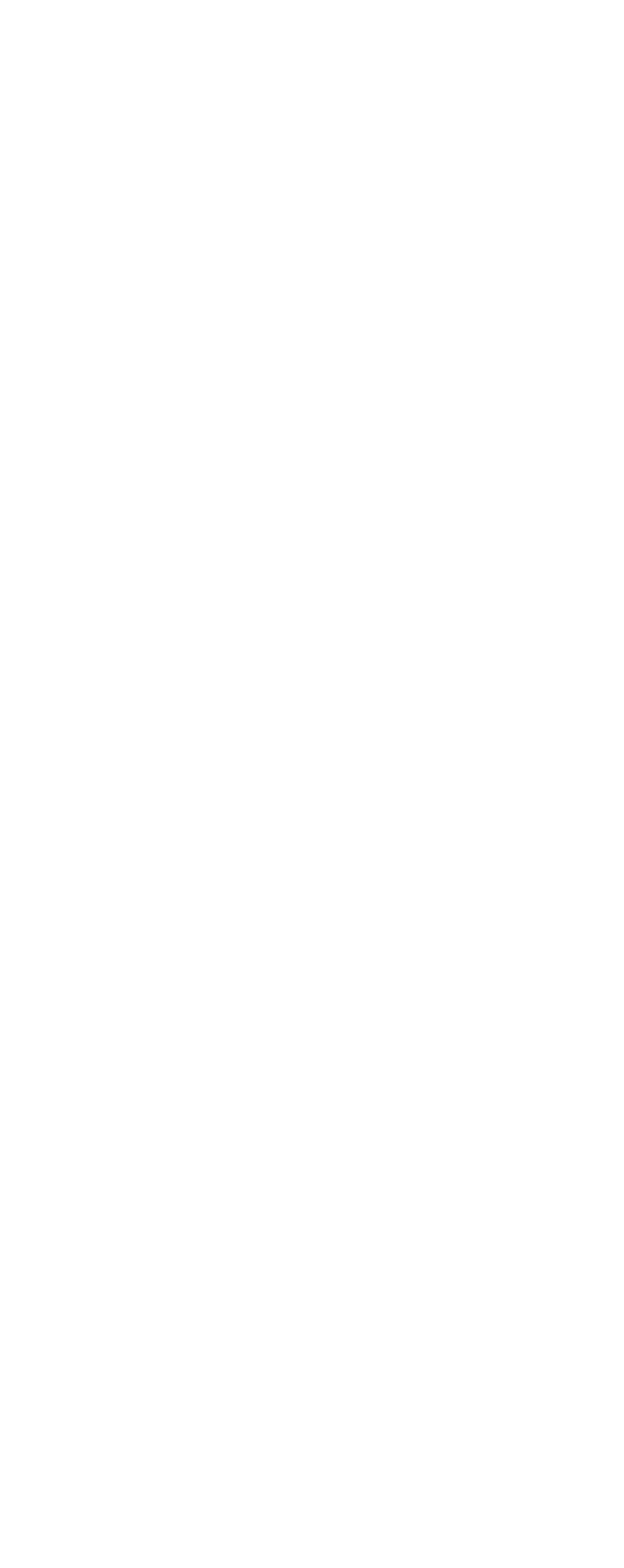



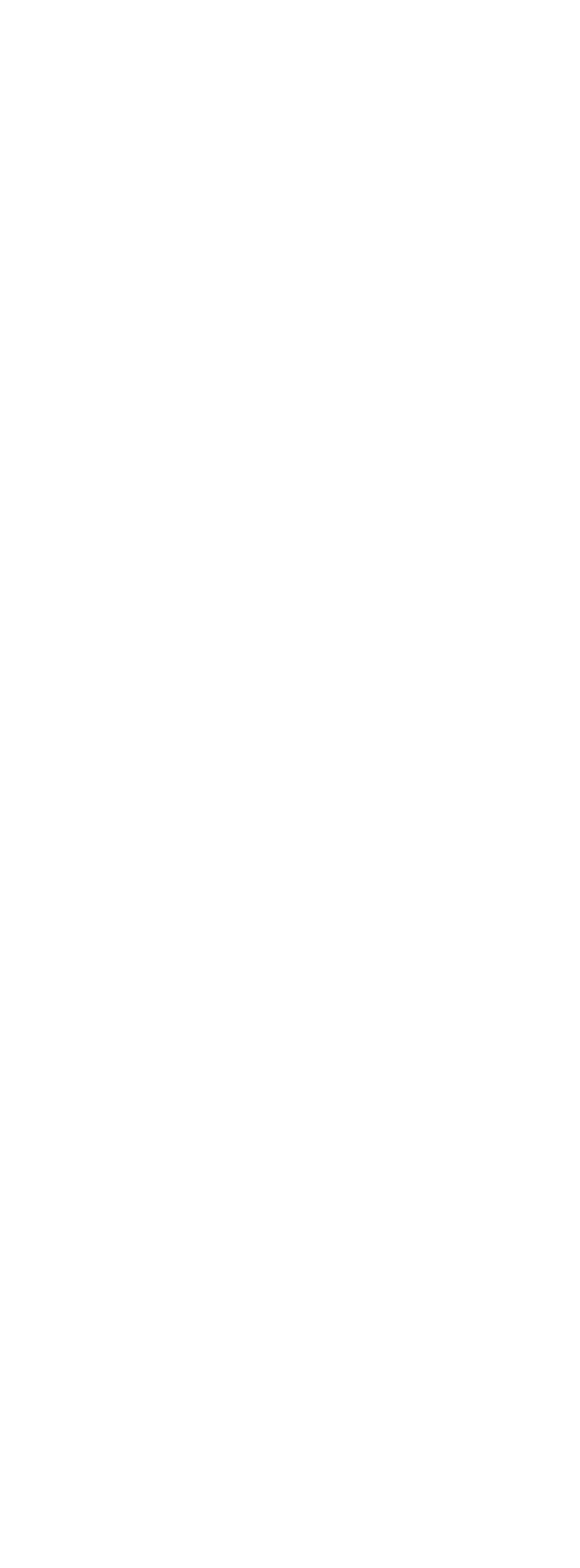


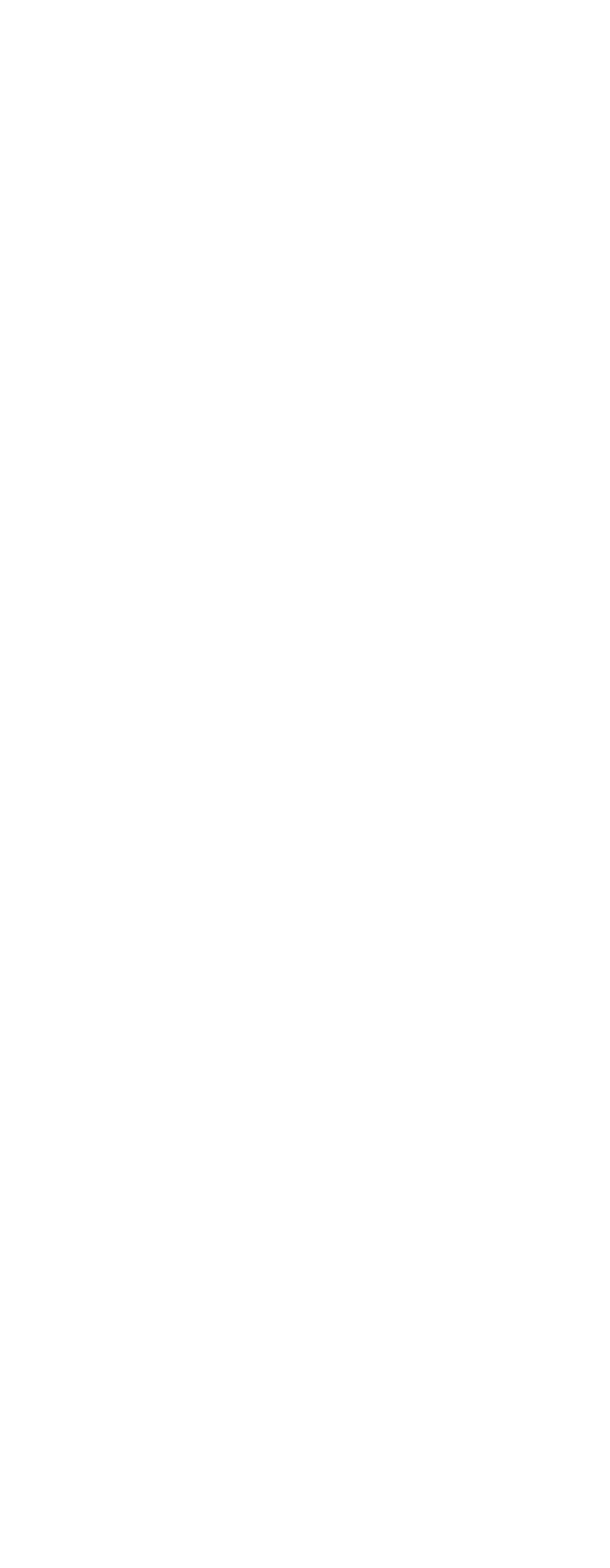



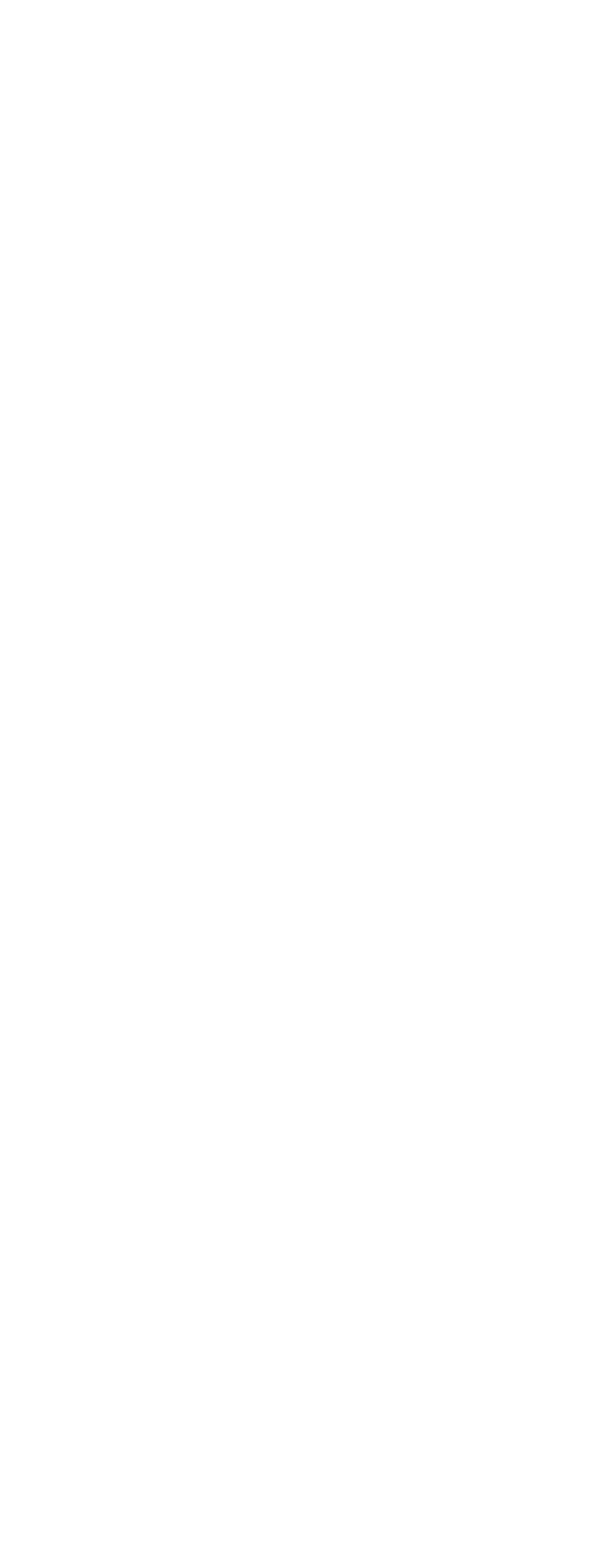


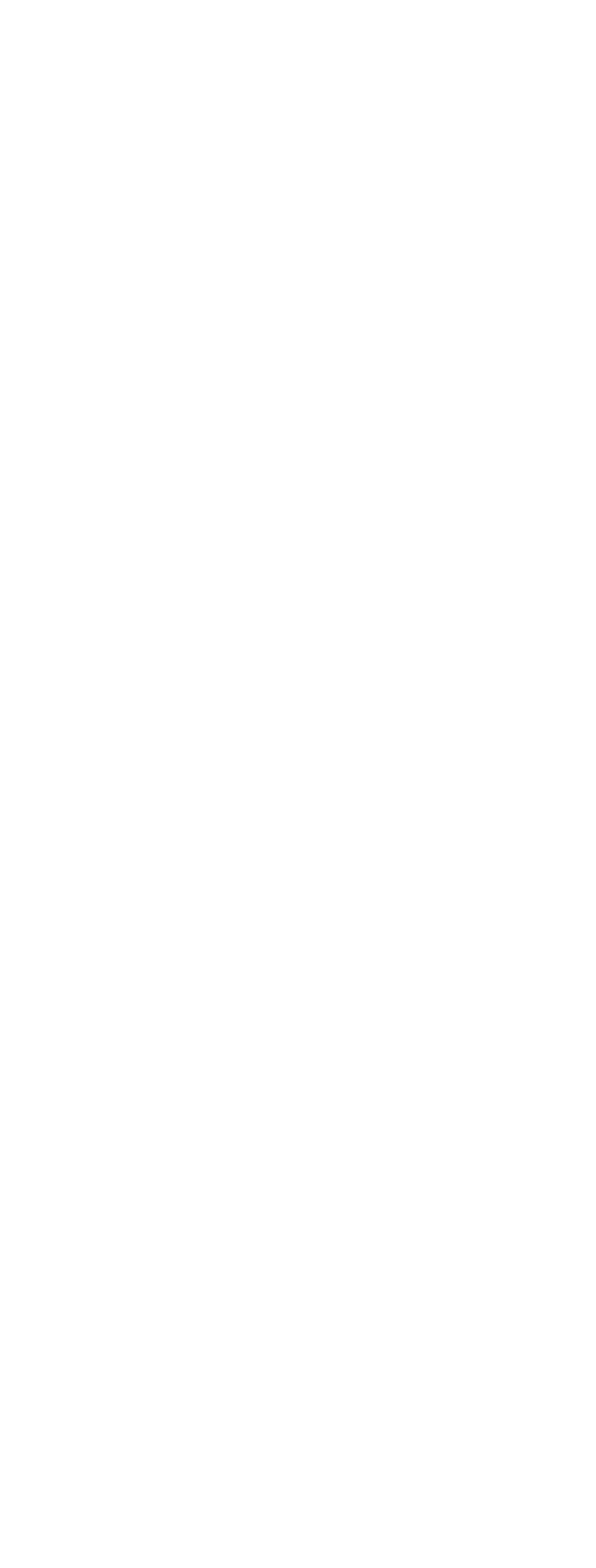



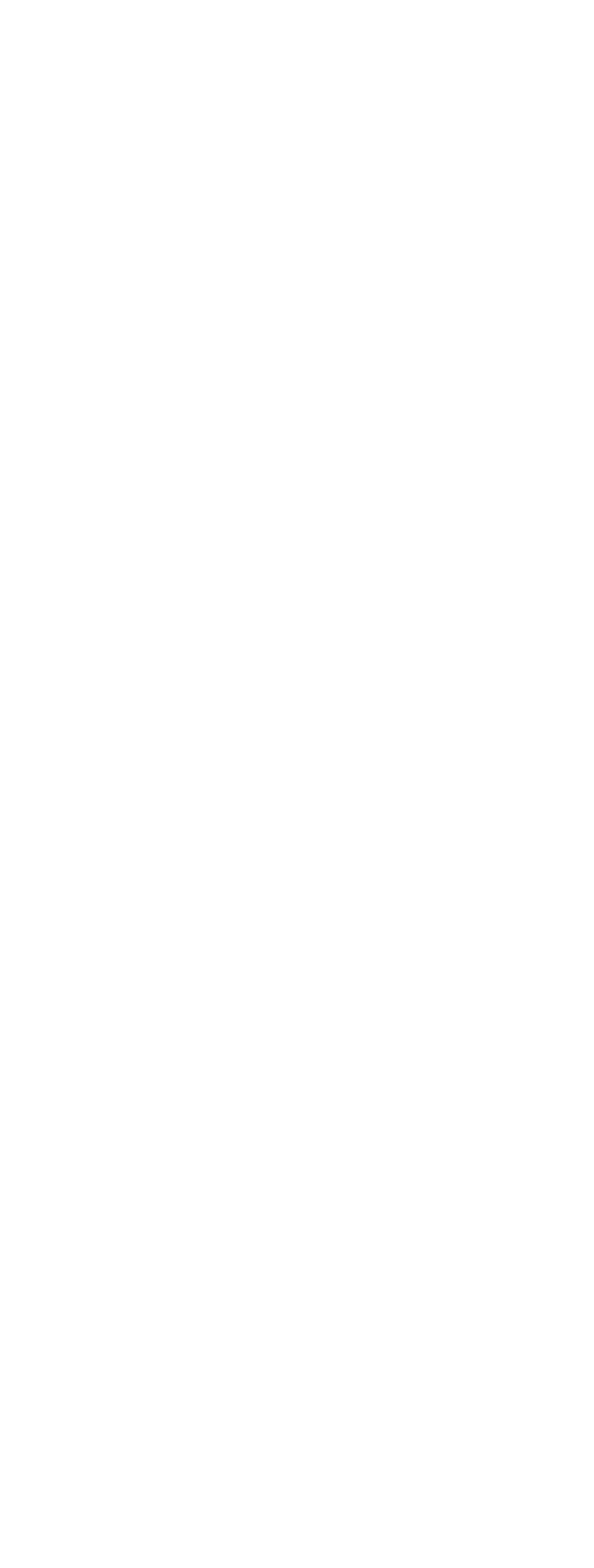


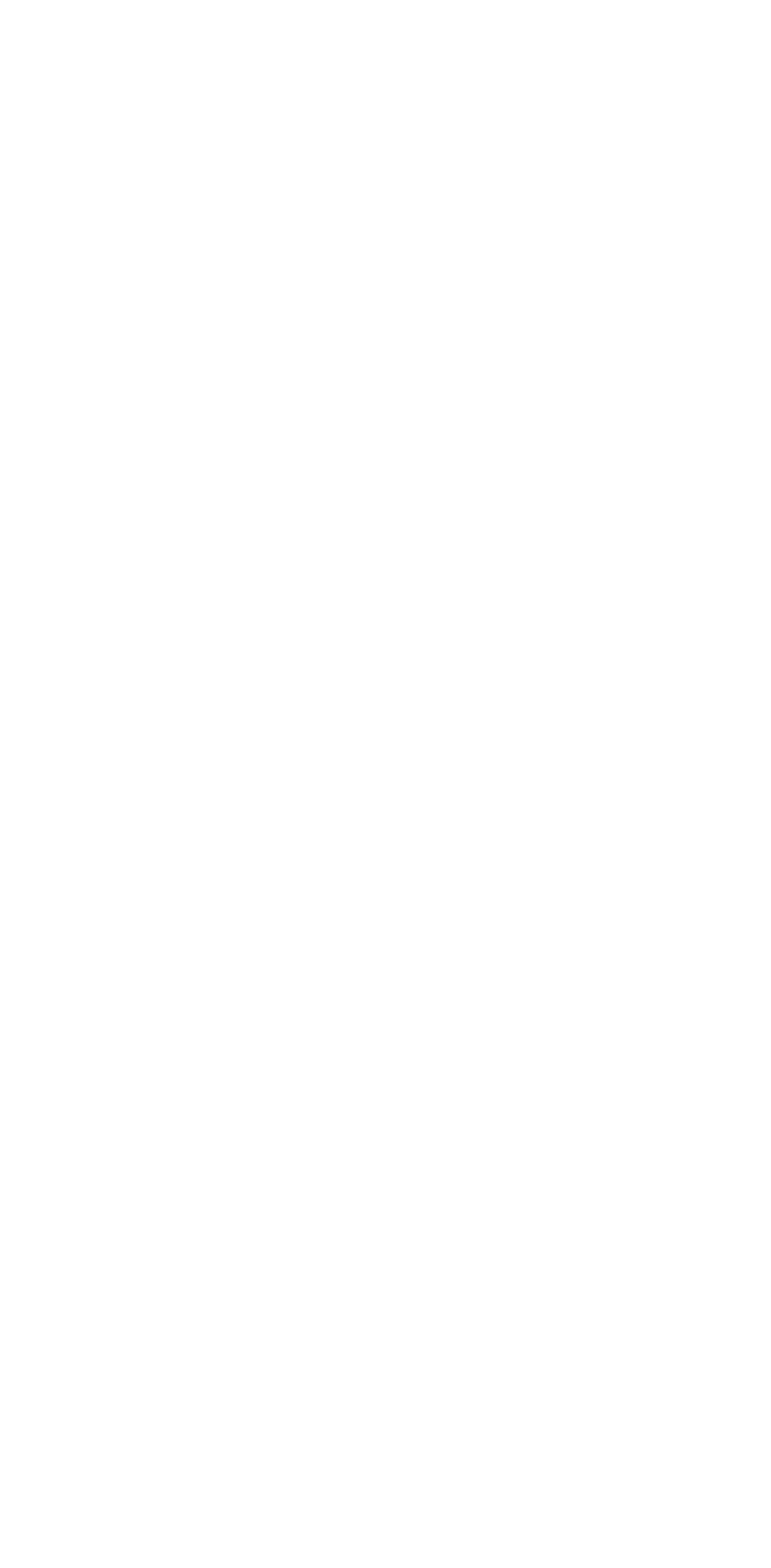


ARKANSAS RIVER BASIN--COntinued

7-1452. SOUTH FORK NINNESCAH RIVER NEAR MURDOCK, KANS.--Continued

Mean specific conductance, water year october 1963 to September 1964 (Continuous conductivity recorder)

\begin{tabular}{|c|c|c|c|c|c|c|c|c|}
\hline \multirow[b]{2}{*}{ Day } & \multicolumn{2}{|c|}{ OCTOBER } & \multicolumn{2}{|c|}{ NOVEMBER } & \multicolumn{2}{|c|}{ DECEMBER } & \multicolumn{2}{|c|}{ JANU ARY } \\
\hline & $\begin{array}{l}\text { Mean } \\
\text { dis- } \\
\text { charge } \\
\text { (cfs) }\end{array}$ & $\begin{array}{l}\text { Specific } \\
\text { conduct- } \\
\text { ance } \\
\text { (micro- } \\
\text { mhos at } \\
25^{\circ} \mathrm{C} \text { ) }\end{array}$ & $\begin{array}{l}\text { Mean } \\
\text { dis- } \\
\text { charge } \\
\text { (cfs) }\end{array}$ & $\begin{array}{l}\text { Specific } \\
\text { conduct- } \\
\text { ance } \\
\text { (micro- } \\
\text { mos at } \\
25^{\circ} \mathrm{C} \text { ) }\end{array}$ & $\begin{array}{l}\text { Mean } \\
\text { dis- } \\
\text { charge } \\
\text { (cfs) }\end{array}$ & $\begin{array}{l}\text { Specific } \\
\text { conduct- } \\
\text { ance } \\
\text { (micro- } \\
\text { mhos at } \\
25^{\circ} \mathrm{C} \text { ) }\end{array}$ & $\begin{array}{l}\text { Mean } \\
\text { dis- } \\
\text { charge } \\
\text { (cfs) }\end{array}$ & $\begin{array}{l}\text { Specific } \\
\text { conduct- } \\
\text { ance } \\
\text { (micro- } \\
\text { mhos at } \\
25^{\circ} \mathrm{C} \text { ) }\end{array}$ \\
\hline $\begin{array}{l}1 \\
2 \\
3 \\
4 \\
5\end{array}$ & & $\begin{array}{l}-- \\
-- \\
-- \\
--\end{array}$ & & $\begin{array}{l}1320 \\
1320 \\
1360 \\
1410 \\
1380\end{array}$ & & $\begin{array}{l}1330 \\
1320 \\
1330 \\
1350 \\
1370\end{array}$ & & $\begin{array}{l}1550 \\
1590 \\
1410 \\
1370 \\
1270\end{array}$ \\
\hline $\begin{array}{r}6 \\
7 \\
8 \\
9 \\
10\end{array}$ & & $\begin{array}{l}-- \\
-- \\
-- \\
--\end{array}$ & & $\begin{array}{l}1370 \\
1380 \\
1380 \\
1380 \\
1380\end{array}$ & & $\begin{array}{l}1380 \\
1380 \\
1380 \\
1350 \\
1330\end{array}$ & & $\begin{array}{l}1240 \\
1230 \\
1250 \\
1320 \\
1280\end{array}$ \\
\hline $\begin{array}{l}11 \\
12 \\
13 \\
14 \\
15\end{array}$ & & $\begin{array}{l}-- \\
-- \\
-- \\
--\end{array}$ & & $\begin{array}{l}1370 \\
1380 \\
1420 \\
1410 \\
1410\end{array}$ & & $\begin{array}{l}1350 \\
1350 \\
1350 \\
1370 \\
1390\end{array}$ & & $\begin{array}{l}1230 \\
1200 \\
1200 \\
1200 \\
1200\end{array}$ \\
\hline $\begin{array}{l}16 \\
17 \\
18 \\
19 \\
20\end{array}$ & & $\begin{array}{l}-- \\
-- \\
-- \\
--\end{array}$ & & $\begin{array}{l}1420 \\
1420 \\
1410 \\
1370 \\
1310\end{array}$ & & $\begin{array}{l}1400 \\
1490 \\
1490 \\
1450 \\
1400\end{array}$ & & $\begin{array}{l}1230 \\
1230 \\
1180 \\
1170 \\
1190\end{array}$ \\
\hline $\begin{array}{l}21 \\
22 \\
23 \\
24 \\
25\end{array}$ & & $\begin{array}{r}-- \\
-- \\
-- \\
1220\end{array}$ & & $\begin{array}{l}1280 \\
1280 \\
1260 \\
1240 \\
1240\end{array}$ & & $\begin{array}{l}1400 \\
1400 \\
1350 \\
1350 \\
1300\end{array}$ & & $\begin{array}{l}1140 \\
1150 \\
1230 \\
1300 \\
1290\end{array}$ \\
\hline $\begin{array}{l}26 \\
27 \\
28 \\
29 \\
30 \\
31\end{array}$ & & $\begin{array}{l}1220 \\
1220 \\
1210 \\
1170 \\
1160 \\
1140\end{array}$ & & $\begin{array}{r}1260 \\
1310 \\
1350 \\
1350 \\
1350 \\
=-\end{array}$ & & $\begin{array}{l}1260 \\
1270 \\
1290 \\
1350 \\
1460 \\
1530\end{array}$ & & $\begin{array}{l}1300 \\
1310 \\
1310 \\
1340 \\
1400 \\
1280\end{array}$ \\
\hline \multicolumn{2}{|c|}{ Monthly mean. } & -- & & -- & & -- & & - \\
\hline & \multicolumn{2}{|c|}{ FEBRUARY } & \multicolumn{2}{|c|}{ MARCH } & \multicolumn{2}{|c|}{ APRIL } & \multicolumn{2}{|c|}{ MAY } \\
\hline $\begin{array}{l}1 \\
2 \\
3 \\
4 \\
5\end{array}$ & & $\begin{array}{l}1230 \\
1200 \\
1260 \\
1280 \\
1280\end{array}$ & & $\begin{array}{l}1350 \\
1350 \\
1320 \\
1310 \\
1310\end{array}$ & & $\begin{array}{r}1310 \\
1290 \\
1190 \\
647 \\
1010\end{array}$ & & $\begin{array}{l}1500 \\
1520 \\
1520 \\
1540 \\
1350\end{array}$ \\
\hline $\begin{array}{r}6 \\
7 \\
8 \\
9 \\
10\end{array}$ & & $\begin{array}{l}1280 \\
1260 \\
1290 \\
1280 \\
1280\end{array}$ & & $\begin{array}{l}\text { A1340 } \\
\text { A1350 } \\
\text { A1350 } \\
\text { A1350 } \\
\text { A1350 }\end{array}$ & & $\begin{array}{l}1160 \\
1230 \\
1260 \\
1300 \\
1400\end{array}$ & & $\begin{array}{r}613 \\
907 \\
1110 \\
1260 \\
1300\end{array}$ \\
\hline $\begin{array}{l}11 \\
12 \\
13 \\
14 \\
15\end{array}$ & & $\begin{array}{l}1280 \\
1280 \\
1440 \\
1560 \\
1490\end{array}$ & & $\begin{array}{l}\text { A1350 } \\
\text { A1350 } \\
\text { A1350 } \\
\text { A1350 } \\
\text { A1350 }\end{array}$ & & $\begin{array}{l}1470 \\
1520 \\
1520 \\
1520 \\
1540\end{array}$ & & $\begin{array}{l}1200 \\
1240 \\
1260 \\
1450 \\
1500\end{array}$ \\
\hline $\begin{array}{l}16 \\
17 \\
18 \\
19 \\
20\end{array}$ & & $\begin{array}{l}1420 \\
1410 \\
1420 \\
1450 \\
1450\end{array}$ & & $\begin{array}{l}\text { A1350 } \\
\text { A1350 } \\
\text { A1350 } \\
\text { A1400 } \\
\text { A1400 }\end{array}$ & & $\begin{array}{l}1600 \\
1610 \\
1560 \\
1530 \\
1440\end{array}$ & & $\begin{array}{l}1220 \\
1360 \\
1490 \\
1370 \\
1540\end{array}$ \\
\hline $\begin{array}{l}21 \\
22 \\
23 \\
24 \\
25\end{array}$ & & $\begin{array}{l}1500 \\
1560 \\
1530 \\
1520 \\
1450\end{array}$ & & $\begin{array}{l}\text { A1400 } \\
\text { A1400 } \\
\text { A1400 } \\
\text { A1400 } \\
\text { A1400 }\end{array}$ & & $\begin{array}{l}1380 \\
1430 \\
1450 \\
1430 \\
1650\end{array}$ & & $\begin{array}{l}1600 \\
1380 \\
1450 \\
1570 \\
1490\end{array}$ \\
\hline $\begin{array}{l}26 \\
27 \\
28 \\
29 \\
30 \\
\mathbf{3 1}\end{array}$ & & $\begin{array}{r}1440 \\
1390 \\
1380 \\
1370 \\
-- \\
--\end{array}$ & & $\begin{array}{l}1450 \\
1460 \\
1400 \\
1400 \\
1400 \\
1380\end{array}$ & & $\begin{array}{r}1710 \\
1710 \\
1700 \\
1630 \\
1520 \\
--\end{array}$ & & $\begin{array}{l}1370 \\
1150 \\
1240 \\
1330 \\
1240 \\
1100\end{array}$ \\
\hline \multicolumn{2}{|c|}{ Monthly mean.. } & -- & & -- & & -- & & - \\
\hline
\end{tabular}

A Estimated. 
ARKANSAS RIVER BASIN--Continued

7-1452. SOUTH FORK NINNESCAH RIVER NEAR MURDOCK, KANS.--Continued

Mean specific conductance, water year October 1963 to September 1964--Continuec (Continuous conductivity recorder)

\begin{tabular}{|c|c|c|c|c|c|c|c|c|}
\hline \multirow[b]{2}{*}{ Day } & \multicolumn{2}{|c|}{ JUNE } & \multicolumn{2}{|c|}{ JULY } & \multicolumn{2}{|c|}{ AUGUST } & \multicolumn{2}{|c|}{ SEPTEMBER } \\
\hline & $\begin{array}{l}\text { Mean } \\
\text { dis- } \\
\text { charge } \\
\text { (cfs) }\end{array}$ & $\begin{array}{l}\text { Specific } \\
\text { conduct- } \\
\text { ance } \\
\text { (micro- } \\
\text { mhos at } \\
25^{\circ} \mathrm{C} \text { ) }\end{array}$ & $\begin{array}{l}\text { Mean } \\
\text { dis- } \\
\text { charge } \\
\text { (cfs) }\end{array}$ & $\begin{array}{l}\text { Specific } \\
\text { conduct- } \\
\text { ance } \\
\text { (micro- } \\
\text { mhos at } \\
25^{\circ} \mathrm{C} \text { ) }\end{array}$ & $\begin{array}{l}\text { Mean } \\
\text { dis- } \\
\text { charge } \\
\text { (cfs) }\end{array}$ & $\begin{array}{l}\text { Specific } \\
\text { conduct- } \\
\text { ance } \\
\text { (micro- } \\
\text { mhos at } \\
25^{\circ} \mathrm{C} \text { ) }\end{array}$ & $\begin{array}{l}\text { Mean } \\
\text { dis- } \\
\text { charge } \\
\text { (cfs) }\end{array}$ & $\begin{array}{l}\text { Specific } \\
\text { conduct- } \\
\text { ance } \\
\text { (micro- } \\
\text { mhos at } \\
25^{\circ} \mathrm{C} \text { ) }\end{array}$ \\
\hline $\begin{array}{l}1 \\
2 \\
3 \\
4 \\
5\end{array}$ & $\begin{array}{l}200 \\
140 \\
110 \\
278 \\
640\end{array}$ & $\begin{array}{r}1220 \\
1230 \\
1240 \\
942 \\
498\end{array}$ & $\begin{array}{r}204 \\
115 \\
87 \\
74 \\
61\end{array}$ & $\begin{array}{l}1830 \\
1160 \\
1560 \\
1710 \\
1860\end{array}$ & $\begin{array}{c}24 \\
15 \\
10 \\
9.0 \\
9.0\end{array}$ & $\begin{array}{l}1930 \\
1990 \\
2020 \\
2020 \\
2080\end{array}$ & $\begin{array}{l}94 \\
74 \\
58 \\
44 \\
39\end{array}$ & $\begin{array}{r}1300 \\
1420 \\
\text { B1640 } \\
\text { B1700 } \\
\text { B1760 }\end{array}$ \\
\hline $\begin{array}{r}6 \\
7 \\
8 \\
9 \\
10\end{array}$ & $\begin{array}{r}126 \\
104 \\
101 \\
98 \\
94\end{array}$ & $\begin{array}{r}878 \\
1100 \\
1220 \\
\mathrm{~B} 1460 \\
\mathrm{~B} 1560\end{array}$ & $\begin{array}{l}52 \\
50 \\
58 \\
55 \\
58\end{array}$ & $\begin{array}{r}1770 \\
1610 \\
1600 \\
\text { B1740 } \\
\text { B1800 }\end{array}$ & $\begin{array}{l}12 \\
15 \\
19 \\
21 \\
26\end{array}$ & $\begin{array}{l}2140 \\
2070 \\
1980 \\
2010 \\
1930\end{array}$ & $\begin{array}{l}42 \\
39 \\
36 \\
36 \\
31\end{array}$ & $\begin{array}{l}\text { B1770 } \\
\text { B1770 } \\
\text { B1800 } \\
\text { B1800 } \\
\text { B1800 }\end{array}$ \\
\hline $\begin{array}{l}11 \\
12 \\
13 \\
14 \\
15\end{array}$ & $\begin{array}{r}104 \\
84 \\
87 \\
94 \\
101\end{array}$ & $\begin{array}{l}\text { B1460 } \\
\text { B1540 } \\
\text { B1530 } \\
\text { B1390 } \\
\text { B1450 }\end{array}$ & $\begin{array}{l}55 \\
55 \\
61 \\
58 \\
50\end{array}$ & $\begin{array}{l}\text { B1770 } \\
\text { B1680 } \\
\text { B1650 } \\
\text { B1730 } \\
\text { B1770 }\end{array}$ & $\begin{array}{l}24 \\
19 \\
24 \\
36 \\
39\end{array}$ & $\begin{array}{l}1930 \\
2000 \\
2000 \\
1810 \\
1860\end{array}$ & $\begin{array}{r}68 \\
84 \\
131 \\
144 \\
104\end{array}$ & $\begin{array}{l}\text { B1750 } \\
\text { B1640 } \\
\text { A1400 } \\
\text { A1350 } \\
\text { A1500 }\end{array}$ \\
\hline $\begin{array}{l}16 \\
17 \\
18 \\
19 \\
20\end{array}$ & $\begin{array}{r}101 \\
101 \\
98 \\
84 \\
74\end{array}$ & $\begin{array}{r}\text { B1440 } \\
\text { B1440 } \\
1460 \\
1500 \\
1600\end{array}$ & $\begin{array}{l}44 \\
44 \\
39 \\
31 \\
28\end{array}$ & $\begin{array}{l}\mathrm{B} 1790 \\
\mathrm{~B} 1820 \\
\mathrm{~B} 1800 \\
\mathrm{~B} 1820 \\
\mathrm{~B} 1910\end{array}$ & $\begin{array}{r}44 \\
130 \\
230 \\
94 \\
101\end{array}$ & $\begin{array}{r}1760 \\
1420 \\
856 \\
1260 \\
1550\end{array}$ & $\begin{array}{l}115 \\
148 \\
135 \\
115 \\
115\end{array}$ & $\begin{array}{l}\text { A1450 } \\
\text { A1350 } \\
\text { A1300 } \\
\text { A1300 } \\
\text { A1350 }\end{array}$ \\
\hline $\begin{array}{l}21 \\
22 \\
23 \\
24 \\
25\end{array}$ & $\begin{array}{r}74 \\
74 \\
104 \\
139 \\
94\end{array}$ & $\begin{array}{l}1560 \\
1590 \\
1460 \\
1500 \\
1290\end{array}$ & $\begin{array}{l}23 \\
23 \\
21 \\
19 \\
17\end{array}$ & $\begin{array}{r}\text { B1910 } \\
\text { B1870 } \\
\text { B1960 } \\
\text { B1870 } \\
1870\end{array}$ & $\begin{array}{l}90 \\
94 \\
87 \\
71 \\
68\end{array}$ & $\begin{array}{l}1460 \\
1210 \\
1430 \\
1570 \\
1700\end{array}$ & $\begin{array}{r}122 \\
131 \\
104 \\
101 \\
87\end{array}$ & $\begin{array}{l}\text { A1300 } \\
\text { A1300 } \\
\text { A1500 } \\
\text { A1500 } \\
\text { A1580 }\end{array}$ \\
\hline $\begin{array}{l}26 \\
27 \\
28 \\
29 \\
30 \\
31\end{array}$ & $\begin{array}{l}61 \\
55 \\
50 \\
44 \\
58 \\
-- \\
\end{array}$ & $\begin{array}{r}1300 \\
1430 \\
1560 \\
1530 \\
1770 \\
--\end{array}$ & $\begin{array}{l}17 \\
28 \\
44 \\
36 \\
33 \\
31\end{array}$ & $\begin{array}{l}1790 \\
1710 \\
1740 \\
1770 \\
1830 \\
1890 \\
\end{array}$ & $\begin{array}{r}58 \\
106 \\
271 \\
148 \\
250 \\
115 \\
\end{array}$ & $\begin{array}{l}1740 \\
1690 \\
1480 \\
1400 \\
1150 \\
1070\end{array}$ & $\begin{array}{r}94 \\
90 \\
87 \\
81 \\
101 \\
-- \\
\end{array}$ & $\begin{array}{r}\text { A1570 } \\
\text { A1550 } \\
\text { A1560 } \\
\text { A1570 } \\
\text { A1500 } \\
-- \\
\end{array}$ \\
\hline \multicolumn{2}{|c|}{ Monthly } & 1190 & -- & 1720 & -- & 1430 & -- & 1470 \\
\hline
\end{tabular}

A Estimated.

B From once daily sample. 


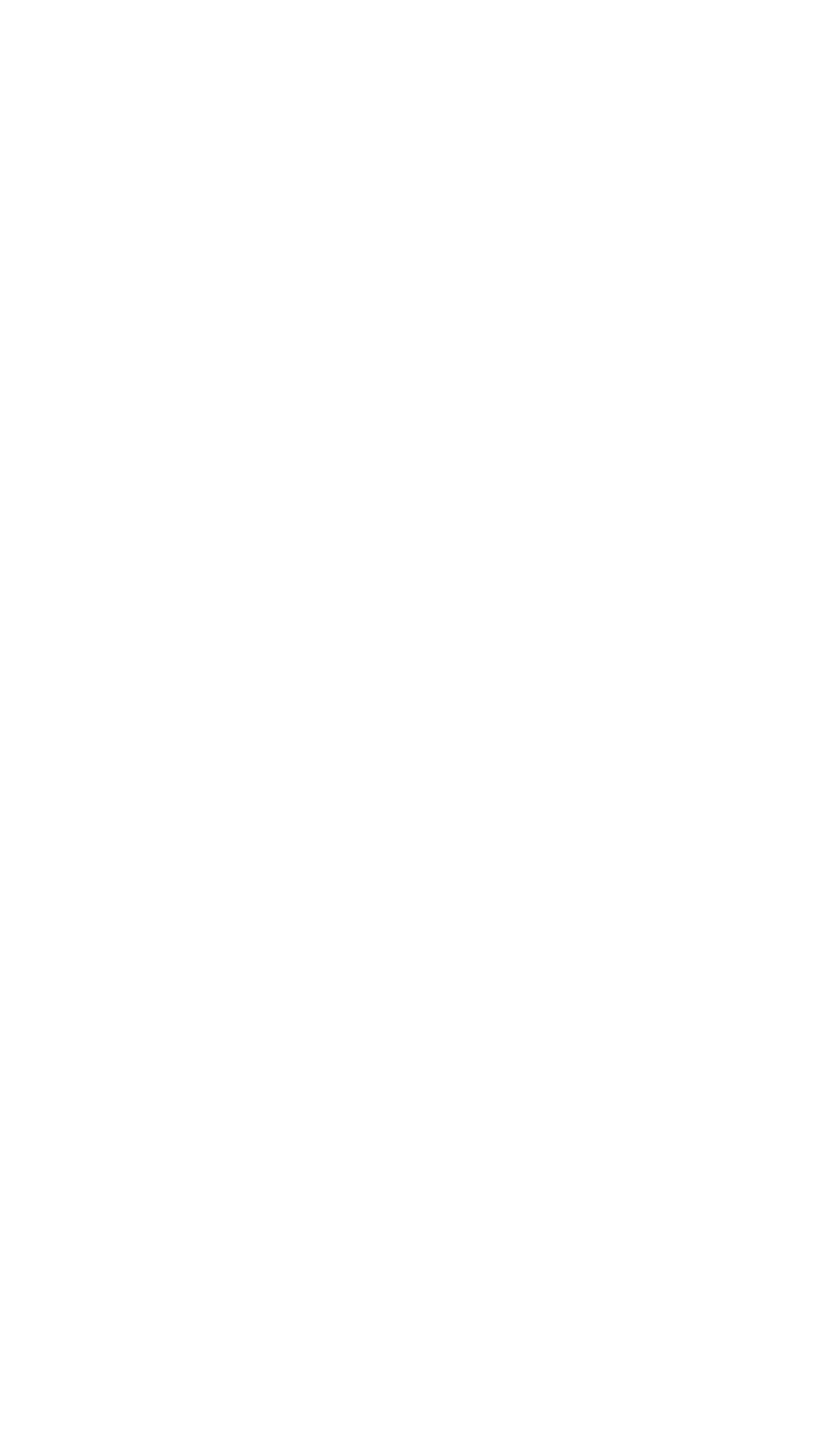




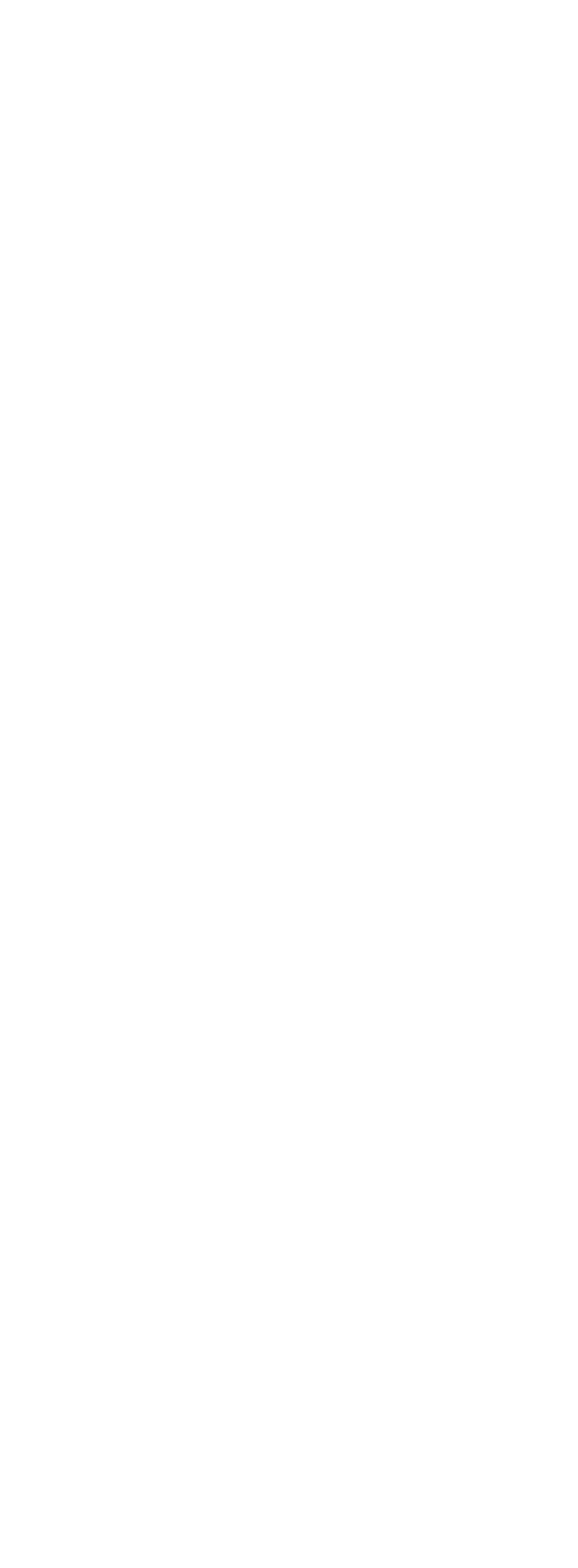




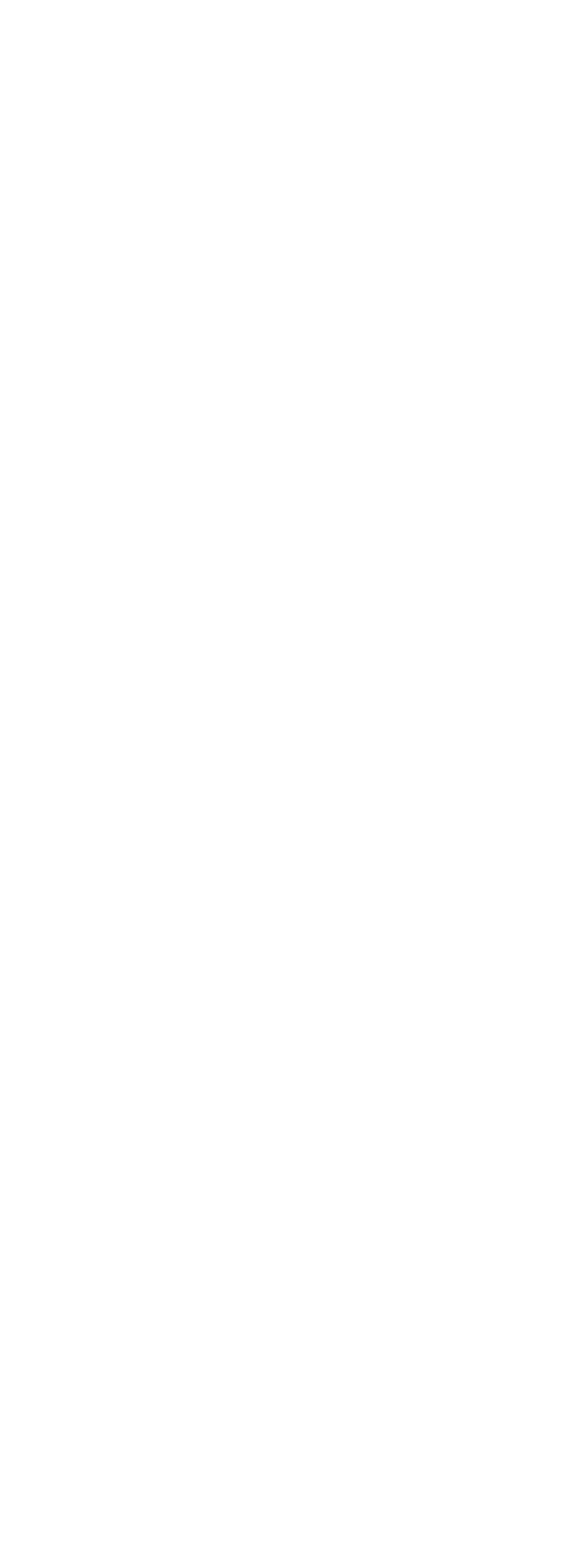




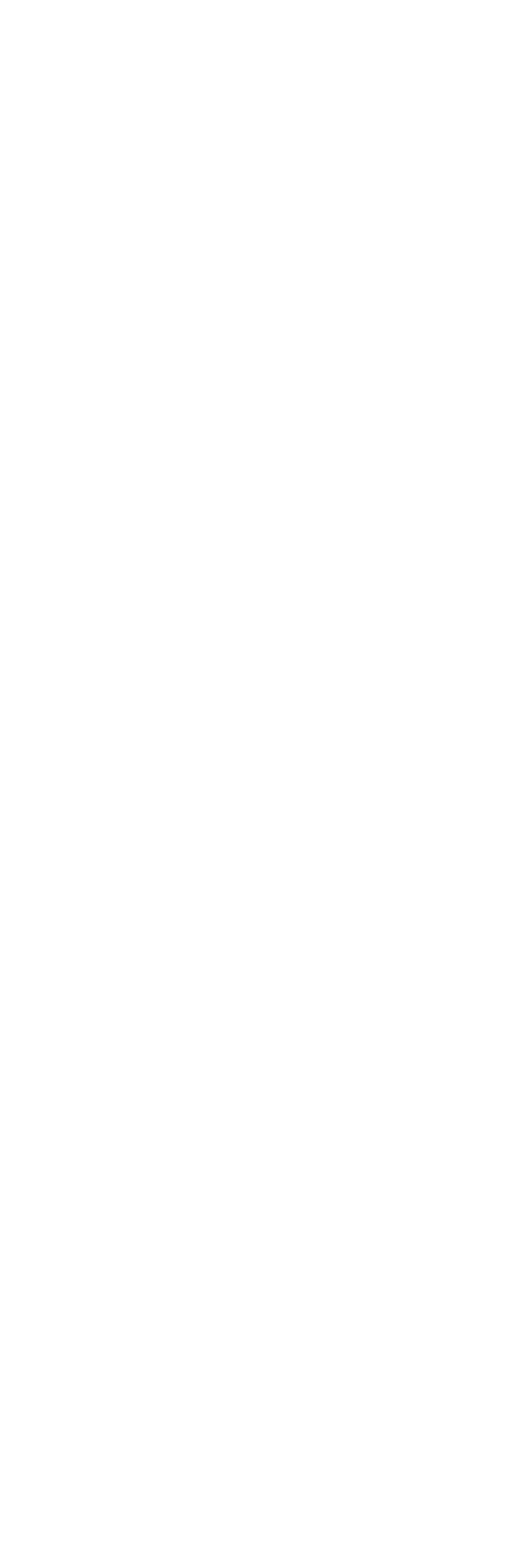




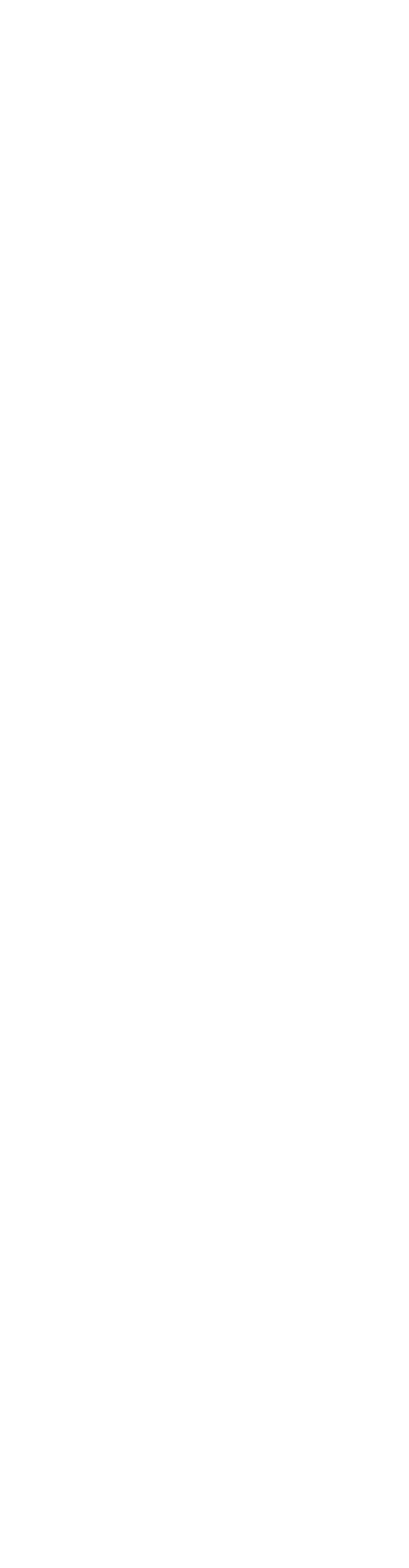

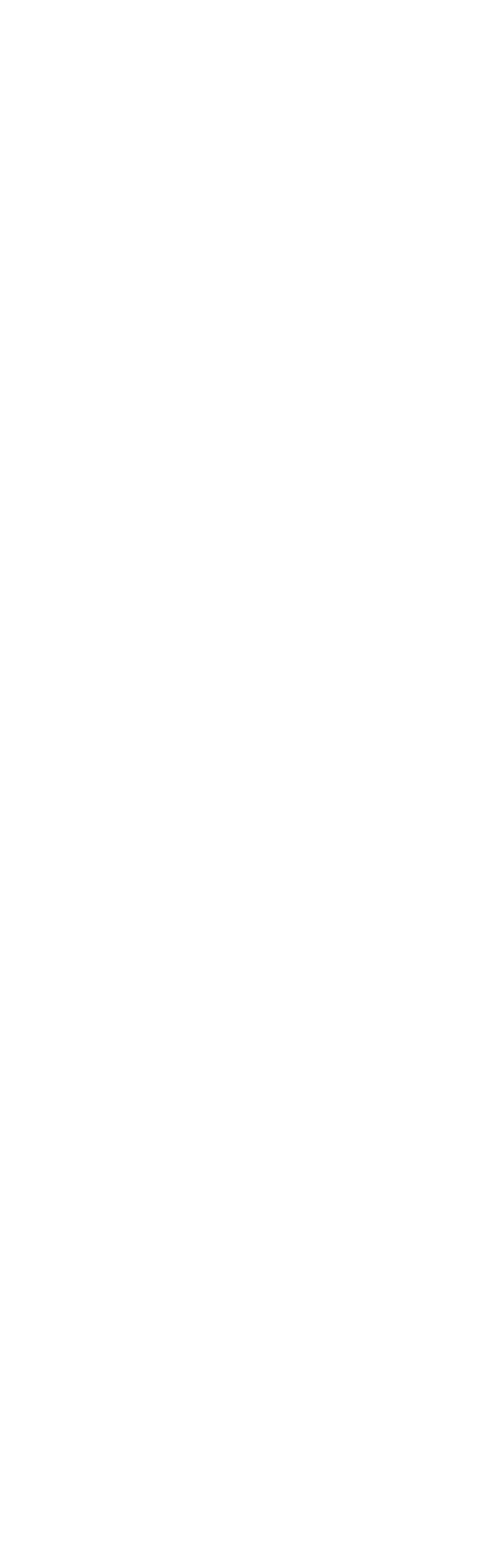




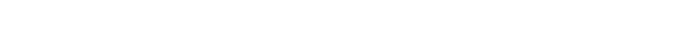

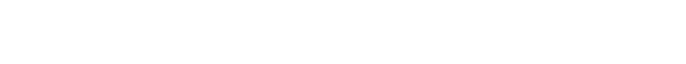

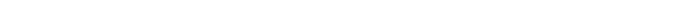

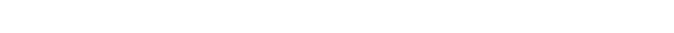

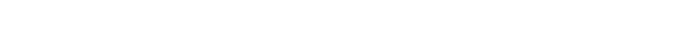

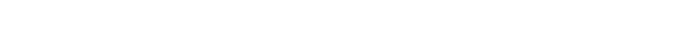

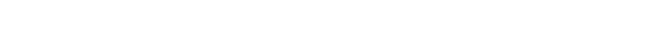

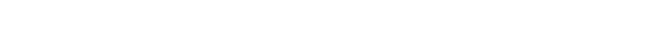

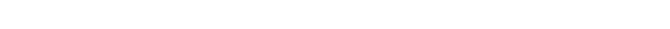

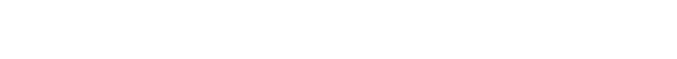

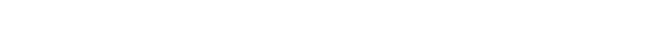

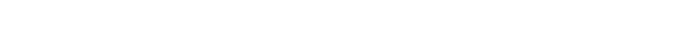

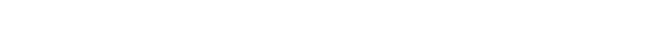

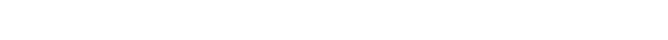

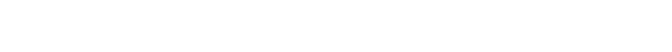

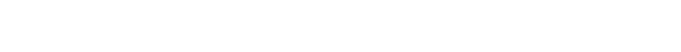

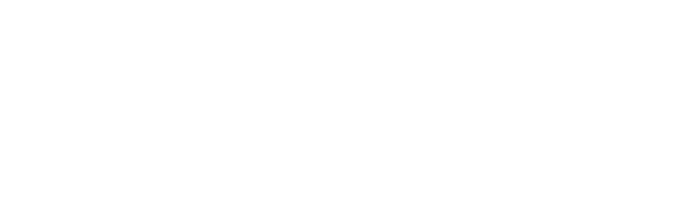




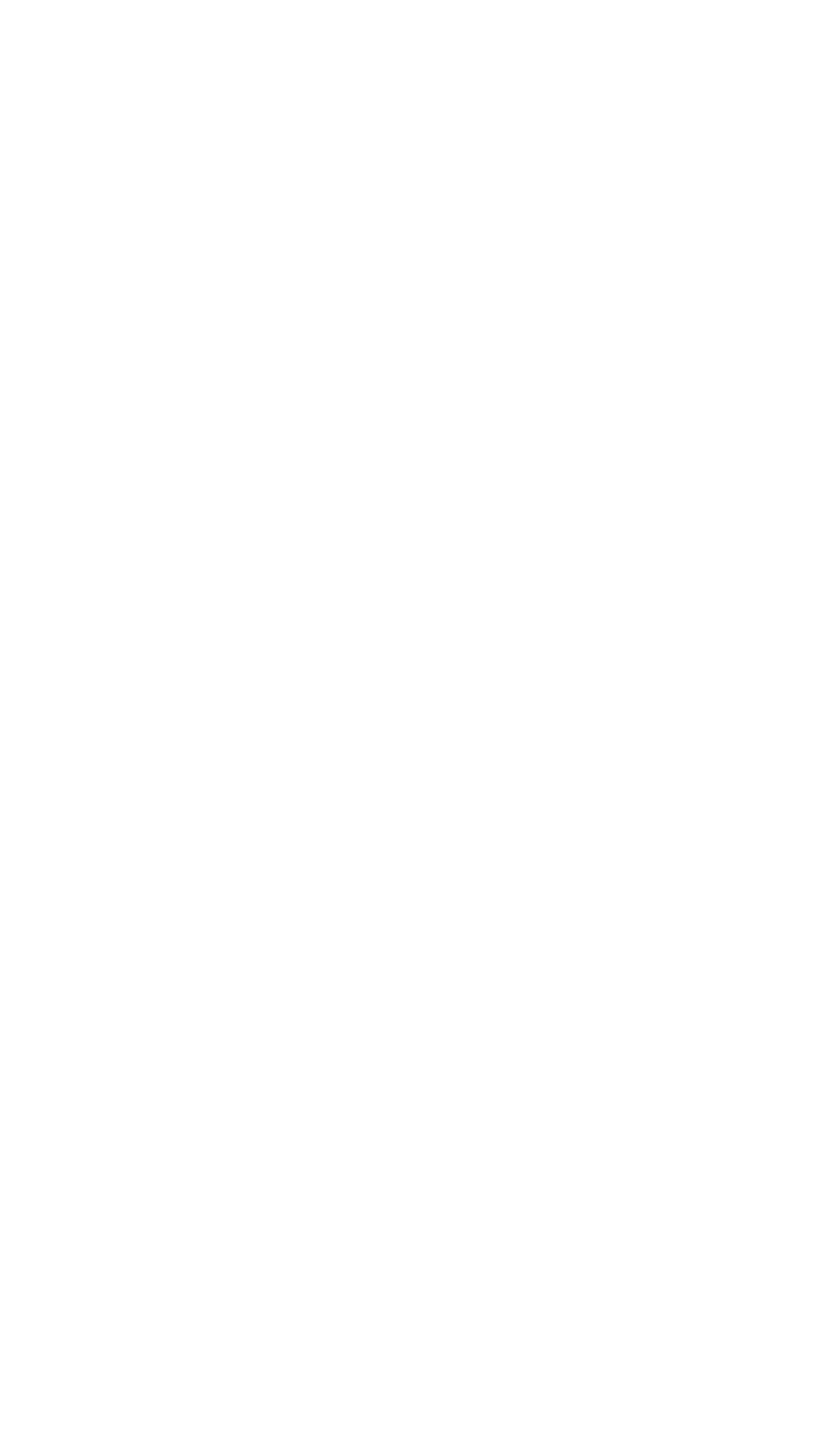




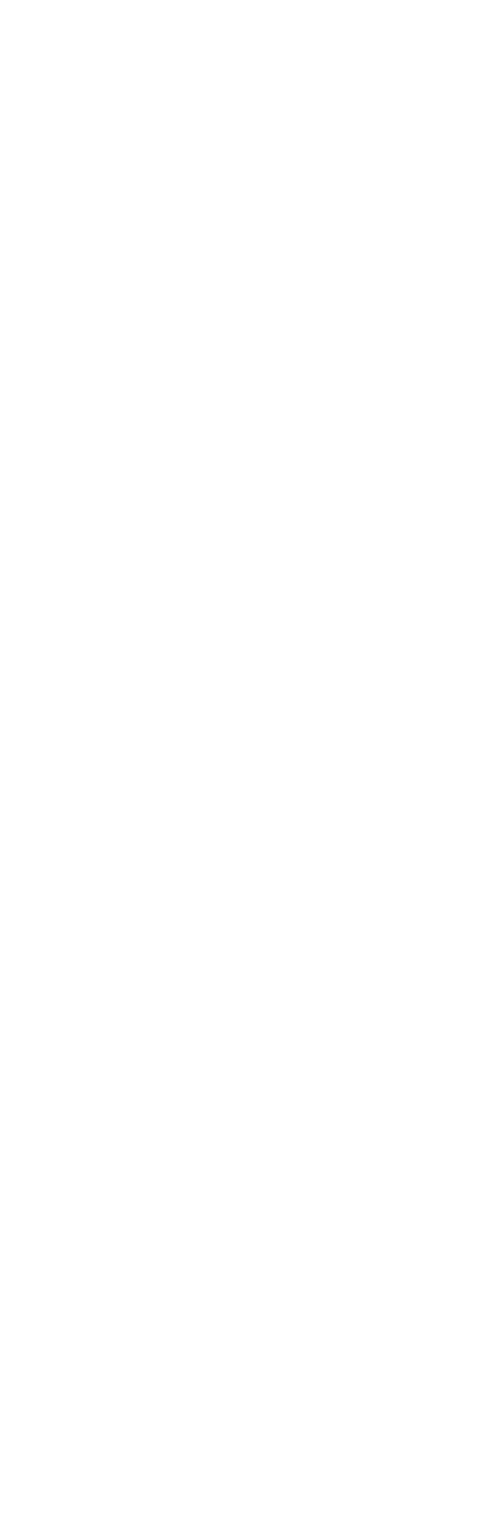


ARKANSAS RIVER BASIN--CONTInued

7-1465. ARKANSAS RIVER AT ARKANSAS CITY, KANS.--Continued

Suspended sediment, water year October 1963 to September 1964 (Where no daily concentrations are reported, loads are estimated)

\begin{tabular}{|c|c|c|c|c|c|c|c|c|c|}
\hline \multirow[b]{3}{*}{ Day } & \multicolumn{3}{|c|}{ OCTOBER } & \multicolumn{3}{|c|}{ NOVEMBER } & \multicolumn{3}{|c|}{ DECEMBER } \\
\hline & \multirow[b]{2}{*}{$\begin{array}{c}\text { Mean } \\
\text { dis- } \\
\text { charge } \\
\text { (cfs) }\end{array}$} & \multicolumn{2}{|c|}{ Suspended sediment } & \multirow[b]{2}{*}{$\begin{array}{c}\text { Mean } \\
\text { dis- } \\
\text { charge } \\
(\mathbf{c f s})\end{array}$} & \multicolumn{2}{|c|}{ Suspended sediment } & \multirow[b]{2}{*}{$\begin{array}{l}\text { Mean } \\
\text { dis- } \\
\text { charge } \\
\text { (cfs) }\end{array}$} & \multicolumn{2}{|c|}{ Suspended sediment } \\
\hline & & $\begin{array}{l}\text { Mean } \\
\text { concen- } \\
\text { tration } \\
\text { (ppm) }\end{array}$ & $\begin{array}{l}\text { Tons } \\
\text { per } \\
\text { day }\end{array}$ & & $\begin{array}{c}\text { Mean } \\
\text { concen- } \\
\text { tration } \\
(\text { ppm })\end{array}$ & $\begin{array}{l}\text { Tons } \\
\text { per } \\
\text { day }\end{array}$ & & $\begin{array}{l}\text { Mean } \\
\text { concen- } \\
\text { tration } \\
\text { (ppm) }\end{array}$ & $\begin{array}{l}\text { Tons } \\
\text { per } \\
\text { day }\end{array}$ \\
\hline $\begin{array}{l}1 \ldots \\
2 \ldots \\
3 \ldots \\
4 \ldots \\
5 \ldots\end{array}$ & $\begin{array}{l}771 \\
969 \\
870 \\
771 \\
740\end{array}$ & $\begin{array}{r}870 \\
1000 \\
1200 \\
910 \\
600\end{array}$ & $\begin{array}{l}1800 \\
2600 \\
2800 \\
1900 \\
1200\end{array}$ & $\begin{array}{l}859 \\
804 \\
750 \\
730 \\
690\end{array}$ & $\begin{array}{l}210 \\
190 \\
190 \\
160 \\
110\end{array}$ & $\begin{array}{l}490 \\
410 \\
380 \\
320 \\
200\end{array}$ & $\begin{array}{l}588 \\
579 \\
579 \\
579 \\
588\end{array}$ & $\begin{array}{l}58 \\
38 \\
44 \\
50 \\
55\end{array}$ & $\begin{array}{l}92 \\
59 \\
69 \\
78 \\
87\end{array}$ \\
\hline $\begin{array}{r}6 \ldots \\
7 \ldots \\
8 \ldots \\
9 \ldots \\
10 \ldots\end{array}$ & $\begin{array}{l}690 \\
651 \\
615 \\
606 \\
570\end{array}$ & $\begin{array}{l}430 \\
270 \\
190 \\
230 \\
210\end{array}$ & $\begin{array}{l}800 \\
470 \\
320 \\
380 \\
320\end{array}$ & $\begin{array}{l}670 \\
651 \\
633 \\
624 \\
615\end{array}$ & $\begin{array}{r}150 \\
160 \\
100 \\
67 \\
76\end{array}$ & $\begin{array}{l}270 \\
280 \\
170 \\
110 \\
130\end{array}$ & $\begin{array}{l}597 \\
606 \\
606 \\
588 \\
579\end{array}$ & $\begin{array}{r}45 \\
74 \\
180 \\
68 \\
--\end{array}$ & $\begin{array}{r}72 \\
120 \\
290 \\
110 \\
80\end{array}$ \\
\hline $\begin{array}{l}11 \ldots \\
12 \ldots \\
13 \ldots \\
14 \ldots \\
15 \ldots\end{array}$ & $\begin{array}{l}552 \\
525 \\
507 \\
480 \\
462\end{array}$ & $\begin{array}{l}180 \\
160 \\
120 \\
130 \\
150\end{array}$ & $\begin{array}{l}270 \\
230 \\
160 \\
170 \\
190\end{array}$ & $\begin{array}{l}606 \\
597 \\
588 \\
588 \\
570\end{array}$ & $\begin{array}{r}61 \\
150 \\
73 \\
92 \\
32\end{array}$ & $\begin{array}{r}100 \\
240 \\
120 \\
150 \\
49\end{array}$ & $\begin{array}{l}534 \\
374 \\
300 \\
250 \\
300\end{array}$ & $\begin{array}{l}-- \\
-- \\
38 \\
85 \\
71\end{array}$ & $\begin{array}{l}60 \\
40 \\
31 \\
57 \\
58\end{array}$ \\
\hline $\begin{array}{l}16 \ldots \\
17 \ldots \\
18 \ldots \\
19 \ldots \\
20 \ldots\end{array}$ & $\begin{array}{r}489 \\
624 \\
680 \\
1880 \\
2910\end{array}$ & $\begin{array}{r}190 \\
290 \\
270 \\
1900 \\
2200\end{array}$ & $\begin{array}{r}250 \\
490 \\
500 \\
9600 \\
17000\end{array}$ & $\begin{array}{l}552 \\
561 \\
552 \\
570 \\
633\end{array}$ & $\begin{array}{l}38 \\
42 \\
41 \\
53 \\
96\end{array}$ & $\begin{array}{r}57 \\
64 \\
61 \\
82 \\
160\end{array}$ & $\begin{array}{l}350 \\
450 \\
400 \\
450 \\
350\end{array}$ & $\begin{array}{l}-- \\
=- \\
=- \\
24\end{array}$ & $\begin{array}{l}50 \\
60 \\
40 \\
40 \\
23\end{array}$ \\
\hline $21 \ldots$ & 2750 & 1400 & 10000 & 782 & 200 & 420 & 300 & - & 20 \\
\hline $\begin{array}{l}22 \ldots \\
23 \ldots \\
24 \ldots \\
25 \ldots\end{array}$ & $\begin{array}{l}2120 \\
2110 \\
3540 \\
3000\end{array}$ & $\begin{array}{l}1100 \\
1200 \\
2200 \\
1800\end{array}$ & $\begin{array}{r}6300 \\
6800 \\
21000 \\
15000\end{array}$ & $\begin{array}{l}760 \\
730 \\
700 \\
660\end{array}$ & $\begin{array}{r}220 \\
130 \\
73 \\
48\end{array}$ & $\begin{array}{r}450 \\
260 \\
140 \\
86\end{array}$ & $\begin{array}{l}280 \\
300 \\
350 \\
450\end{array}$ & $\begin{array}{l}-- \\
--\end{array}$ & $\begin{array}{l}20 \\
20 \\
30 \\
40\end{array}$ \\
\hline $\begin{array}{l}26 \ldots \\
27 \ldots \\
28 \ldots \\
29 \ldots \\
30 \ldots \\
31 \ldots\end{array}$ & $\begin{array}{r}2230 \\
1700 \\
1440 \\
1220 \\
1000 \\
925\end{array}$ & $\begin{array}{r}1200 \\
730 \\
500 \\
400 \\
300 \\
330 \\
\end{array}$ & $\begin{array}{r}7200 \\
3400 \\
1900 \\
1300 \\
810 \\
820 \\
\end{array}$ & $\begin{array}{r}642 \\
624 \\
624 \\
606 \\
597 \\
- \\
\end{array}$ & $\begin{array}{l}58 \\
49 \\
58 \\
48 \\
40 \\
-- \\
\end{array}$ & $\begin{array}{r}100 \\
82 \\
98 \\
78 \\
64 \\
-\cdots \\
\end{array}$ & $\begin{array}{l}500 \\
540 \\
520 \\
550 \\
600 \\
700\end{array}$ & $\begin{array}{l}-- \\
-- \\
-- \\
--\end{array}$ & $\begin{array}{r}50 \\
60 \\
60 \\
70 \\
80 \\
200 \\
\end{array}$ \\
\hline \multirow[t]{2}{*}{ Total } & 38397 & -- & 115980 & 19568 & -- & 5621 & 14737 & -- & 2166 \\
\hline & \multicolumn{3}{|c|}{ JANUARY } & \multicolumn{3}{|c|}{ FEBRUARY } & \multicolumn{3}{|c|}{ MARCH } \\
\hline $\begin{array}{l}1 \ldots \\
2 \ldots \\
3 \ldots \\
4 \ldots \\
5 \ldots\end{array}$ & $\begin{array}{r}800 \\
900 \\
1000 \\
970 \\
1050\end{array}$ & $\begin{array}{r}-- \\
=- \\
220 \\
160\end{array}$ & $\begin{array}{l}200 \\
400 \\
800 \\
580 \\
450\end{array}$ & $\begin{array}{l}677 \\
713 \\
704 \\
722 \\
785\end{array}$ & $\begin{array}{l}72 \\
69 \\
80 \\
73 \\
98\end{array}$ & $\begin{array}{l}130 \\
130 \\
150 \\
140 \\
210\end{array}$ & $\begin{array}{l}528 \\
528 \\
528 \\
534 \\
544\end{array}$ & $\begin{array}{l}42 \\
64 \\
57 \\
58 \\
42\end{array}$ & $\begin{array}{l}60 \\
91 \\
81 \\
84 \\
62\end{array}$ \\
\hline $\begin{array}{r}6 \ldots \\
7 \ldots \\
8 \ldots \\
9 \ldots \\
10 \ldots\end{array}$ & $\begin{array}{l}930 \\
767 \\
740 \\
677 \\
569\end{array}$ & $\begin{array}{r}110 \\
93 \\
97 \\
130 \\
99\end{array}$ & $\begin{array}{l}280 \\
190 \\
190 \\
240 \\
150\end{array}$ & $\begin{array}{l}704 \\
659 \\
632 \\
596 \\
578\end{array}$ & $\begin{array}{l}96 \\
63 \\
66 \\
52 \\
61\end{array}$ & $\begin{array}{r}180 \\
110 \\
110 \\
84 \\
95\end{array}$ & $\begin{array}{l}544 \\
534 \\
552 \\
55 ? \\
569\end{array}$ & $\begin{array}{l}48 \\
42 \\
52 \\
50 \\
55\end{array}$ & $\begin{array}{l}70 \\
61 \\
78 \\
74 \\
84\end{array}$ \\
\hline $\begin{array}{l}11 \ldots \\
12 \ldots \\
13 \ldots \\
14 \ldots \\
15 \ldots\end{array}$ & $\begin{array}{l}500 \\
450 \\
400 \\
450 \\
500\end{array}$ & $\begin{array}{l}=- \\
=- \\
-- \\
--\end{array}$ & $\begin{array}{r}100 \\
100 \\
60 \\
70 \\
80\end{array}$ & $\begin{array}{l}536 \\
520 \\
512 \\
512 \\
536\end{array}$ & $\begin{array}{l}71 \\
80 \\
94 \\
85 \\
85\end{array}$ & $\begin{array}{l}100 \\
110 \\
130 \\
120 \\
120\end{array}$ & $\begin{array}{l}560 \\
544 \\
55 ? \\
534 \\
523\end{array}$ & $\begin{array}{l}37 \\
26 \\
-- \\
--\end{array}$ & $\begin{array}{l}56 \\
38 \\
60 \\
60 \\
60\end{array}$ \\
\hline $\begin{array}{l}16 \ldots \\
17 \ldots \\
18 \ldots \\
19 \ldots \\
20 \ldots\end{array}$ & $\begin{array}{l}550 \\
650 \\
704 \\
767 \\
758\end{array}$ & $\begin{array}{r}-- \\
-- \\
25 \\
140\end{array}$ & $\begin{array}{r}60 \\
70 \\
80 \\
52 \\
290\end{array}$ & $\begin{array}{l}552 \\
578 \\
569 \\
560 \\
560\end{array}$ & $\begin{array}{r}90 \\
110 \\
110 \\
59 \\
48\end{array}$ & $\begin{array}{r}130 \\
170 \\
170 \\
89 \\
72\end{array}$ & $\begin{array}{l}512 \\
499 \\
489 \\
529 \\
560\end{array}$ & $\begin{array}{l}41 \\
24 \\
67 \\
68 \\
73\end{array}$ & $\begin{array}{r}57 \\
32 \\
88 \\
97 \\
110\end{array}$ \\
\hline $\begin{array}{l}21 \ldots \\
22 \ldots \\
23 \ldots \\
24 \ldots \\
25 \ldots\end{array}$ & $\begin{array}{l}677 \\
659 \\
731 \\
758 \\
659\end{array}$ & $\begin{array}{l}140 \\
160 \\
180 \\
150 \\
100\end{array}$ & $\begin{array}{l}260 \\
280 \\
360 \\
310 \\
180\end{array}$ & $\begin{array}{l}552 \\
552 \\
528 \\
520 \\
528\end{array}$ & $\begin{array}{l}41 \\
62 \\
42 \\
59 \\
70\end{array}$ & $\begin{array}{r}61 \\
92 \\
60 \\
83 \\
100\end{array}$ & $\begin{array}{l}641 \\
63 ? \\
663 \\
641 \\
599\end{array}$ & $\begin{array}{l}70 \\
80 \\
55 \\
44 \\
55\end{array}$ & $\begin{array}{r}120 \\
140 \\
99 \\
76 \\
88\end{array}$ \\
\hline $\begin{array}{l}26 \ldots \\
27 \ldots \\
28 \ldots \\
29 \ldots \\
30 \ldots \\
31 \ldots\end{array}$ & $\begin{array}{l}605 \\
587 \\
552 \\
560 \\
560 \\
614\end{array}$ & $\begin{array}{l}72 \\
76 \\
57 \\
80 \\
73 \\
53 \\
\end{array}$ & $\begin{array}{r}120 \\
120 \\
85 \\
120 \\
110 \\
88 \\
\end{array}$ & $\begin{array}{r}520 \\
520 \\
504 \\
520 \\
-- \\
-\end{array}$ & $\begin{array}{l}49 \\
39 \\
63 \\
63 \\
-- \\
--\end{array}$ & $\begin{array}{l}69 \\
55 \\
86 \\
88 \\
-- \\
--\end{array}$ & $\begin{array}{l}569 \\
560 \\
55 ? \\
539 \\
512 \\
50 ?\end{array}$ & $\begin{array}{l}85 \\
66 \\
60 \\
37 \\
43 \\
44\end{array}$ & $\begin{array}{r}130 \\
100 \\
89 \\
54 \\
59 \\
60\end{array}$ \\
\hline Total & 21094 & -- & 6475 & 16949 & -- & 3244 & 17126 & -- & 2418 \\
\hline
\end{tabular}



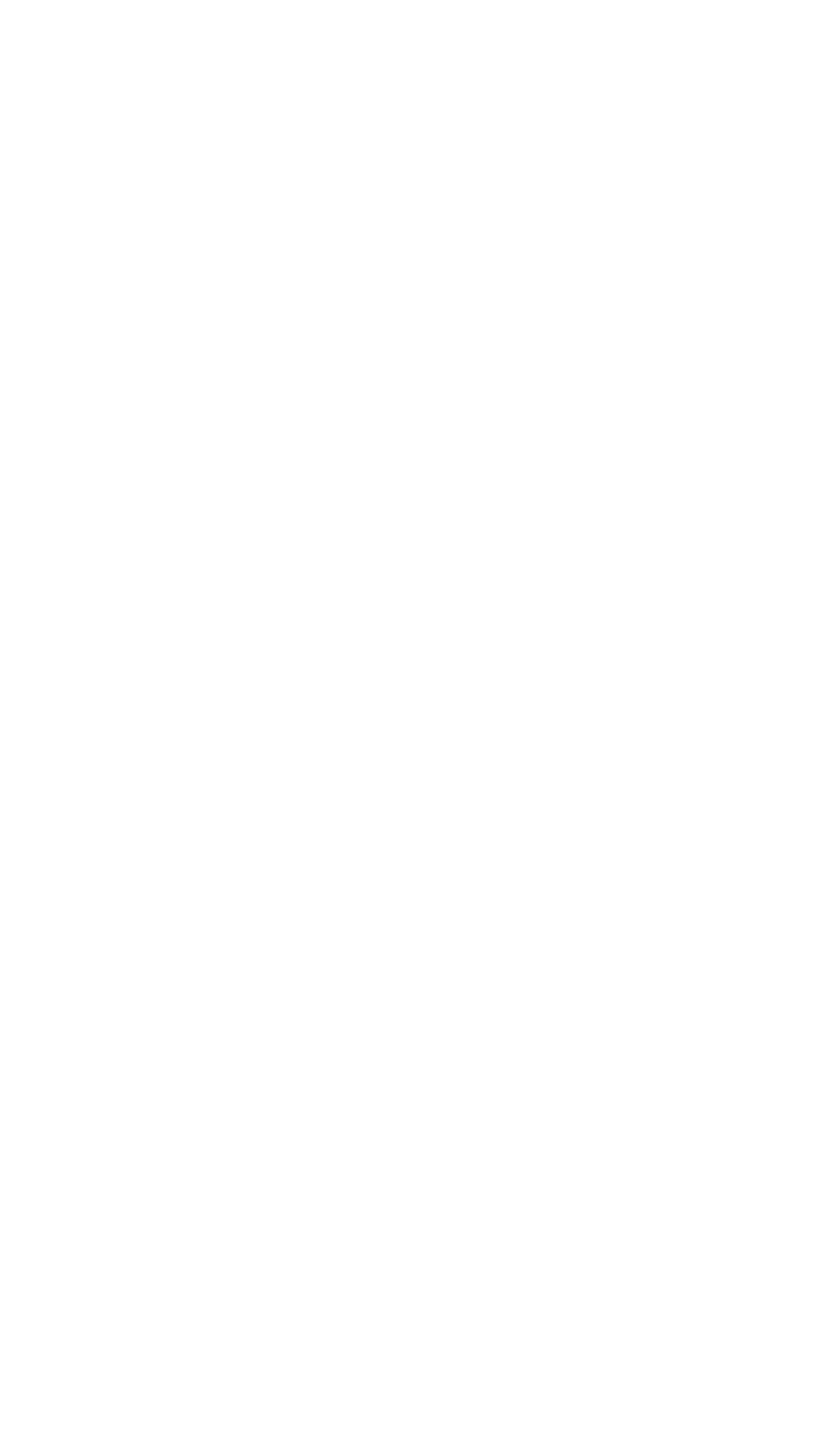


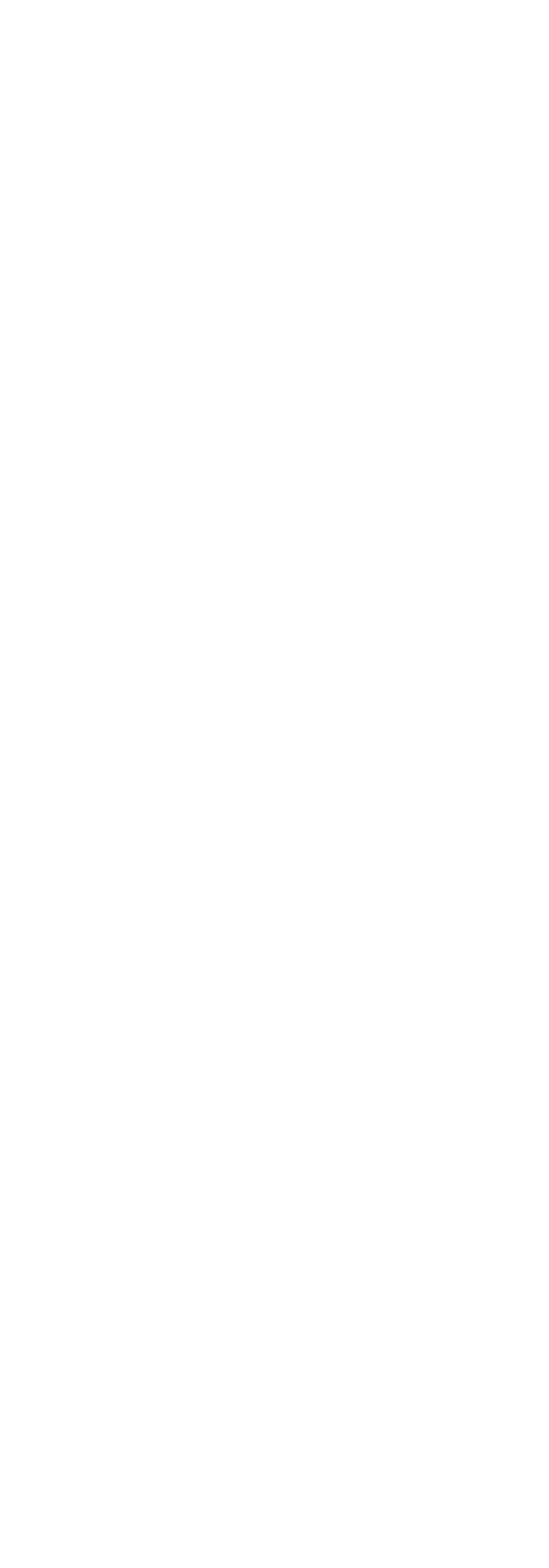

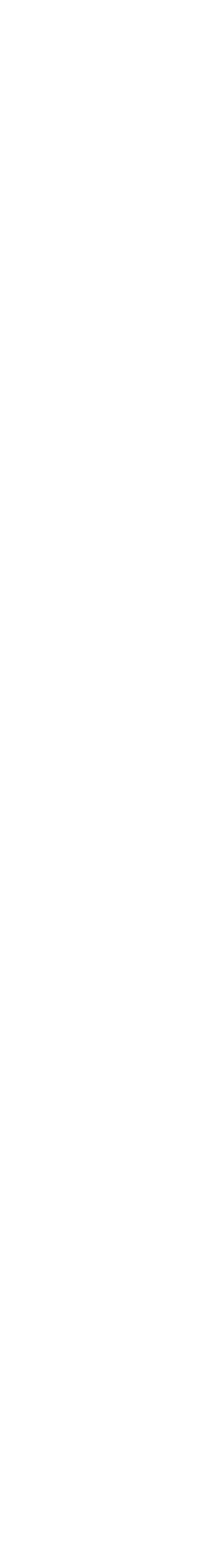




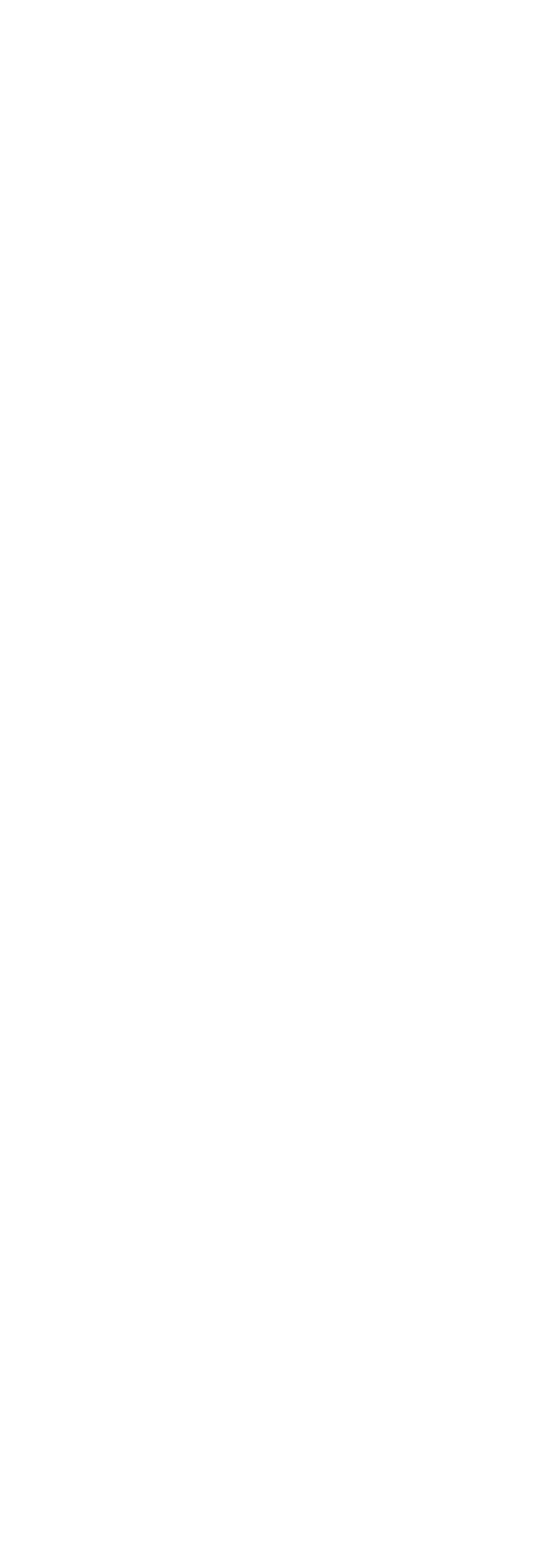




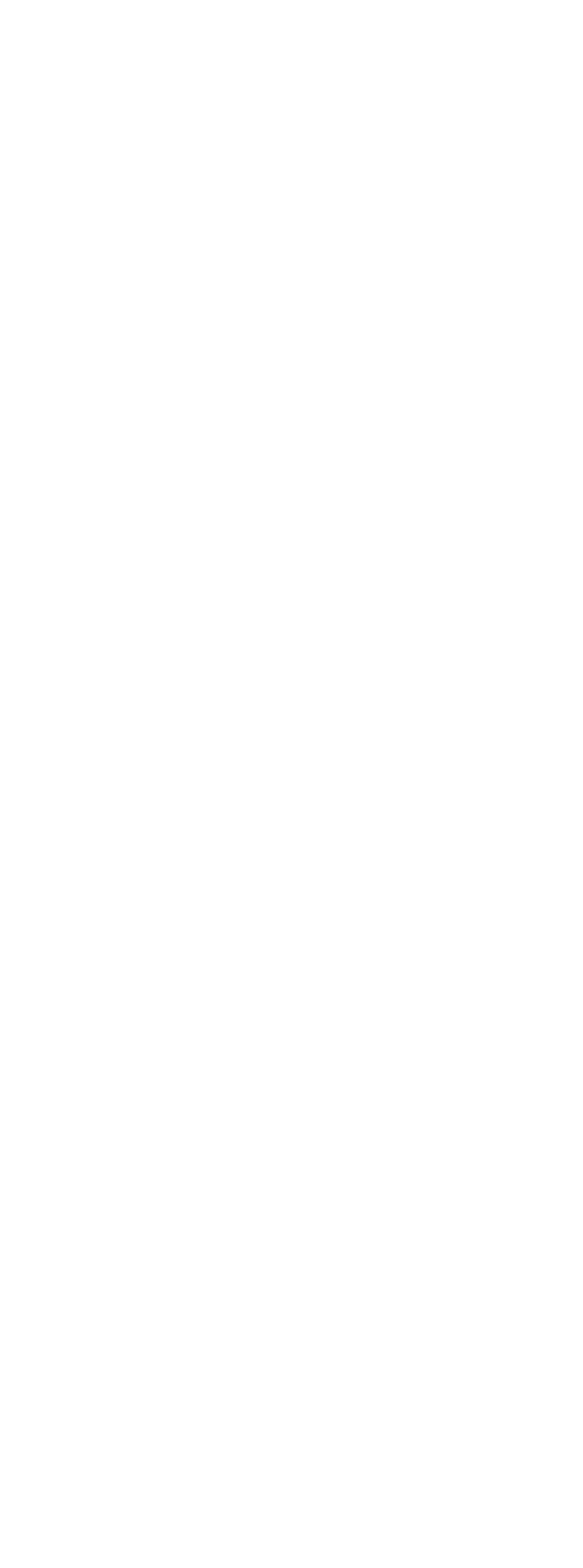




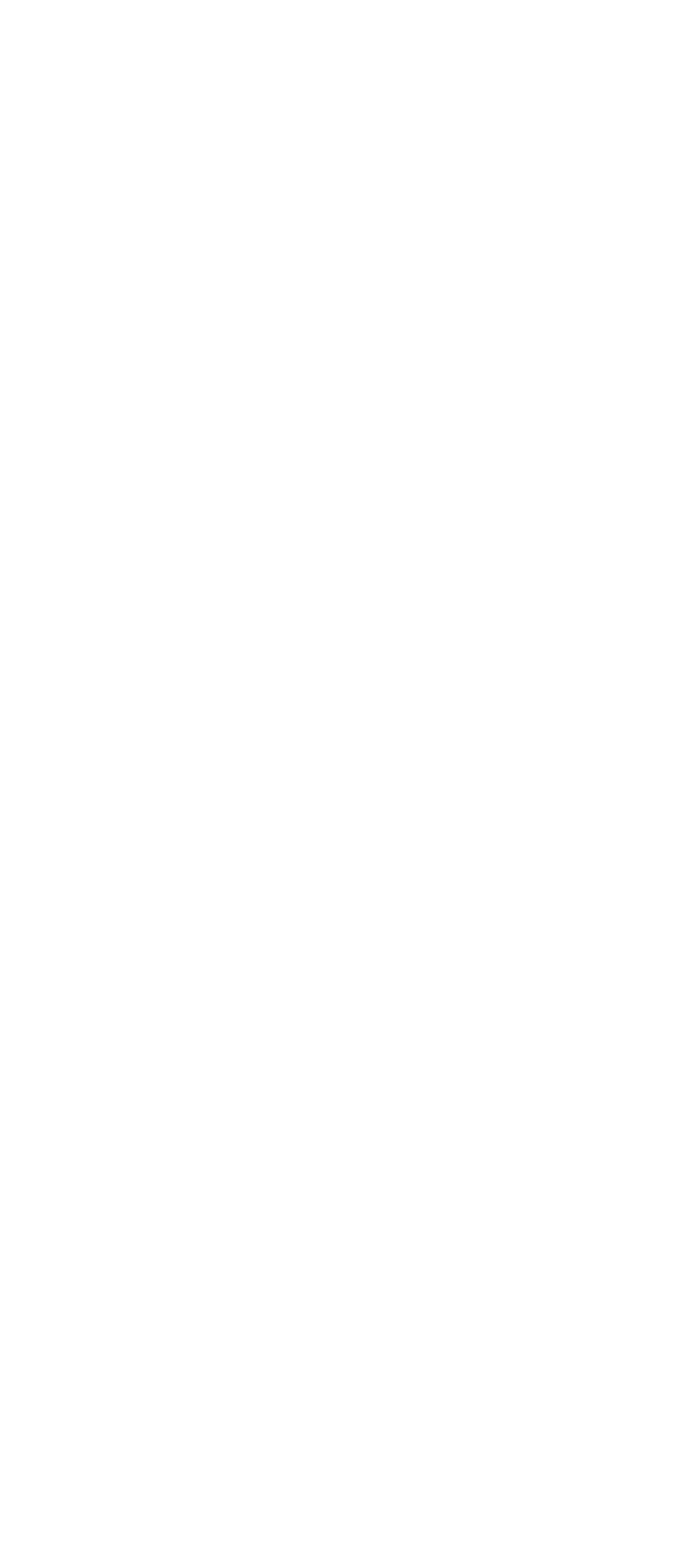




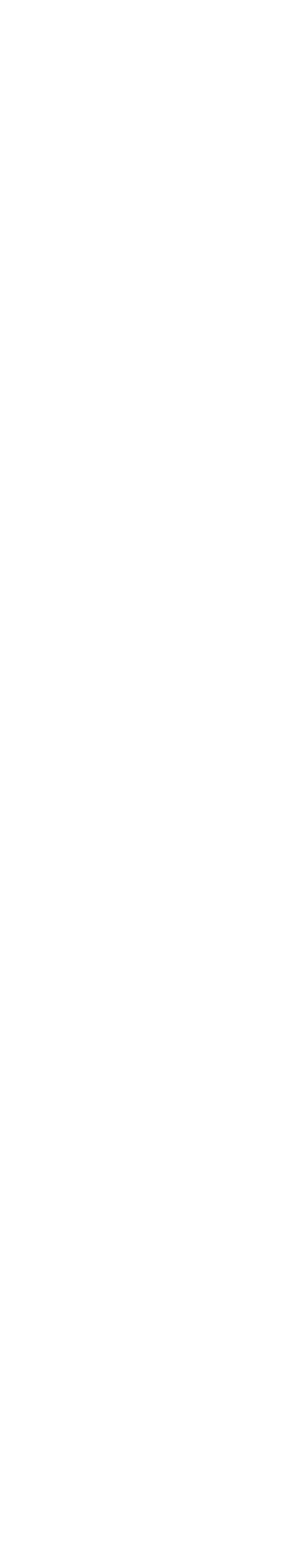




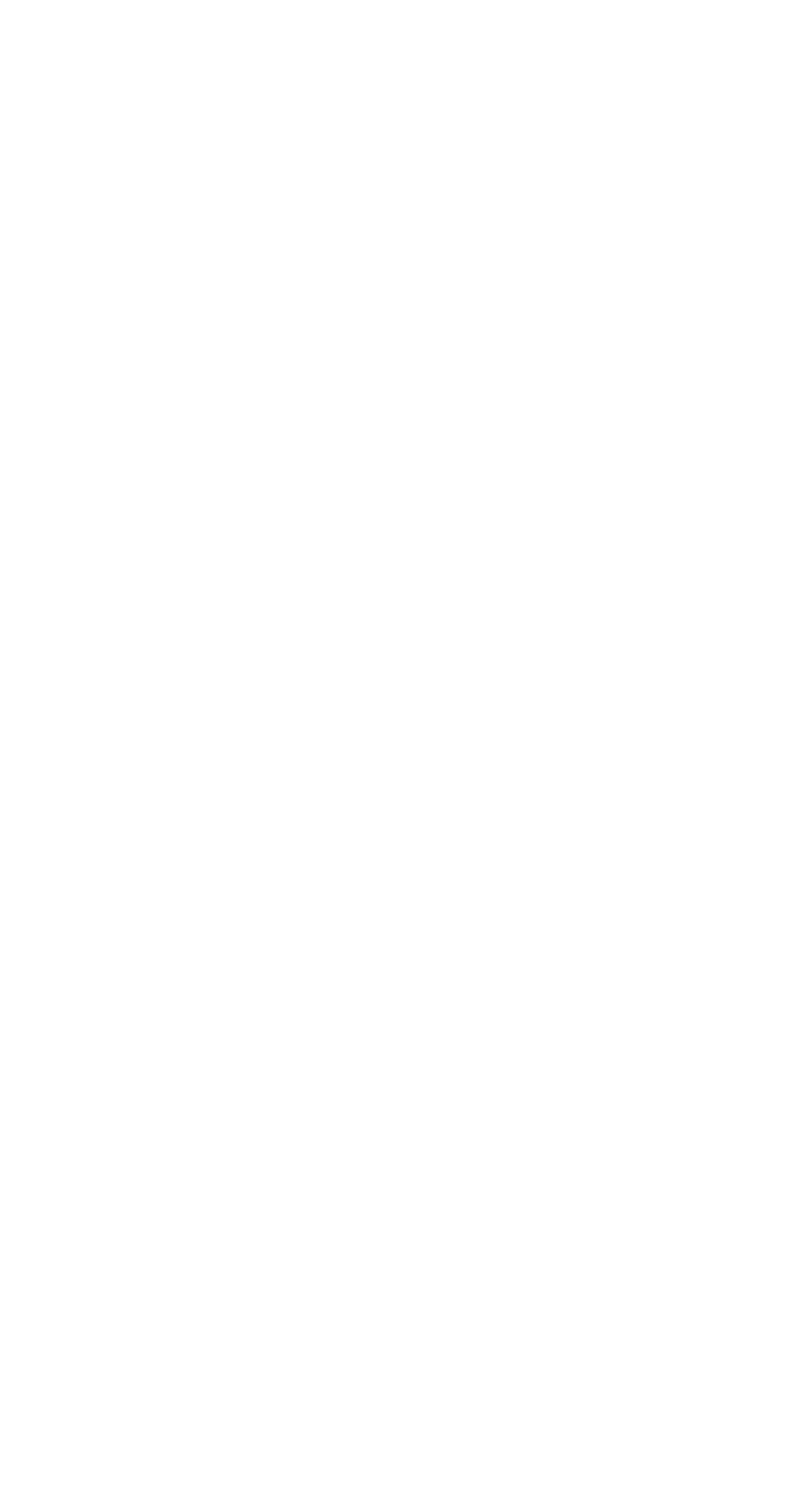




\begin{tabular}{|c|}
\hline 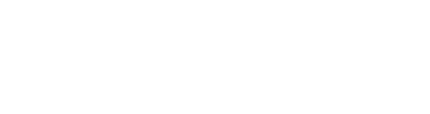 \\
\hline 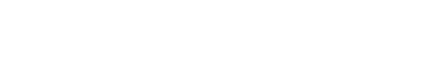 \\
\hline 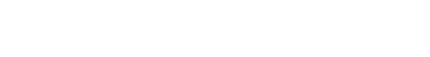 \\
\hline 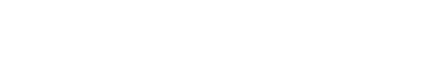 \\
\hline 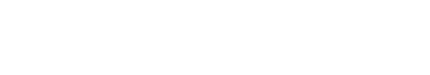 \\
\hline 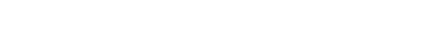 \\
\hline 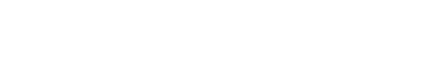 \\
\hline 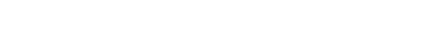 \\
\hline 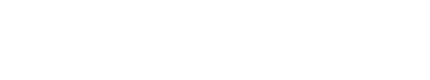 \\
\hline 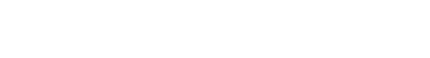 \\
\hline 0001001000010011000 \\
\hline 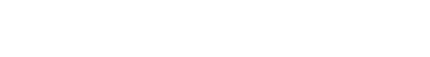 \\
\hline 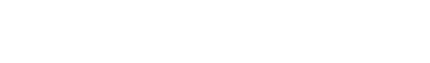 \\
\hline 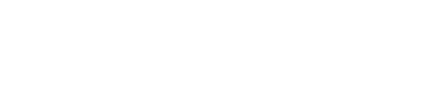 \\
\hline 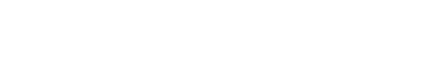 \\
\hline 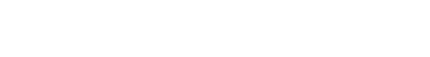 \\
\hline 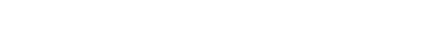 \\
\hline 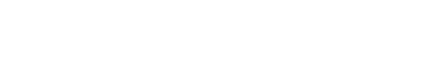 \\
\hline 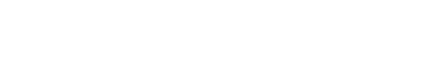 \\
\hline 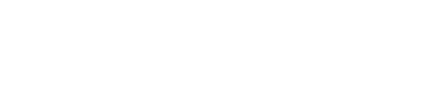 \\
\hline 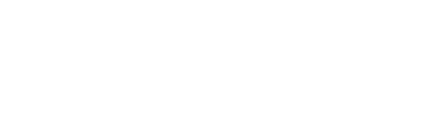 \\
\hline 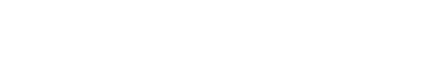 \\
\hline
\end{tabular}


ARKANSAS RIVER BASIN--Continued

7-1470.7. WHI TEWATER RIVER AT TOWANDA, KANS,--Continued

Specific conductance, water year October 1963 to September 1964 (Continuous conductivity recorder)

\begin{tabular}{|c|c|c|c|c|c|c|c|c|c|c|c|c|}
\hline \multirow{3}{*}{ Day } & \multicolumn{4}{|c|}{ OCTOBER } & \multicolumn{4}{|c|}{ NOVEMBER } & \multicolumn{4}{|c|}{ DECENTER } \\
\hline & \multirow{2}{*}{$\begin{array}{l}\text { Mean } \\
\text { dis- } \\
\text { charge } \\
\text { (cfs) }\end{array}$} & \multicolumn{3}{|c|}{$\begin{array}{l}\text { Specific conductance } \\
\left(\text { micromhos at } 25^{\circ} \mathrm{C}\right)\end{array}$} & \multirow{2}{*}{$\begin{array}{l}\text { Mean } \\
\text { dis- } \\
\text { charge } \\
\text { (cfs) }\end{array}$} & \multicolumn{3}{|c|}{$\begin{array}{l}\text { Specific conductance } \\
\text { (micromhos at } 25^{\circ} \mathrm{C} \text { ) }\end{array}$} & \multirow{2}{*}{$\begin{array}{l}\text { Mean } \\
\text { dis- } \\
\text { charge } \\
\text { (cfs) }\end{array}$} & $\begin{array}{l}\text { Speci } \\
\text { (micr }\end{array}$ & $\begin{array}{l}\text { c con } \\
\text { hos a }\end{array}$ & $\begin{array}{l}\text { ctance } \\
\left.25^{\circ} \mathrm{C}\right)\end{array}$ \\
\hline & & Max. & Min. & Mean & & Max. & Min. & Mean & & Max. & Min. & Mean \\
\hline 1 & 8.8 & 2410 & 2250 & 2300 & 9.6 & 1620 & 1480 & 1570 & 13 & 2420 & 1880 & 2380 \\
\hline 2 & 8.8 & 2550 & 2250 & 2390 & 9.6 & 1700 & 1500 & 1670 & 13 & 2390 & 2200 & 2380 \\
\hline 3 & 8.8 & 2570 & 2300 & 2400 & 9.6 & 1790 & 1500 & 1700 & 13 & 2400 & 1990 & 2260 \\
\hline 4 & 8.4 & 2580 & 2300 & 2460 & 9.6 & 1790 & 1620 & 1740 & 13 & 2400 & 2300 & 2360 \\
\hline 5 & 8.0 & 2610 & 2400 & 2470 & 9.6 & 1900 & 1620 & 1750 & 13 & 2390 & 2390 & 2390 \\
\hline 6 & 7.2 & 2600 & 2400 & 2500 & 10 & 1990 & 1800 & 1910 & 13 & 2400 & 2190 & 2290 \\
\hline 7 & 6.8 & 2590 & 2310 & 2440 & 11 & 2020 & 1790 & 1920 & 14 & 2430 & 2100 & 2330 \\
\hline 8 & 6.8 & 2610 & 2310 & 2410 & 11 & 2080 & 1990 & 2050 & 14 & 2420 & 2090 & 2350 \\
\hline 9 & 6 & 2610 & 2380 & 2510 & 11 & 2190 & 2050 & 2140 & 13 & 2400 & 2010 & 2240 \\
\hline 10 & 5.7 & 2640 & 2610 & 2620 & 11 & 2200 & 2040 & 2150 & 13 & 2290 & 2200 & 2240 \\
\hline 11 & 5.1 & 2630 & 2610 & 2590 & 11 & 2200 & 2140 & 2170 & 14 & 2400 & 2290 & 2340 \\
\hline 12 & 4.8 & 2800 & 2600 & 2700 & 10 & 2220 & 2000 & 2220 & 13 & 2500 & 2400 & 2450 \\
\hline 13 & 4.8 & 2790 & 2610 & 2670 & 9.6 & 2310 & 2200 & 2260 & 13 & 2590 & 2460 & 2510 \\
\hline 14 & 4.8 & 2800 & 2650 & 2730 & 8.4 & 2390 & 2250 & 2380 & 13 & 2600 & 2500 & 2580 \\
\hline 15 & 4.8 & 2800 & 2590 & 2770 & 8.8 & 2400 & 2390 & 2400 & 12 & 2600 & 2490 & 2560 \\
\hline 16 & 12 & 2650 & 2410 & 2550 & 9.6 & 2440 & 2390 & 2400 & 12 & 2500 & 2300 & 2460 \\
\hline 17 & 24 & 2580 & 2200 & 2390 & 10 & 2420 & 1800 & 2300 & 12 & 2500 & 2270 & 2400 \\
\hline 18 & 200 & 2800 & 610 & 1260 & 11 & 2400 & 2350 & 2390 & 13 & 2600 & 2550 & 2580 \\
\hline 19 & 40 & 980 & 610 & 818 & 12 & 2390 & 2070 & 2290 & 12 & 2700 & 2370 & 2600 \\
\hline 20 & 26 & 1310 & 980 & 1180 & 18 & 2290 & 1990 & 2160 & 12 & 2700 & 2350 & 2680 \\
\hline 21 & 37 & 1500 & 880 & 1290 & 20 & 2390 & 2150 & 2170 & 12 & 2850 & 2700 & 2820 \\
\hline 22 & 141 & 1400 & 580 & 980 & 20 & 2390 & 2000 & 2260 & 12 & 2830 & 2800 & 2810 \\
\hline 23 & 72 & 1510 & 1080 & 1300 & 22 & 2400 & 2050 & 2260 & 13 & 2800 & 2790 & 2800 \\
\hline 24 & 37 & 1080 & 800 & 903 & 18 & 2220 & 2000 & 2120 & 13 & 2810 & 2800 & 2800 \\
\hline 25 & 26 & 900 & 800 & 875 & 17 & 2200 & 2090 & 2110 & 14 & 2800 & 2500 & 2760 \\
\hline 26 & 20 & 1000 & 900 & 950 & 16 & 2220 & 2050 & 2200 & 15 & 2900 & 2610 & 2710 \\
\hline 27 & 15 & 1210 & 1000 & 1070 & 15 & 2300 & 2200 & 2250 & 16 & 2800 & 2620 & 2700 \\
\hline 28 & 13 & 1270 & 1120 & 1200 & 15 & 2400 & 2200 & 2310 & 17 & 2800 & 2780 & 2790 \\
\hline 29 & 12 & 1380 & 1260 & 1290 & 15 & 2320 & 2200 & 2250 & 16 & 2790 & 2680 & 2750 \\
\hline 30 & 11 & 1500 & 1380 & 1430 & 13 & 2400 & 2180 & 2350 & 16 & 2700 & 2600 & 2650 \\
\hline 31 & 10 & 1500 & 1440 & 1480 & -- & -- & -- & -- & 15 & 2600 & 2400 & 2460 \\
\hline Mont & thly me & $\cdots$ & $\cdots$ & 1360 & -- & -- & -- & 2140 & -- & -- & -- & 2530 \\
\hline & & JAN & ARY & & & FEBR & $A R Y$ & & & MAF & & \\
\hline 1 & 14 & 2600 & 2390 & 2450 & 18 & 2210 & 1800 & 2090 & 14 & 2400 & 2000 & 2230 \\
\hline 2 & 15 & 2610 & 2200 & 2490 & 18 & 2250 & 1750 & 2010 & 13 & 2400 & 2000 & 2150 \\
\hline 3 & 16 & 2590 & 2400 & 2560 & 18 & 2010 & 1730 & 1860 & 14 & 2410 & 2390 & 2400 \\
\hline 4 & 18 & 2580 & 2200 & 2450 & 22 & 2000 & 1700 & 1820 & 14 & 2410 & 2390 & 2400 \\
\hline 5 & 17 & 2480 & 2050 & 2360 & 23 & 2010 & 1700 & 1920 & 17 & 2410 & 2350 & 2350 \\
\hline 6 & 16 & 2400 & 1990 & 2200 & 23 & 2040 & 2000 & 2010 & 14 & 2410 & 2190 & 2290 \\
\hline 7 & 16 & 2420 & 2050 & 2200 & 21 & 2110 & 1900 & 2030 & 14 & 2410 & 2300 & 2360 \\
\hline 8 & 16 & 2410 & 2190 & 2340 & 18 & 2190 & 1900 & 2130 & 13 & 2400 & 2400 & 2400 \\
\hline 9 & 15 & 2400 & 2100 & 2290 & 17 & 2200 & 2040 & 2130 & 14 & 2410 & 2380 & 2390 \\
\hline 10 & 15 & 2450 & 2000 & 2260 & 16 & 2220 & 2200 & 2210 & 15 & 2390 & 2010 & 2250 \\
\hline 11 & 16 & 2420 & 1400 & 2000 & 15 & 2200 & 1810 & 2200 & 15 & 2420 & 2010 & 2250 \\
\hline 12 & 15 & 2410 & 1420 & 1850 & 14 & 2190 & 1800 & 2050 & 14 & 2500 & 2200 & 2420 \\
\hline 13 & 14 & 2410 & 2410 & 2410 & 14 & 2190 & 2000 & 2100 & 14 & 2500 & 2400 & 2480 \\
\hline 14 & 13 & 2550 & 2410 & 2470 & 14 & 2200 & 1810 & 2080 & 14 & 2410 & 2400 & 2410 \\
\hline 15 & 12 & 2600 & 2550 & 2570 & 15 & 2200 & 2100 & 2140 & 13 & 2510 & 2390 & 2450 \\
\hline 16 & 12 & 2600 & 2370 & 2530 & 16 & 2120 & 1900 & 2030 & 13 & 2480 & 2350 & 2390 \\
\hline 17 & 12 & 2550 & 2200 & 2290 & 16 & 2050 & 1900 & 1940 & 13 & 2420 & 2150 & 2400 \\
\hline 18 & 14 & 2420 & 2250 & 2360 & 16 & 2100 & 1600 & 2000 & 13 & 2400 & 2210 & 2380 \\
\hline 19 & 15 & 2600 & 2190 & 2500 & 15 & 2200 & 2100 & 2180 & 14 & 2450 & 2010 & 2290 \\
\hline 20 & 16 & 2600 & 2080 & 2350 & 15 & 2200 & 2150 & 2190 & 17 & 2420 & 2010 & 2420 \\
\hline 21 & 16 & 2580 & 2200 & 2430 & 15 & 2200 & 2150 & 2190 & 18 & 2570 & 2420 & 2500 \\
\hline 22 & 16 & 2590 & 2050 & 2400 & 14 & 2200 & 2000 & 2180 & 17 & 2570 & 2210 & 2500 \\
\hline 23 & 17 & 2590 & 2180 & 2470 & 14 & 2250 & 2000 & 2210 & 17 & 2420 & 2390 & 2390 \\
\hline 24 & 17 & 2500 & 2180 & 2350 & 14 & 2250 & 1990 & 2190 & 17 & 2410 & 2400 & 2400 \\
\hline 25 & 16 & 2390 & 1990 & 2120 & 14 & 2220 & 1850 & 2110 & 16 & 2420 & 2400 & 2410 \\
\hline 26 & 15 & 2230 & 1850 & 2050 & 14 & 2210 & 2190 & 2200 & 16 & 2410 & 2040 & 2260 \\
\hline 27 & 15 & 2090 & 1720 & 1860 & 13 & 2300 & 2210 & 2270 & 15 & 2410 & 2010 & 2270 \\
\hline 28 & 15 & 2200 & 1910 & 2050 & 13 & 2300 & 2300 & 2300 & 14 & 2550 & 2400 & 2470 \\
\hline 29 & 14 & 2090 & 1900 & 1950 & 14 & 2400 & 2250 & 2300 & 13 & 2420 & 2010 & 2300 \\
\hline 30 & 14 & 1950 & 1810 & 1850 & -- & -- & -- & -- & 12 & 2200 & 1900 & 2110 \\
\hline 31 & 16 & 2220 & 1700 & 2050 & -- & -- & -- & - & 12 & 2300 & 1900 & 2060 \\
\hline Mont & thly m & $\ldots$ & $\cdots$ & 2270 & -- & $\infty$ & -- & 2090 & -- & - & -- & 2350 \\
\hline
\end{tabular}


ARKANSAS RIVER BASIN--Continued

7-1470.7. WHITEWATER RIVER AT TOWANDA, KANS.--COntinued

Specific conductance, water year october 1963 to September 1964--Continued (Continuous conductivity recorder)

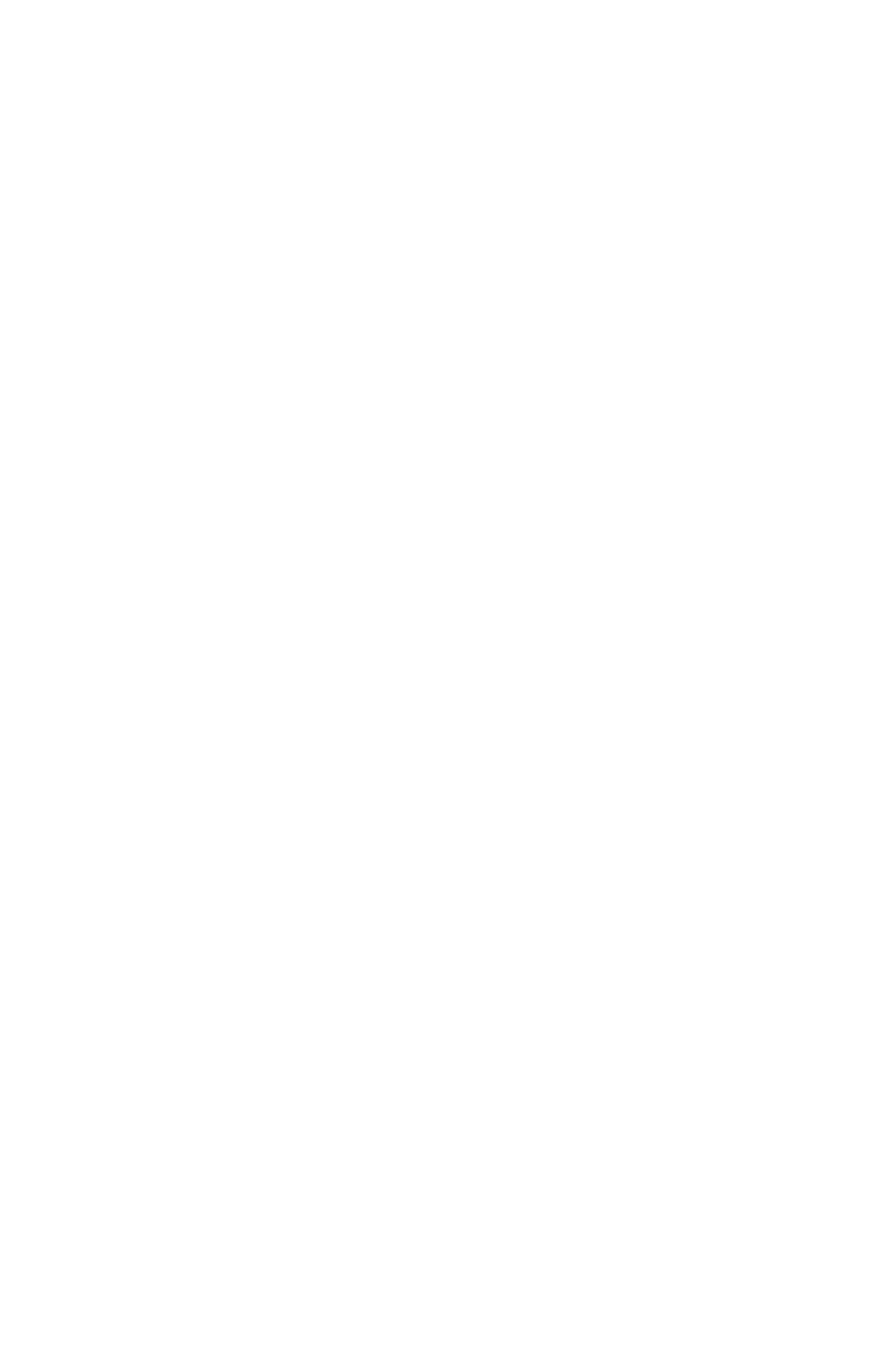

A Includes estimated data for missing days. Represents 100 percent of runoff for water year. 


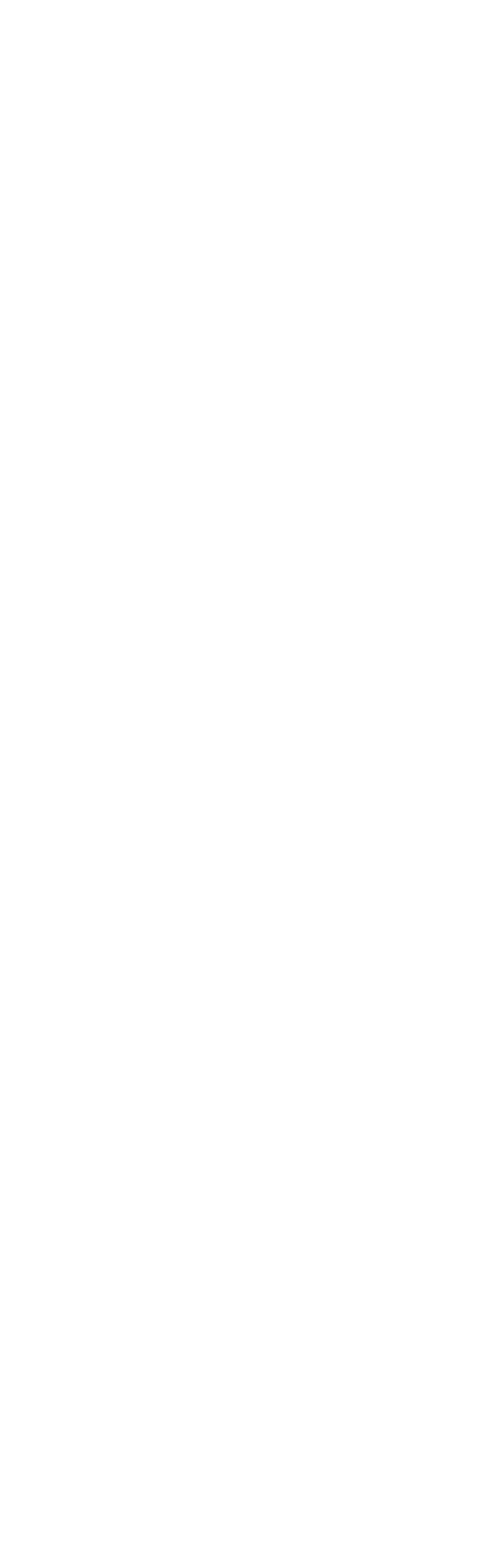




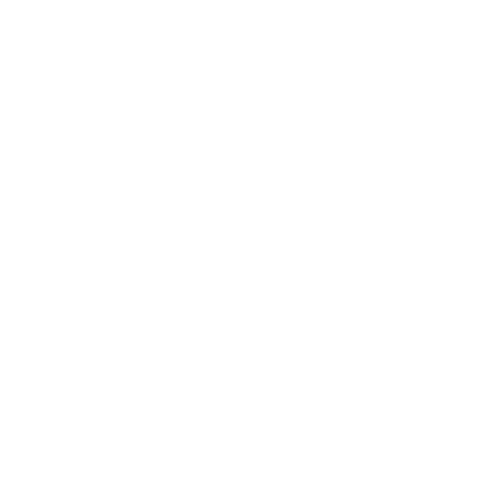

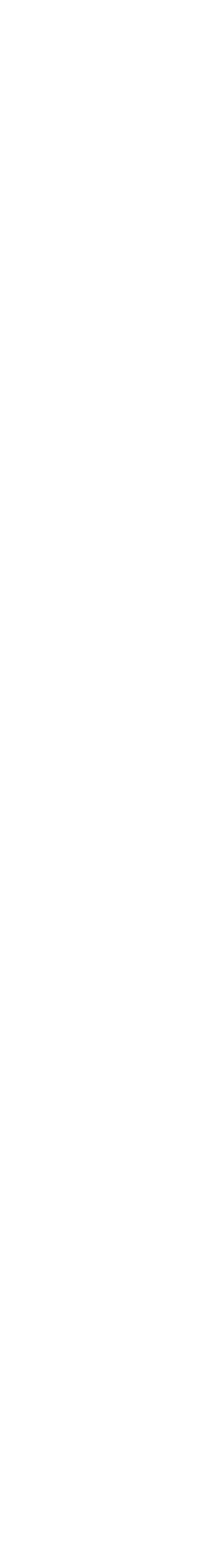



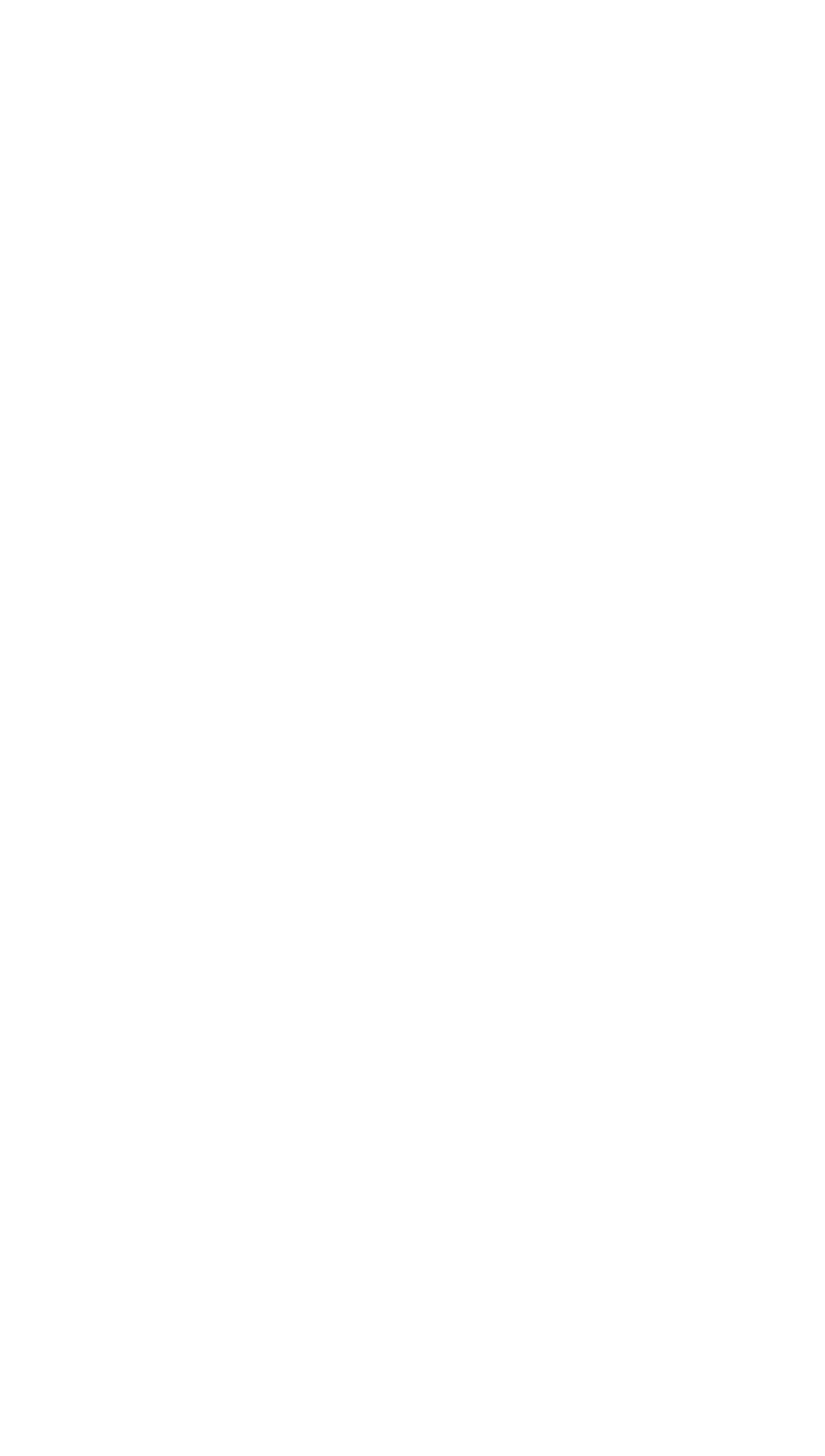


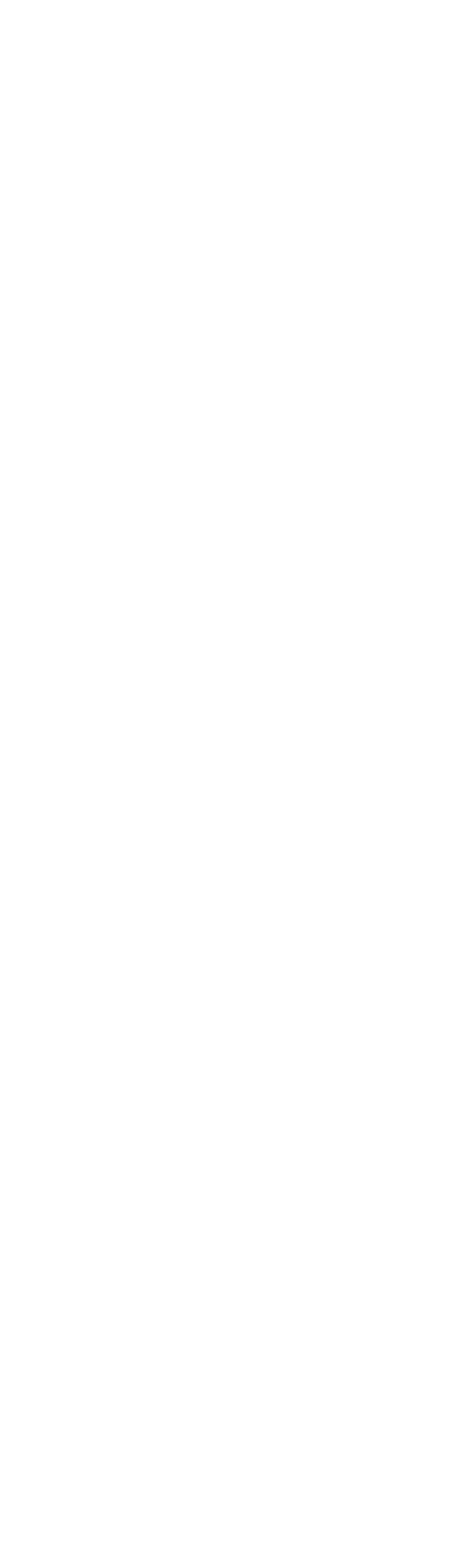

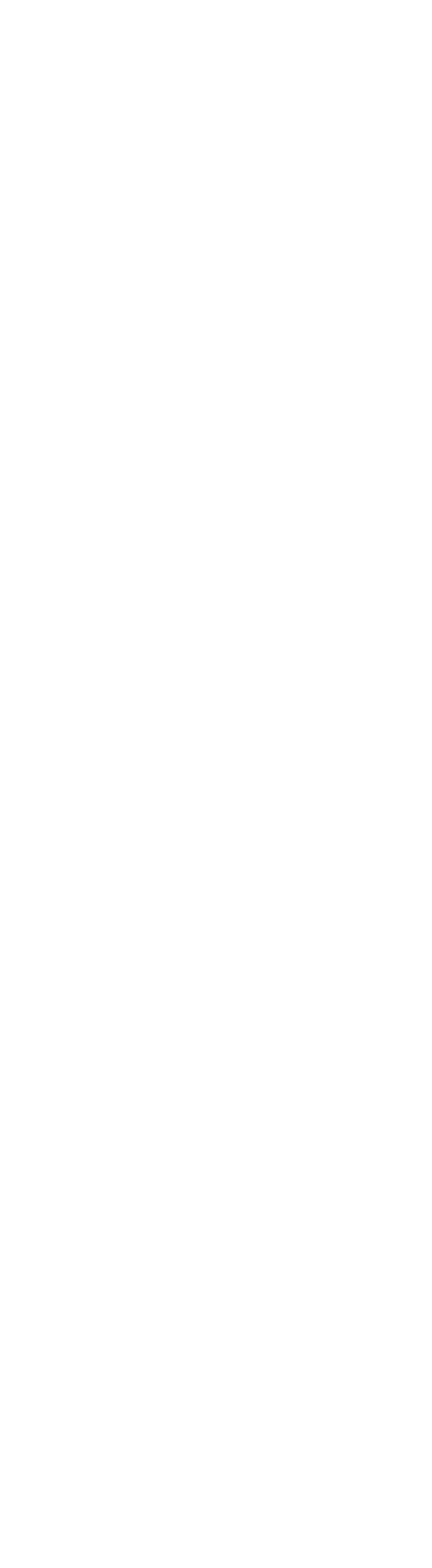




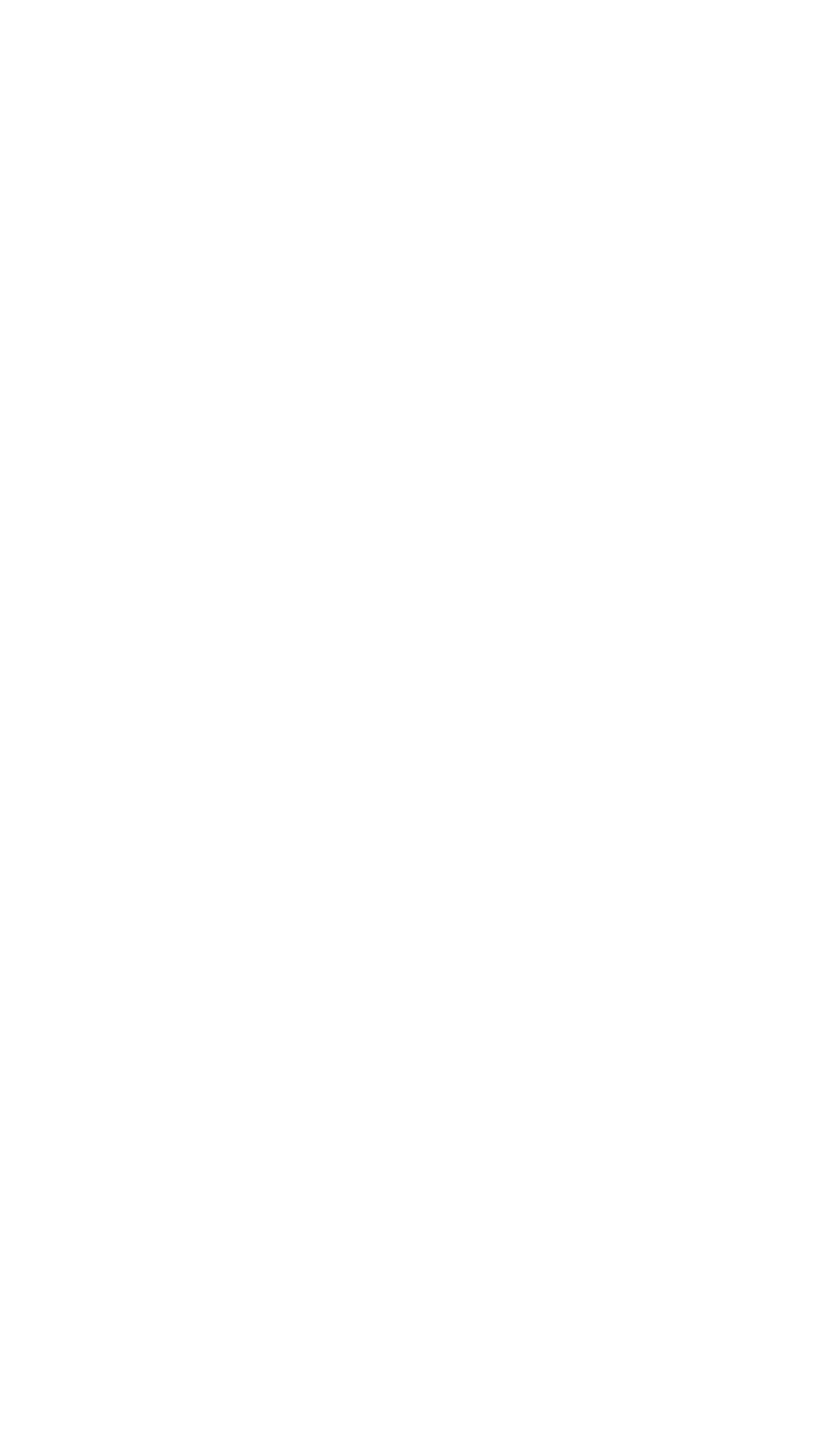




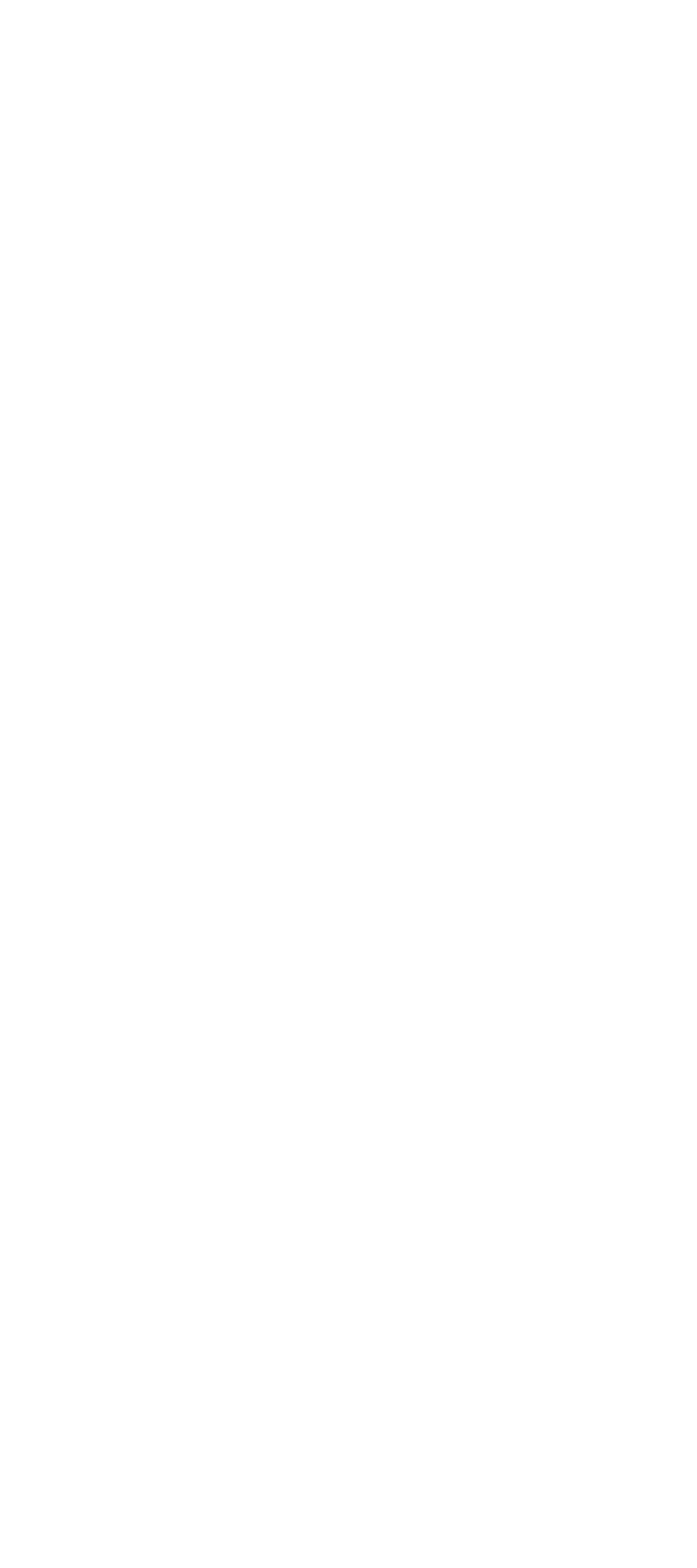




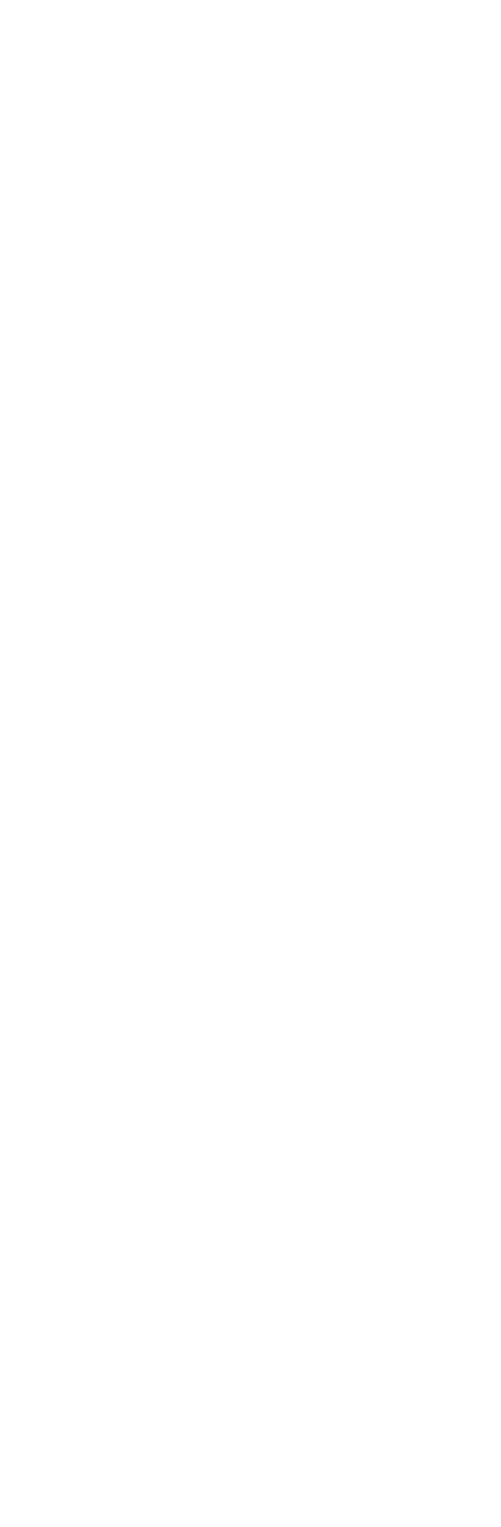


ARKANSAS RIVER BASIN--Continued

7-1478. WALNUT RIVER AT WINFIELD, KANS.--Continued

Suspended sediment, water year October 1963 to September 1964 (Where no daily concentrations are reported, loads are estimated)

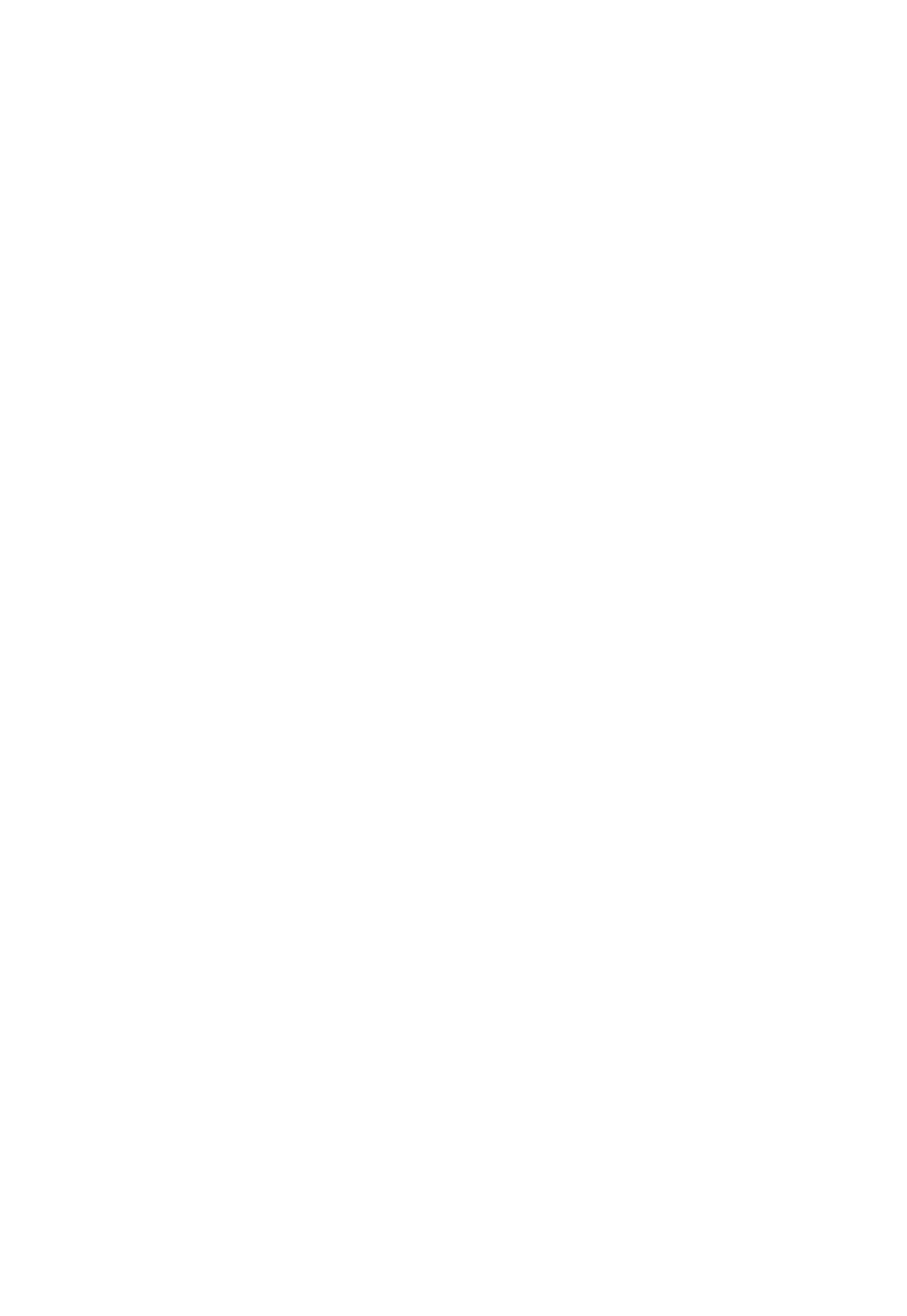


ARKANSAS RIVER BASIN--Continued

7-1478. WALNUT RIVER AT WINFIELD, KANS, --Continued

Suspended sediment, water year October 1963 to September 1964--Continued

(Where no daily concentrations are reported, loads are estimated)

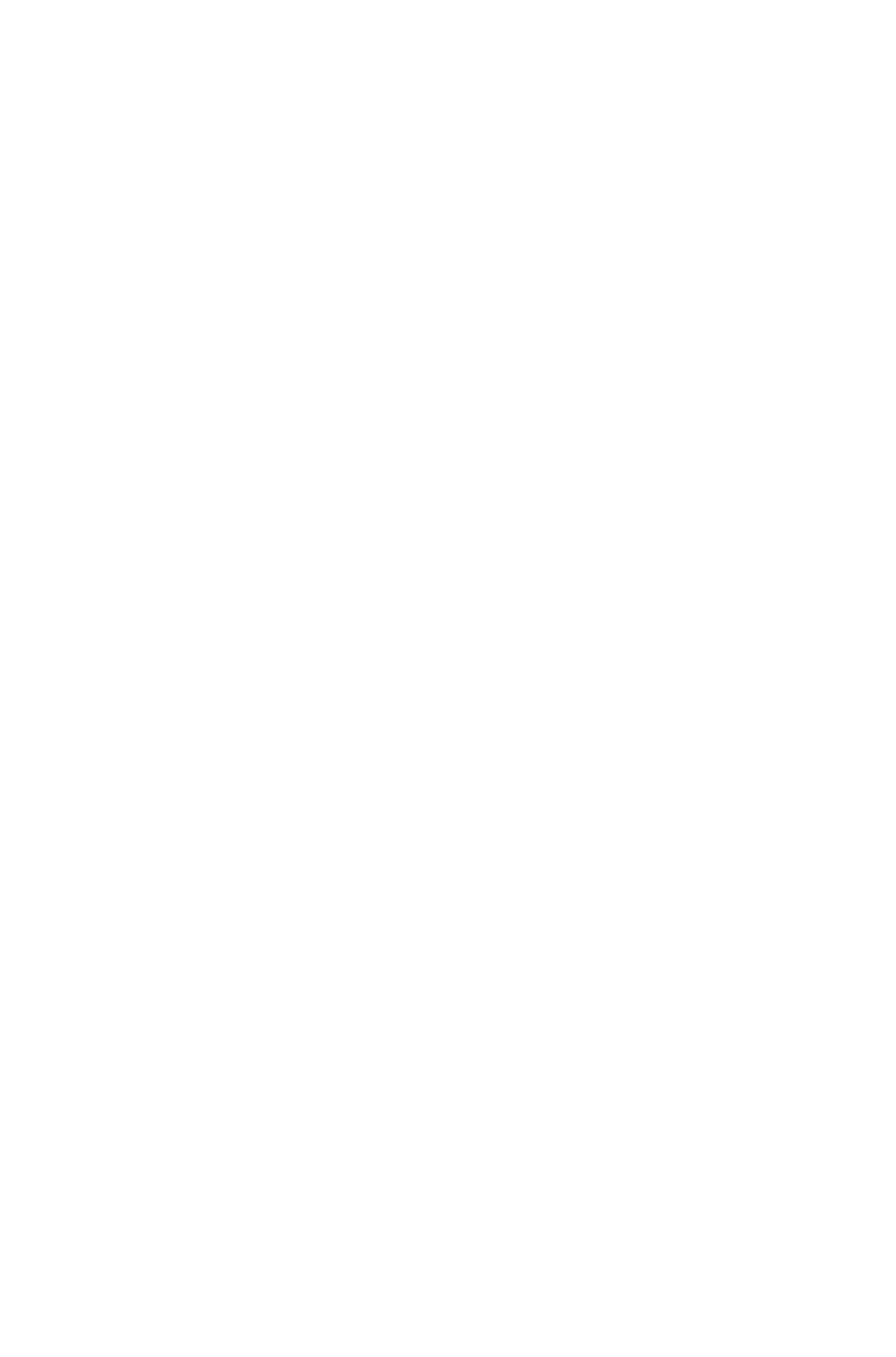

Total load for year (tons)..... 


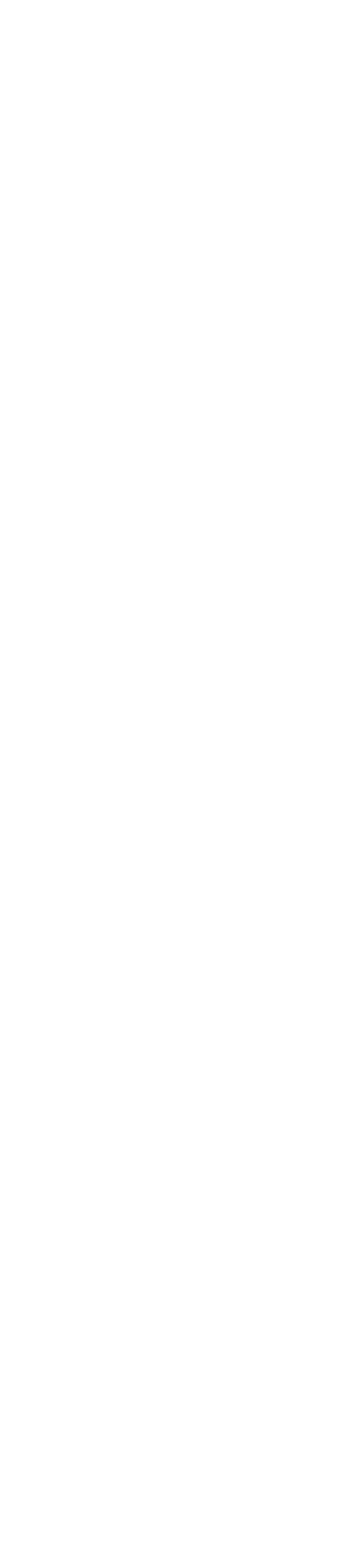




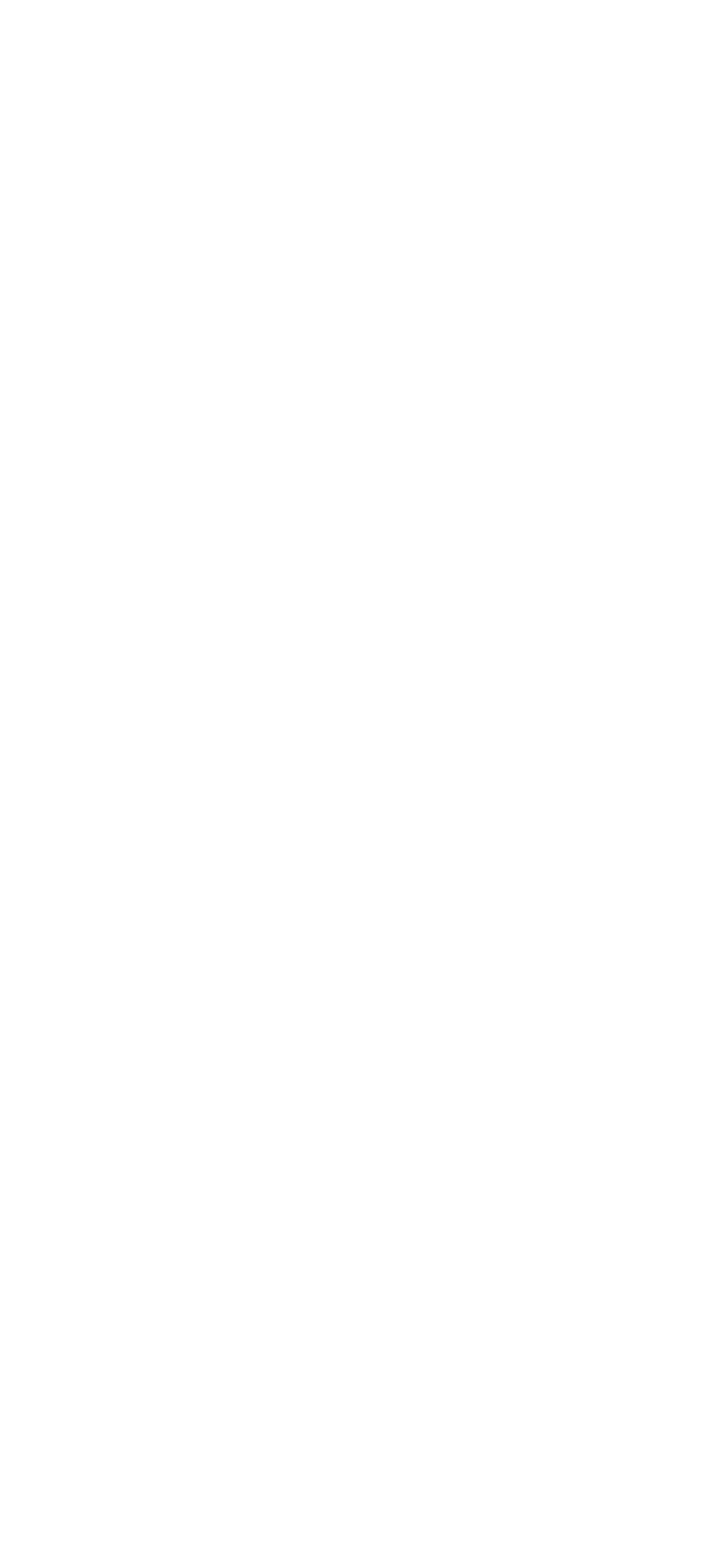




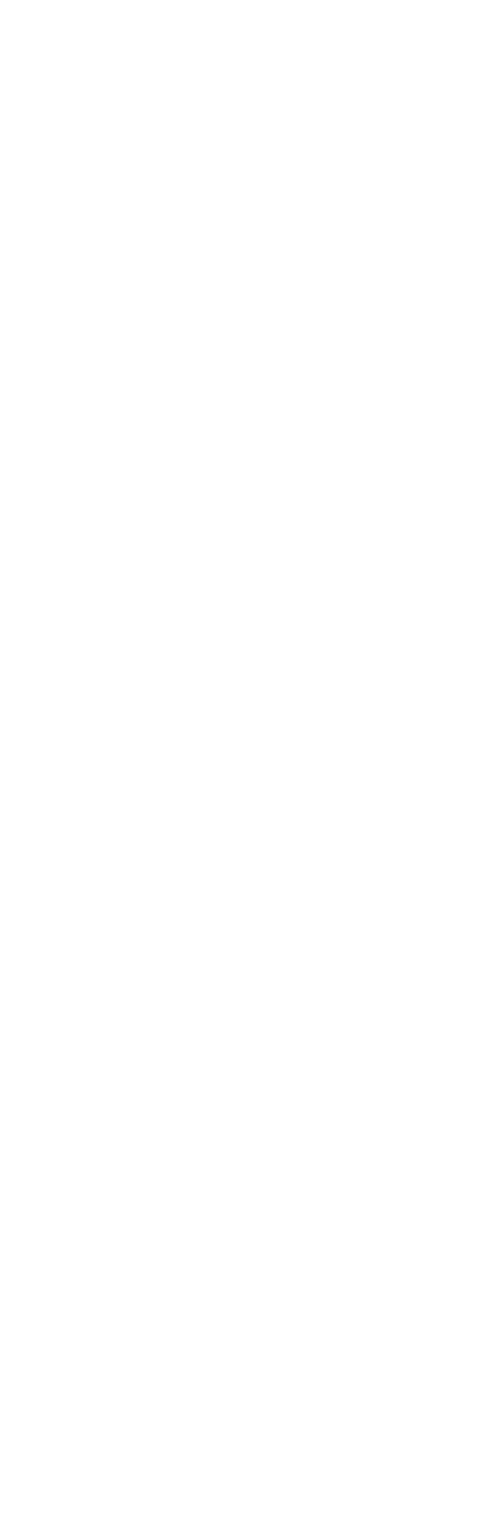


ARKANSAS RIVER BASIN--Continued

7-1515. CHIKASKIA RIVER NEAR CORBIN, KANS.--Continued

Suspended sediment, water year october 1963 to September 1964

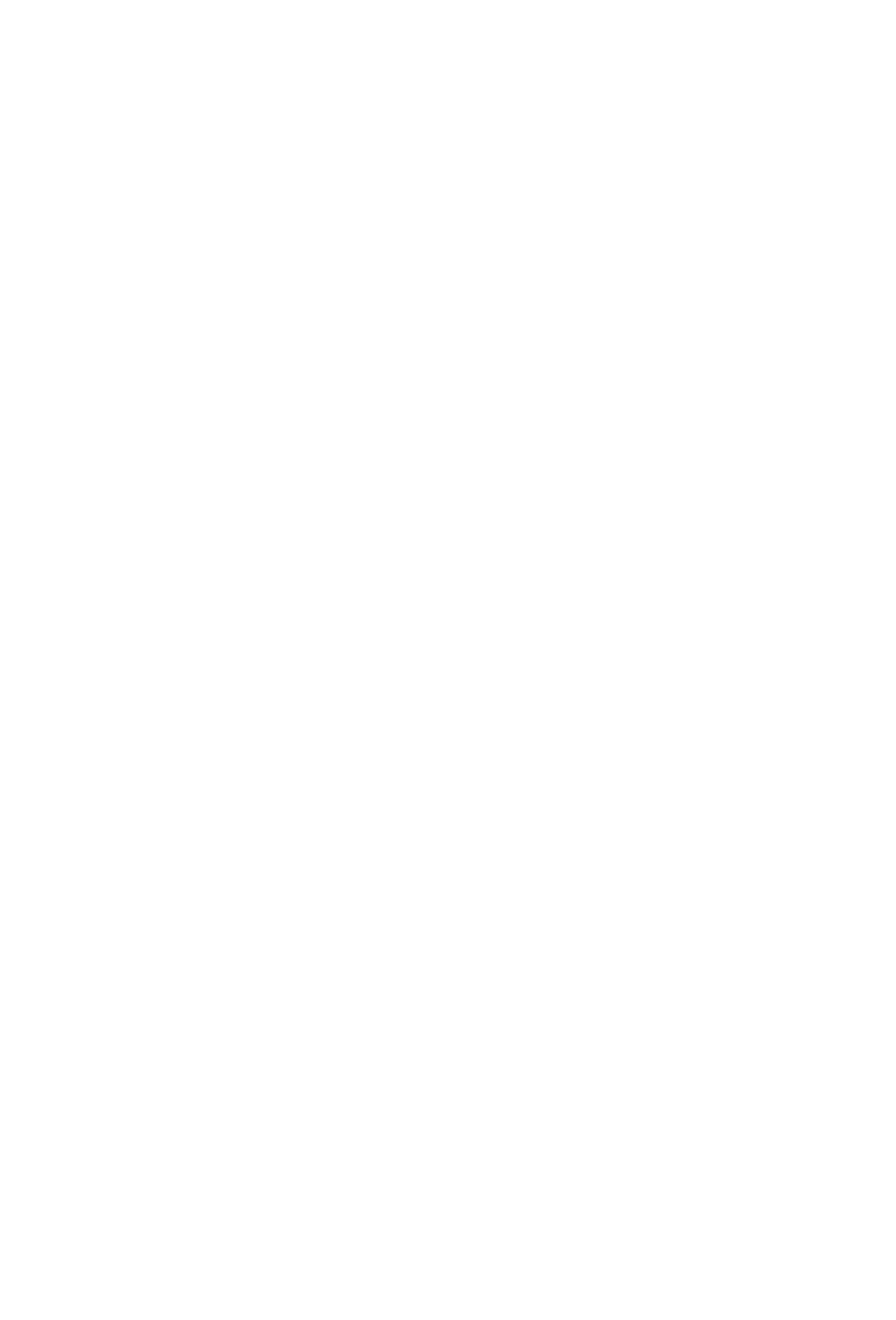

s Computed by subdividing day. 
ARKANSAS RIVER BASIN--Continued

7-1515. CHIKASKIA RIVER NEAR CORBIN, KANS.--Continued

Suspended sediment, water year October 1963 to September 1964--Continued APRIL

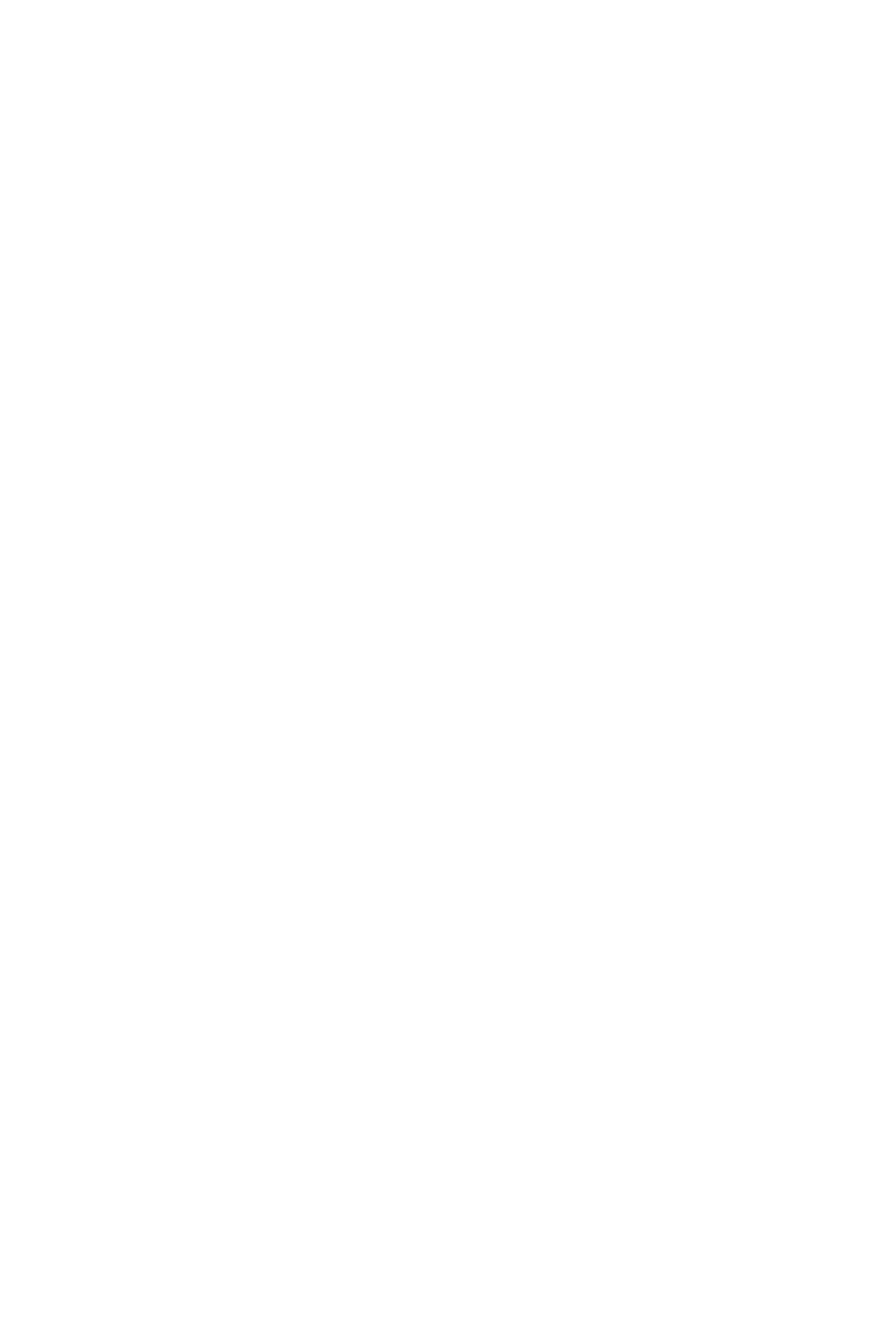

$\therefore$ Computed hy suhdividing day.
Less than 0.50 ton. 

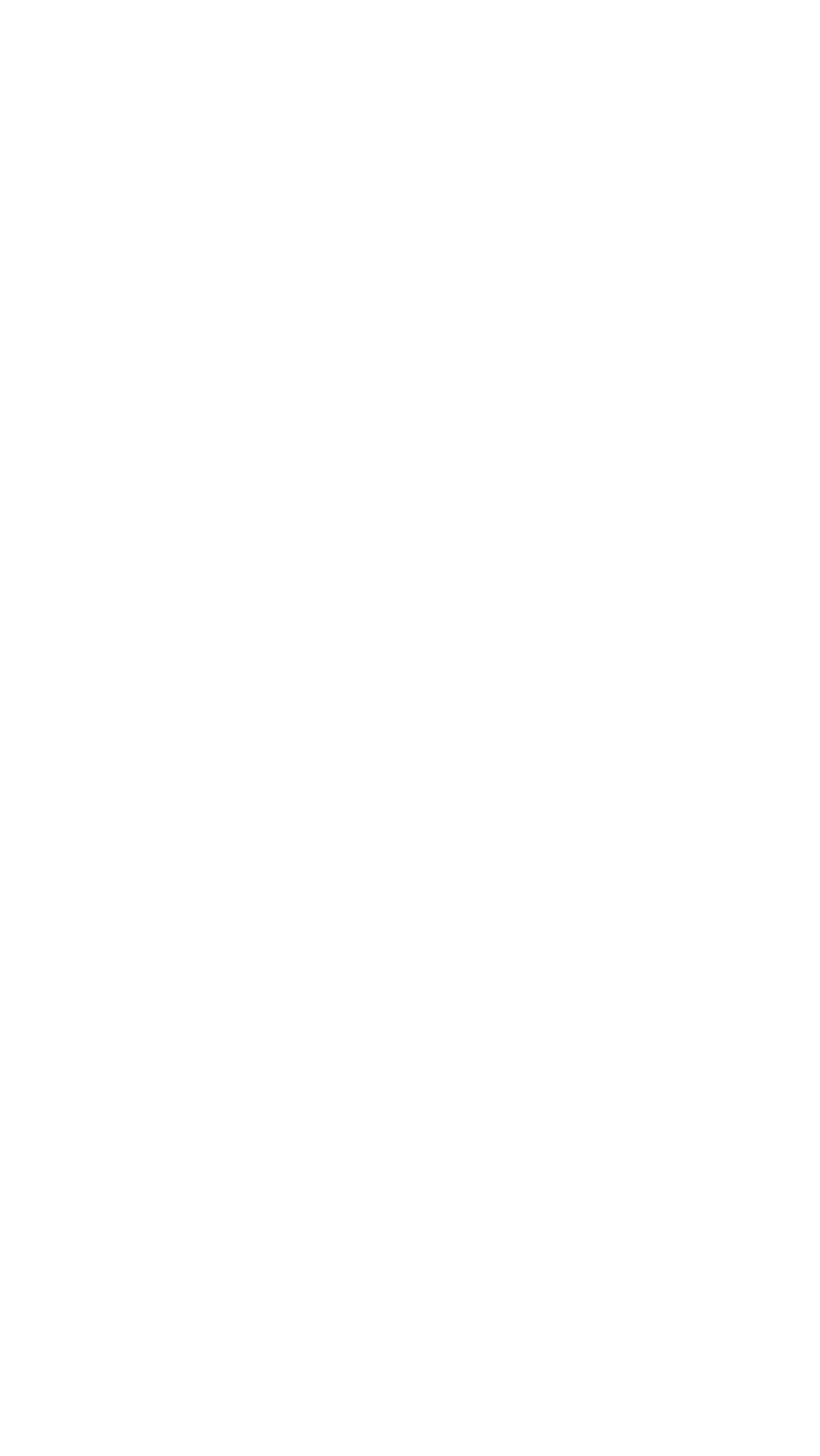


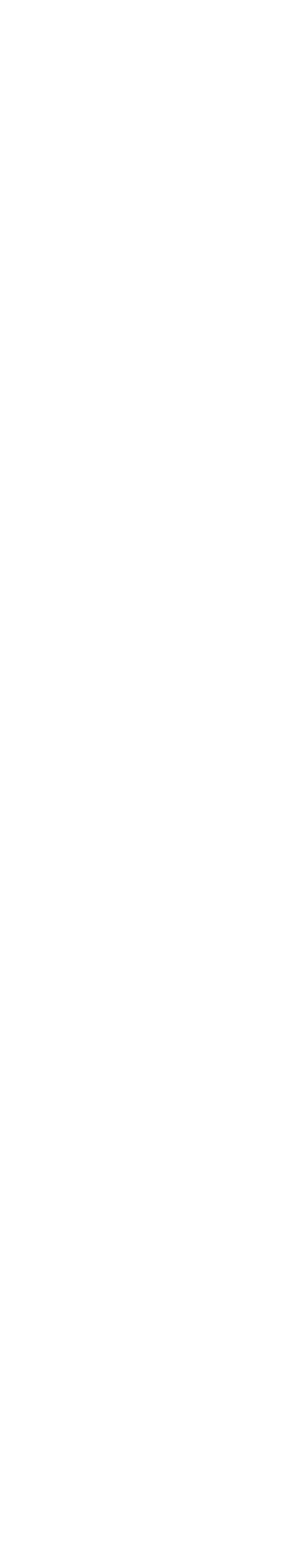

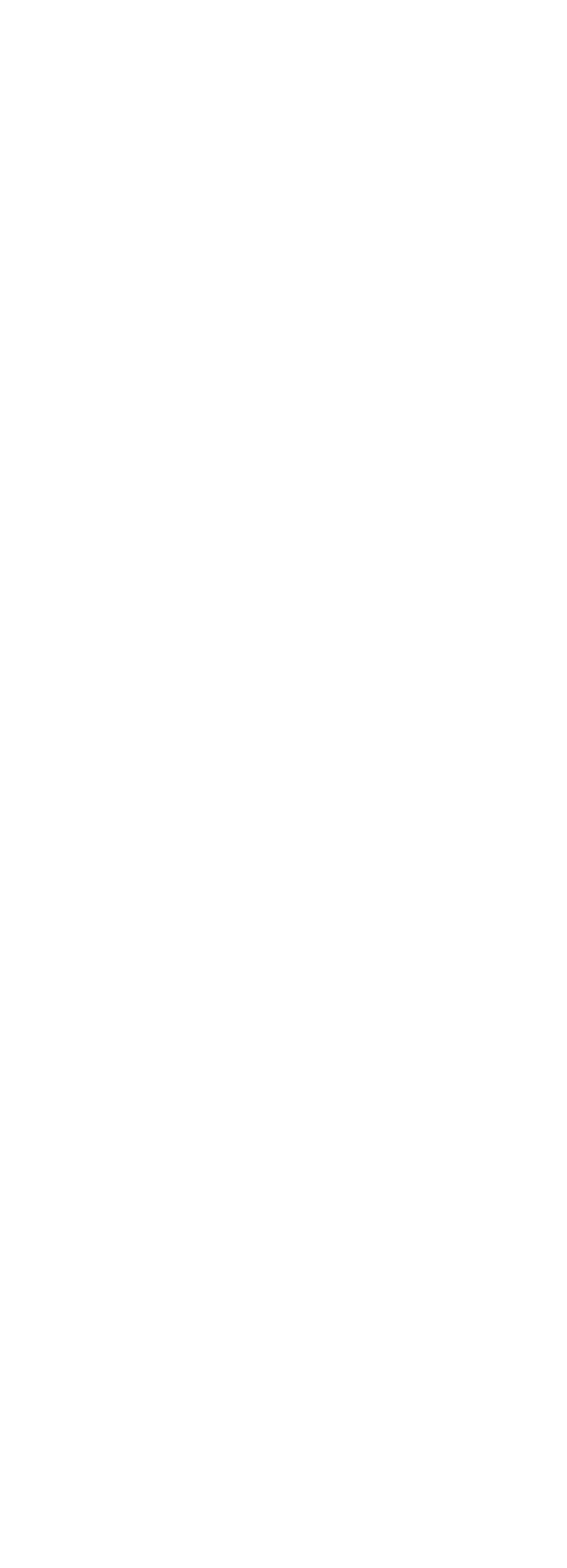




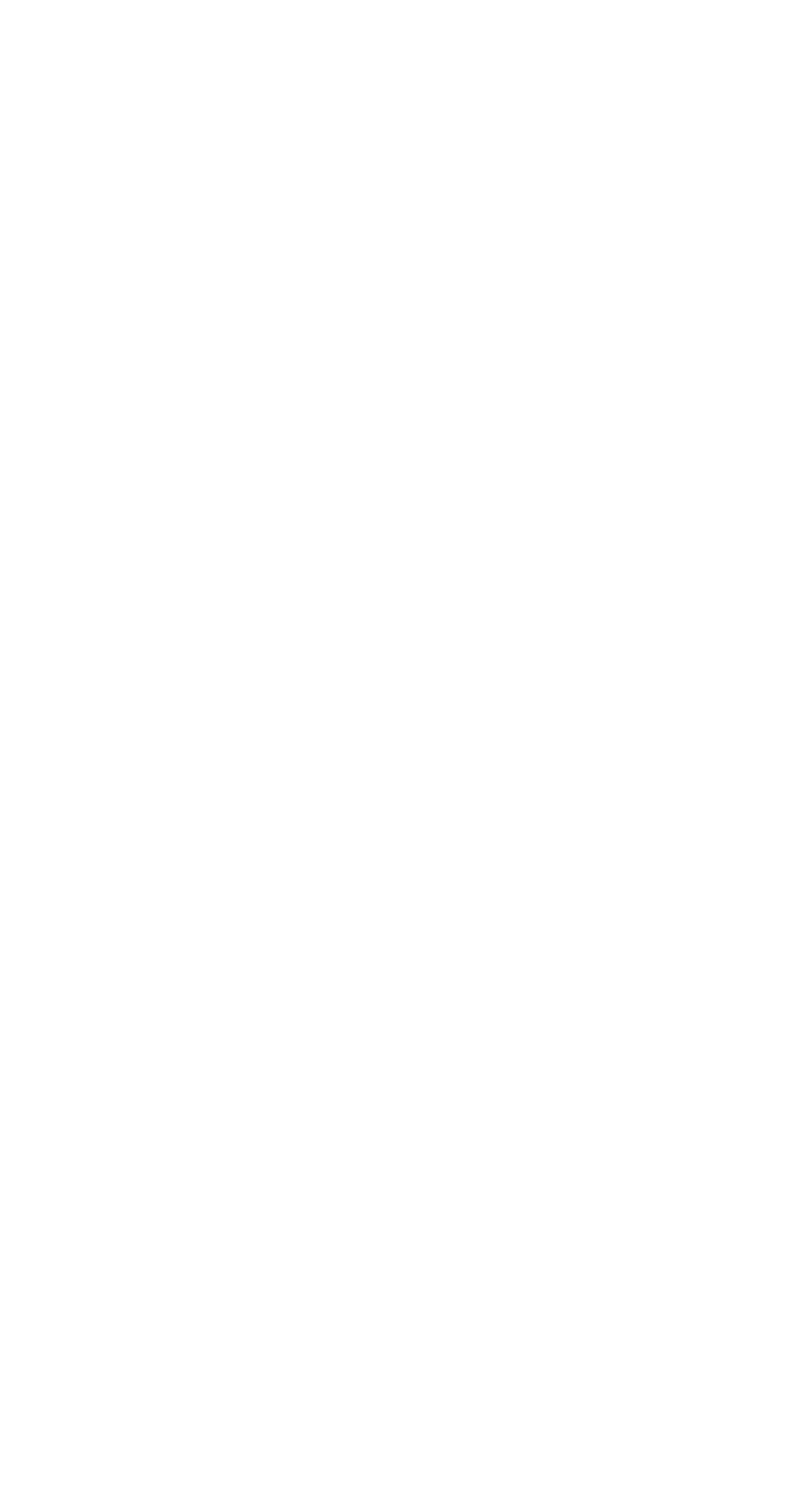



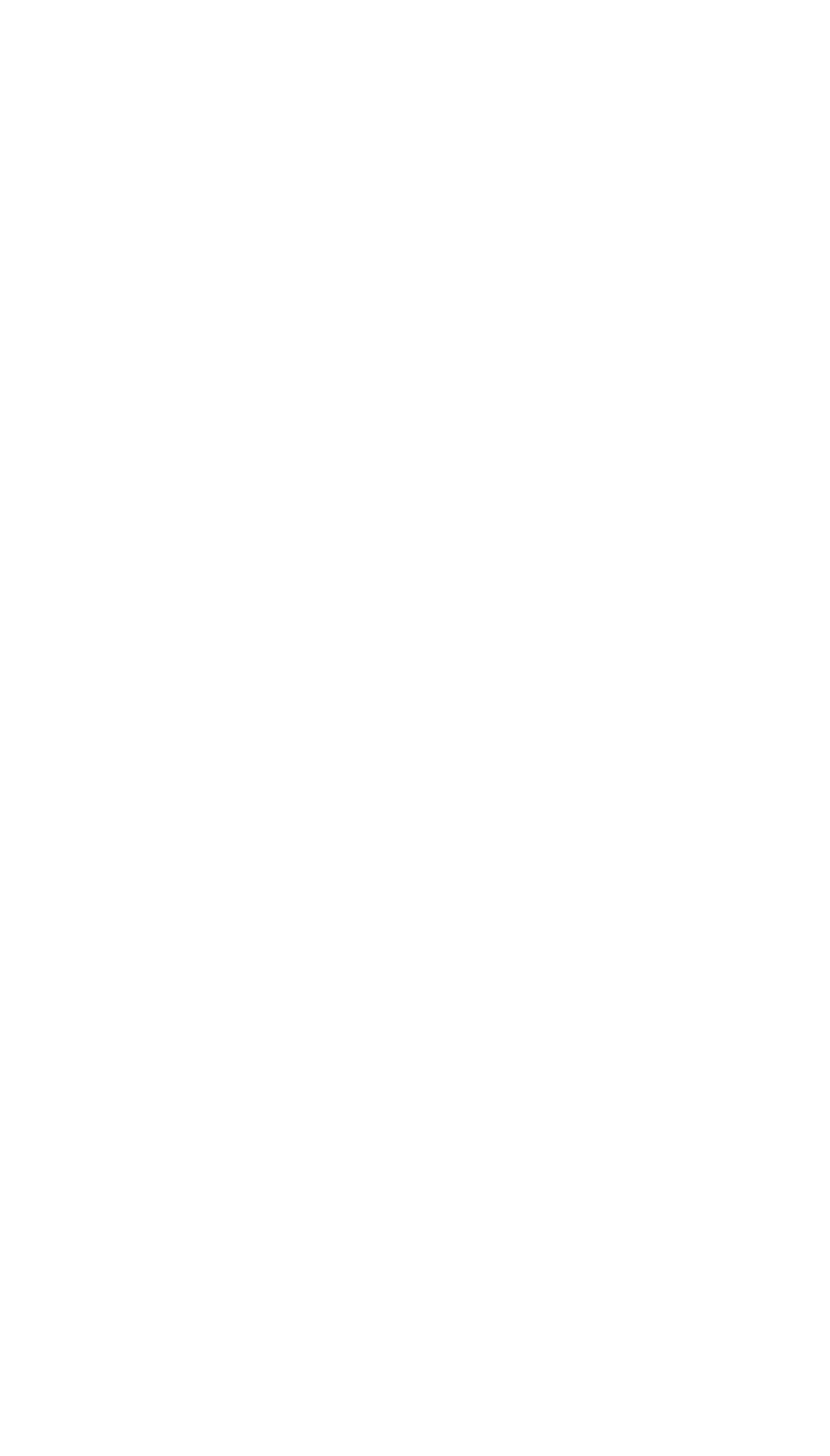


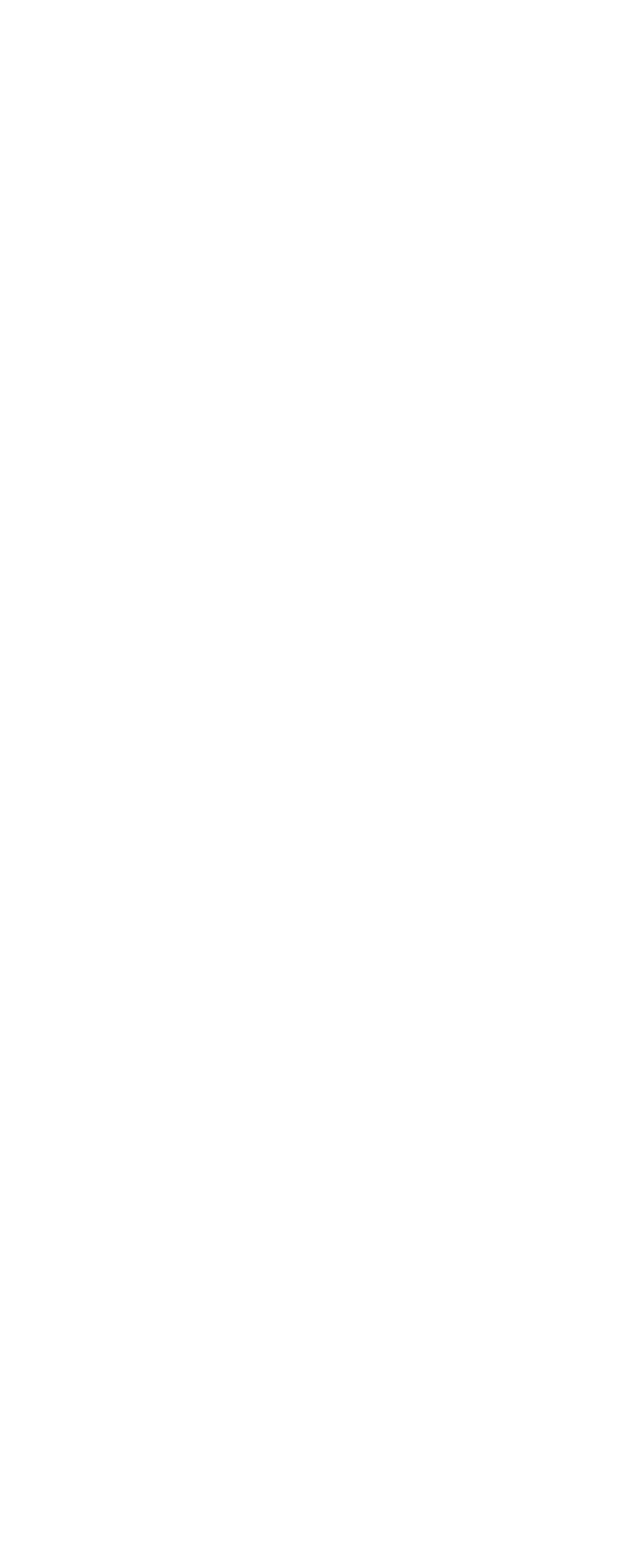




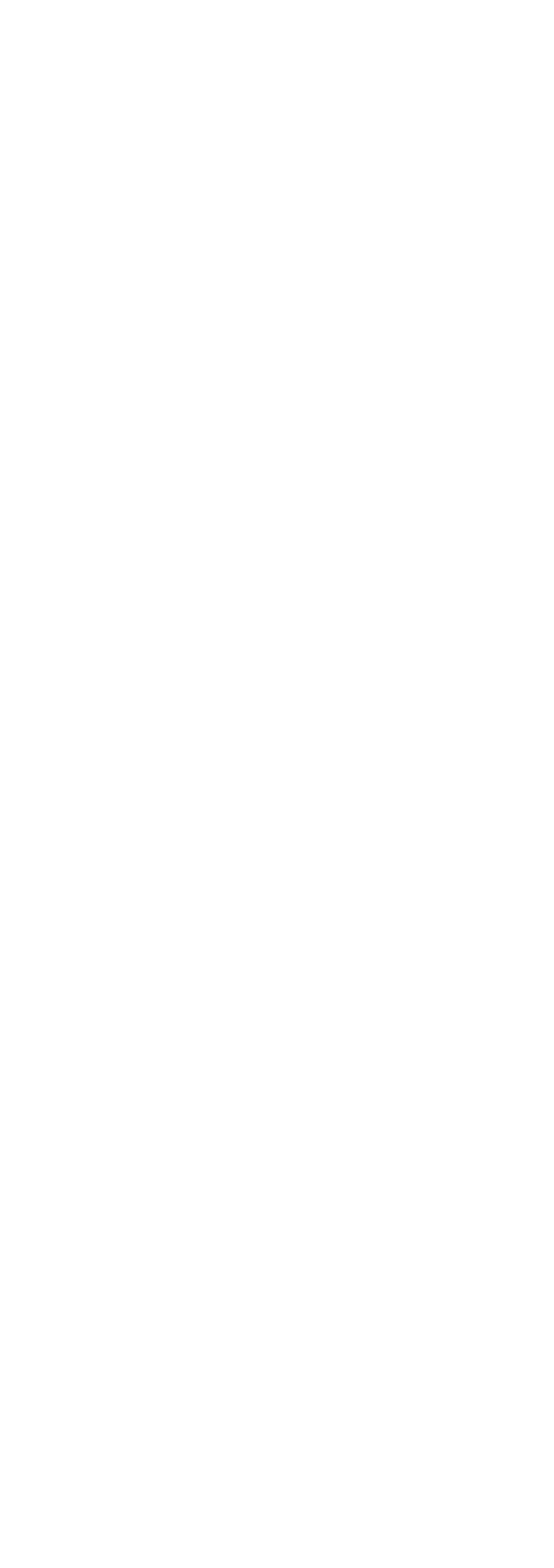




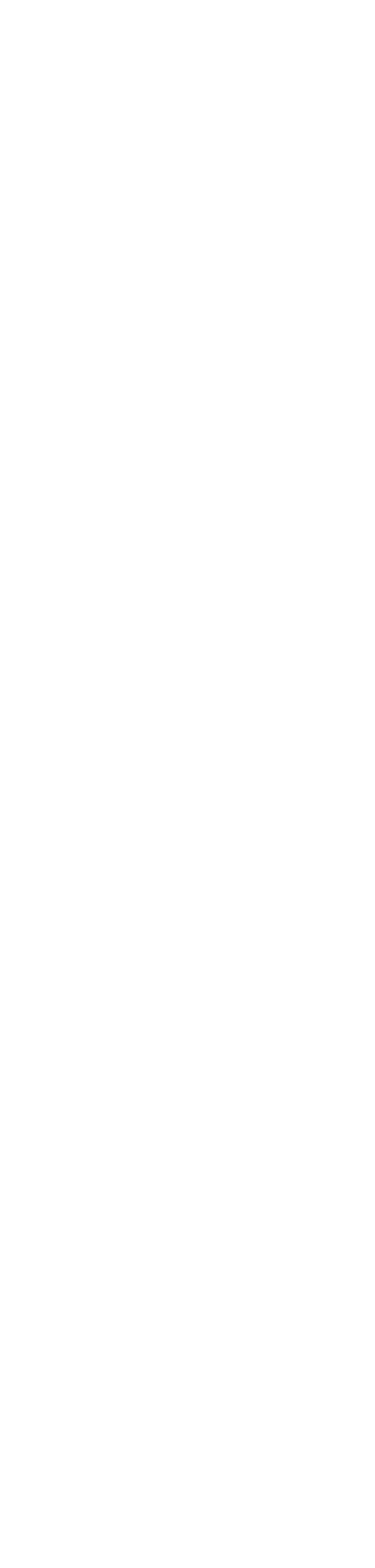

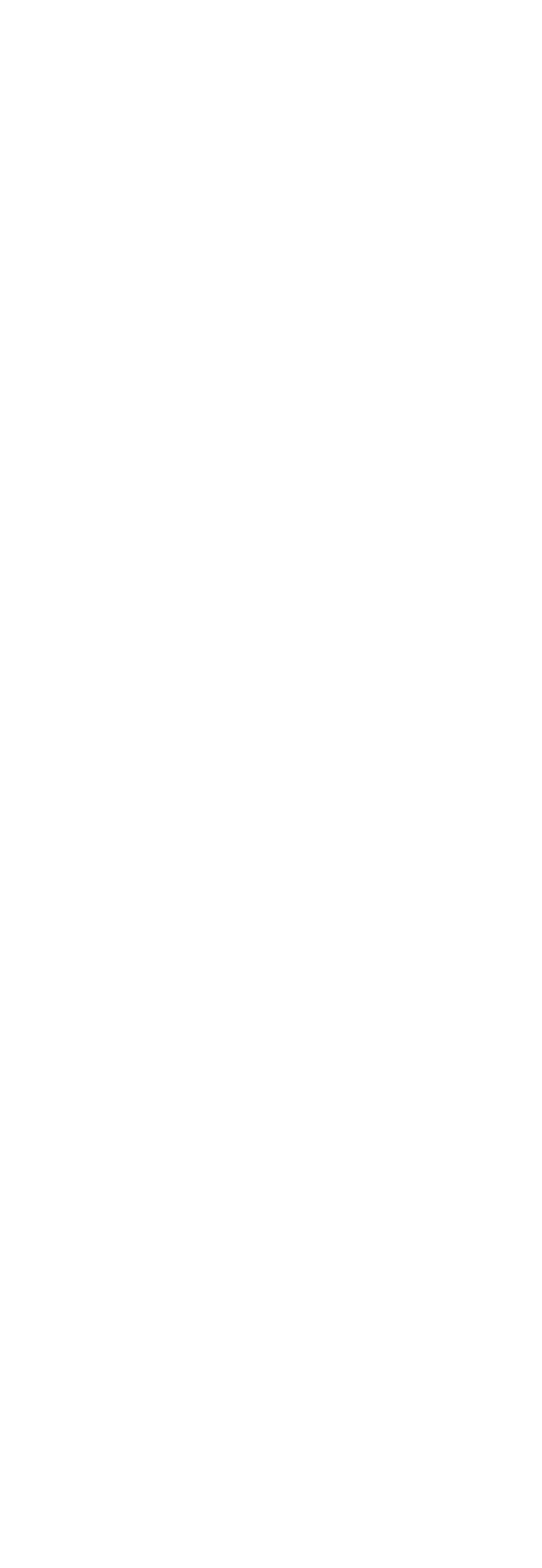




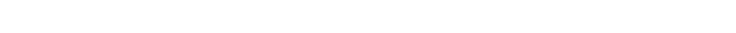

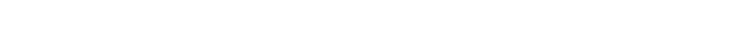

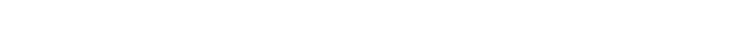

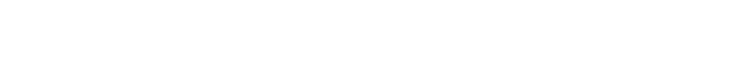

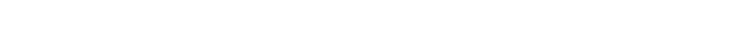

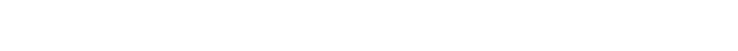

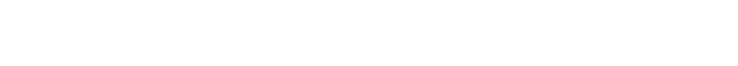

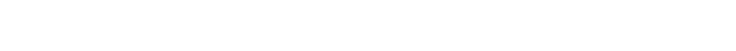
000000000100000000000000070

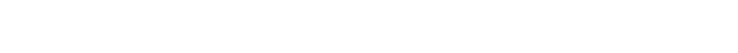

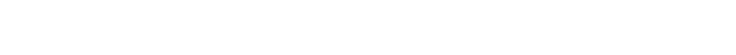

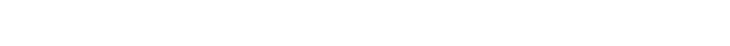

h

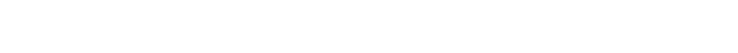

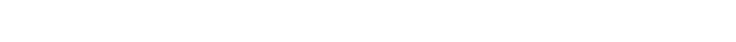

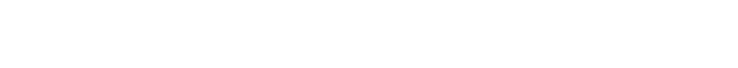

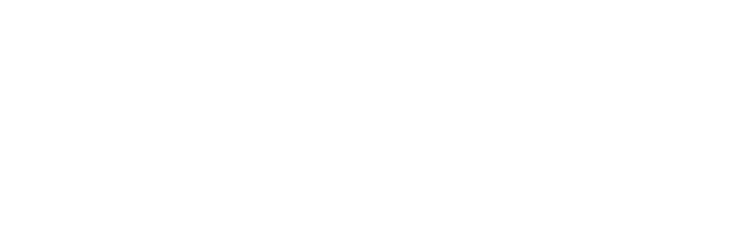




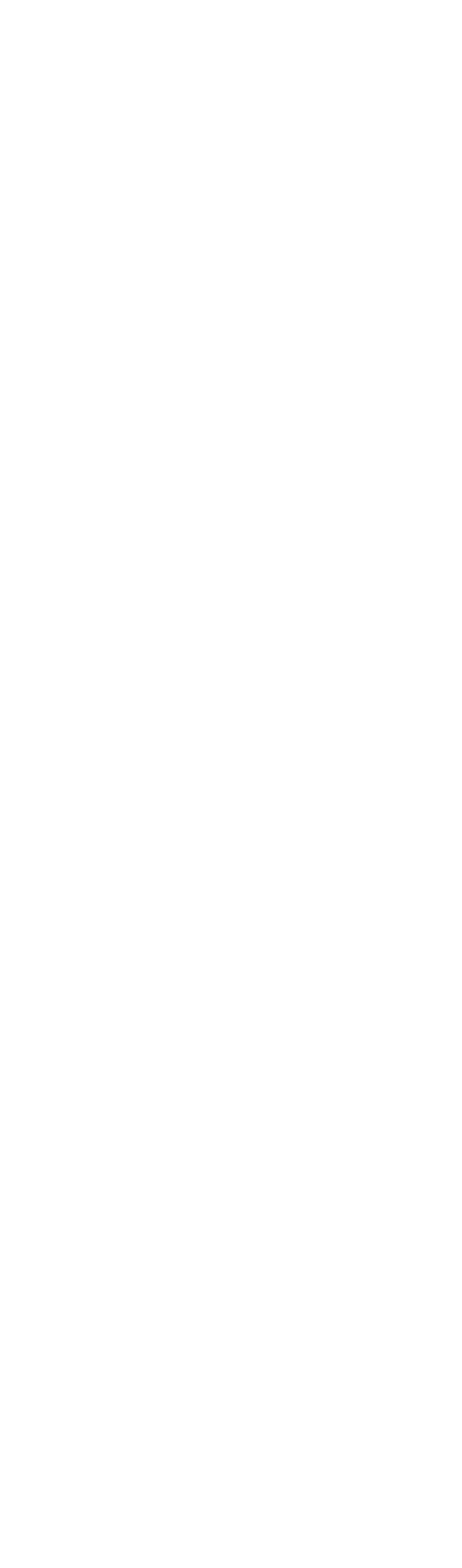




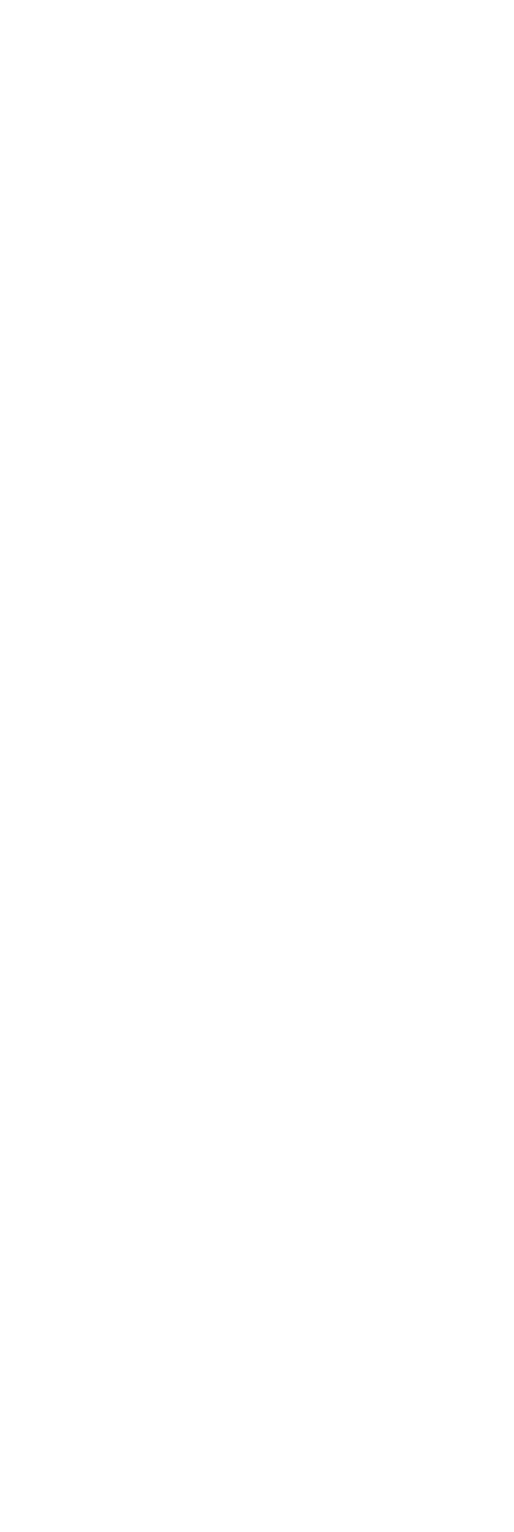




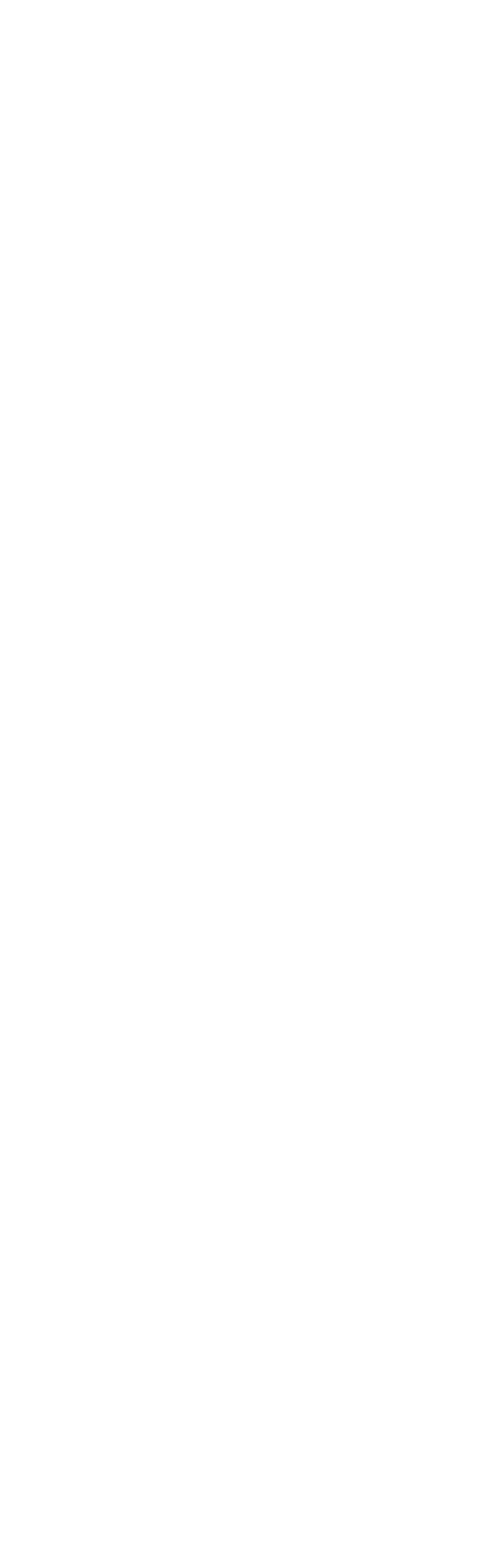




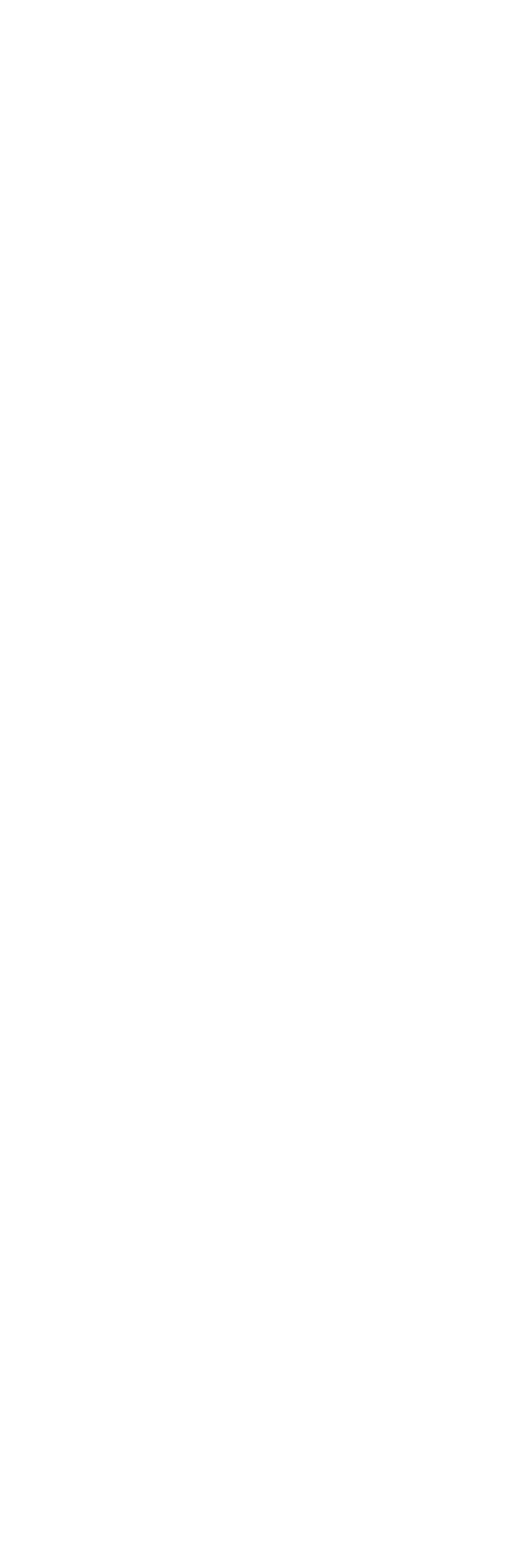




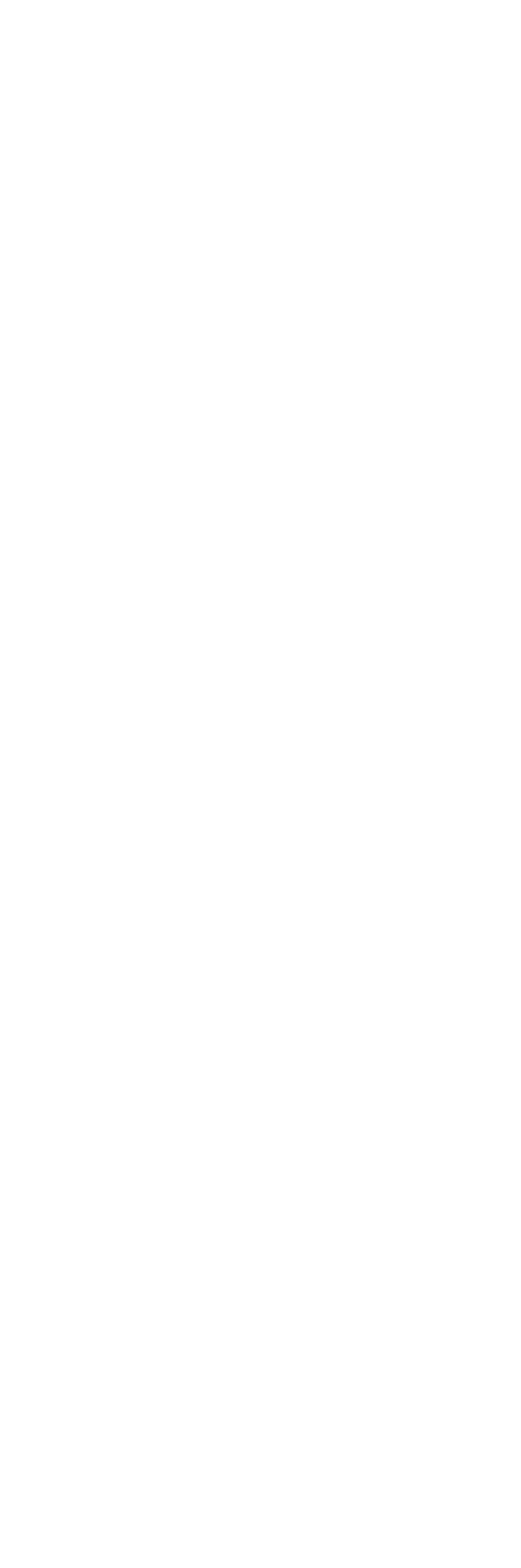




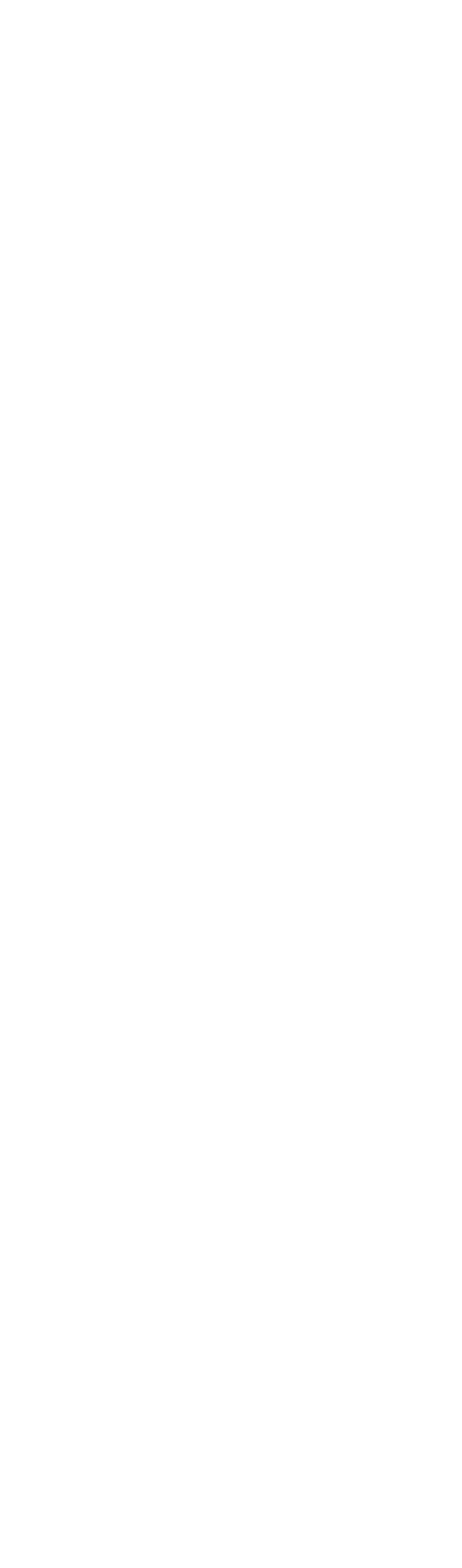




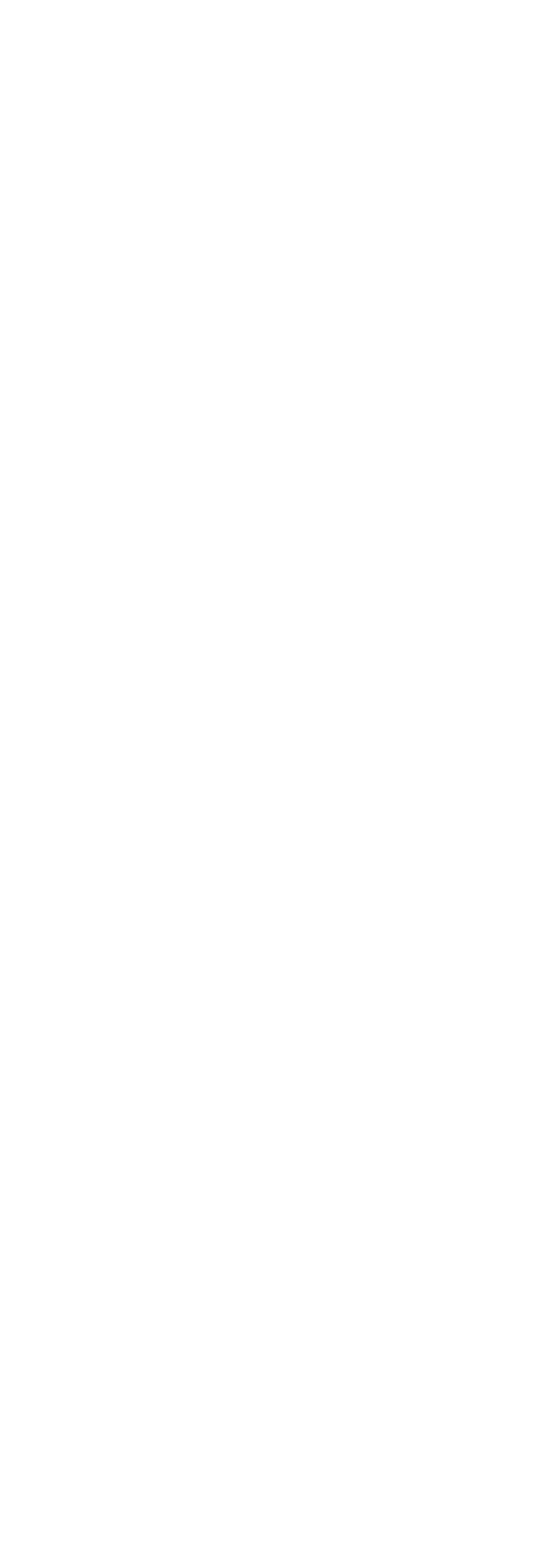




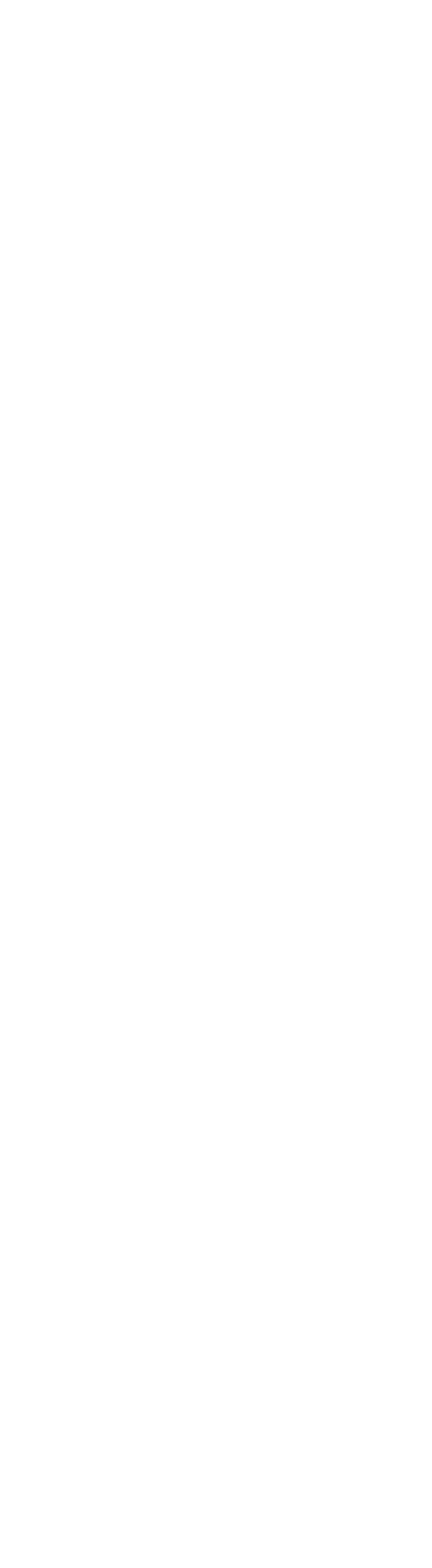

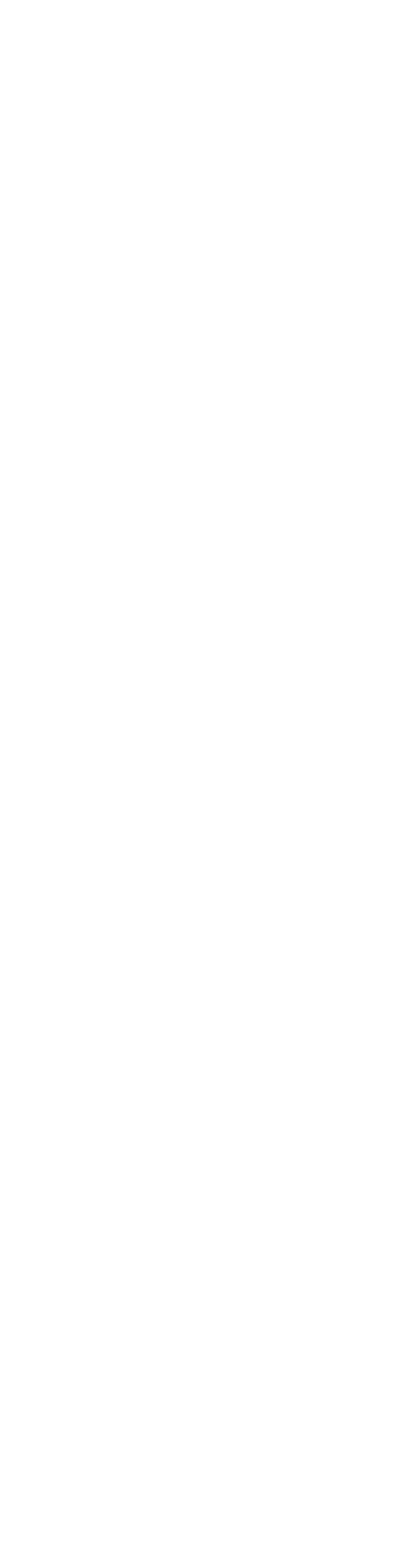




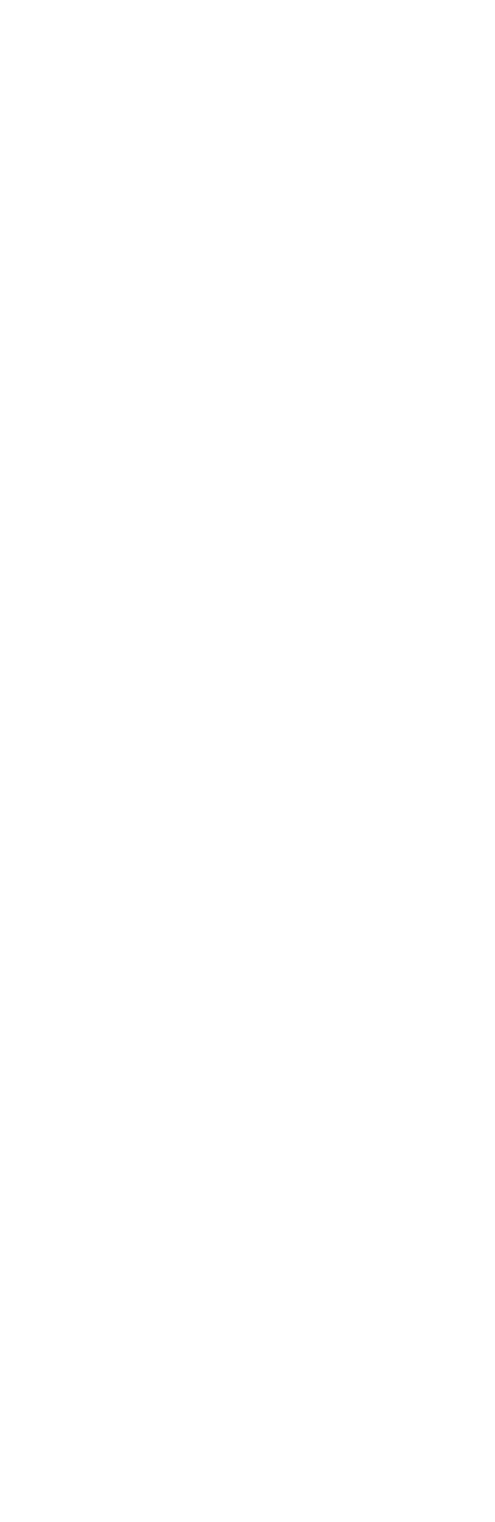




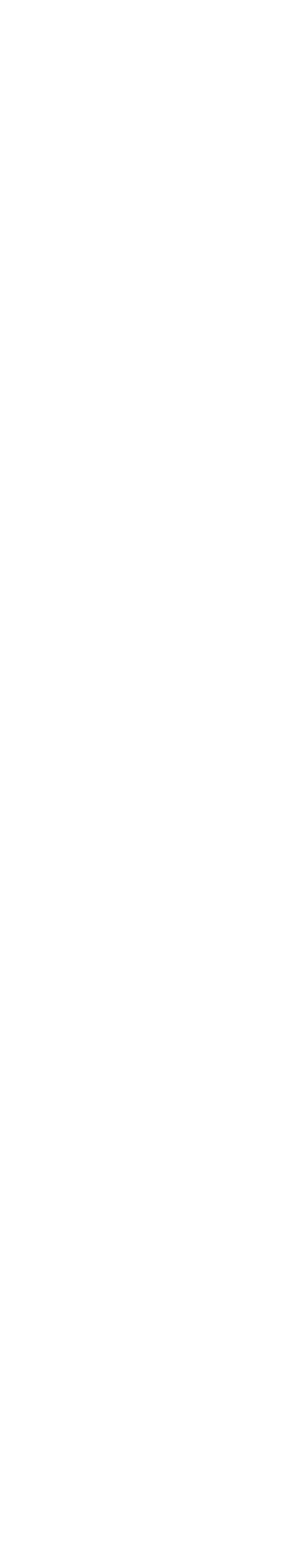

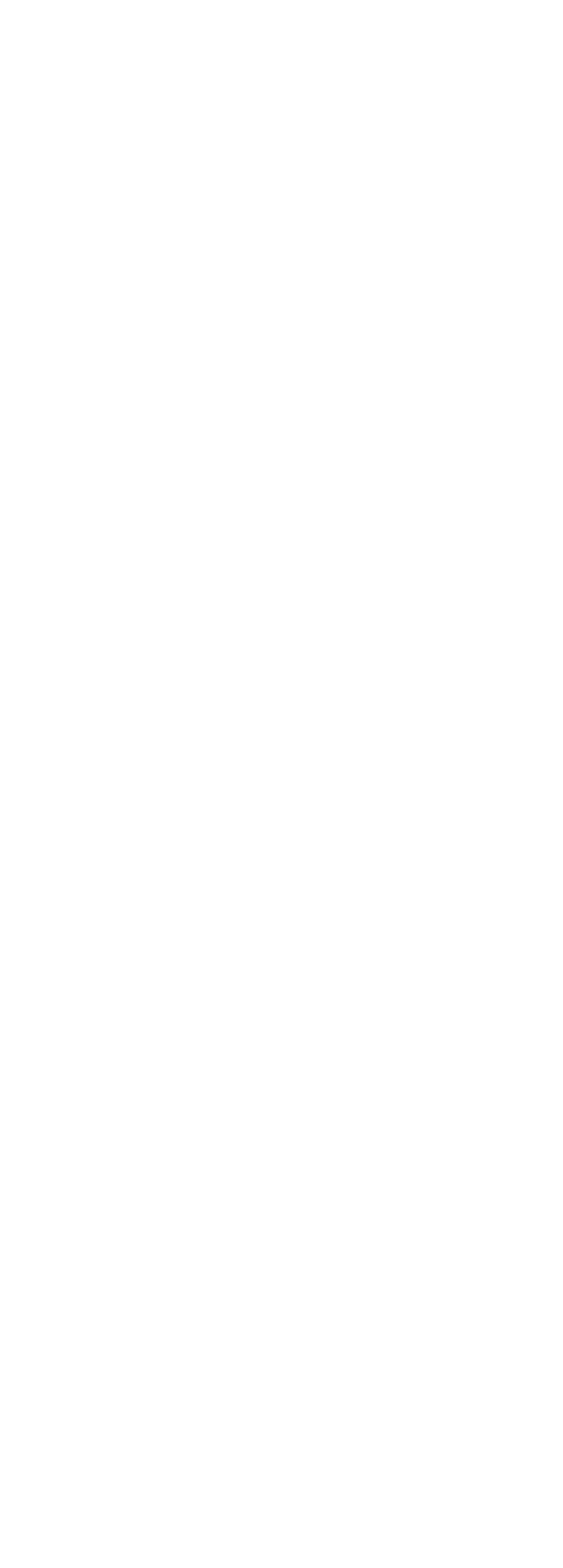




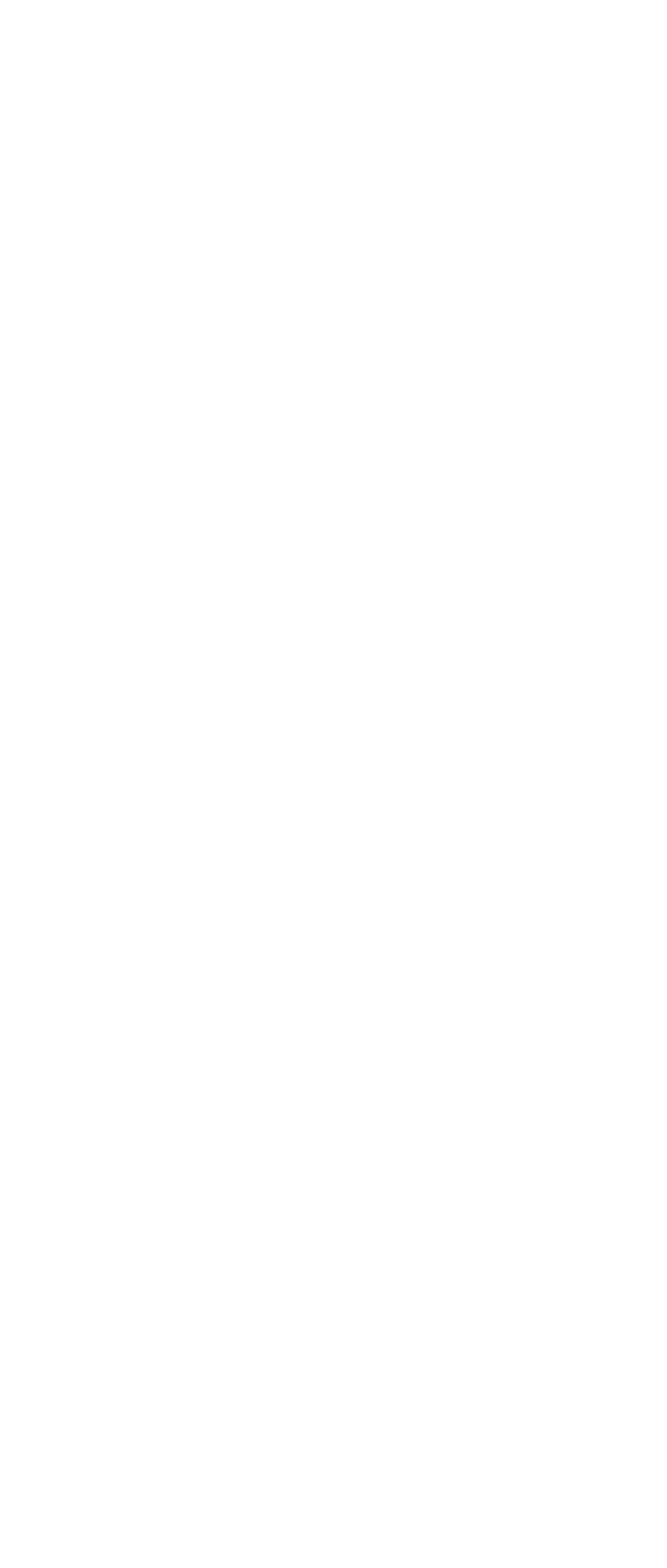




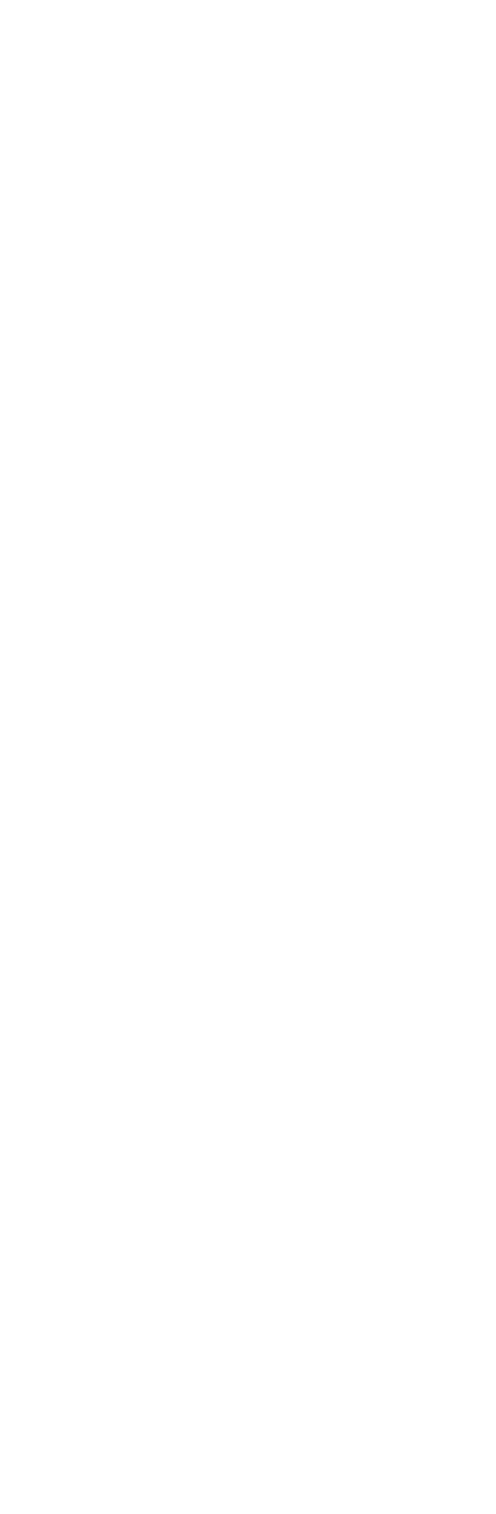




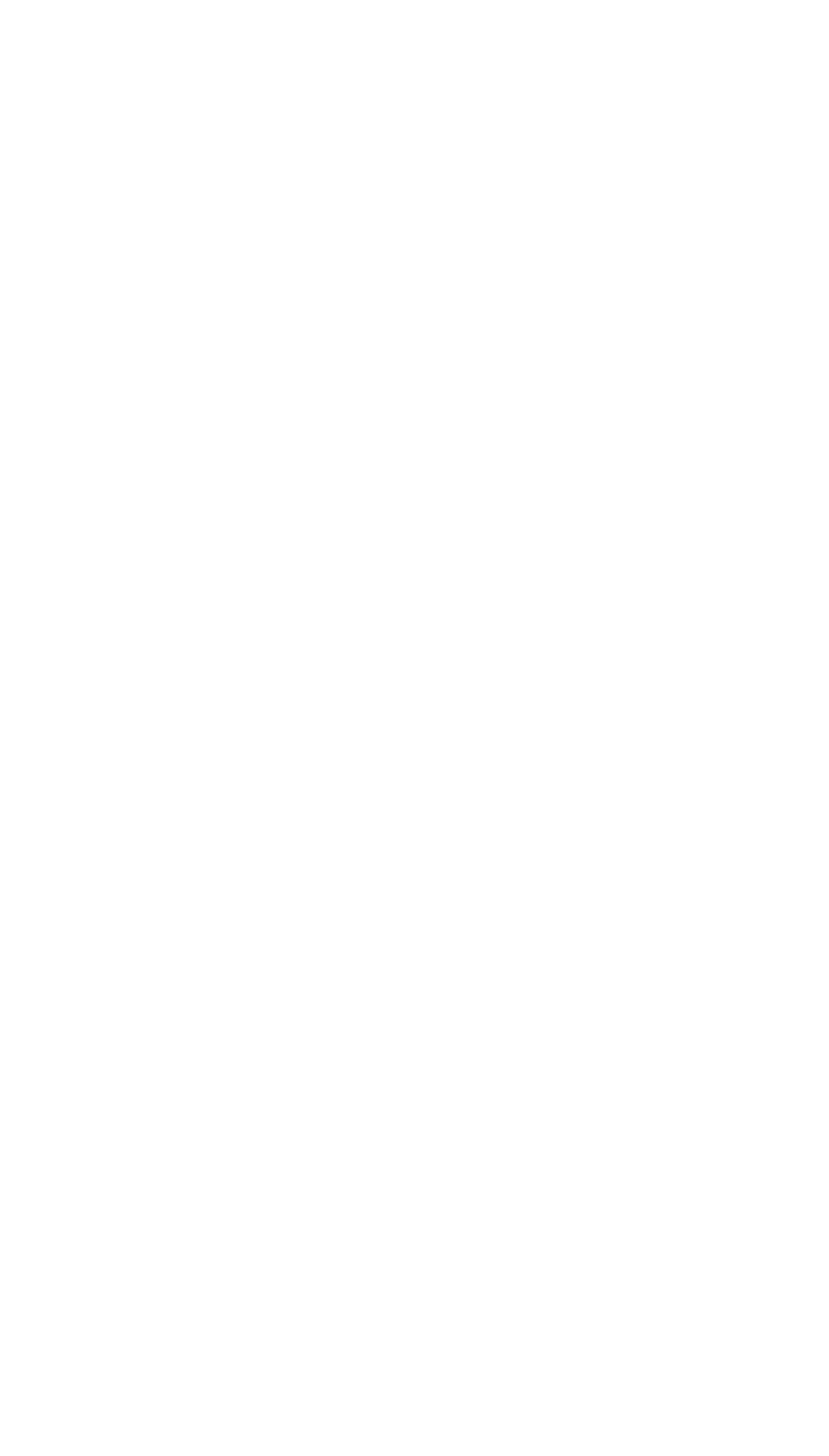




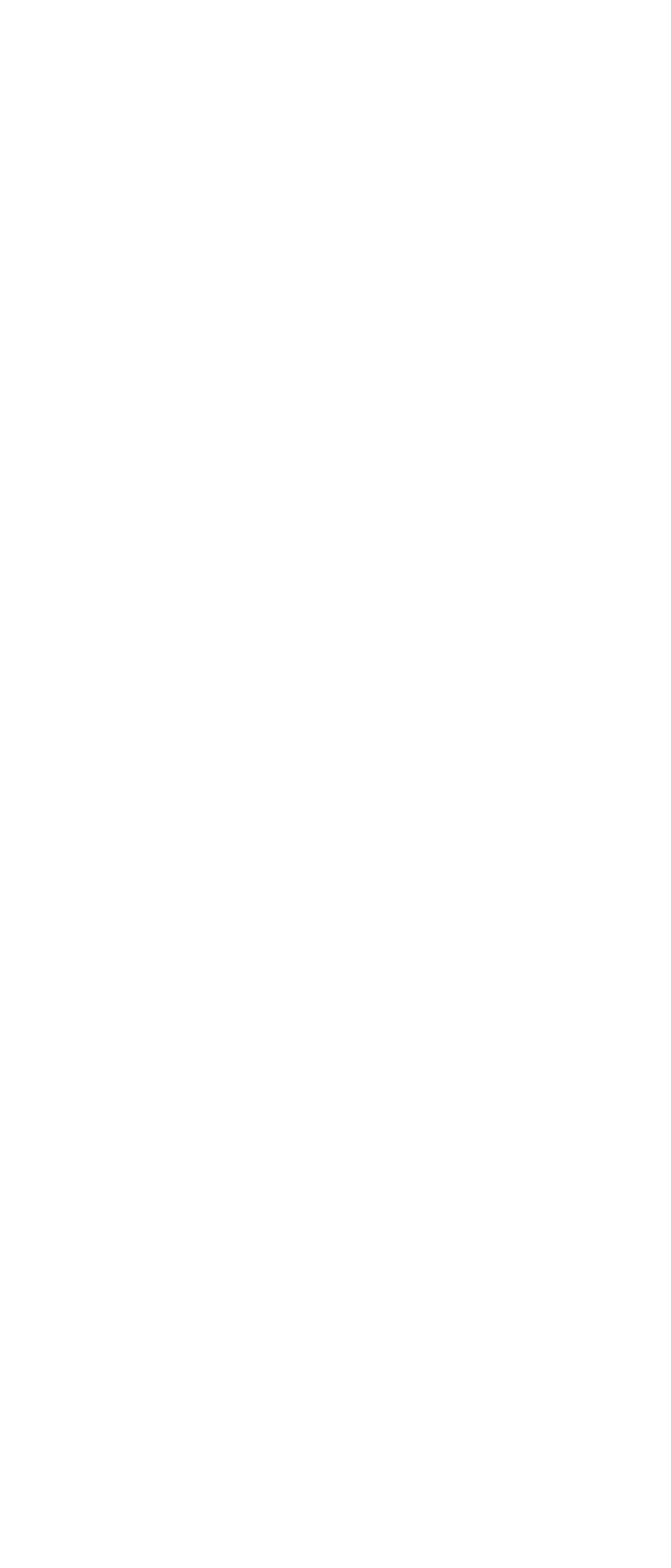




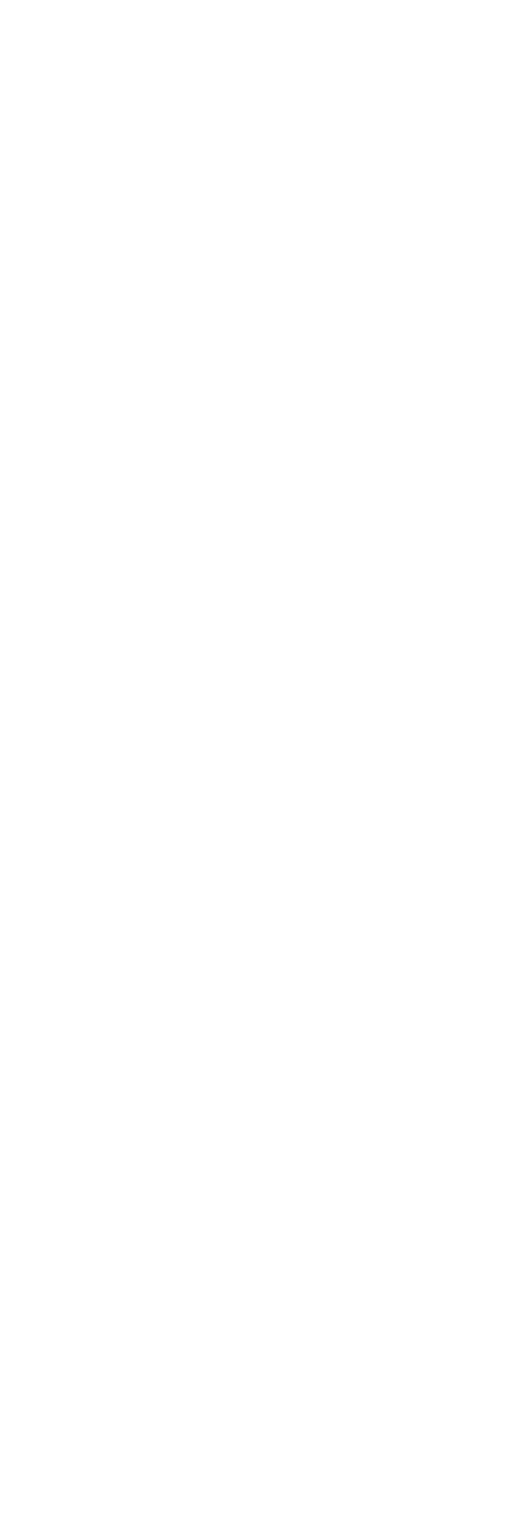



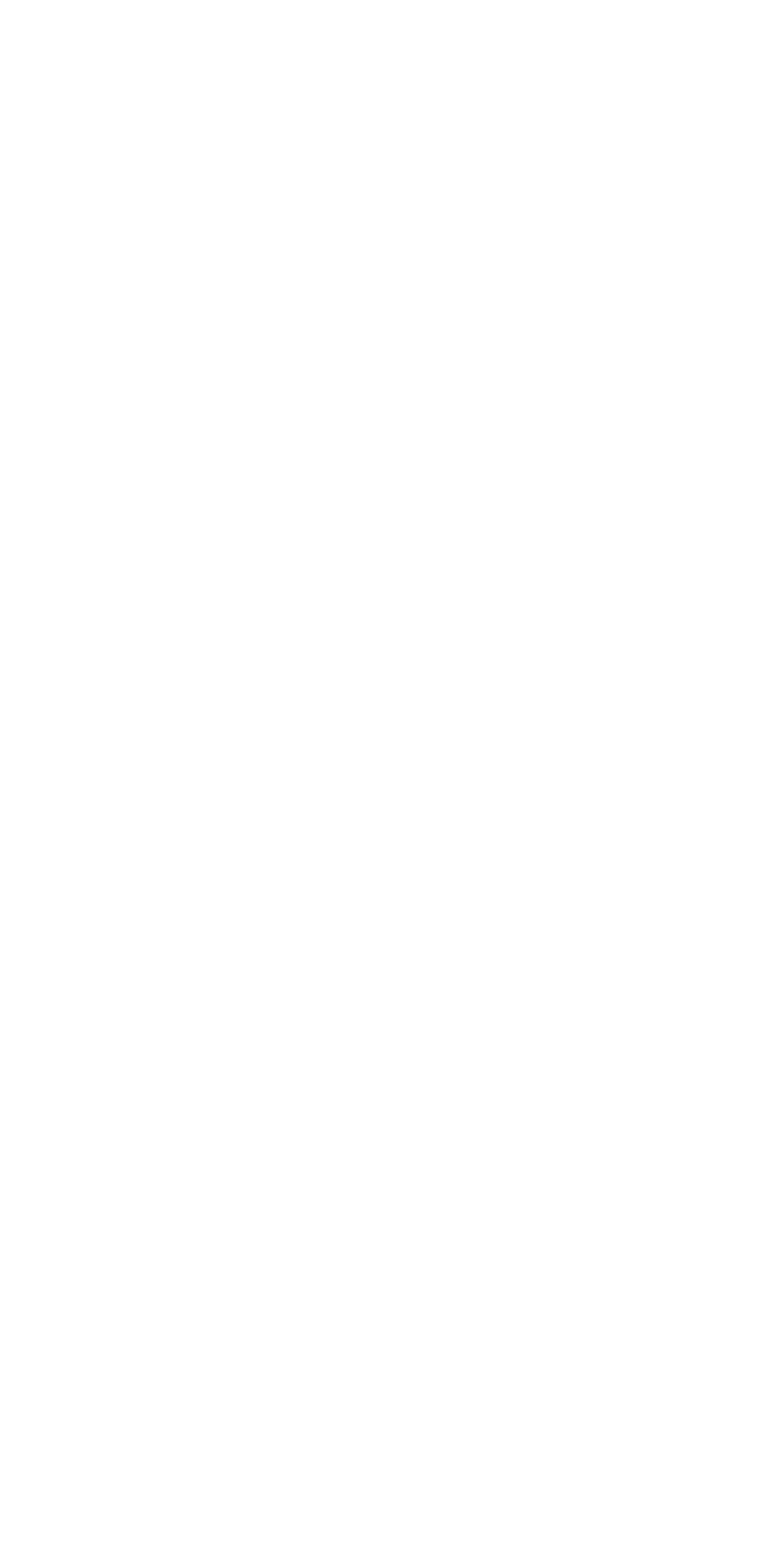

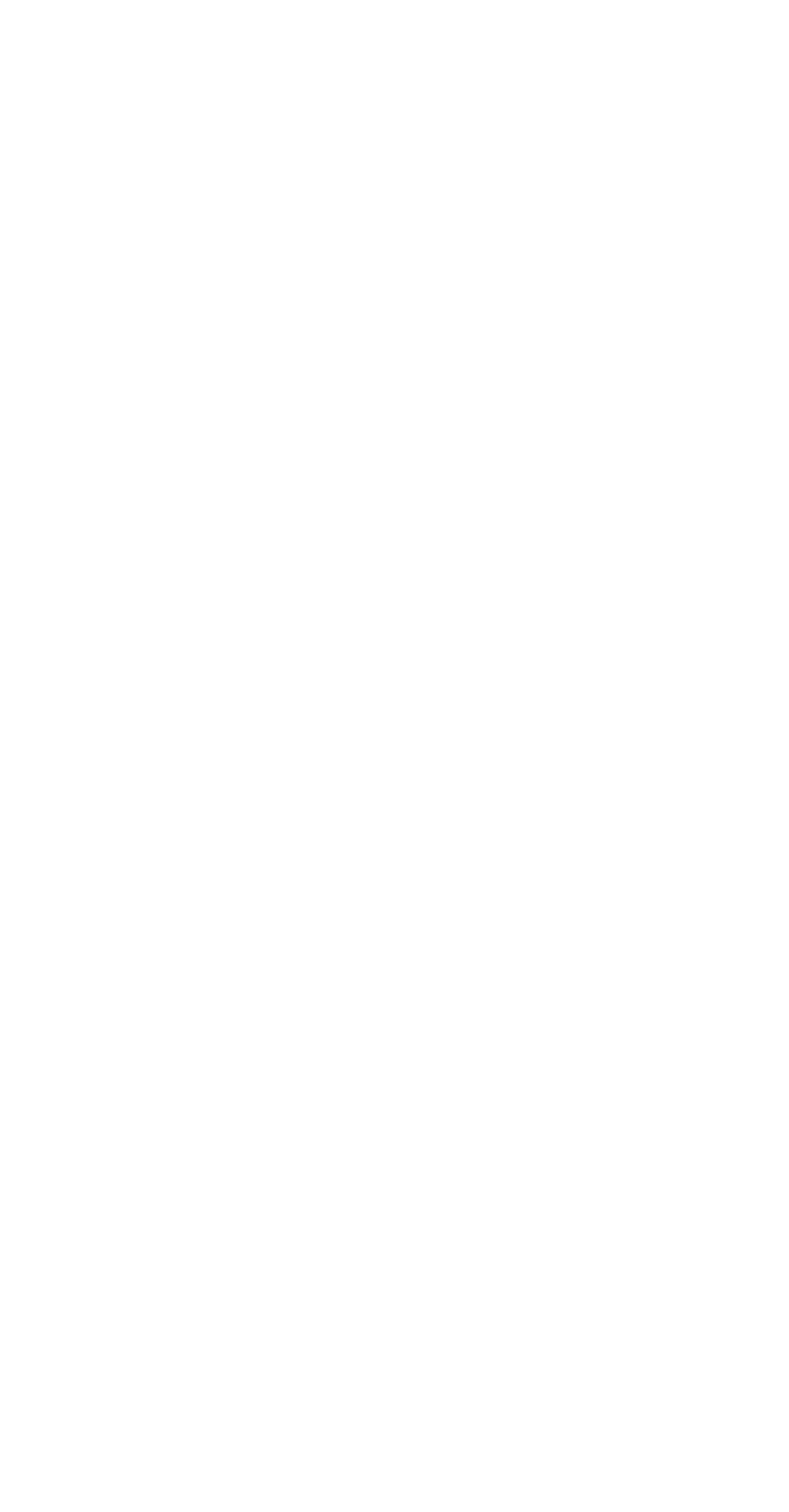

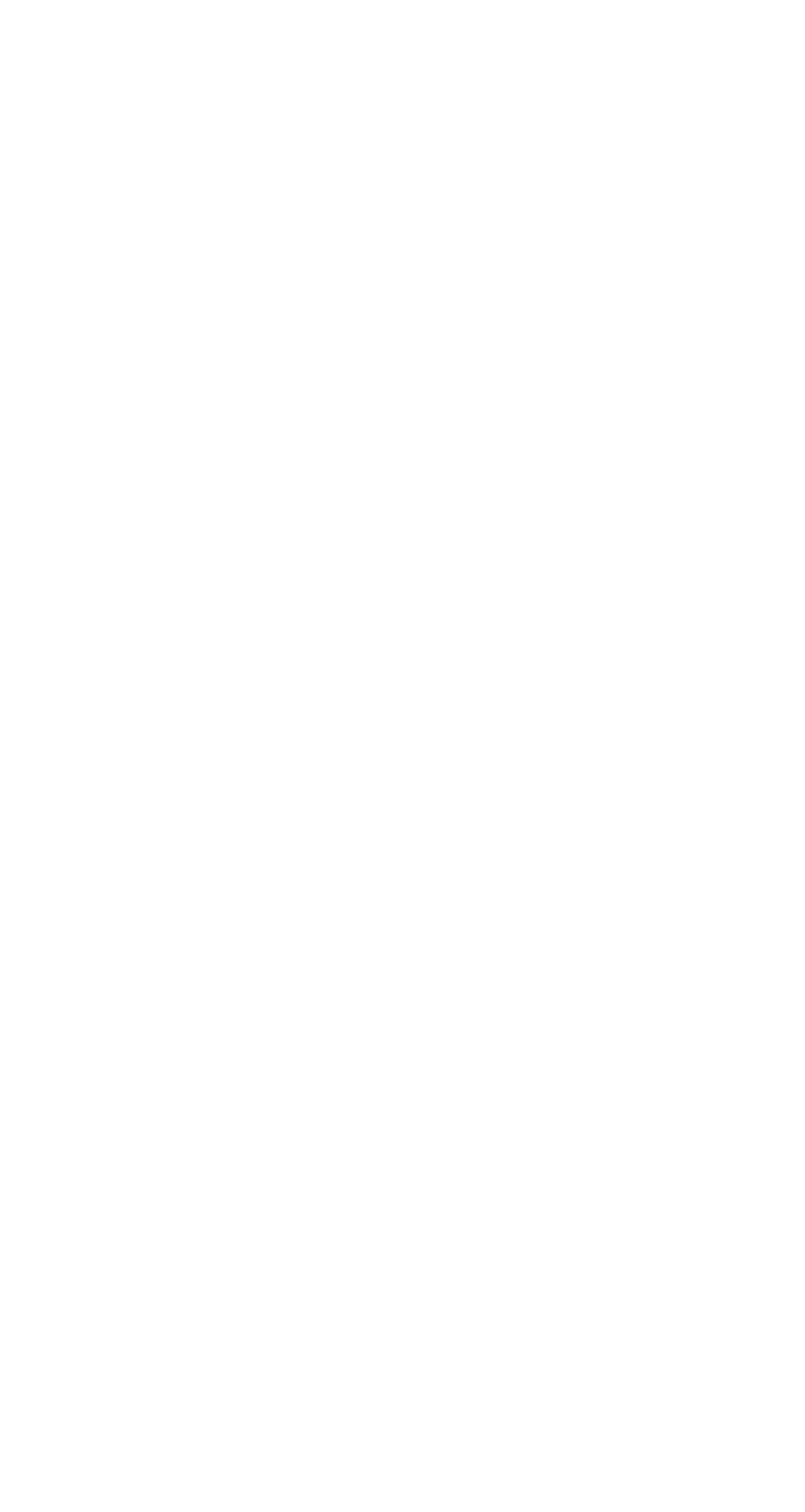


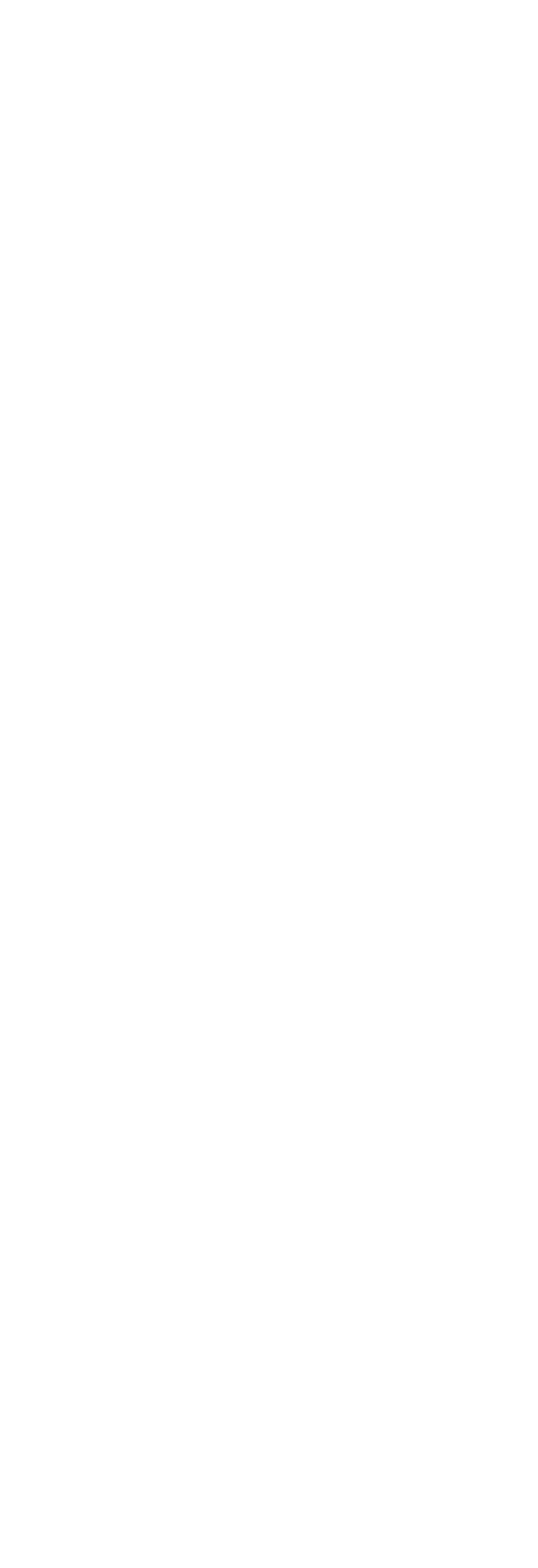




\begin{tabular}{|c|c|}
\hline 国 & 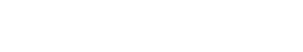 \\
\hline 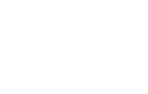 & 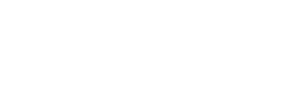 \\
\hline 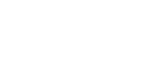 & 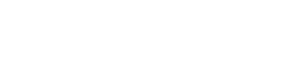 \\
\hline 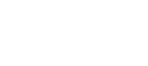 & 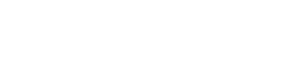 \\
\hline 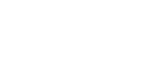 & 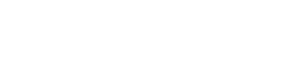 \\
\hline 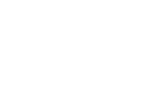 & 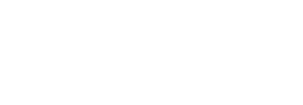 \\
\hline 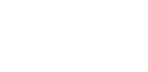 & 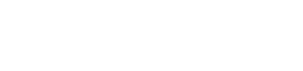 \\
\hline 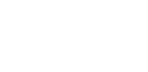 & मே. \\
\hline 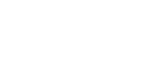 & 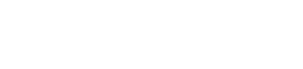 \\
\hline 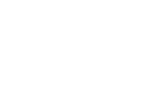 & 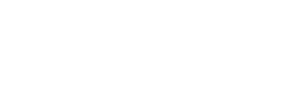 \\
\hline 莺俤 & 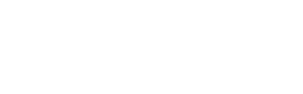 \\
\hline 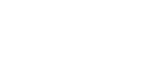 & $00000 \quad 00000 \quad 000$ \\
\hline 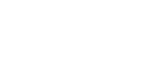 & సีద్లే \\
\hline 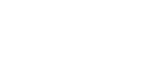 & 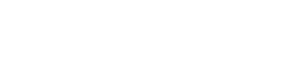 \\
\hline 预 & 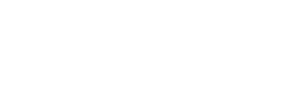 \\
\hline 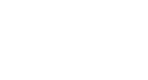 & 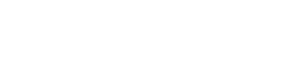 \\
\hline ฮี่ & 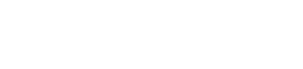 \\
\hline 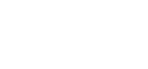 & 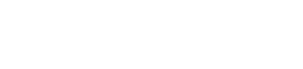 \\
\hline 害恋 & $\begin{array}{r}11 \\
1\end{array}$ \\
\hline 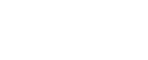 & \\
\hline 串实 & 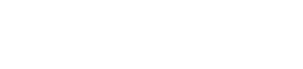 \\
\hline 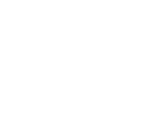 & 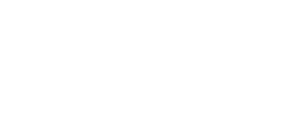 \\
\hline 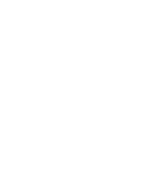 & 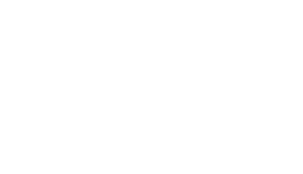 \\
\hline
\end{tabular}

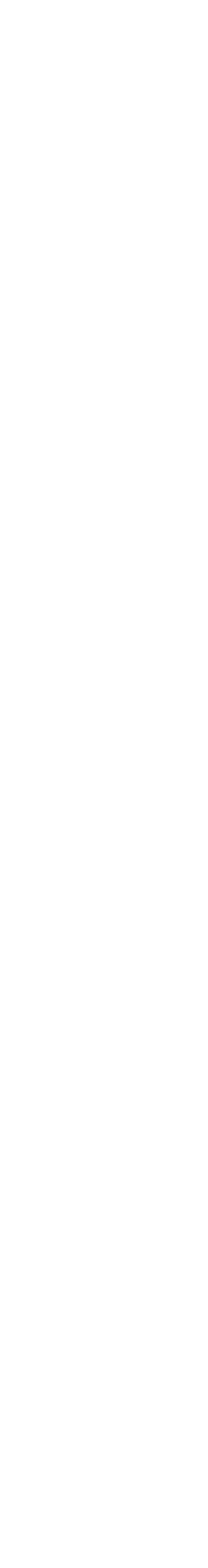



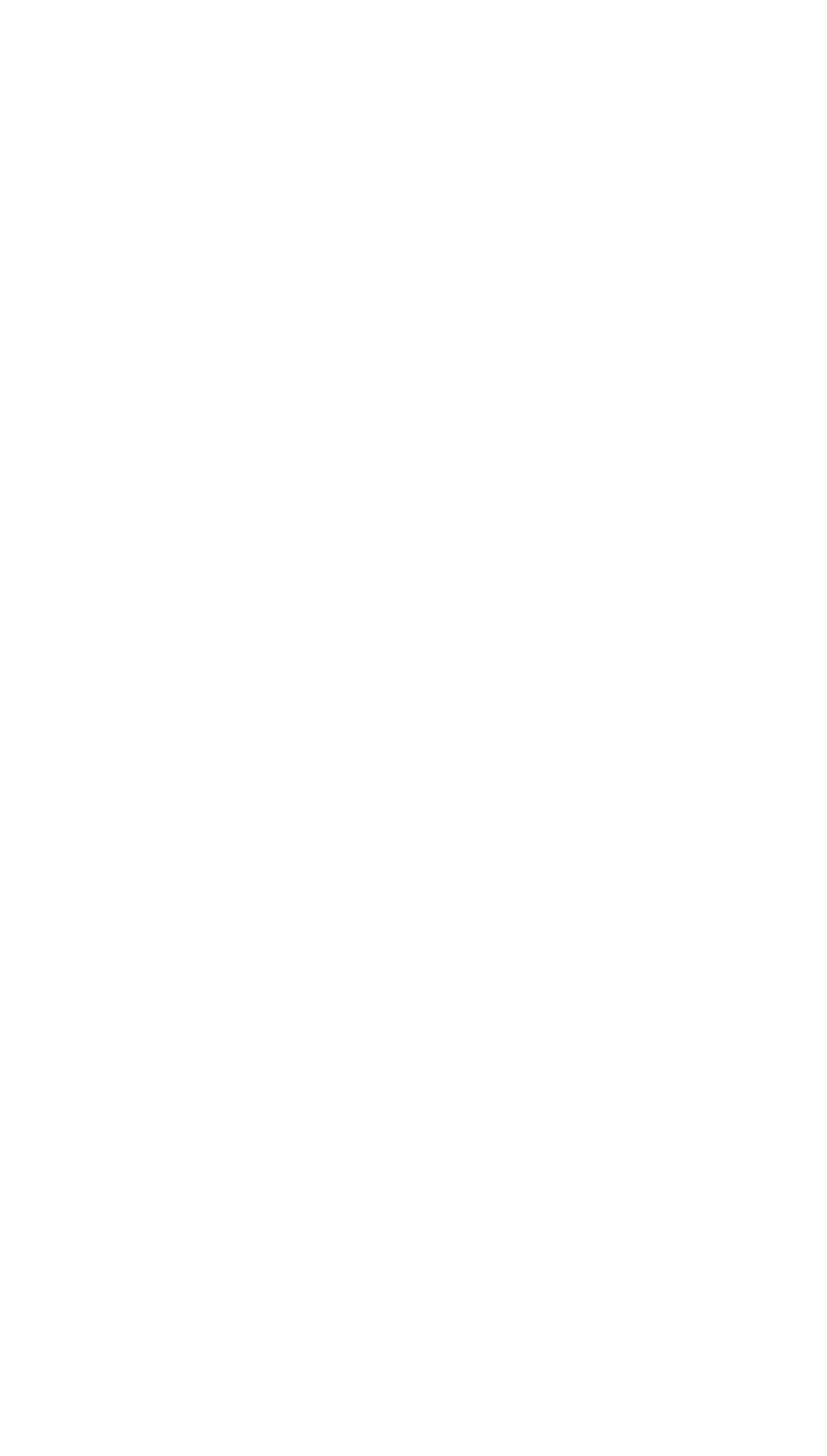


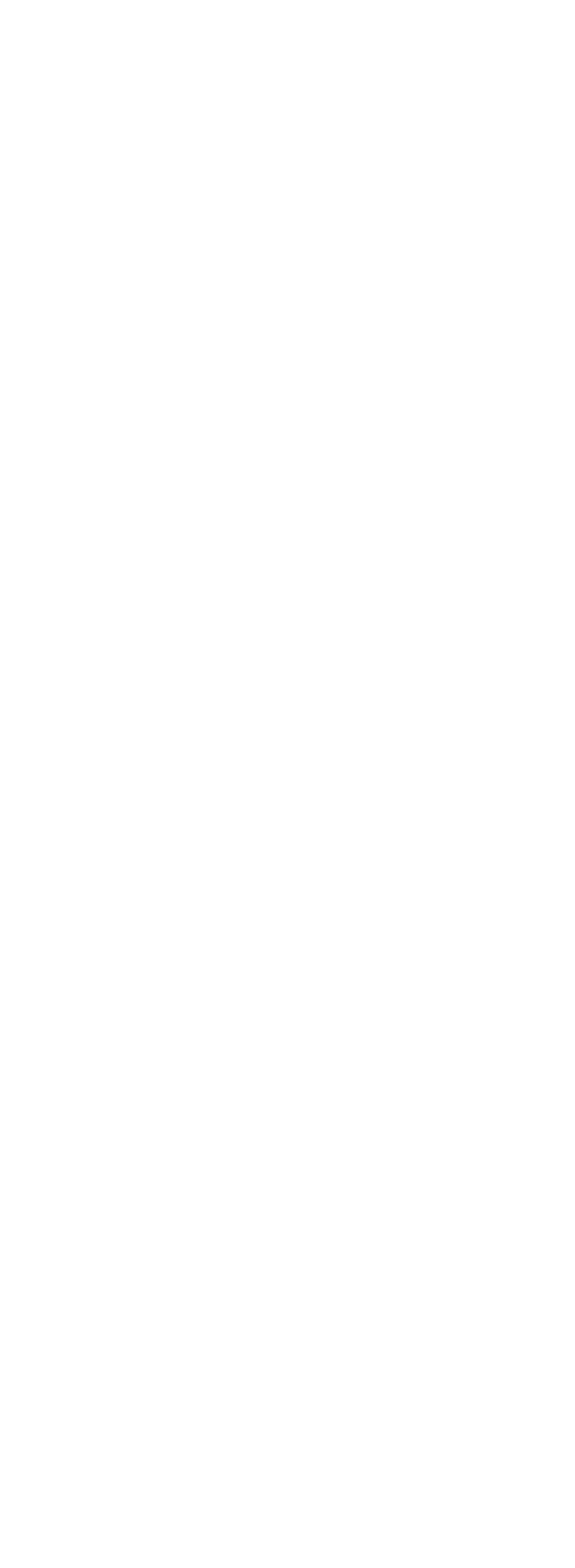




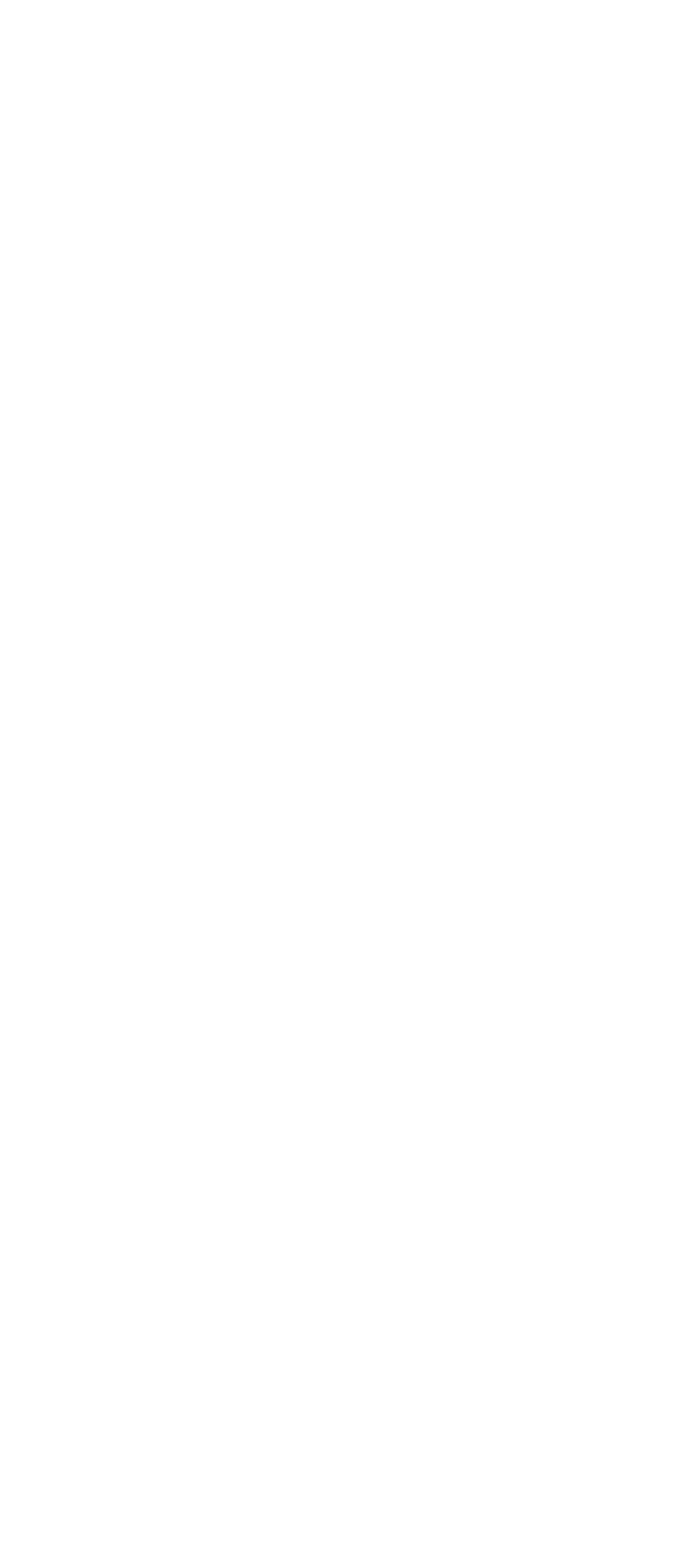




\begin{tabular}{|c|c|c|c|}
\hline $\begin{array}{lllll}1 & 1 & 1 & 1 & 1 \\
1 & 1 & 1\end{array}$ & $i$ & i & $i$ \\
\hline 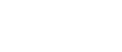 & 离 & 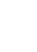 & $i$ \\
\hline m? & $\begin{array}{l}\infty \\
0 \\
0\end{array}$ & $\stackrel{\infty}{\circ}$ & $T$ \\
\hline న్ల & $\bar{N}$ & 8 & 1 \\
\hline 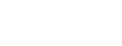 & $\stackrel{8}{\circ}$ & 脀 & 1 \\
\hline 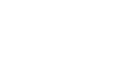 & $\underset{్ ָ}{ }$ & ! & i \\
\hline 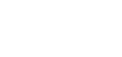 & $\begin{array}{l}m \\
m \\
0 \\
0\end{array}$ & $i$ & $\mathrm{i}$ \\
\hline 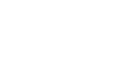 & $\underset{17}{4}$ & 政 & 1 \\
\hline $\begin{array}{llllll} & 1 & 1 & 1 & 1 \\
1 & 1 & 1 & 1\end{array}$ & 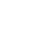 & $i$ & $i$ \\
\hline $\begin{array}{llllll}1 & 1 & 1 & 1 & 1 \\
\end{array}$ & $!$ & 1 & $i$ \\
\hline $1: 11: 1$ & $!$ & $i$ & $i$ \\
\hline$\sharp の$ & $\widetilde{\sim}$ & $\hat{m}$ & $\infty$ \\
\hline 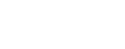 & ชู & \& & 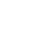 \\
\hline $1: 1: 11$ & ! & 1 & 1 \\
\hline 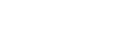 & מู & $\stackrel{\circ}{\circ}$ & $\overrightarrow{\mathrm{G}}$ \\
\hline 11111 & 1 & 1 & 1 \\
\hline ONNลN & $\stackrel{2}{2}$ & 용 & $m$ \\
\hline 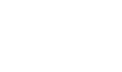 & $\stackrel{\circ}{\circ}$ & : & $\cong$ \\
\hline 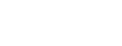 & $\overrightarrow{5}$ & $\therefore$ & $\grave{\infty}$ \\
\hline $111: 1$ & 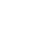 & $i$ & 1 \\
\hline 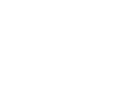 & 1 & 足 & 1 \\
\hline 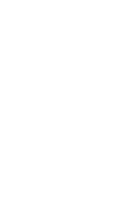 & 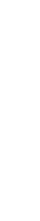 & 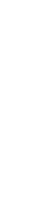 & 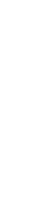 \\
\hline
\end{tabular}

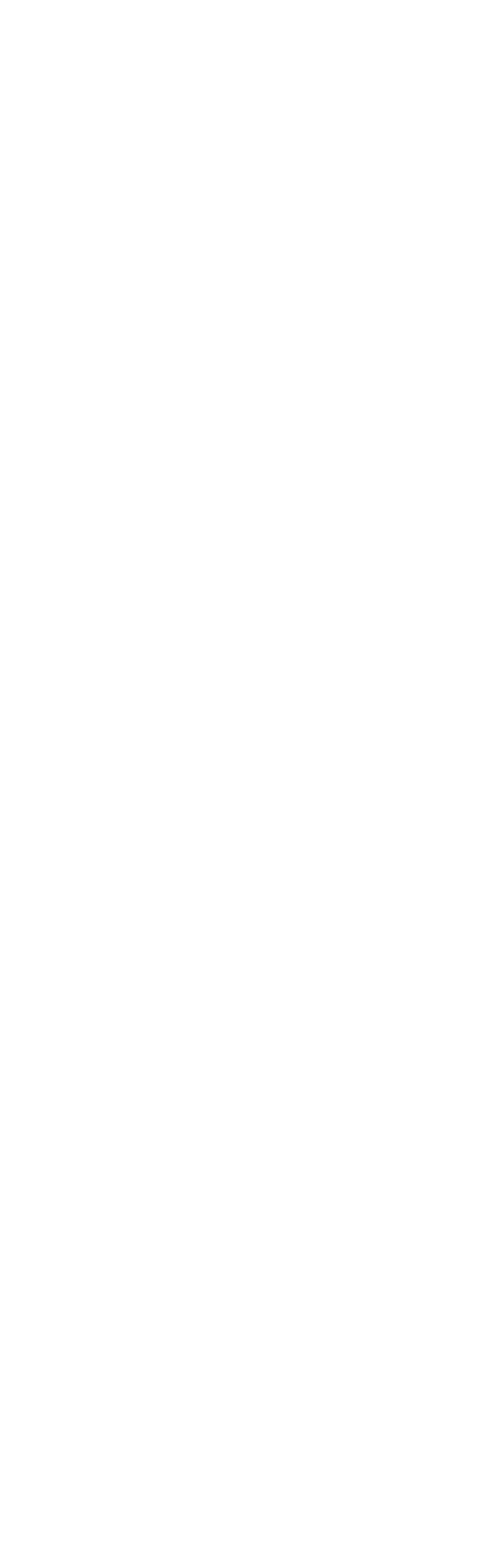




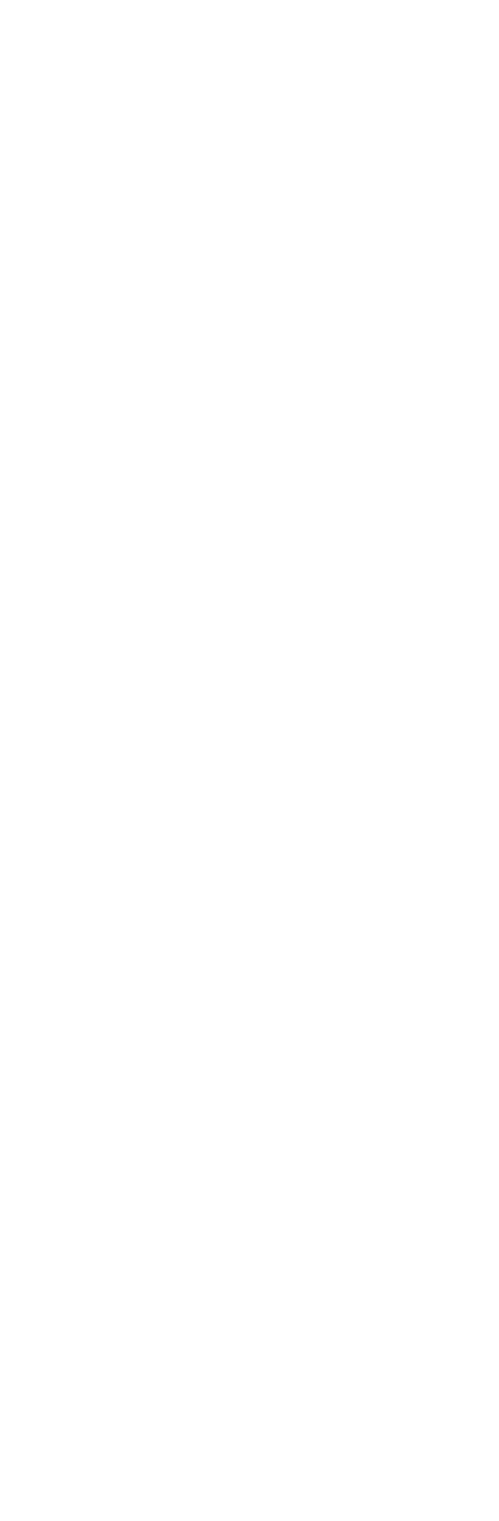




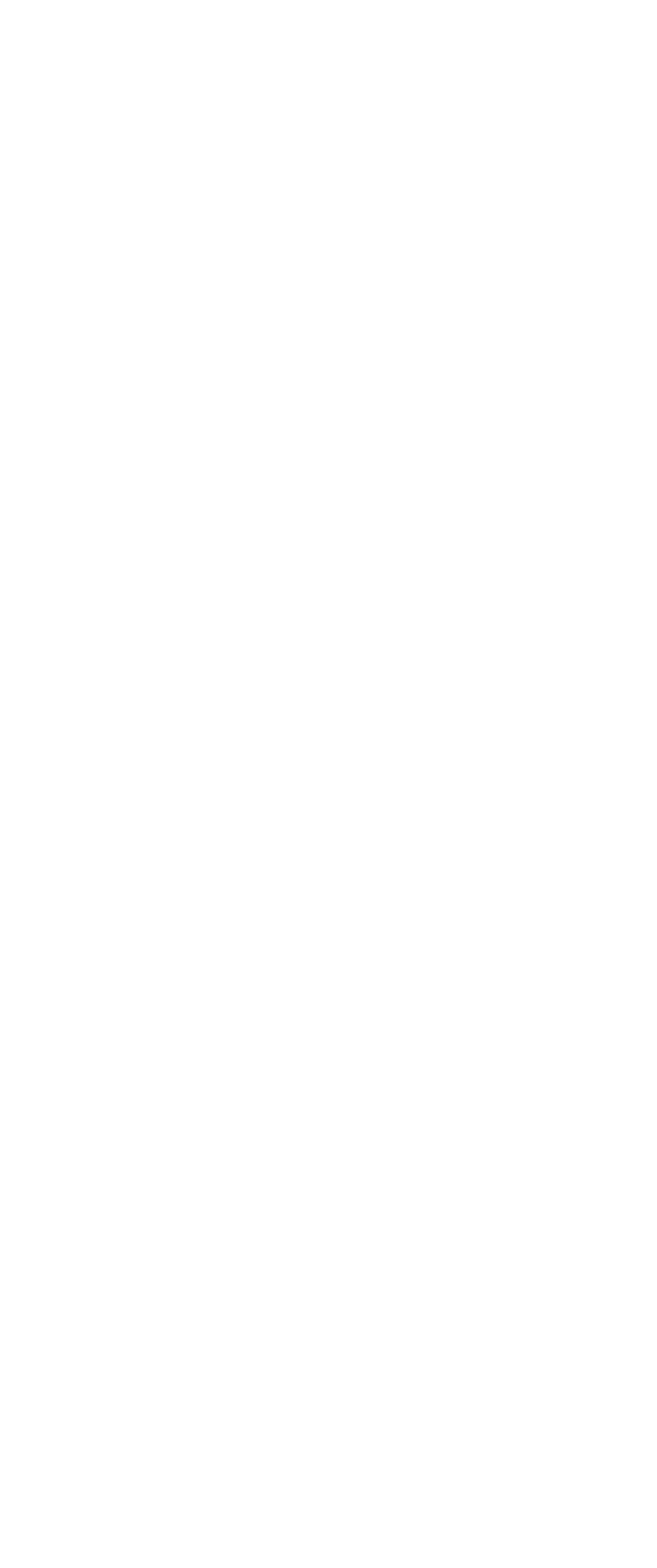




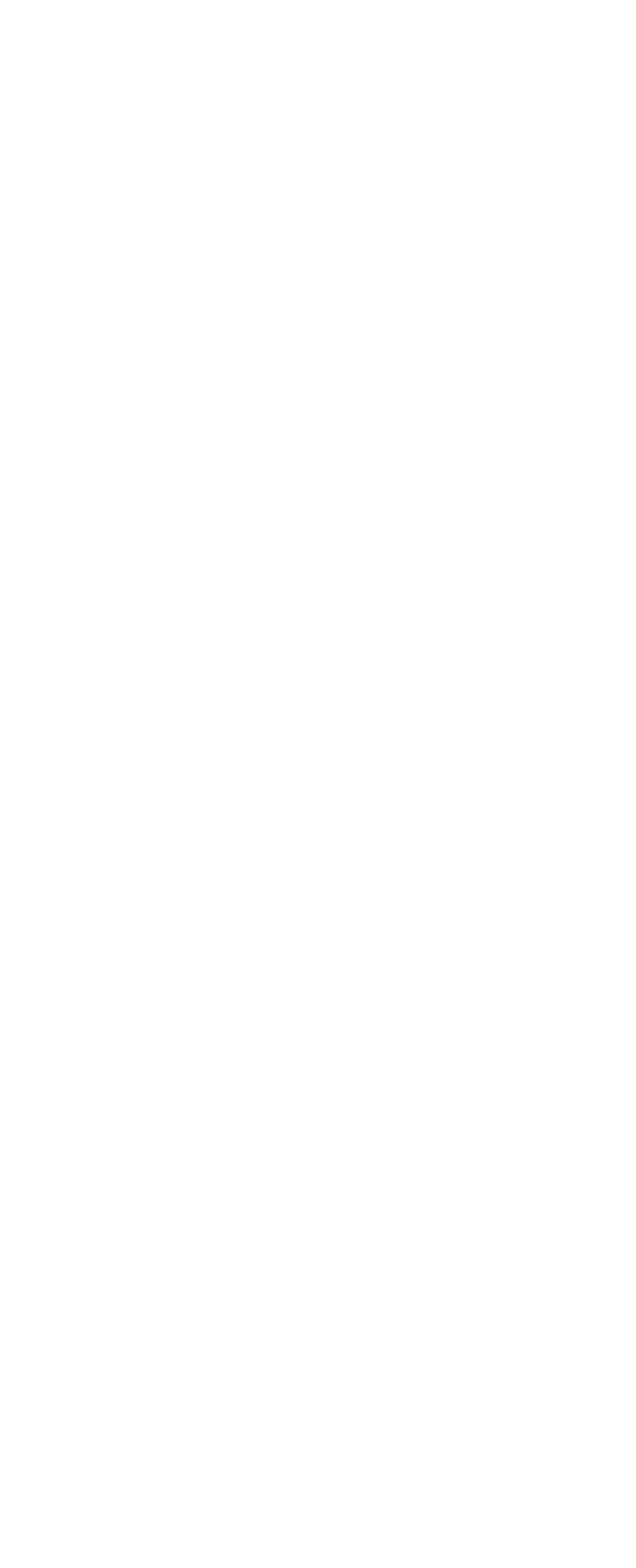



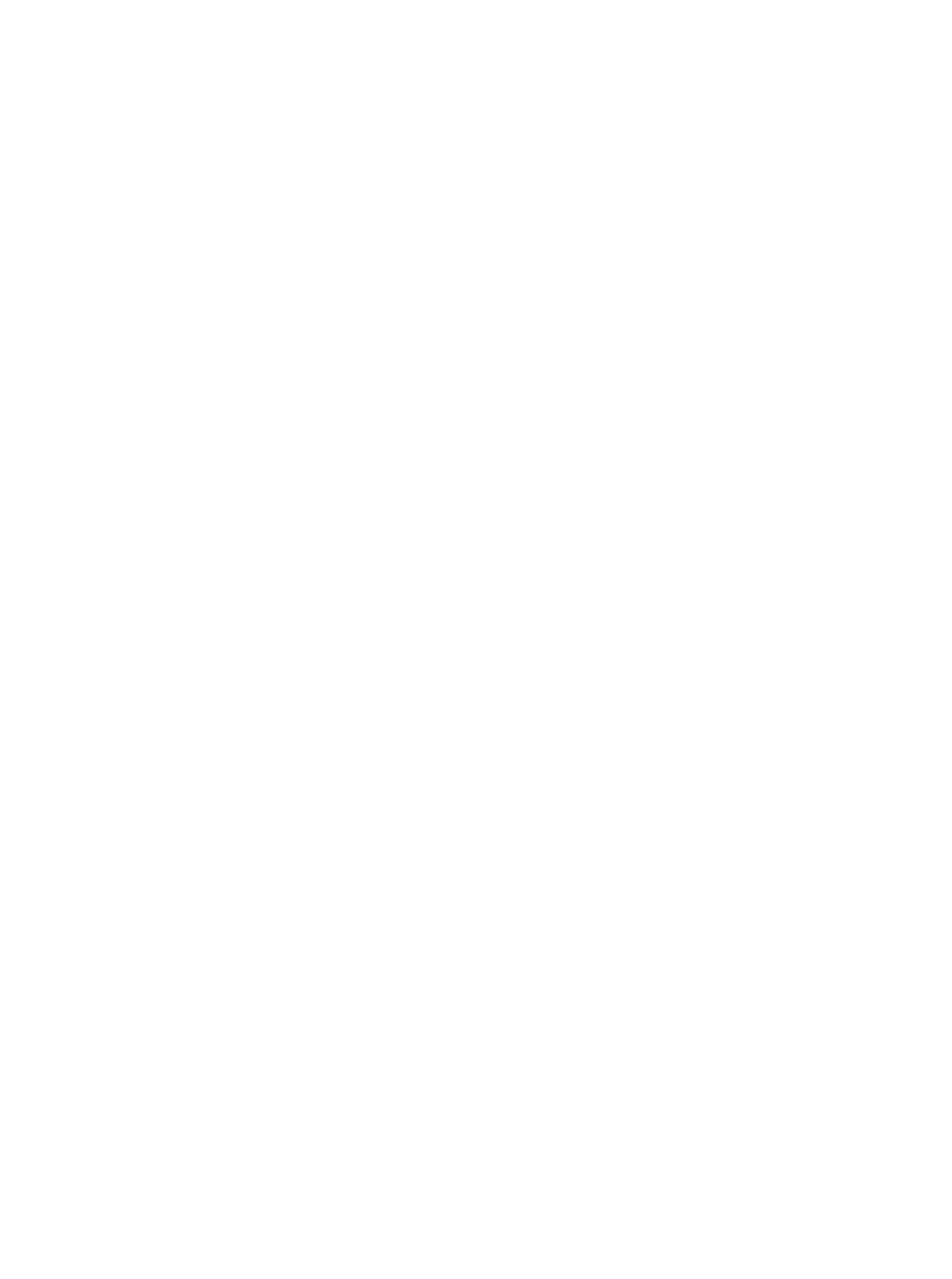
ARKANSAS RIVER BASIN--Continued

7-1864. CENTER CREEK NEAR CARTERVILLE, MO.--Continued

Specific conductance, in micromhos at $25^{\circ} \mathrm{C}$, December 1963 to September 1964

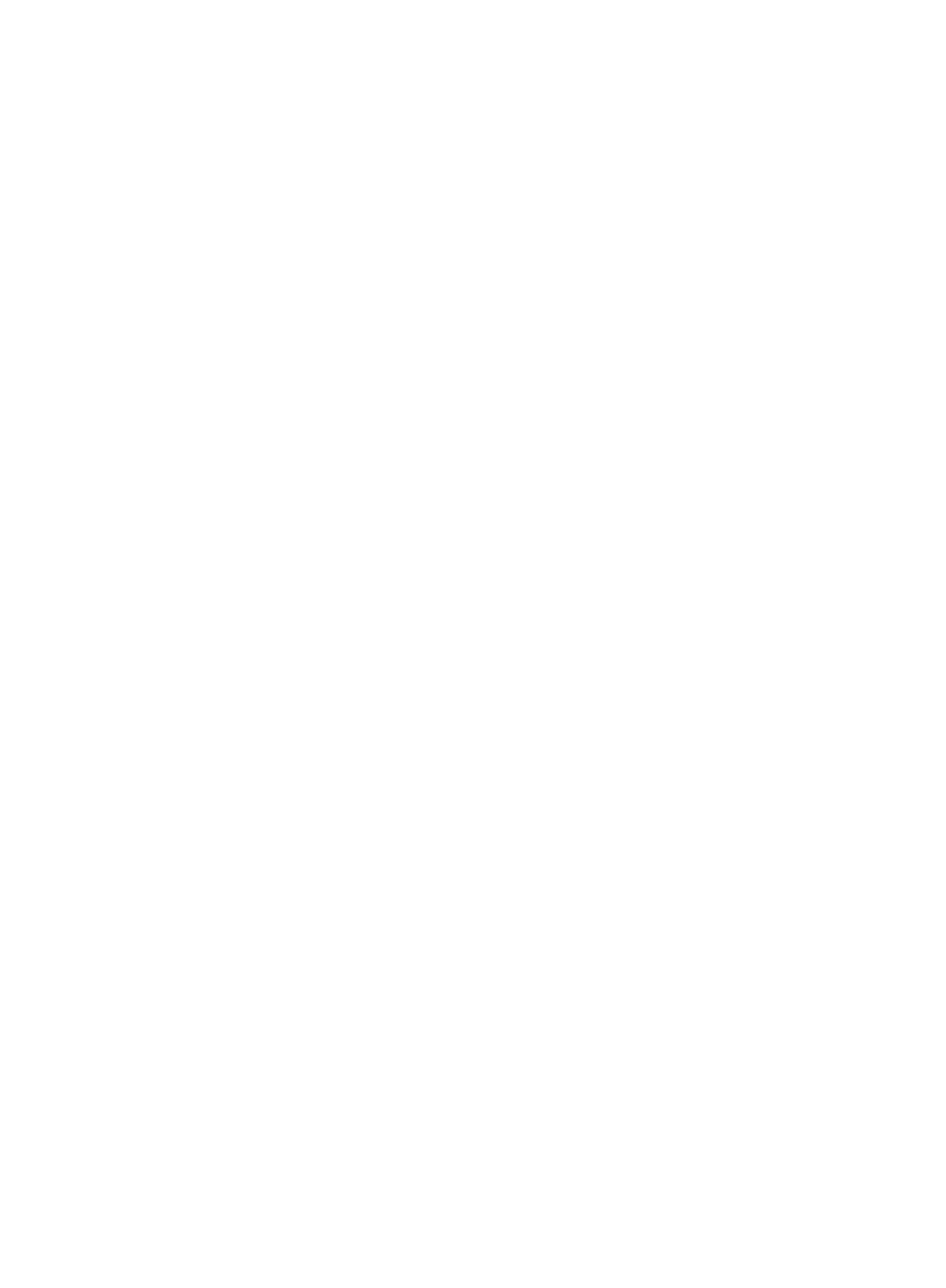

E Estimated. 
ARKANSAS RIVER BASIN--Continued

7-1864. CENTER CREEK NEAR CARTERVILLE, MO.--Continued

Specific conductance, in micromhos at $25^{\circ} \mathrm{C}$, December 1963 to September 1964--Continued

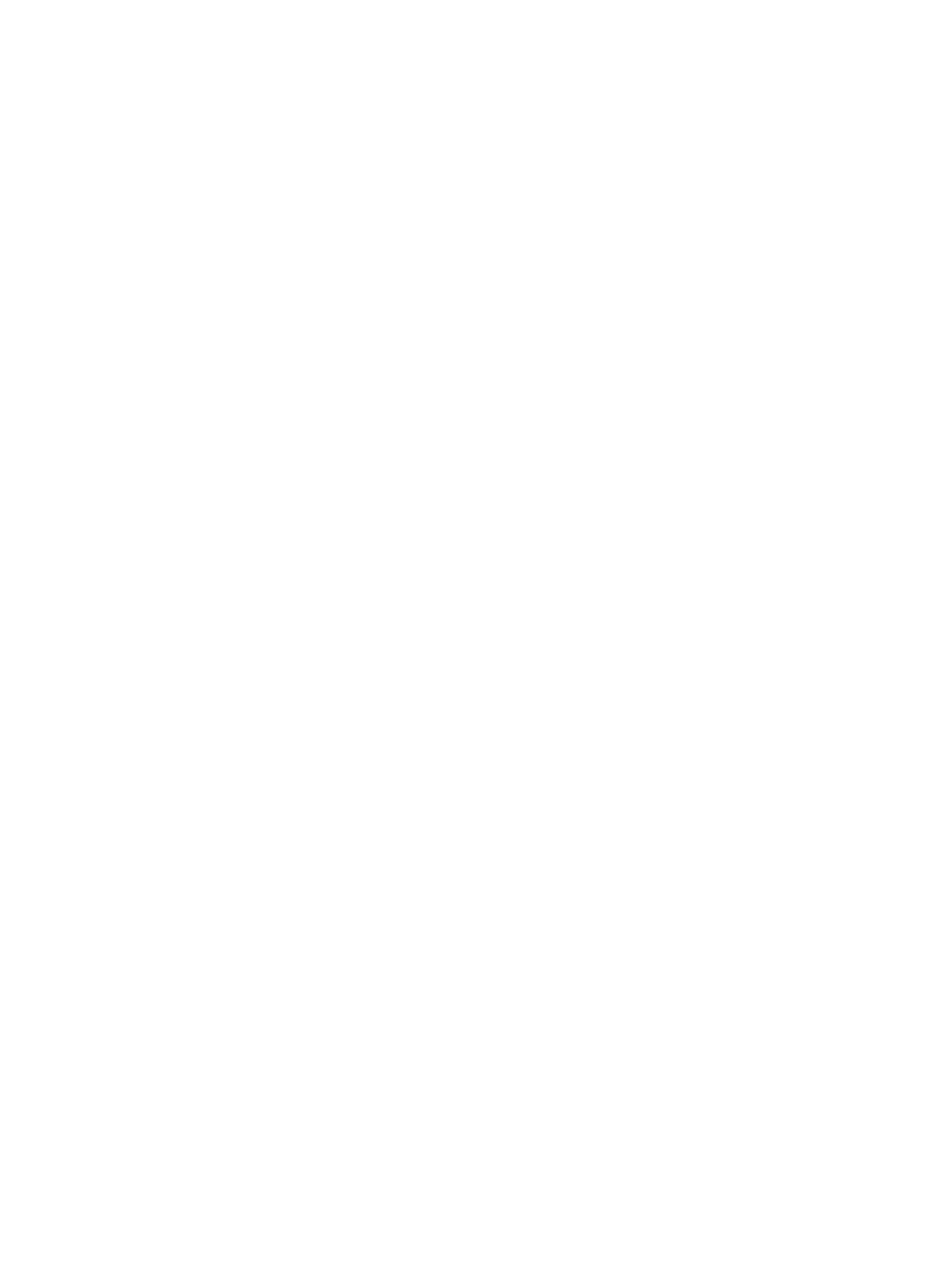

E Estinated. 


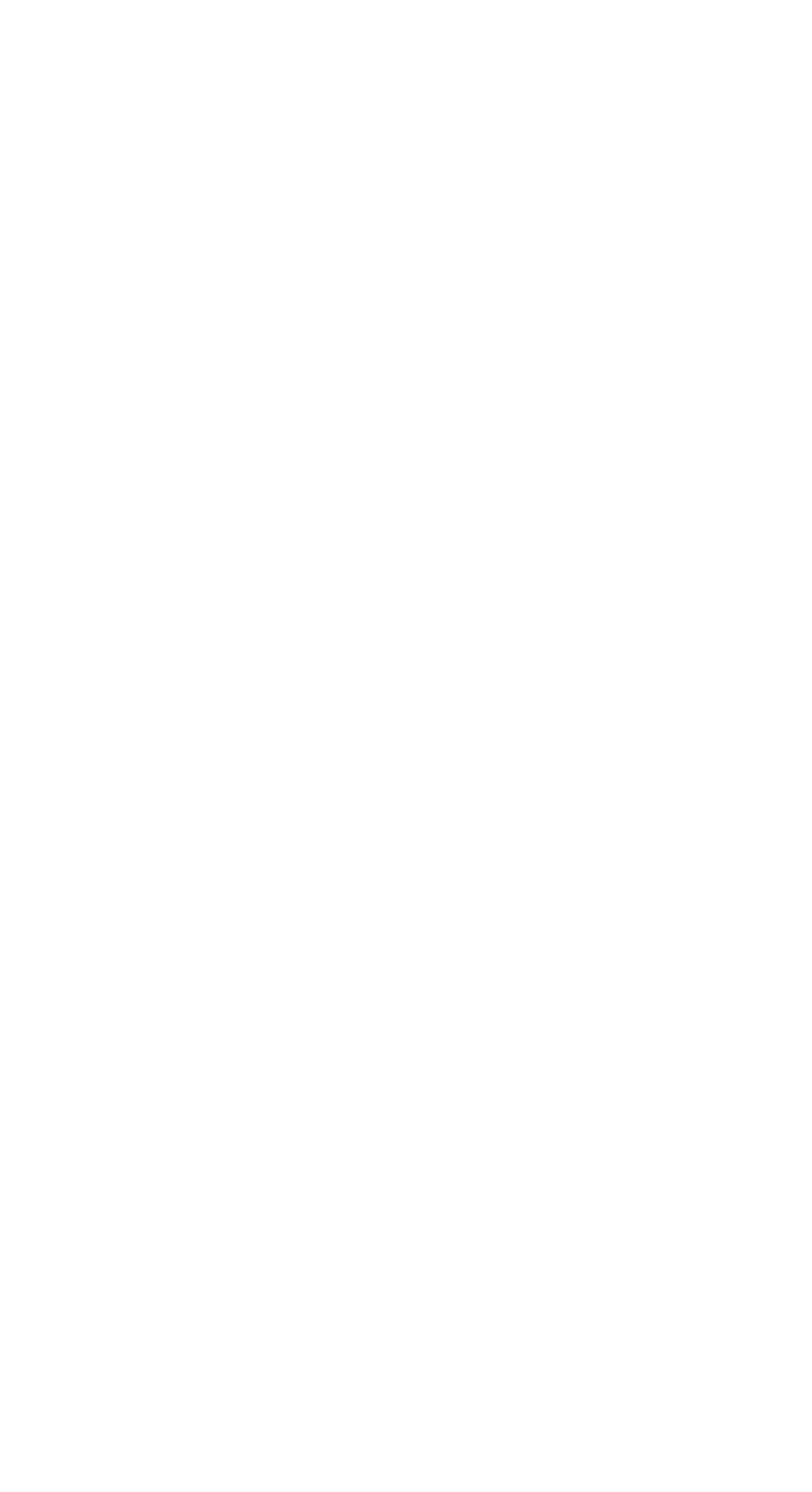




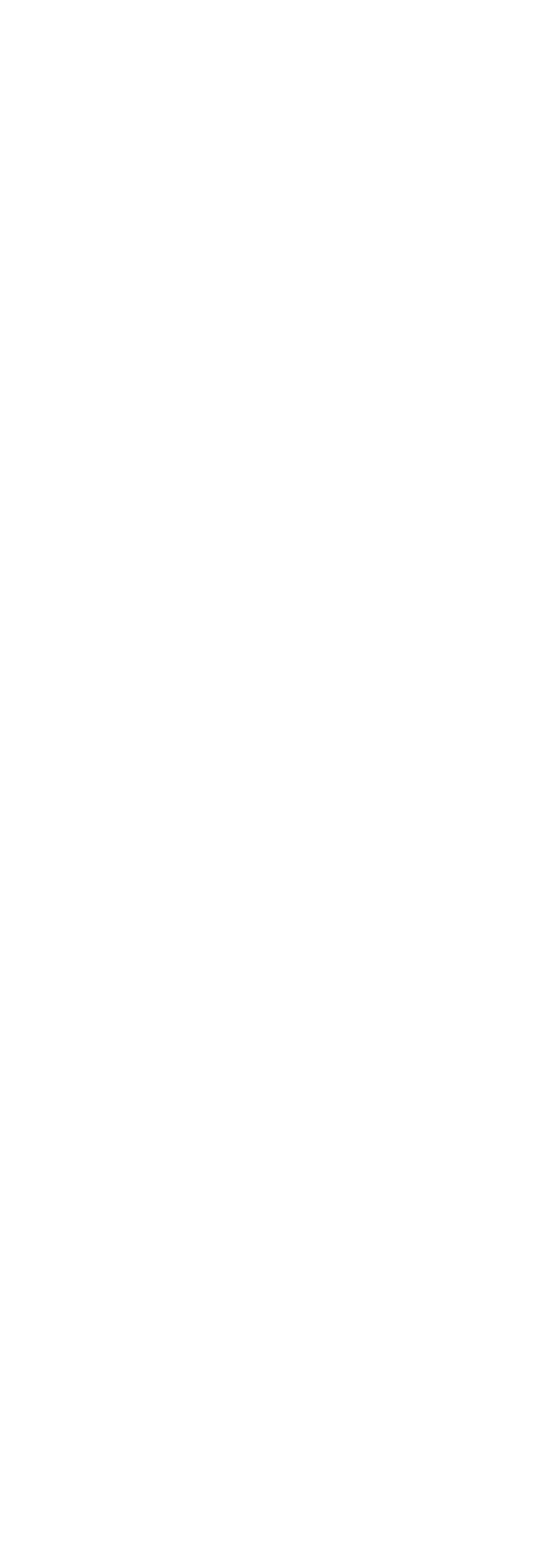




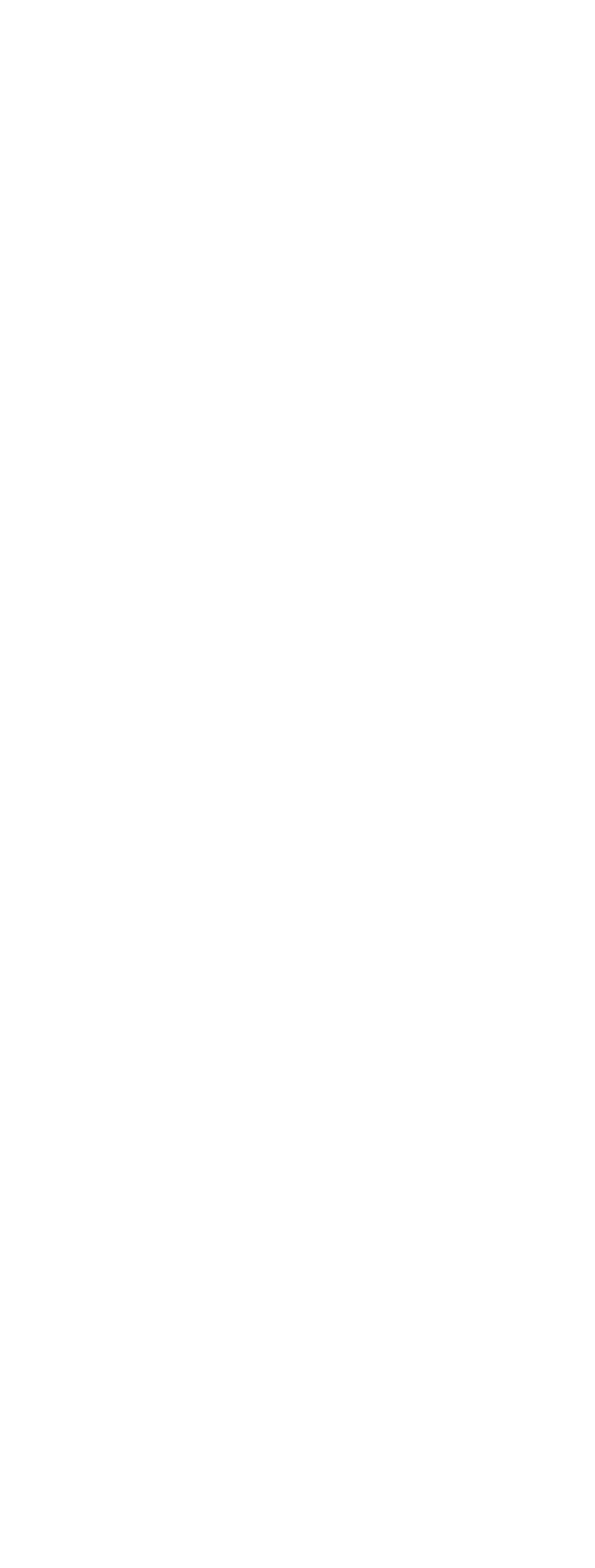




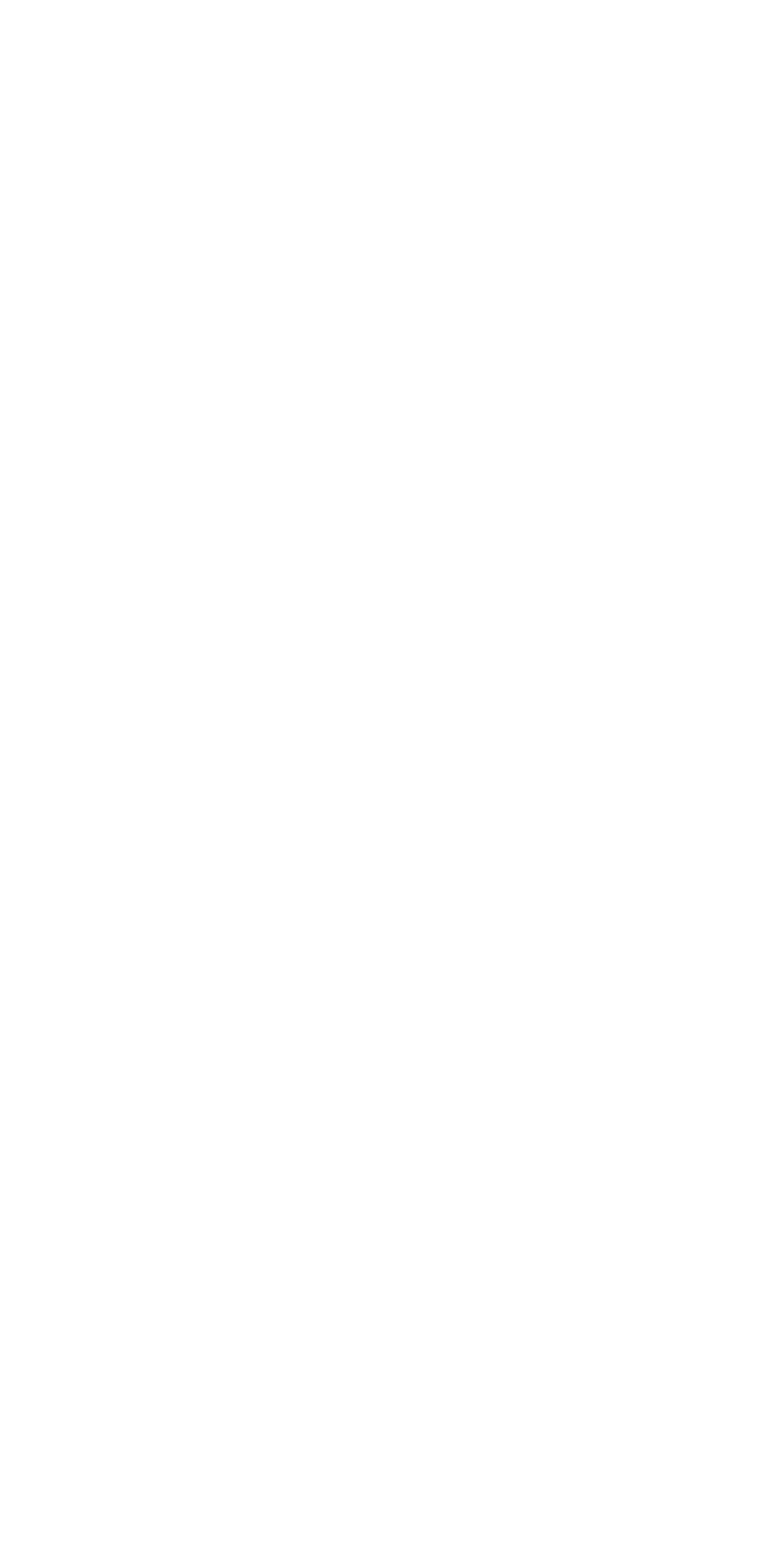




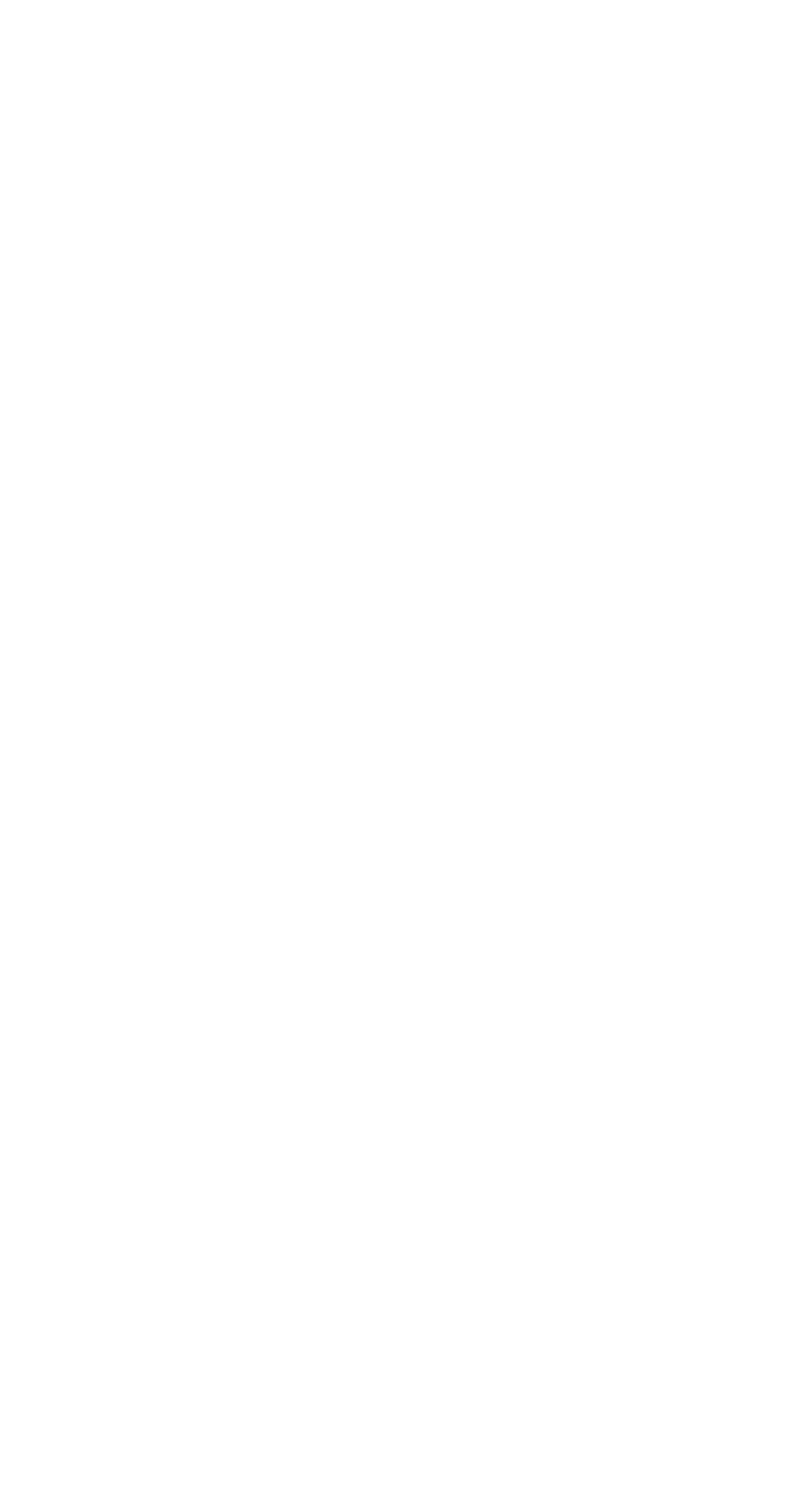




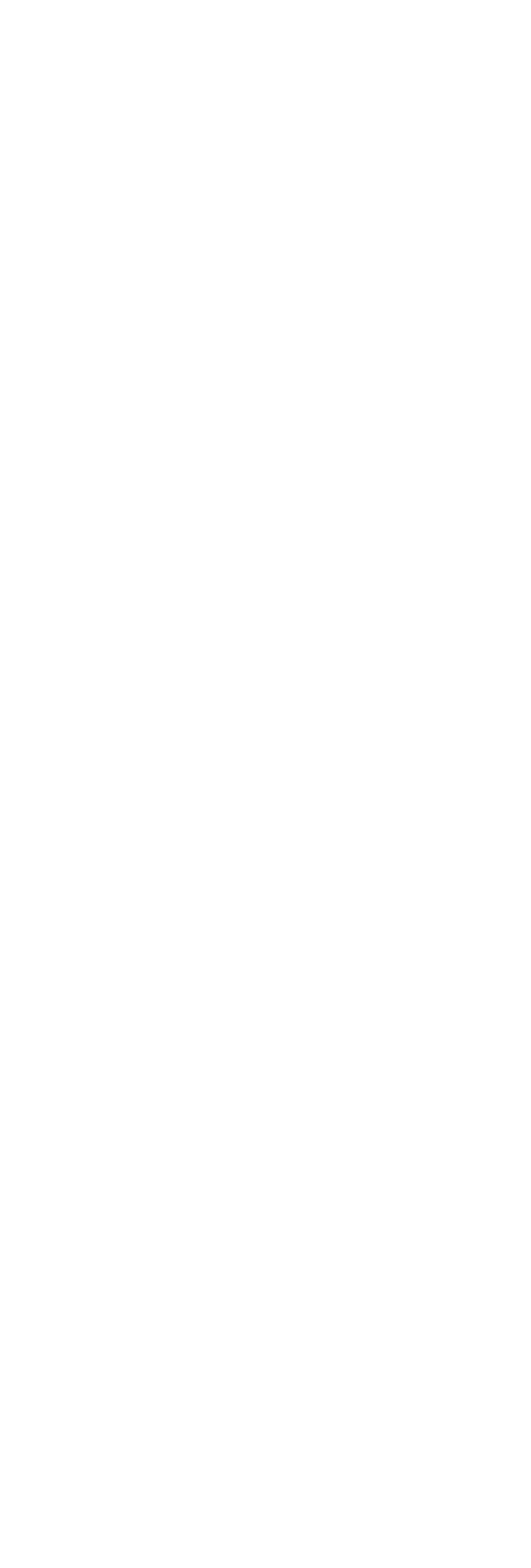




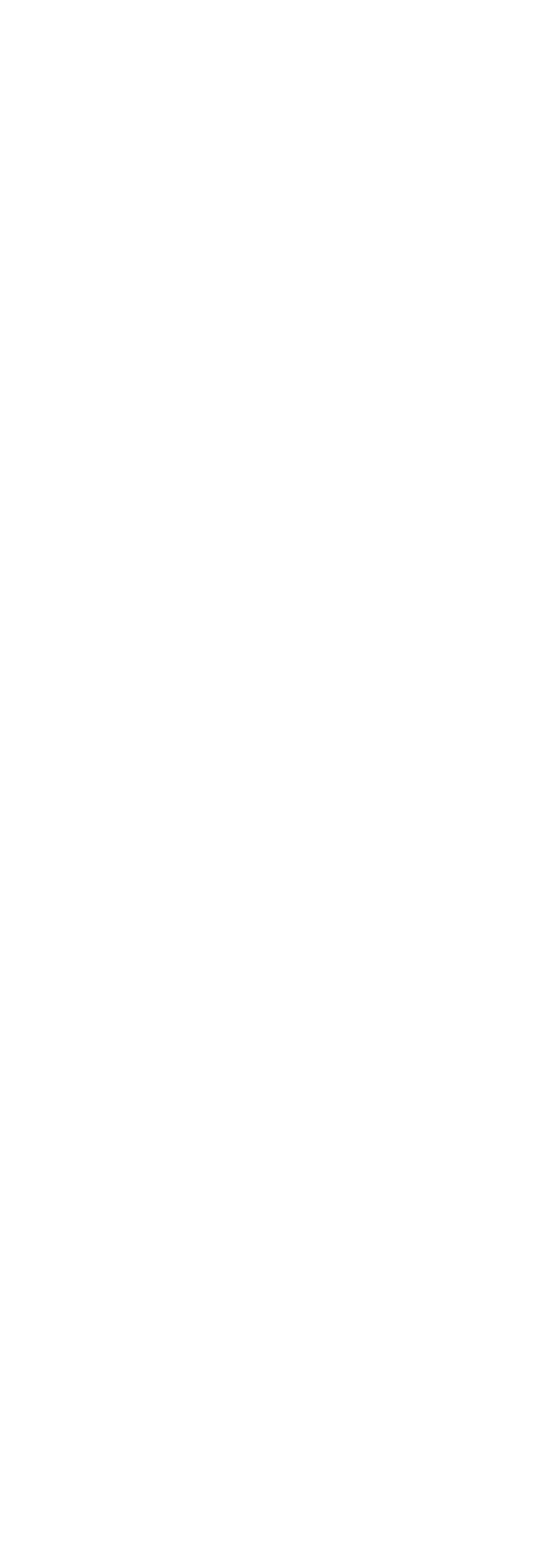




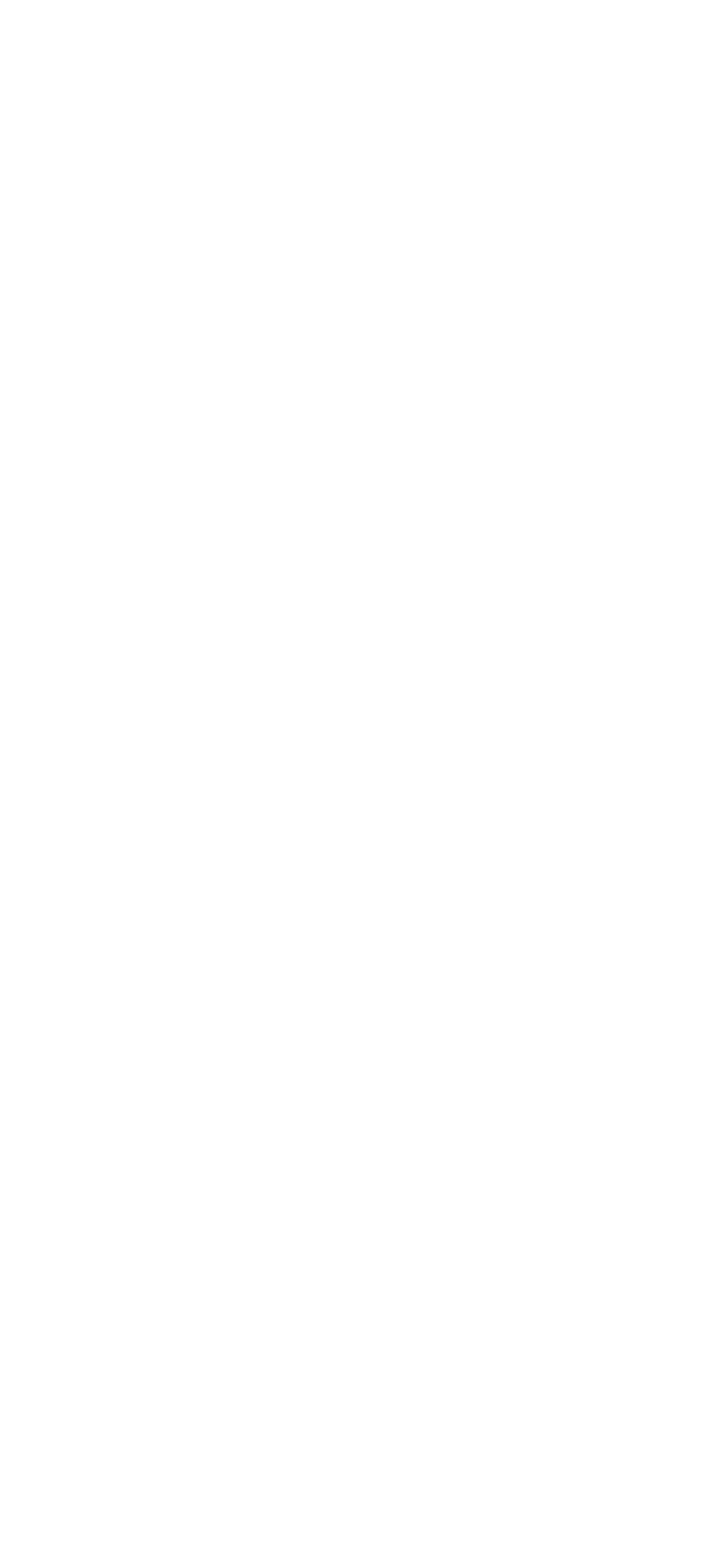




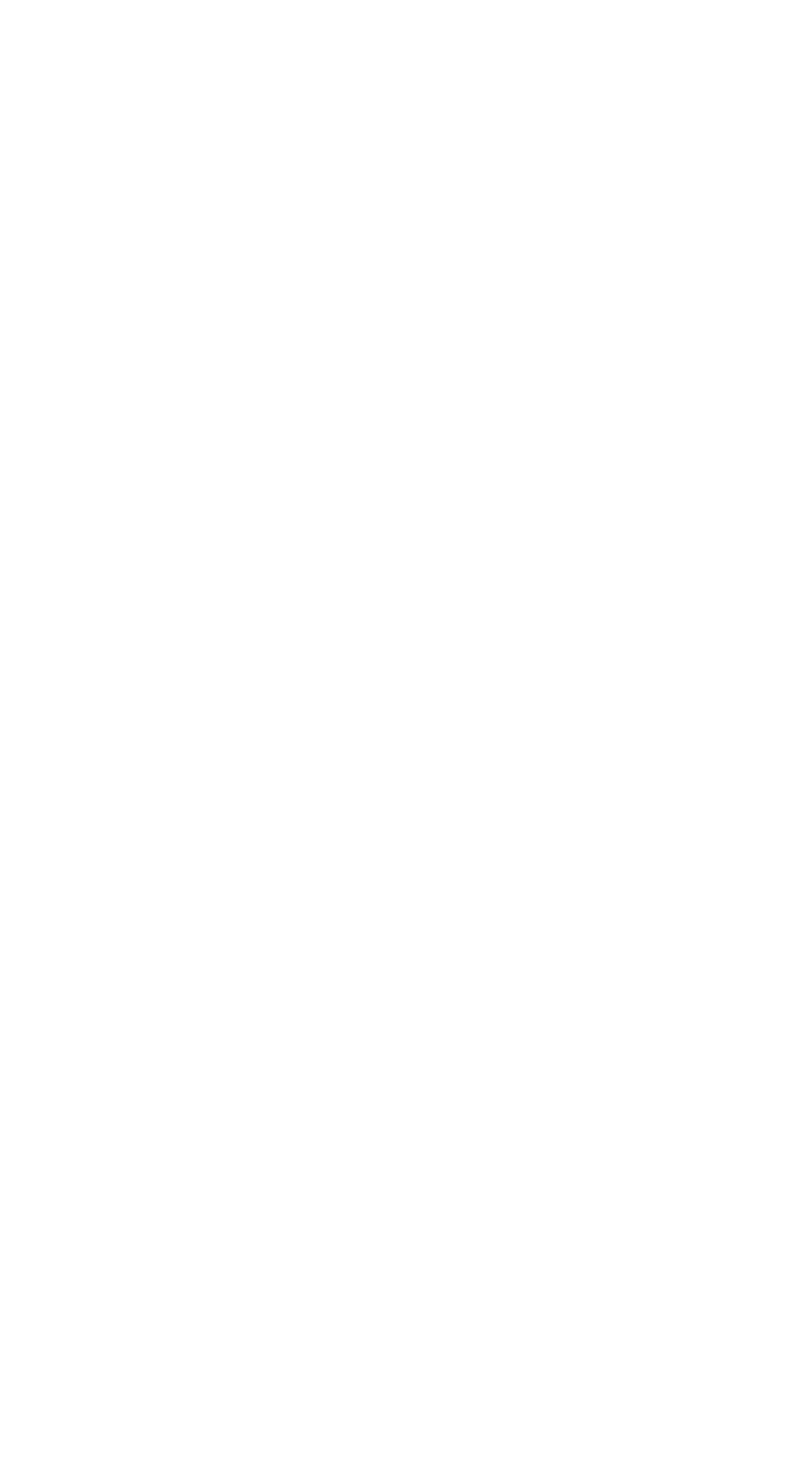




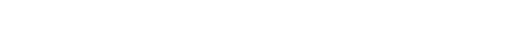

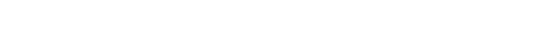

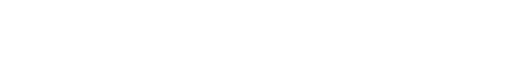

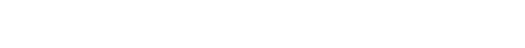

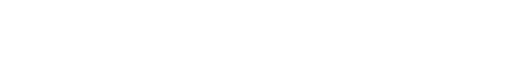

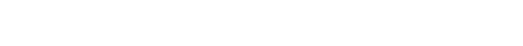

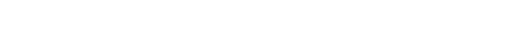

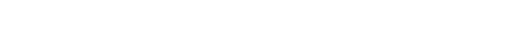

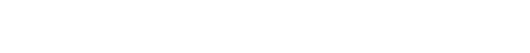

000000000000000000000000

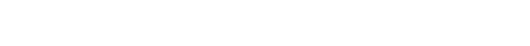

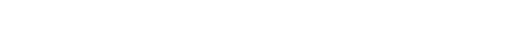

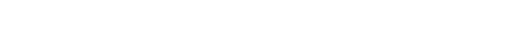

\begin{tabular}{|c|c|c|c|}
\hline 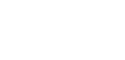 & 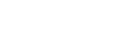 & 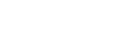 & 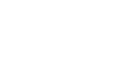 \\
\hline 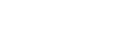 & ஜららの゚の゚ & 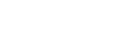 & ద \\
\hline
\end{tabular}

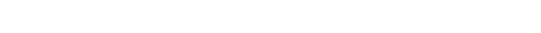

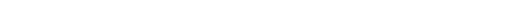

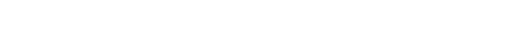

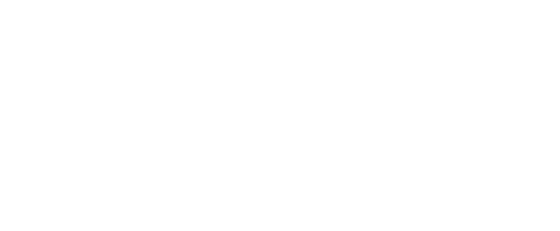




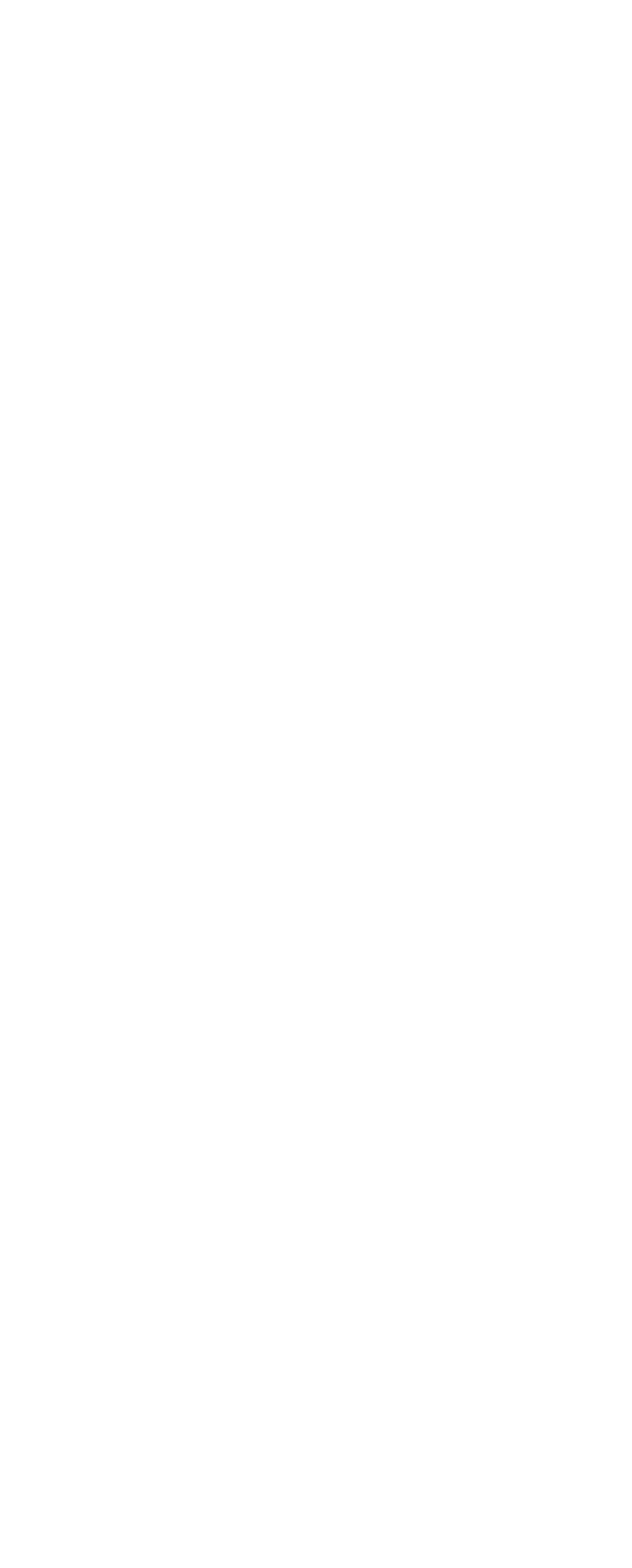




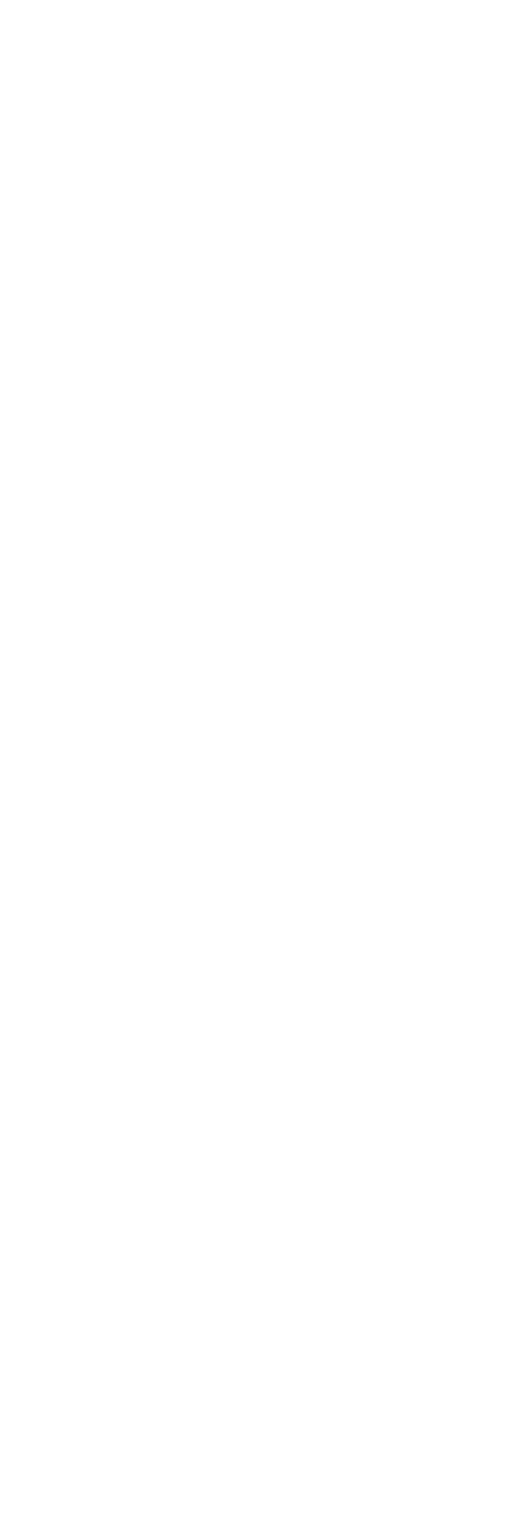

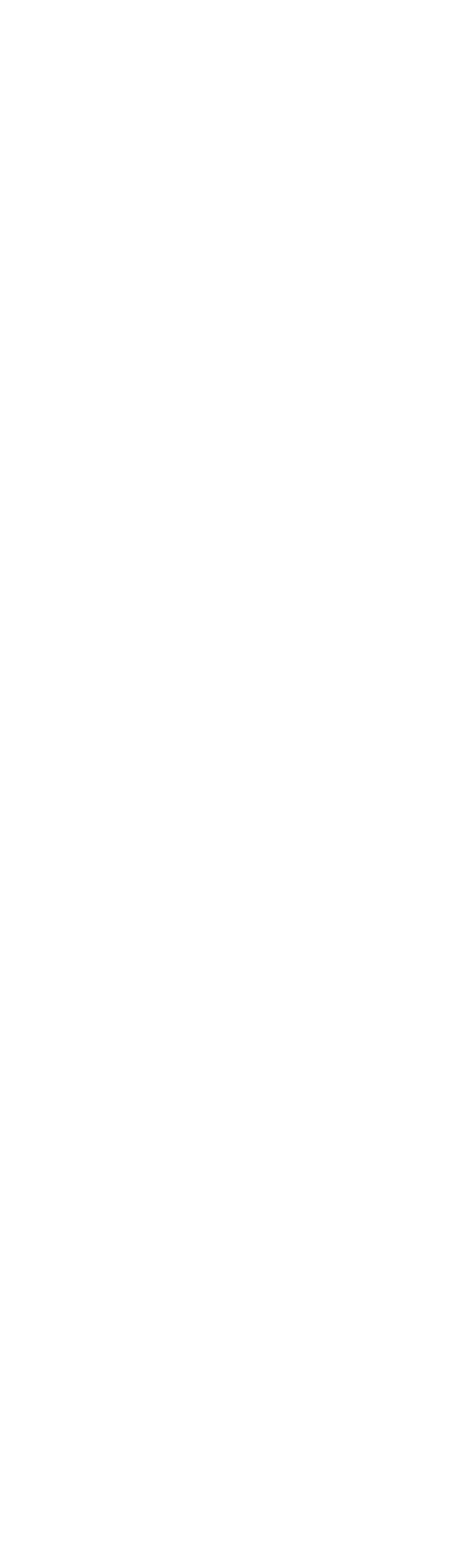




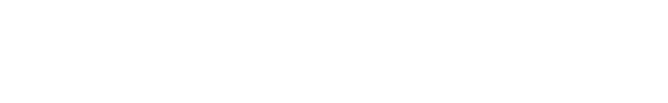

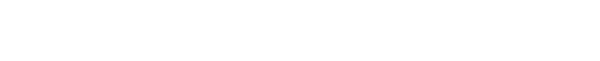

器

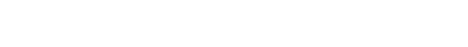

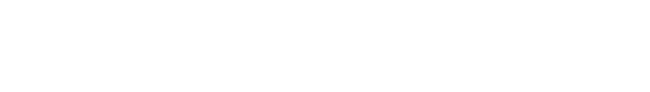

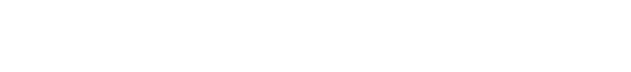

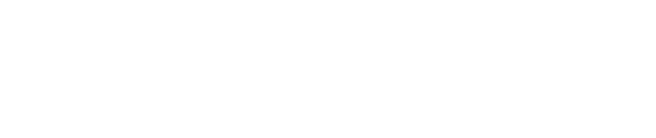
".

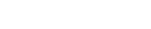
त्व

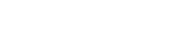
in

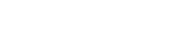

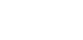
tic on 5 口 o

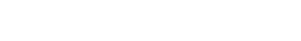

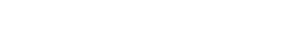

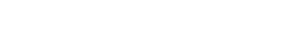
w +

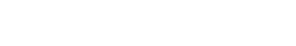

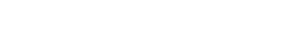

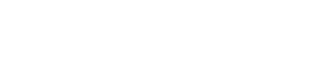

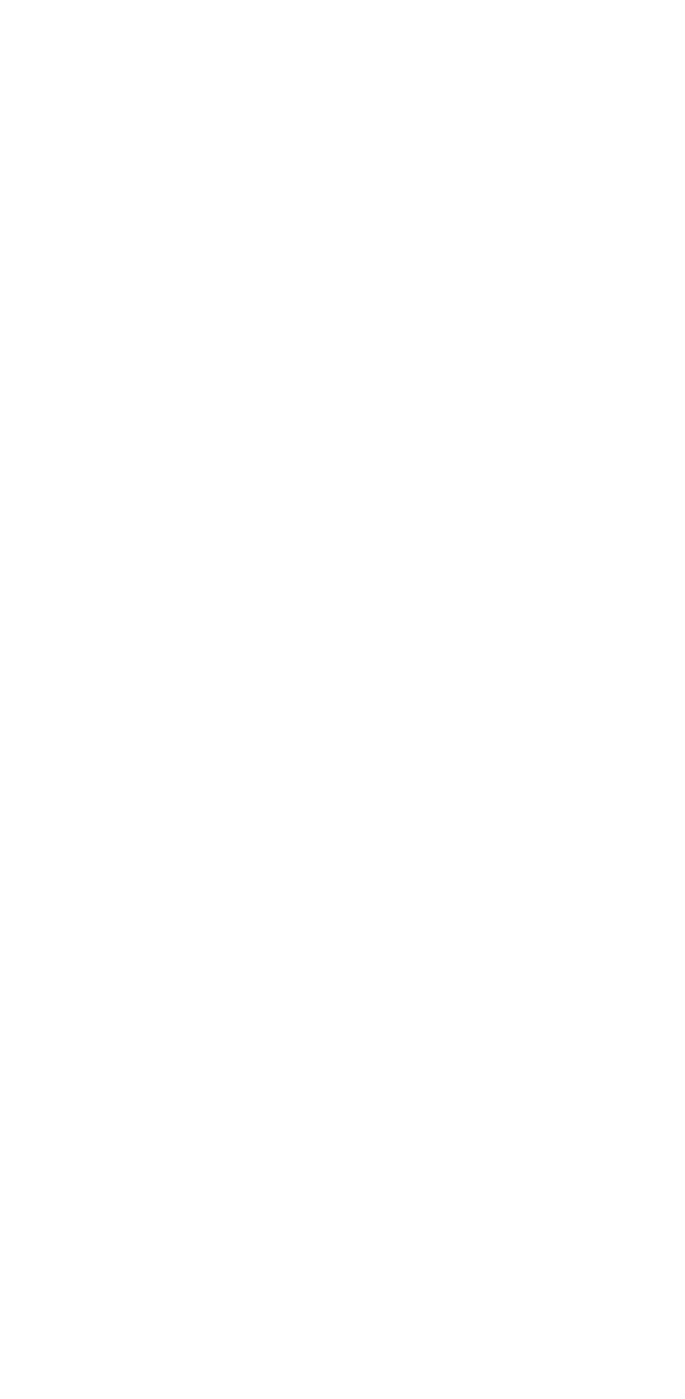




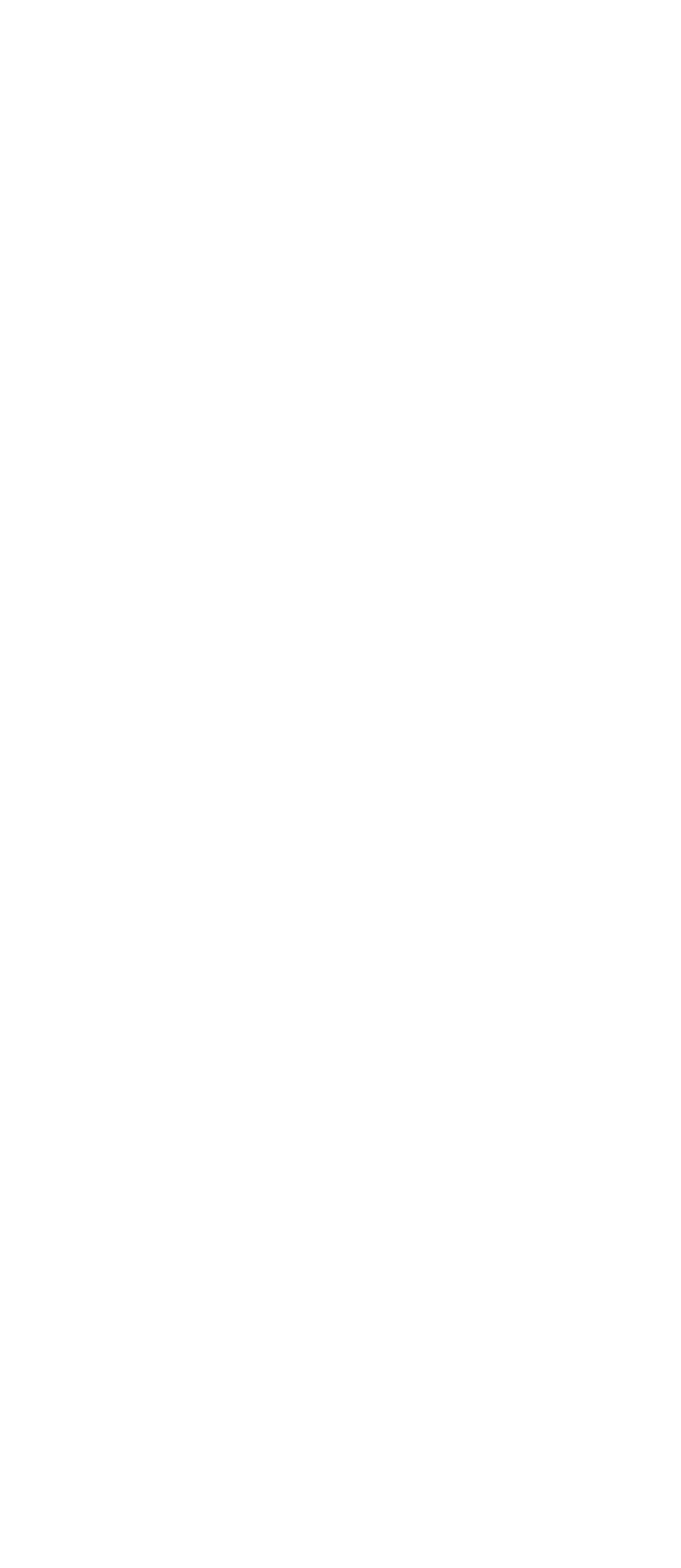




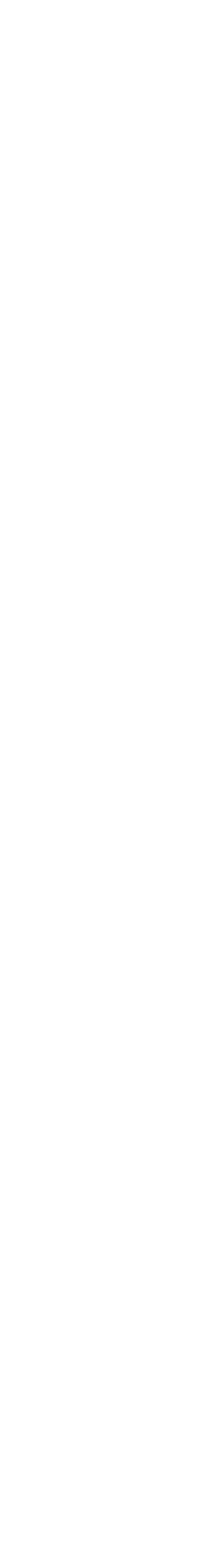

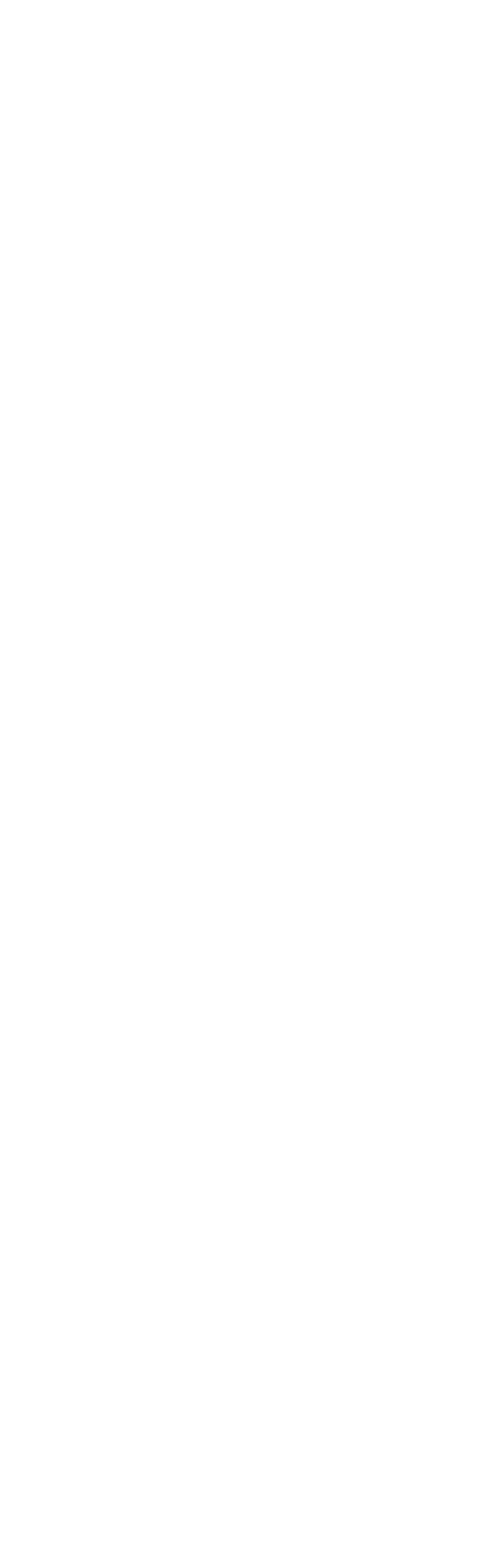




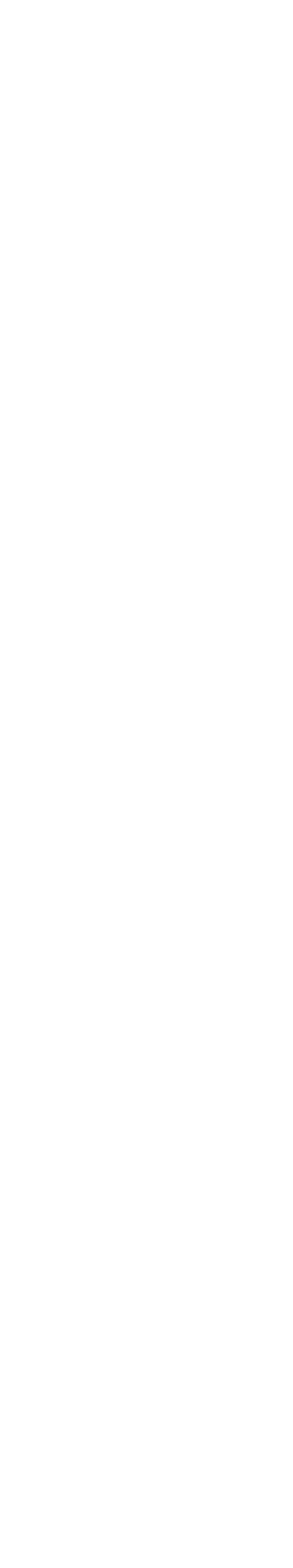

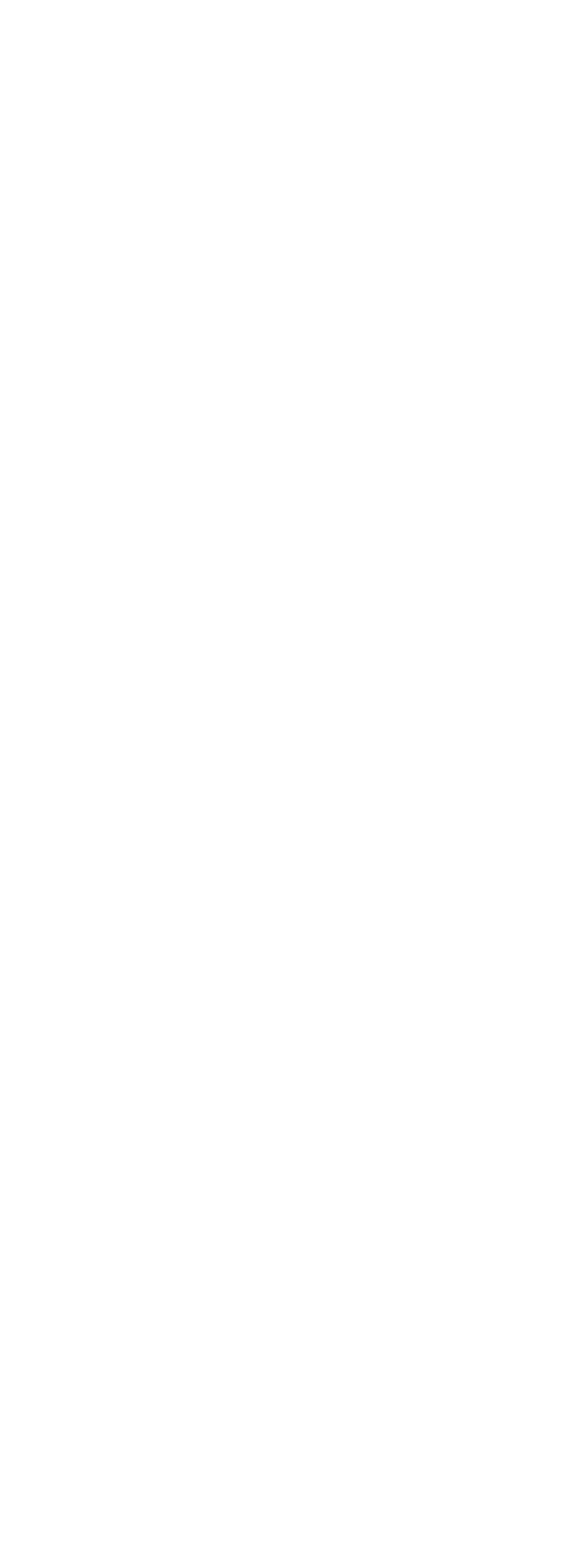




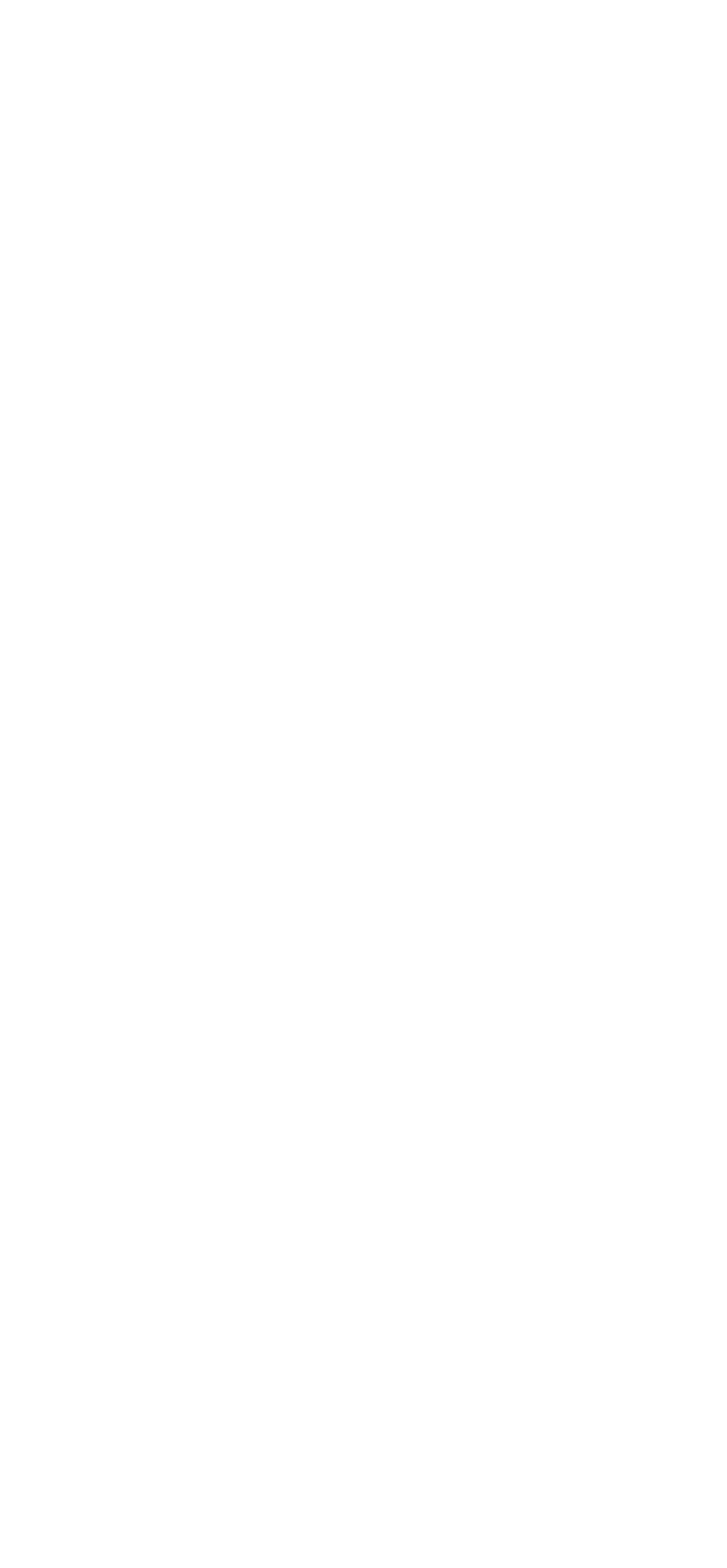



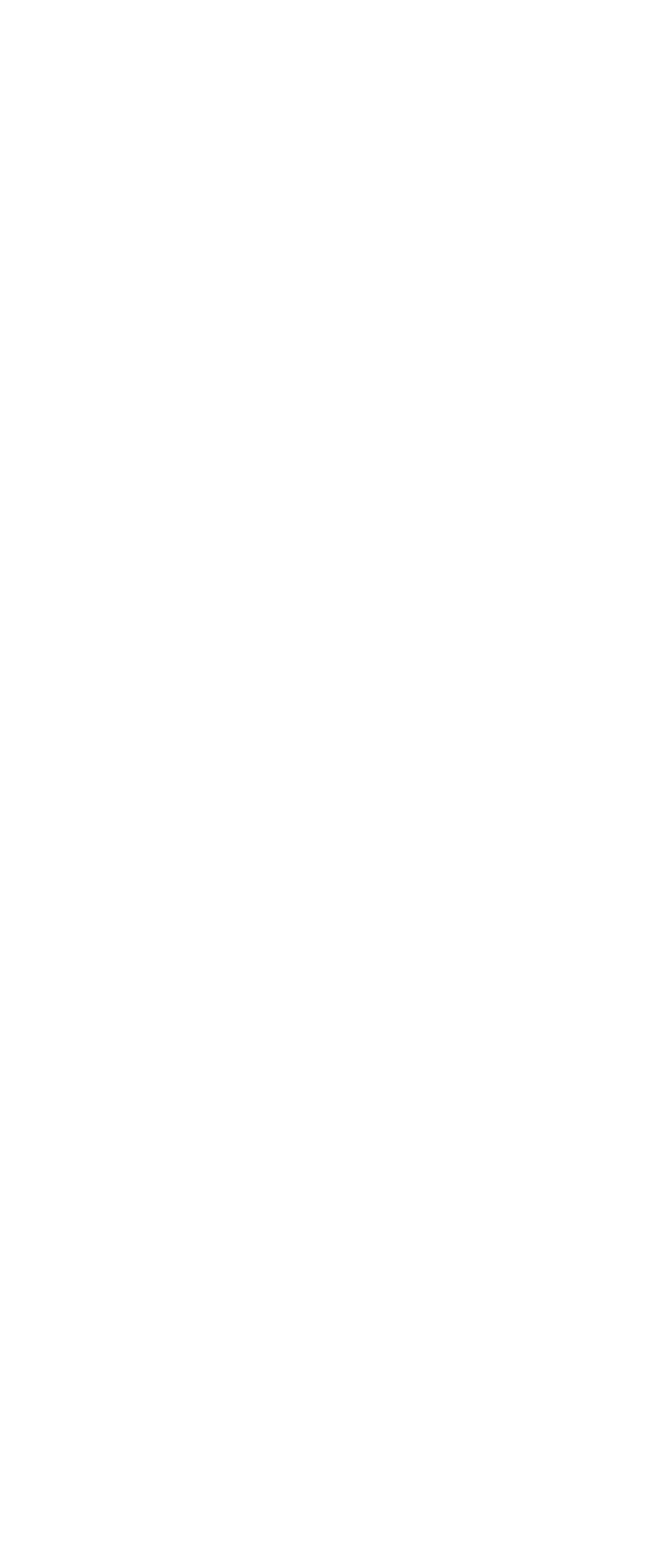


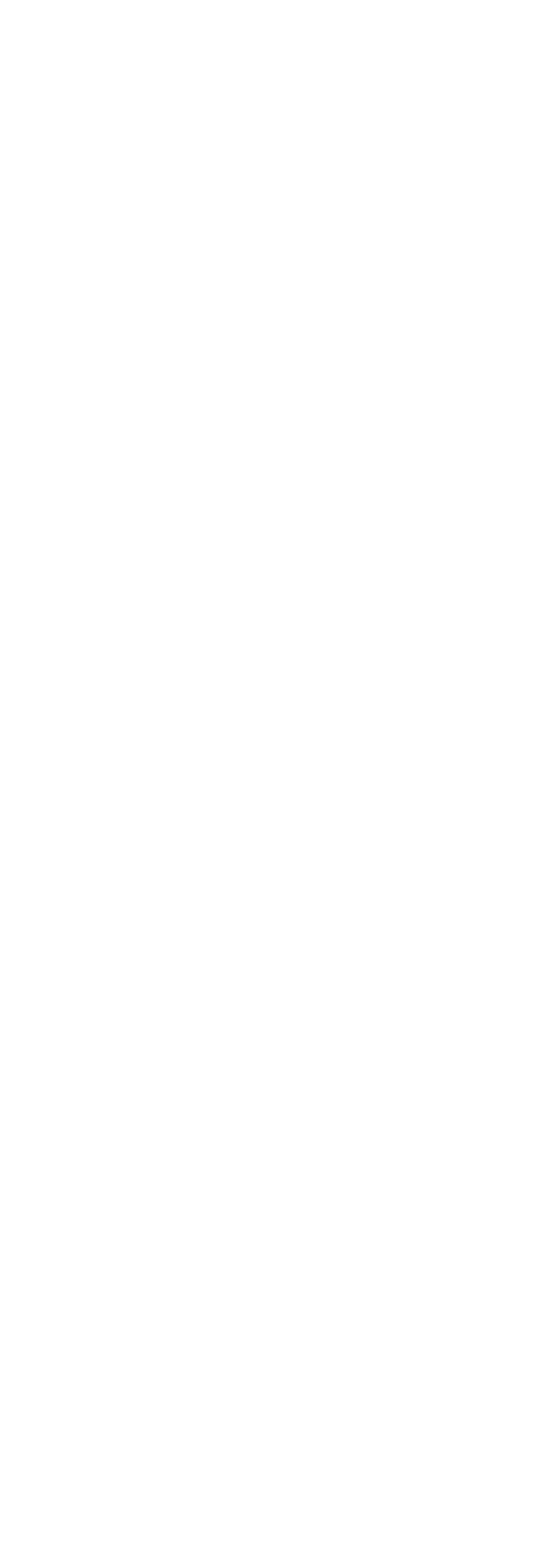




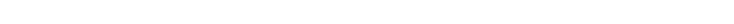

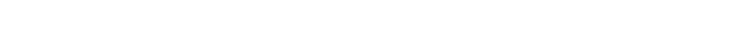

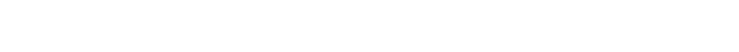

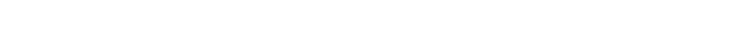
ம்ंம்

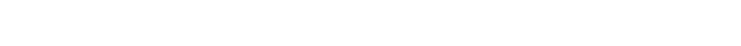

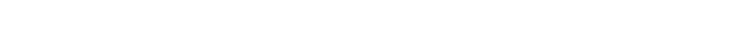

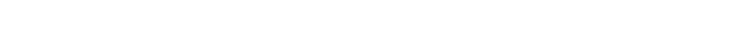

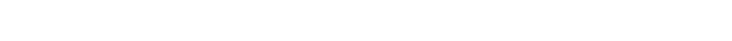

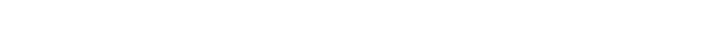

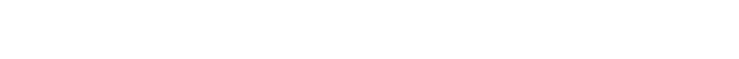

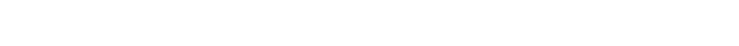
II I I

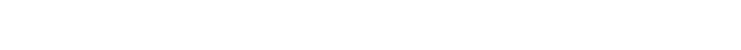

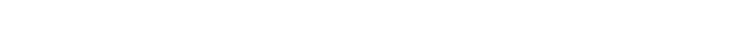

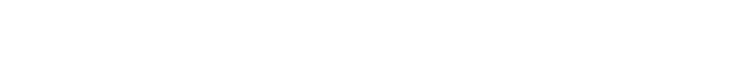

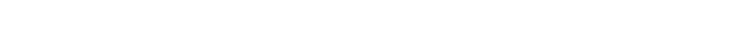

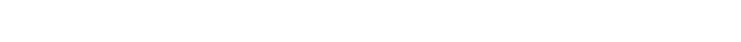




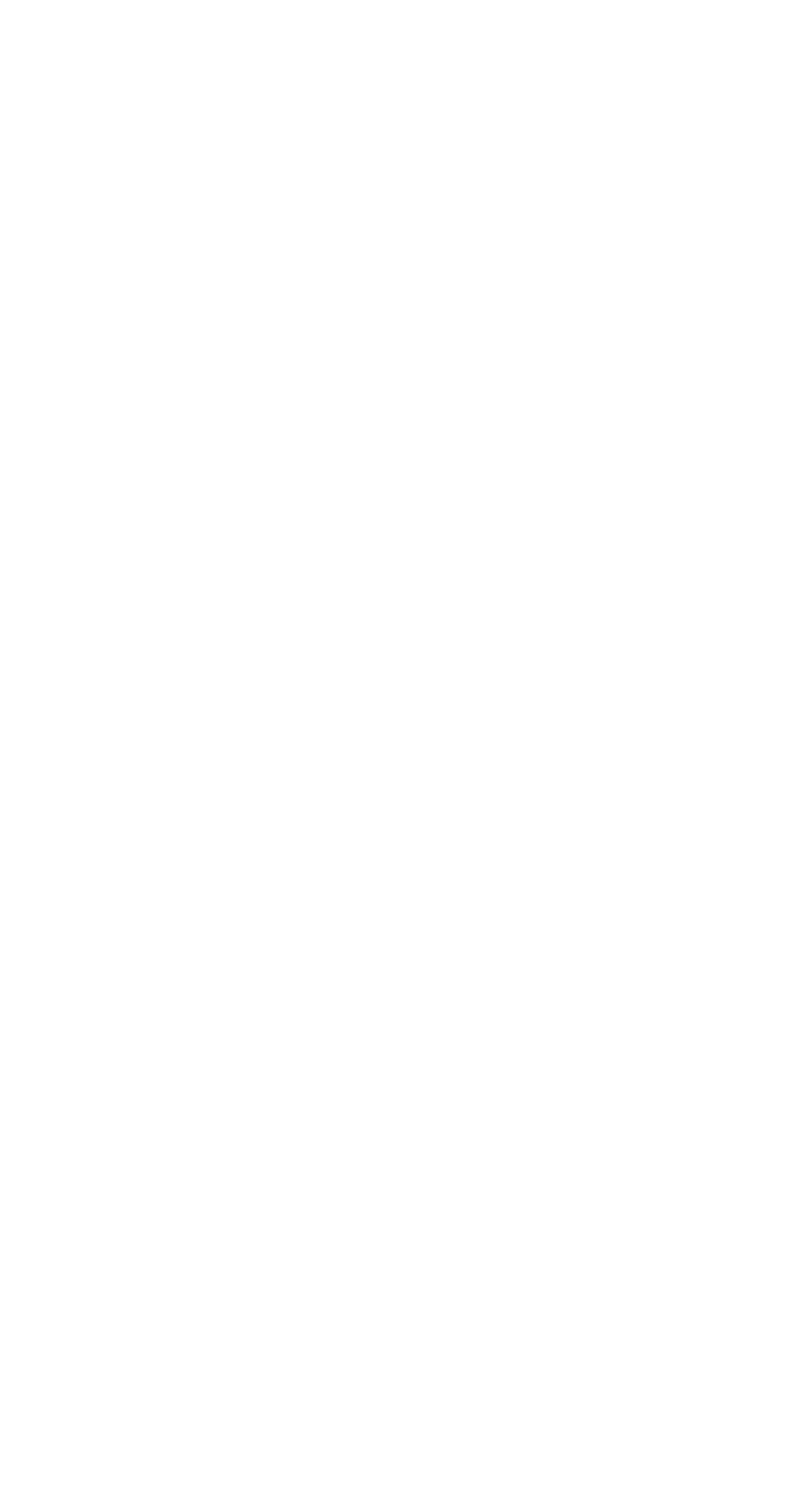




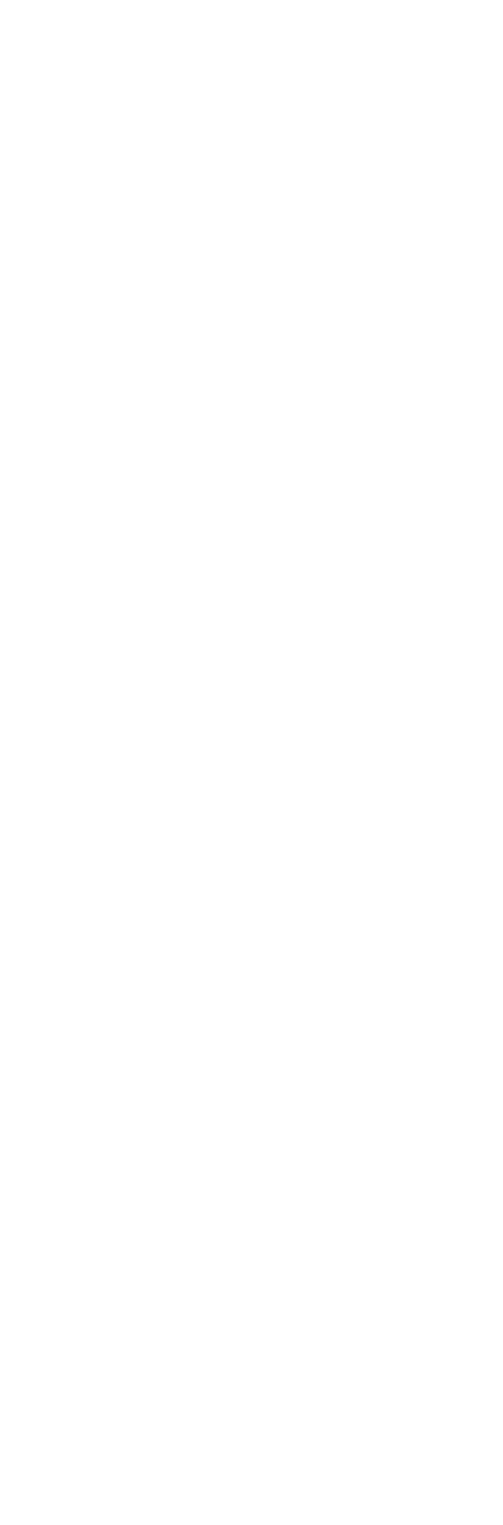




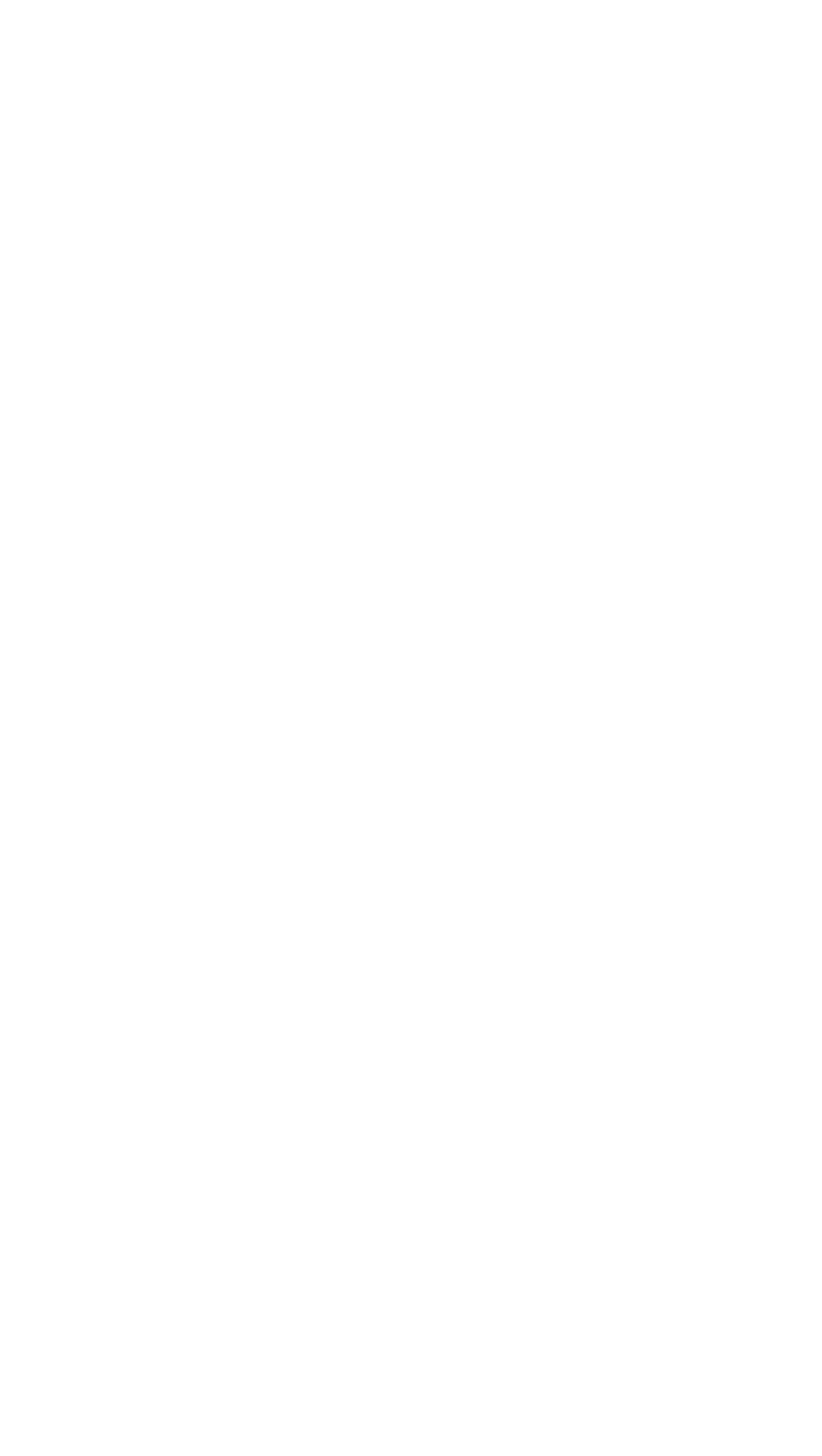




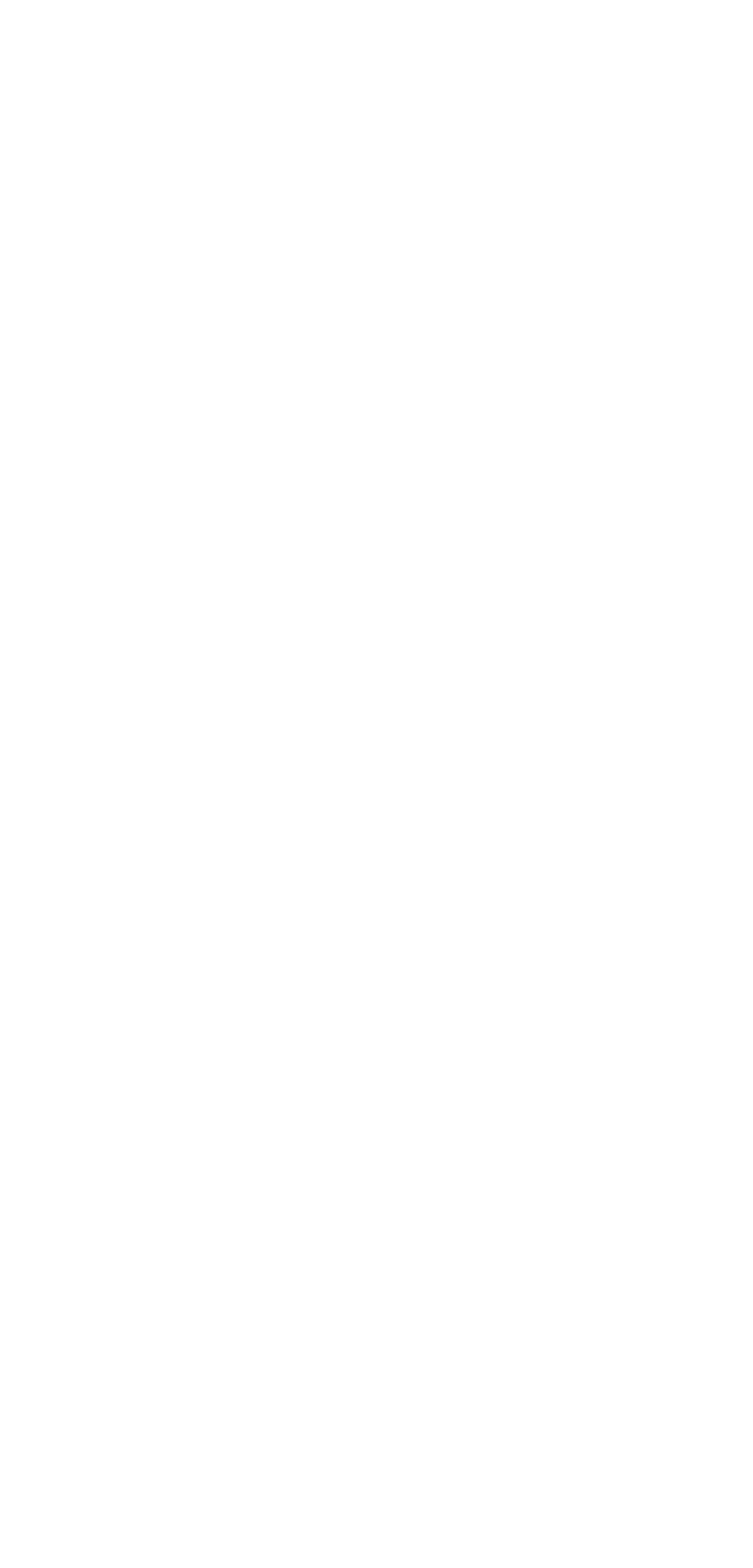




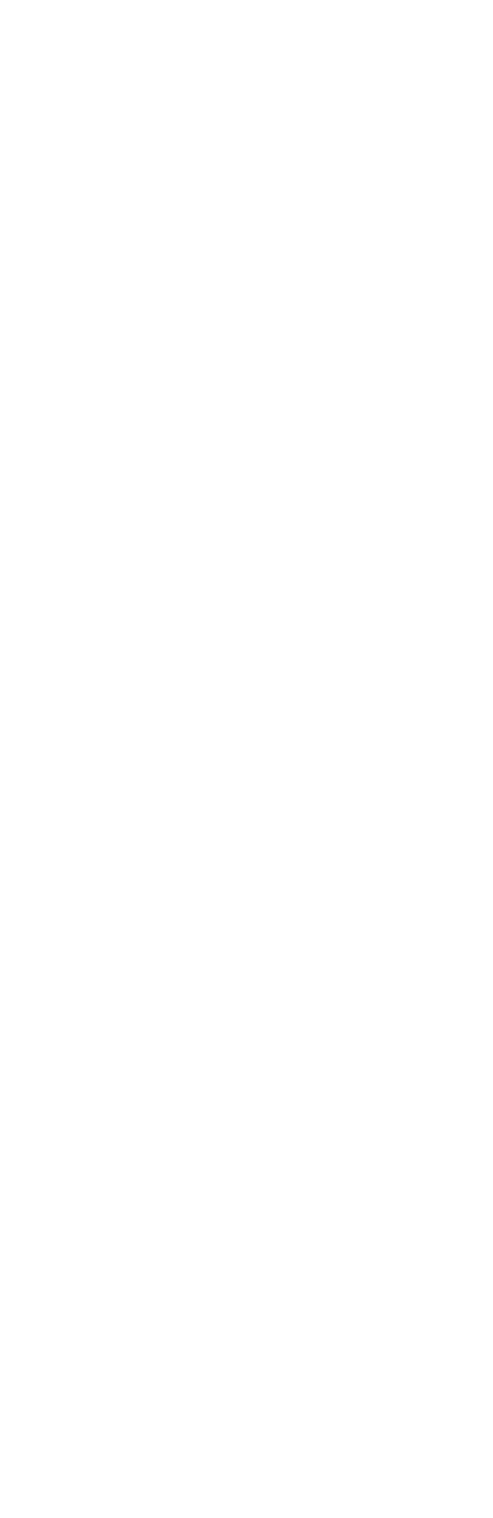




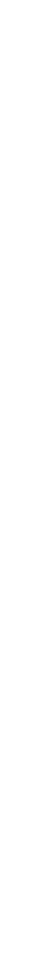$$
\text { 苟 }
$$

\begin{tabular}{|c|c|}
\hline 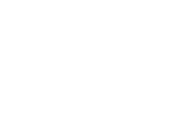 & 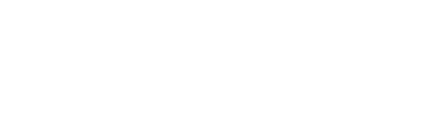 \\
\hline 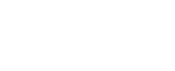 & 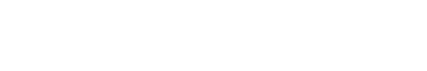 \\
\hline 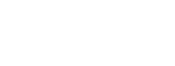 & 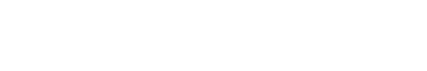 \\
\hline 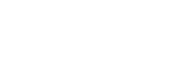 & 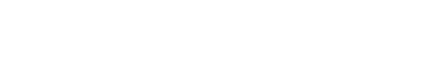 \\
\hline 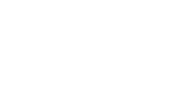 & 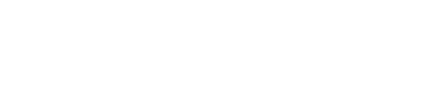 \\
\hline $\begin{array}{lll} & \\
0\end{array}$ & 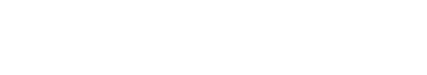 \\
\hline 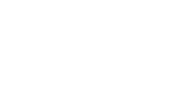 & 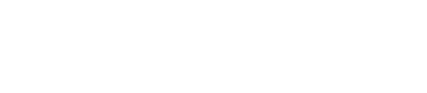 \\
\hline 嵩歖鱼 & 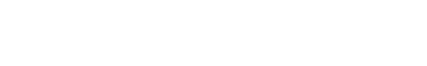 \\
\hline 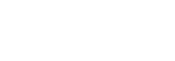 & 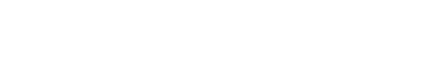 \\
\hline 等 & 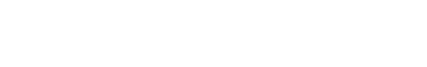 \\
\hline ㅎํㄹำ & 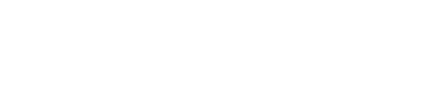 \\
\hline 要 & 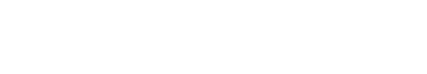 \\
\hline 4. & -00000 00000 \\
\hline 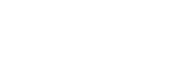 & 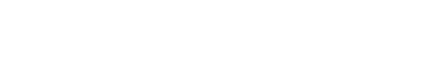 \\
\hline 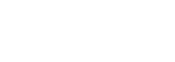 & \\
\hline 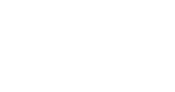 & 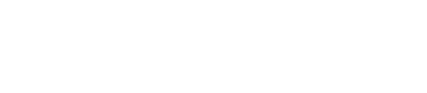 \\
\hline 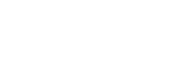 & \\
\hline 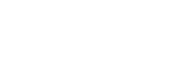 & \\
\hline 㿣胥 & \\
\hline 产高 & 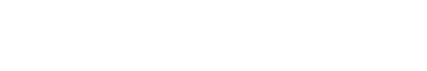 \\
\hline 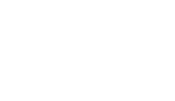 & 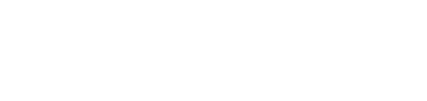 \\
\hline 影嘻 & 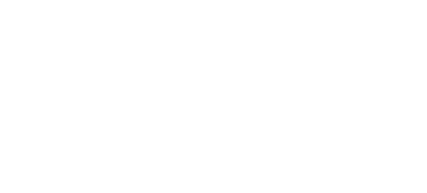 \\
\hline
\end{tabular}




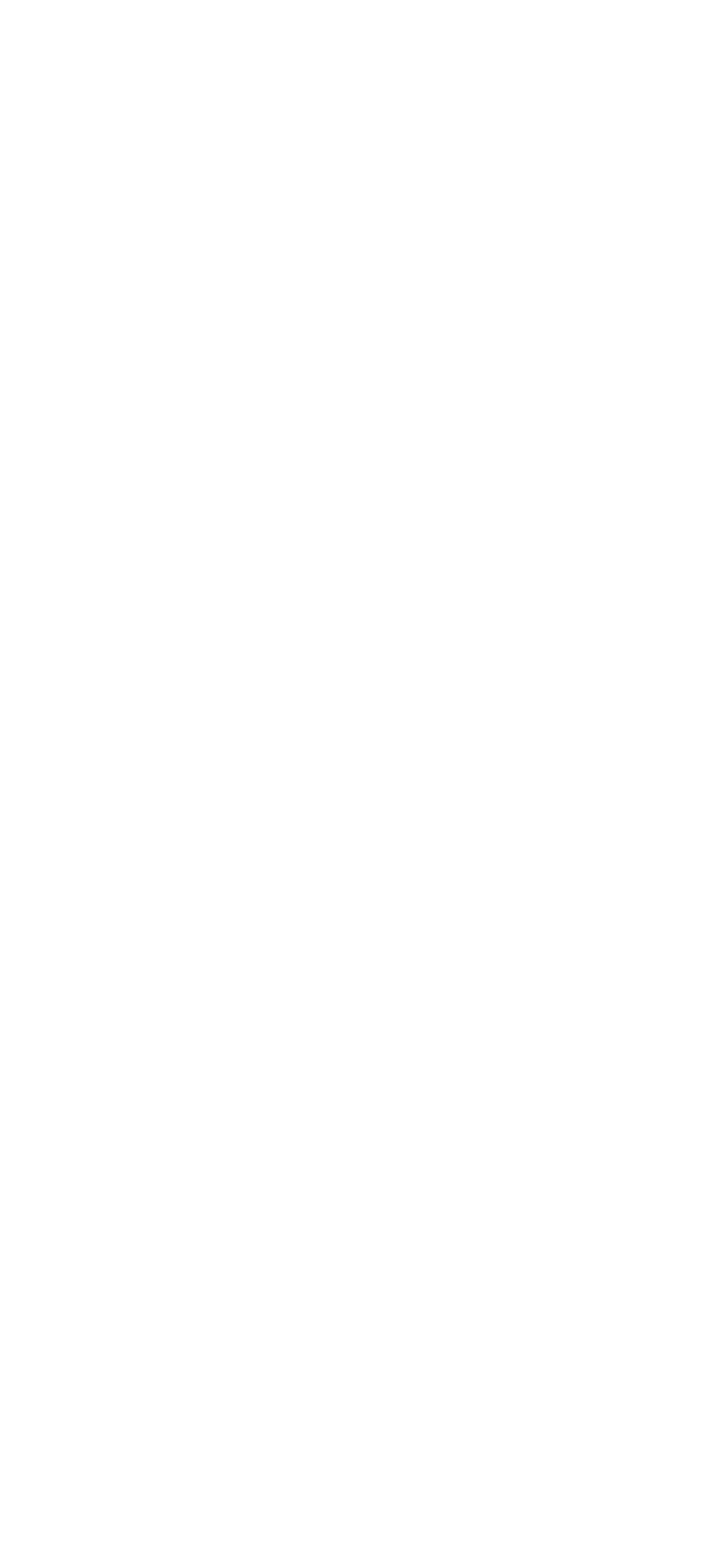




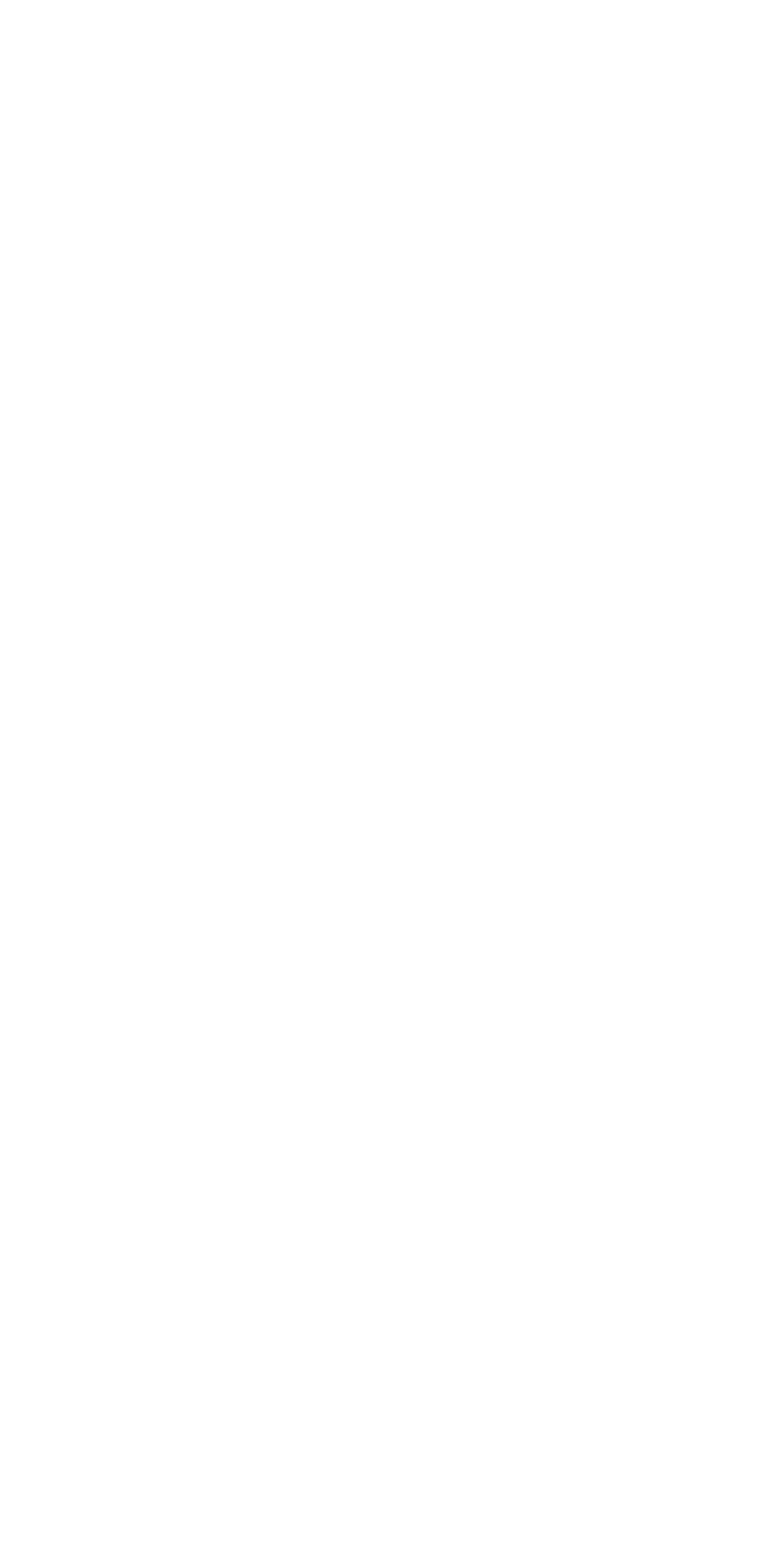




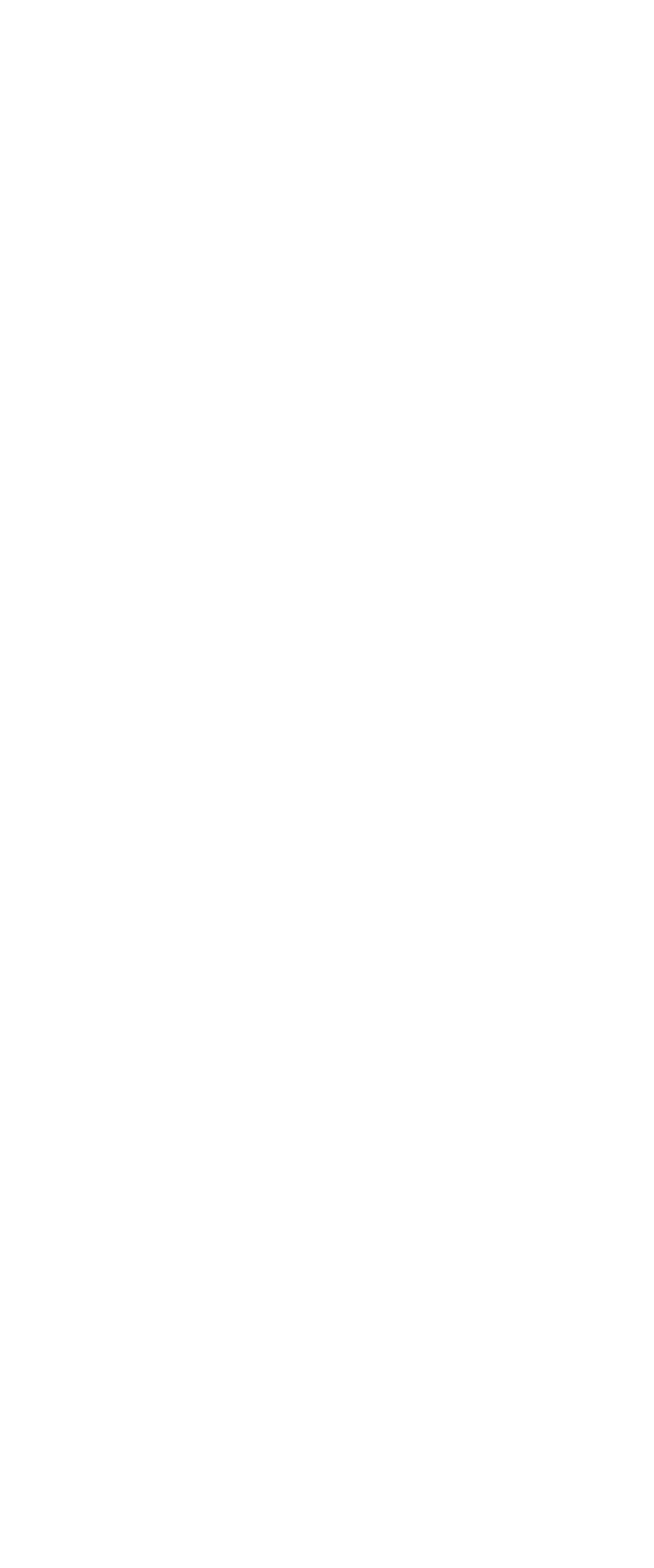


ஸீ亠凶禸்

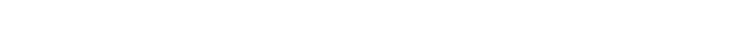

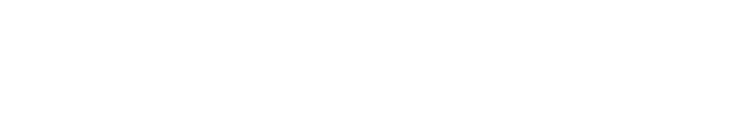

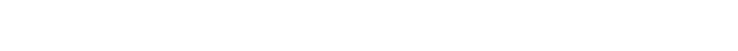

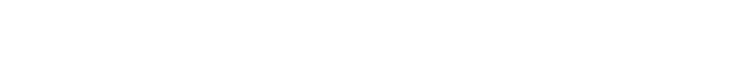

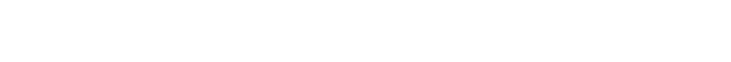

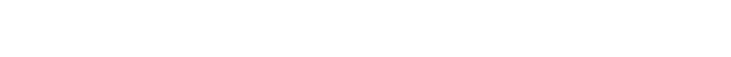

$\overline{1111141111411141111411114111141}$

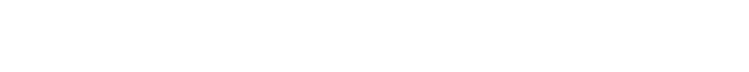

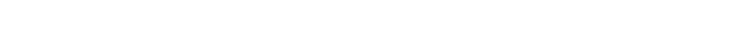

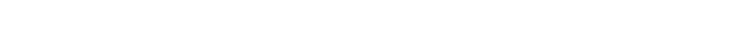

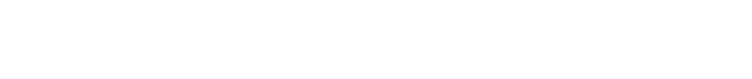

00000 000mn mo000 00N00 0nO00 00070 0h

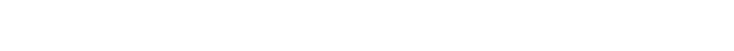

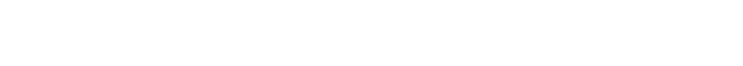

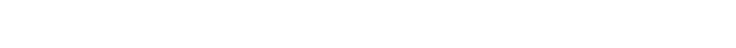

\begin{tabular}{|c|c|c|c|c|c|c|}
\hline حتِ & 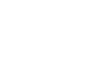 & ๑ & $\because 0^{\circ}=\infty \circ 0$ & ดสำสసี & $\prod_{-\infty}^{m \infty \infty^{\infty}}$ & $\ddot{ન}$ \\
\hline
\end{tabular}

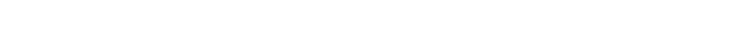

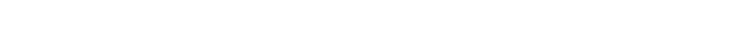

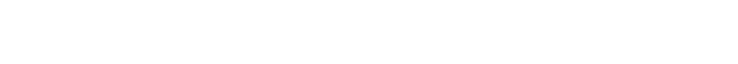

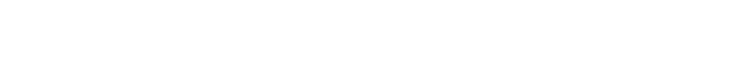

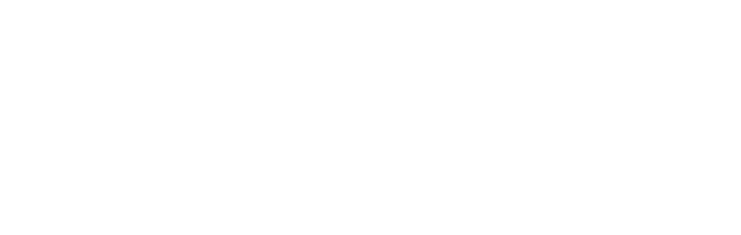




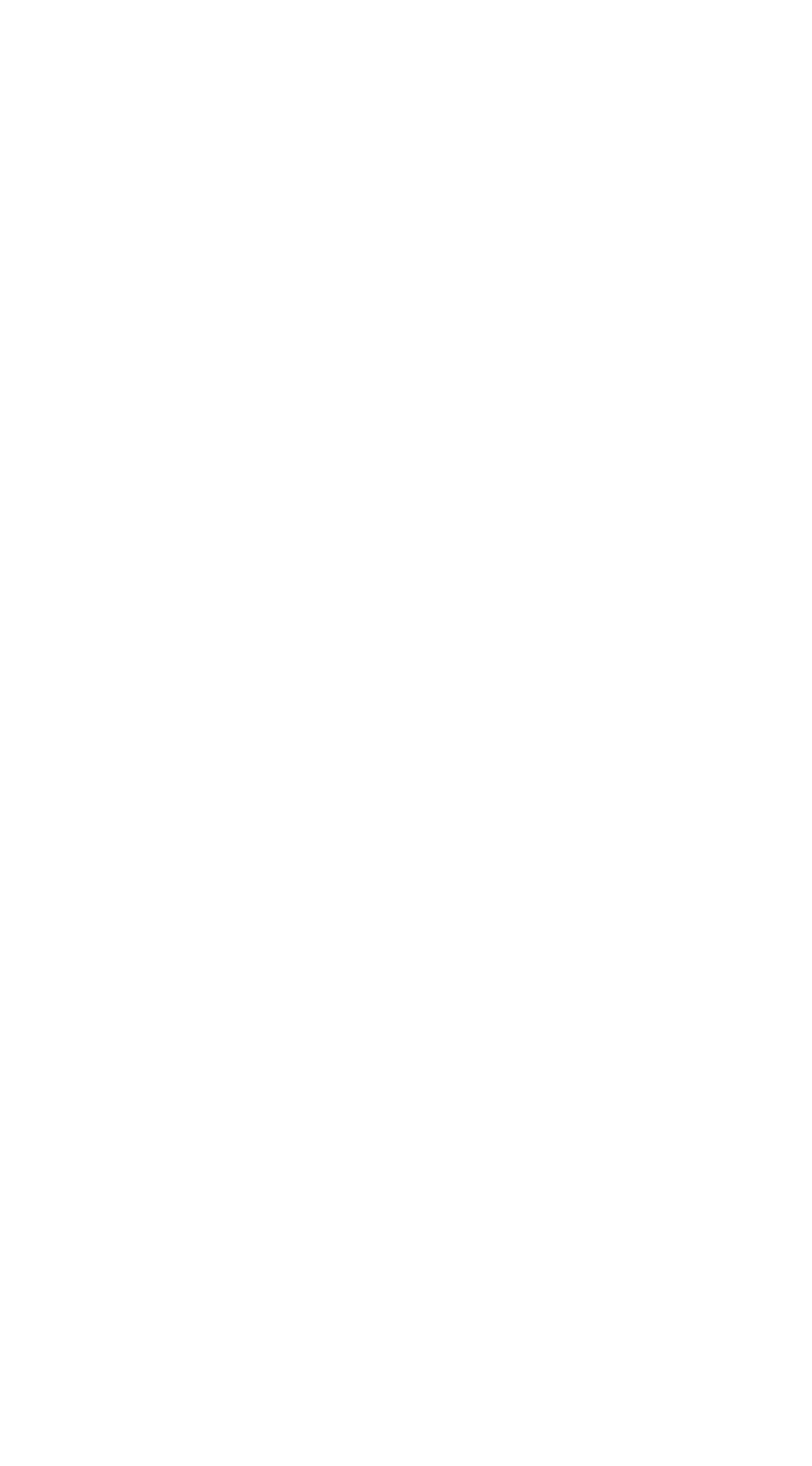




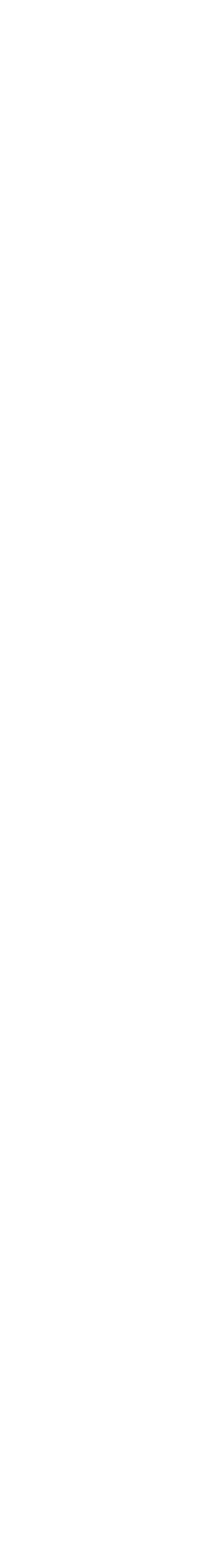

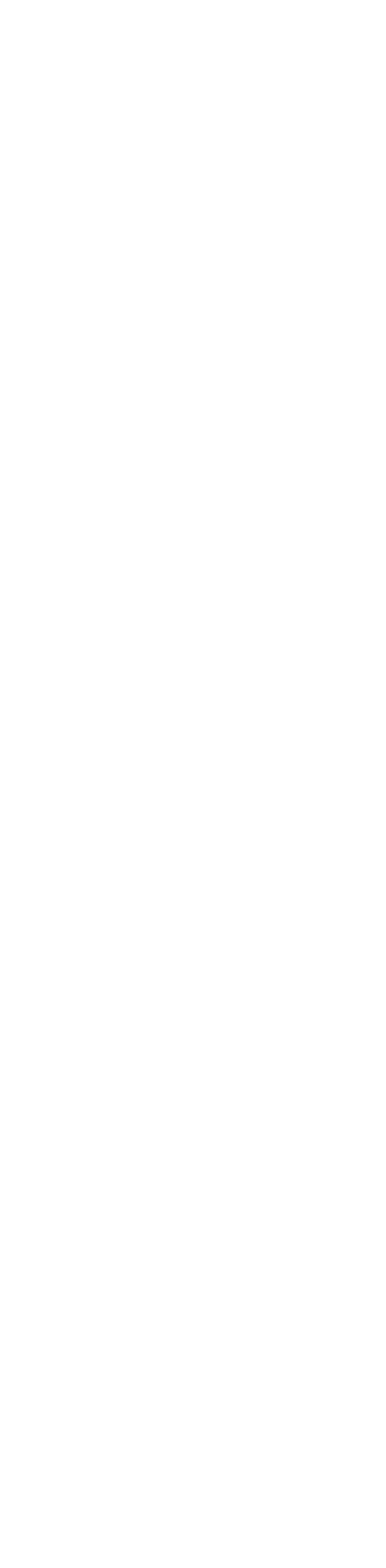




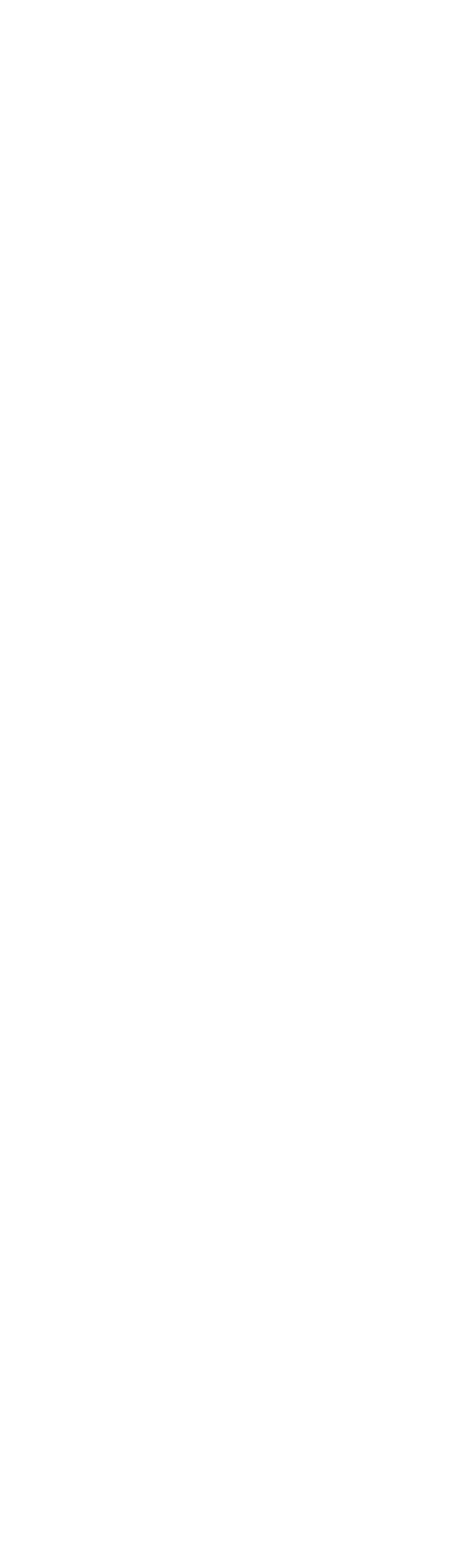

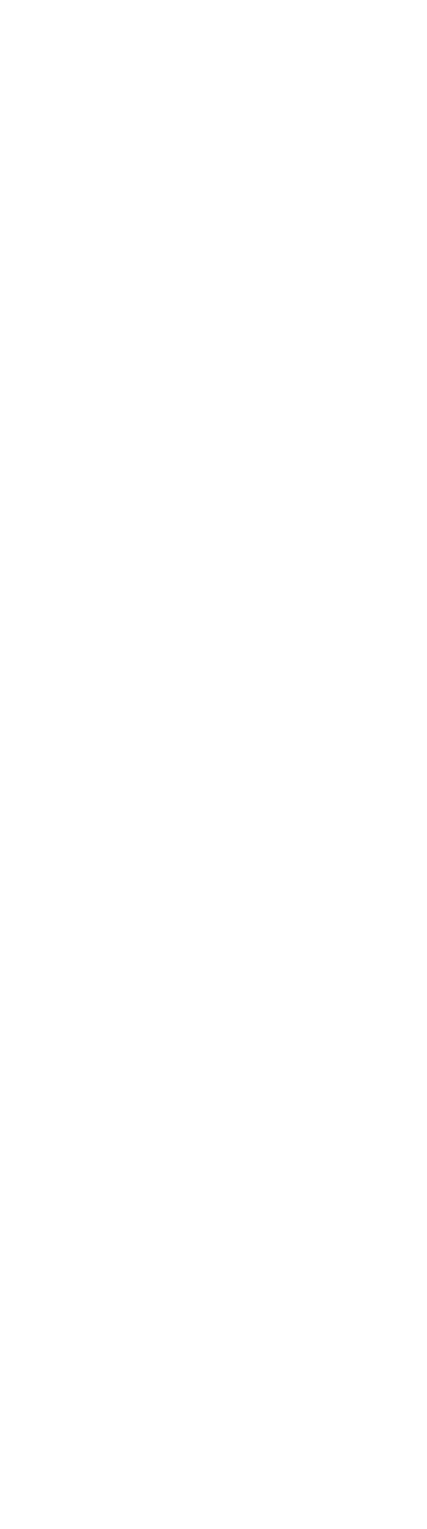




\begin{tabular}{|c|c|}
\hline 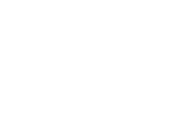 & 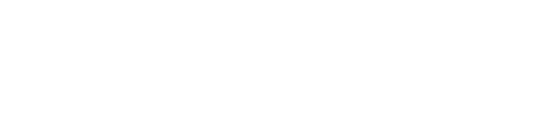 \\
\hline 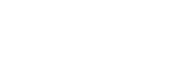 & 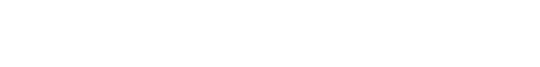 \\
\hline 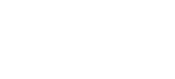 & 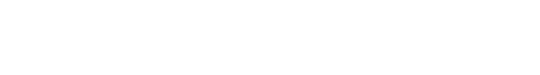 \\
\hline 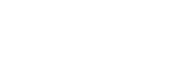 & 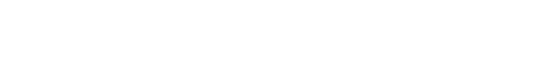 \\
\hline 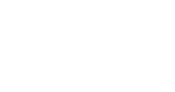 & 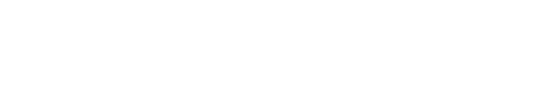 \\
\hline 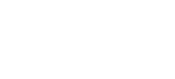 & 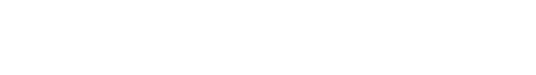 \\
\hline 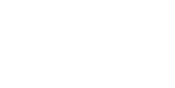 & 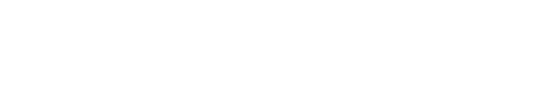 \\
\hline 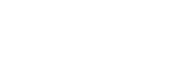 & \\
\hline$\frac{1}{2}$ & 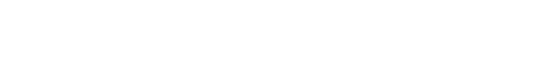 \\
\hline 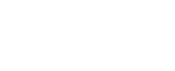 & 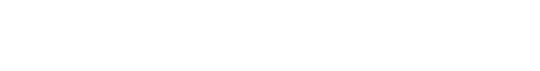 \\
\hline 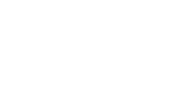 & 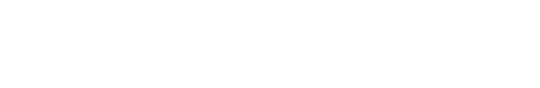 \\
\hline 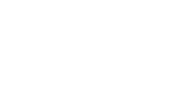 & 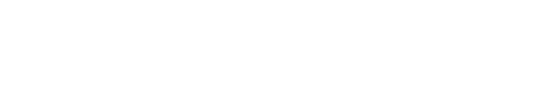 \\
\hline 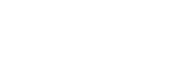 & $\forall+N 0000 \times 000000000000$ \\
\hline 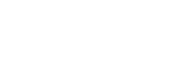 & 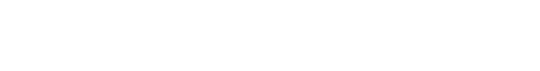 \\
\hline 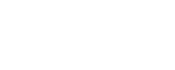 & 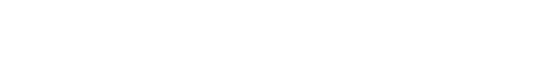 \\
\hline 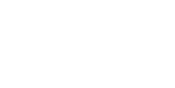 & 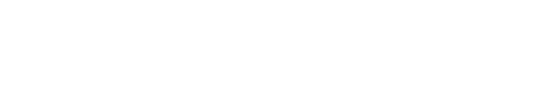 \\
\hline 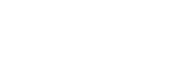 & 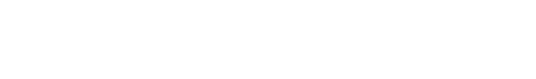 \\
\hline 형영 & 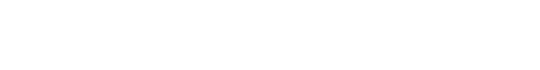 \\
\hline 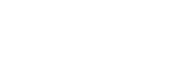 & ب9. \\
\hline 80 & 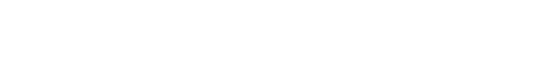 \\
\hline 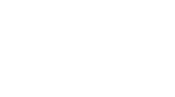 & 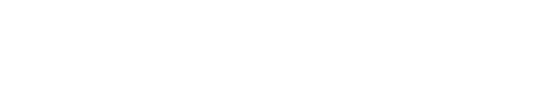 \\
\hline 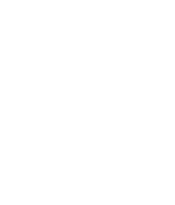 & 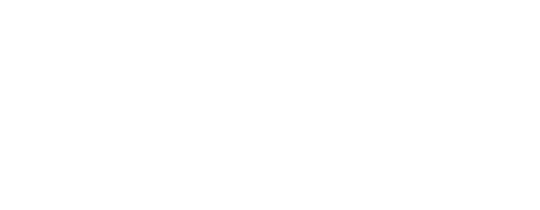 \\
\hline
\end{tabular}




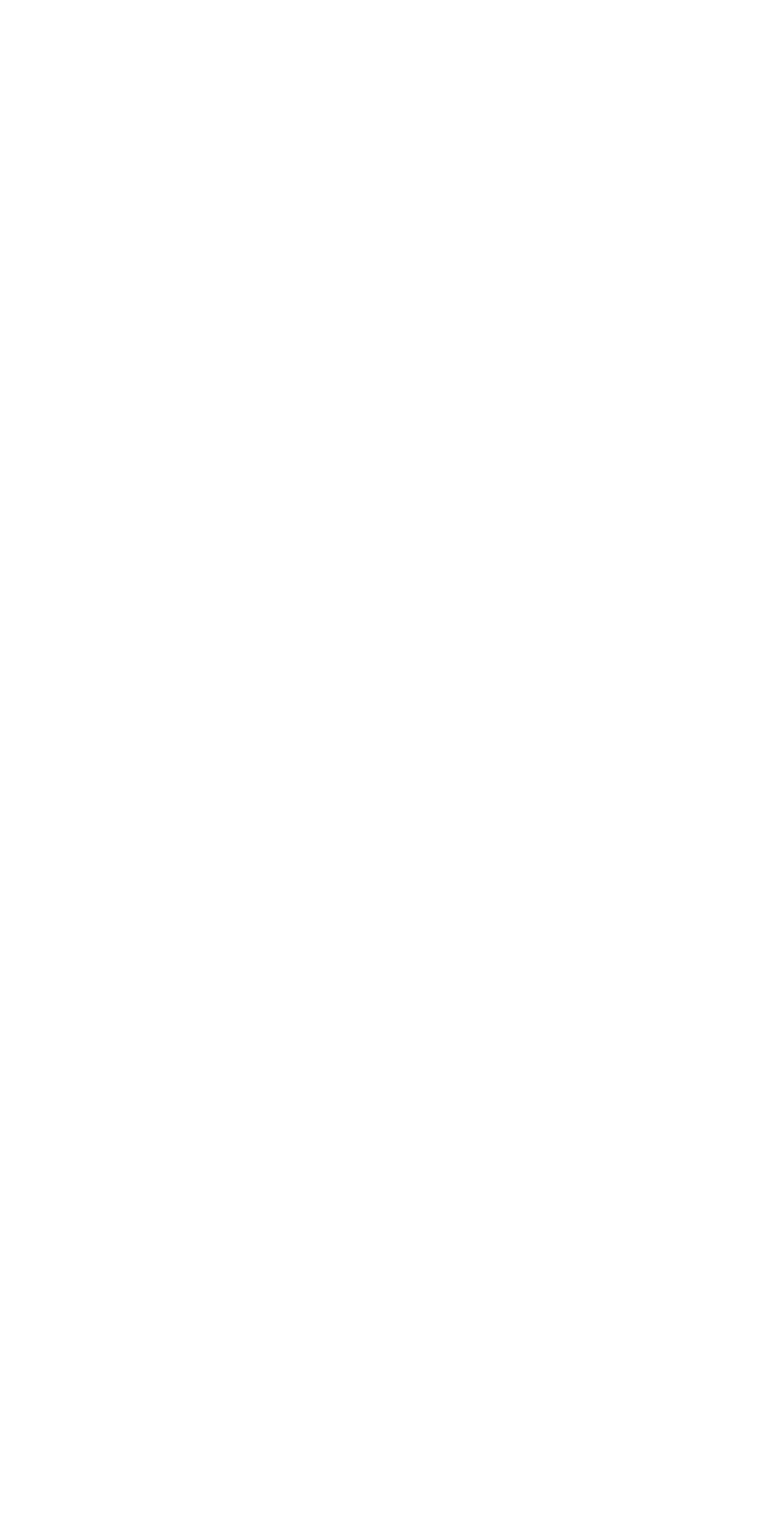



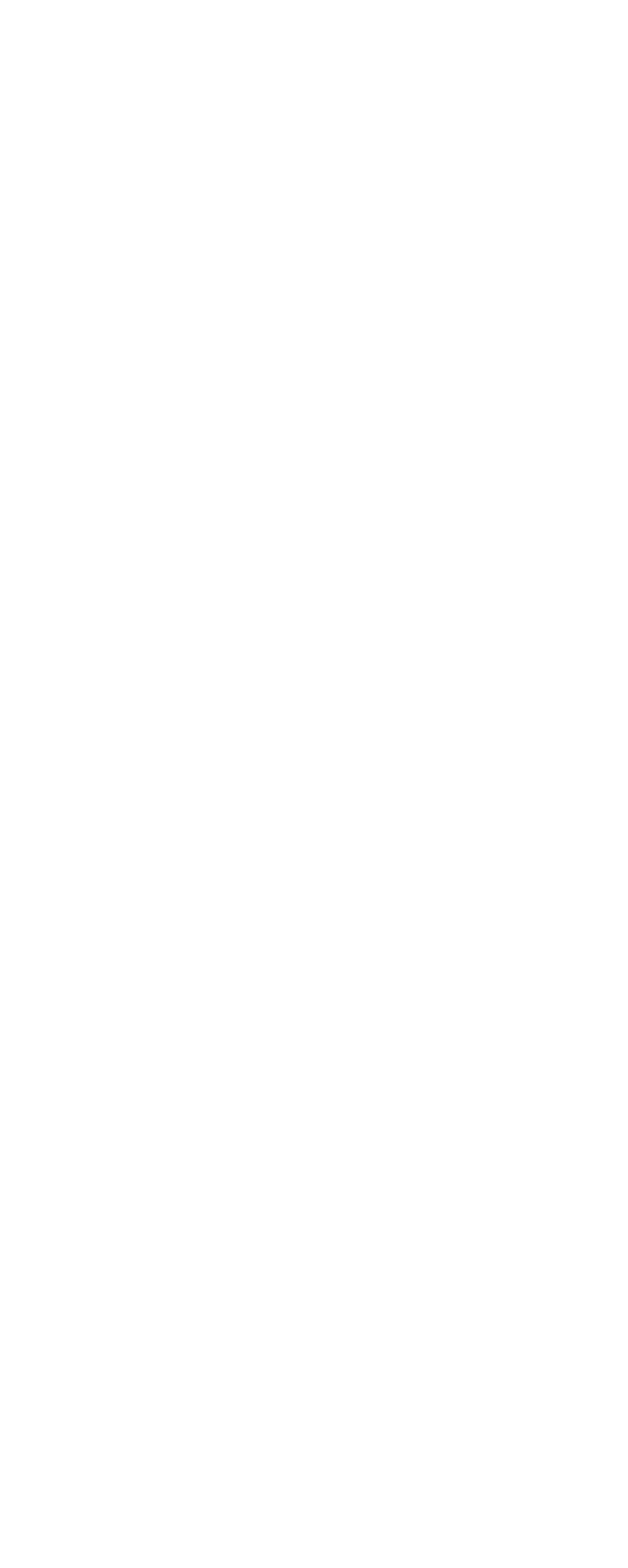

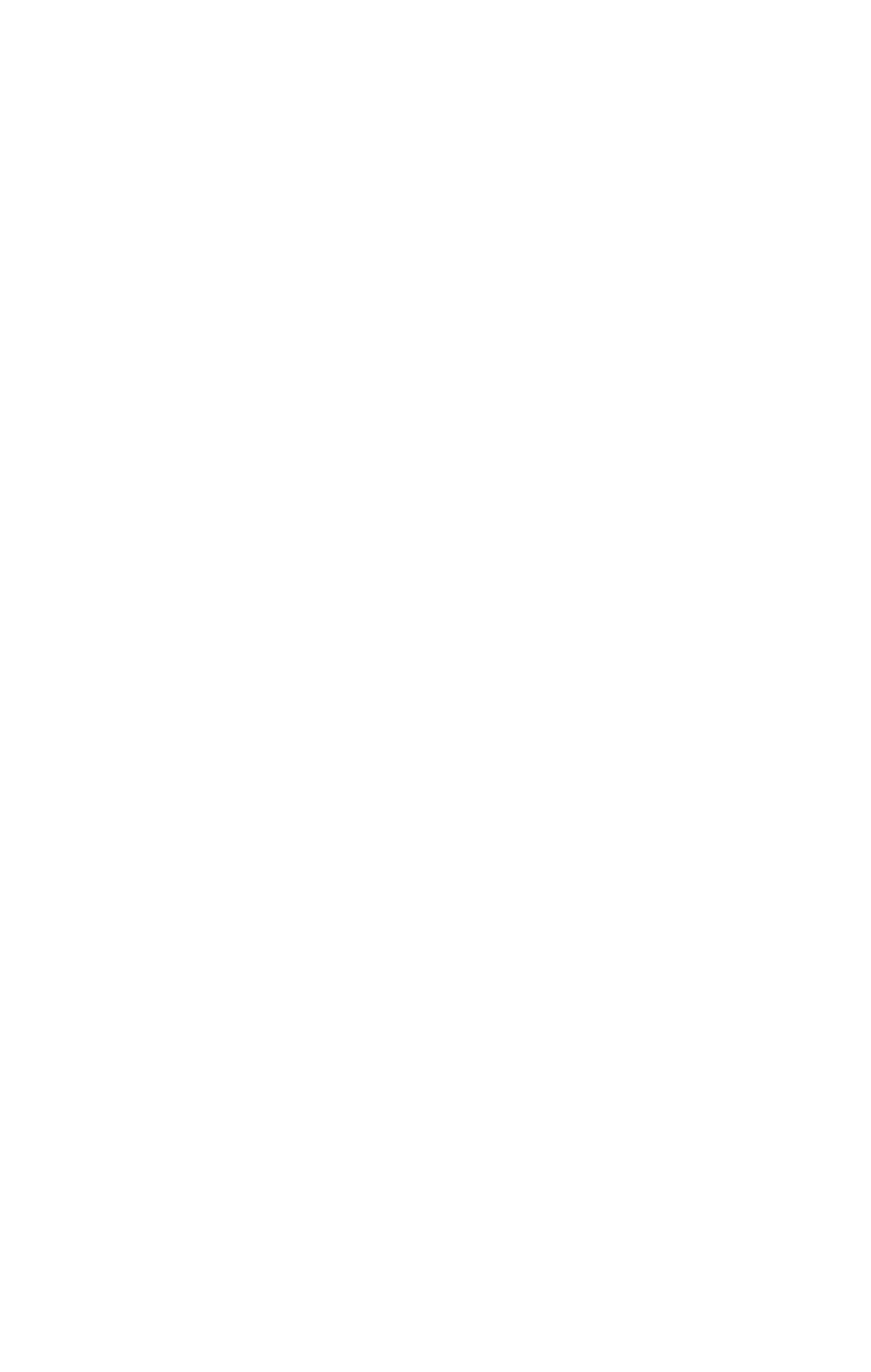


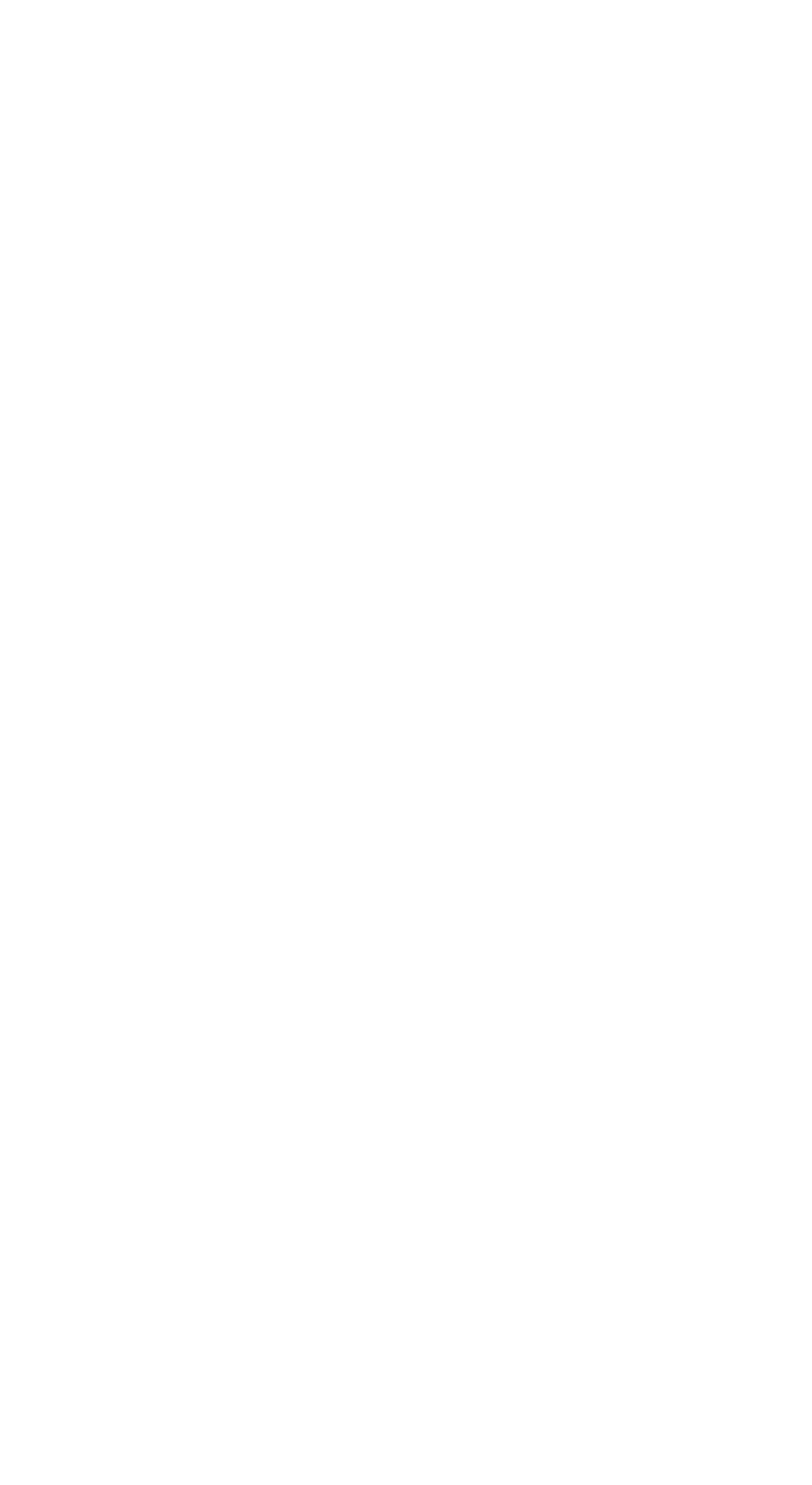




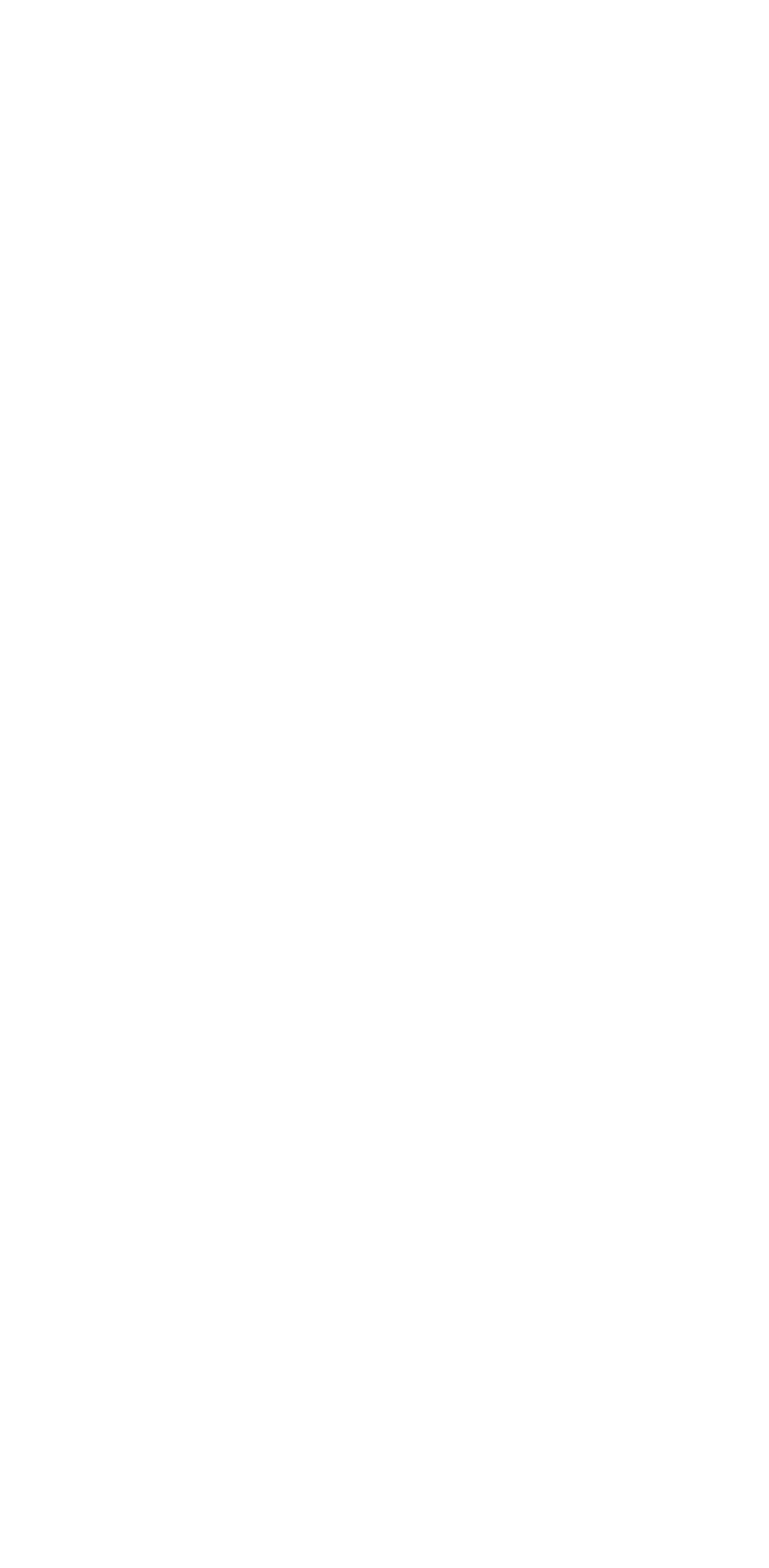




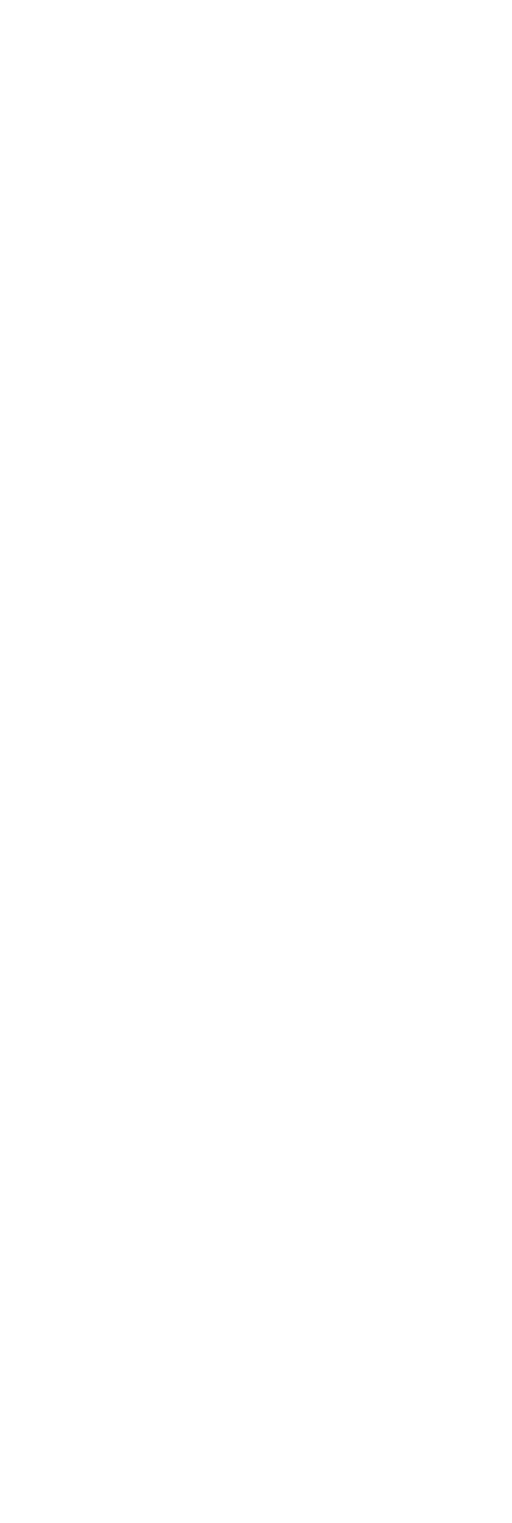




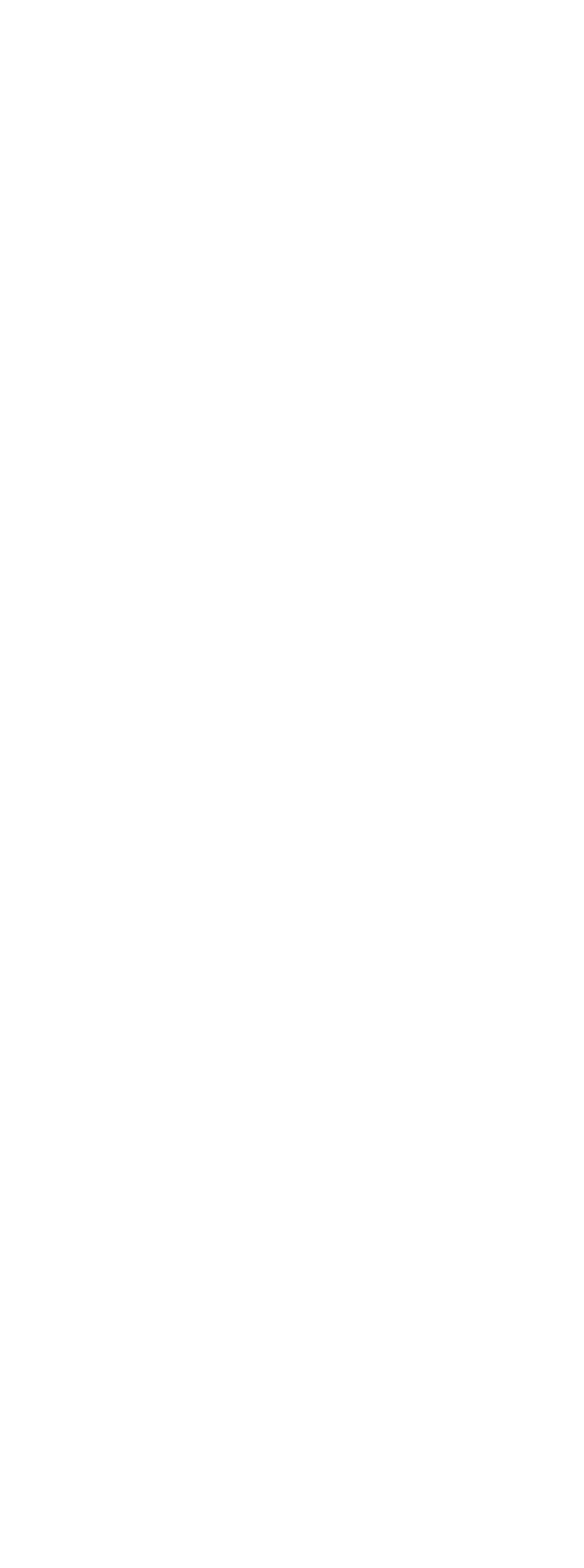




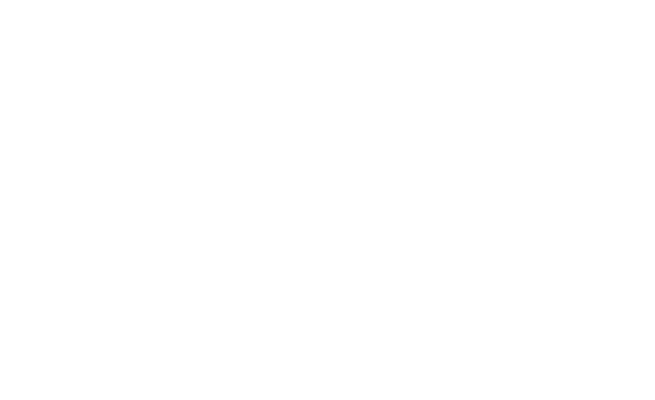

- 䨔:

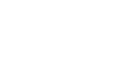

\begin{tabular}{|c|c|c|c|c|}
\hline : & 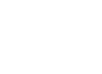 & 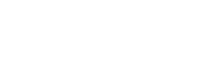 & 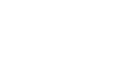 & 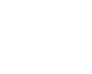 \\
\hline 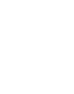 & 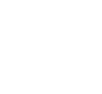 & 뭄욤요 & 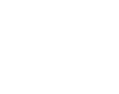 & 용요요 \\
\hline
\end{tabular}

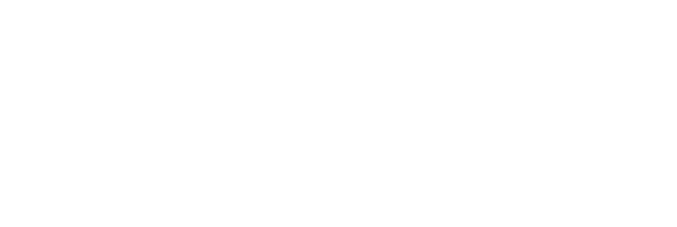
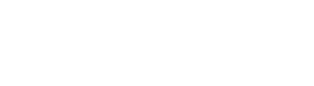

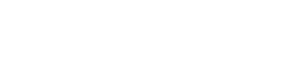
政

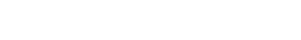

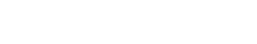

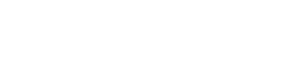
to ou

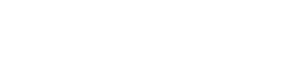

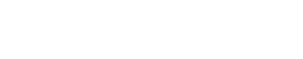

\begin{tabular}{|c|c|}
\hline 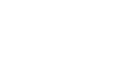 & 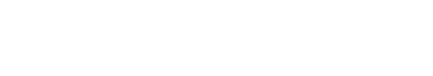 \\
\hline 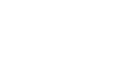 & \\
\hline 量要 & 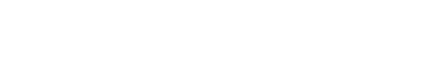 \\
\hline 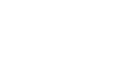 & \\
\hline 竎懸 & \\
\hline 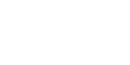 & \\
\hline 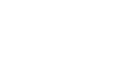 & \\
\hline 高高高 & 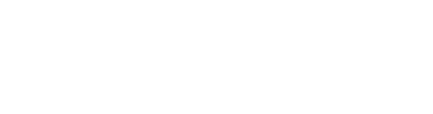 \\
\hline 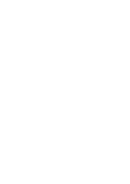 & 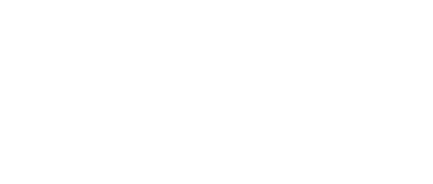 \\
\hline
\end{tabular}




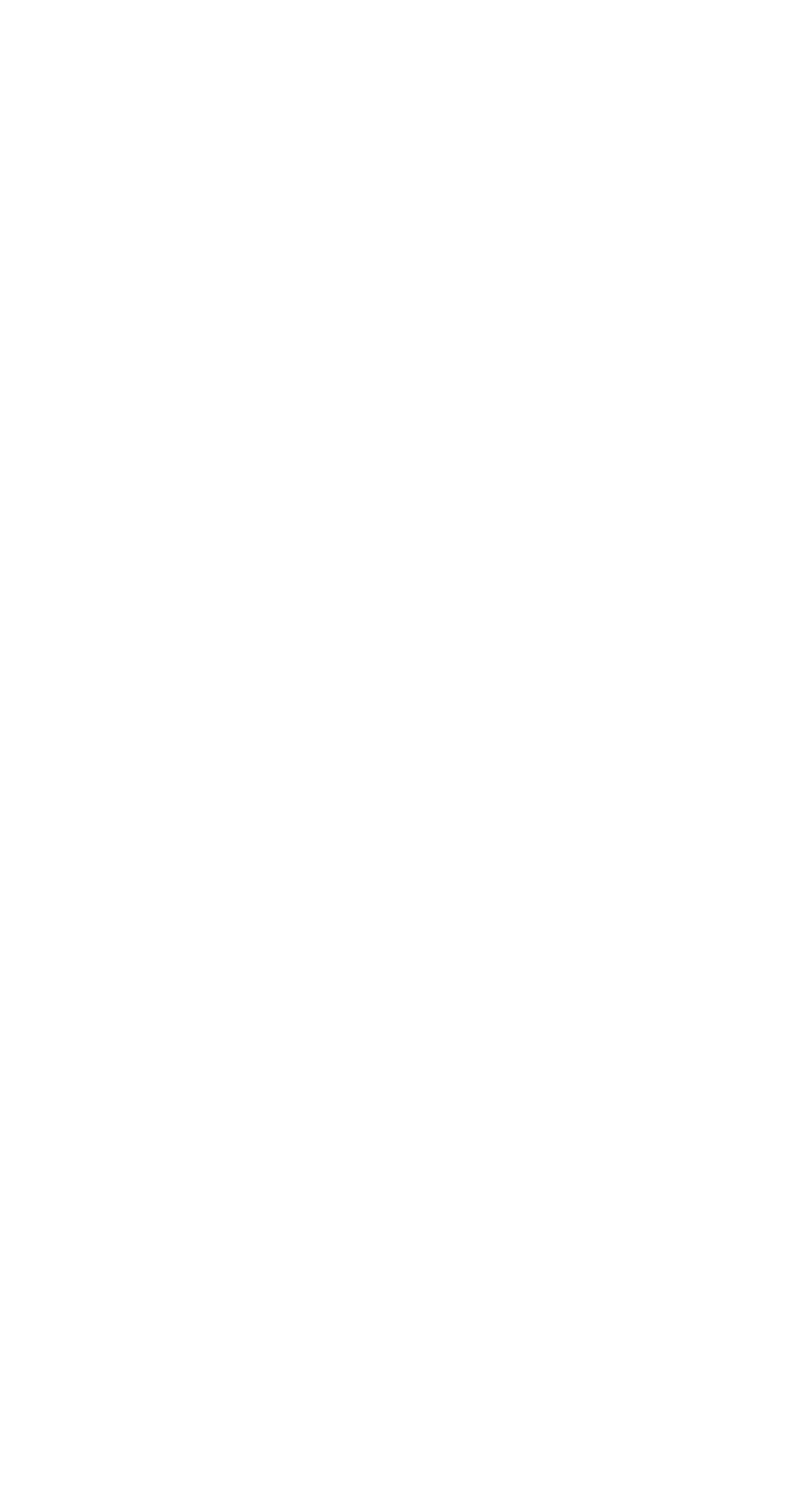




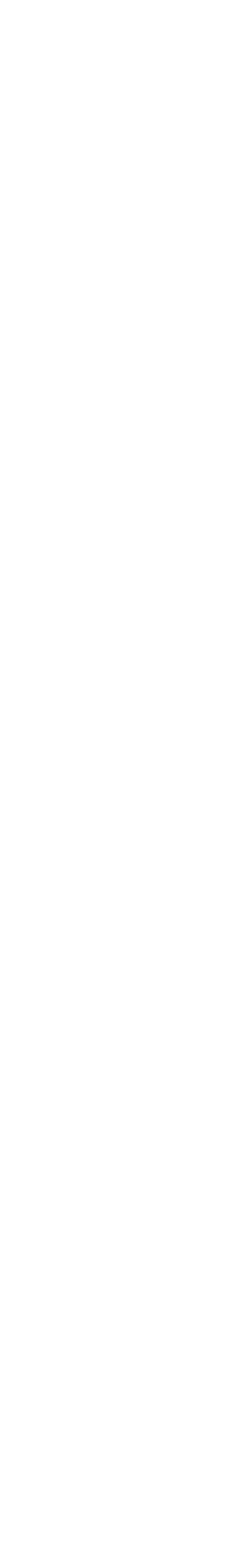

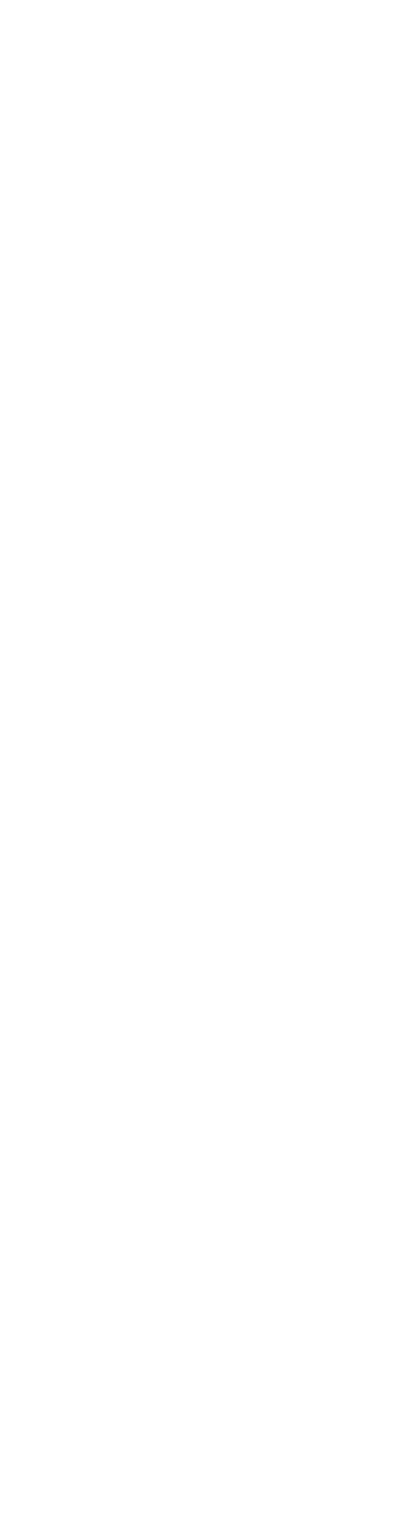




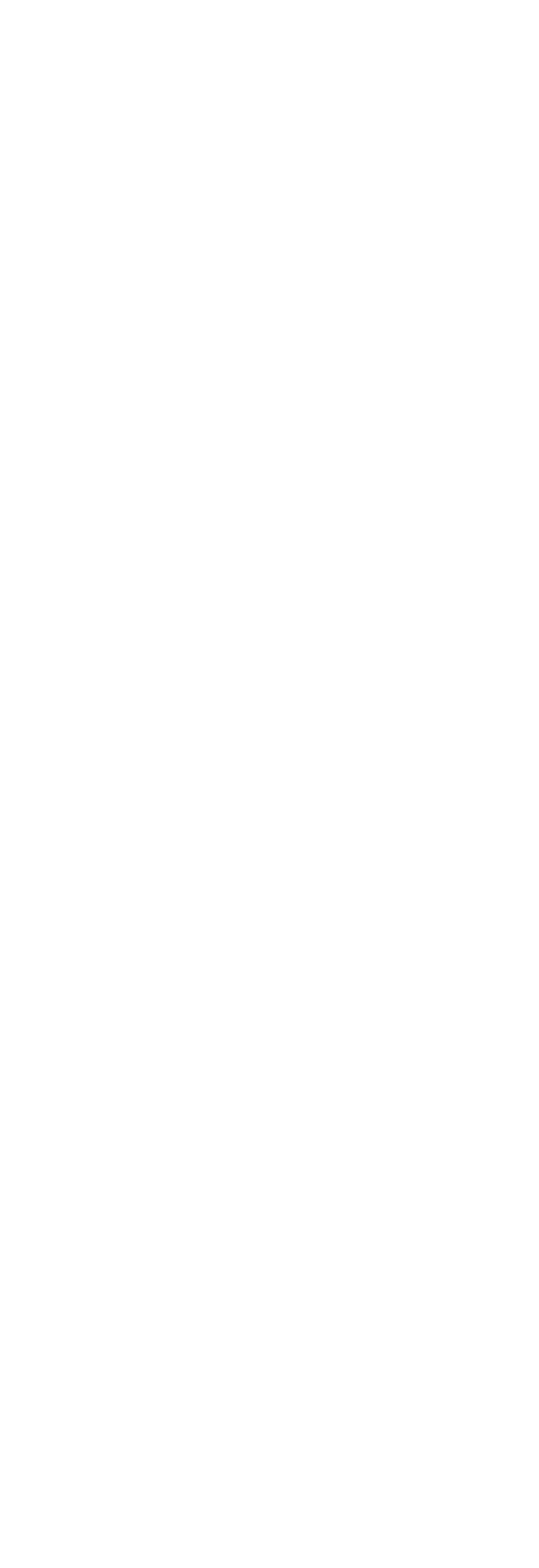




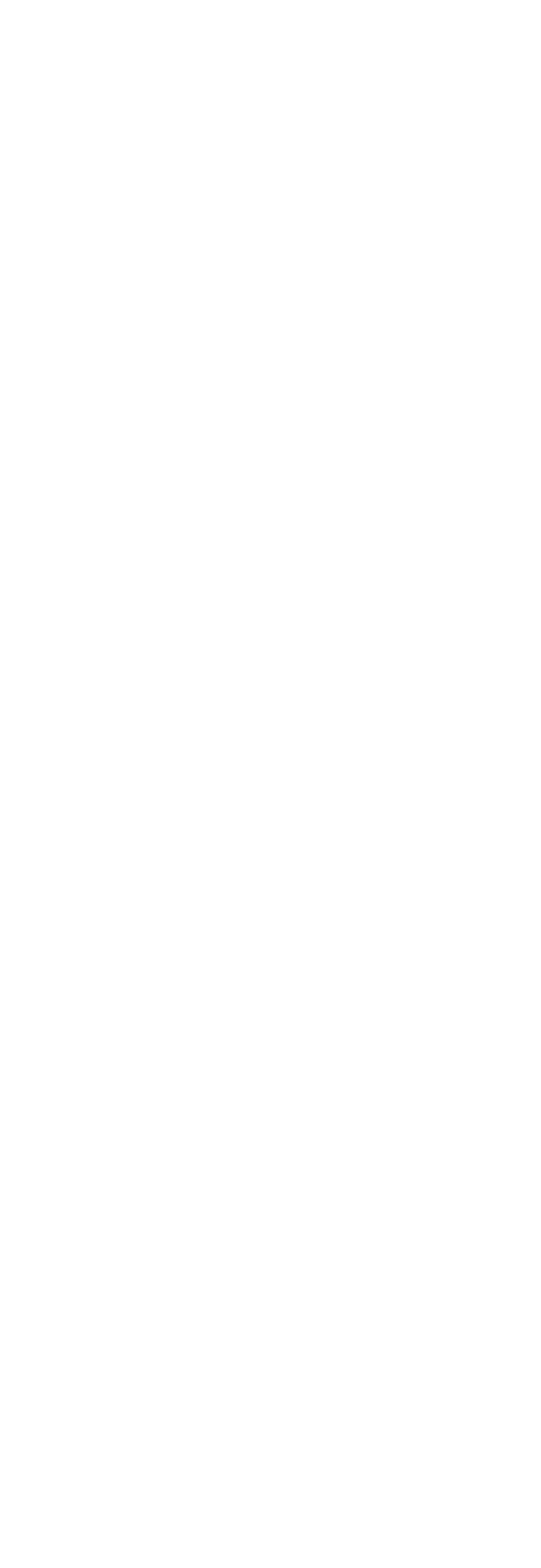




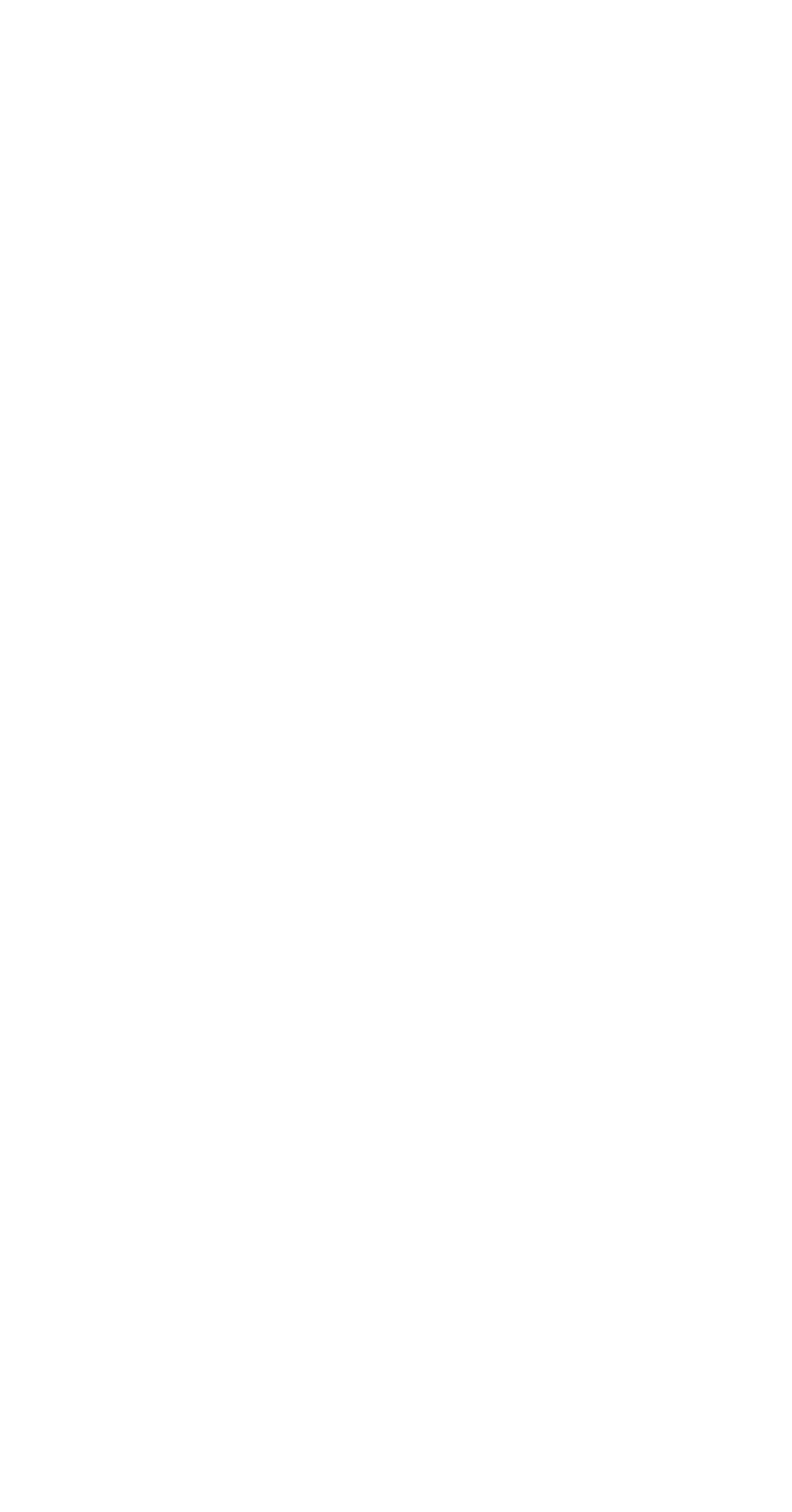



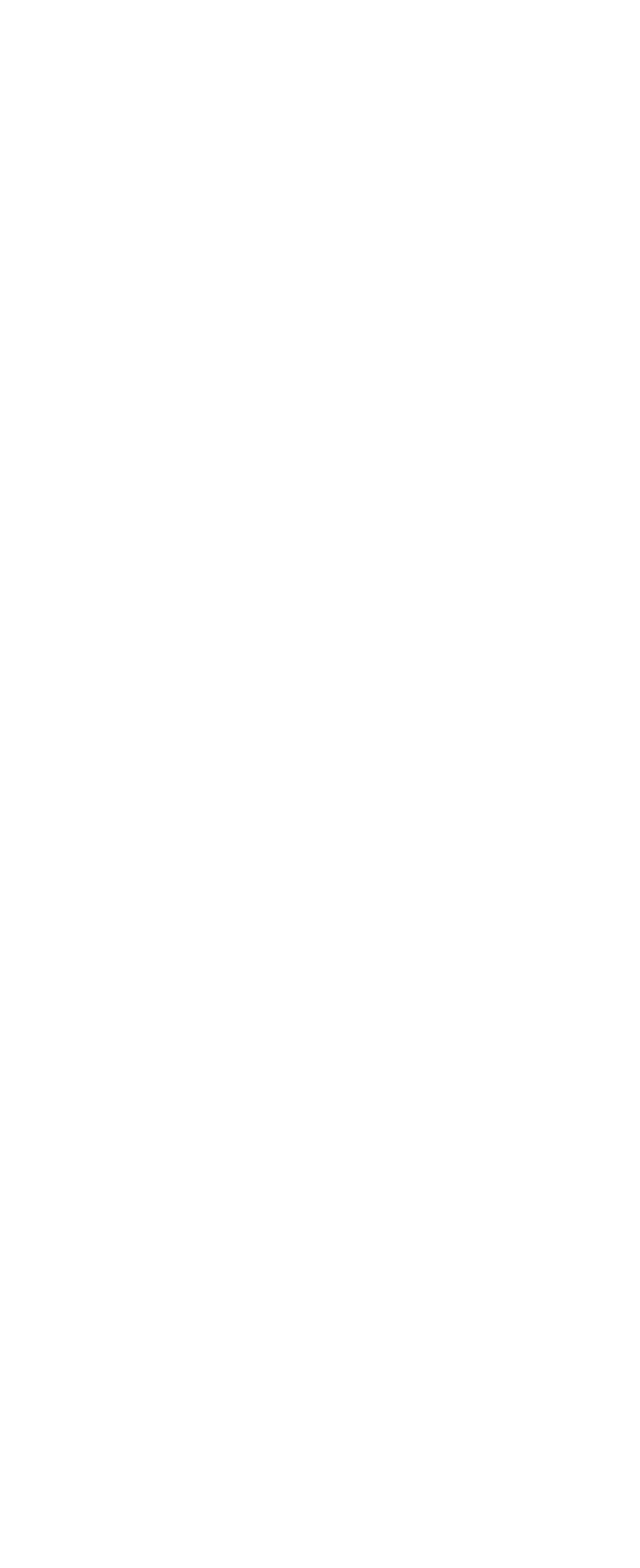


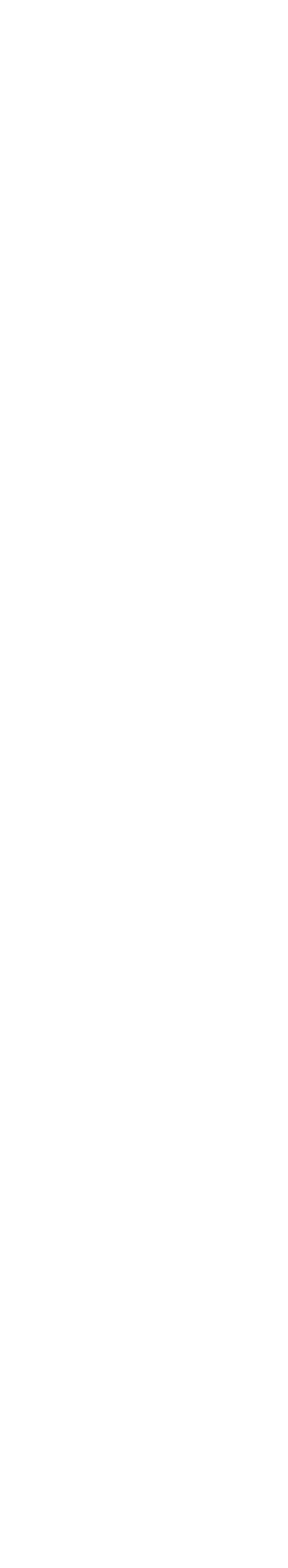

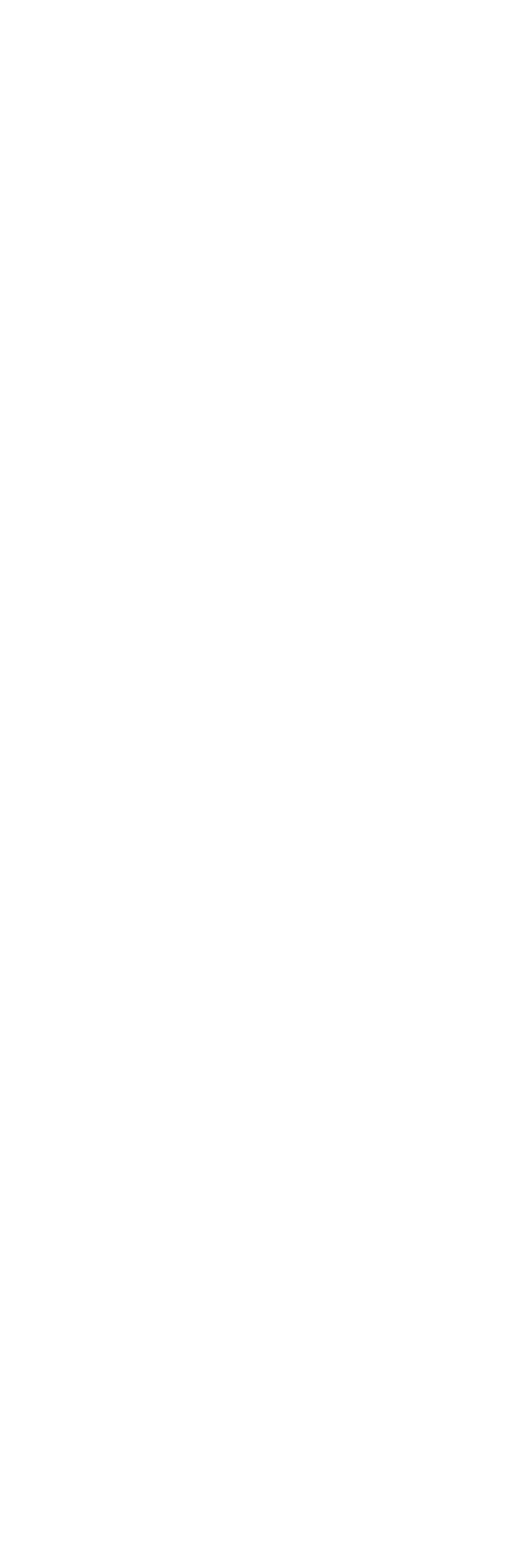




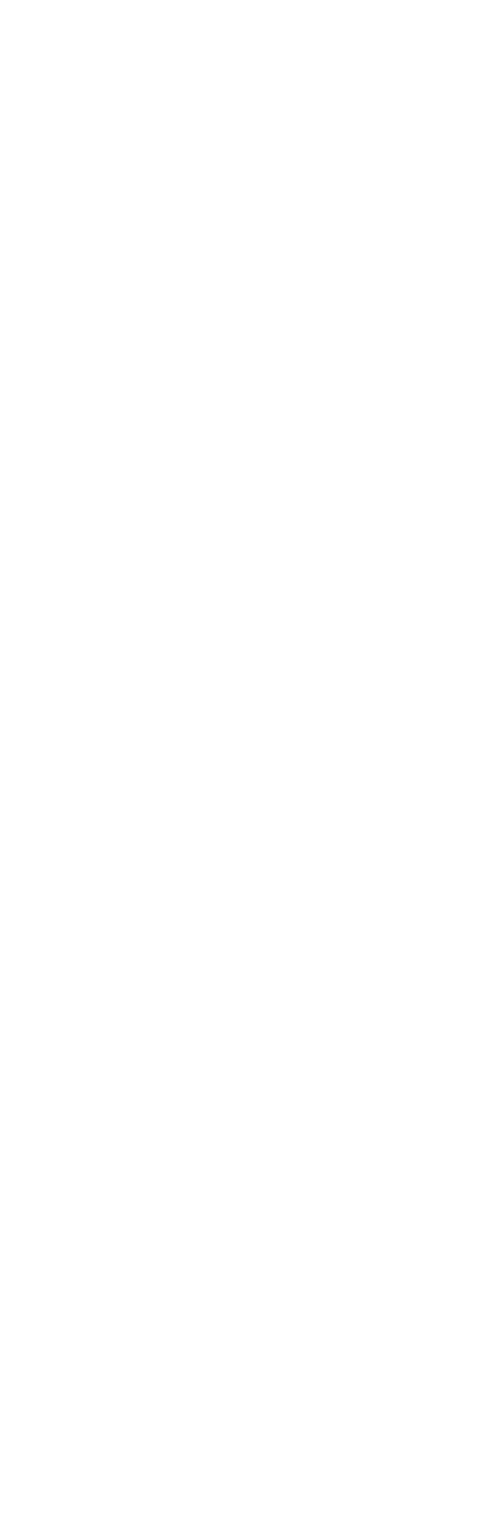




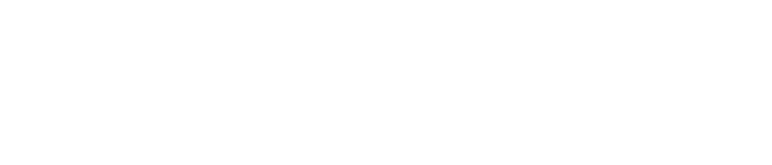

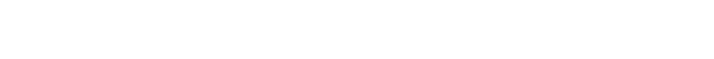

\begin{tabular}{|c|c|c|}
\hline 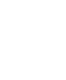 & 客它客齿 & 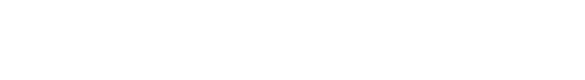 \\
\hline 20 & 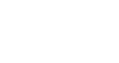 & $i \underset{\infty}{\infty}$ \\
\hline
\end{tabular}

包

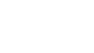

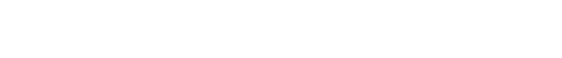

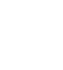

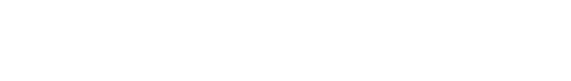

\begin{tabular}{|c|c|}
\hline 宮点可 & \\
\hline 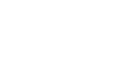 & \\
\hline 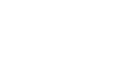 & \\
\hline 递 & 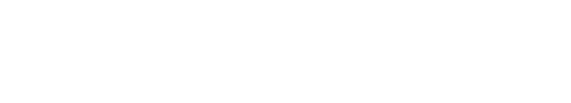 \\
\hline 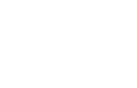 & 1! \\
\hline 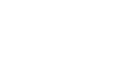 & $11^{+\infty} 11^{+1} 111^{\circ}+11^{0}: 1^{\circ} 011^{\circ}$ \\
\hline 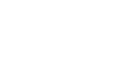 & 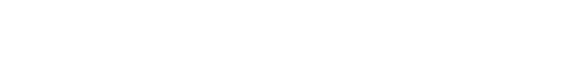 \\
\hline 宫高高区 & \\
\hline 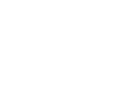 & 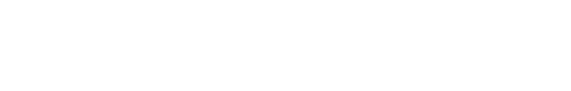 \\
\hline 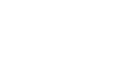 & \\
\hline 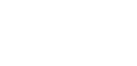 & \\
\hline 跑 & \\
\hline 灵产 & \\
\hline 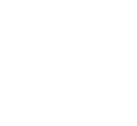 & 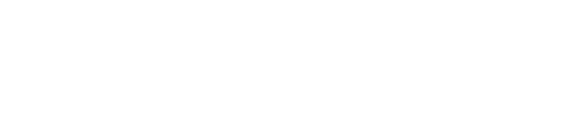 \\
\hline 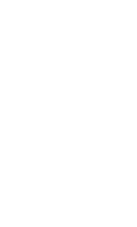 & 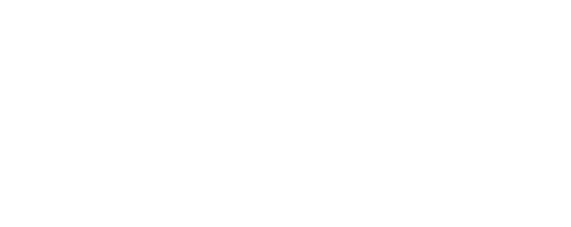 \\
\hline
\end{tabular}




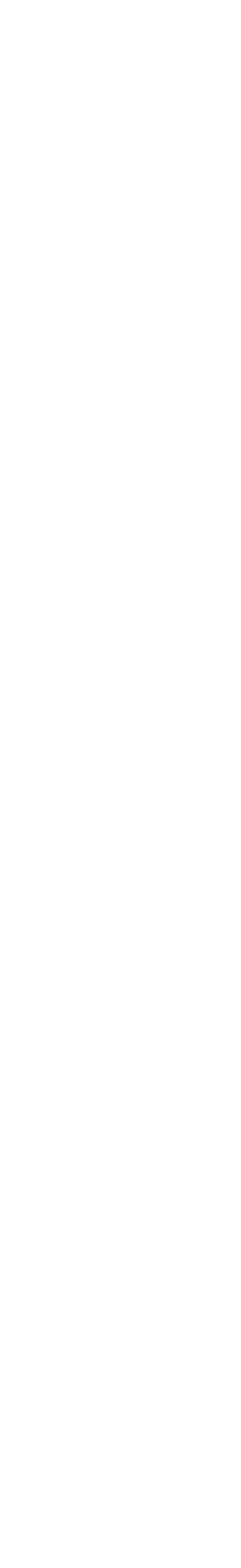




\begin{tabular}{|c|c|}
\hline 类 & 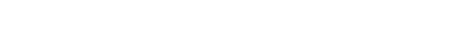 \\
\hline 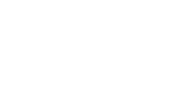 & 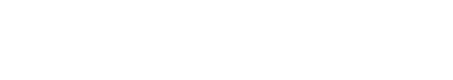 \\
\hline 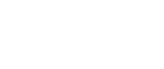 & 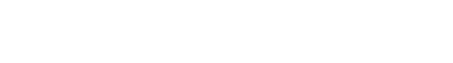 \\
\hline 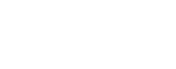 & 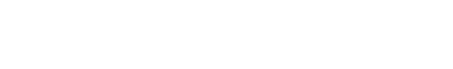 \\
\hline 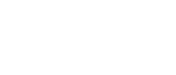 & 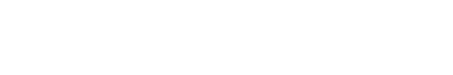 \\
\hline 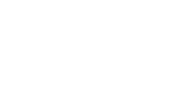 & 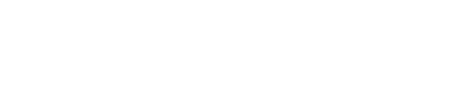 \\
\hline 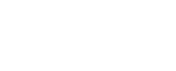 & 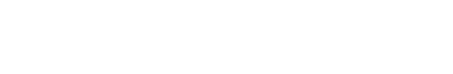 \\
\hline 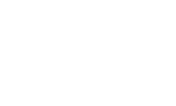 & 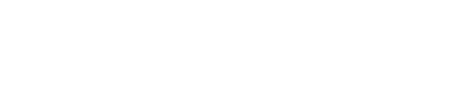 \\
\hline 高点两 & \\
\hline 文旡色 & 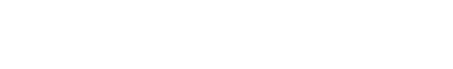 \\
\hline 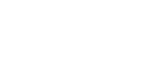 & 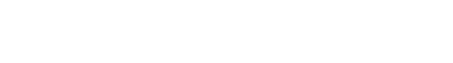 \\
\hline 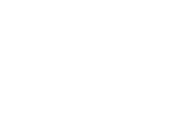 & 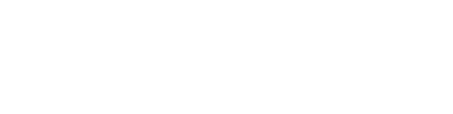 \\
\hline 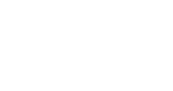 & 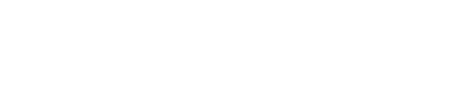 \\
\hline 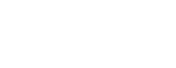 & $000 \mathrm{~m} 00 \mathrm{H} 00000 \mathrm{Nm}$ \\
\hline 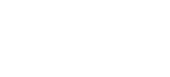 & 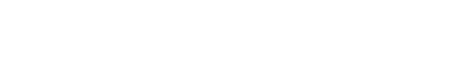 \\
\hline के & 19:11111 11197111 1111111 \\
\hline 镸要 & 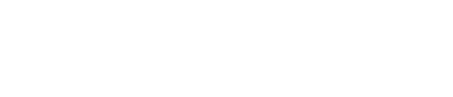 \\
\hline 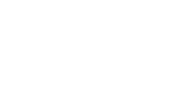 & 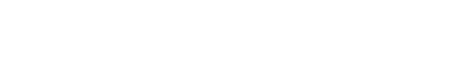 \\
\hline 光息矛 & 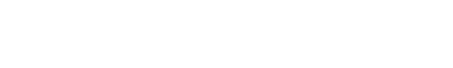 \\
\hline \multicolumn{2}{|l|}{ 造悉 } \\
\hline 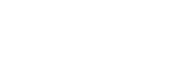 & 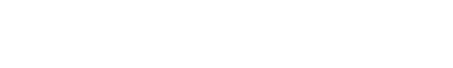 \\
\hline 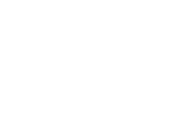 & 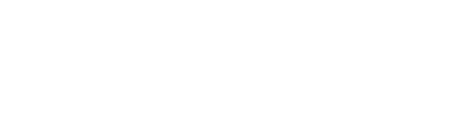 \\
\hline 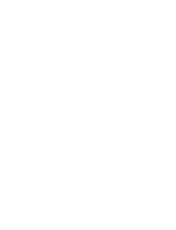 & 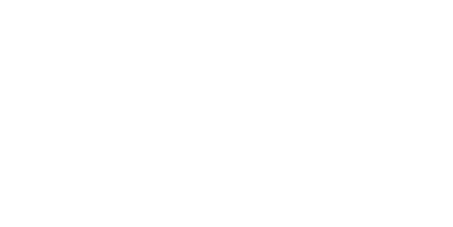 \\
\hline
\end{tabular}




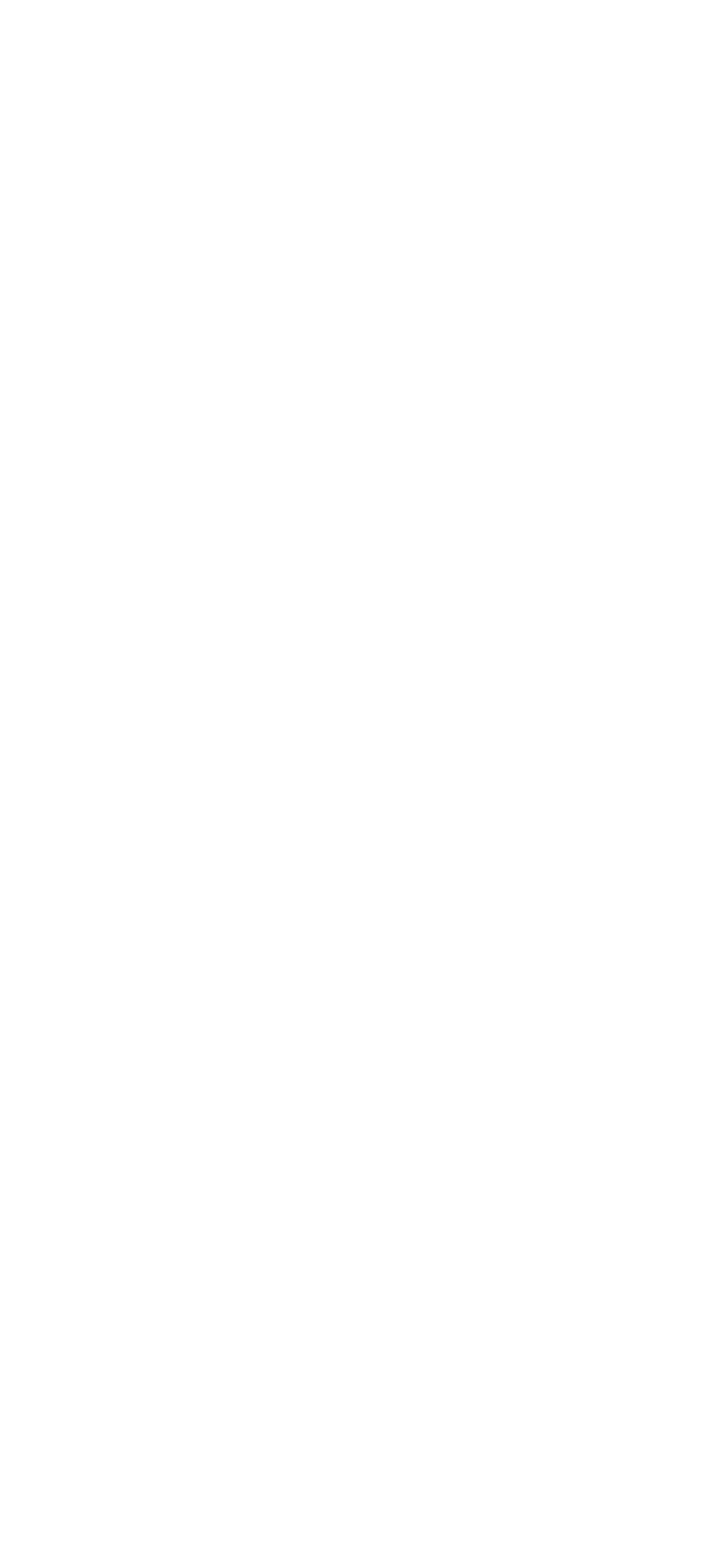




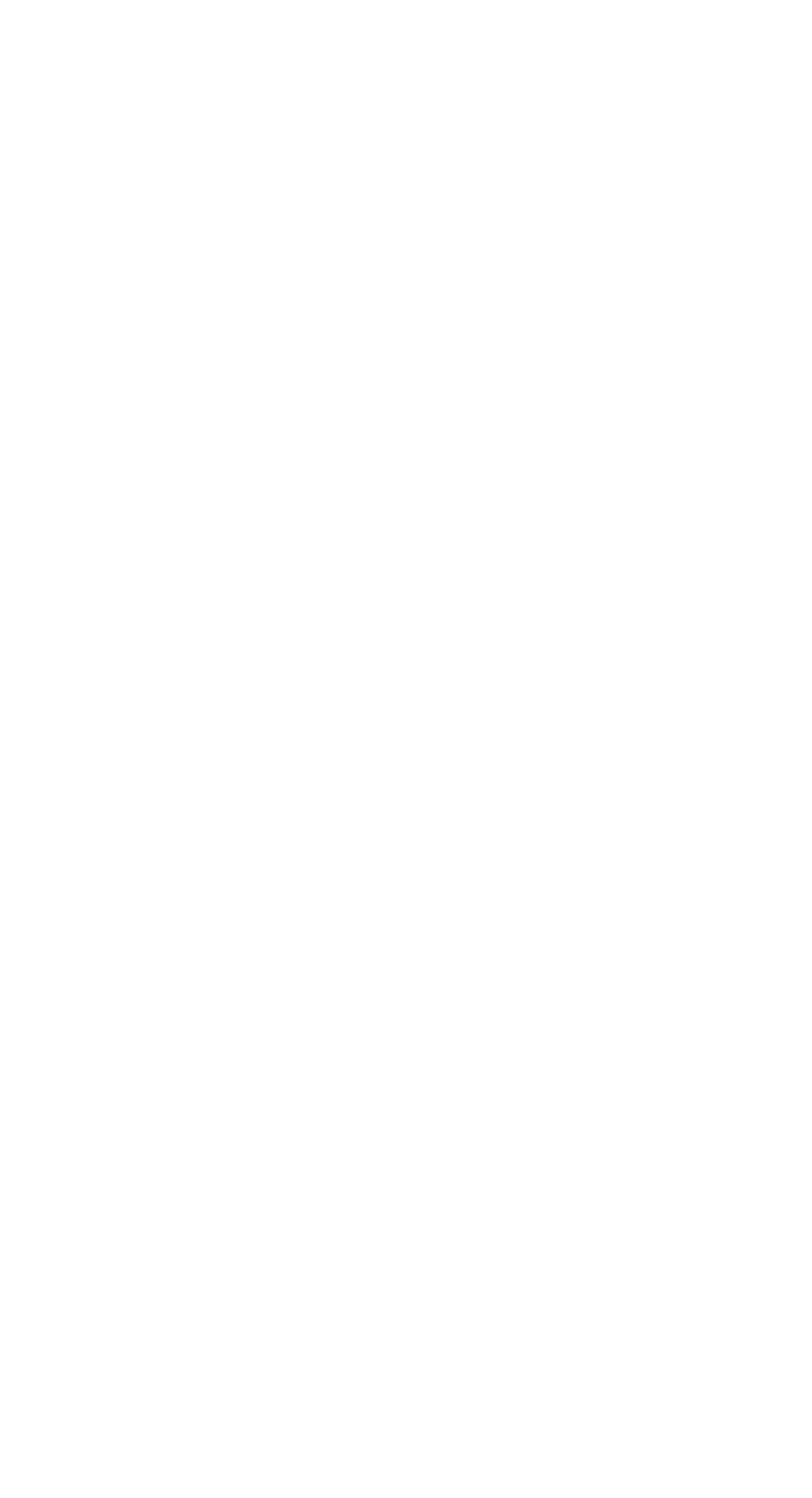




\begin{tabular}{|c|c|c|c|c|c|c|c|c|}
\hline & & & & & $=1$ & & & \\
\hline 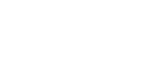 & 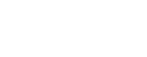 & 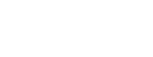 & 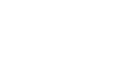 & $\stackrel{0}{\circ}$ & 落落 & & & $\stackrel{6}{\circ}$ \\
\hline 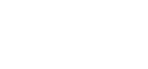 & 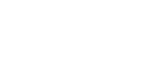 & 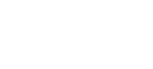 & 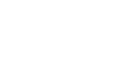 & \& & 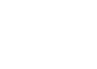 & & & : \\
\hline مُ & 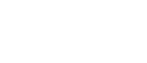 & 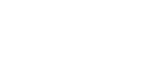 & 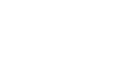 & $\dot{n}$ & 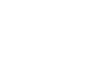 & & & $\begin{array}{ll}0 \\
\\
+\end{array}$ \\
\hline 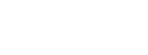 & 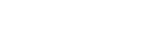 & 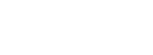 & స̊ำ: & $\stackrel{8}{0}$ & ำలి & & & N \\
\hline : & : & 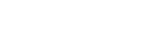 & №nn & & 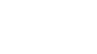 & & & 7 \\
\hline
\end{tabular}

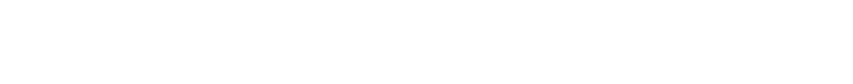

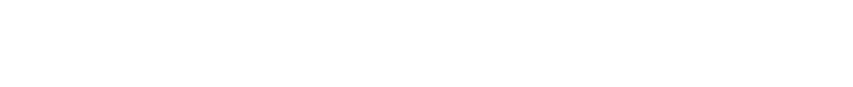

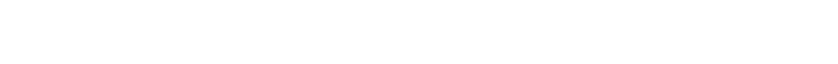

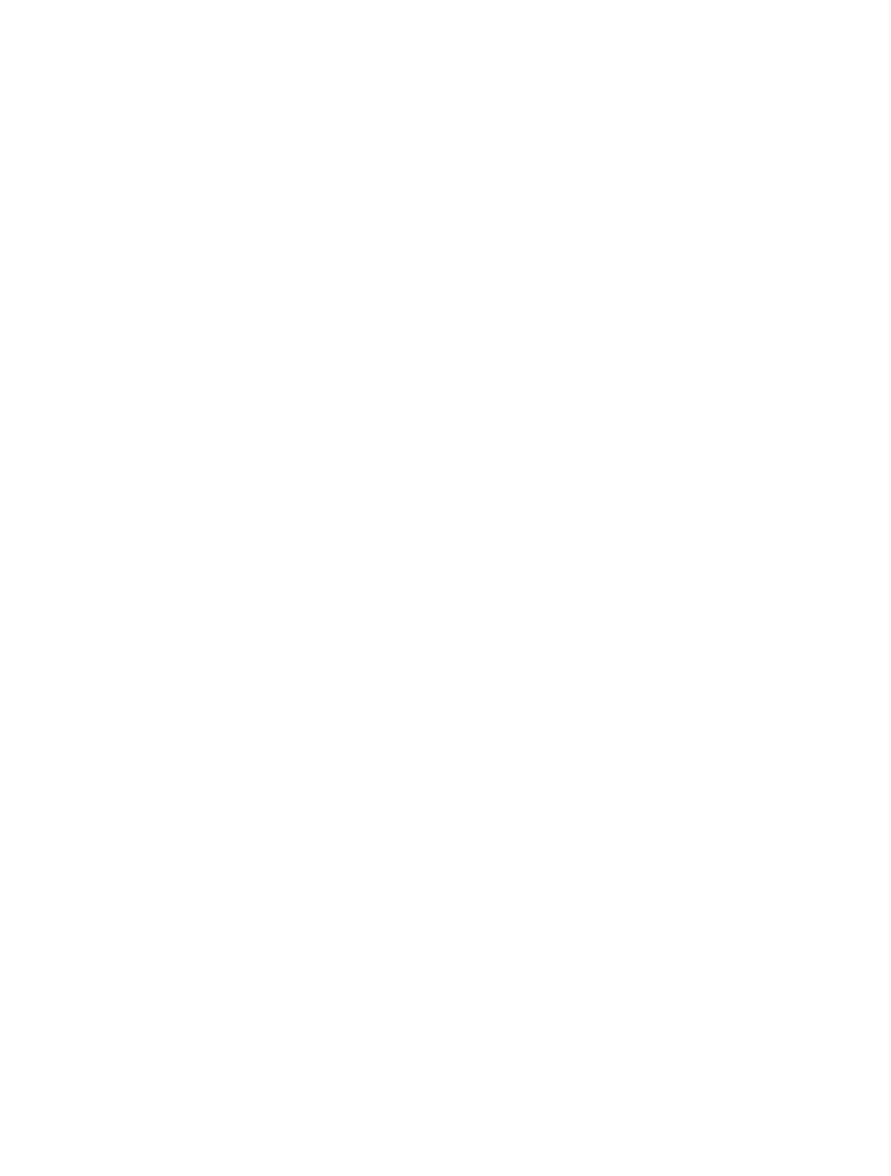




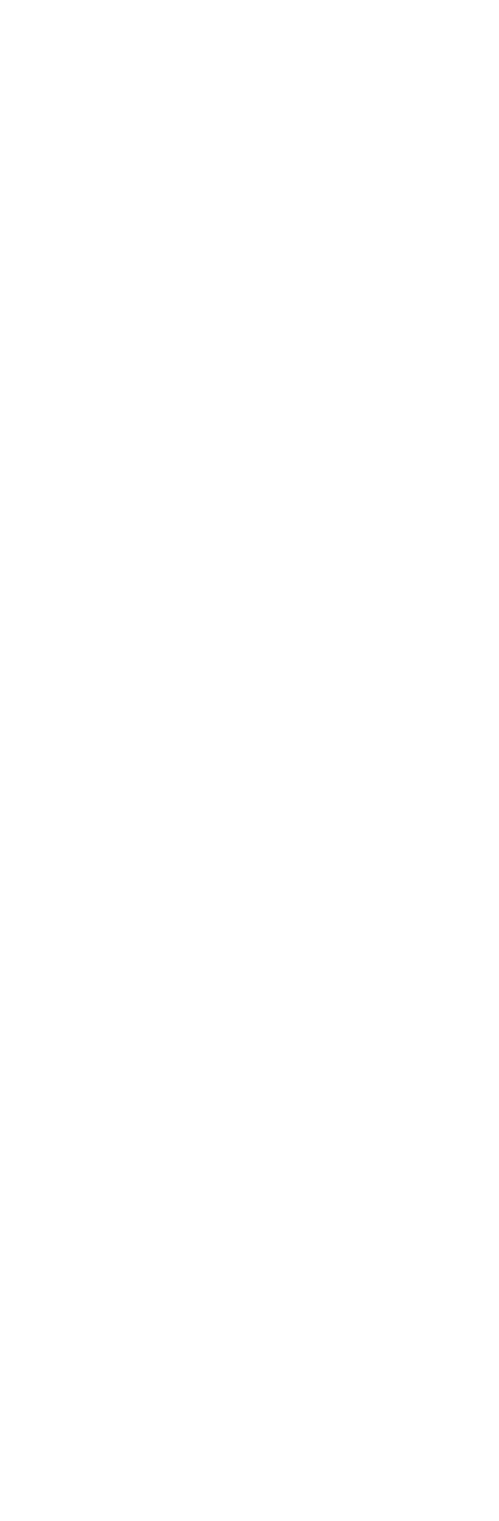




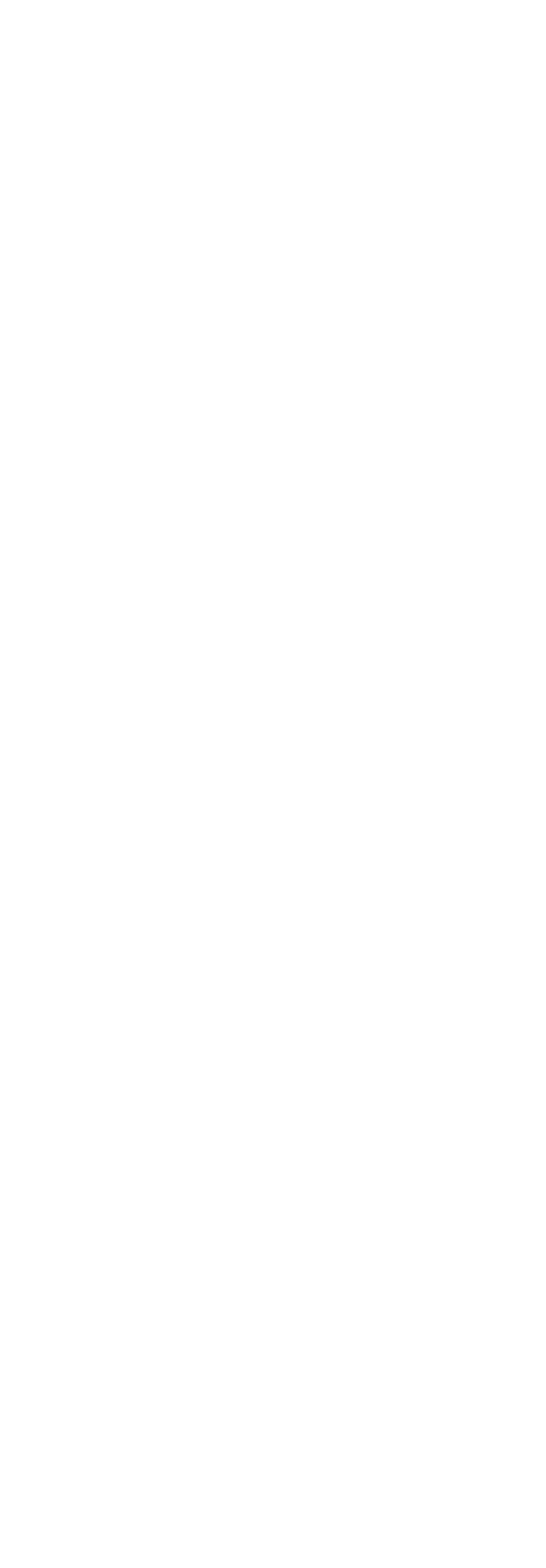




\begin{tabular}{|c|c|c|c|c|c|}
\hline & 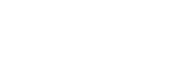 & n:om:m & or & $\nabla$ & \\
\hline & 営 & 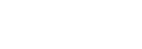 & $0^{\circ}$ & 0 & \\
\hline & 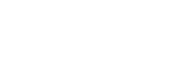 & 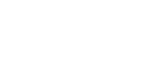 & \% & 2 & \\
\hline & 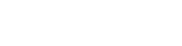 & & & & \\
\hline & 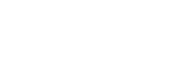 & מNQNAT & $m$ & $\pi$ & i \\
\hline & 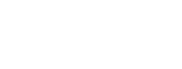 & 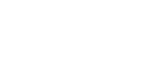 & 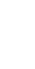 & ล & i \\
\hline 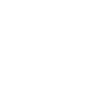 & 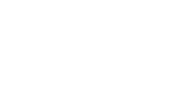 & 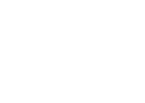 & \& & $g$ & 胥 \\
\hline & 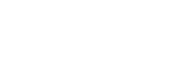 & & & & \\
\hline 吕 & 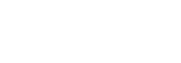 & 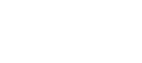 & $\stackrel{\sim}{\pi}$ & ?: & : \\
\hline 兽 & 㲧国 & ㄱ: & $\stackrel{\sim}{\circ}$ & on & ì \\
\hline $\begin{array}{ll}5 & 8 \\
0 & 0 \\
0 & 0 \\
0 & 0 \\
1 & 0 \\
1 & 0 \\
& 0\end{array}$ & 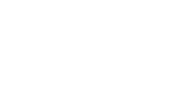 & 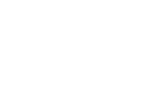 & $\dot{\infty}$ & i) & \\
\hline 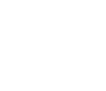 & 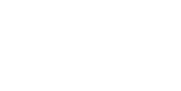 & 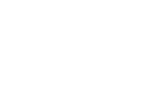 & $\ddot{0}$ & $\therefore$ & \\
\hline 覃 & हैं & 000000 & 0 & 0 & $\circ$ \\
\hline $\begin{array}{ll}7 \\
0 \\
0\end{array}$ & 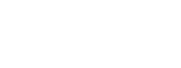 & 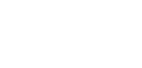 & $\stackrel{2}{n}$ & ล & $\stackrel{8}{8}$ \\
\hline 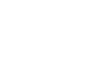 & 产是是 & & & & \\
\hline $\begin{array}{ll}5 & 0 \\
0 & 0 \\
0 & =1\end{array}$ & 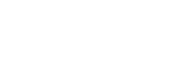 & $\begin{array}{l}\text { N NMO : } \\
\text { HA. }\end{array}$ & $\stackrel{\sim}{4}$ & N. & $\stackrel{\infty}{\infty}$ \\
\hline 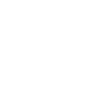 & 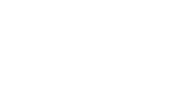 & 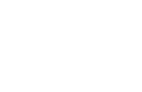 & $\stackrel{\infty}{m}$ & $\overrightarrow{4}$ & \\
\hline 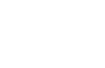 & 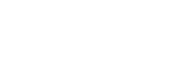 & 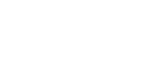 & i. & $\stackrel{9}{-1}$ & \\
\hline & 过炰氖 & 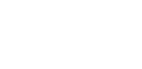 & $\stackrel{\infty}{0}$ & $\stackrel{\infty}{\therefore}$ & ? \\
\hline 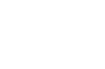 & 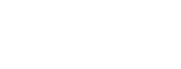 & $\begin{array}{l}815088 \\
0108 \\
0\end{array}$ & $\begin{array}{l}\infty \\
\vdots \\
0\end{array}$ & $\begin{array}{l}\text { İ } \\
\vdots \\
\vdots\end{array}$ & $\begin{array}{l}\text { No } \\
\text { : } \\
0 \\
0\end{array}$ \\
\hline gु & 总离 & $1: 11: 11:$ & 1 & $i$ & $i$ \\
\hline 是 & 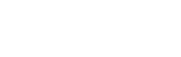 & & & & \\
\hline & 吾危 & 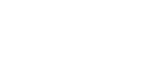 & $\stackrel{m}{0}$ & in: & r \\
\hline & 窟 & 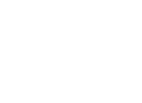 & $i$ & 剠 & \\
\hline & 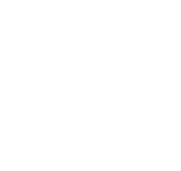 & 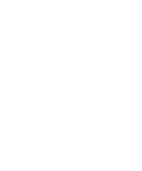 & & $\begin{array}{ll}0 \\
0 \\
0\end{array}$ & \\
\hline
\end{tabular}

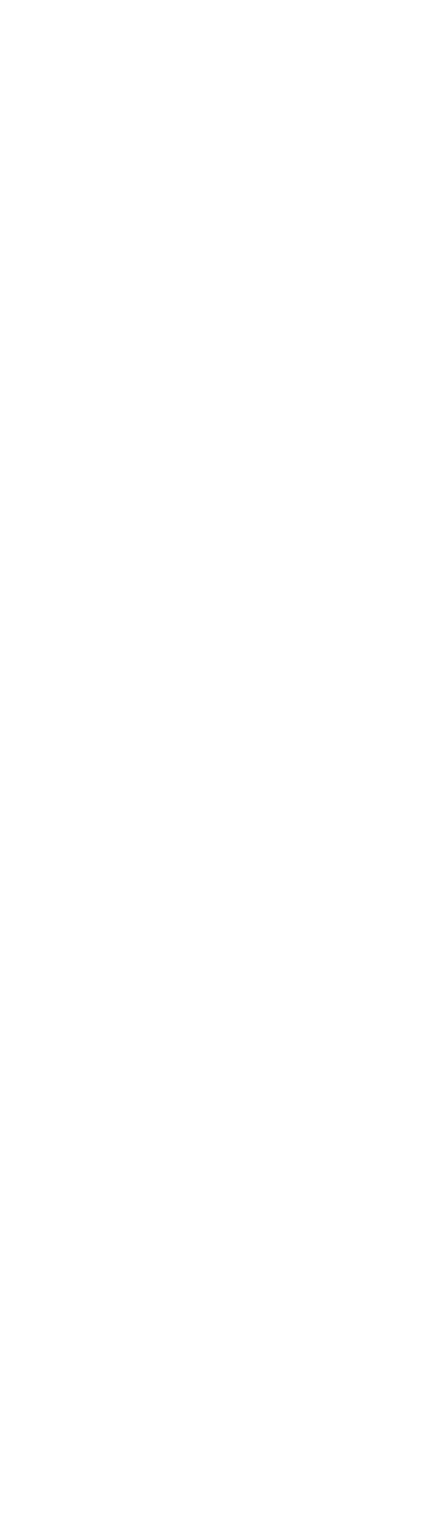




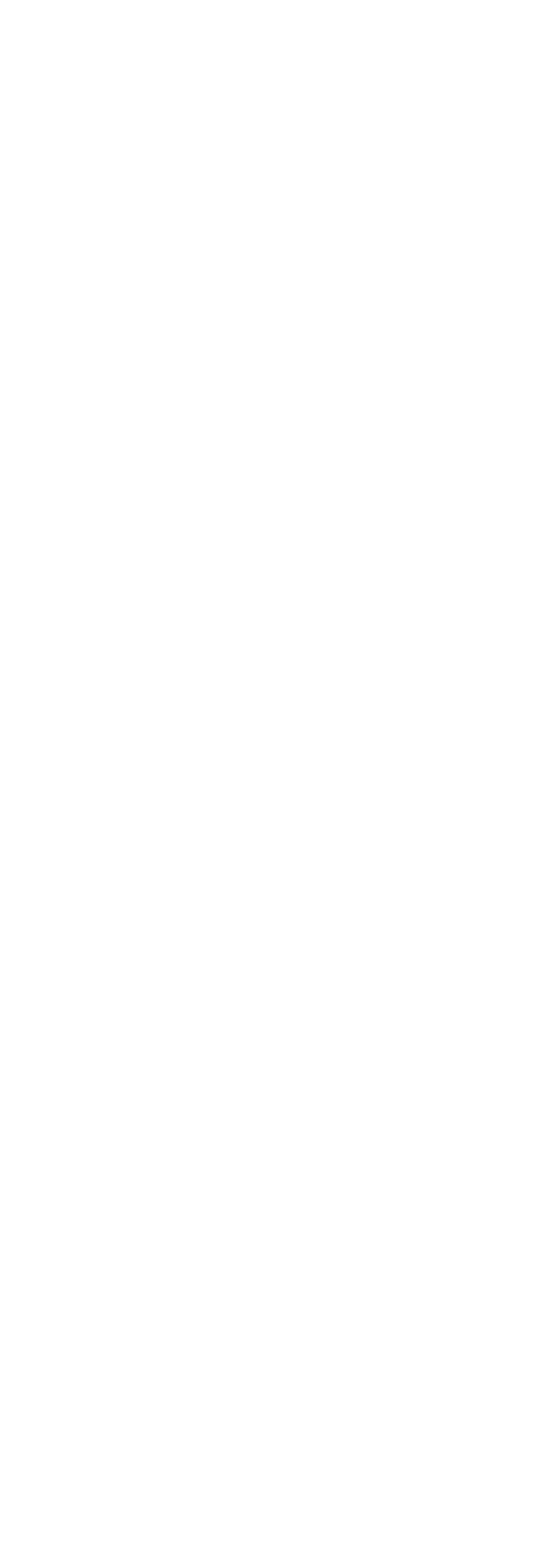


RED RIVER BASIN--Continued

7-3622. SMACKOVER CREEK NEAR NORPHLET, ARK,--Continued

Specific conductance (micromhos at $25^{\circ} \mathrm{C}$ ), and chloride in parts per million,

\begin{tabular}{|c|c|c|c|c|c|c|c|c|}
\hline \multirow[b]{2}{*}{ Day } & \multicolumn{2}{|c|}{ OCTOBER } & \multicolumn{2}{|c|}{ NOVEMBER } & \multicolumn{2}{|c|}{ DECEMBER } & \multicolumn{2}{|c|}{ JANUARY } \\
\hline & $\begin{array}{l}\text { Specific } \\
\text { conduct - } \\
\text { ance } \\
\text { (micro- } \\
\text { mhos at } \\
\left.25^{\circ} \mathrm{C}\right)\end{array}$ & $\begin{array}{l}\text { Chlo- } \\
\text { ride } \\
\text { (C1) }\end{array}$ & $\begin{array}{l}\text { Specific } \\
\text { conduct- } \\
\text { ance } \\
\text { (micro- } \\
\text { mhos at } \\
25^{\circ} \mathrm{C} \text { ) }\end{array}$ & $\begin{array}{l}\text { Chlo- } \\
\text { ride } \\
\text { (Cl) }\end{array}$ & $\begin{array}{l}\text { Specific } \\
\text { conduct - } \\
\text { ance } \\
\text { (micro- } \\
\text { mhos at } \\
25^{\circ} \mathrm{C} \text { ) }\end{array}$ & $\begin{array}{l}\text { Chlo- } \\
\text { ride } \\
\text { (C1) }\end{array}$ & $\begin{array}{l}\text { Specific } \\
\text { conduct - } \\
\text { ance } \\
\text { (micro- } \\
\text { mos at } \\
25^{\circ} \mathrm{C} \text { ) }\end{array}$ & $\begin{array}{l}\text { Chlo- } \\
\text { ride } \\
\text { (C1) }\end{array}$ \\
\hline $\begin{array}{l}1 \\
2 \\
3 \\
4 \\
5\end{array}$ & $\begin{array}{l}25100 \\
25100 \\
25100 \\
23400 \\
21900\end{array}$ & $\begin{array}{l}9550 \\
9550 \\
9550 \\
9000 \\
8400\end{array}$ & $\begin{array}{l}-- \\
-- \\
-- \\
-- \\
--\end{array}$ & $\begin{array}{l}-- \\
z- \\
-- \\
--\end{array}$ & $\begin{array}{l}5540 \\
6270 \\
6010 \\
6170 \\
7490\end{array}$ & $\begin{array}{l}1850 \\
2120 \\
2000 \\
2080 \\
2580\end{array}$ & $\begin{array}{l}4550 \\
5440 \\
5920 \\
5640 \\
5110\end{array}$ & $\begin{array}{l}1400 \\
1750 \\
1900 \\
1780 \\
1600\end{array}$ \\
\hline $\begin{array}{r}6 \\
7 \\
8 \\
9 \\
10\end{array}$ & $\begin{array}{r}23400 \\
25100 \\
-- \\
25100 \\
27000\end{array}$ & $\begin{array}{r}8650 \\
9350 \\
- \\
9650 \\
10300\end{array}$ & $\begin{array}{l}-- \\
-- \\
-- \\
-- \\
--\end{array}$ & $\begin{array}{l}-- \\
-- \\
-- \\
-- \\
--\end{array}$ & $\begin{array}{r}8070 \\
9260 \\
10900 \\
10900 \\
9800\end{array}$ & $\begin{array}{l}2780 \\
3220 \\
3850 \\
3900 \\
3400\end{array}$ & $\begin{array}{r}17400 \\
5350 \\
4170 \\
5780 \\
3100\end{array}$ & $\begin{array}{r}6050 \\
1550 \\
1300 \\
1900 \\
975\end{array}$ \\
\hline $\begin{array}{l}11 \\
12 \\
13 \\
14 \\
15\end{array}$ & $\begin{array}{l}27000 \\
27000 \\
29200 \\
35100 \\
35100\end{array}$ & $\begin{array}{l}10300 \\
10200 \\
11000 \\
13900 \\
14600\end{array}$ & $\begin{array}{r}-- \\
21300 \\
20900\end{array}$ & $\begin{array}{r}-- \\
-- \\
8200 \\
8050\end{array}$ & $\begin{array}{l}4490 \\
3620 \\
1940 \\
1980 \\
1590\end{array}$ & $\begin{array}{r}1450 \\
1180 \\
600 \\
600 \\
500\end{array}$ & $\begin{array}{l}4070 \\
4370 \\
3580 \\
3550 \\
4220\end{array}$ & $\begin{array}{l}1300 \\
1400 \\
1100 \\
1120 \\
1350\end{array}$ \\
\hline $\begin{array}{l}16 \\
17 \\
18 \\
19 \\
20\end{array}$ & $\begin{array}{l}35100 \\
35100 \\
35100 \\
35100 \\
35100\end{array}$ & $\begin{array}{l}14000 \\
14200 \\
14200 \\
14200 \\
14000\end{array}$ & $\begin{array}{l}22500 \\
22700 \\
21800 \\
22600 \\
22000\end{array}$ & $\begin{array}{l}8800 \\
8700 \\
8750 \\
9100 \\
9050\end{array}$ & $\begin{array}{l}1980 \\
2700 \\
3600 \\
3740 \\
4660\end{array}$ & $\begin{array}{r}625 \\
850 \\
1150 \\
1200 \\
1500\end{array}$ & $\begin{array}{l}4720 \\
4420 \\
4510 \\
4500 \\
4420\end{array}$ & $\begin{array}{l}1550 \\
1400 \\
1420 \\
1450 \\
1400\end{array}$ \\
\hline $\begin{array}{l}21 \\
22 \\
23 \\
24 \\
25\end{array}$ & $\begin{array}{l}35100 \\
35100 \\
35200 \\
31900 \\
31900\end{array}$ & $\begin{array}{l}14000 \\
13500 \\
13600 \\
12500 \\
12400\end{array}$ & $\begin{array}{l}20800 \\
19400 \\
12900 \\
11900 \\
10600\end{array}$ & $\begin{array}{l}8250 \\
7700 \\
4800 \\
4300 \\
3850\end{array}$ & $\begin{array}{r}7470 \\
-- \\
4670 \\
5800 \\
6610\end{array}$ & $\begin{array}{r}2550 \\
-- \\
1480 \\
1950 \\
2220\end{array}$ & $\begin{array}{l}4150 \\
4380 \\
4880 \\
5550 \\
4670\end{array}$ & $\begin{array}{l}1300 \\
1400 \\
1580 \\
1800 \\
1500\end{array}$ \\
\hline \multirow[t]{2}{*}{$\begin{array}{l}26 \\
27 \\
28 \\
29 \\
30 \\
31\end{array}$} & $\begin{array}{r}35100 \\
39000 \\
.39000 \\
35100 \\
39000 \\
29200\end{array}$ & $\begin{array}{l}14000 \\
15100 \\
15100 \\
14200 \\
15100 \\
10800\end{array}$ & $\begin{array}{r}7990 \\
4400 \\
5360 \\
10500 \\
6070 \\
--\end{array}$ & $\begin{array}{r}2700 \\
1350 \\
1750 \\
3850 \\
2000 \\
--\end{array}$ & $\begin{array}{l}6060 \\
5130 \\
4250 \\
3560 \\
3890 \\
3740\end{array}$ & $\begin{array}{l}2000 \\
1700 \\
1380 \\
1120 \\
1250 \\
1200\end{array}$ & $\begin{array}{l}4470 \\
4450 \\
4710 \\
4600 \\
4710 \\
7230\end{array}$ & $\begin{array}{l}1400 \\
1420 \\
1500 \\
1480 \\
1550 \\
2350\end{array}$ \\
\hline & \multicolumn{2}{|c|}{ FEBRUARY } & \multicolumn{2}{|c|}{ MARCH } & \multicolumn{2}{|c|}{ APRIL } & \multicolumn{2}{|c|}{ MAY } \\
\hline $\begin{array}{l}1 \\
2 \\
3 \\
4 \\
5\end{array}$ & $\begin{array}{l}6230 \\
5680 \\
5740 \\
5120 \\
6550\end{array}$ & $\begin{array}{l}2020 \\
1850 \\
1850 \\
1620 \\
2180\end{array}$ & $\begin{array}{l}3820 \\
5360 \\
2910 \\
2500 \\
1490\end{array}$ & $\begin{array}{r}1200 \\
1680 \\
875 \\
750 \\
405\end{array}$ & $\begin{array}{l}2310 \\
2510 \\
2890 \\
2740 \\
5040\end{array}$ & $\begin{array}{r}685 \\
750 \\
870 \\
870 \\
1270\end{array}$ & $\begin{array}{l}\mathbf{3 1 7} \\
\mathbf{3 4 2} \\
\mathbf{4 0 7} \\
636 \\
\mathbf{7 6 2}\end{array}$ & $\begin{array}{r}80 \\
90 \\
108 \\
178 \\
215\end{array}$ \\
\hline $\begin{array}{r}6 \\
7 \\
8 \\
9 \\
10\end{array}$ & $\begin{array}{l}4990 \\
3510 \\
3230 \\
3130 \\
4010\end{array}$ & $\begin{array}{r}1550 \\
1080 \\
1000 \\
950 \\
1250\end{array}$ & $\begin{array}{l}1610 \\
1410 \\
1780 \\
2040 \\
2170\end{array}$ & $\begin{array}{l}460 \\
385 \\
525 \\
600 \\
650\end{array}$ & $\begin{array}{r}1620 \\
856 \\
706 \\
753 \\
1060\end{array}$ & $\begin{array}{l}465 \\
242 \\
195 \\
210 \\
308\end{array}$ & $\begin{array}{r}975 \\
1170 \\
1290 \\
1530 \\
3440\end{array}$ & $\begin{array}{r}278 \\
330 \\
375 \\
445 \\
1080\end{array}$ \\
\hline $\begin{array}{l}11 \\
12 \\
13 \\
14 \\
15\end{array}$ & $\begin{array}{l}3730 \\
4960 \\
6350 \\
5310 \\
5620\end{array}$ & $\begin{array}{l}1150 \\
1820 \\
1800 \\
1680 \\
1800\end{array}$ & $\begin{array}{l}1390 \\
1170 \\
1090 \\
1130 \\
1920\end{array}$ & $\begin{array}{l}380 \\
310 \\
295 \\
310 \\
575\end{array}$ & $\begin{array}{l}1730 \\
3330 \\
2210 \\
1400 \\
1250\end{array}$ & $\begin{array}{l}510 \\
820 \\
635 \\
390 \\
355\end{array}$ & $\begin{array}{l}2470 \\
\mathbf{2 4 9 0} \\
2200 \\
2200 \\
\mathbf{2 3 2 0}\end{array}$ & $\begin{array}{l}750 \\
775 \\
650 \\
675 \\
700\end{array}$ \\
\hline $\begin{array}{l}16 \\
17 \\
18 \\
19 \\
20\end{array}$ & $\begin{array}{l}4280 \\
3150 \\
3020 \\
2990 \\
3250\end{array}$ & $\begin{array}{r}1320 \\
950 \\
925 \\
900 \\
1020\end{array}$ & $\begin{array}{l}1580 \\
1370 \\
1410 \\
2140 \\
2560\end{array}$ & $\begin{array}{l}440 \\
385 \\
395 \\
650 \\
800\end{array}$ & $\begin{array}{l}1460 \\
1700 \\
1990 \\
2140 \\
2280\end{array}$ & $\begin{array}{l}\mathbf{4 1 5} \\
\mathbf{4 9 5} \\
\mathbf{5 8 5} \\
\mathbf{6 3 0} \\
\mathbf{6 7 5}\end{array}$ & $\begin{array}{l}3840 \\
2890 \\
3030 \\
3580 \\
3240\end{array}$ & $\begin{array}{r}1200 \\
875 \\
950 \\
1100 \\
1000\end{array}$ \\
\hline $\begin{array}{l}21 \\
22 \\
23 \\
24 \\
25\end{array}$ & $\begin{array}{l}3500 \\
3240 \\
3030 \\
3160 \\
\mathbf{3 4 8 0}\end{array}$ & $\begin{array}{r}1100 \\
9750 \\
925 \\
975 \\
1080\end{array}$ & $\begin{array}{l}1670 \\
1300 \\
1640 \\
1850 \\
2320\end{array}$ & $\begin{array}{l}440 \\
360 \\
465 \\
550 \\
725\end{array}$ & $\begin{array}{r}2300 \\
5860 \\
2210 \\
822 \\
660\end{array}$ & $\begin{array}{r}685 \\
1500 \\
650 \\
228 \\
182\end{array}$ & $\begin{array}{l}3830 \\
4080 \\
8040 \\
5110 \\
5260\end{array}$ & $\begin{array}{l}1200 \\
1280 \\
2650 \\
1580 \\
1680\end{array}$ \\
\hline $\begin{array}{l}26 \\
27 \\
28 \\
29 \\
30 \\
31\end{array}$ & $\begin{array}{r}3870 \\
5250 \\
4620 \\
4170 \\
-- \\
--\end{array}$ & $\begin{array}{r}1220 \\
1680 \\
1450 \\
1300 \\
-- \\
--\end{array}$ & $\begin{array}{r}2370 \\
2350 \\
1750 \\
1700 \\
1700 \\
--\end{array}$ & $\begin{array}{r}825 \\
700 \\
490 \\
480 \\
480 \\
--\end{array}$ & $\begin{array}{c}459 \\
275 \\
253 \\
302 \\
288 \\
--\end{array}$ & $\begin{array}{r}122 \\
70 \\
60 \\
75 \\
70 \\
--\end{array}$ & $\begin{array}{l}5210 \\
4790 \\
5620 \\
5000 \\
5740 \\
6880\end{array}$ & $\begin{array}{l}1650 \\
1480 \\
1800 \\
1580 \\
1820 \\
2200\end{array}$ \\
\hline
\end{tabular}


RED RIVER BASIN--COntinued

7-3622. SMACKOVER CREEK NEAR NORPHLET, ARK,--Continued

Specific conductance (micromhos at $25^{\circ} \mathrm{C}$ ), and chloride in parts per million, water year October 1963 to September 1964--Continued

\begin{tabular}{|c|c|c|c|c|c|c|c|c|}
\hline \multirow[b]{2}{*}{ Day } & \multicolumn{2}{|c|}{ JUNE } & \multicolumn{2}{|c|}{ JULY } & \multicolumn{2}{|c|}{ AUGUST } & \multicolumn{2}{|c|}{ SEPTEMBEF } \\
\hline & $\begin{array}{l}\text { Specific } \\
\text { conduct - } \\
\text { ance } \\
\text { (micro- } \\
\text { mhos at } \\
25^{\circ} \mathrm{C} \text { ) }\end{array}$ & $\begin{array}{l}\text { Chlo- } \\
\text { ride } \\
\text { (C1) }\end{array}$ & $\begin{array}{l}\text { Specific } \\
\text { conduct- } \\
\text { ance } \\
\text { (micro- } \\
\text { mhos at } \\
25^{\circ} \mathrm{C} \text { ) }\end{array}$ & $\begin{array}{l}\text { Chlo- } \\
\text { ride } \\
\text { (Cl) }\end{array}$ & $\begin{array}{l}\text { Specif ic } \\
\text { conduct- } \\
\text { ance } \\
\text { (micro- } \\
\text { mhos at } \\
25^{\circ} \mathrm{C} \text { ) }\end{array}$ & $\begin{array}{l}\text { Chlo- } \\
\text { ride } \\
\text { (Cl) }\end{array}$ & $\begin{array}{l}\text { Specific } \\
\text { conduct - } \\
\text { ance } \\
\text { (micro- } \\
\text { mhos at } \\
25^{\circ} \mathrm{C} \text { ) }\end{array}$ & $\begin{array}{l}\text { Chlo- } \\
\text { ride } \\
(C 1)\end{array}$ \\
\hline $\begin{array}{l}1 \\
2 \\
3 \\
4 \\
5\end{array}$ & $\begin{array}{l}7730 \\
6340 \\
5260 \\
5060 \\
4850\end{array}$ & $\begin{array}{l}2420 \\
1980 \\
1650 \\
1600 \\
1520\end{array}$ & $\begin{array}{r}11500 \\
7580 \\
6790 \\
7030 \\
6210\end{array}$ & $\begin{array}{l}3900 \\
2450 \\
2180 \\
2300 \\
1980\end{array}$ & $\begin{array}{l}24600 \\
24300 \\
24100 \\
26200 \\
22700\end{array}$ & $\begin{array}{l}8800 \\
8700 \\
8600 \\
9500 \\
8100\end{array}$ & $\begin{array}{l}8070 \\
7570 \\
7180 \\
7370 \\
7610\end{array}$ & $\begin{array}{l}2650 \\
2450 \\
2320 \\
2400 \\
2480\end{array}$ \\
\hline $\begin{array}{r}6 \\
7 \\
8 \\
9 \\
10\end{array}$ & $\begin{array}{l}4780 \\
4400 \\
3620 \\
4780 \\
4700\end{array}$ & $\begin{array}{l}1520 \\
1350 \\
1120 \\
1520 \\
1480\end{array}$ & $\begin{array}{r}6100 \\
5870 \\
5740 \\
6240 \\
10600\end{array}$ & $\begin{array}{l}1950 \\
1880 \\
1800 \\
2000 \\
3600\end{array}$ & $\begin{array}{l}25500 \\
25500 \\
26400 \\
22800 \\
21100\end{array}$ & $\begin{array}{l}9250 \\
9300 \\
9700 \\
8100 \\
7500\end{array}$ & $\begin{array}{r}10300 \\
11300 \\
6900 \\
6180 \\
6430\end{array}$ & $\begin{array}{l}3500 \\
3800 \\
2150 \\
1980 \\
2050\end{array}$ \\
\hline $\begin{array}{l}11 \\
12 \\
13 \\
14 \\
15\end{array}$ & $\begin{array}{l}3790 \\
3640 \\
4700 \\
5180 \\
4900\end{array}$ & $\begin{array}{l}1150 \\
1100 \\
1500 \\
1650 \\
1550\end{array}$ & $\begin{array}{l}11100 \\
13200 \\
11400 \\
12400 \\
13100\end{array}$ & $\begin{array}{l}3750 \\
4500 \\
3750 \\
4150 \\
4150\end{array}$ & $\begin{array}{l}27300 \\
20800 \\
22400 \\
18300 \\
18200\end{array}$ & $\begin{array}{r}10200 \\
7450 \\
8150 \\
6500 \\
6450\end{array}$ & $\begin{array}{l}6570 \\
7400 \\
6720 \\
5490 \\
5350\end{array}$ & $\begin{array}{l}2150 \\
2420 \\
2180 \\
1700 \\
1650\end{array}$ \\
\hline $\begin{array}{l}16 \\
17 \\
18 \\
19 \\
20\end{array}$ & $\begin{array}{l}5050 \\
7100 \\
6900 \\
5700 \\
6110\end{array}$ & $\begin{array}{l}1600 \\
2320 \\
2220 \\
1800 \\
1980\end{array}$ & $\begin{array}{l}15600 \\
15600 \\
16000 \\
17500 \\
16500\end{array}$ & $\begin{array}{l}5400 \\
5350 \\
5550 \\
6150 \\
5700\end{array}$ & $\begin{array}{l}18100 \\
13300 \\
13100 \\
10300 \\
10100\end{array}$ & $\begin{array}{l}6400 \\
4500 \\
4500 \\
3400 \\
3400\end{array}$ & $\begin{array}{l}6220 \\
8200 \\
4130 \\
1110 \\
1270\end{array}$ & $\begin{array}{r}2000 \\
2720 \\
1240 \\
320 \\
370\end{array}$ \\
\hline $\begin{array}{l}21 \\
22 \\
23 \\
24 \\
25\end{array}$ & $\begin{array}{r}6140 \\
5890 \\
5680 \\
12200 \\
7980\end{array}$ & $\begin{array}{l}2000 \\
1880 \\
1820 \\
4100 \\
2550\end{array}$ & $\begin{array}{l}15400 \\
19200 \\
18500 \\
20500 \\
18400\end{array}$ & $\begin{array}{l}5300 \\
6850 \\
6500 \\
7300 \\
6500\end{array}$ & $\begin{array}{r}8040 \\
13400 \\
11200 \\
11600 \\
11800\end{array}$ & $\begin{array}{l}2650 \\
4700 \\
3700 \\
4000 \\
4000\end{array}$ & $\begin{array}{l}1700 \\
2130 \\
2670 \\
3240 \\
3330\end{array}$ & $\begin{array}{r}510 \\
640 \\
820 \\
1000 \\
1020\end{array}$ \\
\hline $\begin{array}{l}26 \\
27 \\
28 \\
29 \\
30 \\
31\end{array}$ & $\begin{array}{r}4600 \\
4640 \\
4850 \\
5050 \\
-- \\
--\end{array}$ & $\begin{array}{r}1400 \\
1450 \\
1550 \\
1650 \\
-- \\
--\end{array}$ & $\begin{array}{l}20100 \\
19700 \\
21800 \\
21700 \\
20800 \\
22400\end{array}$ & $\begin{array}{l}7200 \\
7000 \\
7900 \\
7900 \\
7550 \\
8100\end{array}$ & $\begin{array}{r}10500 \\
12400 \\
11200 \\
10100 \\
8780 \\
8300\end{array}$ & $\begin{array}{l}3550 \\
4300 \\
3800 \\
3400 \\
2900 \\
2700\end{array}$ & $\begin{array}{r}3620 \\
4070 \\
9620 \\
6170 \\
3790 \\
--\end{array}$ & $\begin{array}{r}1130 \\
1280 \\
3300 \\
3450 \\
1150 \\
--\end{array}$ \\
\hline
\end{tabular}




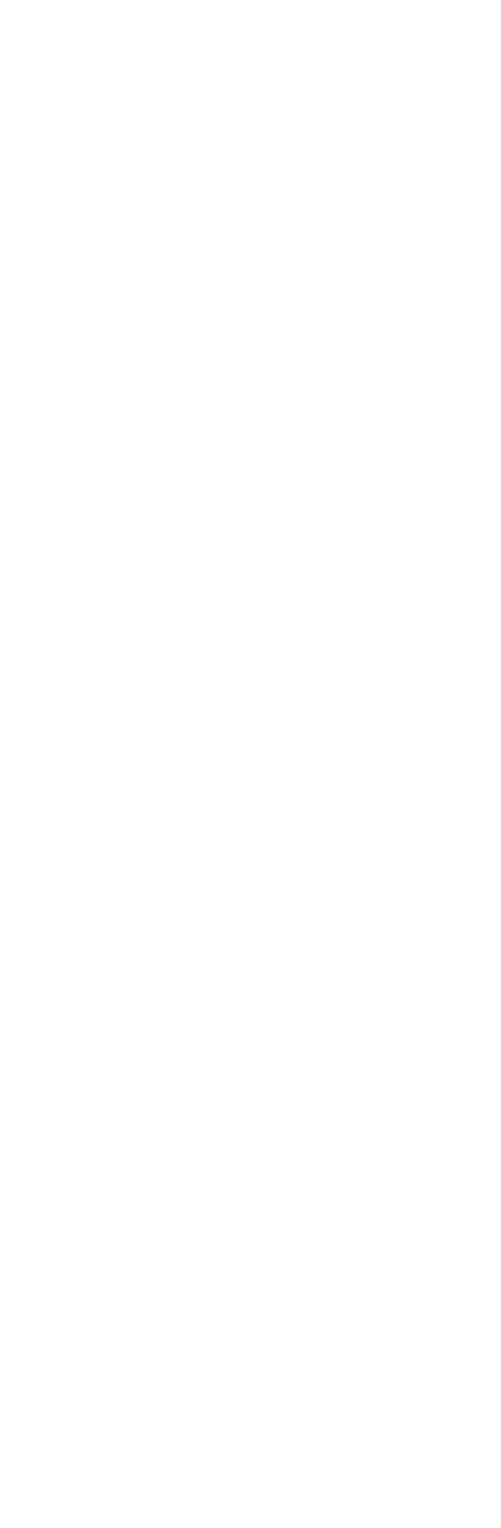




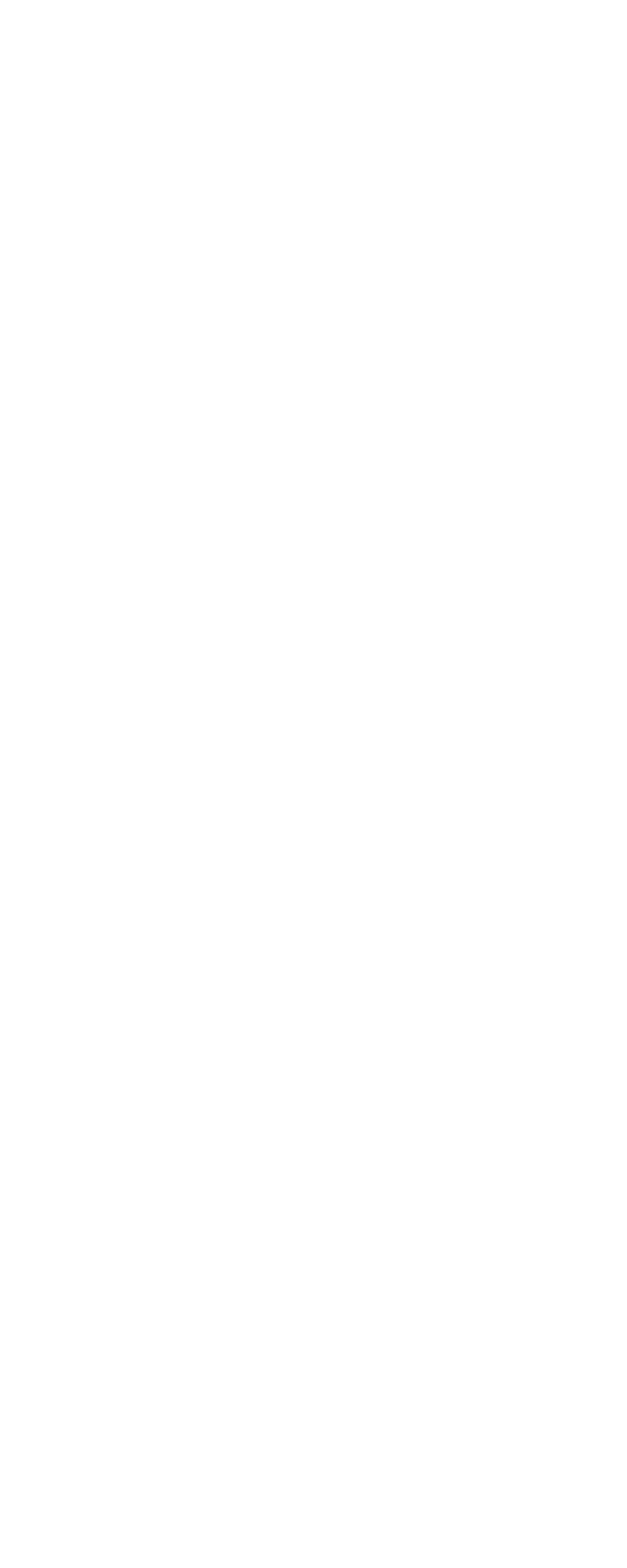




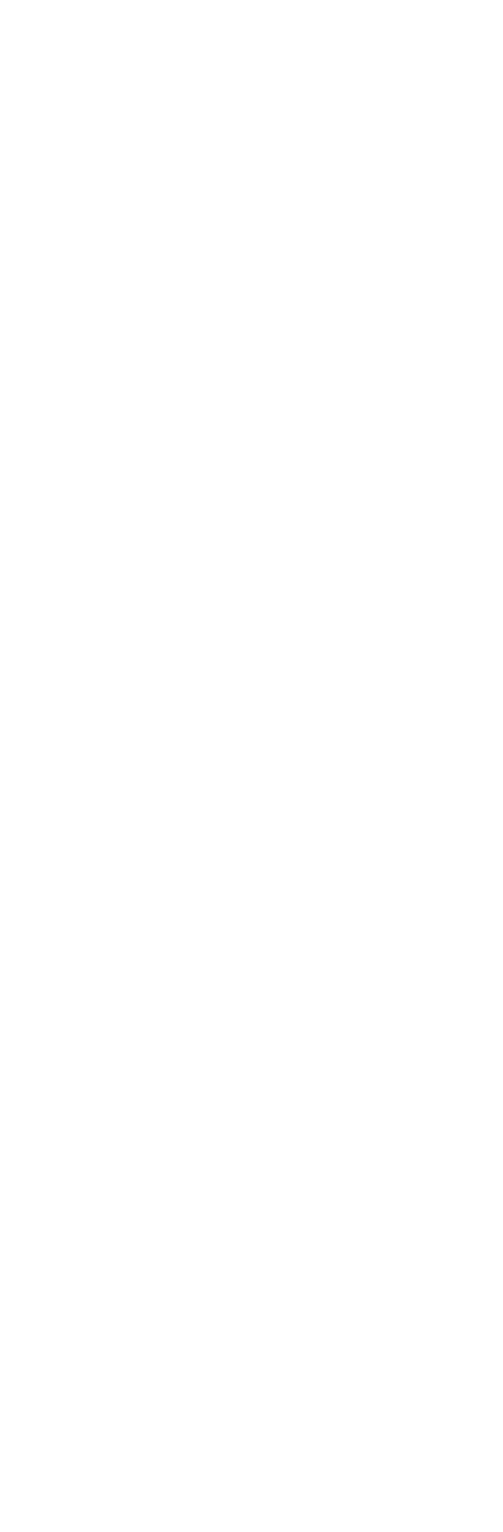




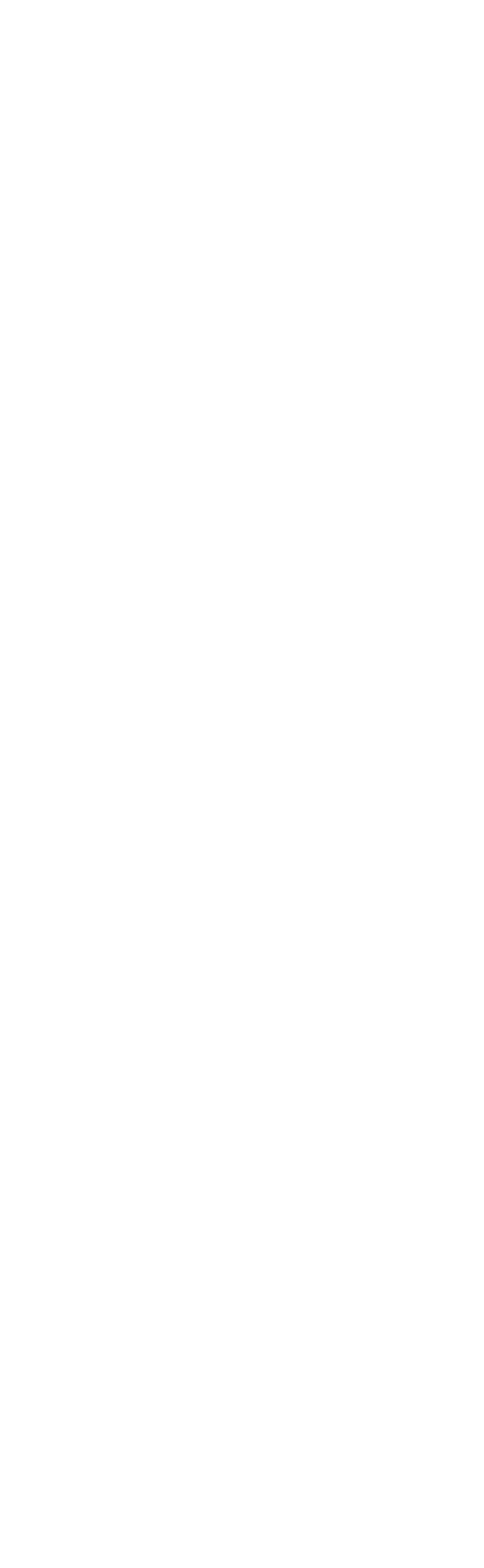




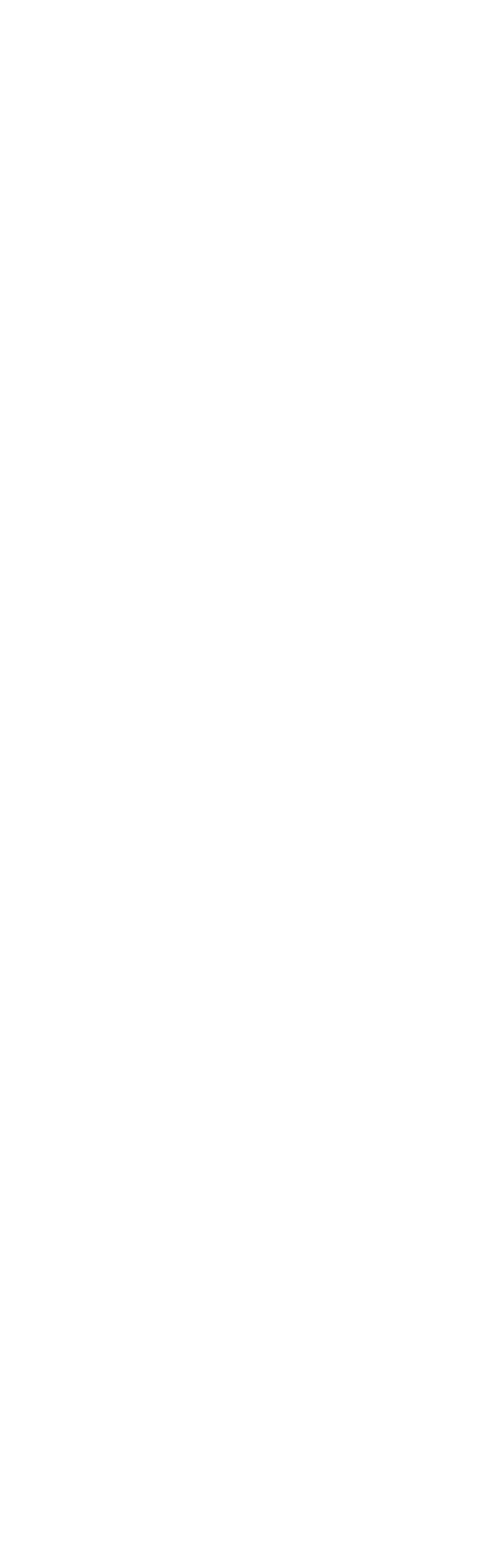




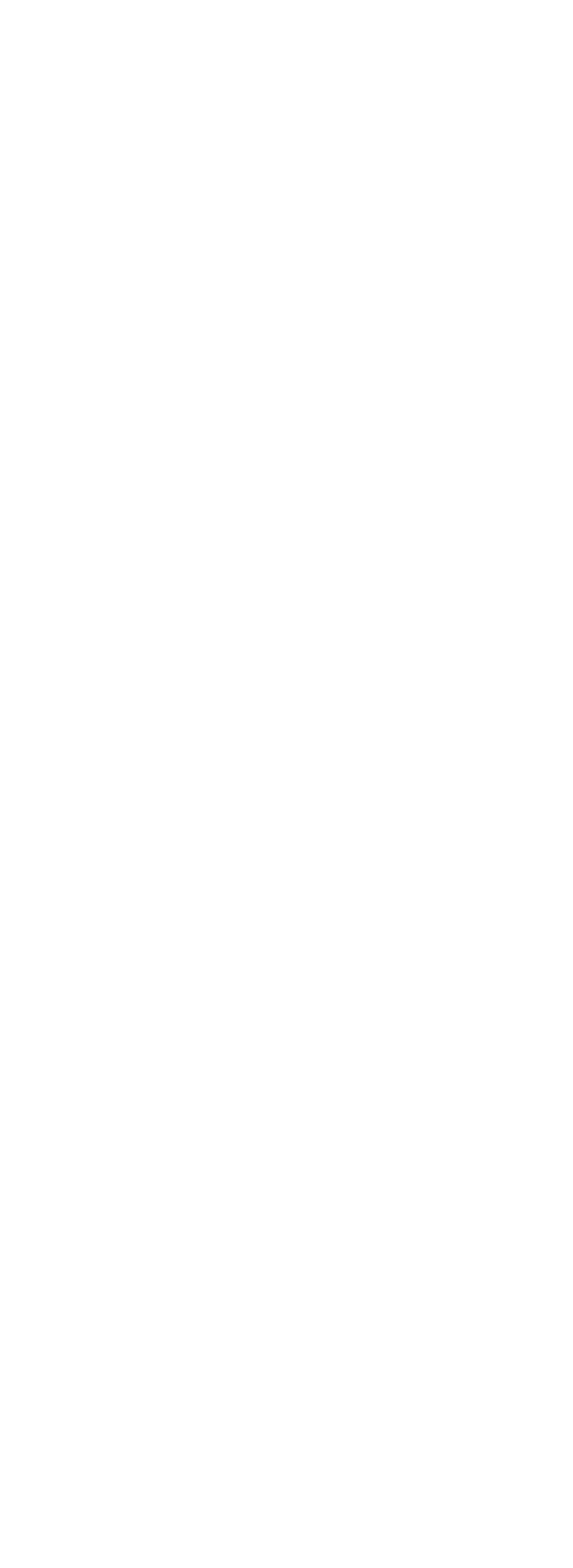




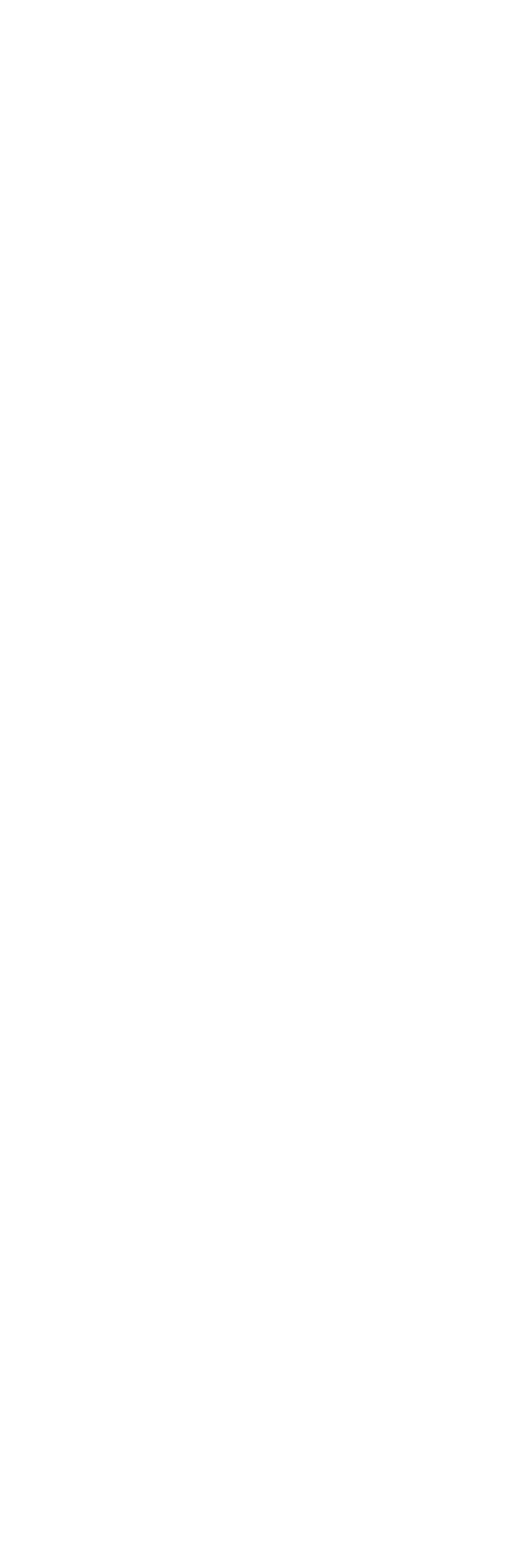




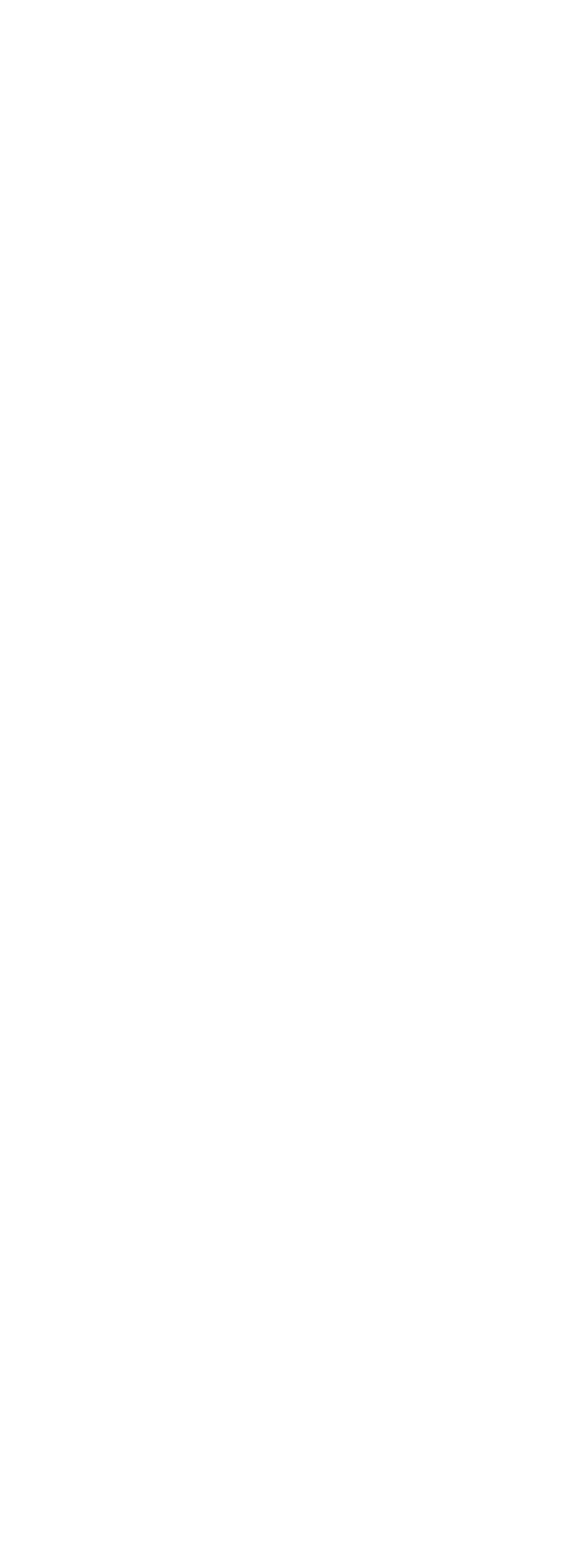




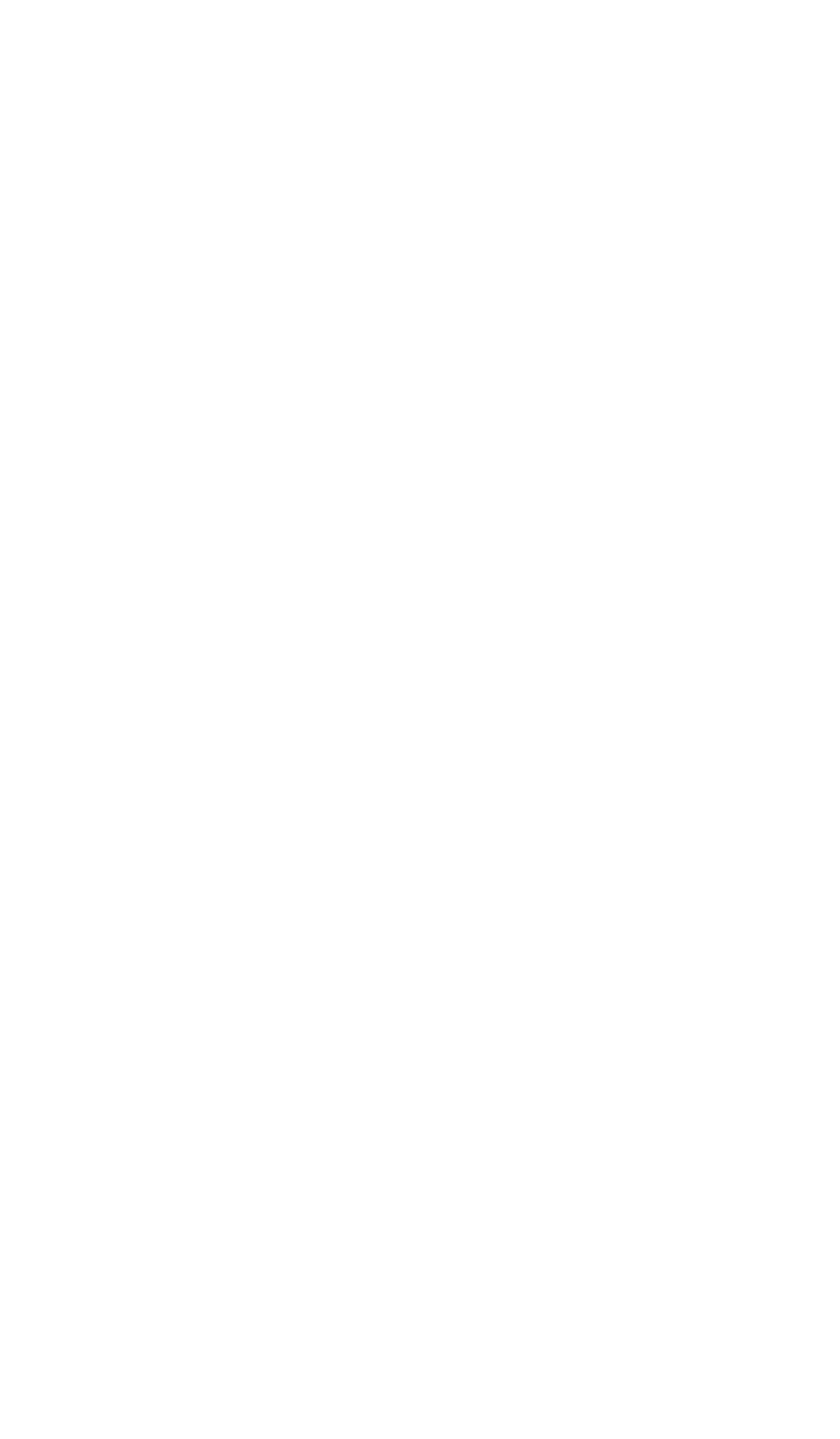




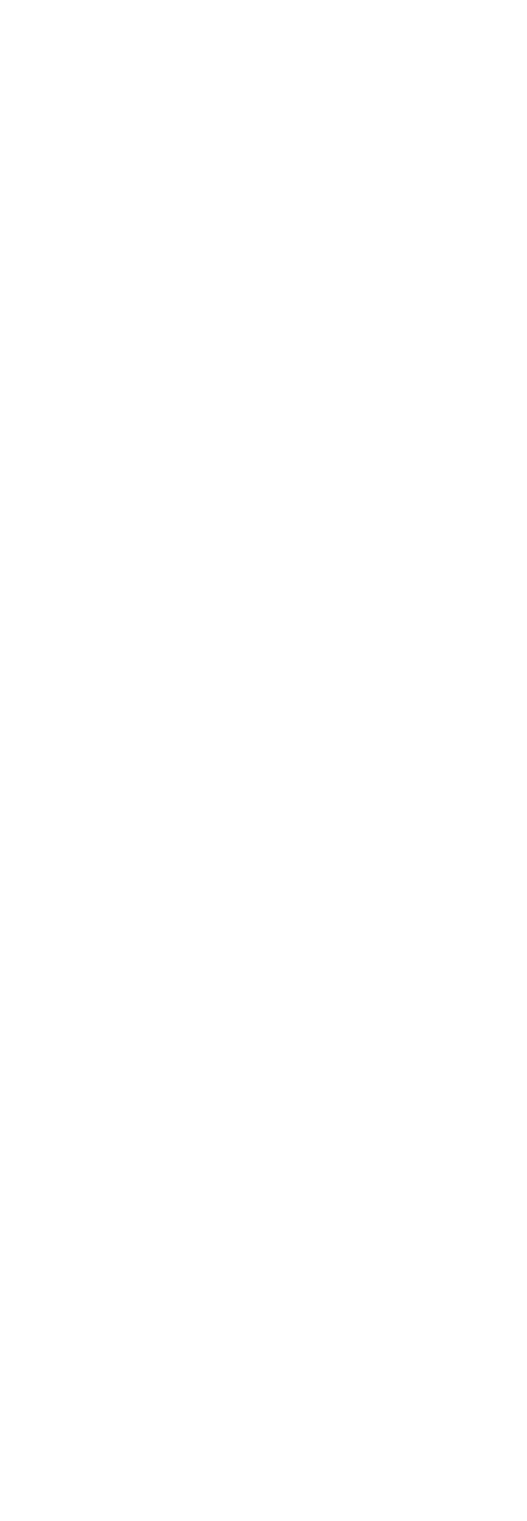




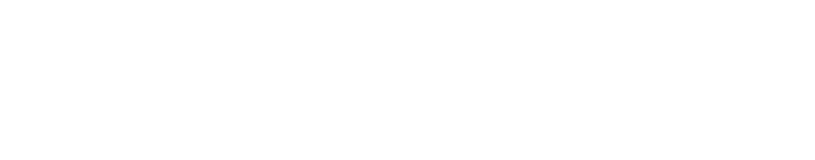

\begin{tabular}{|c|c|c|c|c|c|c|c|c|c|}
\hline 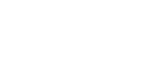 & $\stackrel{\infty}{0}_{0}^{\circ}$ & i & ㄱ & i & $0^{\circ}$ & $\stackrel{*}{*}:$ & $\stackrel{3}{-1}$ & $\stackrel{\circ}{i} \mid$ & $i$ \\
\hline 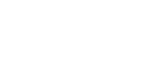 & H & $m$ & ส & i & $\stackrel{\infty}{\sim}$ & $\bar{\infty}=7$ & $\stackrel{\infty}{\infty}$ & $\sim^{\infty}$ & $\underset{\sim}{\infty}$ \\
\hline
\end{tabular}

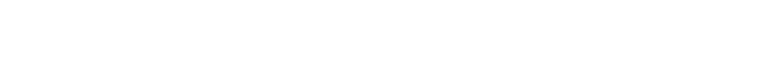

\begin{tabular}{|c|c|c|c|c|c|c|c|c|c|c|}
\hline 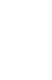 & 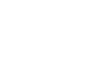 & $\begin{array}{l}8 \\
0 \\
0\end{array}$ & $i$ & $i$ & $i$ & $\dot{\sim}$ & : & $\begin{array}{l}\text { \& } \\
\text { ํ. }\end{array}$ & $\because 1$ & 1 \\
\hline 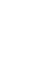 & 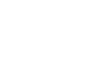 & : & 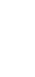 & $\begin{array}{l}\text { 离 } \\
\text { 吕 }\end{array}$ & 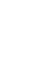 & \% & ஓ্ণু & $\$$ & $\overbrace{0}^{\infty}$ & \% \\
\hline
\end{tabular}

\begin{tabular}{|c|c|c|c|c|c|c|c|c|c|c|}
\hline & 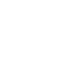 & ت & 1 & $\stackrel{\infty}{\sim}$ & $\cong$ & $\stackrel{\circ}{\dot{m}}$ & $\stackrel{\infty}{\infty} \dot{x}^{\prime}$ & $\stackrel{\text { ن }}{\circ}$ & $\stackrel{N}{*}:$ & 1 \\
\hline & 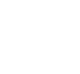 & $\because$ & I & i & i & $\stackrel{0}{\circ}$ & ?! & $\stackrel{0}{\circ}$ & ?: & 1 \\
\hline
\end{tabular}

\begin{tabular}{|c|c|c|c|c|c|c|c|c|c|}
\hline 호ㅇㅝㅜㅇ & $\dot{\infty}$ & $\Rightarrow$ & ה & $\overrightarrow{4}$ & $ت$ & $\dddot{9} \cong$ & స & Fم & $\stackrel{7}{7}$ \\
\hline 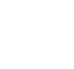 & $\sigma_{0}^{\pi}$ & $i$ & $\stackrel{\infty}{\stackrel{\infty}{~}}$ & เั๊ & & $\stackrel{్}{0}^{1}$ & 户े & $\stackrel{\infty}{1}^{1}$ & $i$ \\
\hline
\end{tabular}

\begin{tabular}{|c|c|c|c|c|c|c|c|c|c|}
\hline 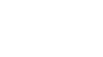 & $\sigma^{\circ}$ & & $\stackrel{\circ}{\circledR}$ & เั้ & $\stackrel{m}{m}$ & $\stackrel{\leftrightarrow}{-1}^{1}$ & 요 & $\underset{\infty}{\infty} 1$ & \\
\hline 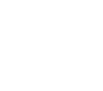 & กิ & 요 & $\circ$ & $\circ$ & $\infty$ & 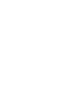 & $*$ & 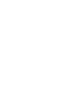 & 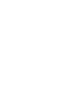 \\
\hline 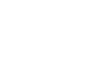 & $\hat{i}$ & 1 & i⿱ & 1 & 1 & 11 & 1 & 11 & 1 \\
\hline 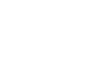 & $\begin{array}{l}\infty \\
\infty \\
\infty\end{array}$ & i & 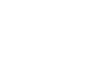 & 1 & $\approx$ & $m^{\prime \prime}$ & $\Xi$ & $y^{\prime}$ & 1 \\
\hline 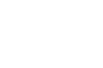 & $\vec{n}$ & 1 & $i$ & 1 & $\stackrel{\infty}{-i}$ & î! & $\stackrel{\dot{i}}{ }$ & $\overbrace{i}^{3}:$ & 1 \\
\hline 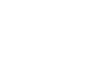 & $\stackrel{\sim}{*}$ & 1 & 1 & $i$ & $\stackrel{\infty}{\infty}$ & $\begin{array}{lll}\infty & 1 \\
\infty & 1\end{array}$ & $\ddot{n}$ & $\stackrel{\infty}{\infty}_{\infty}^{0} 1$ & 1 \\
\hline 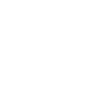 & สี & $\hat{N}$ & क & $\vec{n}$ & $\grave{a}$ & $\overrightarrow{N i}$ & $\hat{\mathbf{N}}$ & ส 1 & 1 \\
\hline 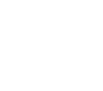 & 迥 & $\begin{array}{l}5 \\
\dot{\infty} \\
\end{array}$ & ఖ్ & $\stackrel{\circ}{-i}$ & $\begin{array}{l}\overrightarrow{1} \\
\dot{0}\end{array}$ & $\stackrel{\infty}{\rightarrow}: 1$ & 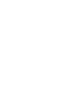 & $\underset{7}{7}$ & న్ \\
\hline 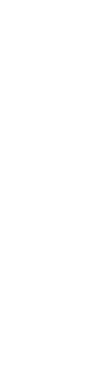 & 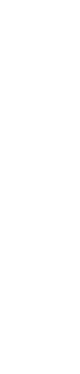 & 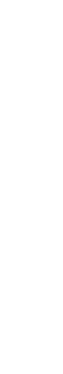 & 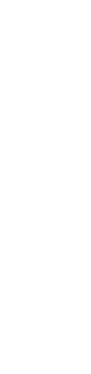 & 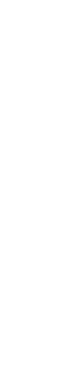 & 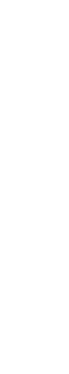 & 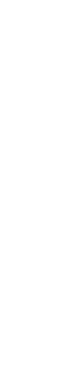 & 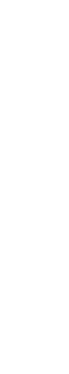 & 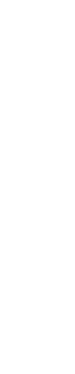 & 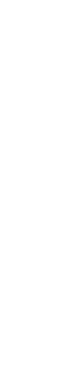 \\
\hline 咅 & 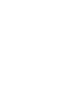 & 욕 & $\Rightarrow$ & \& & $\begin{array}{l}N \\
\dot{d} \\
\stackrel{\dot{d}}{n}\end{array}$ & $\begin{array}{l}\text { 여 } \\
\vdots \\
g\end{array}$ & $\begin{array}{l}N \\
\dot{a} \\
\stackrel{a}{n}\end{array}$ & $\begin{array}{l}=90 \\
\Rightarrow 9 \\
0 \\
5 \\
5\end{array}$ & $\sqrt{4}$ \\
\hline
\end{tabular}




\begin{tabular}{|c|c|c|c|c|c|c|c|c|c|c|c|c|}
\hline & 평 & $\mid \begin{array}{l}\infty \\
i \infty \\
i \infty\end{array}$ & is & $\stackrel{0}{\infty} \underset{0}{\infty}$ & $\tilde{i}$ & $\stackrel{\sim}{\pi}$ & $\ddot{m}$ & $\stackrel{0}{\dot{0}}$ & $\stackrel{m}{0}$ & $\stackrel{?}{\infty}$ & $\stackrel{?}{\dot{0}}$ & \\
\hline & 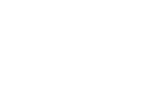 & $\stackrel{\infty}{\stackrel{-1}{0}}$ & : & $\underset{\sim}{\infty}=$ & 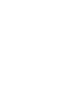 & 总 & 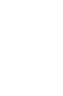 & $\ddot{\Xi}$ & $\bar{\infty}$ & $\bar{\infty}$ & 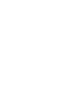 & \\
\hline & 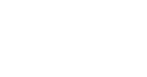 & $i$ & $\stackrel{\pi}{i}$ & 11 & 1 & 1 & 1 & 1 & 1 & $i$ & 1 & \\
\hline & 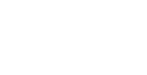 & $\Rightarrow$ & ส & $\approx^{\infty}$ & $\vec{\infty}$ & $\vec{\infty}$ & $\stackrel{\infty}{\sim}$ & 10 & 0 & $N$ & r & \\
\hline & 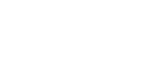 & 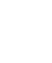 & $\vec{\lambda}$ & D̊ & 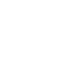 & $\stackrel{\Delta}{\Delta}$ & $\stackrel{\Phi}{\oplus}$ & $\ddot{\varphi}$ & $\bar{n}$ & 9 & $\mathbb{N}$ & \\
\hline & 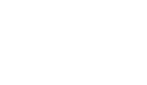 & $i$ & $\overrightarrow{5}$ & 11 & $i$ & 1 & 1 & 1 & 1 & 1 & $i$ & \\
\hline & 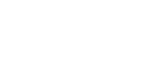 & $\vec{\infty}$ & 今) & is & $i$ & $i$ & $\begin{array}{l}\stackrel{0}{0} \\
\stackrel{0}{0} \\
0\end{array}$ & $\stackrel{\infty}{\infty}$ & i & $\overrightarrow{6}$ & in & \\
\hline 竞 & 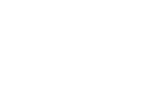 & 1 & ๆ & 1 i & 1 & 1 & i & i & 1 & i & i & \\
\hline $\begin{array}{ll}E & 0 \\
& 1 \\
1 & 1 \\
0 & 0 \\
0 & 0\end{array}$ & 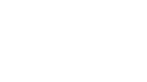 & 1 & : & 11 & 1 & 1 & 1 & i & 1 & i & 1 & \\
\hline 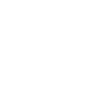 & 웛 & $\underset{\sim}{\mathbb{N}}$ & 勇 & $\vec{n}$ & ల్ల్ల & $\stackrel{\circ}{\stackrel{7}{7}}$ & \&્్ํ & $\begin{array}{l}0 \\
\text { in }\end{array}$ & $\stackrel{\square}{\infty}$ & $\stackrel{\sim}{\sim}$ & $\stackrel{4}{\circ:}$ & \\
\hline 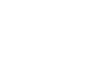 & 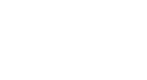 & 1 & $\ddot{m}$ & ${ }^{1} 1$ & 1 & 1 & 1 & 1 & 1 & $i$ & 1 & \\
\hline 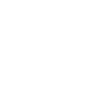 & 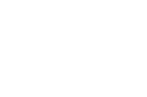 & $\stackrel{1}{1}$ & $N$ & 19 & 0 & 0 & 0 & $\nexists$ & $\stackrel{\infty}{\sim}$ & $\vec{N}$ & P्म & \\
\hline $\begin{array}{ll}7 & 7 \\
0 & 7 \\
0 & 1\end{array}$ & 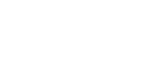 & 1 & $\stackrel{\infty}{a}$ & 11 & $i$ & 1 & 1 & $i$ & i & 1 & 1 & \\
\hline 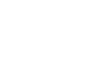 & 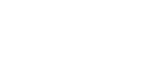 & 1 & $\stackrel{\sim}{\prime}$ & $\infty^{\prime}$ & 1 & $i$ & 1 & 1 & 1 & 1 & i & \\
\hline 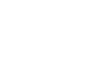 & 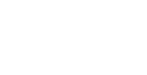 & 1 & $\stackrel{m}{i}$ & 11 & 1 & $i$ & 1 & $i$ & 1 & $i$ & 1 & \\
\hline 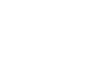 & 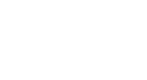 & 1 & $\stackrel{\infty}{\infty}$ i. & 11 & 1 & 1 & $i$ & 1 & 1 & $i$ & $i$ & \\
\hline 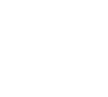 & 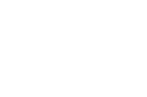 & $i$ & $\hat{ส}$ & i i & ஓ్లి & 1 & 1 & i & ల్ల & I & $i$ & \\
\hline 离 & 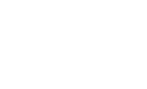 & $\stackrel{0}{0}$ & $\stackrel{m}{0}$ & $\stackrel{\stackrel{m}{+} 1}{+1}$ & $\stackrel{\circ}{?}$ & $\fallingdotseq$ & $\fallingdotseq$ & $\stackrel{0}{\dddot{1}}$ & 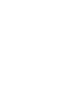 & : & $?$ & \\
\hline & 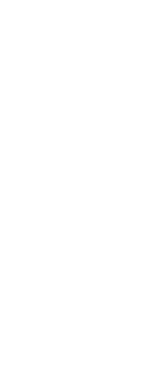 & 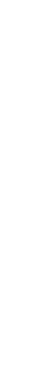 & 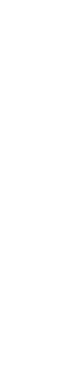 & 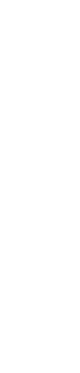 & 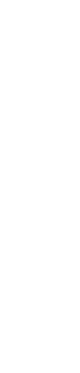 & 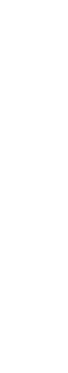 & 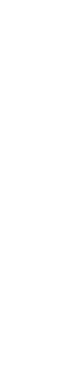 & 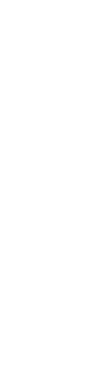 & 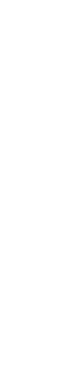 & 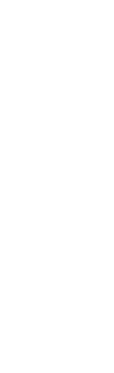 & 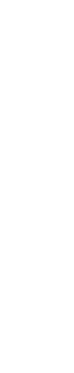 & \\
\hline & $\stackrel{8}{ \pm}$ & $\begin{array}{l}7 \\
0\end{array}$ & N & $\begin{array}{l}\text { 육 } \\
0 \\
5 \\
5\end{array}$ & $\Rightarrow$ & 7 & $\overline{9}$ & $\exists$ & 요 & $\overrightarrow{7}$ & $\Rightarrow$ & \\
\hline
\end{tabular}




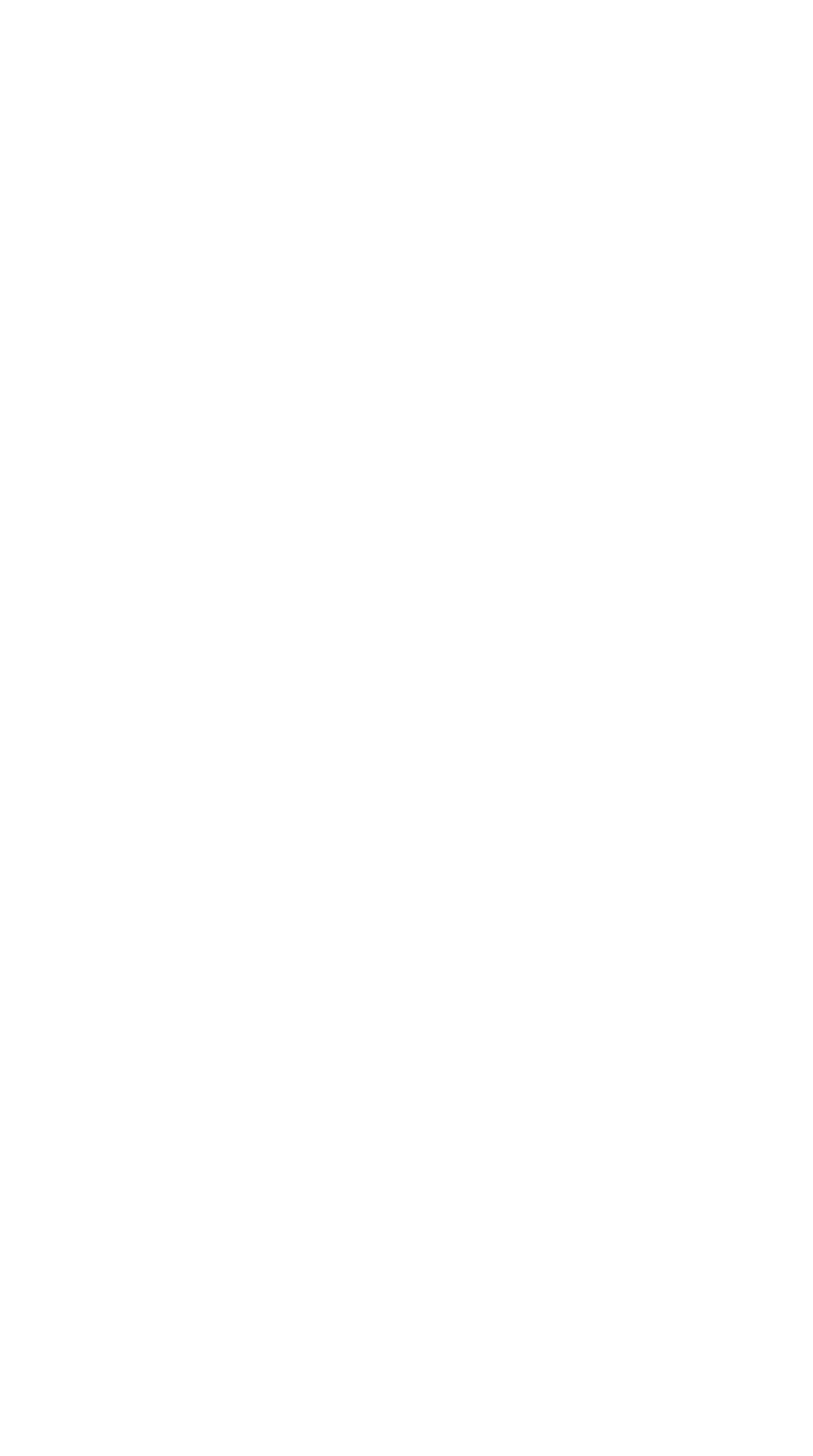




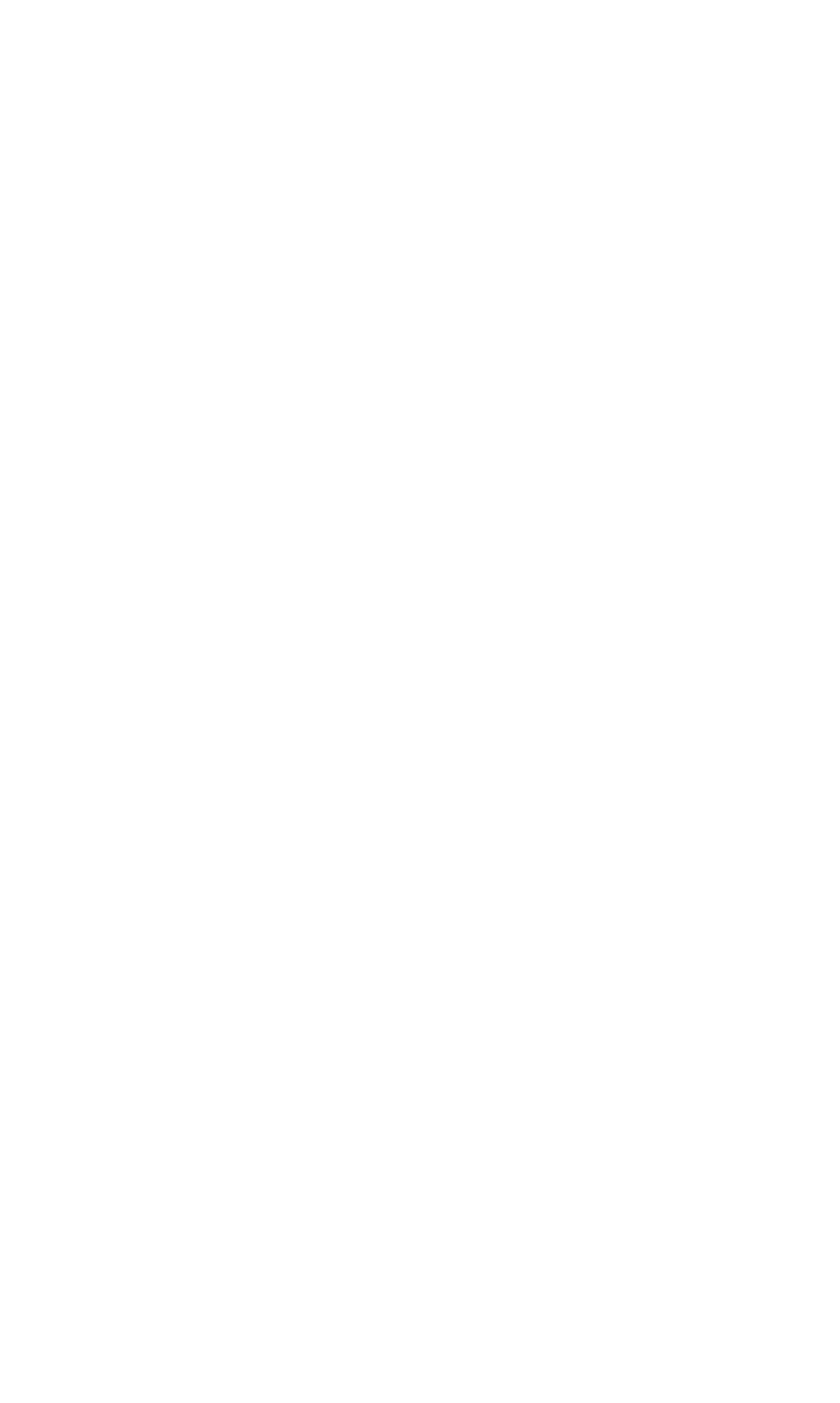




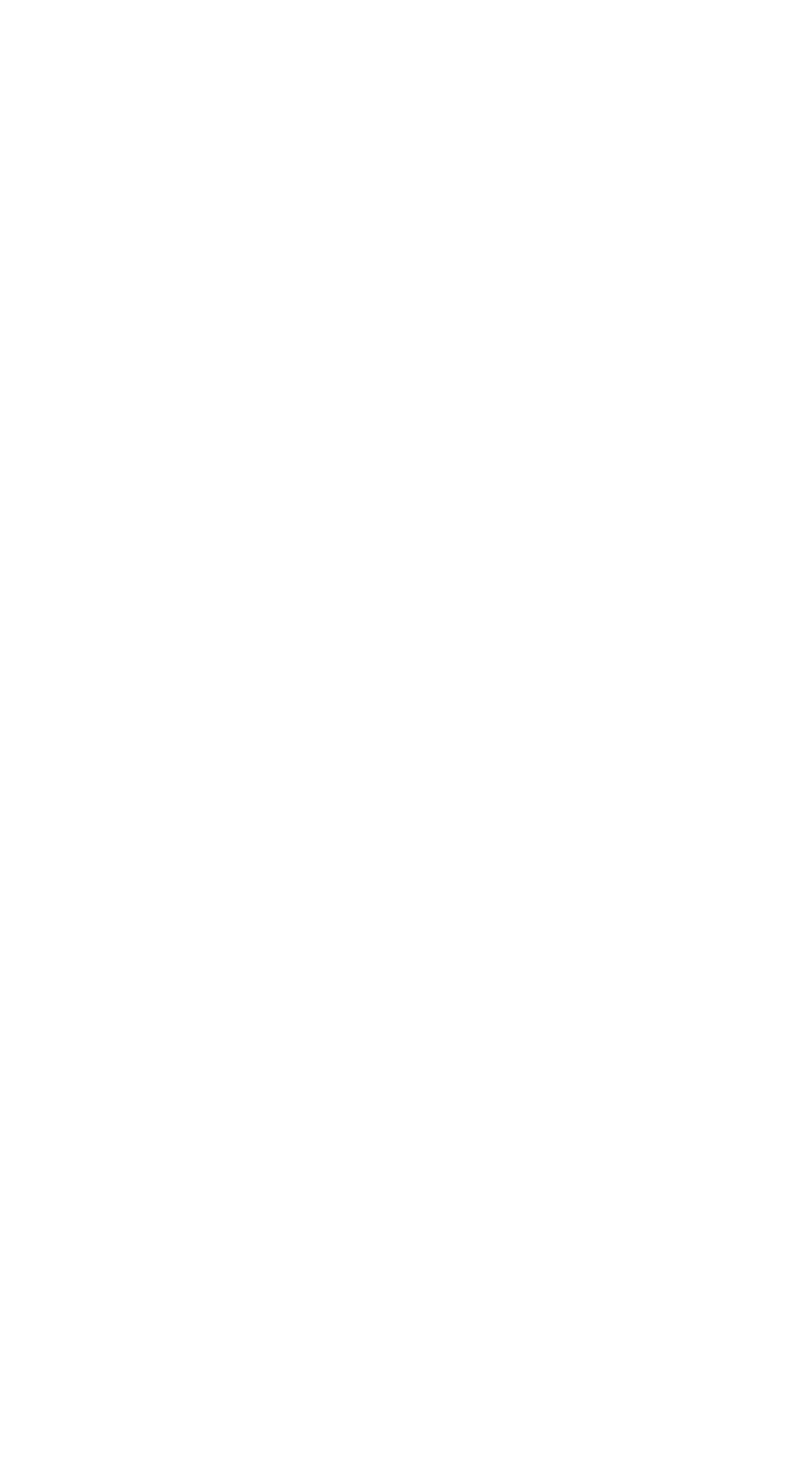




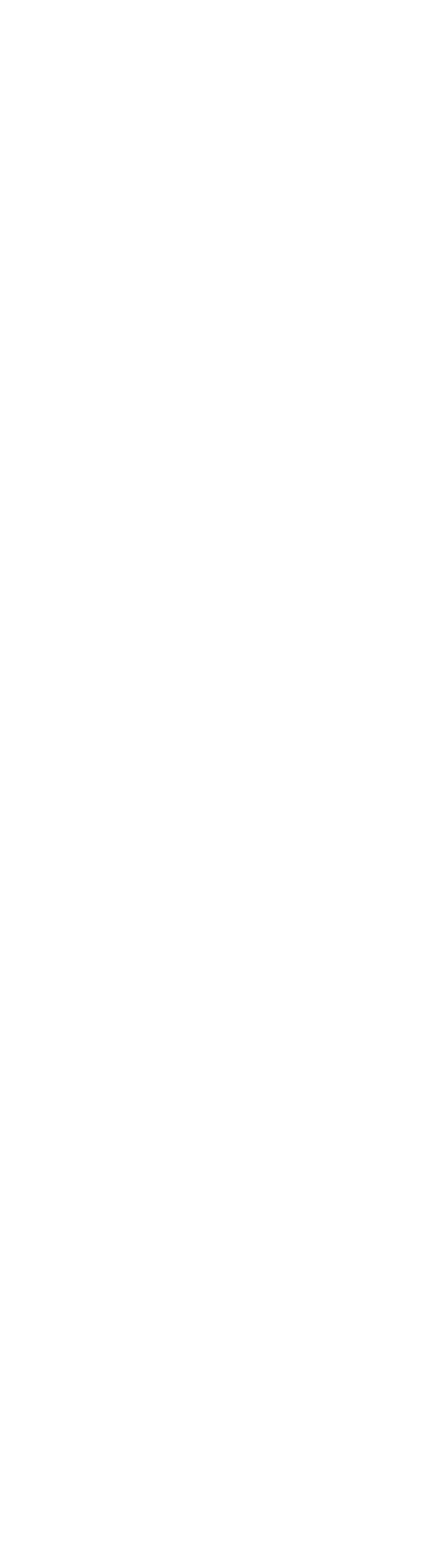

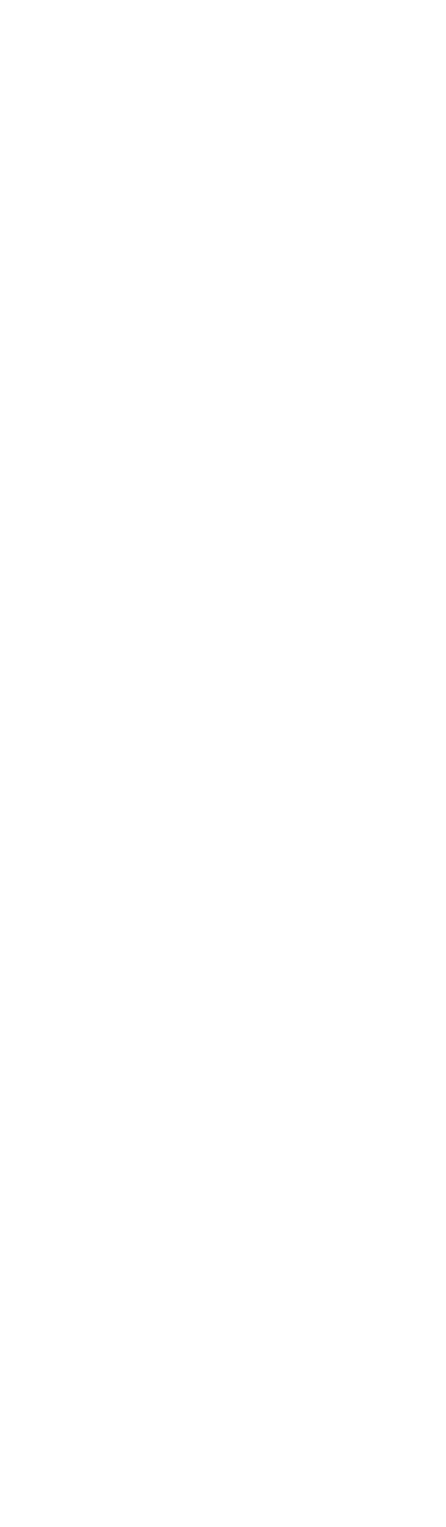




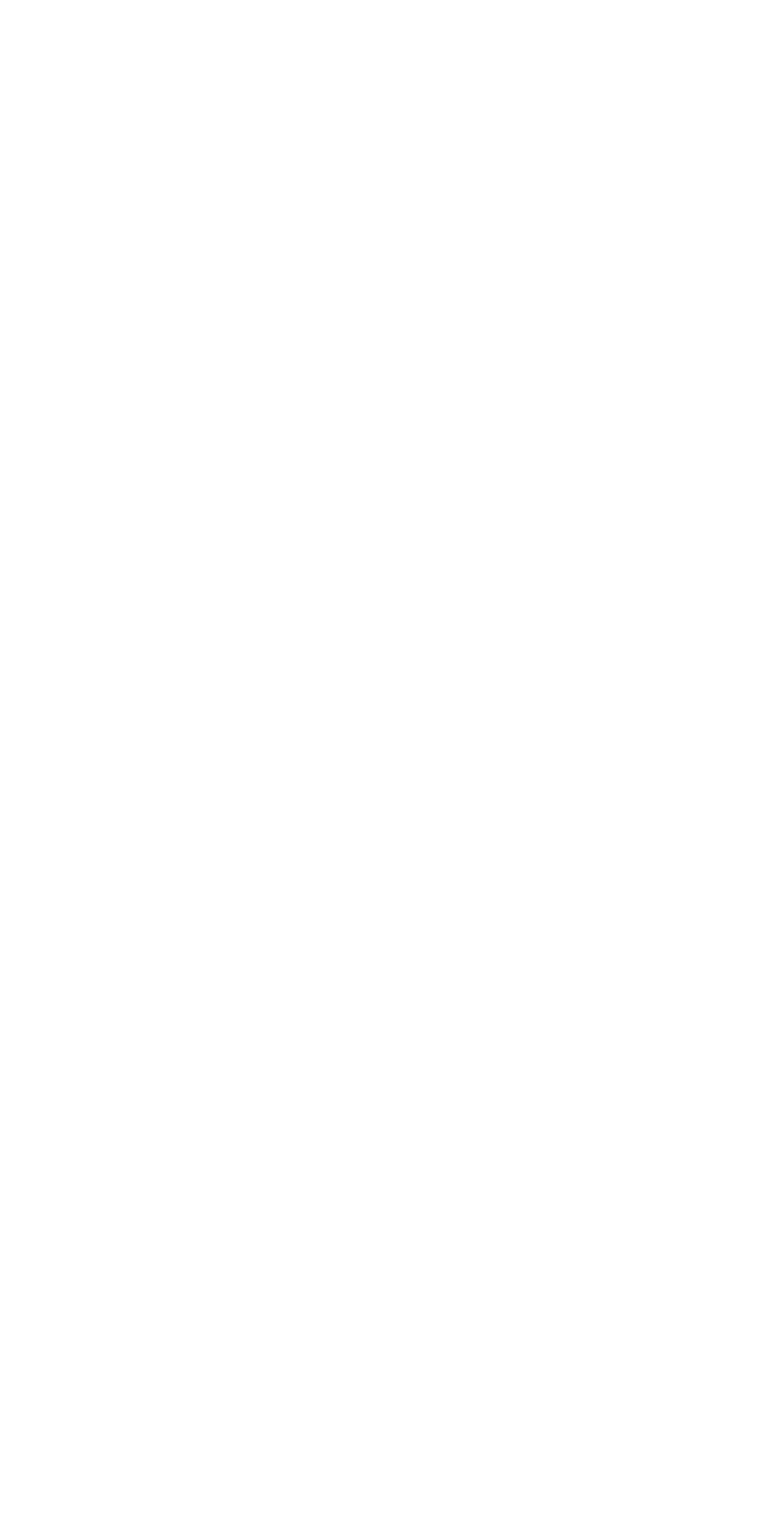




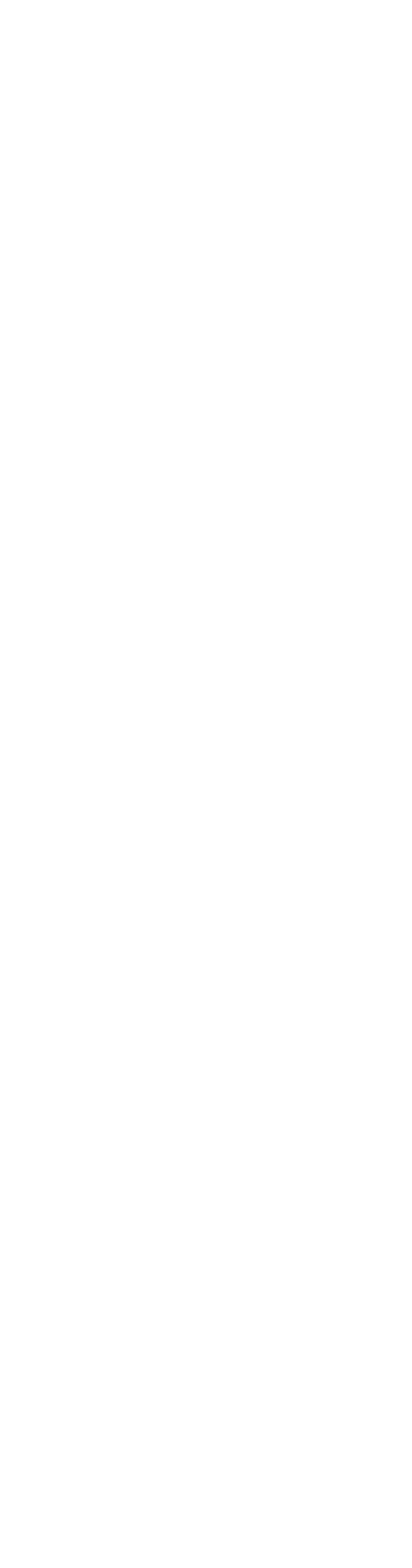

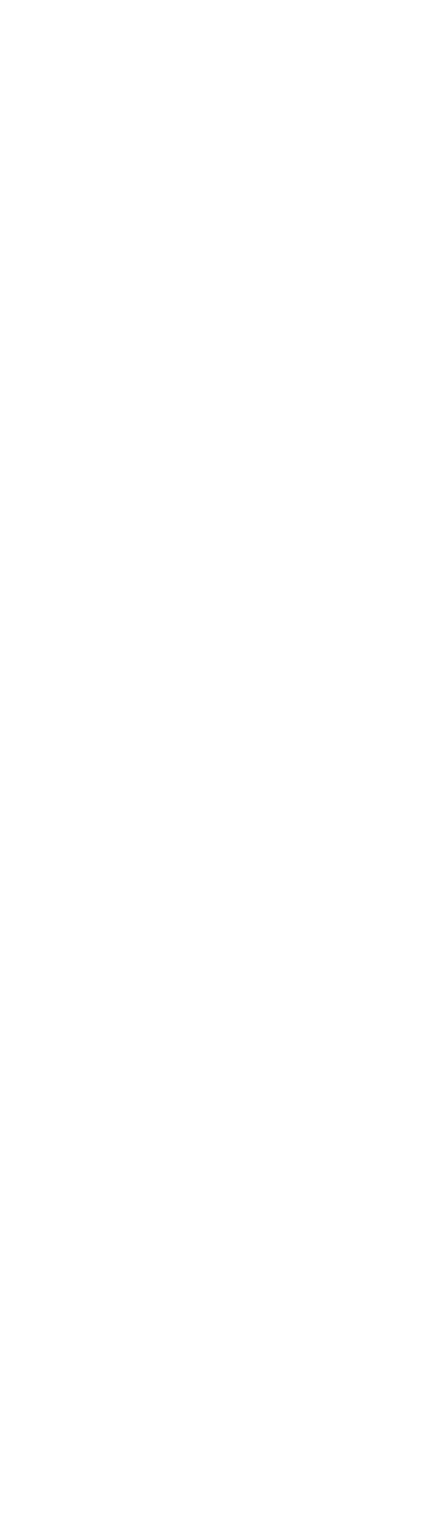




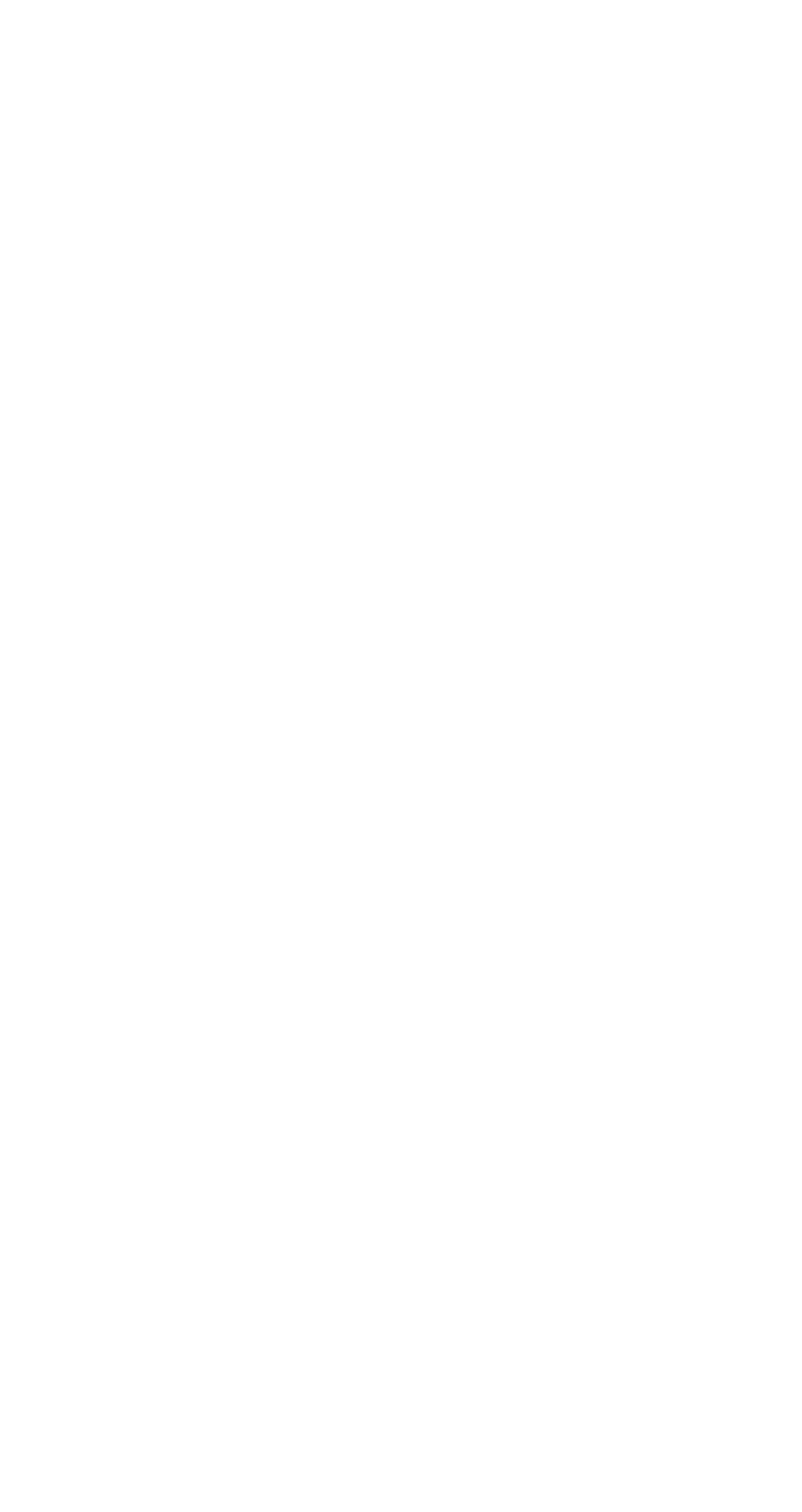




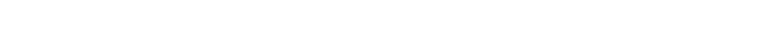
mạm

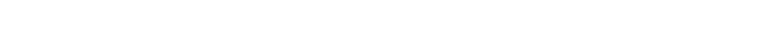

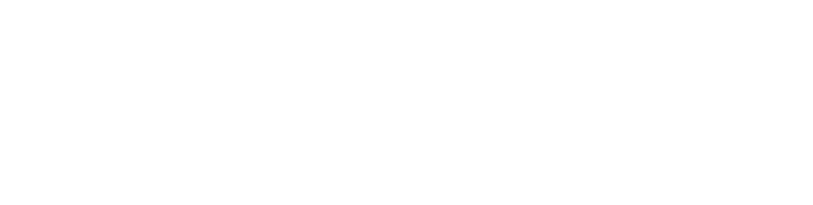

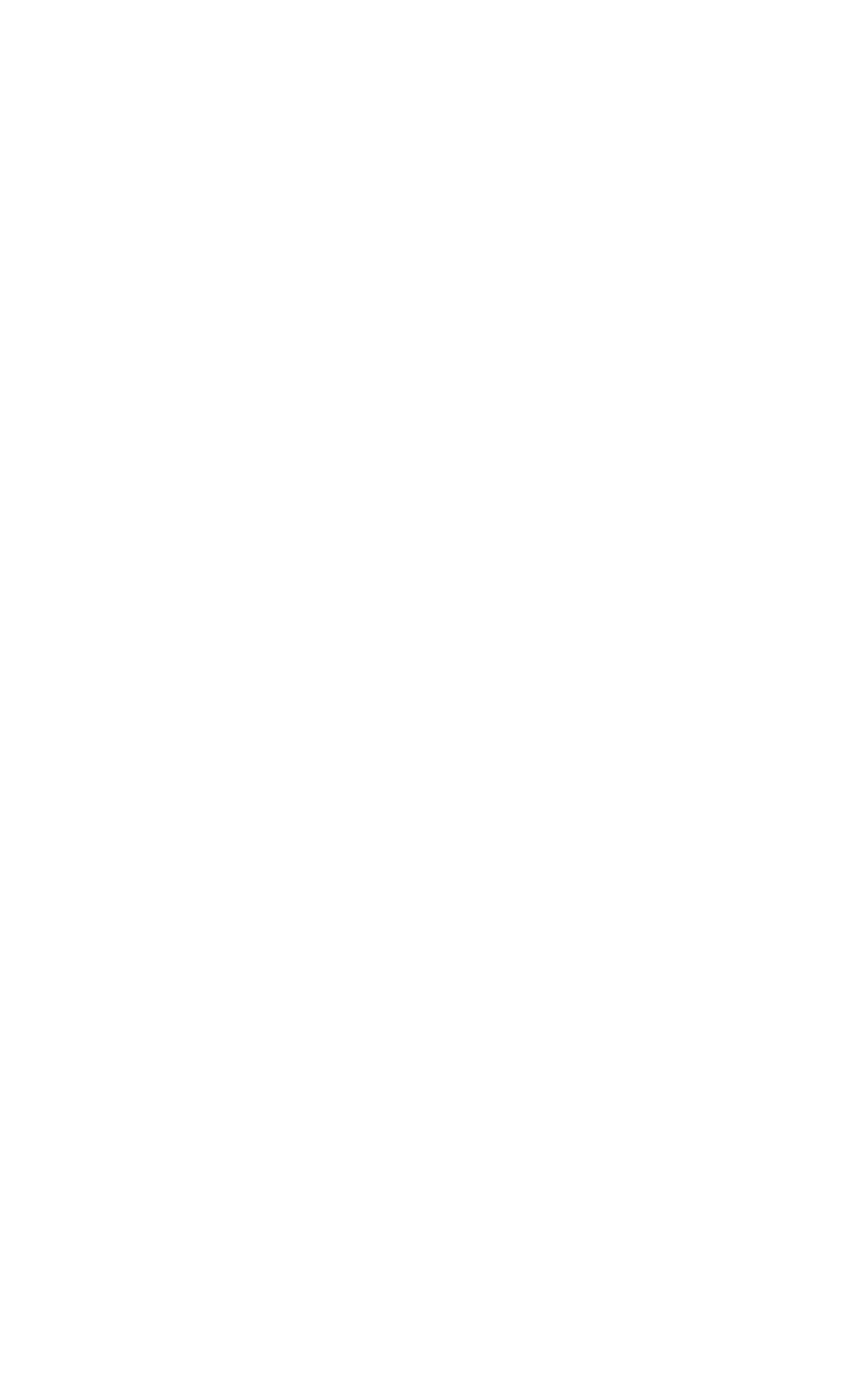




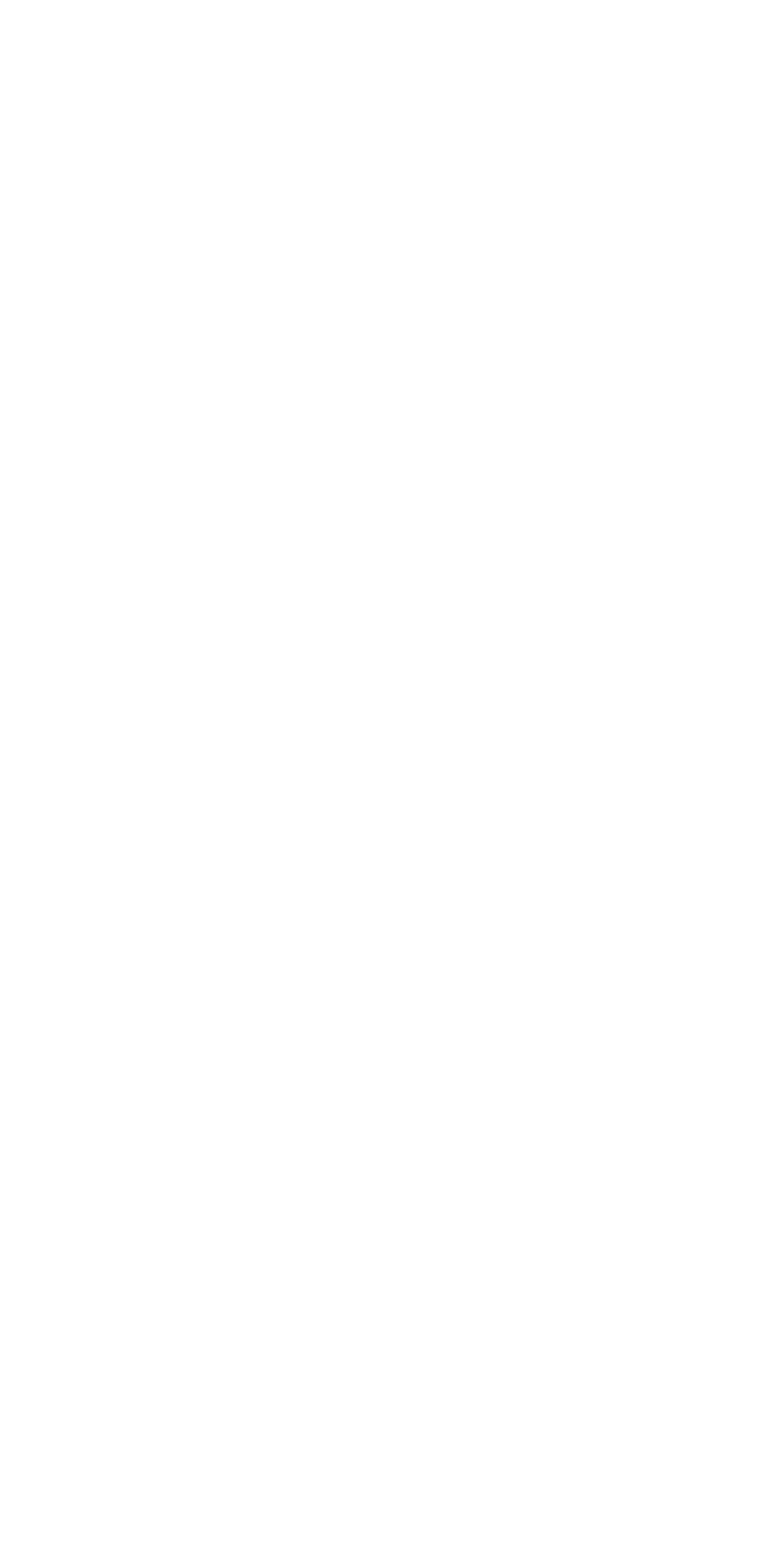




\begin{tabular}{|c|c|c|c|c|c|c|c|c|c|c|}
\hline 옹유육유 & 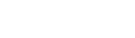 & 윽 떤 & $\underset{1}{n}$ & 오규 & 유용요 & 요 & ᄋ i & लै। & ฟี & 1 \\
\hline けீல் & 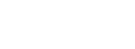 & ๑ீ: & $\ddot{0}$ & 10. & $\begin{array}{l}\text { जo } \\
000\end{array}$ & $\stackrel{0}{0}$ & $\because 1$ & : & $\stackrel{?}{0}$ & i \\
\hline 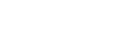 & 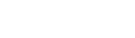 & 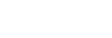 & $\ddot{n}$ & $\mathscr{m}$ & 俞尔品 & $\stackrel{\infty}{*}$ & ชึก & $\stackrel{*}{*}$ & is & I \\
\hline$\because 0$ & ${ }^{4}$ & $\stackrel{-10}{-10}$ & $\varphi$ & $\varphi \%$ & $?$ & $\because$ & $\because 1$ & $\begin{array}{l}\dot{0} \\
\dot{0}\end{array}$ & $\because$ & I \\
\hline NO0000 & $00 \mathrm{HOOO}$ & 00000 & 0 & 00 & 000 & 0 & 01 & - & 0 & i \\
\hline 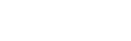 & 윰육윰을 & 썩윰육유 & ㄱ & 00 & $\cos$ & $\sigma$ & $\infty 1$ & क) & 욱 & 1 \\
\hline 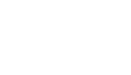 & 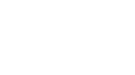 & ஜேッீ" & in & i். & نं & "ं & mi & $\begin{array}{l}0 \\
0\end{array}$ & i & i \\
\hline 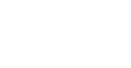 & ㅁํㅇํํㅇํㅇ & 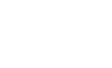 & $\%$ & ठํ? & ํํㅇㅇㅇㅇ & $\stackrel{0}{\circ}$ & 눙 ! & $\begin{array}{l}2 \\
0 \\
0\end{array}$ & i & i \\
\hline ్ㅜ유유 & 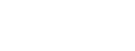 & 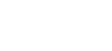 & 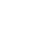 & लू है & సूल & $\vec{m}$ & है ! & लें & $\stackrel{\infty}{m}$ & I \\
\hline ๓?\%? & ๑ฺ ฺฺ & $\because N \because \div \%$ & ? & $\because 0$ & m! & $?$ & ? I & $\because$ & $\ddot{0}$ & ? \\
\hline$\because 0$ & $\because-$ & :0.ㅁ․ & $\because$ & $\because \because$ & $\because \because 0$ & $\because$ & $\because 1$ & $\dot{0}$ & $\ddot{0}$ & $\stackrel{*}{0}$ \\
\hline का & $\begin{array}{l}\varphi \oplus \infty \\
\infty\end{array}$ & 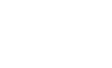 & $\stackrel{\infty}{\infty}$ & म் & חथ & $\stackrel{+}{*}$ & 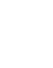 & $\infty$ & $\because$ & $\stackrel{\bullet}{-1}$ \\
\hline प̣: & 우 & 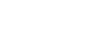 & i & 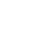 & ज़् & $\stackrel{\circ}{\text { N }}$ & $\underset{-1}{0} 1$ & ì & $\infty$ & $\stackrel{0}{\dot{0}}$ \\
\hline 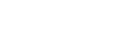 & 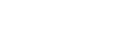 & 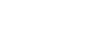 & $\stackrel{\infty}{\rightarrow}$ & $\underset{710}{0}$ & 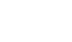 & $\cong$ & $\stackrel{1}{\rightarrow} !$ & 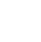 & ت্ & กั \\
\hline & & & in & $\begin{array}{l}0.1 \\
\text { in }\end{array}$ & & $\ddot{\pi}$ & $\stackrel{\varphi}{ }+1$ & in & 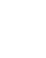 & i \\
\hline min & $\ddot{\circ}$ & $\ddot{\infty}$ & $\because$ & ザ & 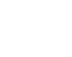 & $\because$ & $\stackrel{+}{*} 1$ & $\stackrel{+}{\circ}$ & $\stackrel{0}{i}$ & 구 \\
\hline & & $\because 9$ กั & $\because$ & $\varphi$ & กัะ & in & $? !$ & $\dot{0}$ & $\because$ & - \\
\hline 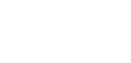 & 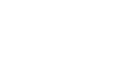 & 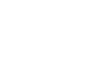 & $\because$ & ஸें & लั่ं & $\stackrel{\infty}{\infty}$ & 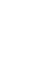 & is & $\infty$ & $\stackrel{m}{-1}$ \\
\hline 융 & 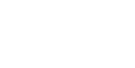 & 옹ํㅇㅇㅇㅇㅇㅛ. & \% & ชิํㅇ․ & ๕ั0. & $\dddot{8}$ & 51 & $\begin{array}{l}9 \\
0 \\
0\end{array}$ & $\stackrel{\circ}{\circ}$ & ஸे \\
\hline 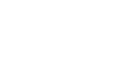 & ำ & 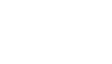 & 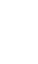 & 폴 & N.m & $m$ & ${ }^{1}$ & 오 & $m$ & จ \\
\hline 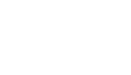 & 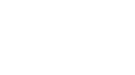 & 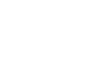 & 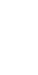 & مீ: & 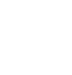 & लू & न พู้ & i & ช్ & 1 \\
\hline 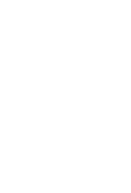 & 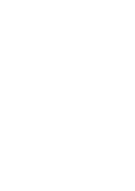 & 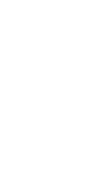 & 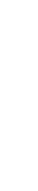 & 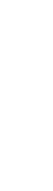 & 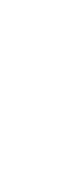 & : & 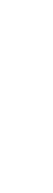 & 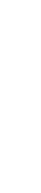 & 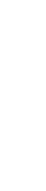 & 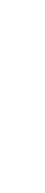 \\
\hline
\end{tabular}




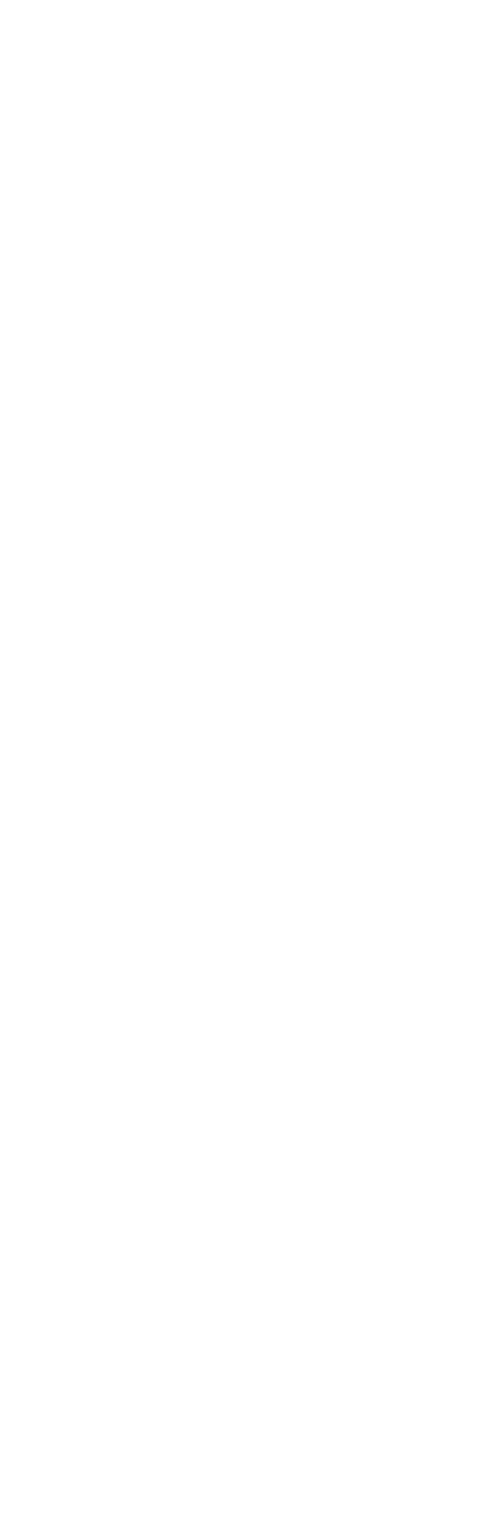




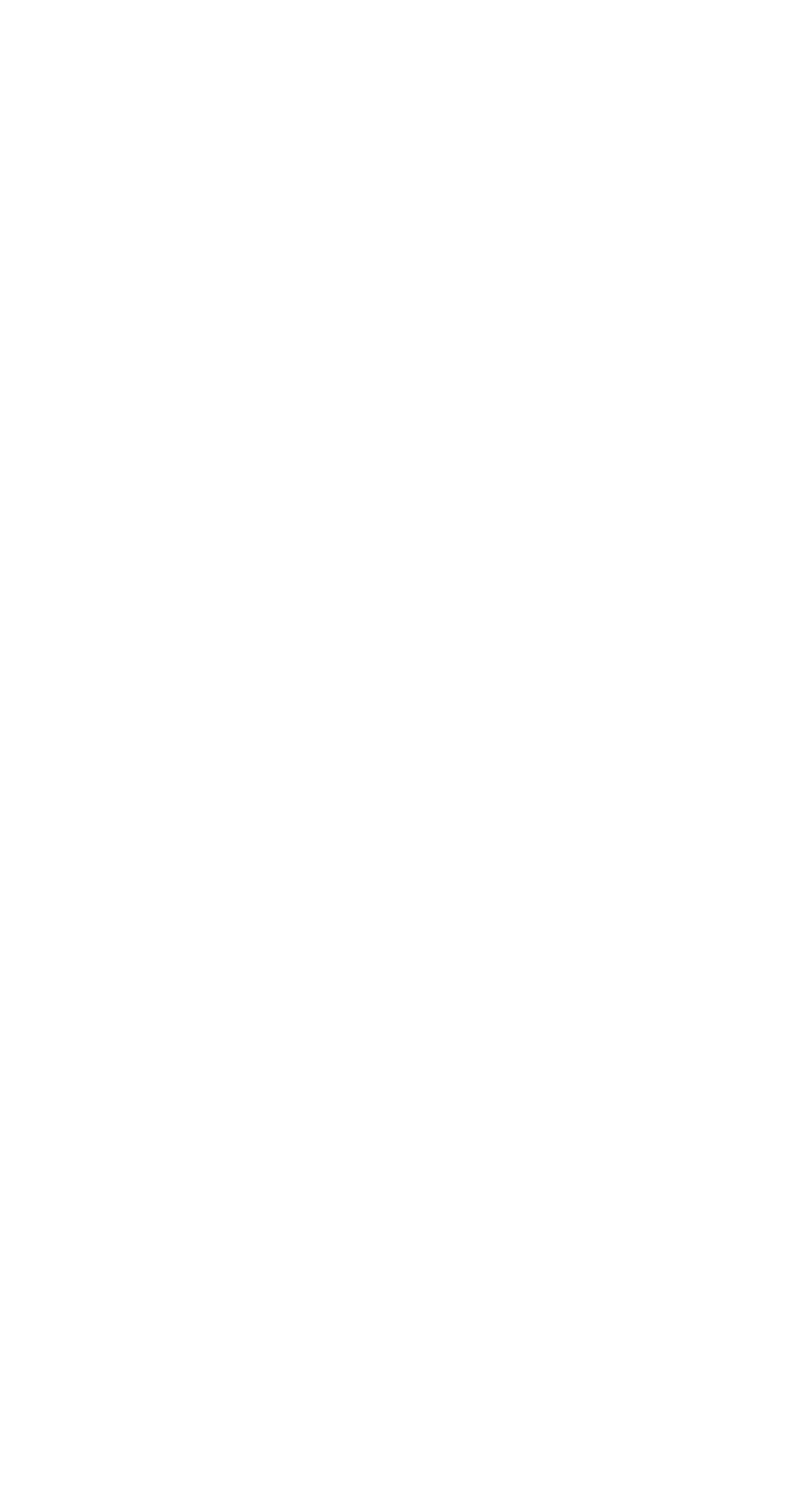



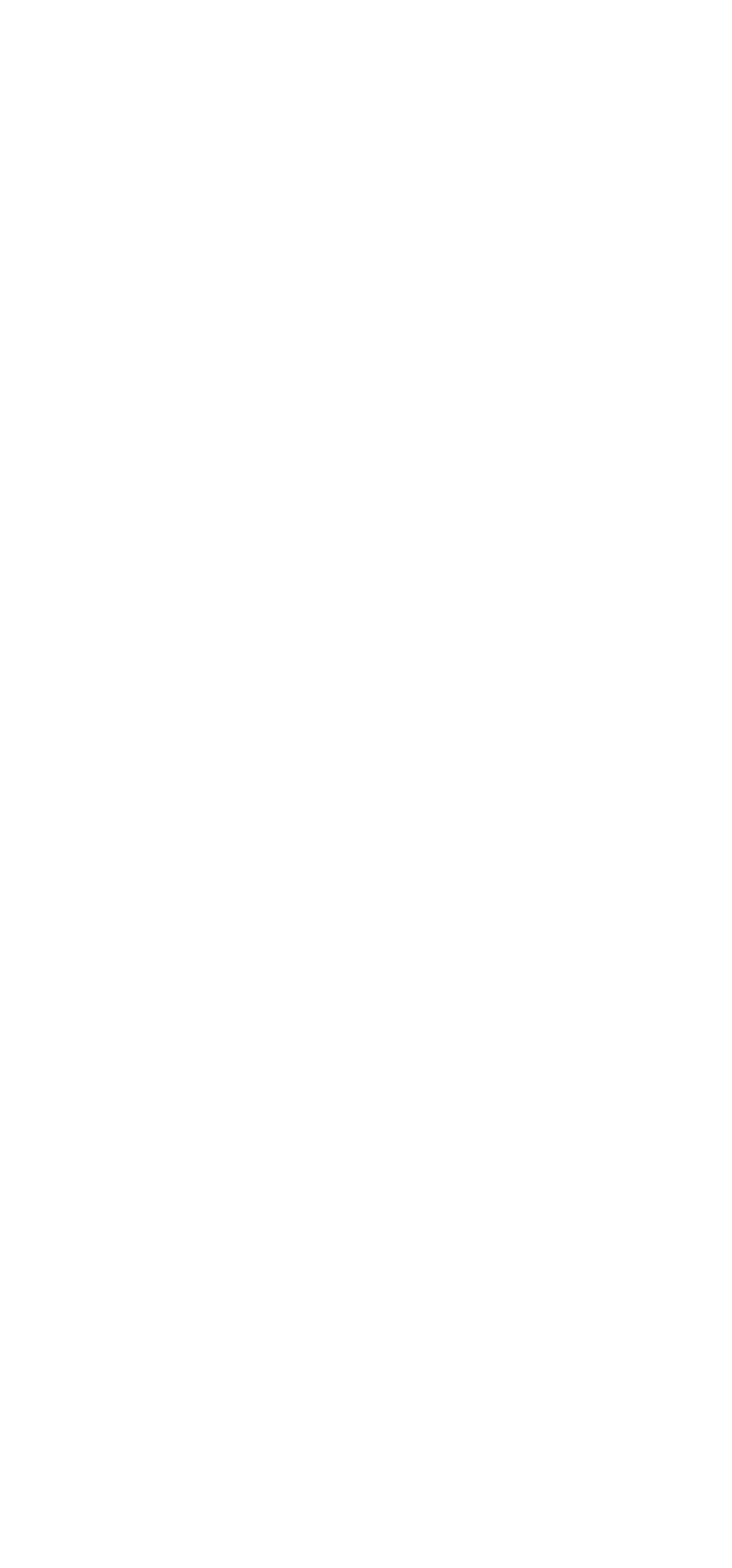


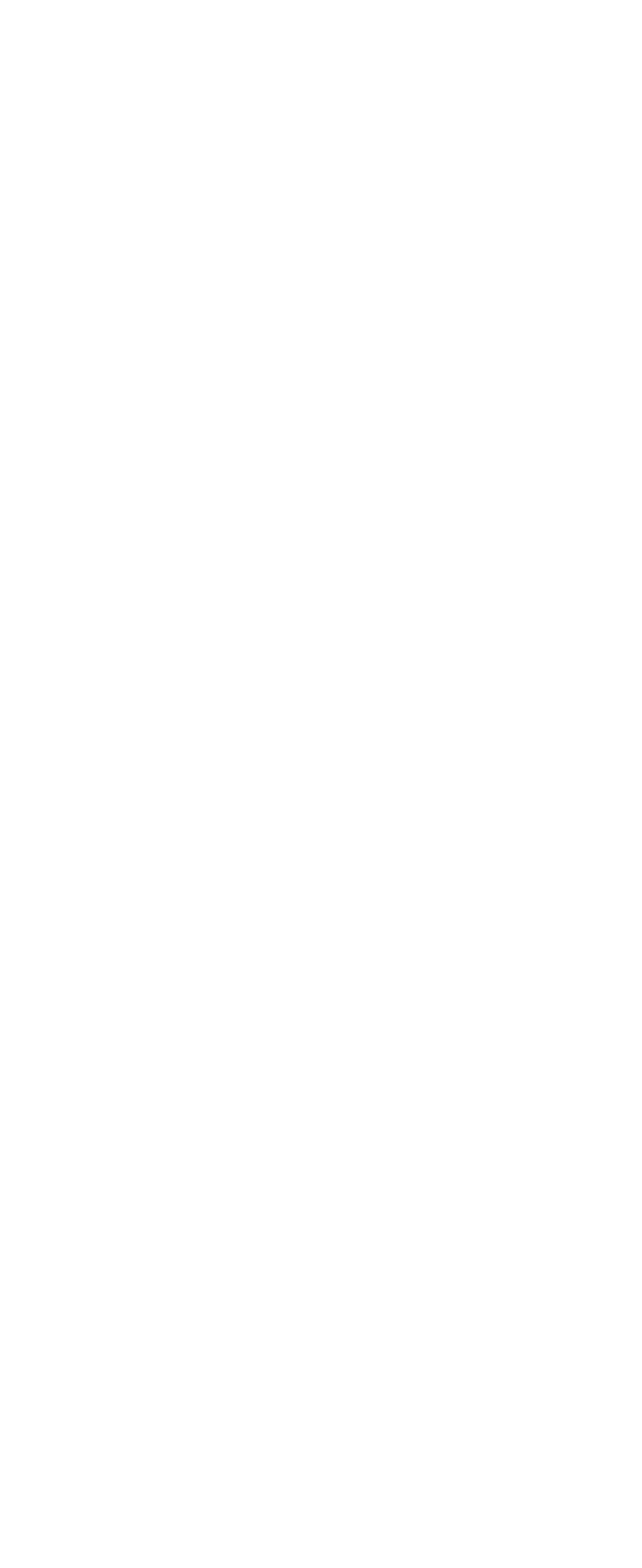




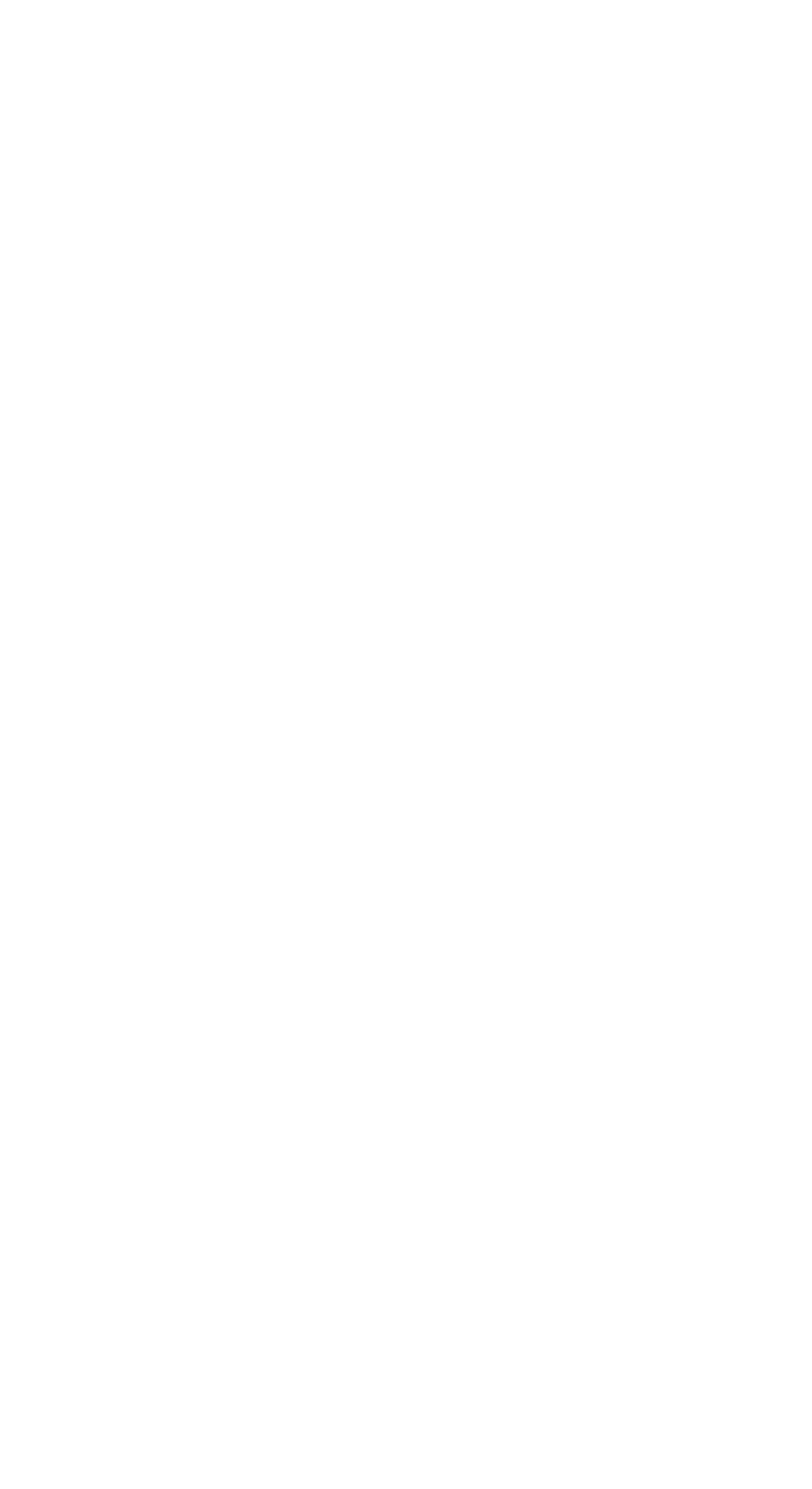




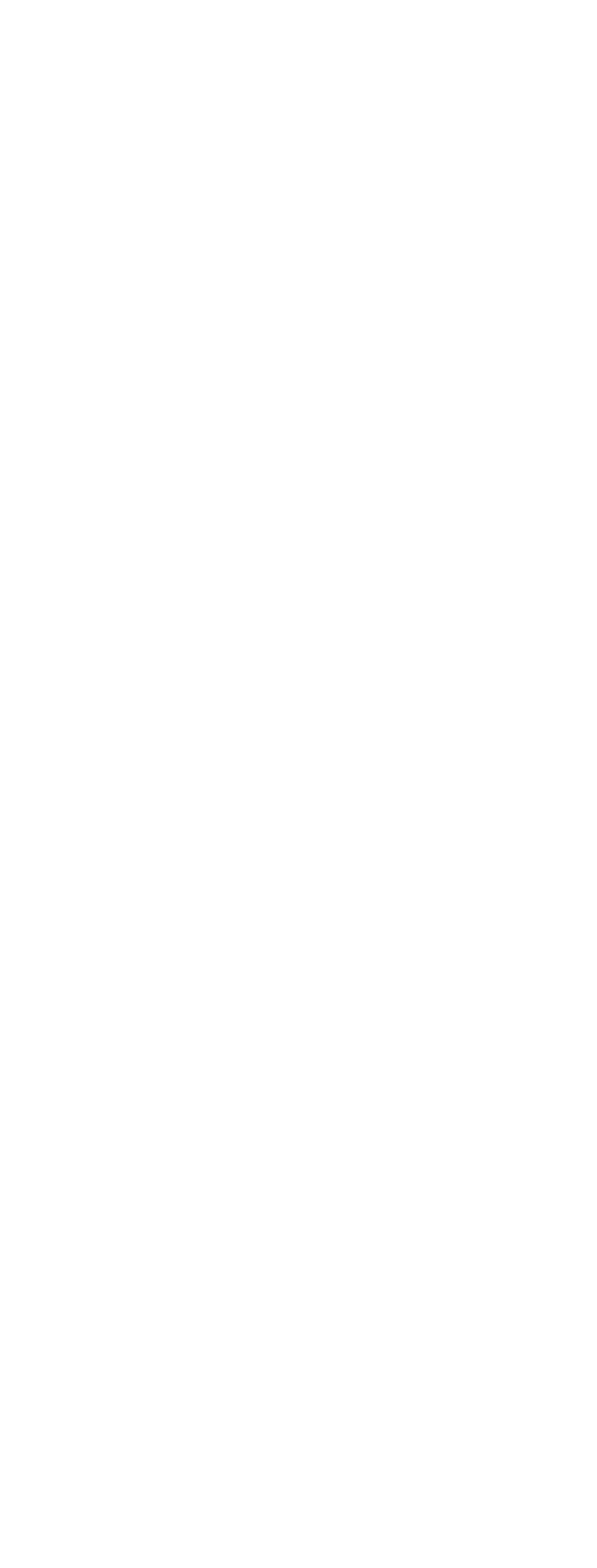



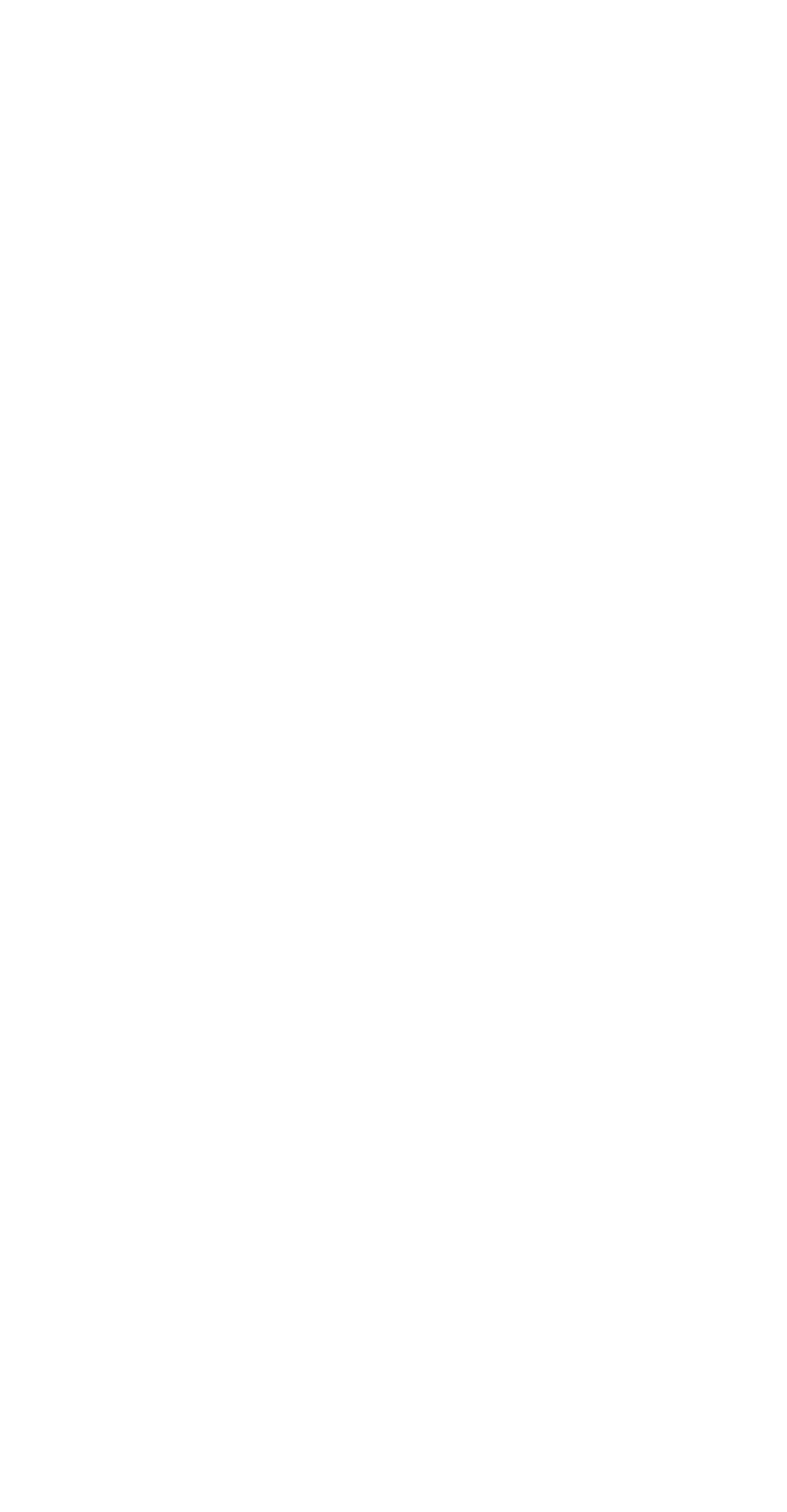


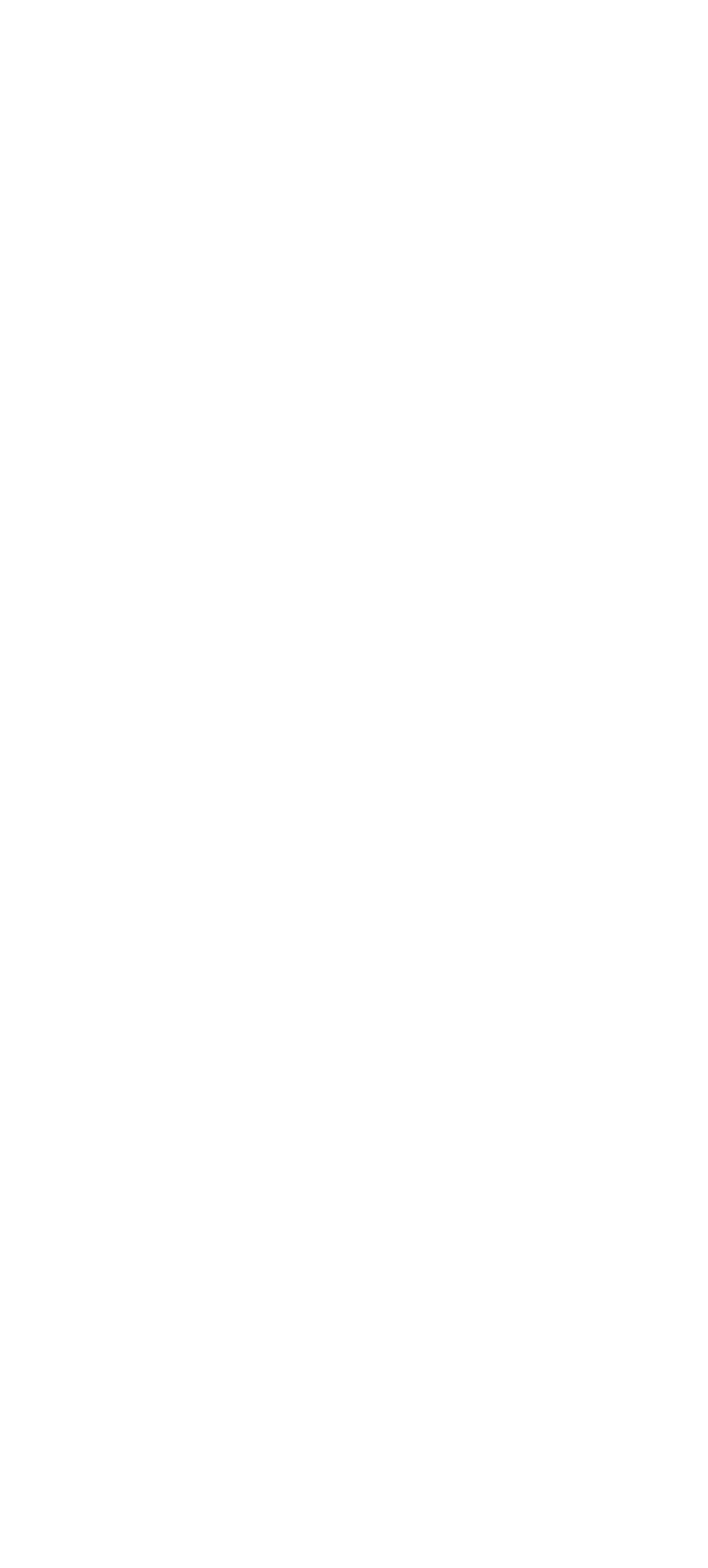




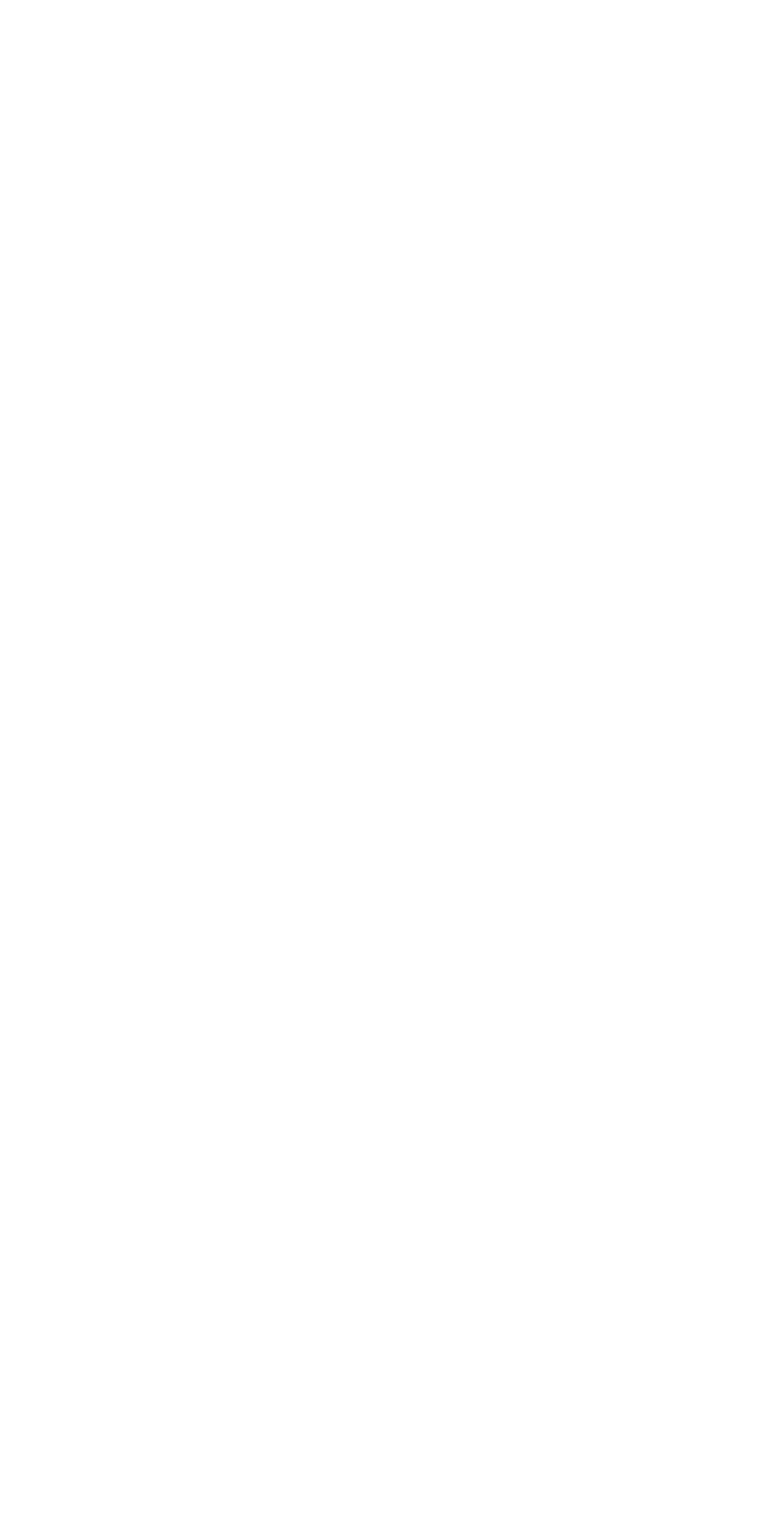




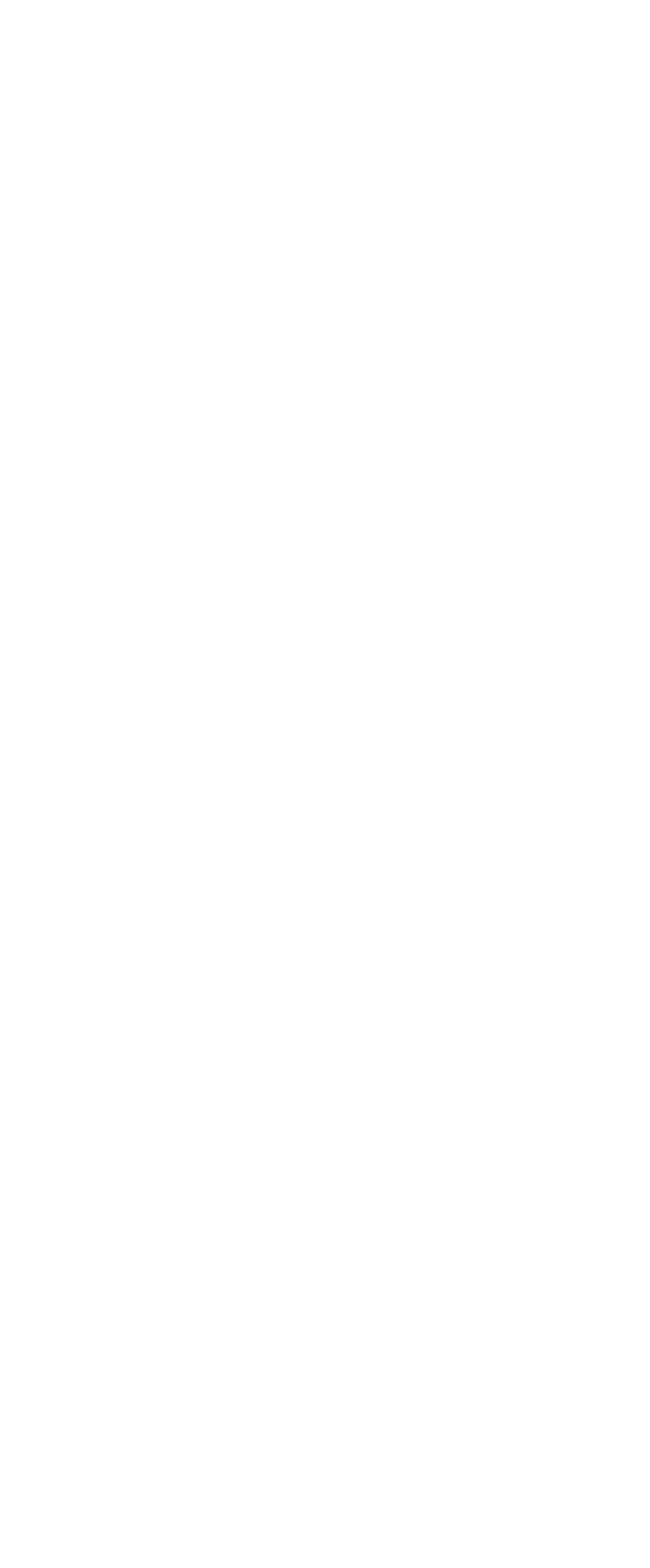




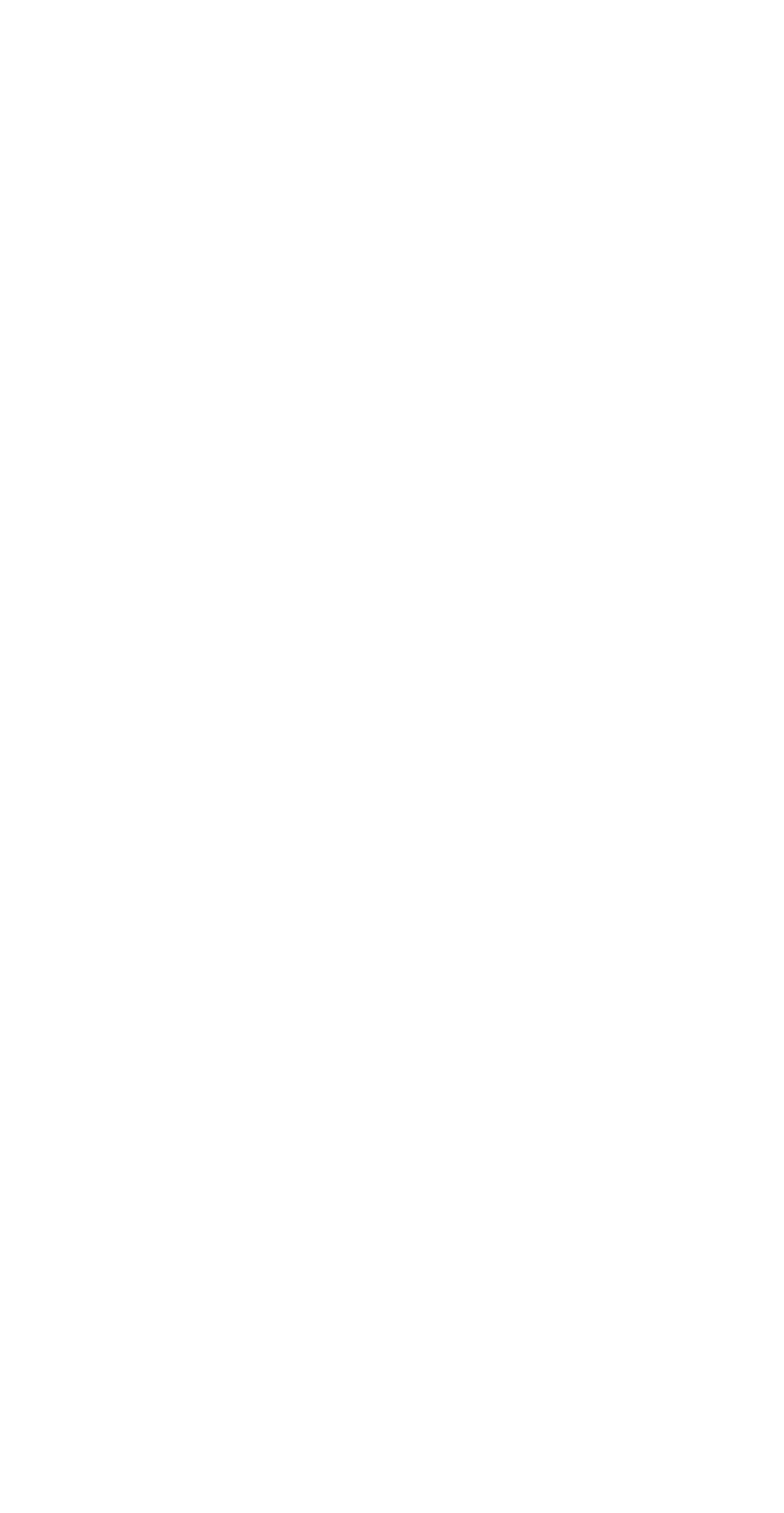




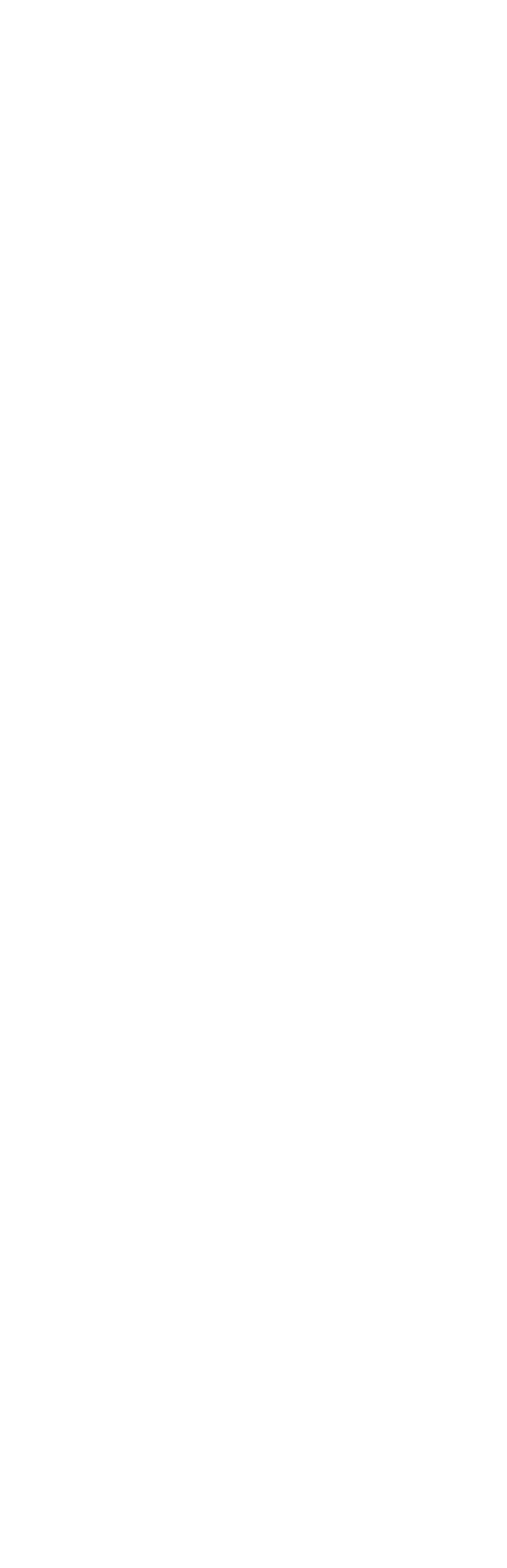




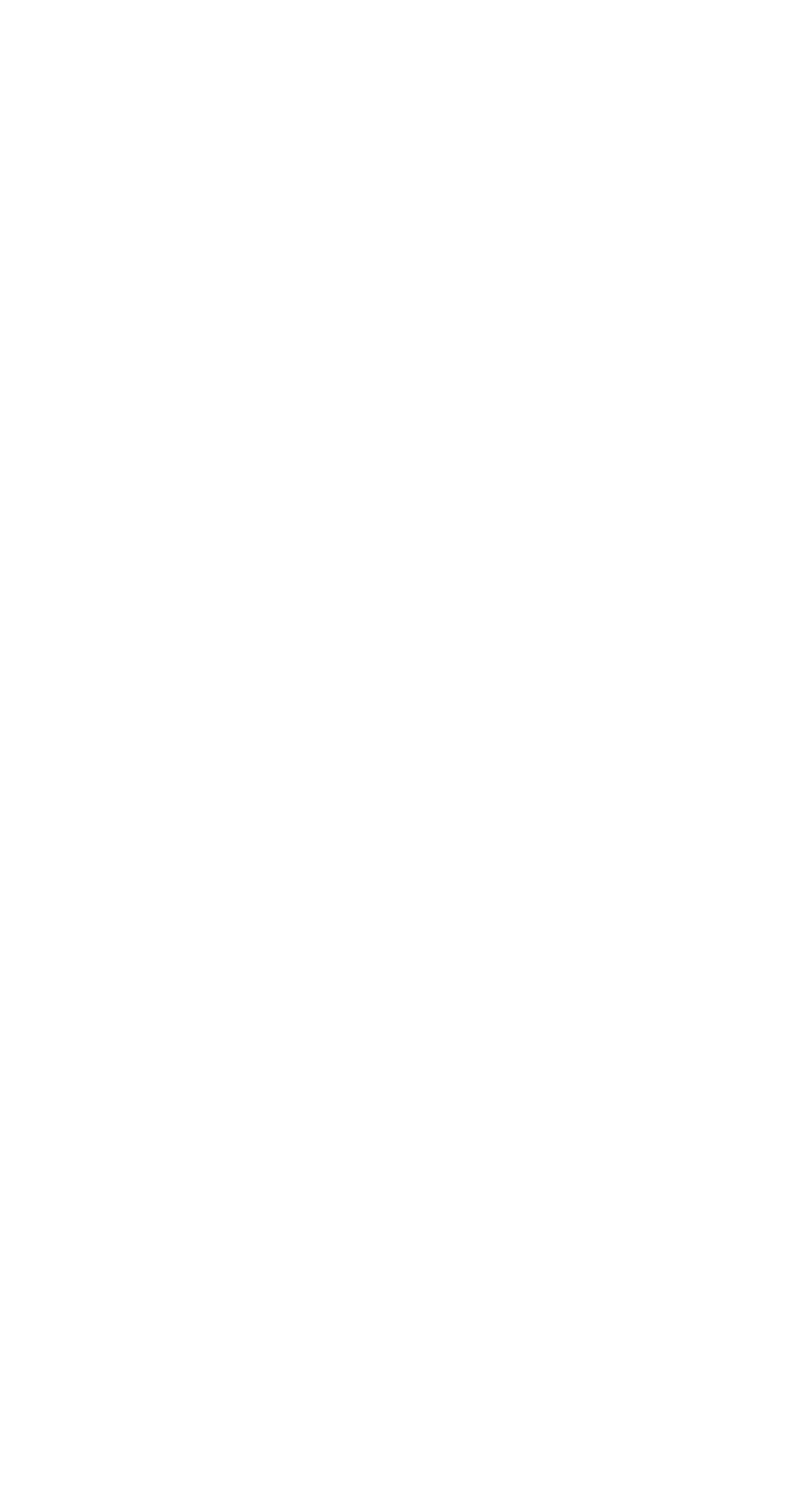




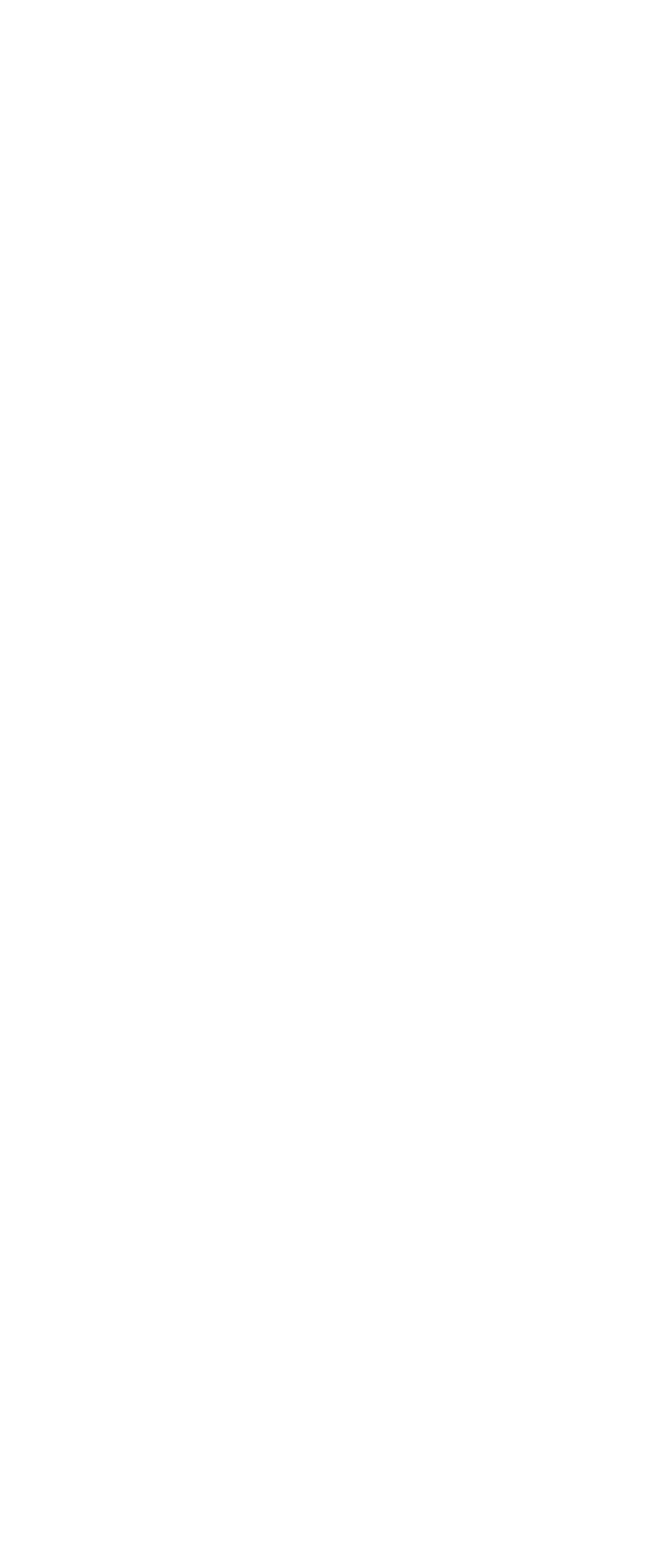




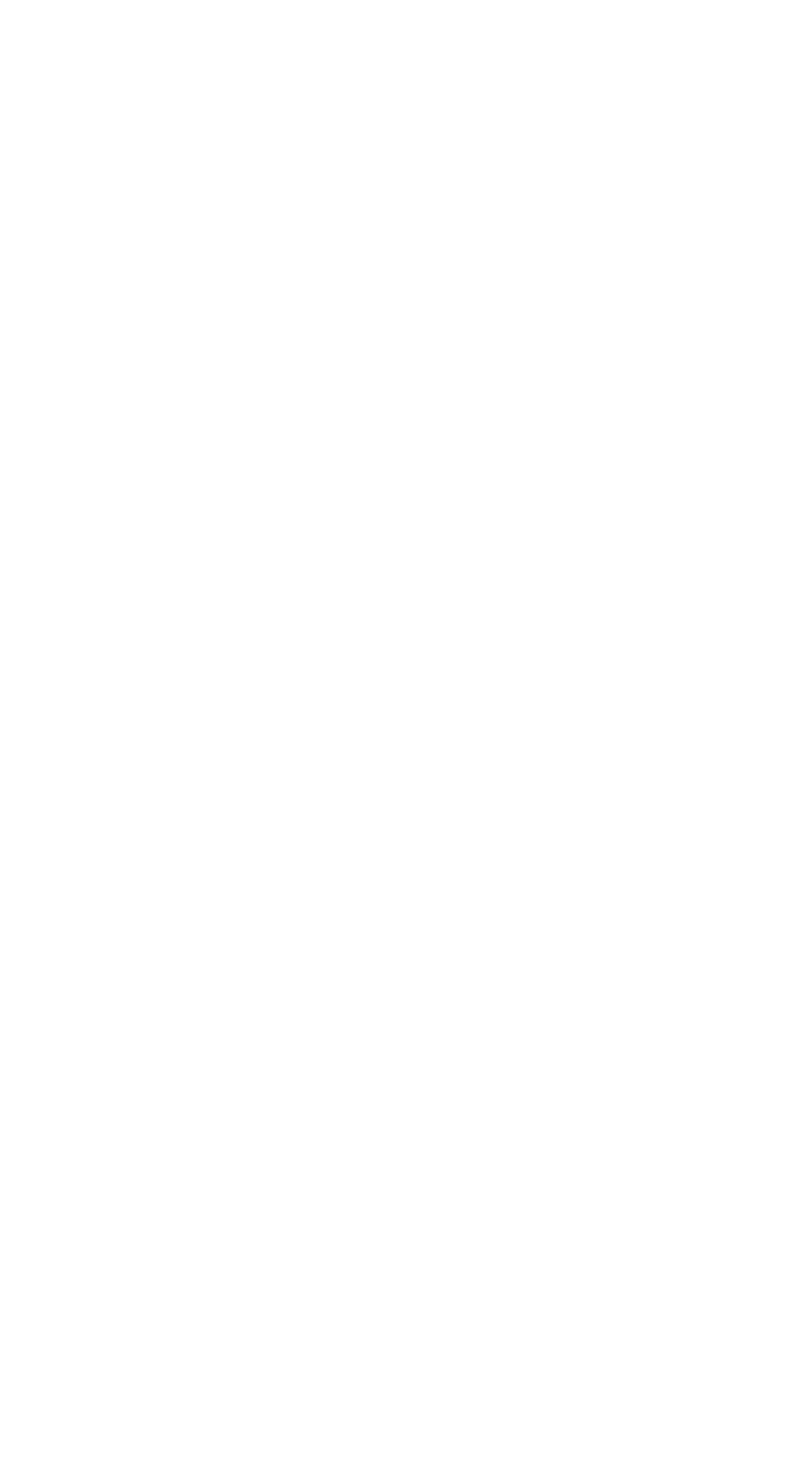




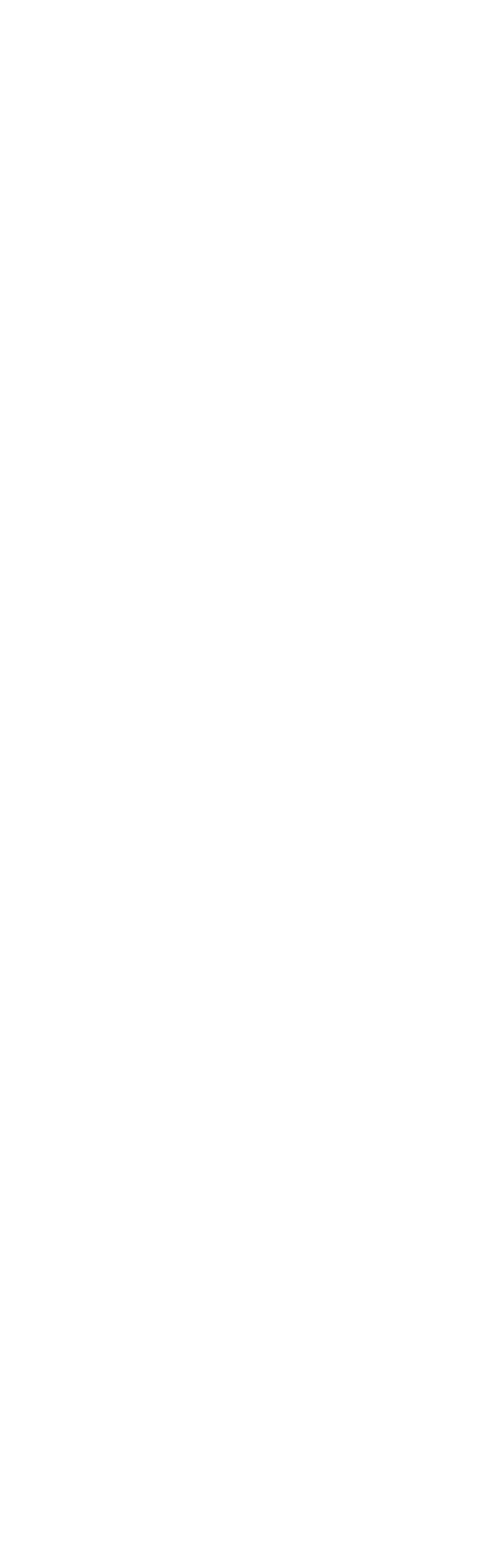




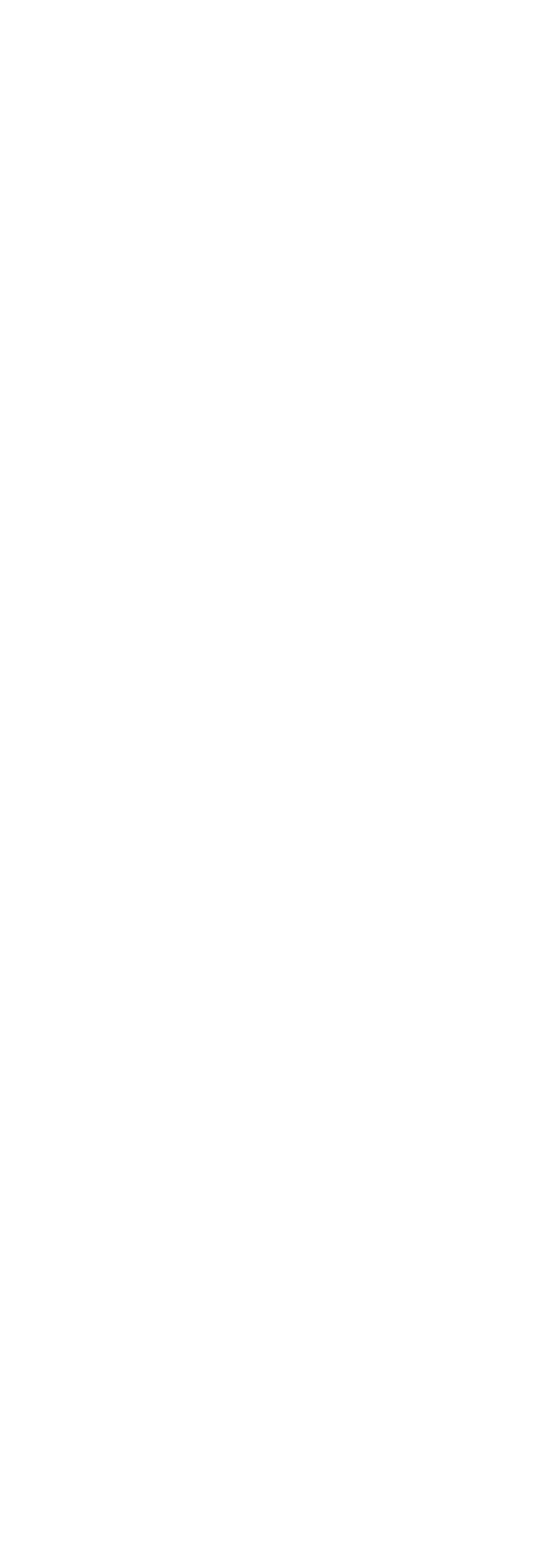


PART 8. WESTERN GULF OF MEXICO BASINS

MERMENTAU RIVER BASIN

8-124. MERMENTAU RIVER AT LAKE ARTHUR, LA.

LOCATION,--At bridge on state Highway 14, 0.5 mile east of Lake Arthur, Jefferson Davis Parish.

RECORDS AVAILABLE,--Chemical analyses: January 1949 to September 1964.

Water temperatures: October 1949 to September 1951, September 1959 to september 1964. EXTREMES, 1963-64.--Specific conductance: Maximum daily, 734 micromhos Dec. 2; minimum daily, 68 micromhos Mar. 14-16.

water temperatures: Maximum, $89^{\circ} \mathrm{F}$ May 22 , July 8 ; minimum, $38^{\circ} \mathrm{F}$ Dec. 23.

EXTREMES, 1949-64.--Specif ic conductance: Maximum daily, 6,330 micromhos June 30, 1952; water temperatures (1959-64): Maximum, $90^{\circ} \mathrm{F}$ July 24, 1962; minimum, $33^{\circ} \mathrm{F}$ Jan, 12,1962

Specific conductance and chloride, in parts per million, water year October 1963 to september 1964

\begin{tabular}{|c|c|c|c|c|c|c|c|c|}
\hline \multirow[b]{2}{*}{ Day } & \multicolumn{2}{|c|}{ OCTOBER } & \multicolumn{2}{|c|}{ NOVEMBER } & \multicolumn{2}{|c|}{ DECEMBER } & \multicolumn{2}{|c|}{ JANUARY } \\
\hline & $\begin{array}{l}\text { Specific } \\
\text { conduct- } \\
\text { ance } \\
\text { (micro- } \\
\text { mhos at } \\
25^{\circ} \mathrm{C} \text { ) }\end{array}$ & $\begin{array}{l}\text { Chlo- } \\
\text { ride } \\
\text { (C1) }\end{array}$ & $\begin{array}{c}\text { Specific } \\
\text { conduct- } \\
\text { ance } \\
\text { (micro- } \\
\text { mhos at } \\
25^{\circ} \mathrm{C} \text { ) }\end{array}$ & $\begin{array}{l}\text { Chlo- } \\
\text { ride } \\
\text { (C1) }\end{array}$ & $\begin{array}{l}\text { Specific } \\
\text { conduct- } \\
\text { ance } \\
\text { (micro- } \\
\text { mhos at } \\
25^{\circ} \mathrm{C} \text { ) }\end{array}$ & $\begin{array}{l}\text { Chlo- } \\
\text { ride } \\
\text { (C1) }\end{array}$ & $\begin{array}{c}\text { Specif ic } \\
\text { conduct - } \\
\text { ance } \\
\text { (micro- } \\
\text { mhos at } \\
25^{\circ} \mathrm{C} \text { ) }\end{array}$ & $\begin{array}{l}\text { Chlo- } \\
\text { ride } \\
\text { (Cl) }\end{array}$ \\
\hline $\begin{array}{l}\mathbf{1} \\
2 \\
3 \\
\mathbf{4} \\
\mathbf{5}\end{array}$ & $\begin{array}{l}138 \\
139 \\
139 \\
143 \\
143\end{array}$ & $\begin{array}{l}16 \\
-- \\
-- \\
-- \\
--\end{array}$ & $\begin{array}{l}154 \\
153 \\
153 \\
154 \\
153\end{array}$ & $\begin{array}{l}-- \\
=- \\
-- \\
--\end{array}$ & $\begin{array}{l}610 \\
734 \\
613 \\
571 \\
472\end{array}$ & $\begin{array}{r}\overline{193} \\
-- \\
\overline{121}\end{array}$ & $\begin{array}{l}184 \\
178 \\
177 \\
180 \\
179\end{array}$ & $\begin{array}{l}-- \\
-- \\
--\end{array}$ \\
\hline $\begin{array}{r}6 \\
7 \\
8 \\
9 \\
10\end{array}$ & $\begin{array}{l}141 \\
145 \\
141 \\
143 \\
166\end{array}$ & $\begin{array}{l}-- \\
-- \\
-- \\
-- \\
--\end{array}$ & $\begin{array}{l}155 \\
153 \\
157 \\
152 \\
153\end{array}$ & $\begin{array}{l}-- \\
20 \\
-- \\
--\end{array}$ & $\begin{array}{l}336 \\
239 \\
239 \\
189 \\
190\end{array}$ & $\begin{array}{l}-- \\
-- \\
-- \\
--\end{array}$ & $\begin{array}{l}135 \\
131 \\
277 \\
228 \\
170\end{array}$ & $\begin{array}{l}-- \\
66 \\
49 \\
--\end{array}$ \\
\hline $\begin{array}{l}11 \\
12 \\
13 \\
14 \\
15\end{array}$ & $\begin{array}{l}147 \\
146 \\
148 \\
152 \\
147\end{array}$ & $\begin{array}{l}17 \\
-- \\
18 \\
--\end{array}$ & $\begin{array}{l}160 \\
165 \\
166 \\
174 \\
189\end{array}$ & $\begin{array}{l}21 \\
-- \\
-\overline{26} \\
30\end{array}$ & $\begin{array}{l}249 \\
249 \\
245 \\
246 \\
247\end{array}$ & $\begin{array}{l}-- \\
=- \\
-- \\
--\end{array}$ & $\begin{array}{l}155 \\
156 \\
154 \\
124 \\
126\end{array}$ & $\begin{array}{l}-- \\
30 \\
-- \\
--\end{array}$ \\
\hline $\begin{array}{l}16 \\
17 \\
18 \\
19 \\
20\end{array}$ & $\begin{array}{l}145 \\
146 \\
146 \\
147 \\
159\end{array}$ & $\begin{array}{l}-- \\
-- \\
-- \\
--\end{array}$ & $\begin{array}{l}208 \\
198 \\
205 \\
209 \\
194\end{array}$ & $\begin{array}{l}=- \\
=- \\
=-\end{array}$ & $\begin{array}{l}303 \\
197 \\
187 \\
161 \\
183\end{array}$ & $\begin{array}{l}73 \\
43 \\
-- \\
-- \\
--\end{array}$ & $\begin{array}{l}102 \\
109 \\
121 \\
136 \\
126\end{array}$ & $\begin{array}{l}-- \\
21 \\
--\end{array}$ \\
\hline $\begin{array}{l}21 \\
22 \\
23 \\
24 \\
25\end{array}$ & $\begin{array}{l}151 \\
150 \\
149 \\
148 \\
148\end{array}$ & $\begin{array}{l}-- \\
-- \\
-- \\
--\end{array}$ & $\begin{array}{l}214 \\
188 \\
205 \\
203 \\
244\end{array}$ & $\begin{array}{l}=- \\
=- \\
=- \\
=-\end{array}$ & $\begin{array}{l}173 \\
173 \\
163 \\
165 \\
164\end{array}$ & $\begin{array}{l}-\overline{37} \\
-- \\
--\end{array}$ & $\begin{array}{r}124 \\
118 \\
98 \\
100 \\
111\end{array}$ & $\begin{array}{l}24 \\
-- \\
-- \\
--\end{array}$ \\
\hline $\begin{array}{l}26 \\
27 \\
28 \\
29 \\
30 \\
31\end{array}$ & $\begin{array}{l}149 \\
148 \\
152 \\
153 \\
152 \\
153\end{array}$ & $\begin{array}{l}-- \\
18 \\
-- \\
-- \\
--\end{array}$ & $\begin{array}{c}272 \\
301 \\
472 \\
493 \\
600 \\
--\end{array}$ & $\begin{array}{c}54 \\
-- \\
-- \\
155 \\
--\end{array}$ & $\begin{array}{l}154 \\
153 \\
189 \\
190 \\
187 \\
187\end{array}$ & $\begin{array}{l}-- \\
-- \\
-- \\
40 \\
--\end{array}$ & $\begin{array}{r}103 \\
91 \\
91 \\
93 \\
93 \\
81\end{array}$ & $\begin{array}{l}-- \\
-- \\
-- \\
-- \\
--\end{array}$ \\
\hline
\end{tabular}


MERMENTAU RIVER BASIN--COntinued

8-124. MERMENTAU RIVER AT LAKE ARTHUR, LA.--Continued

Specific conductance and chloride, in parts per million,

water year October 1963 to September 1964--Continued

\begin{tabular}{|c|c|c|c|c|c|c|c|c|}
\hline \multirow[b]{2}{*}{ Day } & \multicolumn{2}{|c|}{ FEBRUARY } & \multicolumn{2}{|c|}{ MARCH } & \multicolumn{2}{|c|}{ APRI L } & \multicolumn{2}{|c|}{ MAY } \\
\hline & $\begin{array}{l}\text { Specific } \\
\text { conduct- } \\
\text { ance } \\
\text { (micro- } \\
\text { mhos at } \\
25^{\circ} \mathrm{C} \text { ) }\end{array}$ & $\begin{array}{l}\text { Chlo- } \\
\text { ride } \\
\text { (C1) }\end{array}$ & $\begin{array}{l}\text { Specific } \\
\text { conduct - } \\
\text { ance } \\
\text { (micro- } \\
\text { mhos at } \\
25^{\circ} \mathrm{C} \text { ) }\end{array}$ & $\begin{array}{l}\text { Chlo- } \\
\text { ride } \\
\text { (Cl) }\end{array}$ & $\begin{array}{l}\text { Specif ic } \\
\text { conduct - } \\
\text { ance } \\
\text { (micro- } \\
\text { mhos at } \\
25^{\circ} \mathrm{C} \text { ) }\end{array}$ & $\begin{array}{l}\text { Chlo- } \\
\text { ride } \\
\text { (CI) }\end{array}$ & $\begin{array}{l}\text { Specific } \\
\text { conduct- } \\
\text { ance } \\
\text { (micro- } \\
\text { mhos at } \\
25^{\circ} \mathrm{C} \text { ) }\end{array}$ & $\begin{array}{l}\text { Chlo- } \\
\text { ride } \\
\text { (Cl) }\end{array}$ \\
\hline $\begin{array}{l}1 \\
2 \\
3 \\
4 \\
5\end{array}$ & $\begin{array}{l}108 \\
105 \\
103 \\
102 \\
100\end{array}$ & $\begin{array}{l}-- \\
-- \\
-- \\
--\end{array}$ & $\begin{array}{l}223 \\
137 \\
110 \\
149 \\
120\end{array}$ & $\begin{array}{l}51 \\
-- \\
-- \\
-- \\
--\end{array}$ & $\begin{array}{l}77 \\
80 \\
93 \\
88 \\
84\end{array}$ & $\begin{array}{l}-- \\
-- \\
--\end{array}$ & $\begin{array}{l}120 \\
114 \\
114 \\
172 \\
171\end{array}$ & $=$ \\
\hline $\begin{array}{r}6 \\
7 \\
8 \\
9 \\
10\end{array}$ & $\begin{array}{r}100 \\
126 \\
106 \\
\overline{104}\end{array}$ & $\begin{array}{l}-- \\
23 \\
-- \\
--\end{array}$ & $\begin{array}{r}115 \\
95 \\
86 \\
80 \\
77\end{array}$ & $\begin{array}{l}=- \\
=- \\
=- \\
=-\end{array}$ & \begin{tabular}{|r|}
87 \\
81 \\
109 \\
111 \\
86
\end{tabular} & $16^{--}$ & $\begin{array}{l}165 \\
164 \\
135 \\
134 \\
135\end{array}$ & $\begin{array}{l}- \\
- \\
-\end{array}$ \\
\hline $\begin{array}{l}11 \\
12 \\
13 \\
14 \\
15\end{array}$ & $\begin{array}{l}105 \\
107 \\
108 \\
109 \\
109\end{array}$ & $\begin{array}{l}-- \\
-- \\
-- \\
--\end{array}$ & $\begin{array}{l}76 \\
81 \\
77 \\
68 \\
68\end{array}$ & $\begin{array}{l}-- \\
-- \\
-- \\
--\end{array}$ & $\begin{array}{r}88 \\
87 \\
104 \\
97 \\
89\end{array}$ & $15^{--}$ & $\begin{array}{r}105 \\
10 € \\
102 \\
9 \varepsilon \\
103\end{array}$ & $\begin{array}{l}- \\
- \\
\overline{1} \\
-\end{array}$ \\
\hline $\begin{array}{l}16 \\
17 \\
18 \\
19 \\
20\end{array}$ & $\begin{array}{l}107 \\
106 \\
113 \\
111 \\
199\end{array}$ & $\begin{array}{l}-- \\
-- \\
20 \\
20 \\
--\end{array}$ & $\begin{array}{r}68 \\
101 \\
103 \\
100 \\
102\end{array}$ & $\begin{array}{l}-- \\
18 \\
--\end{array}$ & $\begin{array}{l}90 \\
93 \\
92 \\
94 \\
94\end{array}$ & $\begin{array}{l}-- \\
=- \\
=- \\
--\end{array}$ & $\begin{array}{l}10 \Xi \\
104 \\
10 € \\
10 € \\
10 €\end{array}$ & $\begin{array}{l}- \\
- \\
- \\
-\end{array}$ \\
\hline $\begin{array}{l}21 \\
22 \\
23 \\
24 \\
25\end{array}$ & $\begin{array}{l}200 \\
327 \\
329 \\
331 \\
331\end{array}$ & $\begin{array}{l}-- \\
-- \\
-- \\
--\end{array}$ & $\begin{array}{l}153 \\
154 \\
139 \\
136 \\
122\end{array}$ & $\begin{array}{l}30 \\
31 \\
-- \\
-- \\
--\end{array}$ & $\begin{array}{r}101 \\
98 \\
119 \\
119 \\
94\end{array}$ & $19^{15}$ & $\begin{array}{l}10 E \\
111 \\
112 \\
111 \\
121\end{array}$ & $\begin{array}{l}- \\
\overline{1} \\
\mathbf{1}\end{array}$ \\
\hline \multirow[t]{2}{*}{$\begin{array}{l}26 \\
27 \\
28 \\
29 \\
30 \\
31\end{array}$} & $\begin{array}{r}311 \\
312 \\
288 \\
226 \\
-- \\
--\end{array}$ & $\begin{array}{l}-- \\
-- \\
-- \\
-- \\
--\end{array}$ & \begin{tabular}{r|}
121 \\
84 \\
88 \\
88 \\
84 \\
81
\end{tabular} & $\begin{array}{l}-- \\
-- \\
-- \\
-- \\
--\end{array}$ & $\begin{array}{r}97 \\
69 \\
88 \\
93 \\
122 \\
--\end{array}$ & $\begin{array}{r}-\overline{1} \\
-- \\
-- \\
-- \\
--\end{array}$ & $\begin{array}{l}12 \overline{1} \\
139 \\
127 \\
129 \\
134\end{array}$ & $\begin{array}{l}- \\
- \\
- \\
-\end{array}$ \\
\hline & \multicolumn{2}{|c|}{ JUNE } & \multicolumn{2}{|c|}{ JULY } & \multicolumn{2}{|c|}{ AUGUST } & \multicolumn{2}{|c|}{ SEPTEMBER } \\
\hline $\begin{array}{l}1 \\
2 \\
3 \\
4 \\
5\end{array}$ & $\begin{array}{l}138 \\
141 \\
142 \\
145 \\
141\end{array}$ & $\begin{array}{l}-- \\
=- \\
=- \\
--\end{array}$ & $\begin{array}{l}275 \\
275 \\
197 \\
215 \\
227\end{array}$ & $\begin{array}{l}-- \\
57 \\
-- \\
-- \\
--\end{array}$ & $\begin{array}{l}292 \\
293 \\
243 \\
250 \\
251\end{array}$ & $46^{--}$ & $\begin{array}{l}180 \\
181 \\
180 \\
180 \\
182\end{array}$ & $\begin{array}{l}2 \\
- \\
-\end{array}$ \\
\hline $\begin{array}{r}6 \\
7 \\
8 \\
9 \\
10\end{array}$ & $\begin{array}{l}142 \\
142 \\
149 \\
140 \\
143\end{array}$ & $\begin{array}{l}-- \\
\overline{20} \\
-- \\
--\end{array}$ & $\begin{array}{l}238 \\
196 \\
206 \\
223 \\
197\end{array}$ & $\begin{array}{l}-- \\
-- \\
=- \\
--\end{array}$ & $\begin{array}{l}241 \\
241 \\
240 \\
240 \\
241\end{array}$ & $\begin{array}{l}-- \\
=- \\
=- \\
--\end{array}$ & $\begin{array}{l}181 \\
184 \\
185 \\
186 \\
193\end{array}$ & $\begin{array}{l}\overline{-} \\
\overline{2}\end{array}$ \\
\hline $\begin{array}{l}11 \\
12 \\
13 \\
14 \\
15\end{array}$ & $\begin{array}{l}156 \\
180 \\
158 \\
157 \\
169\end{array}$ & $\begin{array}{l}-- \\
-- \\
-- \\
--\end{array}$ & $\begin{array}{l}196 \\
197 \\
206 \\
231 \\
229\end{array}$ & $\begin{array}{l}29 \\
-- \\
-- \\
-- \\
--\end{array}$ & $\begin{array}{l}239 \\
238 \\
239 \\
248 \\
248\end{array}$ & $\begin{array}{l}=- \\
=- \\
--\end{array}$ & $\begin{array}{l}185 \\
197 \\
190 \\
193 \\
192\end{array}$ & $\begin{array}{l}- \\
- \\
-\end{array}$ \\
\hline $\begin{array}{l}16 \\
17 \\
18 \\
19 \\
20\end{array}$ & $\begin{array}{l}198 \\
194 \\
201 \\
211 \\
228\end{array}$ & $\begin{array}{l}-- \\
-- \\
-- \\
45\end{array}$ & $\begin{array}{l}226 \\
219 \\
224 \\
220 \\
236\end{array}$ & $\begin{array}{l}=- \\
=- \\
\overline{35}\end{array}$ & $\begin{array}{l}250 \\
248 \\
260 \\
258 \\
279\end{array}$ & $39^{--}$ & $\begin{array}{l}190 \\
190 \\
19 . \\
205 \\
205\end{array}$ & $\begin{array}{l}- \\
\\
\end{array}$ \\
\hline $\begin{array}{l}21 \\
22 \\
23 \\
24 \\
25\end{array}$ & $\begin{array}{l}232 \\
251 \\
275 \\
284 \\
299\end{array}$ & $\begin{array}{l}-- \\
\overline{--} \\
\overline{66}\end{array}$ & $\begin{array}{l}227 \\
227 \\
223 \\
225 \\
217\end{array}$ & $\begin{array}{l}-- \\
\overline{--} \\
34 \\
--\end{array}$ & $\begin{array}{l}279 \\
214 \\
189 \\
172 \\
173\end{array}$ & $\begin{array}{l}-- \\
-- \\
--\end{array}$ & $\begin{array}{l}262 \\
390 \\
393 \\
187 \\
18 .\end{array}$ & $\begin{array}{l}5 \\
8 \\
-\end{array}$ \\
\hline $\begin{array}{l}26 \\
27 \\
28 \\
29 \\
\mathbf{3 0} \\
\mathbf{3 1}\end{array}$ & $\begin{array}{r}322 \\
338 \\
339 \\
355 \\
383 \\
--\end{array}$ & $\begin{array}{l}71 \\
-- \\
-- \\
\overline{86} \\
--\end{array}$ & $\begin{array}{l}215 \\
238 \\
236 \\
263 \\
262 \\
299\end{array}$ & $\begin{array}{l}-- \\
\overline{37} \\
-- \\
-\overline{48}\end{array}$ & $\begin{array}{l}162 \\
161 \\
163 \\
177 \\
175 \\
182\end{array}$ & $24^{--}$ & $\begin{array}{r}160 \\
160 \\
172 \\
170 \\
141 \\
-\infty\end{array}$ & $\begin{array}{l}- \\
- \\
2 \\
-\end{array}$ \\
\hline
\end{tabular}




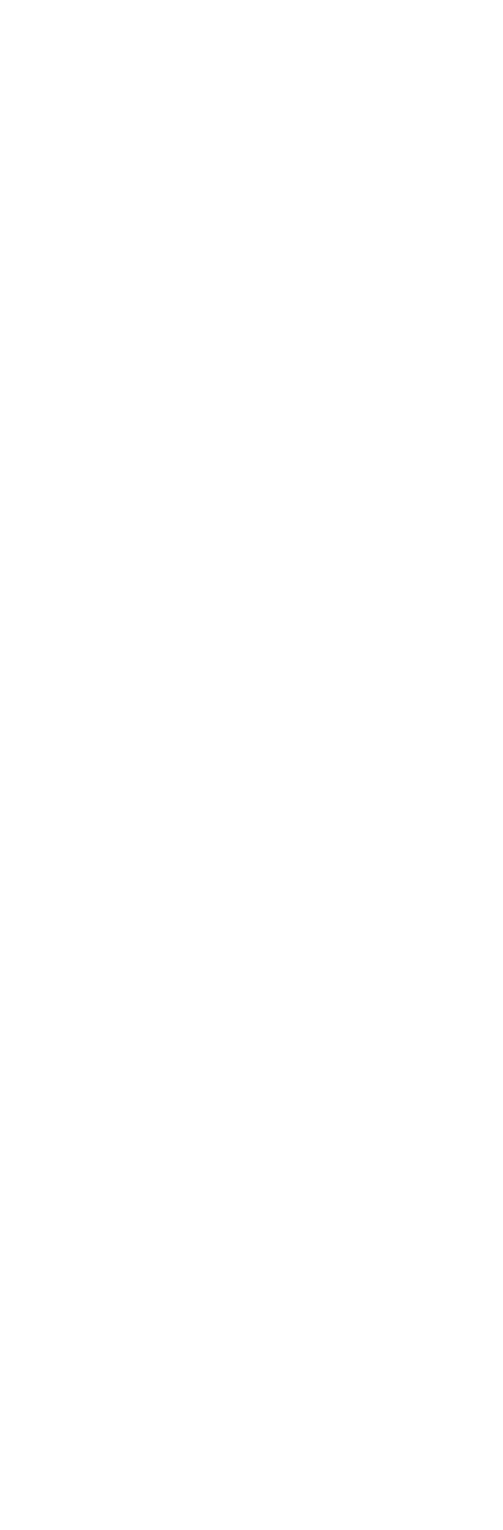




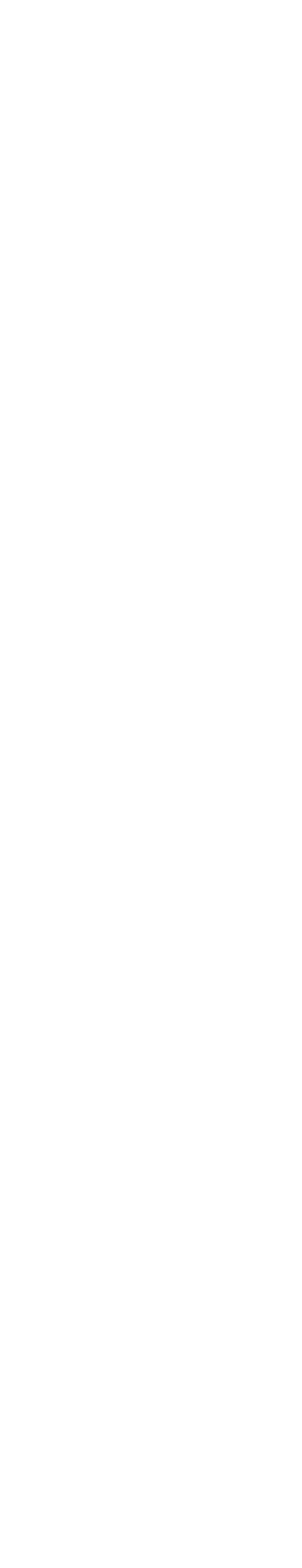

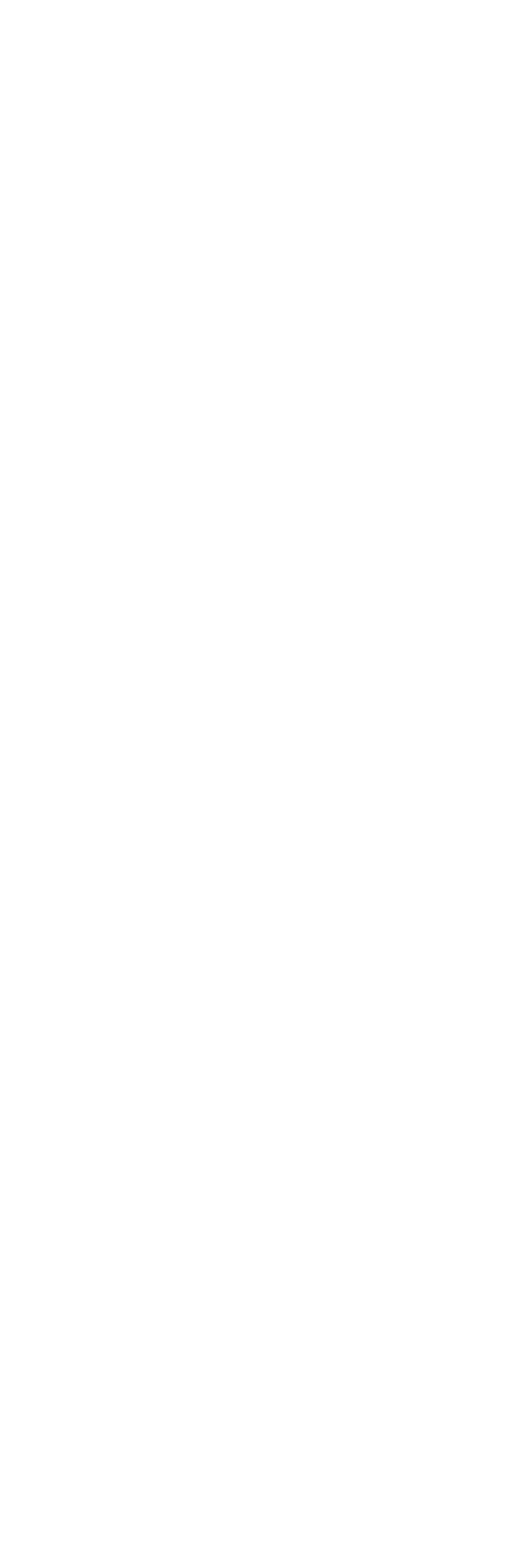




\begin{tabular}{|c|c|c|c|c|c|c|}
\hline の & 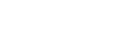 & 905 & 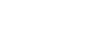 & : & 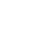 & I \\
\hline 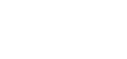 & 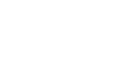 & 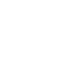 & $\begin{array}{l}\infty \\
\infty \\
\text { ம }\end{array}$ & क्ष & ?ొ & i \\
\hline 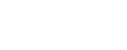 & กับั & ज्ञ & "थ: & ली & is & I \\
\hline ோ & 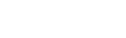 & 요유 & 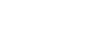 & $\stackrel{*}{*}$ & $\ddot{~}$ & i \\
\hline 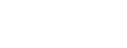 & 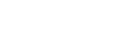 & 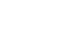 & 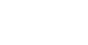 & 8 & क & 1 \\
\hline 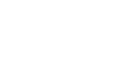 & 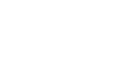 & ตัตั & จุล์ & సิ & i) & i \\
\hline ตำำำ & เทีคำ & ఈำ. & & ஸे. & $i$ & i \\
\hline 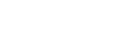 & 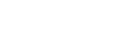 & 苍怘突 & 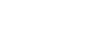 & N & लें & I \\
\hline & & & ¿ & -1 & N & N. \\
\hline ข. ! ! & 19119. & 111 & $\because$ & 1 & 1 & $i$ \\
\hline 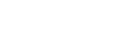 & 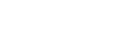 & 독요 & $\underset{\sim-1}{\infty} \underset{\sim}{\infty} \underset{\sim}{\infty} \infty$ & $\begin{array}{c}\mathbf{v} \\
-1 \\
-1\end{array}$ & $\stackrel{+}{-4}$ & 5ే \\
\hline 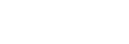 & 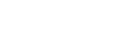 & ๓ొఱొ సี & "ొ & $\begin{array}{l}\infty \\
\infty\end{array}$ & 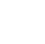 & ผ \\
\hline 000 & 00 & 0 & 00 & 0 & 0 & 0 \\
\hline 유유 유요 & 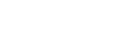 & ติำ ถู & चธक्ष & ลี & $=-1$ & -1 \\
\hline 111 & 1 1 | & 111 & $\begin{array}{ll}1 & 1\end{array}$ & $i$ & I & 1 \\
\hline 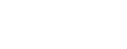 & 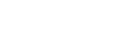 & 舟年年 & 촘ํำ & $\mathfrak{N}$ & 하 & 8 \\
\hline 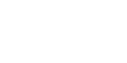 & 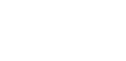 & & & ? & : & is \\
\hline ニ⿻日 & 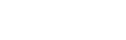 & ज出 & $\underset{\sim}{\infty} \mathbb{\sim}$ & $=$ & $=$ & $\rightarrow$ \\
\hline$\underset{-1}{\infty}=$ & 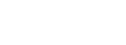 & & ir & $\overrightarrow{4}$ & গ్త| & 工 \\
\hline 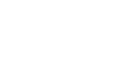 & 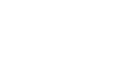 & क्ष & ஸ்ற் & i] & लै- & ! \\
\hline 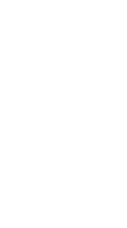 & 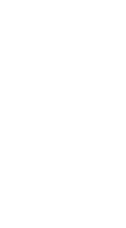 & 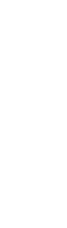 & $\begin{array}{l}\vdots: \vdots: \\
\vdots \vdots \\
\vdots \\
\vdots \\
\vdots\end{array}$ & 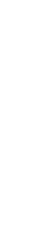 & 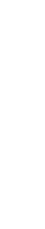 & 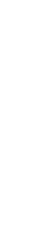 \\
\hline
\end{tabular}

\begin{tabular}{|c|c|}
\hline 崤 & 1:1 11 in \\
\hline$\overline{\mathrm{m}}$ & $\begin{array}{lllllllllll}1 & 1 & 1 & 1 & \infty & 0 & 1 & 1 & 0 & 0 & 0\end{array}$ \\
\hline 요 & 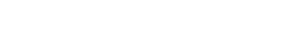 \\
\hline i & 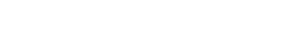 \\
\hline$\stackrel{\infty}{\infty}$ & 1 11 1 \\
\hline$\hat{\imath}$ & 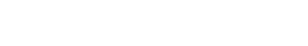 \\
\hline$\stackrel{\circ}{\circ}$ & 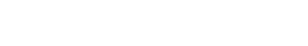 \\
\hline$\stackrel{\sim}{\sim}$ & $1: 11 \% 5$ \\
\hline 4 & 111 100 \\
\hline$\stackrel{2}{2}$ & 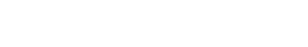 \\
\hline ง & 1:1 跑 \\
\hline$\overline{\mathrm{N}}$ & 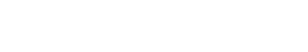 \\
\hline i & 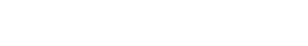 \\
\hline$\underline{a}$ & 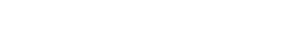 \\
\hline$\stackrel{\infty}{=}$ & 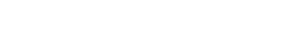 \\
\hline 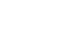 & 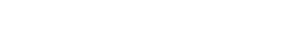 \\
\hline$\simeq$ & 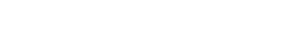 \\
\hline 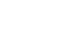 & 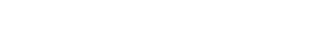 \\
\hline 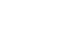 & 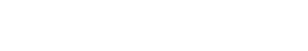 \\
\hline$\stackrel{m}{-}$ & \begin{tabular}{|l|llll:lllll}
1 & 1 & 1 & 0 & 0 & 0 & 1 & 0 & 0 & 1 \\
\end{tabular} \\
\hline$\cong$ & 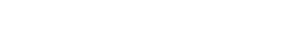 \\
\hline$=$ & 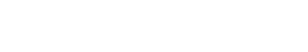 \\
\hline 으 & 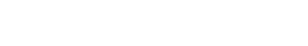 \\
\hline$a$ & 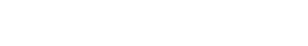 \\
\hline$\infty$ & 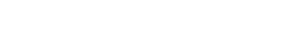 \\
\hline s & 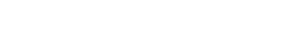 \\
\hline$\circ$ & ili 1 io \\
\hline in & 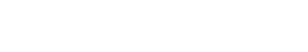 \\
\hline$\nabla$ & 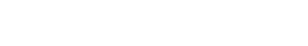 \\
\hline$m$ & 11: $11:$ in \\
\hline n & 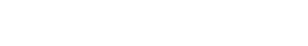 \\
\hline- & 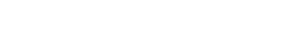 \\
\hline 总 & 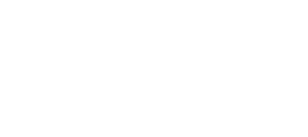 \\
\hline
\end{tabular}




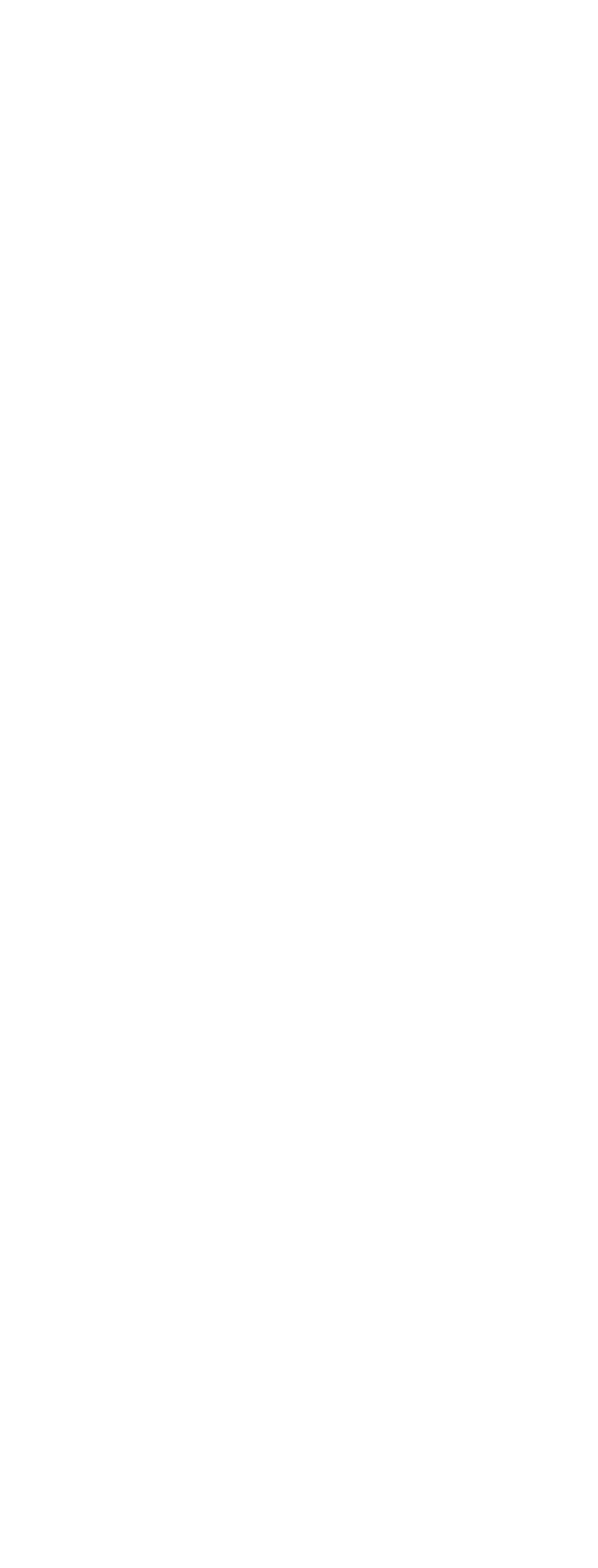




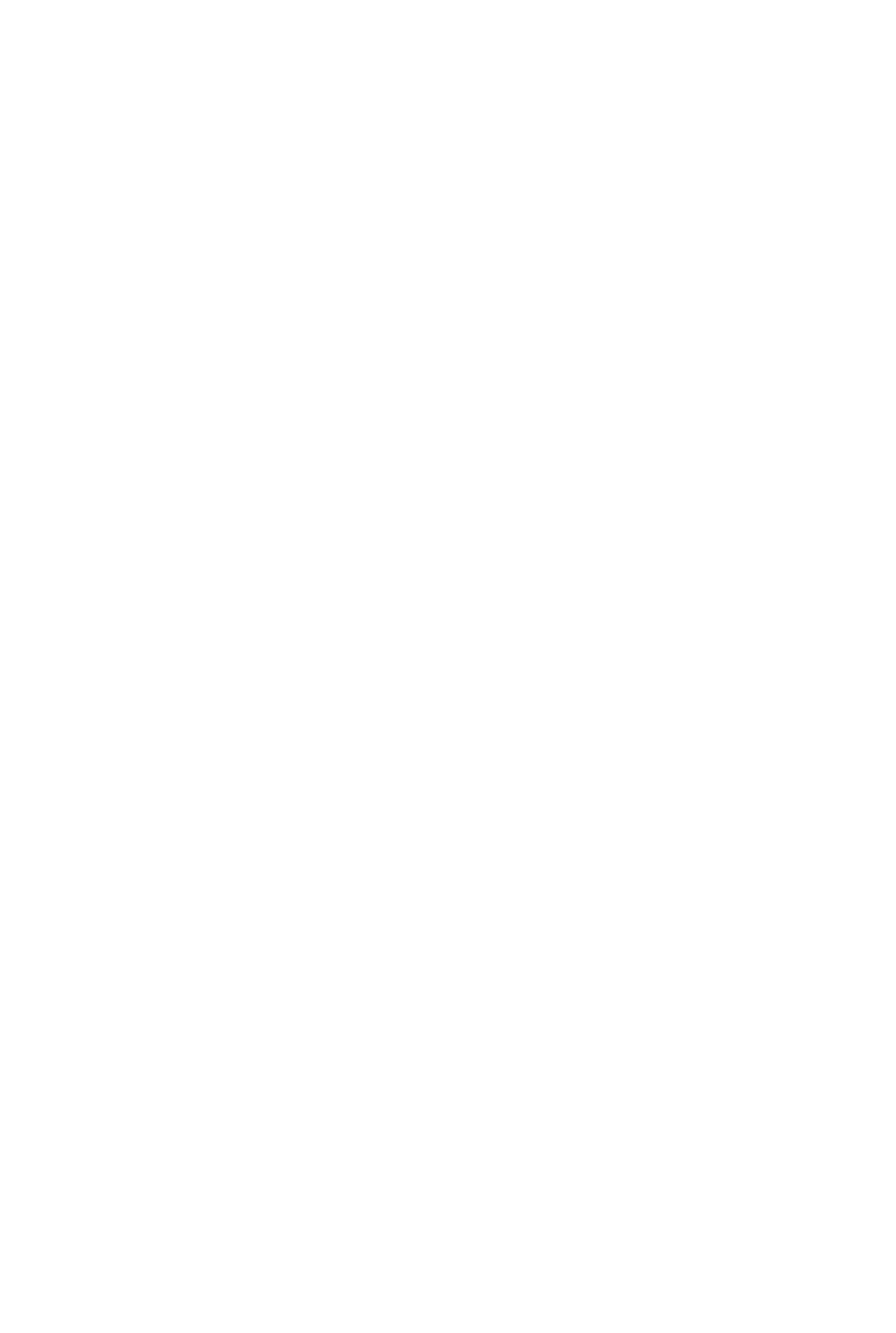


(2)

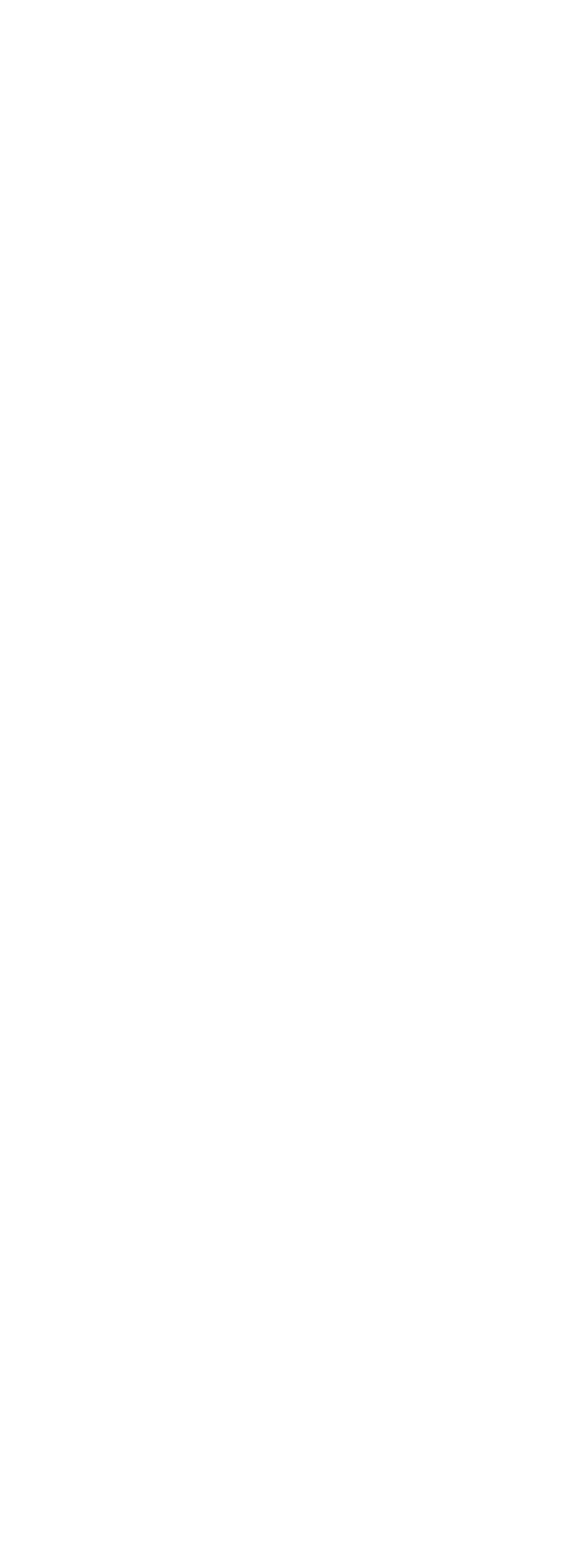




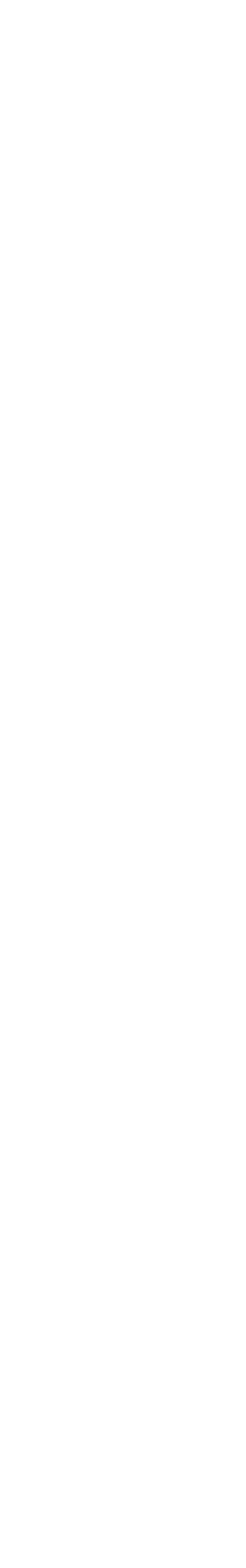

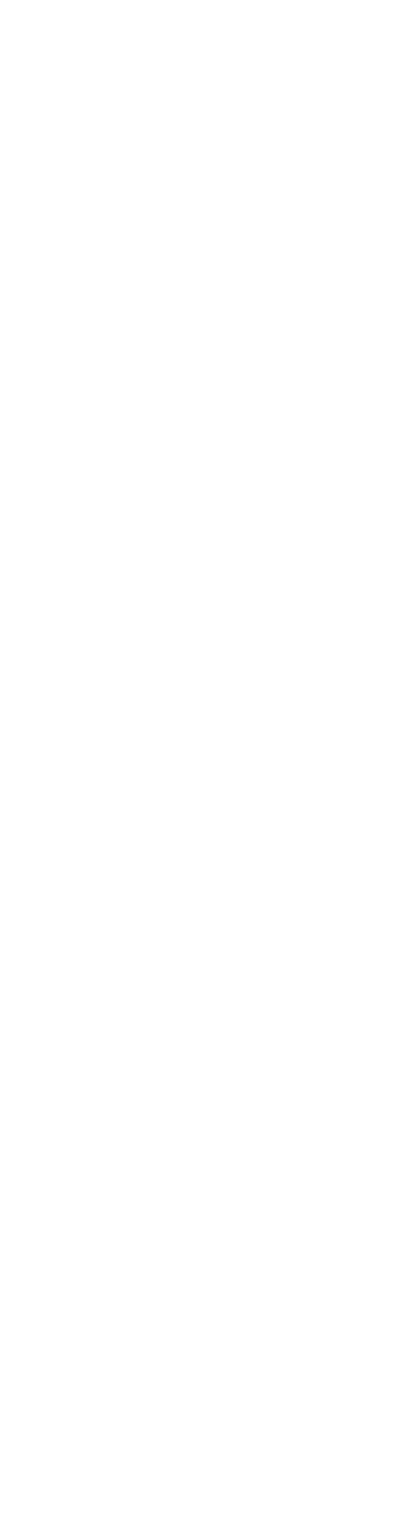




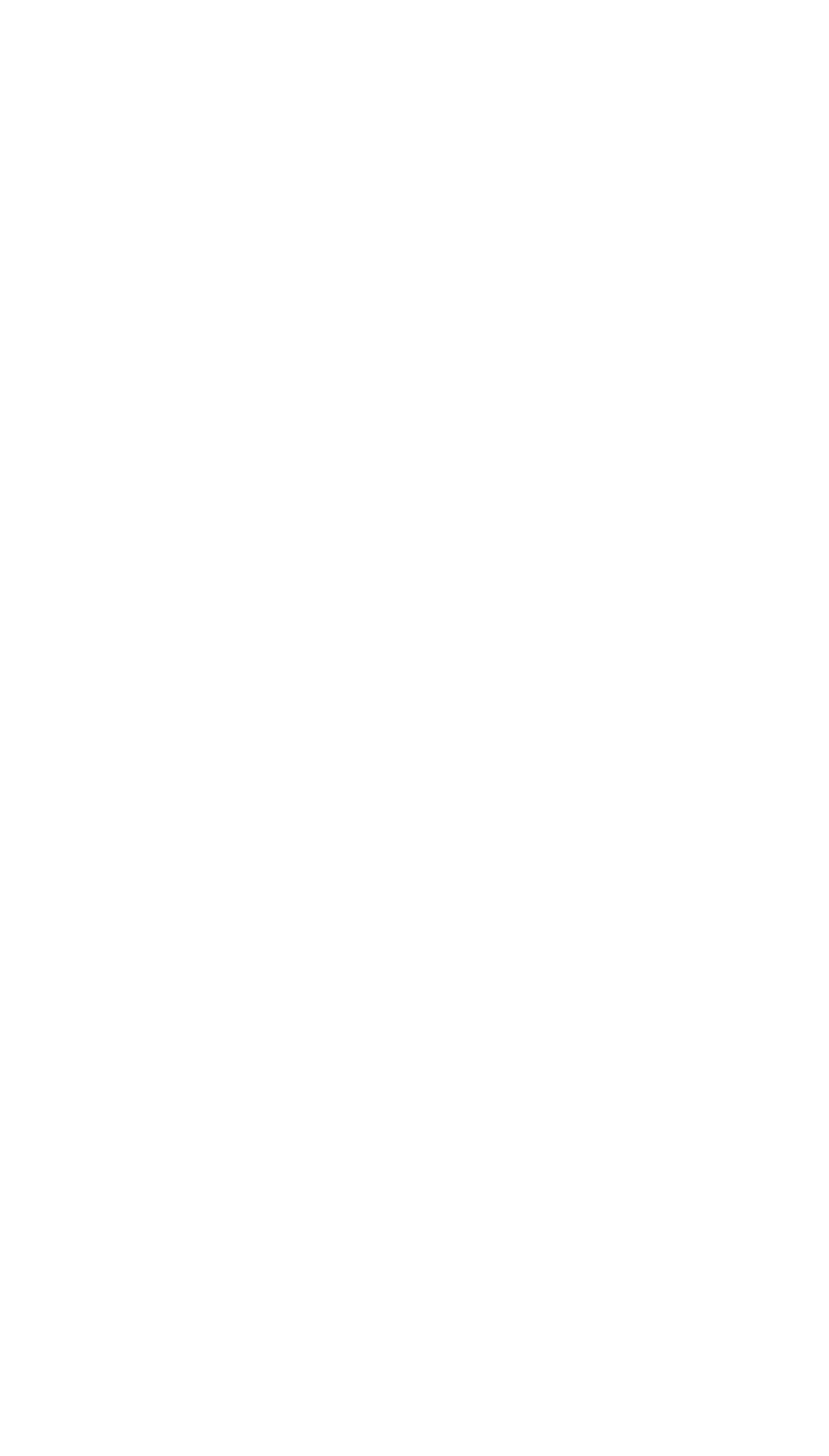




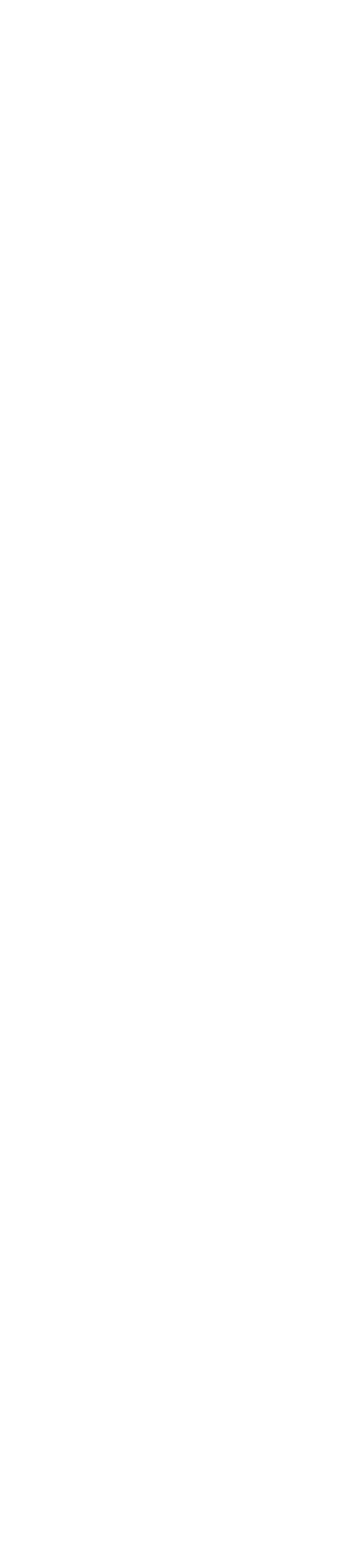

\begin{tabular}{|c|c|c|c|c|c|}
\hline \multicolumn{2}{|c|}{ 岀 案 } & ถึกต & ตร์ถ & 면 & $\stackrel{m}{\infty}$ \\
\hline & $\bar{m}$ & $\begin{array}{lll}0 & 0 \\
\end{array}$ & in 1 is & 101 & $\tilde{\infty} \approx 1$ \\
\hline & 8 & $\operatorname{mog}_{0 \rightarrow \infty}$ & 于q & 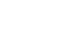 & ஹ®゙ \\
\hline & 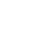 & โธกตำ & of fo & 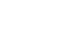 & 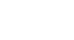 \\
\hline & $\stackrel{\infty}{\sim}$ & 옹ํㅁ & 只与品 & $\approx \sim_{\infty}^{ \pm}$ & 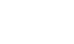 \\
\hline & $\hat{N}$ & बin & $\vec{n} \underset{f}{n} \mathfrak{n}$ & โลก & ๓ूç \\
\hline & $\stackrel{2}{2}$ & iñ & テ洋 & $\underset{100}{a}$ & 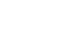 \\
\hline & $\stackrel{\sim}{\sim}$ & Dinn & ån & $\stackrel{2}{2} \div$ & 士品 \\
\hline & N & $\sin =$ & 证すo & mº & 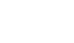 \\
\hline & กี & 모ำ & ingin & $\stackrel{2}{2} \underset{\sim}{2-\infty}$ & 士क口 \\
\hline & N & 5000 & 于括 & నก๊ & $\cos _{0}^{2}$ \\
\hline & $\bar{N}$ & :ำ & J于? & $\stackrel{n}{\sim 2} \vec{\infty}$ & 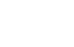 \\
\hline & $\stackrel{N}{2}$ & 趈F & 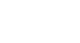 & $\approx \approx \sim$ & to \\
\hline & $\stackrel{9}{2}$ & 0.75 & is Fo & $\stackrel{2}{2} \therefore \infty$ & ロேト \\
\hline & $\stackrel{\infty}{-}$ & 9.9 & $\tilde{c} \sigma^{\infty}$ & 过足 & 묘요 \\
\hline & 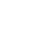 & $\begin{array}{l}989 \\
089\end{array}$ & 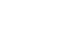 & ก\%० & $\stackrel{2}{a n}$ \\
\hline t & 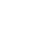 & 중 & 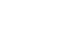 & ำส & ${ }_{\infty}^{\infty} \sum_{\infty}^{\infty}$ \\
\hline & $\underline{2}$ & Rำ & 离于出 & ๑ேत & 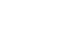 \\
\hline & \pm & 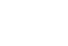 & ตั & Ð & $\mathbb{\infty}_{\infty}^{\infty} \mathbb{\infty}$ \\
\hline & $\stackrel{m}{-}$ & ㅇำ & 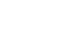 & 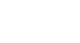 & 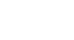 \\
\hline & $\simeq$ & 요숌ำ & gFo & 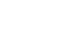 & 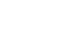 \\
\hline & $=$ & 只唄出 & gF F in & \$0 1 & $\begin{array}{ccc}n & 0 & 0 \\
\infty & \infty & \infty \\
\end{array}$ \\
\hline & 으 & ㅇ:o & $F_{j} \sigma_{j n}^{n}$ & Oad & 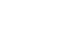 \\
\hline & $\alpha$ & त्: & F Fin & in 2 ! & $\begin{array}{l}\text { J } \\
\infty \\
0\end{array}$ \\
\hline & $\infty$ & $\therefore 8 g$ & 舫于 & Tin & $\begin{array}{lll}n \\
\infty\end{array}$ \\
\hline & $n$ & 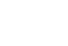 & fon & ํำ & 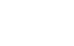 \\
\hline & 0 & 2:0 & F⿻ำ & ำ & $\begin{array}{l}+ \\
\infty\end{array}$ \\
\hline & $n$ & $\begin{array}{l}900 \\
0.5\end{array}$ & 乎品苟 & ๑ோ & 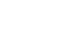 \\
\hline & $\nabla$ & 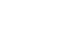 & Jง กี & $5 \approx 2$ & $\approx \tilde{\infty}$ \\
\hline & $m$ & 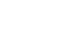 & ซึีต & 똥요 & 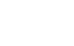 \\
\hline & $\sim$ & 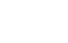 & F舟品 & 몽요 & 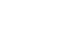 \\
\hline & - & 0.90 & 要品 & ㅁㅇㅇ & 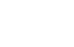 \\
\hline & & 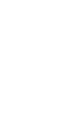 & 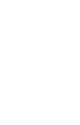 & 它: & 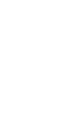 \\
\hline
\end{tabular}




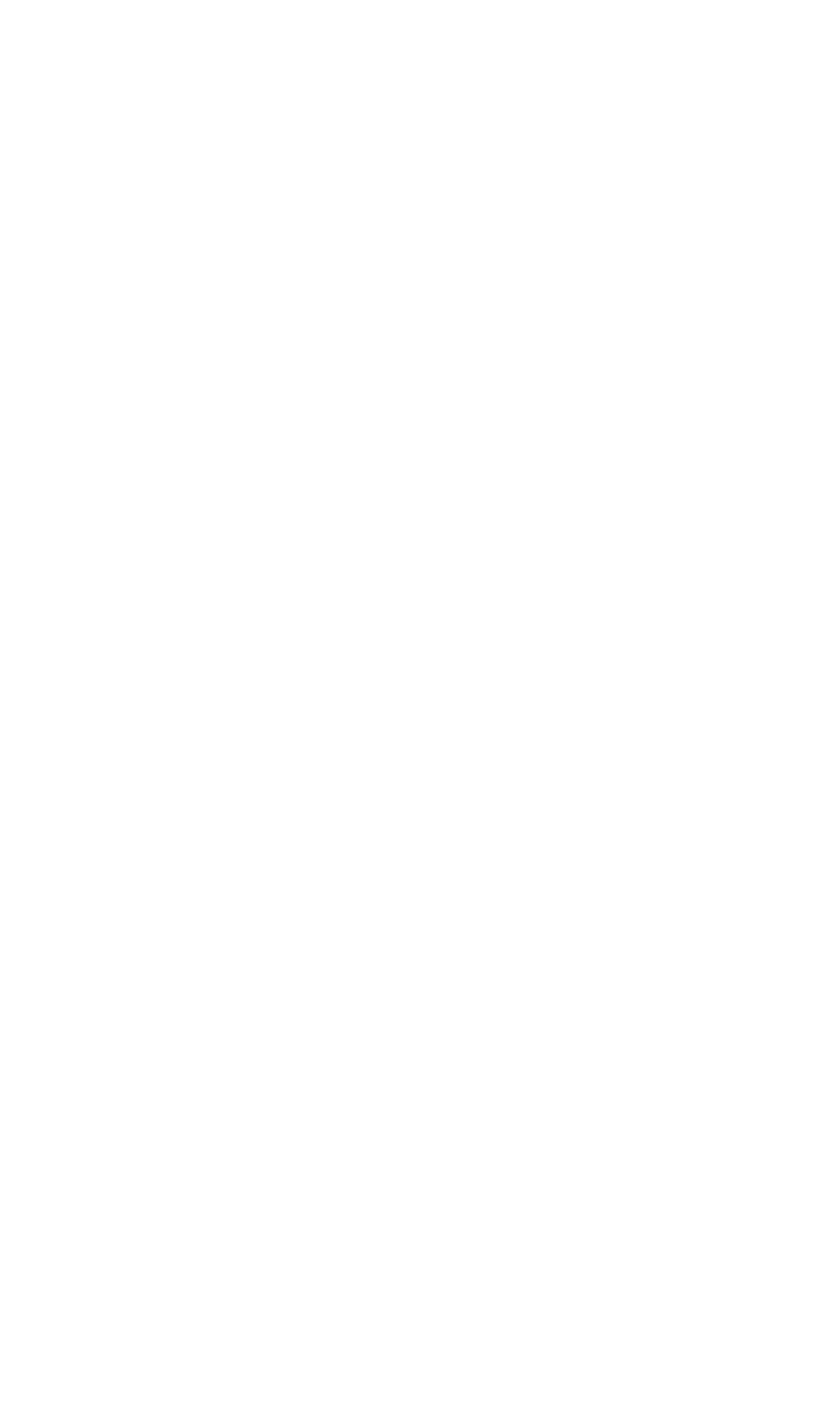




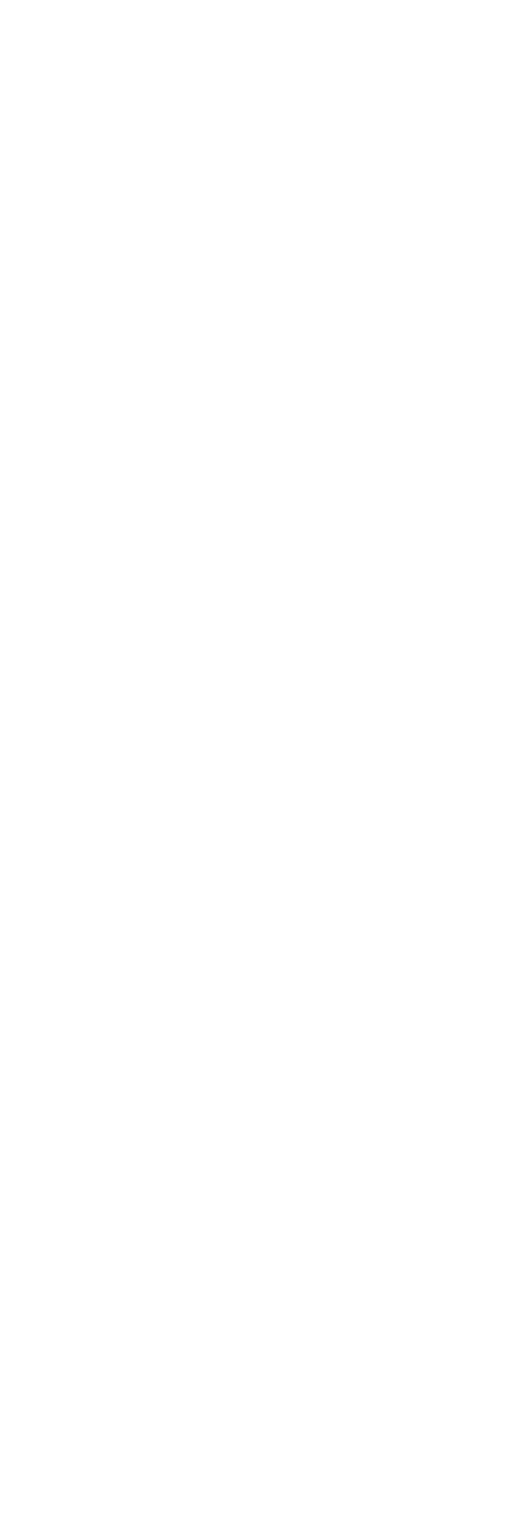




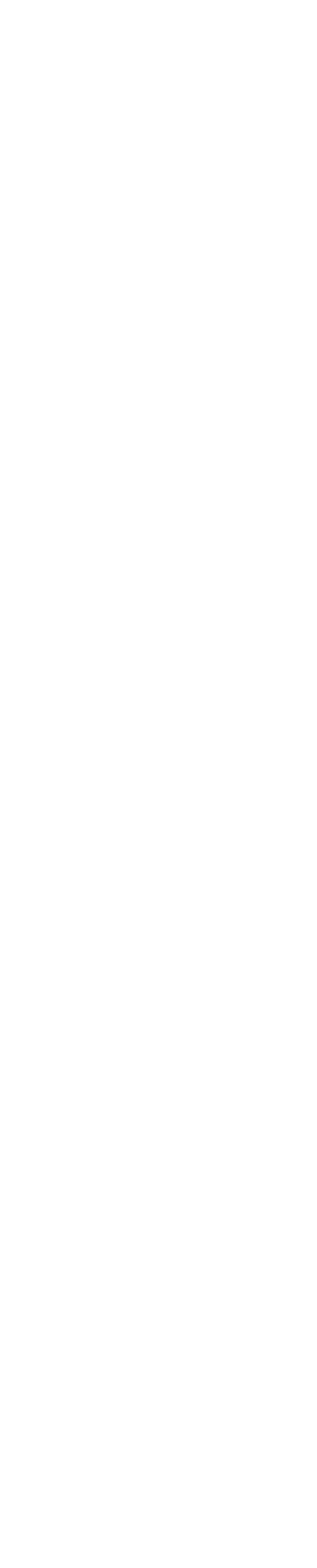

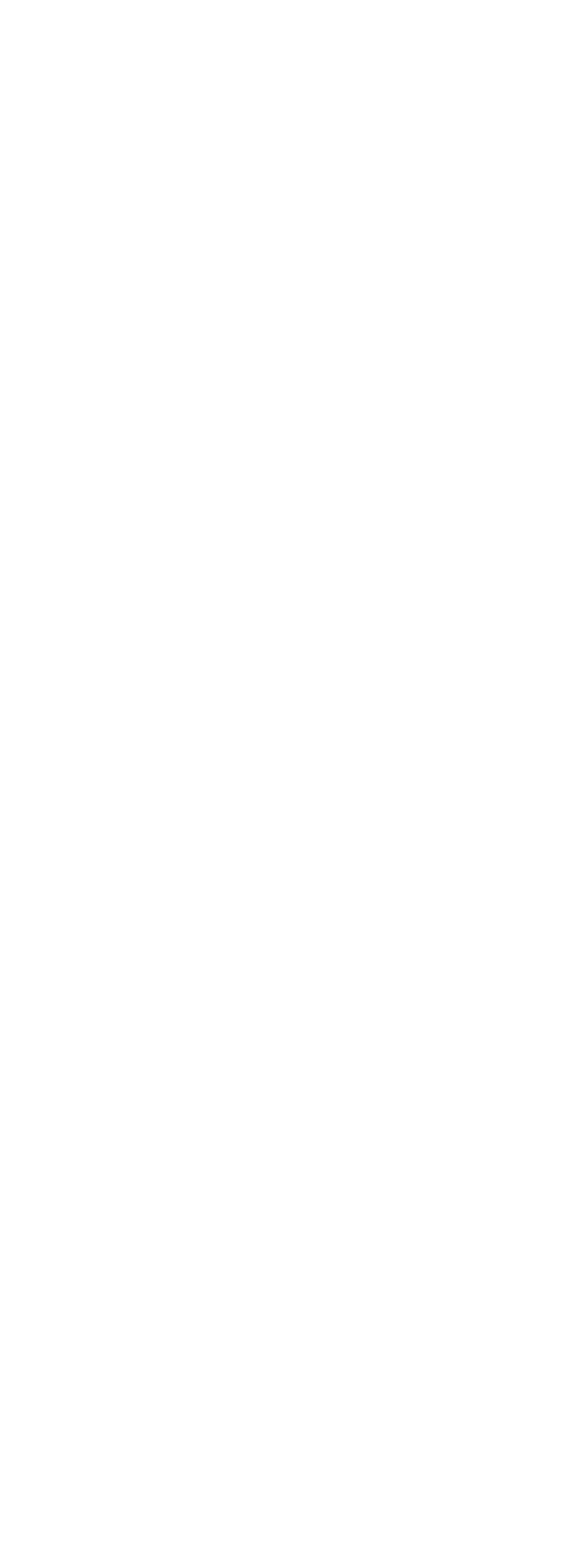




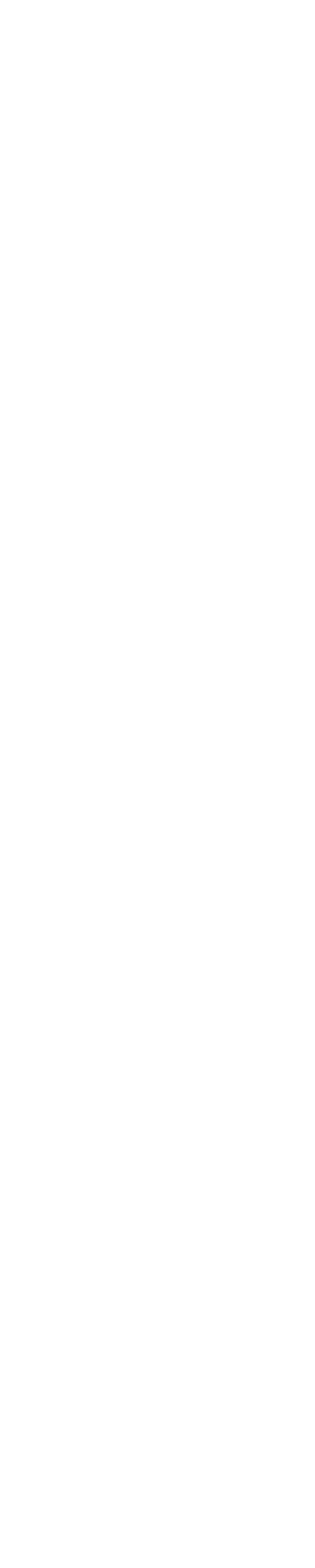

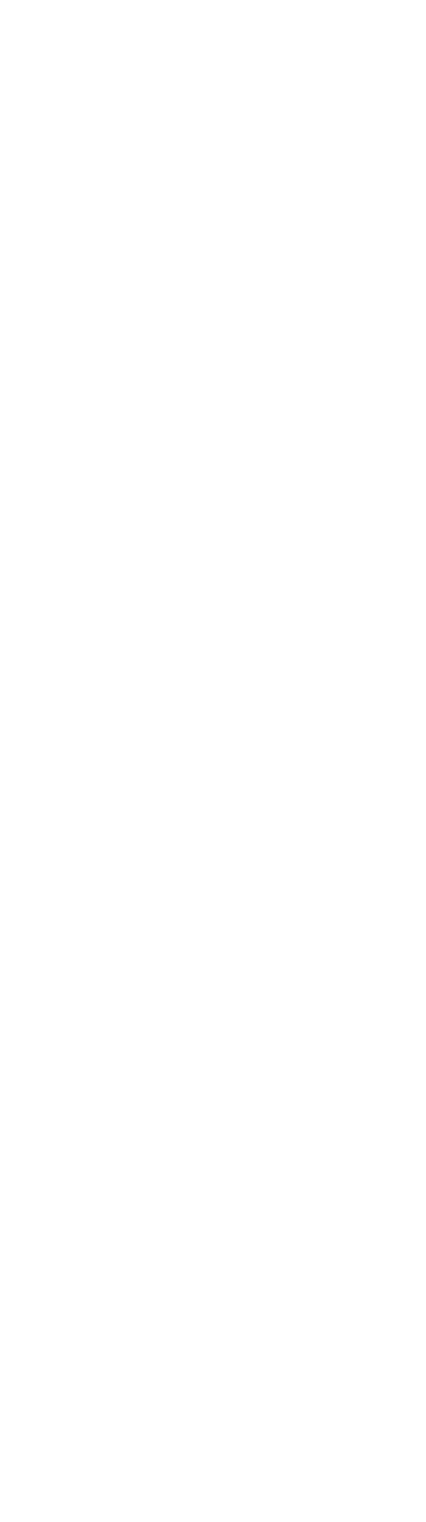




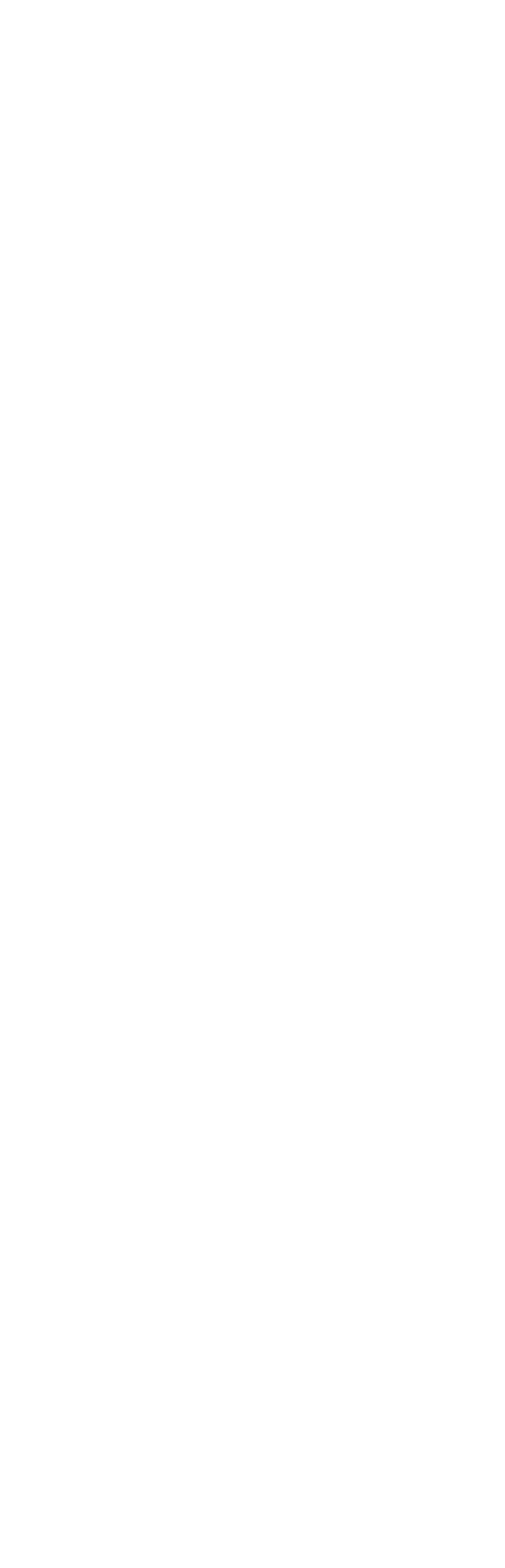


TRINITY RIVER BASIN--Continued

8-503. ELM FORK TRINITY RIVER NEAR MUENSTER, TEX.--Continued

Suspended sediment, water year october 1963 to September 1964

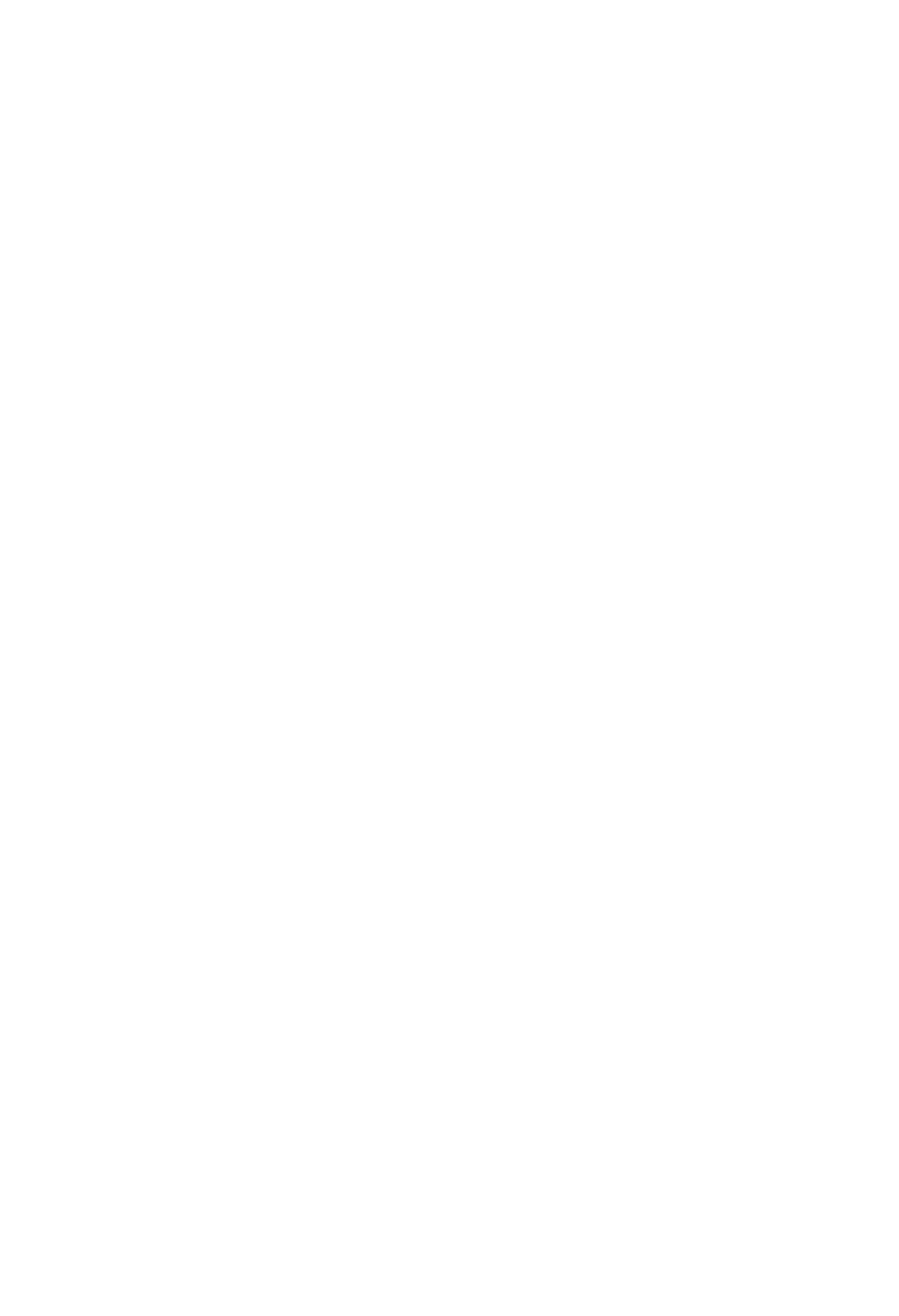

Estimated.

$T$ Less than 0.50 ton.

C Composite period. 
TRINITY RIVER BASIN--Continued

8-503. ELM FORK TRINITY RIVER NEAR MUENSTER, TEX.--Continu?d

Suspended sediment, water year October 1963 to September 1964--Continued

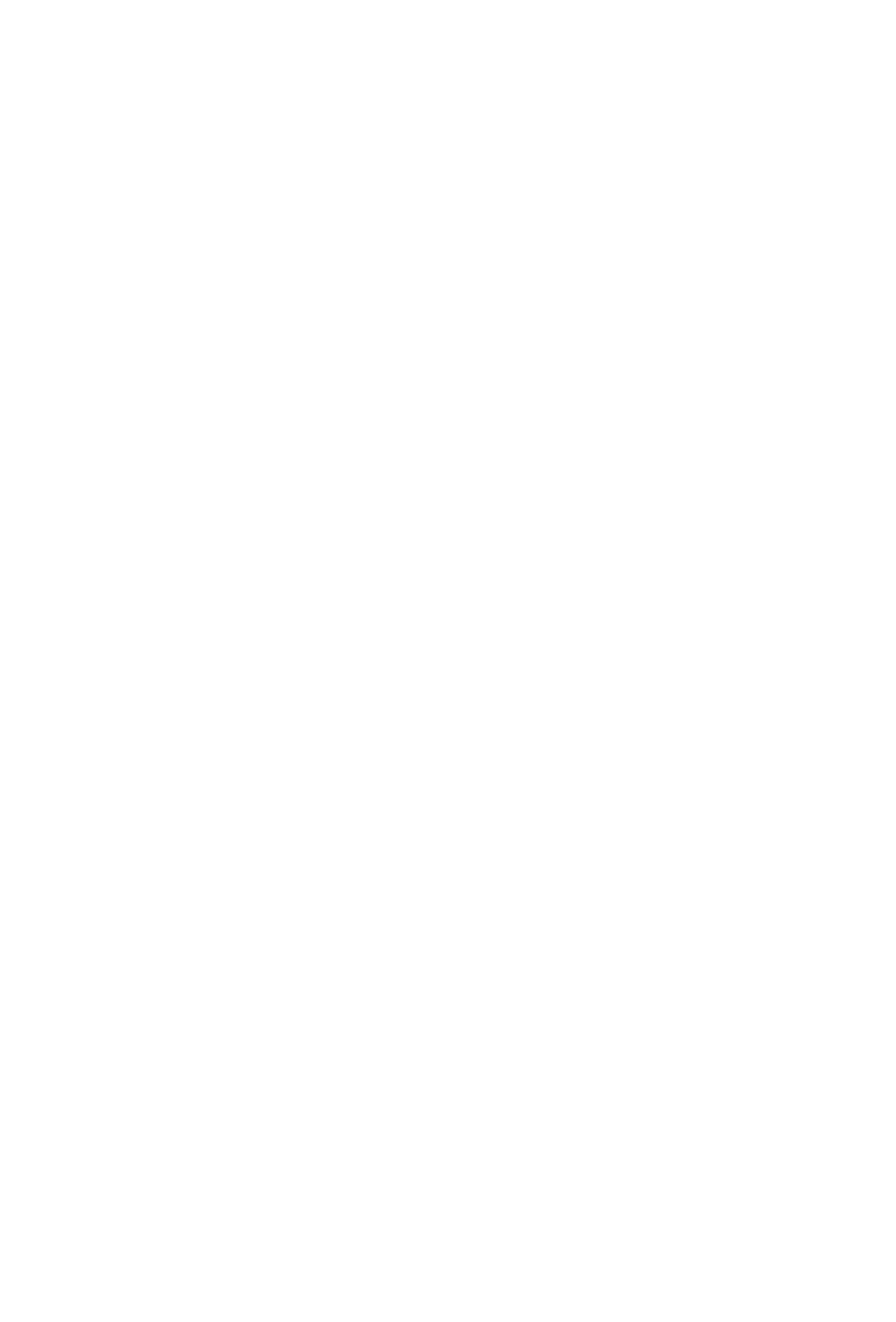

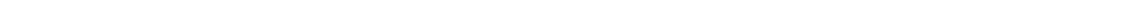

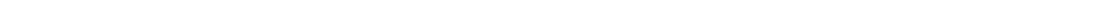




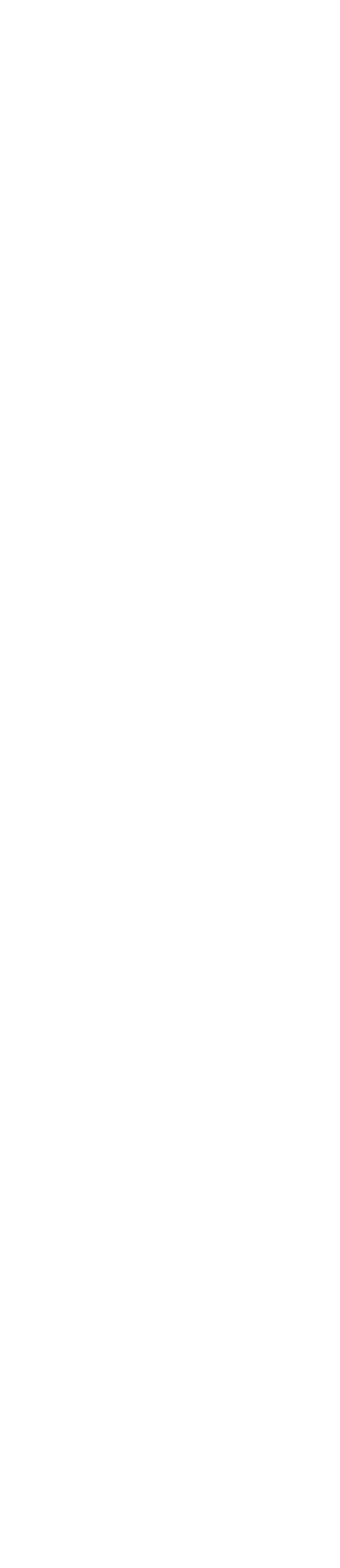




\begin{tabular}{|c|c|}
\hline 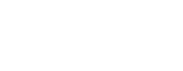 & 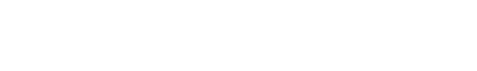 \\
\hline$\frac{\pi}{2}$ & 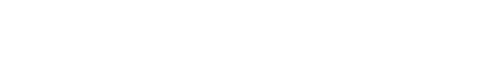 \\
\hline 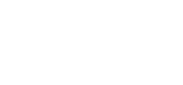 & 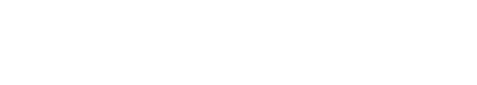 \\
\hline 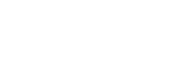 & 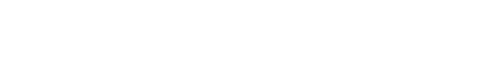 \\
\hline 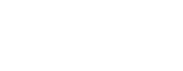 & 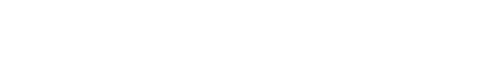 \\
\hline 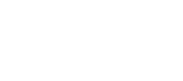 & 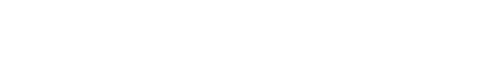 \\
\hline 善点票 & 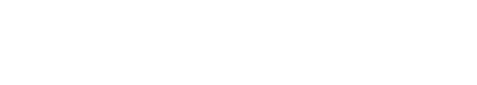 \\
\hline 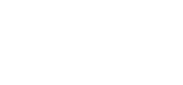 & 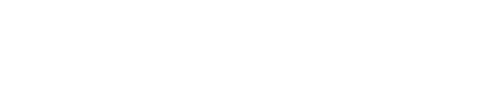 \\
\hline 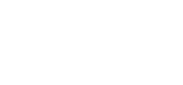 & 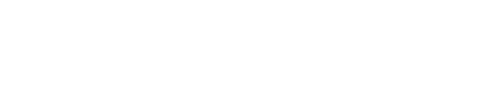 \\
\hline 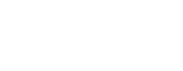 & 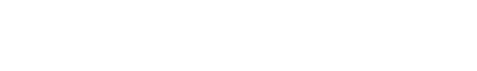 \\
\hline 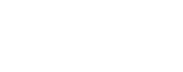 & 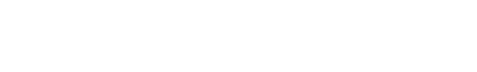 \\
\hline 飬害画 & 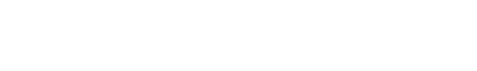 \\
\hline $\begin{array}{l}\text { 害 } \\
\text { 응 }\end{array}$ & 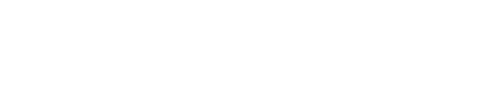 \\
\hline 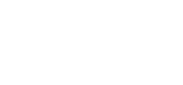 & 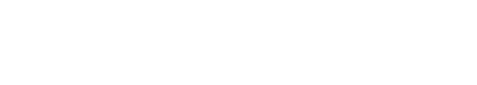 \\
\hline 亩 & 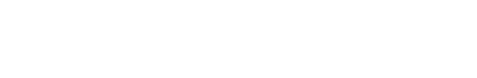 \\
\hline 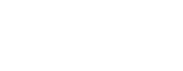 & 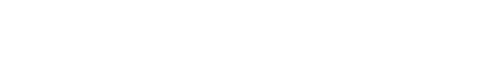 \\
\hline 量焉 & 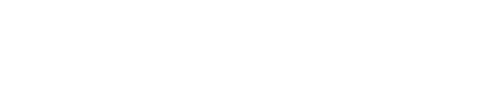 \\
\hline 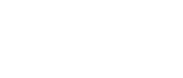 & 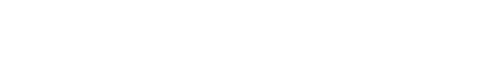 \\
\hline 경웡음 & 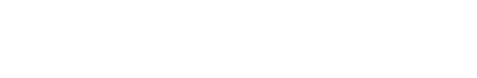 \\
\hline 总要 & \\
\hline 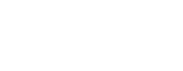 & 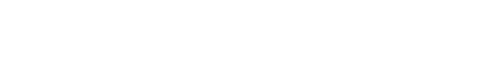 \\
\hline 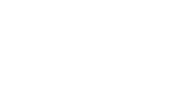 & 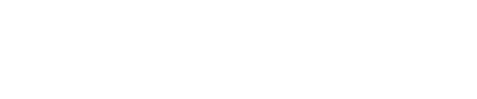 \\
\hline & 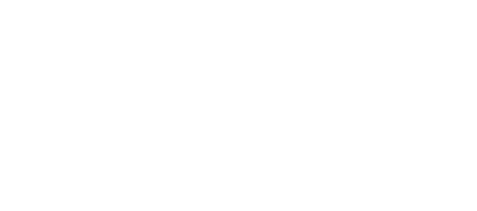 \\
\hline
\end{tabular}




\begin{tabular}{|c|c|c|c|}
\hline 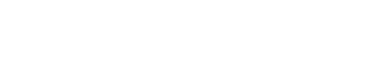 & $\begin{array}{l}q \\
: \\
0\end{array}$ & $\stackrel{\circ}{i}$ & $\stackrel{9}{i}$ \\
\hline mañ & $\therefore$ & $\because$ & \\
\hline 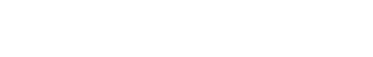 & F⿱ & : & 1 \\
\hline 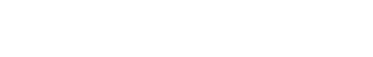 & \begin{tabular}{|l|}
$m$ \\
$\dot{n}$
\end{tabular} & $\underset{4}{4}$ & $\mathrm{i}$ \\
\hline 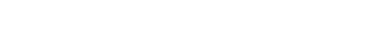 & N & D & 1 \\
\hline 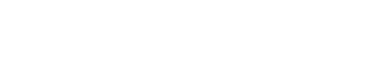 & $I^{2}$ & 宫 & $\mathrm{T}$ \\
\hline 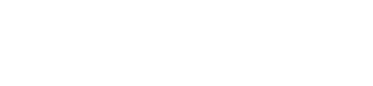 & $\stackrel{\tilde{D}}{-\infty}$ & i & 1 \\
\hline 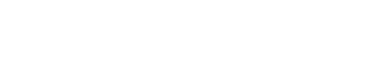 & \begin{tabular}{|l|}
$\frac{2}{4}$ \\
\\
\end{tabular} & 1 & 1 \\
\hline 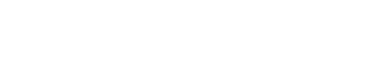 & is & 品 & 1 \\
\hline 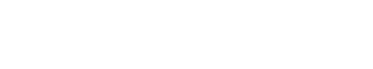 & \begin{tabular}{|c|}
$\dot{a}$ \\
\end{tabular} & के & $\ddot{5}$ \\
\hline 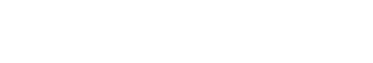 & สี & ₹ & in \\
\hline 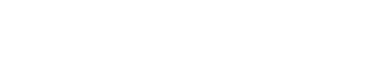 & 1 & i & i \\
\hline 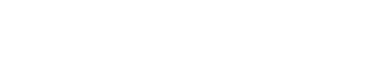 & ร & 品 & to \\
\hline 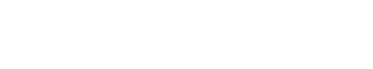 & 2 & $\overrightarrow{\mathcal{N}}$ & $\vec{x}$ \\
\hline 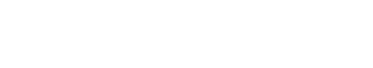 & 节 & $\underset{T}{T}$ & ब్ల \\
\hline 1111111 1 1111111 & 1 & $i$ & $i$ \\
\hline 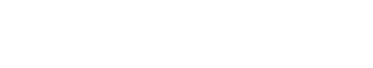 & $\because$ & Э & ผี \\
\hline 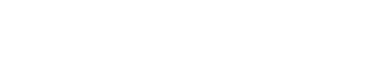 & $\dot{0} \dot{0}$ & $\stackrel{\infty}{0}$ & $\Rightarrow$ \\
\hline 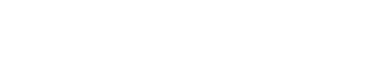 & in & \& & \pm \\
\hline m & 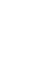 & $\mathbb{I}$ & ֻ \\
\hline 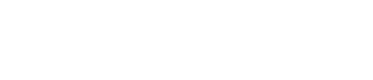 & 1 & $\stackrel{\infty}{\infty}$ & 1 \\
\hline 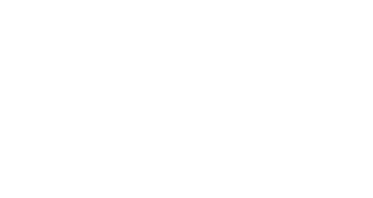 & & 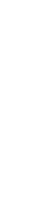 & 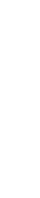 \\
\hline
\end{tabular}



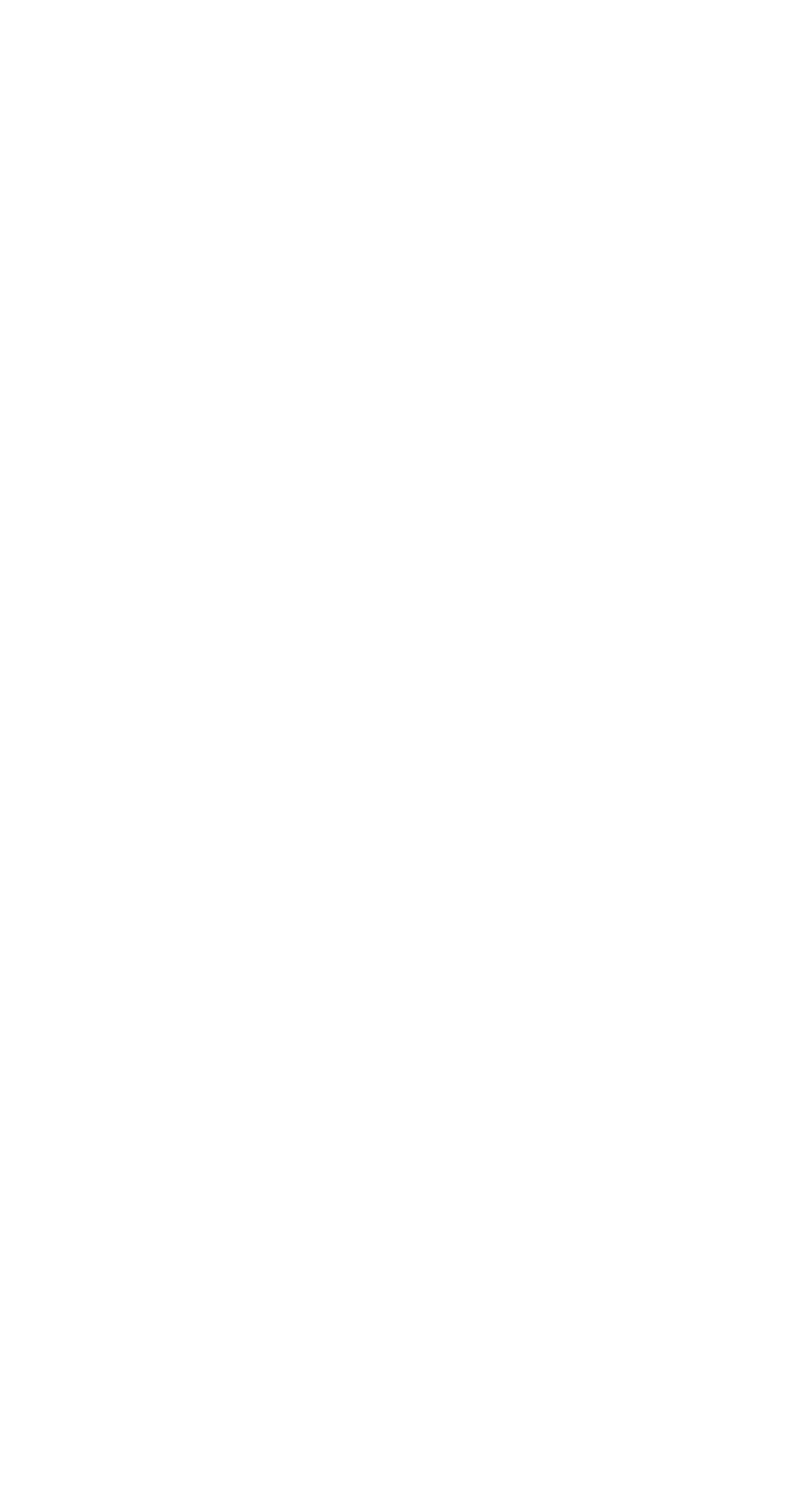
TRINITY RIVER BASIN--COntinued

8-632. PIN OAK CREEK NEAR HUBBARD, TEX.

LOCATION,--At gaging station (on right bank) at bridge on State Highway $171,5.8 \mathrm{miles}$ southeast of Hubbard, Hill County, and 9 miles upstream from Elm Creek.

DRAINAGE AREA. --17.6 square miles.

RECORDS AVAILABLE. --Water temperatures: January 1957 to September 1960

Sediment records: October 1956 to September 1960, September 1962 to September 1964.

EXTREMES, 1963-64.--Sediment concentrations: Maximum dai1y, 820 ppm Sept. 17; mininum

daily, no flow on

Sedimennt loads: Maximum daily, 64 tons Sept. 17; minimum daily, 0 tons on many days. EXTREMES, 1956-60, 1962-64.--Sediment concentrations: Maximum daily, 5, 160 ppm June 4, 1957; minimum daily, no flow on many days.

Sediment loads: Maximum daily, 12,200 tons Apr. 20, 1957; minimum daily, 0 tons on many days.

Suspended sediment, water year October 1963 to September 1964 (Flow occurred only on days indicated)

\begin{tabular}{|c|c|c|c|c|c|}
\hline \multirow[b]{2}{*}{ Date } & \multirow[b]{2}{*}{$\begin{array}{l}\text { Time } \\
(24 \mathrm{hr})\end{array}$} & \multirow[b]{2}{*}{$\begin{array}{l}\text { Water } \\
\text { tem- } \\
\text { per- } \\
\text { ature } \\
\left({ }^{\circ} \mathrm{F}\right)\end{array}$} & \multirow[b]{2}{*}{$\begin{array}{l}\text { Discharge } \\
\text { (cfs) }\end{array}$} & \multicolumn{2}{|c|}{ Suspended sedime it } \\
\hline & & & & $\begin{array}{c}\text { Mean } \\
\text { concen- } \\
\text { tration } \\
\text { (ppm) }\end{array}$ & $\begin{array}{c}\text { Discharge } \\
\text { (tons per day) }\end{array}$ \\
\hline Jan. $31,1964 \ldots \ldots \ldots \ldots \ldots$ & & & 0.1 & 750 & $\mathbf{T}$ \\
\hline $\operatorname{rota1} \ldots \ldots \ldots \ldots \ldots \ldots$ & & & 0.1 & -- & $\mathbf{T}$ \\
\hline Mar. $19 \ldots \ldots \ldots \ldots \ldots \ldots$ & & & 5.3 & 405 & $\mathrm{~s}$ \\
\hline $\operatorname{Total} \ldots \ldots \ldots \ldots \ldots \ldots$ & & & 5.3 & $\sim$ & 7 \\
\hline $\begin{array}{l}\text { Sept. }{ }^{16} \ldots \ldots \ldots \ldots \ldots \ldots \ldots \ldots \ldots \\
\text { Sept. } 17 \ldots \ldots \ldots \ldots \ldots \ldots \ldots\end{array}$ & & & $\begin{array}{l}14 \\
25\end{array}$ & $\begin{array}{l}730 \\
820\end{array}$ & $\begin{array}{l}53 \\
64\end{array}$ \\
\hline Total.......... & & & 39 & -- & 117 \\
\hline
\end{tabular}

Total discharge for year (cfs-days) $\ldots \ldots \ldots \ldots \ldots \ldots \ldots \ldots \ldots \ldots \ldots \ldots \ldots \ldots \ldots \ldots \ldots 44$

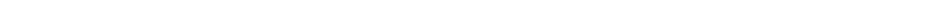

S Computed by subdividing day.

$T$ Less than 0.50 ton. 


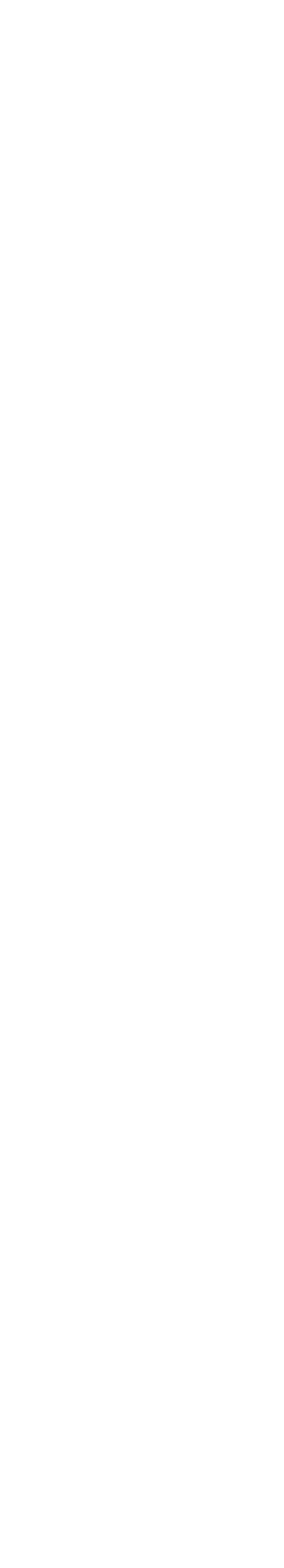




\begin{tabular}{|c|c|c|}
\hline & 四 & 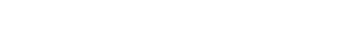 \\
\hline & 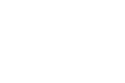 & 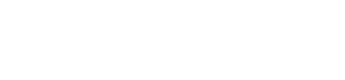 \\
\hline & 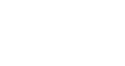 & 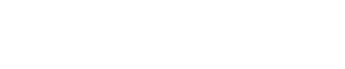 \\
\hline : & 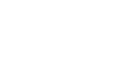 & 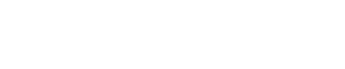 \\
\hline 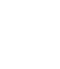 & 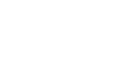 & 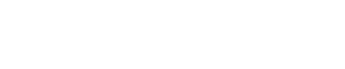 \\
\hline ä & 总台宗 & 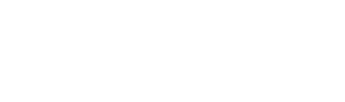 \\
\hline 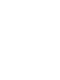 & 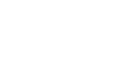 & 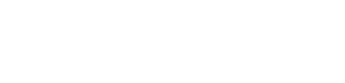 \\
\hline 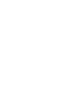 & 密器尊 & 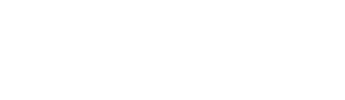 \\
\hline & 官蔗高 & \\
\hline & 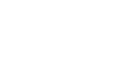 & 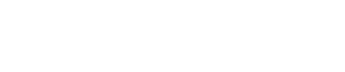 \\
\hline & 家部画 & 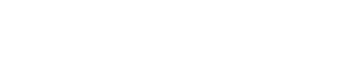 \\
\hline & & $\mathrm{DN}$ \\
\hline
\end{tabular}

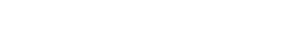

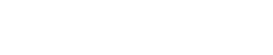

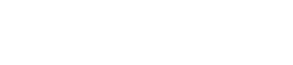

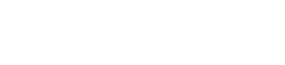
O 5

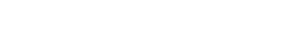

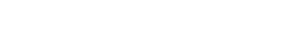
ơ

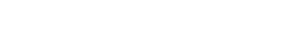

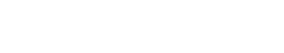




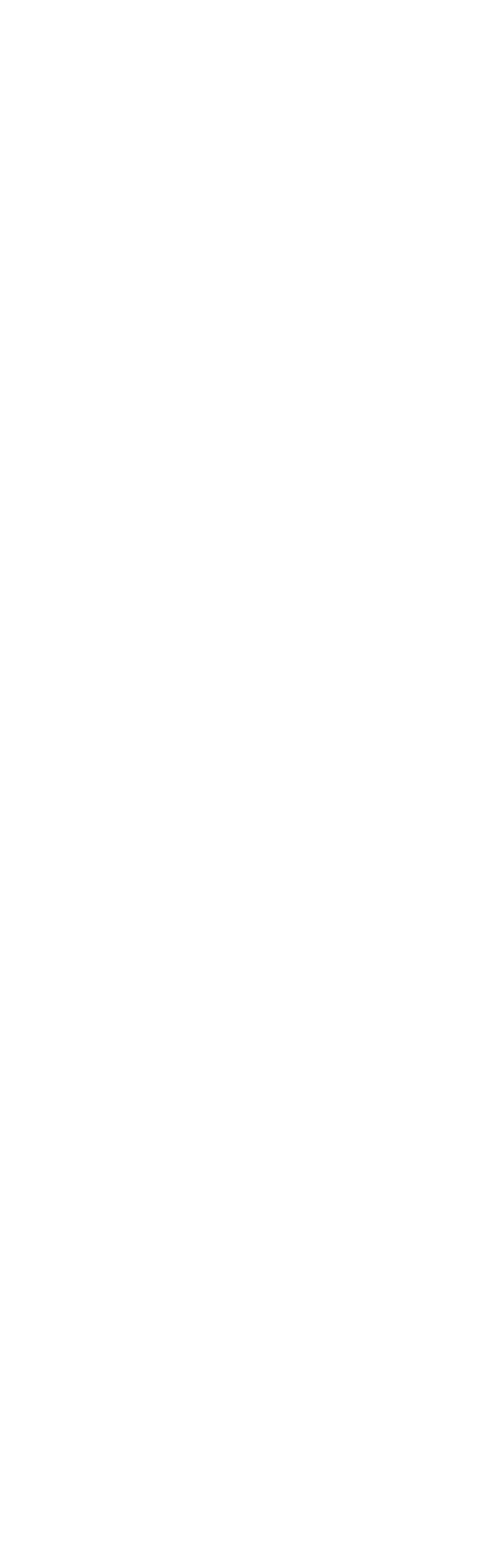




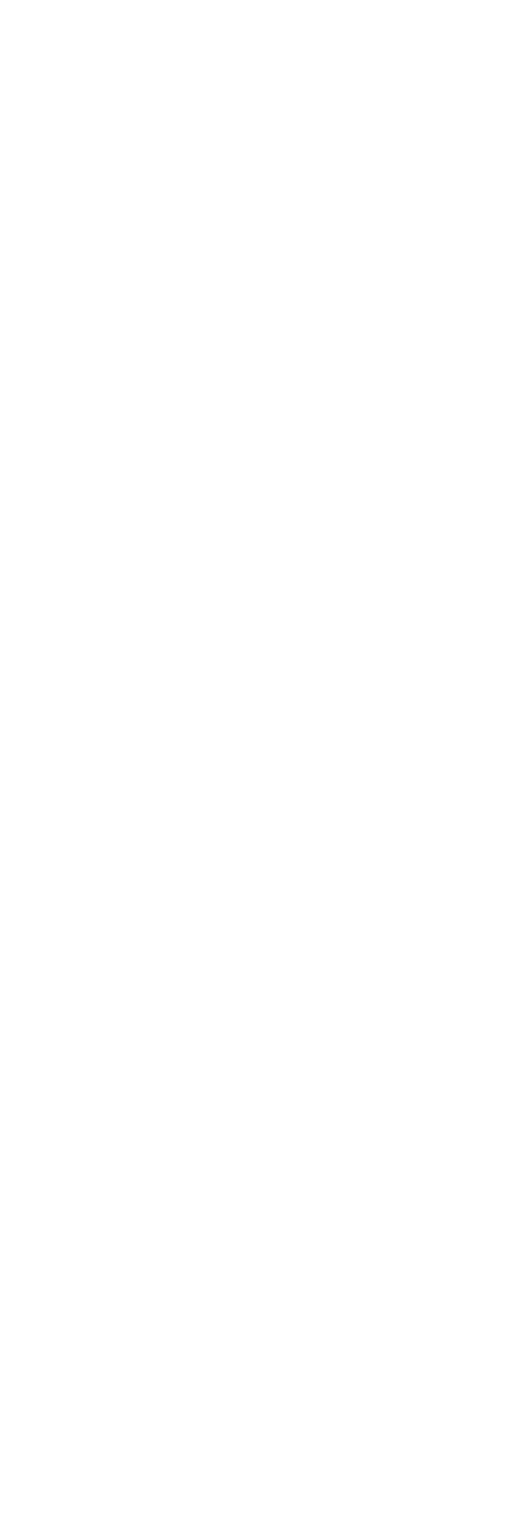




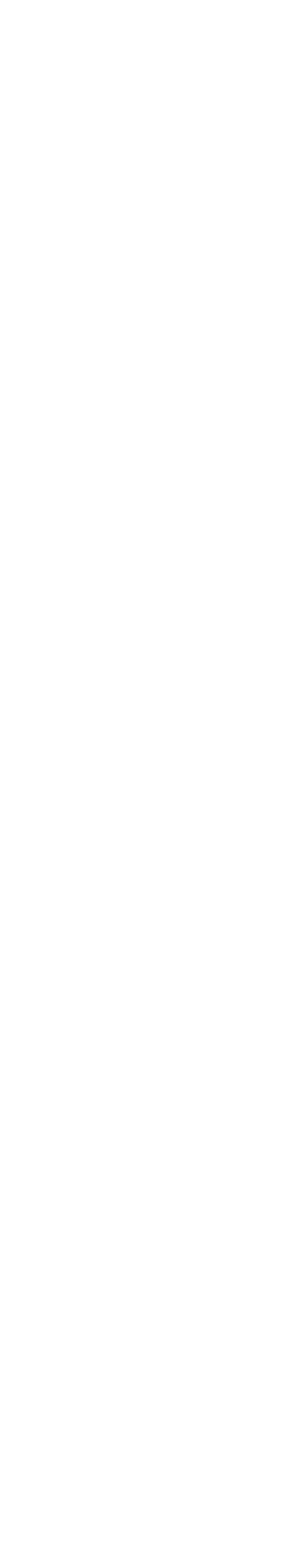

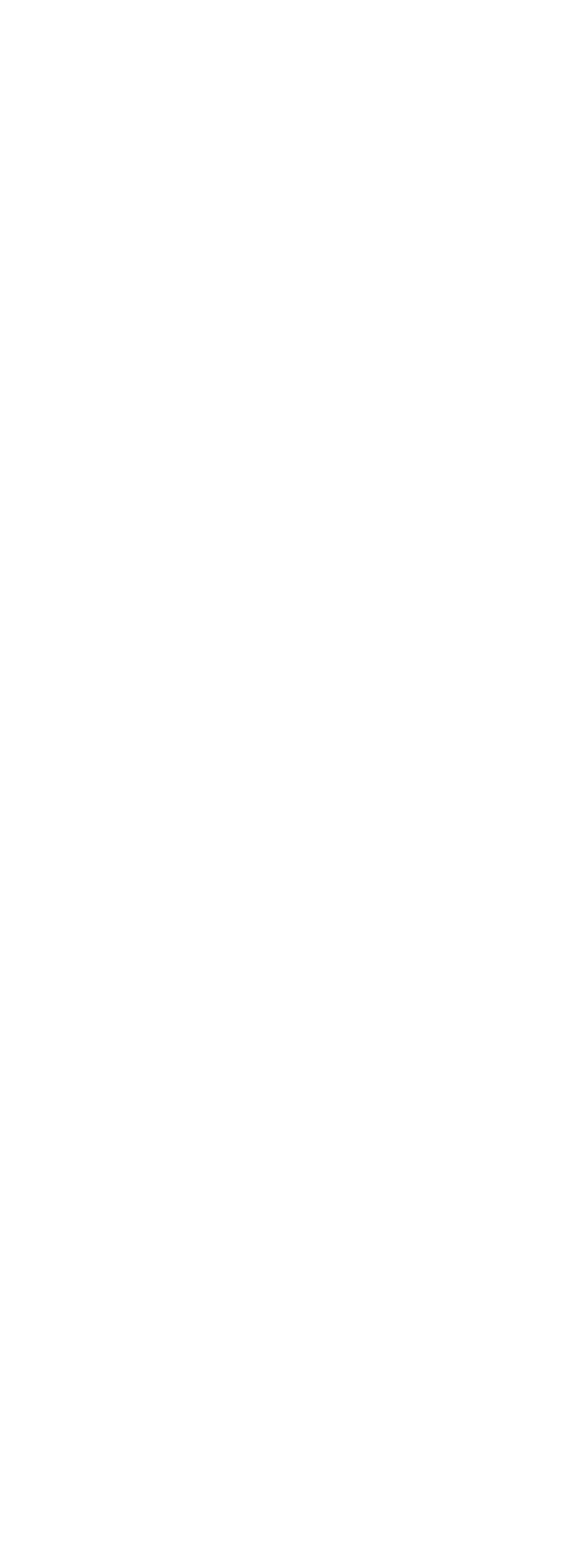




\begin{tabular}{|c|c|c|}
\hline 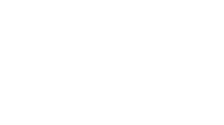 & 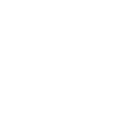 & \\
\hline 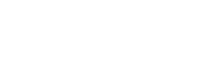 & 응유유츄 & $\stackrel{8}{\circ}$ \\
\hline 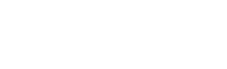 & ற주ㅇㅠㅠ: & 8 \\
\hline 0 N0000 800 & $0000 \pi$ & \% \\
\hline 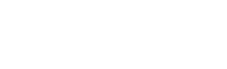 & 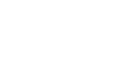 & 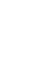 \\
\hline 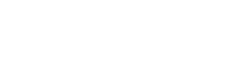 & 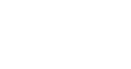 & : \\
\hline 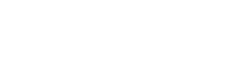 & 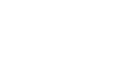 & क्ष్ \\
\hline 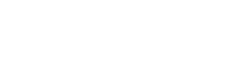 & $\begin{array}{lllll} & 1 & 1 & 1 & 1 \\
\end{array}$ & 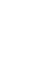 \\
\hline 1111119.11 & 11119 & 1 \\
\hline 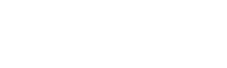 & 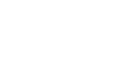 & 今. \\
\hline 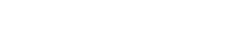 & 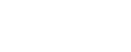 & i in \\
\hline 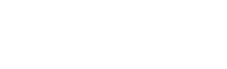 & 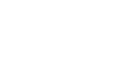 & की \\
\hline $1: 1111: 11$ & 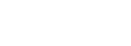 & 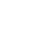 \\
\hline 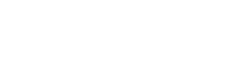 & 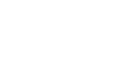 & 연 \\
\hline 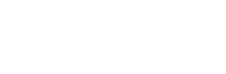 & 유유요 & 4 \\
\hline 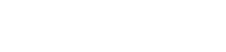 & 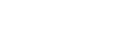 & f \\
\hline 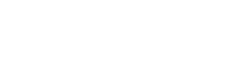 & 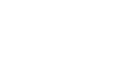 & $:$ \\
\hline 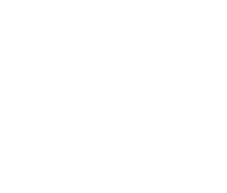 & $\vdots$ & 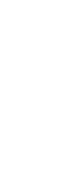 \\
\hline
\end{tabular}

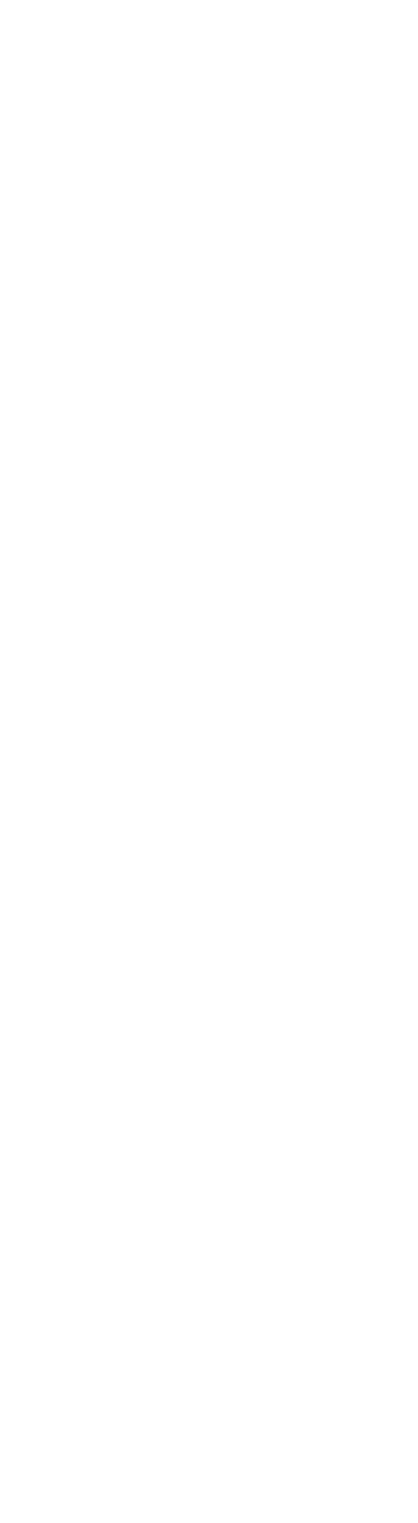




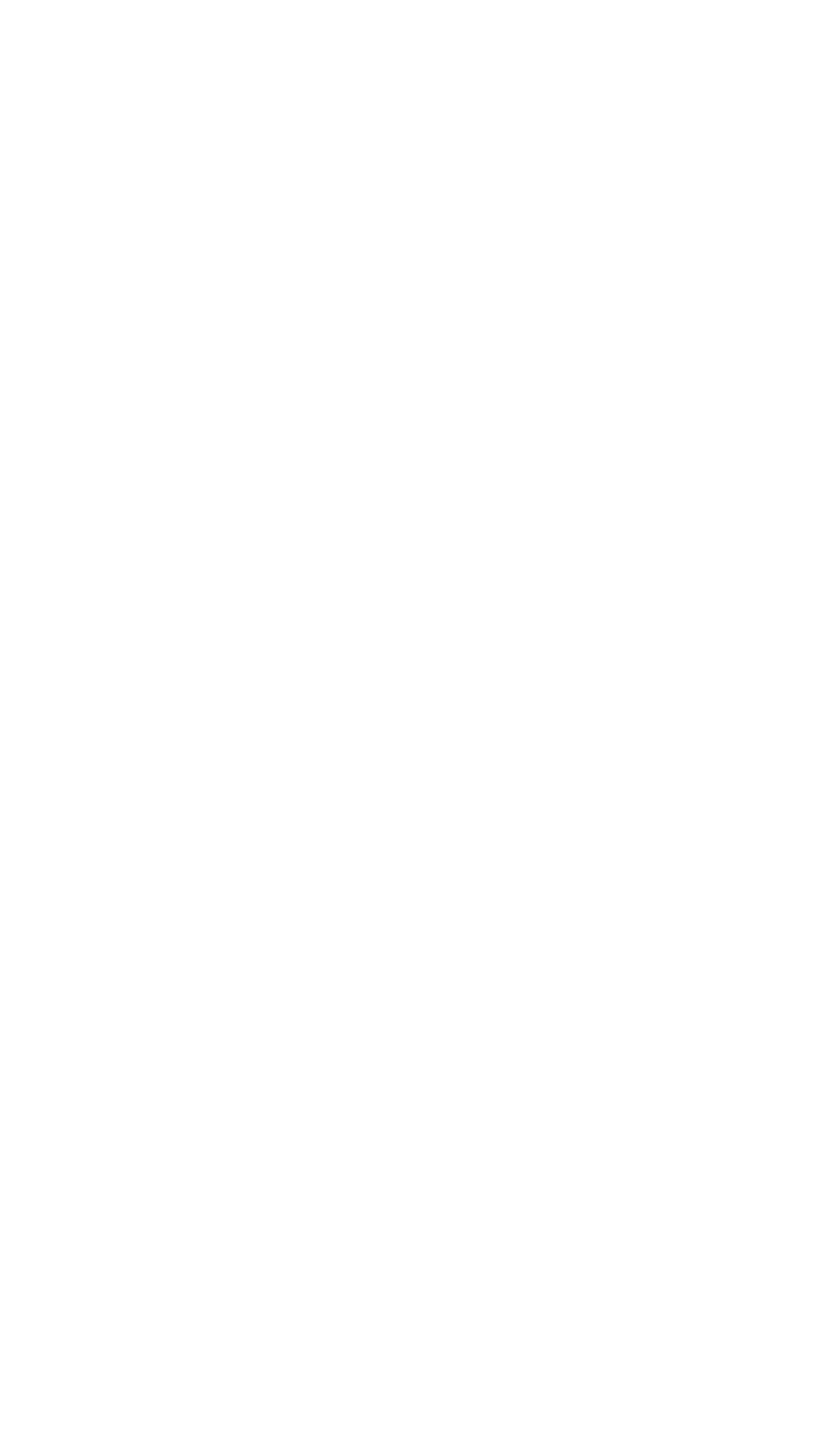



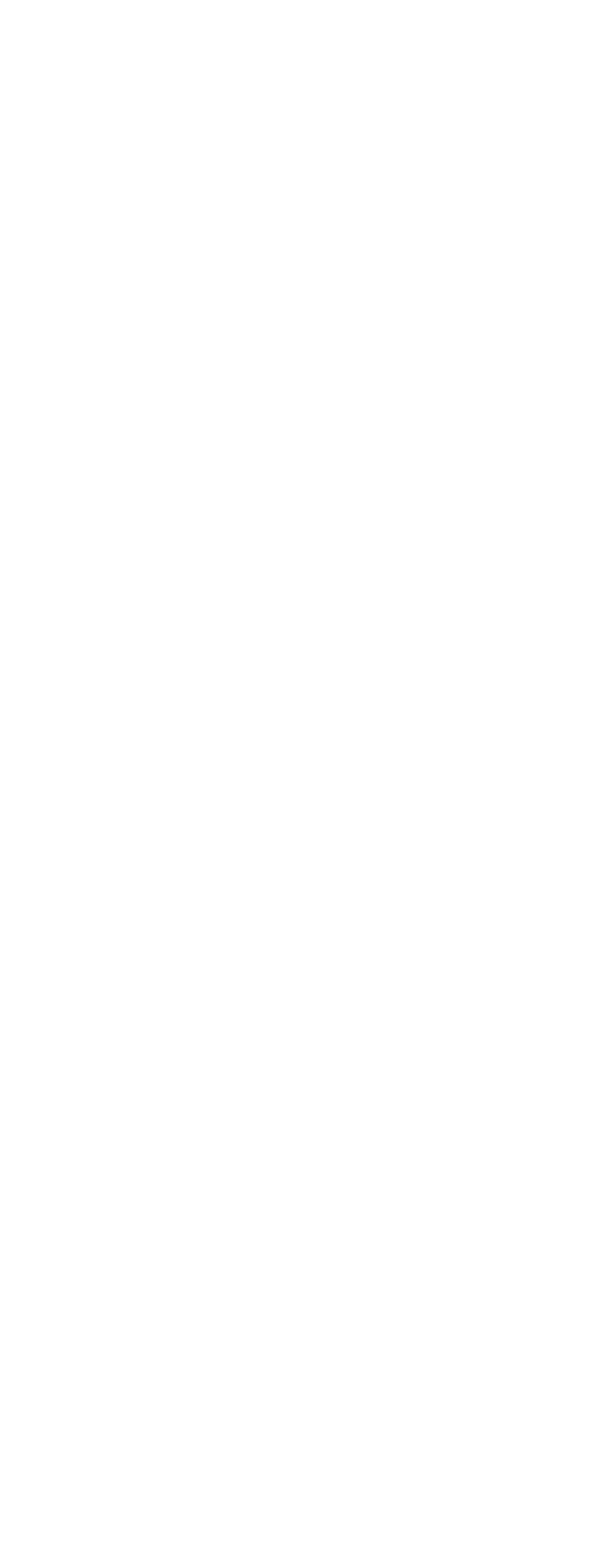


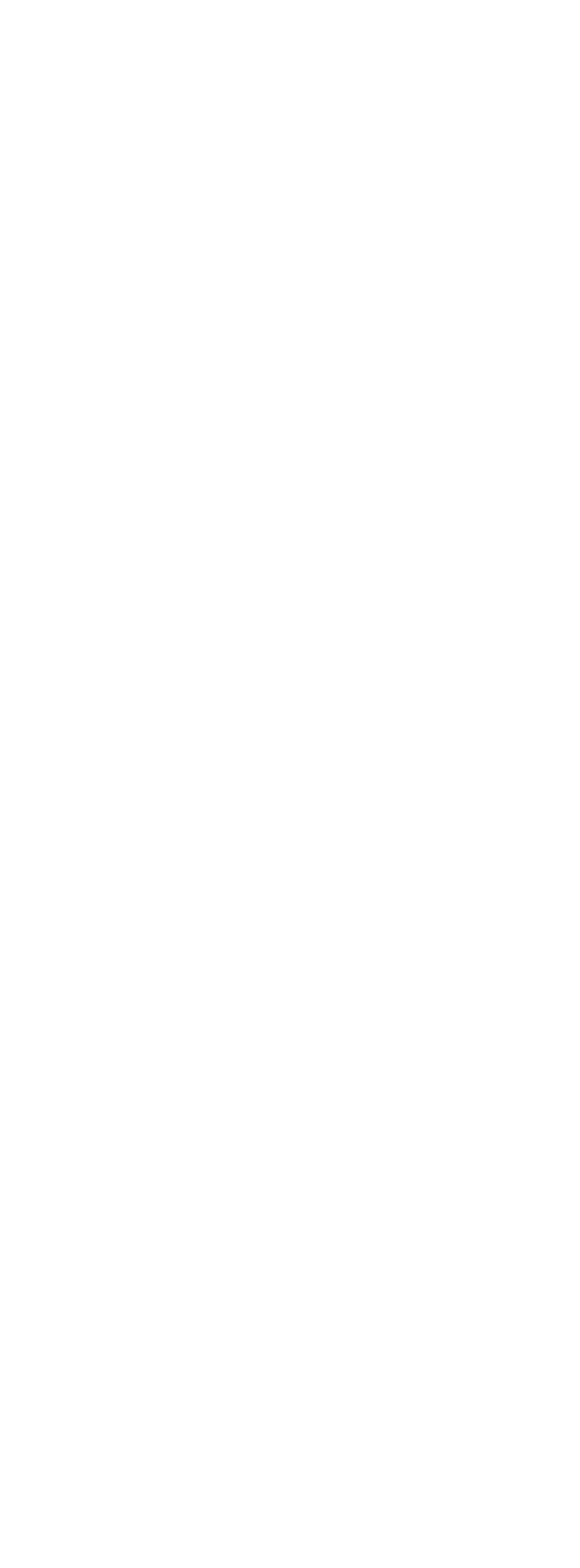




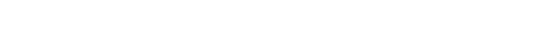

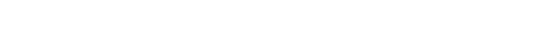

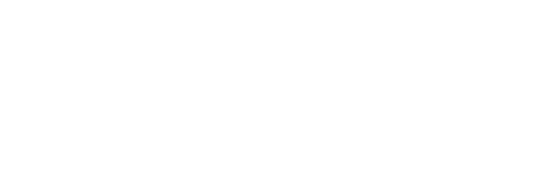

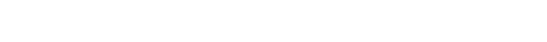

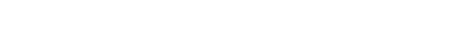

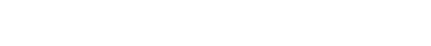

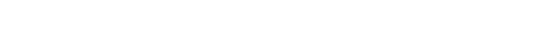

$e_{\infty}^{4}$

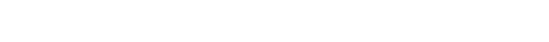

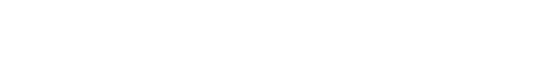
भु్ન

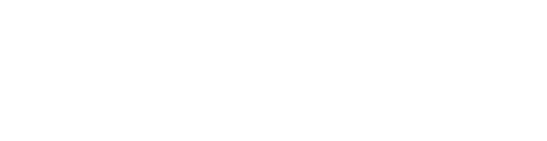

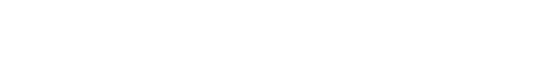

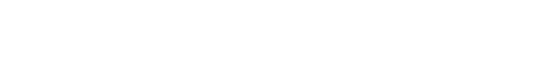

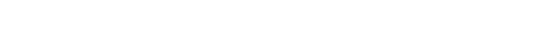

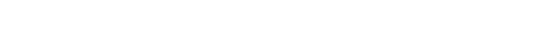

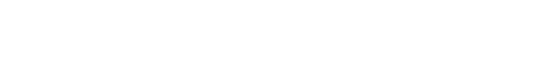

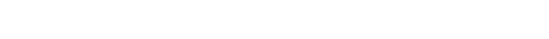

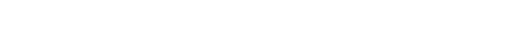

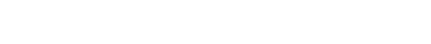

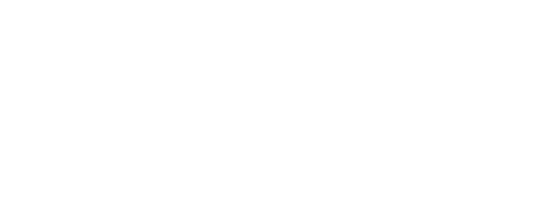

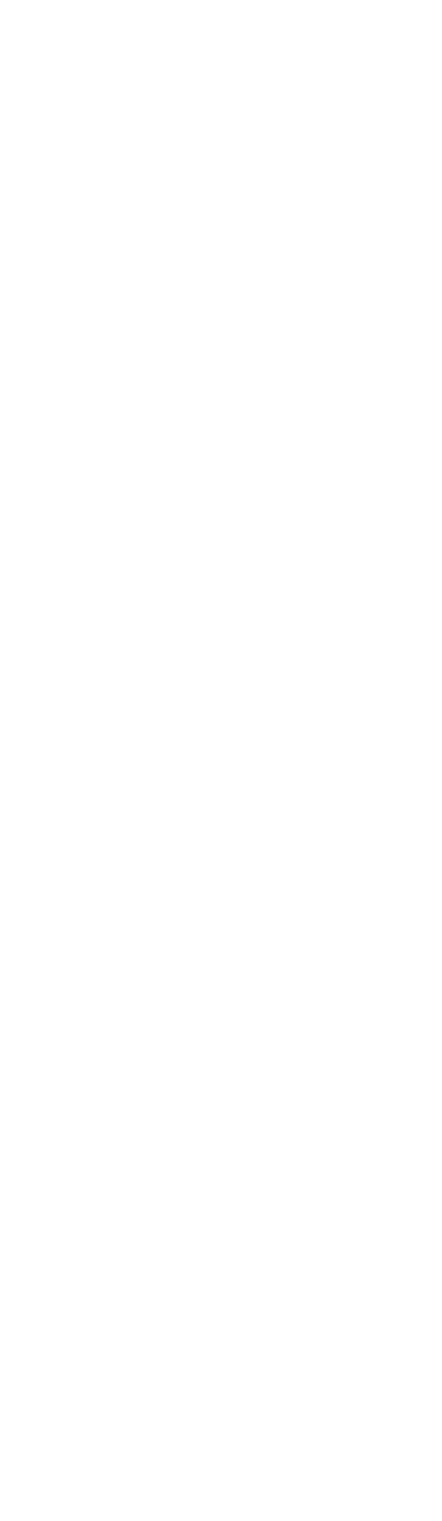




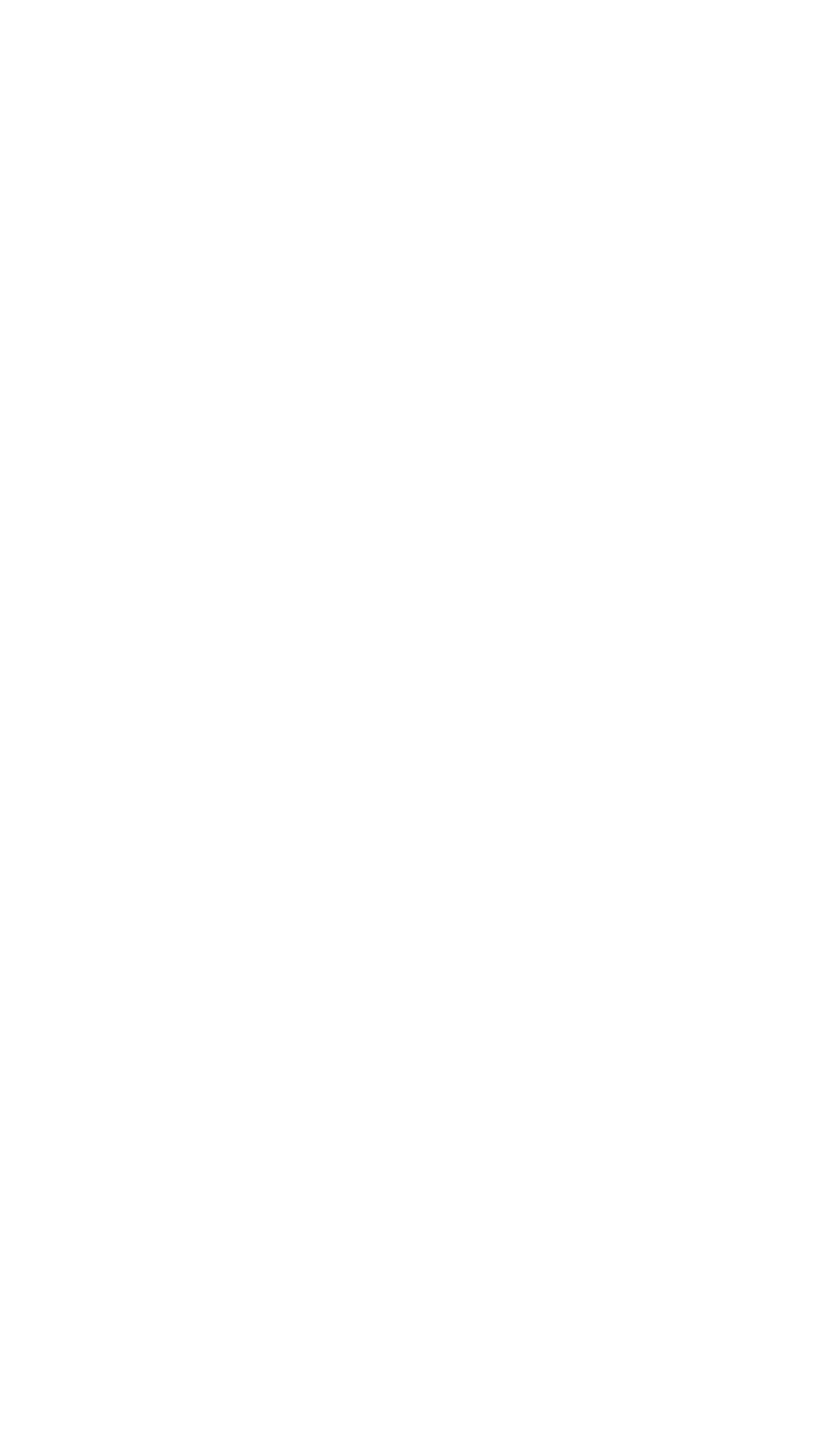




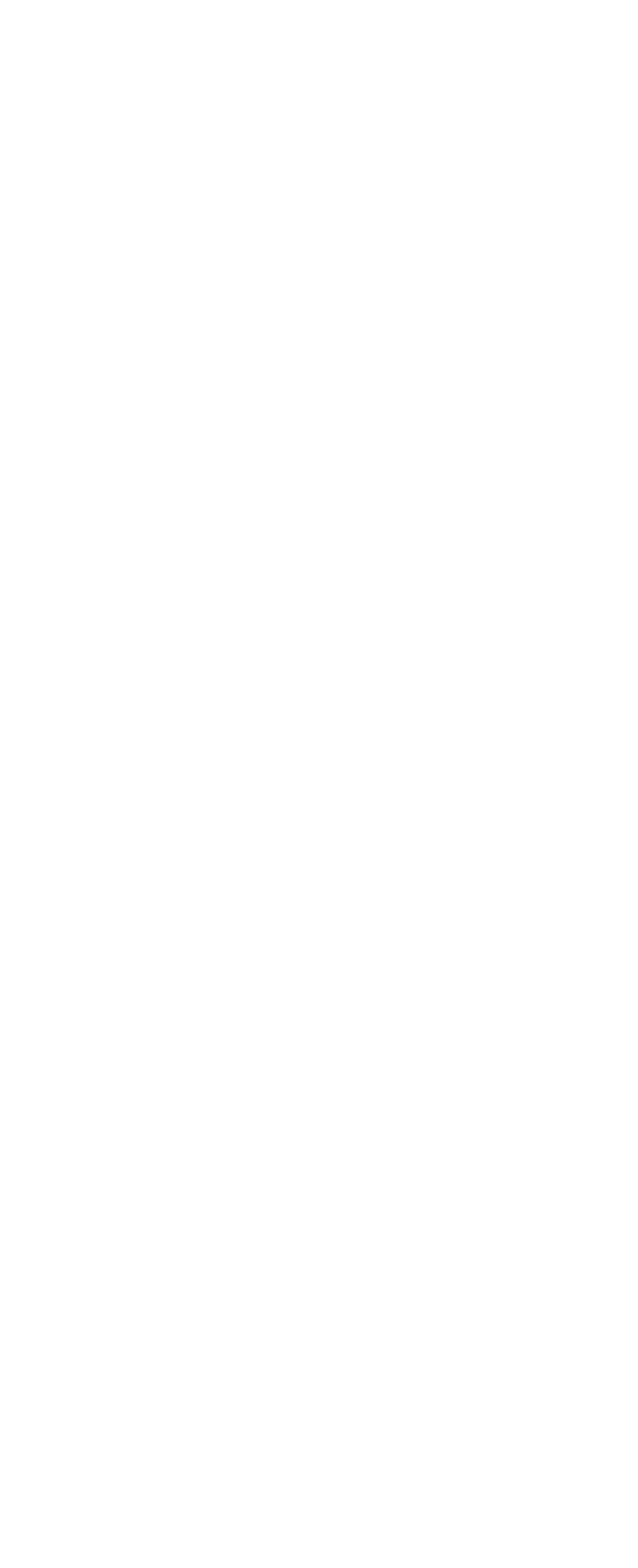




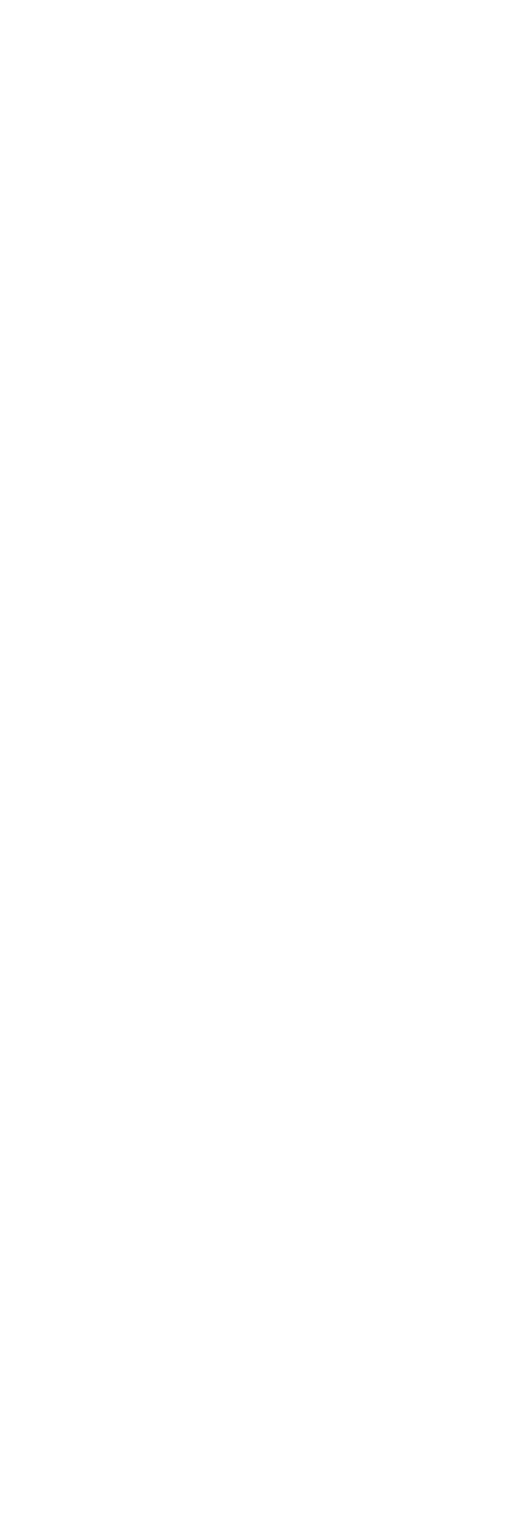




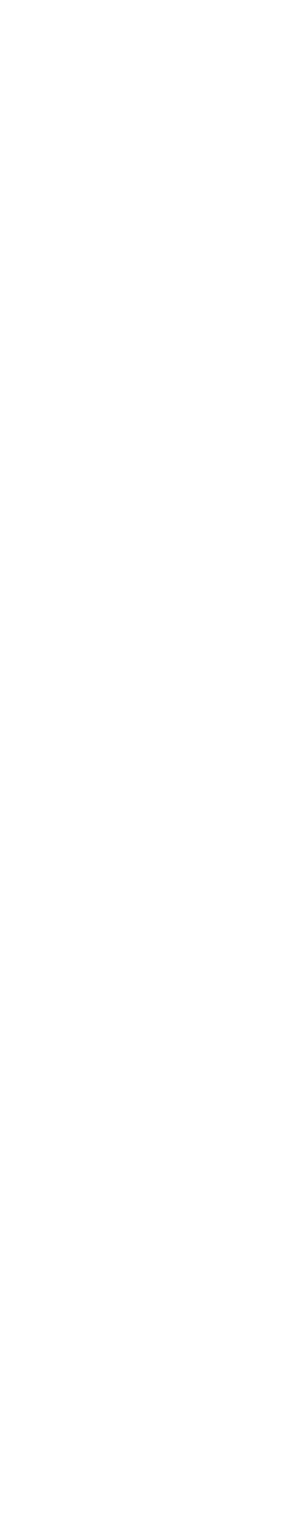

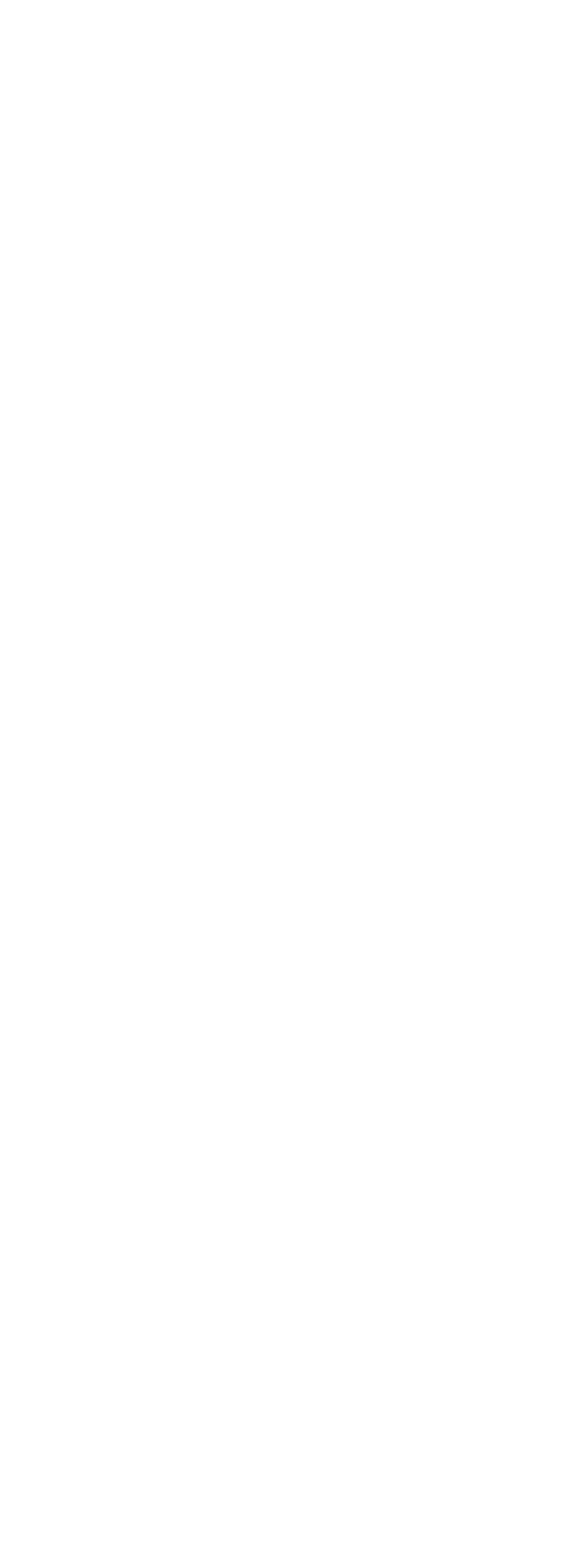




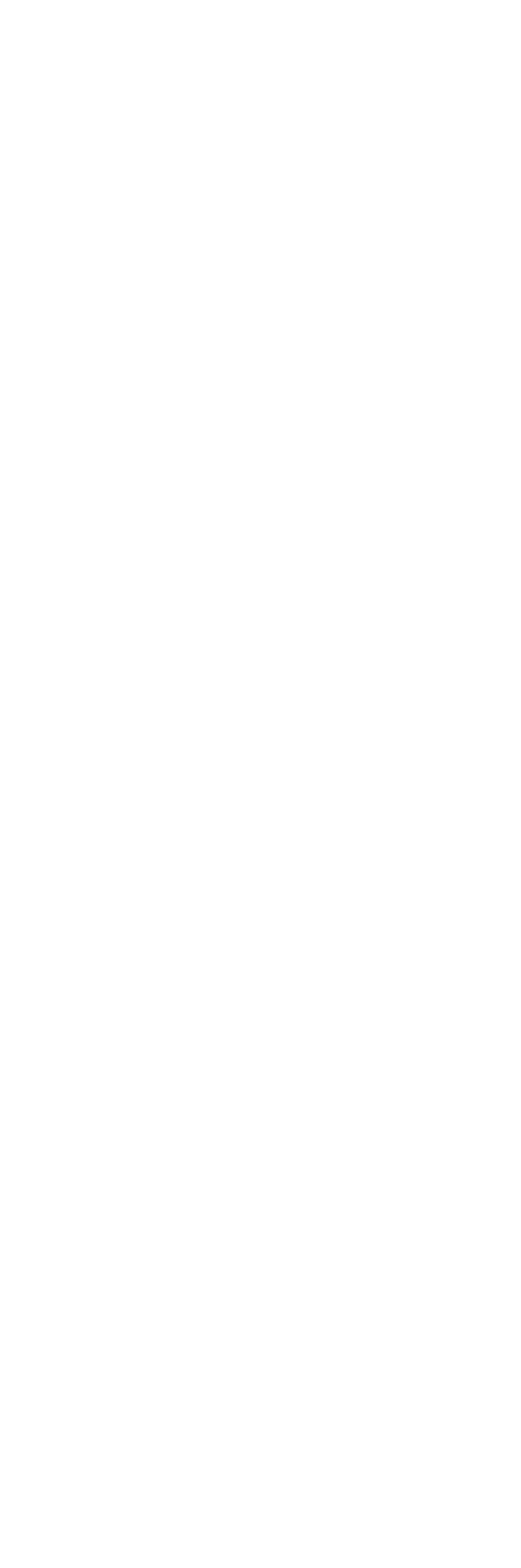




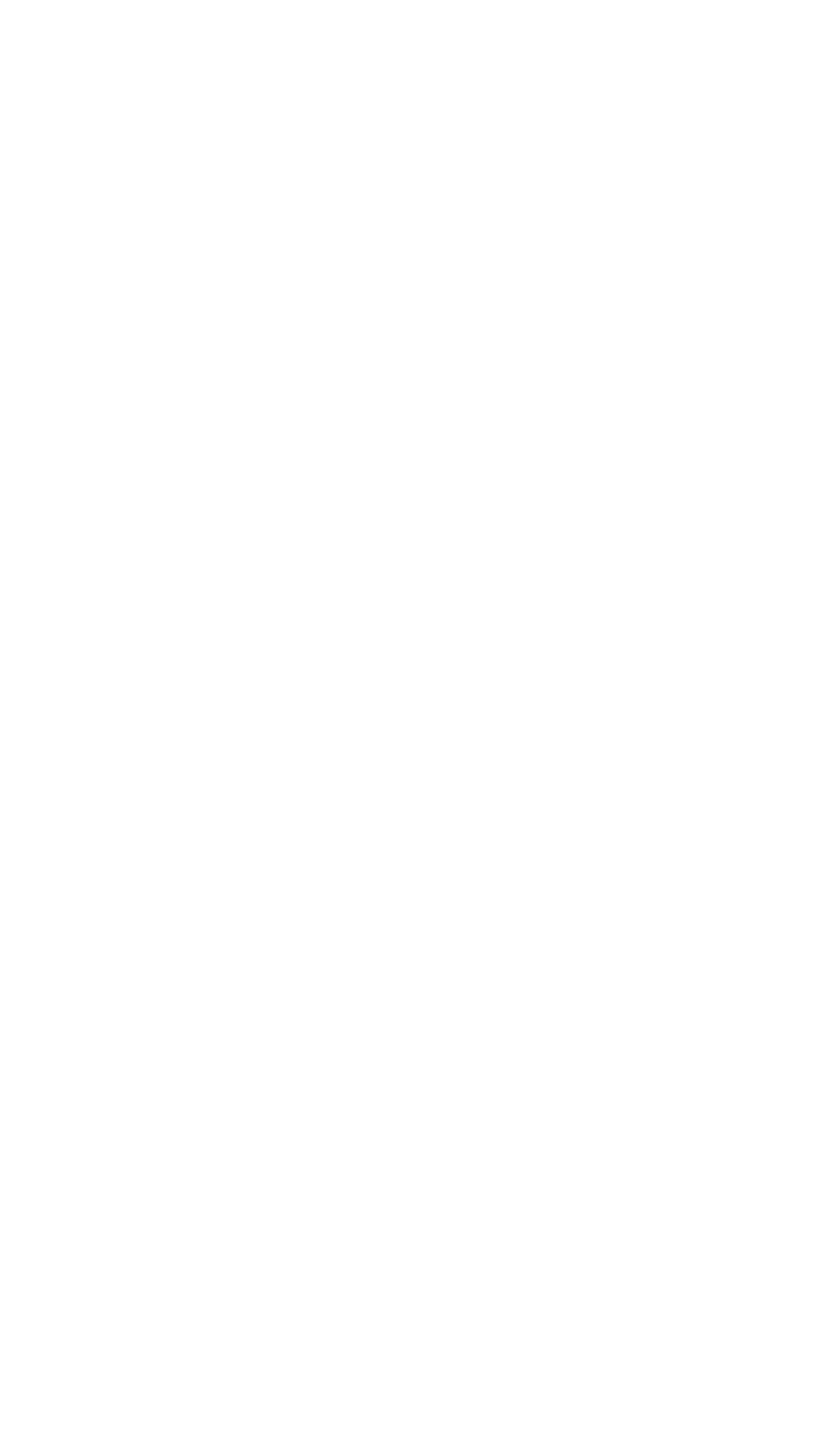




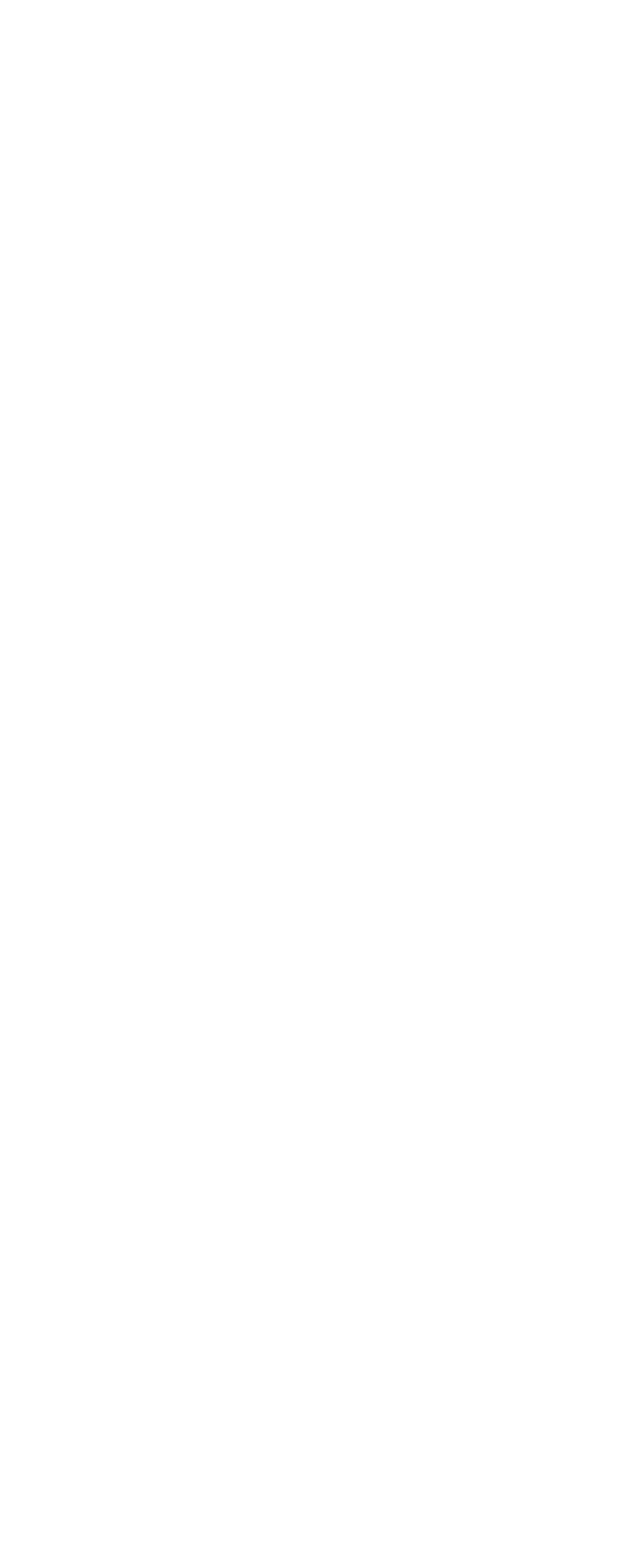




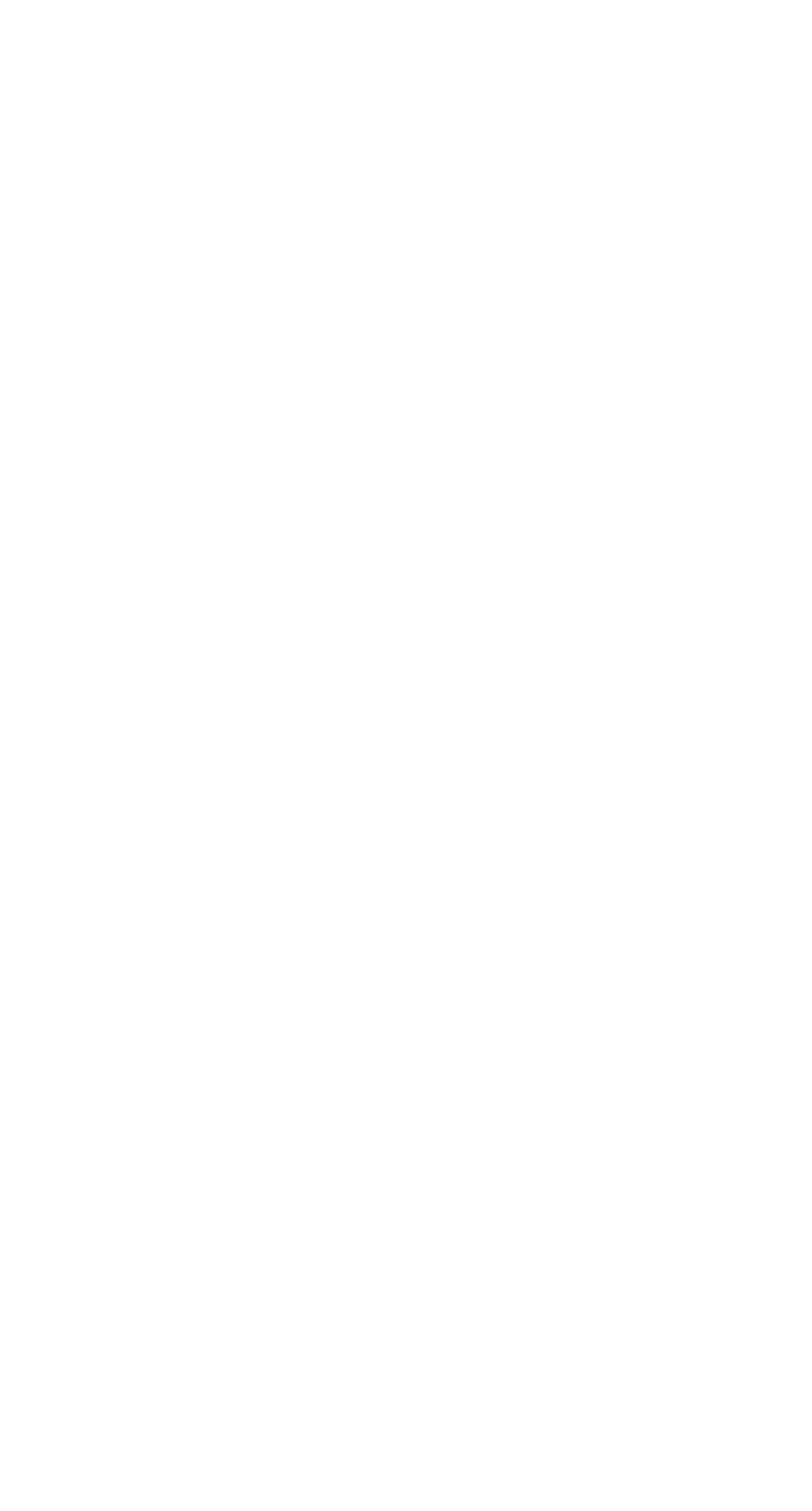




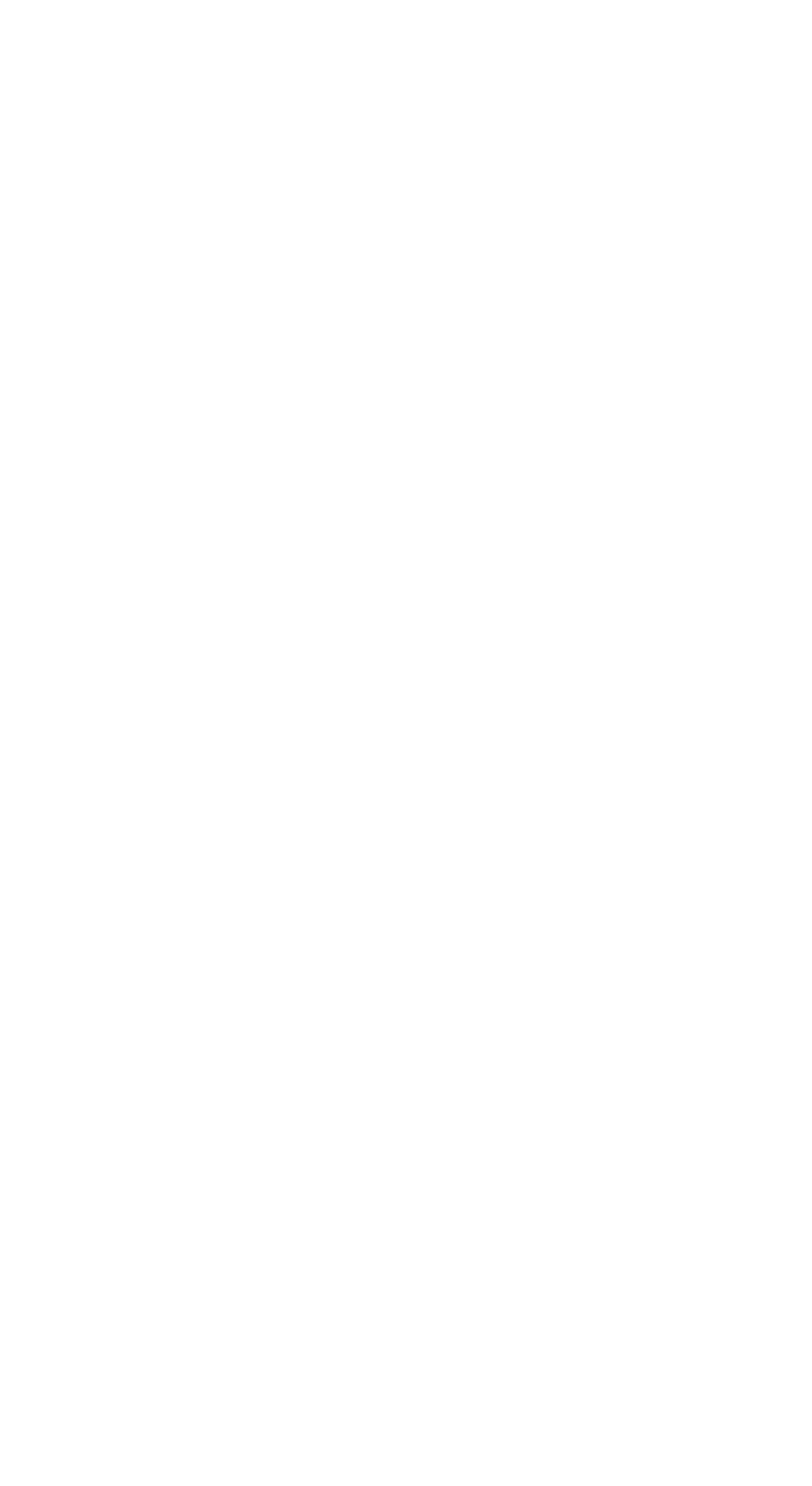




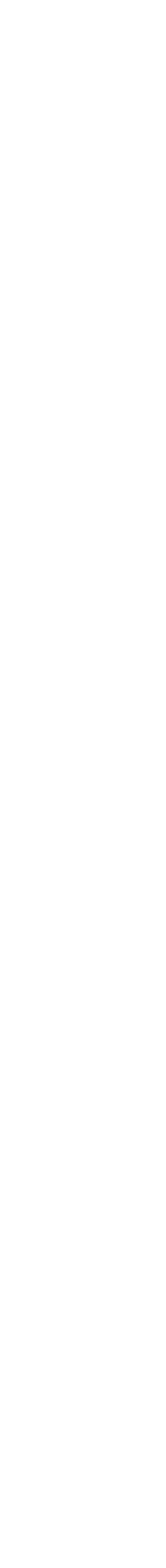




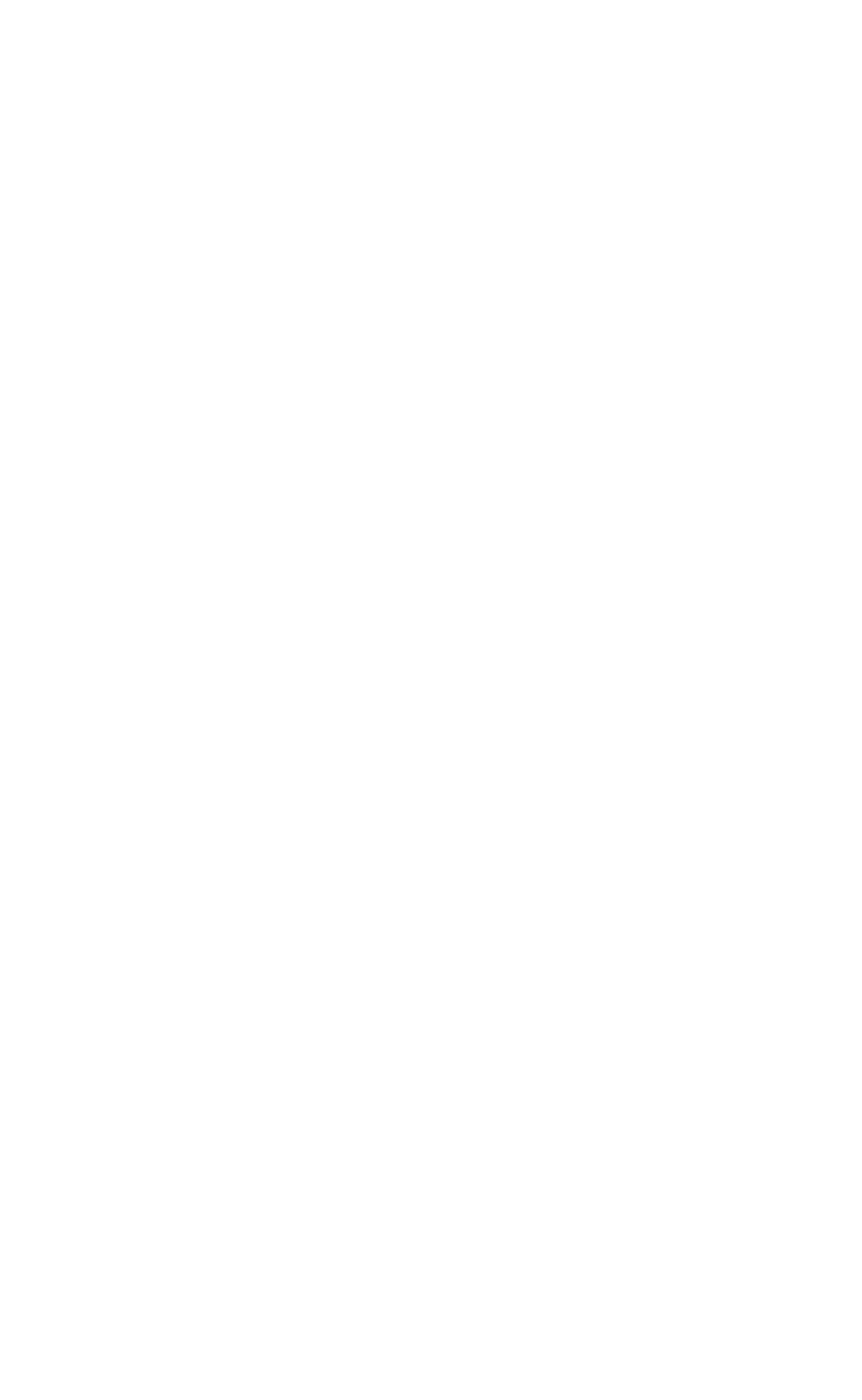




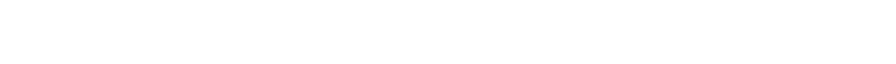

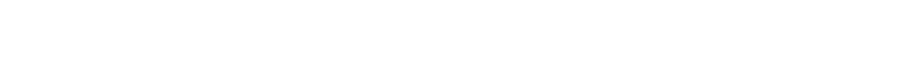

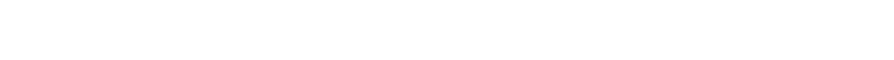

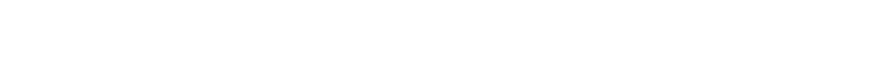

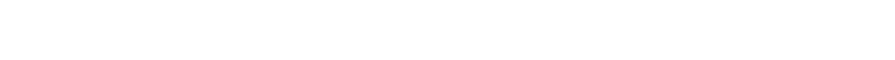

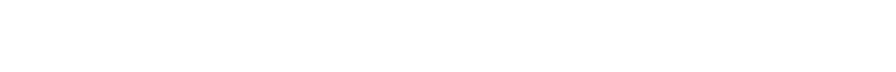

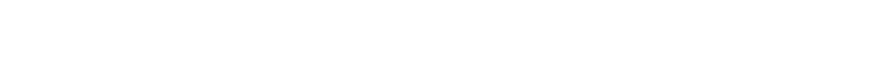

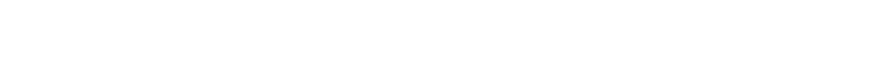

苛

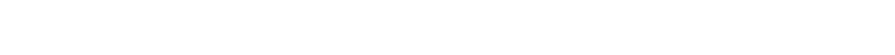

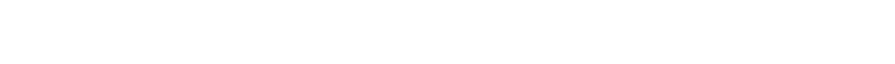




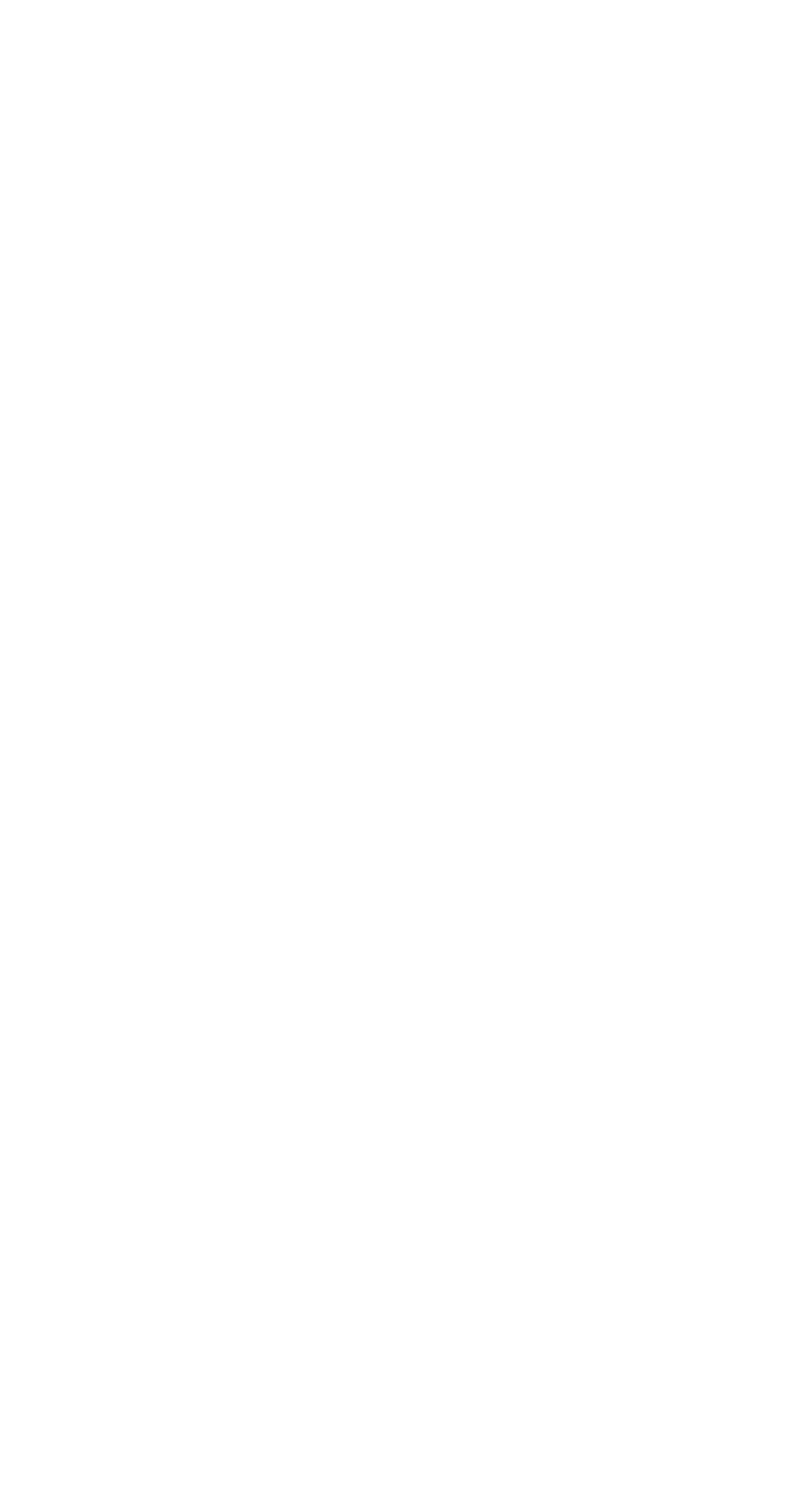




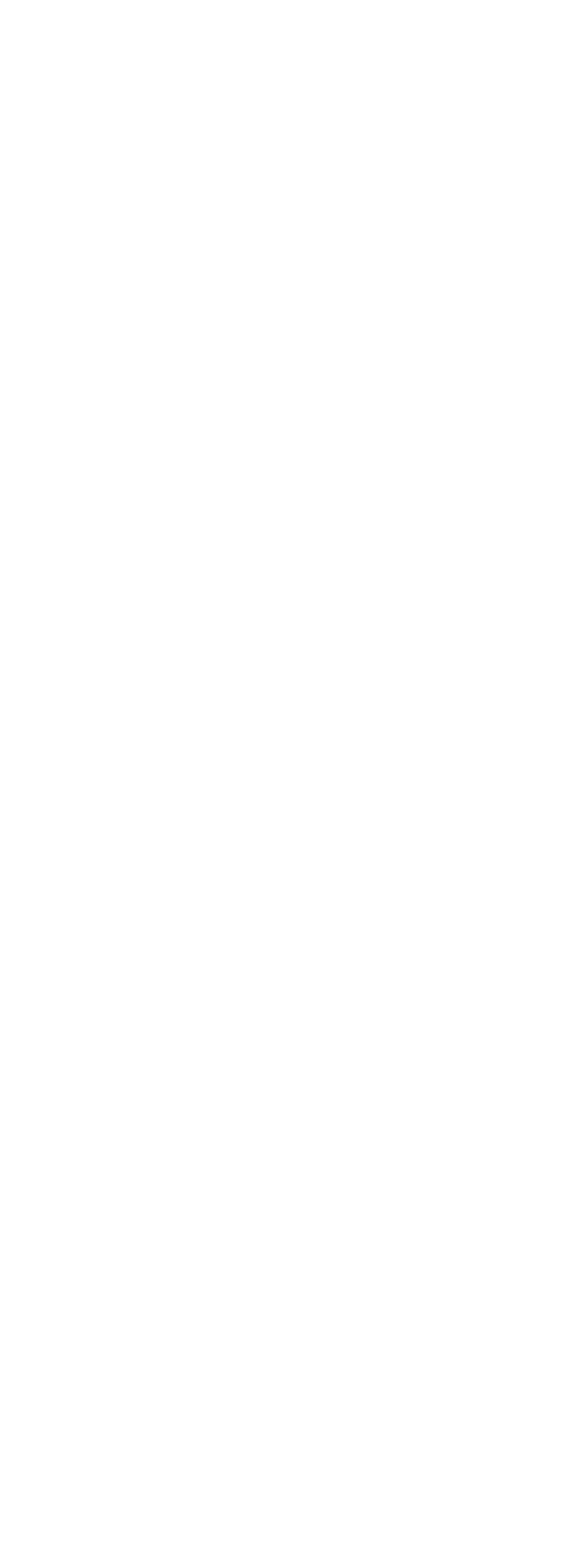




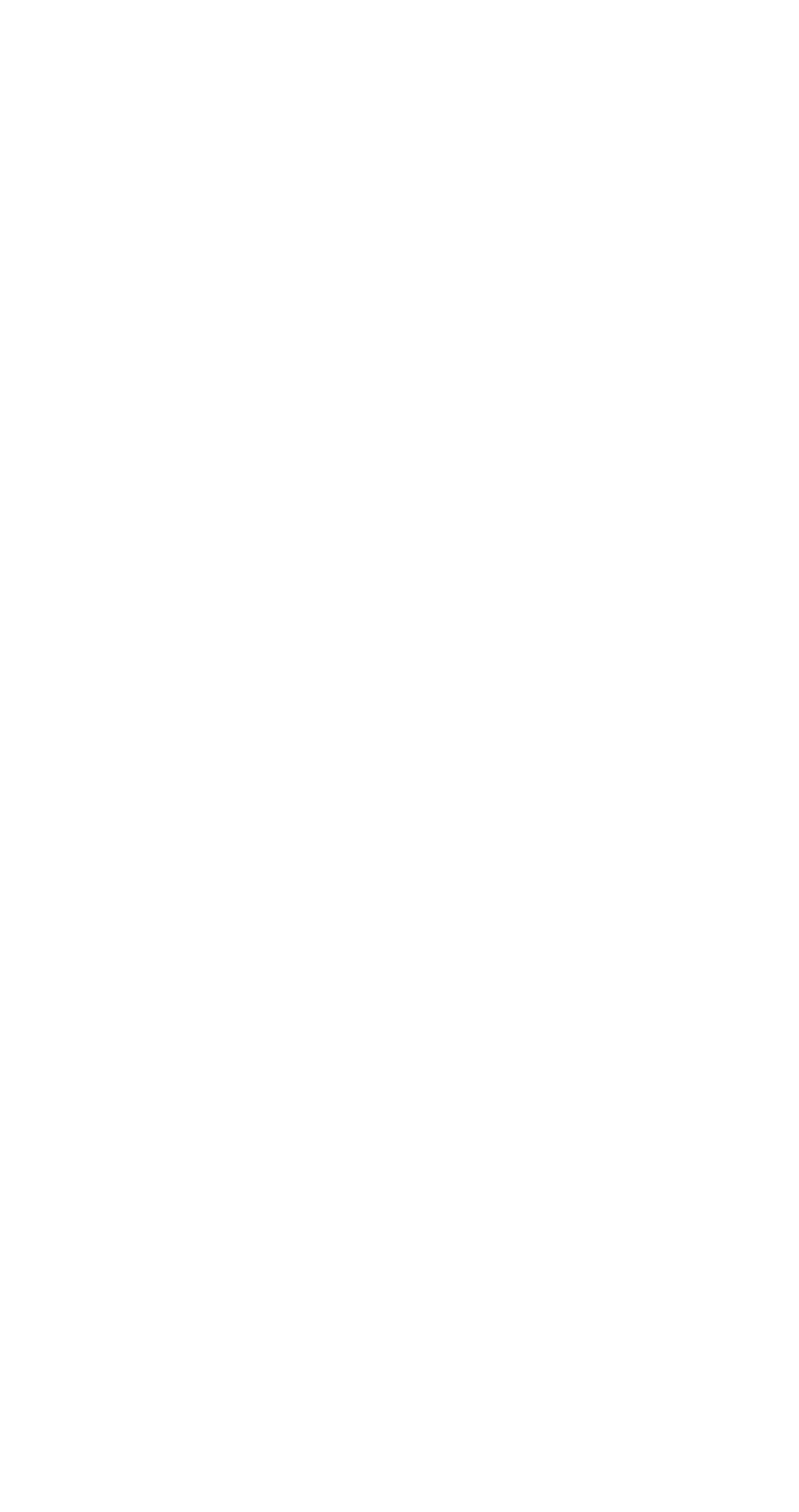




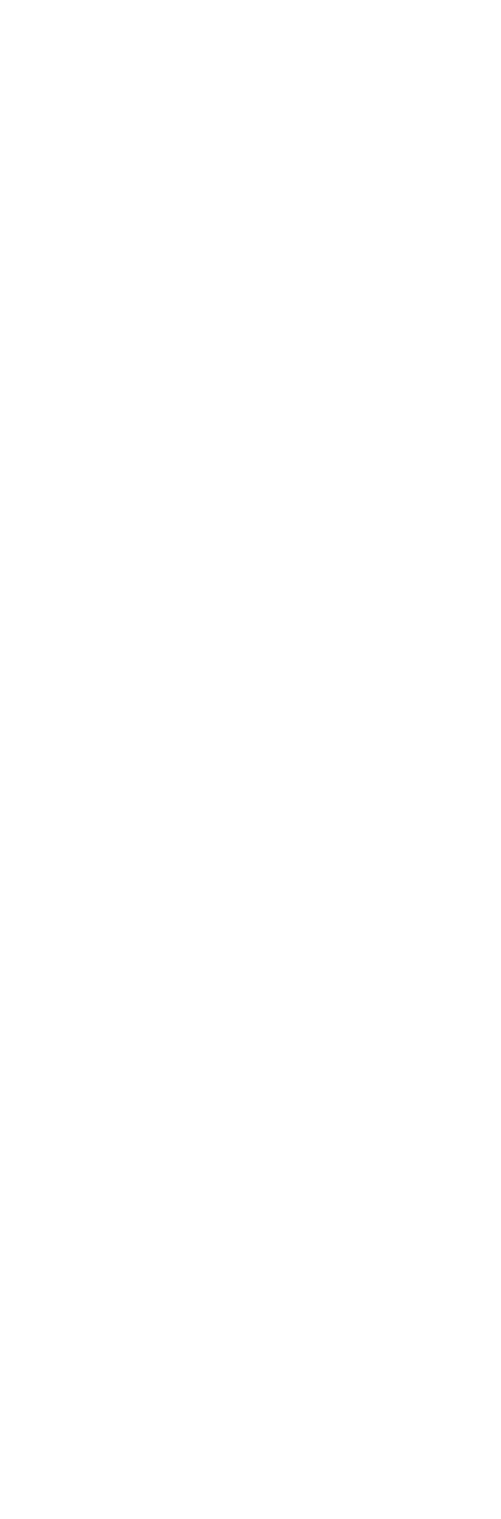




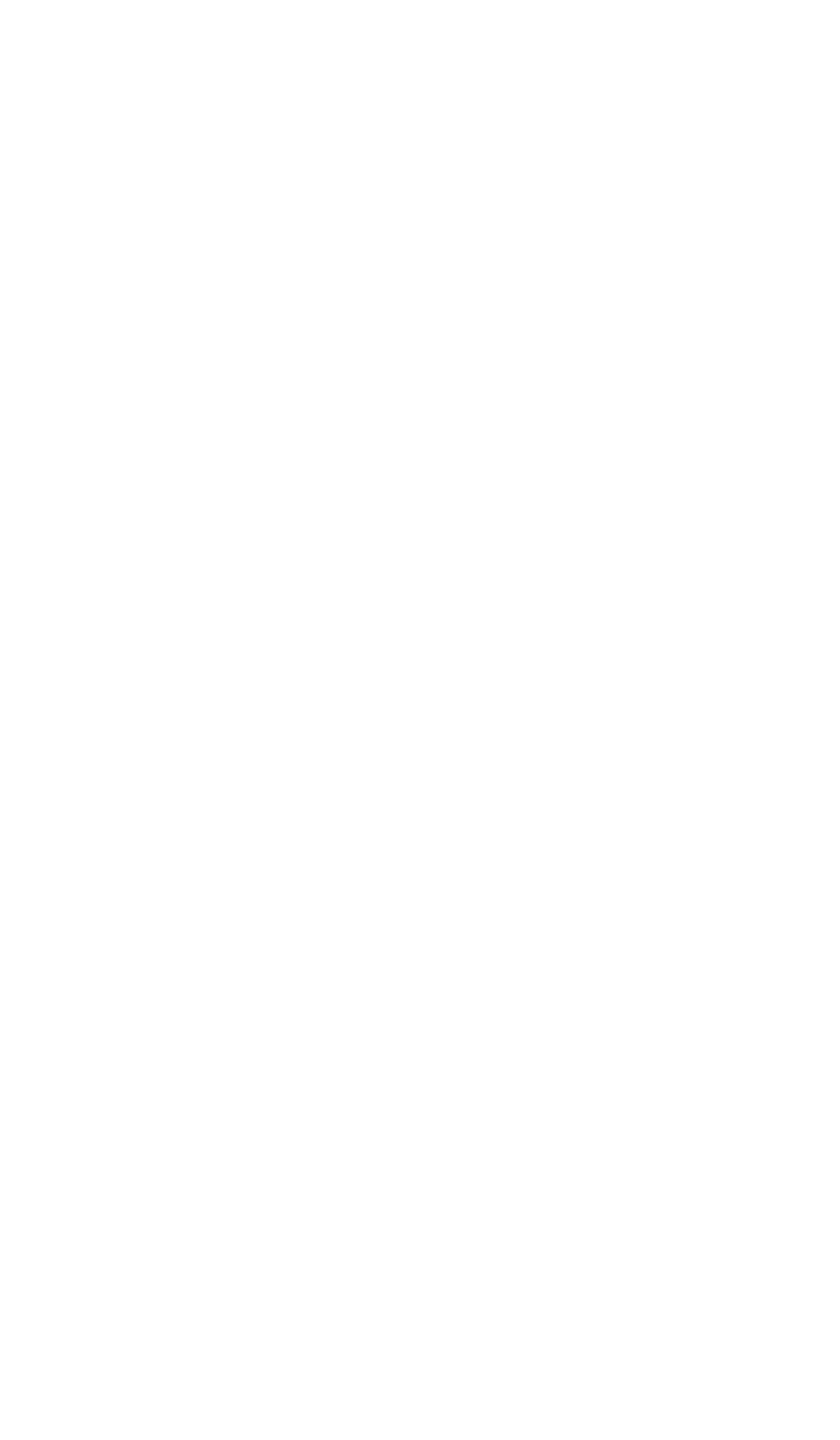




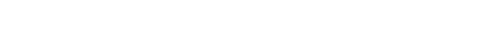

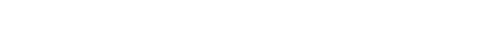

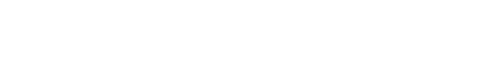

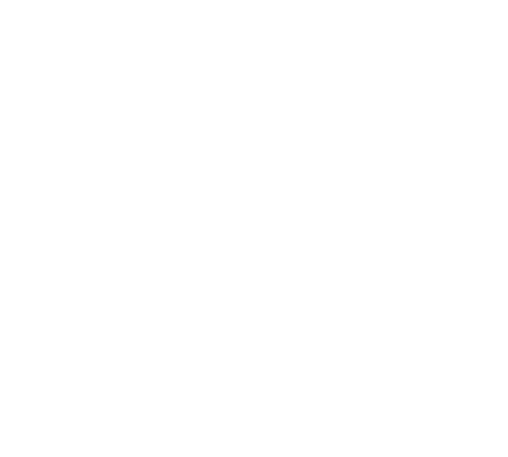

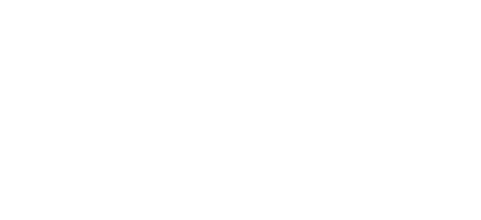

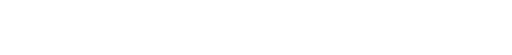

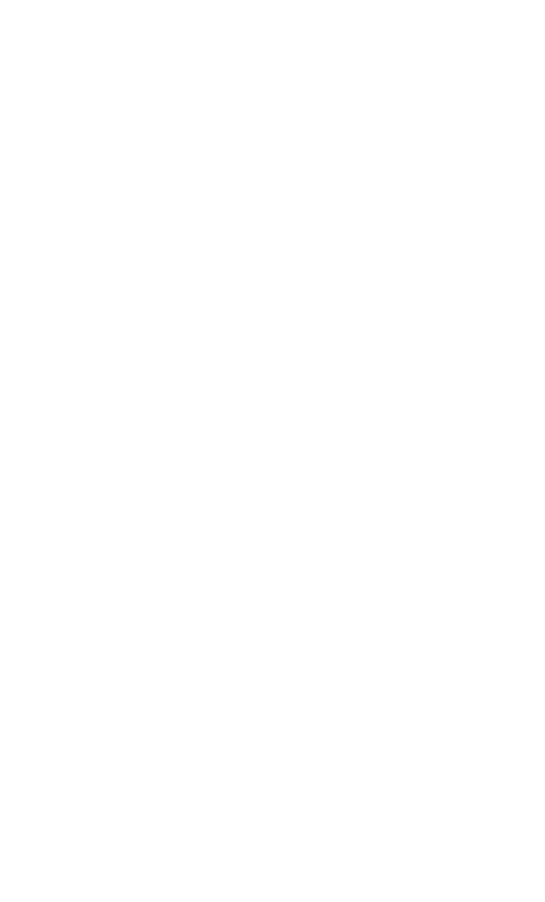

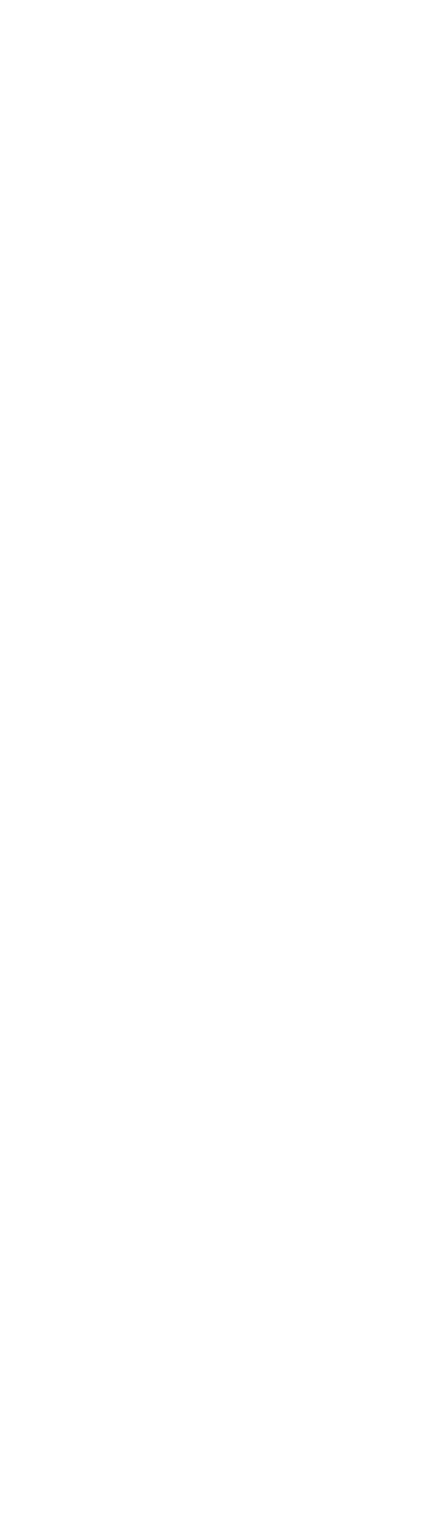




\begin{tabular}{|c|c|}
\hline 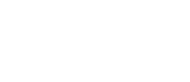 & 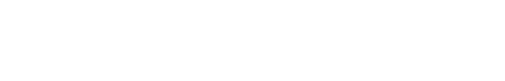 \\
\hline 녕 & 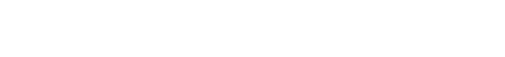 \\
\hline 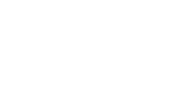 & 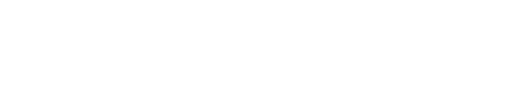 \\
\hline 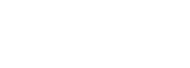 & 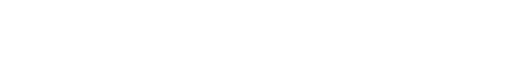 \\
\hline 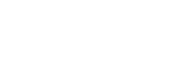 & 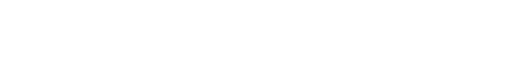 \\
\hline 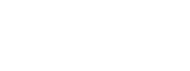 & 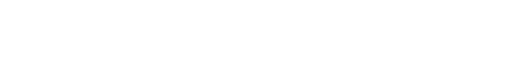 \\
\hline 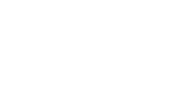 & 势 \\
\hline 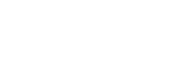 & 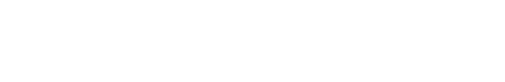 \\
\hline 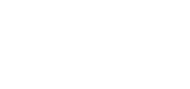 & 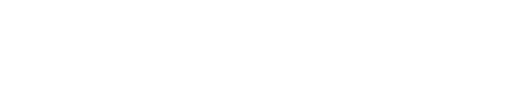 \\
\hline 嵩㤐鱼 & \\
\hline$\frac{1}{\bar{z}}$ & 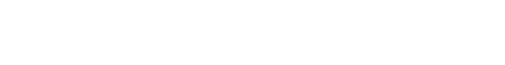 \\
\hline 产咅国 & \\
\hline 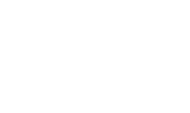 & 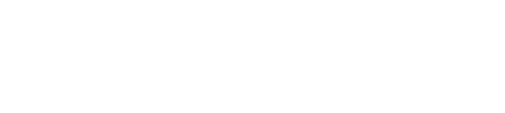 \\
\hline 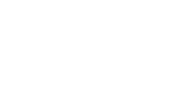 & 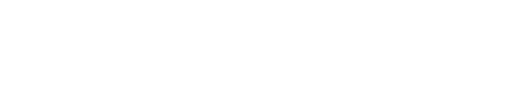 \\
\hline 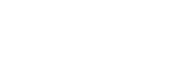 & 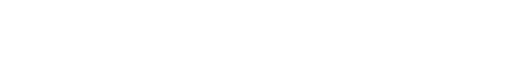 \\
\hline 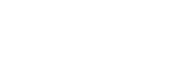 & 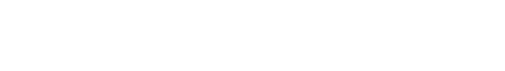 \\
\hline 量要 & 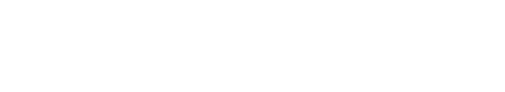 \\
\hline 言引包 & \\
\hline 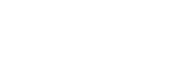 & 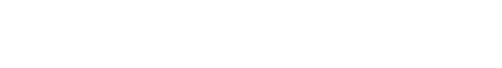 \\
\hline 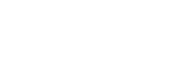 & 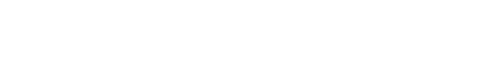 \\
\hline 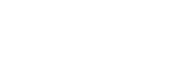 & \\
\hline 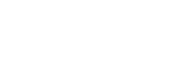 & बन. \\
\hline 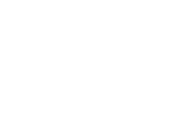 & 我 \\
\hline 胥 & 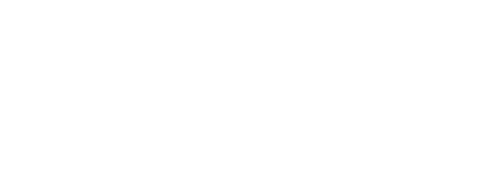 \\
\hline
\end{tabular}




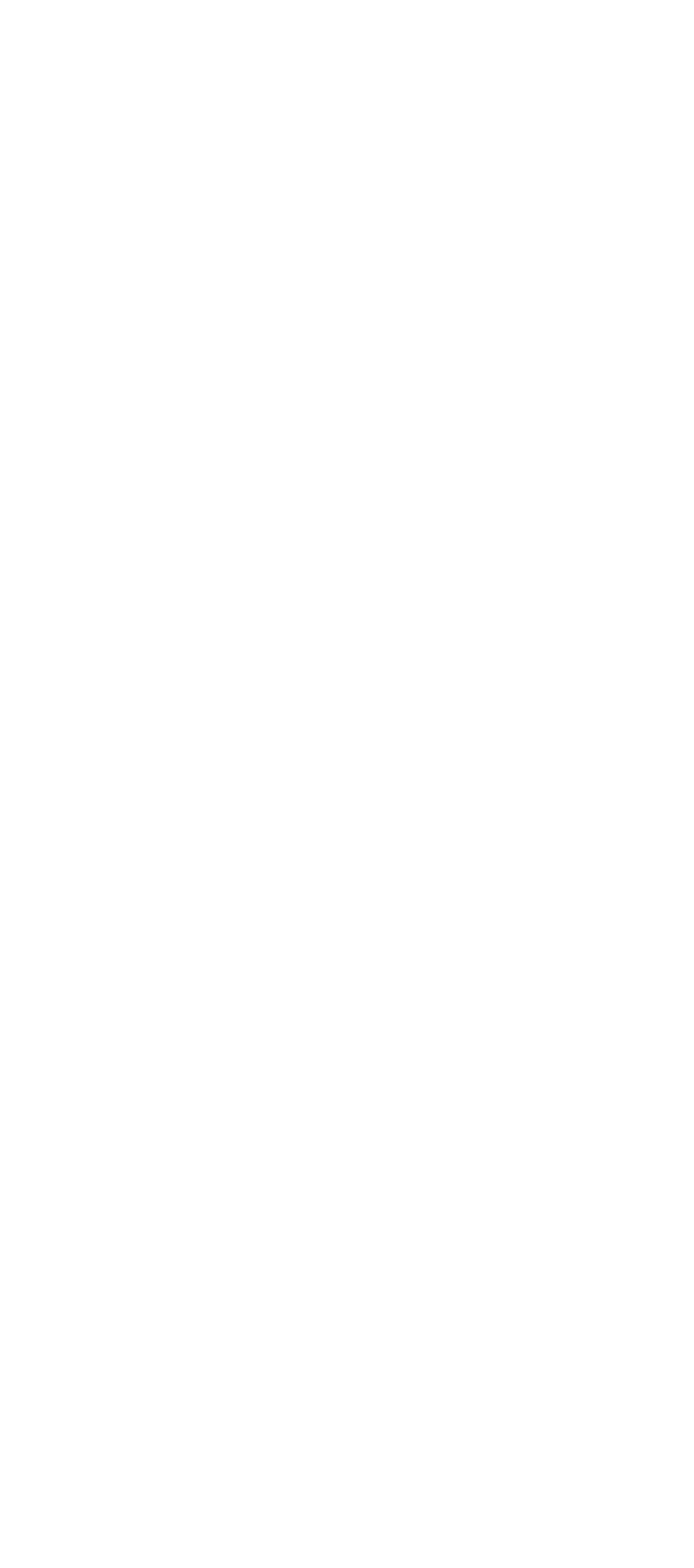




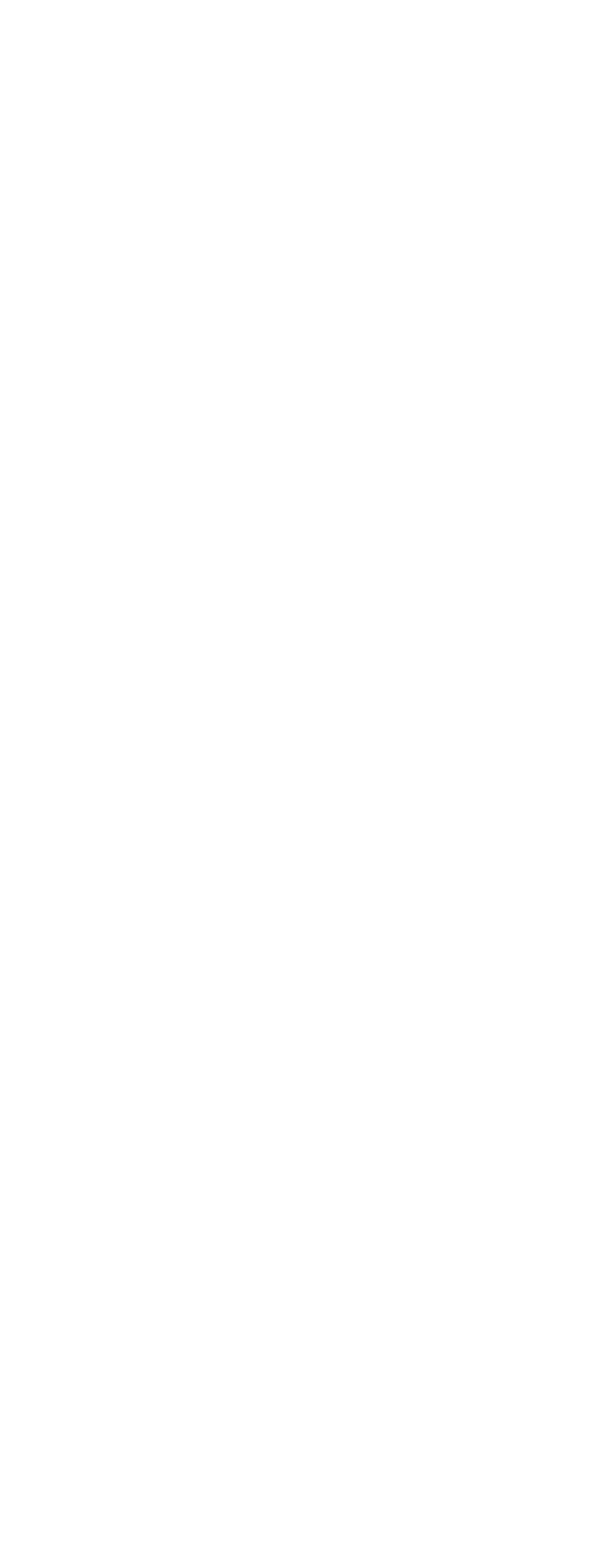




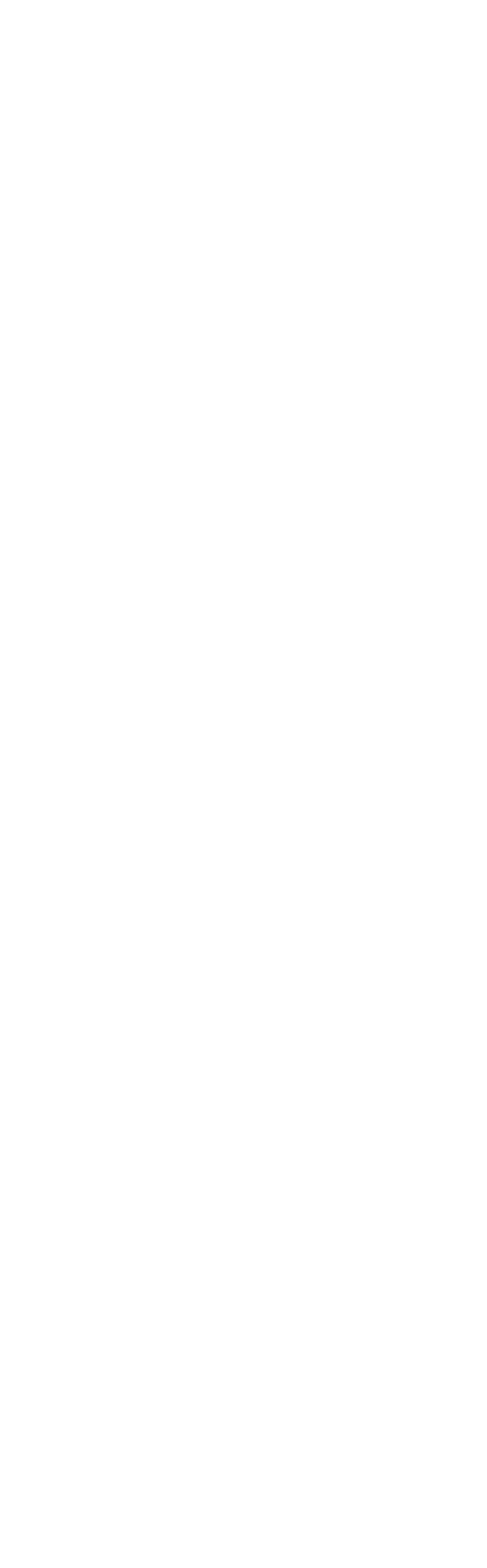




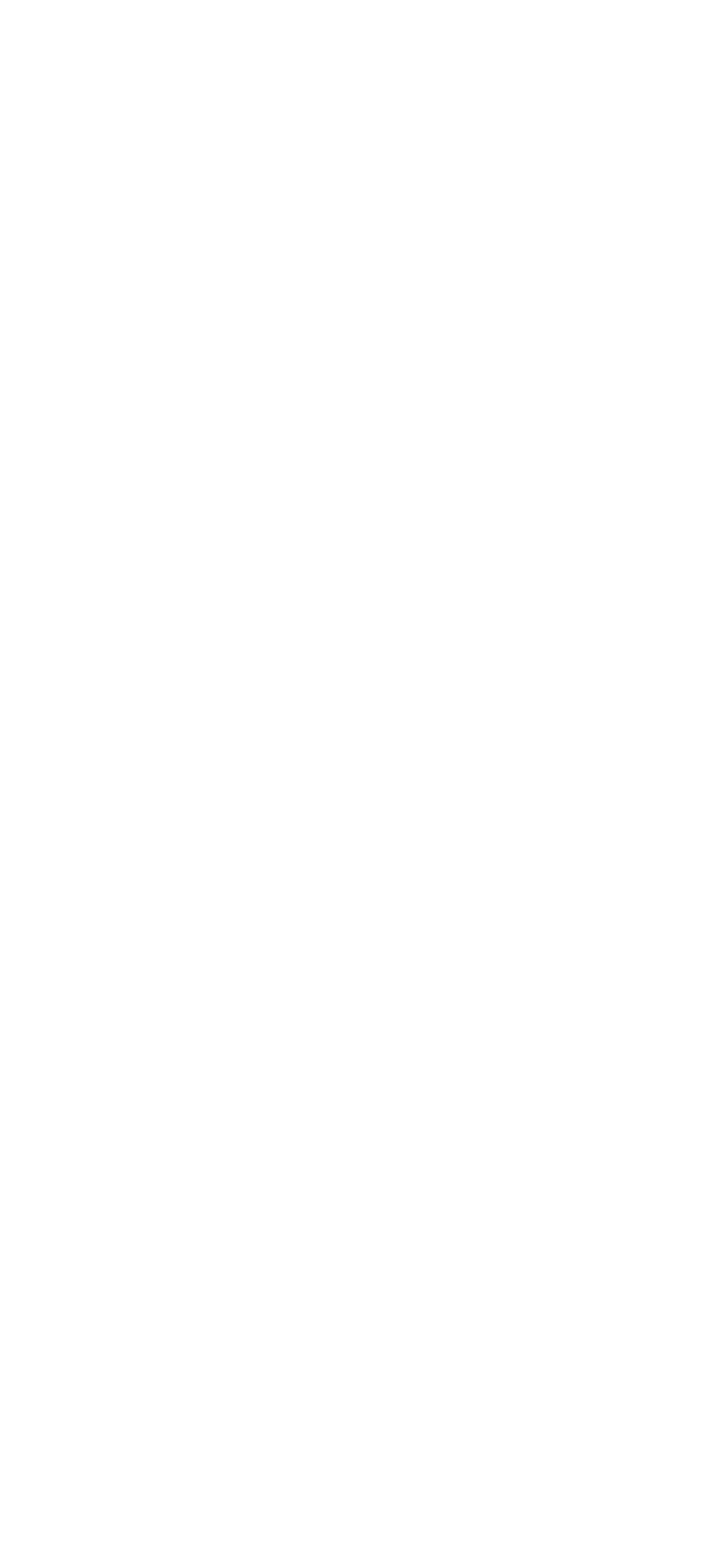




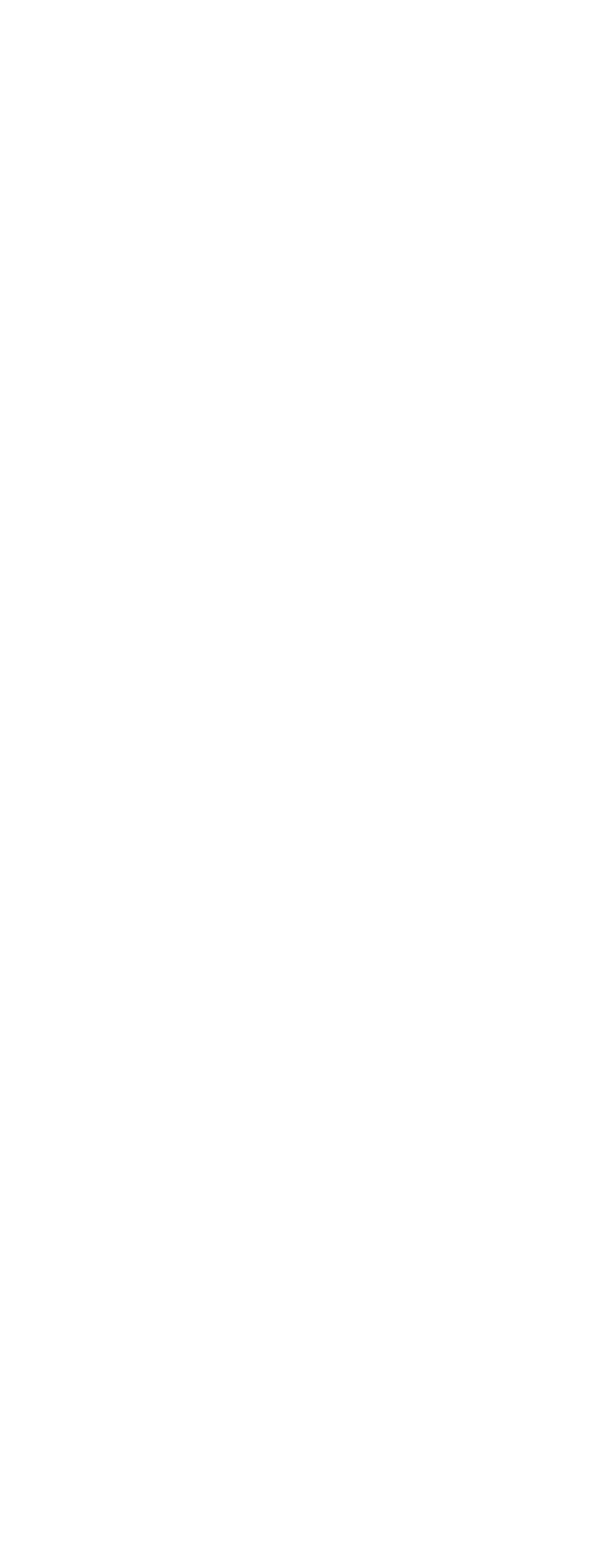




\begin{tabular}{|c|c|c|c|c|c|}
\hline & 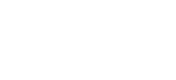 & 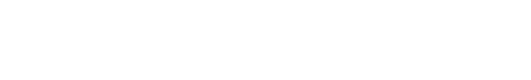 & & & \\
\hline & 视 & 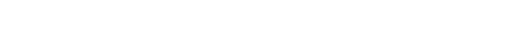 & 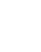 & $\stackrel{3}{i}$ & \\
\hline & 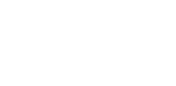 & 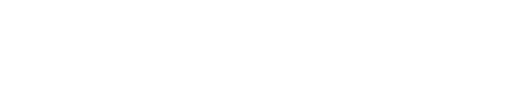 & 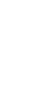 & 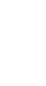 & \\
\hline & 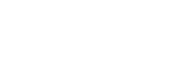 & 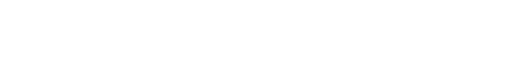 & 今 & \& & i \\
\hline & 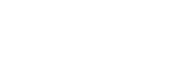 & 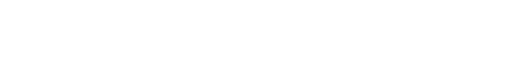 & ờํ. & 品 & \\
\hline & 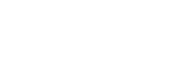 & 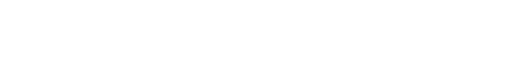 & จิ & : & i \\
\hline & 总总要 & N० & 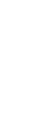 & 1 & 1 \\
\hline & 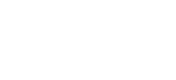 & 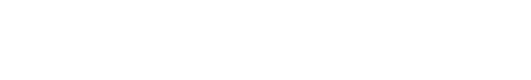 & 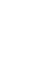 & $i$ & I \\
\hline 总 & 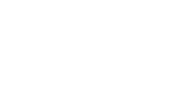 & 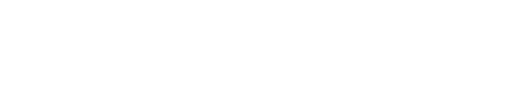 & 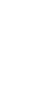 & 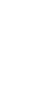 & ! \\
\hline & 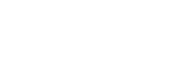 & $\stackrel{\leftrightarrow}{\ddot{m}}$ & & & \\
\hline 络 & 产草国 & & & & \\
\hline 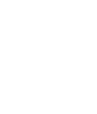 & 赵司 & 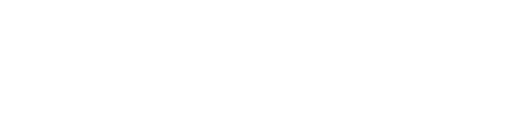 & : & 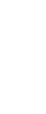 & 总 \\
\hline 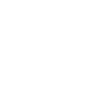 & 荧官 & 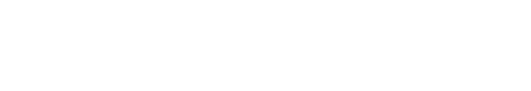 & 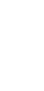 & 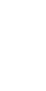 & $\infty$ \\
\hline 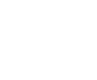 & 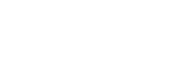 & 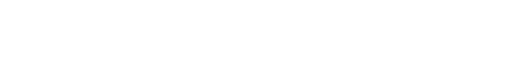 & 蛋 & 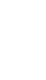 & 0 \\
\hline 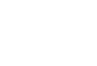 & 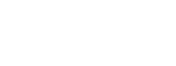 & 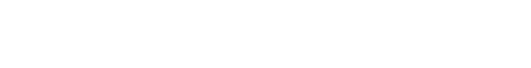 & 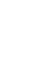 & 1 & 1 \\
\hline 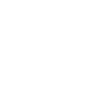 & 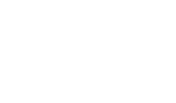 & 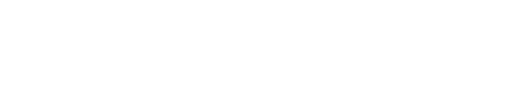 & ㅇํำ & 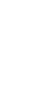 & 雇 \\
\hline ఇ & 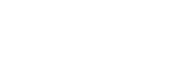 & & & & \\
\hline & 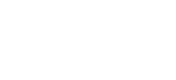 & 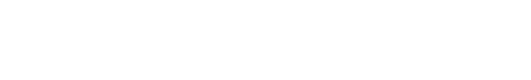 & $\stackrel{0}{:}$ & 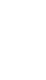 & $\stackrel{\leftrightarrow}{\sim}$ \\
\hline & 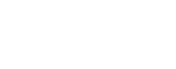 & 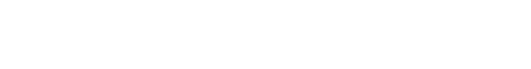 & ํํํ & : & $m$ \\
\hline & 总㲧 & & & & \\
\hline & 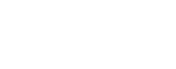 & นำㅉำ & 와 & 요 & $\stackrel{0}{i}$ \\
\hline & 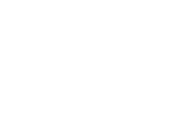 & 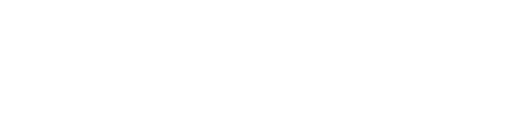 & $i$ & $\overrightarrow{9}$ & $i$ \\
\hline & 总喏 & 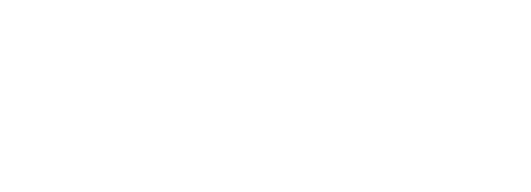 & & 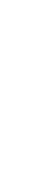 & 这 \\
\hline
\end{tabular}




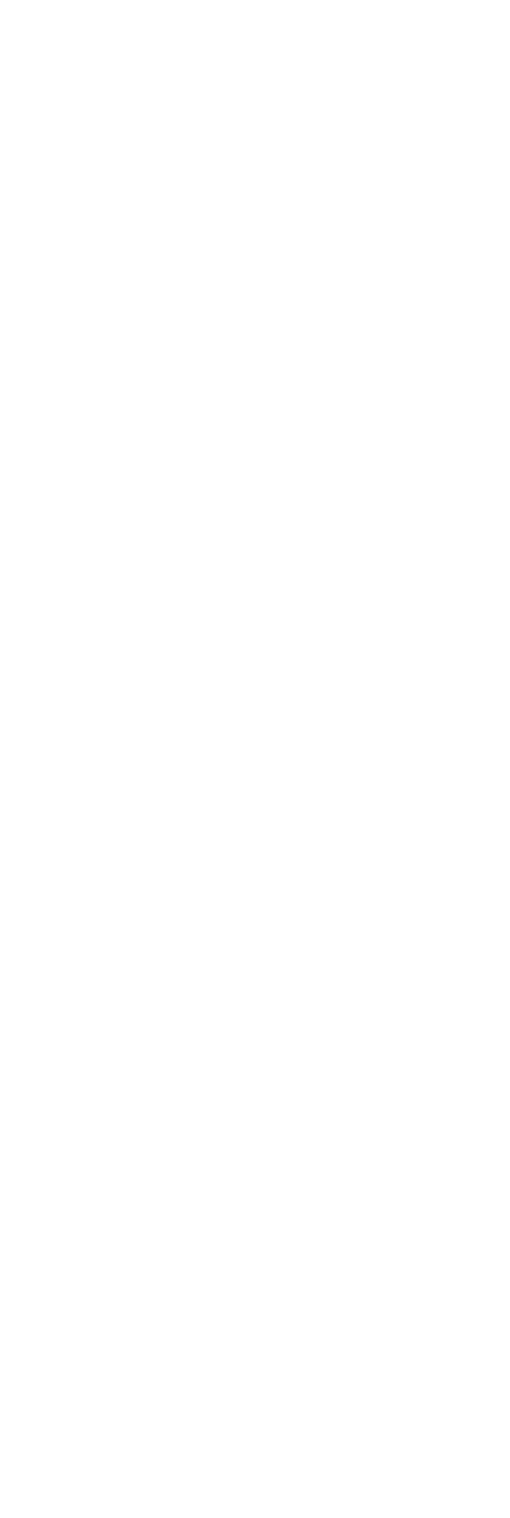




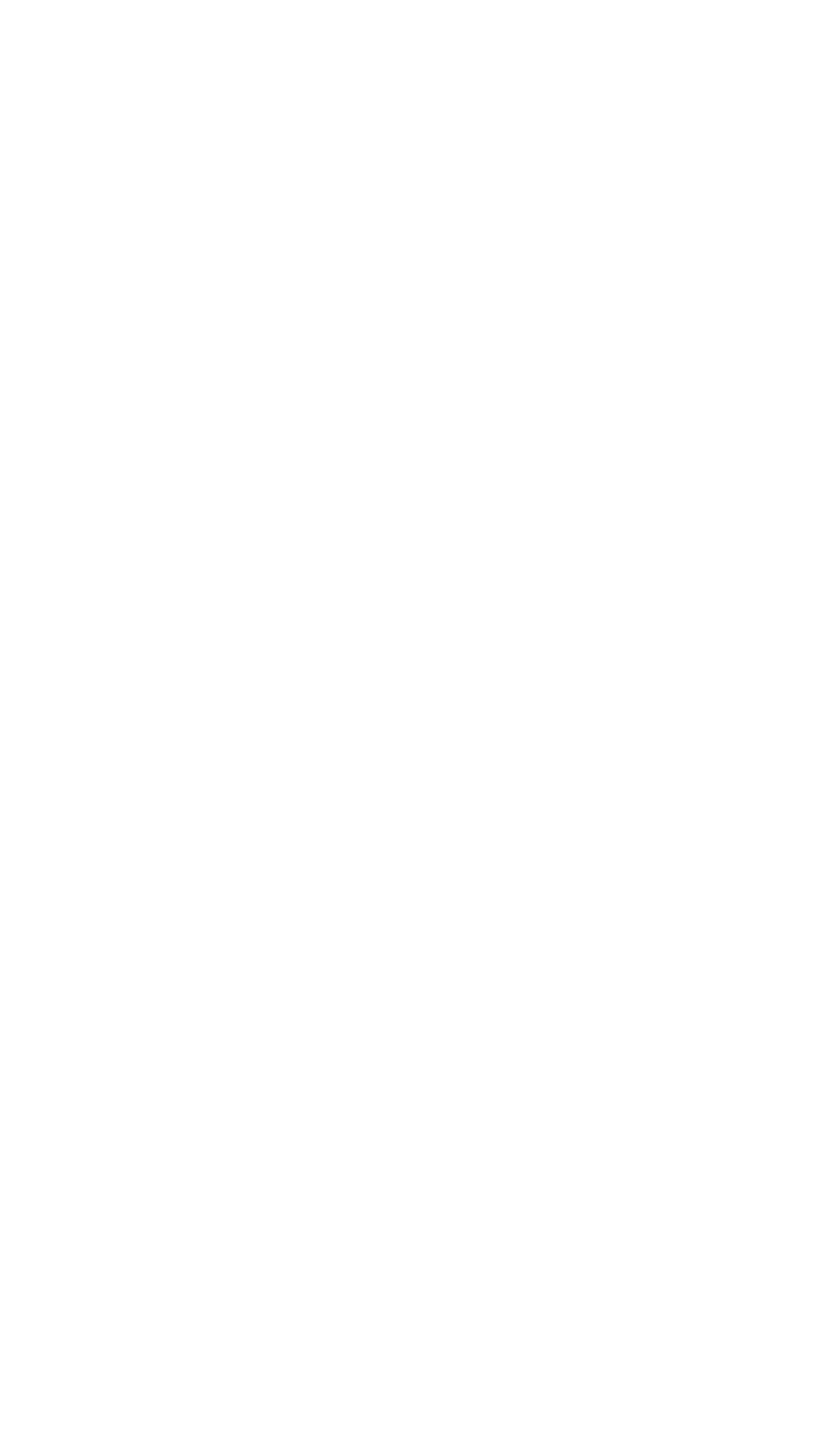




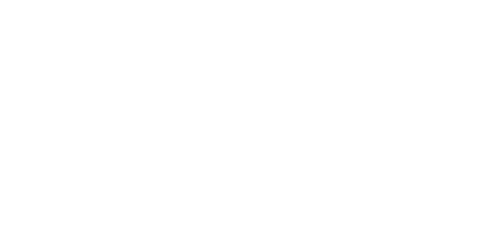

\begin{tabular}{|c|c|c|c|c|}
\hline 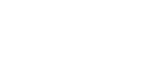 & 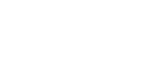 & $\stackrel{\infty}{\infty} \stackrel{\circ}{\circ}^{\circ}$ & 구 & $\overrightarrow{\text { Ṅ }}$ \\
\hline 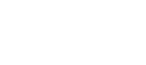 & 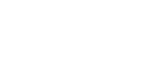 & $\begin{array}{l}6 \\
\text { लू J }\end{array}$ & ? & $\begin{array}{l}0 \\
\infty \\
10 \\
-1\end{array}$ \\
\hline 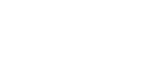 & 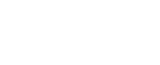 & 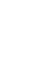 & $\underset{\infty}{\$}$ & $\stackrel{8}{6}$ \\
\hline
\end{tabular}

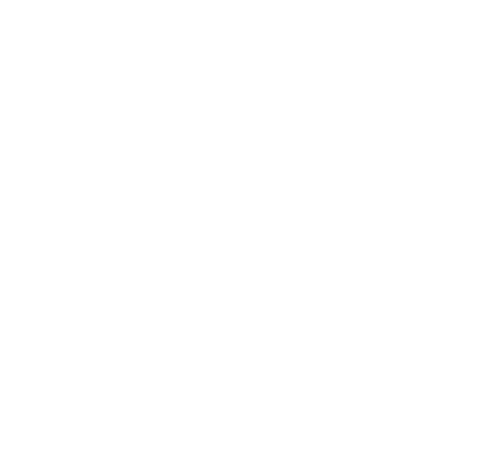

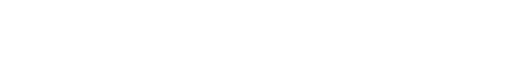

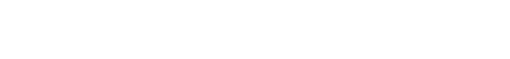

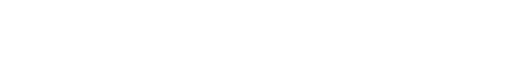

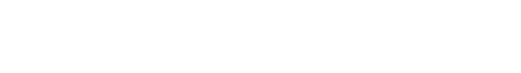

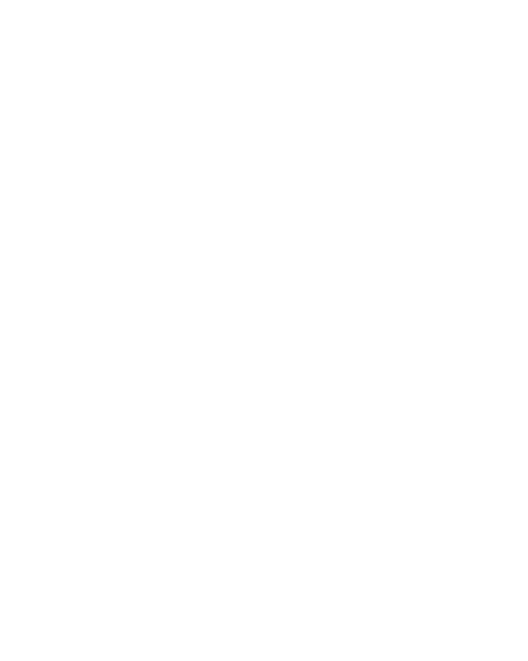

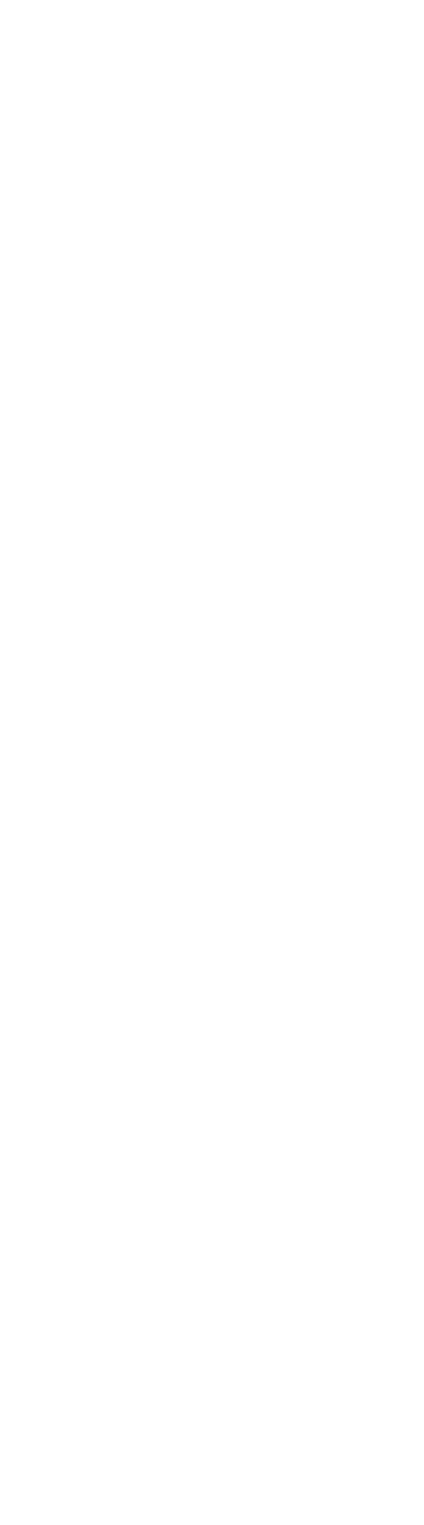




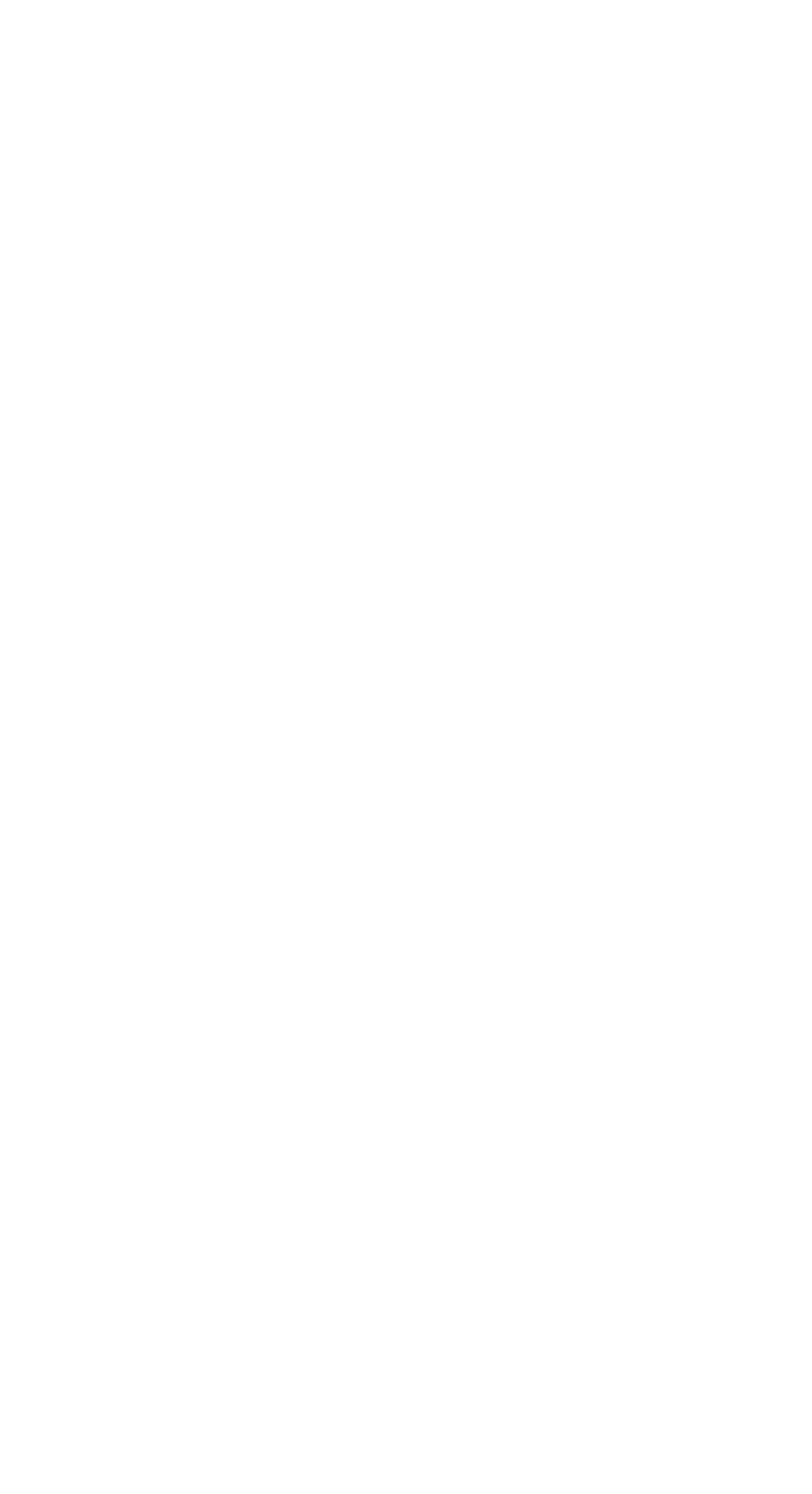




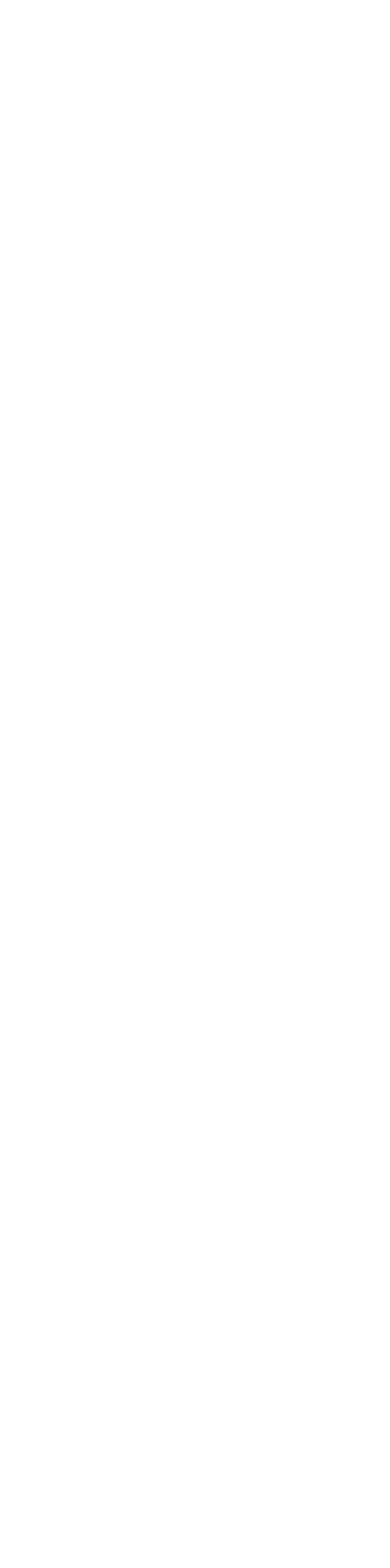

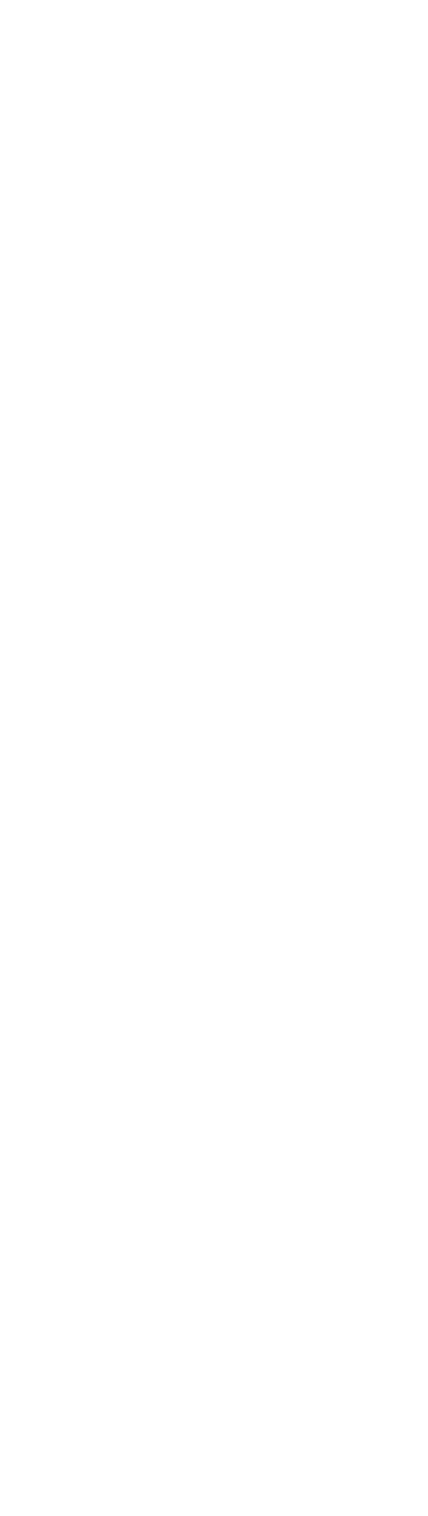




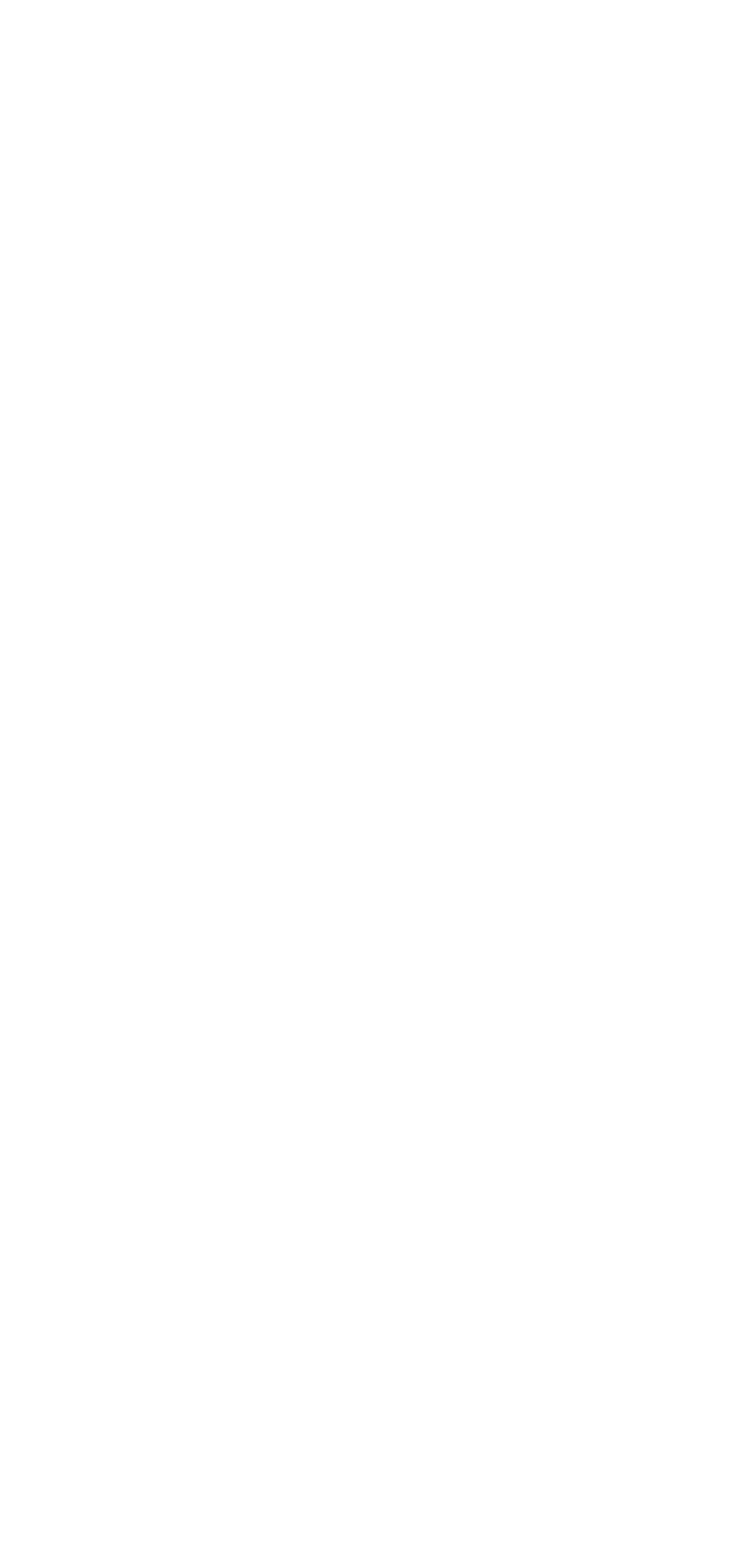




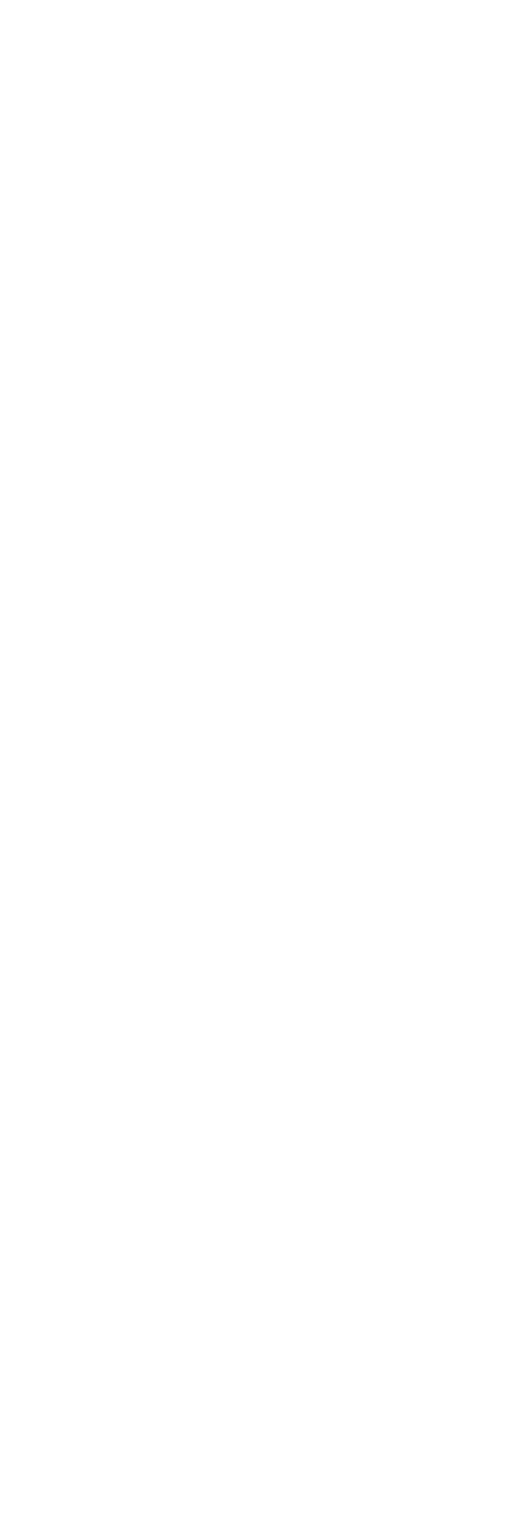




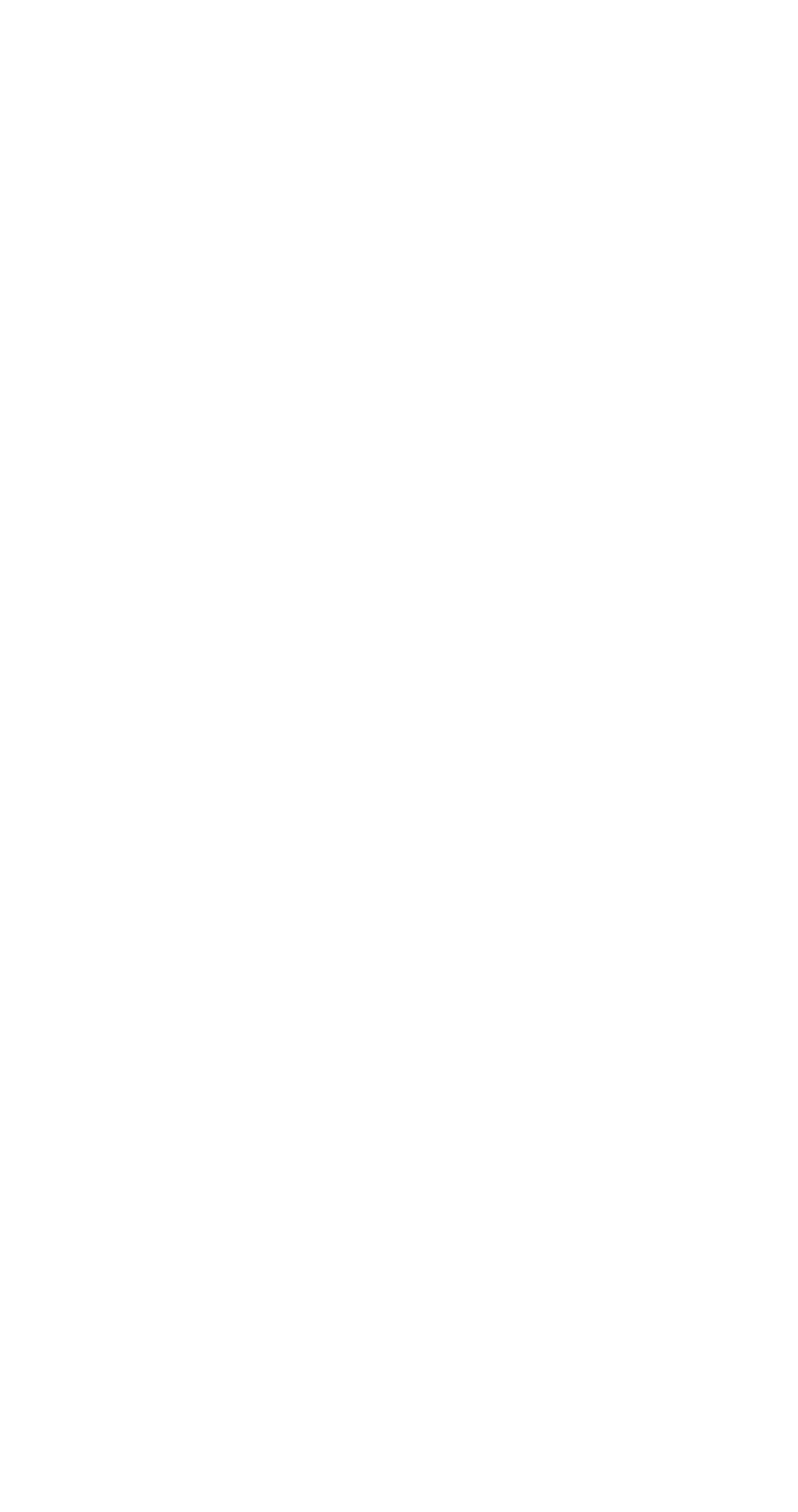



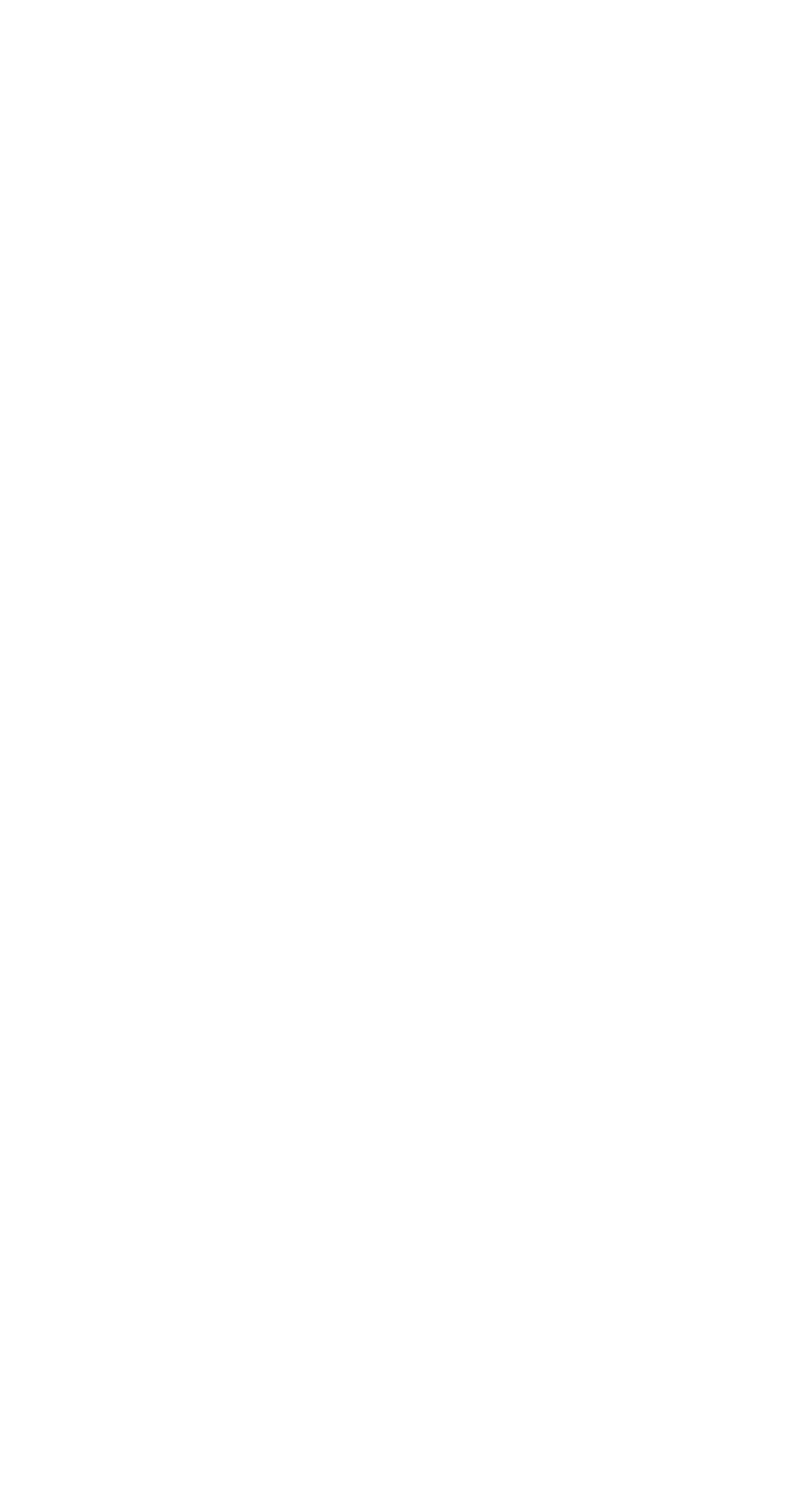


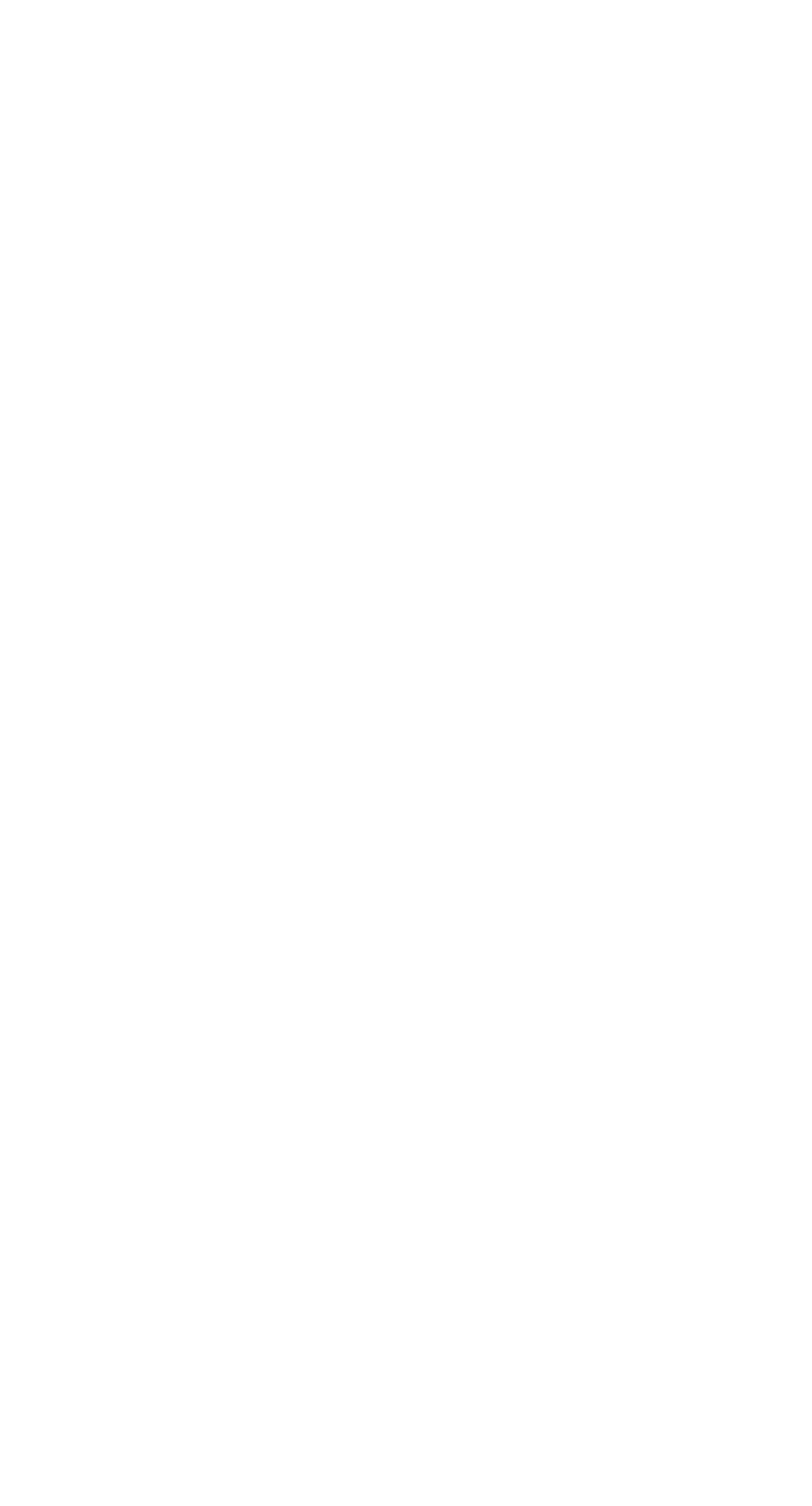




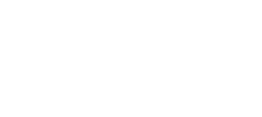

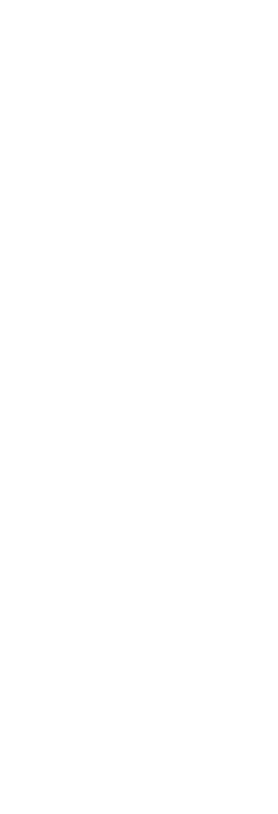

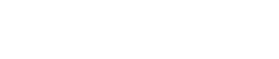

\begin{tabular}{l|l|l|l} 
& & & \\
\hline 0
\end{tabular}

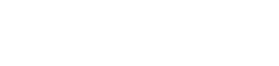

$\begin{array}{lllll}1111111 & 11\end{array}$

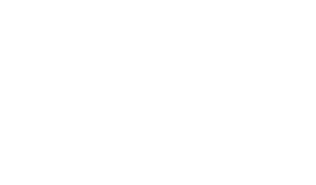

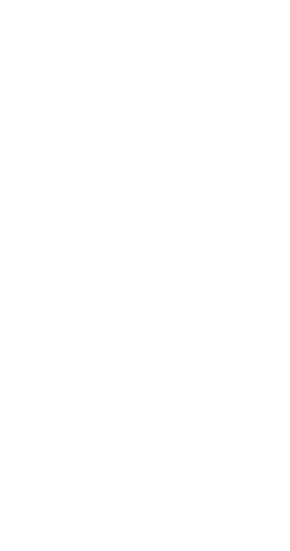

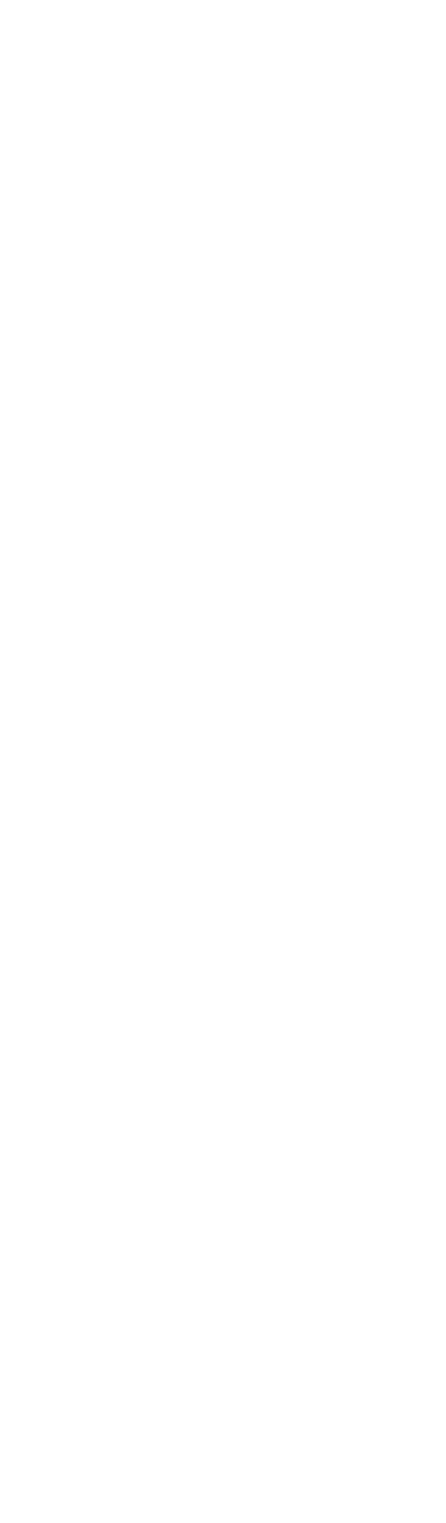




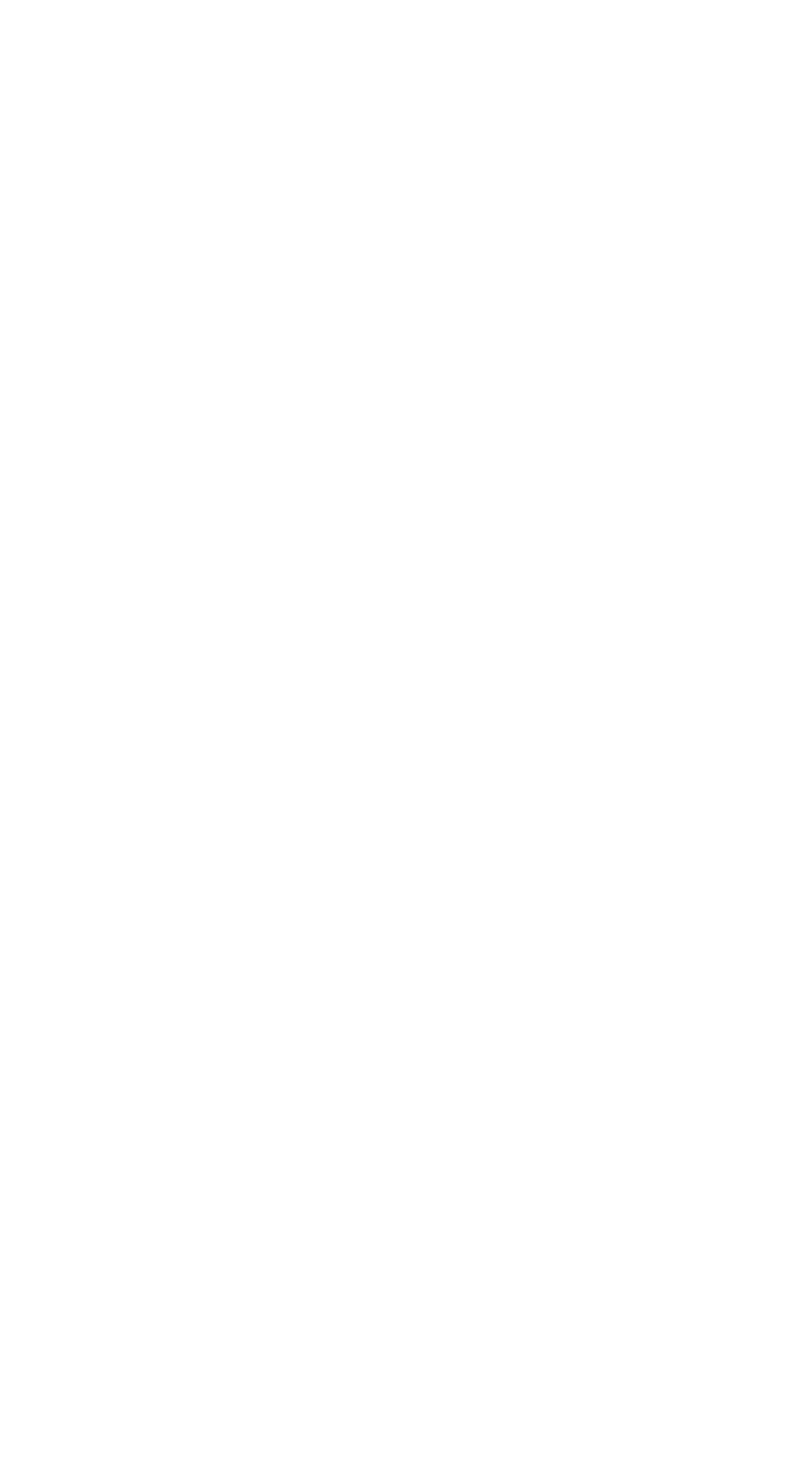




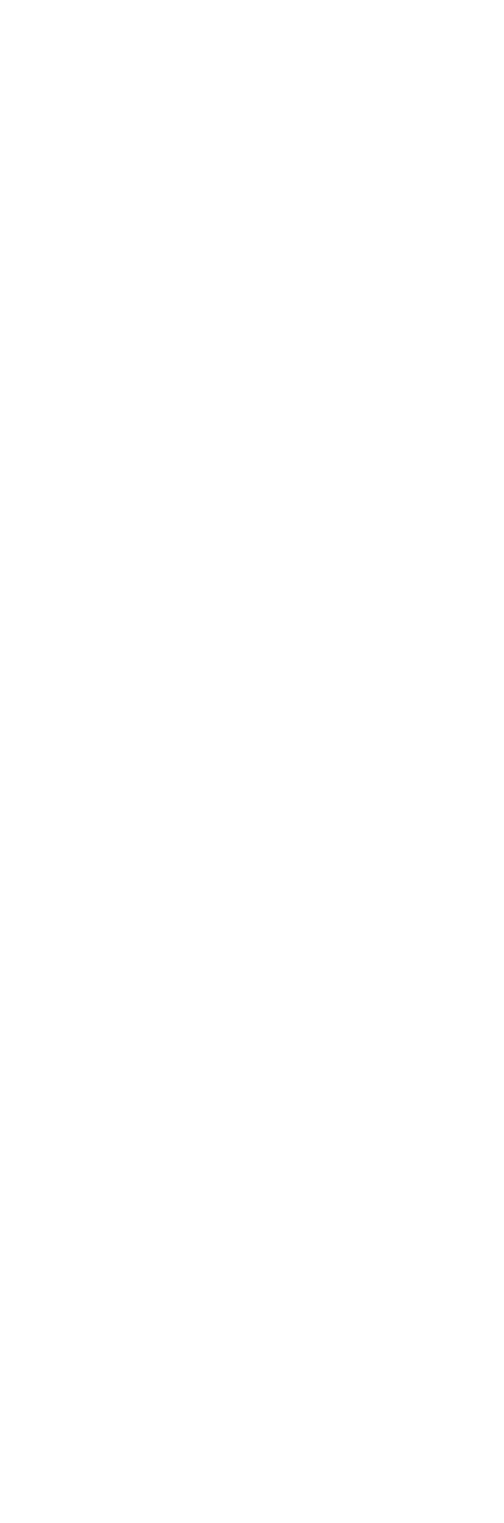




\begin{tabular}{|c|c|c|}
\hline 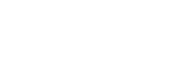 & $11114: 11114$ & $\because 09 \infty \% \pi$ \\
\hline 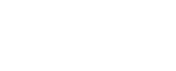 & 111114111111 & mang \\
\hline 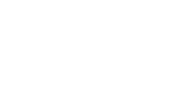 & 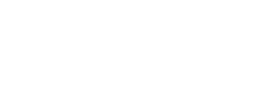 & 111111 \\
\hline$\frac{\pi}{2}$ & 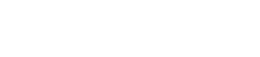 & 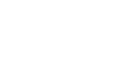 \\
\hline 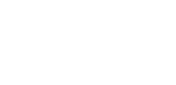 & 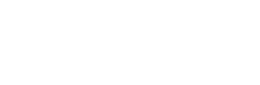 & 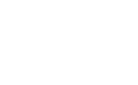 \\
\hline 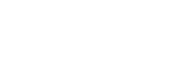 & 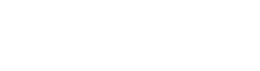 & 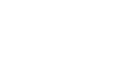 \\
\hline 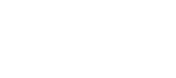 & 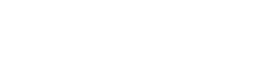 & 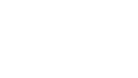 \\
\hline 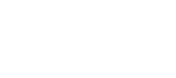 & 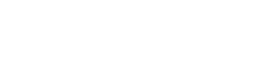 & 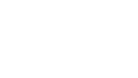 \\
\hline 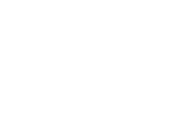 & 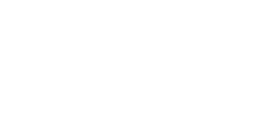 & 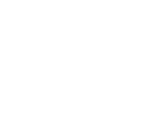 \\
\hline 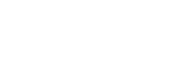 & 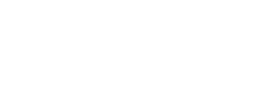 & 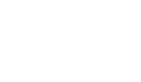 \\
\hline 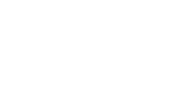 & 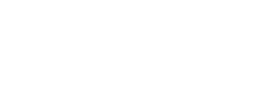 & 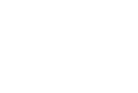 \\
\hline 嵩点㑹 & $\vec{a}$ & \\
\hline 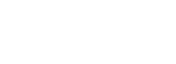 & 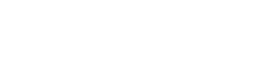 & 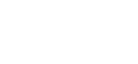 \\
\hline 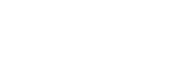 & 11119314i & 111711 \\
\hline 递 & 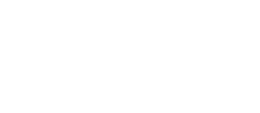 & 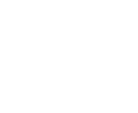 \\
\hline 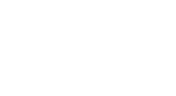 & 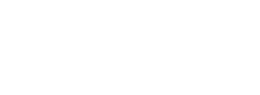 & 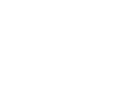 \\
\hline 亩 & 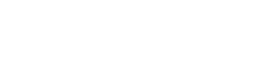 & 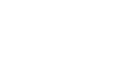 \\
\hline 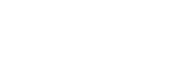 & 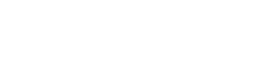 & $\begin{array}{c}711111 \\
0\end{array}$ \\
\hline 害 & 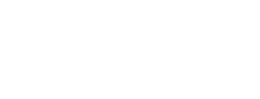 & 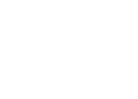 \\
\hline 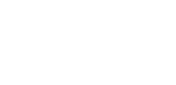 & 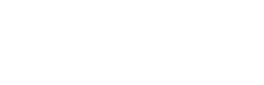 & 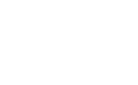 \\
\hline 过慁可 & 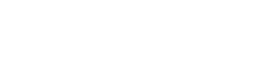 & 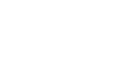 \\
\hline 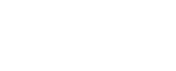 & 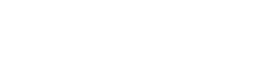 & 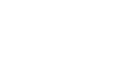 \\
\hline 赵 & بִ & 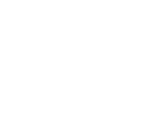 \\
\hline & 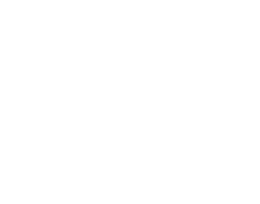 & 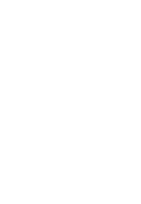 \\
\hline
\end{tabular}




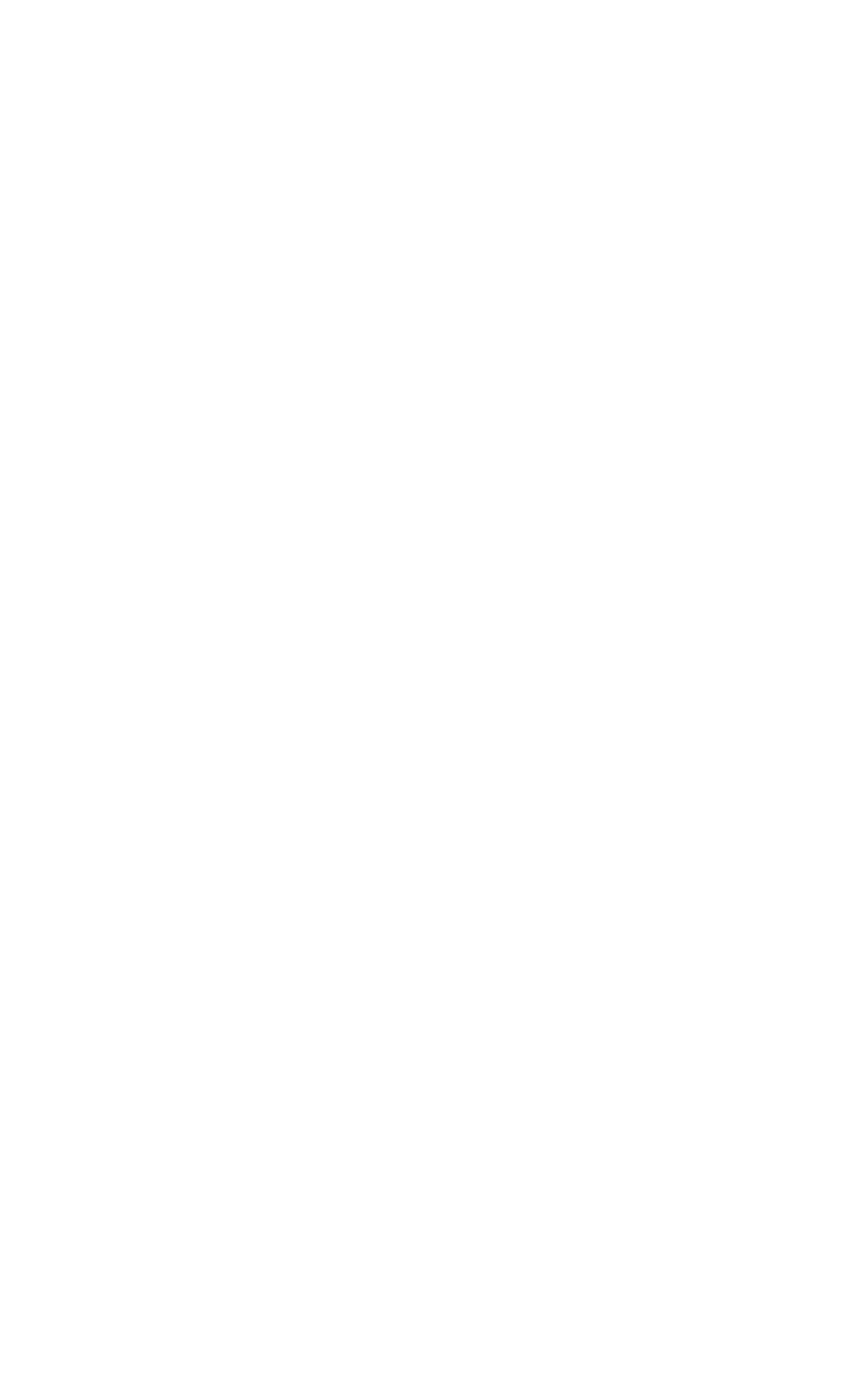




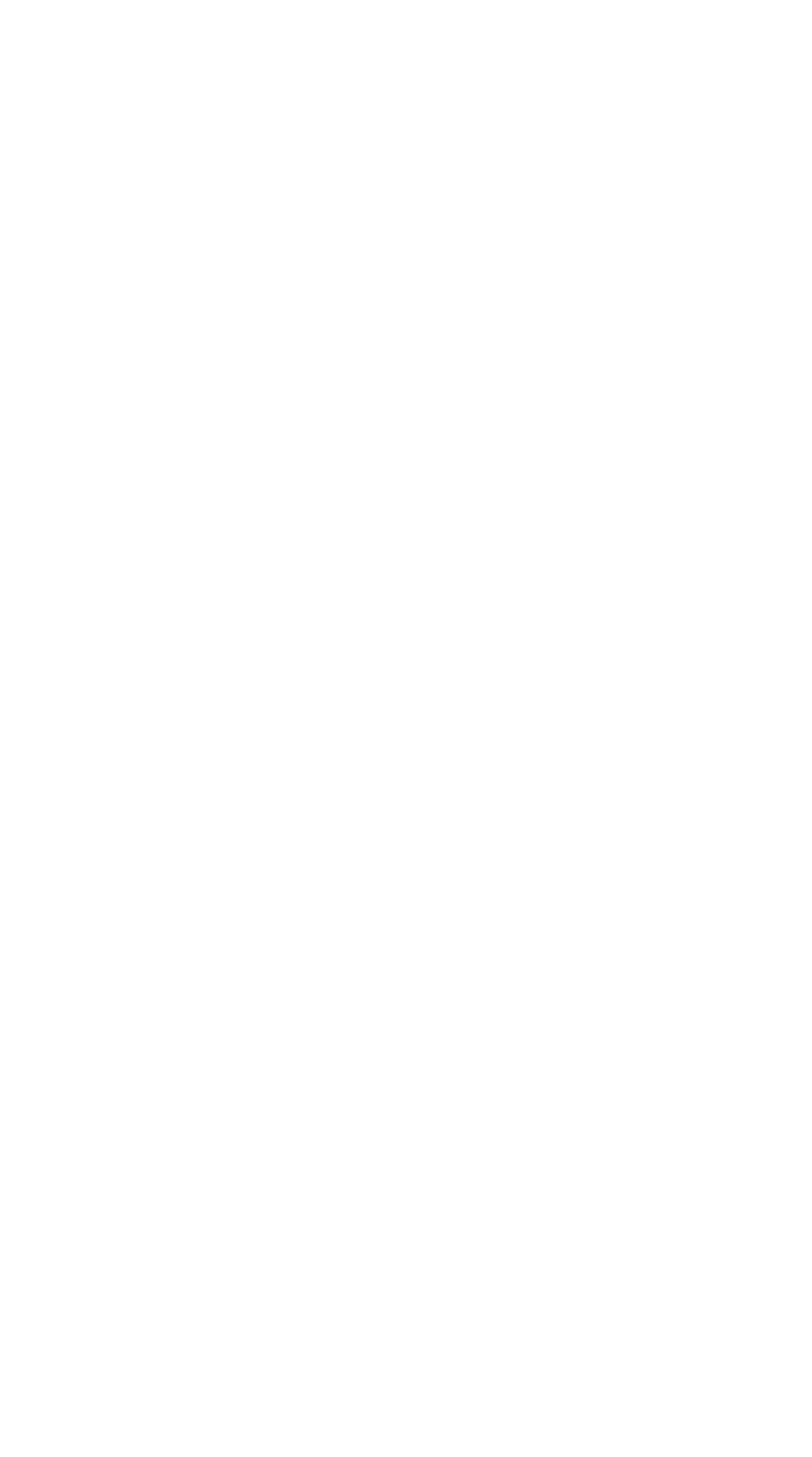




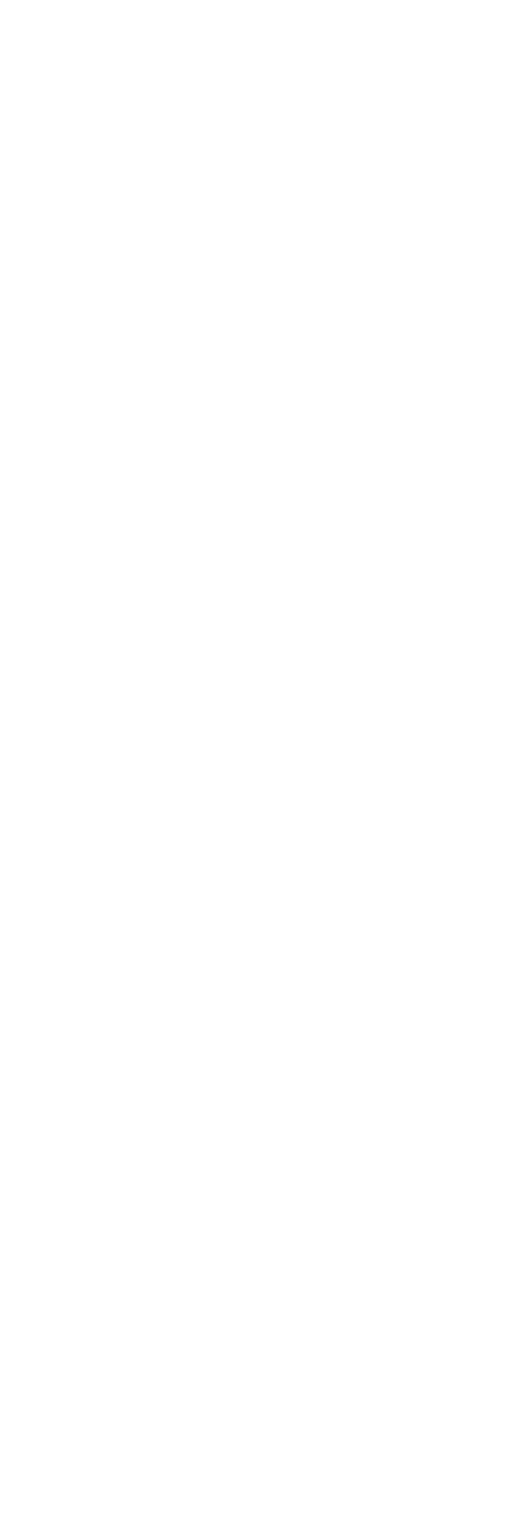




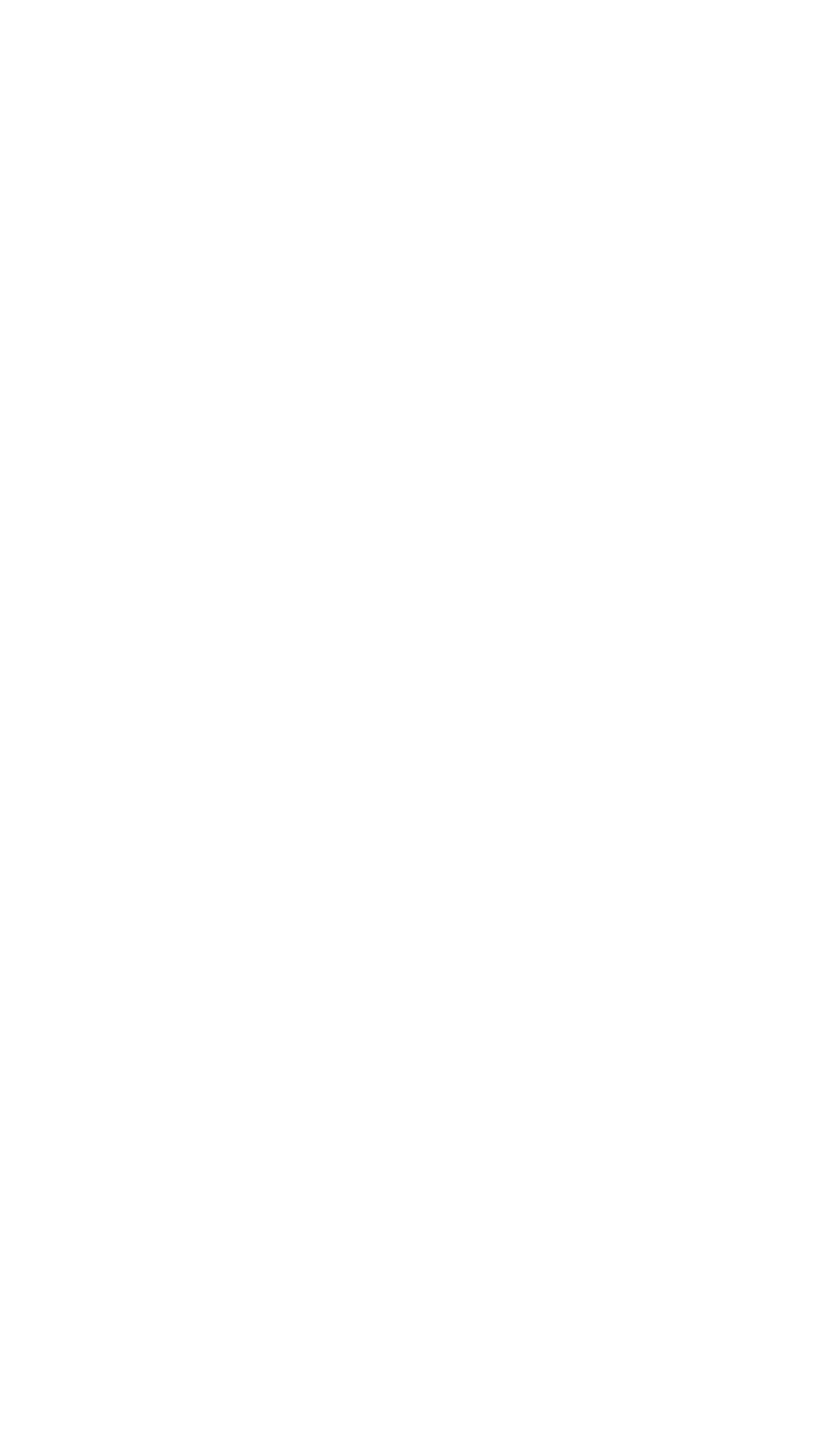




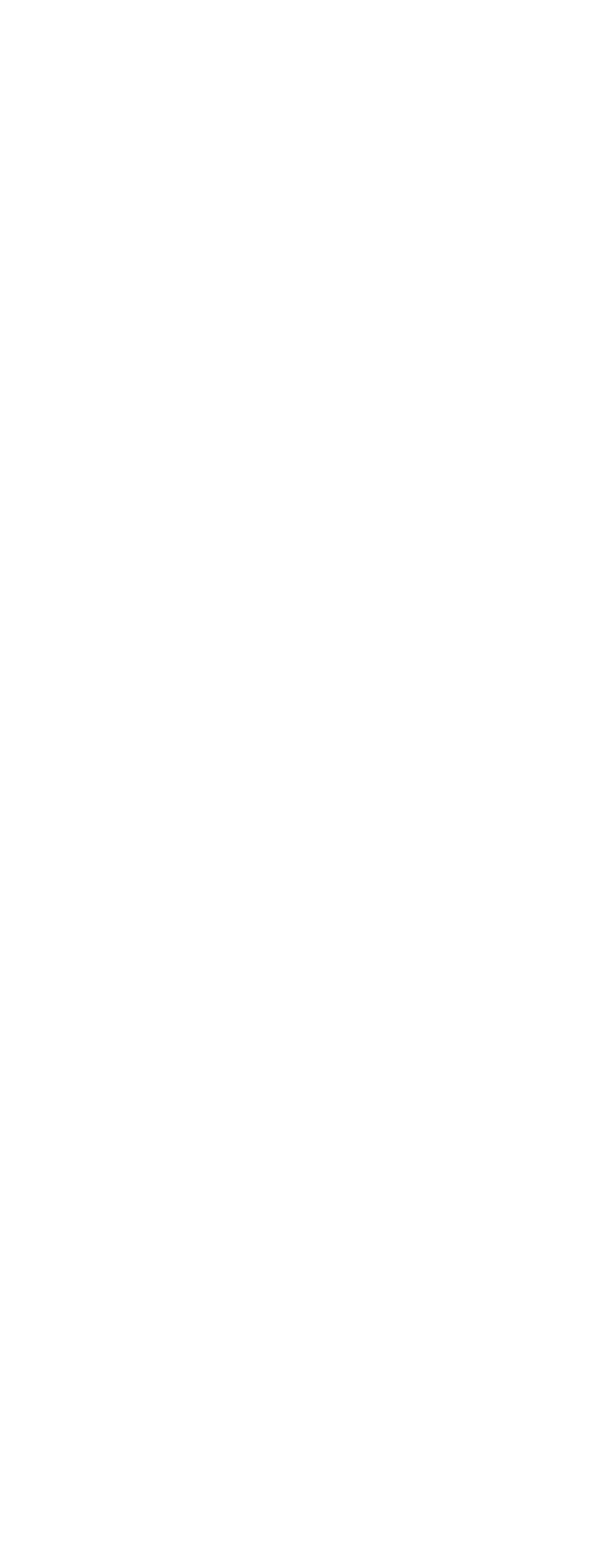



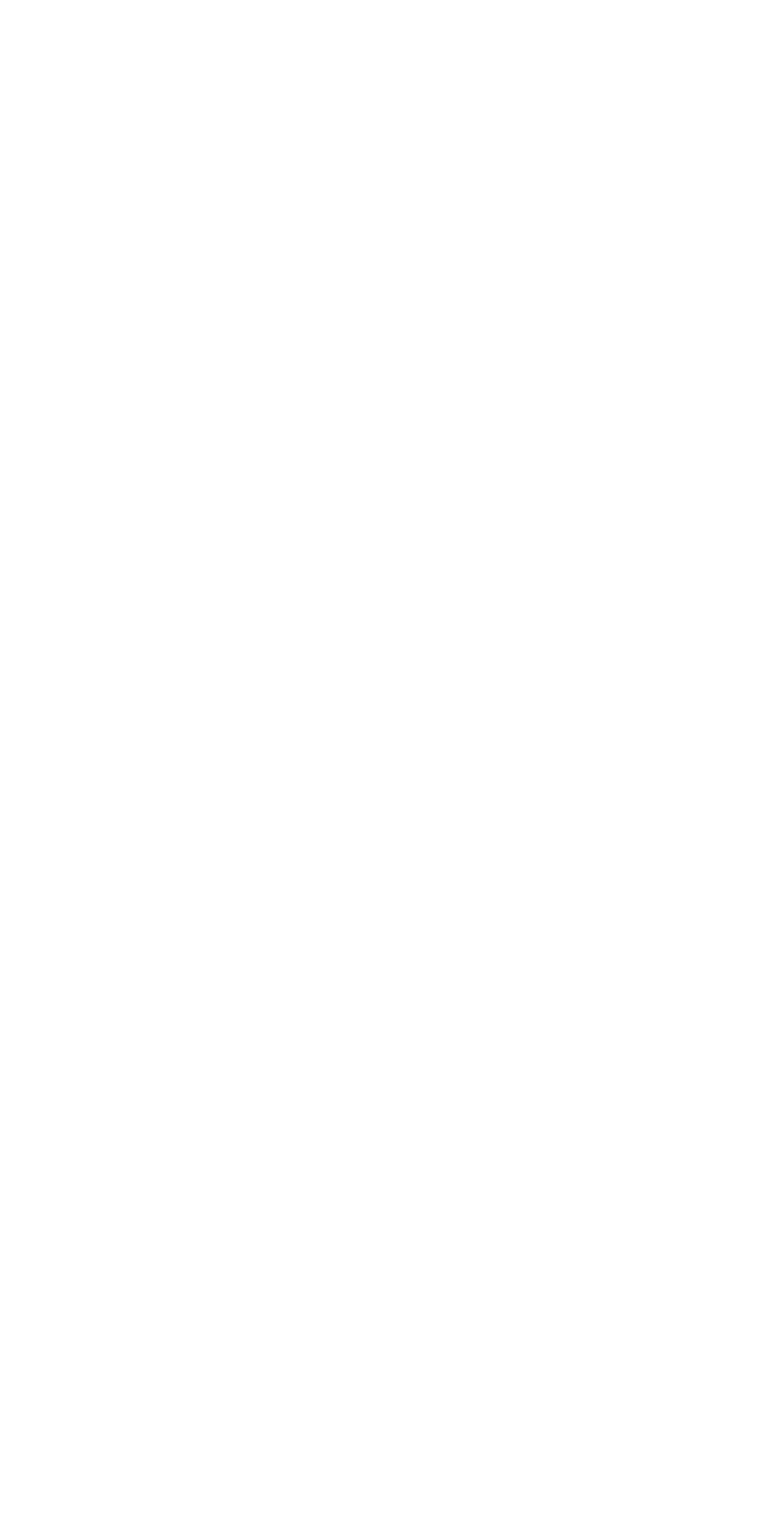


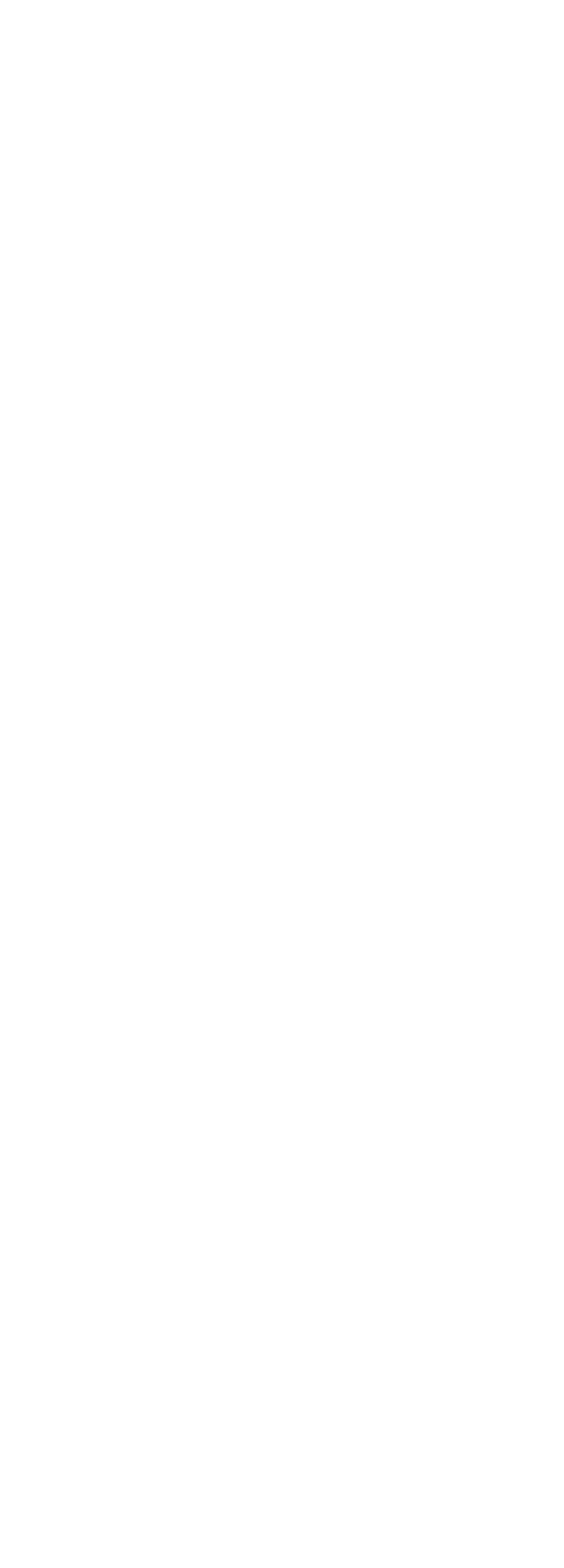




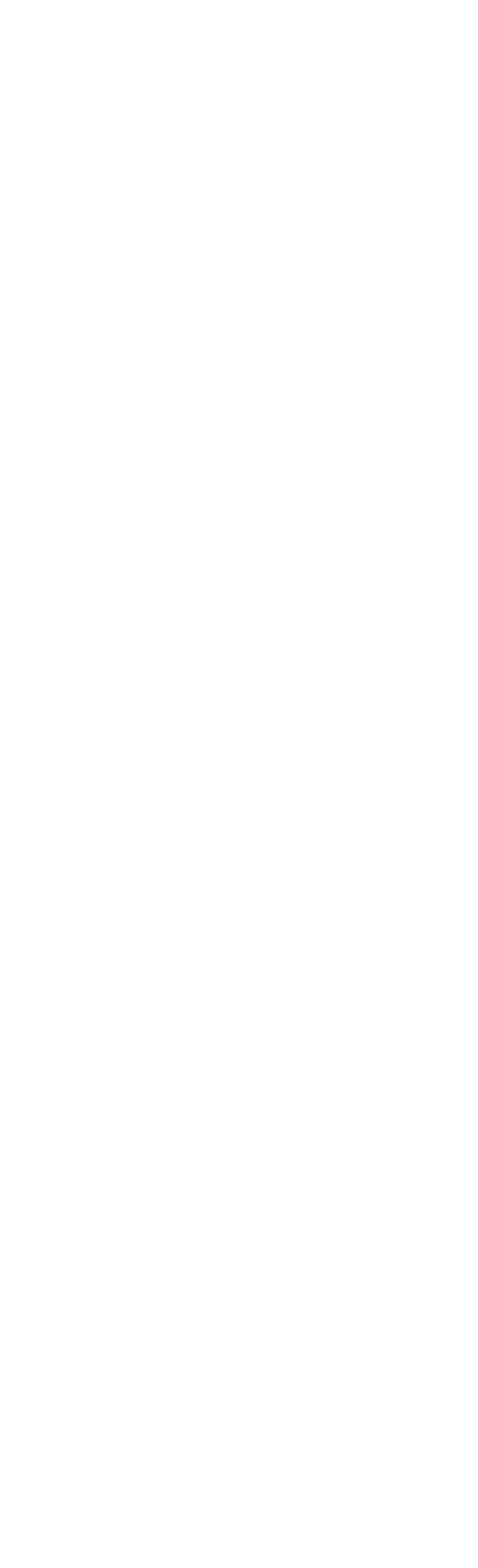

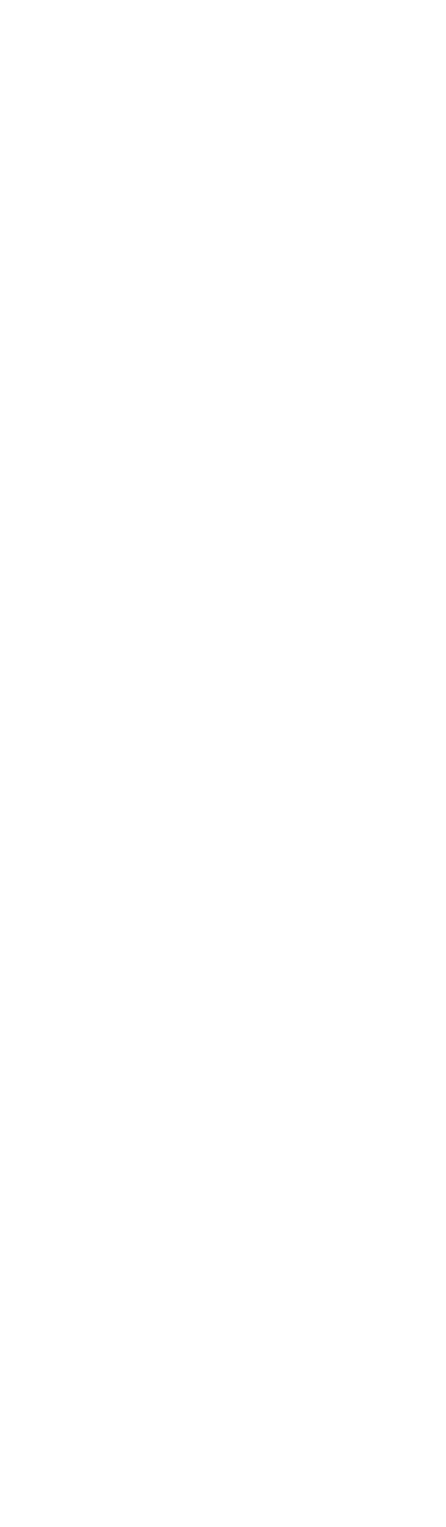




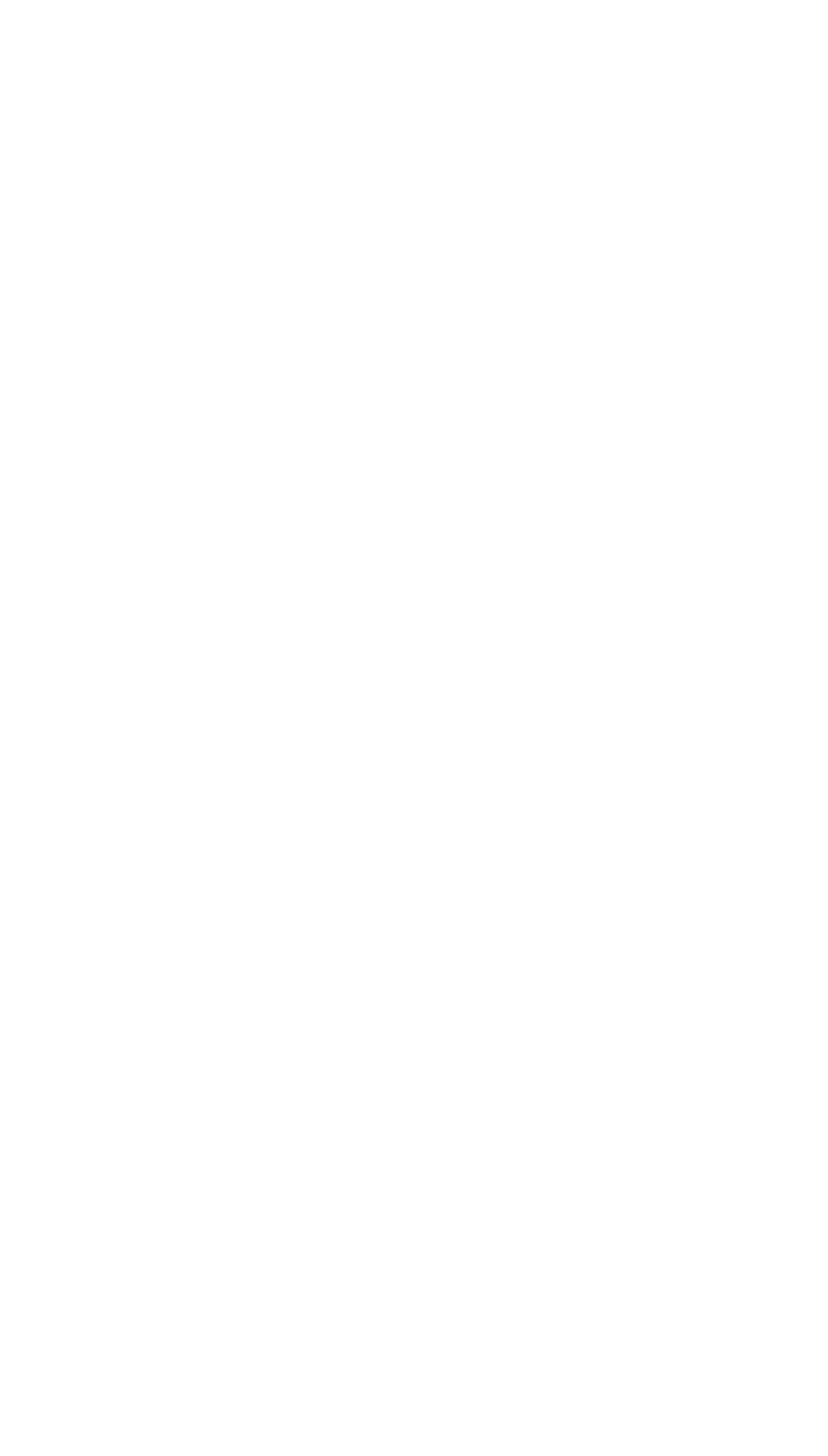




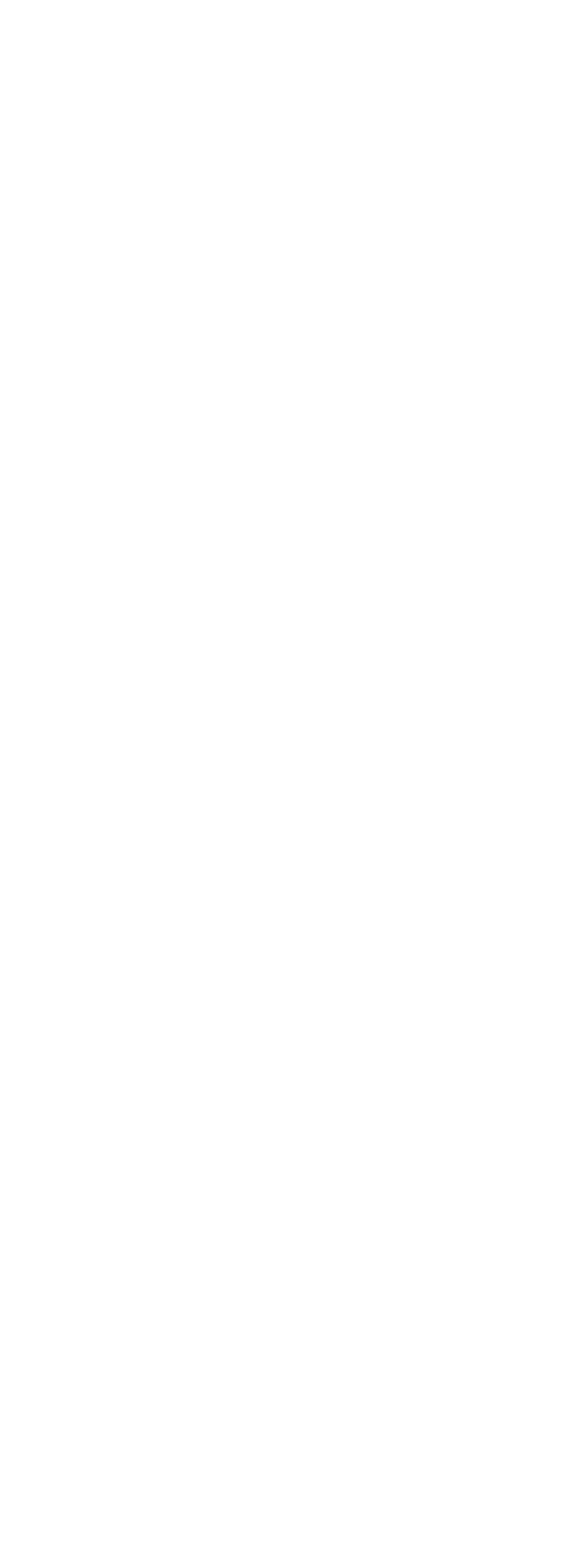




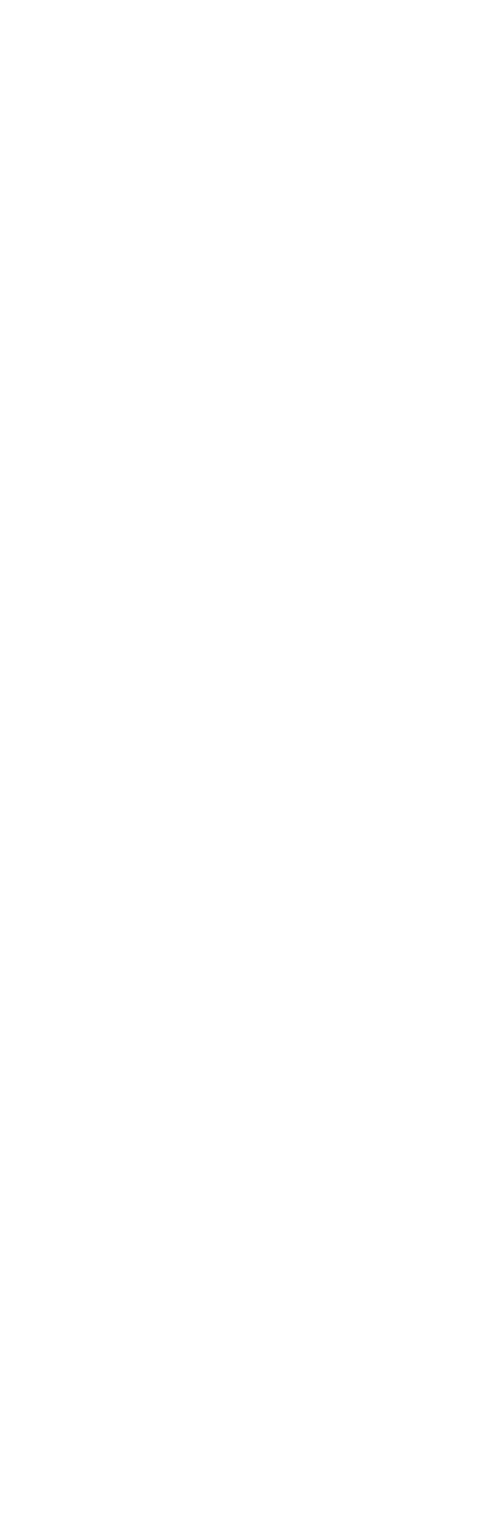




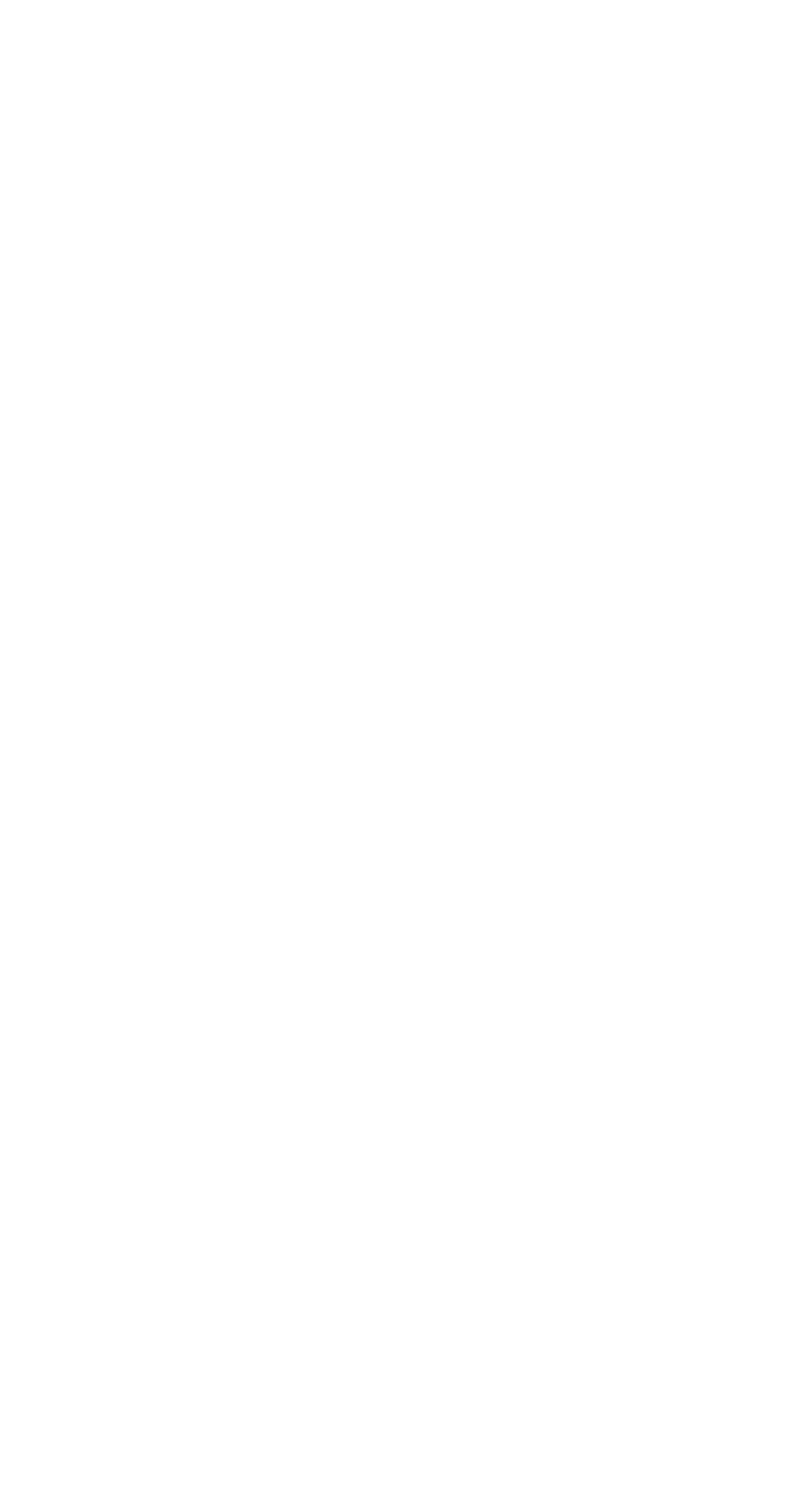




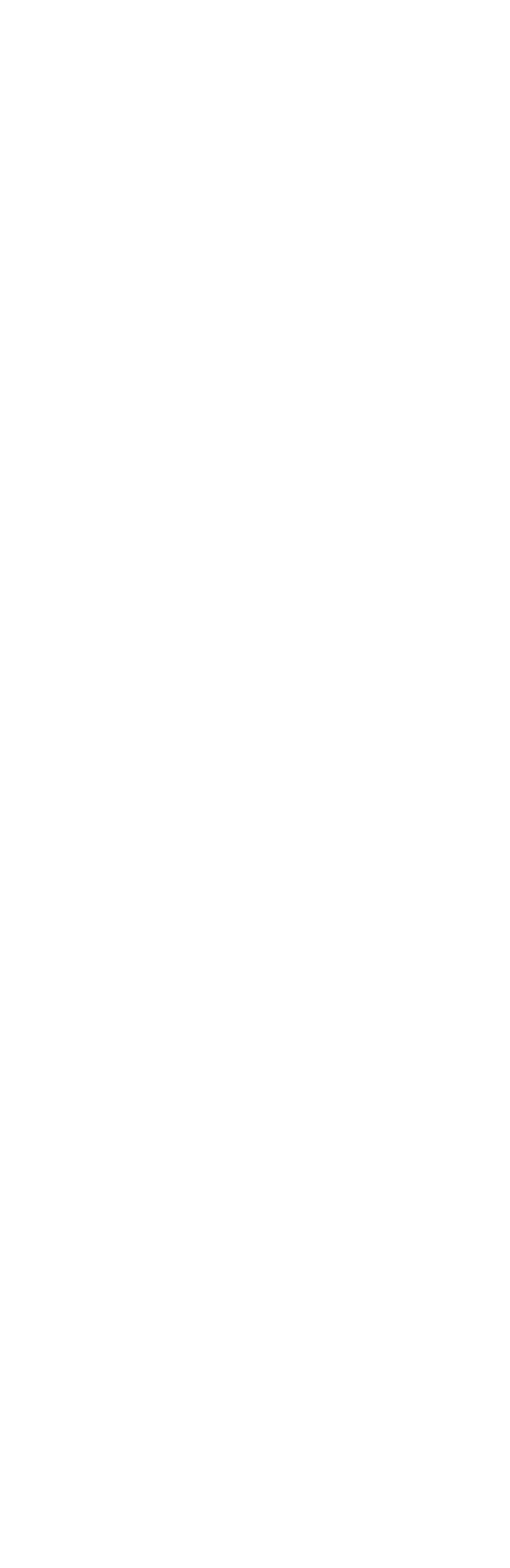




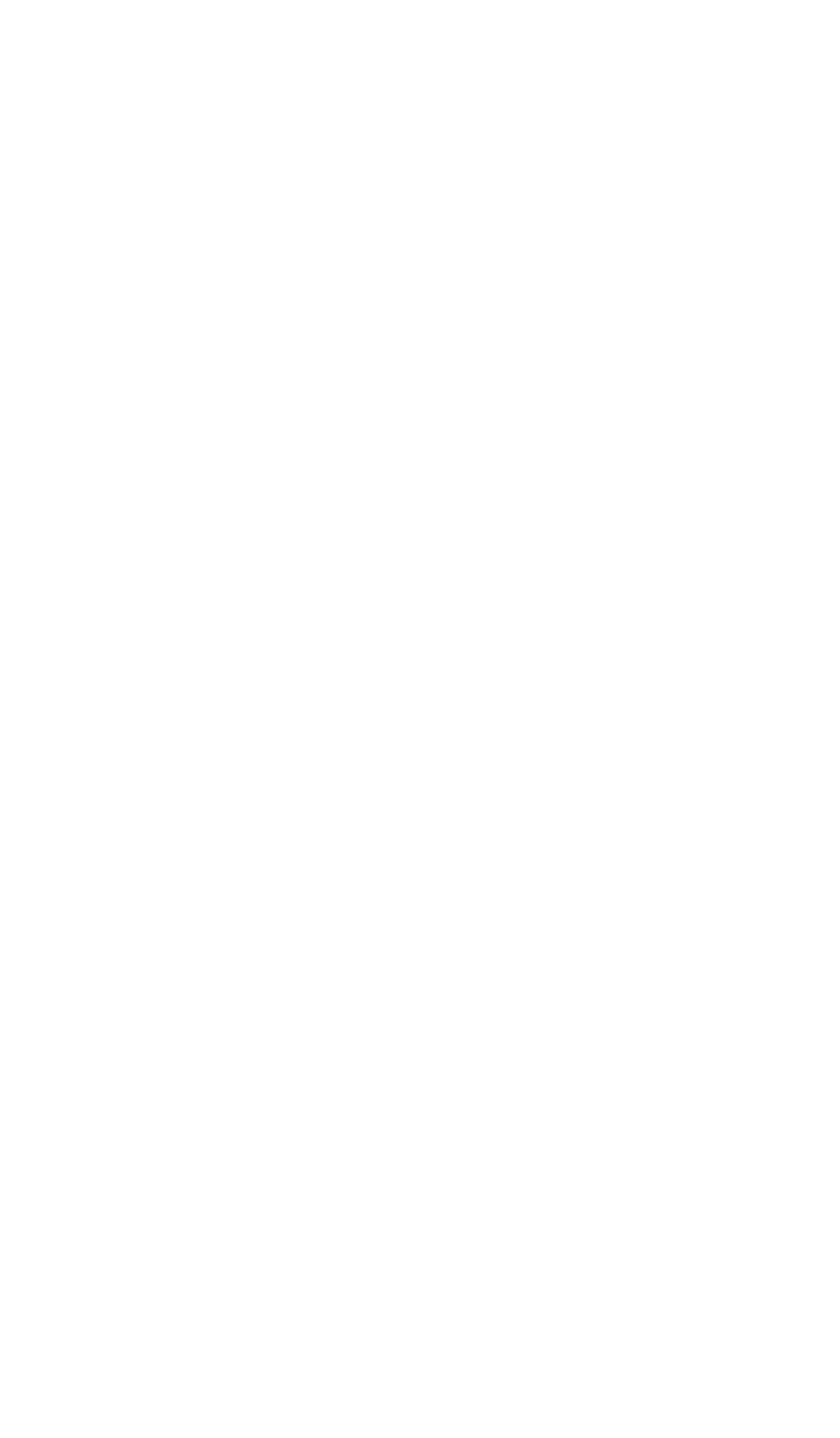




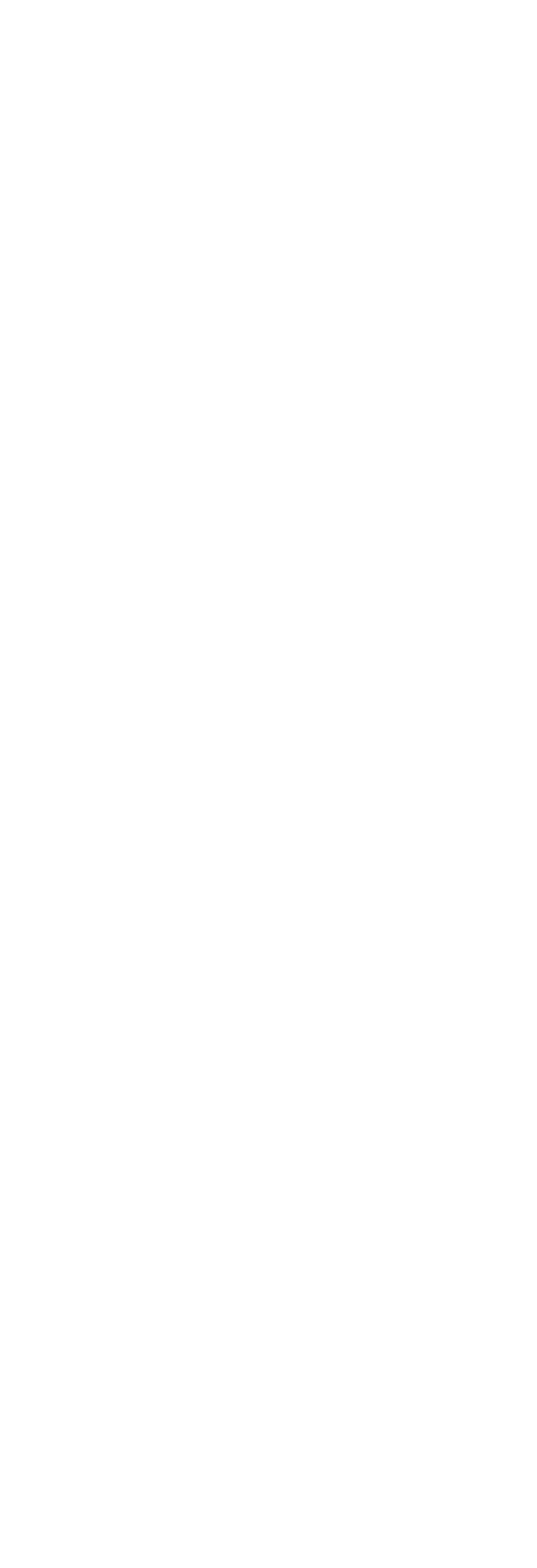




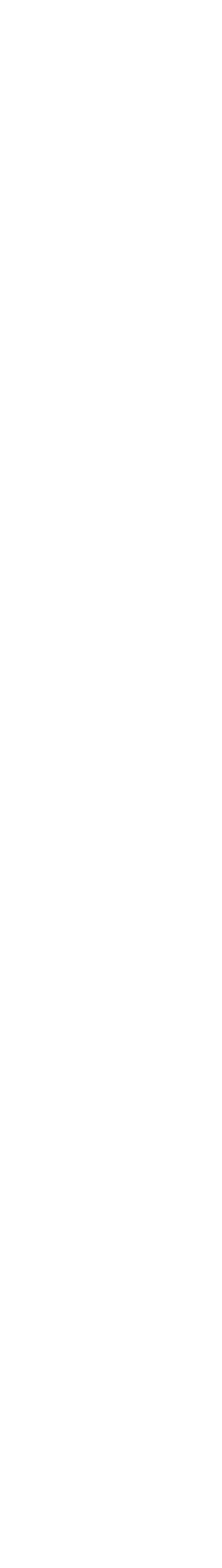

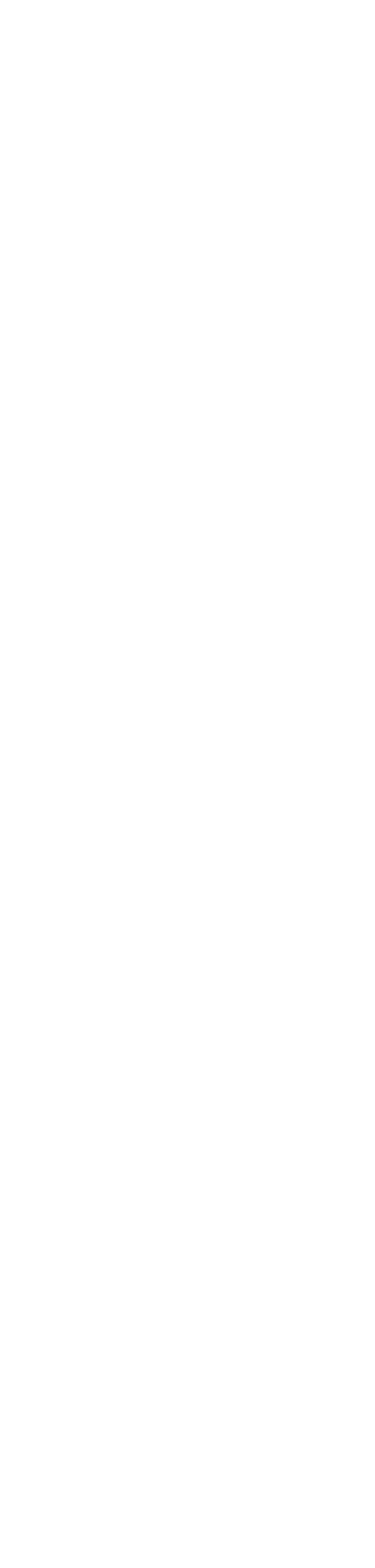




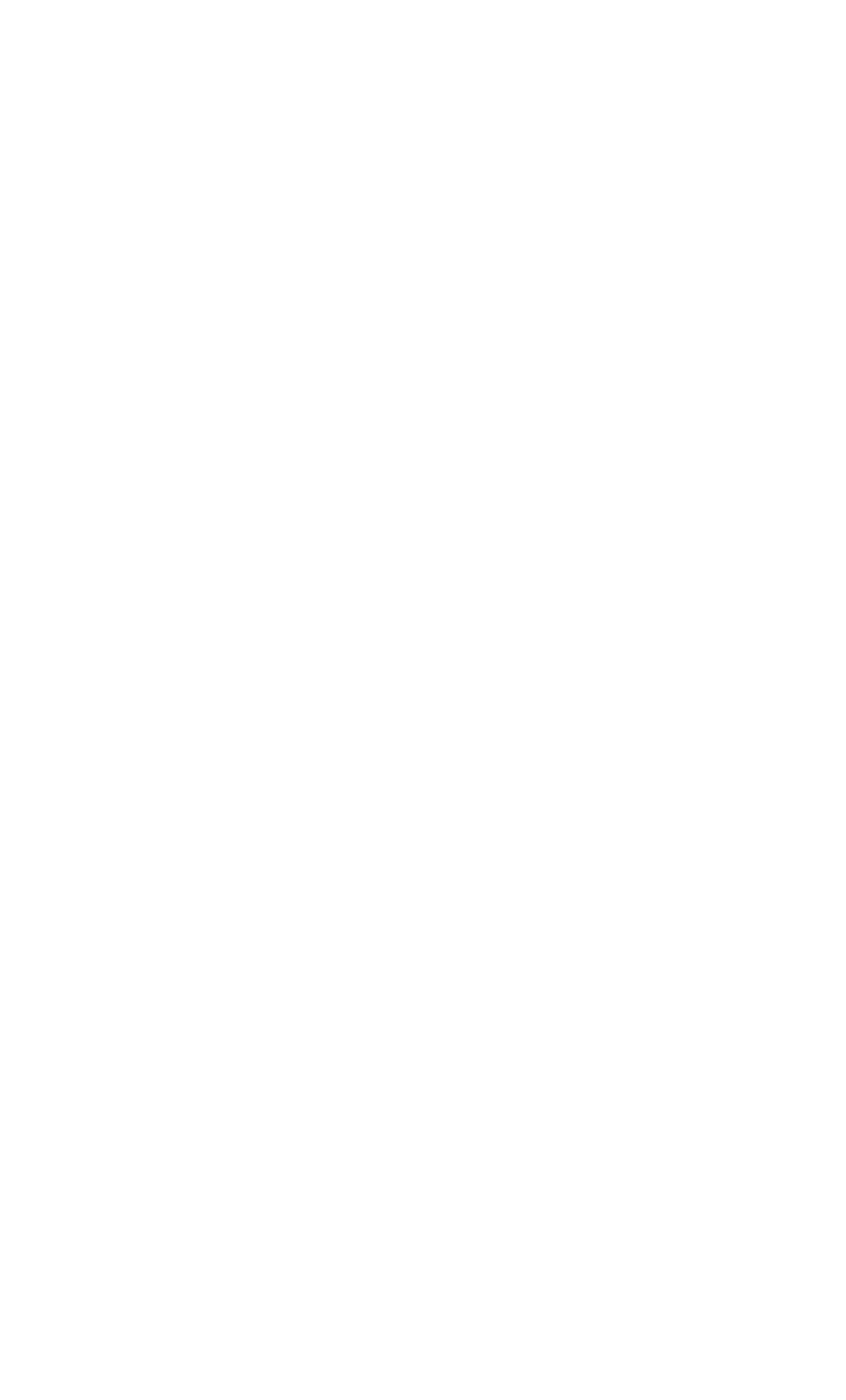




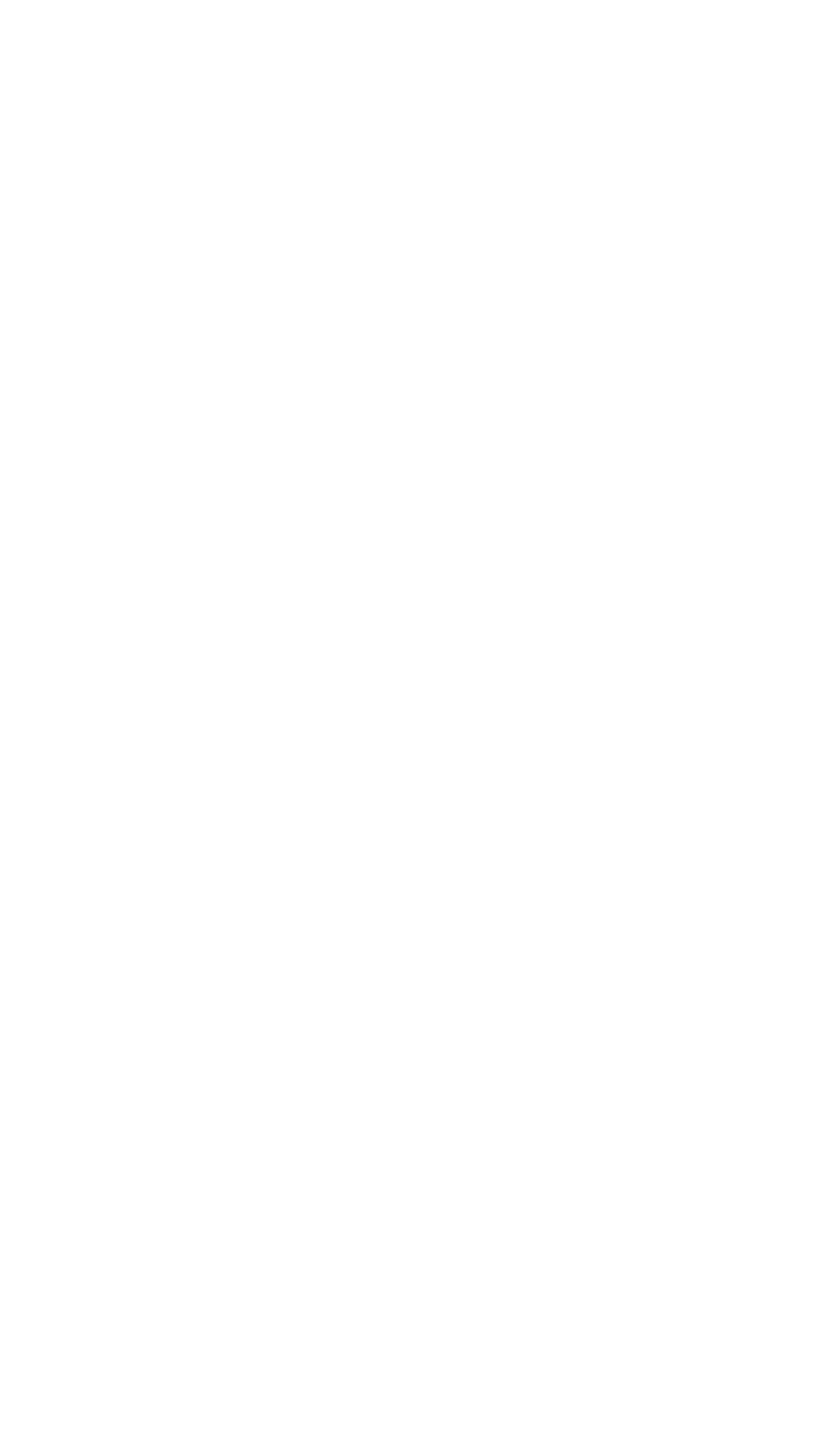




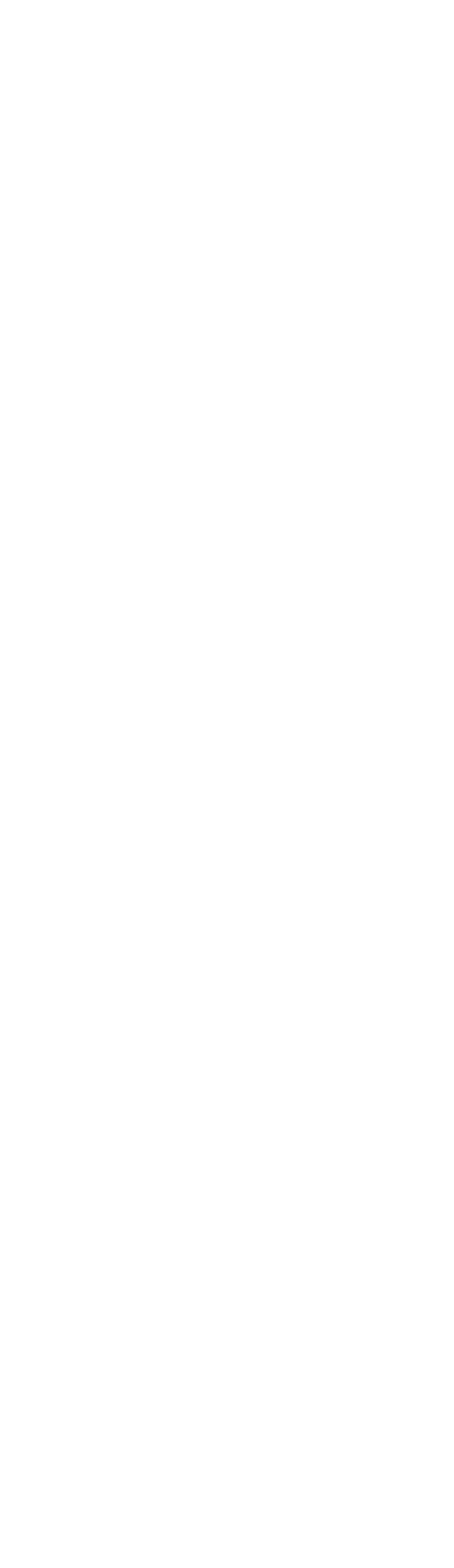

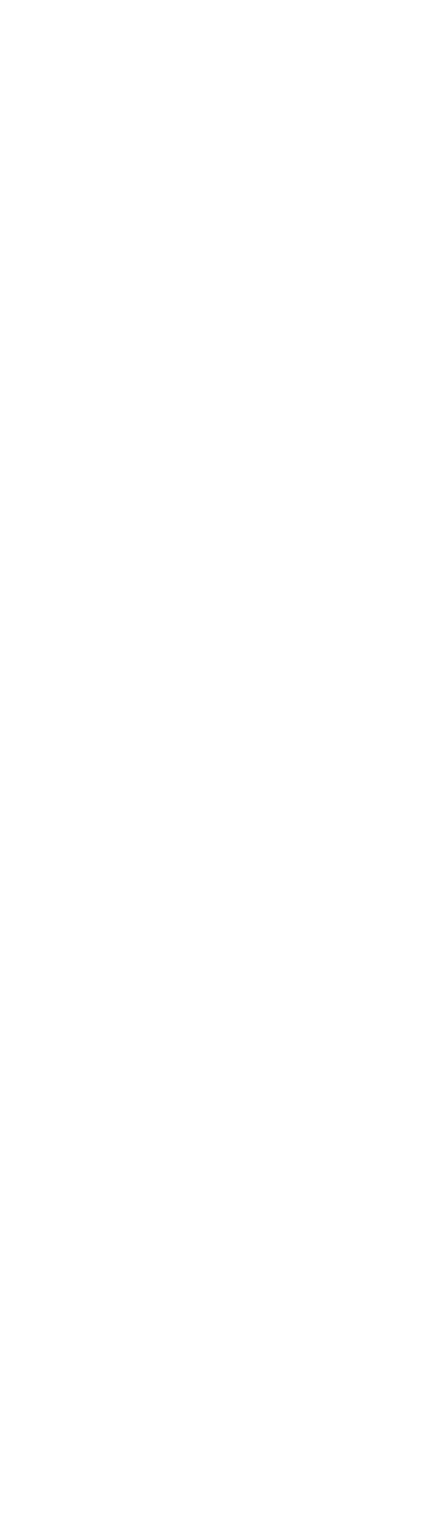




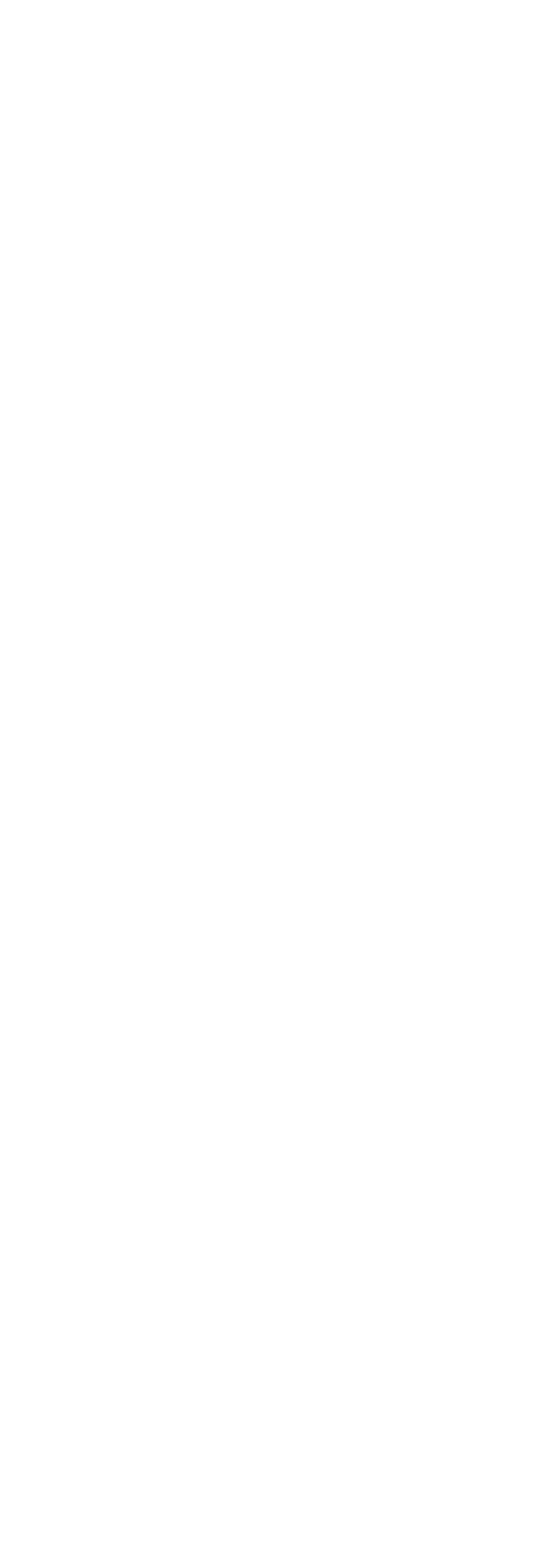




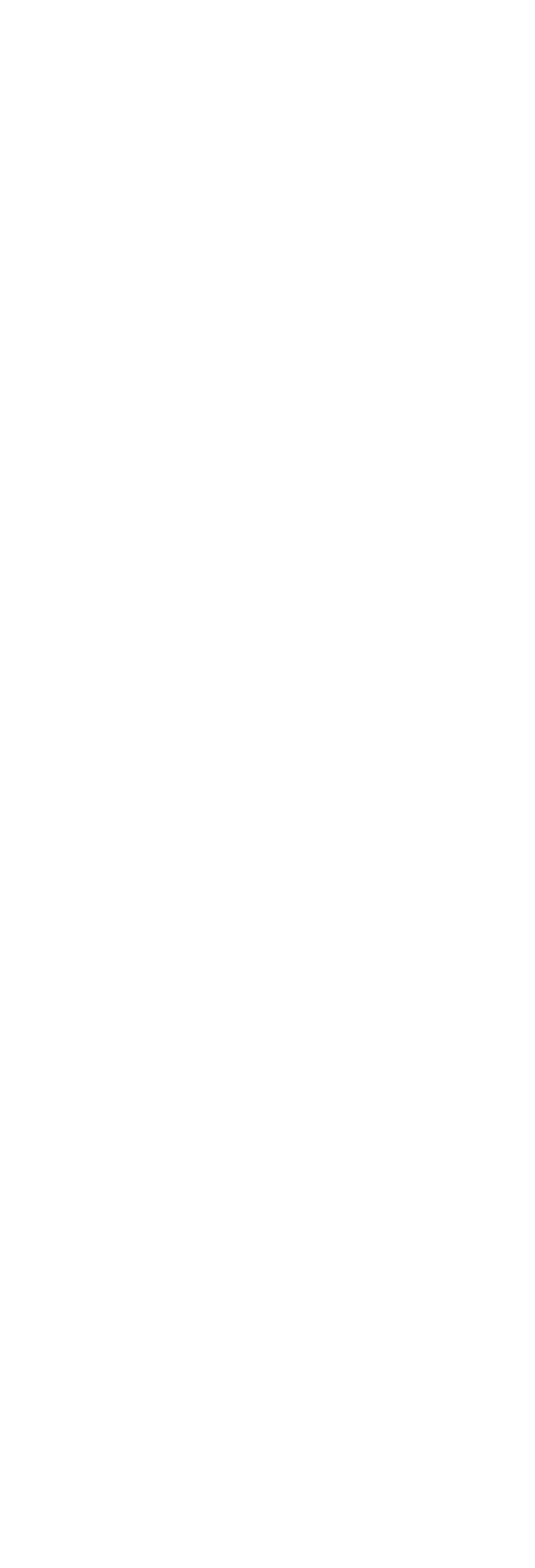




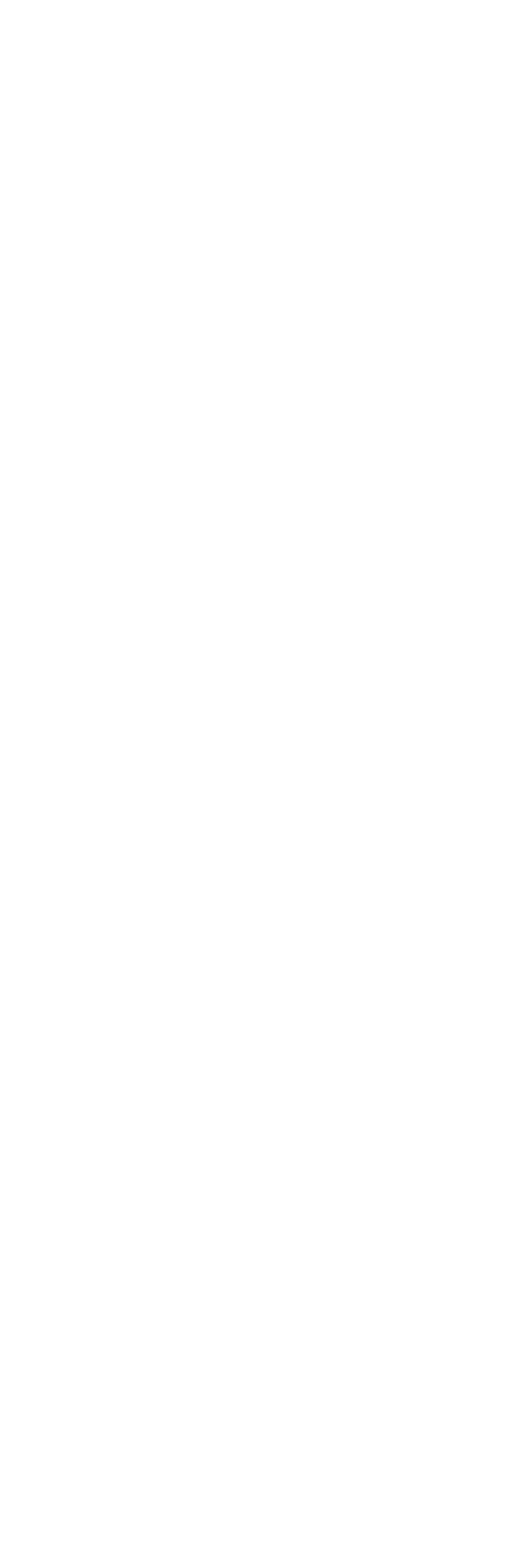




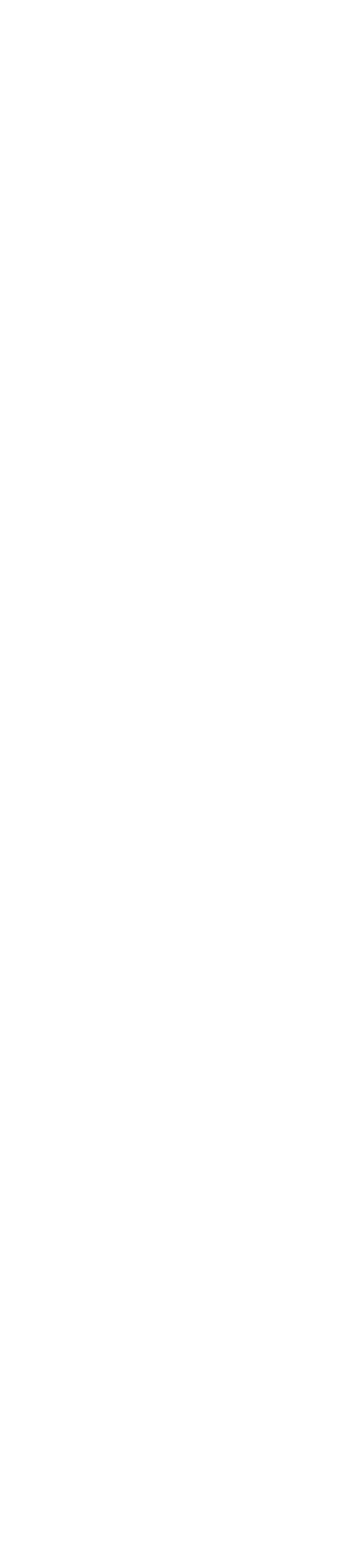

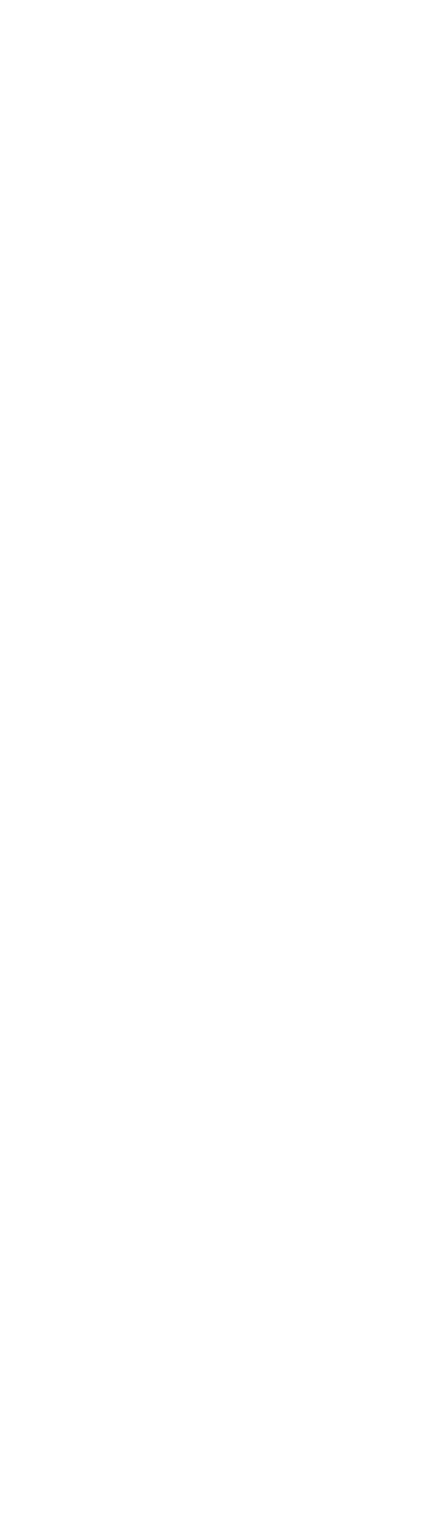




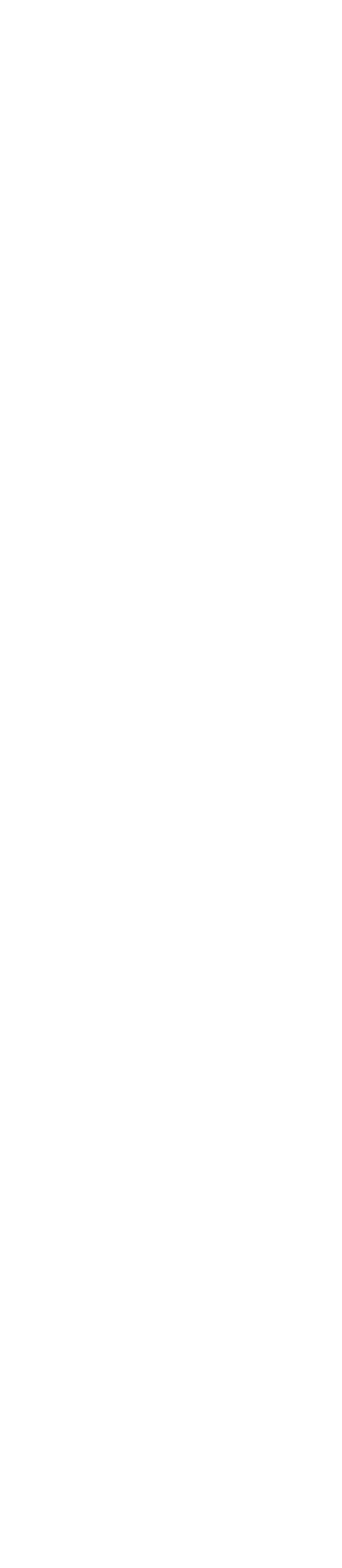

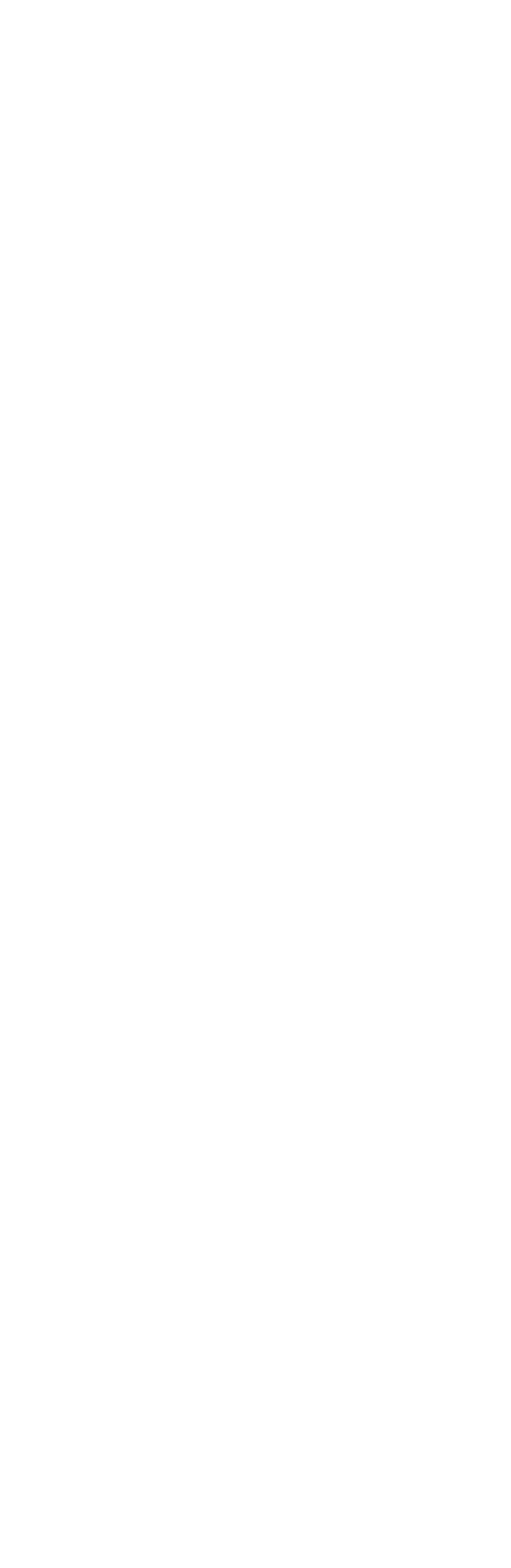




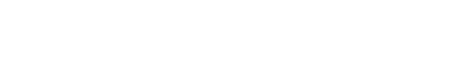

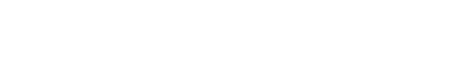

\begin{tabular}{|c|c|c|}
\hline 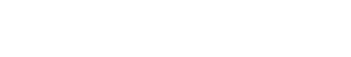 & $\stackrel{\circ}{i}$ & \begin{tabular}{l|l} 
& 1
\end{tabular} \\
\hline 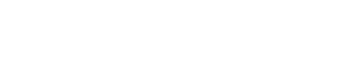 & 8 & : \\
\hline 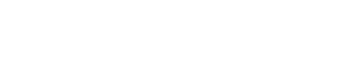 & & \\
\hline
\end{tabular}

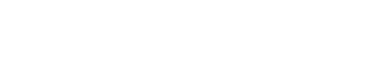

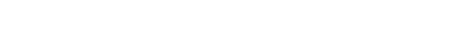

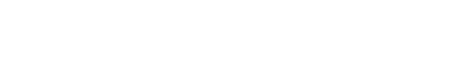

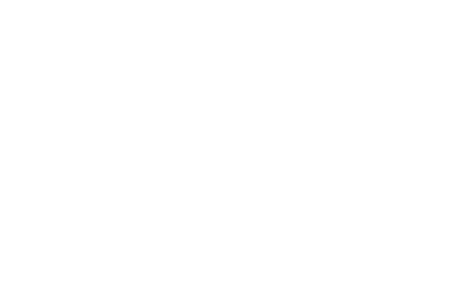

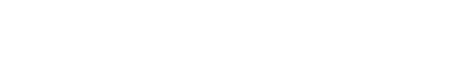

\begin{tabular}{ll|l|l|l|}
\hline 000000000000000 & 0 & 0 & n
\end{tabular}

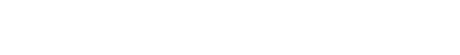

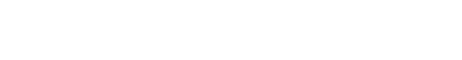

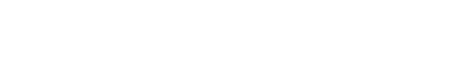

\begin{tabular}{|c|c|c|}
\hline 펌유 & શ & \\
\hline 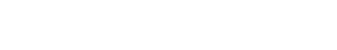 & & \\
\hline
\end{tabular}

\begin{tabular}{|c|c|}
\hline 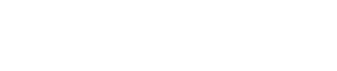 & 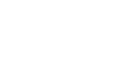 \\
\hline 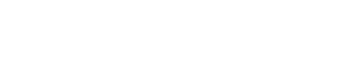 & 节 \\
\hline 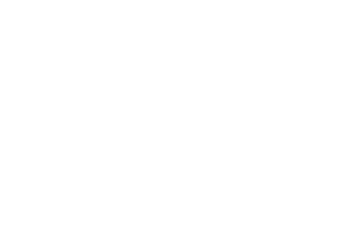 & 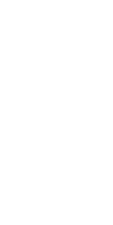 \\
\hline
\end{tabular}

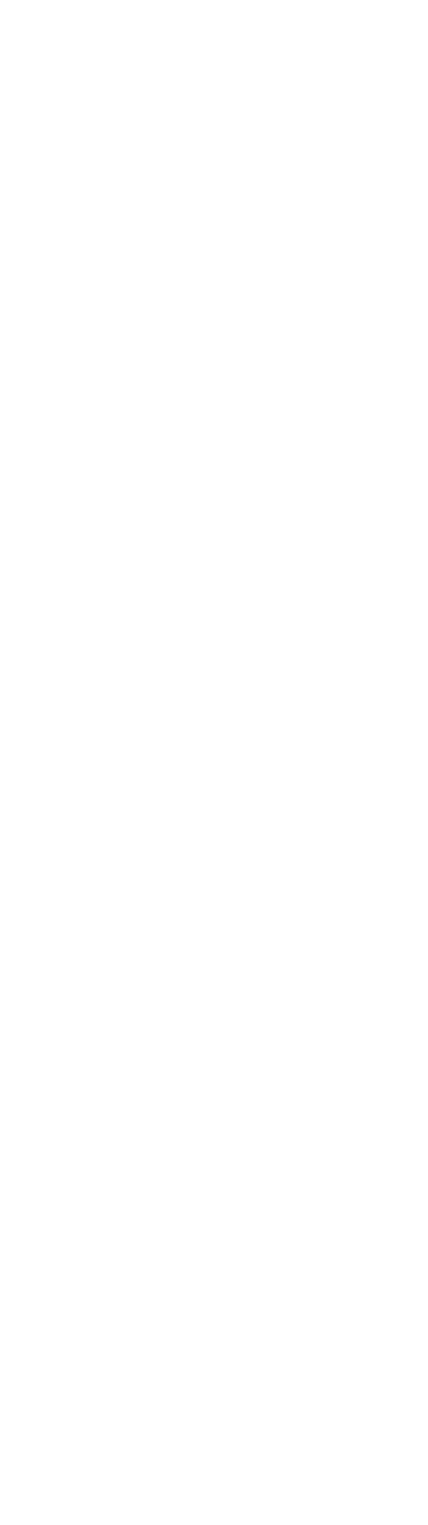




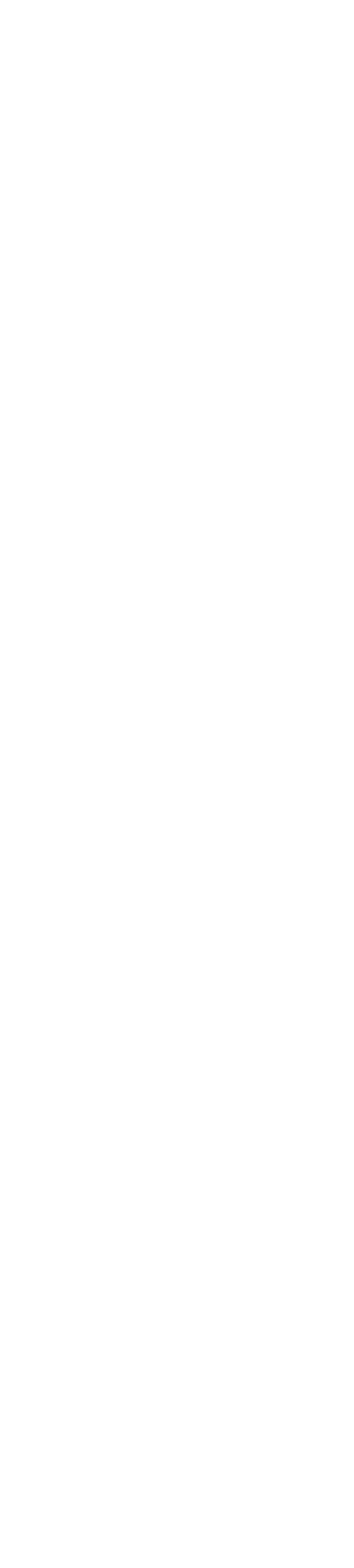

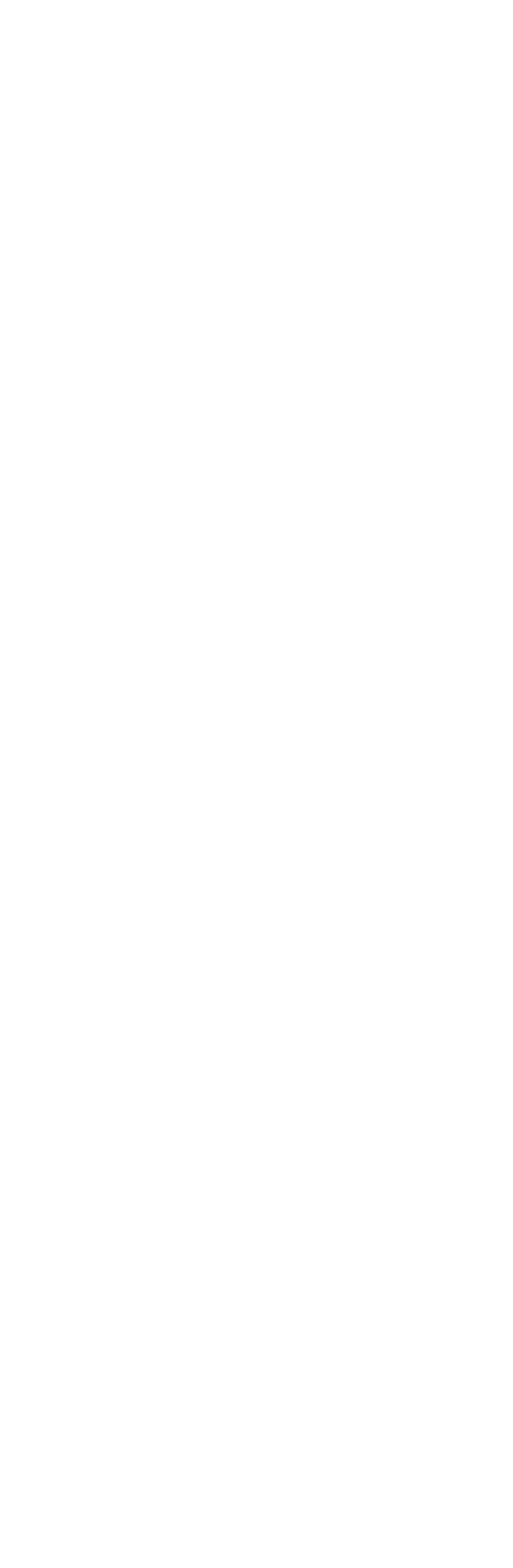




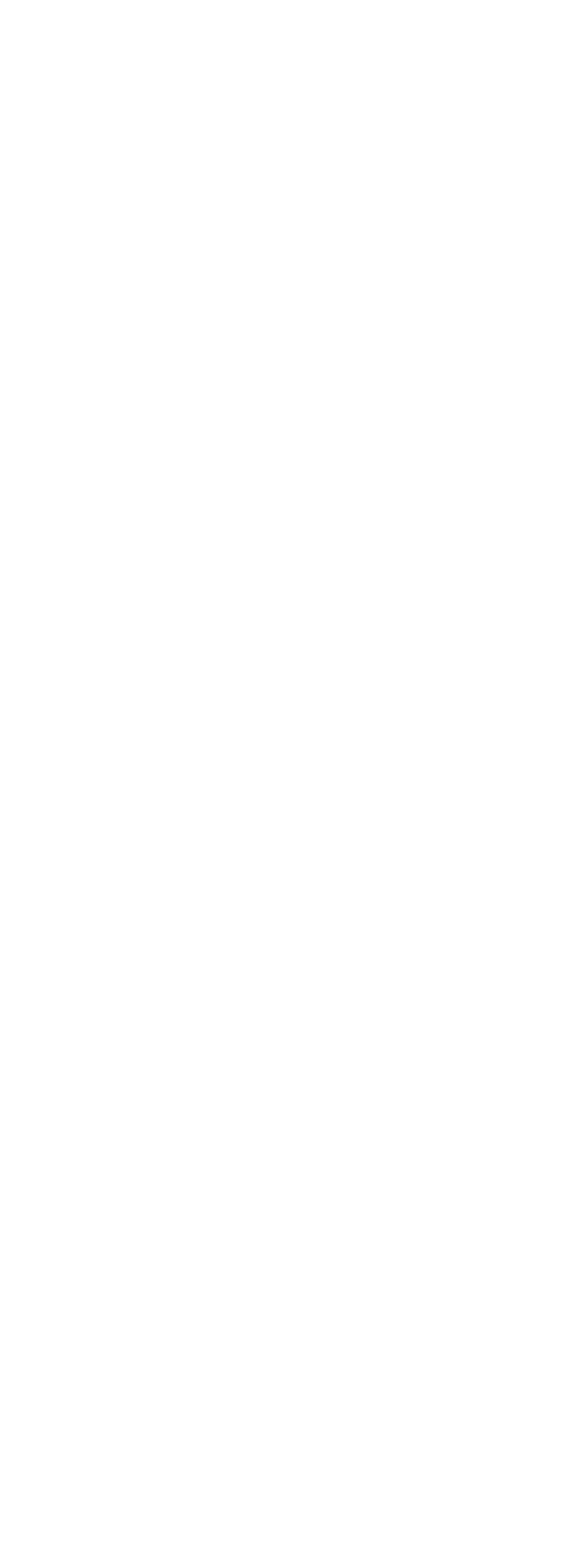




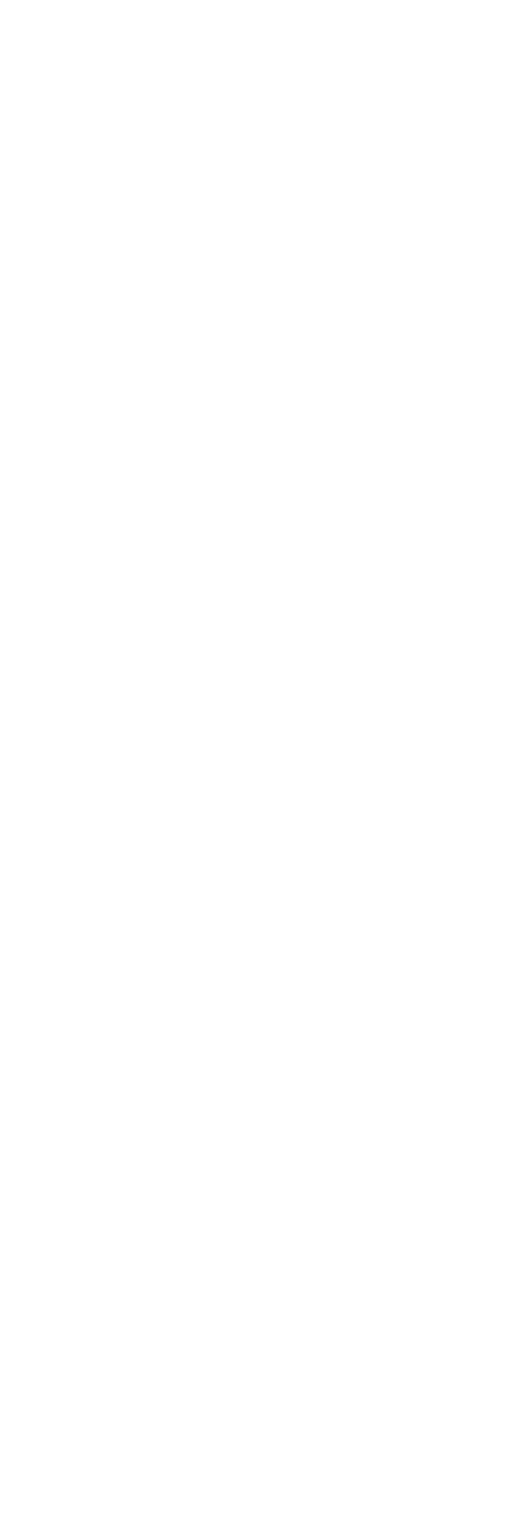




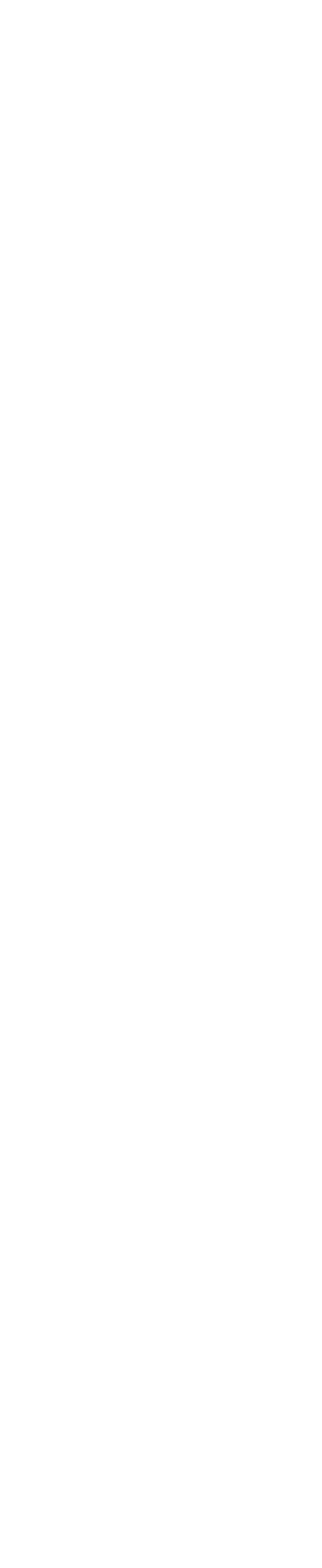

\begin{tabular}{|c|c|}
\hline 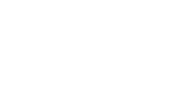 & $\tilde{\widetilde{\pi}}$ \\
\hline 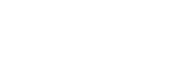 & 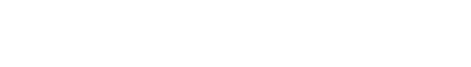 \\
\hline 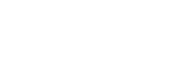 & 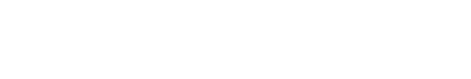 \\
\hline 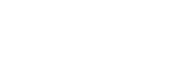 & 诵 \\
\hline 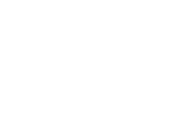 & 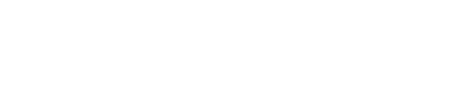 \\
\hline 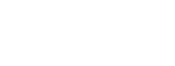 & 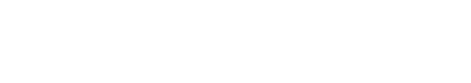 \\
\hline 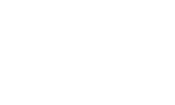 & 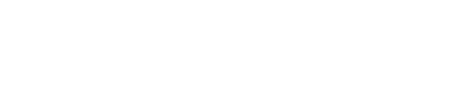 \\
\hline 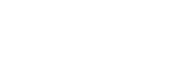 & \\
\hline 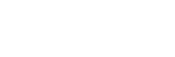 & 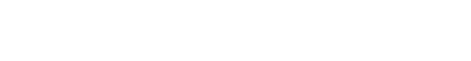 \\
\hline 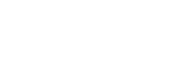 & 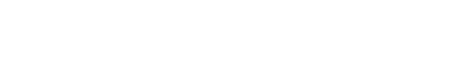 \\
\hline 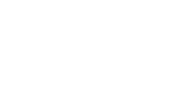 & 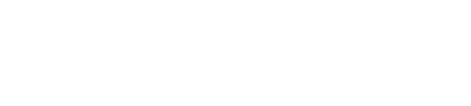 \\
\hline 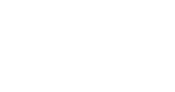 & 북군ㅁำ \\
\hline 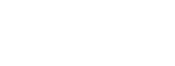 & 000000000000000000 \\
\hline 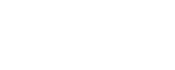 & 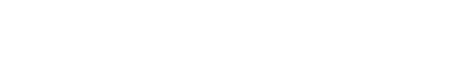 \\
\hline 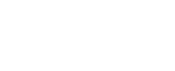 & 60 \\
\hline 裻要 & 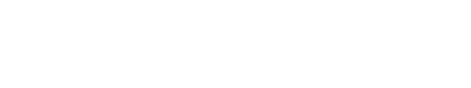 \\
\hline 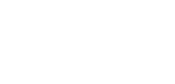 & 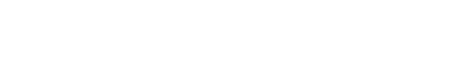 \\
\hline 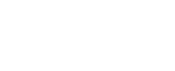 & 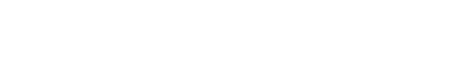 \\
\hline 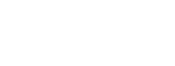 & \\
\hline 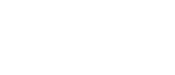 & 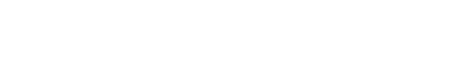 \\
\hline 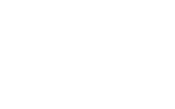 & 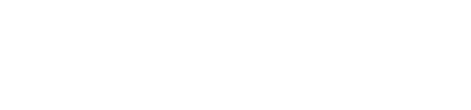 \\
\hline 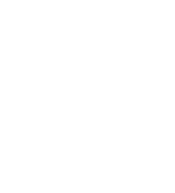 & 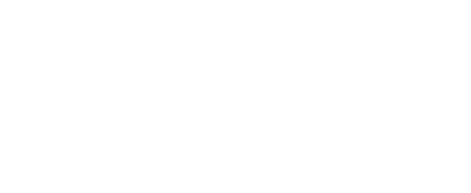 \\
\hline
\end{tabular}




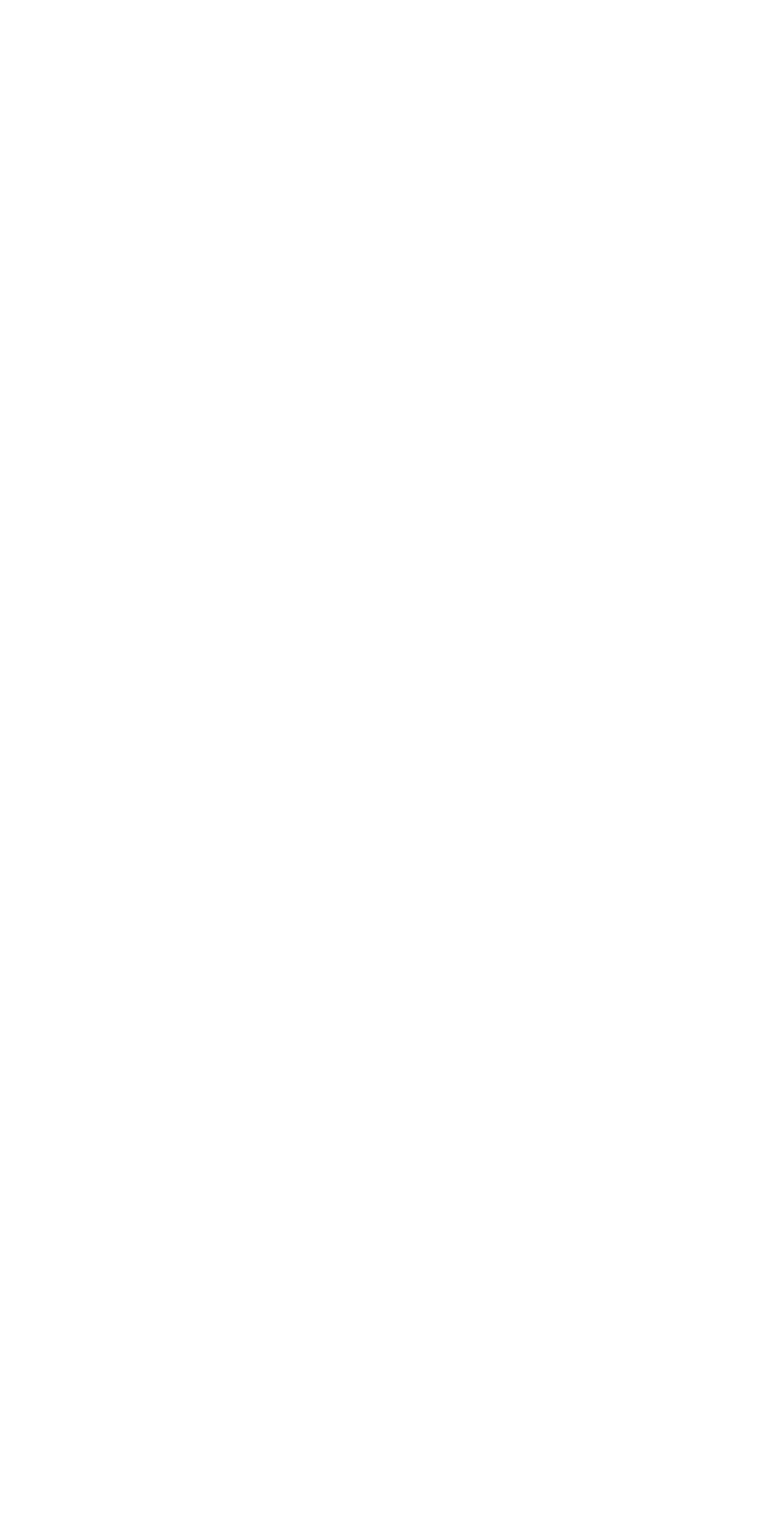




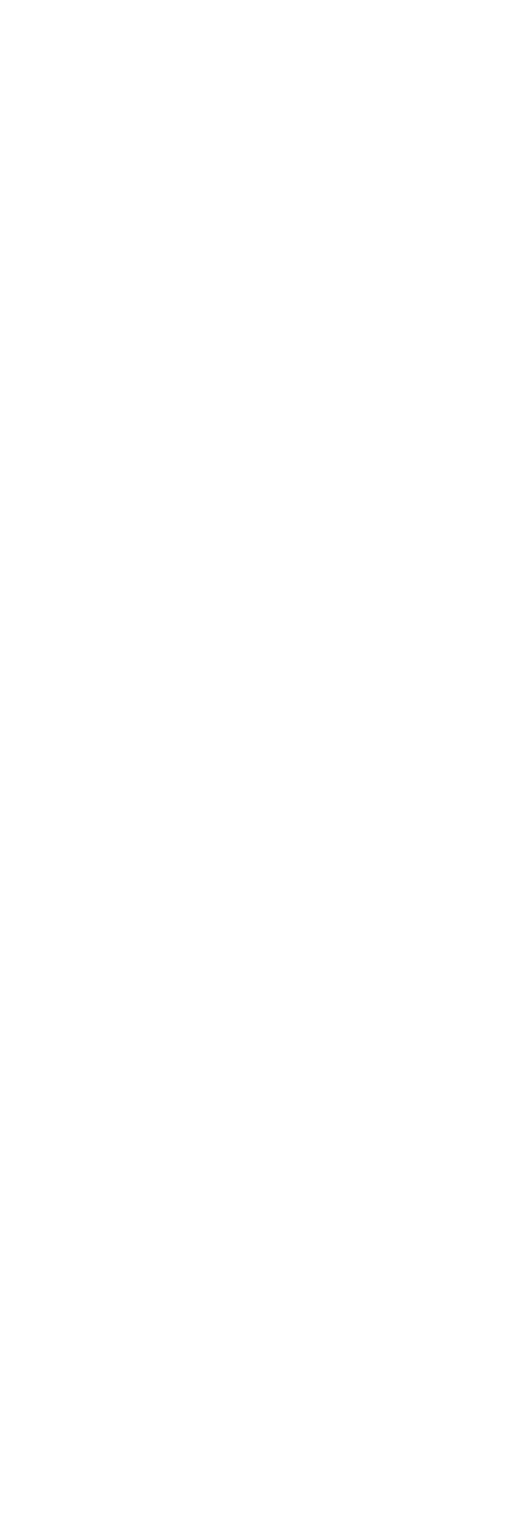




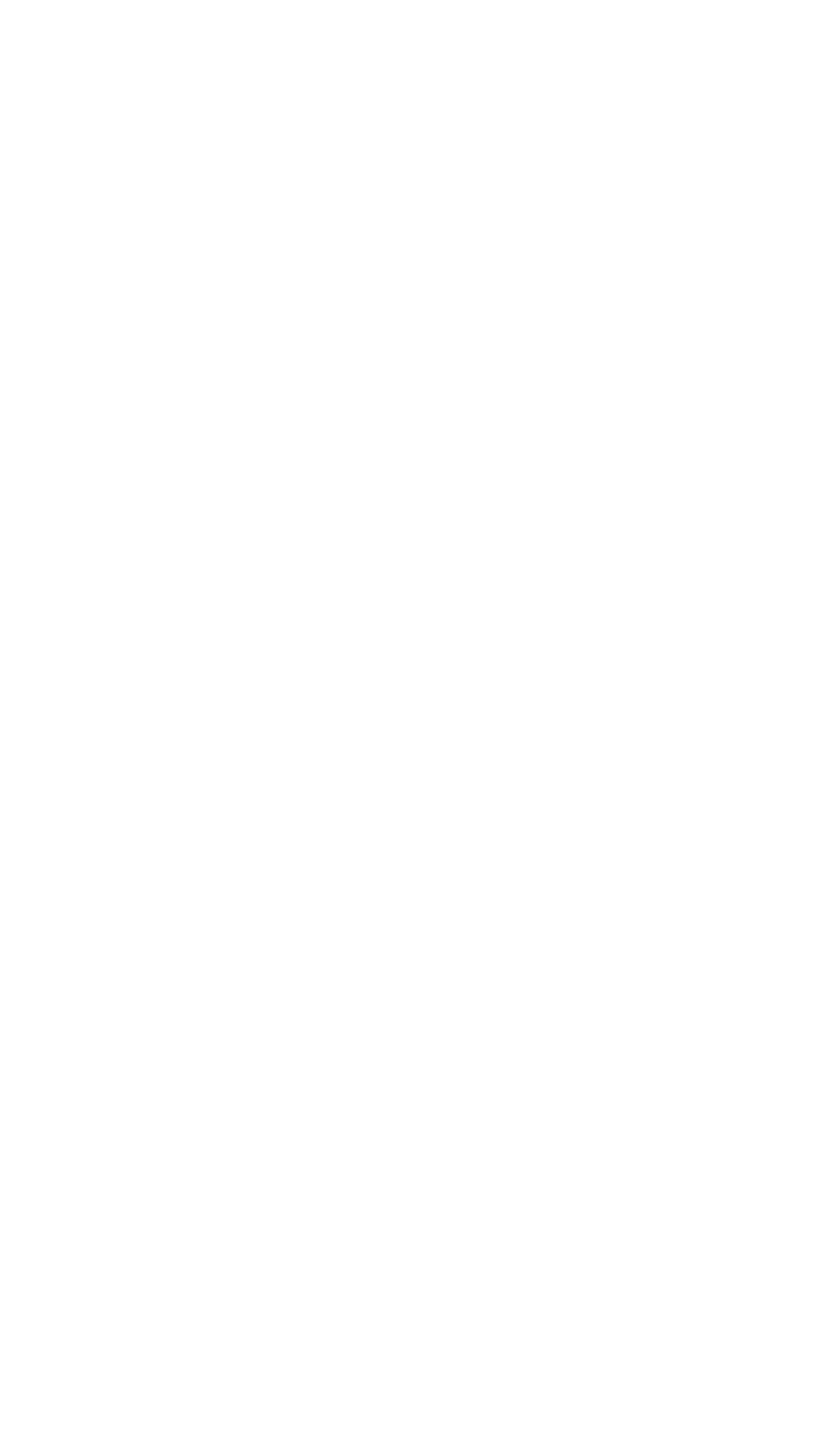




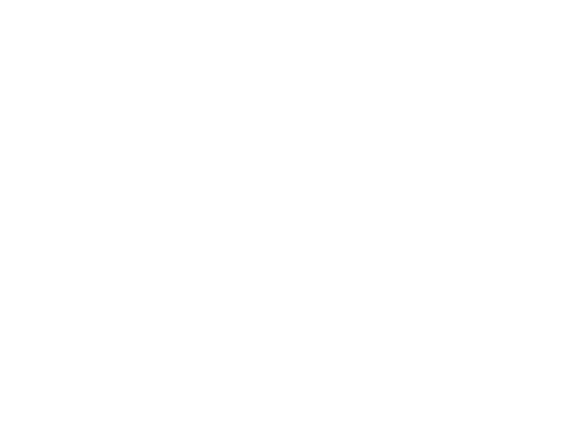

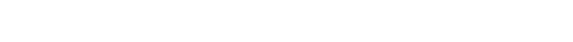

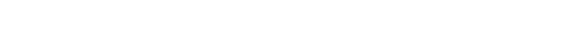

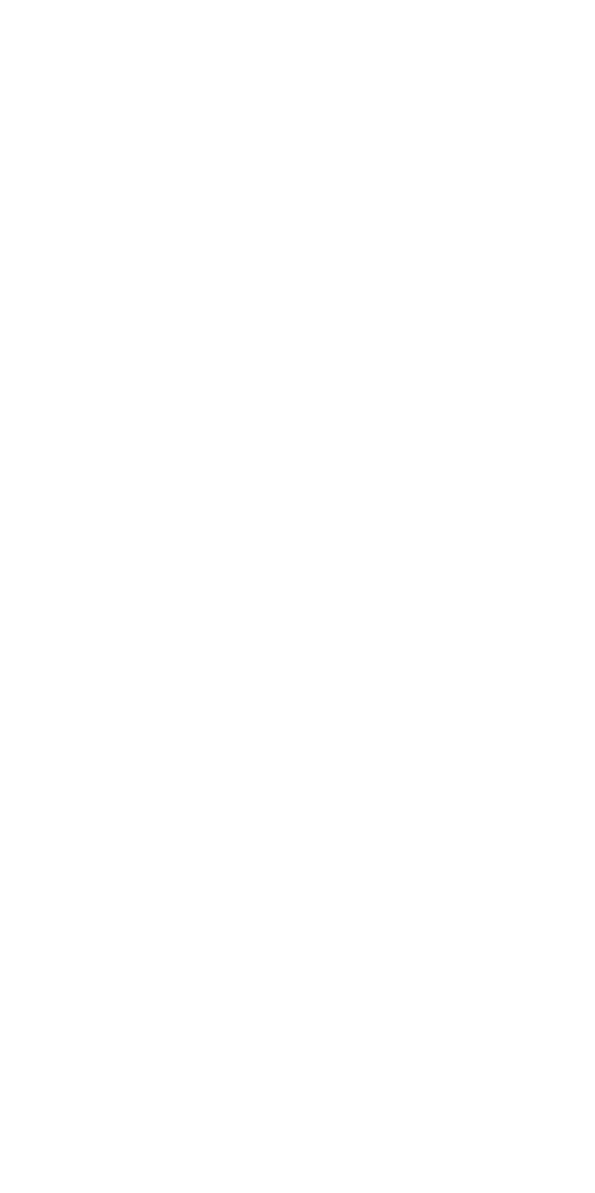

\begin{tabular}{|c|c|c|c|c|}
\hline & nחm & 式药。 & $\vec{R} \tilde{\alpha}$ & ڤ్ \\
\hline & 919 & in $1:$ & 101 & $1: 1$ \\
\hline & ⿱屮凵心 & ס & 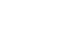 & 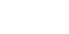 \\
\hline & ming & $n \approx n t$ & $\stackrel{\sim}{\sim} \sim \underset{\infty}{\tilde{N}}$ & జ \\
\hline & ning & 絽 & $\stackrel{20}{\infty}$ & $\stackrel{\substack{\infty \\
\infty}}{ }$ \\
\hline & กㅇㅇ & 415 & 용 & $\mathscr{\infty}_{\infty \pm 0}^{\circ}$ \\
\hline & 요 09 & 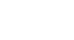 & 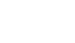 & : \\
\hline & nog & in i⿱ & 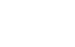 & Ton \\
\hline & 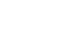 & 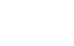 & 120 & 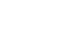 \\
\hline & 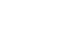 & n1 10 & $\stackrel{\infty}{\sim \infty}$ & 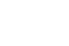 \\
\hline & กุ๊ & ถ & $\stackrel{n}{n} \infty_{\infty}^{\infty}$ & 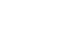 \\
\hline & \pm 50 & 岳的白 & 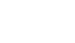 & $\prod_{\infty}^{\infty} \infty$ \\
\hline & $\therefore \rightarrow 00$ & กิ nี & $\stackrel{n \infty}{n}=$ & 声品 \\
\hline & naf & 品告吉 & $m \sim-\infty$ & 声策品 \\
\hline & nof & 워요 & REN & 品变 \\
\hline & 웋 & 马ำ & $\because \approx N$ & 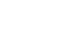 \\
\hline כ) & ㅇ:a & 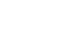 & Q尺N & 邑 \\
\hline & $\begin{array}{r}10 \% \\
\therefore 89 \\
\end{array}$ & 9 กิก & 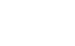 & $\begin{array}{l}2 \\
\infty \\
\infty\end{array}$ \\
\hline & № & J & 무 & 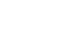 \\
\hline & R未og & 요 & aro & $\begin{array}{l}0 \\
\infty \\
\infty\end{array} \infty$ \\
\hline & กตู & 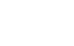 & ofm & 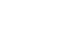 \\
\hline & on in & in 40 & 다요 & 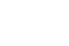 \\
\hline & rofin & ofin & : & 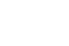 \\
\hline & ID m & in $\approx \overrightarrow{0}$ & $5 \curvearrowleft N$ & ๑ \\
\hline & nis: & こ至す & $a$ & 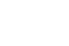 \\
\hline & ㅇon & $\vec{n}$ 出。 & $R_{i}^{n}=$ & $\begin{array}{l}\infty \\
\infty \\
\infty \\
\infty\end{array}$ \\
\hline & nas & Bn n & $\begin{array}{l}a n \infty \\
0 \\
0\end{array}$ & $\underbrace{\infty}_{\infty} \begin{array}{c}\infty \\
\infty\end{array}$ \\
\hline & nos.t. & Sin no & 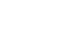 & : \\
\hline & nop & ginn & D゚̊ & 冓 \\
\hline & man & gano & 思な゚ & 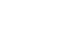 \\
\hline & No: & gon & 品N & ‡ \\
\hline & 중 & gूn & 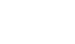 & $\stackrel{5}{\infty} \approx$ \\
\hline$\overline{2}$ & 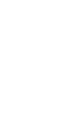 & 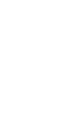 & 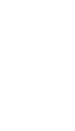 & 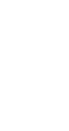 \\
\hline
\end{tabular}




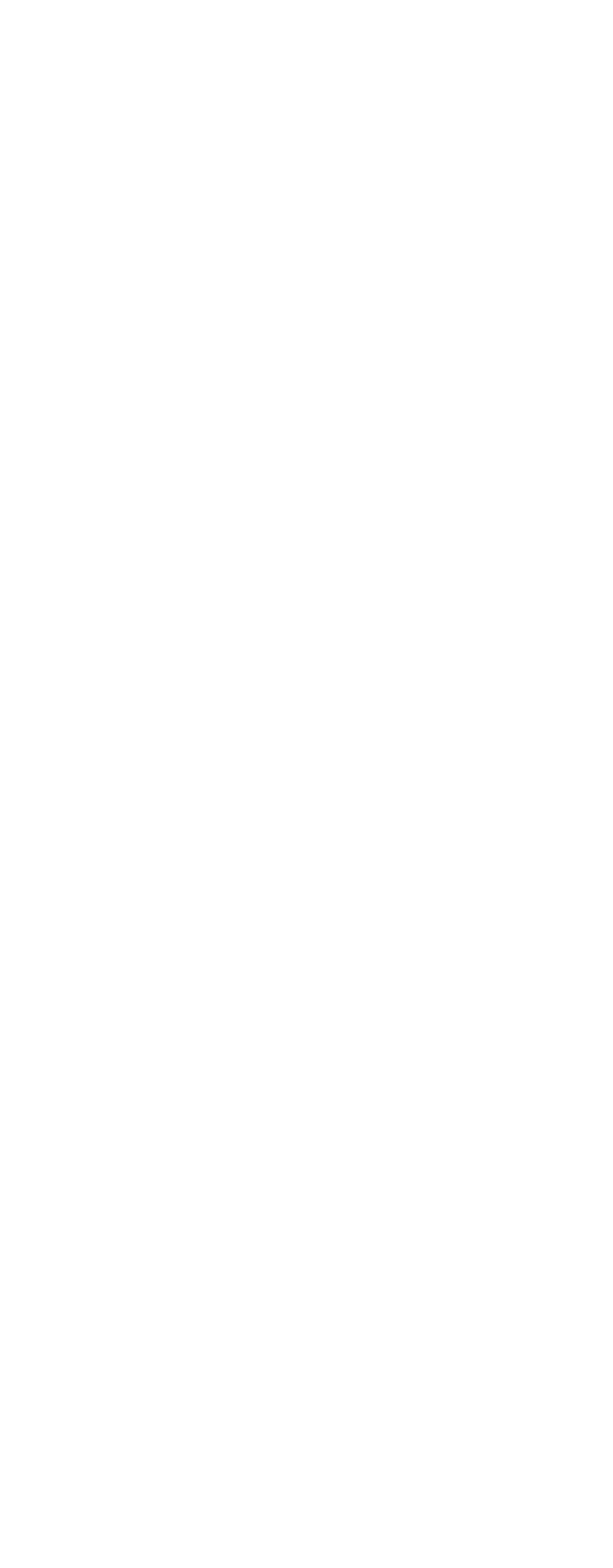




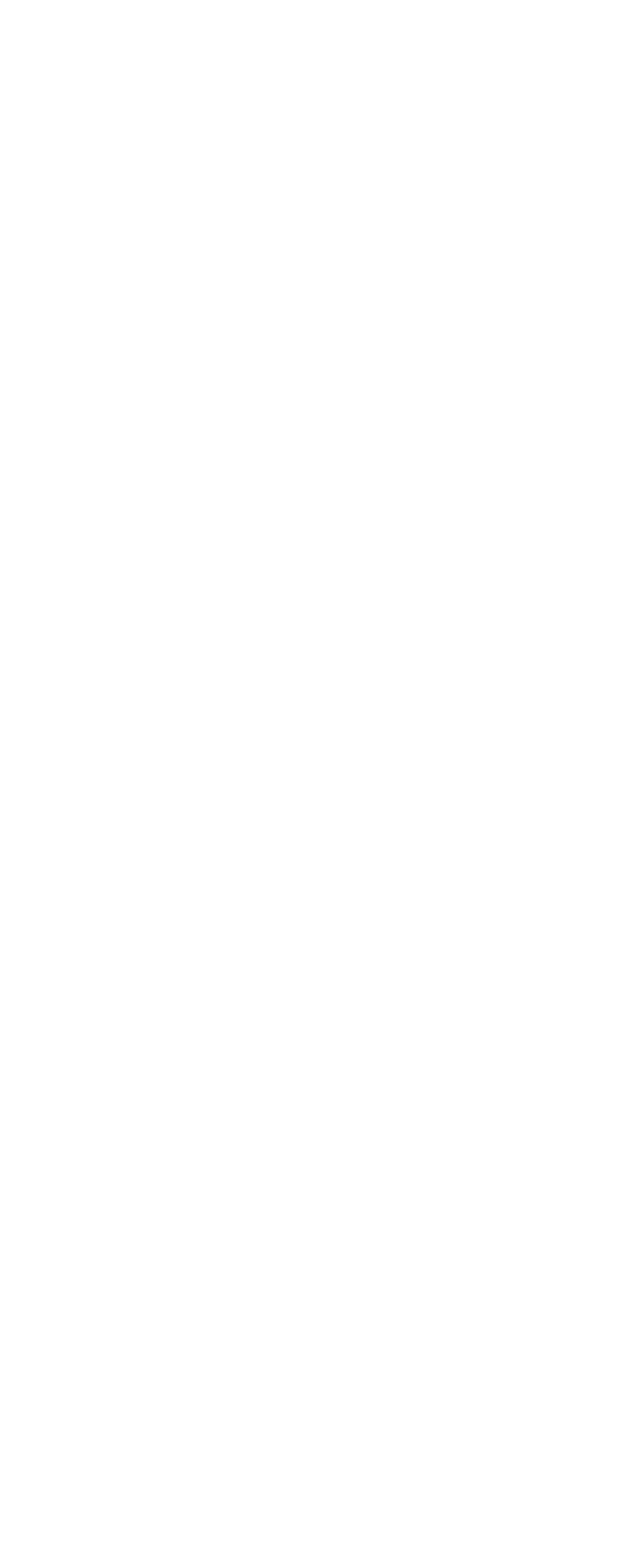




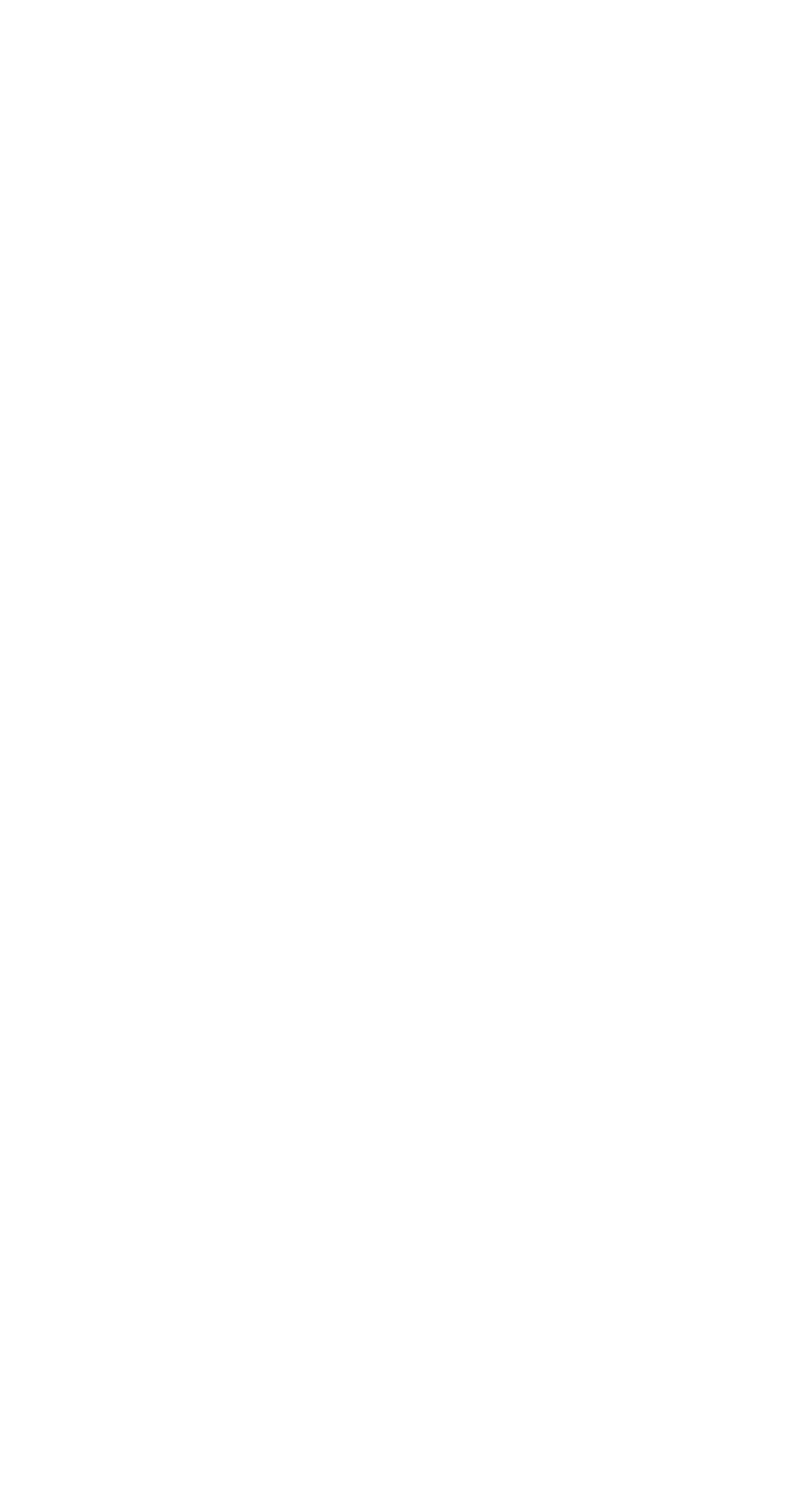




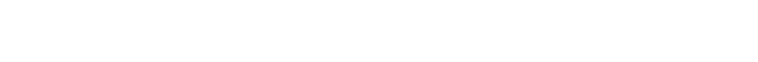

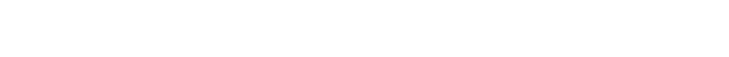

\begin{tabular}{|c|c|c|c|c|c|c|c|c|c|}
\hline 1 & r. & $\bullet$. & $!$ & 1 $\%$ & m. & 1 & $m$ & & ल. \\
\hline$\infty$ & $\hat{m}$ & F & $\therefore$ & \& \& \& & ฟ & $\dddot{7}$ & ले & in & 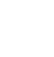 \\
\hline 苛 & $\stackrel{\infty}{\Phi}$ & 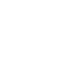 & $\stackrel{\infty}{\sim}$ & $\stackrel{\infty}{\mathbb{N}} \underset{\sim}{\infty} \stackrel{\infty}{\sim} \underset{]}{\infty}$ & 芯 & 웅 & $\stackrel{\mathscr{D}}{\mathscr{D}}$ & $\stackrel{\pi}{\pi}$ & 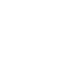 \\
\hline i & 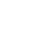 & 今े & 1 & 1 ले ڤ్ & ભొ & 1 & $\stackrel{\infty}{m}$ & 1 & ले. \\
\hline
\end{tabular}

\begin{tabular}{|c|c|c|c|c|c|c|c|c|}
\hline$i$ & $\tilde{\aleph}$ & 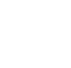 & & $1:$ & & 1 & $\stackrel{\infty}{N}$ & I స్ูิ \\
\hline$i$ & : & $\dot{\dot{m}} \stackrel{\sim}{+}$ & & $1 \stackrel{n}{i} \stackrel{0}{0}$ & & I & $\Rightarrow$ & $1=\stackrel{+}{\infty}$ \\
\hline i & ?. & $\%$ & & 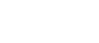 & 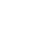 & i & r. & $1 \quad 0.0$ \\
\hline
\end{tabular}

\begin{tabular}{|c|c|c|c|c|c|c|c|c|}
\hline I & a & $\exists \cong$ & $\ddot{N}$ & $\cong$ & \& & 욤 & 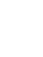 & $\approx \pm 0$ \\
\hline 1 & $\tilde{ల}$ & $\ddot{a} q$ & $i$ & $1 \%$ & $\tilde{~}$ & 1 & N & i \& \& జ \\
\hline 8 & $\stackrel{్}{\$}$ & $\underset{N}{\mathbb{N}}$ & 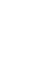 & 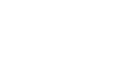 & d. & $\stackrel{\circ}{\circ}$ & $\stackrel{\infty}{\infty}$ & 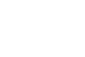 \\
\hline
\end{tabular}

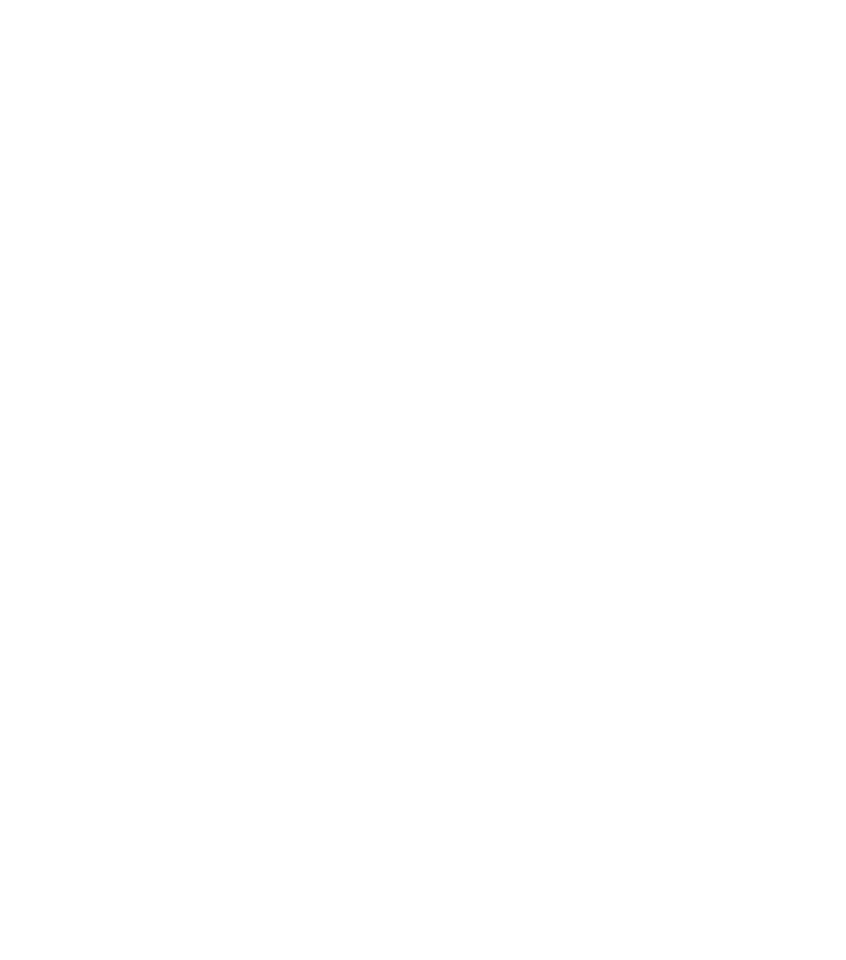




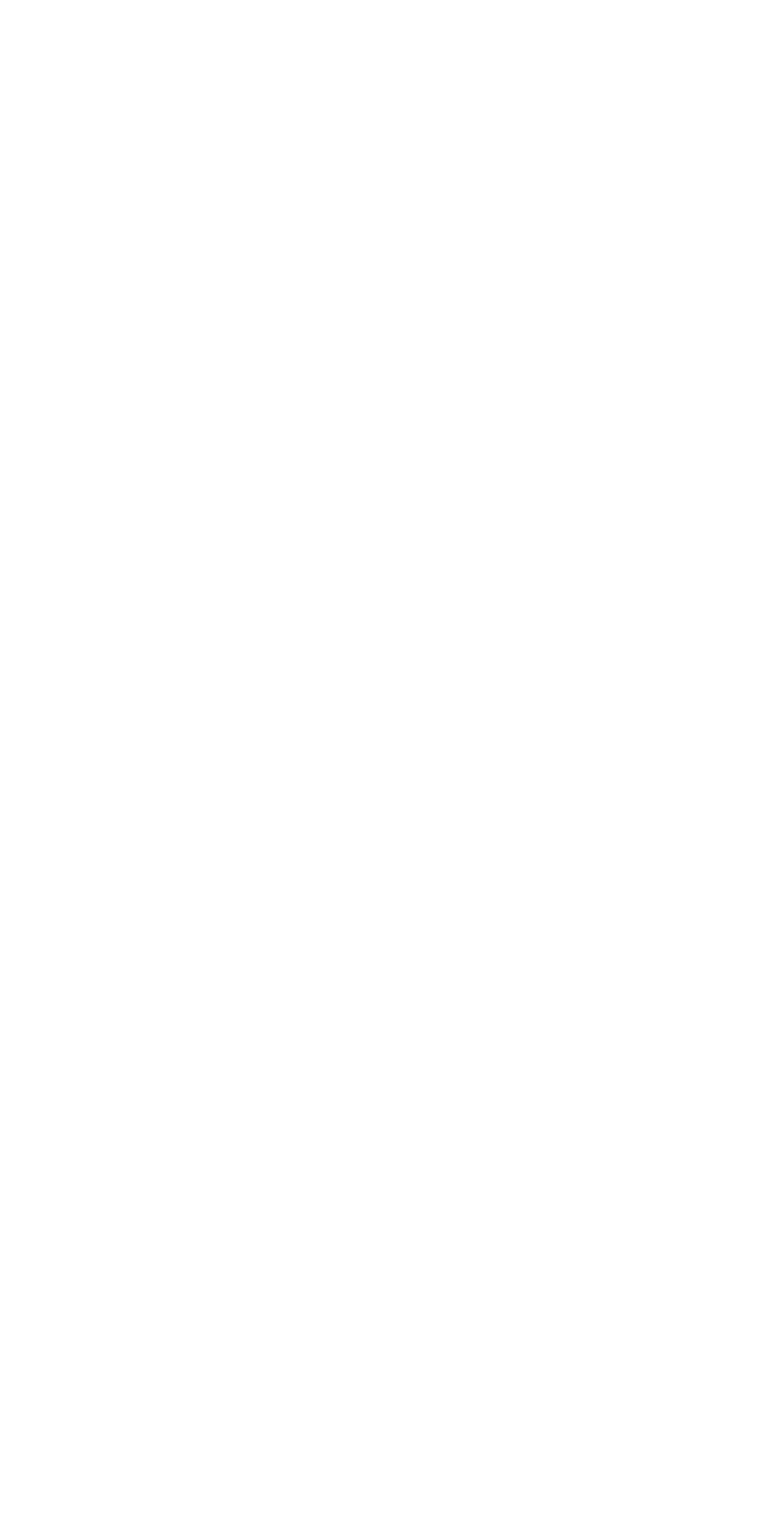




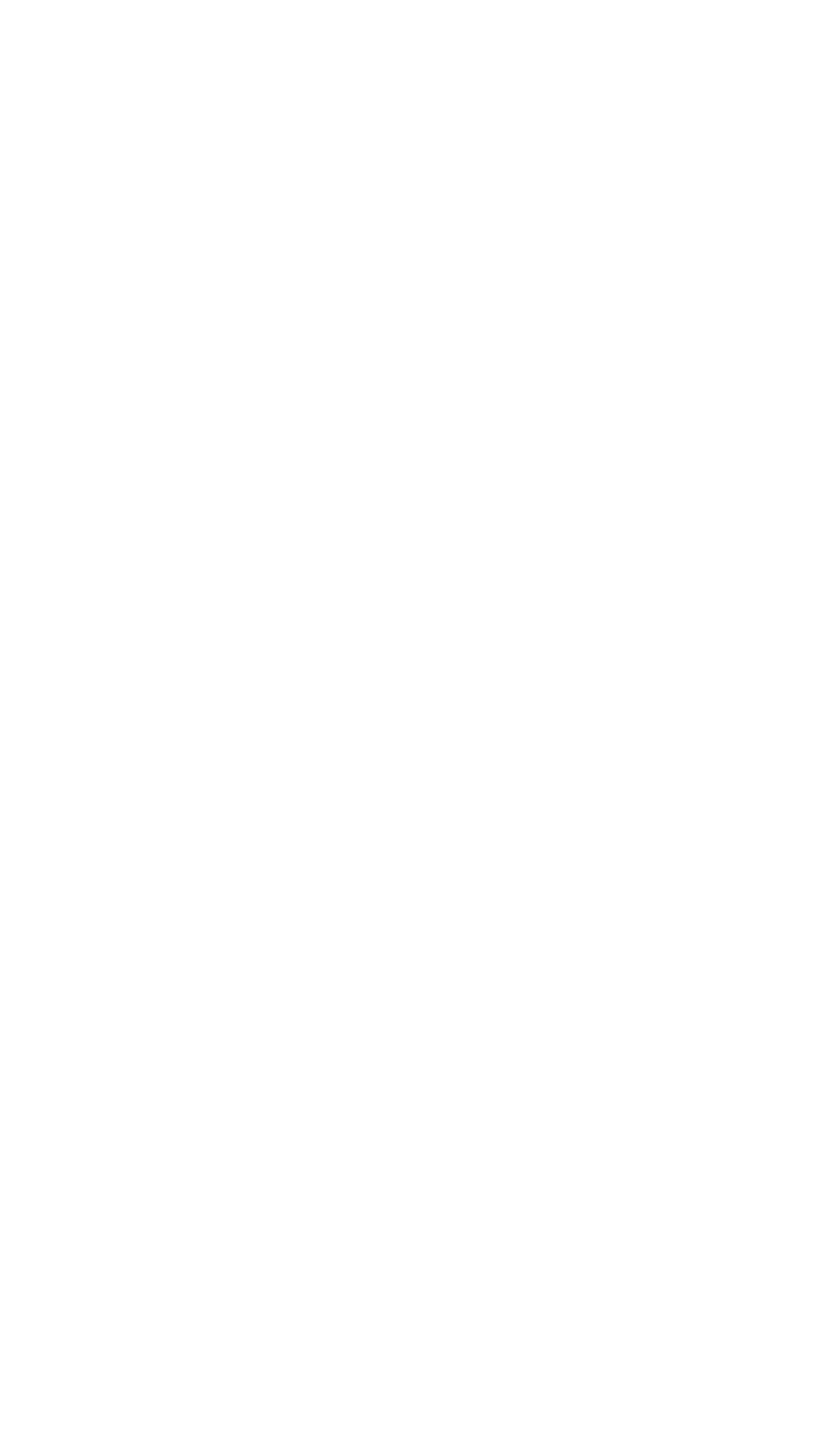




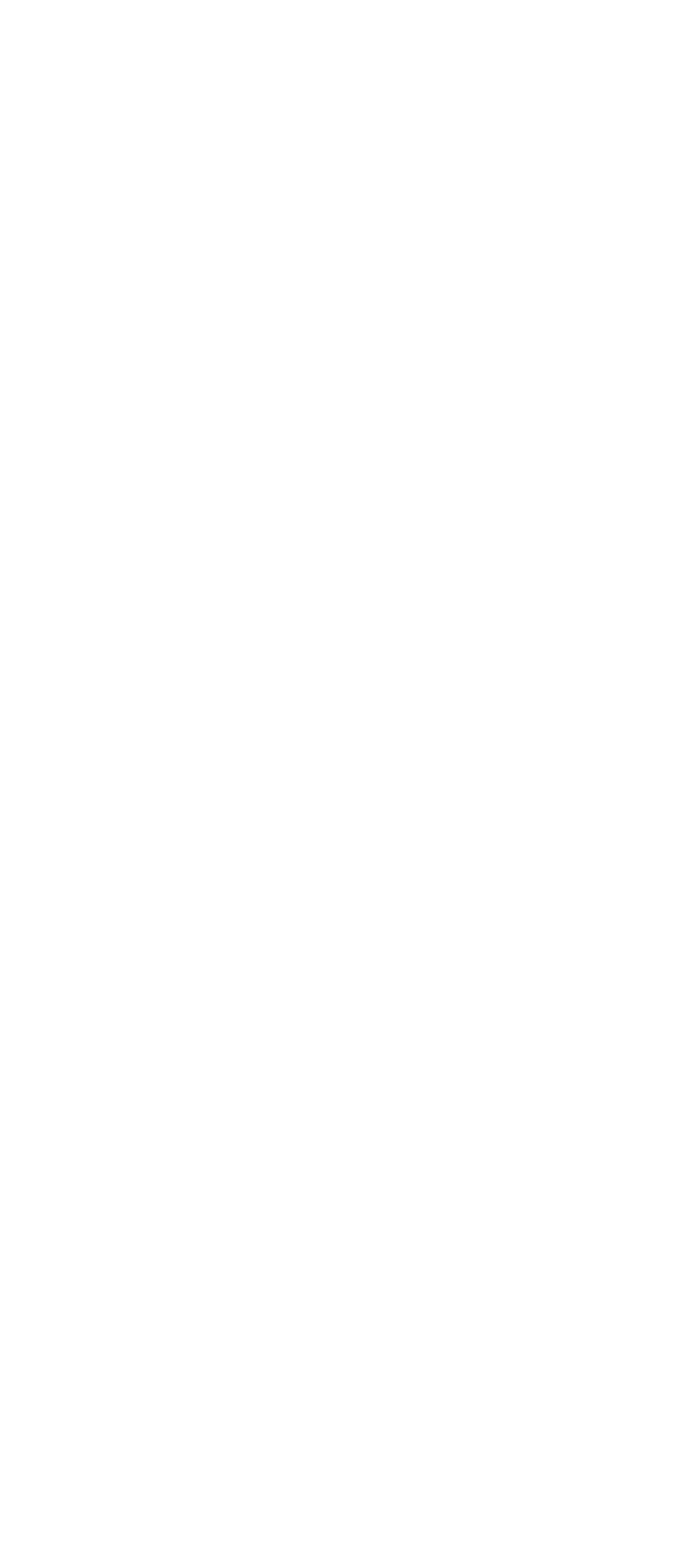




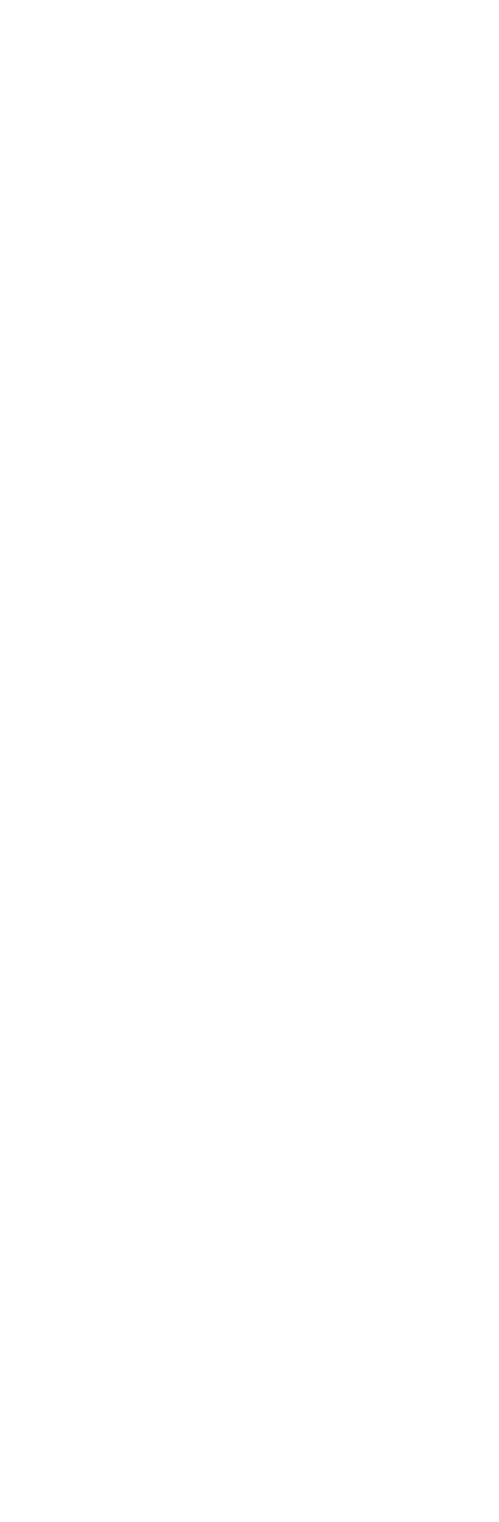




\begin{tabular}{|c|c|}
\hline 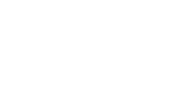 & 18 \\
\hline 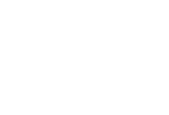 & 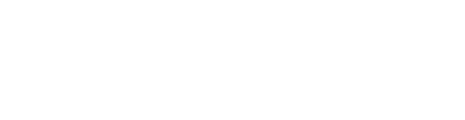 \\
\hline 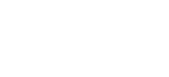 & 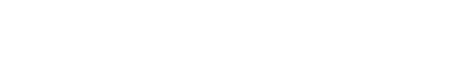 \\
\hline 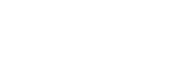 & 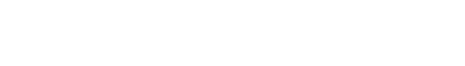 \\
\hline 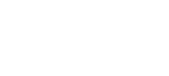 & 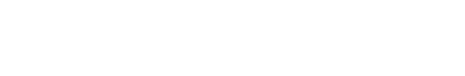 \\
\hline 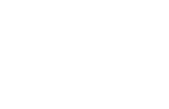 & 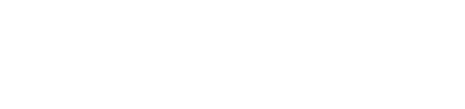 \\
\hline 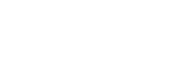 & 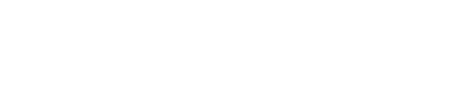 \\
\hline 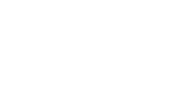 & 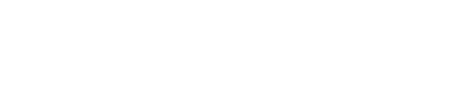 \\
\hline 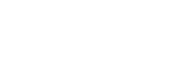 & 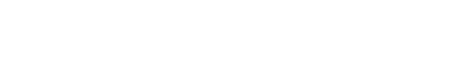 \\
\hline 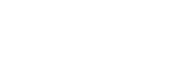 & 1911110111114111111 \\
\hline 昜产 & 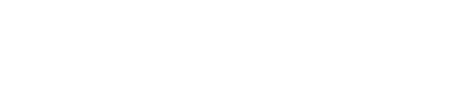 \\
\hline 要 & 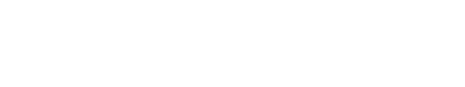 \\
\hline 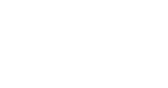 & 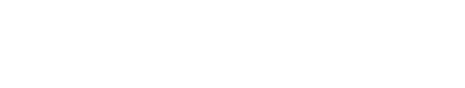 \\
\hline 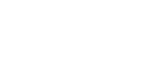 & 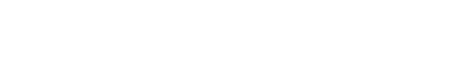 \\
\hline 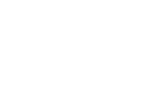 & 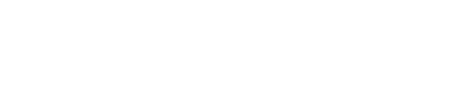 \\
\hline 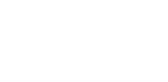 & \\
\hline 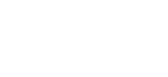 & 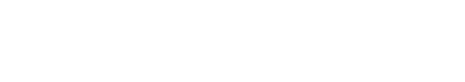 \\
\hline 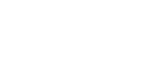 & 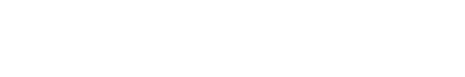 \\
\hline 器嵒 & \\
\hline 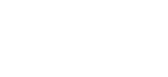 & 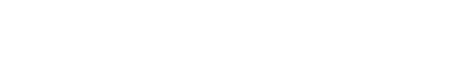 \\
\hline 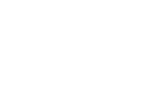 & 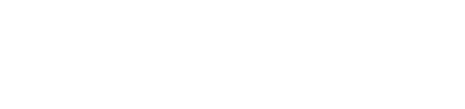 \\
\hline 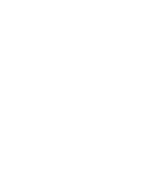 & 促: \\
\hline
\end{tabular}




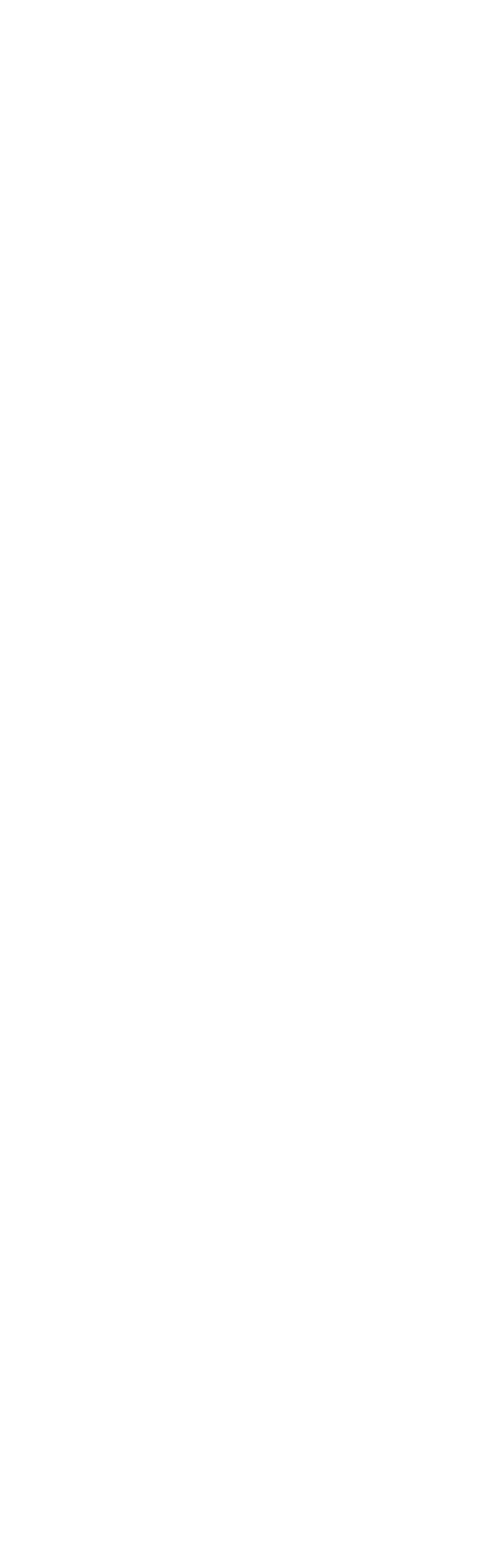

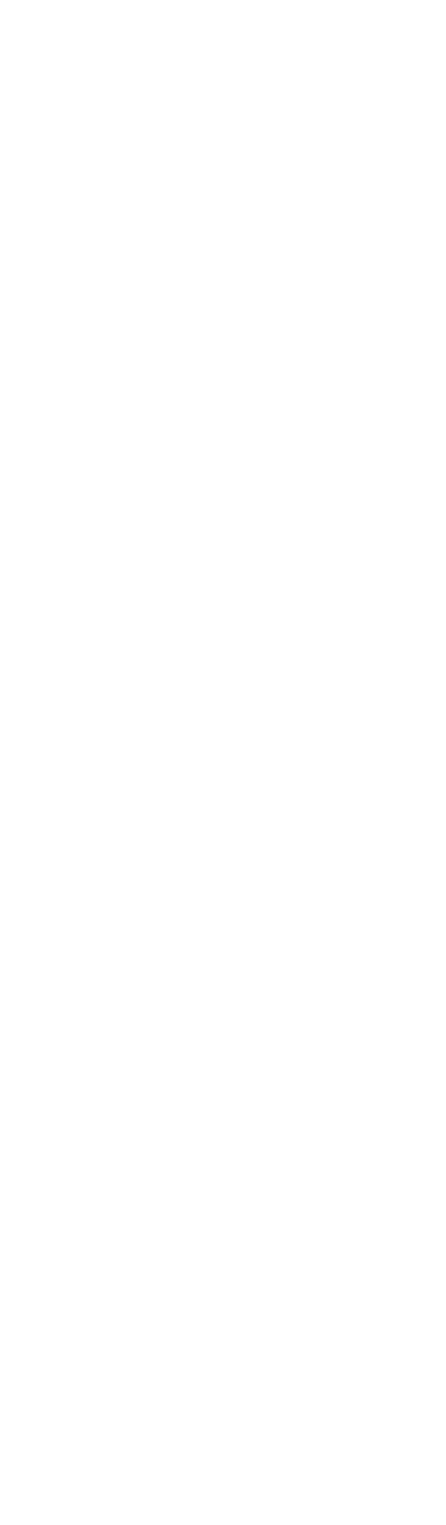




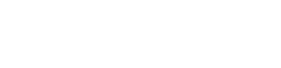

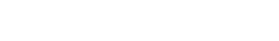
70\%

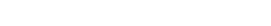

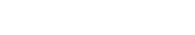
象息

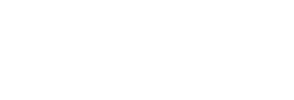

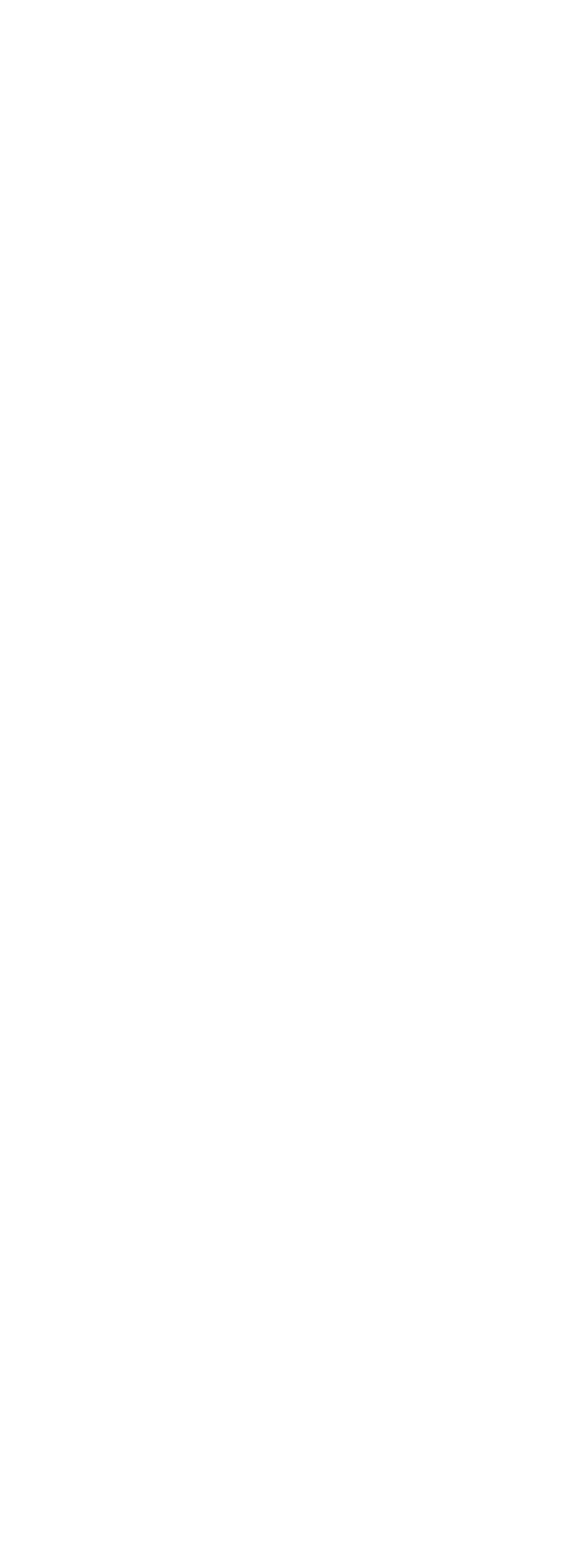




\begin{tabular}{|c|c|c|c|}
\hline 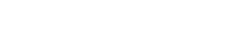 & \begin{tabular}{lll}
$n$ \\
\hdashline \\
\hdashline
\end{tabular} & $\therefore$ & \\
\hline 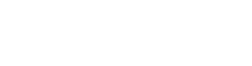 & 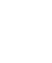 & 装 & \\
\hline मुम & \begin{tabular}{|l|l|}
$m$ \\
+ \\
+
\end{tabular} & : & \\
\hline 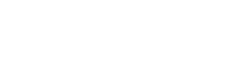 & 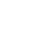 & 㐫 & 1 \\
\hline 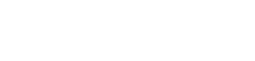 & \begin{tabular}{|l|}
$\infty$ \\
$m$ \\
$m$
\end{tabular} & बे & $\mathrm{i}$ \\
\hline 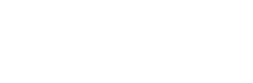 & & 1 & 1 \\
\hline สุ๊ & $\begin{array}{c}\ddot{N} \\
i \\
\end{array}$ & 1 & 1 \\
\hline 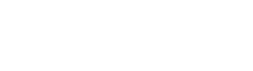 & $\frac{\pi}{2}$ & 品 & 1 \\
\hline 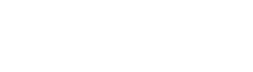 & $\stackrel{0}{0}$ & $\approx$ & $\ddot{\circ}$ \\
\hline 11919111117 & 1 & $i$ & ! \\
\hline 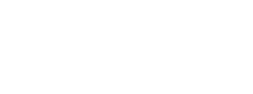 & 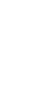 & สี & $\vec{\infty}^{\circ}$ \\
\hline 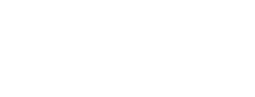 & 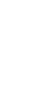 & ज़| & : \\
\hline 00000000000 & N & $\pi$ & $\circ$ \\
\hline 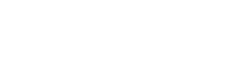 & 然 & ๙ิ & + \\
\hline 1111111111 & 1 & 1 & 1 \\
\hline 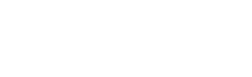 & กิ & ป্ & is \\
\hline 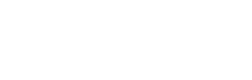 & $\infty$ & 의 & $\stackrel{\circ}{i}$ \\
\hline 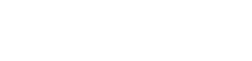 & 8 & 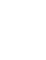 & - \\
\hline 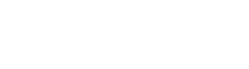 & $\approx$ & $\because$ & $\stackrel{m}{0}$ \\
\hline 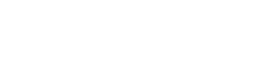 & 1 & : & i \\
\hline 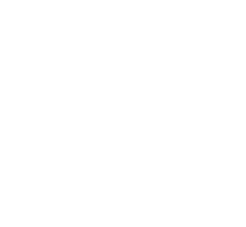 & 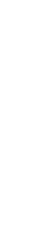 & 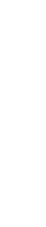 & 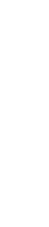 \\
\hline
\end{tabular}

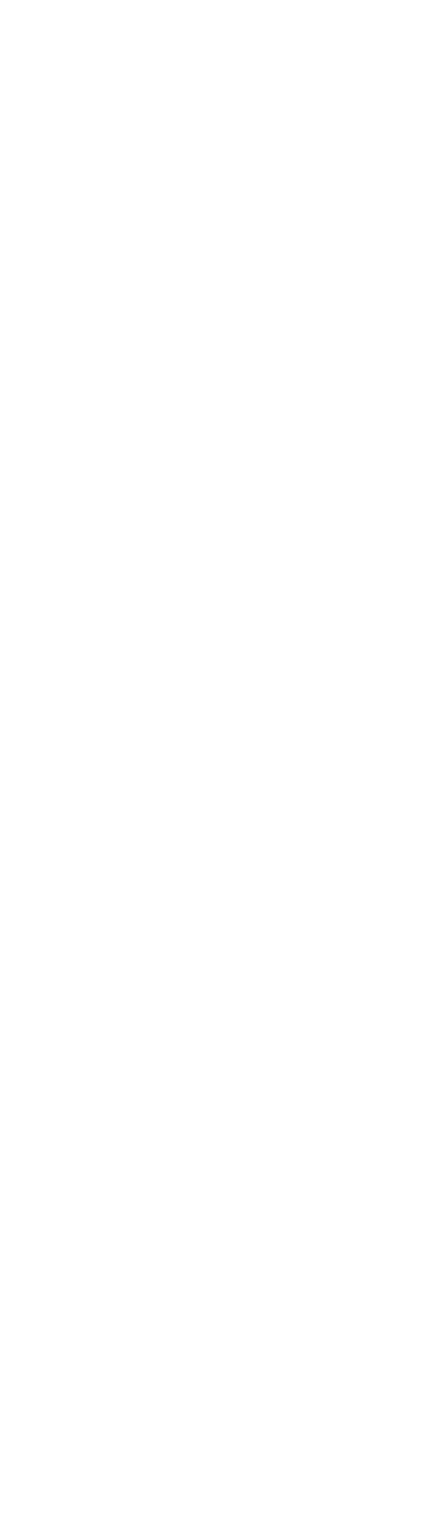




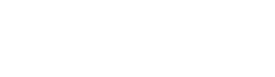

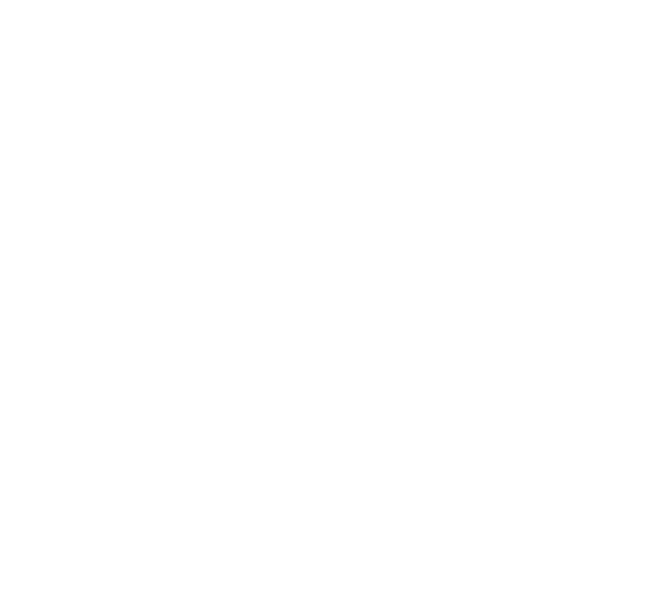

\begin{tabular}{|l|l|}
\hline 安若西 & \\
\hline
\end{tabular}

\begin{tabular}{|c|c|}
\hline 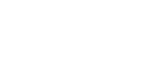 & 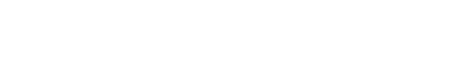 \\
\hline
\end{tabular}

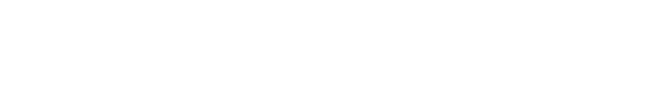

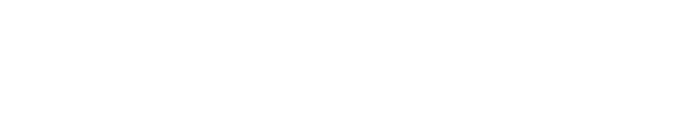

\begin{tabular}{|c|c|c|}
\hline 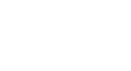 & 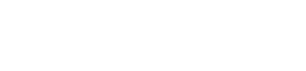 & 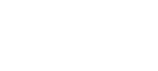 \\
\hline 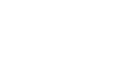 & 00000000000000 & 00000 \\
\hline$\frac{1}{1}$ & 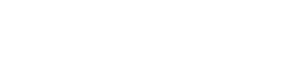 & 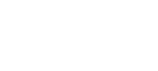 \\
\hline 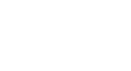 & $111111 \quad 1114111$ & $1111^{2} 11$ \\
\hline 轰要 & 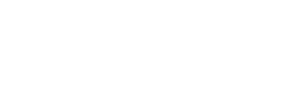 & 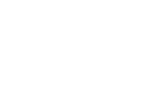 \\
\hline 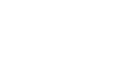 & 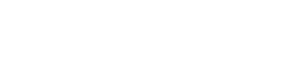 & 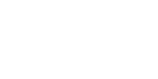 \\
\hline 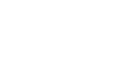 & 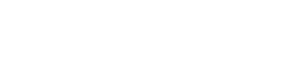 & 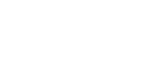 \\
\hline 跑 & & \\
\hline 造产 & 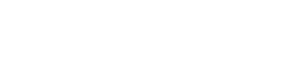 & 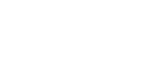 \\
\hline 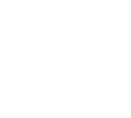 & 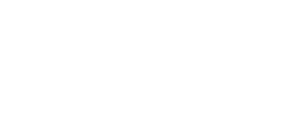 & 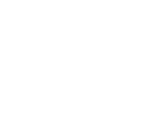 \\
\hline 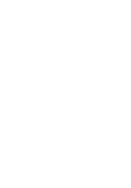 & 思 & 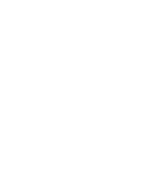 \\
\hline
\end{tabular}




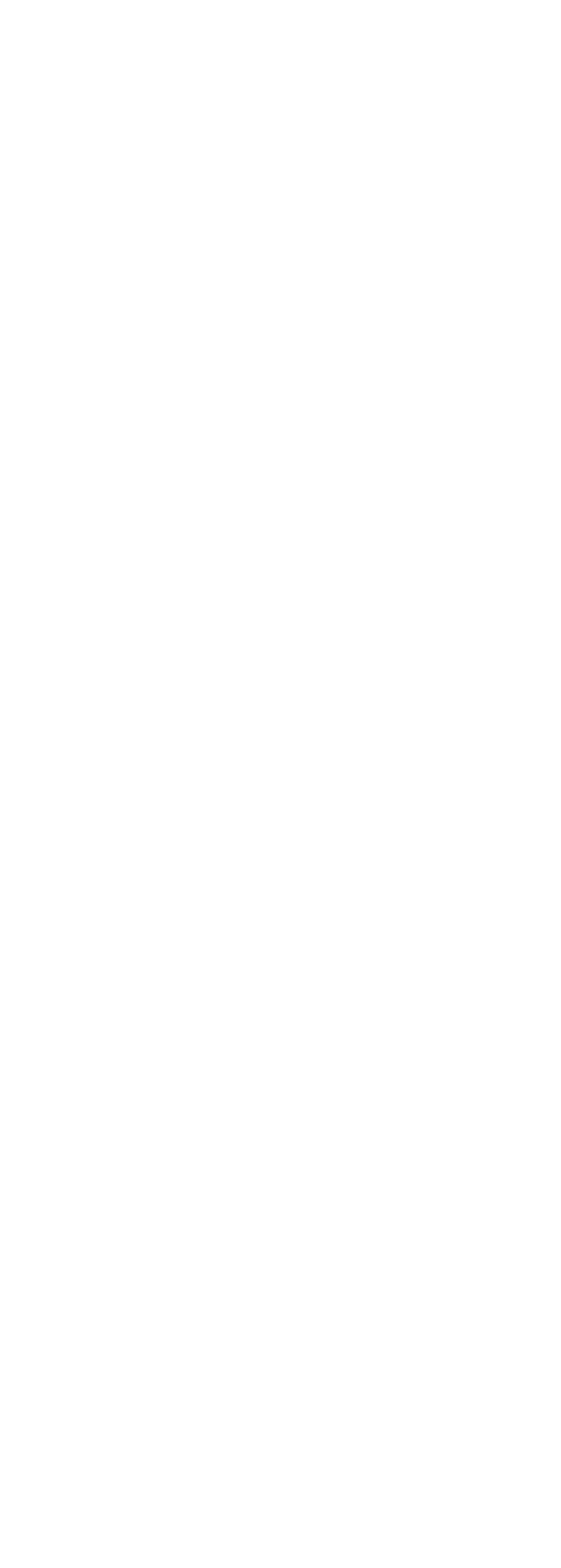




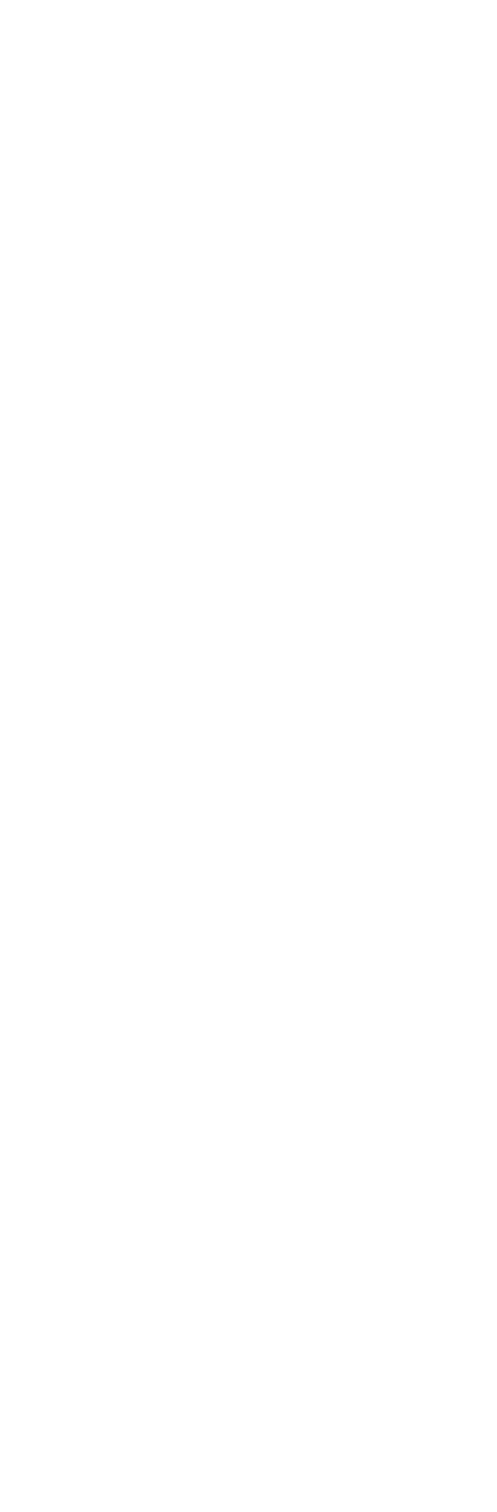




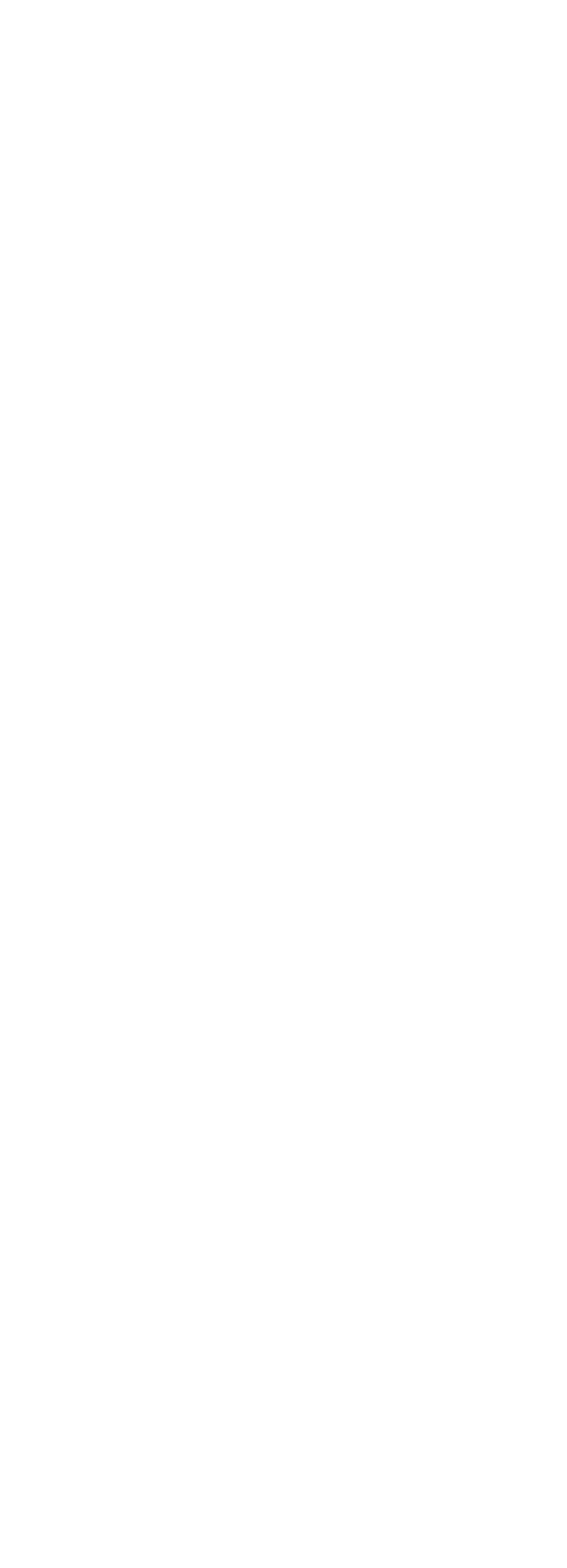




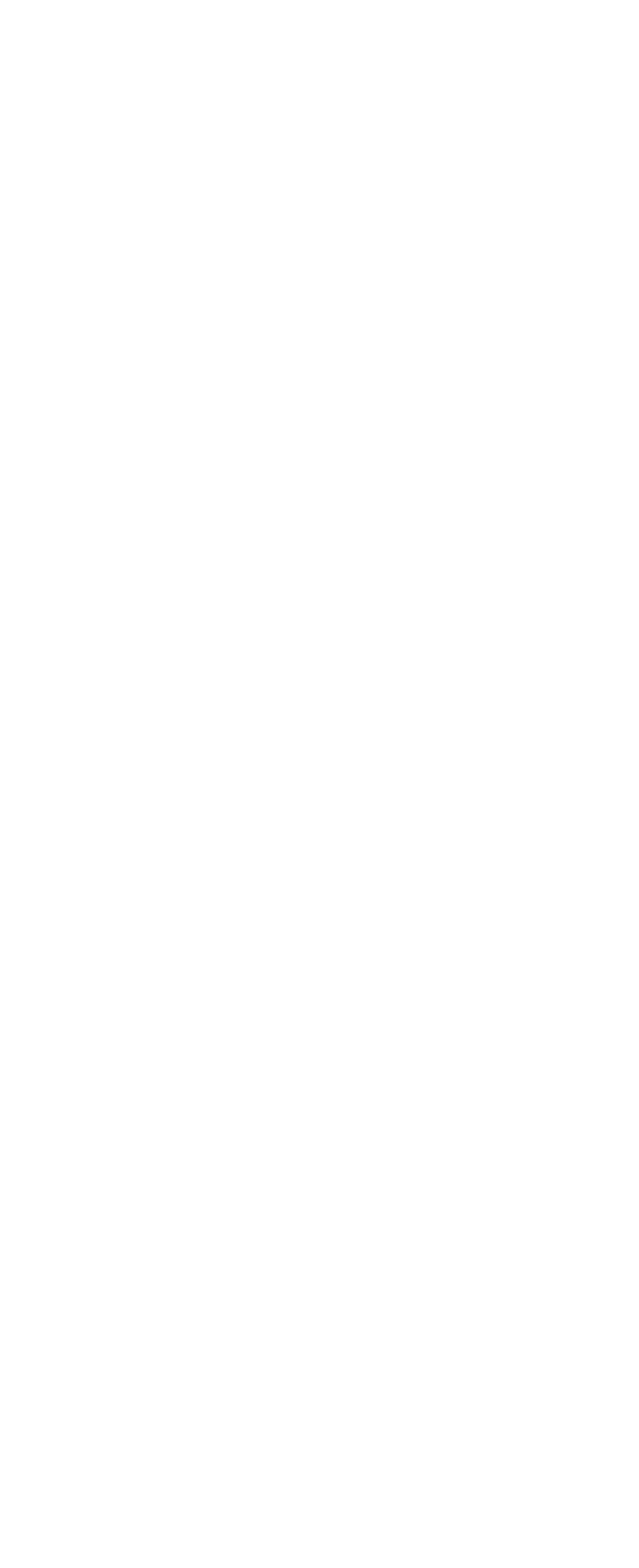




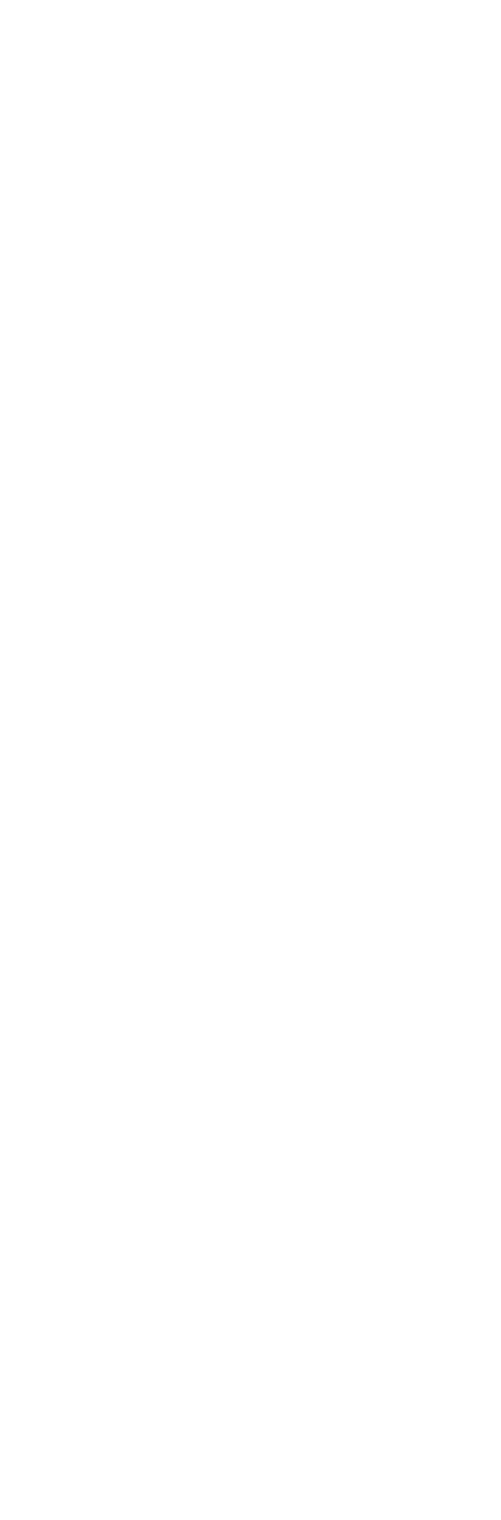




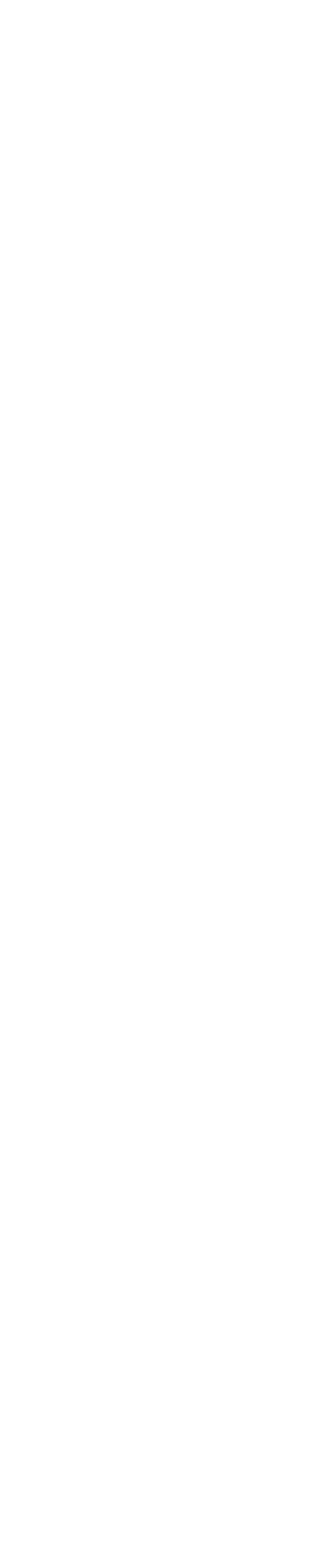

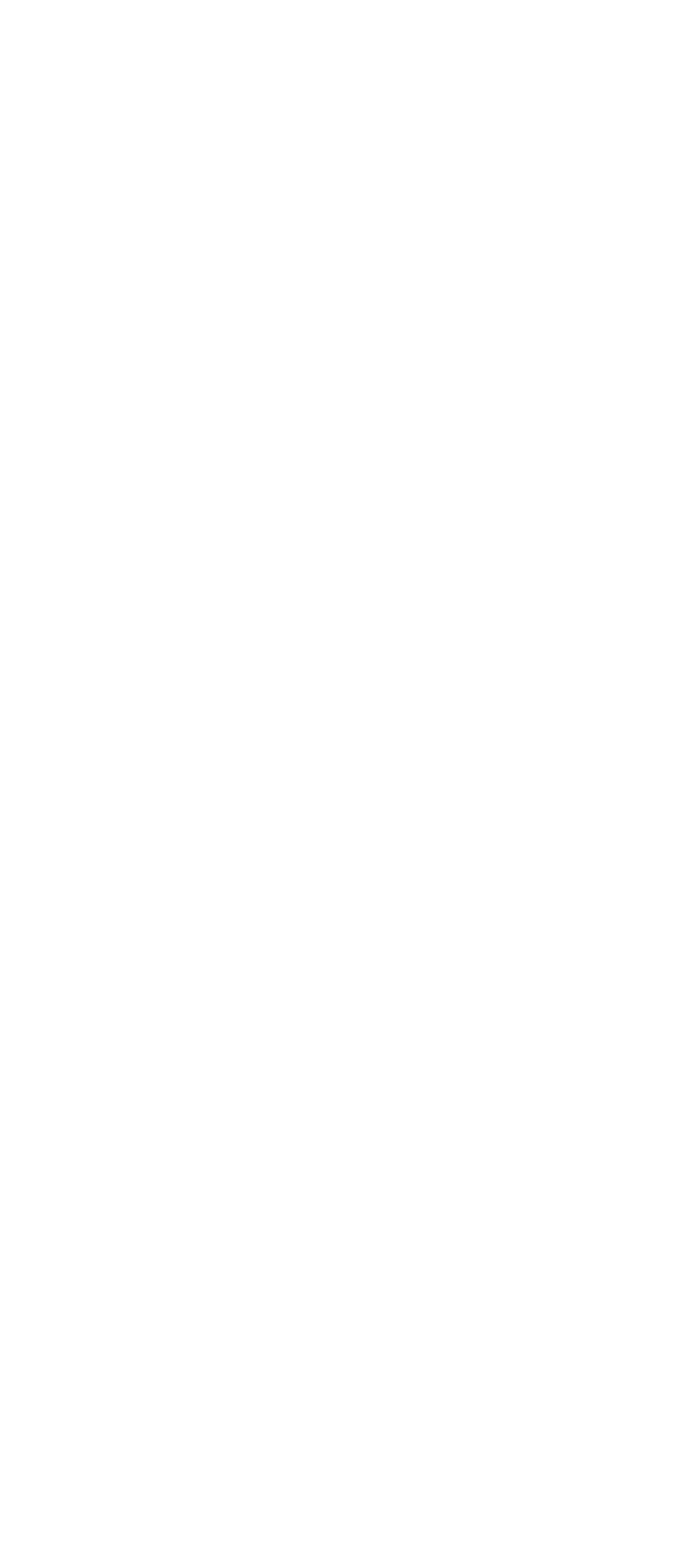



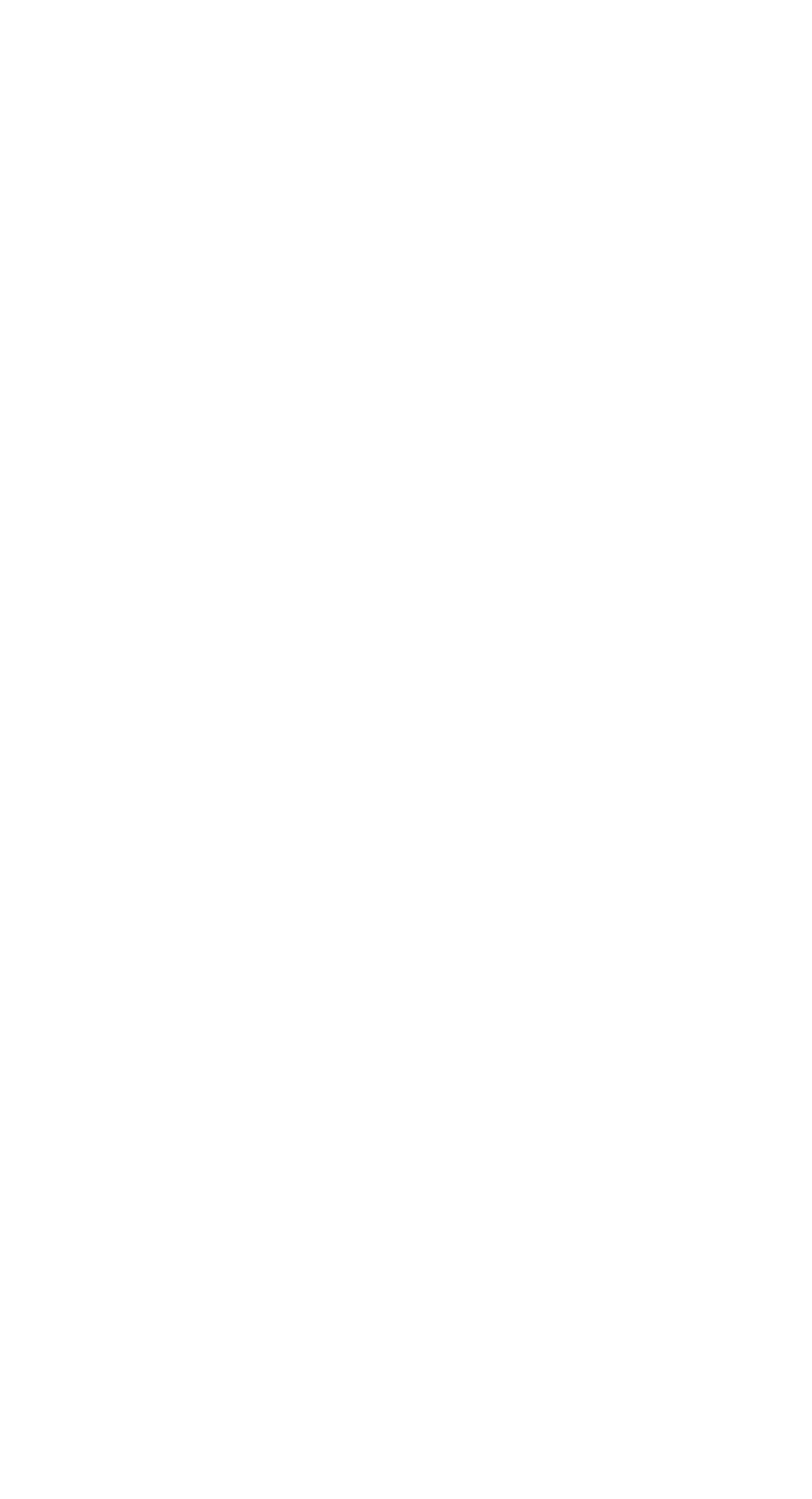


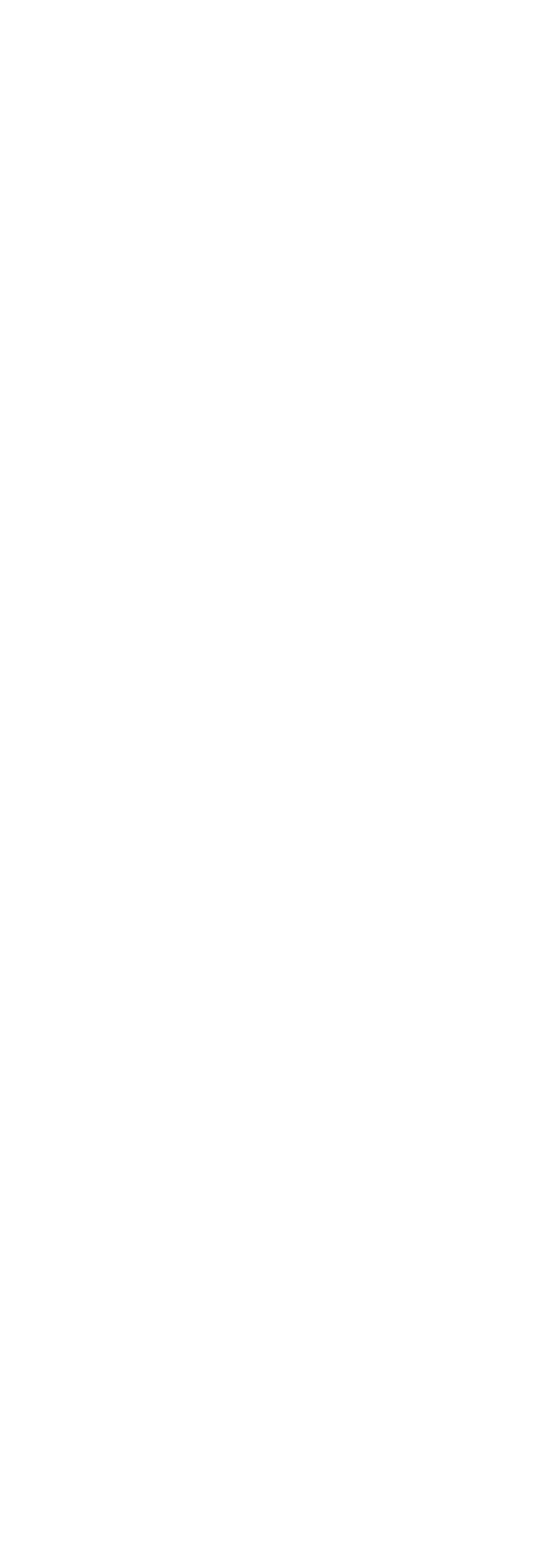




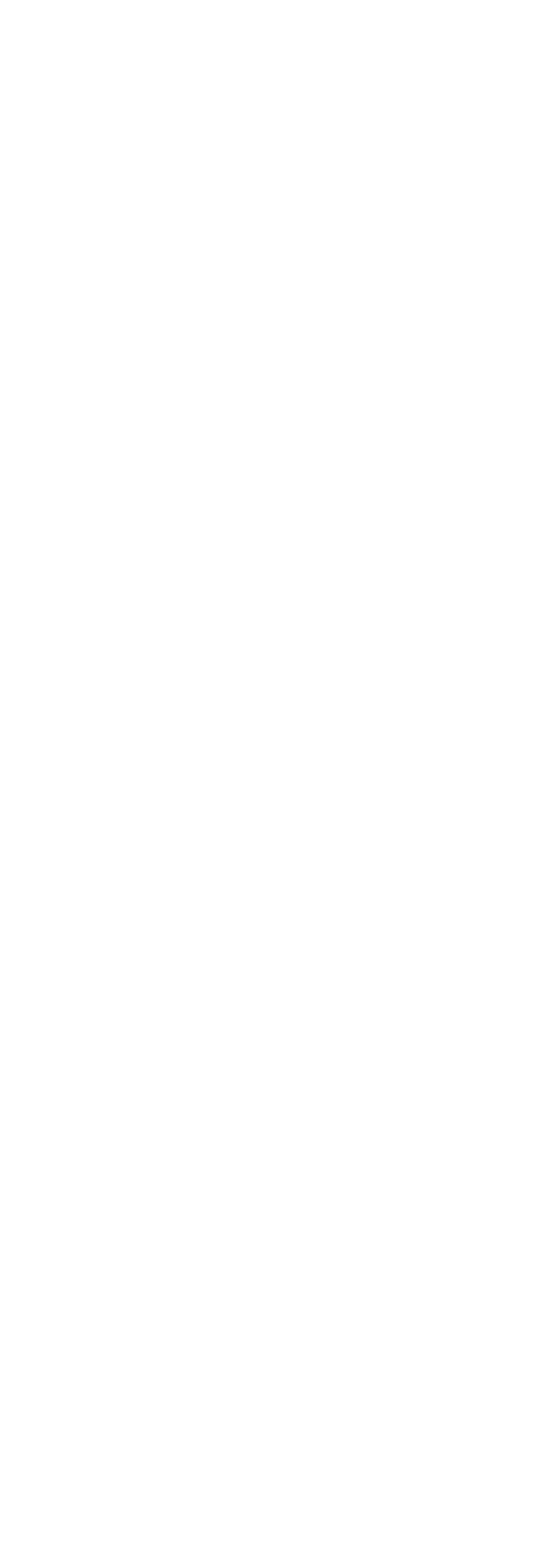




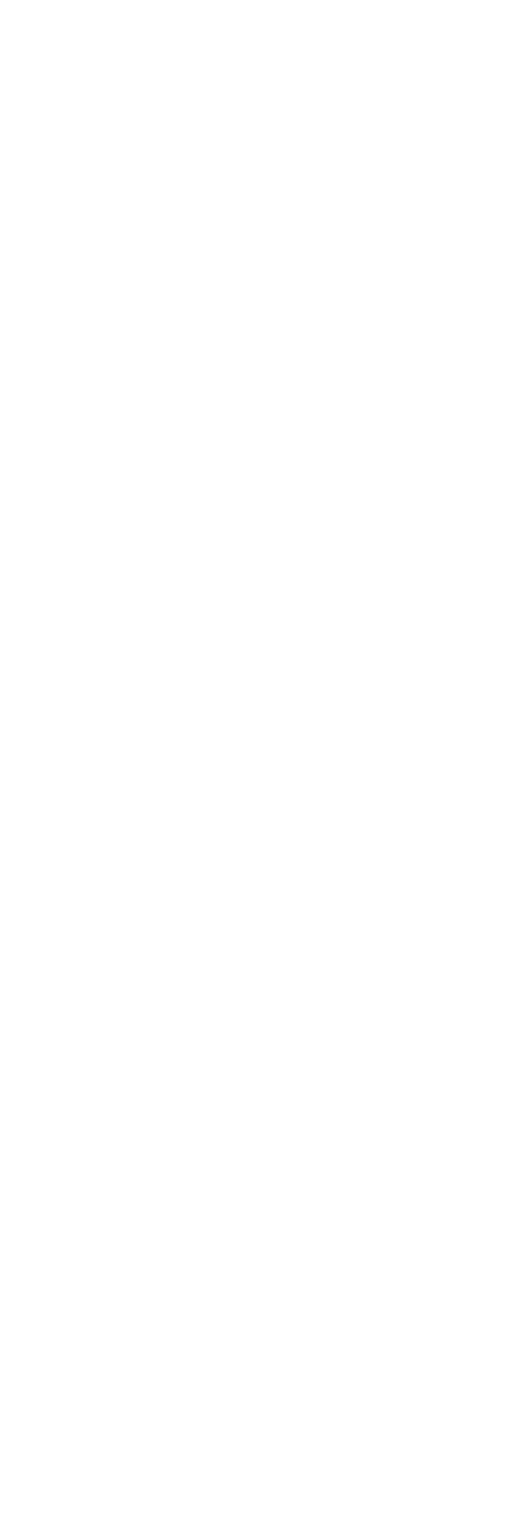




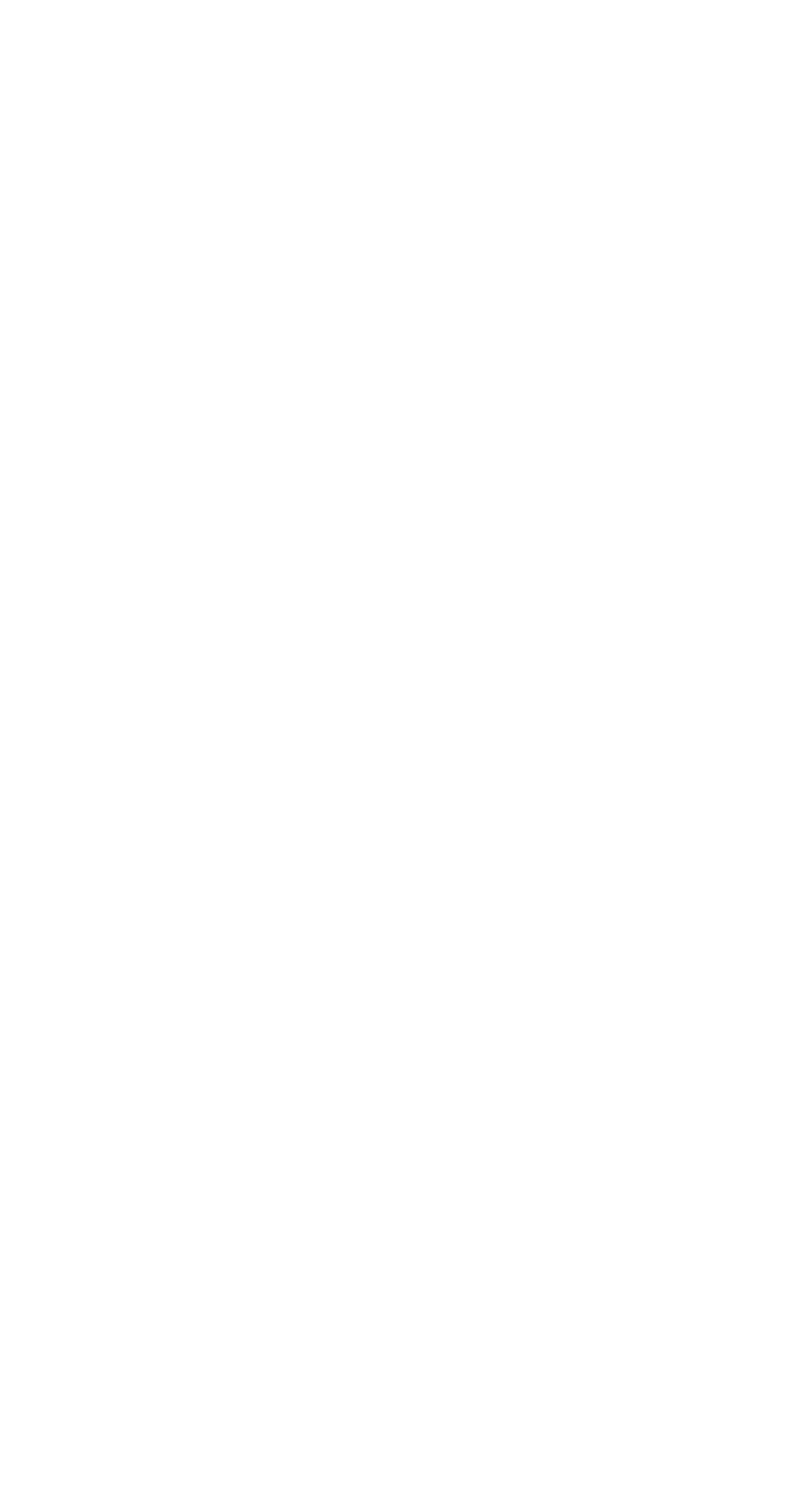




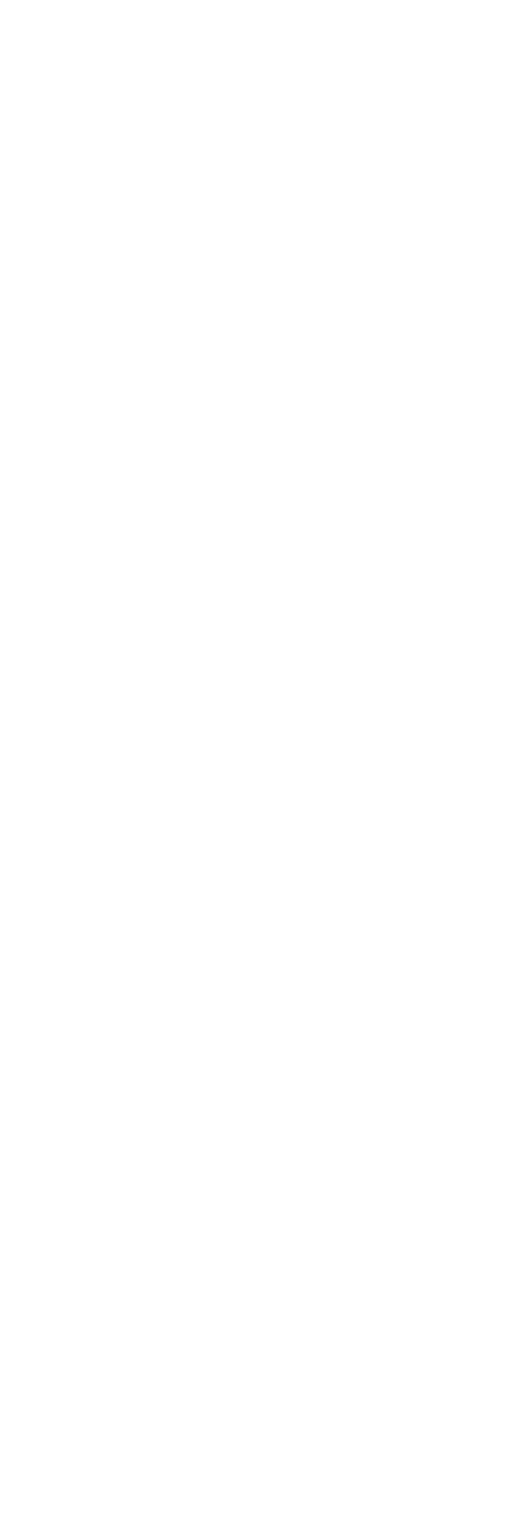




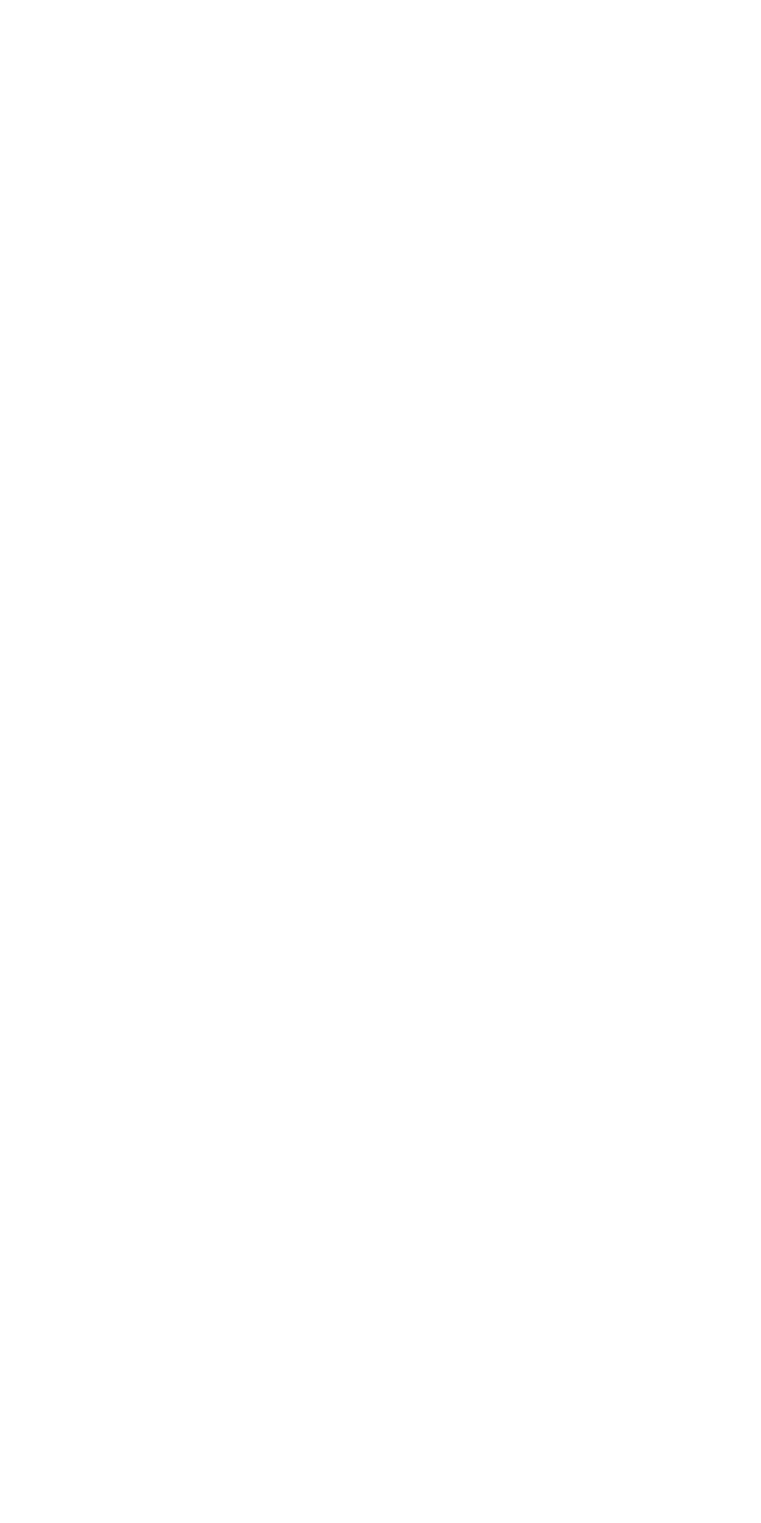


COLORADO RIVER BASIN--Continued

8-1610. COLORADO RIVER AT COLUMBUS, TEX.--Continued

\begin{tabular}{|c|c|c|c|c|c|c|c|c|c|c|}
\hline \multirow[b]{3}{*}{ Day } & \multicolumn{3}{|c|}{ OCTOBER } & \multicolumn{3}{|c|}{ NOVEMBER } & \multicolumn{4}{|c|}{ DECEMBER } \\
\hline & \multirow[b]{2}{*}{$\begin{array}{c}\text { Mean } \\
\text { dis- } \\
\text { charge } \\
\text { (cfs) }\end{array}$} & \multicolumn{2}{|c|}{ Suspended sediment } & \multirow[b]{2}{*}{$\begin{array}{c}\text { Mean } \\
\text { dis- } \\
\text { charge } \\
\text { (cfs) }\end{array}$} & \multicolumn{2}{|c|}{ Suspended sediment } & \multirow[b]{2}{*}{$\begin{array}{l}\text { Mean } \\
\text { dis- } \\
\text { charge } \\
\text { (cfs) }\end{array}$} & \multicolumn{3}{|c|}{ Suspended sediment } \\
\hline & & $\begin{array}{c}\text { Mean } \\
\text { concen- } \\
\text { tration } \\
\text { (ppm) }\end{array}$ & $\begin{array}{l}\text { Tons } \\
\text { per } \\
\text { day }\end{array}$ & & $\begin{array}{c}\text { Mean } \\
\text { concen- } \\
\text { tration } \\
(\mathrm{ppm})\end{array}$ & $\begin{array}{l}\text { Tons } \\
\text { per } \\
\text { day }\end{array}$ & & $\begin{array}{l}\text { Mean } \\
\text { concen- } \\
\text { tration } \\
\text { (ppm) }\end{array}$ & & $\begin{array}{l}\text { Tons } \\
\text { per } \\
\text { day }\end{array}$ \\
\hline $\begin{array}{l}1 \ldots \\
2 \ldots \\
3 \ldots \\
4 \ldots \\
5 \ldots\end{array}$ & $\begin{array}{l}960 \\
840 \\
830 \\
720 \\
700\end{array}$ & $\begin{array}{l}26 \\
23 \\
34 \\
29 \\
31\end{array}$ & $\begin{array}{l}67 \\
52 \\
76 \\
56 \\
59\end{array}$ & $\begin{array}{l}188 \\
188 \\
188 \\
202 \\
209\end{array}$ & $\begin{array}{l}32 \\
38 \\
35 \\
25 \\
17\end{array}$ & $\begin{array}{l}16 \\
19 \\
18 \\
14 \\
10\end{array}$ & $\begin{array}{l}167 \\
160 \\
148 \\
142 \\
142\end{array}$ & $\begin{array}{l}38 \\
31 \\
28 \\
26 \\
34\end{array}$ & & $\begin{array}{l}17 \\
13 \\
11 \\
10 \\
13\end{array}$ \\
\hline $\begin{array}{r}6 \ldots \\
7 \ldots \\
9 \ldots \\
10 \ldots\end{array}$ & $\begin{array}{l}660 \\
660 \\
651 \\
588 \\
561\end{array}$ & $\begin{array}{l}39 \\
51 \\
28 \\
22 \\
22\end{array}$ & $\begin{array}{l}69 \\
91 \\
49 \\
35 \\
33\end{array}$ & $\begin{array}{l}195 \\
181 \\
181 \\
230 \\
254\end{array}$ & $\begin{array}{l}37 \\
47 \\
31 \\
47 \\
44\end{array}$ & $\begin{array}{l}19 \\
23 \\
15 \\
29 \\
30\end{array}$ & $\begin{array}{l}142 \\
136 \\
118 \\
112 \\
124\end{array}$ & $\begin{array}{l}32 \\
31 \\
26 \\
32 \\
28\end{array}$ & & $\begin{array}{r}12 \\
11 \\
8 \\
10 \\
9\end{array}$ \\
\hline $\begin{array}{l}11 \ldots \\
12 \ldots \\
13 \ldots \\
14 \ldots \\
15 \ldots\end{array}$ & $\begin{array}{l}579 \\
570 \\
489 \\
408 \\
358\end{array}$ & $\begin{array}{l}22 \\
29 \\
25 \\
25 \\
28\end{array}$ & $\begin{array}{l}34 \\
45 \\
33 \\
28 \\
27\end{array}$ & $\begin{array}{l}399 \\
286 \\
223 \\
195 \\
195\end{array}$ & $\begin{array}{l}-- \\
42 \\
26 \\
16 \\
23\end{array}$ & $\begin{array}{r}50 \\
32 \\
16 \\
8 \\
12\end{array}$ & $\begin{array}{l}130 \\
124 \\
154 \\
202 \\
202\end{array}$ & $\begin{array}{l}28 \\
32 \\
22 \\
17 \\
21\end{array}$ & & $\begin{array}{r}10 \\
11 \\
9 \\
9 \\
11\end{array}$ \\
\hline $\begin{array}{l}16 \ldots \\
17 \ldots \\
18 \ldots \\
19 \ldots \\
20 \ldots\end{array}$ & $\begin{array}{l}326 \\
302 \\
286 \\
286 \\
278\end{array}$ & $\begin{array}{l}32 \\
35 \\
24 \\
48 \\
54\end{array}$ & $\begin{array}{l}28 \\
29 \\
19 \\
37 \\
41\end{array}$ & $\begin{array}{l}195 \\
195 \\
195 \\
188 \\
195\end{array}$ & $\begin{array}{l}18 \\
25 \\
40 \\
24 \\
24\end{array}$ & $\begin{array}{l}9 \\
13 \\
21 \\
12 \\
13\end{array}$ & $\begin{array}{l}216 \\
209 \\
195 \\
181 \\
209\end{array}$ & $\begin{array}{l}29 \\
11 \\
29 \\
12 \\
15\end{array}$ & & $\begin{array}{r}17 \\
6 \\
15 \\
6 \\
8\end{array}$ \\
\hline $\begin{array}{l}21 \ldots \\
22 \ldots \\
23 \ldots \\
24 \ldots \\
25 \ldots\end{array}$ & $\begin{array}{c}270 \\
254 \\
246 \\
230 \\
230\end{array}$ & $\begin{array}{l}35 \\
38 \\
42 \\
45 \\
35\end{array}$ & $\begin{array}{l}26 \\
26 \\
28 \\
28 \\
22\end{array}$ & $\begin{array}{l}188 \\
223 \\
202 \\
181 \\
160\end{array}$ & $\begin{array}{l}18 \\
19 \\
21 \\
18 \\
16\end{array}$ & $\begin{array}{r}9 \\
11 \\
11 \\
9 \\
7\end{array}$ & $\begin{array}{l}209 \\
195 \\
181 \\
160 \\
154\end{array}$ & $\begin{array}{l}21 \\
31 \\
13 \\
-12\end{array}$ & $E$ & $\begin{array}{r}12 \\
16 \\
6 \\
4 \\
5\end{array}$ \\
\hline $\begin{array}{l}26 \ldots \\
27 \ldots \\
28 \ldots \\
29 \ldots \\
30 \ldots \\
31 \ldots\end{array}$ & $\begin{array}{l}223 \\
223 \\
216 \\
209 \\
202 \\
188\end{array}$ & $\begin{array}{l}35 \\
57 \\
34 \\
40 \\
36 \\
18\end{array}$ & $\begin{array}{r}21 \\
34 \\
20 \\
23 \\
20 \\
9\end{array}$ & $\begin{array}{l}148 \\
154 \\
209 \\
188 \\
174 \\
\end{array}$ & $\begin{array}{l}19 \\
23 \\
50 \\
47 \\
37 \\
--\end{array}$ & $\begin{array}{r}8 \\
10 \\
28 \\
24 \\
17 \\
--\end{array}$ & $\begin{array}{l}148 \\
154 \\
154 \\
148 \\
160 \\
154\end{array}$ & $\begin{array}{l}17 \\
20 \\
24 \\
26 \\
17 \\
13\end{array}$ & & $\begin{array}{r}7 \\
8 \\
10 \\
10 \\
7 \\
5\end{array}$ \\
\hline \multirow[t]{2}{*}{ Total } & 13543 & -- & 1165 & 6109 & -- & 513 & 5030 & -- & & 306 \\
\hline & \multicolumn{3}{|c|}{ JANUARY } & \multicolumn{3}{|c|}{ FEBRUARY } & \multicolumn{4}{|c|}{ MARCH } \\
\hline $\begin{array}{l}1 \ldots \\
2 \ldots \\
3 \ldots \\
4 \ldots \\
5 \ldots\end{array}$ & $\begin{array}{l}136 \\
130 \\
130 \\
124 \\
124\end{array}$ & $\begin{array}{l}9 \\
11 \\
14 \\
18 \\
14\end{array}$ & $\begin{array}{l}3 \\
4 \\
5 \\
6 \\
5\end{array}$ & $\begin{array}{l}399 \\
480 \\
366 \\
435 \\
453\end{array}$ & $\begin{array}{l}44 \\
59 \\
42 \\
76 \\
52\end{array}$ & $\begin{array}{l}47 \\
76 \\
42 \\
89 \\
64\end{array}$ & $\begin{array}{r}230 \\
1940 \\
2960 \\
1410 \\
670\end{array}$ & $\begin{array}{r}33 \\
900 \\
1300 \\
930 \\
340\end{array}$ & 5 & $\begin{array}{r}20 \\
8400 \\
10000 \\
3500 \\
620\end{array}$ \\
\hline $\begin{array}{r}6 \ldots \\
7 \ldots \\
8 \ldots \\
10 \ldots\end{array}$ & $\begin{array}{l}124 \\
118 \\
118 \\
112 \\
118\end{array}$ & $\begin{array}{l}18 \\
19 \\
15 \\
14 \\
10\end{array}$ & $\begin{array}{l}6 \\
6 \\
5 \\
4 \\
3\end{array}$ & $\begin{array}{l}507 \\
462 \\
350 \\
294 \\
302\end{array}$ & $\begin{array}{l}58 \\
52 \\
79 \\
79 \\
50\end{array}$ & $\begin{array}{l}79 \\
65 \\
75 \\
63 \\
41\end{array}$ & $\begin{array}{l}444 \\
342 \\
310 \\
270 \\
326\end{array}$ & $\begin{array}{l}55 \\
61 \\
80 \\
68 \\
32\end{array}$ & & $\begin{array}{l}66 \\
56 \\
67 \\
50 \\
28\end{array}$ \\
\hline $\begin{array}{l}11 \ldots \\
12 \ldots \\
13 \ldots \\
14 \ldots \\
15 \ldots\end{array}$ & $\begin{array}{l}118 \\
174 \\
230 \\
188 \\
174\end{array}$ & $\begin{array}{l}10 \\
12 \\
21 \\
12\end{array}$ & $\begin{array}{r}3 \\
5 \\
7 \\
11 \\
6\end{array}$ & $\begin{array}{l}254 \\
230 \\
216 \\
195 \\
209\end{array}$ & $\begin{array}{l}98 \\
66 \\
58 \\
32 \\
38\end{array}$ & $\begin{array}{l}67 \\
41 \\
34 \\
17 \\
21\end{array}$ & $\begin{array}{l}435 \\
334 \\
246 \\
202 \\
167\end{array}$ & $\begin{array}{l}60 \\
72 \\
60 \\
51 \\
87\end{array}$ & & $\begin{array}{l}70 \\
65 \\
40 \\
28 \\
39\end{array}$ \\
\hline $\begin{array}{l}16 \ldots \\
17 \ldots \\
18 \ldots \\
19 \ldots \\
20 \ldots\end{array}$ & $\begin{array}{l}230 \\
238 \\
223 \\
209 \\
209\end{array}$ & $\begin{array}{l}22 \\
30 \\
17 \\
29 \\
34\end{array}$ & $\begin{array}{l}14 \\
19 \\
10 \\
16 \\
19\end{array}$ & $\begin{array}{l}181 \\
174 \\
174 \\
160 \\
148\end{array}$ & $\begin{array}{l}-- \\
44 \\
42 \\
26 \\
42\end{array}$ & $\begin{array}{l}20 \\
21 \\
20 \\
11 \\
17\end{array}$ & $\begin{array}{r}142 \\
130 \\
118 \\
1550 \\
3220\end{array}$ & $\begin{array}{r}79 \\
52 \\
42 \\
430 \\
1200\end{array}$ & $\left\{\begin{array}{l}5 \\
5\end{array}\right.$ & $\begin{array}{r}30 \\
18 \\
13 \\
2600 \\
10000\end{array}$ \\
\hline $\begin{array}{l}21 \ldots \\
22 \ldots \\
23 \ldots \\
24 \ldots \\
25 \ldots\end{array}$ & $\begin{array}{l}209 \\
246 \\
254 \\
230 \\
209\end{array}$ & $\begin{array}{l}45 \\
52 \\
55 \\
56 \\
22\end{array}$ & $\begin{array}{l}25 \\
35 \\
38 \\
35 \\
12\end{array}$ & $\begin{array}{l}181 \\
181 \\
167 \\
202 \\
382\end{array}$ & $\begin{array}{l}42 \\
45 \\
-- \\
38 \\
57\end{array}$ & $\begin{array}{l}21 \\
22 \\
20 \\
21 \\
59\end{array}$ & $\begin{array}{r}1610 \\
740 \\
570 \\
471 \\
390\end{array}$ & $\begin{array}{r}670 \\
410 \\
98 \\
100 \\
62\end{array}$ & & $\begin{array}{r}2900 \\
820 \\
150 \\
130 \\
65\end{array}$ \\
\hline $\begin{array}{l}26 \ldots \\
27 \ldots \\
28 \ldots \\
29 \ldots \\
30 \ldots \\
31 \ldots\end{array}$ & $\begin{array}{l}188 \\
181 \\
167 \\
154 \\
202 \\
374\end{array}$ & $\begin{array}{l}12 \\
17 \\
19 \\
19 \\
22 \\
37\end{array}$ & $\begin{array}{l}6 \\
8 \\
9 \\
8 \\
12 \\
37\end{array}$ & $\begin{array}{r}278 \\
286 \\
278 \\
254 \\
-- \\
--\end{array}$ & $\begin{array}{l}50 \\
50 \\
40 \\
36 \\
-- \\
--\end{array}$ & $\begin{array}{l}38 \\
39 \\
30 \\
25 \\
-- \\
--\end{array}$ & $\begin{array}{l}310 \\
254 \\
223 \\
195 \\
160 \\
148\end{array}$ & $\begin{array}{l}58 \\
45 \\
40 \\
48 \\
50 \\
50\end{array}$ & & $\begin{array}{l}49 \\
31 \\
24 \\
25 \\
22 \\
20\end{array}$ \\
\hline Total & 5641 & -- & 382 & 8198 & -- & 1185 & 20517 & -- & & 39946 \\
\hline
\end{tabular}

E Estimated.

$S$ Computed by subdividing day. 
COLORADO RIVER BASIN--Continued

8-1610. COLORADO RIVER AT COLUMBUS, TEX.--Continued

Suspended sediment, water year October 1963 to September 1964--Continued

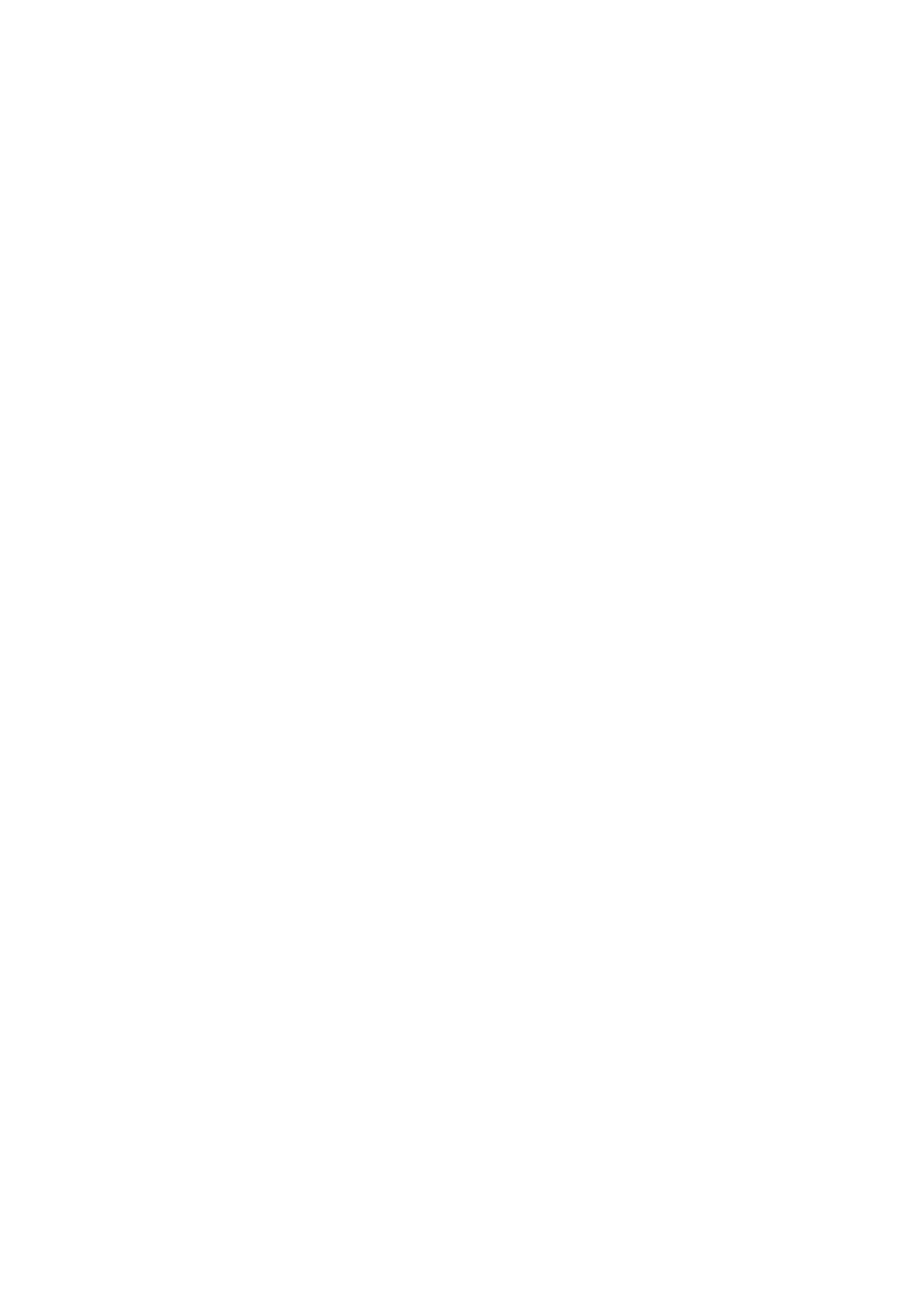

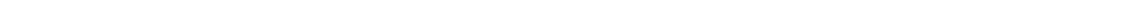

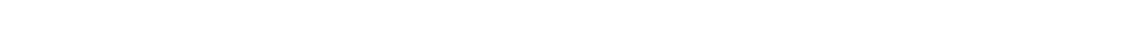
E Est1mated.

S Computed by subdividing day. 


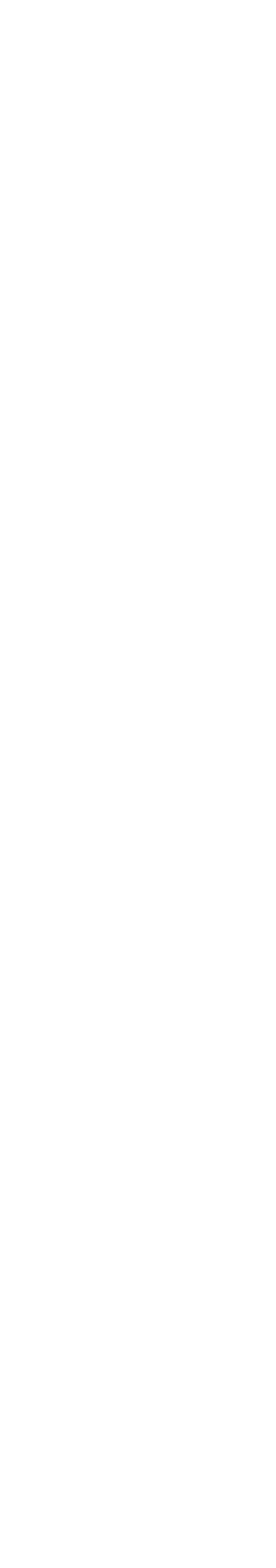




\begin{tabular}{|c|c|c|c|c|}
\hline & $\therefore \rightarrow \infty$ & & \\
\hline \multicolumn{2}{|c|}{ 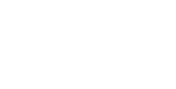 } & 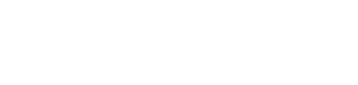 & 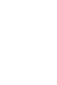 & : \\
\hline \multicolumn{2}{|r|}{ 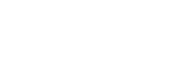 } & 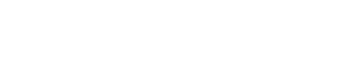 & $\stackrel{m}{-i}$ & \begin{tabular}{l|l}
\multirow{4}{*}{$: 1$} & 1
\end{tabular} \\
\hline \multirow{2}{*}{ 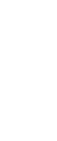 } & 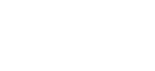 & 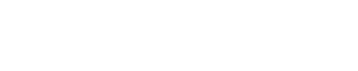 & 9 & 9 \\
\hline & 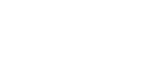 & 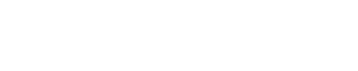 & 品 & 電: \\
\hline \multirow{3}{*}{ 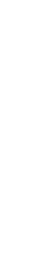 } & 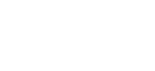 & 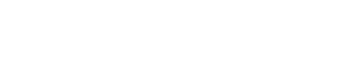 & $\mid \begin{array}{l}9 \\
9 \\
010\end{array}$ & $1: 1$ \\
\hline & 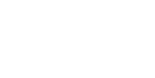 & 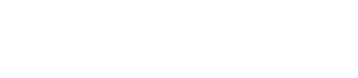 & \begin{tabular}{|c|c|} 
\\
$\vdots$ \\
0 \\
\end{tabular} & $1: 1$ \\
\hline & 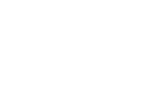 & 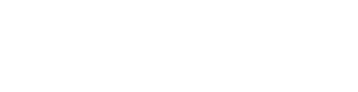 & 串 & $\begin{array}{ll} \\
\end{array}$ \\
\hline \multicolumn{5}{|c|}{ 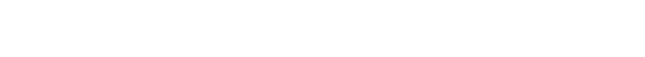 } \\
\hline \multirow[t]{2}{*}{ 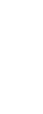 } & 公题题 & 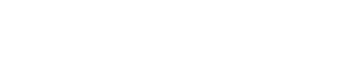 & $\stackrel{M}{4}$ & $\stackrel{9}{\circ}: \stackrel{9}{-1}$ \\
\hline & 욜 & 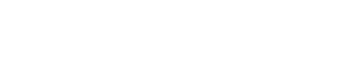 & $\begin{array}{ll}n \\
\\
0\end{array}$ & $\stackrel{0}{0}: \because$ \\
\hline & 遌 & 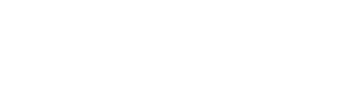 & $\ddot{6}$ & $: \exists$ \\
\hline 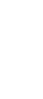 & 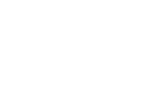 & 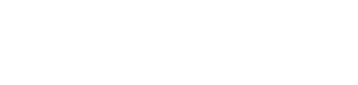 & ¥ & $9:$ \\
\hline & 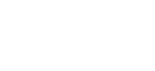 & 0000000000000000 & 0 & 0.0 \\
\hline & 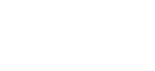 & สె. & 禺 & : จ \\
\hline & 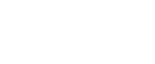 & 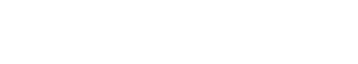 & 1 & \begin{tabular}{l|l}
1 & 1
\end{tabular} \\
\hline & 害要 & 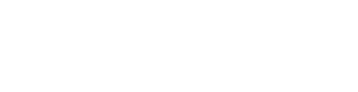 & q & $F$ \\
\hline \multirow[t]{2}{*}{ : } & 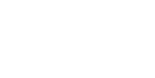 & 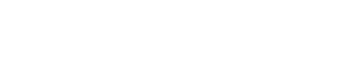 & I & $\therefore$ ज्ञ \\
\hline & 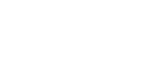 & 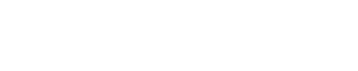 & 5 & \begin{tabular}{l|l|l|}
0 & \pm \\
\end{tabular} \\
\hline & E⿸\zh14 & & & \\
\hline & 耪总 & 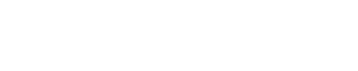 & 2 & i) \\
\hline \multicolumn{2}{|c|}{ 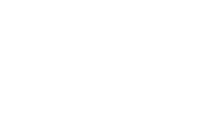 } & 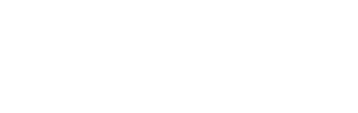 & i & $\begin{array}{lll}0 & 1 \\
0 & 1\end{array}$ \\
\hline \multicolumn{2}{|r|}{ 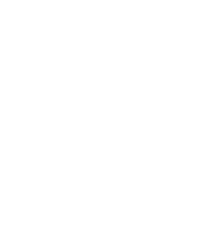 } & 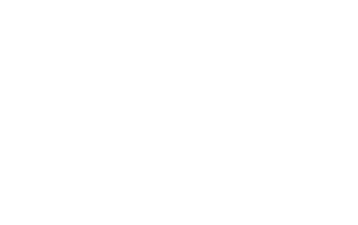 & 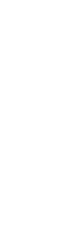 & 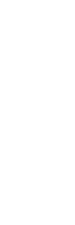 \\
\hline
\end{tabular}




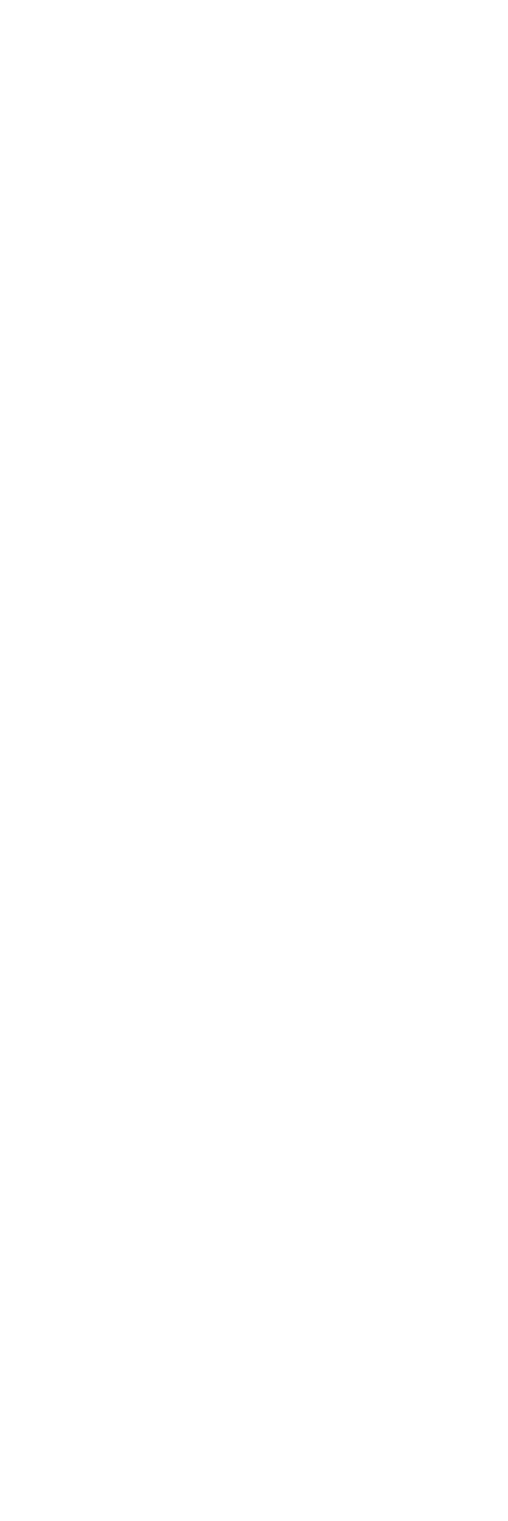




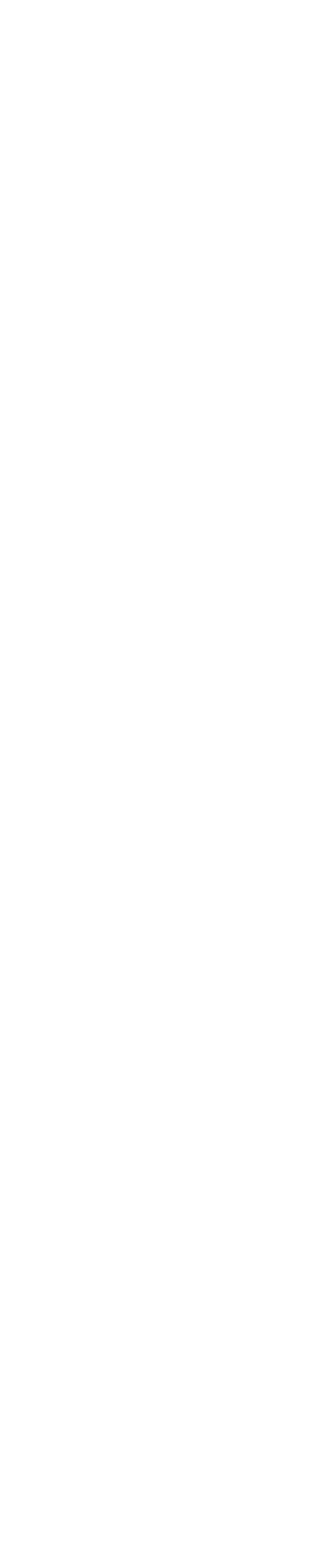

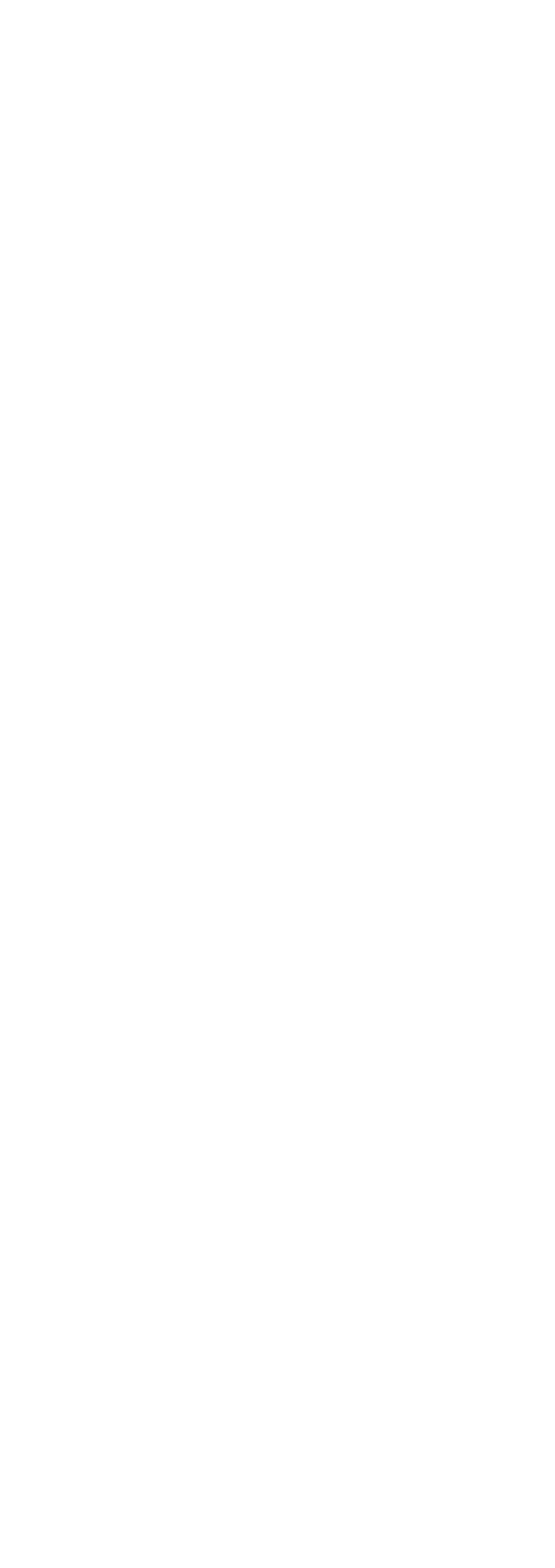




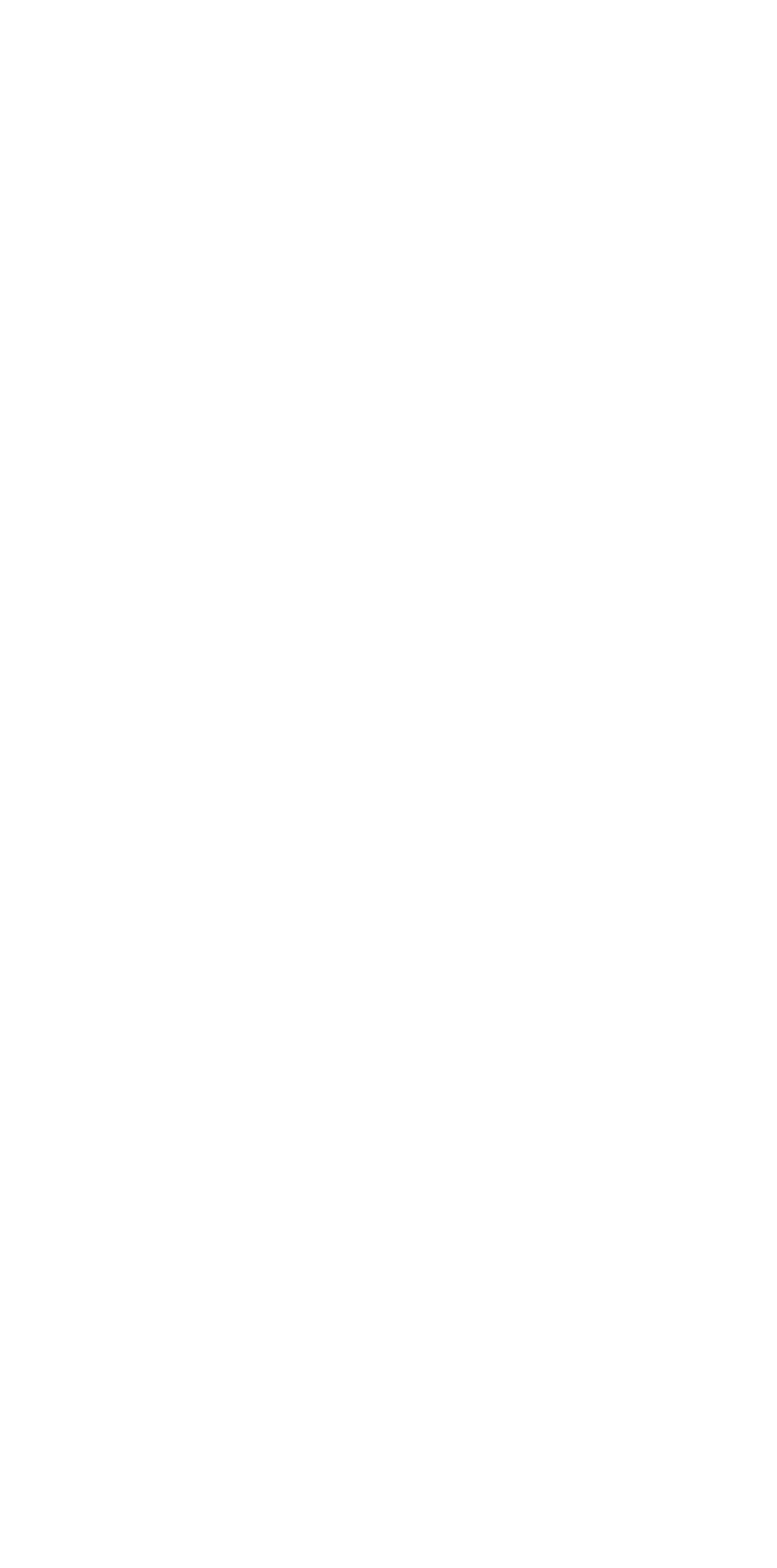




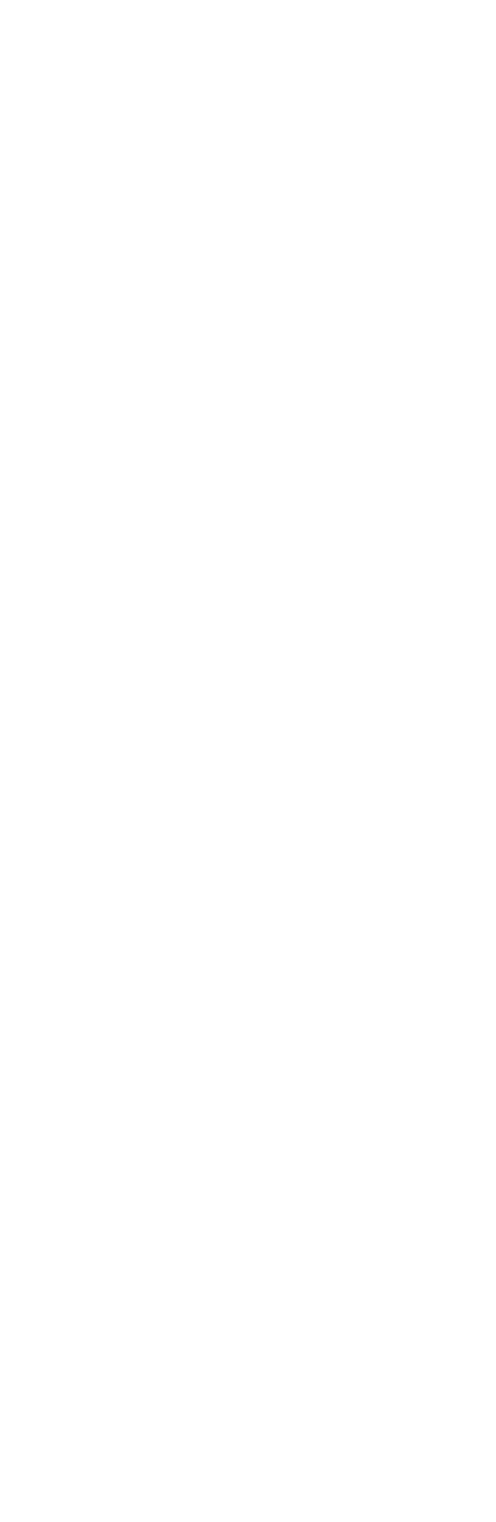




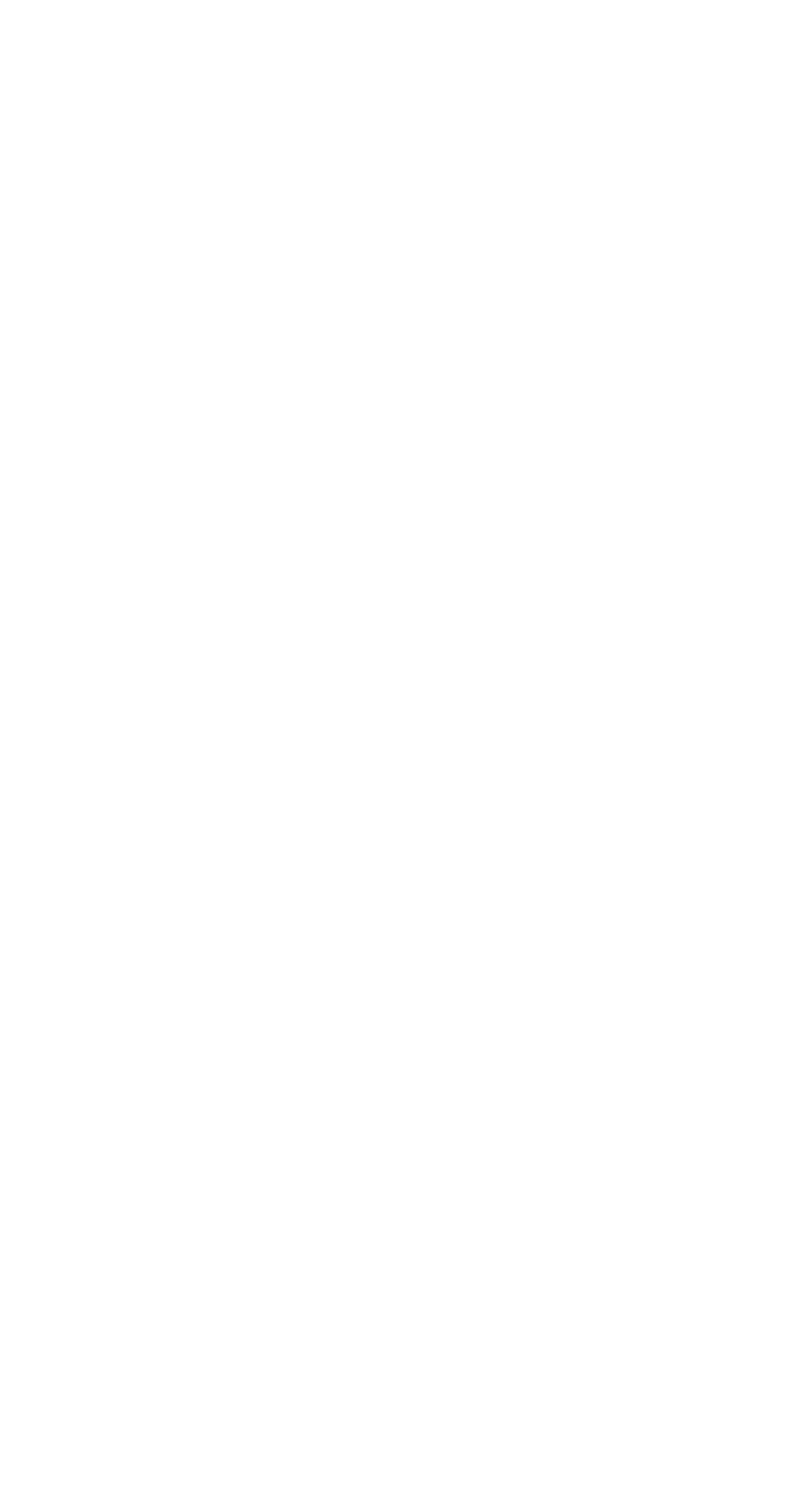



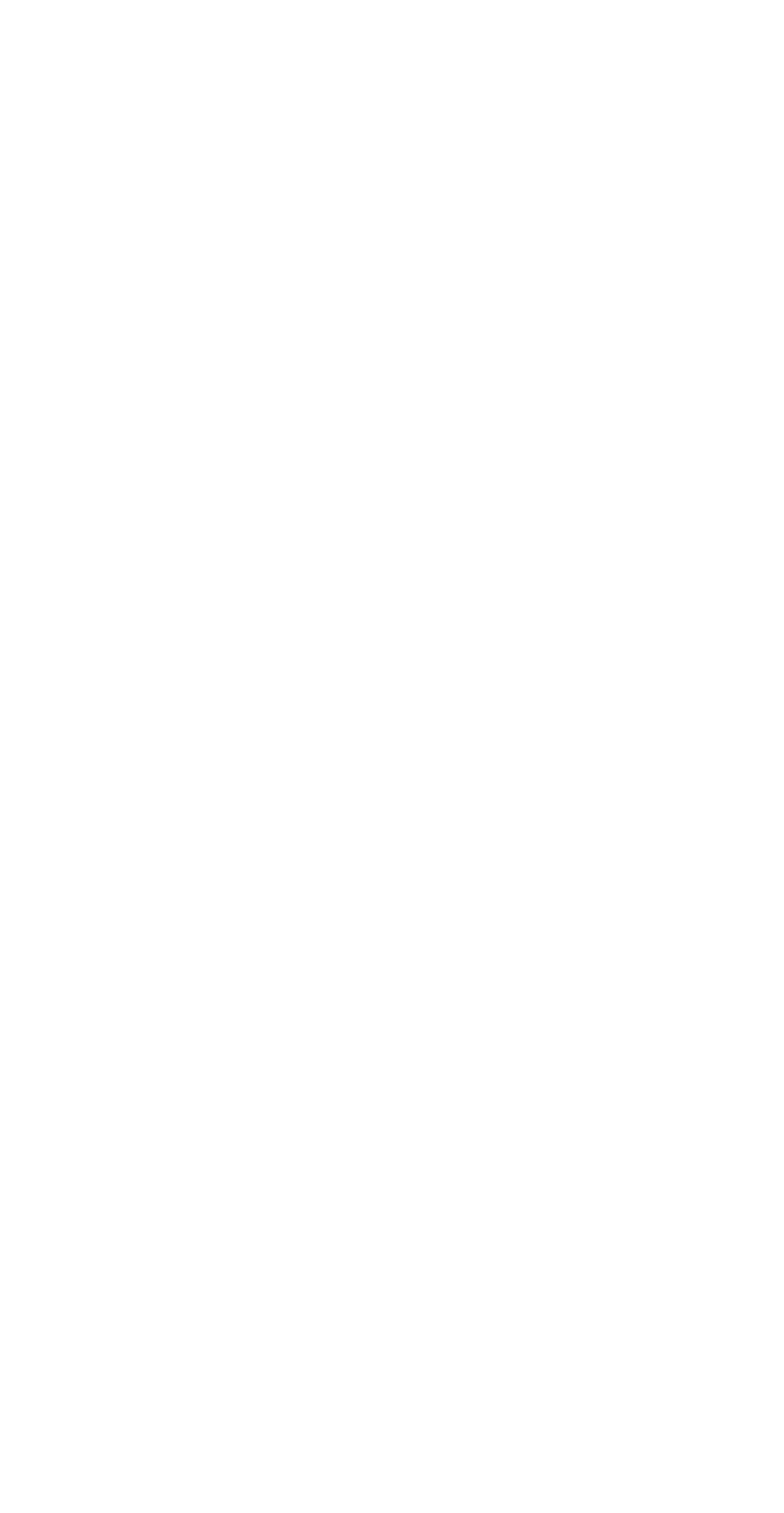


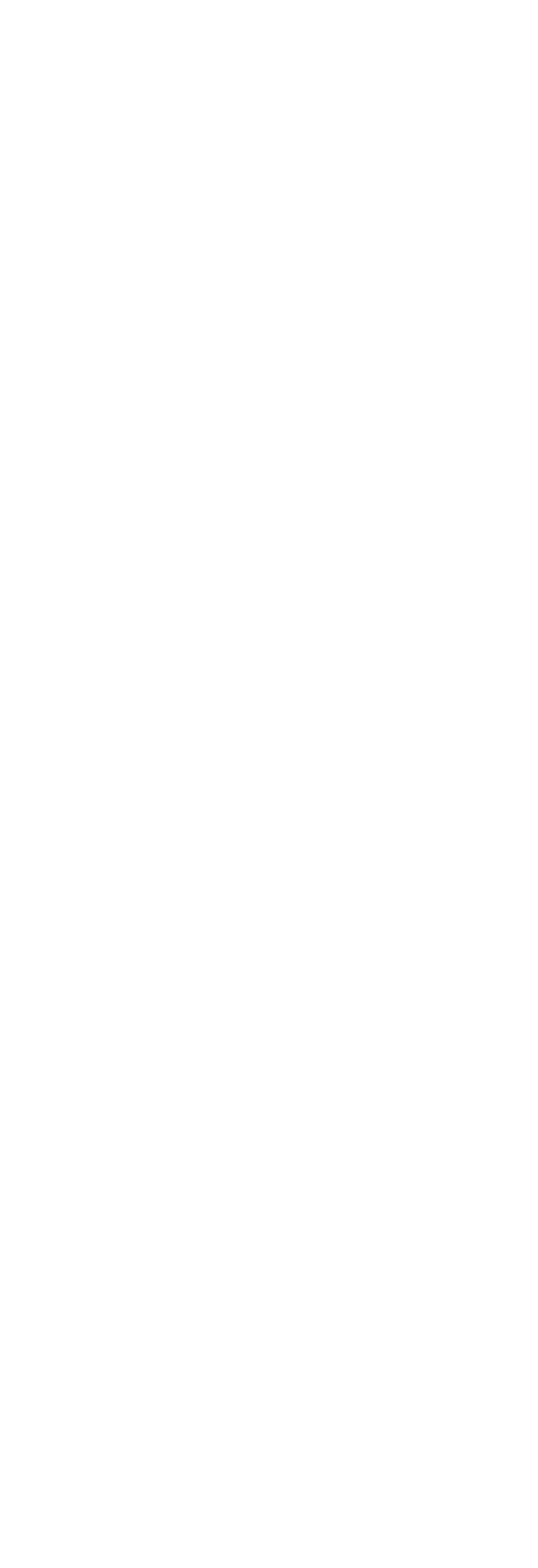




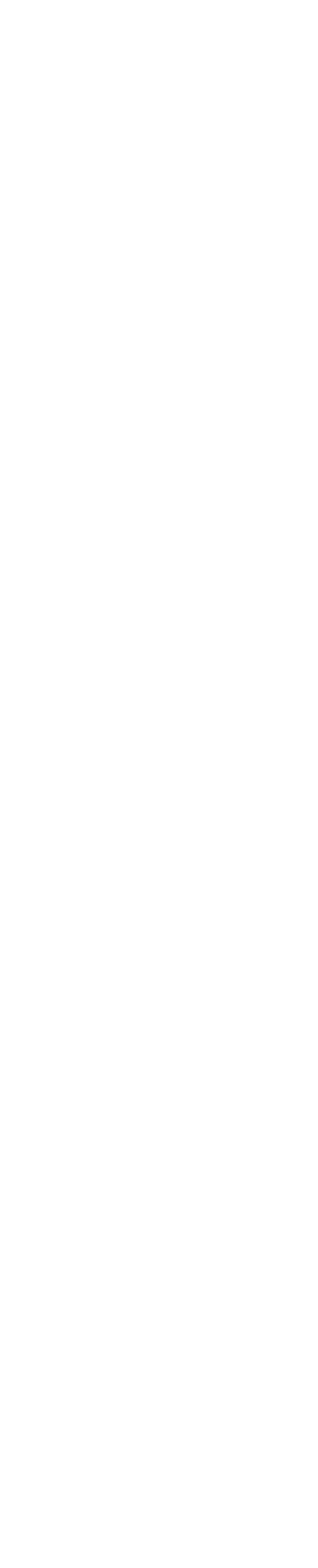

\begin{tabular}{|c|c|}
\hline 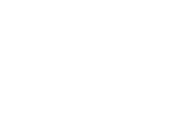 & 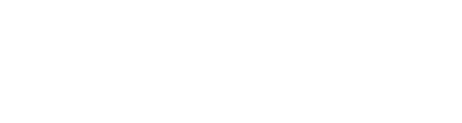 \\
\hline 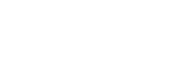 & 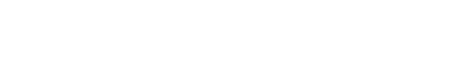 \\
\hline 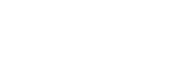 & 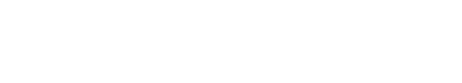 \\
\hline 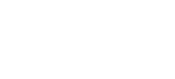 & 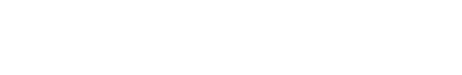 \\
\hline 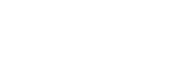 & 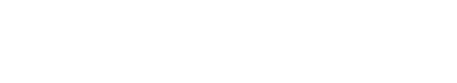 \\
\hline 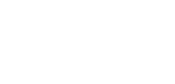 & 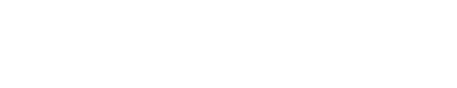 \\
\hline 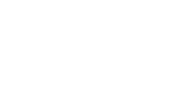 & 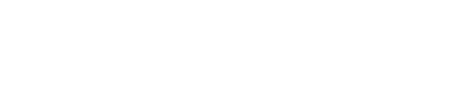 \\
\hline 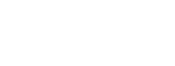 & \\
\hline 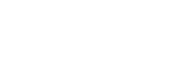 & 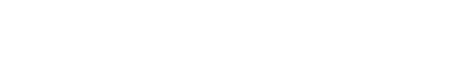 \\
\hline 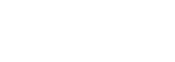 & $\begin{array}{llll}0 & 0.1111 & 10.10 .11^{m} & 11^{m} \\
0 & 0.1\end{array}$ \\
\hline 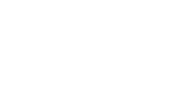 & 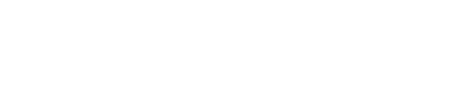 \\
\hline 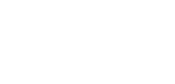 & 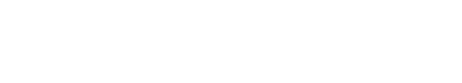 \\
\hline í & $00000000000 \$ 000 \quad 000$ \\
\hline 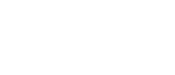 & 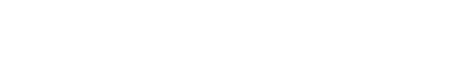 \\
\hline 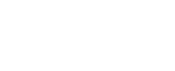 & 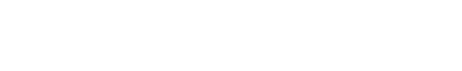 \\
\hline 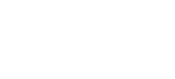 & 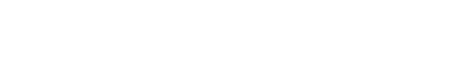 \\
\hline 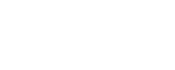 & 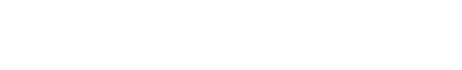 \\
\hline 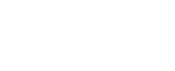 & 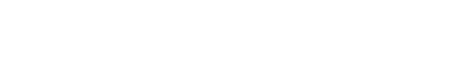 \\
\hline 80 & \\
\hline 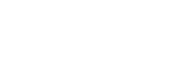 & 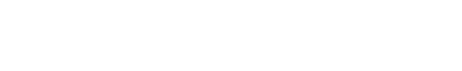 \\
\hline รั: & 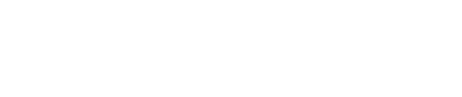 \\
\hline 8 & 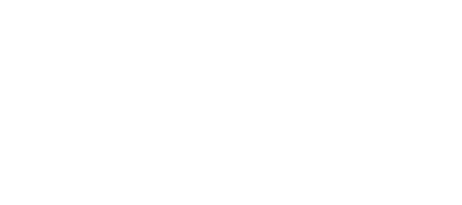 \\
\hline
\end{tabular}




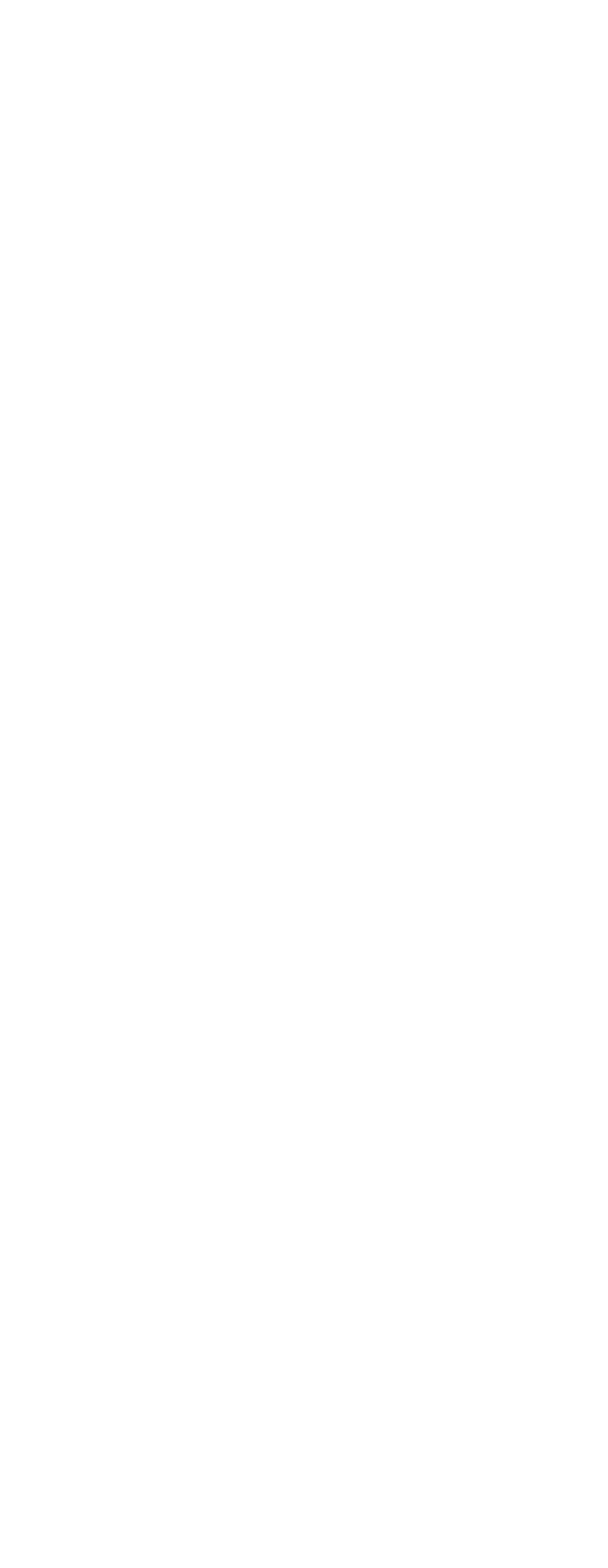




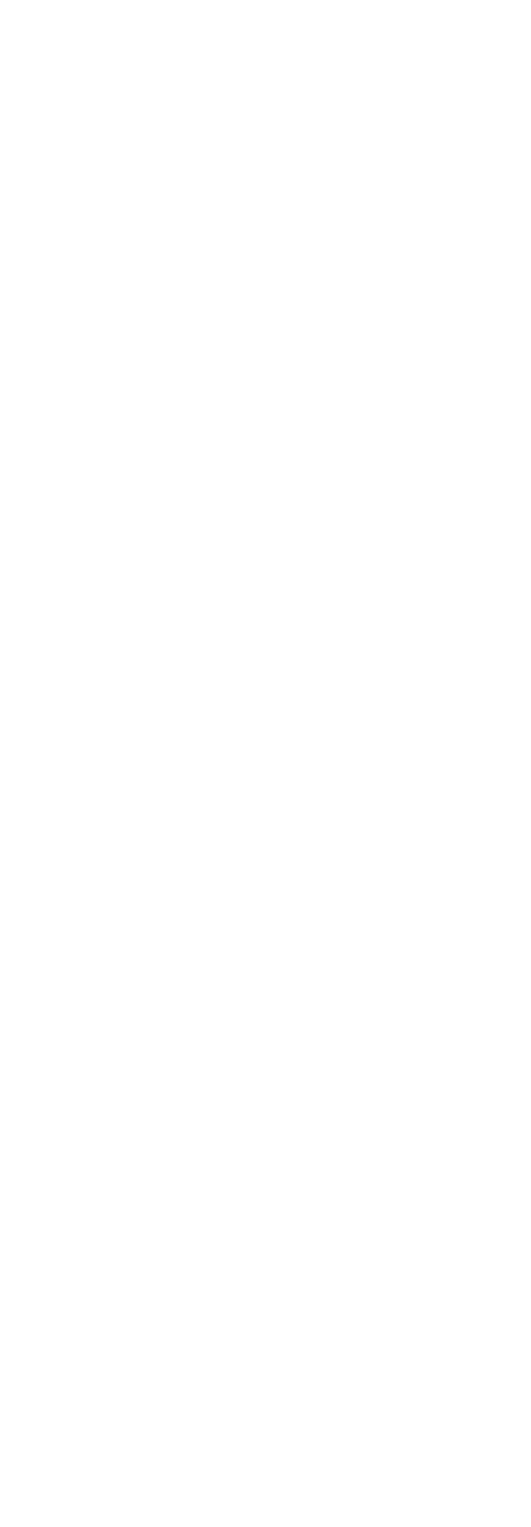




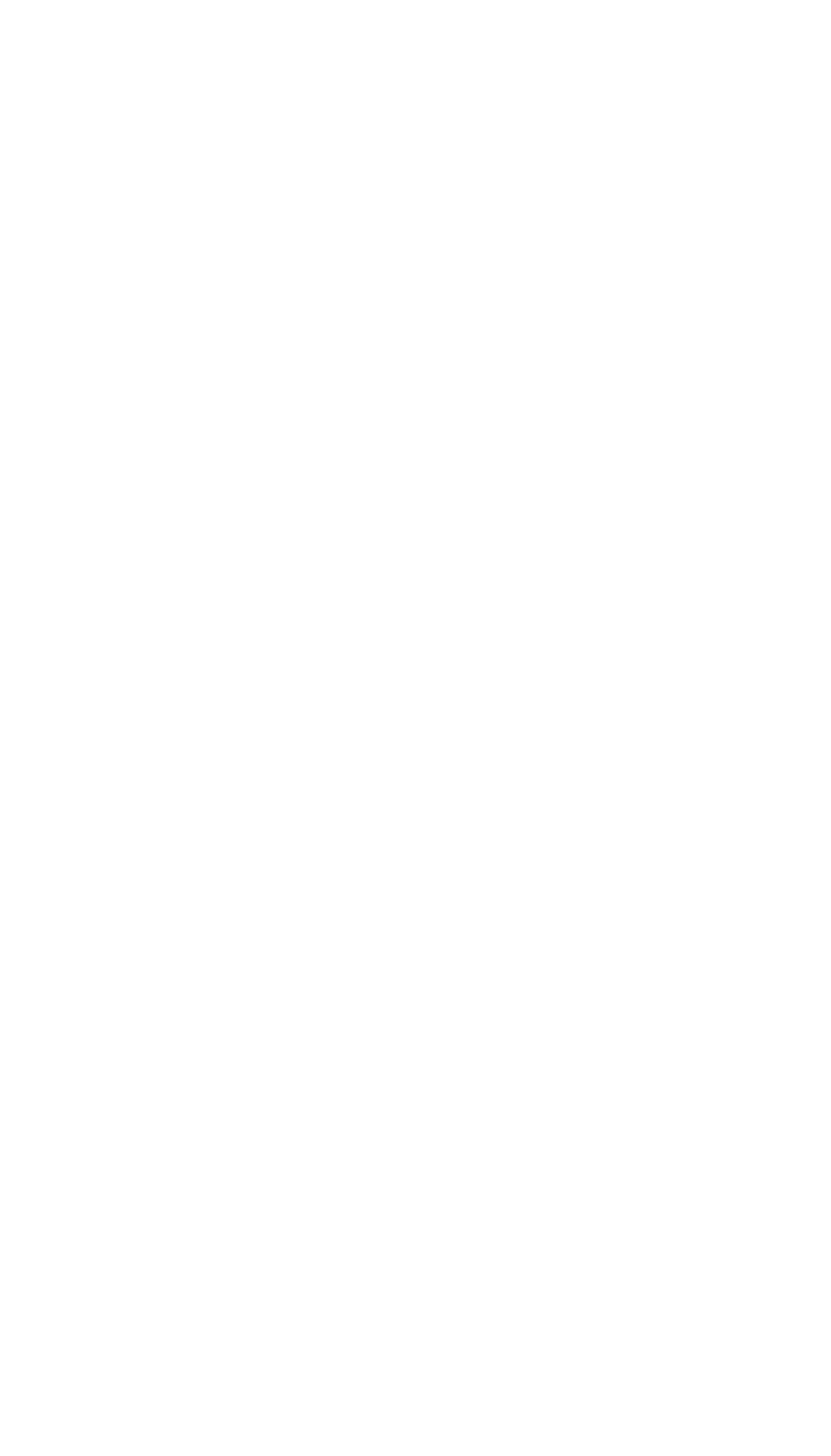




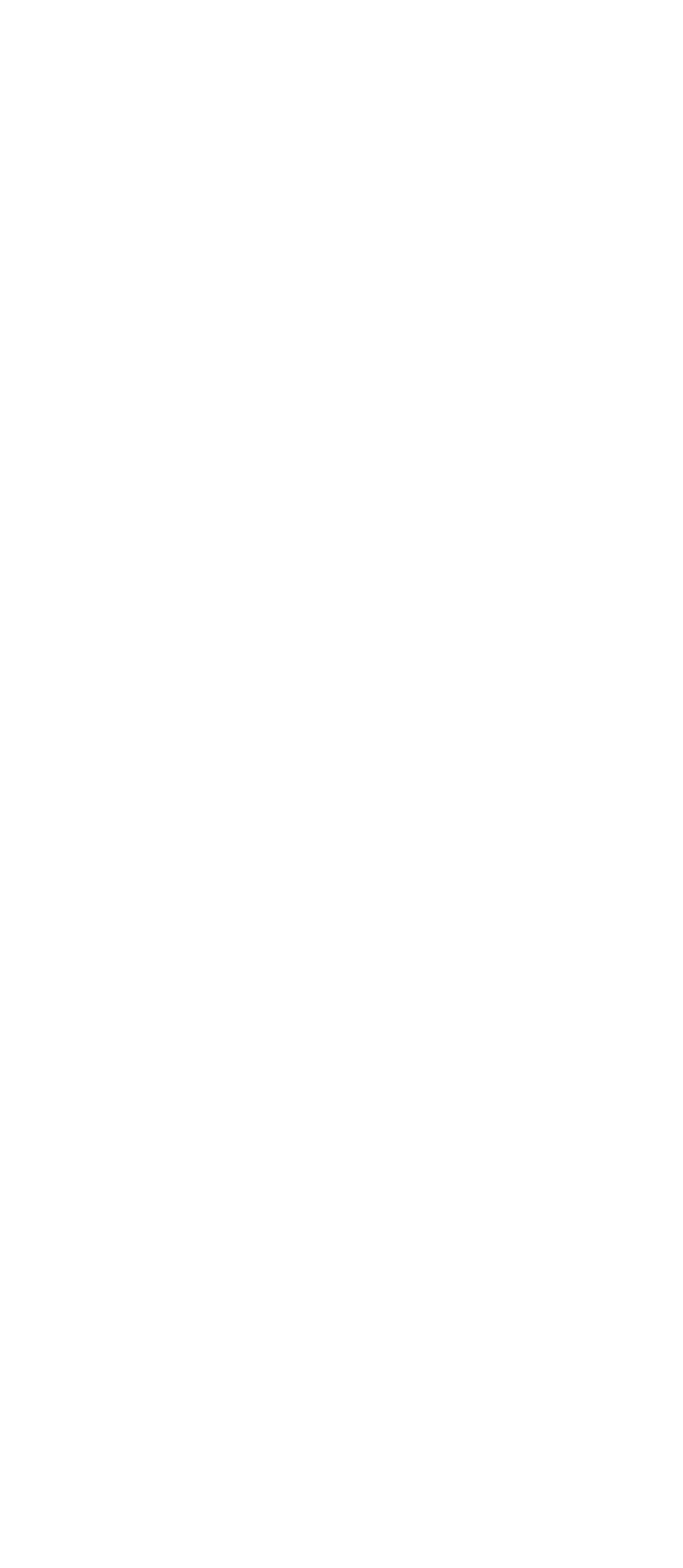




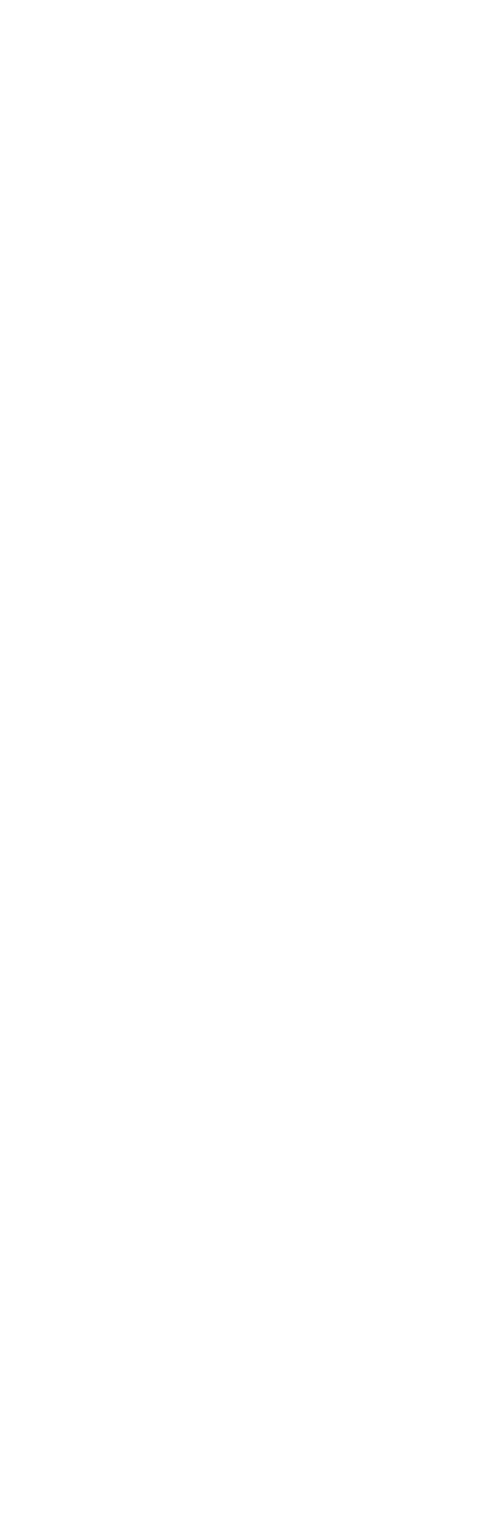




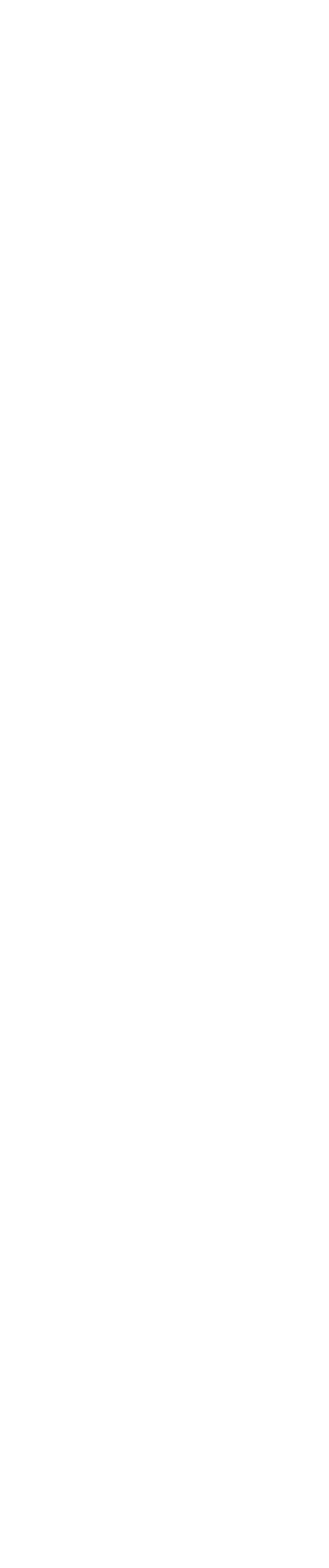

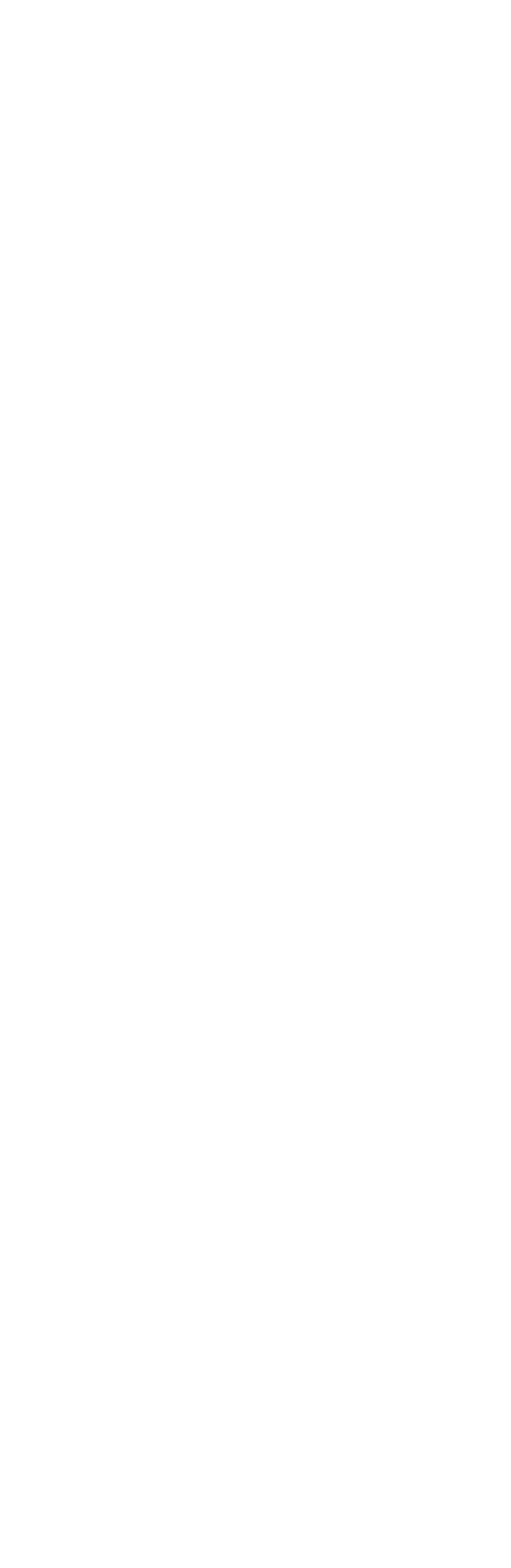




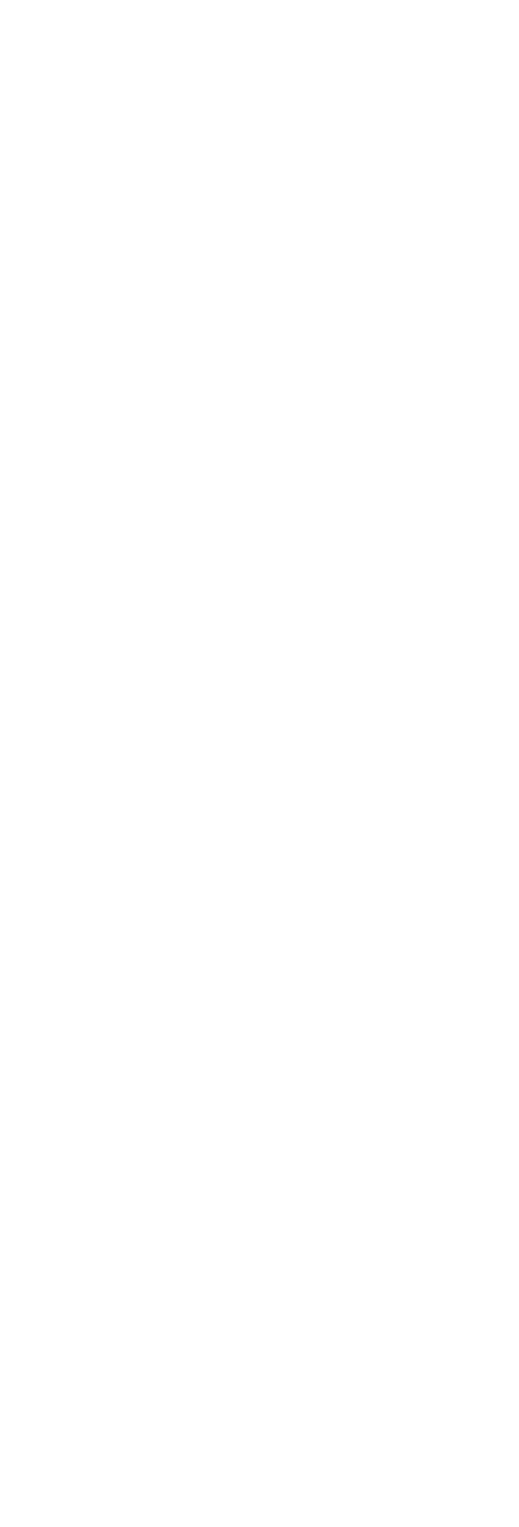




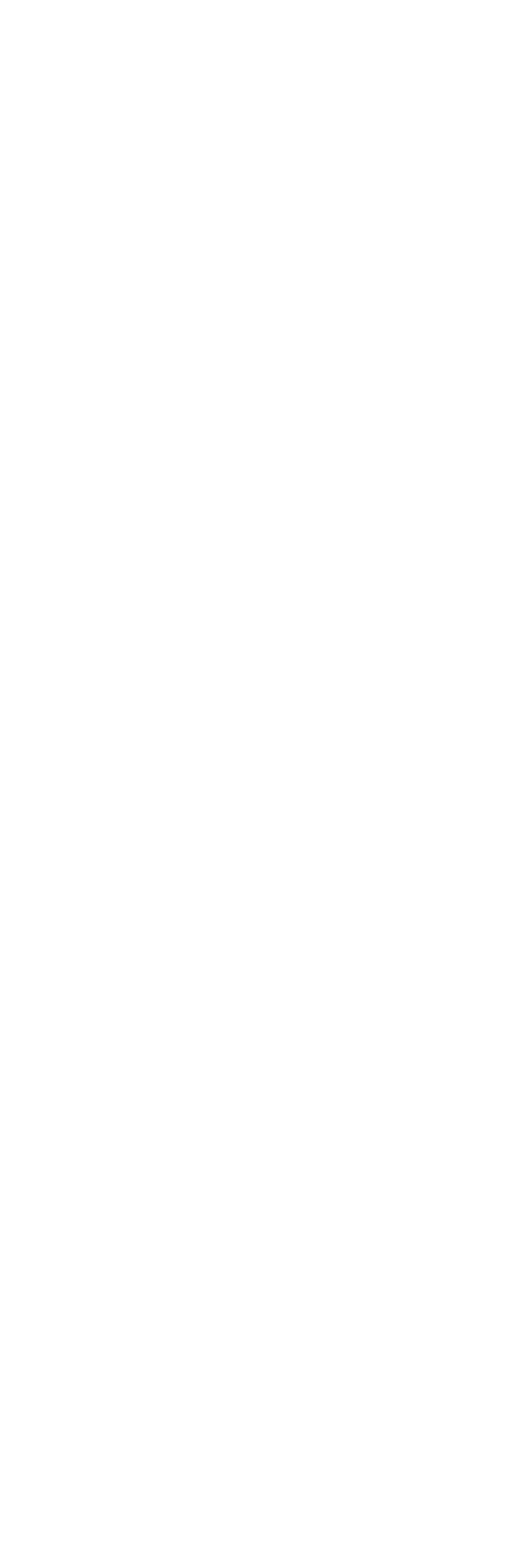




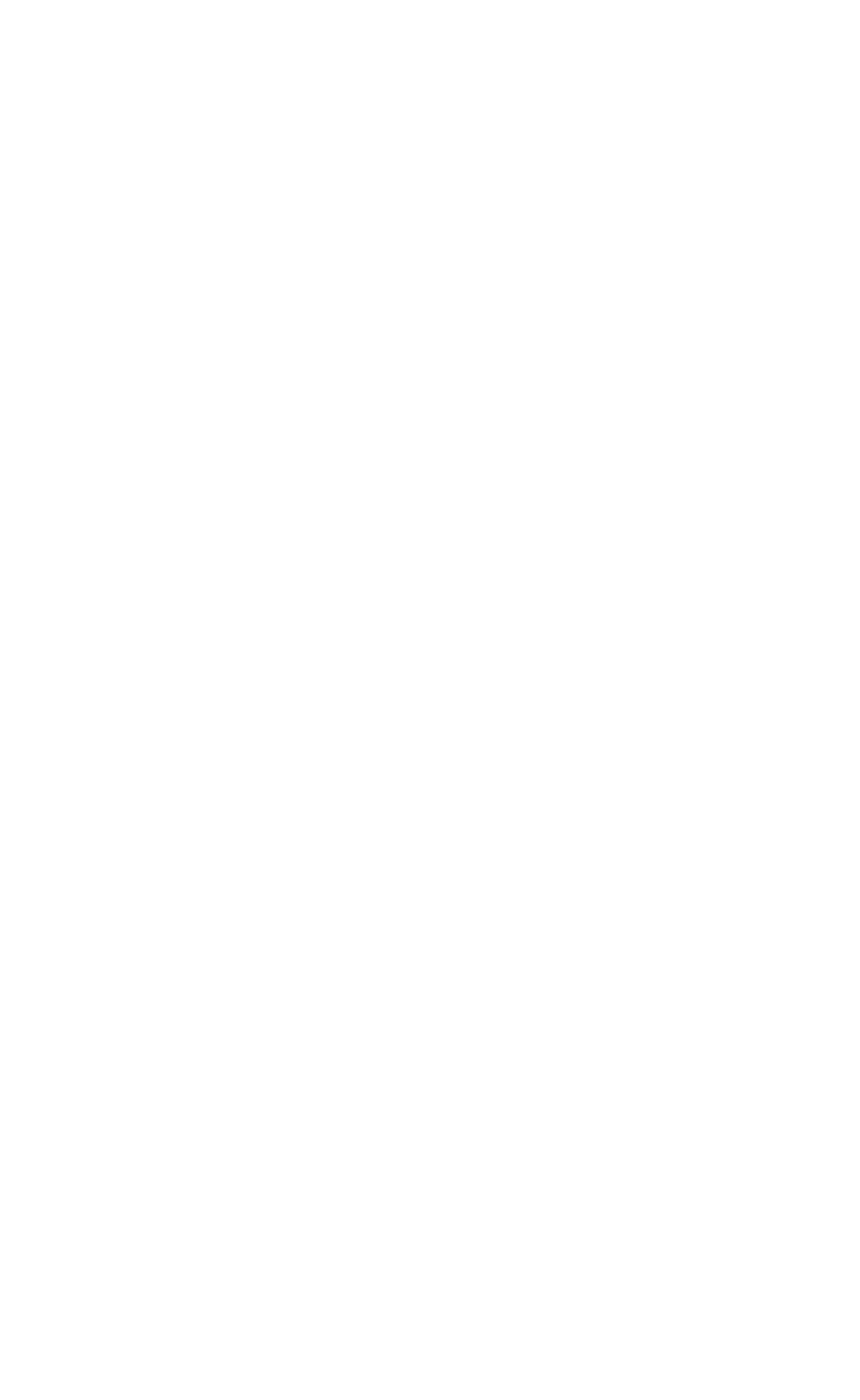




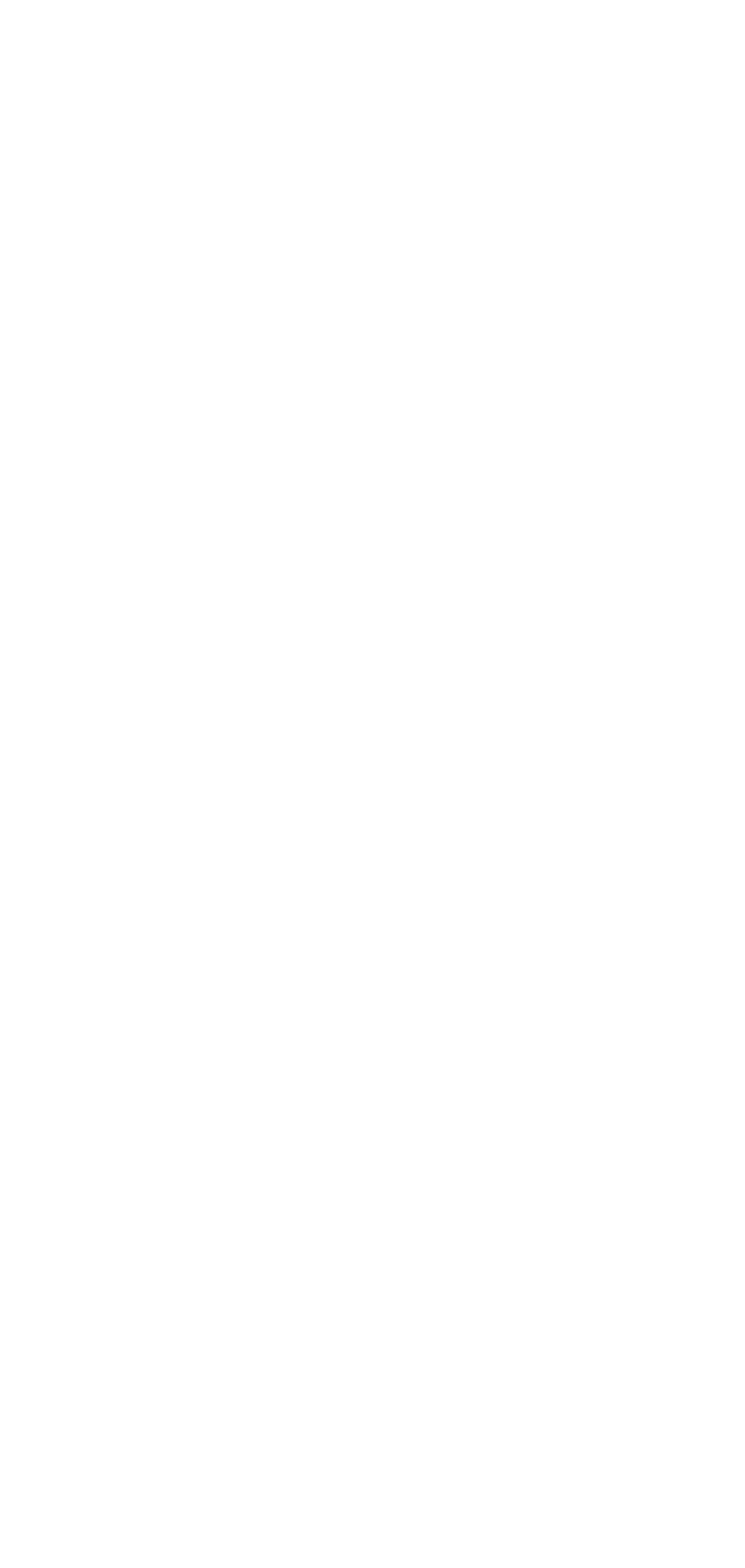




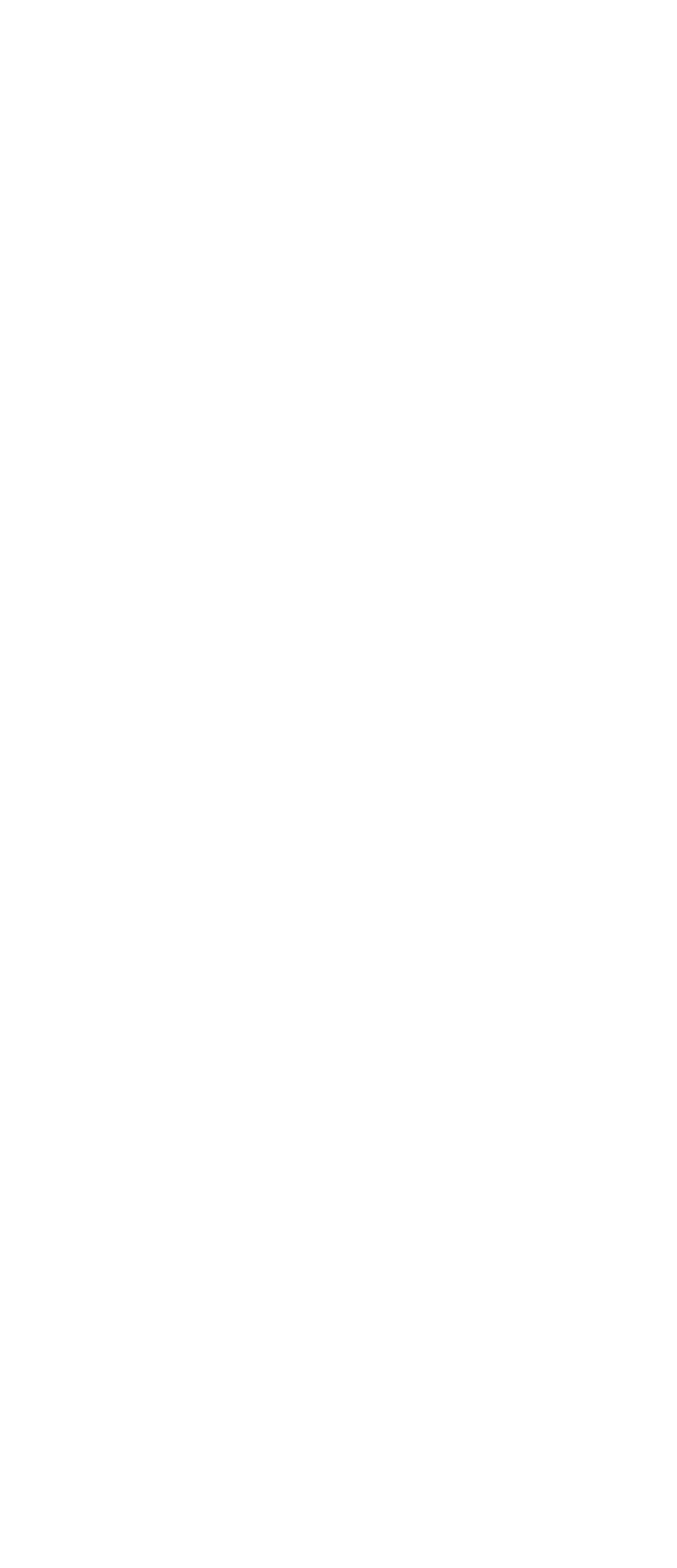




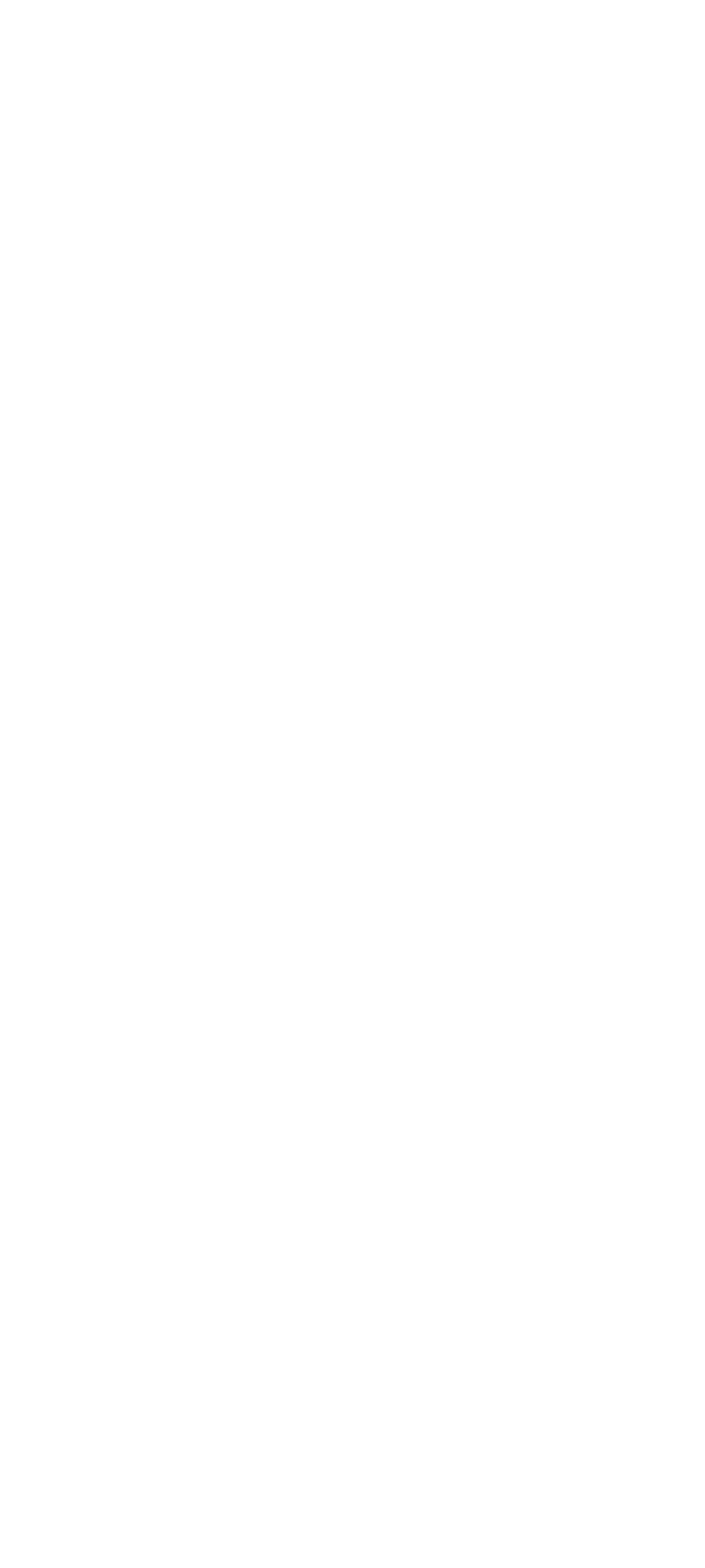




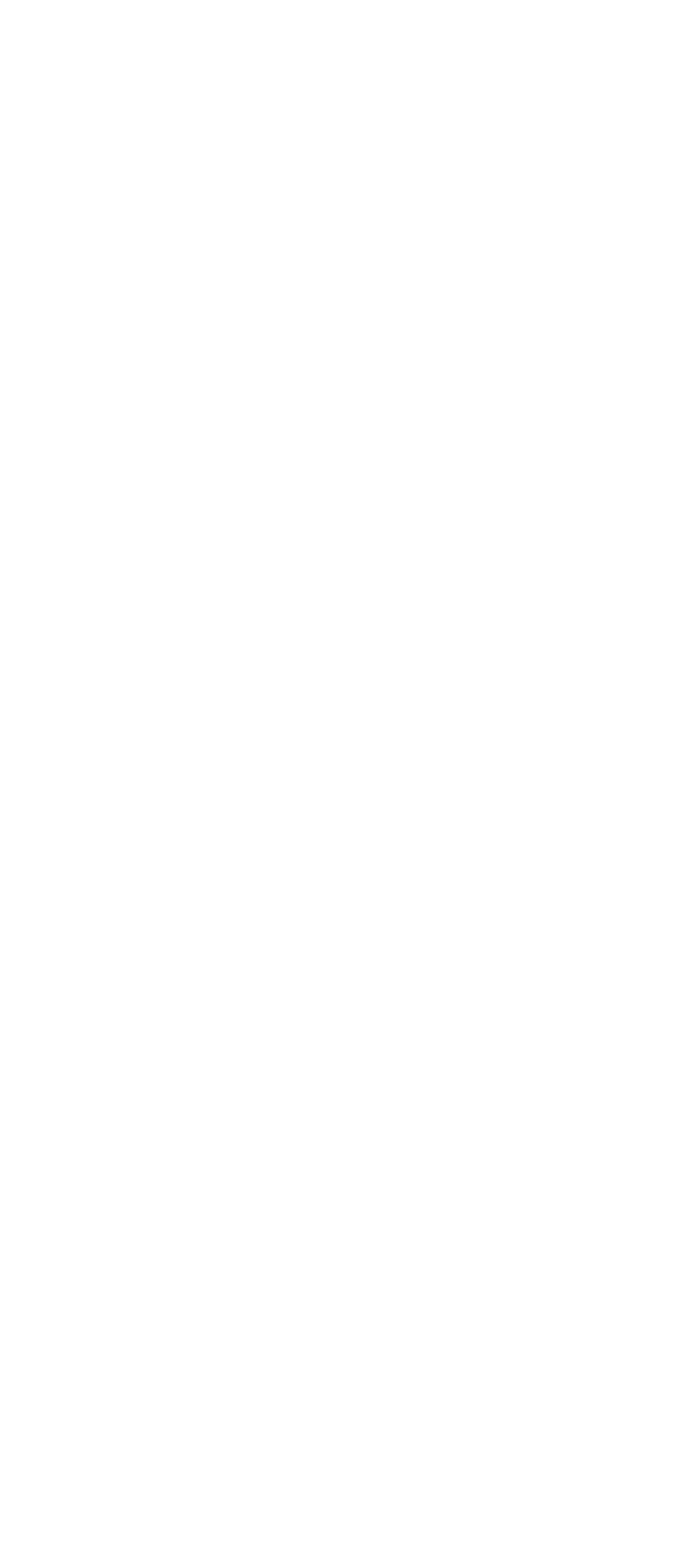




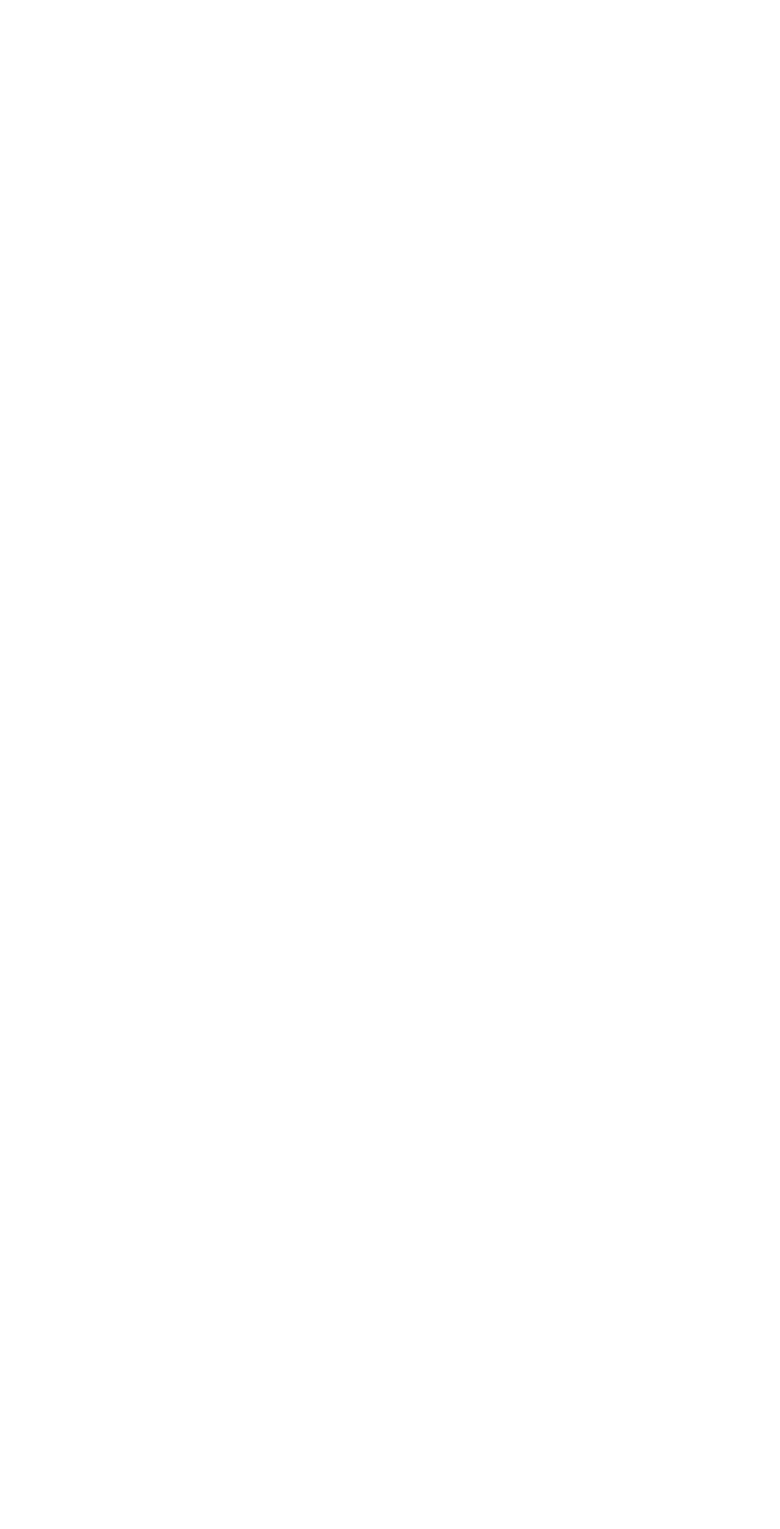


RIO GRANDE BASIN--Continued

8-2865. RIO CHAMA ABOVE ABIQUIU RESERVOIR, N. MEX.--Contin'red

Suspended sediment, water year october 1963 to September 1964

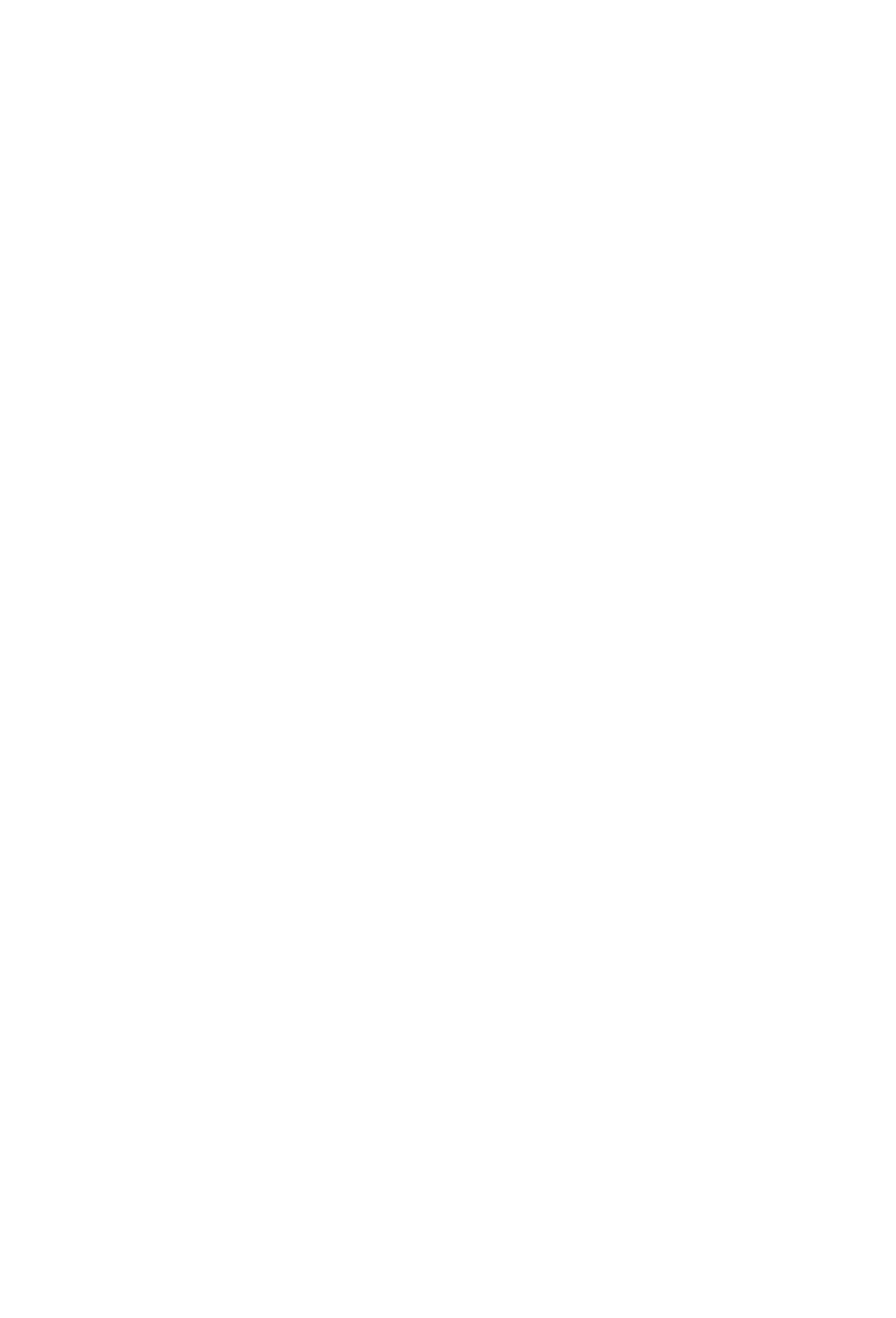

S Computed by subdividing day.

$J$ Computed from partiy estimated-concentration graph and subdividing day. 
RIO GRANDE BASIN--Continued

8-2865. RIO CHAMA ABOVE ABIQUTU RESERVOIR, N. MEX.--Continued

Suspended sediment, water year October 1963 to September 1964--Continued

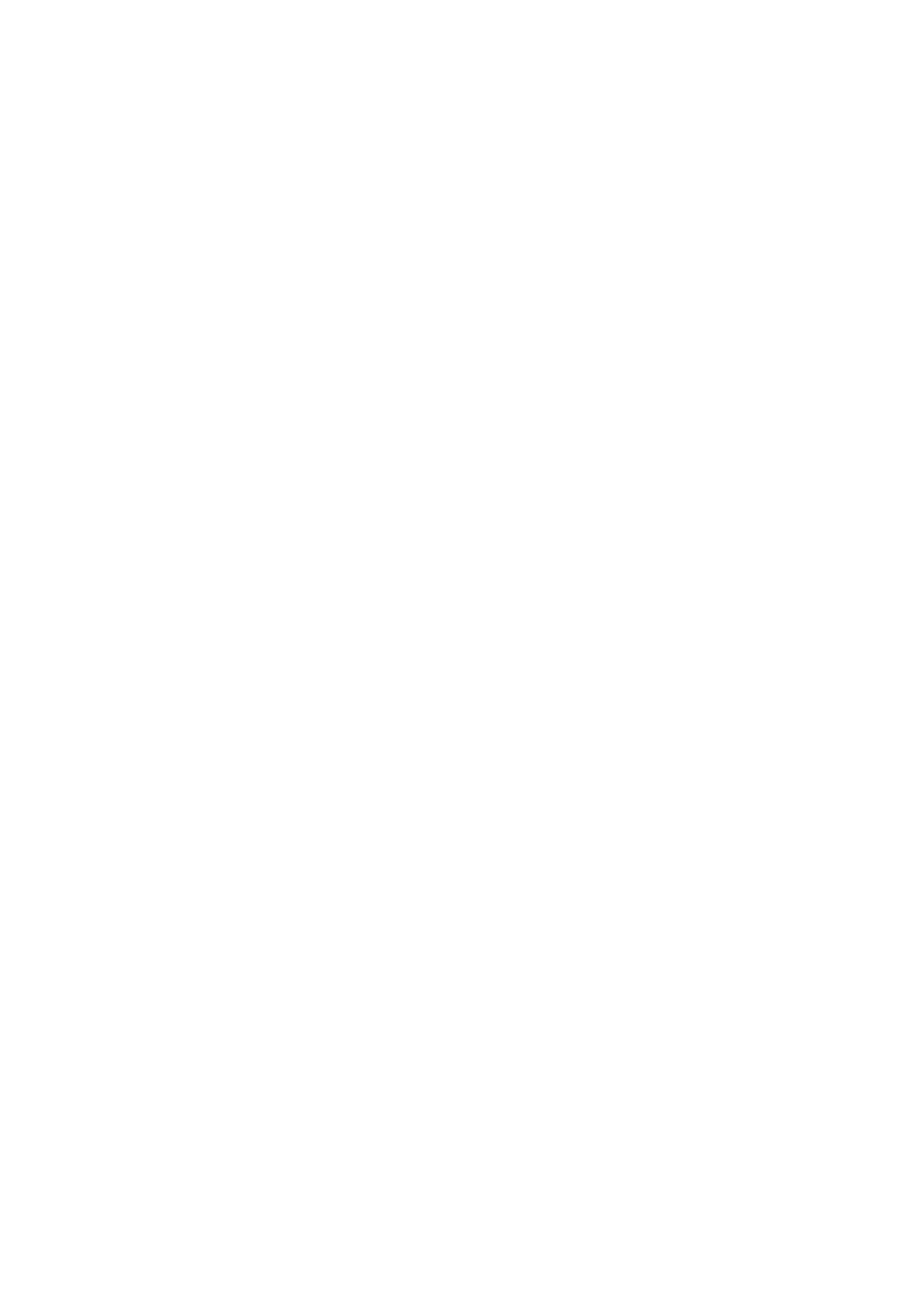

S Computed by subdividing day.

A Computed from partly estimated-concentration graph.

$J$ Computed from partly estimated-

A Computed from partly estimated-concentration
B Computed from estimated-concentration graph. 


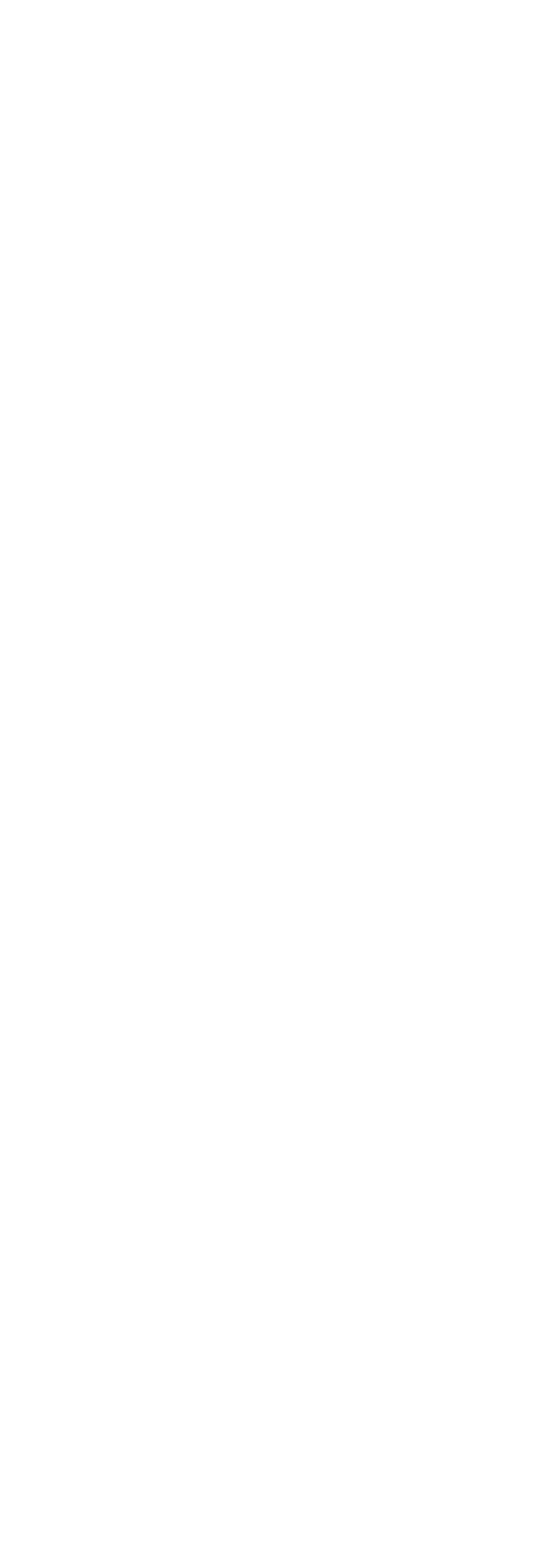




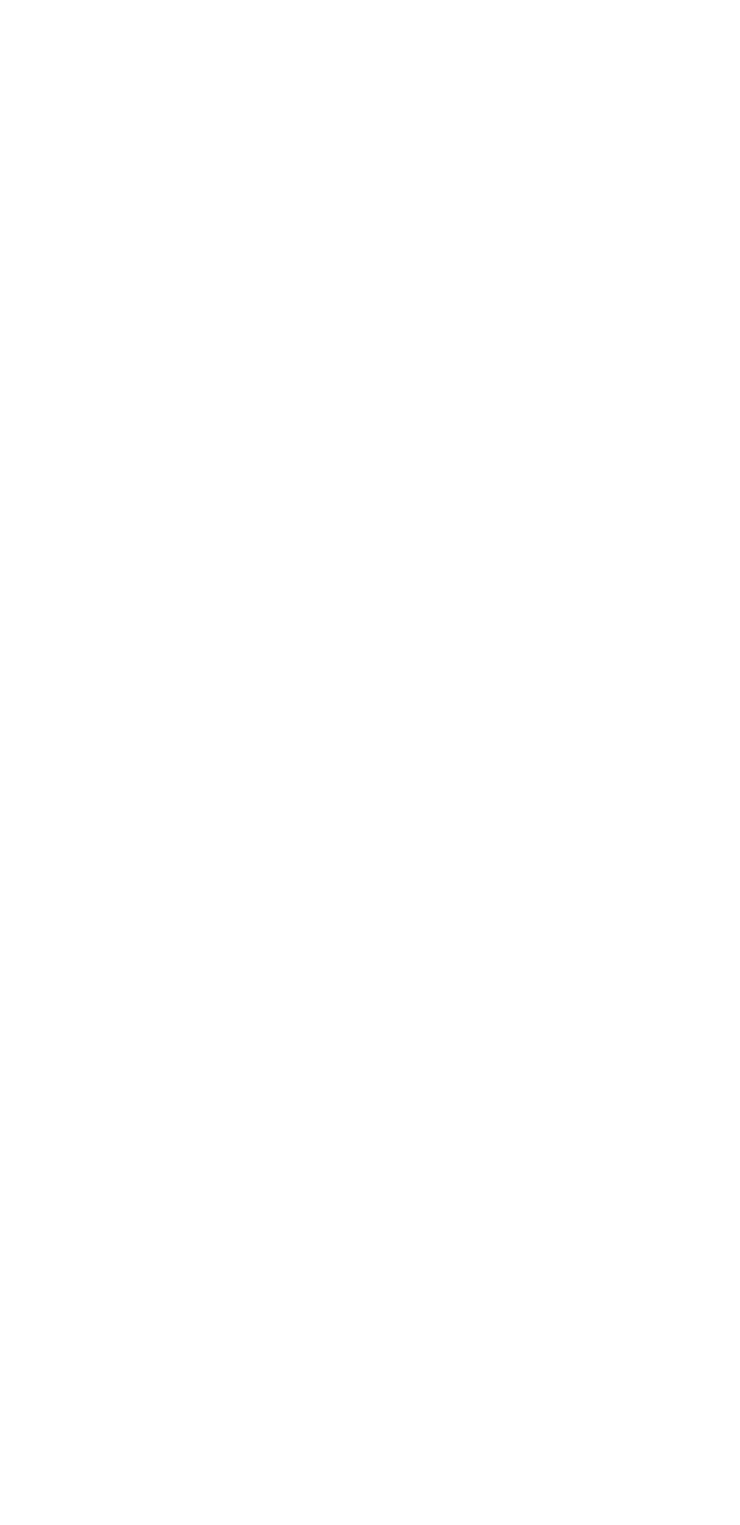


RIO GRANDE BASIN--Continued

8-2870. RIO CHAMA BELOW ABIQUIU DAM, N. MEX.--Continued

Suspended sediment, water year October 1963 to september $1 \& 64$

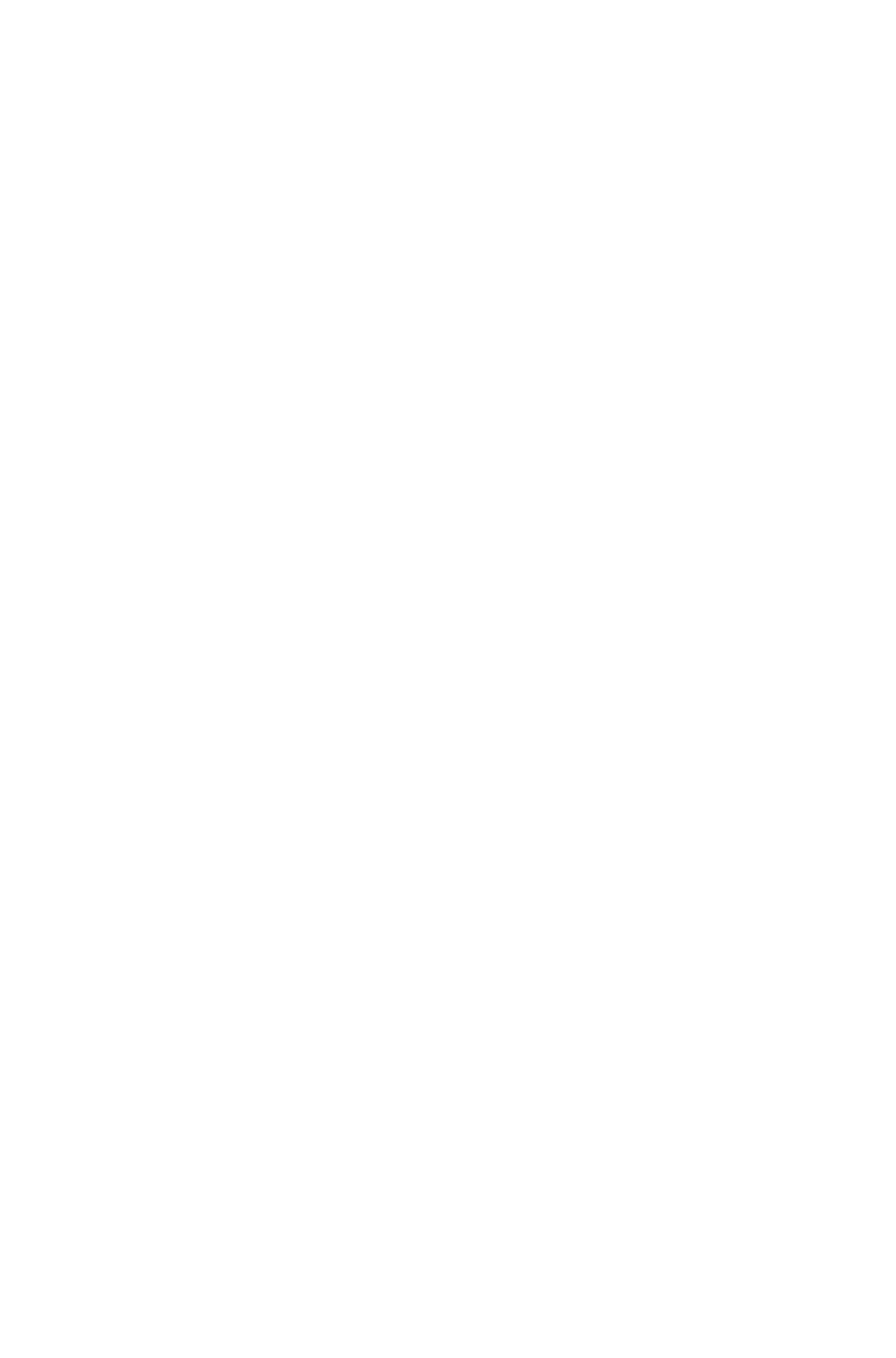


RIO GRANDE BASIN--Continued

8 2870. RIO CHAMA BELOW ABIQUIU DAM, N. MEX.--Continued

Suspended sediment, water year October 1963 to September 1964--Continued

\begin{tabular}{|c|c|c|c|c|c|c|c|c|c|}
\hline \multirow[b]{3}{*}{ Day } & \multicolumn{3}{|c|}{ APRIL } & \multicolumn{3}{|c|}{ MAY } & \multicolumn{3}{|c|}{ JUNE } \\
\hline & \multirow[b]{2}{*}{$\begin{array}{c}\text { Mean } \\
\text { dis- } \\
\text { charge } \\
\text { (cfs) }\end{array}$} & \multicolumn{2}{|c|}{ Suspended sediment } & \multirow[b]{2}{*}{$\begin{array}{c}\text { Mean } \\
\text { dis- } \\
\text { charge } \\
\text { (cfs) }\end{array}$} & \multicolumn{2}{|c|}{ Suspended sediment } & \multirow[b]{2}{*}{$\begin{array}{c}\text { Mean } \\
\text { dis- } \\
\text { charge } \\
(\mathrm{cfs})\end{array}$} & \multicolumn{2}{|c|}{ Suspended sediment } \\
\hline & & $\begin{array}{c}\text { Mean } \\
\text { concen- } \\
\text { tration } \\
\text { (ppm) }\end{array}$ & $\begin{array}{l}\text { Tons } \\
\text { per } \\
\text { day }\end{array}$ & & $\begin{array}{c}\text { Mean } \\
\text { concen- } \\
\text { tration } \\
(\text { ppm) }\end{array}$ & $\begin{array}{c}\text { Tons } \\
\text { per } \\
\text { day }\end{array}$ & & $\begin{array}{c}\text { Mean } \\
\text { concen- } \\
\text { tration } \\
(\text { ppm) }\end{array}$ & $\begin{array}{c}\text { Tons } \\
\text { per } \\
\text { day }\end{array}$ \\
\hline $\begin{array}{l}1 \ldots \\
2 \cdots \\
3 \ldots \\
4 \ldots \\
5 \ldots\end{array}$ & $\begin{array}{l}238 \\
299 \\
289 \\
235 \\
123\end{array}$ & $\begin{array}{r}680 \\
670 \\
380 \\
-- \\
--\end{array}$ & $\begin{array}{l}440 \\
540 \\
300 \\
360 \\
610\end{array}$ & $\begin{array}{l}452 \\
577 \\
595 \\
779 \\
759\end{array}$ & $\begin{array}{l}300 \\
250 \\
240 \\
530 \\
410\end{array}$ & $\begin{array}{r}370 \\
390 \\
390 \\
1100 \\
840\end{array}$ & $\begin{array}{l}539 \\
595 \\
490 \\
437 \\
380\end{array}$ & $\begin{array}{r}160 \\
90 \\
160 \\
210 \\
90\end{array}$ & $\begin{array}{l}230 \\
150 \\
210 \\
250 \\
100\end{array}$ \\
\hline $\begin{array}{r}6 \ldots \\
7 \ldots \\
9 \ldots \\
10 \ldots\end{array}$ & $\begin{array}{r}83 \\
122 \\
95 \\
122 \\
109\end{array}$ & $\begin{array}{l}1800 \\
3600 \\
1900 \\
4900 \\
6500\end{array}$ & $\begin{array}{r}540 \\
1200 \\
480 \\
1600 \\
1900\end{array}$ & $\begin{array}{l}739 \\
830 \\
851 \\
862 \\
873\end{array}$ & $\begin{array}{l}320 \\
340 \\
270 \\
220 \\
190\end{array}$ & $\begin{array}{l}640 \\
760 \\
620 \\
510 \\
450\end{array}$ & $\begin{array}{l}268 \\
235 \\
225 \\
245 \\
314\end{array}$ & $\begin{array}{l}90 \\
80 \\
60 \\
50 \\
50\end{array}$ & $\begin{array}{l}65 \\
51 \\
36 \\
33 \\
42\end{array}$ \\
\hline $\begin{array}{l}11 \ldots \\
12 \ldots \\
13 \ldots \\
14 \ldots \\
15 \ldots\end{array}$ & $\begin{array}{r}97 \\
98 \\
104 \\
104 \\
146\end{array}$ & $\begin{array}{r}1600 \\
210 \\
180 \\
130 \\
200\end{array}$ & $\begin{array}{r}420 \\
56 \\
51 \\
37 \\
79\end{array}$ & $\begin{array}{r}873 \\
867 \\
939 \\
1040 \\
1060\end{array}$ & $\begin{array}{l}220 \\
280 \\
340 \\
430 \\
430\end{array}$ & $\begin{array}{r}520 \\
660 \\
860 \\
1200 \\
1200\end{array}$ & $\begin{array}{l}340 \\
225 \\
204 \\
202 \\
165\end{array}$ & $\begin{array}{l}60 \\
70 \\
60 \\
50 \\
80\end{array}$ & $\begin{array}{l}55 \\
43 \\
33 \\
27 \\
36\end{array}$ \\
\hline $\begin{array}{l}16 \ldots \\
17 \ldots \\
18 \ldots \\
19 \ldots \\
20 \ldots\end{array}$ & $\begin{array}{l}266 \\
277 \\
440 \\
535 \\
415\end{array}$ & $\begin{array}{l}480 \\
230 \\
290 \\
340 \\
420\end{array}$ & $\begin{array}{l}380 \\
170 \\
350 \\
490 \\
470\end{array}$ & $\begin{array}{r}1050 \\
1050 \\
911 \\
878 \\
973\end{array}$ & $\begin{array}{l}400 \\
320 \\
300 \\
260 \\
250\end{array}$ & $\begin{array}{r}1100 \\
910 \\
740 \\
620 \\
660\end{array}$ & $\begin{array}{r}176 \\
104 \\
88 \\
74 \\
51\end{array}$ & $\begin{array}{l}-- \\
-- \\
-- \\
z- \\
--\end{array}$ & $\begin{array}{r}450 \\
200 \\
50 \\
30 \\
20\end{array}$ \\
\hline $\begin{array}{l}21 \ldots \\
22 \ldots \\
23 \ldots \\
24 \ldots \\
25 \ldots\end{array}$ & $\begin{array}{l}243 \\
159 \\
230 \\
434 \\
522\end{array}$ & $\begin{array}{l}430 \\
250 \\
260 \\
290 \\
400\end{array}$ & $\begin{array}{l}280 \\
110 \\
160 \\
400 \\
560\end{array}$ & $\begin{array}{r}1080 \\
1110 \\
1060 \\
894 \\
830\end{array}$ & $\begin{array}{l}230 \\
190 \\
150 \\
120 \\
100\end{array}$ & $\begin{array}{l}670 \\
570 \\
430 \\
290 \\
220\end{array}$ & $\begin{array}{l}40 \\
38 \\
36 \\
35 \\
35\end{array}$ & $\begin{array}{l}=- \\
z- \\
z- \\
--\end{array}$ & $\begin{array}{l}10 \\
10 \\
10 \\
10 \\
10\end{array}$ \\
\hline $\begin{array}{l}26 \ldots \\
27 \ldots \\
28 \ldots \\
29 \ldots \\
30 \ldots \\
31 \ldots \\
\end{array}$ & $\begin{array}{r}518 \\
526 \\
455 \\
314 \\
311 \\
-\end{array}$ & $\begin{array}{r}370 \\
220 \\
260 \\
260 \\
270 \\
- \\
\end{array}$ & $\begin{array}{r}520 \\
310 \\
320 \\
220 \\
230 \\
--\end{array}$ & $\begin{array}{l}851 \\
830 \\
944 \\
996 \\
825 \\
530 \\
\end{array}$ & $\begin{array}{l}110 \\
220 \\
240 \\
130 \\
110 \\
110 \\
\end{array}$ & $\begin{array}{l}250 \\
490 \\
610 \\
350 \\
250 \\
160 \\
\end{array}$ & $\begin{array}{r}35 \\
35 \\
35 \\
40 \\
448 \\
--\end{array}$ & $\begin{array}{r}-- \\
=- \\
=- \\
1500 \\
-\end{array}$ & $\begin{array}{r}10 \\
10 \\
10 \\
10 \\
2500 \\
-\end{array}$ \\
\hline \multirow[t]{2}{*}{ Total } & 7909 & -- & 13583 & 26908 & -- & 18830 & 6134 & -- & 4701 \\
\hline & \multicolumn{3}{|c|}{ JULY } & \multicolumn{3}{|c|}{ AUGUST } & \multicolumn{3}{|c|}{ SEPTEMBER } \\
\hline $\begin{array}{l}1 \ldots \\
2 \cdots \\
3 \ldots \\
4 \ldots \\
5 \bullet\end{array}$ & $\begin{array}{l}444 \\
444 \\
437 \\
433 \\
270\end{array}$ & $\begin{array}{r}450 \\
950 \\
1700 \\
2400 \\
3000\end{array}$ & $\begin{array}{l}540 \\
1100 \\
1900 \\
2800 \\
2100\end{array}$ & $\begin{array}{l}212 \\
232 \\
562 \\
700 \\
471\end{array}$ & $\begin{array}{r}-- \\
10000 \\
8200 \\
5100\end{array}$ & $\begin{array}{r}6100 \\
4300 \\
18000 \\
16000 \\
6500\end{array}$ & $\begin{array}{r}31 \\
29 \\
408 \\
550 \\
471\end{array}$ & $\begin{array}{r}210 \\
400 \\
1900 \\
440 \\
--\end{array}$ & $\begin{array}{r}18 \\
31 \\
4200 \\
890 \\
450\end{array}$ \\
\hline $\begin{array}{c}6 \ldots \\
7 \ldots \\
8 \ldots \\
9 \ldots \\
10 \ldots\end{array}$ & $\begin{array}{r}38 \\
20 \\
17 \\
32 \\
105\end{array}$ & $\begin{array}{r}3000 \\
2500 \\
3400 \\
7300 \\
24000\end{array}$ & $\begin{array}{r}310 \\
130 \\
160 \\
1500 \\
7900\end{array}$ & $\begin{array}{l}166 \\
148 \\
174 \\
210 \\
192\end{array}$ & $\begin{array}{r}21000 \\
14000 \\
0 \\
11000\end{array}$ & $\begin{array}{l}8300 \\
5400 \\
6200 \\
7500 \\
5600\end{array}$ & $\begin{array}{r}474 \\
455 \\
267 \\
68 \\
60\end{array}$ & $\begin{array}{r}-- \\
1200 \\
2400 \\
900\end{array}$ & $\begin{array}{l}440 \\
430 \\
430 \\
440 \\
150\end{array}$ \\
\hline $\begin{array}{l}11 \ldots \\
12 \ldots \\
13 \ldots \\
14 \ldots \\
15 \ldots\end{array}$ & $\begin{array}{r}21 \\
36 \\
226 \\
42 \\
22\end{array}$ & $\begin{array}{r}=- \\
21000 \\
23000 \\
840\end{array}$ & $\begin{array}{r}260 \\
340 \\
12000 \\
2600 \\
50\end{array}$ & $\begin{array}{l}186 \\
679 \\
800 \\
920 \\
800\end{array}$ & $\begin{array}{r}3500 \\
33000 \\
19000 \\
1000 \\
--\end{array}$ & $\begin{array}{r}1700 \\
77000 \\
41000 \\
2500 \\
4700\end{array}$ & $\begin{array}{l}59 \\
54 \\
52 \\
51 \\
66\end{array}$ & $\begin{array}{l}400 \\
400 \\
350 \\
360 \\
260\end{array}$ & $\begin{array}{l}64 \\
58 \\
49 \\
50 \\
46\end{array}$ \\
\hline $\begin{array}{l}16 \ldots \\
17 \ldots \\
18 \ldots \\
19 \ldots \\
20 \ldots\end{array}$ & $\begin{array}{l}24 \\
31 \\
32 \\
44 \\
52\end{array}$ & $\begin{array}{r}5100 \\
1600 \\
-- \\
10000\end{array}$ & $\begin{array}{r}330 \\
130 \\
160 \\
1000 \\
1400\end{array}$ & $\begin{array}{r}400 \\
215 \\
204 \\
83 \\
60\end{array}$ & $\begin{array}{r}14000 \\
4100 \\
9400 \\
3300\end{array}$ & $\begin{array}{r}12000 \\
8100 \\
2200 \\
2100 \\
530\end{array}$ & $\begin{array}{r}120 \\
134 \\
56 \\
52 \\
79\end{array}$ & $\begin{array}{r}2000 \\
1100 \\
930 \\
850 \\
980\end{array}$ & $\begin{array}{l}800 \\
410 \\
140 \\
120 \\
220\end{array}$ \\
\hline $\begin{array}{l}21 \ldots \\
22 \ldots \\
23 \ldots \\
24 \ldots \\
25 \ldots\end{array}$ & $\begin{array}{r}53 \\
62 \\
80 \\
120 \\
236\end{array}$ & $\begin{array}{r}5400 \\
2000 \\
11000 \\
32000 \\
\end{array}$ & $\begin{array}{r}770 \\
340 \\
3900 \\
11000 \\
6200\end{array}$ & $\begin{array}{l}61 \\
97 \\
89 \\
86 \\
77\end{array}$ & $\begin{array}{r}3700 \\
3100 \\
1600 \\
750 \\
680\end{array}$ & $\begin{array}{l}610 \\
810 \\
380 \\
170 \\
140\end{array}$ & $\begin{array}{r}104 \\
192 \\
183 \\
172 \\
82\end{array}$ & $\begin{array}{l}6200 \\
3800 \\
3000 \\
1500 \\
2200\end{array}$ & $\begin{array}{r}1600 \\
1900 \\
1500 \\
700 \\
490\end{array}$ \\
\hline $\begin{array}{l}26 \ldots \\
27 \ldots \\
28 \ldots \\
29 \ldots \\
30 \ldots \\
31 \ldots\end{array}$ & $\begin{array}{r}46 \\
68 \\
88 \\
90 \\
158 \\
188 \\
\end{array}$ & $\begin{array}{r}22000 \\
34000 \\
7200 \\
38000 \\
24000\end{array}$ & $\begin{array}{r}500 \\
5700 \\
3200 \\
1700 \\
19000 \\
12000 \\
\end{array}$ & $\begin{array}{l}46 \\
37 \\
37 \\
33 \\
43 \\
32\end{array}$ & $\begin{array}{r}1000 \\
390 \\
390 \\
400 \\
350 \\
260\end{array}$ & $\begin{array}{r}120 \\
39 \\
39 \\
36 \\
41 \\
22 \\
\end{array}$ & $\begin{array}{l}42 \\
35 \\
33 \\
29 \\
26 \\
\end{array}$ & $\begin{array}{r}-- \\
1200 \\
1200 \\
1500 \\
--\end{array}$ & $\begin{array}{r}140 \\
90 \\
110 \\
94 \\
100 \\
-- \\
\end{array}$ \\
\hline Total & 3759 & - & 101020 & 8052 & - & 238137 & 4434 & -- & 16160 \\
\hline
\end{tabular}

E Estinated.

B Computed from estimated-consen-

$S$ Computed from partly estimated-concentration graph. tration graph. 


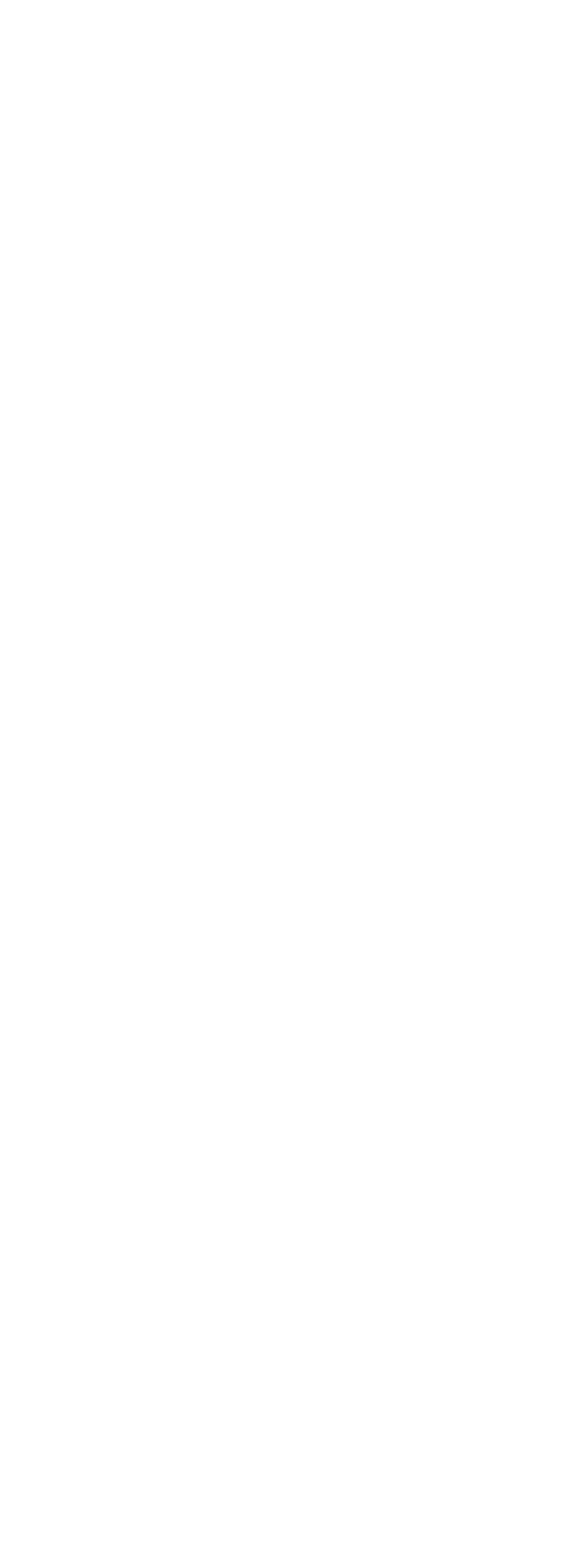




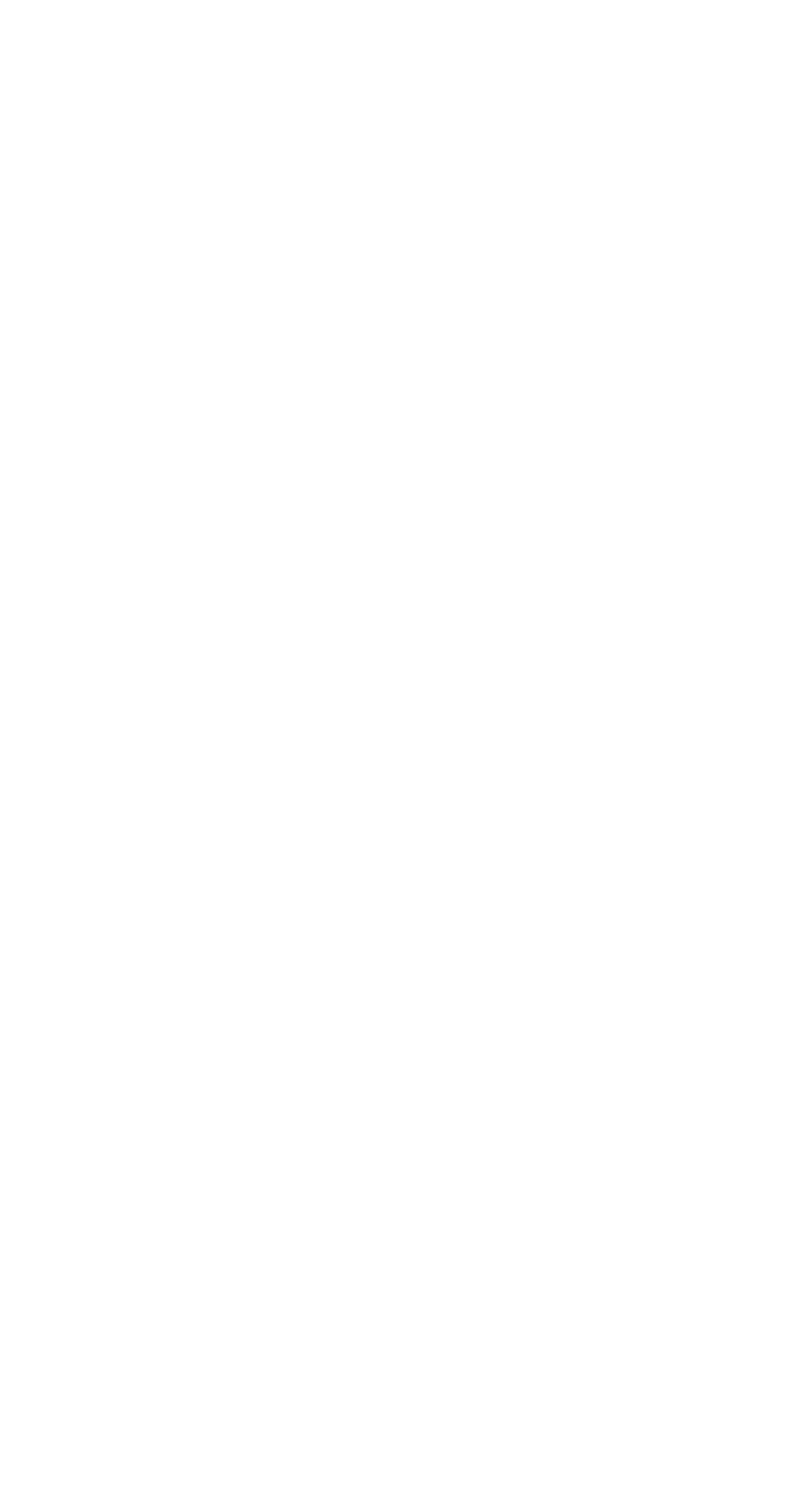




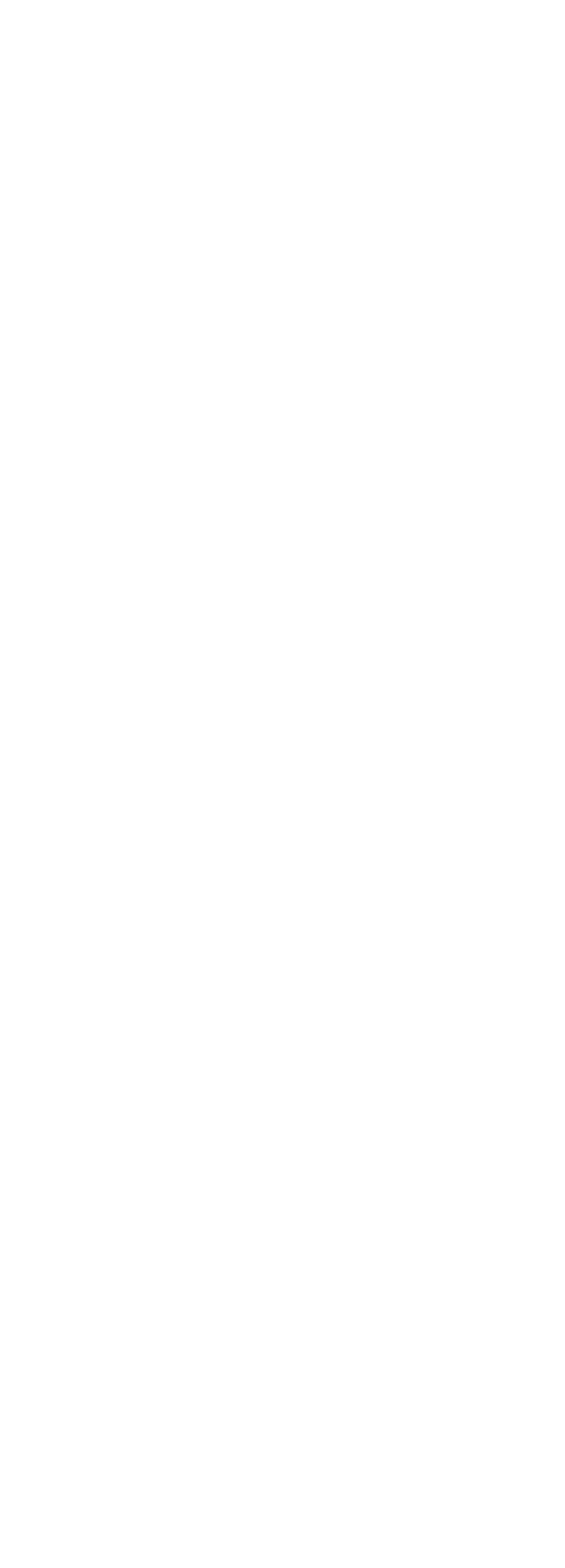




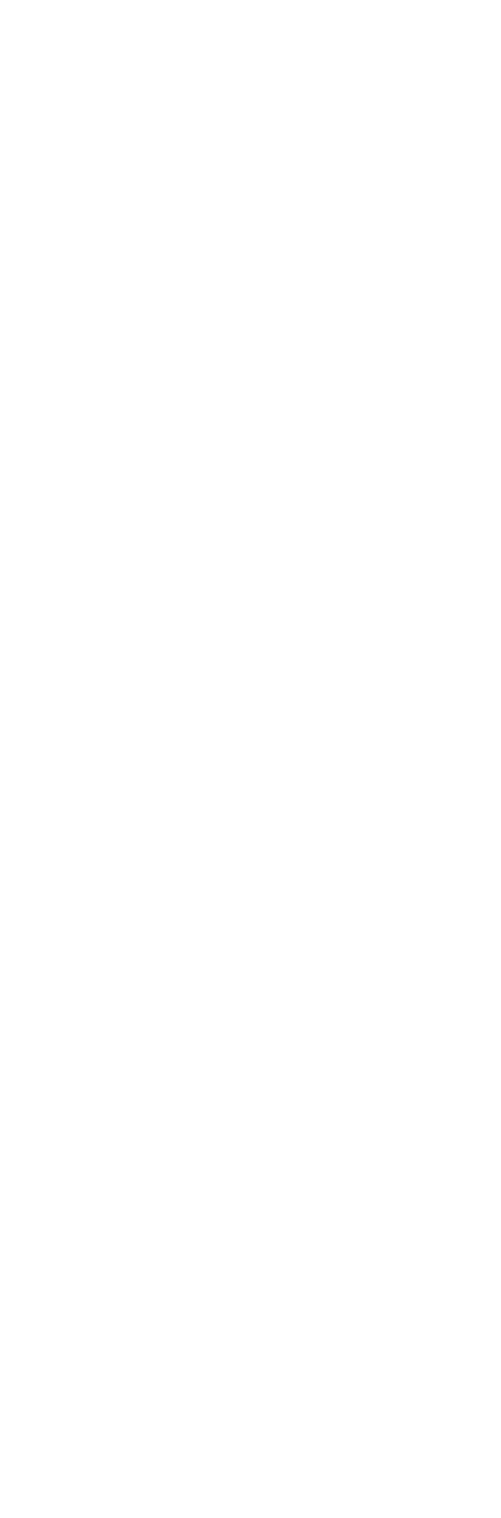


RIO GRANDE BASIN--Continued

8-2900. RIO CHAMA NEAR CHAMITA, N. MEX.--Continued

Suspended sediment, water year October 1963 to September 1964

\begin{tabular}{|c|c|c|c|c|c|c|c|c|c|}
\hline \multirow[b]{2}{*}{ Day } & \multicolumn{3}{|c|}{ OCTOBER } & \multicolumn{3}{|c|}{ NOVEMBER } & \multicolumn{3}{|c|}{ DECEMBER } \\
\hline & $\begin{array}{c}\text { Mean } \\
\text { dis- } \\
\text { charge } \\
\text { (cfs) }\end{array}$ & $\begin{array}{l}\text { Mean } \\
\text { concen- } \\
\text { tration } \\
(\text { ppm) }\end{array}$ & $\begin{array}{c}\text { Tons } \\
\text { per } \\
\text { day }\end{array}$ & $\begin{array}{c}\text { Mean } \\
\text { dis- } \\
\text { charge } \\
\text { (cfs) }\end{array}$ & $\begin{array}{l}\text { Mean } \\
\text { concen- } \\
\text { tration } \\
\text { (ppm) }\end{array}$ & $\begin{array}{c}\text { Tons } \\
\text { per } \\
\text { day }\end{array}$ & $\begin{array}{c}\text { Mean } \\
\text { dis- } \\
\text { charge } \\
\text { (cfs) }\end{array}$ & $\begin{array}{c}\text { Mean } \\
\text { concen- } \\
\text { tration } \\
\text { (ppm) }\end{array}$ & $\begin{array}{l}\text { Tons } \\
\text { per } \\
\text { day }\end{array}$ \\
\hline $\begin{array}{l}1 \ldots \\
2 \ldots \\
3 \ldots \\
4 \ldots \\
5 \ldots\end{array}$ & $\begin{array}{l}7.9 \\
7.3 \\
6.4 \\
5.4 \\
4.8\end{array}$ & $\begin{array}{l}60 \\
60 \\
50 \\
50 \\
30\end{array}$ & $\begin{array}{l}1 \\
1 \\
1 \\
1 \\
T\end{array}$ & $\begin{array}{l}31 \\
24 \\
22 \\
22 \\
34\end{array}$ & $\begin{array}{l}250 \\
210 \\
180 \\
190 \\
230\end{array}$ & $\begin{array}{l}21 \\
14 \\
11 \\
11 \\
22\end{array}$ & $\begin{array}{l}62 \\
59 \\
52 \\
52 \\
52\end{array}$ & $\begin{array}{l}160 \\
110 \\
200 \\
260 \\
730\end{array}$ & $\begin{array}{r}27 \\
18 \\
28 \\
37 \\
100\end{array}$ \\
\hline $\begin{array}{r}6 \ldots \\
7 \ldots \\
8 \ldots \\
10 \ldots\end{array}$ & $\begin{array}{l}4.8 \\
6.0 \\
5.4 \\
5.4 \\
5.7\end{array}$ & $\begin{array}{l}30 \\
90 \\
50 \\
20 \\
40\end{array}$ & $\begin{array}{l}M \\
1 \\
1 \\
T \\
1\end{array}$ & $\begin{array}{l}38 \\
40 \\
45 \\
42 \\
44\end{array}$ & $\begin{array}{r}180 \\
230 \\
1400 \\
600 \\
450\end{array}$ & $\begin{array}{r}18 \\
25 \\
170 \\
68 \\
53\end{array}$ & $\begin{array}{l}57 \\
52 \\
54 \\
57 \\
60\end{array}$ & $\begin{array}{l}320 \\
390 \\
150 \\
290 \\
190\end{array}$ & $\begin{array}{l}49 \\
55 \\
15 \\
45 \\
31\end{array}$ \\
\hline $\begin{array}{l}16 \ldots \\
17 \ldots \\
18 \ldots \\
19 \ldots \\
20 .\end{array}$ & $\begin{array}{c}6.4 \\
6.4 \\
6.0 \\
10 \\
20\end{array}$ & $\begin{array}{r}20 \\
20 \\
20 \\
180 \\
600\end{array}$ & $\begin{array}{r}M \\
T \\
T \\
5 \\
33\end{array}$ & $\begin{array}{l}820 \\
820 \\
747 \\
494 \\
187\end{array}$ & $\begin{array}{r}1600 \\
1100 \\
860 \\
600 \\
830\end{array}$ & $\begin{array}{r}3600 \\
2400 \\
1700 \\
800 \\
420\end{array}$ & $\begin{array}{l}48 \\
50 \\
51 \\
52 \\
52\end{array}$ & $\begin{array}{l}220 \\
310 \\
250 \\
240 \\
220\end{array}$ & $\begin{array}{l}29 \\
42 \\
34 \\
34 \\
31\end{array}$ \\
\hline $\begin{array}{l}21 \ldots \\
22 \ldots \\
23 \ldots \\
24 \ldots \\
25 \ldots\end{array}$ & $\begin{array}{l}25 \\
39 \\
34 \\
44 \\
50\end{array}$ & $\begin{array}{r}360 \\
830 \\
990 \\
1000 \\
1100\end{array}$ & $\begin{array}{r}24 \\
87 \\
91 \\
120 \\
140\end{array}$ & $\begin{array}{r}116 \\
102 \\
92 \\
87 \\
82\end{array}$ & $\begin{array}{l}\mathbf{6 2 0} \\
\mathbf{5 5 0} \\
\mathbf{7 1 0} \\
430 \\
390\end{array}$ & $\begin{array}{r}190 \\
150 \\
180 \\
100 \\
86\end{array}$ & $\begin{array}{l}51 \\
50 \\
45 \\
47 \\
48\end{array}$ & $\begin{array}{r}190 \\
140 \\
70 \\
100 \\
160\end{array}$ & $\begin{array}{r}26 \\
19 \\
9 \\
13 \\
21\end{array}$ \\
\hline Total & \multicolumn{3}{|c|}{ JANUARY } & \multicolumn{3}{|c|}{ FEBRUARY } & \multicolumn{3}{|c|}{ MARCH } \\
\hline $\begin{array}{l}1 \ldots \\
2 \ldots \\
3 \ldots \\
4 \ldots \\
5 \ldots\end{array}$ & $\begin{array}{l}65 \\
65 \\
60 \\
55 \\
60\end{array}$ & $\begin{array}{r}140 \\
80 \\
90 \\
150 \\
140\end{array}$ & $\begin{array}{l}25 \\
14 \\
15 \\
22 \\
23\end{array}$ & $\begin{array}{l}60 \\
44 \\
45 \\
75 \\
65\end{array}$ & $\begin{array}{r}160 \\
60 \\
120 \\
160 \\
150\end{array}$ & $\begin{array}{r}26 \\
7 \\
17 \\
32 \\
26\end{array}$ & $\begin{array}{l}73 \\
85 \\
87 \\
85 \\
78\end{array}$ & $\begin{array}{r}80 \\
110 \\
160 \\
220 \\
80\end{array}$ & $\begin{array}{l}16 \\
25 \\
38 \\
50 \\
17\end{array}$ \\
\hline $\begin{array}{r}6 \ldots \\
7 \ldots \\
8 \ldots \\
9 \ldots \\
10 .\end{array}$ & $\begin{array}{l}58 \\
56 \\
54 \\
50 \\
50\end{array}$ & $\begin{array}{r}140 \\
100 \\
70 \\
70 \\
110\end{array}$ & $\begin{array}{r}22 \\
15 \\
10 \\
9 \\
15\end{array}$ & $\begin{array}{l}56 \\
54 \\
65 \\
70 \\
75\end{array}$ & $\begin{array}{r}80 \\
70 \\
240 \\
220 \\
220\end{array}$ & $\begin{array}{l}12 \\
10 \\
42 \\
42 \\
51\end{array}$ & $\begin{array}{l}80 \\
82 \\
92 \\
82 \\
87\end{array}$ & $\begin{array}{r}50 \\
70 \\
90 \\
140 \\
150\end{array}$ & $\begin{array}{l}11 \\
15 \\
22 \\
31 \\
35\end{array}$ \\
\hline $\begin{array}{l}11 \ldots \\
12 \ldots \\
13 \ldots \\
14 \ldots \\
15 \ldots\end{array}$ & $\begin{array}{l}50 \\
50 \\
45 \\
50 \\
55\end{array}$ & $\begin{array}{r}100 \\
100 \\
90 \\
70 \\
110\end{array}$ & $\begin{array}{r}14 \\
14 \\
11 \\
9 \\
16\end{array}$ & $\begin{array}{l}73 \\
80 \\
70 \\
66 \\
60\end{array}$ & $\begin{array}{r}150 \\
130 \\
90 \\
90 \\
120\end{array}$ & $\begin{array}{l}30 \\
28 \\
17 \\
16 \\
19\end{array}$ & $\begin{array}{l}82 \\
85 \\
85 \\
82 \\
82\end{array}$ & $\begin{array}{r}220 \\
110 \\
130 \\
70 \\
100\end{array}$ & $\begin{array}{l}49 \\
25 \\
30 \\
15 \\
22\end{array}$ \\
\hline $\begin{array}{l}16 \ldots \\
17 . \\
18 . \\
19 . \\
20 .\end{array}$ & $\begin{array}{l}65 \\
75 \\
90 \\
80 \\
61\end{array}$ & $\begin{array}{r}80 \\
110 \\
160 \\
250 \\
220\end{array}$ & $\begin{array}{l}14 \\
22 \\
39 \\
54 \\
36\end{array}$ & $\begin{array}{l}56 \\
56 \\
53 \\
58 \\
60\end{array}$ & $\begin{array}{r}110 \\
90 \\
70 \\
70 \\
70\end{array}$ & $\begin{array}{l}17 \\
14 \\
10 \\
11 \\
11\end{array}$ & $\begin{array}{r}85 \\
92 \\
95 \\
109 \\
119\end{array}$ & $\begin{array}{r}120 \\
100 \\
90 \\
100 \\
530\end{array}$ & $\begin{array}{r}28 \\
25 \\
23 \\
29 \\
170\end{array}$ \\
\hline $\begin{array}{l}21 \ldots \\
22 \ldots \\
23 \ldots \\
24 \ldots \\
25 \ldots\end{array}$ & $\begin{array}{l}63 \\
66 \\
66 \\
50 \\
50\end{array}$ & $\begin{array}{r}230 \\
170 \\
100 \\
70 \\
80\end{array}$ & $\begin{array}{l}39 \\
30 \\
18 \\
12 \\
14\end{array}$ & $\begin{array}{l}62 \\
60 \\
65 \\
66 \\
68\end{array}$ & $\begin{array}{l}90 \\
90 \\
70 \\
70 \\
60\end{array}$ & $\begin{array}{l}15 \\
15 \\
12 \\
12 \\
11\end{array}$ & $\begin{array}{l}119 \\
131 \\
145 \\
150 \\
174\end{array}$ & $\begin{array}{r}1600 \\
1800 \\
680 \\
710 \\
720\end{array}$ & $\begin{array}{l}530 \\
630 \\
270 \\
290 \\
340\end{array}$ \\
\hline
\end{tabular}

S Computed by subdividing day.

K Computed from estirated-concentration

$T$ Less than 0.50 ton. graph and subdividing day.

A Computed from partiy estimated-concentration graph. M Less than 0.50 ton, computed from esti-

B Computed from estimated concentration graph.

$J$ Computed from partly estimated-concentration

graph and subdividing day. 
RIO GRANDE BASIN--Continued

8-2900. RIO CHAMA NEAR CHAMITA, N. MEX.--Continued

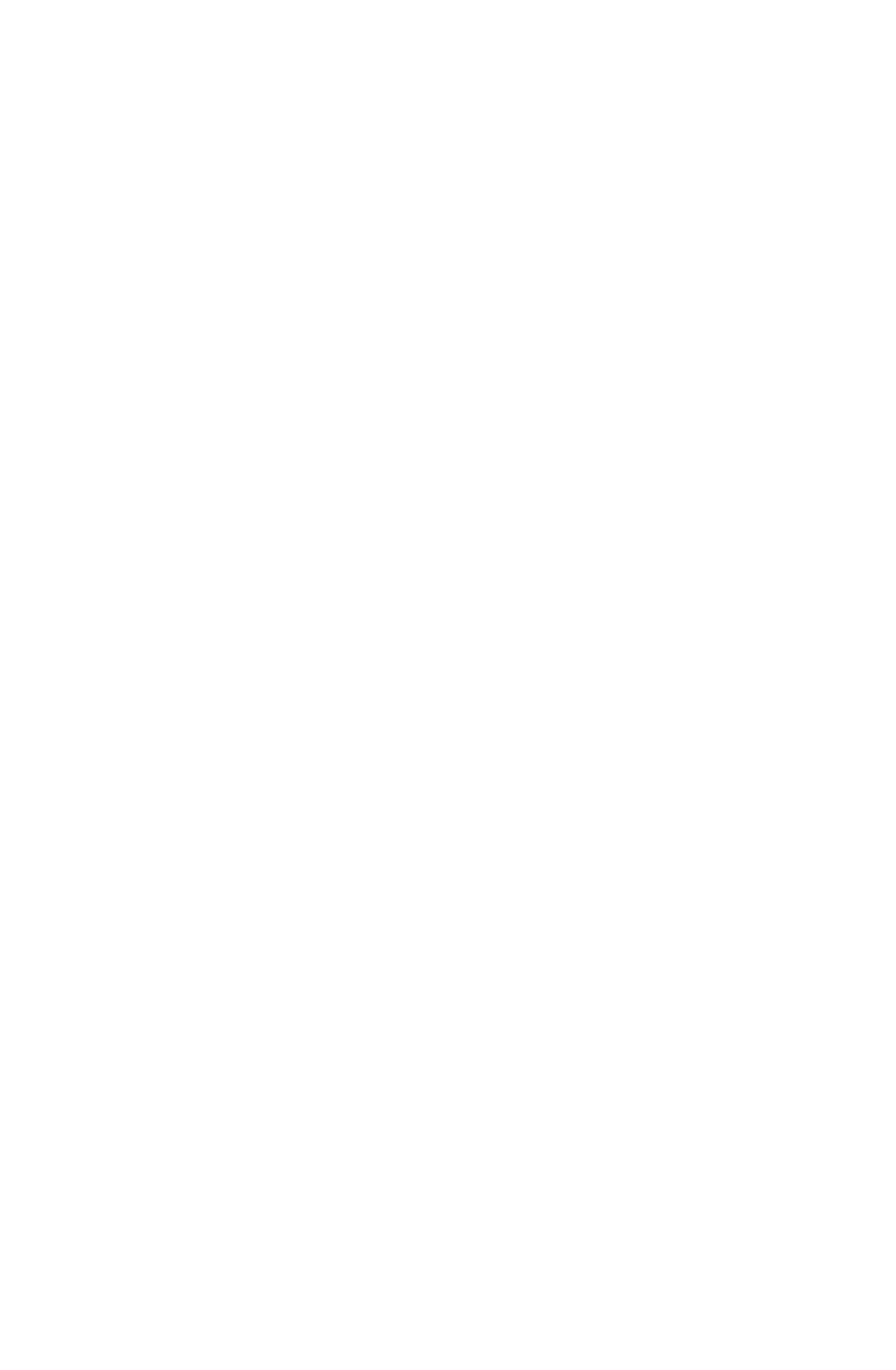

E Estimated.

$S$ Computed by subdividing day.

$T$ Less than 0.50 ton.

A Computed from partiy estimated-concentration graph.

B Computed from estimated-concentration graph.
J Computed from partly estimated-concentration graph and subdividing day.

$K$ Computed from estimated-concentration graph and subdividing day.

M Less than 0.50 ton, computed from estimated-concentration graph. 


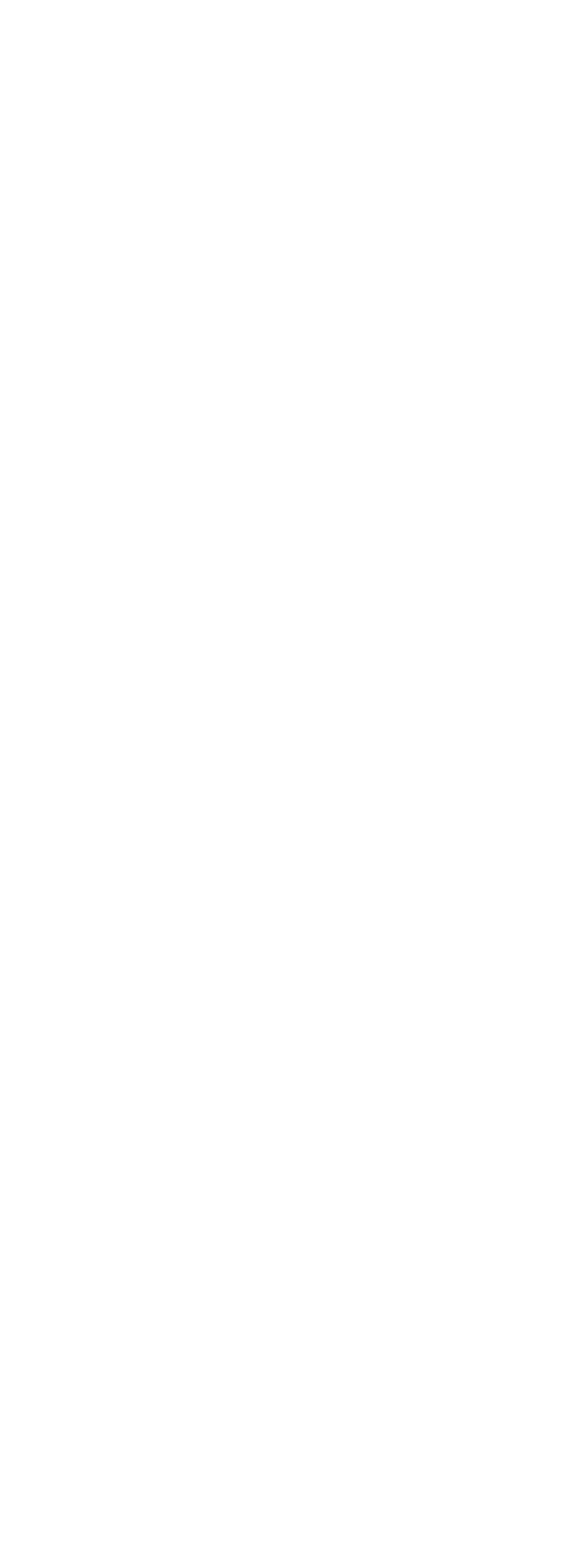




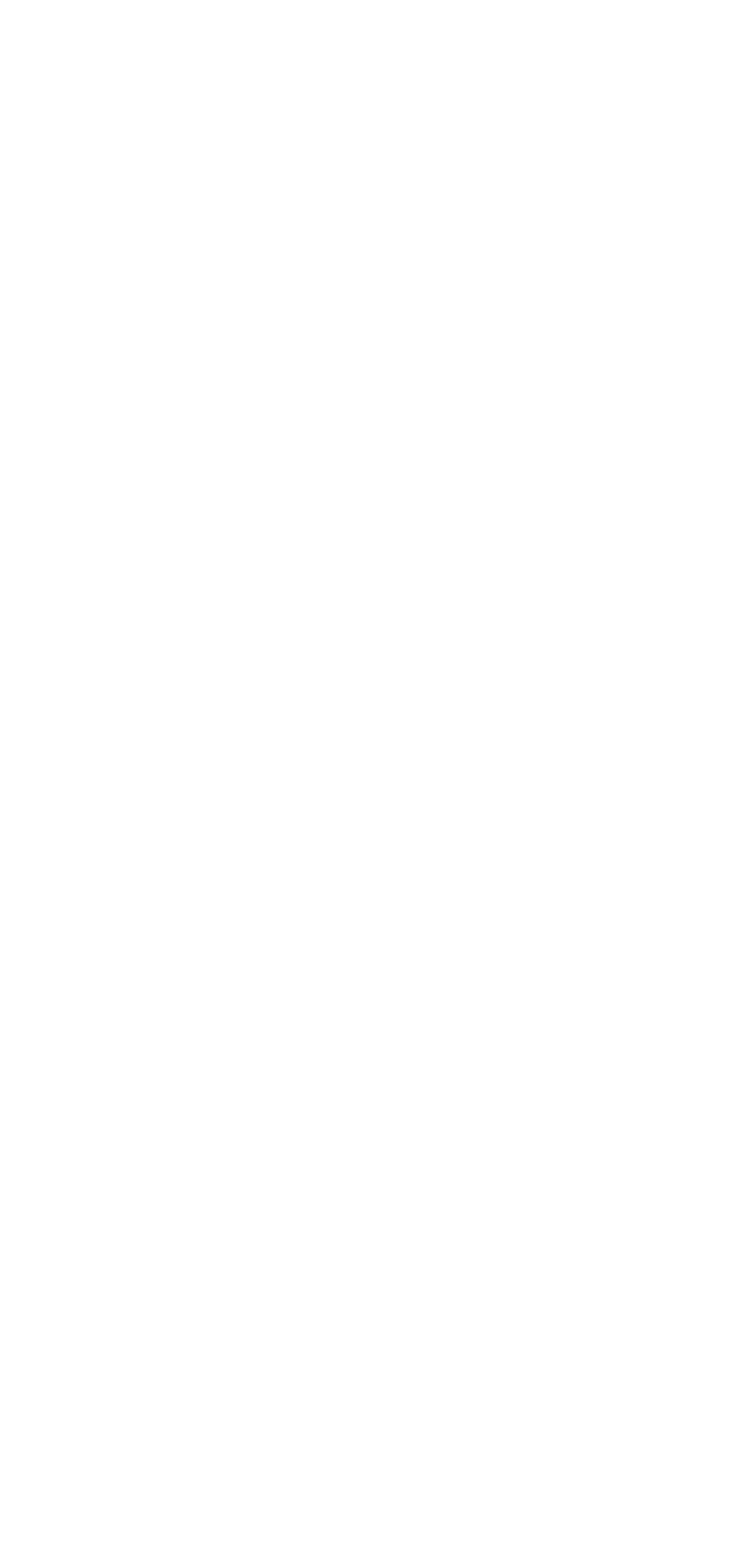


on



吕

政

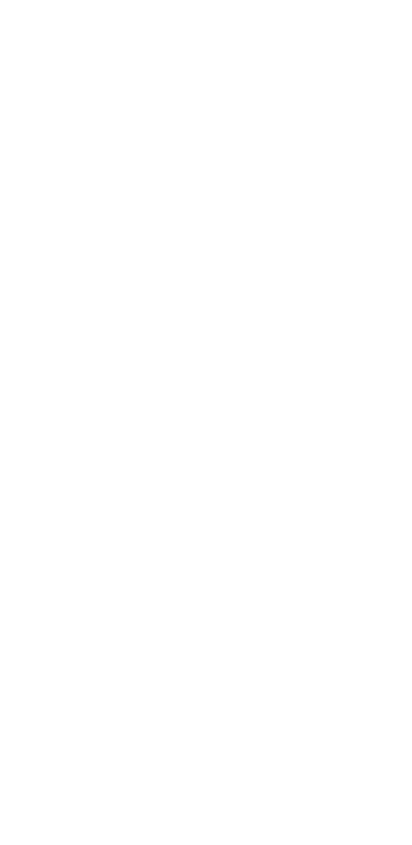

\begin{tabular}{|c|c|c|}
\hline & : & 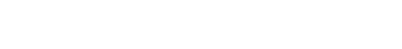 \\
\hline & 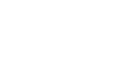 & 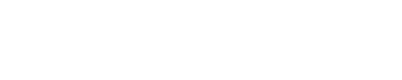 \\
\hline & 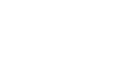 & 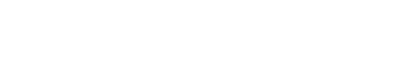 \\
\hline 屯 & 这客娄 & 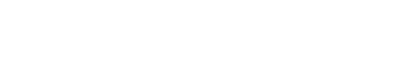 \\
\hline 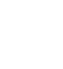 & 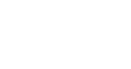 & 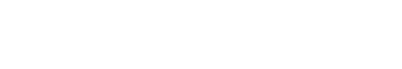 \\
\hline 8 & 总宫票 & 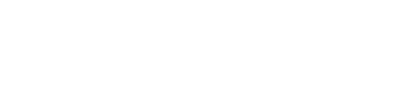 \\
\hline (1) & 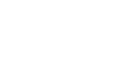 & 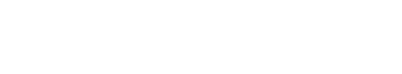 \\
\hline 要 & 票台范 & 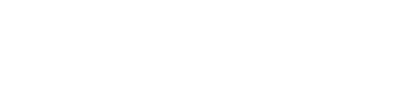 \\
\hline & 宮号苗 & \\
\hline & 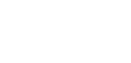 & 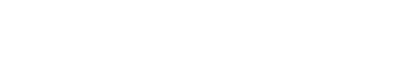 \\
\hline & 高要画 & 1111119011119091111 \\
\hline & 형을 & 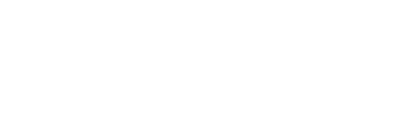 \\
\hline & 葠递 & 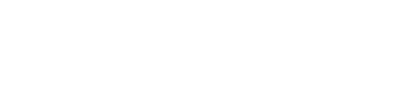 \\
\hline & ชู่ & \\
\hline & 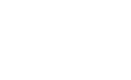 & 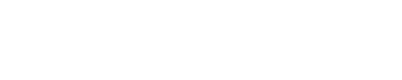 \\
\hline & 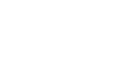 & 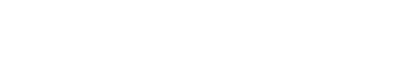 \\
\hline & 费夶 & 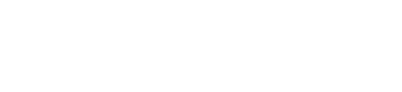 \\
\hline & 运要要要 & 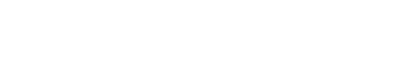 \\
\hline & 光慁焉 & 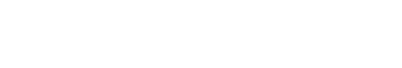 \\
\hline & 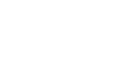 & \\
\hline & 娄莌 & 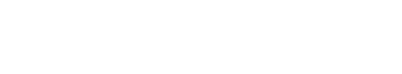 \\
\hline & 害总 & 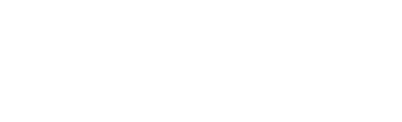 \\
\hline & 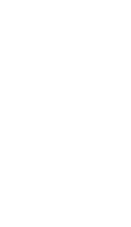 & 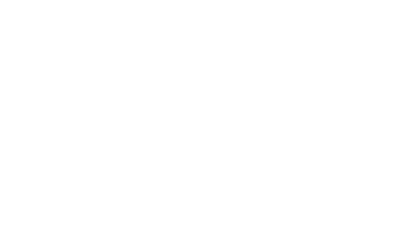 \\
\hline
\end{tabular}




\begin{tabular}{|c|c|c|c|c|c|}
\hline 舟舟品: & 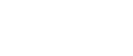 & 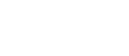 & $\stackrel{9}{\therefore}$ & $\stackrel{q}{2}$ & \\
\hline 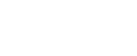 & 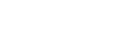 & 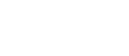 & $\underset{N}{N}$ & $\stackrel{m}{\circ}$ & \\
\hline 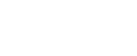 & $\ddot{4} \div$ & ๑9:9:०० & $\infty$ & $\stackrel{?}{0}$ & \\
\hline 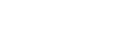 & 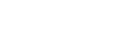 & 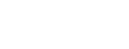 & न & 9 & ! \\
\hline 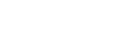 & 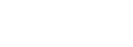 & 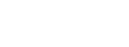 & $\underset{-1}{7}$ & $\stackrel{\infty}{\sim}$ & 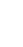 \\
\hline 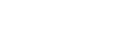 & 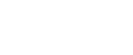 & 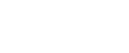 & 总 & i & 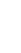 \\
\hline 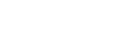 & 屯ี. & 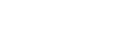 & m & 1 & 1 \\
\hline 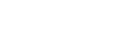 & 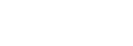 & 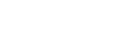 & ปู & กิ & i \\
\hline 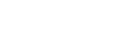 & றด̣ & ๑ด : & $\because$ & $\ddot{0}$ & 1 \\
\hline 111911 & 111111 & 111111 & 1 & 1 & i \\
\hline ח̊ & 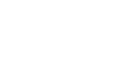 & 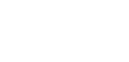 & $\stackrel{0}{0}$ & $\stackrel{m}{i}$ & a \\
\hline 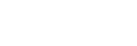 & Шే: & 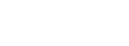 & 8 & 8 & $\begin{array}{ll}10 \\
\infty\end{array}$ \\
\hline 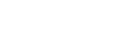 & స్జ & 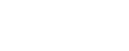 & 疍 & ๘్త & $\frac{1}{2}$ \\
\hline $\begin{array}{lllllll}1 & 1 & 1 & 1 & 1 & 1\end{array}$ & 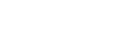 & $1911: 11$ & 1 & $!$ & 1 \\
\hline ํㅠㅁำ & 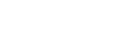 & 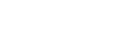 & Aี & i & m \\
\hline 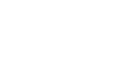 & 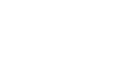 & $\because \stackrel{\infty}{\infty}:$ & $\stackrel{0}{i}$ & $\dddot{?}$ & 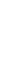 \\
\hline எే & 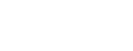 & 영영영ㅁㅇㅇ & 4 & $\mathscr{f}$ & 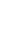 \\
\hline 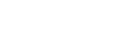 & กสุ & 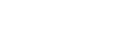 & สี & กั & m \\
\hline 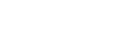 & 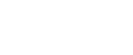 & 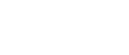 & 1 & $\stackrel{\infty}{\text { ஸ }}$ & 1 \\
\hline ลั & pi & 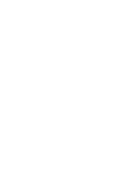 & 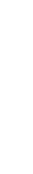 & 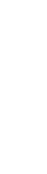 & $\mid$ \\
\hline 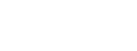 & 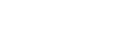 & 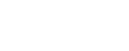 & 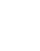 & & \\
\hline
\end{tabular}




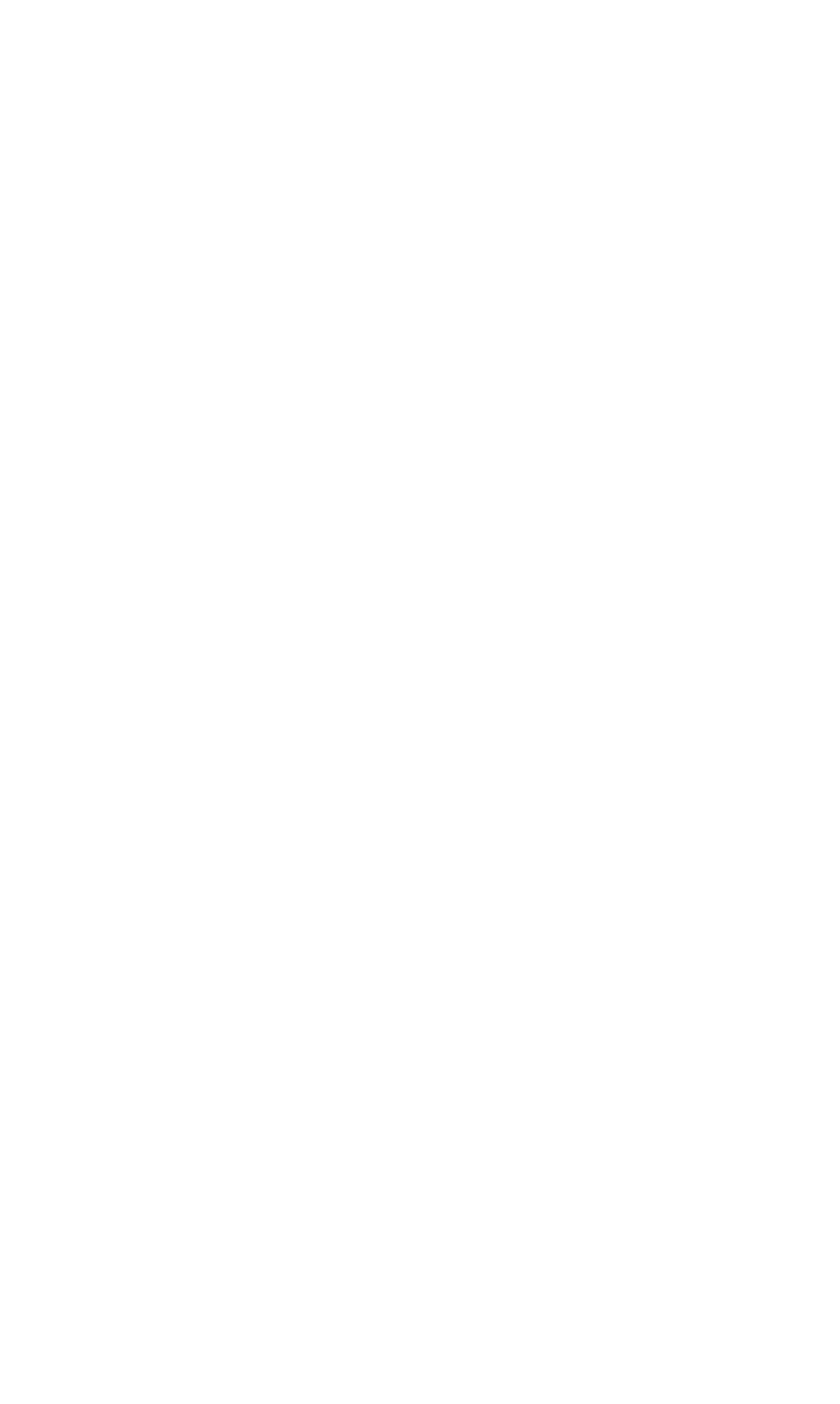


RIO GRANDE BASIN--Continued

8-3130. RIO GRANDE AT OTOWI BRIDGE, NEAR SAN ILDEFONSO, N. MEX.--Continued Suspended Sediment, water year October 1963 to September 1964

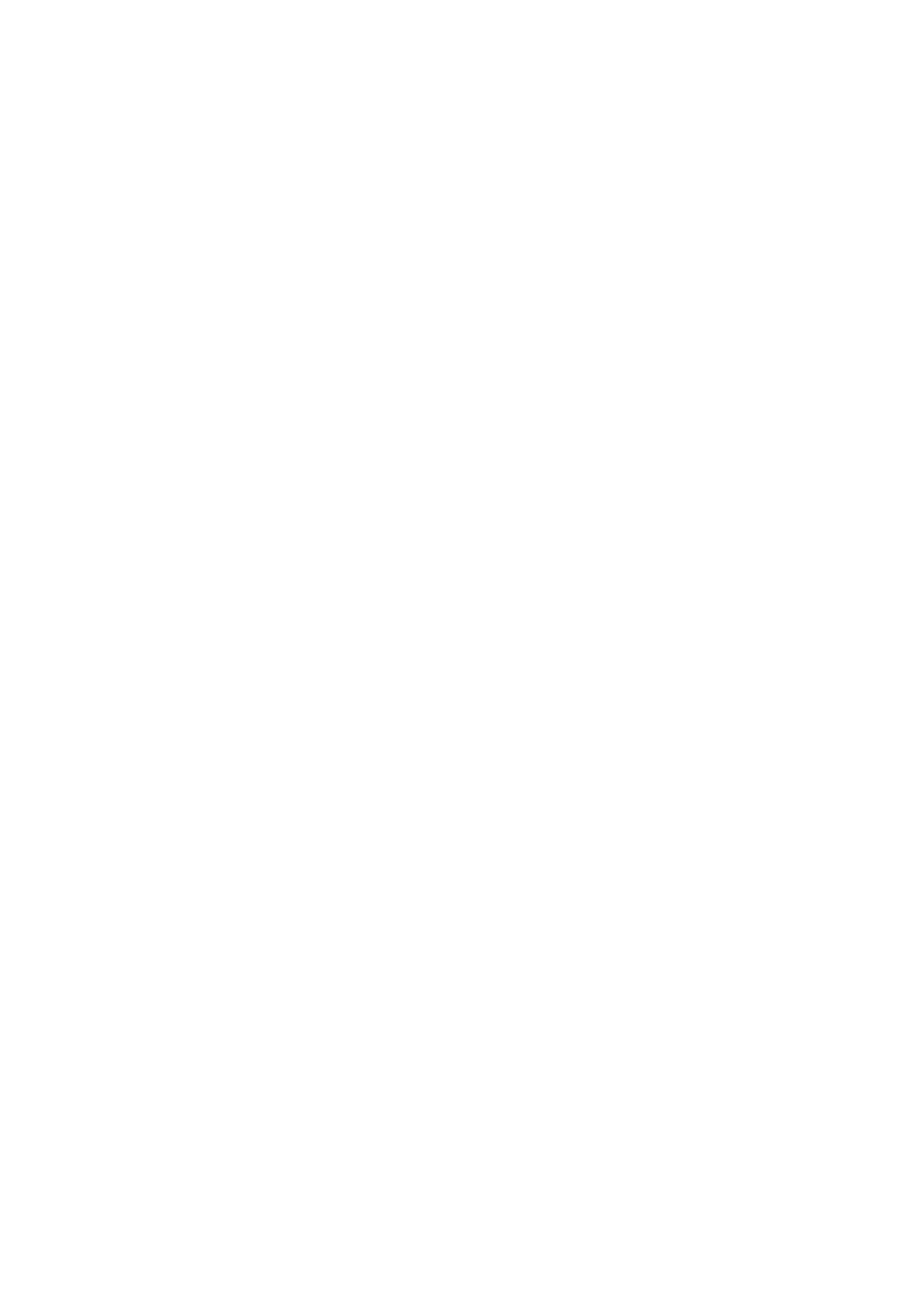

s Computed by subdividing day.

B Computed from estimated-concentration graph. 
RIO GRANDE BASIN--Continued

8-3130. RIO GRANDE AT OTOWI BRIDGE, NEAR SAN ILDEFONSO, N. MEX.--Continued

Suspended sediment, water year October 1963 to September 1964--Continued

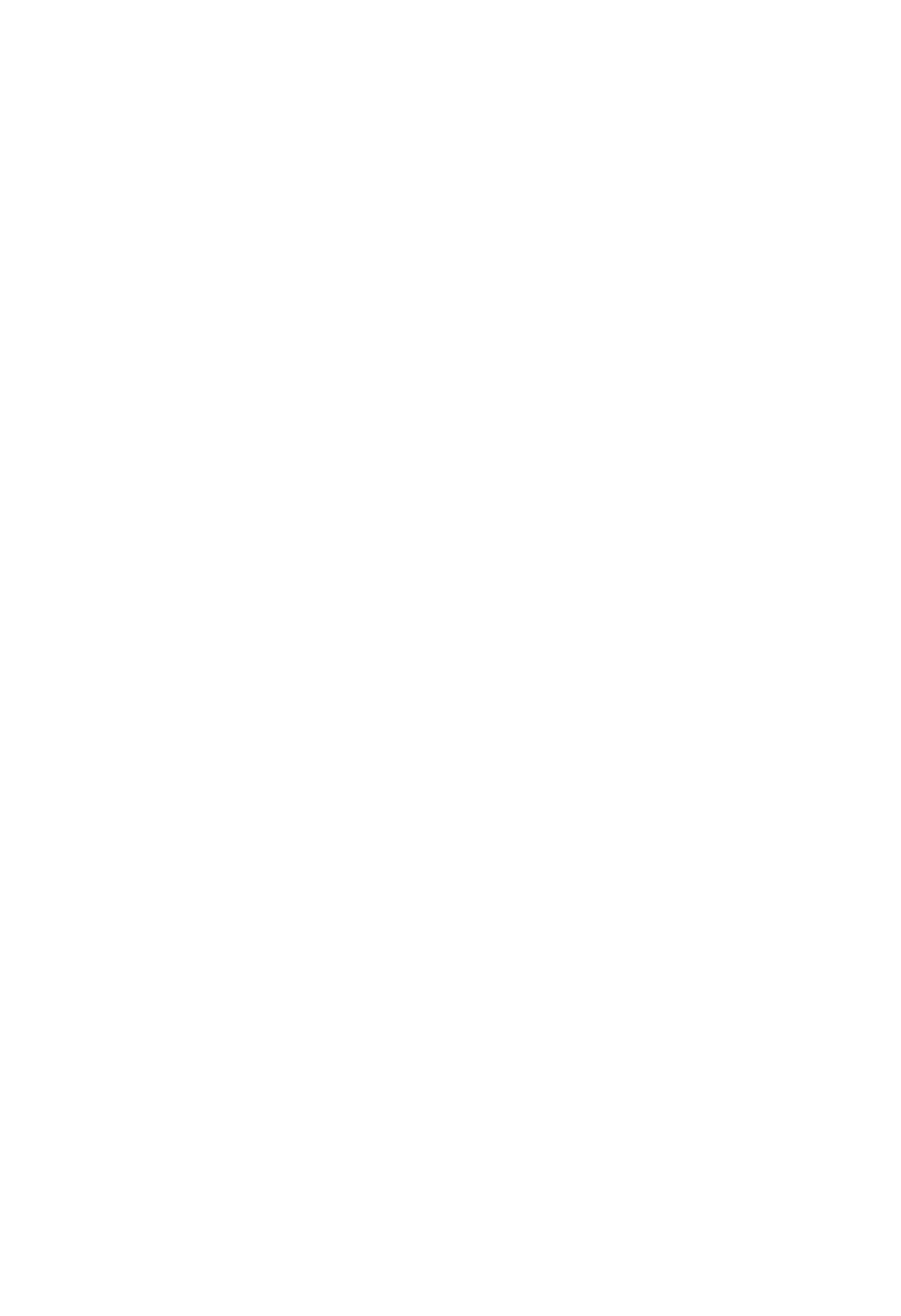

s Computed by subdividing day.

C Composite period. 


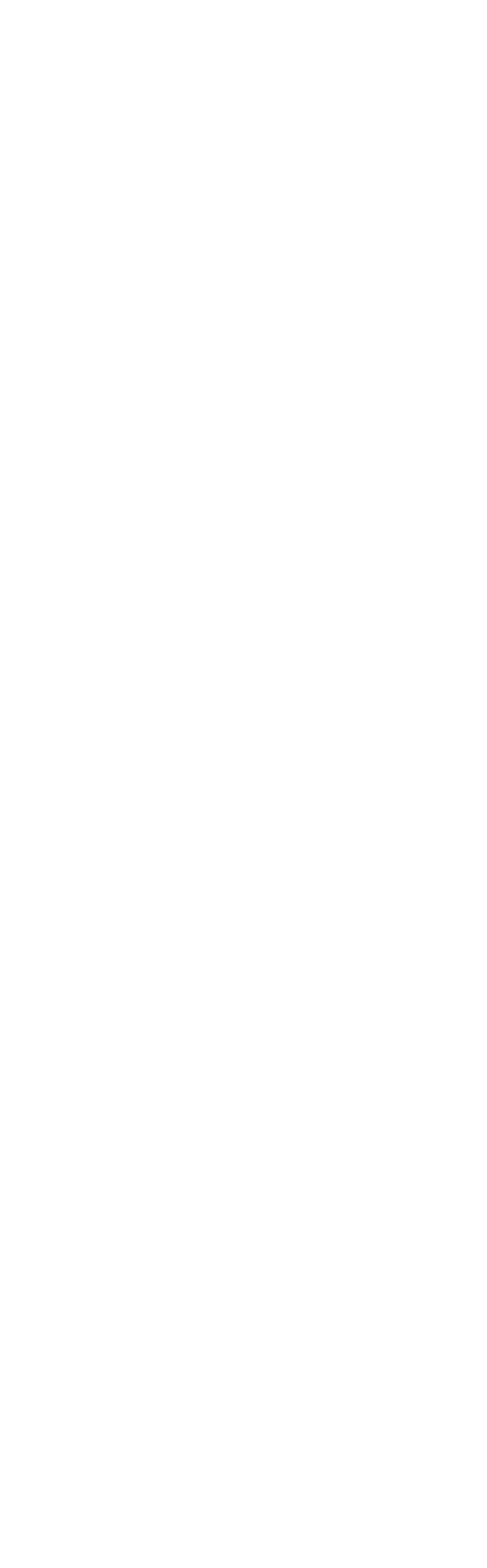




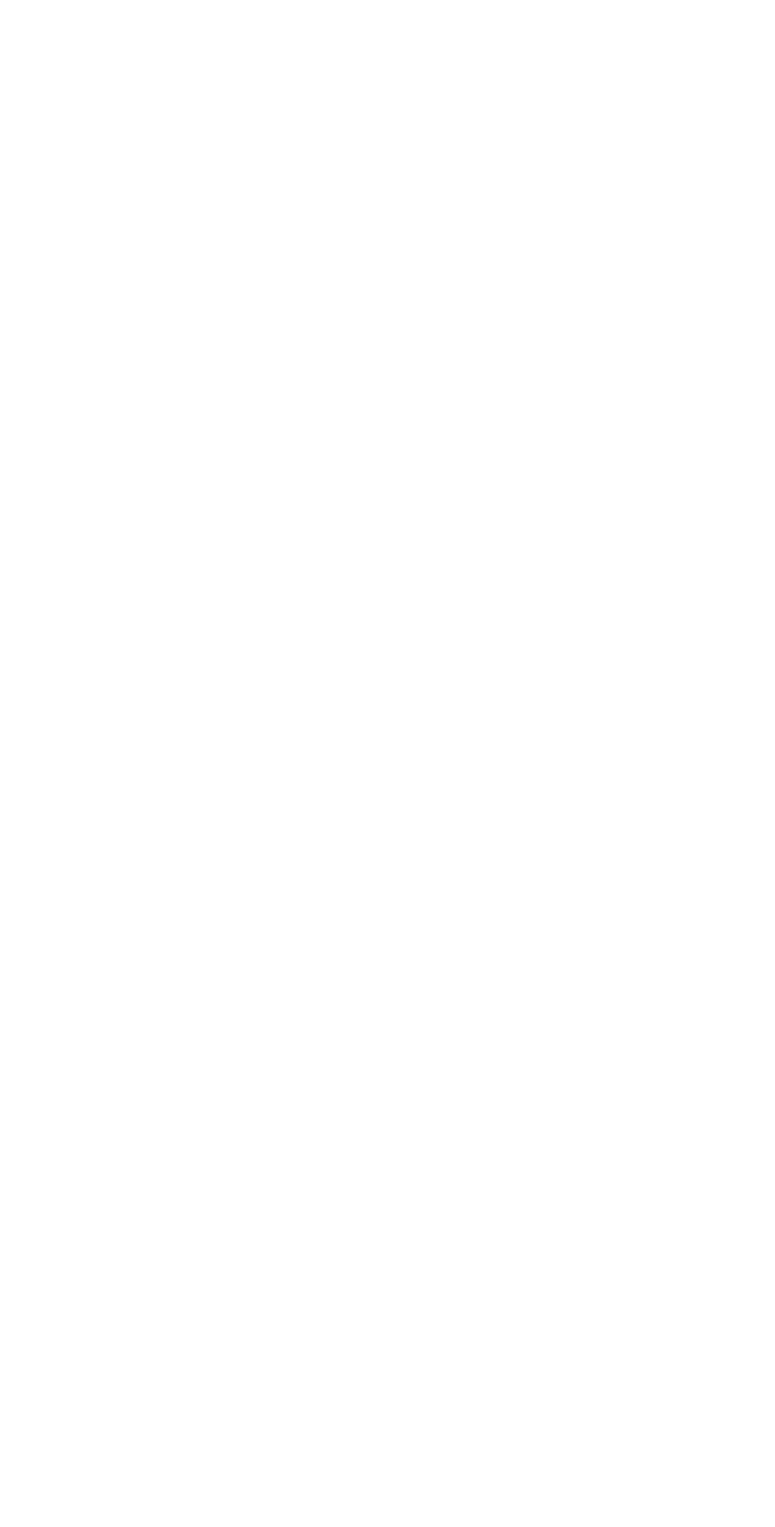


RIO GRANDE BASIN--Continued

8-3180. GALISTEO CREEK AT DOMINGO, N. MEX.--Continued

Suspended sediment, water year October 1963 to September 1964

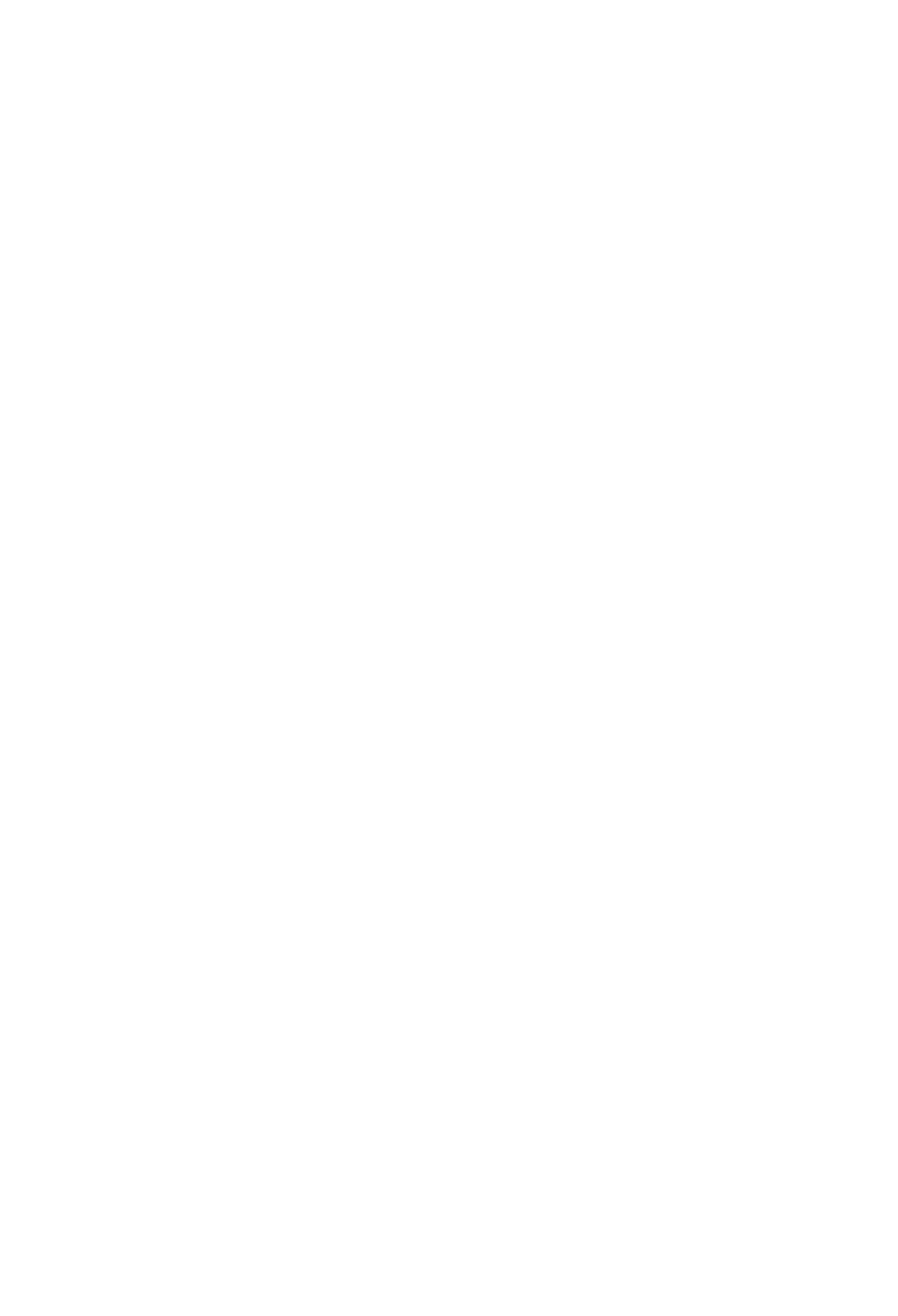

$S$ Computed by subdividing day.

T Less than 0.50 ton.

C Composite period. 
RIO GRANDE BASIN--Continued

8-3180. GALISTEO CREEK AT DOMINGO, N. MEX.--Continued

Suspended sediment, water year october 1963 to September 1964--Continued

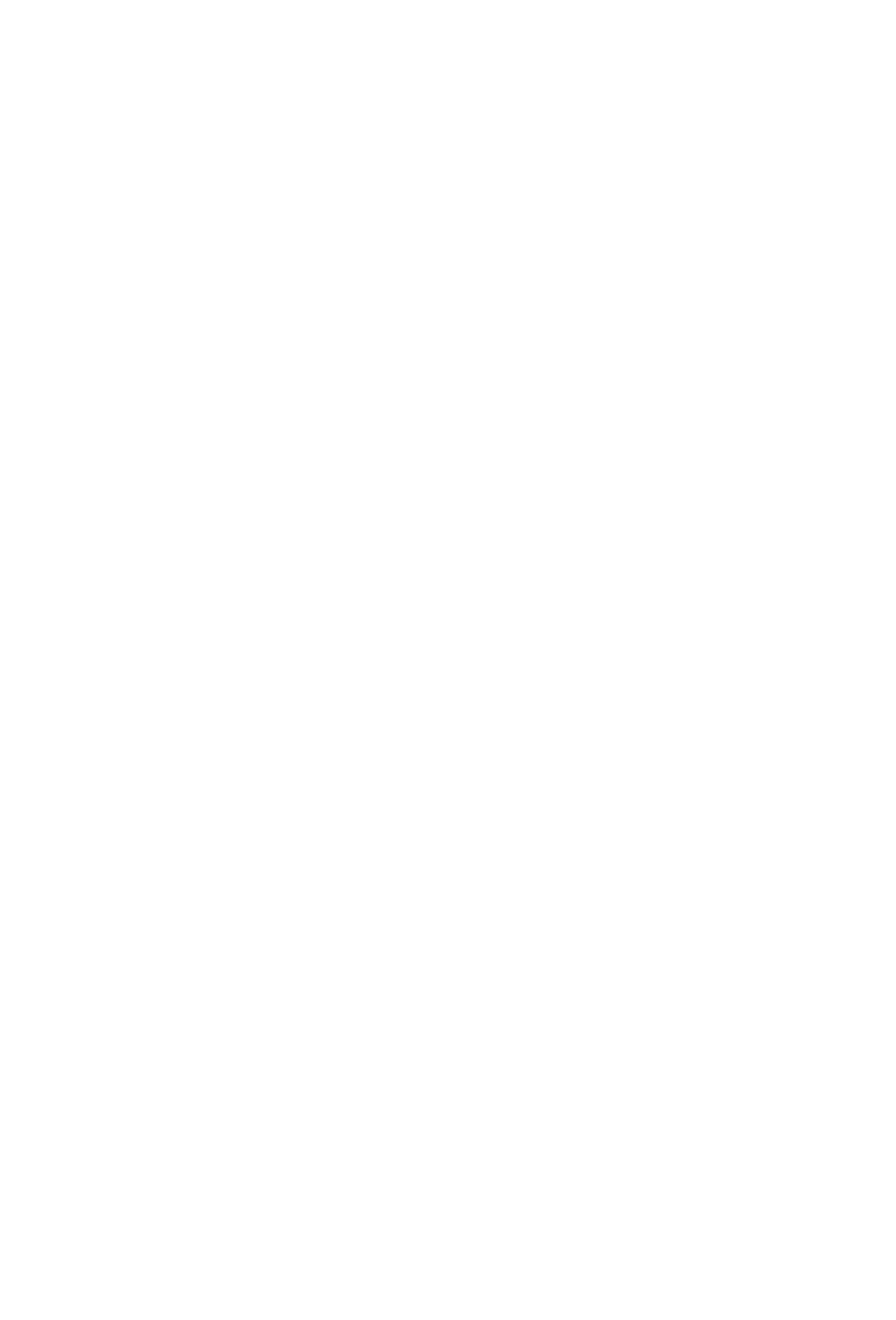

$S$ Computed by subdividing day.

$\mathrm{T}$ Less than 0.50 ton. 


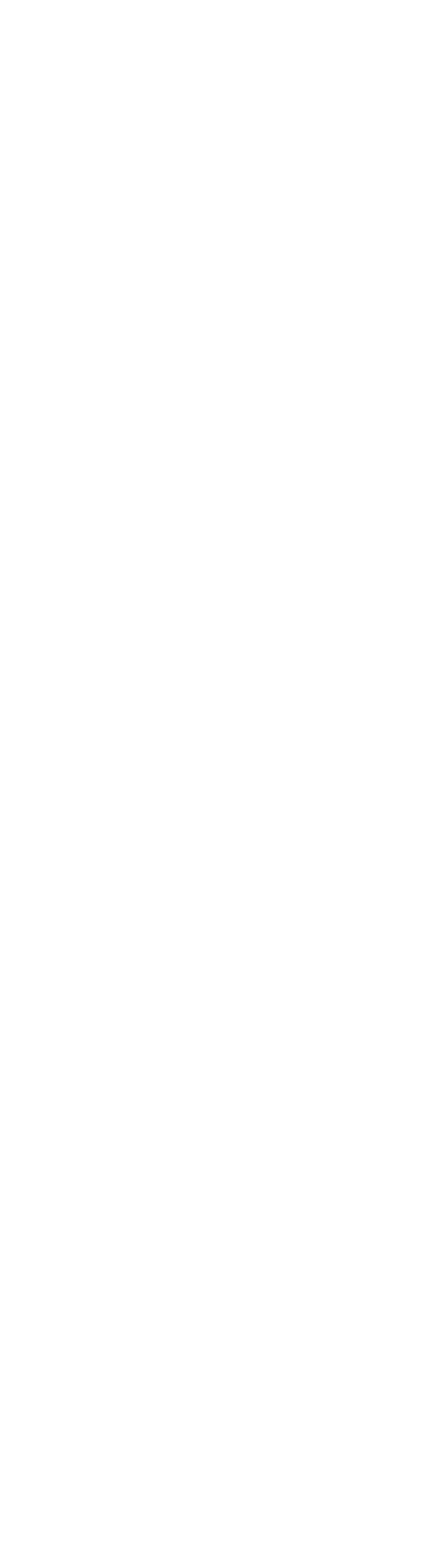




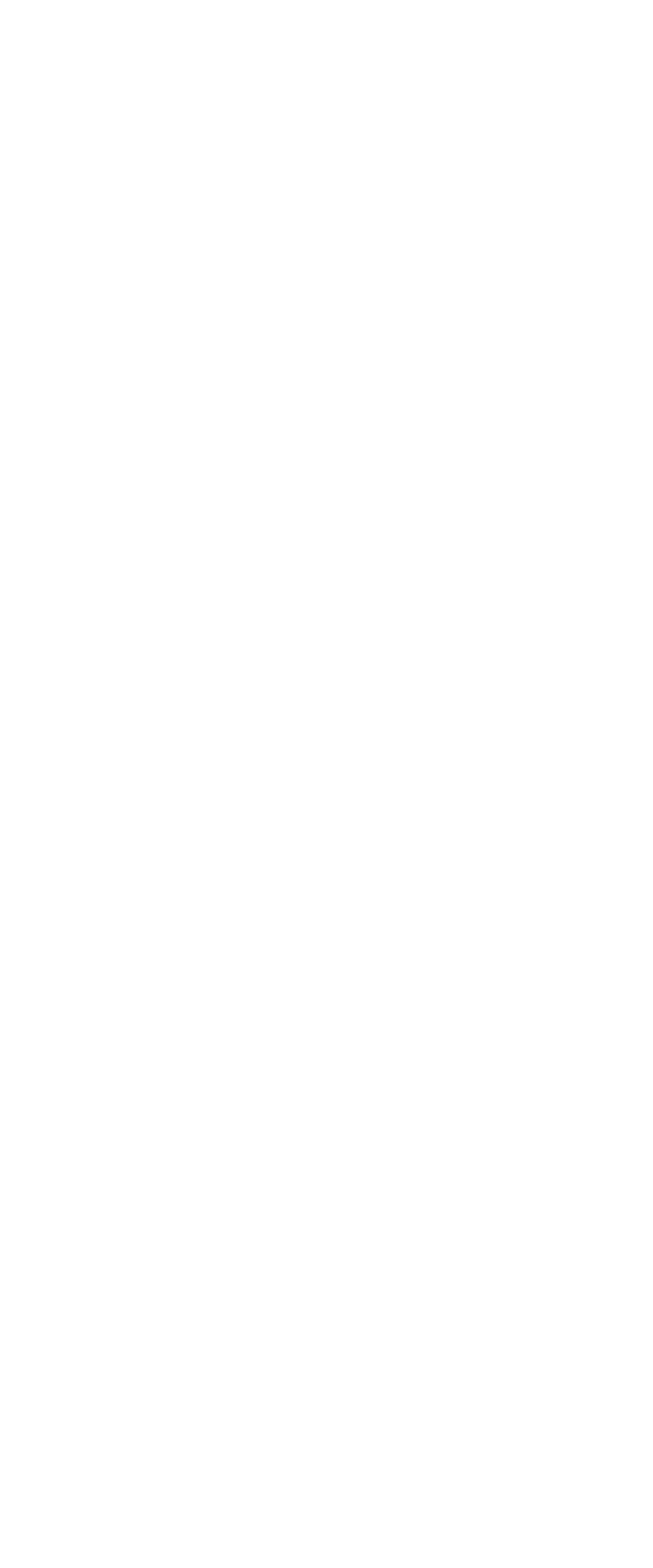




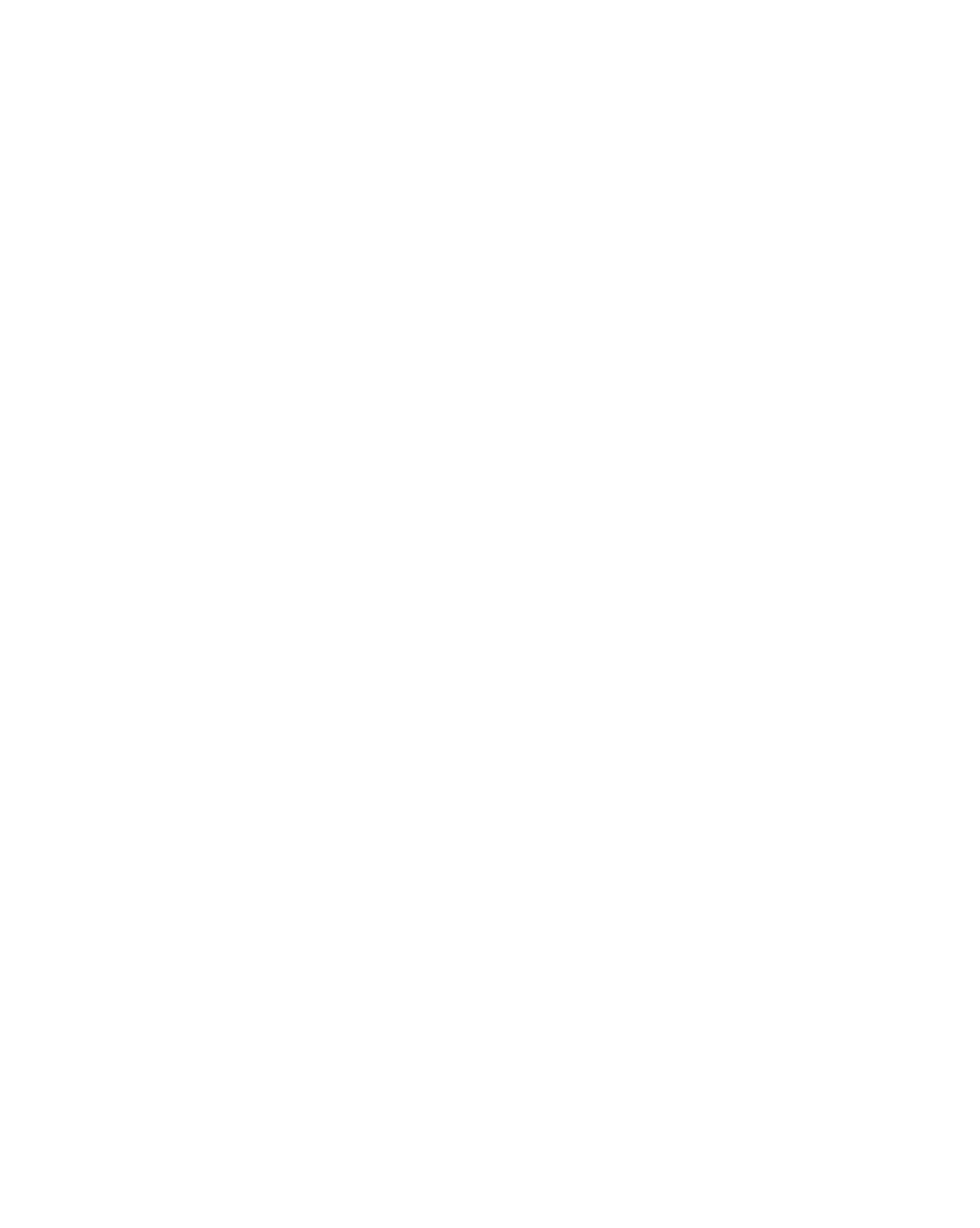



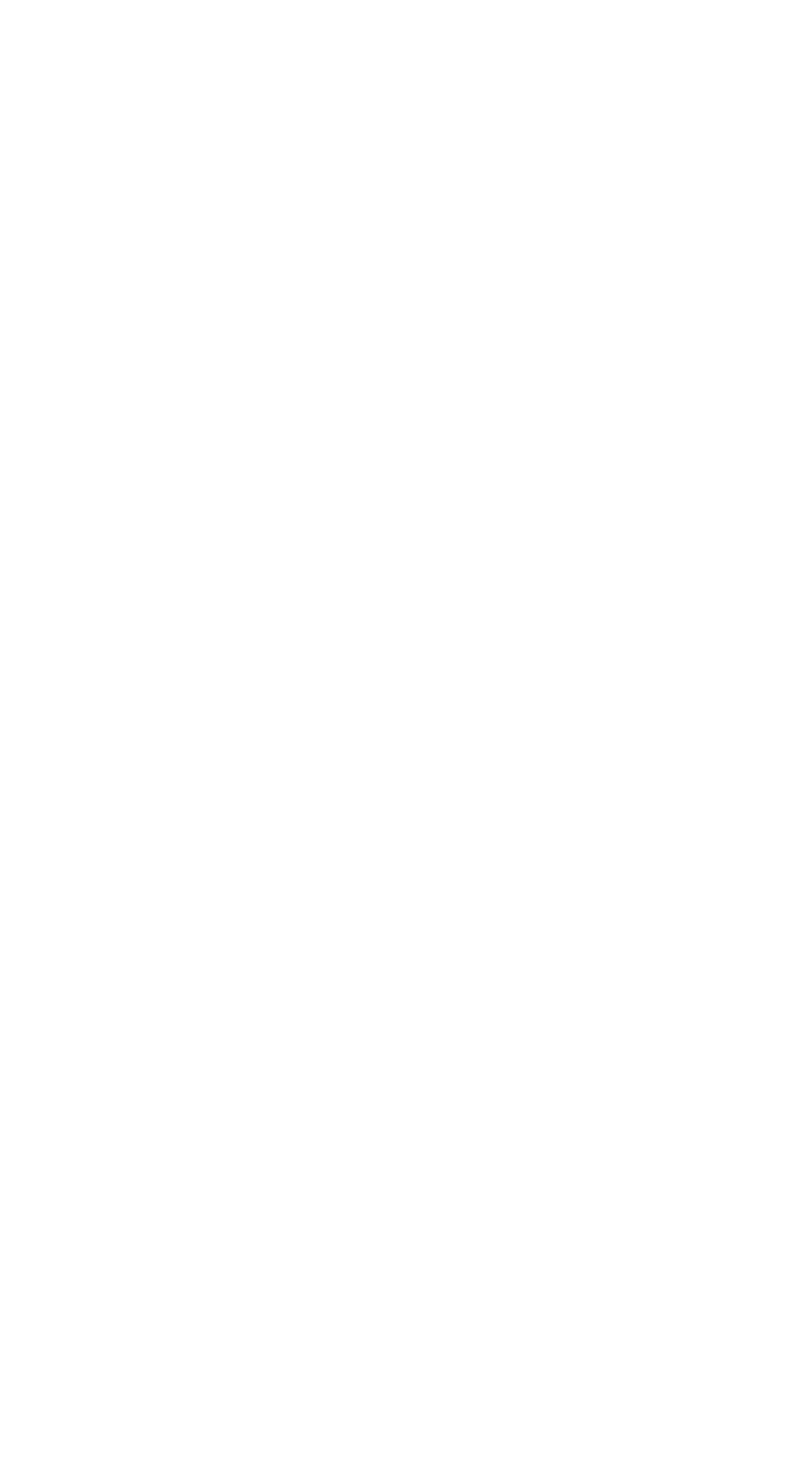
RIO GRANDE BASIN--Continued

8-3295. RIO GRANDE NEAR BERNALILLO, N. MEX,--Continued

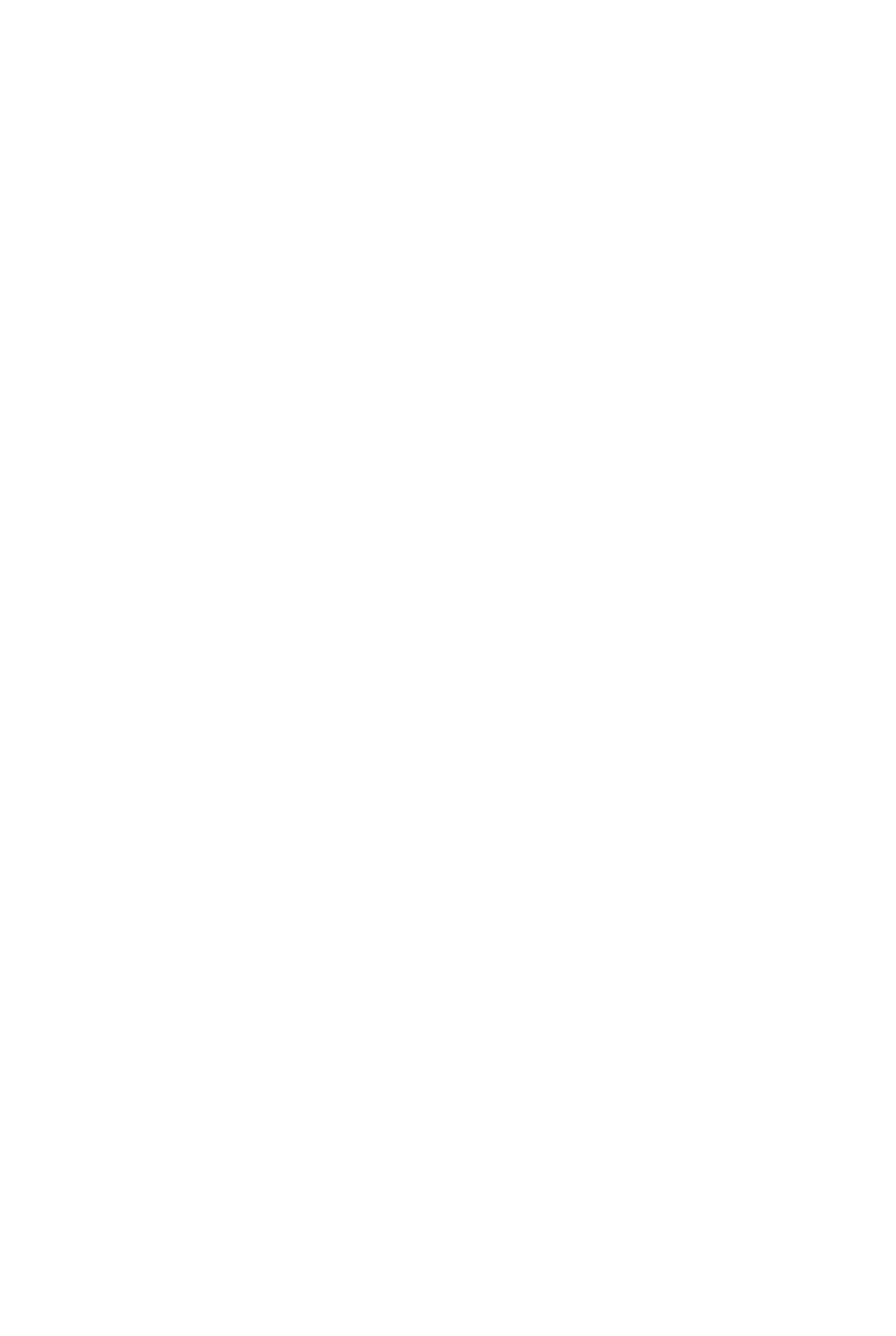

E Estimated.

S Computed by subdividing day.

B Computed from estimated-concentration graph. 
RIO GRANDE BASIN--Continued

8-3295. RIO GRANDE NEAR BERNALILLO, N. MEX.--Continued

Suspended sediment, water year October 1963 to September 1964--Continued

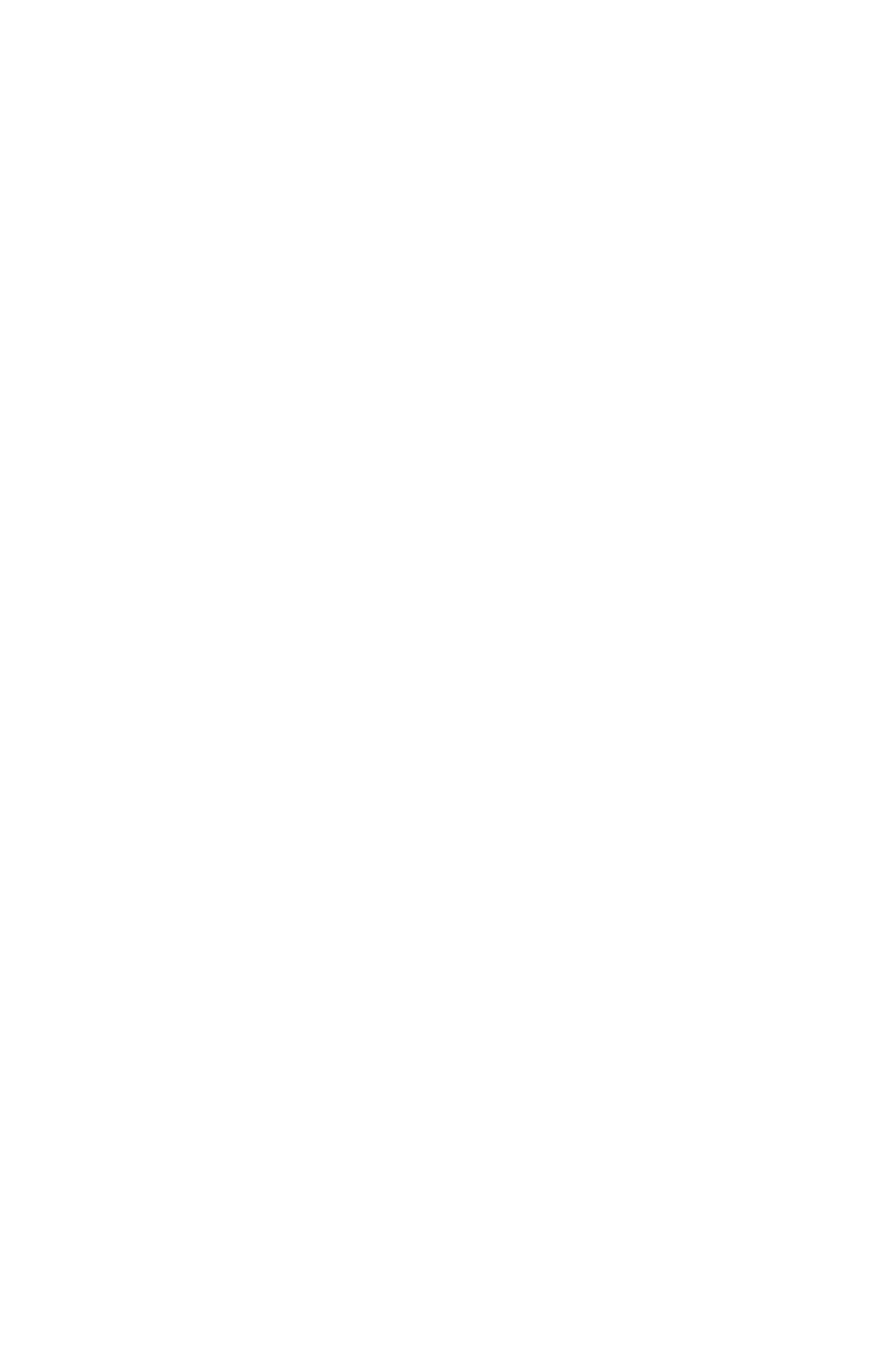

E Estimated.

$S$ Computed by subdividing day.

B Computed from estimated-concentration graph.

$K$ Computed from estimated-concentration graph and subdividing day. 


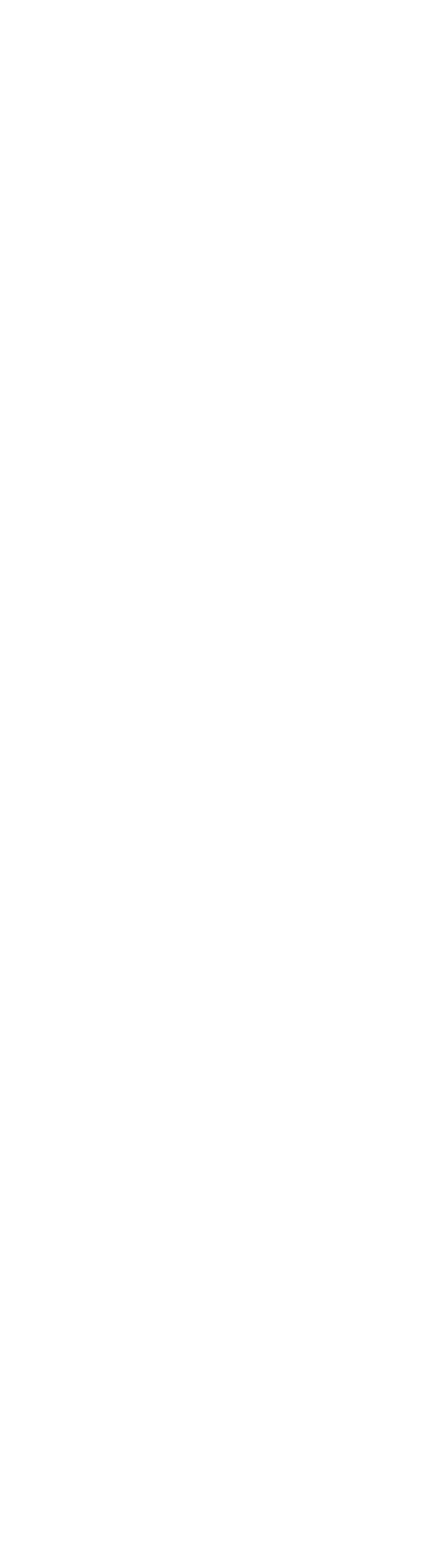




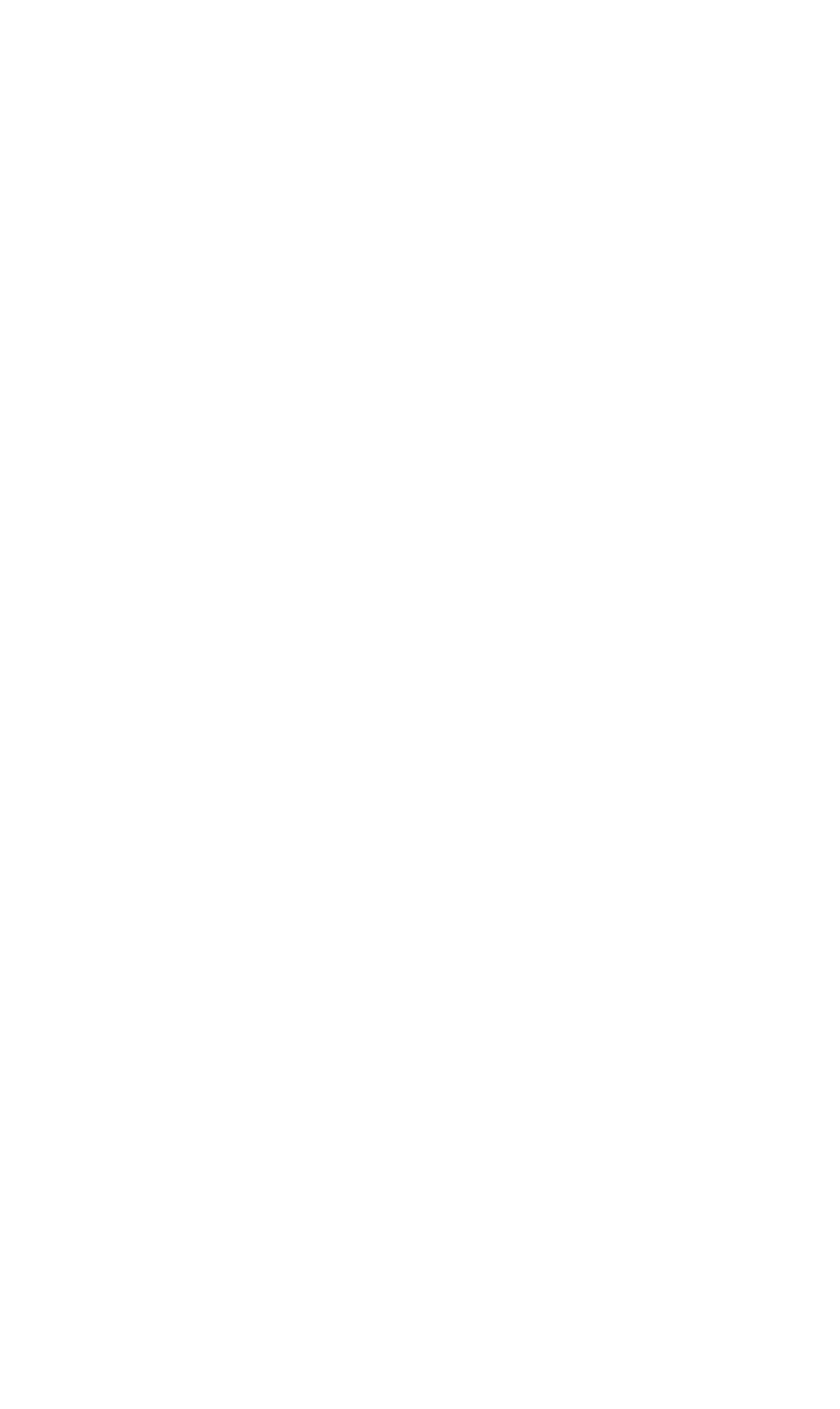




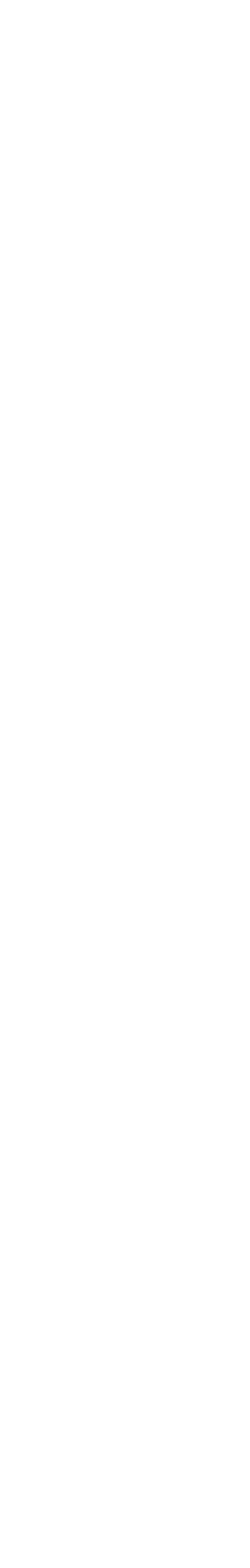




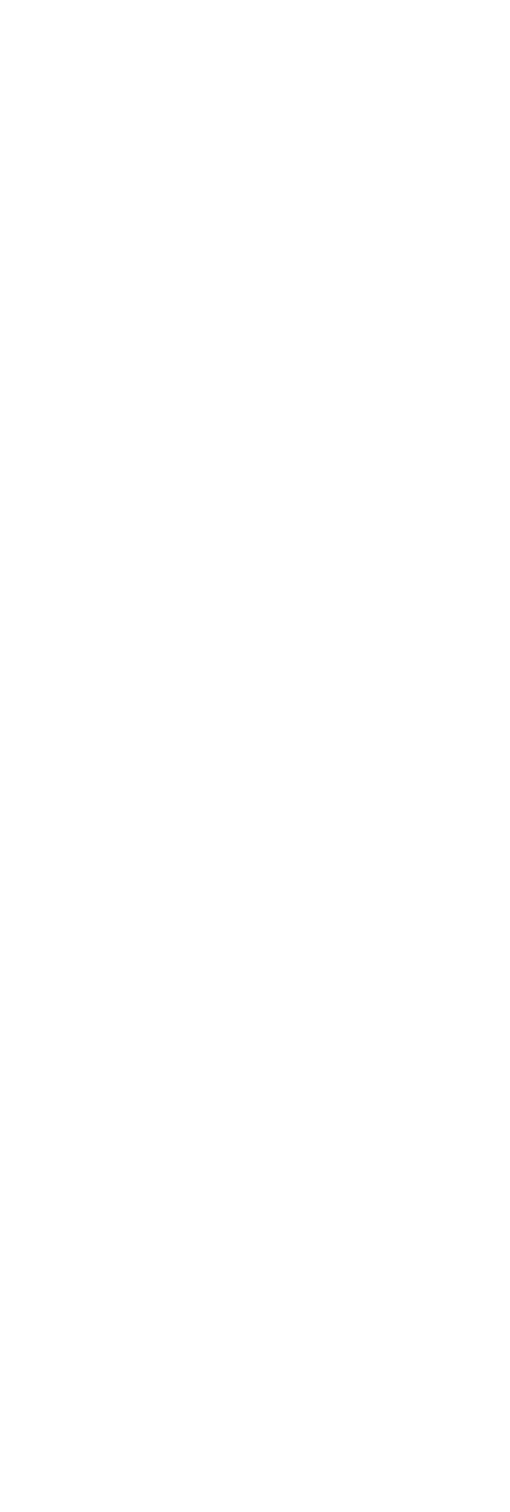


RIO GRANDE BASIN--Continued

8-3320. RIO GRANDE NEAR BERNARDO, N. MEX.--Continued

Suspended sediment, water year October 1963 to September 1964

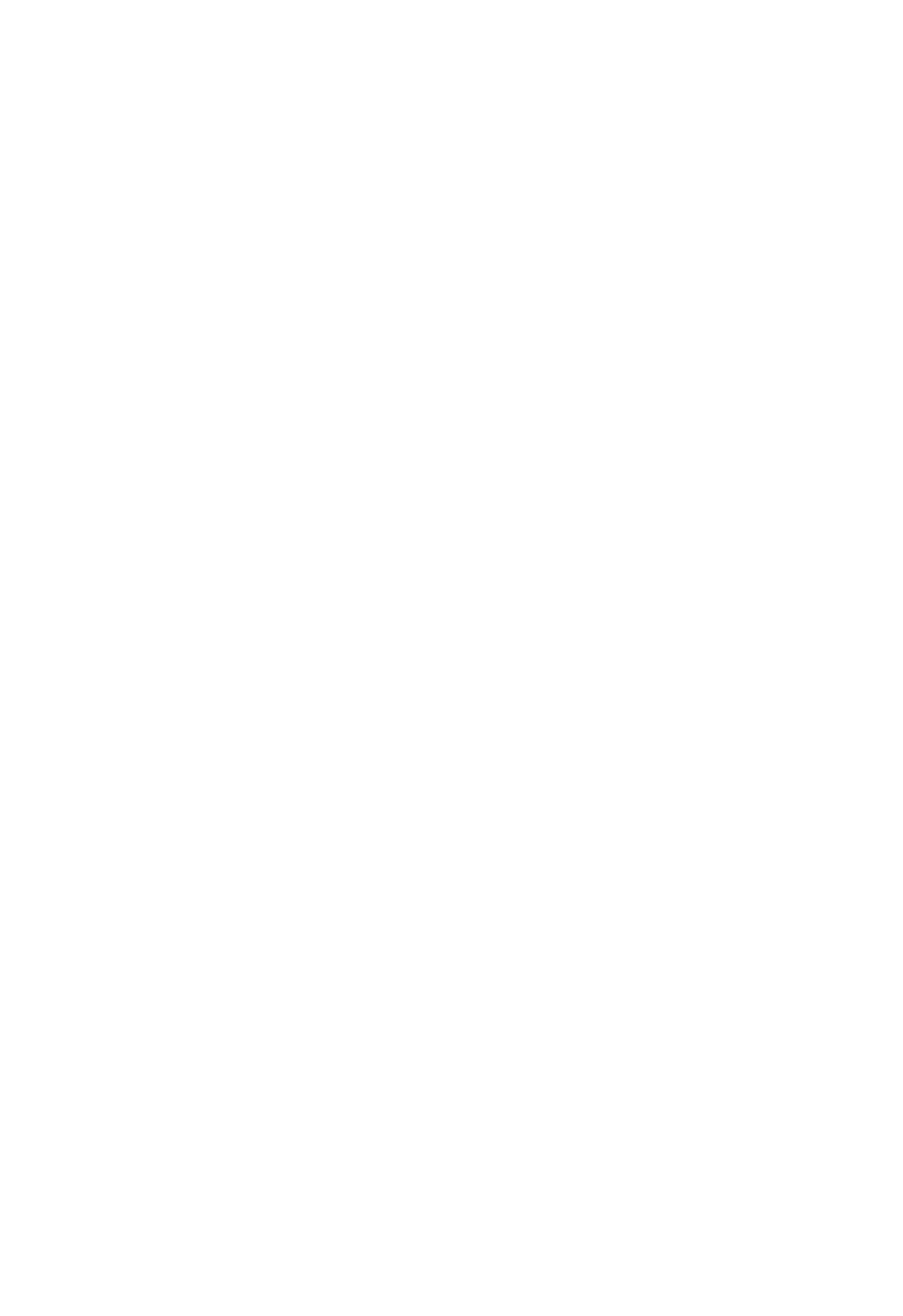

S Computed by subdividing day.

B Computed from estimated-concentration graph. 
RIO GRANDE BASIN--Continued

8-3320. RIO GRANDE NEAR BERNARDO, N. MEX.--Continued

Suspended sediment, water year October 1963 to September 1964--Continued

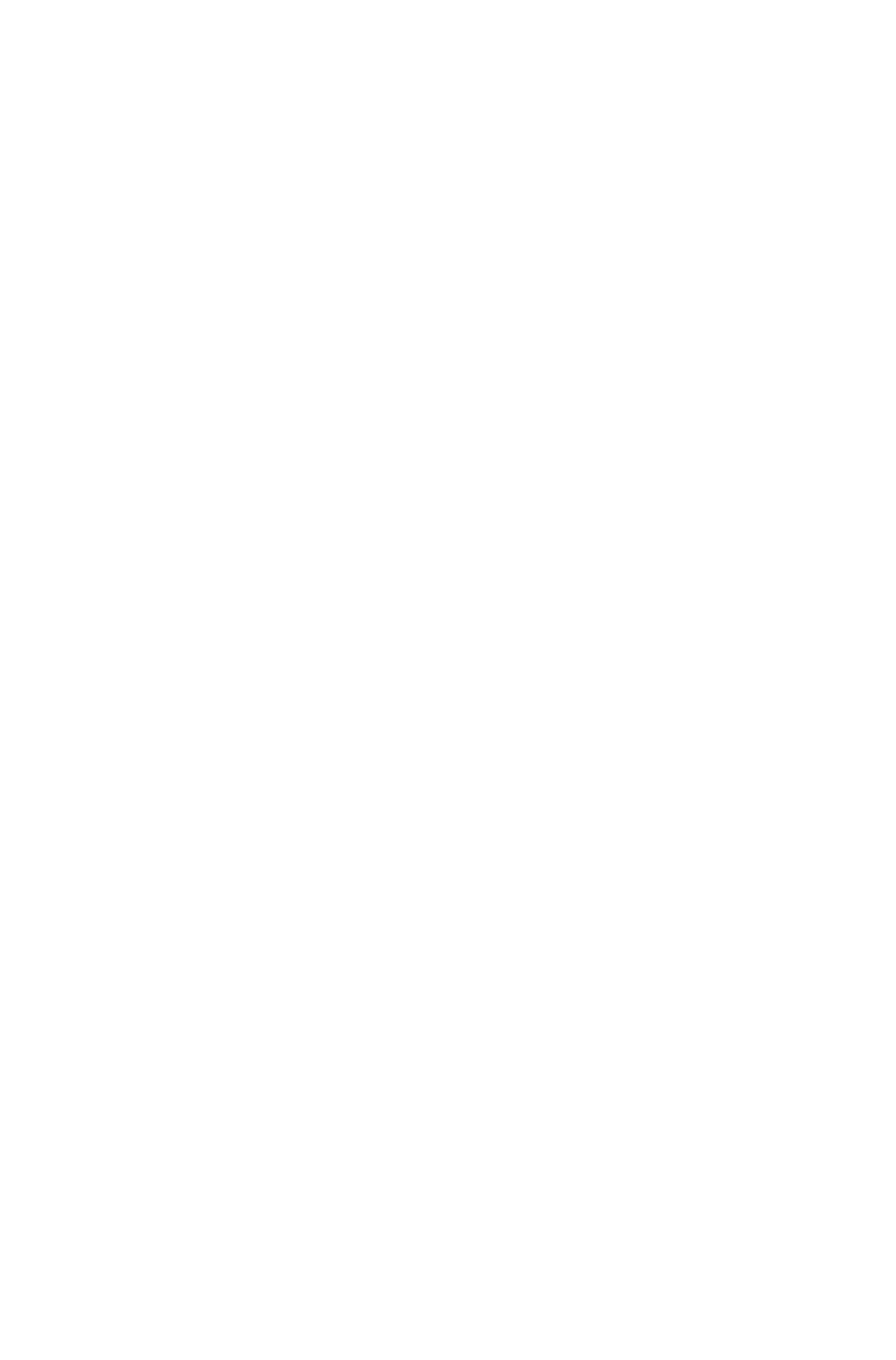

S Computed by subdividing day.

$T$ Less than 0.50 ton.

B Computed from estimated-concentration graph. 


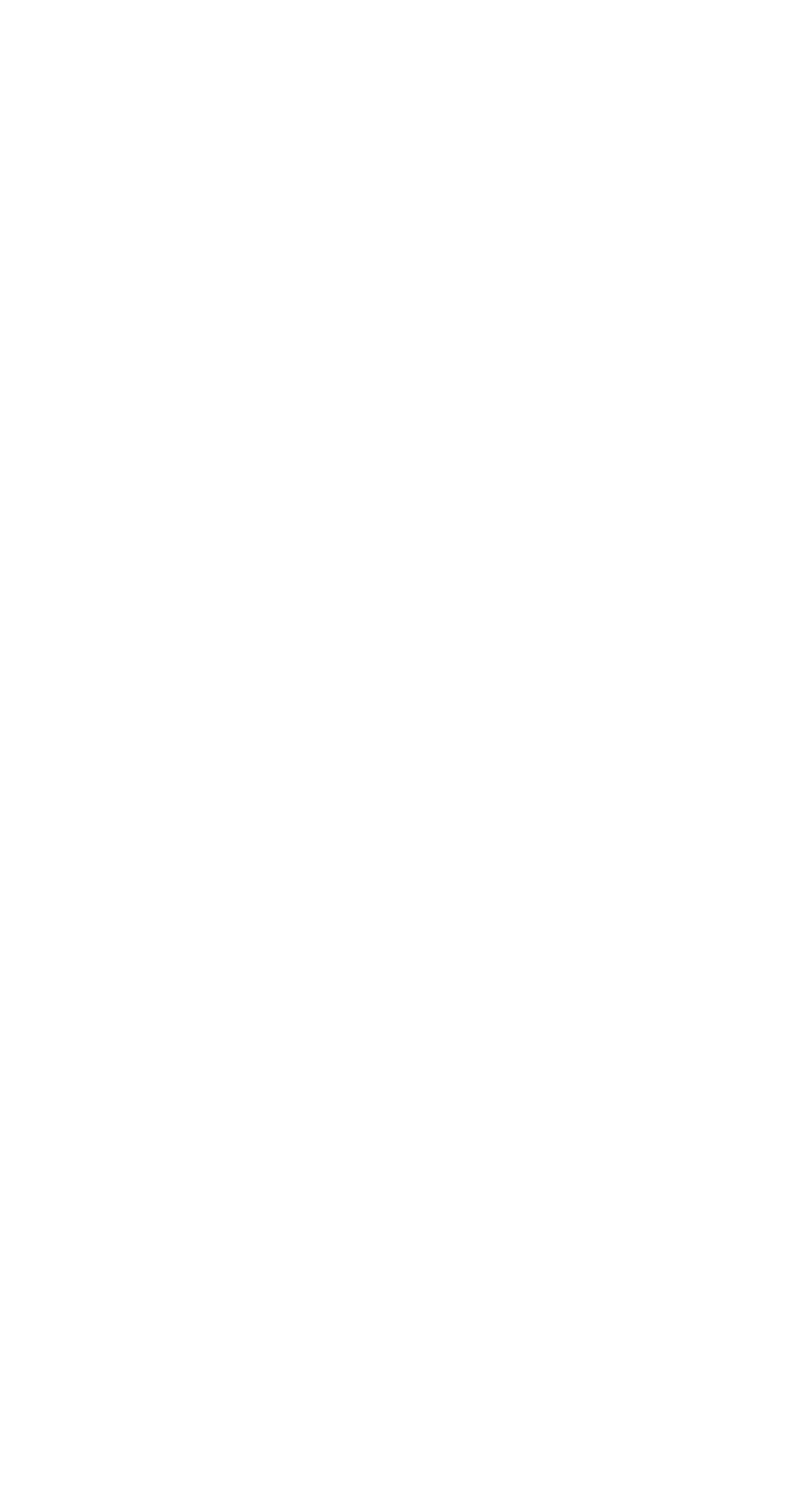




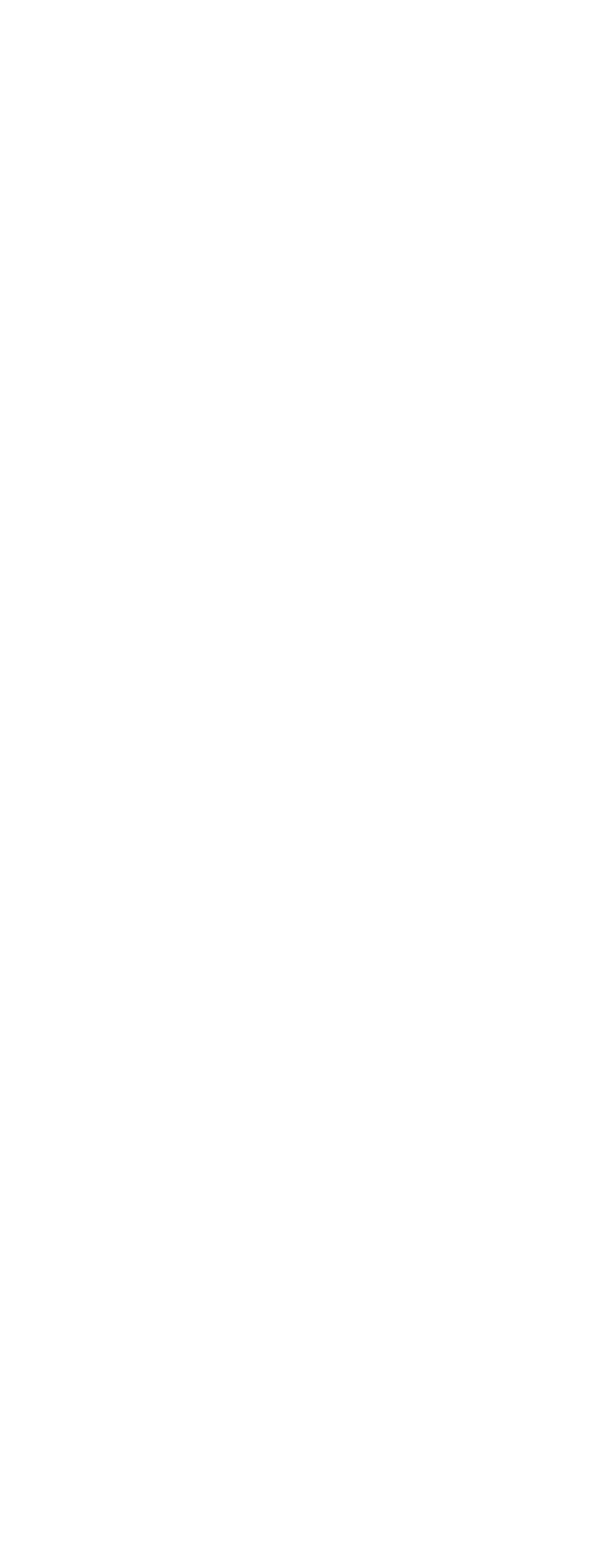


RIO GRANDE BASIN--Continued

8-3530. RIO PUERCO NEAR BERNARDO, N. MEX.--Continued

Suspended sediment, water year October 1963 to September 1964 (Where no daily concentrations are reported, loads are estimated)

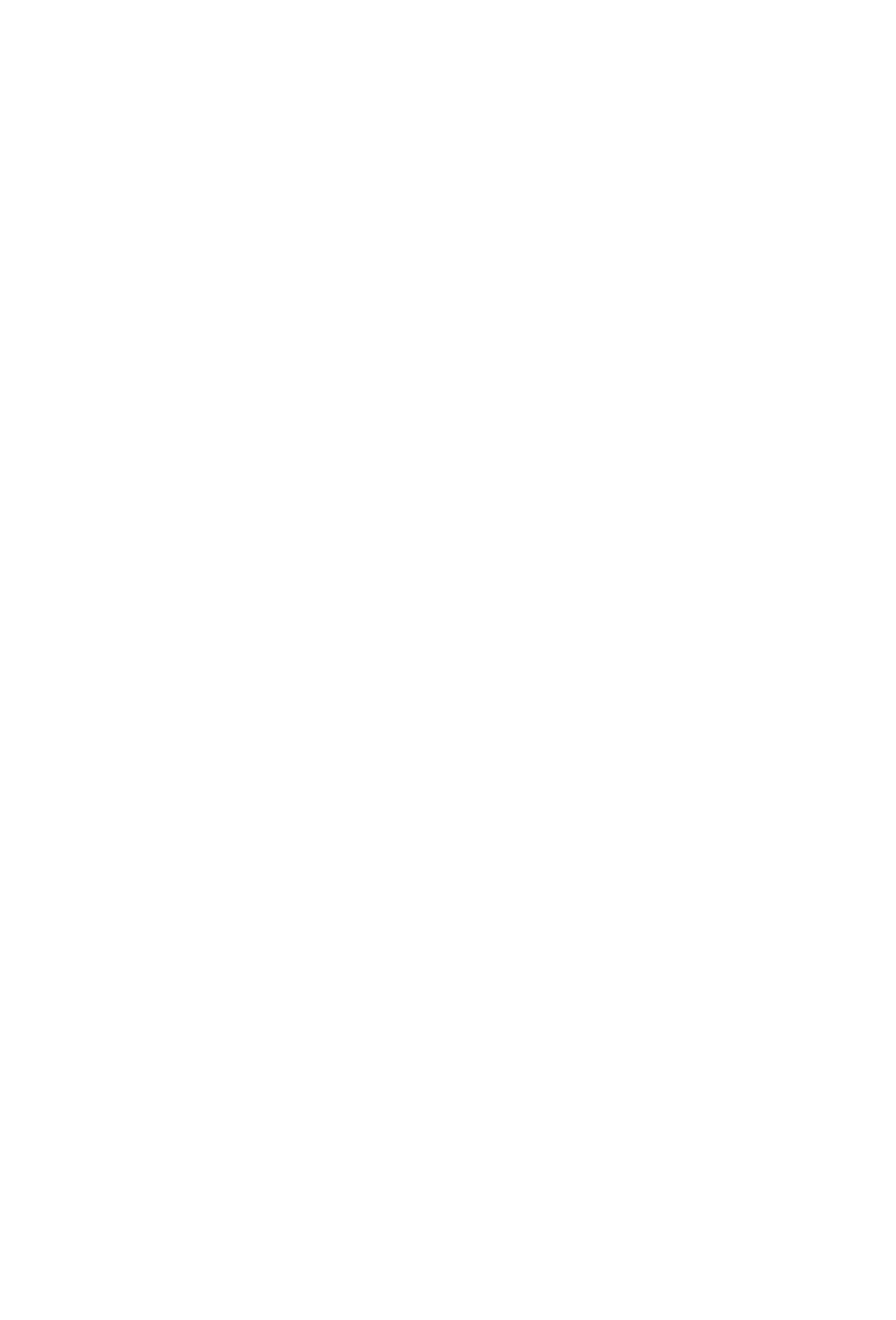

S Computed by subdividing day.

$\mathrm{K}$ Computed from estimated-concentration graph and subdividing day. 
RIO GRANDE BASIN--Continued

8-3530. RIO PUERCO NEAR BERNARDO, N. MEX.--Continued

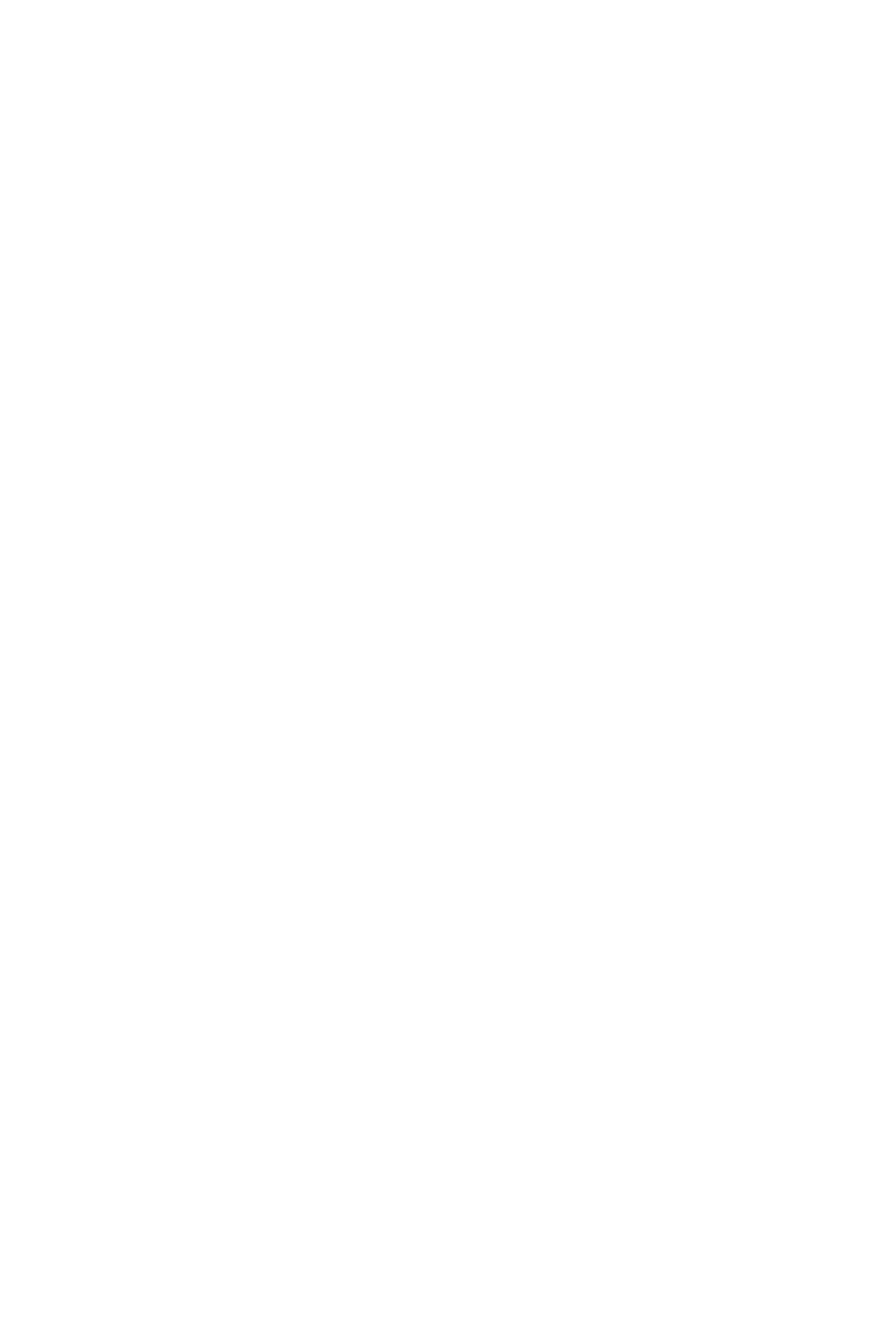

$S$ Computed by subdividing day.

B Computed from estimated-concentration graph.

$K$ Computed from estimated-concentration graph and subdividing day. 


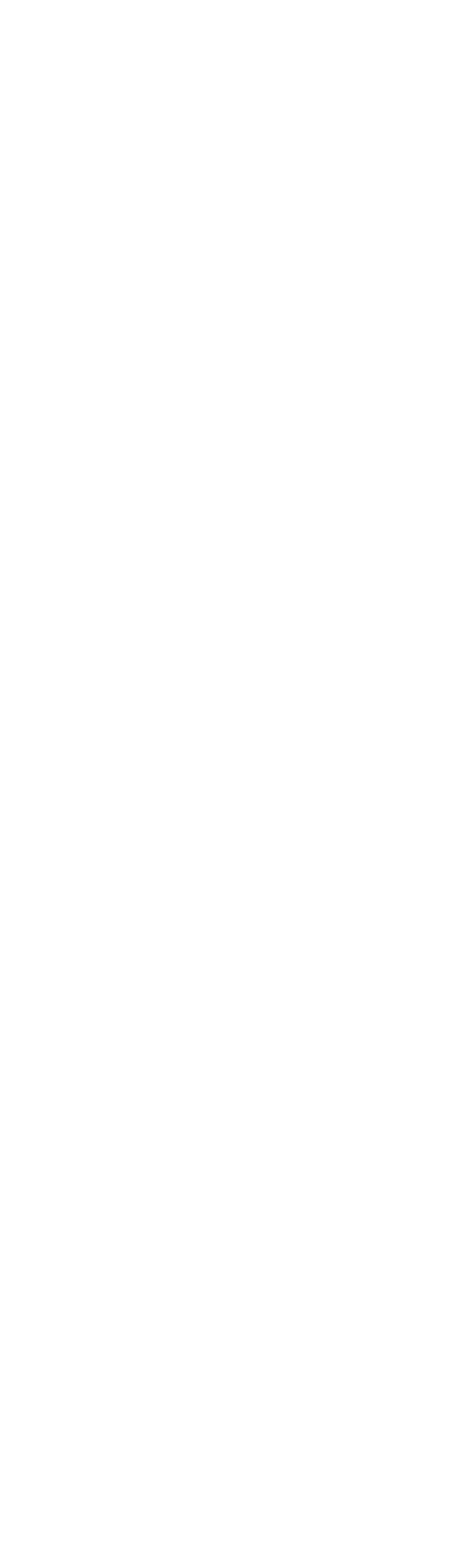




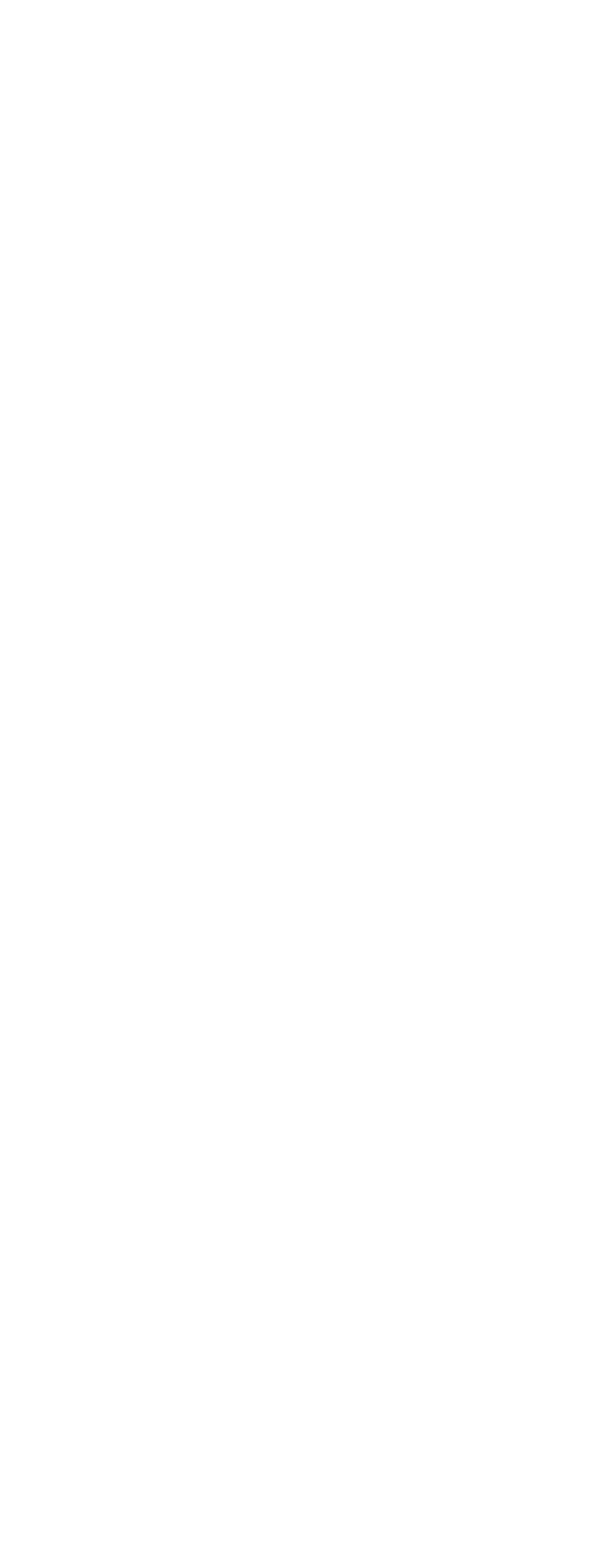




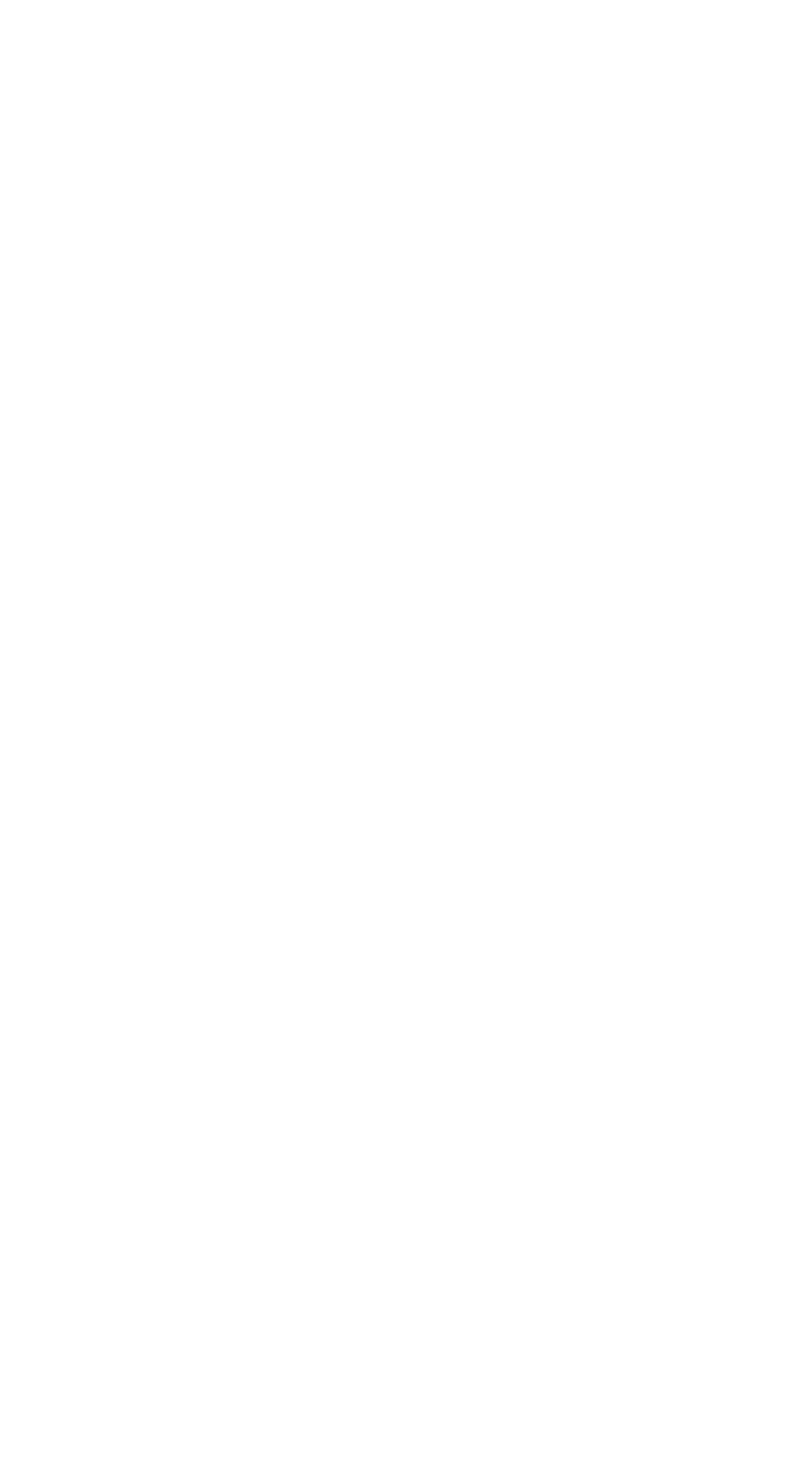


RIO GRANDE BASIN--Continued

8-3548. RIO GRANDE CONVEYANCE CHANNEL AT SAN ACACIA, N. MEX.--Cortinued

Suspended sediment, water year October 1963 to September 1964

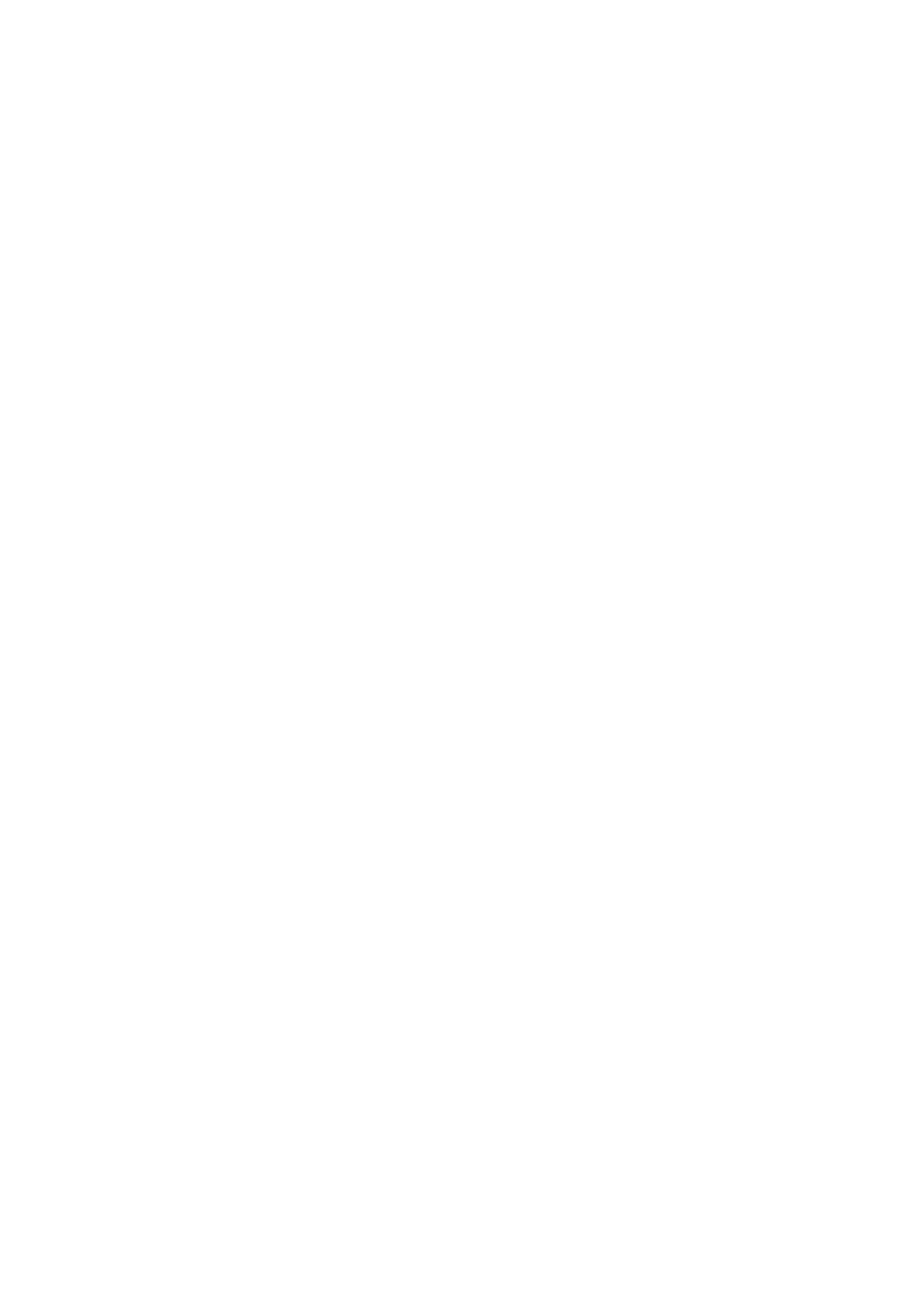

S Computed by subdividing day. 
RIO GRANDE BASIN--Continued

8-3548. RIO GRANDE CONVEYANCE CHANNEL AT SAN ACACIA, N. MEX.--Continued

Suspended sediment, water year October 1963 to September 1964--Continued

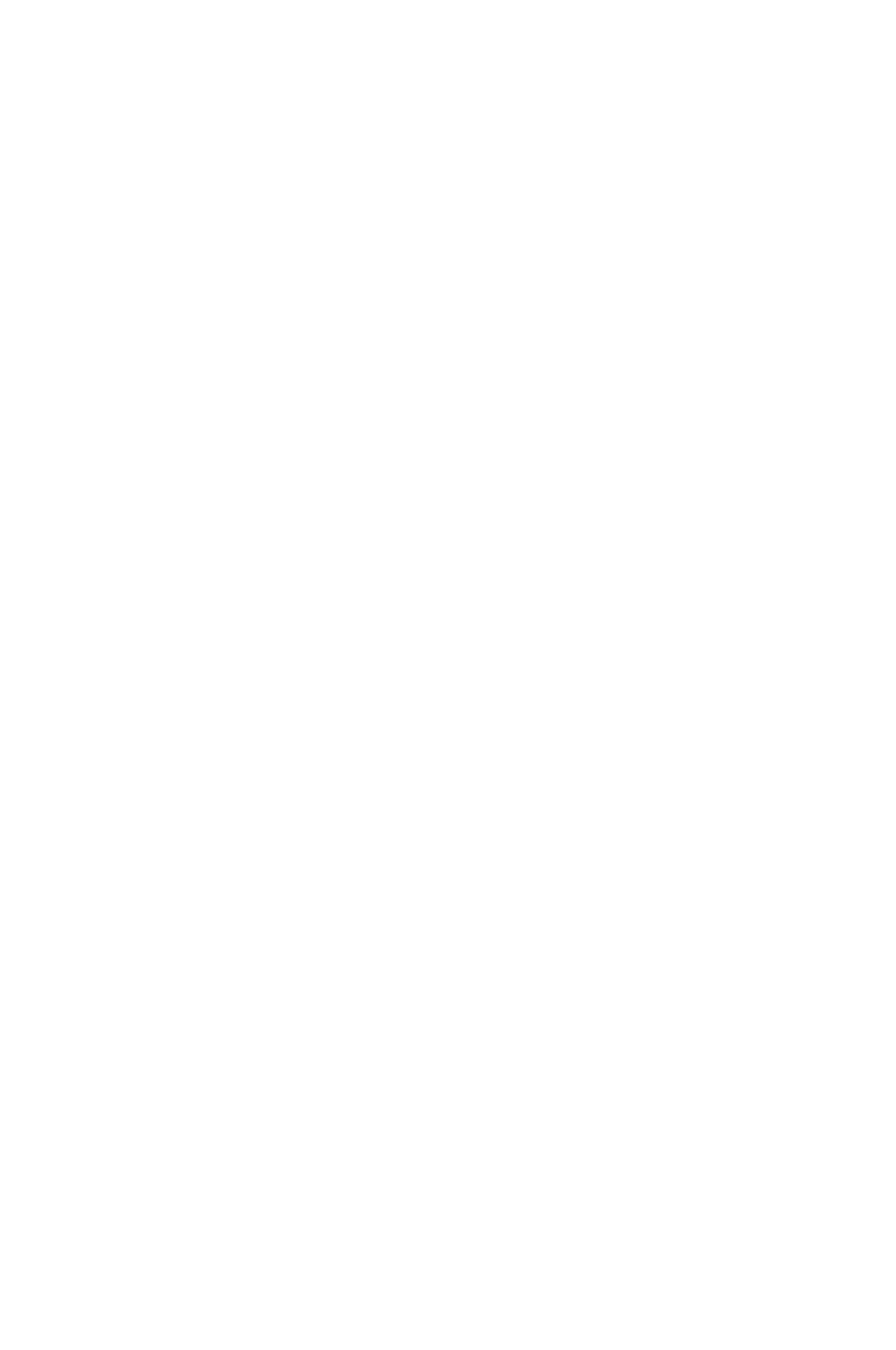




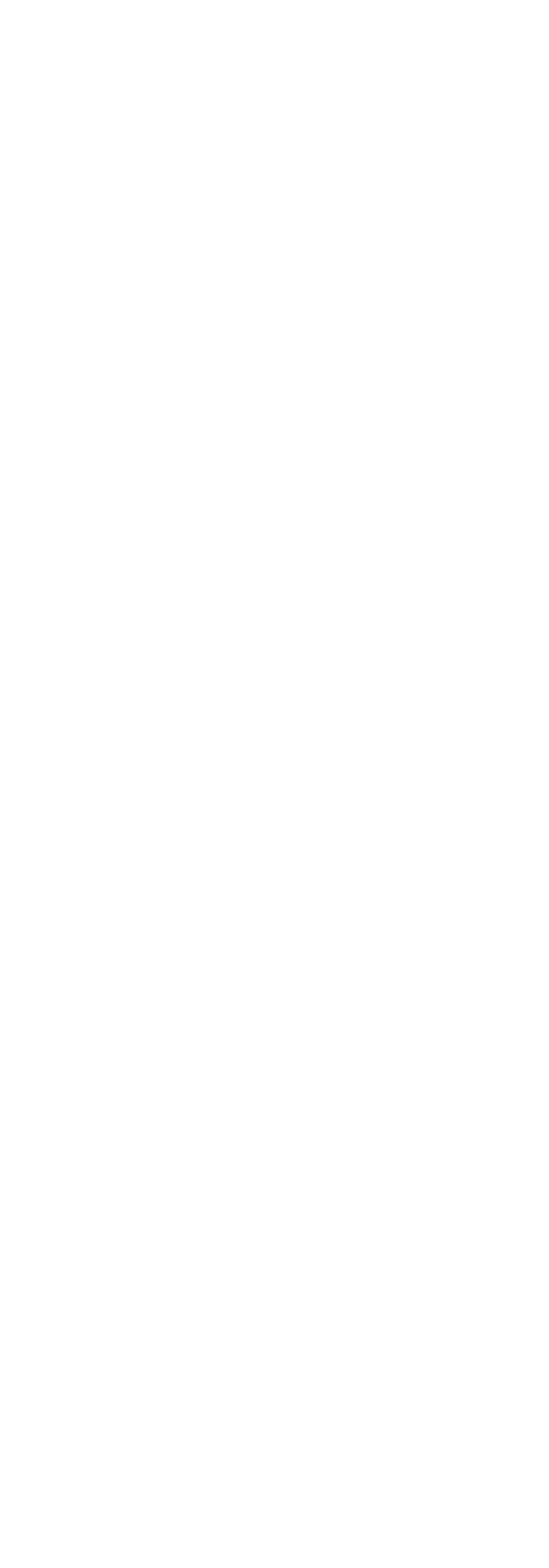




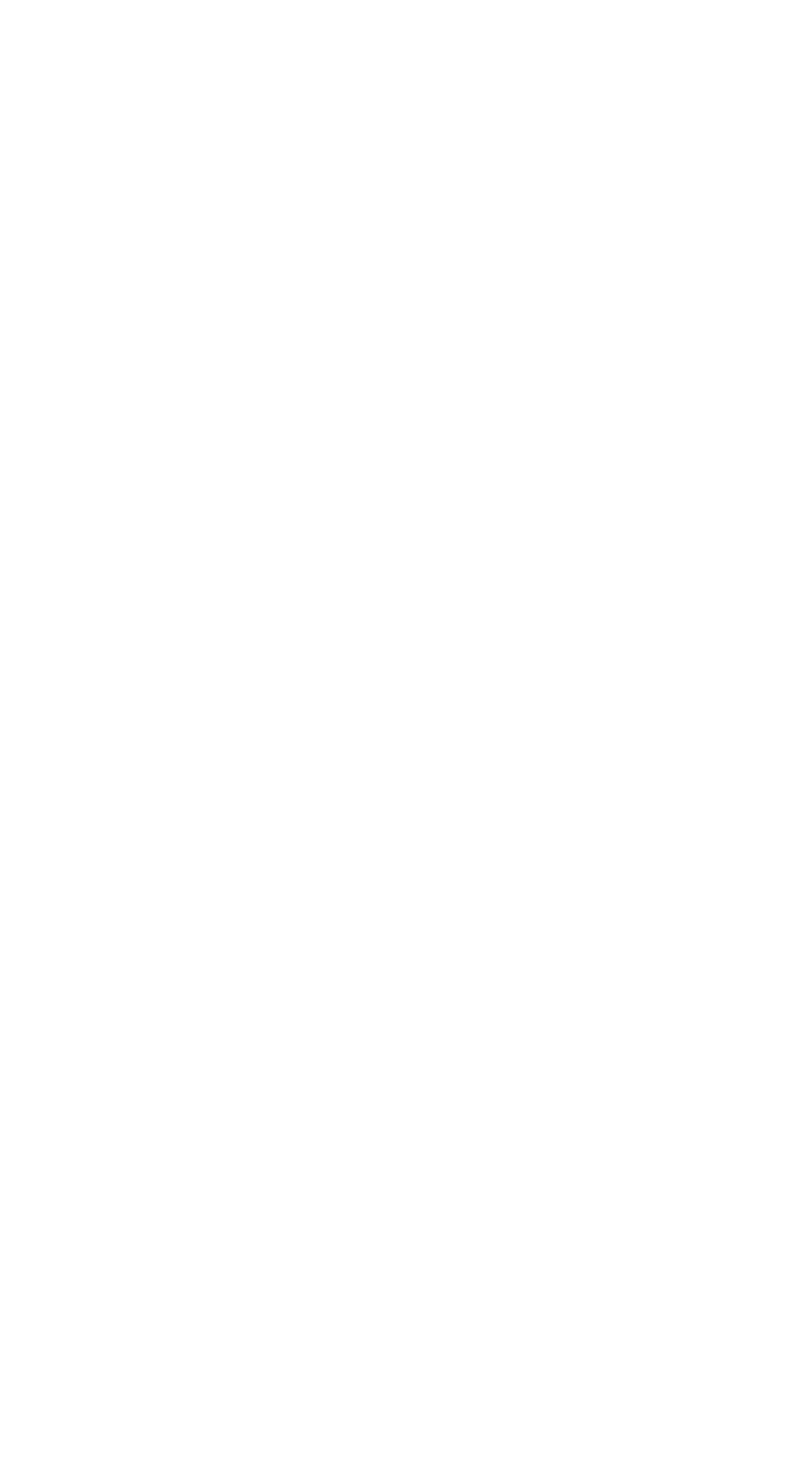


RIO GRANDE BASIN--Continued

8-3549. RIO GRANDE FLOODWAY AT SAN ACACIA, N. MEX.--Continued

Suspended sediment, water year October 1963 to september 1964

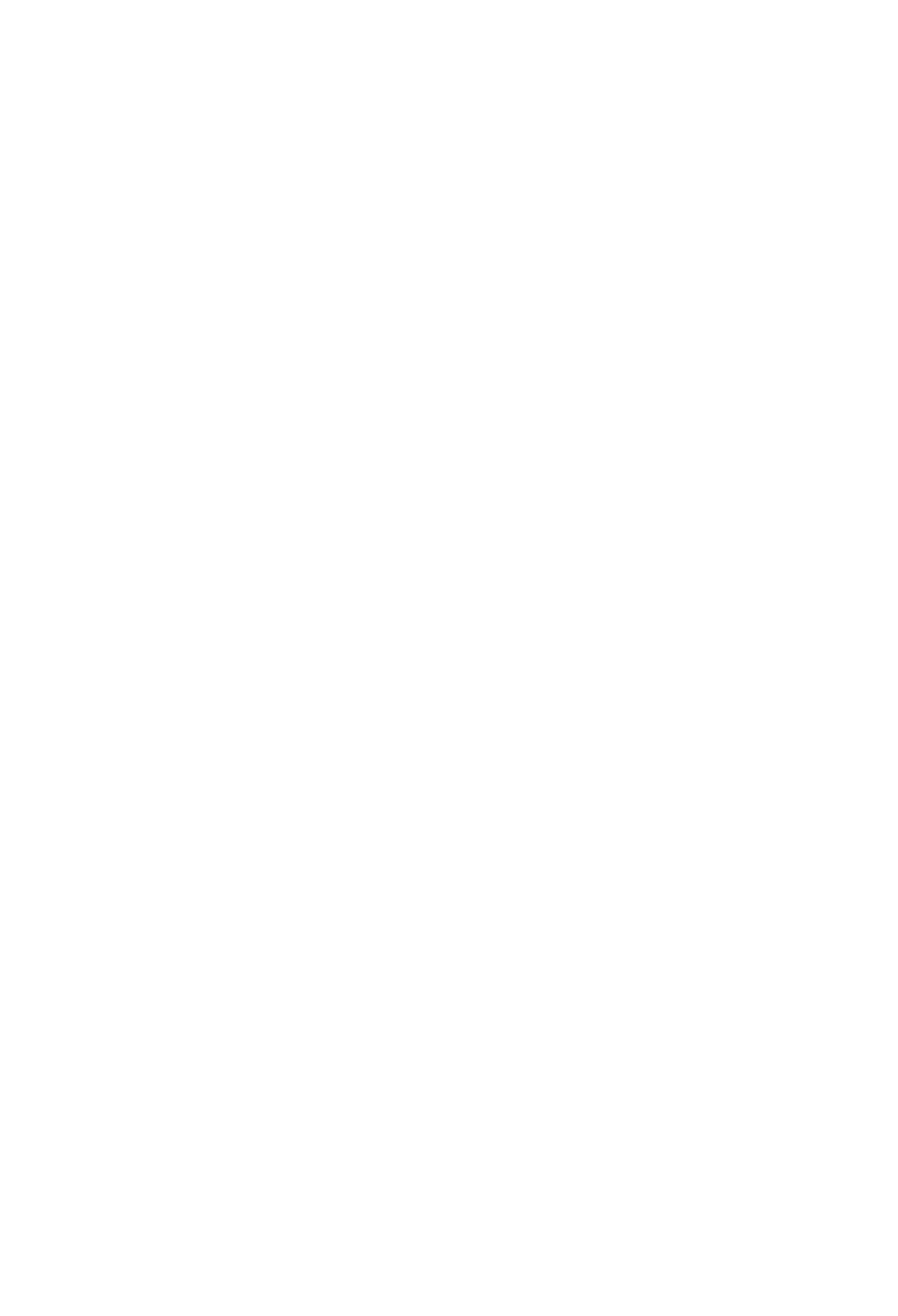

S Computed by subdividing day.

$T$ Less than 0.50 ton. 
RIO GRANDE BASIN--Continued

8-3549. RIO GRANDE FLOODWAY AT SAN ACACIA, N. MEX,--Continued

Suspended sediment, water year october 1963 to september 1964--Continued

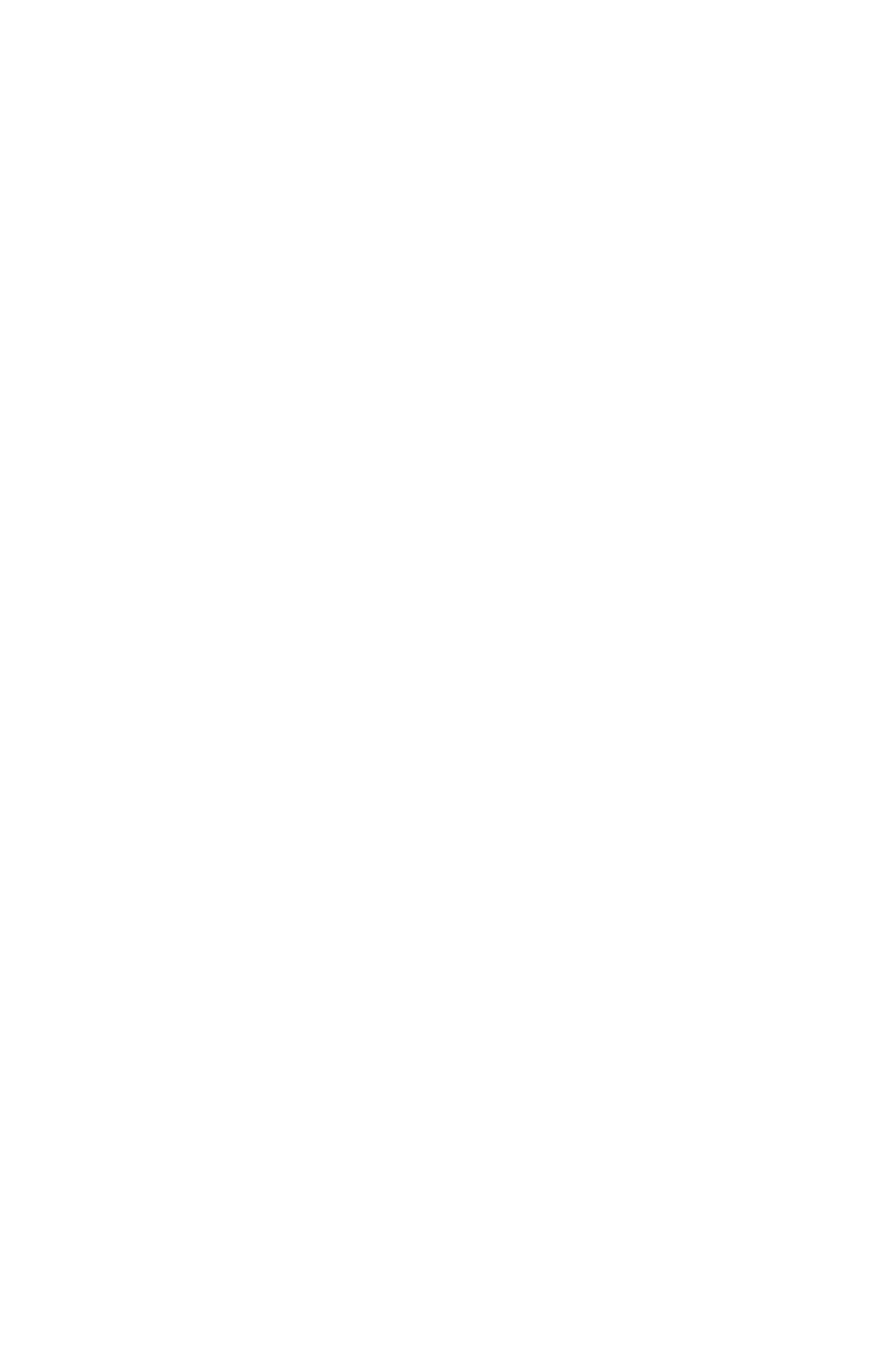




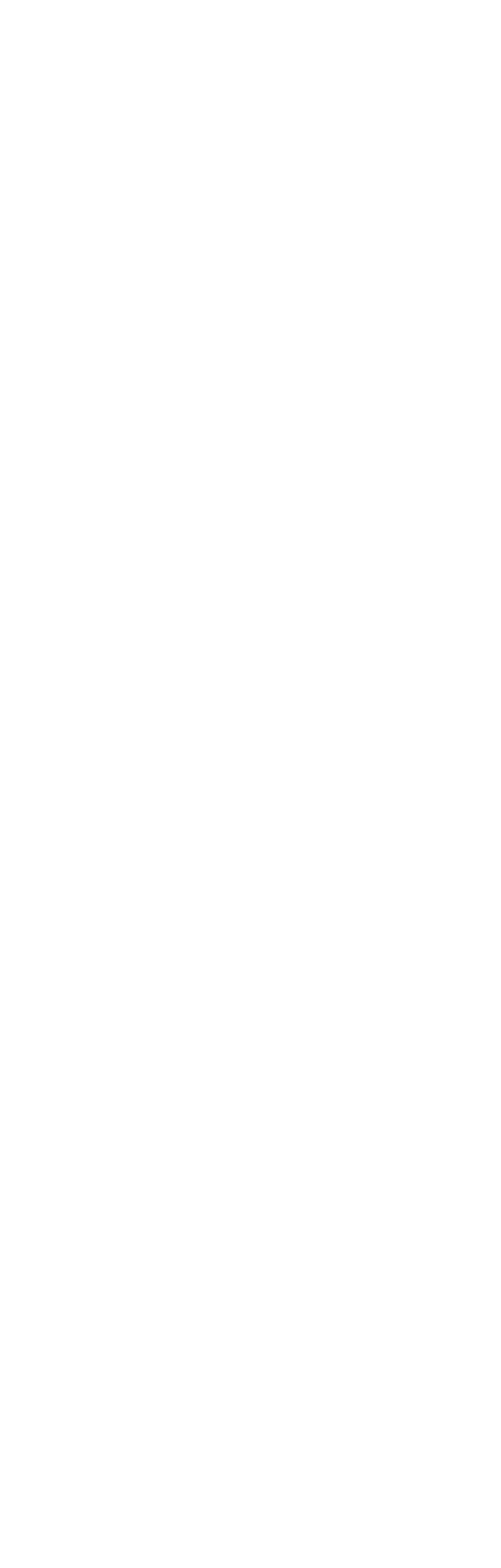




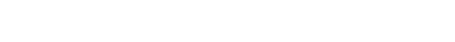
我!

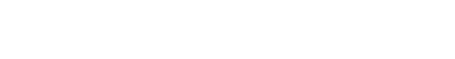

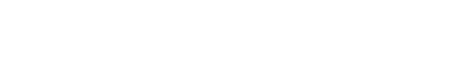

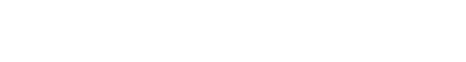

\begin{tabular}{|c|c|c|}
\hline \multirow{2}{*}{\multicolumn{2}{|c|}{ 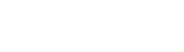 }} & \\
\hline & & $t_{0}$ \\
\hline \multicolumn{2}{|c|}{ 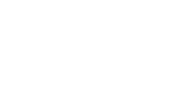 } & 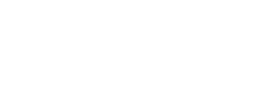 \\
\hline \multicolumn{2}{|c|}{ 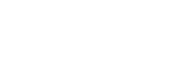 } & 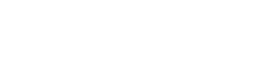 \\
\hline \multirow{2}{*}{ 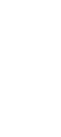 } & 言高高齿 & 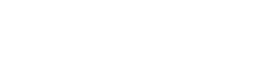 \\
\hline & 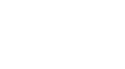 & 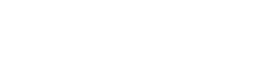 \\
\hline \multirow{3}{*}{ 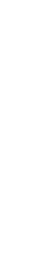 } & $\overbrace{0}^{\infty}$ & 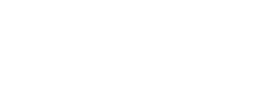 \\
\hline & 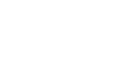 & 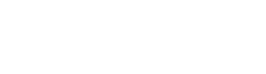 \\
\hline & 学总总 & 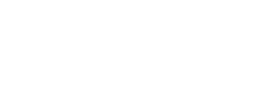 \\
\hline \multicolumn{2}{|r|}{ 的吠鱼 } & 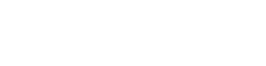 \\
\hline \multicolumn{2}{|r|}{ 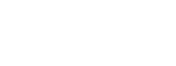 } & 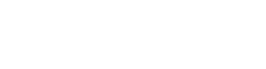 \\
\hline \multicolumn{2}{|r|}{ 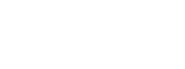 } & $1: 111110111$ \\
\hline \multicolumn{2}{|r|}{ 吳 } & 1品 $10: 1: 11$ \\
\hline \multicolumn{2}{|r|}{ 善象 } & 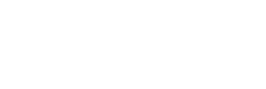 \\
\hline \multicolumn{2}{|c|}{ में } & $000000<\infty$ \\
\hline \multicolumn{2}{|c|}{ 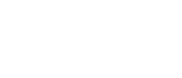 } & 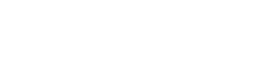 \\
\hline \multicolumn{2}{|c|}{ 就塄異 } & $1_{0}^{1} \begin{array}{lllllll}1 & 1 & 1 & 1 & 1 & 1 & 1 \\
\end{array}$ \\
\hline \multicolumn{2}{|r|}{ 悬要 } & 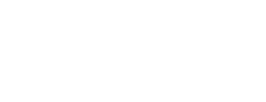 \\
\hline \multicolumn{2}{|r|}{ 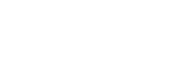 } & 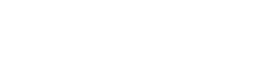 \\
\hline \multicolumn{2}{|r|}{ ฮี่ } & 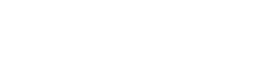 \\
\hline \multicolumn{2}{|r|}{ 总焉 } & $\begin{array}{c}18 \\
0 \\
0\end{array} 11110111111$ \\
\hline \multicolumn{2}{|r|}{ 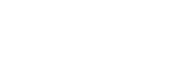 } & $17: 1: 1111111$ \\
\hline \multicolumn{2}{|r|}{ 氧要 } & 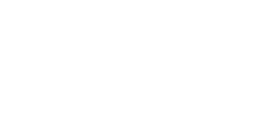 \\
\hline \multicolumn{2}{|r|}{ 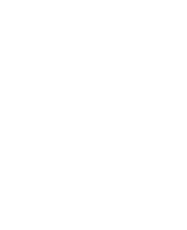 } & 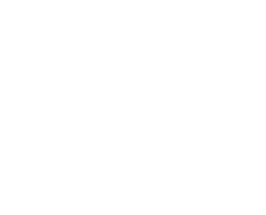 \\
\hline
\end{tabular}




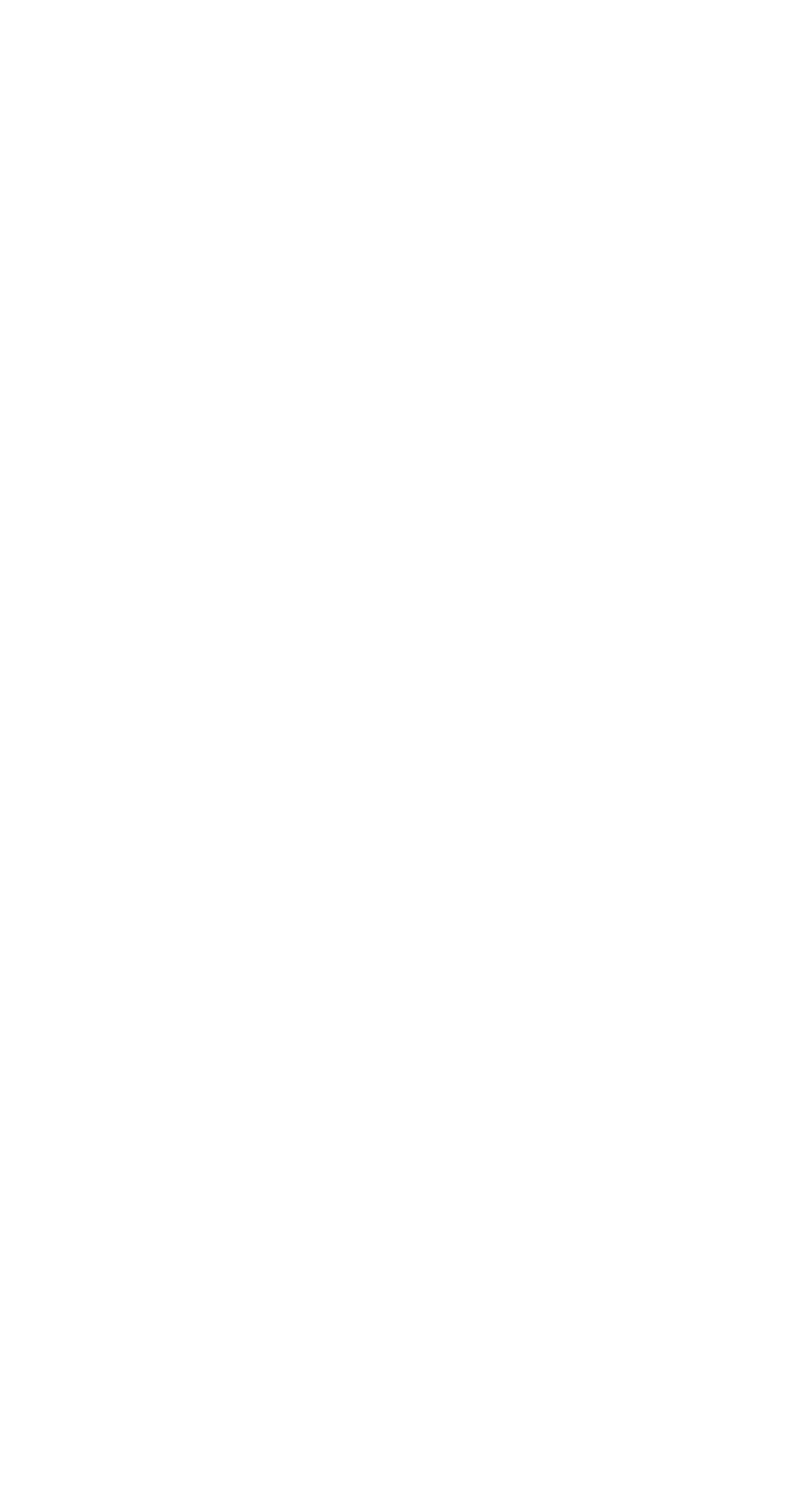




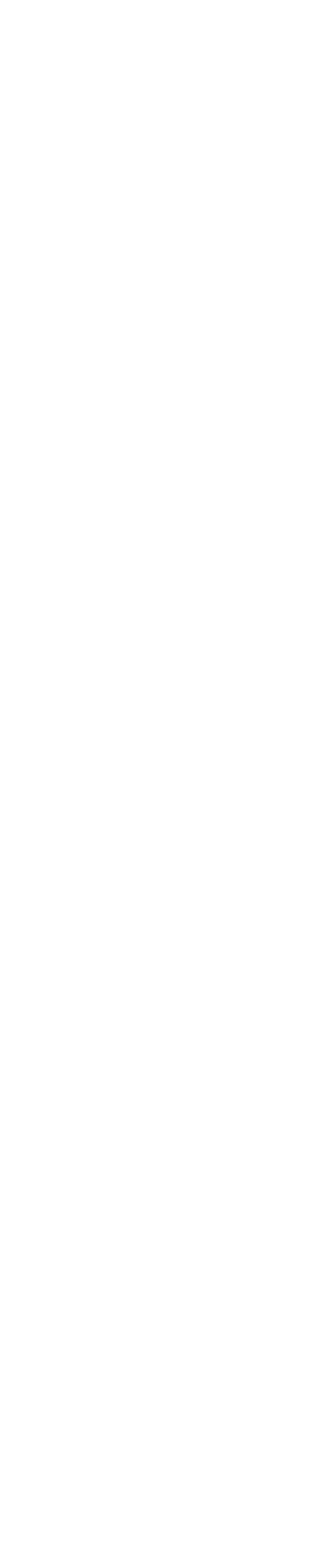

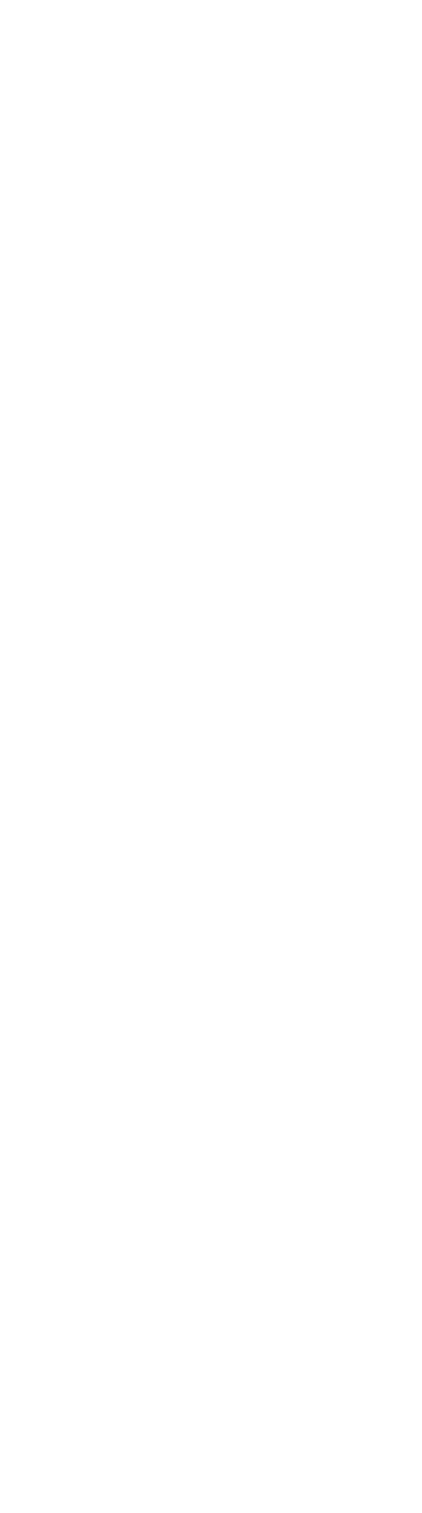


RIO GRANDE BASIN--Continued

8-3583. RIO GRANDE CONVEYANCE CHANNEL AT SAN MARCIAL, N. MEX.--Continued Suspended sediment, water year October 1963 to September 1964

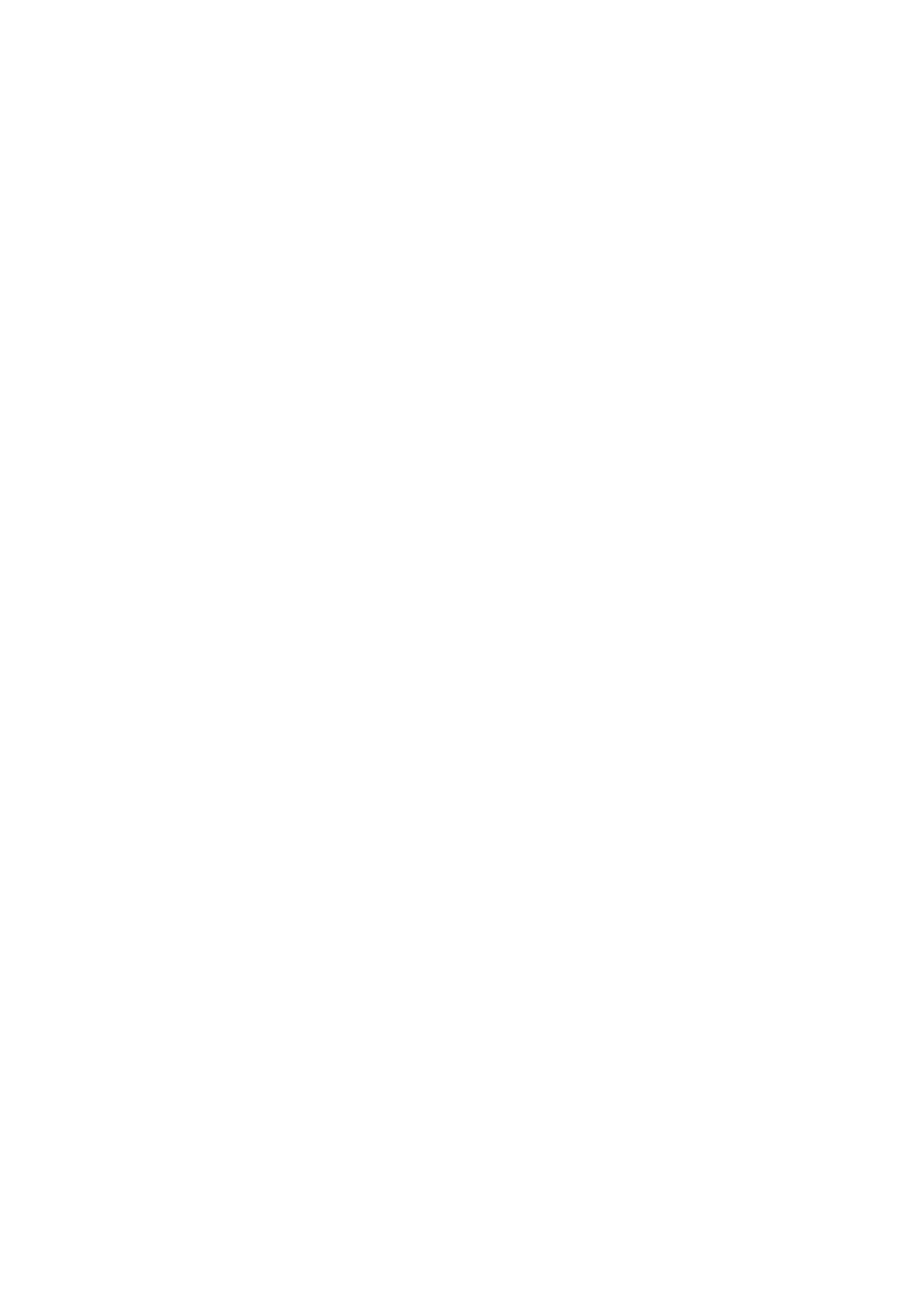

$S$ Computed by subdividing day

B Computed from estimated-concentration graph. 
RIO GRANDE BASIN--Continued

8-3583. RIO GRANDE CONVEYANCE CHANNEL AT SAN MARCIAL, N. MEX.--Continued

Suspended sediment, water year actober 1963 to september 1964--Continued

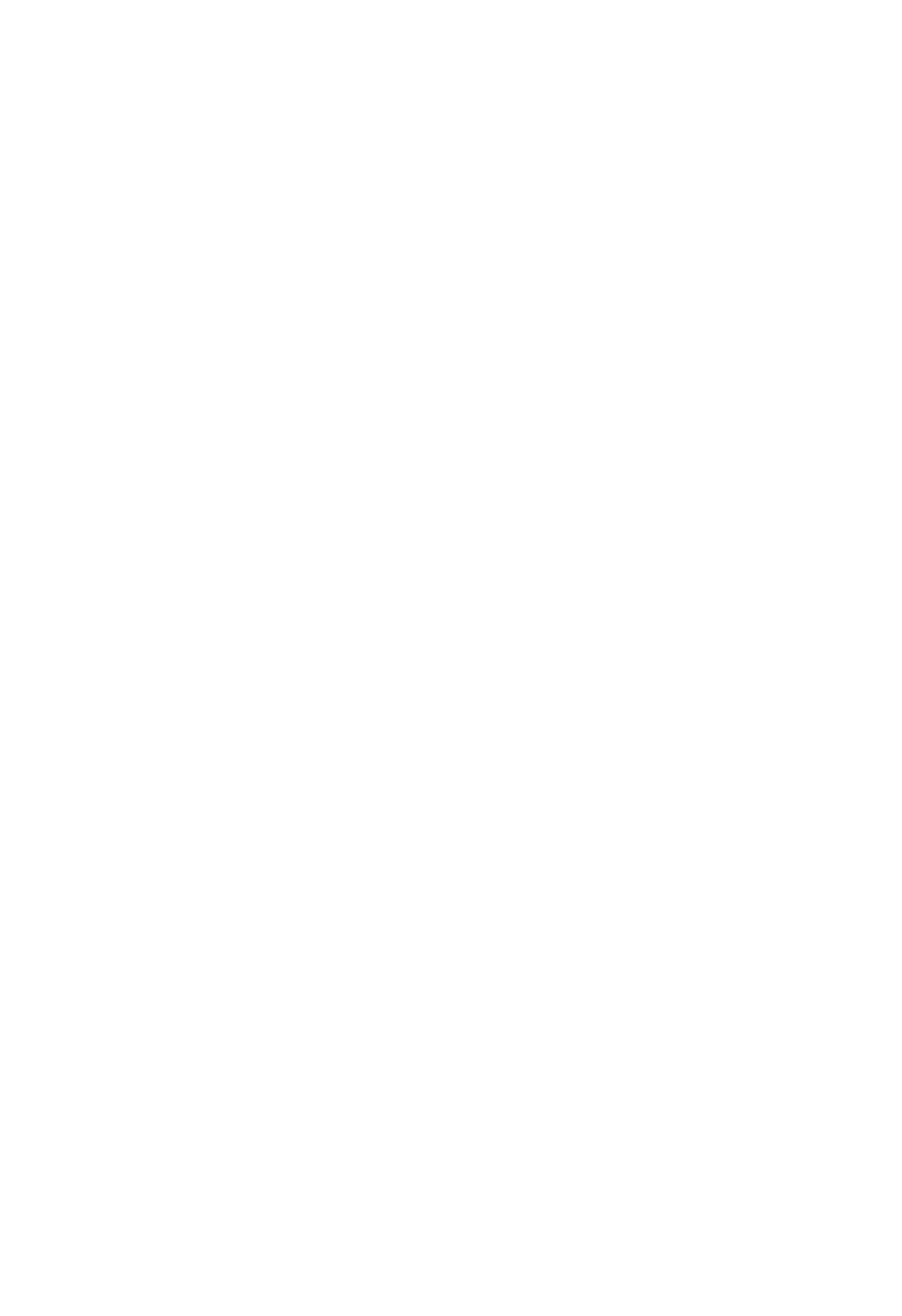

$S$ Computed by subdividing day.

$T$ Less than 0.50 ton.

B Computed from estimated-concentration graph.
$K$ Computed from estimated-concentration graph and subdividing day. 


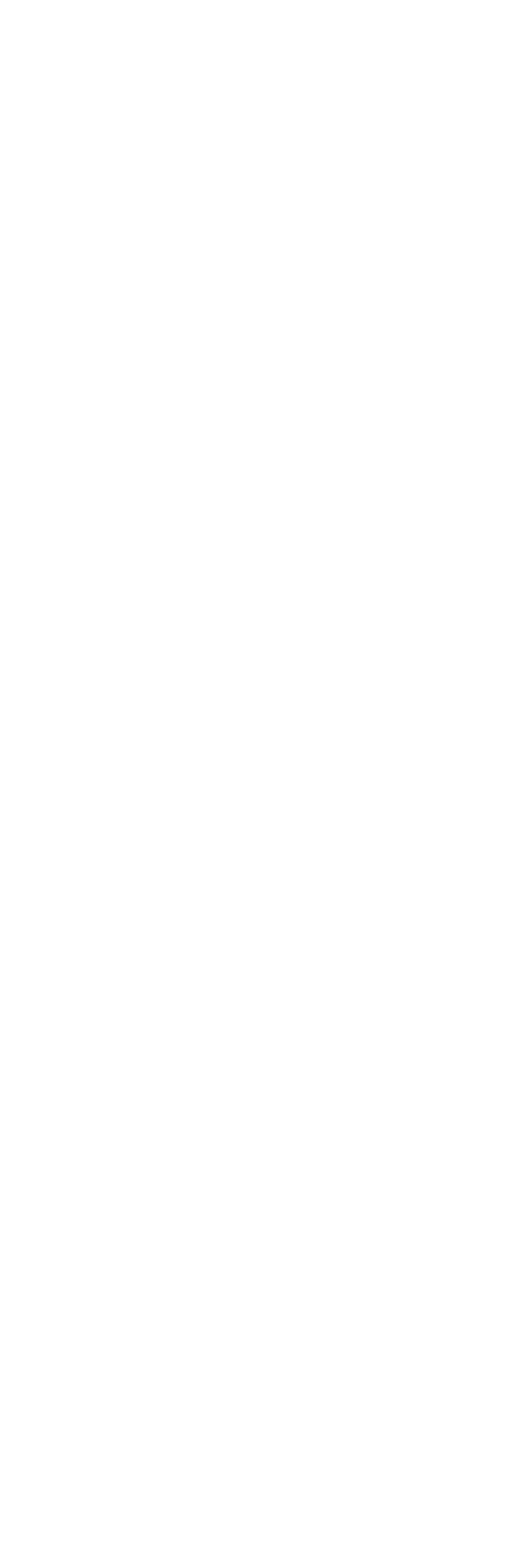




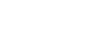

궁웡

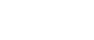

\begin{tabular}{|c|c|c|c|}
\hline & 혐 & 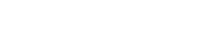 & \begin{tabular}{ll|}
0 & 0 \\
& 0 \\
\end{tabular} \\
\hline & 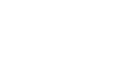 & 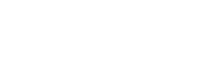 & 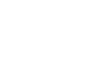 \\
\hline & 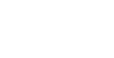 & & \\
\hline \$્⿷ & 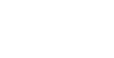 & & \\
\hline 象 & 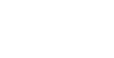 & 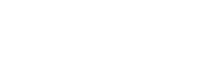 & $\mid \begin{array}{ll}\stackrel{5}{0} & 1 \\
0 & i n\end{array}$ \\
\hline
\end{tabular}

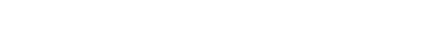
to

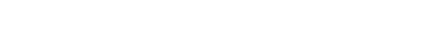

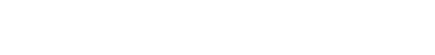

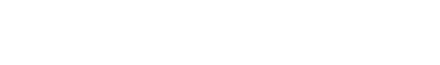

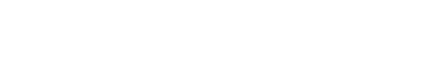
in 管 on

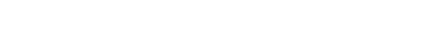

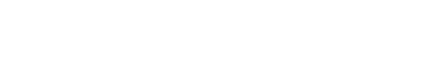

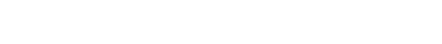

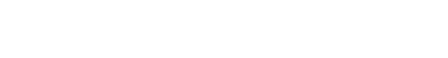

\begin{tabular}{|c|c|c|}
\hline 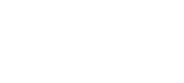 & & \\
\hline 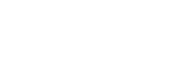 & & \\
\hline 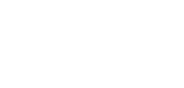 & 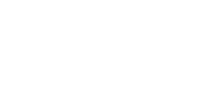 & $\begin{array}{cc}0 \\
0.0\end{array}$ \\
\hline 安部鱼 & 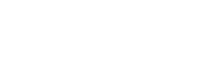 & \\
\hline 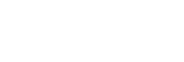 & 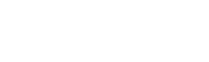 & 1 \\
\hline 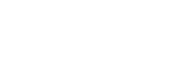 & 9.1140110 .11 & 11 \\
\hline 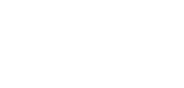 & 吕:18 11811 & \\
\hline 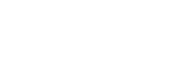 & 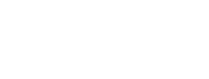 & $:$ \\
\hline is & & \\
\hline 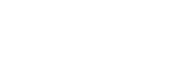 & 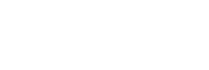 & 票 \\
\hline 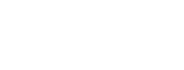 & 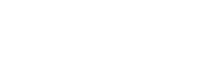 & i \\
\hline 謇要 & 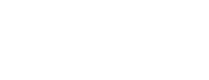 & 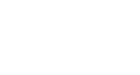 \\
\hline 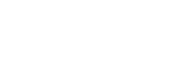 & 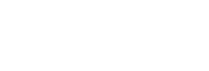 & $\mid \begin{array}{lll}* & 0 & 0\end{array}$ \\
\hline 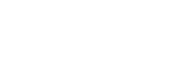 & 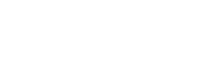 & 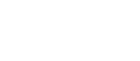 \\
\hline 咆垔 & 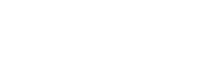 & \\
\hline 焉递 & $\begin{array}{lllll}511 & 1 & 1 & 8 & 1\end{array}$ & \\
\hline 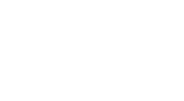 & 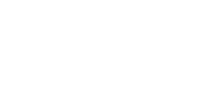 & 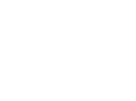 \\
\hline 粍 & 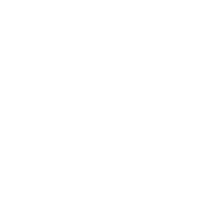 & 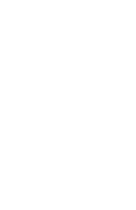 \\
\hline
\end{tabular}



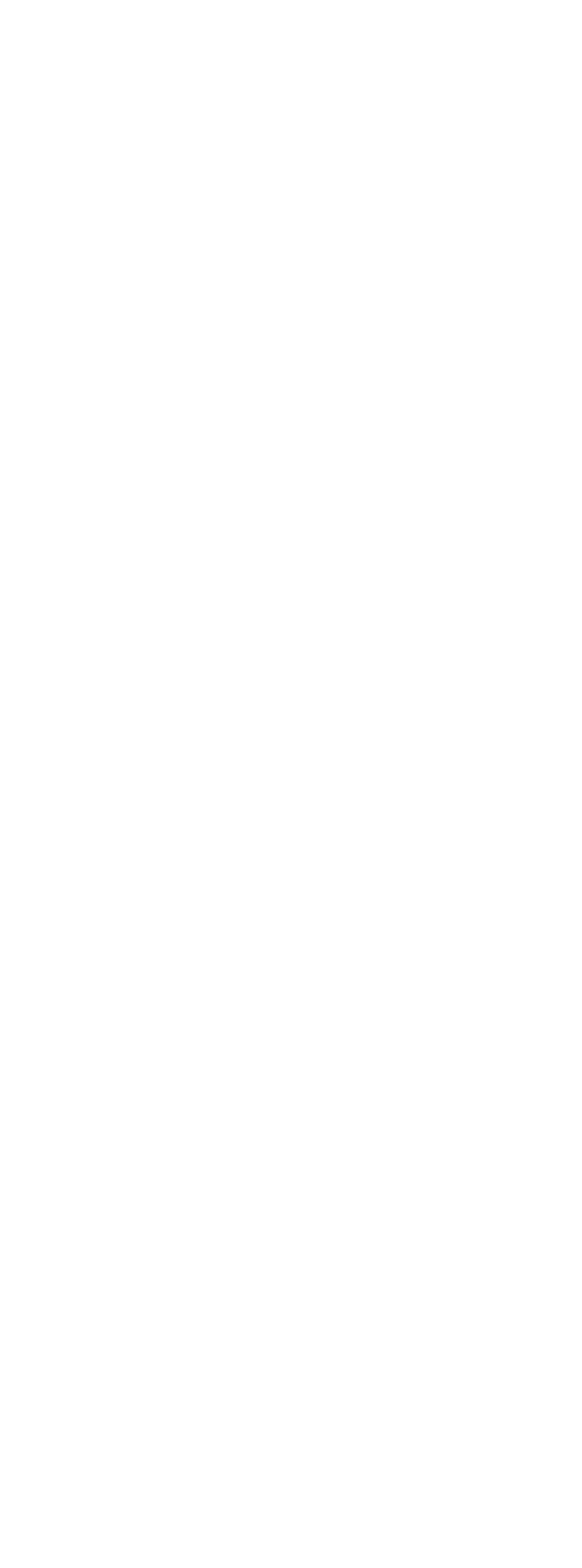
RIO GRANDE BASIN--Continued

8-3584. RIO GRANDE FLOODWAY AT SAN MARCIAL, N. MEX.--Continued

Suspended sediment, water year October 1963 to september 1964 (Where no daily concentrations are reported, loads are estimated)

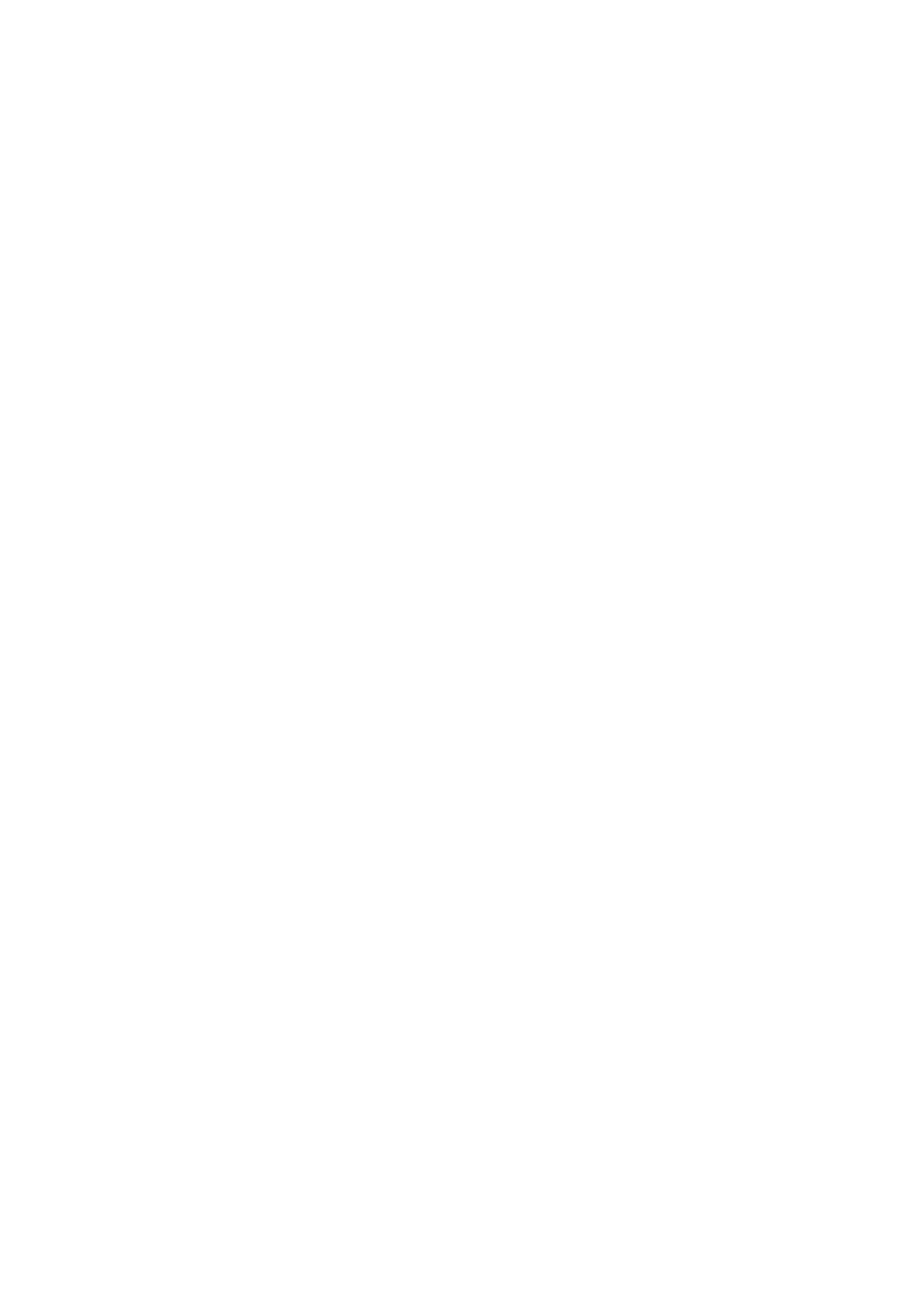



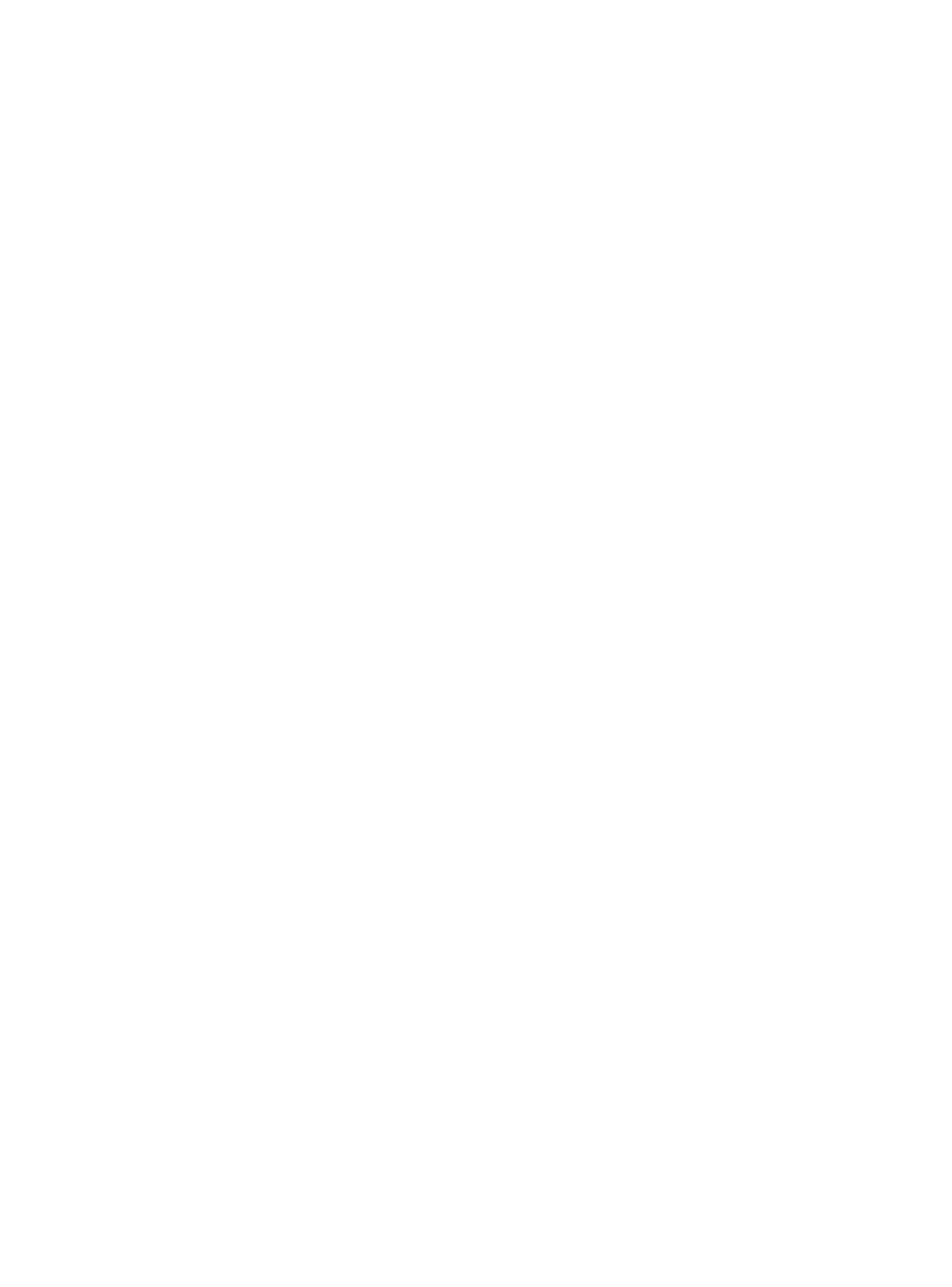


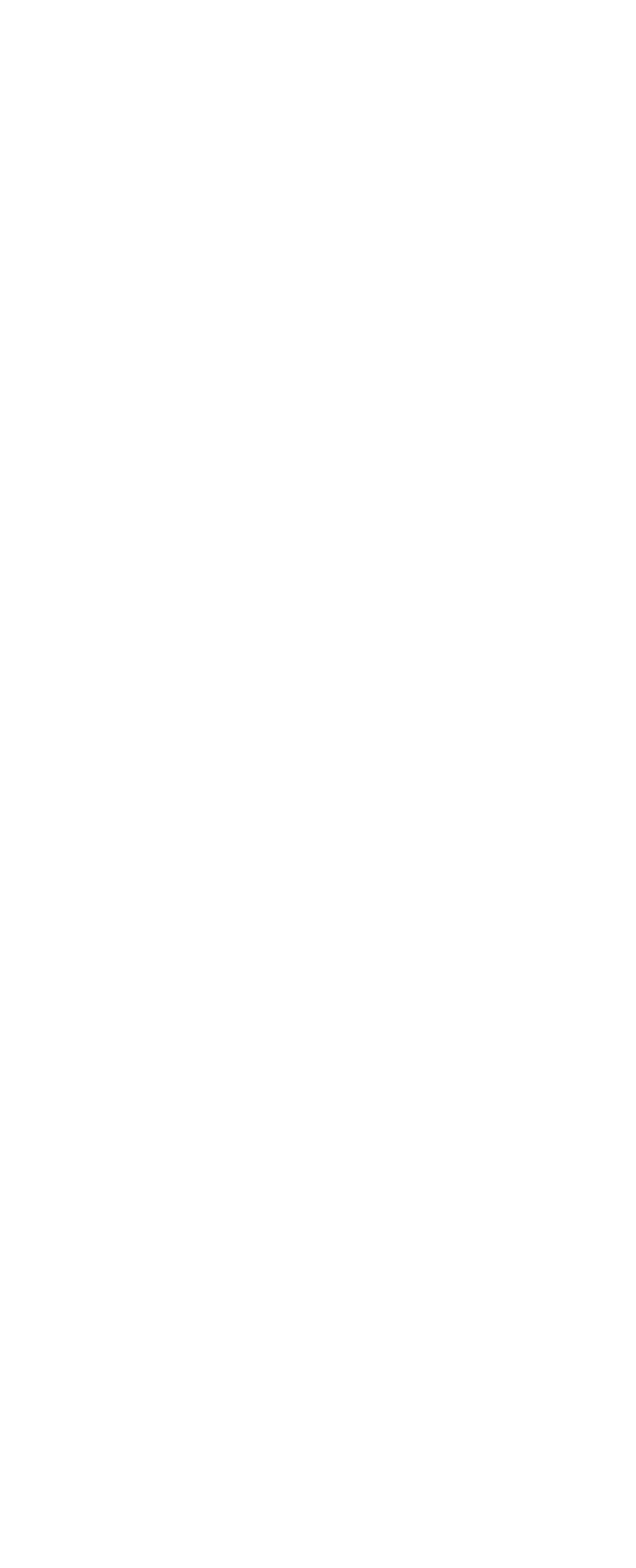




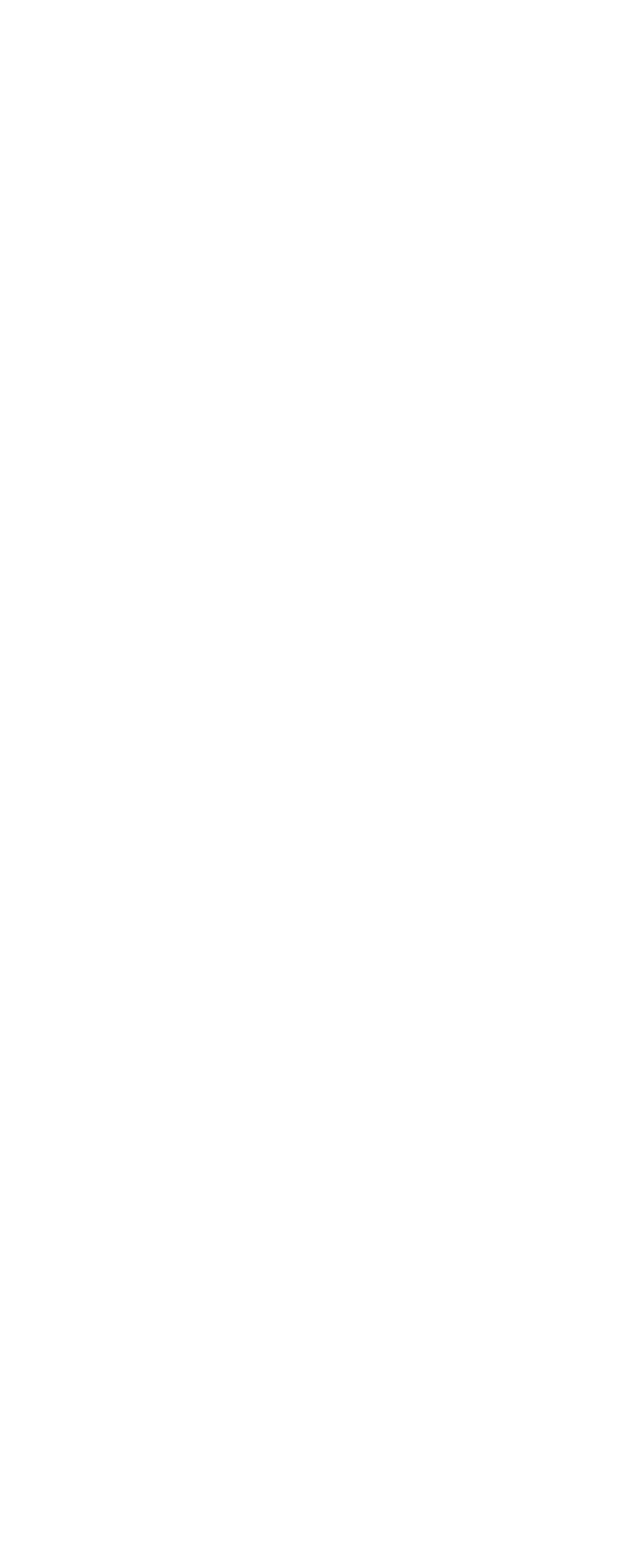




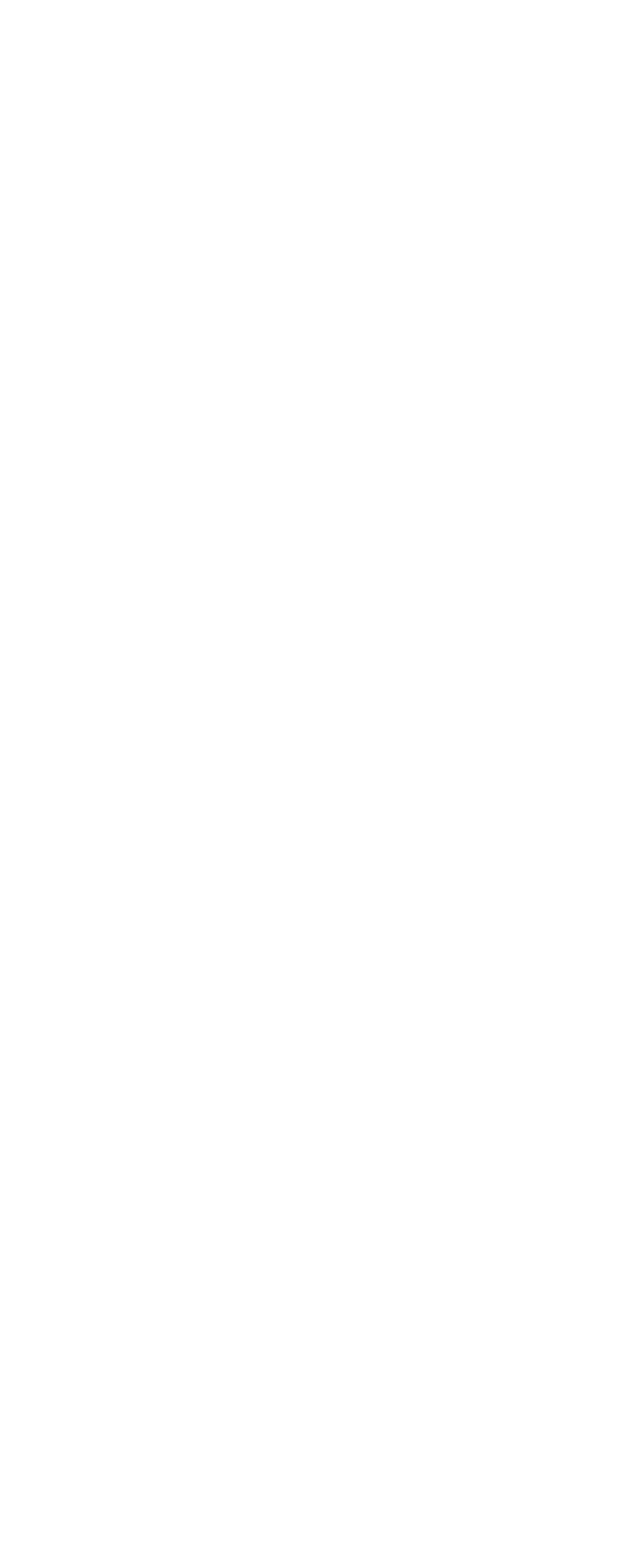




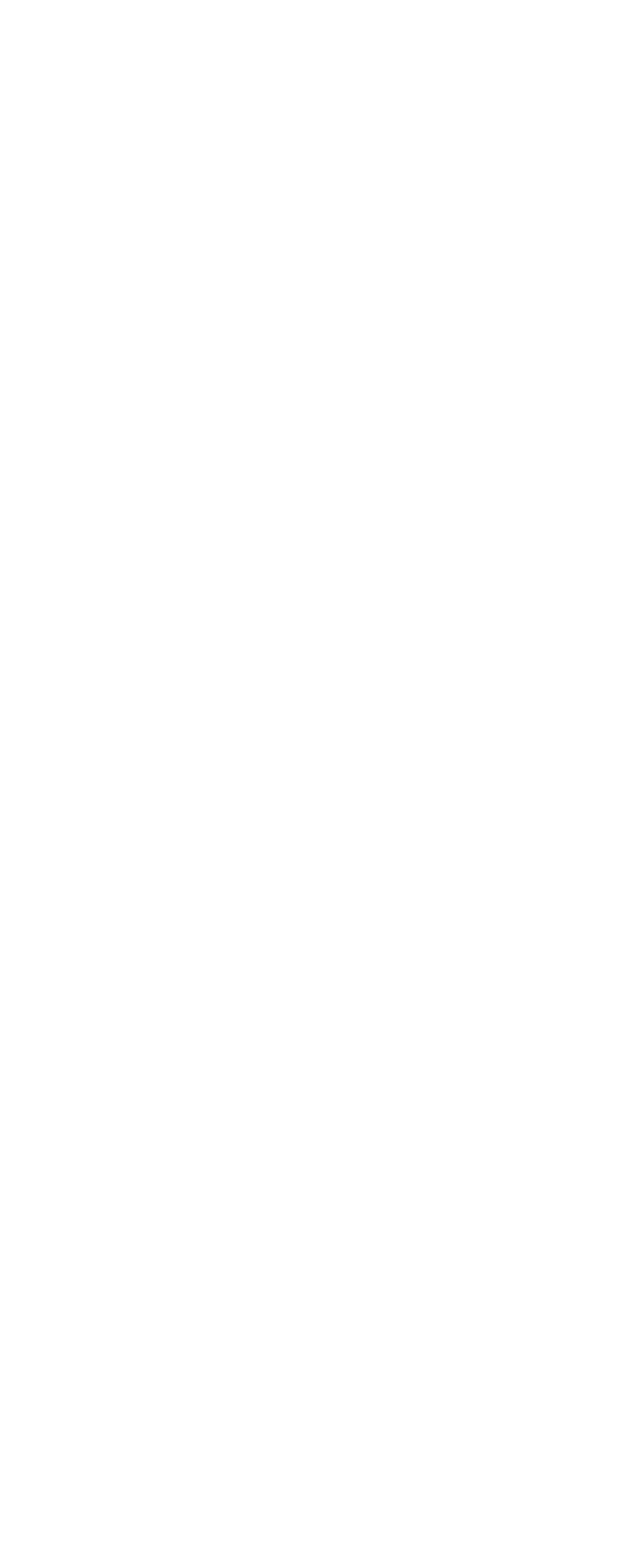




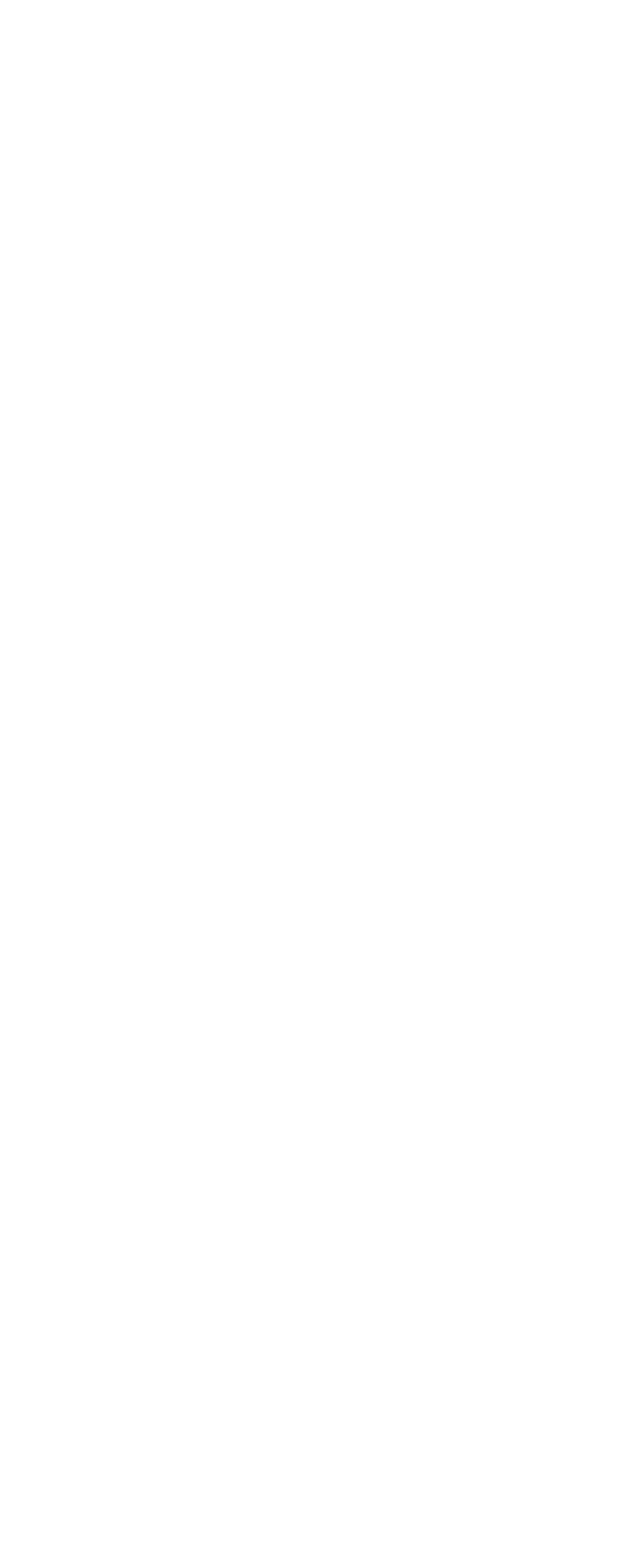




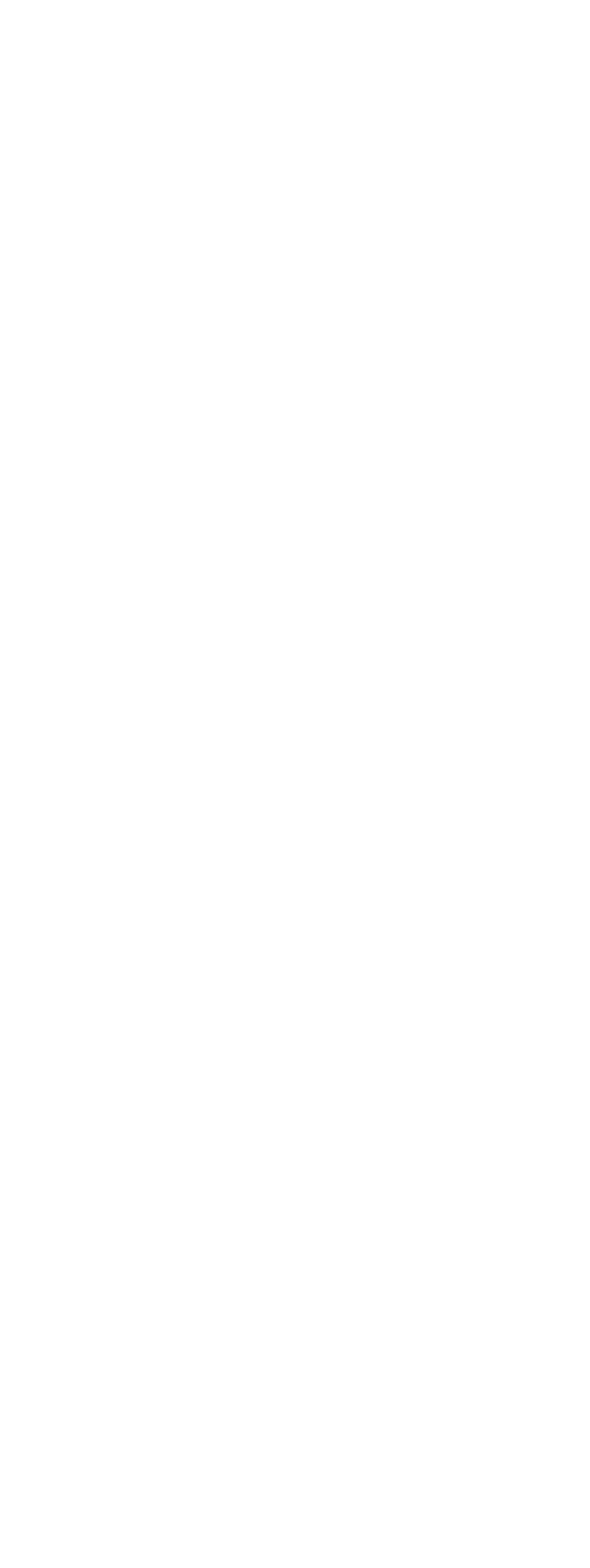




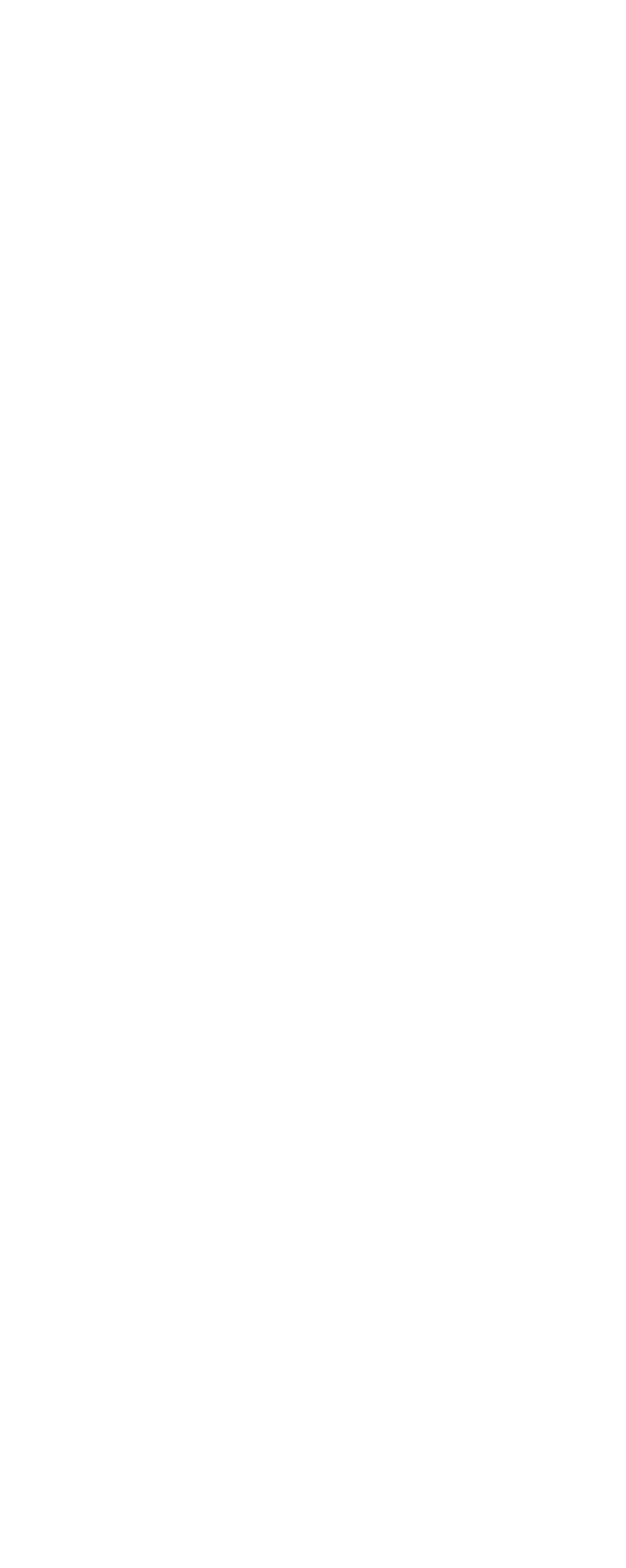




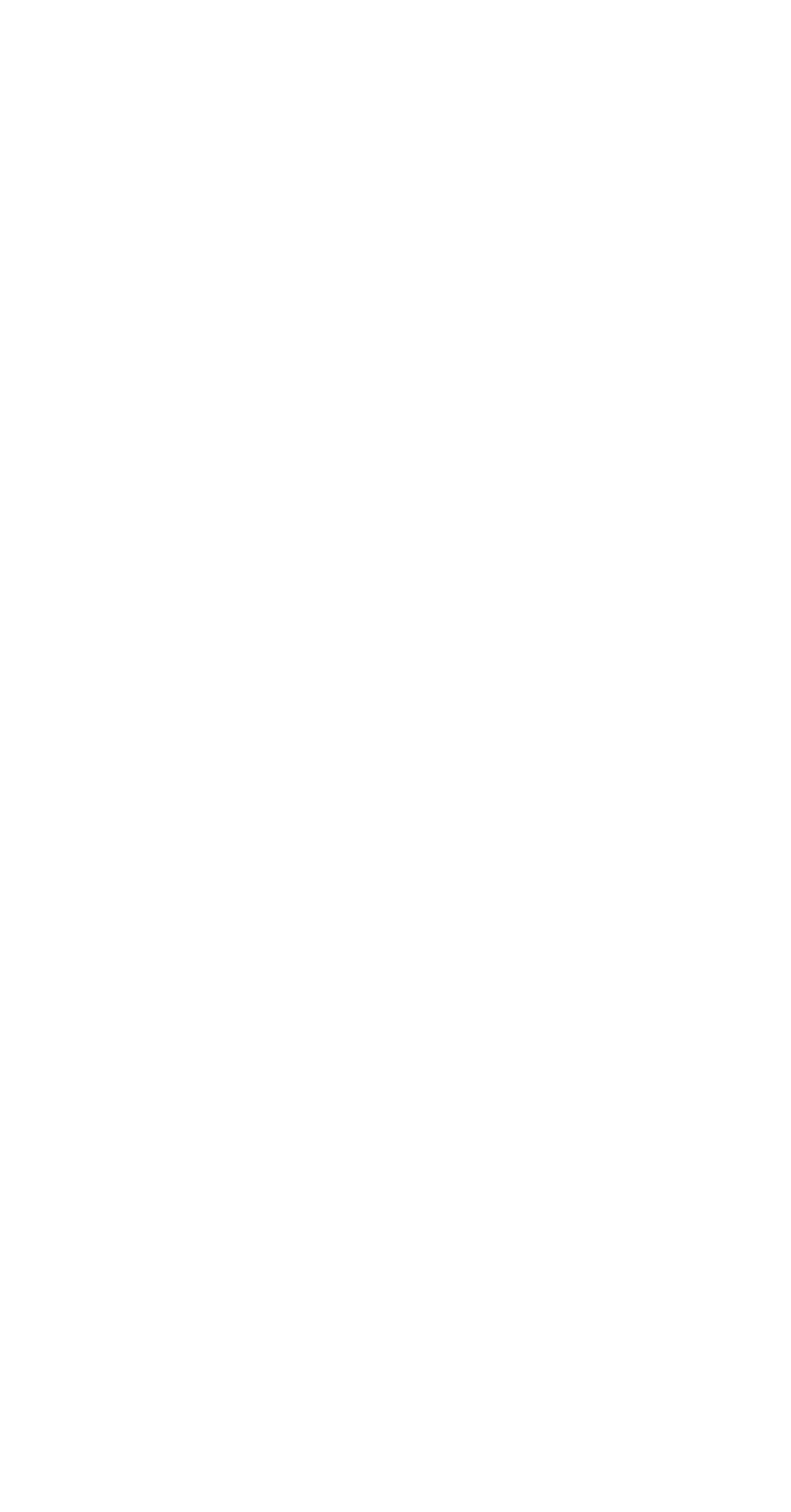


RIO GRANDE BASIN--Continued

8-3830. PECOS RIVER AT SANTA ROSA, N. MEX.--Continued

Suspended sediment, water year October 1963 to September 1964 (Where no daily concentrations are reported, loads are estimated)

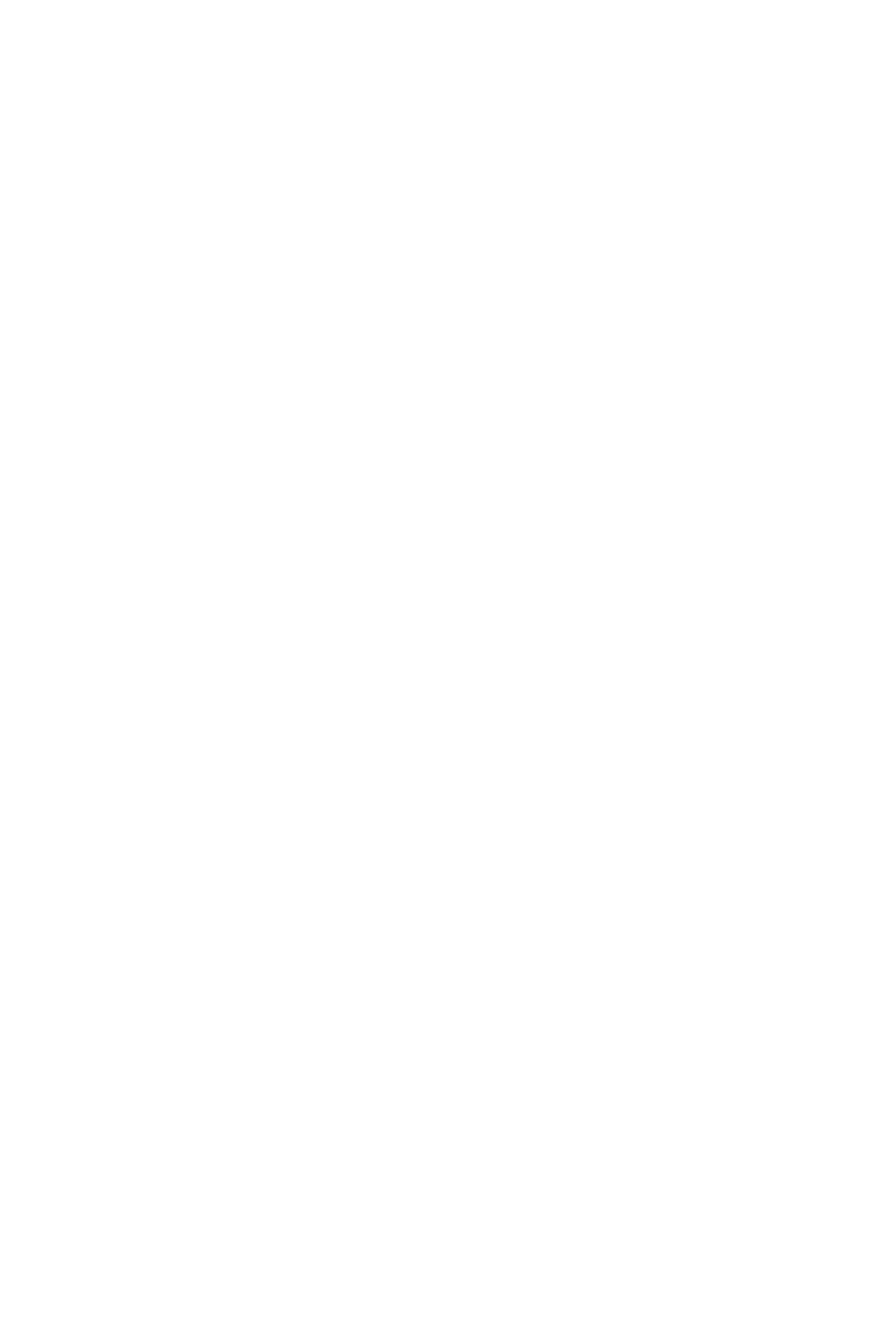

S Computed by subdividing day.

B Computed from estimated-concentration graph.

C Composite period. 
RIO GRANDE BASIN--Continued

8-3830. PECOS RIVER AT SANTA ROSA, N. MEX.--Continued

Suspended sediment, water year October 1963 to September 1964--Continued

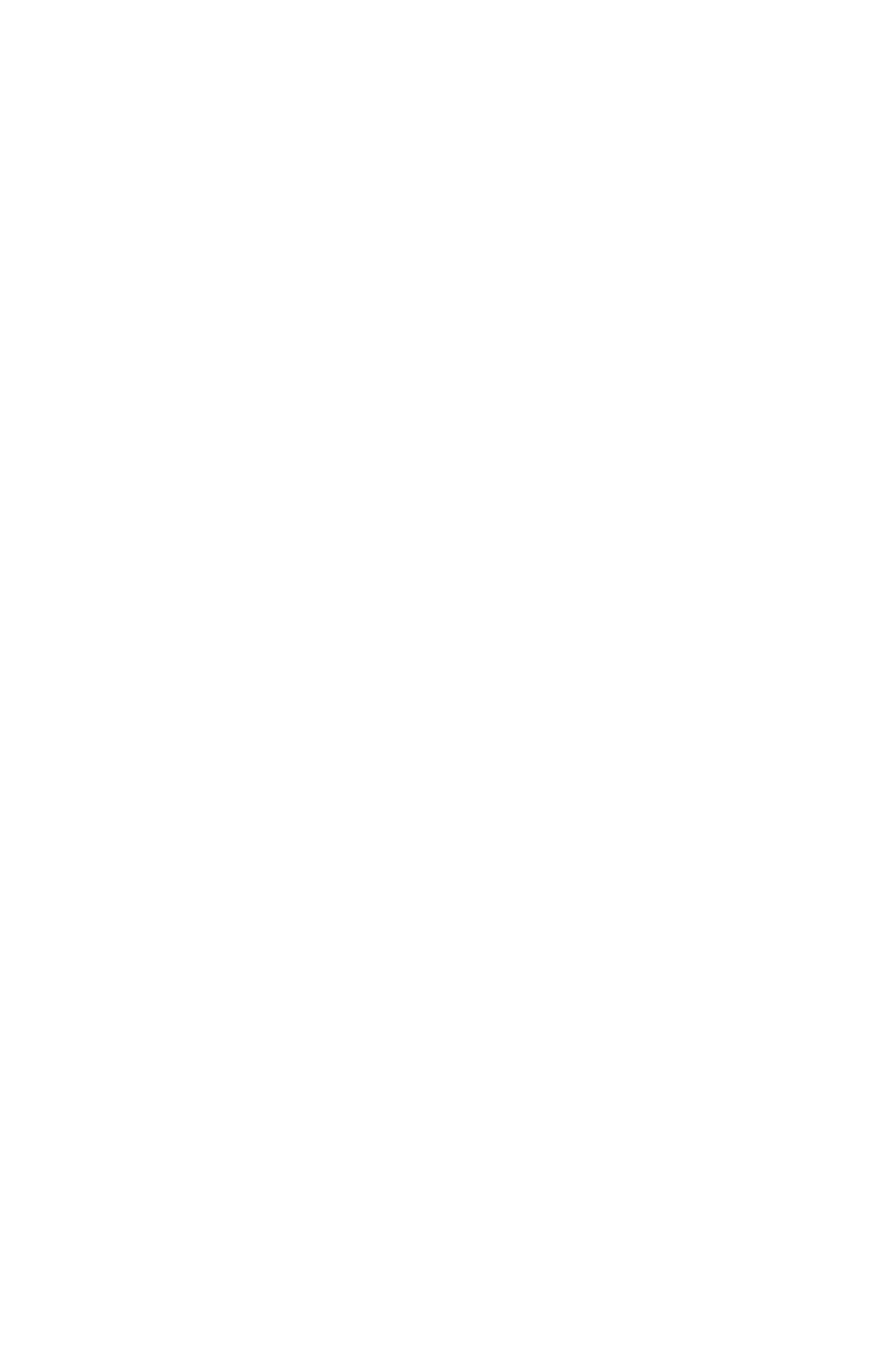




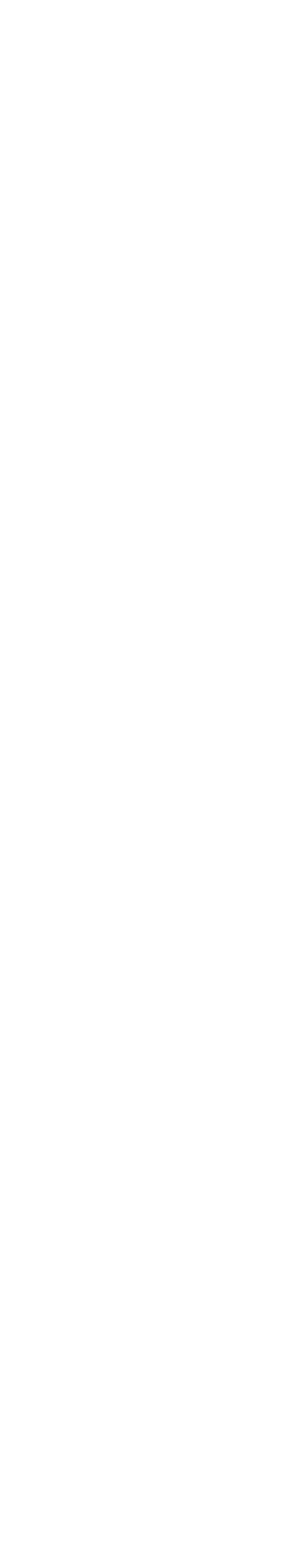




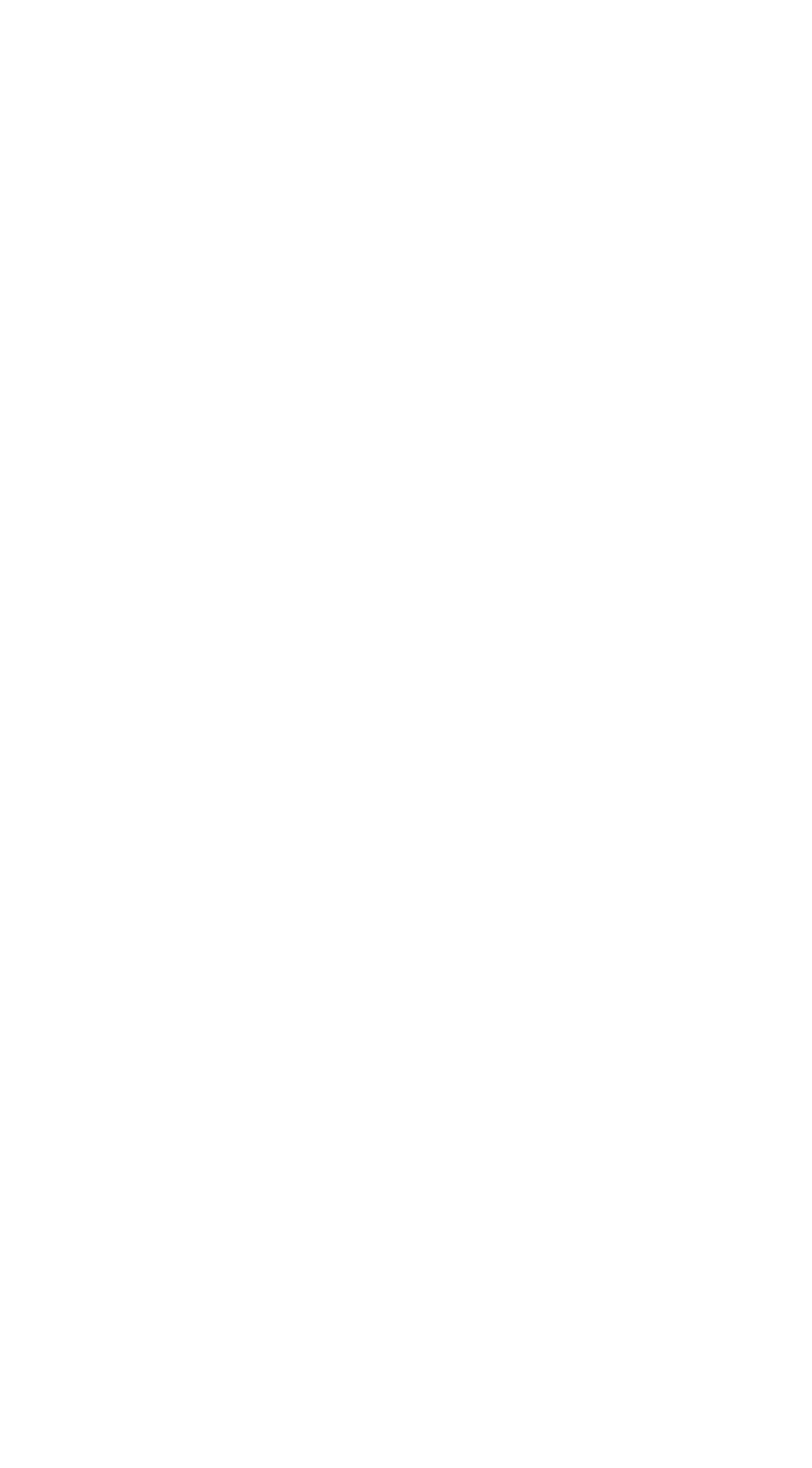




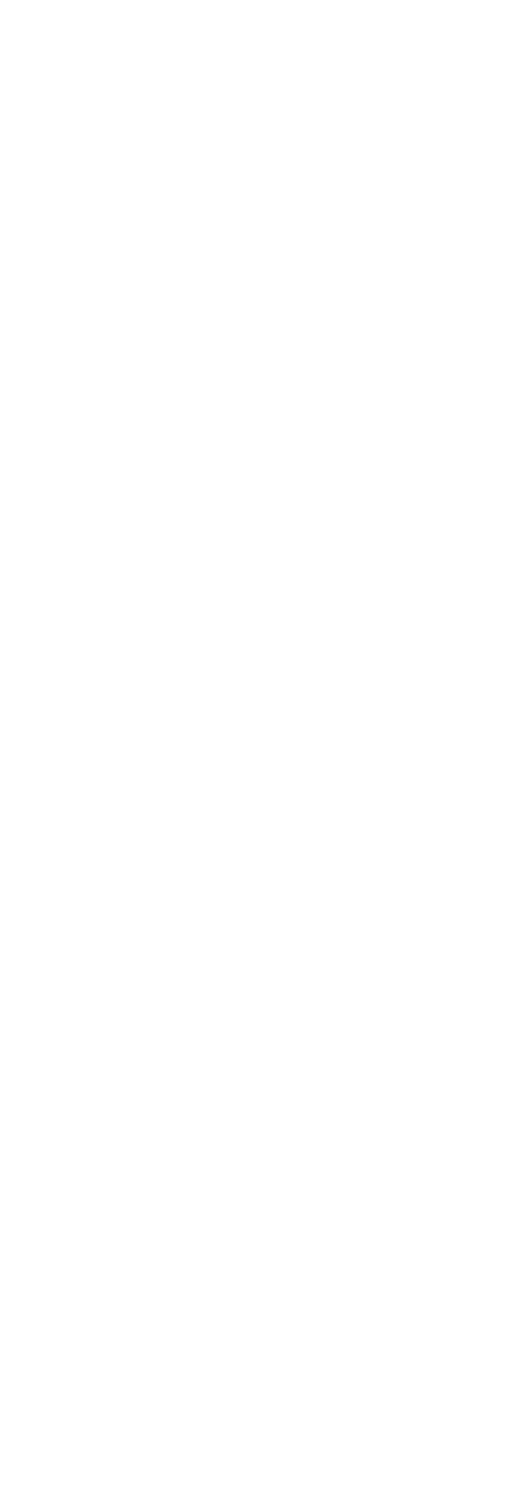




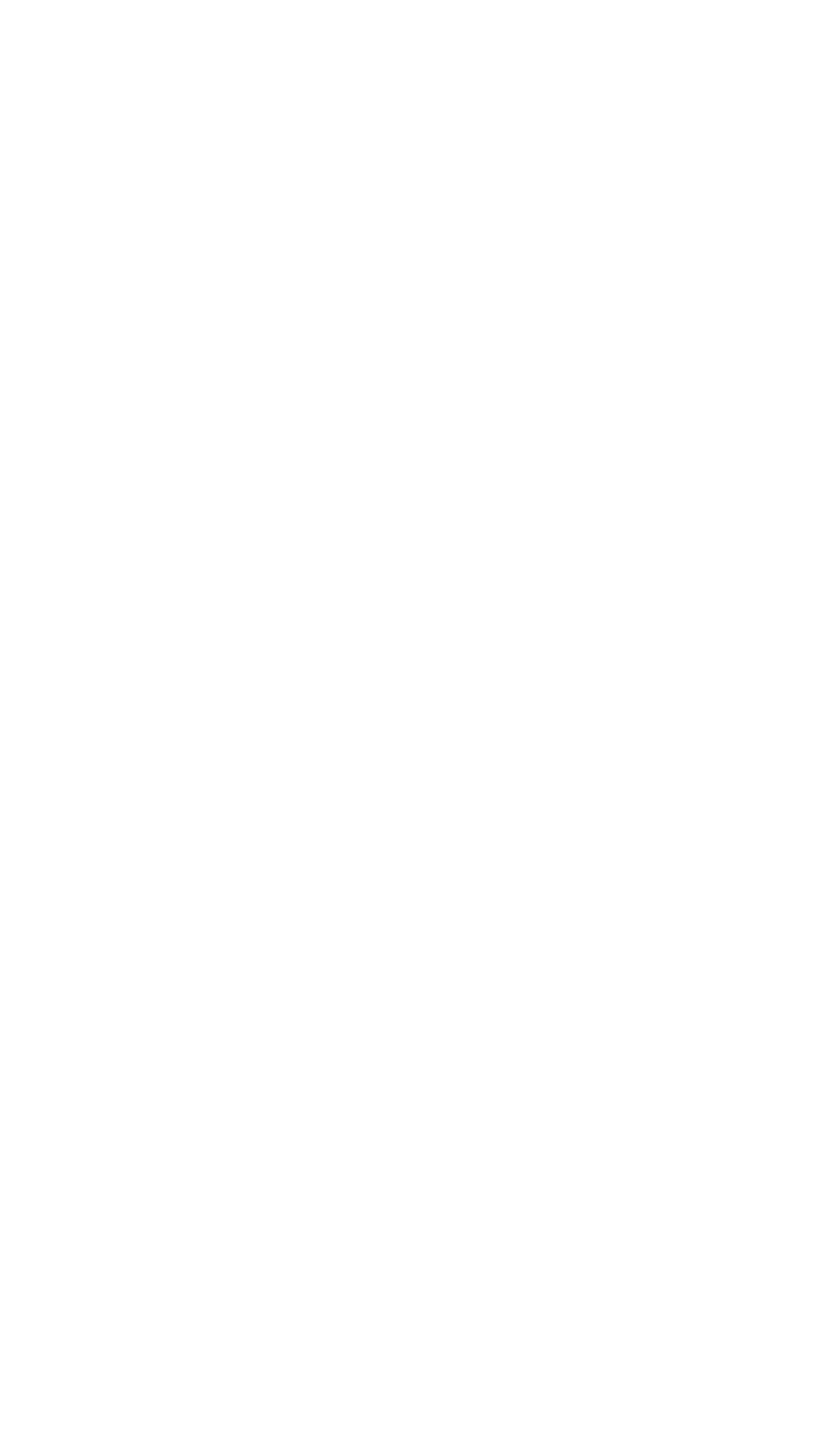




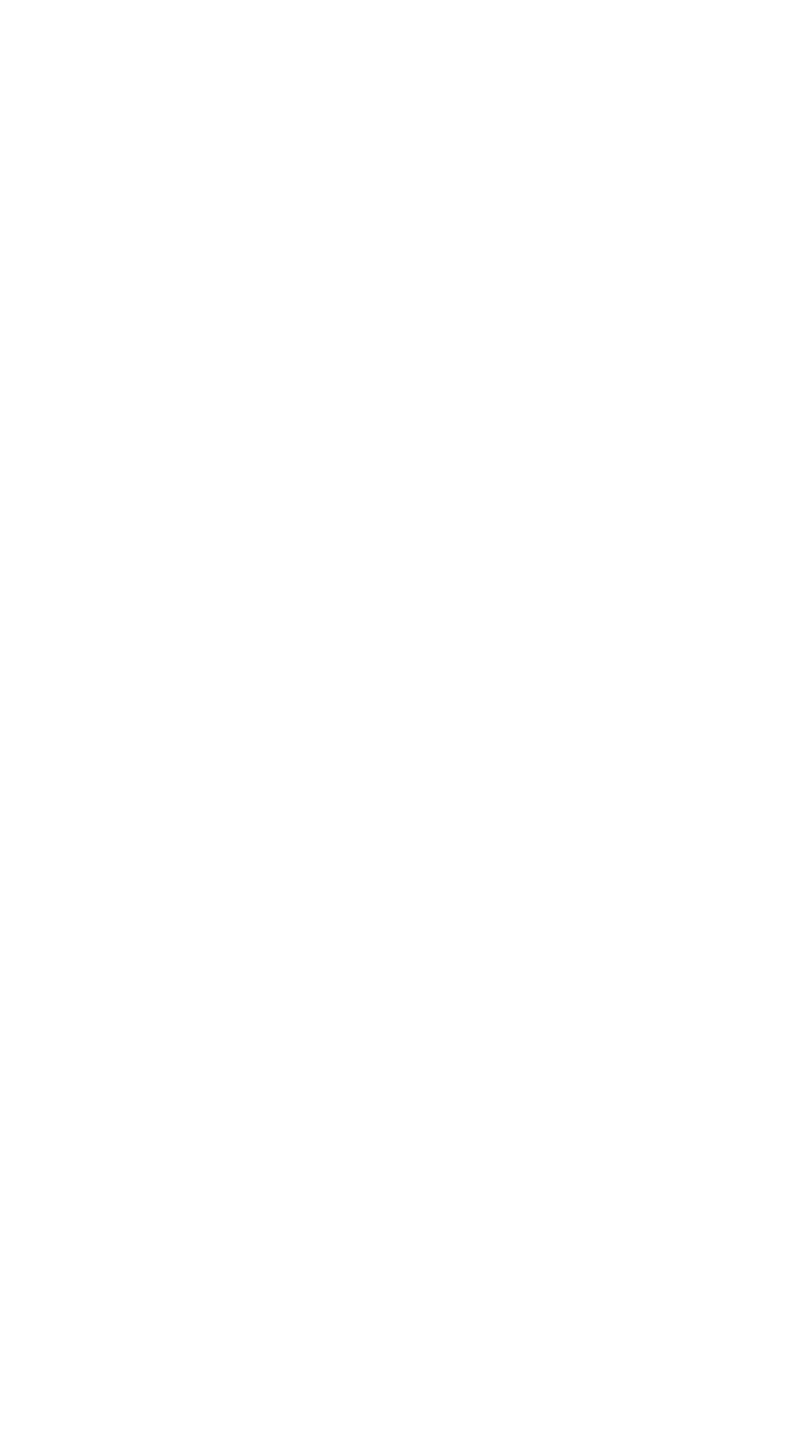




\begin{tabular}{|c|c|}
\hline 圆 & 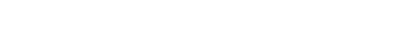 \\
\hline 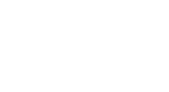 & 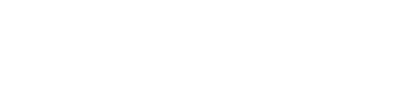 \\
\hline 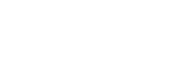 & 71 \\
\hline
\end{tabular}

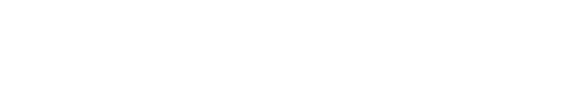

臿

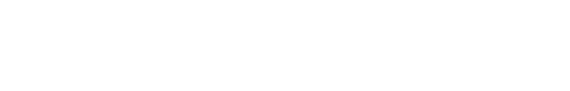

萧

政

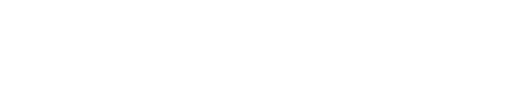

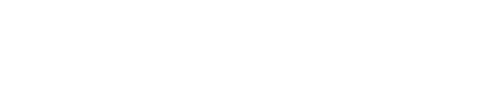

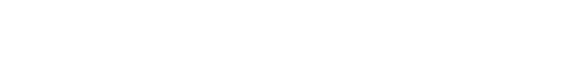

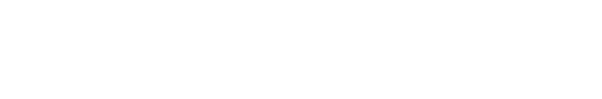

\begin{tabular}{|c|c|}
\hline 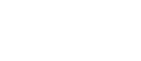 & 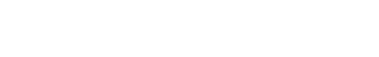 \\
\hline 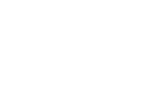 & 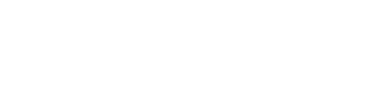 \\
\hline 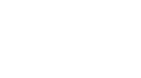 & 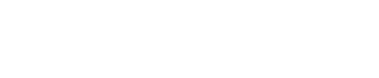 \\
\hline 立产里记 & 00000000000000000 \\
\hline 亩就高㥼 & 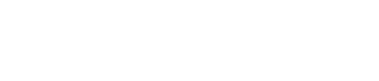 \\
\hline 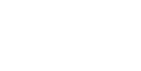 & 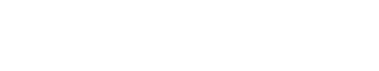 \\
\hline 害璦 & 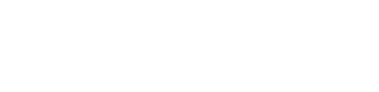 \\
\hline 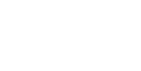 & $1: 111: 114: 1 \quad 10100011$ \\
\hline 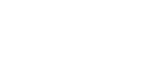 & 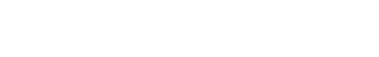 \\
\hline 挽 & 111111 11111 \\
\hline 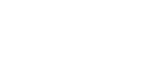 & H:HA HH:H \\
\hline 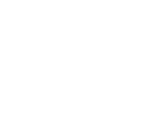 & 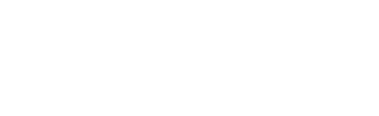 \\
\hline 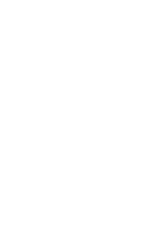 & 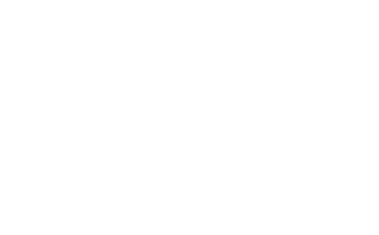 \\
\hline
\end{tabular}




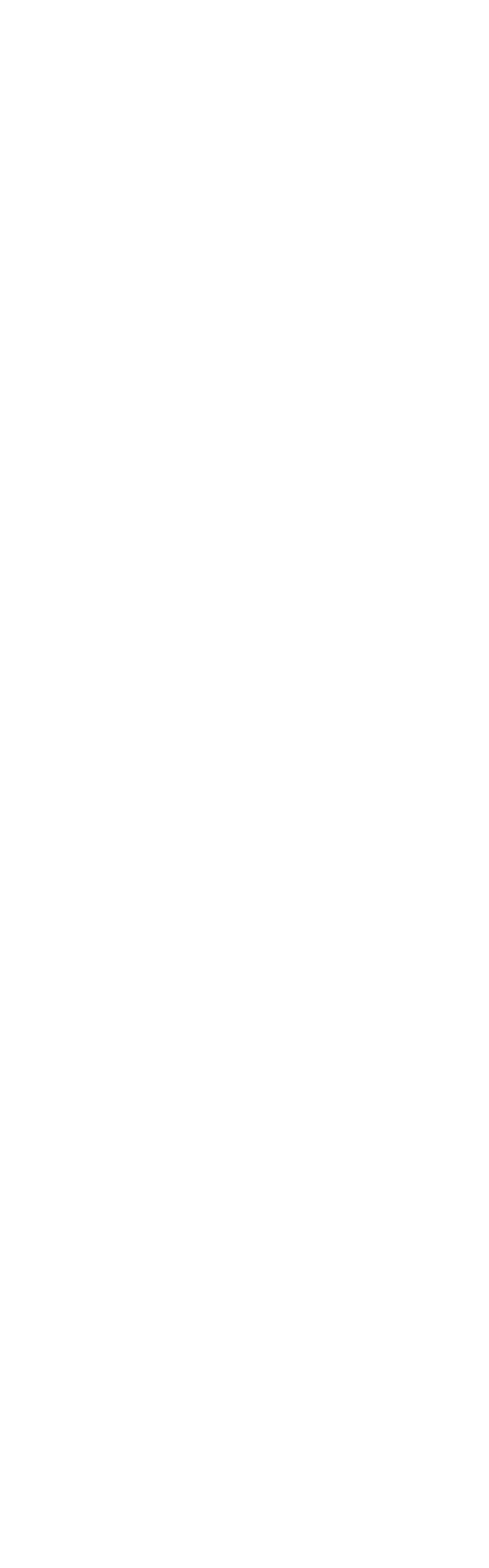




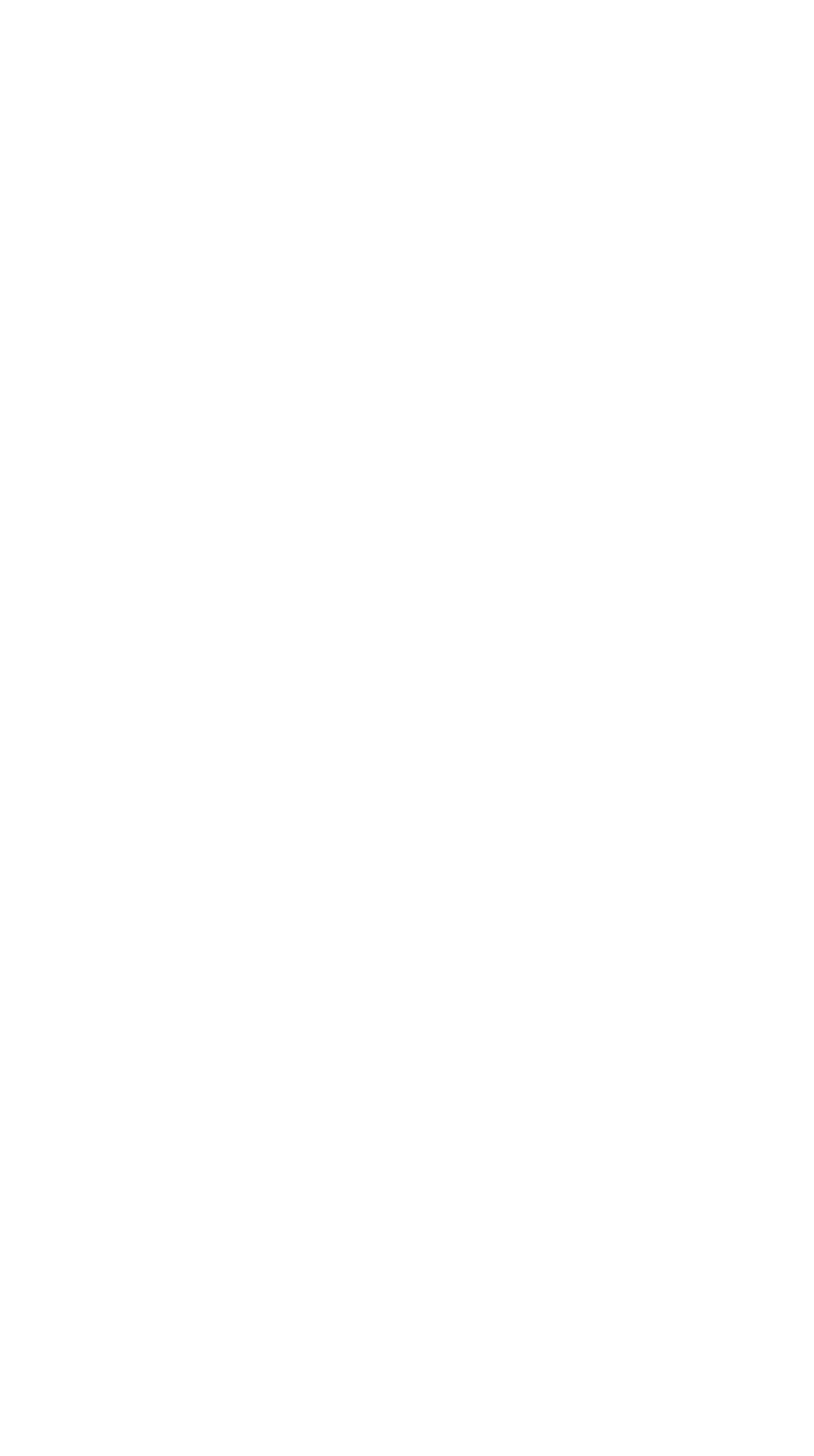




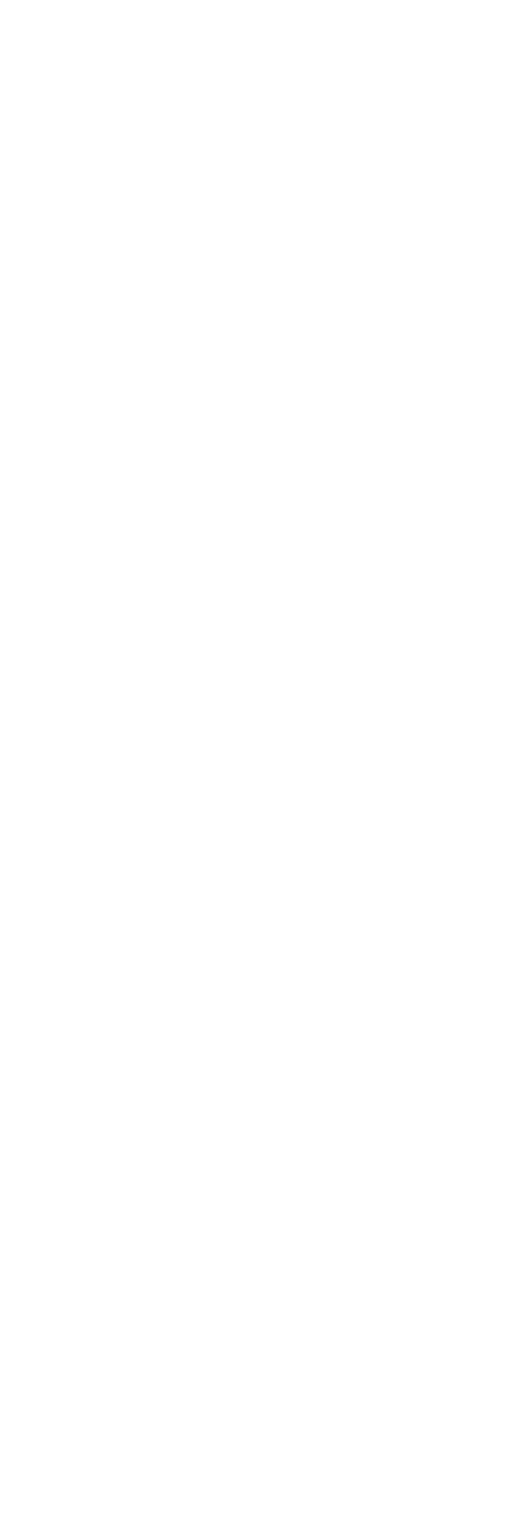


RIO GRANDE BASIN--Continued

8-3965. PECOS RIVER NEAR ARTESIA, N. MEX.--Continued

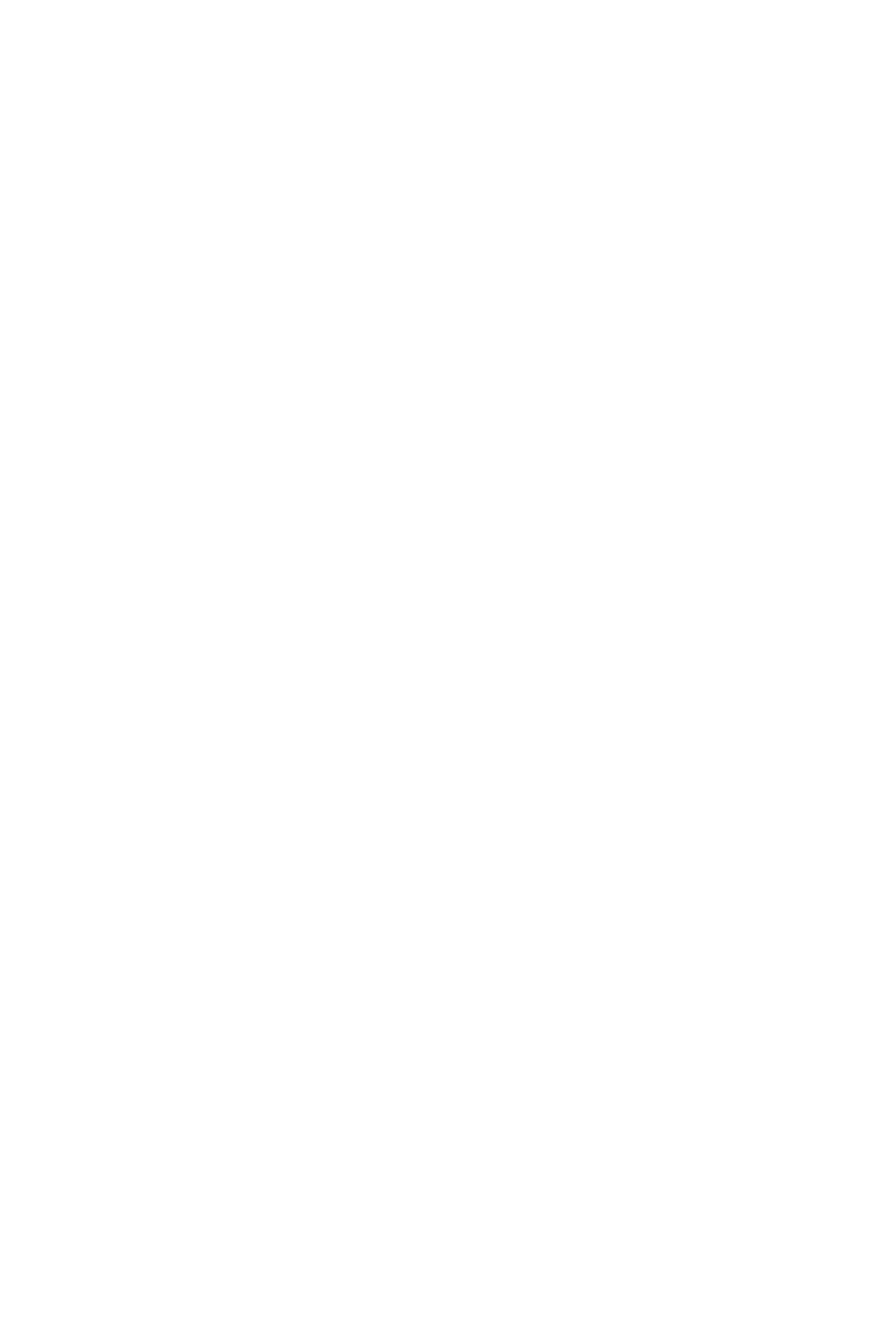

B Computed from estimated-concentration graph.

C Composite period. 
RIO GRANDE BASIN--Continued

8-3965. PECOS RIVER NEAR ARTESIA, N. MEX,--Continued

Suspended sediment, water year October 1963 to September 1964--Continued

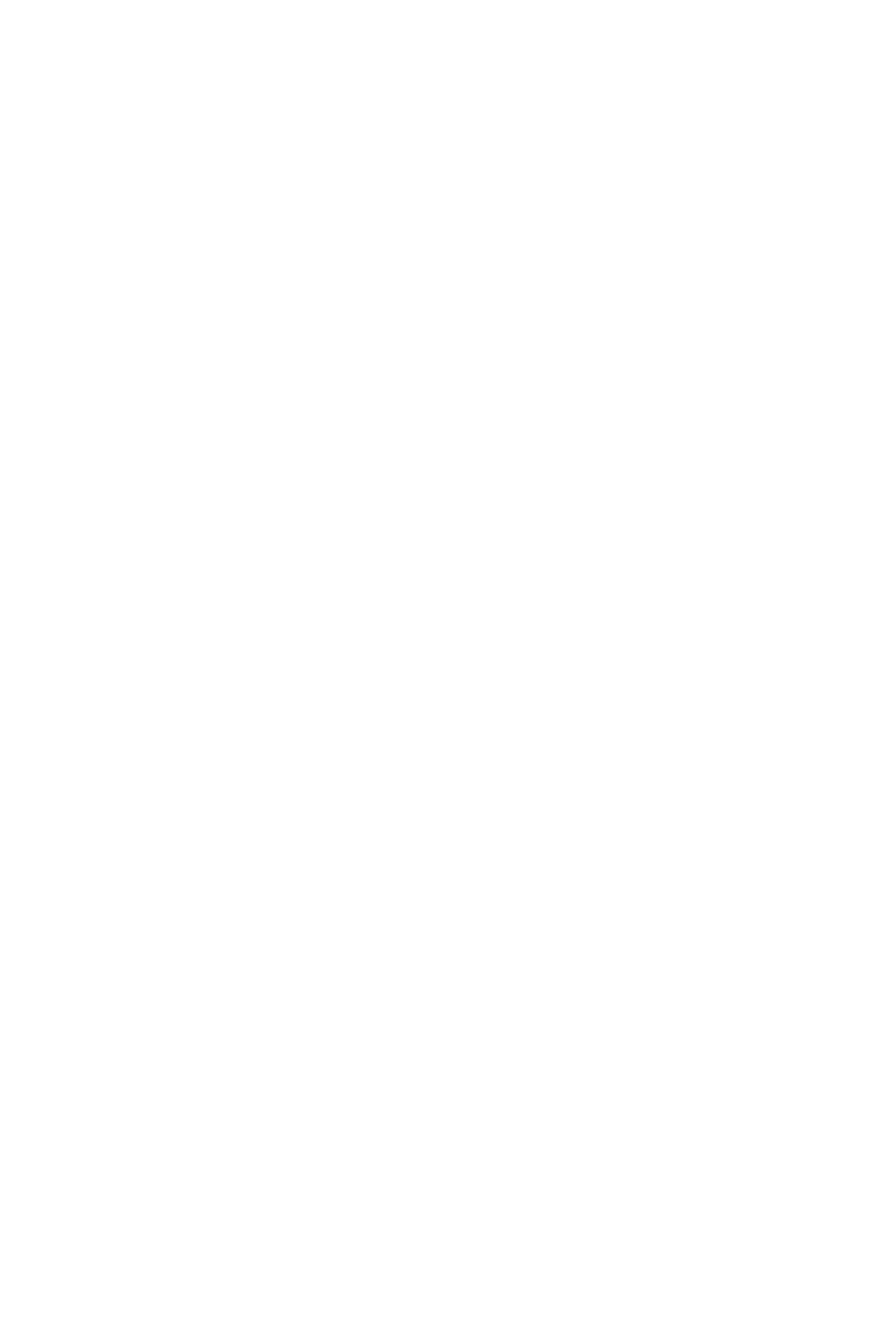

$E$ Estimated.

S Computed by subdividing day.

$T$ Less than 0.50 ton.

C Composite period. 


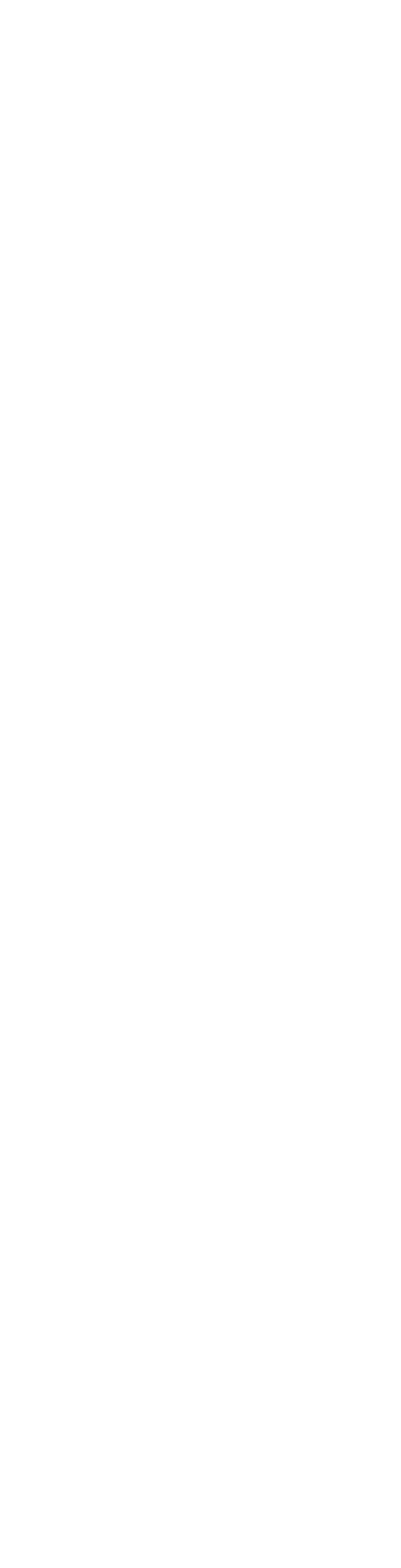


RIO GRANDE BASIN--Continued

8-3985. RIO PENASCO AT DAYTON, N, MEX.

LOCATION.--At gaging station at abandoned diversion dam, $1 \mathrm{mile}$ northeast of old Dayton railway station, 3.5 miles upstream from mouth, and 7 miles southeast of Artesia, Eddy County.

DRAINAGE AREA.--1,070 square miles, approximately.

RECORDS AVAILABLE.--Sediment records: September ig61 to September 1964

EXTREMES, 1963-64.--Sediment concentrations: Maximum daily, 1,300 ppm J jne 14; minimum daily, no flow on many days.

Sediment loads: Maximum daily, 2,400 tons June 13; minimum daily, 0 tons on many days.

EXTREAIES, 1951-64.--Sediment concentrations: Maximum daily, $30,000 \mathrm{ppm}$ Oct. 7 , 1954; minimum daily, no flow on many days.

Sediment loads: Maximum daily, 600,000 tons Oct. 7, 1954; minimum daily, 0 tons on many days.

REMARKS,--Records of specific conductance of daily samples and water temperatures available in district office at A1buquerque, $N$. Mex. Flow occurred on1y on days indicated.

Suspended sediment, water year October 1963 to September 1964

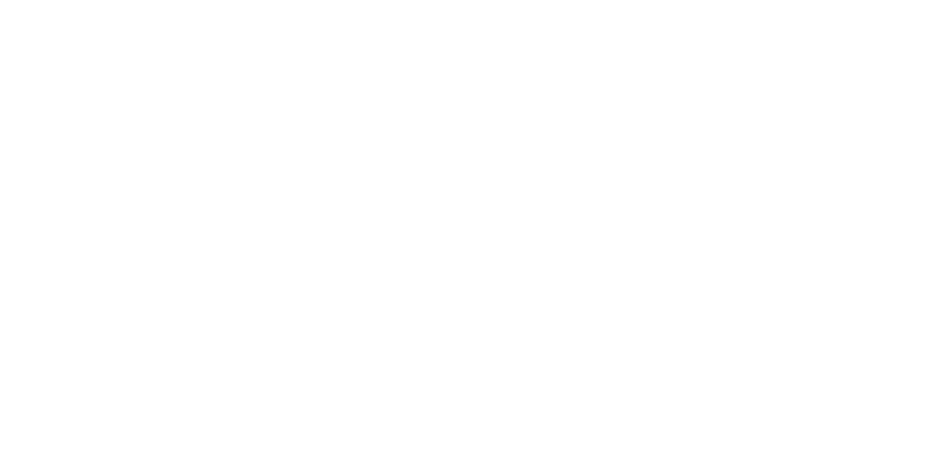

E Estimated.

S Computed by subdividing day.

$\mathrm{K}$ Computed from estimated concentration graph and subdividing day. 


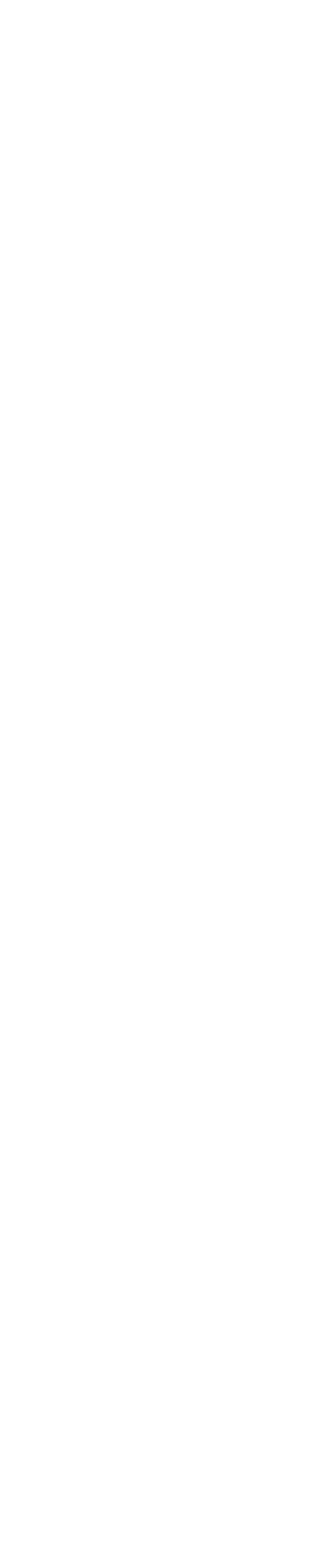



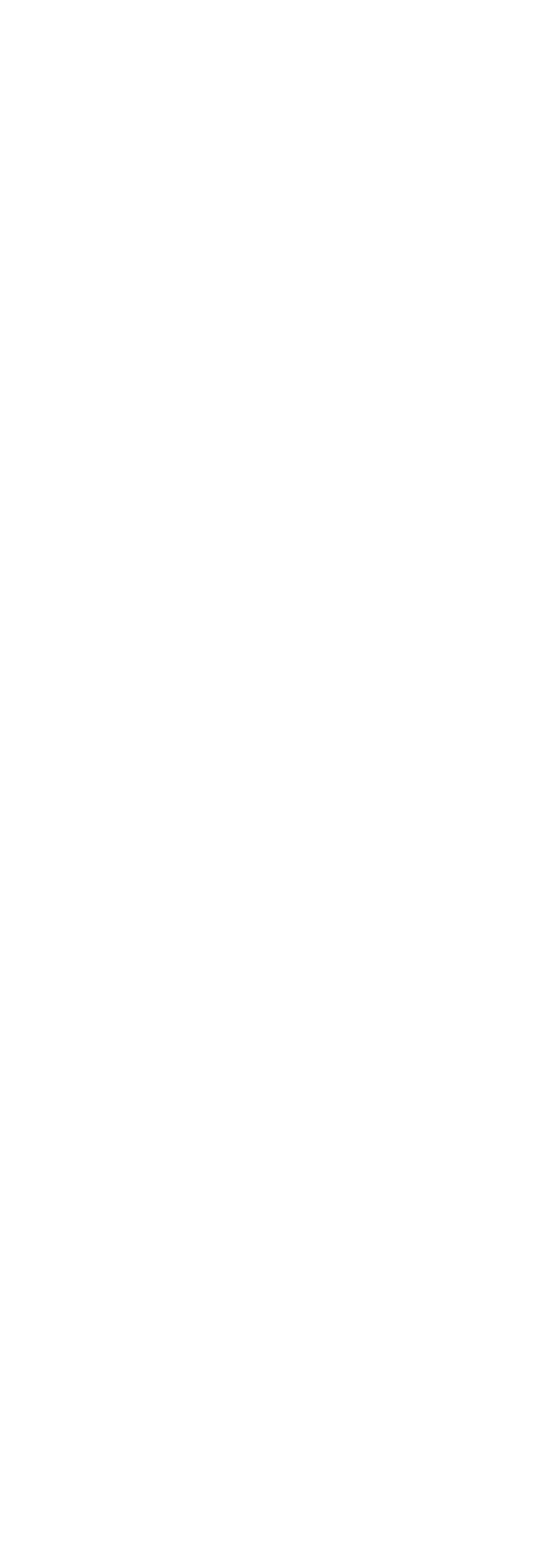

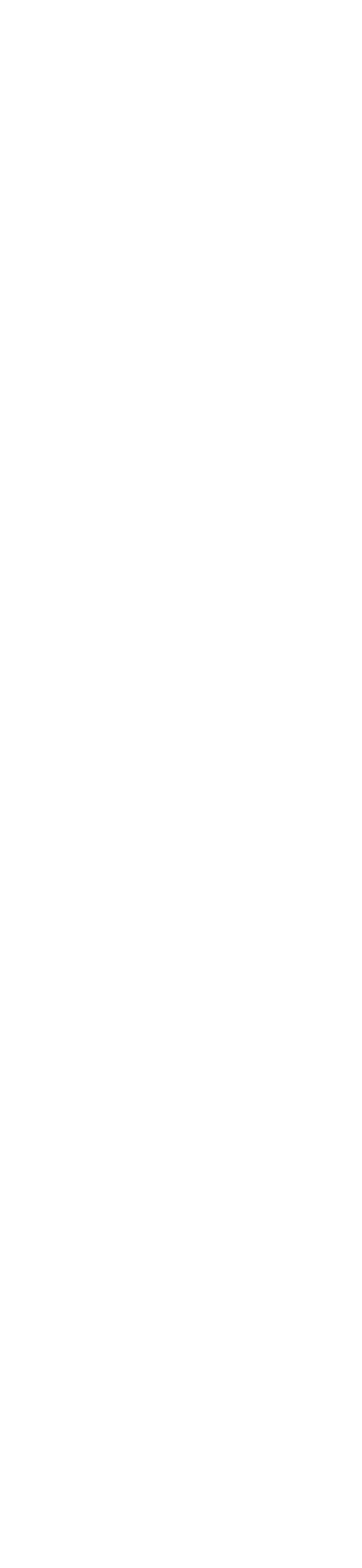




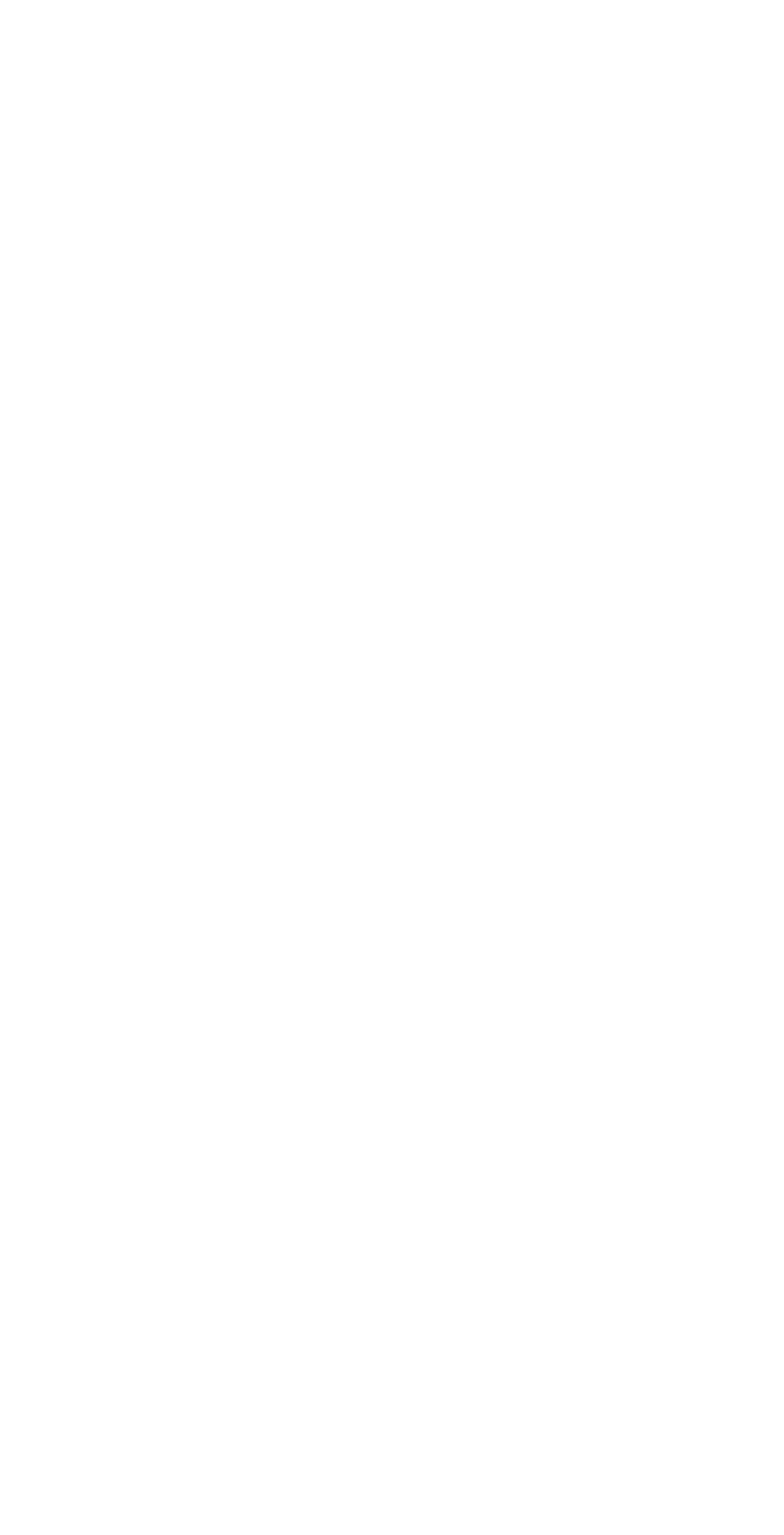




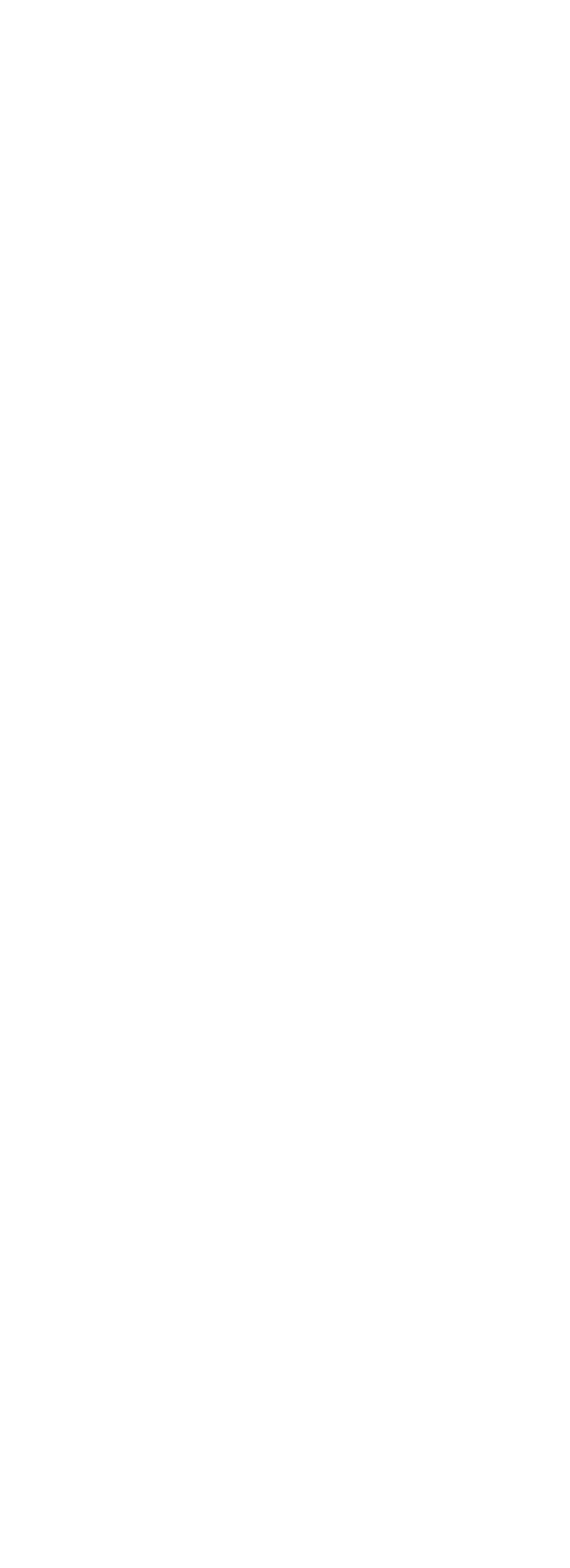




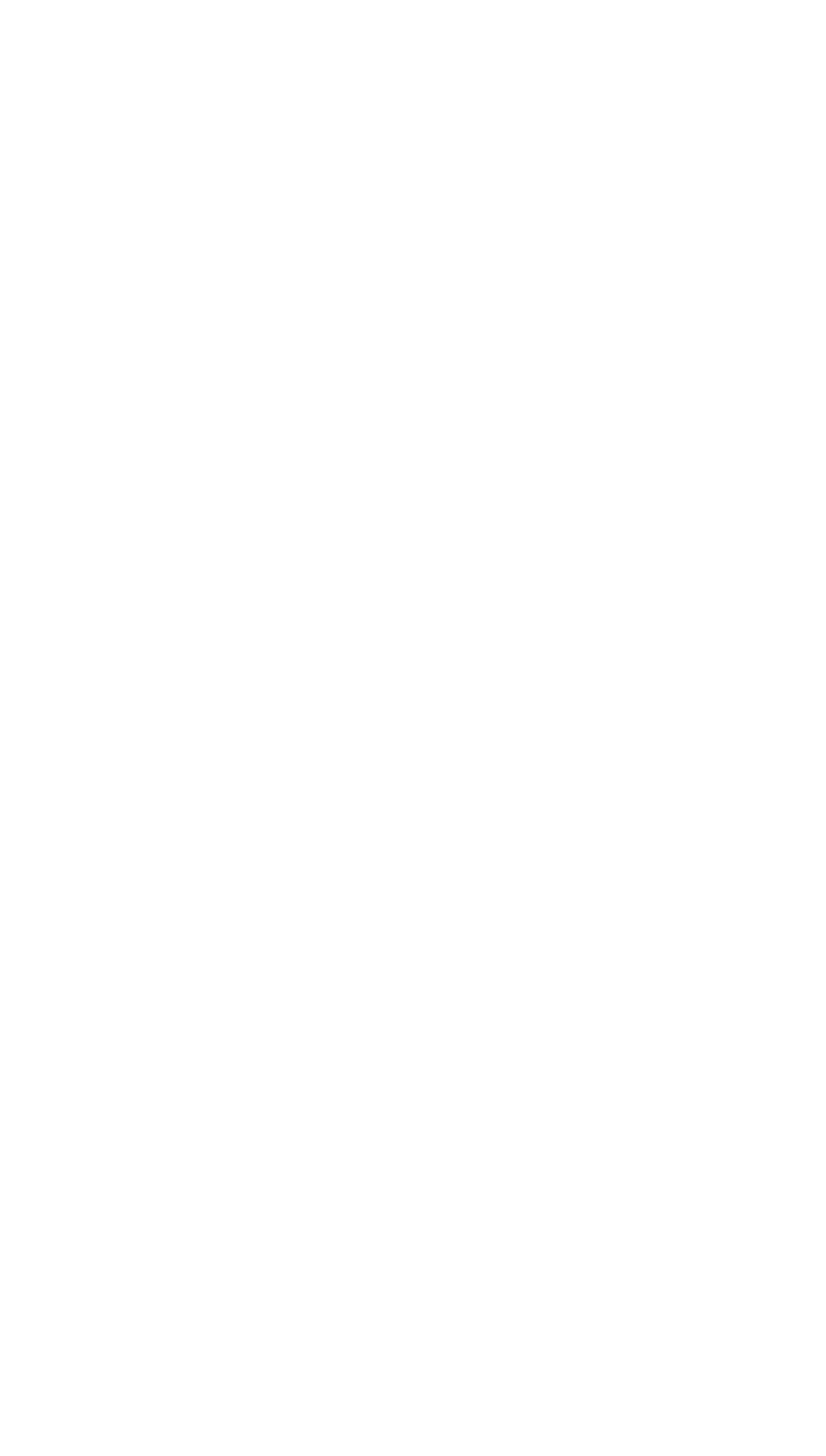




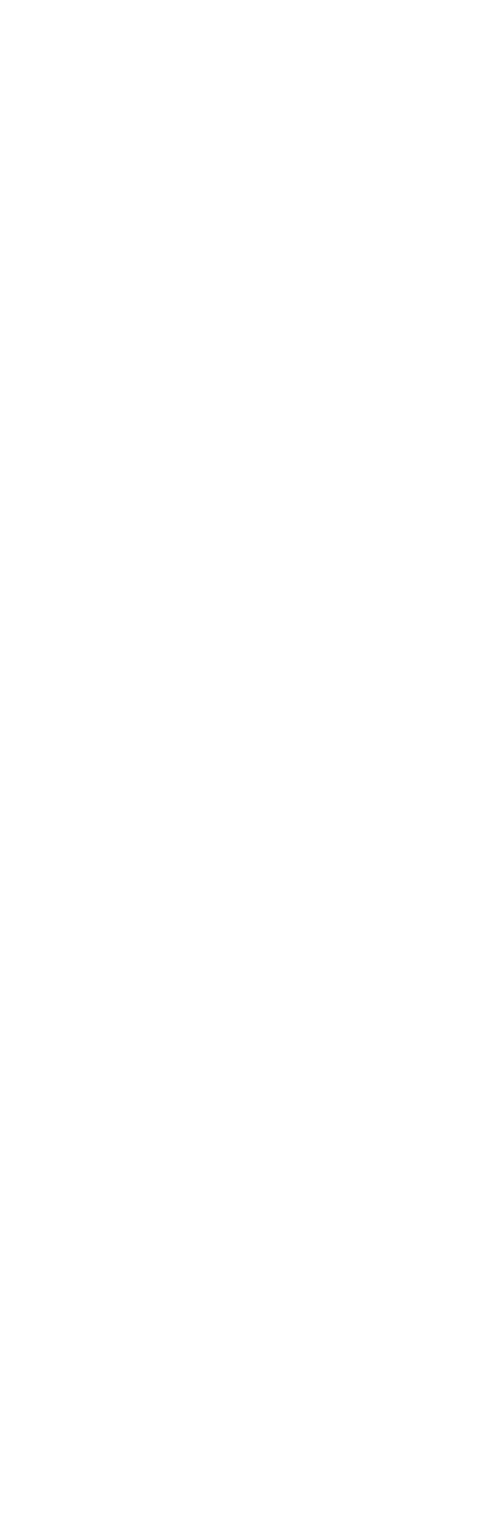




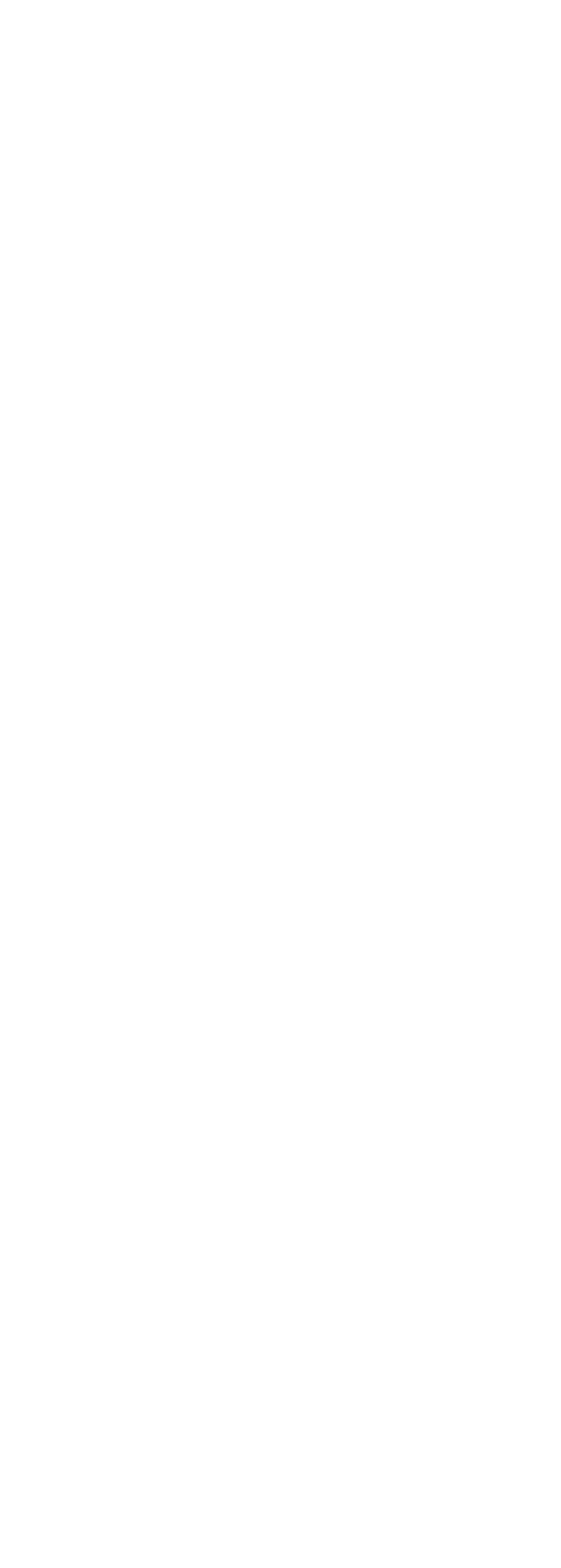




\begin{tabular}{|c|c|}
\hline 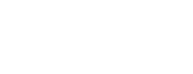 & ن \\
\hline 要 & بgen \\
\hline 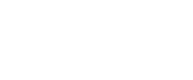 & 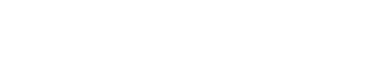 \\
\hline 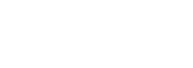 & 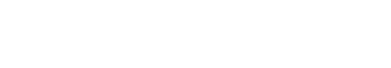 \\
\hline : & 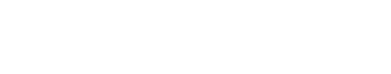 \\
\hline 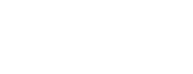 & 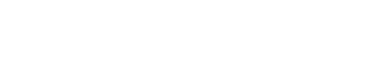 \\
\hline 总 哭 & 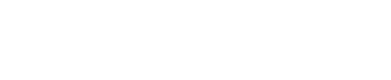 \\
\hline 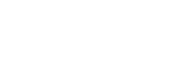 & 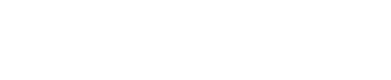 \\
\hline 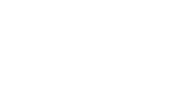 & 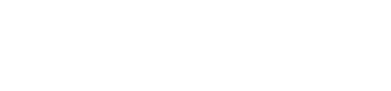 \\
\hline 宫点鱼 & 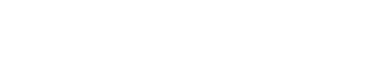 \\
\hline 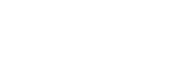 & 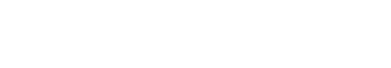 \\
\hline 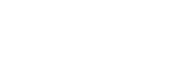 & 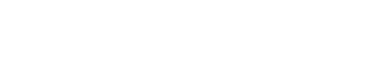 \\
\hline 害司 & 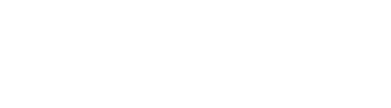 \\
\hline 密。 & 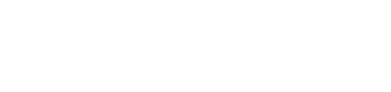 \\
\hline दुँ & \\
\hline 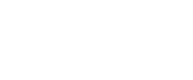 & 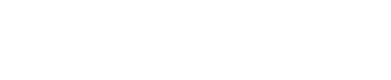 \\
\hline 官守慁区 & 1:1:10 1:1:1:11 \\
\hline 糸蛋 & 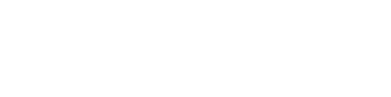 \\
\hline 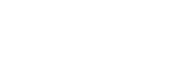 & 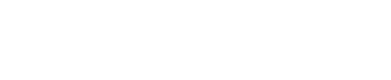 \\
\hline 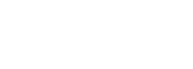 & 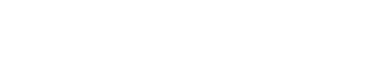 \\
\hline 占离 & $\begin{array}{l}111150 \\
0\end{array}$ \\
\hline 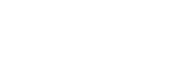 & 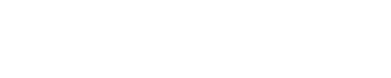 \\
\hline 造 & 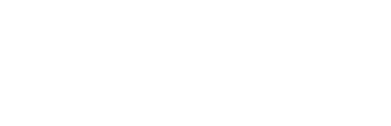 \\
\hline 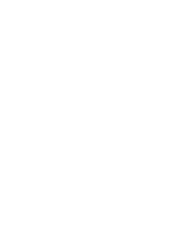 & 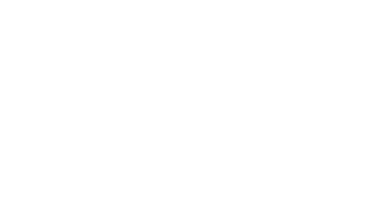 \\
\hline
\end{tabular}




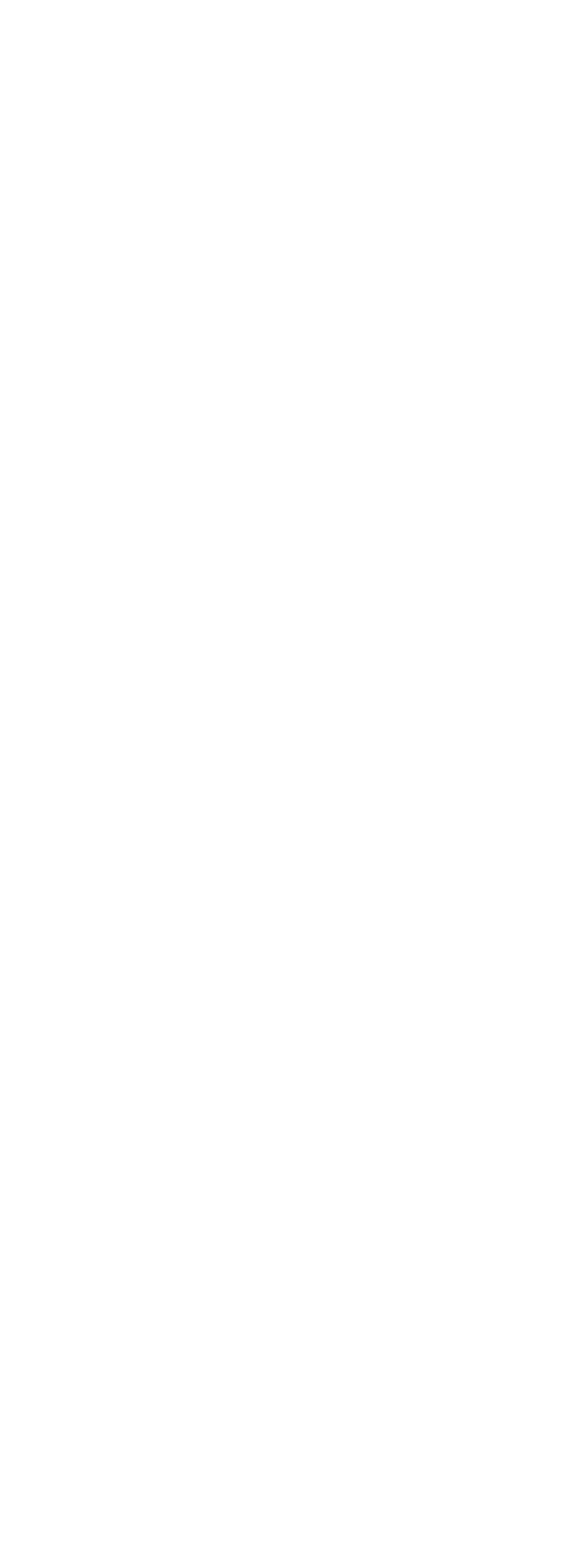




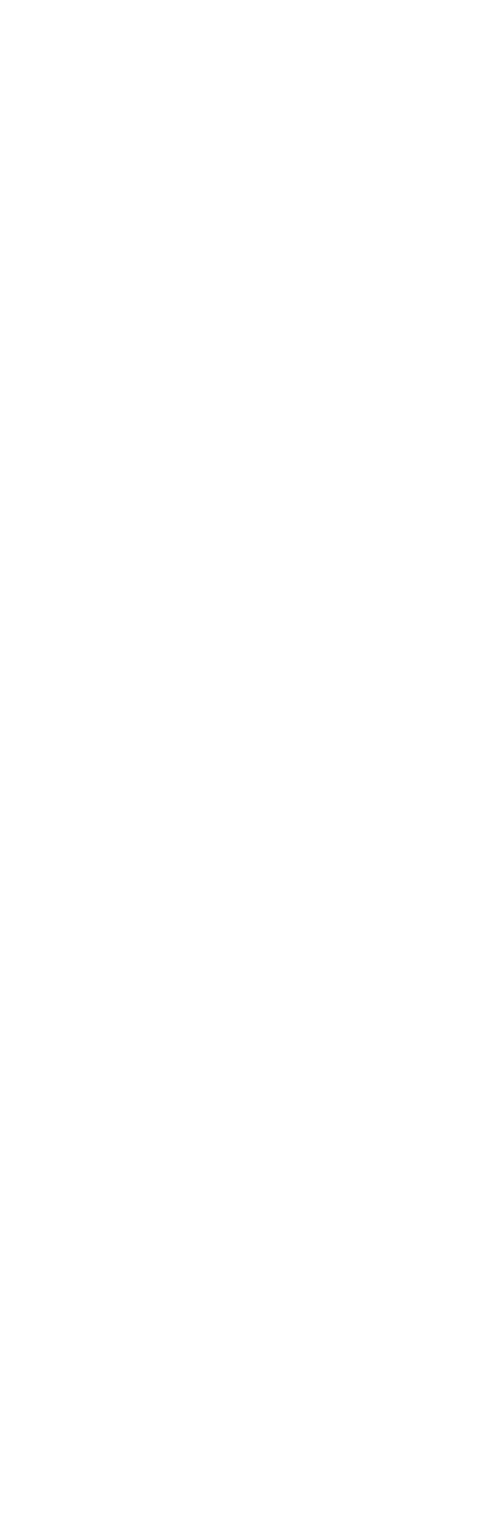




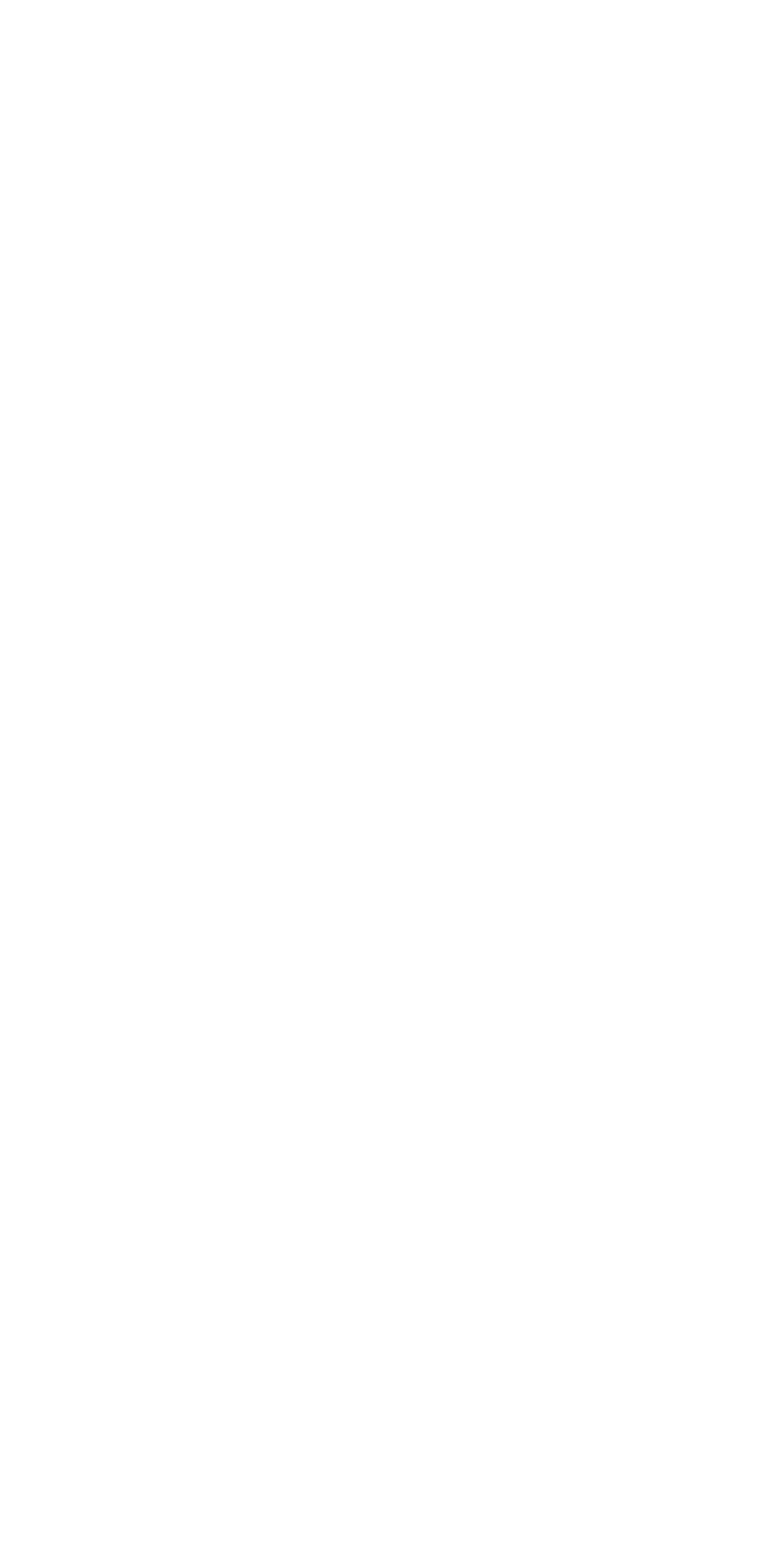




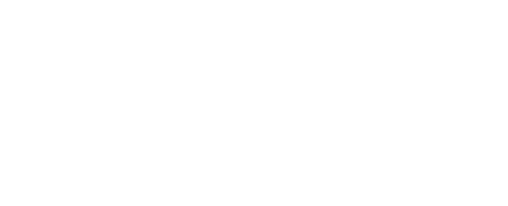

\begin{tabular}{|c|c|c|c|c|}
\hline कींब & त̄ & 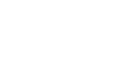 & $\stackrel{9}{2}$ & $\stackrel{\mathscr{n}}{*}$ \\
\hline 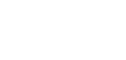 & 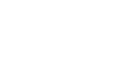 & 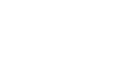 & $\stackrel{\infty}{a}$ & \begin{tabular}{l} 
P \\
\multirow{H}{*}{} \\
N
\end{tabular} \\
\hline 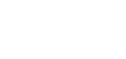 & 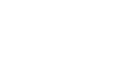 & 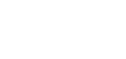 & 今) & $\underset{\substack{0 \\
0}}{\circ}$ \\
\hline
\end{tabular}

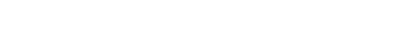

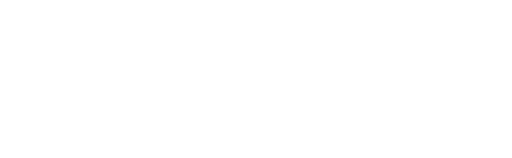

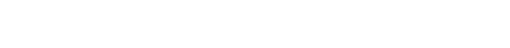

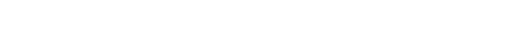
1109111 11:

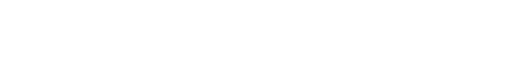

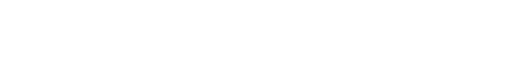

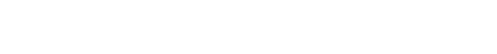

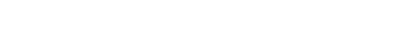

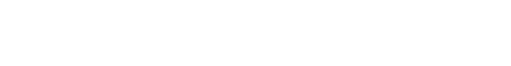

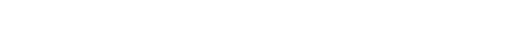

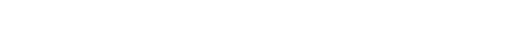

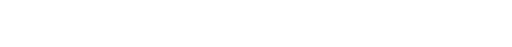

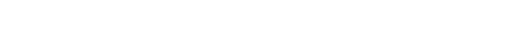

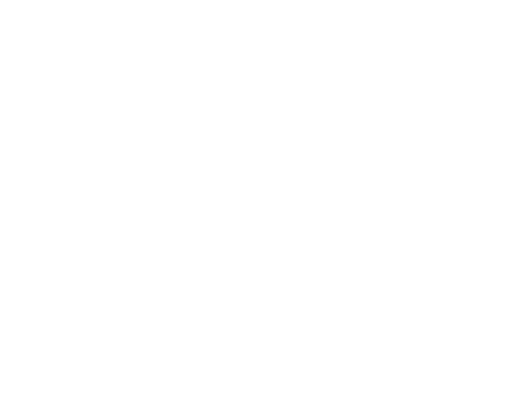

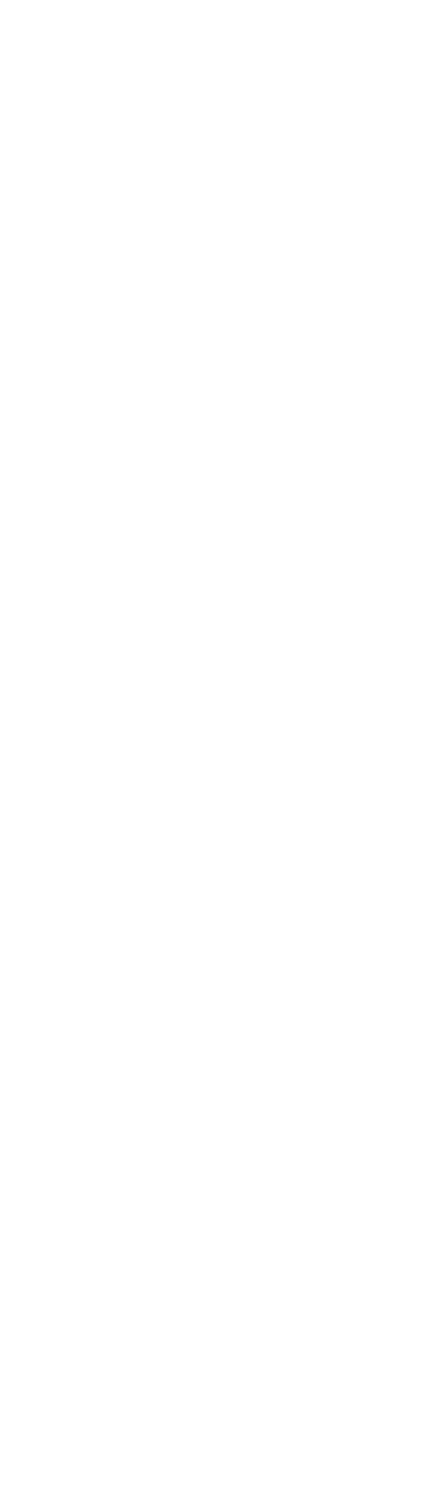


(2)

\begin{tabular}{|c|c|}
\hline 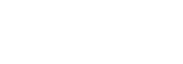 & 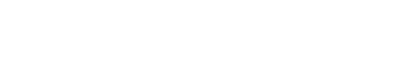 \\
\hline & 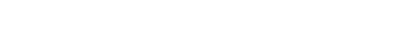 \\
\hline 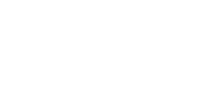 & 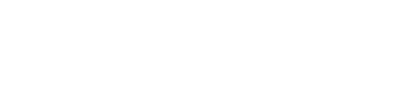 \\
\hline 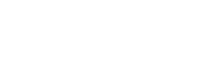 & 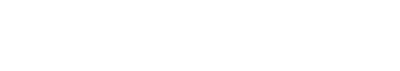 \\
\hline 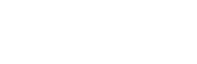 & 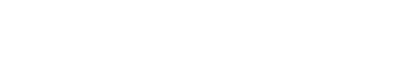 \\
\hline 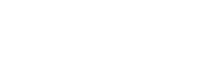 & 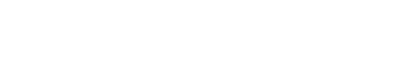 \\
\hline 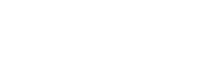 & 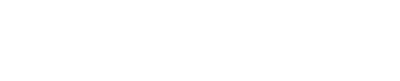 \\
\hline 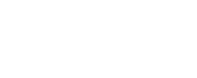 & 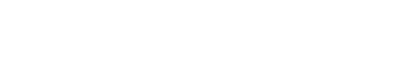 \\
\hline 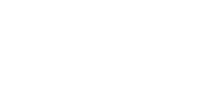 & 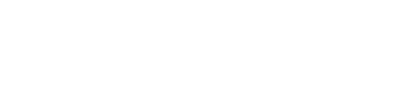 \\
\hline 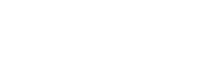 & 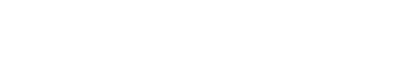 \\
\hline 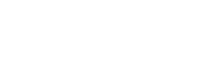 & 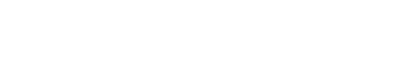 \\
\hline 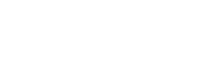 & 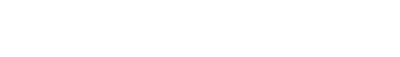 \\
\hline 递 & 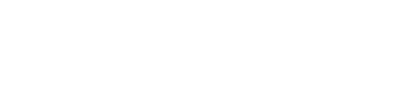 \\
\hline 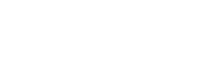 & 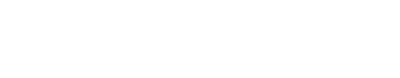 \\
\hline 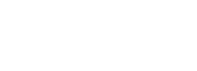 & \\
\hline 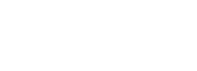 & 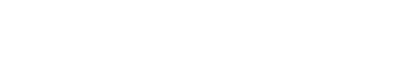 \\
\hline 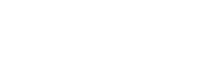 & 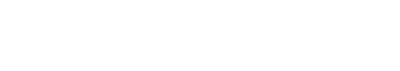 \\
\hline 害愛 & 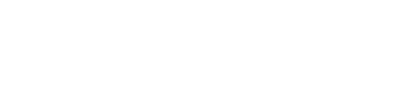 \\
\hline 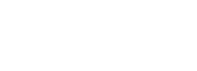 & 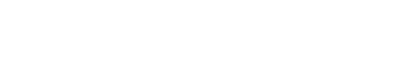 \\
\hline 秀罥要 & 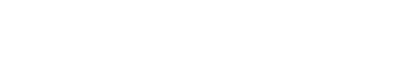 \\
\hline 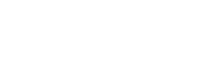 & $\begin{array}{l}14000 \\
0\end{array}$ \\
\hline 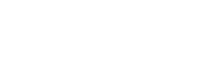 & 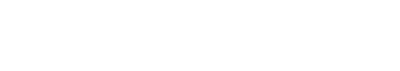 \\
\hline รั. & 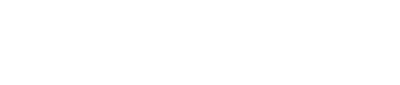 \\
\hline & 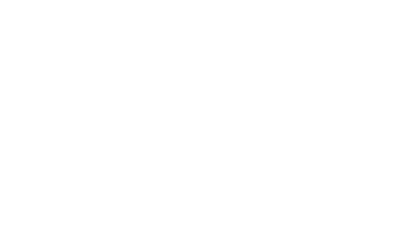 \\
\hline
\end{tabular}




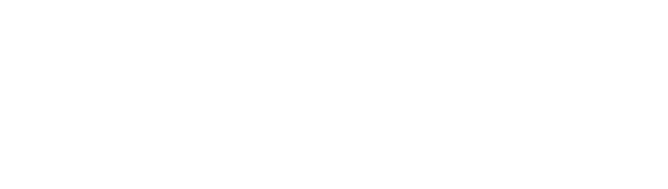

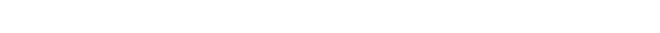

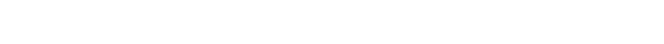

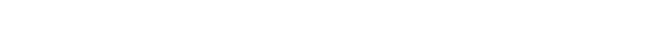

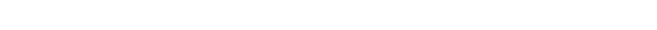

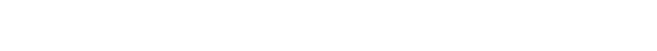

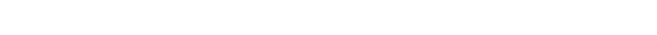

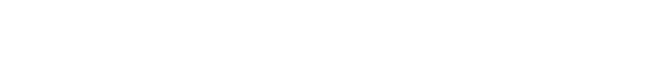

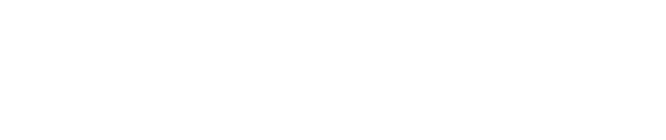

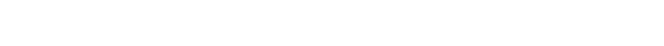

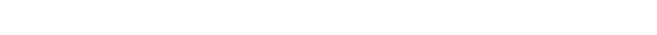
111119911191111911190911111

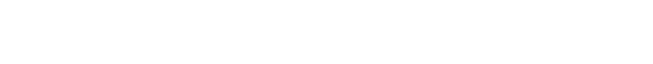

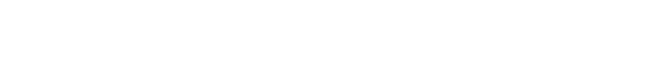

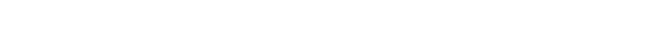
1114114

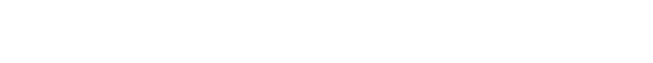

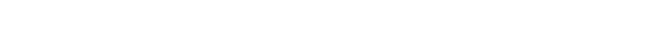

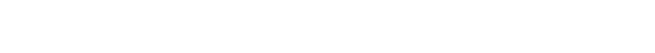

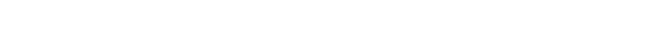

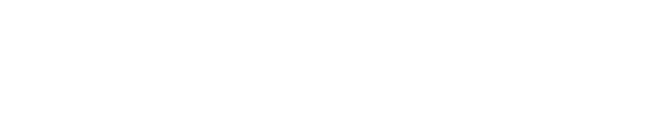

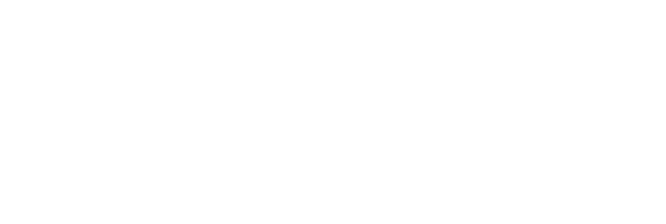




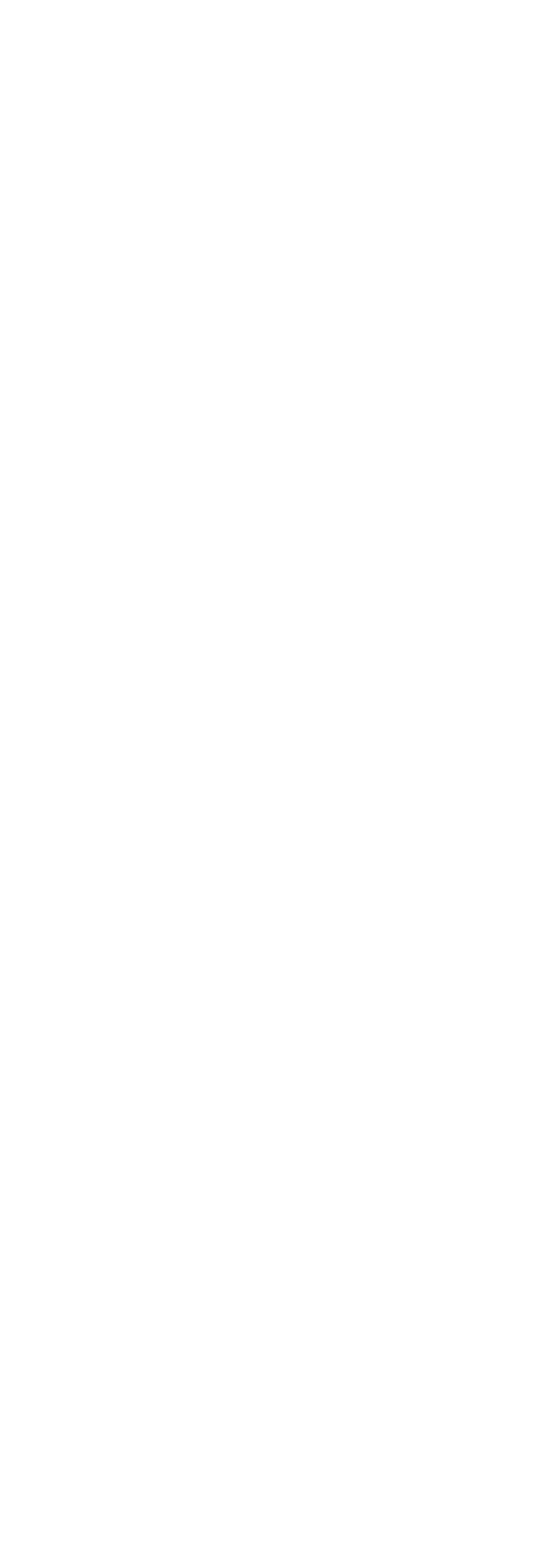




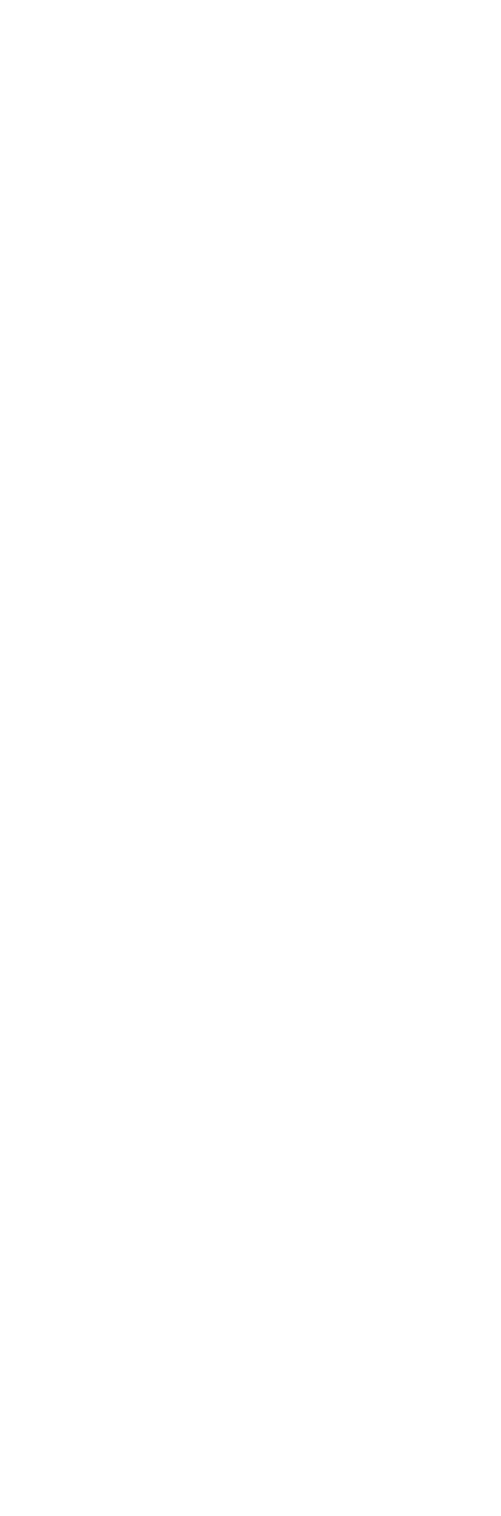




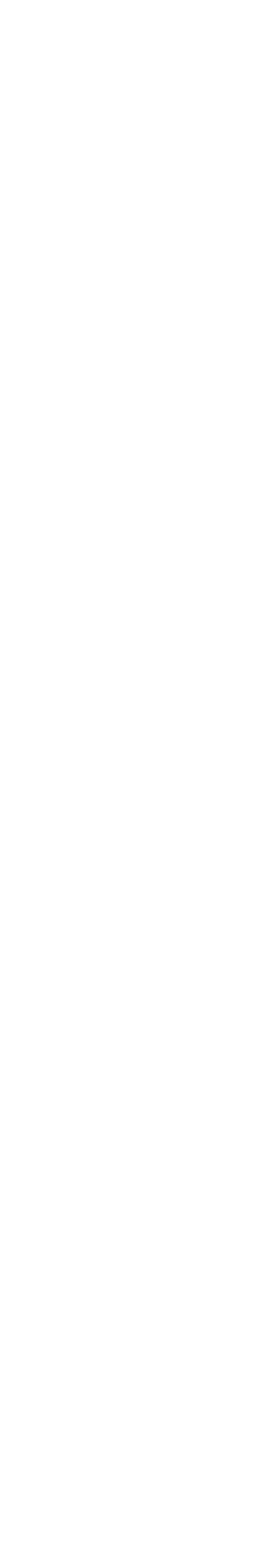

\begin{tabular}{|c|c|c|}
\hline 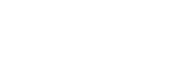 & 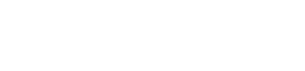 & \\
\hline 产 & 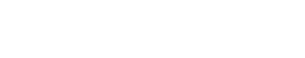 & \\
\hline 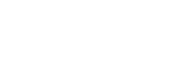 & 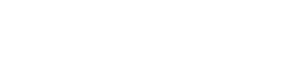 & 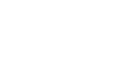 \\
\hline 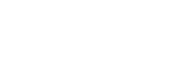 & & \\
\hline 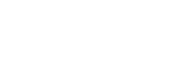 & 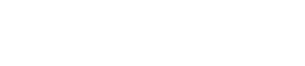 & \\
\hline 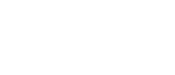 & 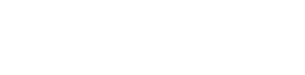 & 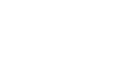 \\
\hline 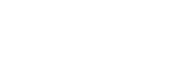 & 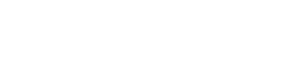 & \\
\hline 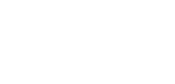 & 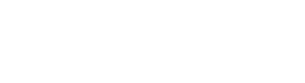 & \\
\hline 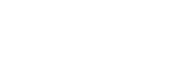 & 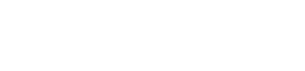 & 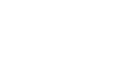 \\
\hline 宫 & & \\
\hline 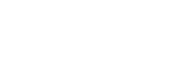 & & \\
\hline 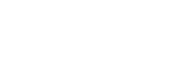 & & \\
\hline 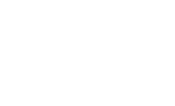 & 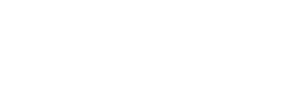 & 总品总 \\
\hline 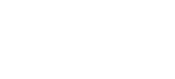 & 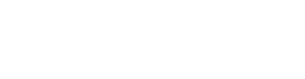 & 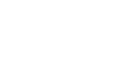 \\
\hline 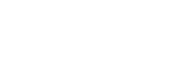 & 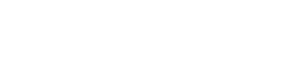 & $\because=9$ \\
\hline 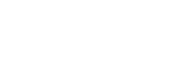 & DH:告: & \\
\hline 唇 & 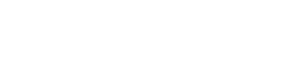 & : ڤ్ \\
\hline 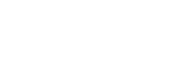 & & \\
\hline 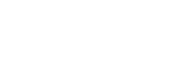 & 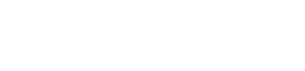 & 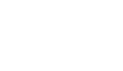 \\
\hline 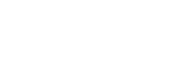 & 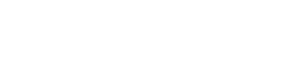 & \\
\hline 害蛋 & & \\
\hline 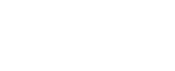 & 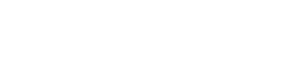 & $=:$ \\
\hline 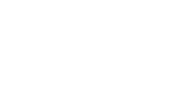 & 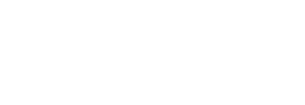 & ले \\
\hline 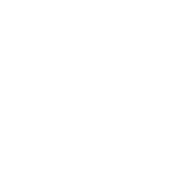 & 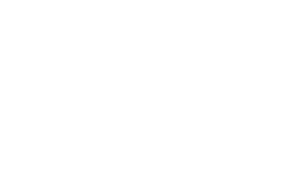 & 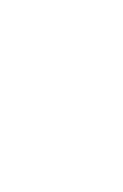 \\
\hline
\end{tabular}




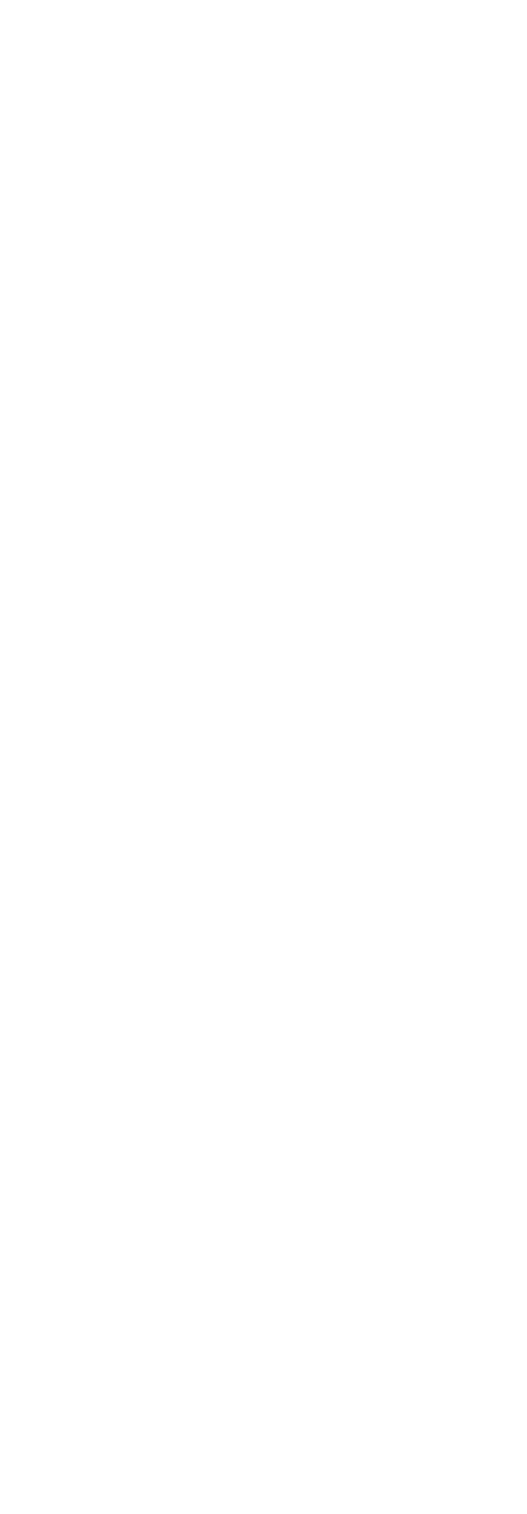




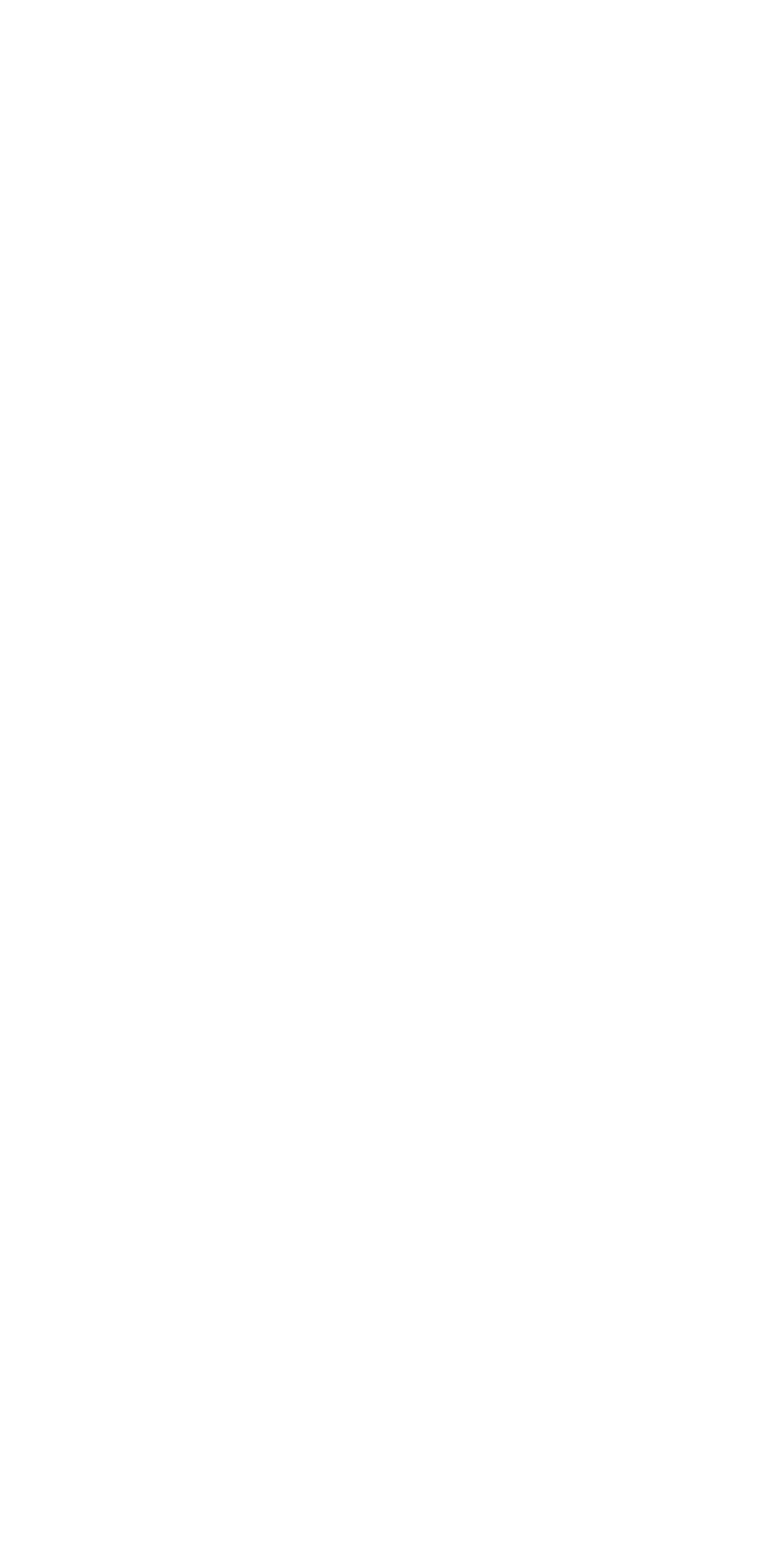



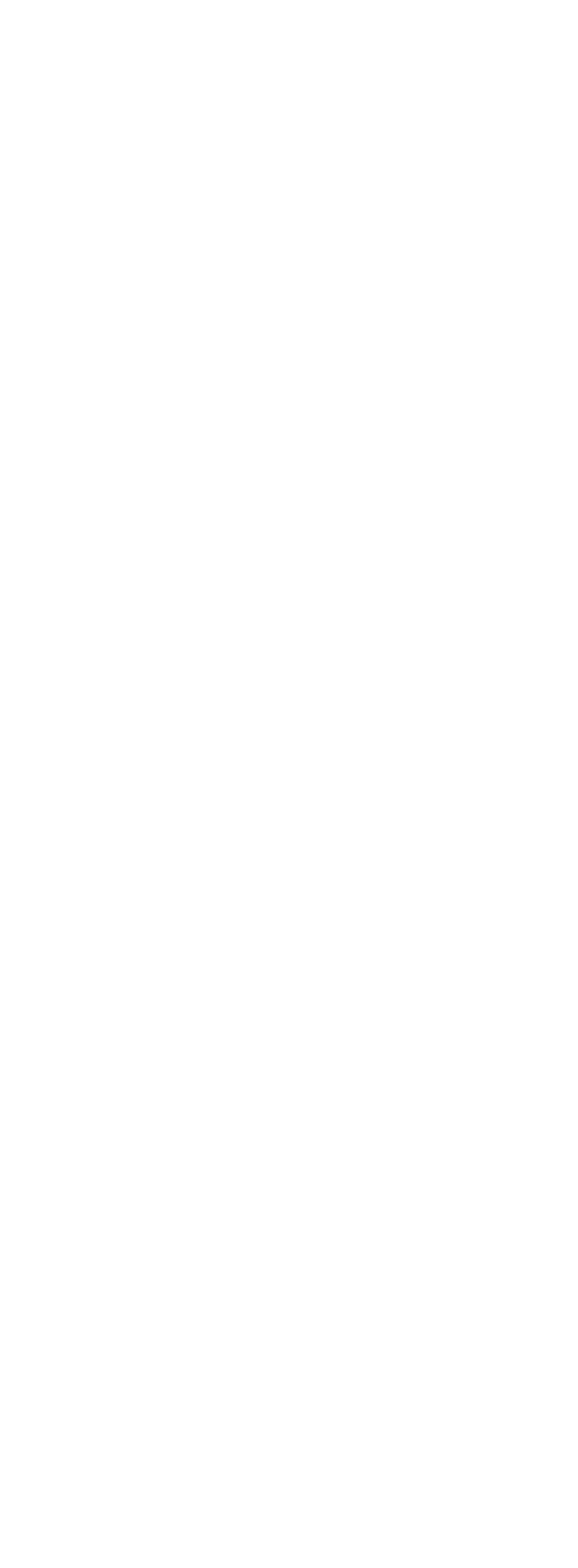


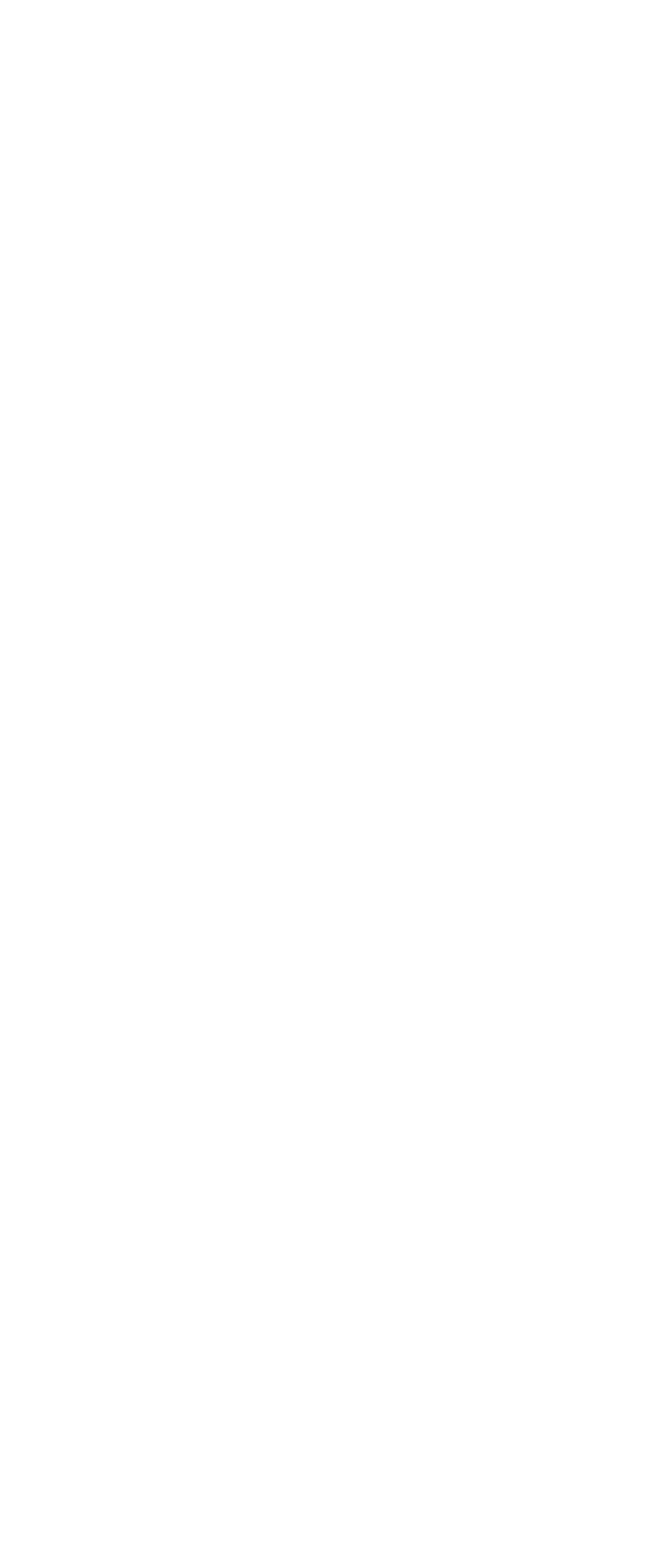




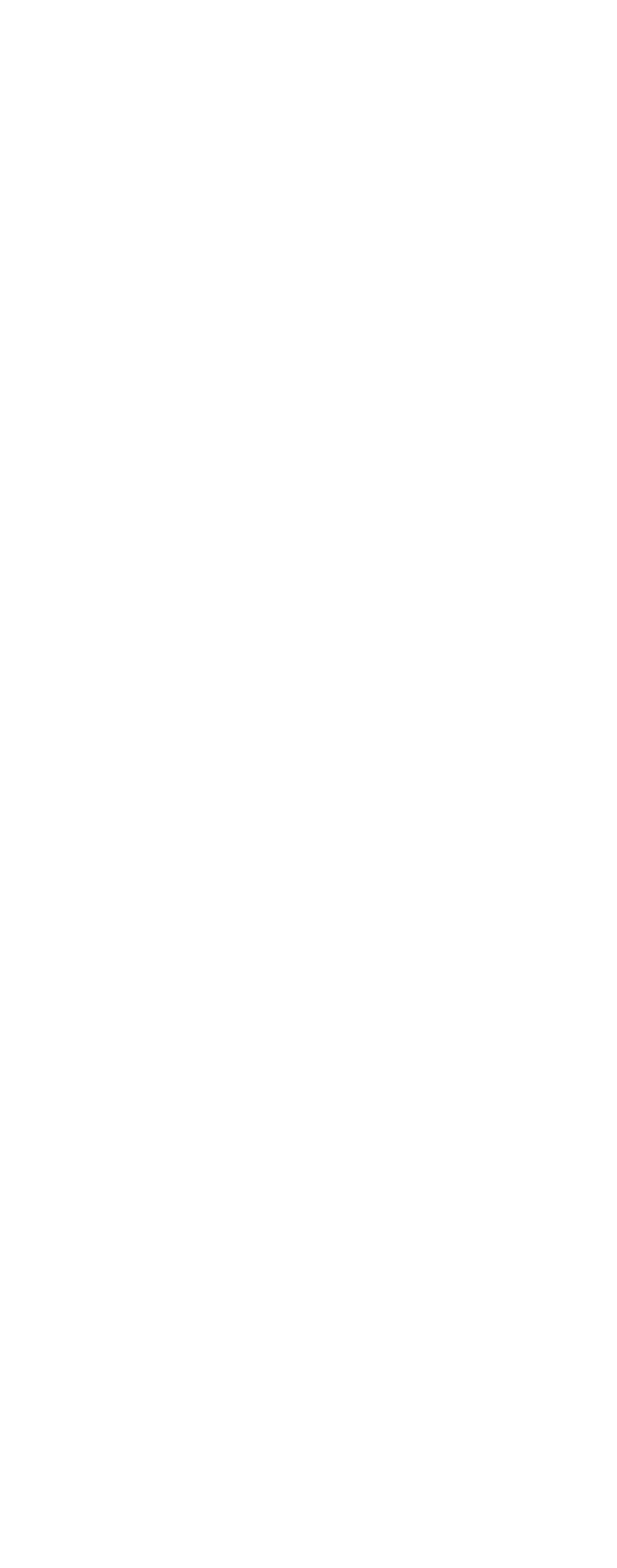




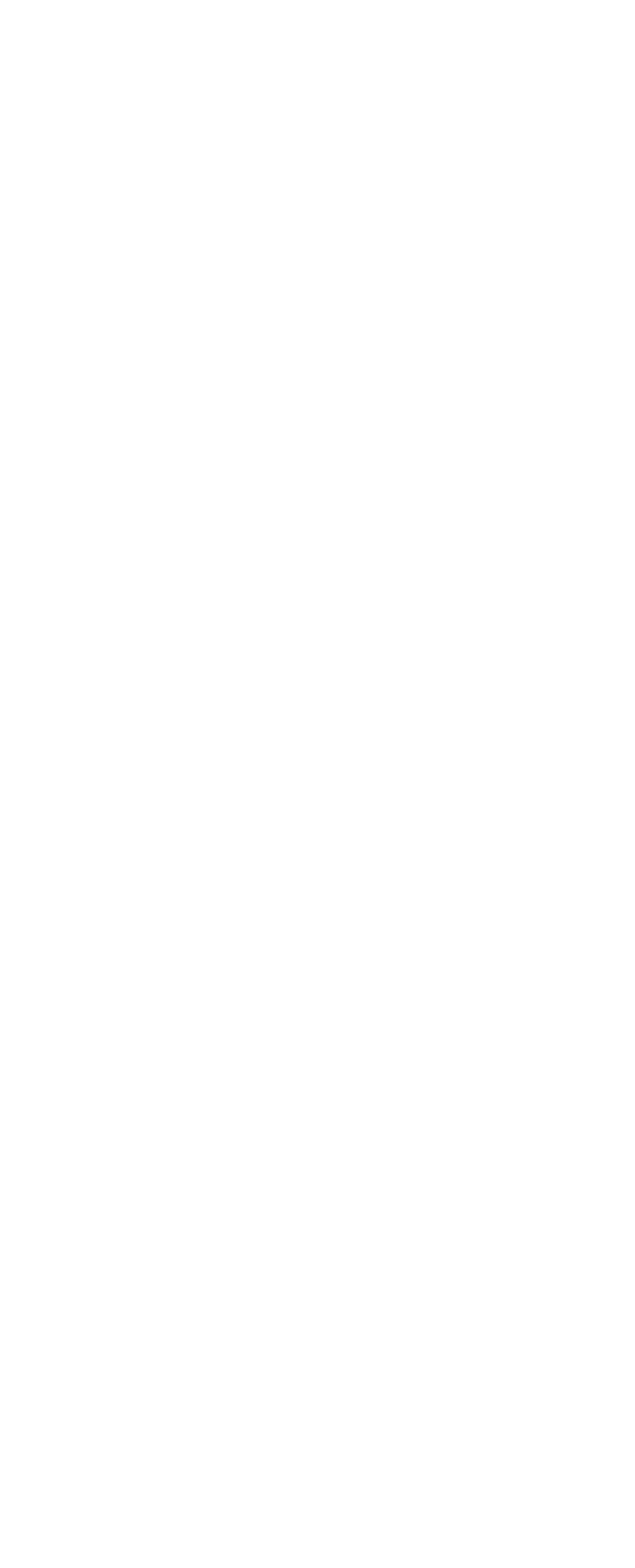




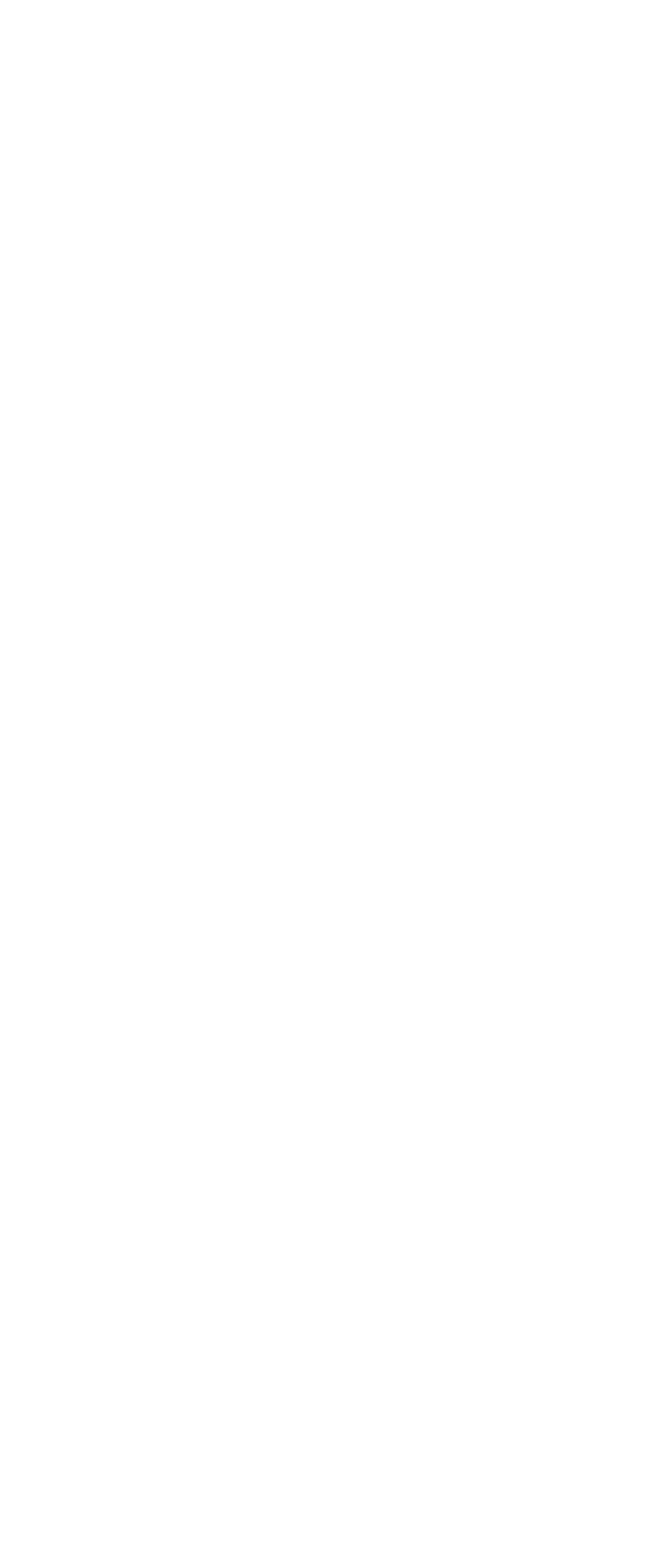




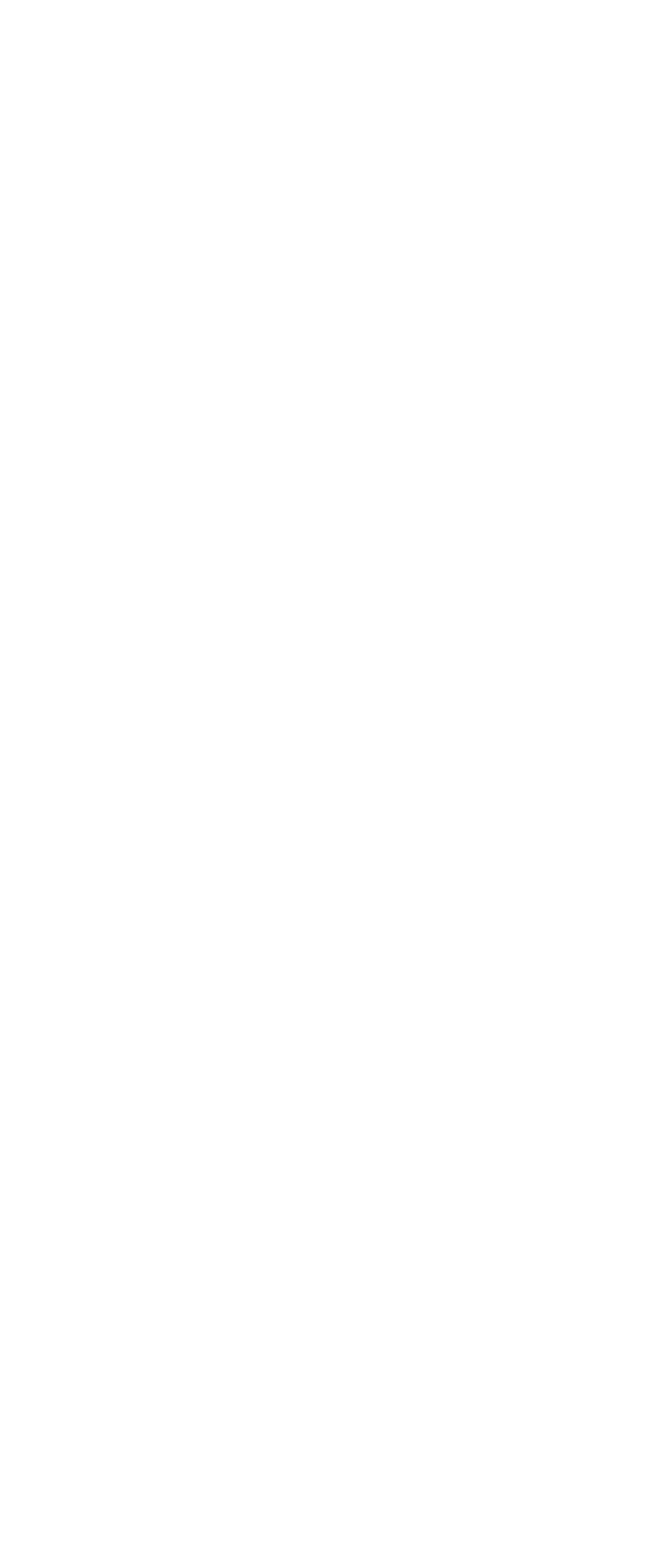




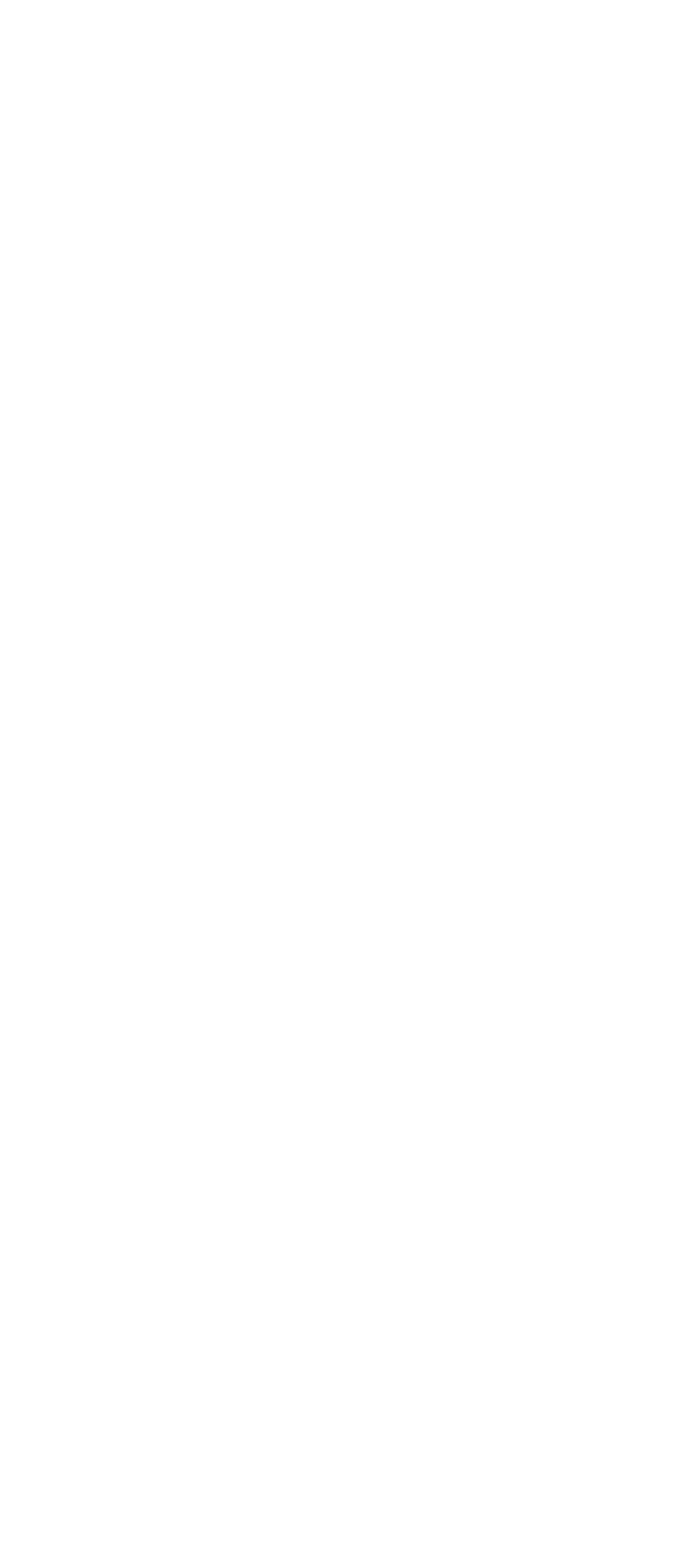




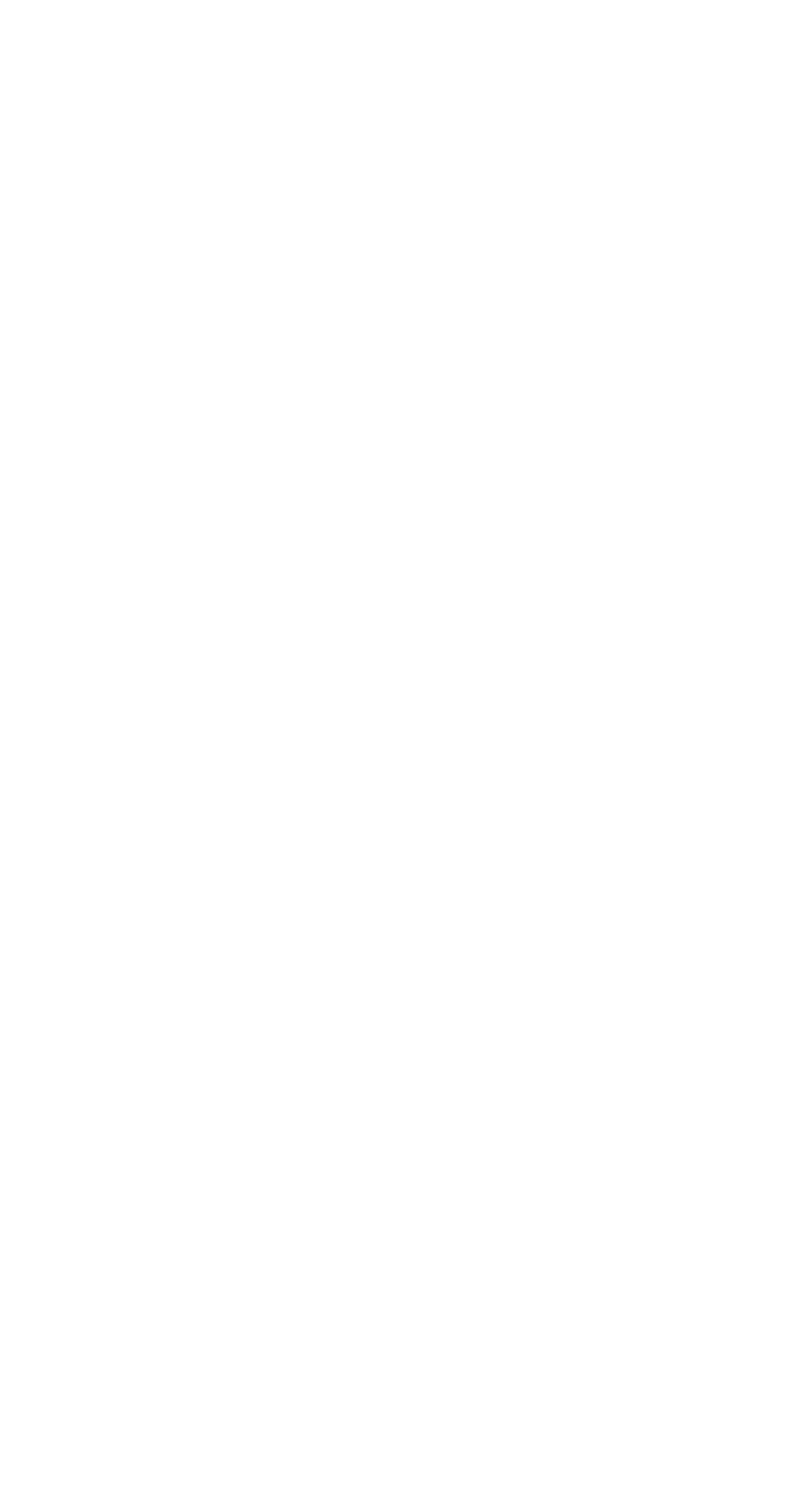




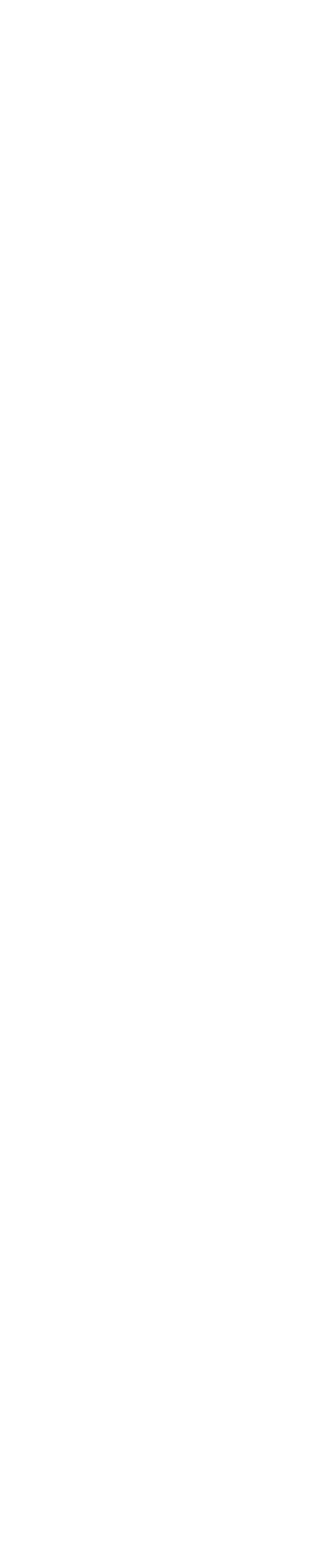




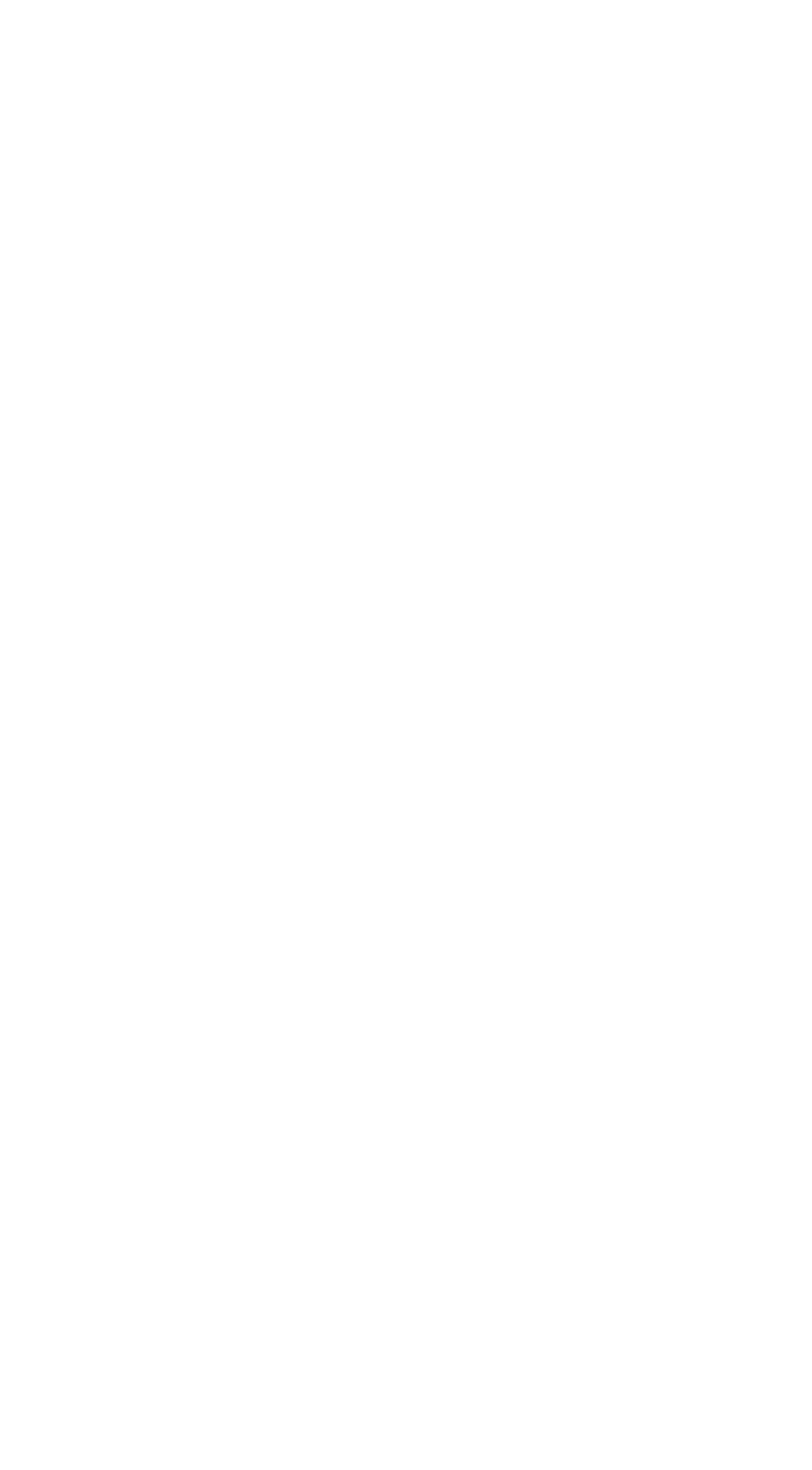




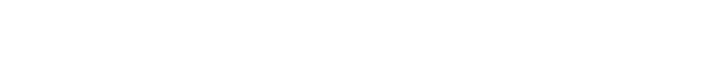

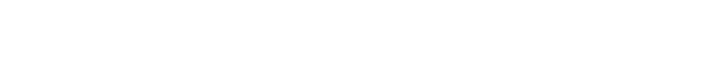

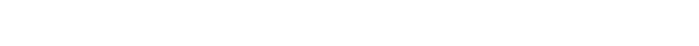

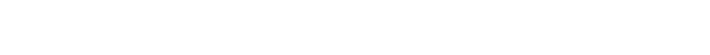

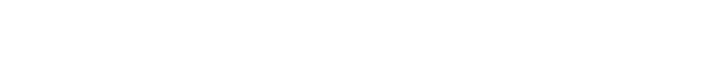

ก

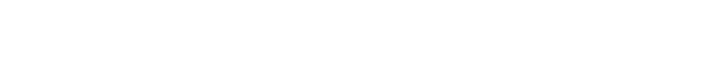

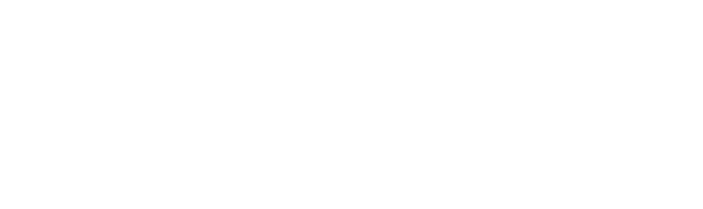

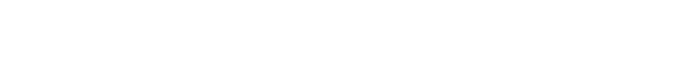

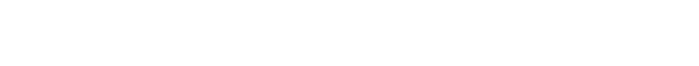

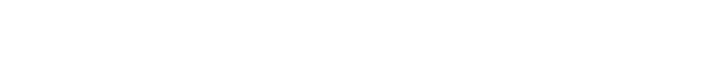

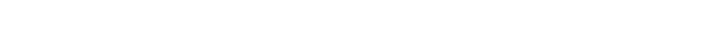

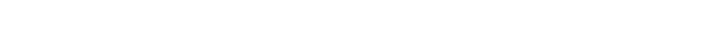
๓

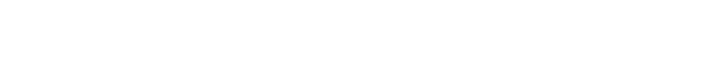

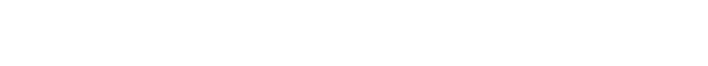

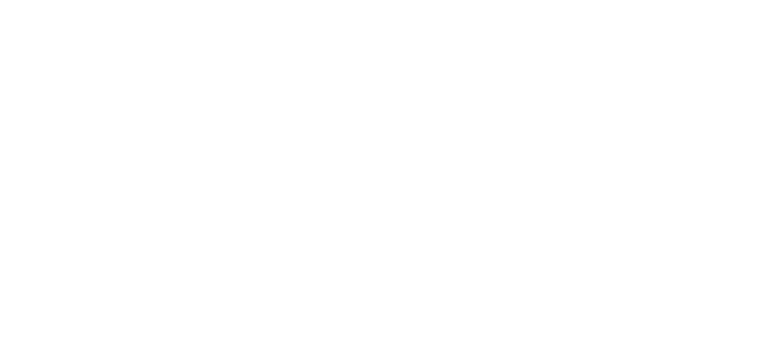




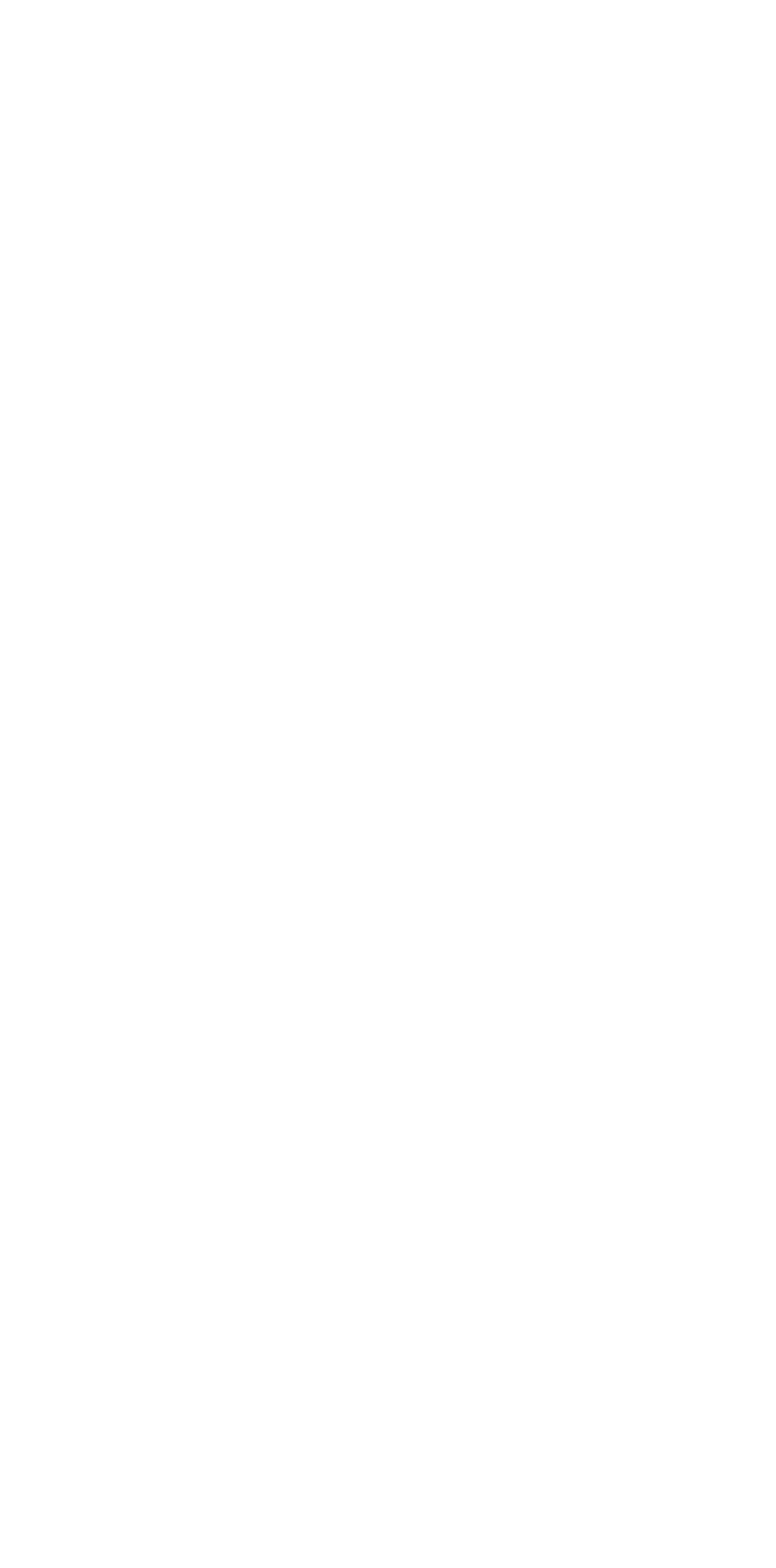




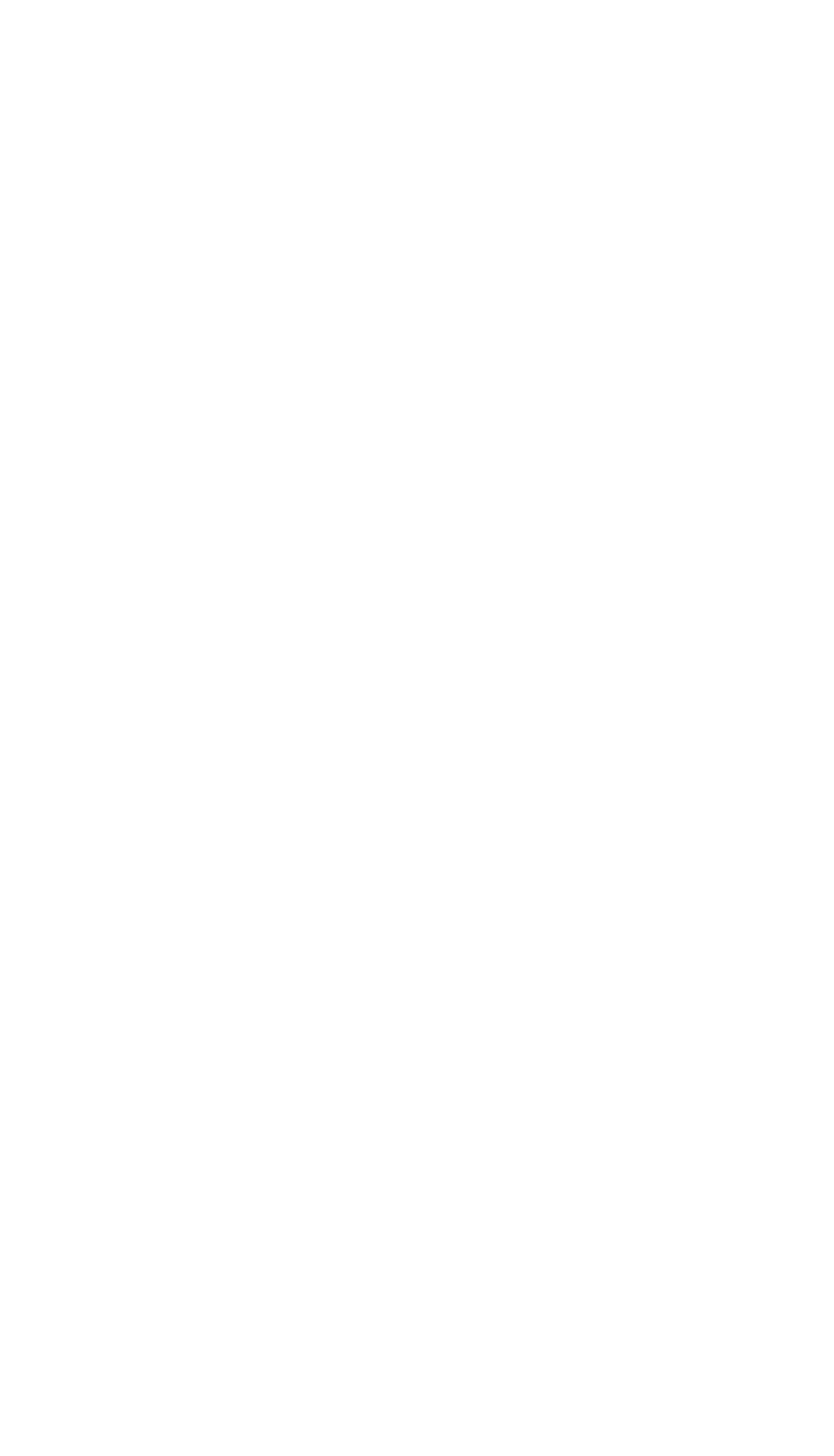




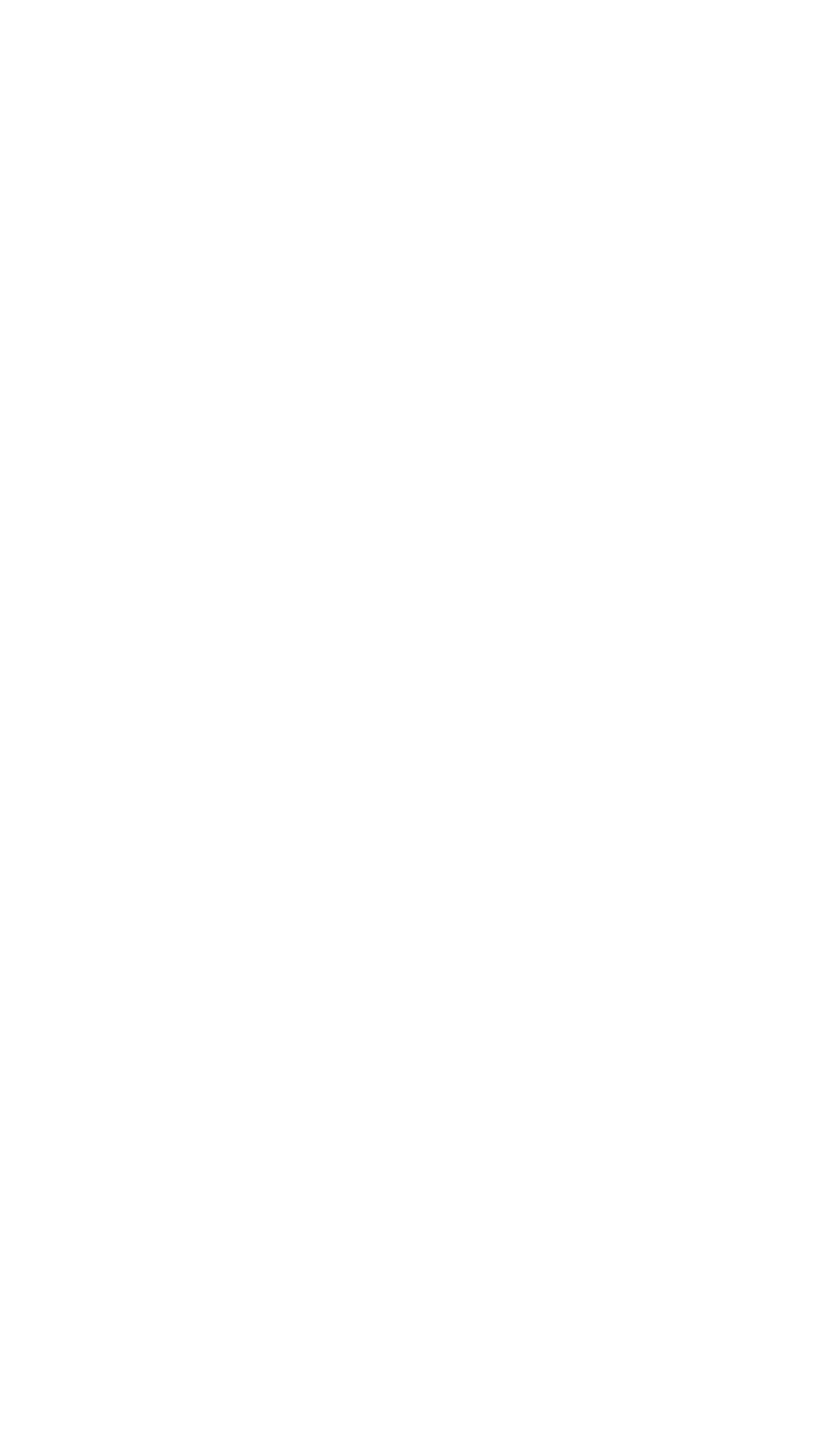




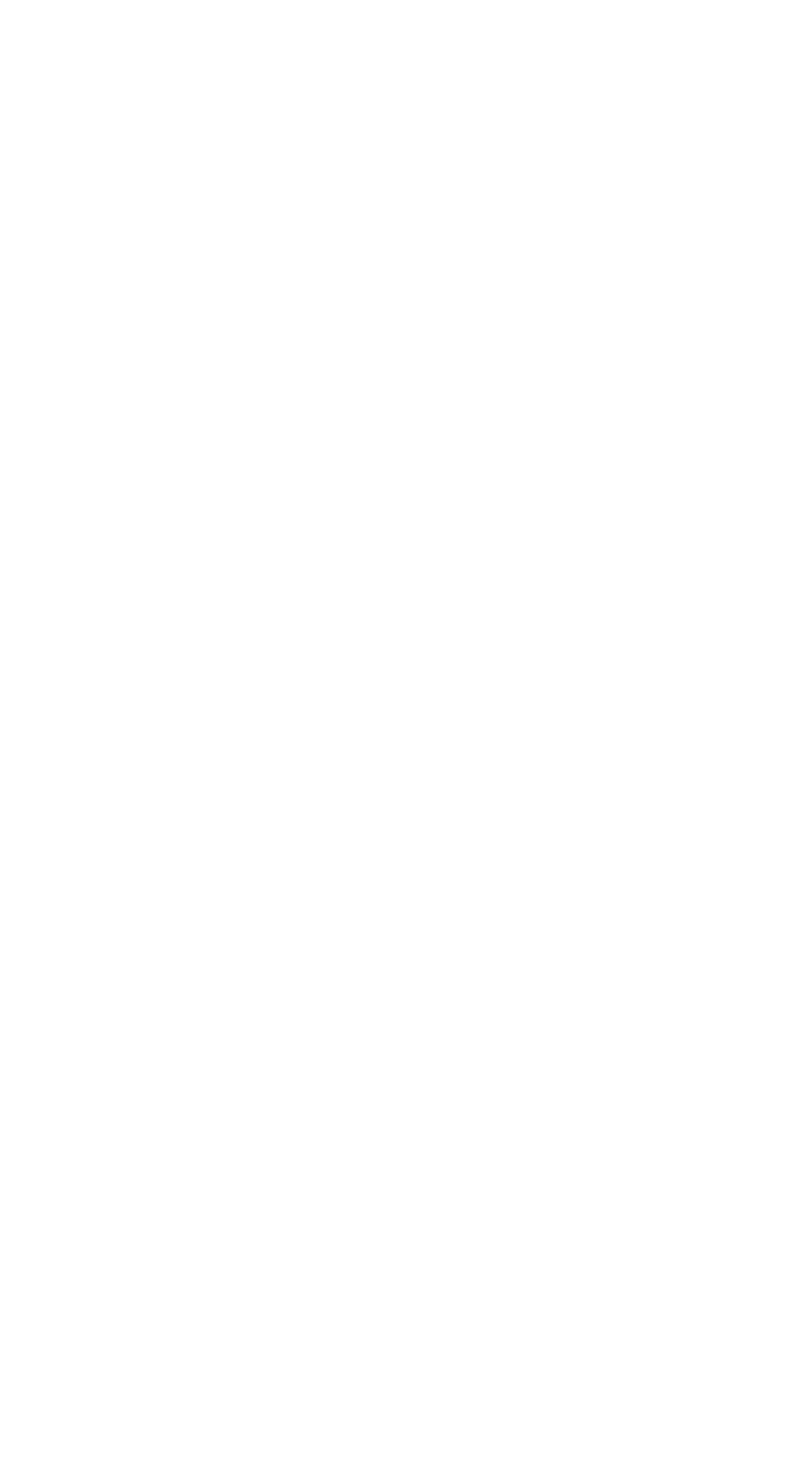




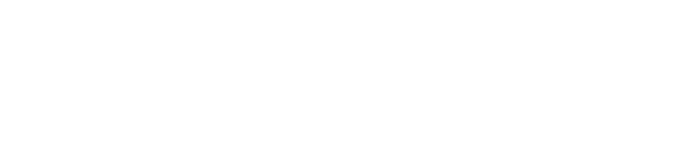

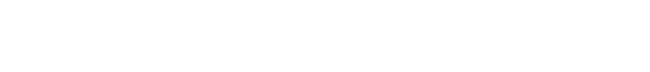

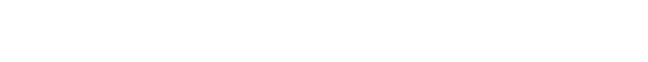

00000000000000000000

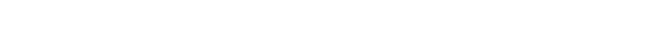

ఫ.

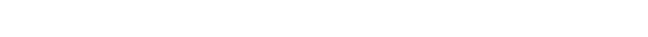

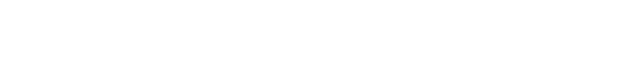

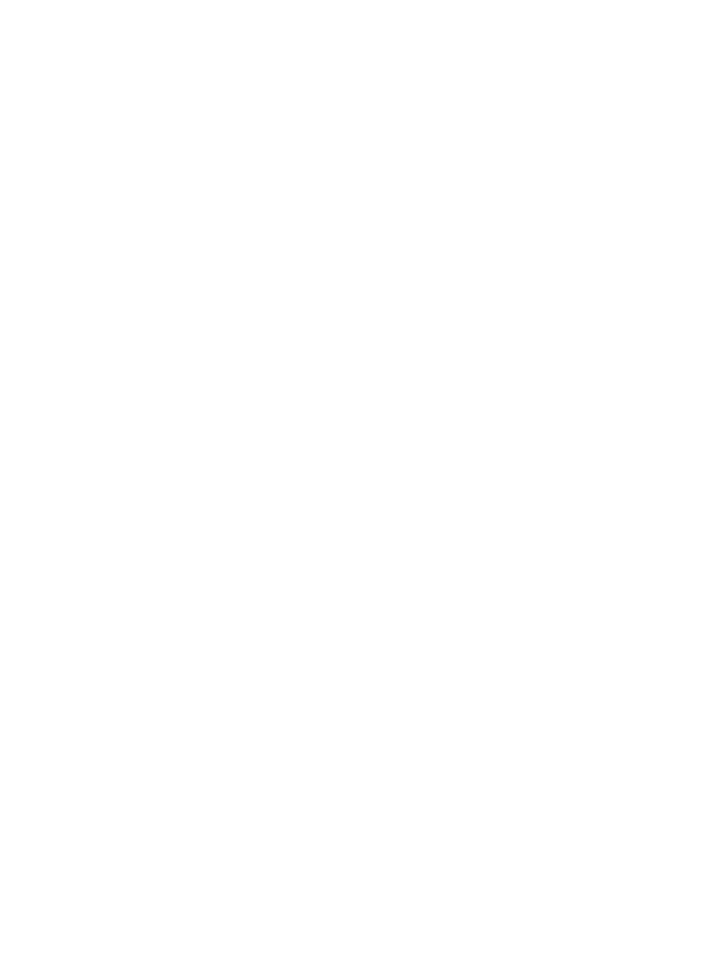




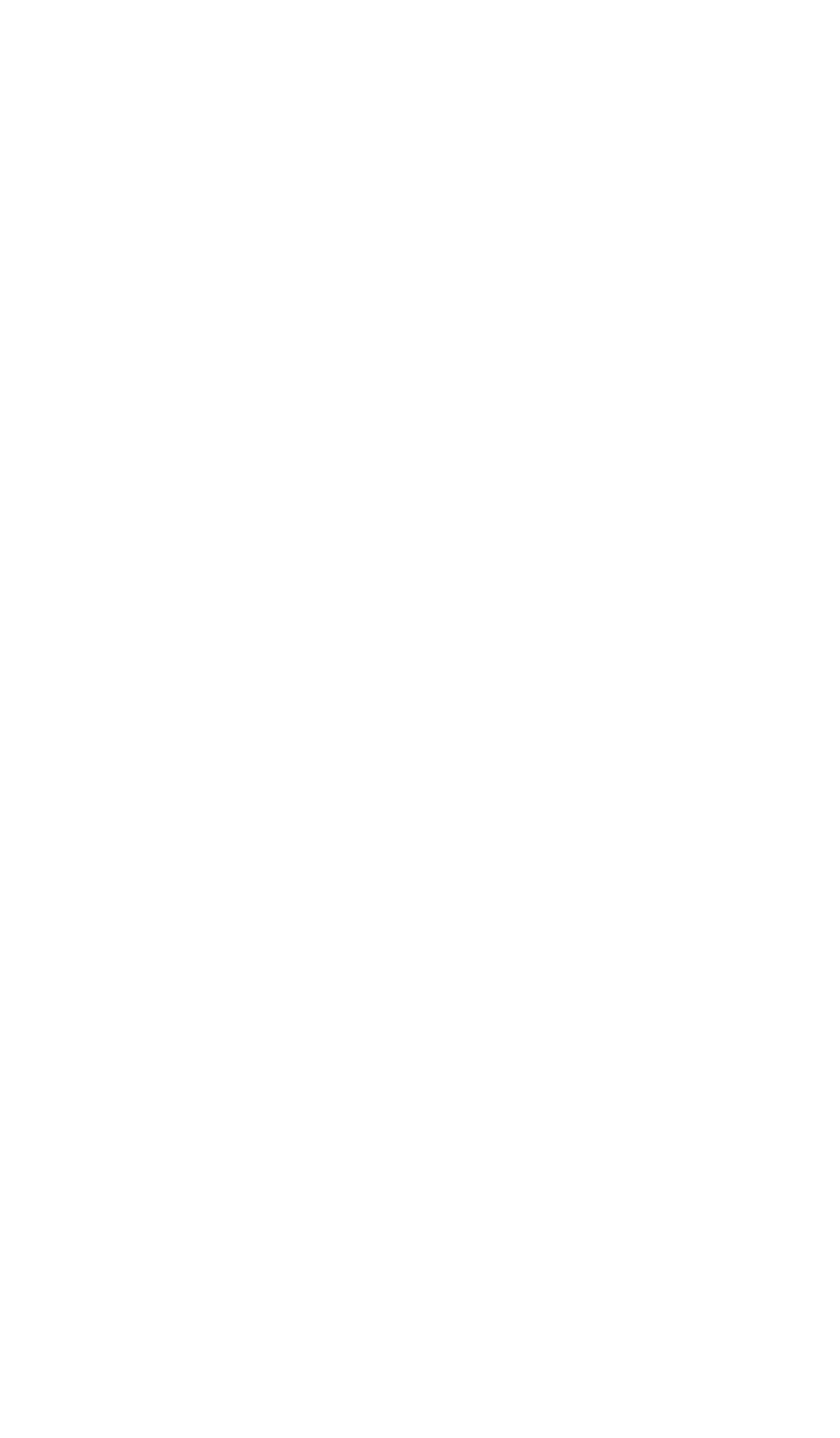




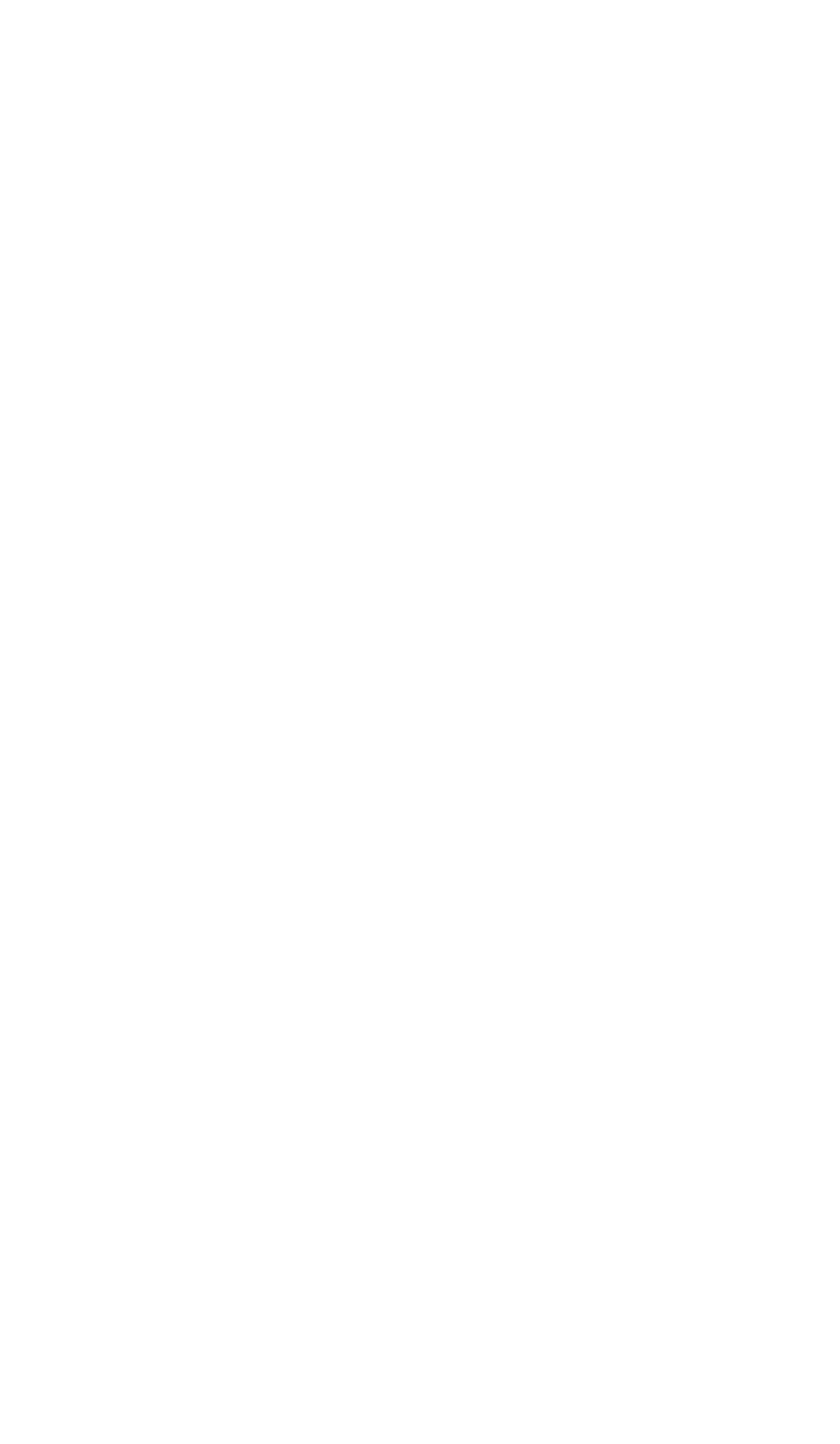




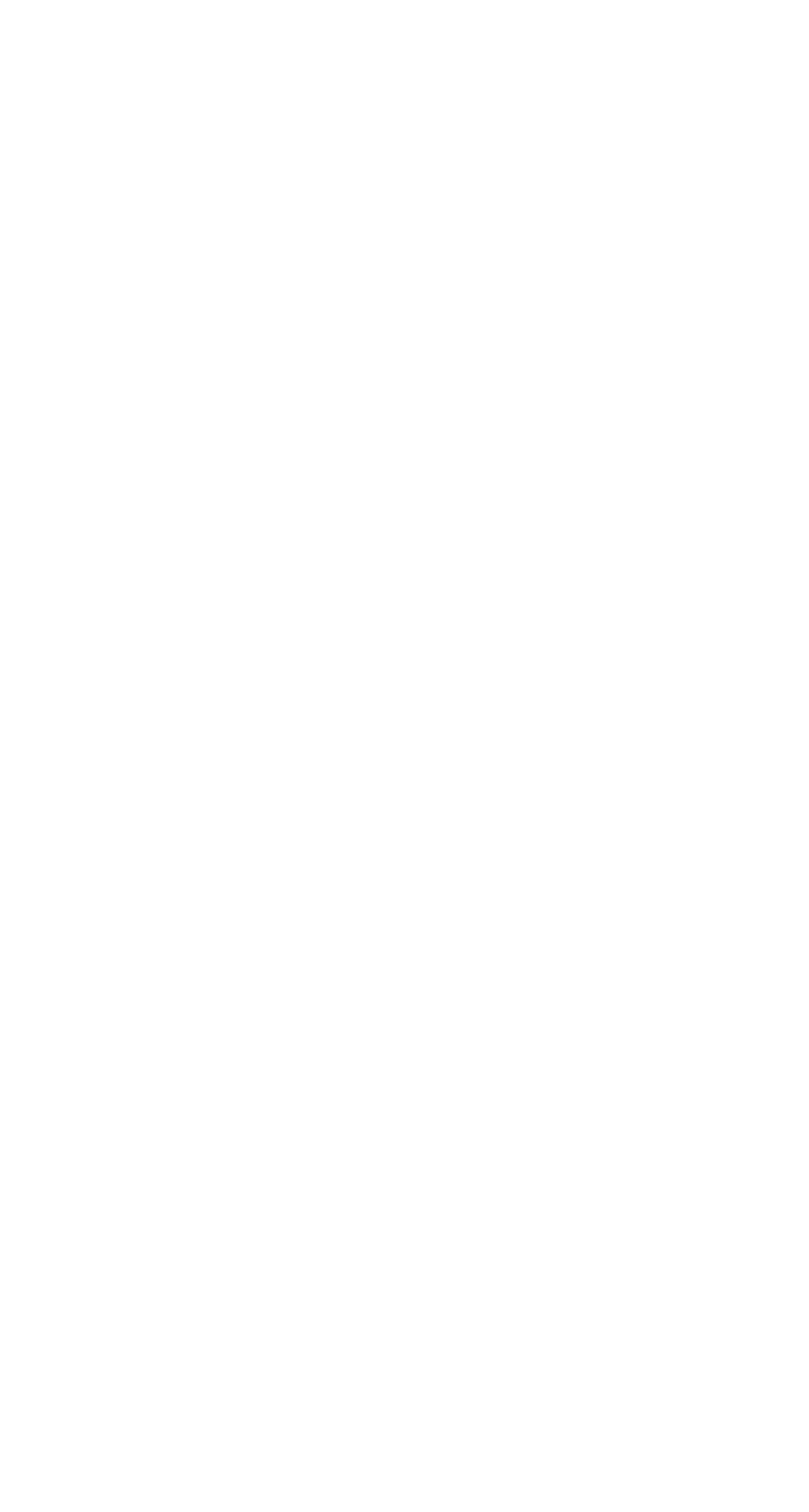




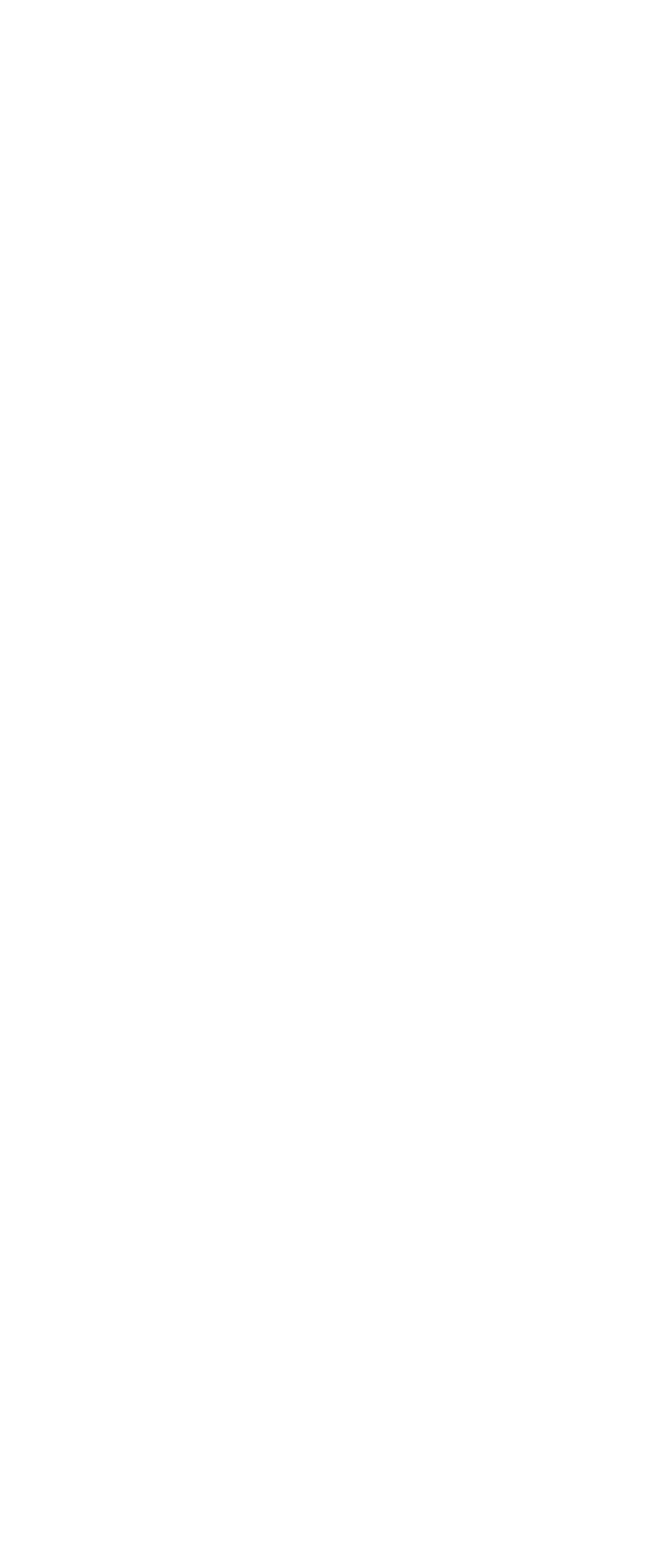




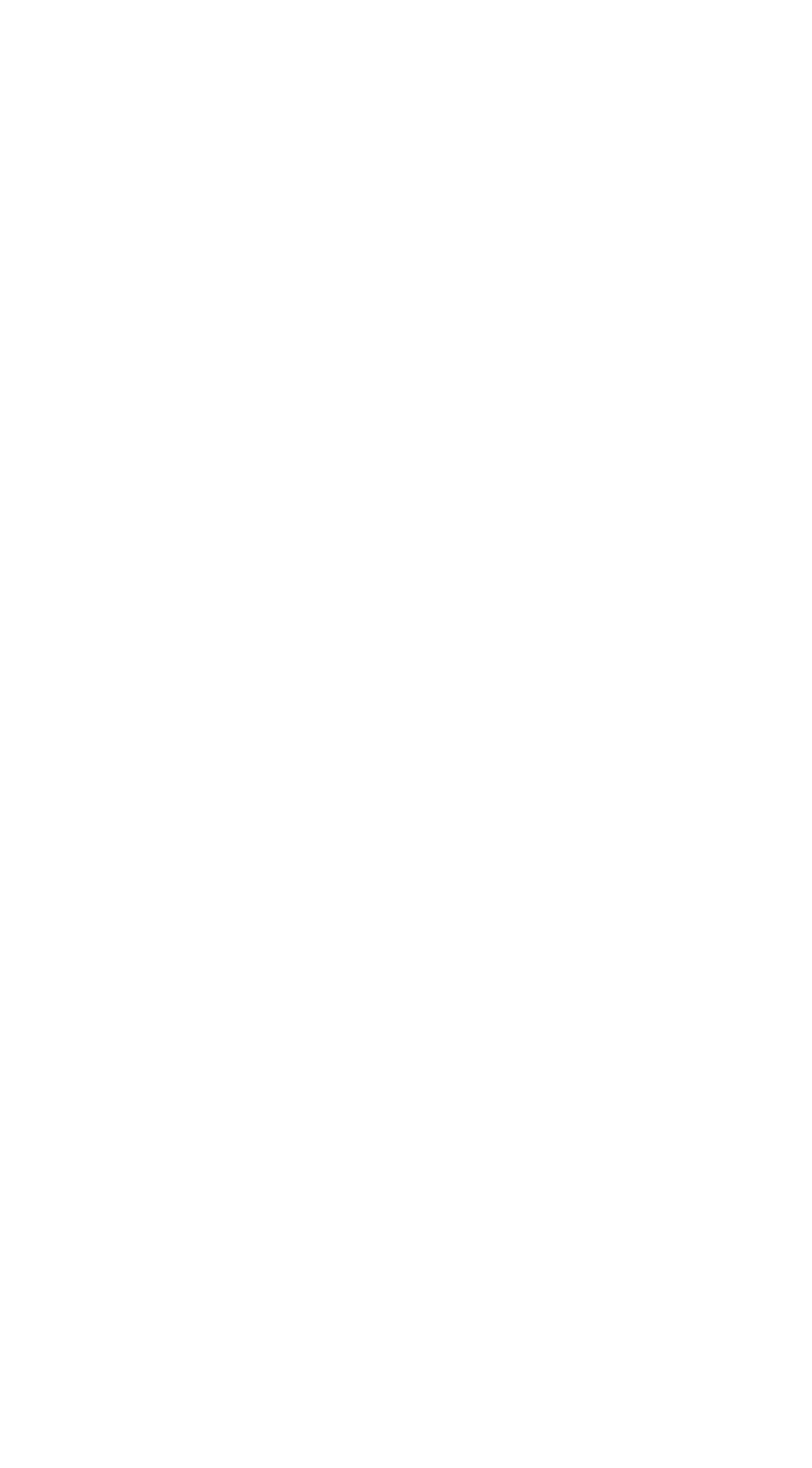




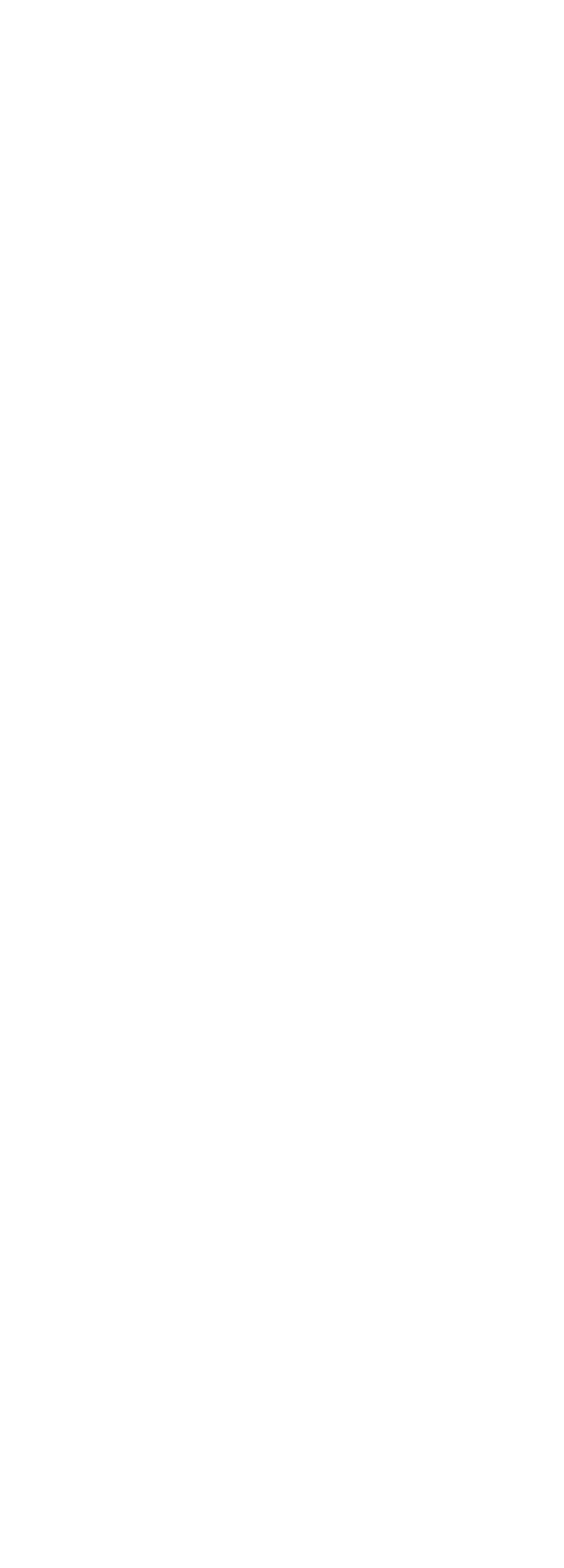


RIO GRANDE BASIN--Continued

PECOS RIVER SEEPAGE INVESTIGATION--Continued

MALAGA BEND EXPERIMENTAL SALINITY ALLEVIATION PROJECT, EDDY COUNTY, N, MEX.

A series of discharge measurements was made to study gains and losses on the pecos River from the gaging station "near Malaga" to the gaging station "at pierce Canyon Crossing, near Malaga". The only diversion in this reach is Moutray's pump which was in operation during this investigation. Samples were collected for chemical analyses at the following measuring sites. The site numbers are used to establish the downstream order of the measurements and have no relationship to river miles. Locations were obtained from U.S. Geological Survey Plan and Profile maps of the Malaga Bend Area. Previous investigations with samples collected for chemical analyses were made at measuring sites within the reach during $1956-60,1962-63$ water years.

Chemical analyses, in parts per million, water year october 1963 to September 1964

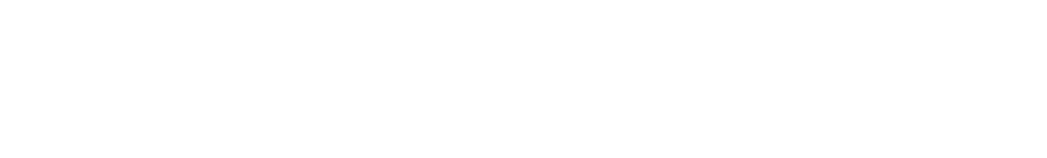

Site No. 333.5. pecos River NE $\frac{1}{4} W_{4}^{\frac{1}{4}}$ sec.19, T.24 S., R.29 E., near Malaga (regular gage)

\begin{tabular}{|l|l|l|l|l|l|l}
\hline Aug. 13, $1964 \ldots \ldots \ldots \ldots \ldots \ldots$ & 1310 & 88 & 8.16 & 1760 & 7850 & \\
\hline
\end{tabular}

Site No. 334. pecos River $\mathrm{SE}_{4}^{1} \mathrm{NE}_{4}^{\frac{1}{4}} \mathrm{sec} .17$, T.24 S., R.29 E., at Fishing Rock Crossing

\begin{tabular}{|c|c|c|c|c|c|c|}
\hline 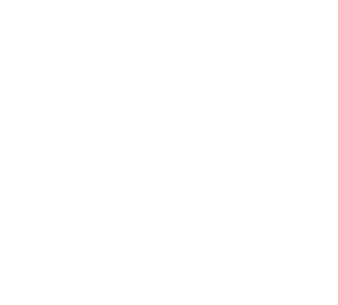 & $\begin{array}{l}1725 \\
1540 \\
1610 \\
1500 \\
1350 \\
1405 \\
1545 \\
1135 \\
1155 \\
1410 \\
1243 \\
1345 \\
1305 \\
1305 \\
1540\end{array}$ & $\begin{array}{l}72 \\
72 \\
70 \\
70 \\
62 \\
60 \\
60 \\
54 \\
48 \\
50 \\
46 \\
44 \\
47 \\
46 \\
44\end{array}$ & $\begin{array}{l}26 \\
24 \\
23 \\
21 \\
43 \\
50 \\
43 \\
43 \\
39 \\
52 \\
66 \\
70 \\
56 \\
56 \\
56\end{array}$ & $\begin{array}{l}1760 \\
2430 \\
2660 \\
2680 \\
2890 \\
2080 \\
2010 \\
2140 \\
2300 \\
1900 \\
1720 \\
1640 \\
1600 \\
1540 \\
1560\end{array}$ & $\begin{array}{r}7850 \\
9900 \\
10700 \\
10800 \\
11500 \\
9050 \\
883 ? \\
937 ? \\
9720 \\
8479 \\
7760 \\
7489 \\
7389 \\
7173 \\
7220\end{array}$ & $\begin{array}{l}-- \\
-- \\
-- \\
-- \\
-- \\
-- \\
-- \\
-- \\
-- \\
-- \\
--\end{array}$ \\
\hline 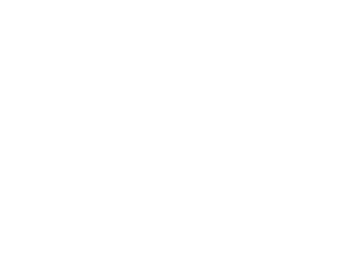 & $\begin{array}{l}1345 \\
1400 \\
1410 \\
1343 \\
1435 \\
1210 \\
1445 \\
1400 \\
1355 \\
1215 \\
1515 \\
1530 \\
1415\end{array}$ & $\begin{array}{l}42 \\
48 \\
50 \\
-46 \\
48 \\
48 \\
56 \\
58 \\
61 \\
60 \\
66 \\
68 \\
74\end{array}$ & $\begin{array}{l}58 \\
26 \\
23 \\
39 \\
43 \\
32 \\
25 \\
26 \\
20 \\
18 \\
17 \\
18 \\
18\end{array}$ & $\begin{array}{l}1520 \\
1740 \\
2300 \\
1940 \\
1560 \\
1580 \\
2040 \\
2380 \\
2120 \\
2690 \\
2910 \\
2590 \\
2610\end{array}$ & $\begin{array}{r}7073 \\
7760 \\
9459 \\
8490 \\
7323 \\
7273 \\
8809 \\
984 ? \\
9049 \\
10809 \\
11609 \\
1050 ? \\
10703\end{array}$ & $\begin{array}{l}-- \\
-- \\
-- \\
-- \\
-- \\
-- \\
-\end{array}$ \\
\hline 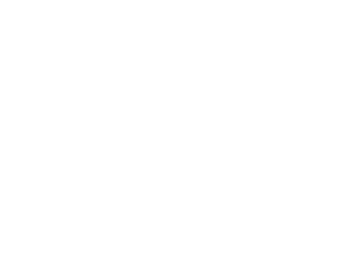 & $\begin{array}{l}1550 \\
1425 \\
1345 \\
1525 \\
1410 \\
1410 \\
1450 \\
1347 \\
1500 \\
1345 \\
1330 \\
1200 \\
1410\end{array}$ & $\begin{array}{l}70 \\
70 \\
68 \\
77 \\
82 \\
82 \\
82 \\
84 \\
87 \\
84 \\
89 \\
86 \\
84\end{array}$ & $\begin{array}{c}20 \\
17 \\
15 \\
14 \\
14 \\
13 \\
14 \\
13 \\
13 \\
13 \\
12 \\
11 \\
9.8\end{array}$ & $\begin{array}{l}2860 \\
2790 \\
3180 \\
2990 \\
2990 \\
2960 \\
3010 \\
3140 \\
1270 \\
2840 \\
3230 \\
3610 \\
3600\end{array}$ & $\begin{array}{r}1130\} \\
11207 \\
1290 ? \\
12003 \\
11907 \\
1180 ? \\
1200 ? \\
1240 ? \\
573 ? \\
1130 ? \\
1250 ? \\
1340 ? \\
1380 ?\end{array}$ & $\begin{array}{l}-- \\
-- \\
-- \\
-- \\
-- \\
-- \\
-- \\
--\end{array}$ \\
\hline 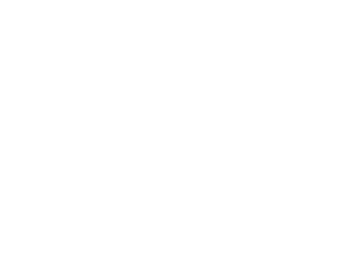 & $\begin{array}{l}1350 \\
1410 \\
1310 \\
1110 \\
0730 \\
1000 \\
1200 \\
1400 \\
1440 \\
1600 \\
1800 \\
2000 \\
2200\end{array}$ & $\begin{array}{l}87 \\
87 \\
88 \\
86 \\
78 \\
81 \\
84 \\
86 \\
86 \\
87 \\
86 \\
84 \\
84\end{array}$ & $\begin{array}{c}9.8 \\
9.5 \\
9.5 \\
10.2 \\
9.21 \\
10.9 \\
10.3 \\
10.0 \\
9.95 \\
9.8 \\
9.6 \\
10.1 \\
12.0\end{array}$ & $\begin{array}{r}3810 \\
3750 \\
3650 \\
- \\
-- \\
3730 \\
3730 \\
3780 \\
3760 \\
3760 \\
3780 \\
3740 \\
3750\end{array}$ & $\begin{array}{r}14407 \\
14307 \\
1370 ? \\
-- \\
14007 \\
14007 \\
14207 \\
14207 \\
14207 \\
14207 \\
14107 \\
10107\end{array}$ & $\begin{array}{r}=- \\
=- \\
=- \\
1.006 \\
1.006 \\
1.006 \\
1.006 \\
1.006 \\
1.006 \\
1.006 \\
1.006\end{array}$ \\
\hline
\end{tabular}


RIO GRANDE BASIN--Continued

PECOS RIVER SEEPAGE INVESTIGATION--Continued

MALAGA BEND EXPERINENTAL SALINITY ALLEVIATION PROJECT, EDDY COUNTY, N. MEX.--Continued

Chemical analyses, in parts per million, water year October 1963 to September 1964--Continued

\begin{tabular}{|c|c|c|c|c|c|c|}
\hline Date & $\begin{array}{l}\text { Time } \\
(24 \mathrm{hr})\end{array}$ & $\begin{array}{l}\text { Tem- } \\
\text { per- } \\
\text { a- } \\
\text { ture } \\
\left({ }^{\circ} F\right)\end{array}$ & $\begin{array}{c}\text { Discharge } \\
\text { (cfs) }\end{array}$ & $\begin{array}{l}\text { Chlo- } \\
\text { ride } \\
\text { (C1) }\end{array}$ & $\begin{array}{l}\text { Specific } \\
\text { conduct- } \\
\text { ance } \\
\text { (micro- } \\
\text { mhos at } \\
25^{\circ} \mathrm{C} \text { ) }\end{array}$ & $\begin{array}{l}\text { Den- } \\
\text { sity } \\
\text { g/ml } \\
\text { at } \\
20^{\circ} \mathrm{C}\end{array}$ \\
\hline
\end{tabular}

Site No. 334. Pecos River, at Fishing Rock Crossing--Continued

\begin{tabular}{|c|c|c|c|c|c|c|}
\hline 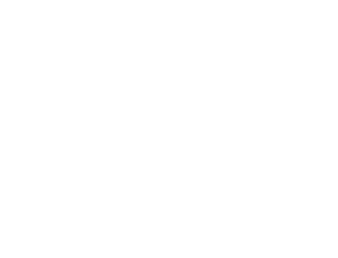 & $\begin{array}{l}0002 \\
0200 \\
0400 \\
0600 \\
0800 \\
1000 \\
1325 \\
1455 \\
1410 \\
1415 \\
1500 \\
0935 \\
1305\end{array}$ & $\begin{array}{l}82 \\
81 \\
80 \\
79 \\
80 \\
82 \\
89 \\
87 \\
85 \\
83 \\
82 \\
75 \\
72\end{array}$ & $\begin{array}{r}11.2 \\
10.2 \\
9.8 \\
9.6 \\
9.4 \\
9.3 \\
9.0 \\
9.5 \\
9.2 \\
9.2 \\
9.0 \\
12 \\
13\end{array}$ & $\begin{array}{l}3710 \\
3710 \\
3630 \\
3550 \\
3510 \\
3510 \\
3800 \\
3650 \\
3930 \\
3830 \\
4100 \\
1180 \\
2200\end{array}$ & $\begin{array}{r}14000 \\
14000 \\
13800 \\
13500 \\
13400 \\
13400 \\
14100 \\
13900 \\
14700 \\
14600 \\
15200 \\
5440 \\
9080\end{array}$ & $\begin{array}{r}1.006 \\
1.006 \\
1.006 \\
1.005 \\
1.005 \\
1.005 \\
-- \\
-- \\
-- \\
-- \\
--\end{array}$ \\
\hline
\end{tabular}

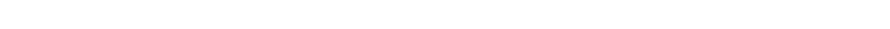

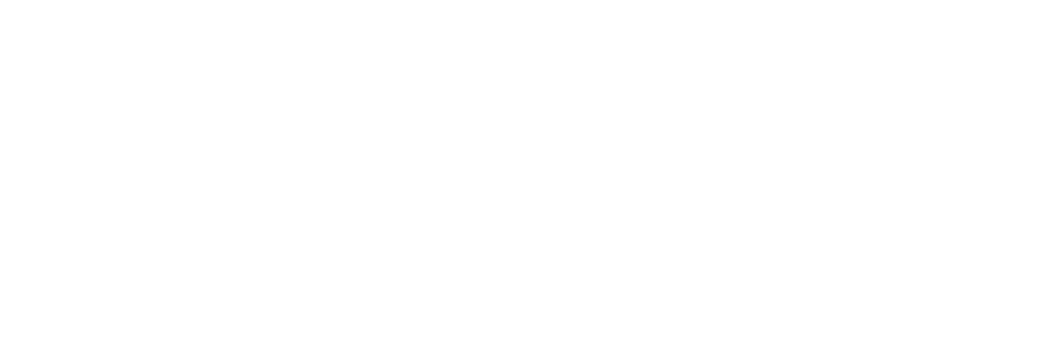

Site No. 337. Moutray's pump NW $\frac{1}{4} \mathrm{NW} \frac{1}{4} \mathrm{sec.21}, \mathrm{T} .24$ S., R.29 E., from left bank

\begin{tabular}{|c|c|c|c|c|c|c|}
\hline 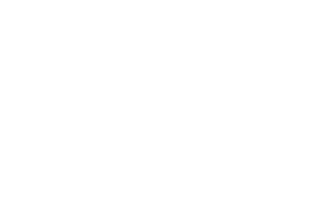 & $\begin{array}{l}0935 \\
1050 \\
1230 \\
1430 \\
1620 \\
1630 \\
1843 \\
2030 \\
2235\end{array}$ & $\begin{array}{l}\overline{77} \\
81 \\
86 \\
89 \\
88 \\
88 \\
86 \\
84 \\
82\end{array}$ & $\begin{array}{l}-- \\
5.8 \\
5.8 \\
5.7 \\
-\overline{--} \\
5.6 \\
5.4 \\
5.2 \\
5.0\end{array}$ & $\begin{array}{r}-- \\
5460 \\
5460 \\
5410 \\
\overline{5510} \\
5460 \\
5360 \\
5510\end{array}$ & $\begin{array}{r}-\overline{--} \\
18800 \\
18800 \\
18900 \\
18900 \\
18900 \\
18500 \\
18800\end{array}$ & $\begin{array}{l}1 . \overline{-} \\
1.008 \\
1.008 \\
1.008 \\
1.008 \\
1.008 \\
1.008 \\
1.008\end{array}$ \\
\hline 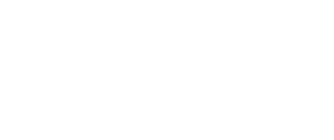 & $\begin{array}{l}0045 \\
0245 \\
0435 \\
0635 \\
0850 \\
1000 \\
1037\end{array}$ & $\begin{array}{l}80 \\
79 \\
78 \\
77 \\
79 \\
79 \\
82\end{array}$ & $\begin{array}{l}4.9 \\
4.7 \\
4.6 \\
4.4 \\
4.2 \\
4.0\end{array}$ & $\begin{array}{r}\mathbf{5 4 1 0} \\
\mathbf{5 3 6 0} \\
\mathbf{5 3 6 0} \\
\mathbf{5 3 6 0} \\
\mathbf{5 4 1 0} \\
\mathbf{5 4 6 0}\end{array}$ & $\begin{array}{l}18600 \\
1850 \\
18700 \\
18700 \\
18800 \\
18900\end{array}$ & $\begin{array}{r}1.008 \\
1.008 \\
1.008 \\
1.008 \\
1.008 \\
1.008\end{array}$ \\
\hline
\end{tabular}

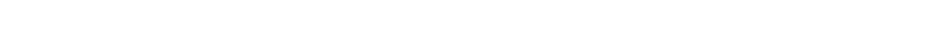

\begin{tabular}{|c|c|c|c|c|c|c|}
\hline 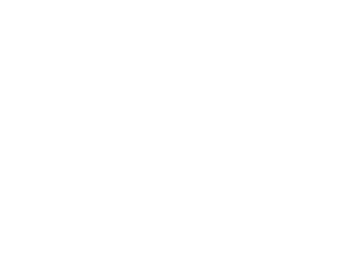 & $\begin{array}{l}1630 \\
1410 \\
1505 \\
1342 \\
1340 \\
1350 \\
1455 \\
1040 \\
1145 \\
1355 \\
1230 \\
1330 \\
1105\end{array}$ & $\begin{array}{l}75 \\
74 \\
75 \\
72 \\
64 \\
62 \\
63 \\
56 \\
48 \\
52 \\
46 \\
44 \\
46\end{array}$ & $\begin{array}{l}31 \\
30 \\
25 \\
19 \\
29 \\
39 \\
32 \\
32 \\
39 \\
47 \\
61 \\
58 \\
55\end{array}$ & $\begin{array}{l}2990 \\
3650 \\
4100 \\
4590 \\
3900 \\
2810 \\
2770 \\
2870 \\
3130 \\
2730 \\
2420 \\
2250 \\
2160\end{array}$ & $\begin{array}{r}11500 \\
13500 \\
14800 \\
15900 \\
14300 \\
11300 \\
11000 \\
11400 \\
12100 \\
10900 \\
9900 \\
9200 \\
9040\end{array}$ & 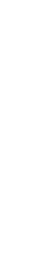 \\
\hline 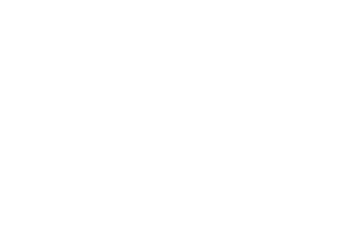 & $\begin{array}{l}1335 \\
1425 \\
1335 \\
1345 \\
1355 \\
1140 \\
1405 \\
1200 \\
1430 \\
1350 \\
1335 \\
1155\end{array}$ & $\begin{array}{l}46 \\
46 \\
42 \\
48 \\
52 \\
51 \\
45 \\
49 \\
59 \\
61 \\
63 \\
62\end{array}$ & $\begin{array}{l}54 \\
55 \\
55 \\
18 \\
20 \\
35 \\
39 \\
37 \\
22 \\
32 \\
20 \\
21\end{array}$ & $\begin{array}{l}2200 \\
2260 \\
2080 \\
2820 \\
3640 \\
3140 \\
2160 \\
2260 \\
3110 \\
3470 \\
3340 \\
3660\end{array}$ & $\begin{array}{r}9040 \\
9290 \\
8740 \\
10900 \\
13400 \\
11600 \\
9020 \\
9300 \\
11900 \\
13000 \\
12500 \\
13600\end{array}$ & $\begin{array}{l}-\square \\
-- \\
-- \\
-\square \\
-- \\
-- \\
-\square \\
--\end{array}$ \\
\hline
\end{tabular}


RIO GRANDE BASIN--Continued

PECOS RIVER SEEPAGE INVESTIGATION--Continued

MALAGA BEND EXPERIMENTAL SALINITY ALLEVIATION PROJECT, EDDY COUNTY, N. MEX.--Continued Chemical analyses, in parts per million, water year October 1963 to September 1964--Continued

\begin{tabular}{|c|c|c|c|c|c|c|}
\hline Date & $\begin{array}{l}\text { Time } \\
(24 \mathrm{hr})\end{array}$ & $\begin{array}{c}\text { Tem- } \\
\text { per- } \\
\text { a- } \\
\text { ture } \\
\left({ }^{\circ} \mathrm{F}\right)\end{array}$ & $\begin{array}{c}\text { Discharge } \\
\text { (cfs) }\end{array}$ & $\begin{array}{l}\text { Chlo- } \\
\text { ride } \\
\text { (Cl) }\end{array}$ & $\begin{array}{c}\text { Specific } \\
\text { conduct- } \\
\text { ance } \\
\text { (micro- } \\
\text { mhos at } \\
25^{\circ} \mathrm{C} \text { ) }\end{array}$ & $\begin{array}{c}\text { Den- } \\
\text { sity } \\
\mathrm{g} / \mathrm{ml} \\
\mathrm{at} \\
20^{\circ} \mathrm{C}\end{array}$ \\
\hline
\end{tabular}

Site No. 337.2. Pecos River, near well U.S.G.S. 11--Continued

\begin{tabular}{|c|c|c|c|c|c|c|}
\hline 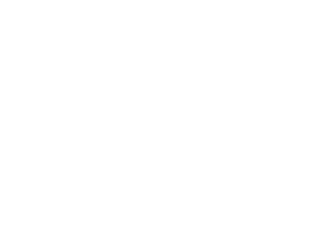 & $\begin{array}{l}1455 \\
1510 \\
1348 \\
1535 \\
1355 \\
1315 \\
1505 \\
1400 \\
1350 \\
1413 \\
1325 \\
1430\end{array}$ & $\begin{array}{l}69 \\
72 \\
77 \\
72 \\
73 \\
70 \\
79 \\
85 \\
87 \\
83 \\
87 \\
88\end{array}$ & $\begin{array}{l}15 \\
18 \\
14 \\
18 \\
12 \\
6.6 \\
12 \\
12 \\
9.5 \\
15 \\
15 \\
9.0\end{array}$ & $\begin{array}{l}3980 \\
3980 \\
4080 \\
4100 \\
4350 \\
4550 \\
4170 \\
4490 \\
4540 \\
4550 \\
4890 \\
2820\end{array}$ & $\begin{array}{l}14600 \\
14400 \\
14700 \\
14700 \\
15700 \\
16300 \\
15400 \\
16200 \\
16400 \\
16400 \\
17400 \\
10500\end{array}$ & $\begin{array}{l}=- \\
=- \\
=- \\
= \\
= \\
= \\
= \\
=\end{array}$ \\
\hline 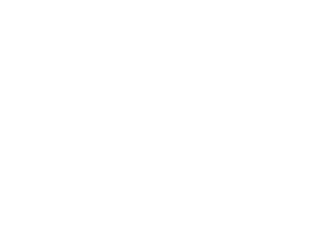 & $\begin{array}{l}1330 \\
1315 \\
1140 \\
1355 \\
1330 \\
1355 \\
1400 \\
1410 \\
1031 \\
1110 \\
1230 \\
1445\end{array}$ & $\begin{array}{l}86 \\
89 \\
89 \\
87 \\
90 \\
91 \\
91 \\
90 \\
82 \\
83 \\
89 \\
89\end{array}$ & $\begin{array}{l}12 \\
10 \\
4.0 \\
5.8 \\
3.5 \\
5.8 \\
6.2 \\
6.11 \\
1.80 \\
5.27 \\
5.0 \\
5.0\end{array}$ & $\begin{array}{r}3980 \\
4950 \\
5800 \\
6340 \\
6680 \\
6390 \\
7420 \\
-- \\
6860 \\
6880 \\
6880\end{array}$ & $\begin{array}{r}14400 \\
1730 C \\
1990 C \\
2140 C \\
2240 C \\
2170 C \\
2410 C \\
2260 C \\
2260 C \\
2260 C\end{array}$ & $\begin{array}{r}-- \\
-- \\
-- \\
\overline{--} \\
1.010 \\
1.010 \\
1.010\end{array}$ \\
\hline 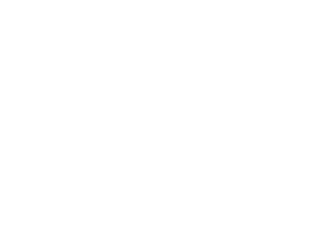 & $\begin{array}{l}1635 \\
1725 \\
1835 \\
2035 \\
2242 \\
0050 \\
0255 \\
0445 \\
0640 \\
0855 \\
1043 \\
1130\end{array}$ & $\begin{array}{l}88 \\
95 \\
85 \\
82 \\
81 \\
79 \\
78 \\
76 \\
77 \\
80 \\
84 \\
87\end{array}$ & $\begin{array}{l}4.8 \\
4.92 \\
4.7 \\
4.7 \\
4.6 \\
4.5 \\
4.5 \\
4.5 \\
4.4 \\
4.3 \\
4.3 \\
5.30\end{array}$ & $\begin{array}{r}6810 \\
7760 \\
6610 \\
6610 \\
6710 \\
6760 \\
6830 \\
6660 \\
6660 \\
7130 \\
-\end{array}$ & $\begin{array}{r}22400 \\
- \\
25300 \\
22000 \\
22000 \\
22200 \\
22400 \\
22600 \\
22000 \\
22000 \\
23400 \\
\end{array}$ & $\begin{array}{r}1.010 \\
1.011 \\
1.010 \\
1.010 \\
1.010 \\
1.010 \\
1.010 \\
1.010 \\
1.010 \\
1.010 \\
\end{array}$ \\
\hline 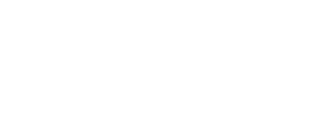 & $\begin{array}{l}1400 \\
1435 \\
1350 \\
1400 \\
1420 \\
1040 \\
1245\end{array}$ & $\begin{array}{l}92 \\
89 \\
87 \\
86 \\
83 \\
78 \\
73\end{array}$ & $\begin{array}{r}4.8 \\
6.2 \\
4.8 \\
5.8 \\
2.5 \\
7.5 \\
15\end{array}$ & $\begin{array}{l}6880 \\
6880 \\
7430 \\
6840 \\
7910 \\
\mathbf{3 4 4 0} \\
\mathbf{4 4 8 0}\end{array}$ & $\begin{array}{l}22900 \\
22600 \\
24100 \\
22700 \\
25600 \\
12100 \\
15600\end{array}$ & $\begin{array}{l}=- \\
=- \\
= \\
=\end{array}$ \\
\hline
\end{tabular}

Site No. 338. Pecos River NE $\frac{1}{4} \mathrm{NE}_{4}^{1}$ sec.20 T.24 S., R.29 E., at 10wer wading station

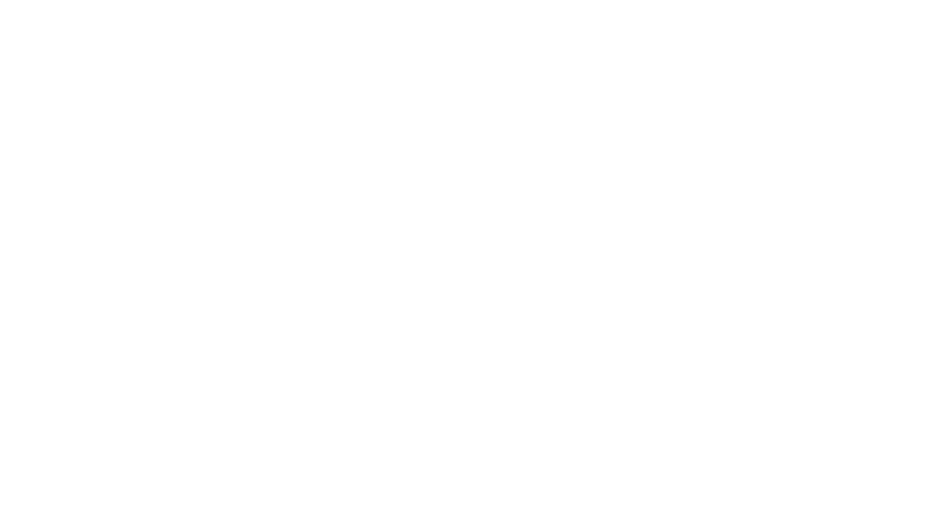


RIO GRANDE BASIN--Contínued

PECOS RIVER SEEPAGE INVESTIGATION--CONTInUEd

MALAGA BEND EXPERIMENTAL SALINITY ALLEVIATION PROJECT, EDDY COUNTY, N. MEX.--Continued

Chemical analyses, in parts per million, water year October 1963 to September 1964--Continued

\begin{tabular}{|c|c|c|c|c|c|c|}
\hline Date & $\begin{array}{l}\text { Time } \\
(24 \mathrm{hr})\end{array}$ & $\begin{array}{l}\text { Tem- } \\
\text { per- } \\
\text { a- } \\
\text { ture } \\
\left({ }^{\circ} \mathrm{F}\right)\end{array}$ & $\begin{array}{c}\text { Discharge } \\
(\mathrm{cfs})\end{array}$ & $\begin{array}{l}\text { Chlo- } \\
\text { ride } \\
\text { (C1) }\end{array}$ & $\begin{array}{c}\text { Specific } \\
\text { conduct- } \\
\text { ance } \\
\text { (micro- } \\
\text { mhos at } \\
25^{\circ} \mathrm{C} \text { ) }\end{array}$ & $\begin{array}{l}\text { Den- } \\
\text { sity } \\
\text { g/ml } \\
\text { at } \\
20^{\circ} \mathrm{C}\end{array}$ \\
\hline
\end{tabular}

Site No. 338. pecos River, at lower wading station--Continued

\begin{tabular}{|c|c|c|c|c|c|}
\hline 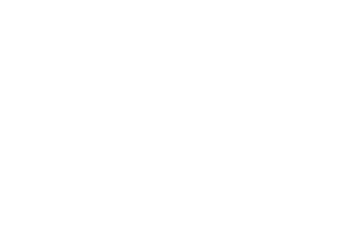 & $\begin{array}{l}1435 \\
1440 \\
1305 \\
1505 \\
1340 \\
1250 \\
1435 \\
1335 \\
1330 \\
1435 \\
1308 \\
1410\end{array}$ & $\begin{array}{l}68 \\
71 \\
75 \\
72 \\
73 \\
69 \\
80 \\
85 \\
88 \\
85 \\
87 \\
85\end{array}$ & $\begin{array}{l}-- \\
-- \\
-- \\
-- \\
=- \\
-- \\
=- \\
=- \\
--\end{array}$ & $\begin{array}{l}4500 \\
4320 \\
5260 \\
5160 \\
5530 \\
5460 \\
5360 \\
5210 \\
5680 \\
5430 \\
5700 \\
3880\end{array}$ & $\begin{array}{l}16000 \\
15200 \\
18000 \\
17700 \\
18900 \\
18800 \\
18700 \\
18200 \\
19300 \\
18800 \\
19700 \\
13400\end{array}$ \\
\hline 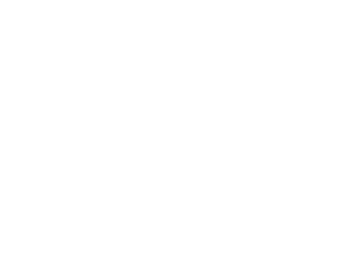 & $\begin{array}{l}1310 \\
1250 \\
1120 \\
1335 \\
1310 \\
1330 \\
1410 \\
1340 \\
1405 \\
1315 \\
1345 \\
1405 \\
1245 \\
1210\end{array}$ & $\begin{array}{l}86 \\
89 \\
86 \\
89 \\
90 \\
90 \\
92 \\
95 \\
-8 \\
87 \\
89 \\
84 \\
82 \\
72\end{array}$ & 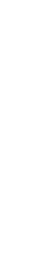 & $\begin{array}{r}4840 \\
6030 \\
7180 \\
8790 \\
7910 \\
8160 \\
9280 \\
9280 \\
8790 \\
9750 \\
8940 \\
14100 \\
3830 \\
4820\end{array}$ & $\begin{array}{l}16900 \\
20200 \\
23600 \\
27900 \\
25700 \\
26400 \\
29200 \\
28800 \\
27700 \\
30300 \\
28200 \\
41300 \\
13200 \\
17000\end{array}$ \\
\hline
\end{tabular}

Site No. 338.2, Pecos River NW $\frac{1}{4} S W \frac{1}{6}$ sec.27, T.24 S., R. 29 E., at Pierce Canyon Crossing (regular gage)

\begin{tabular}{|c|c|c|c|c|c|}
\hline 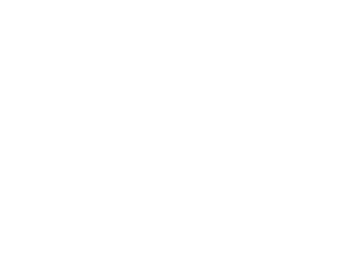 & $\begin{array}{l}1320 \\
1335 \\
1135 \\
1320 \\
1315 \\
1325 \\
1440 \\
1503 \\
1105 \\
1320 \\
1208 \\
1305 \\
1045\end{array}$ & $\begin{array}{l}70 \\
73 \\
70 \\
70 \\
64 \\
60 \\
61 \\
56 \\
48 \\
49 \\
46 \\
43 \\
45\end{array}$ & $\begin{array}{l}30 \\
29 \\
26 \\
21 \\
29 \\
43 \\
35 \\
36 \\
37 \\
48 \\
59 \\
63 \\
54\end{array}$ & $\begin{array}{l}3270 \\
4270 \\
4470 \\
4870 \\
4810 \\
3380 \\
3070 \\
3390 \\
3550 \\
3130 \\
2600 \\
2460 \\
2340\end{array}$ & $\begin{array}{r}12000 \\
15200 \\
15800 \\
16900 \\
16700 \\
12800 \\
11800 \\
12700 \\
13100 \\
12000 \\
10400 \\
10000 \\
9630\end{array}$ \\
\hline 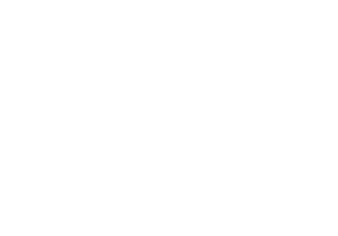 & $\begin{array}{l}1143 \\
1407 \\
1310 \\
1320 \\
1333 \\
1125 \\
1340 \\
1135 \\
1335 \\
1125 \\
1250 \\
1055\end{array}$ & $\begin{array}{l}46 \\
43 \\
41 \\
49 \\
50 \\
51 \\
46 \\
49 \\
56 \\
58 \\
60 \\
59\end{array}$ & $\begin{array}{l}59 \\
53 \\
54 \\
25 \\
19 \\
35 \\
47 \\
31 \\
17 \\
27 \\
17 \\
16\end{array}$ & $\begin{array}{l}2240 \\
2280 \\
2240 \\
2840 \\
4070 \\
3230 \\
2320 \\
2390 \\
3410 \\
3960 \\
3760 \\
4340\end{array}$ & $\begin{array}{r}9290 \\
9370 \\
9200 \\
11000 \\
14900 \\
12500 \\
9530 \\
9710 \\
13000 \\
14100 \\
13800 \\
15400\end{array}$ \\
\hline 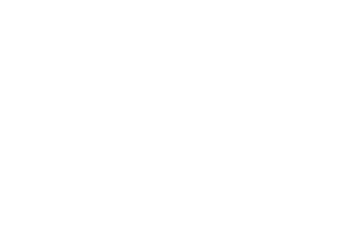 & $\begin{array}{l}1400 \\
1420 \\
1210 \\
1445 \\
1327 \\
1125 \\
1410 \\
1250 \\
1140 \\
1400 \\
1252 \\
1350\end{array}$ & $\begin{array}{l}68 \\
7 \mathbf{7 8} \\
71 \\
70 \\
68 \\
76 \\
80 \\
79 \\
77 \\
82 \\
88\end{array}$ & $\begin{array}{l}17 \\
14 \\
10 \\
19 \\
17 \\
9.1 \\
12 \\
15 \\
8.5 \\
15 \\
12 \\
8.5\end{array}$ & $\begin{array}{l}4970 \\
4520 \\
4990 \\
4970 \\
4890 \\
5040 \\
5700 \\
5310 \\
5310 \\
5750 \\
6220 \\
1640\end{array}$ & $\begin{array}{r}17200 \\
16000 \\
17300 \\
17300 \\
17300 \\
17800 \\
19800 \\
18600 \\
18700 \\
19800 \\
21000 \\
6780\end{array}$ \\
\hline 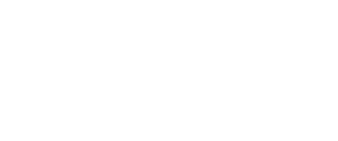 & $\begin{array}{l}1200 \\
1155 \\
1055 \\
1145 \\
1220 \\
1315 \\
1120\end{array}$ & $\begin{array}{l}79 \\
88 \\
85 \\
82 \\
86 \\
86 \\
85\end{array}$ & $\begin{array}{l}14 \\
11 \\
6.3 \\
4.8 \\
5.8 \\
5.3 \\
6.3\end{array}$ & $\begin{array}{l}4530 \\
5650 \\
6580 \\
7760 \\
8790 \\
8840 \\
8400\end{array}$ & $\begin{array}{l}15500 \\
19200 \\
22200 \\
25300 \\
28100 \\
28200 \\
27000\end{array}$ \\
\hline
\end{tabular}


RIO GRANDE BASIN--Continued

PECOS RIVER SEEPAGE INVESTIGATION--CONtinued

MALAGA BEND EXPERIMENTAL SALINITY ALLEVIATION PROJECT, EDDY COUNTY, N. MEX.--Continued

Chemical analyses, in parts per million, water year October 1963 to September 1964--Cont inued

\begin{tabular}{|c|c|c|c|c|c|c|}
\hline Date & $\begin{array}{l}\text { Time } \\
(24 \mathrm{hr})\end{array}$ & $\begin{array}{l}\text { Tem- } \\
\text { per- } \\
\text { a- } \\
\text { ture } \\
\left({ }^{\circ} \mathrm{F}\right)\end{array}$ & $\begin{array}{c}\text { Discharge } \\
\text { (cfs) }\end{array}$ & $\begin{array}{l}\text { ChIo- } \\
\text { ride } \\
\text { (CI) }\end{array}$ & $\begin{array}{c}\text { Specific } \\
\text { conduct- } \\
\text { ance } \\
\text { (micrs- } \\
\text { mos at } \\
25^{\circ} \mathrm{C} \text { ) }\end{array}$ & $\begin{array}{c}\text { Den- } \\
\text { sity } \\
\mathrm{g} / \mathrm{mI} \\
\text { at } \\
20^{\circ} \mathrm{C}\end{array}$ \\
\hline
\end{tabular}

Site No. 338.2. pecos River, at Pierce Canyon Crossing (reguIar gage)--Continued

\begin{tabular}{|c|c|c|c|c|c|}
\hline 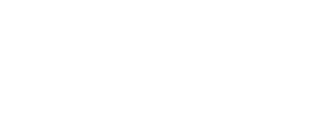 & $\begin{array}{l}1220 \\
1350 \\
1255 \\
1325 \\
1340 \\
1145 \\
1155\end{array}$ & $\begin{array}{l}86 \\
87 \\
84 \\
82 \\
82 \\
80 \\
73\end{array}$ & $\begin{array}{r}6.3 \\
7.4 \\
5.3 \\
5.5 \\
4.0 \\
9.6 \\
17\end{array}$ & $\begin{array}{r}8750 \\
9080 \\
8790 \\
10300 \\
10600 \\
1620 \\
4430\end{array}$ & $\begin{array}{r}28000 \\
28600 \\
27900 \\
31600 \\
32600 \\
6680 \\
15400\end{array}$ \\
\hline
\end{tabular}

Site No. 340. Pecos River Nw $\frac{1}{4} \mathrm{SE}_{4}^{\frac{1}{4}}$ sec.6, T.25 S., R.29 E., above Reed's pump

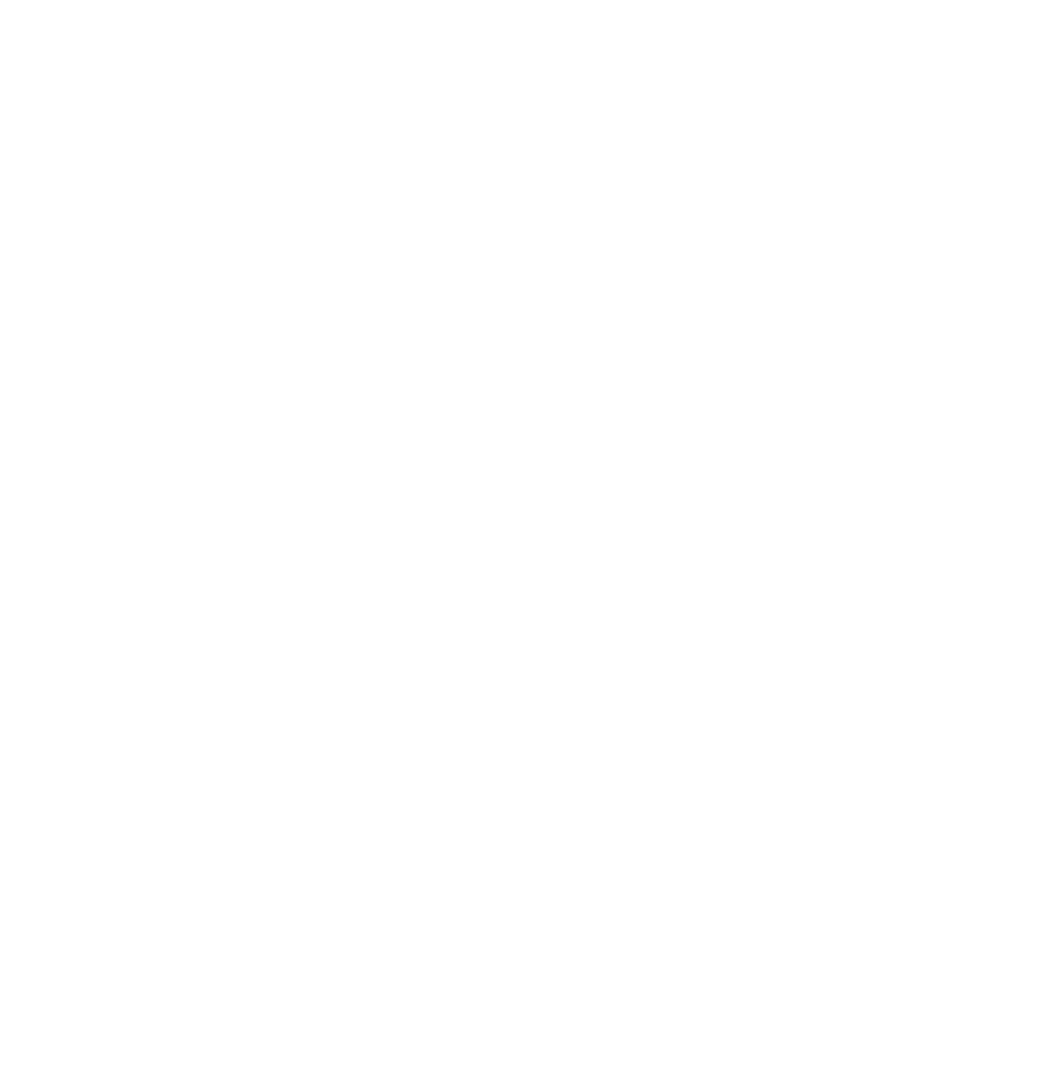




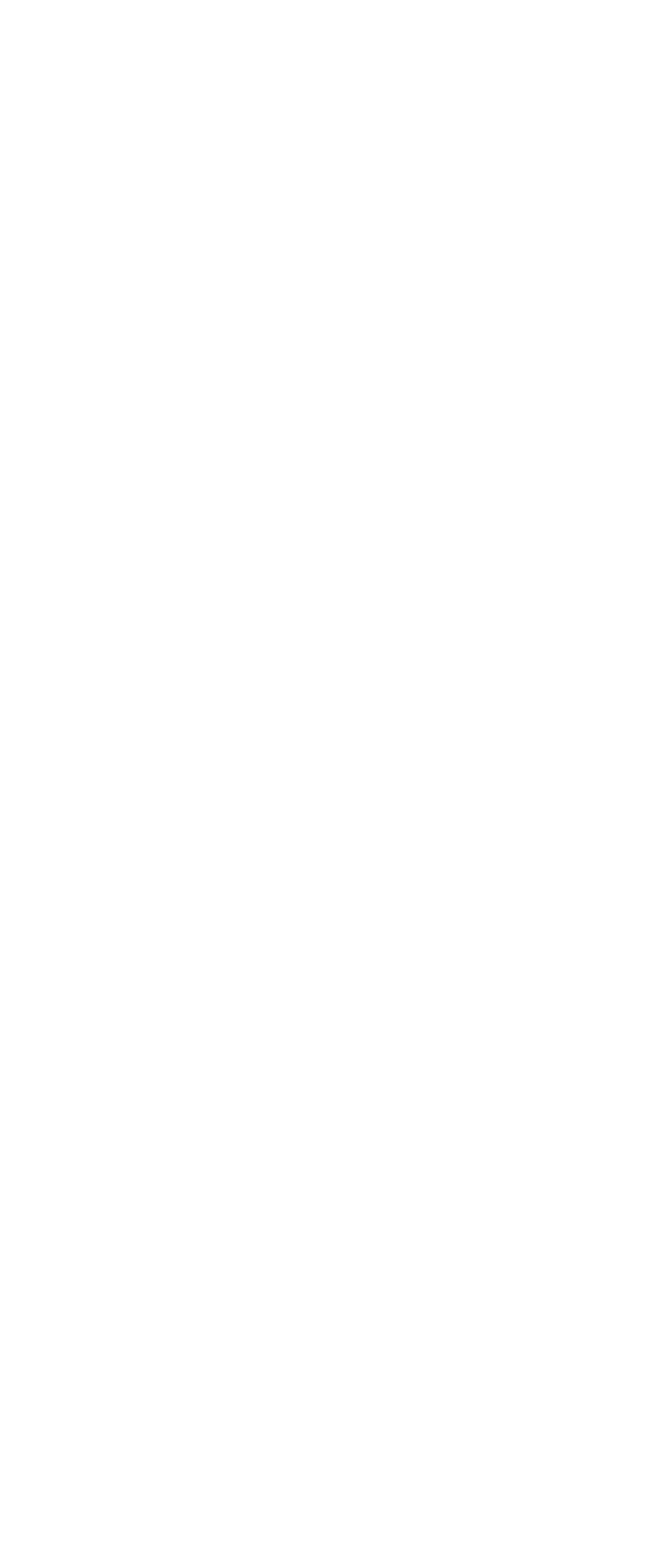




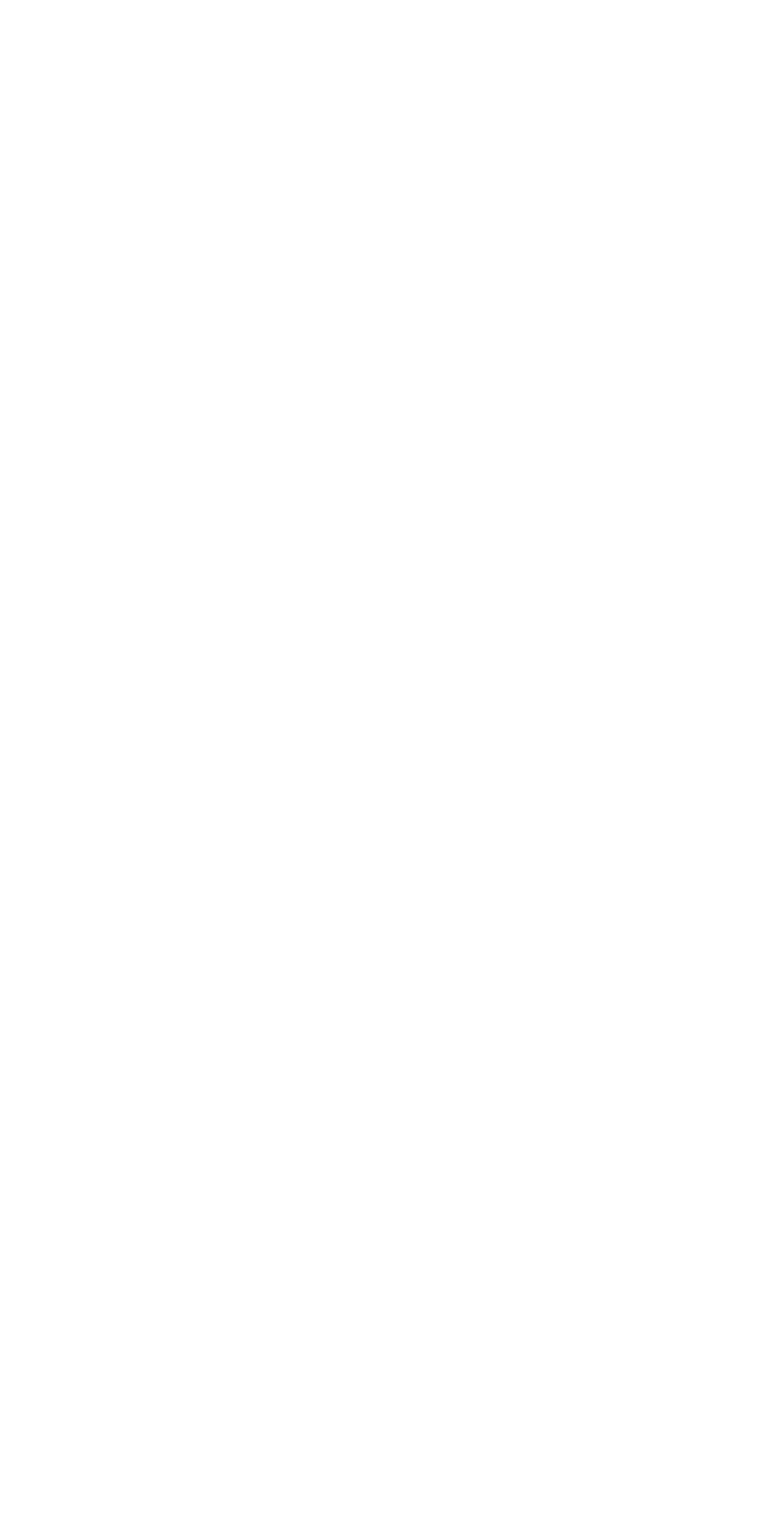




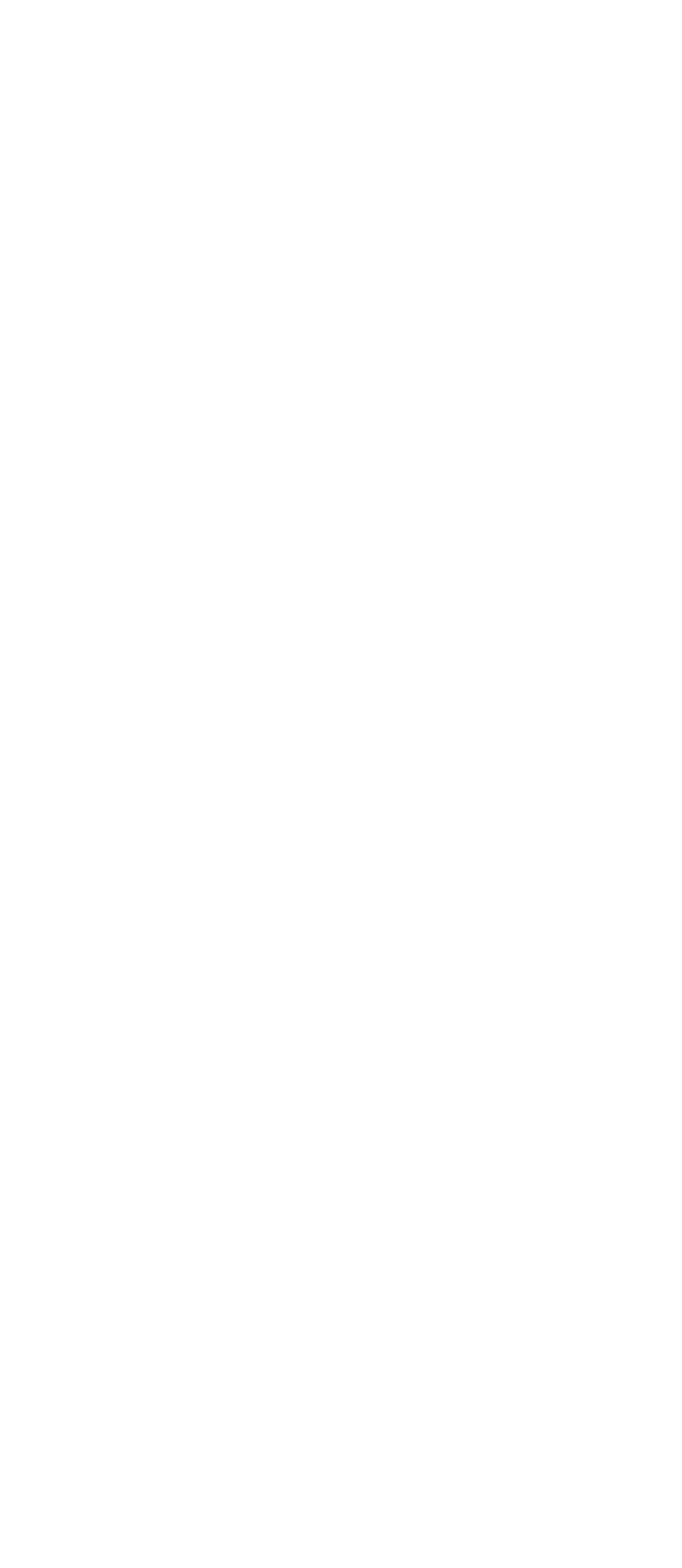




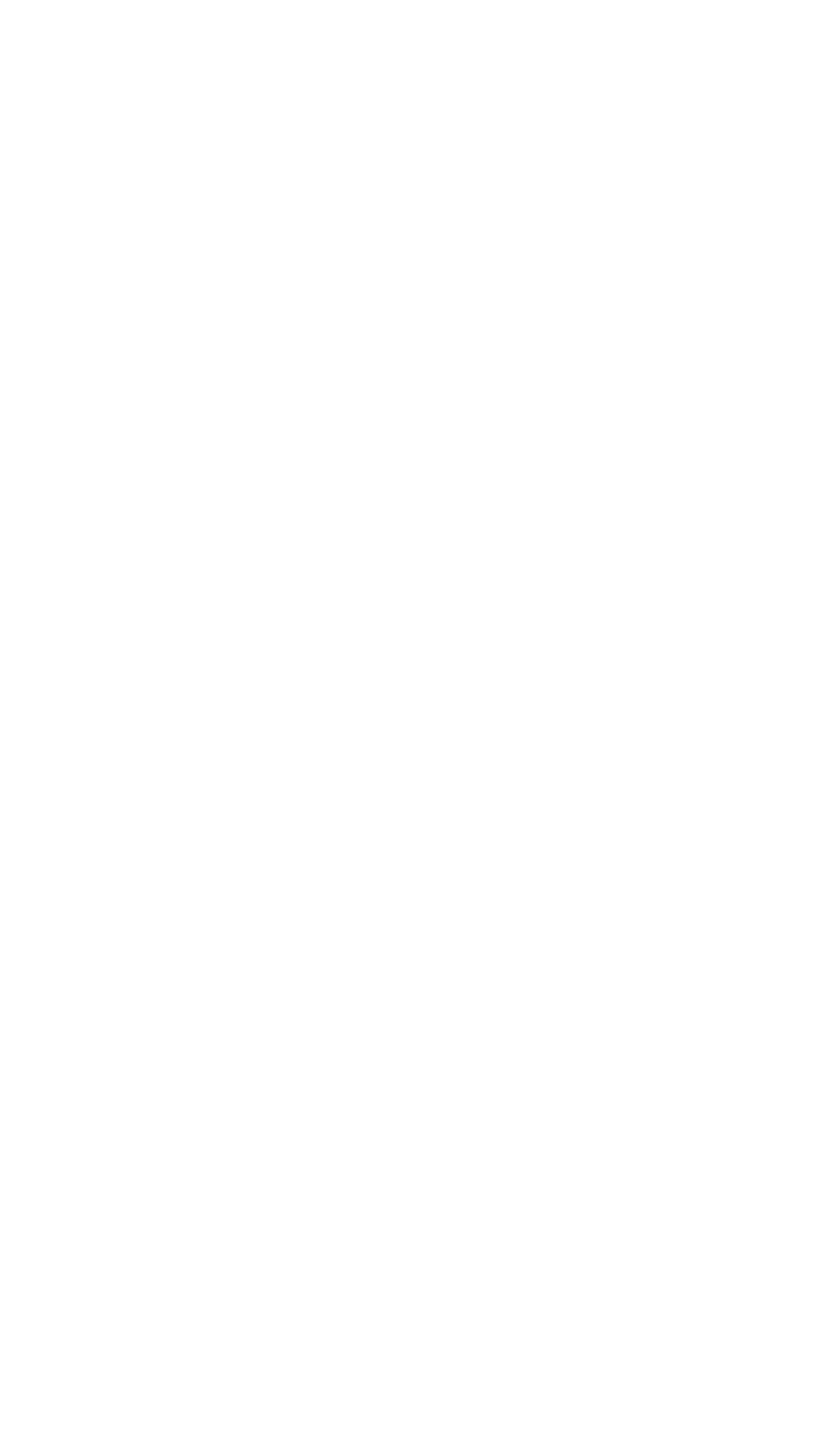




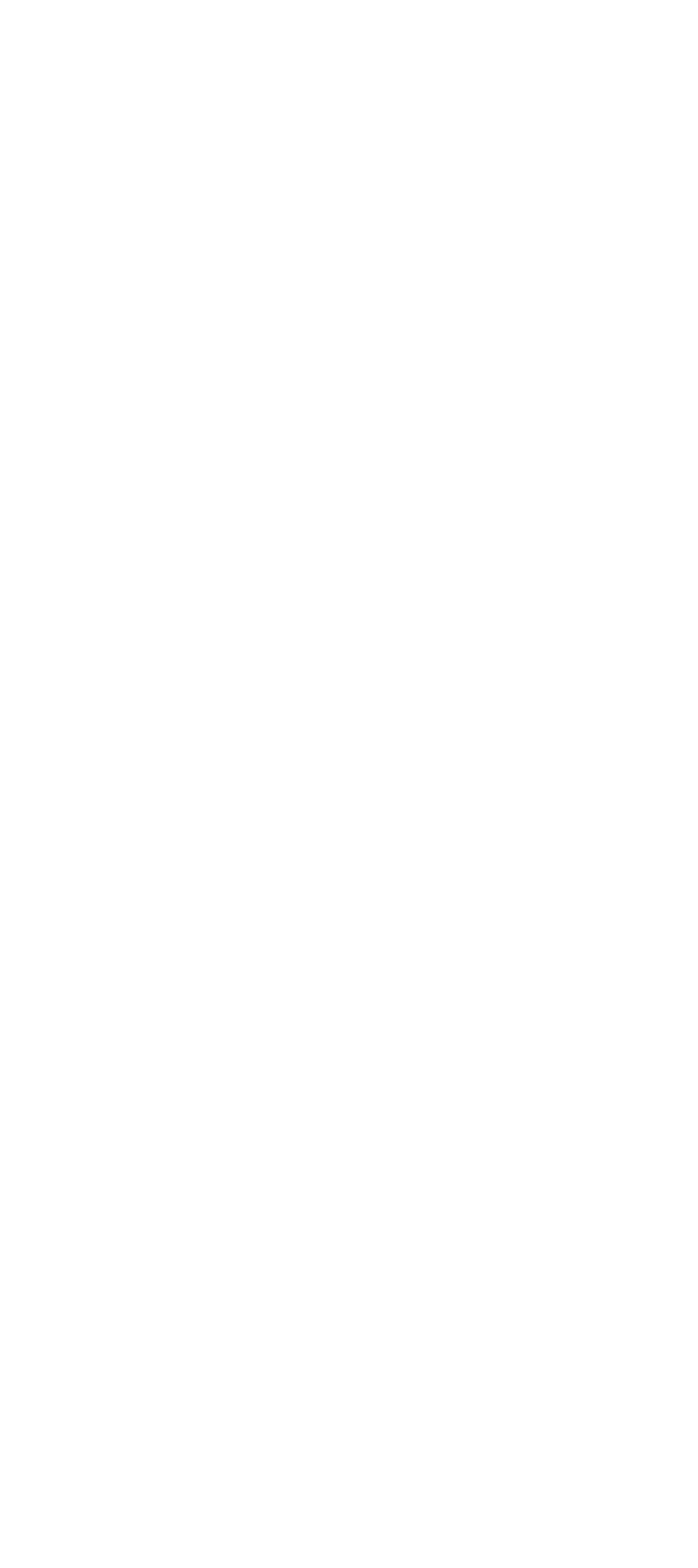




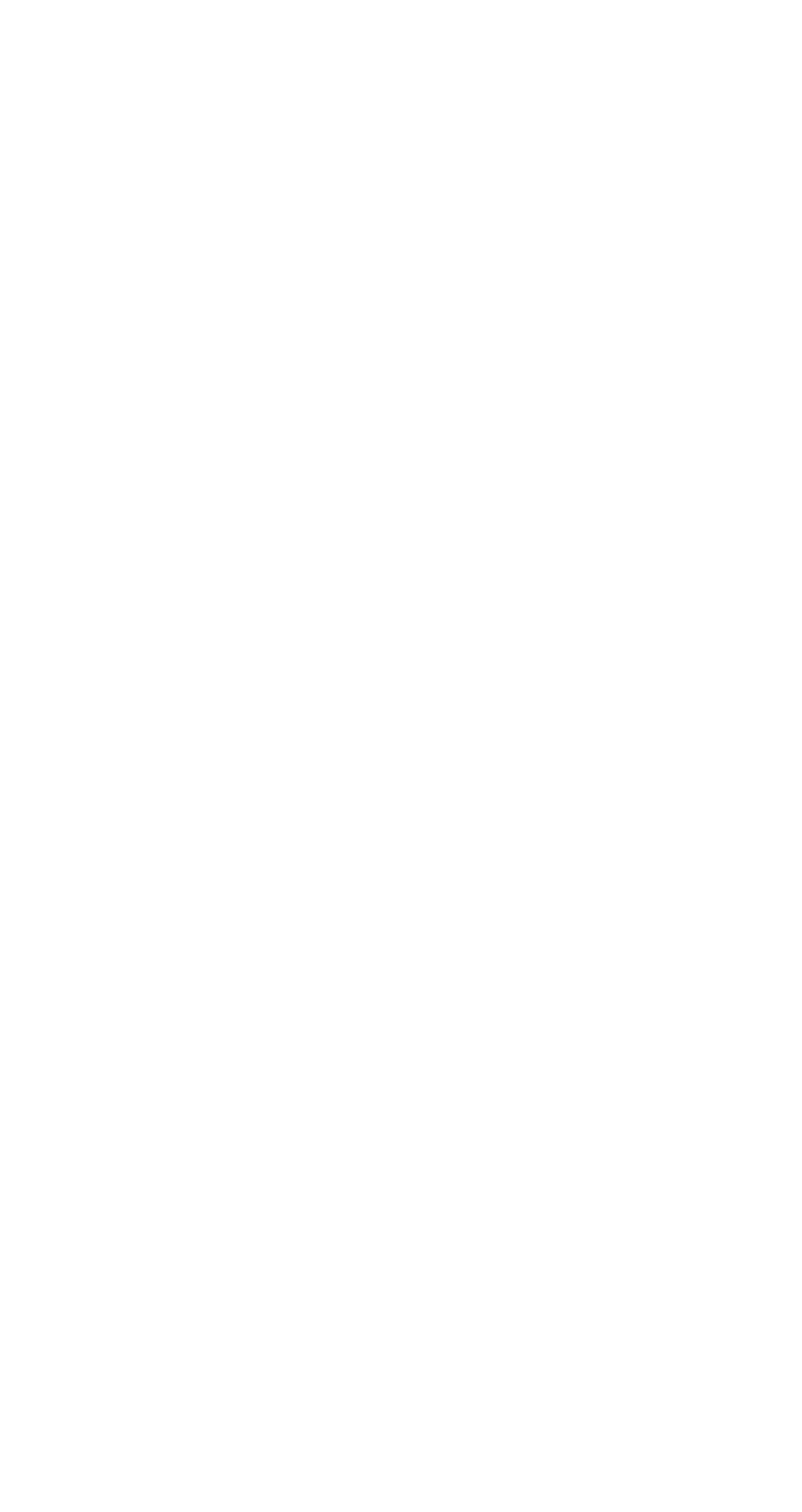




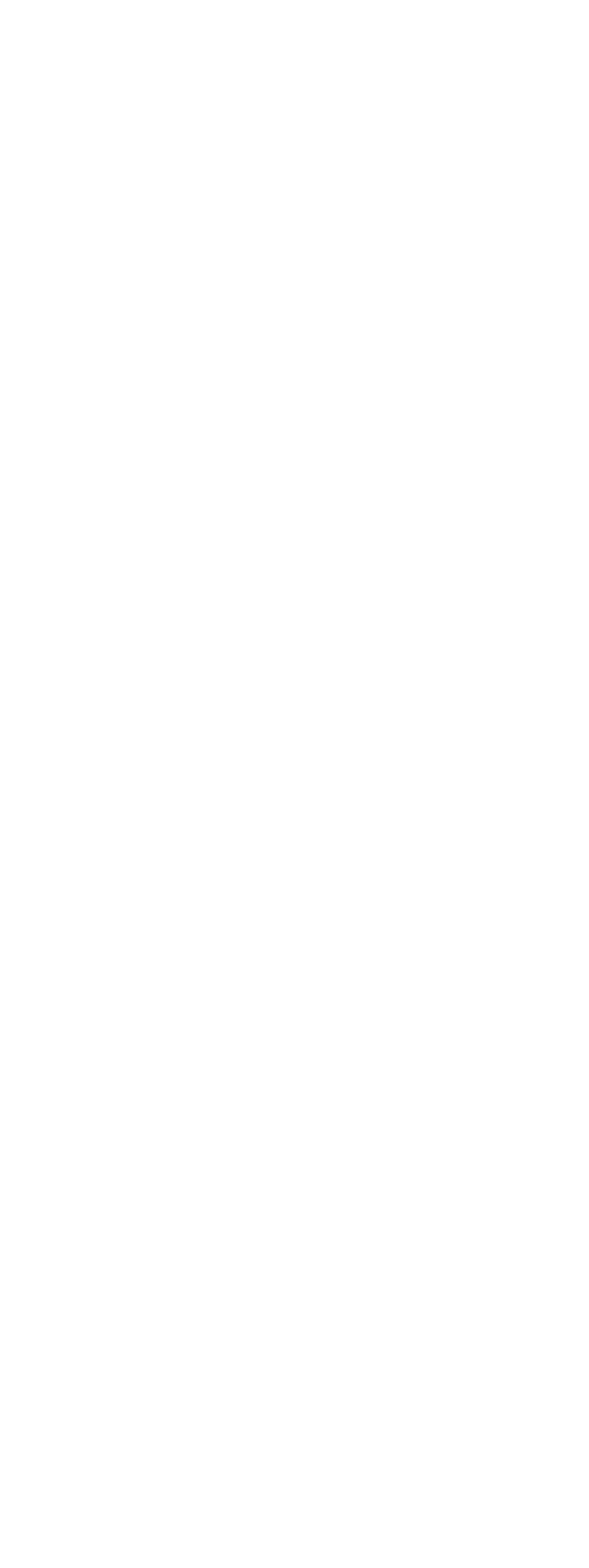




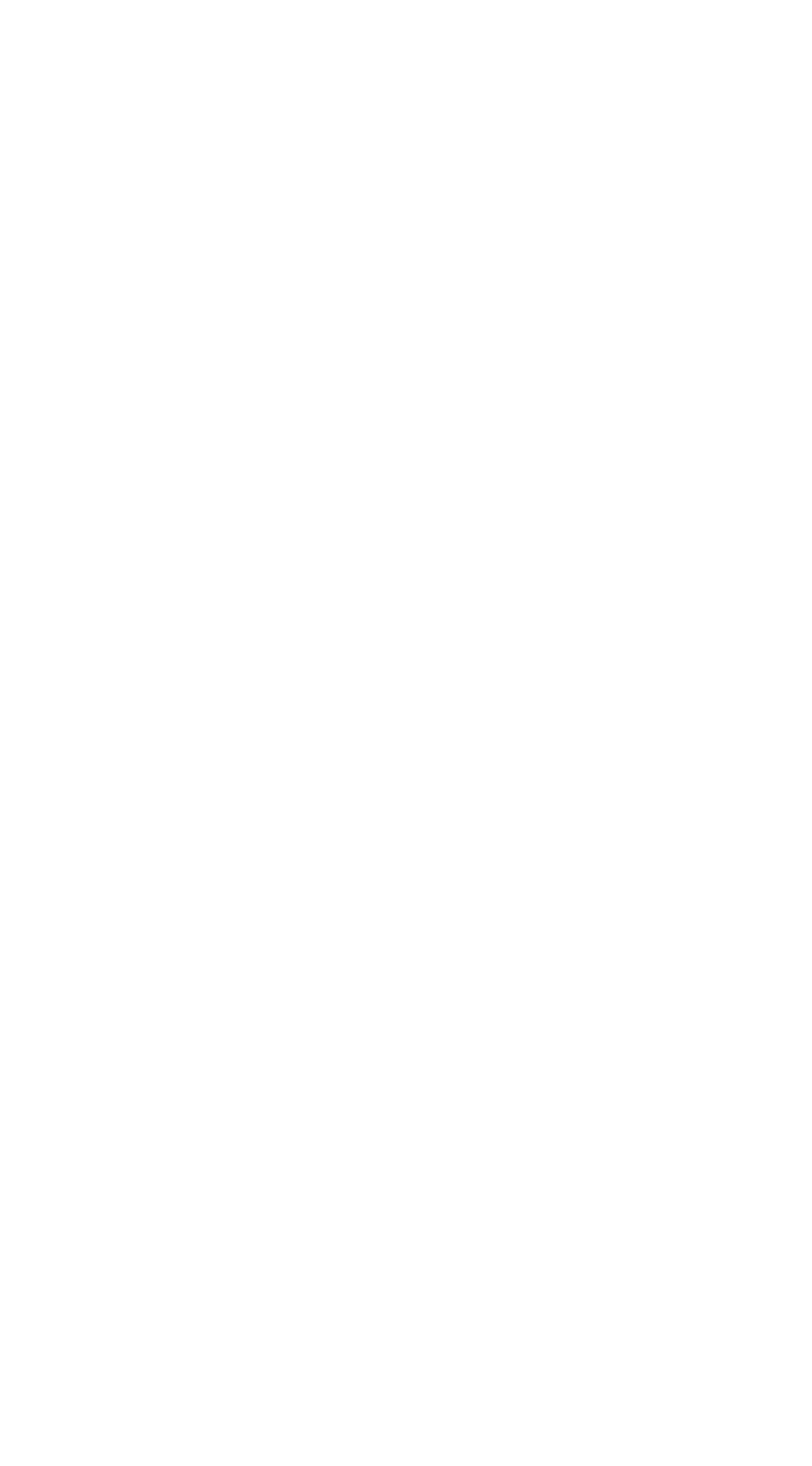




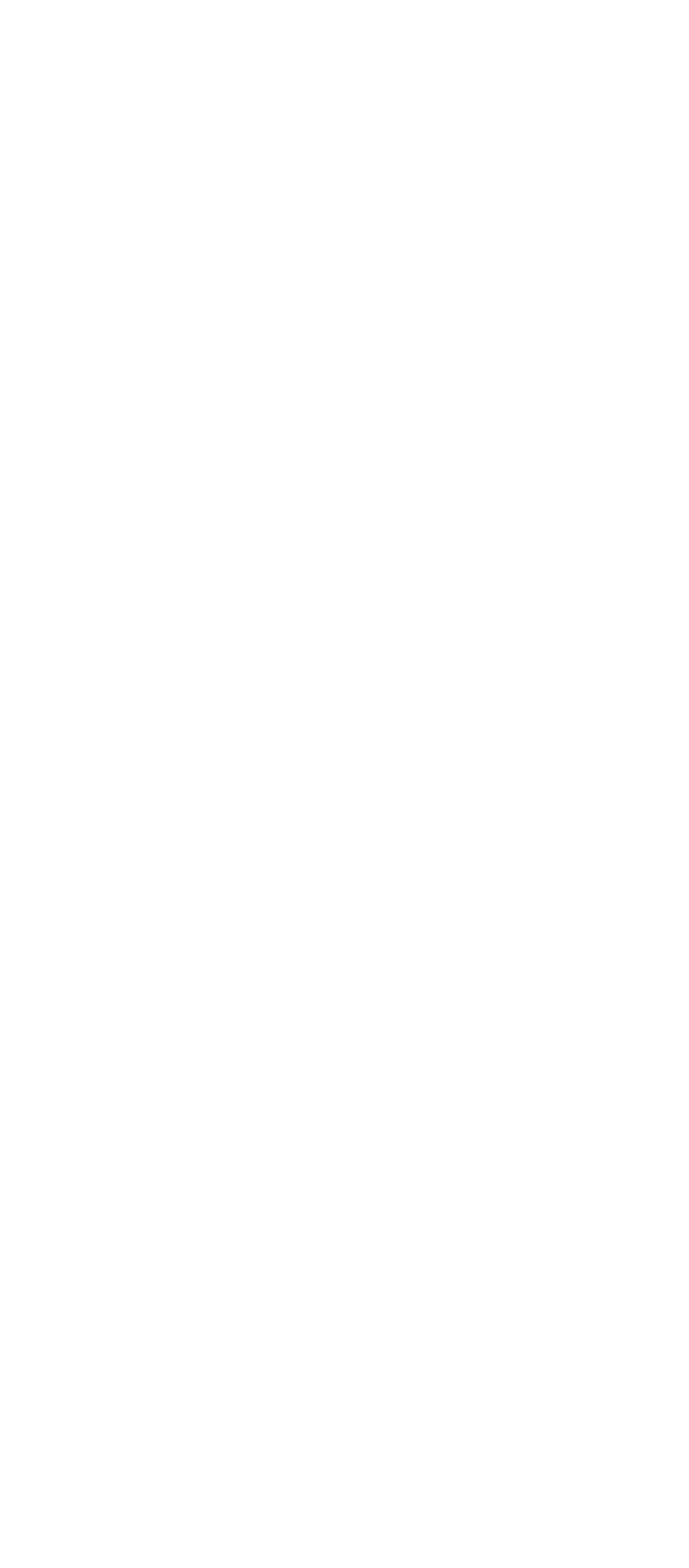




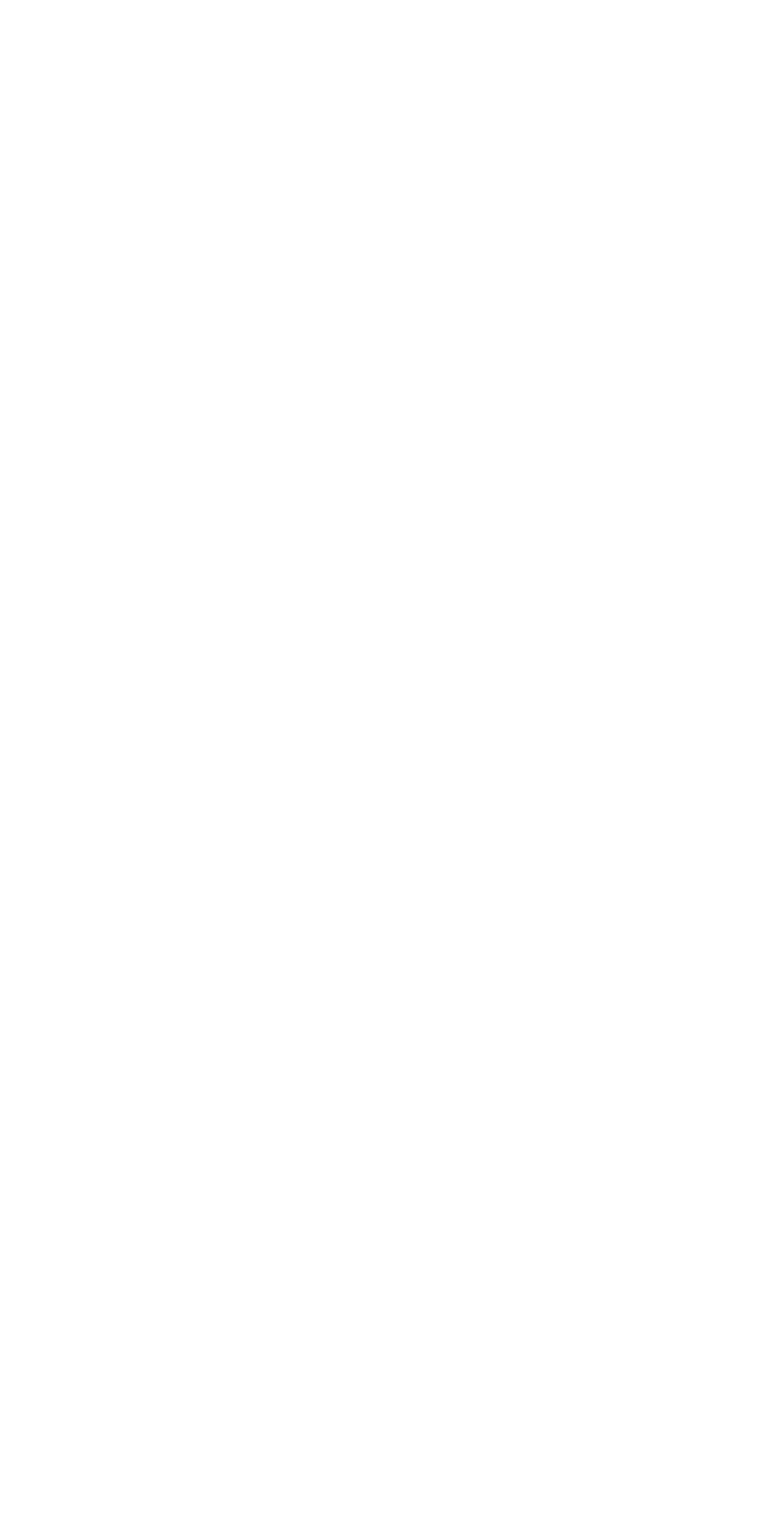




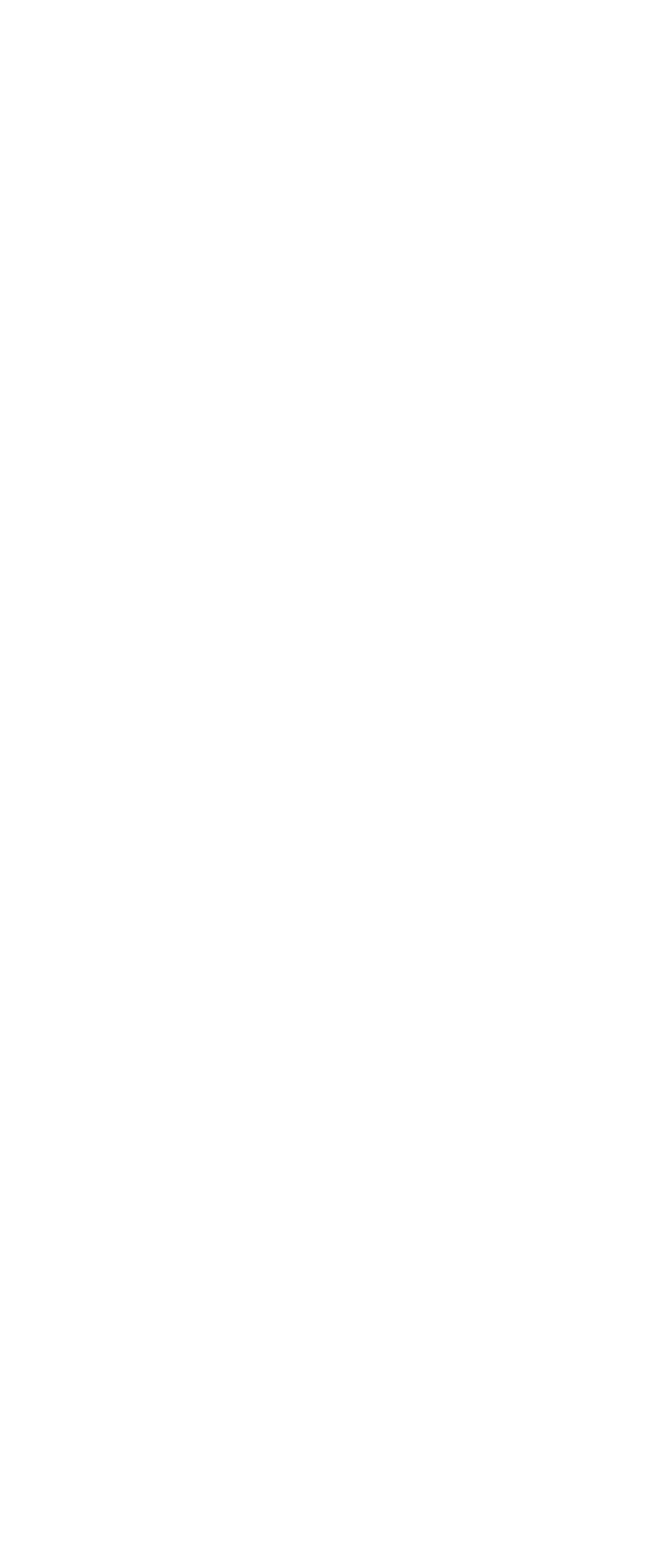




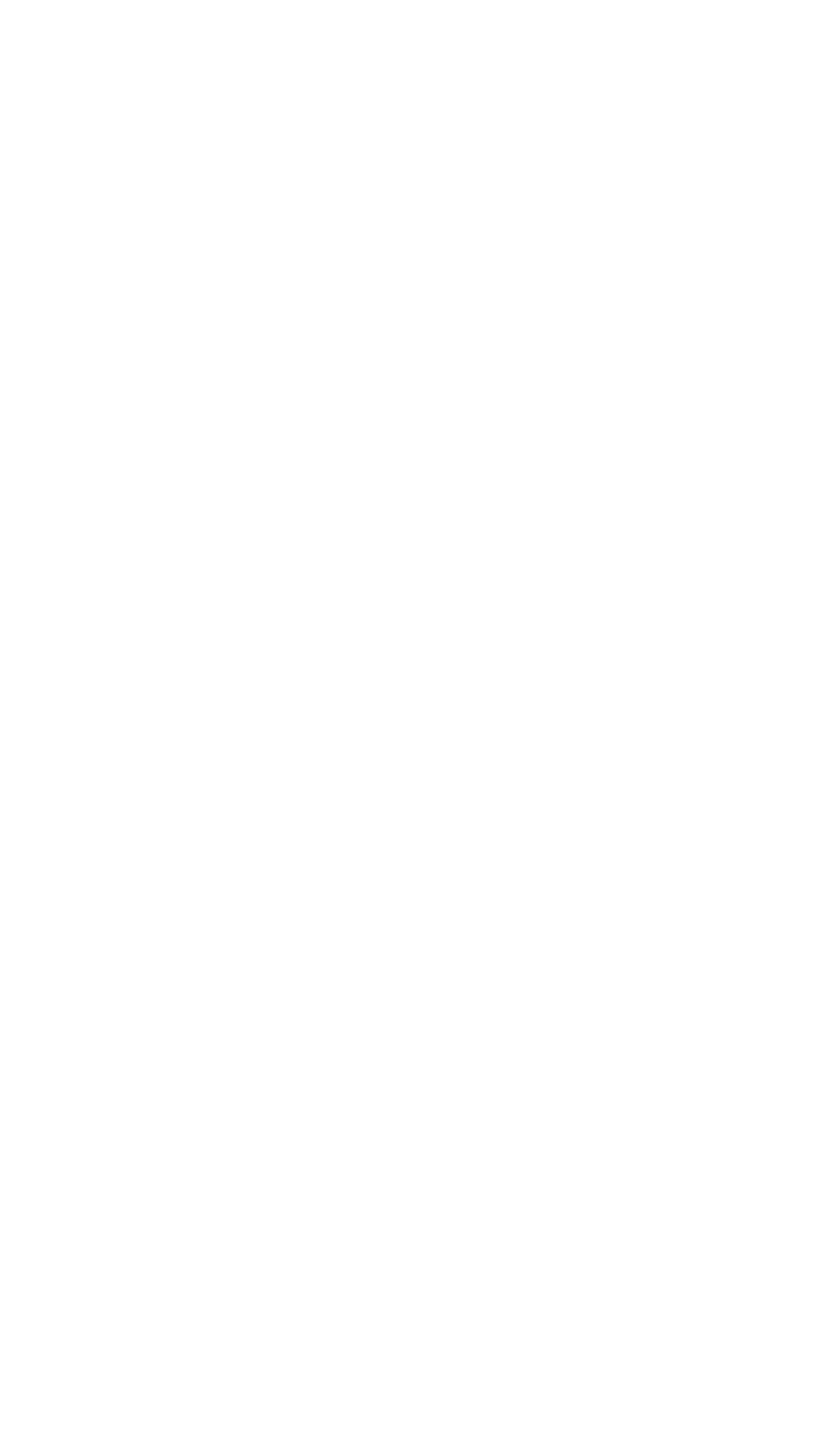




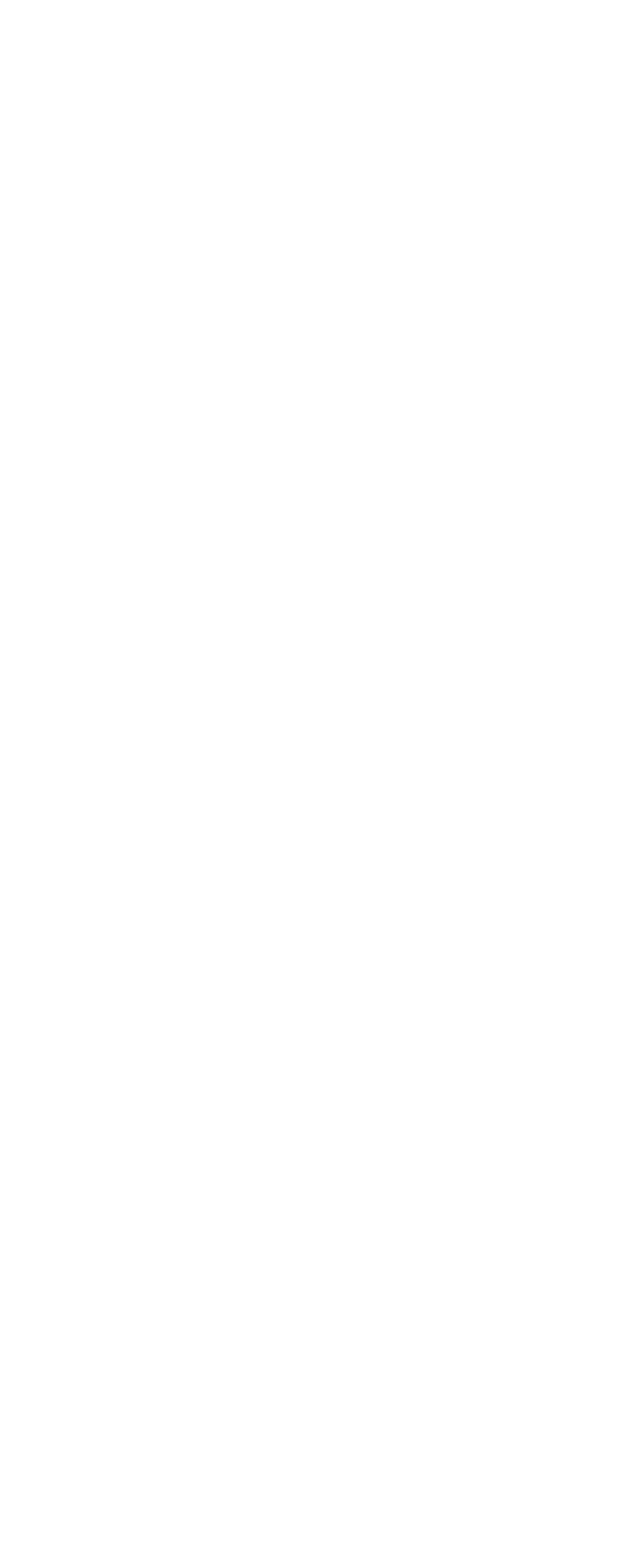




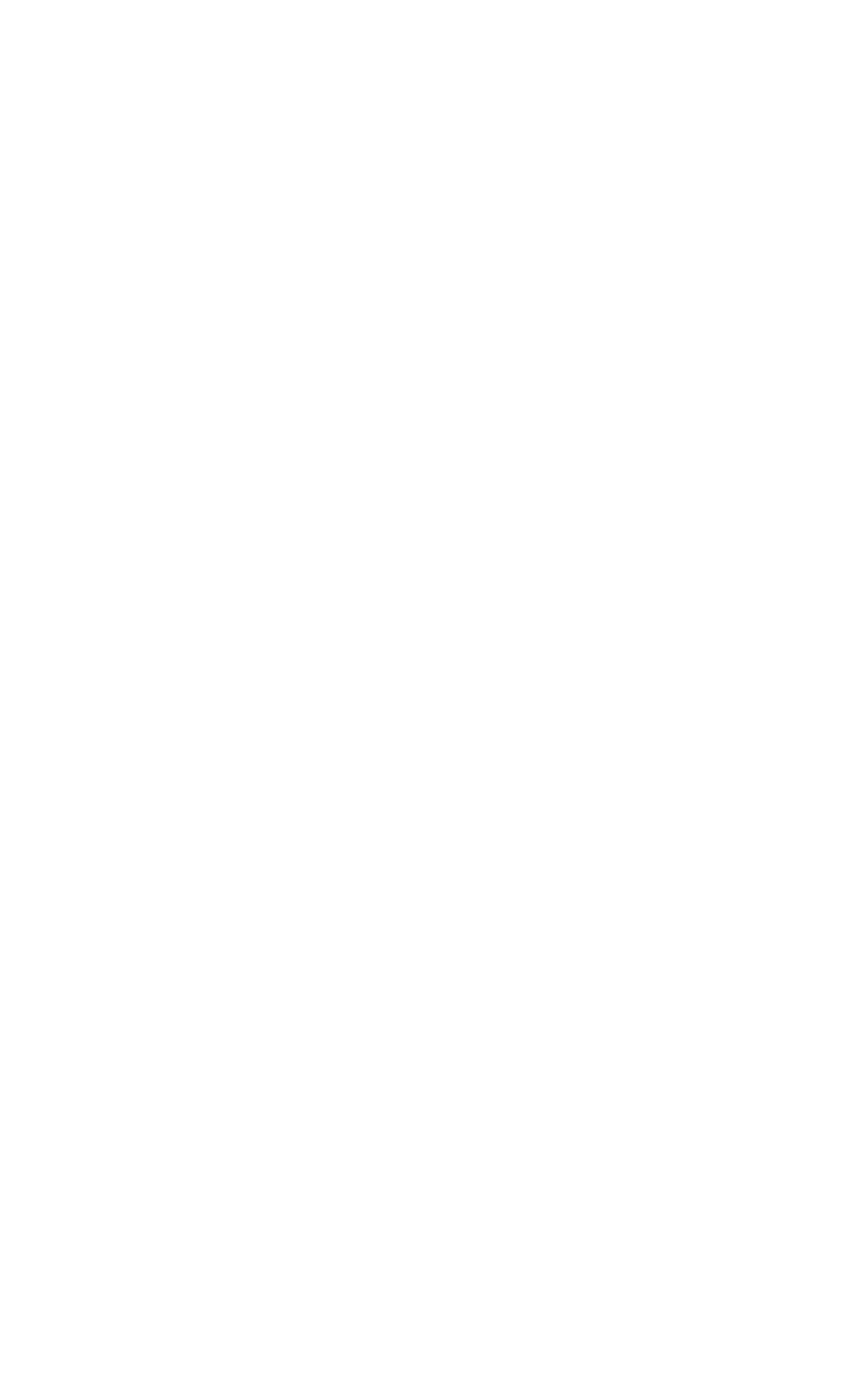




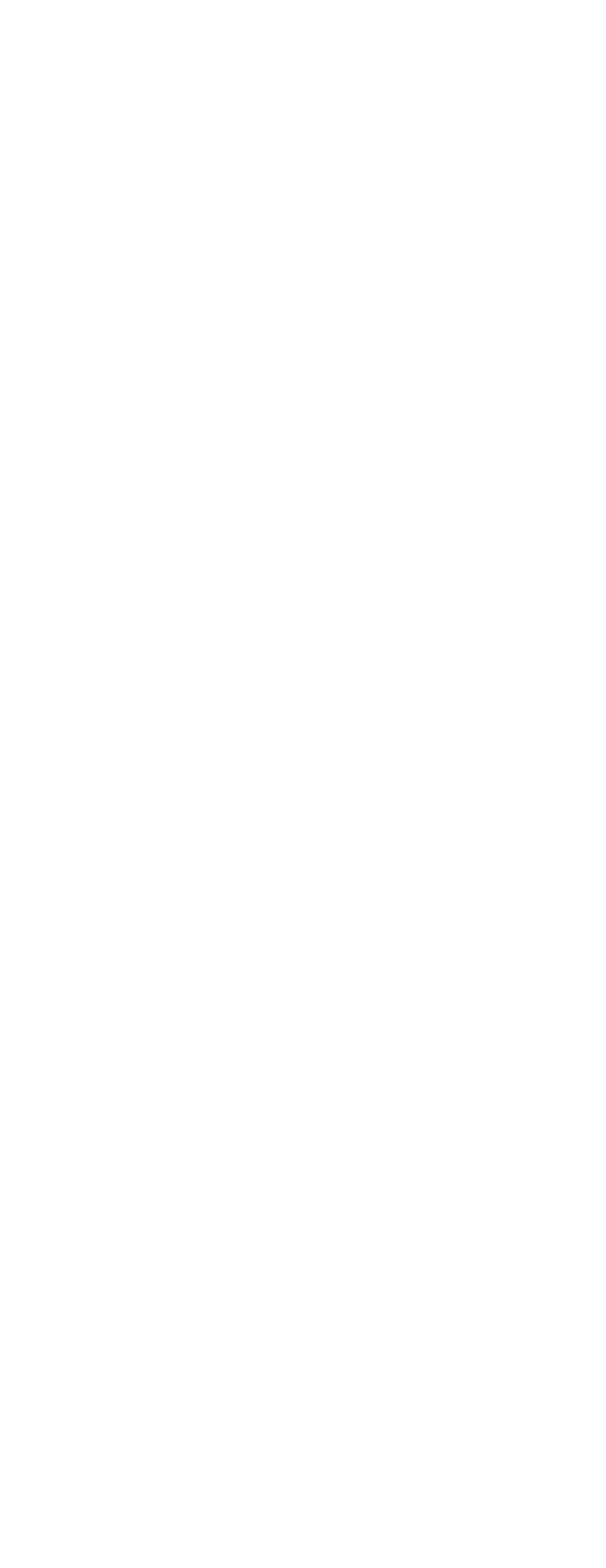




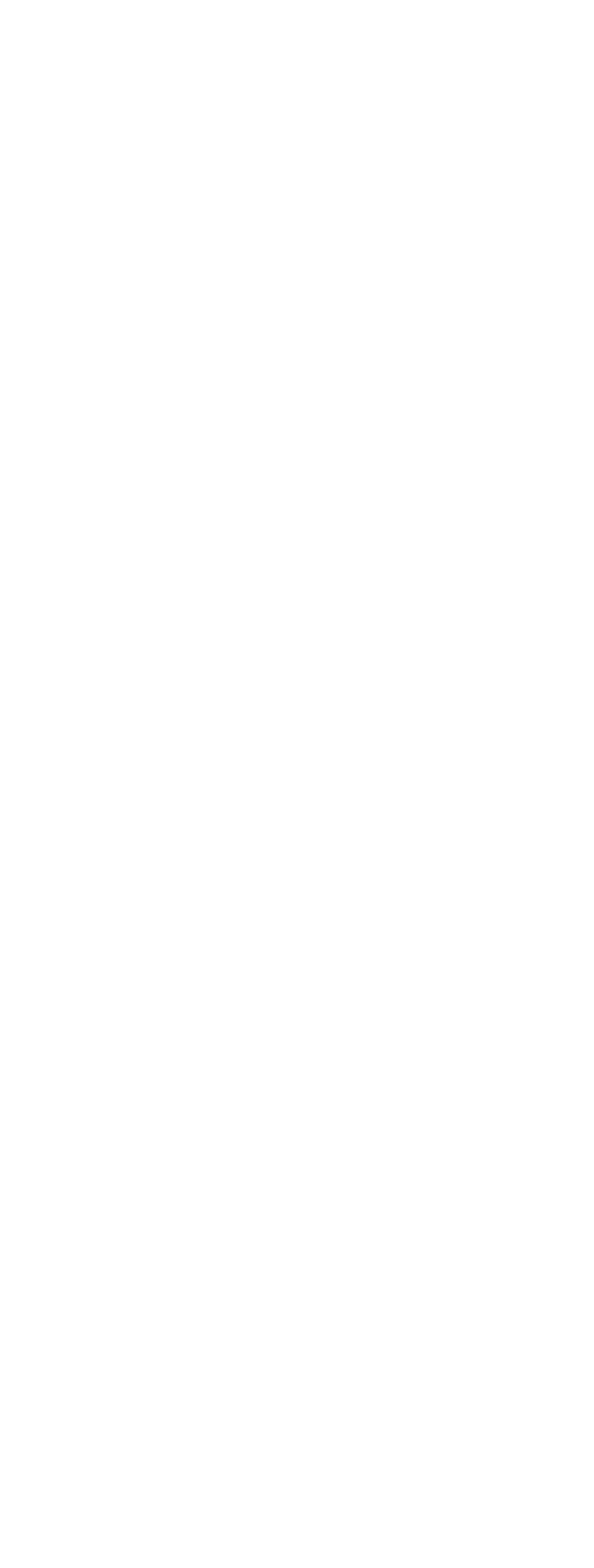


MISCELLANEOUS ANALYSES OF STREAMS IN WESTERN GULF OF MEXICO BASINS--Continued

Chloride, in parts per million, and specific conductance, micromhos at $25^{\circ} \mathrm{C}$, water year October 1963 to September 1964

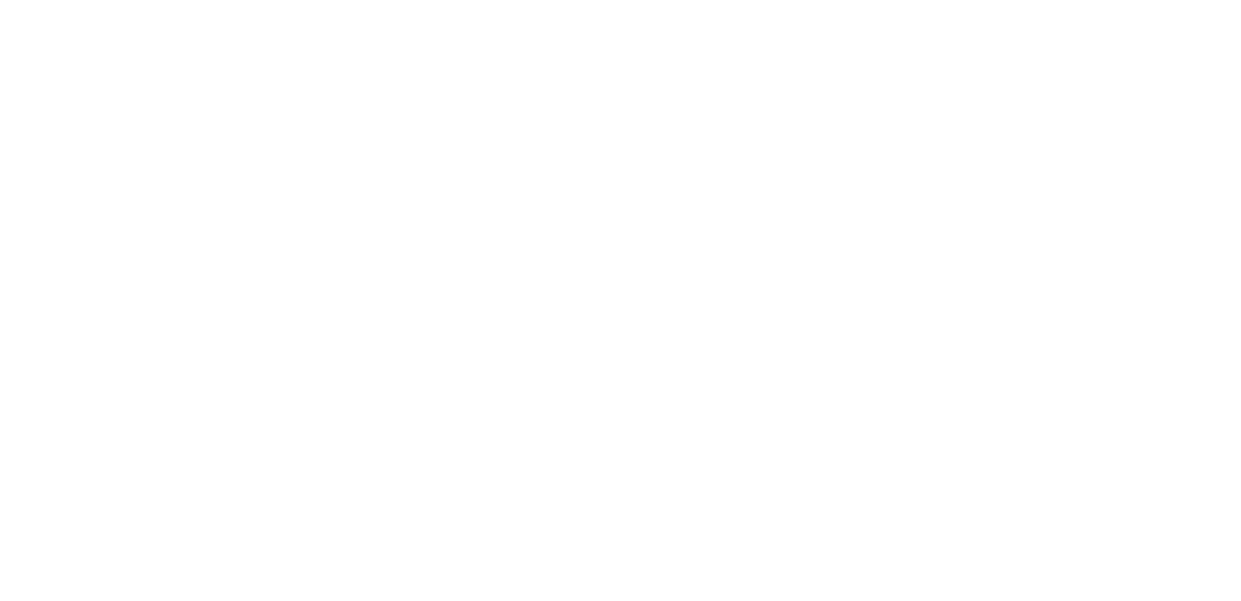

BIG CREEK AT FARM ROAD 762, NEAR GUY, TEX.

\begin{tabular}{|c|c|c|c|}
\hline $\begin{array}{l}\text { Oct. } 15,1963 \ldots \ldots \ldots \ldots \\
\text { Nov. } 21, \ldots \ldots \ldots \ldots \\
\text { Dec. } 6 \ldots \ldots \ldots \\
\text { Jan. } 14,1964 \ldots \ldots \ldots \\
\text { Feb. } 4 \ldots \ldots \ldots \ldots \ldots\end{array}$ & $\begin{array}{r}1.8 \\
2.0 \\
2.2 \\
1.8 \\
770\end{array}$ & $\begin{array}{r}292 \\
180 \\
138 \\
172 \\
3,000\end{array}$ & $\begin{array}{l}1,500 \\
1,110 \\
1,050 \\
1,110 \\
9,470\end{array}$ \\
\hline 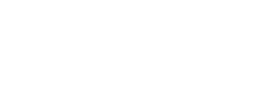 & $\begin{array}{l}550 \\
440 \\
220 \\
660 \\
440\end{array}$ & $\begin{array}{r}3,630 \\
13,000 \\
151 \\
9,150 \\
4,280\end{array}$ & $\begin{array}{r}11,100 \\
33,400 \\
793 \\
25,100 \\
13,000\end{array}$ \\
\hline 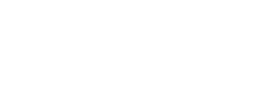 & $\begin{array}{r}440 \\
5.5 \\
-\overline{7.7} \\
4.4\end{array}$ & $\begin{array}{l}880 \\
178 \\
198 \\
242 \\
232\end{array}$ & $\begin{array}{l}3,120 \\
1,150 \\
1,120 \\
1,270 \\
1,170\end{array}$ \\
\hline 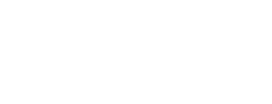 & $\begin{array}{c}100 \\
22 \\
35 \\
7.7 \\
44\end{array}$ & $\begin{array}{r}78 \\
98 \\
118 \\
292 \\
242\end{array}$ & $\begin{array}{r}550 \\
661 \\
713 \\
1,360 \\
1,210\end{array}$ \\
\hline
\end{tabular}

BIG CREEK AT FARM ROAD 1994, NEAR GUY, TEX.

\begin{tabular}{|c|c|c|c|}
\hline $\begin{array}{l}\text { Oct. } 15,1963 \ldots \ldots \ldots \ldots \\
\text { Nov. } 21 \ldots \ldots \ldots \ldots \ldots \\
\text { Dec. } 6 \ldots \ldots \ldots \\
\text { Jan. } 14,1964 \ldots \ldots \ldots \ldots \\
\text { Feb. } 4 \ldots \ldots \ldots \ldots \ldots\end{array}$ & $\begin{array}{r}\text { l. } \\
1.3 \\
1.3 \\
660^{.9}\end{array}$ & $\begin{array}{r}310 \\
190 \\
119 \\
140 \\
11\end{array}$ & $\begin{array}{r}1,550 \\
1,120 \\
770 \\
983 \\
205\end{array}$ \\
\hline 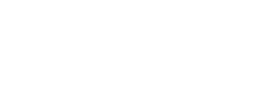 & $\begin{array}{r}440 \\
330 \\
175 \\
1,100 \\
330\end{array}$ & $\begin{array}{r}16 \\
16 \\
9.0 \\
4.7 \\
6.7\end{array}$ & $\begin{array}{l}289 \\
190 \\
158 \\
398 \\
242\end{array}$ \\
\hline $\begin{array}{l}\text { Mar. } 4 \ldots \ldots \ldots \\
\text { Mar. } 17 \ldots \ldots \ldots \\
\text { Apr. } 21 \ldots \ldots \ldots \\
\text { Apr } 23 \ldots \ldots \ldots \\
\text { May } 19 \ldots \ldots \ldots\end{array}$ & $\begin{array}{l}330 \\
2.2 \\
20 \\
2.2 \\
1.6\end{array}$ & $\begin{array}{l}7.9 \\
121 \\
198 \\
225 \\
280\end{array}$ & $\begin{array}{r}166 \\
893 \\
1,090 \\
1,200 \\
1,290\end{array}$ \\
\hline 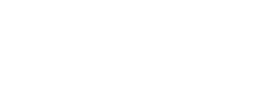 & $\begin{array}{l}88 \\
18 \\
10 \\
4.4 \\
33\end{array}$ & $\begin{array}{l}150 \\
102 \\
122 \\
308 \\
255\end{array}$ & $\begin{array}{r}822 \\
669 \\
723 \\
1,460 \\
1,360\end{array}$ \\
\hline
\end{tabular}


MISCELlaNEOUS ANALYSES OF STREAMS IN WESTERN GULF OF MEXICO BASINS--Continued

Chloride, in parts per milion, and specific conductance, micromhos at $25^{\circ} \mathrm{C}$, water year October 1963 to September 1964

\begin{tabular}{l|c|c|c|c}
\hline Date of collection & Discharge (cfs estimated) & Chloride (ppm) & $\begin{array}{l}\text { Specific conductance } \\
\text { (micromhos at } 25^{\circ} \mathrm{C} \text { ) }\end{array}$ \\
\hline
\end{tabular}

BRAZOS RIVER BASIN--Continued

COW CREEK AT KITTY NASH ROAD, 8 MILES NORTHEAST OF DAMON, TEX.

\begin{tabular}{|c|c|c|c|}
\hline $\begin{array}{ll}\text { Oct. } & 15,1963 \ldots \ldots \ldots \\
\text { Nov. } & 21 \ldots \ldots \ldots \ldots \\
\text { Dec. } & 6 \ldots \ldots \ldots \ldots \\
\text { Jan. } 14,1964 \ldots \ldots \\
\text { Feb. } & 4 \ldots \ldots \ldots \ldots \\
\end{array}$ & $\begin{array}{c}0.4 \\
.1 \\
.7 \\
550\end{array}$ & $\begin{array}{r}645 \\
200 \\
61 \\
65 \\
15\end{array}$ & $\begin{array}{r}2,510 \\
1,000 \\
410 \\
418 \\
187\end{array}$ \\
\hline 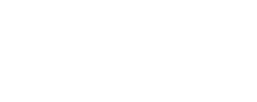 & $\begin{array}{l}330 \\
220 \\
.2 \\
.--\end{array}$ & $\begin{array}{l}23 \\
21 \\
29 \\
71 \\
73\end{array}$ & $\begin{array}{l}188 \\
199 \\
208 \\
464 \\
576\end{array}$ \\
\hline 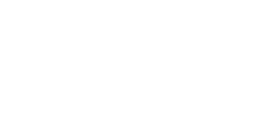 & $\begin{array}{r}.2 \\
22.3 \\
2.2 \\
130\end{array}$ & $\begin{array}{r}95 \\
100 \\
30 \\
258 \\
121 \\
16\end{array}$ & $\begin{array}{r}663 \\
723 \\
278 \\
1,280 \\
772 \\
209\end{array}$ \\
\hline
\end{tabular}

VARNER CREEK 2 MILES NORTH OF WEST COLUMBIA, TEX. ABOUT 3 MILES ABOTE MOUTH.

\begin{tabular}{|c|c|c|c|}
\hline $\begin{array}{l}\text { Oct. } 15,1963 \ldots \ldots \ldots \\
\text { Nov. } 21 \ldots \ldots \ldots \ldots \ldots \\
\text { Dec. } 6 \ldots \ldots \ldots \ldots \ldots \\
\text { Jan. } 14,1964 \ldots \ldots \ldots \ldots \\
\text { Feb. } 4 \ldots \ldots \ldots \ldots \ldots\end{array}$ & $\begin{array}{l}0 . \overline{07} \\
1.3 \\
.04 \\
4.4\end{array}$ & $\begin{array}{r}880 \\
12,100 \\
150 \\
1,550 \\
17\end{array}$ & $\begin{array}{r}3,070 \\
30,100 \\
689 \\
4,880 \\
166\end{array}$ \\
\hline $\begin{array}{ll}\text { Feb. } & 5 \ldots \ldots \ldots \\
\text { Feb. } & 6 \ldots \ldots \ldots \ldots \\
\text { Mar. } & 17 \ldots \ldots \ldots \ldots \\
\text { Apr. } & 21 \\
\text { Apr. } & 23 \ldots \ldots \ldots\end{array}$ & $\begin{array}{r}330 \\
88 \\
.2 \\
\quad-- \\
--\end{array}$ & $\begin{array}{r}10 \\
21 \\
232 \\
136 \\
9,150\end{array}$ & $\begin{array}{r}134 \\
186 \\
1,060 \\
879 \\
23,700\end{array}$ \\
\hline $\begin{array}{l}\text { May } 19 \ldots \ldots \ldots \\
\text { June } 16 \ldots \ldots \ldots \\
\text { July } 15 \ldots \ldots \ldots \\
\text { Aug. } 18 \ldots \ldots \ldots \\
\text { Sept. } 17 \ldots \ldots \ldots\end{array}$ & $\begin{array}{r}.1 \\
1.1 \\
1.9 \\
22\end{array}$ & $\begin{array}{r}740 \\
315 \\
121 \\
1,550 \\
163\end{array}$ & $\begin{array}{r}2,590 \\
1,260 \\
551 \\
4,810 \\
691\end{array}$ \\
\hline
\end{tabular}

VARNER CREEK AT STATE HIGHWAY 35, AT EAST COLUMBIA, TEX.

\begin{tabular}{|c|c|c|c|}
\hline $\begin{array}{ll}\text { Oct. } & 15,1963 \ldots \ldots \ldots \\
\text { Nov. } & 21 \ldots \ldots \ldots \ldots \\
\text { Dec. } & 6, \ldots \ldots \ldots \ldots \\
\text { Jan. } 14,1964 \ldots \ldots \\
\text { Feb. } 4 \ldots \ldots \ldots\end{array}$ & $\begin{array}{r}0.1 \\
1.4 \\
130^{.1}\end{array}$ & $\begin{array}{l}310 \\
188 \\
285 \\
305 \\
137\end{array}$ & $\begin{array}{r}1,530 \\
910 \\
1,170 \\
1,540 \\
617\end{array}$ \\
\hline $\begin{array}{ll}\text { Feb. } & 5 \\
\text { Feb. } & 61 \ldots \ldots \ldots \ldots \\
\text { Mar. } & 17 \ldots \ldots \ldots \\
\text { Apr. } & 21 \\
\text { Apr. } & 23 \ldots \ldots\end{array}$ & $\begin{array}{r}550 \\
130 \\
-- \\
-\overline{.2}\end{array}$ & $\begin{array}{r}61 \\
25 \\
308 \\
298 \\
298\end{array}$ & $\begin{array}{r}325 \\
202 \\
1,560 \\
1,500 \\
1,490\end{array}$ \\
\hline $\begin{array}{l}\text { May } 19, \ldots \ldots \ldots \ldots \\
\text { June } 16 \ldots \ldots \ldots \\
\text { July } 15 \ldots \ldots \ldots \\
\text { Aug. } 18 \ldots \ldots \ldots \\
\text { Sept. } 17 \ldots \ldots \ldots\end{array}$ & $\begin{array}{l}2.1 \\
1.1 \\
1.5 \\
66\end{array}$ & $\begin{array}{r}348 \\
2,600 \\
545 \\
462 \\
910\end{array}$ & $\begin{array}{l}1,710 \\
7,830 \\
2,080 \\
1,670 \\
3,040\end{array}$ \\
\hline
\end{tabular}

WEST COLUMBIA OIL FIELD WASTE DISCHARGE DITCH, ABOUT 2 MILES NORTH OF WEST COLUMBIA, TEX.

\begin{tabular}{|c|c|c|c|}
\hline $\begin{array}{l}\text { Nov. } 21,1963 \ldots \ldots \ldots \\
\text { Dec. } 6 \ldots \ldots \ldots \\
\text { Jan. } 14,1964 \ldots \ldots \ldots \ldots \\
\text { Feb. } 4 \ldots \ldots \ldots \ldots \ldots \\
\text { Feb. } 5 \ldots \ldots \ldots \ldots\end{array}$ & $\begin{array}{r}0.01 \\
.01 \\
44^{--} \\
110^{--}\end{array}$ & $\begin{array}{r}\mathbf{7}, 780 \\
6,000 \\
3,550 \\
575 \\
\mathbf{3 2 8}\end{array}$ & $\begin{array}{r}20,800 \\
16,500 \\
10,100 \\
2,020 \\
1,260\end{array}$ \\
\hline $\begin{array}{l}\text { Feb. } 6 \ldots \ldots \ldots \ldots \ldots \\
\text { Mar. } 17 \ldots \ldots \ldots \ldots \ldots \\
\text { June } 16 \ldots \ldots \ldots \ldots \ldots \\
\text { July } 15 \ldots \ldots \ldots \ldots \ldots \\
\text { Aug. } 18 \ldots \ldots \ldots \ldots \ldots \\
\text { Sept. } 17 \ldots \ldots \ldots \ldots \ldots\end{array}$ & $\frac{22}{33^{. \overline{1}}}$ & $\begin{array}{r}532 \\
37,300 \\
2,050 \\
2,650 \\
6,850 \\
1,030\end{array}$ & $\begin{array}{r}1,920 \\
75,400 \\
6,970 \\
7,920 \\
18,200 \\
3,290\end{array}$ \\
\hline
\end{tabular}




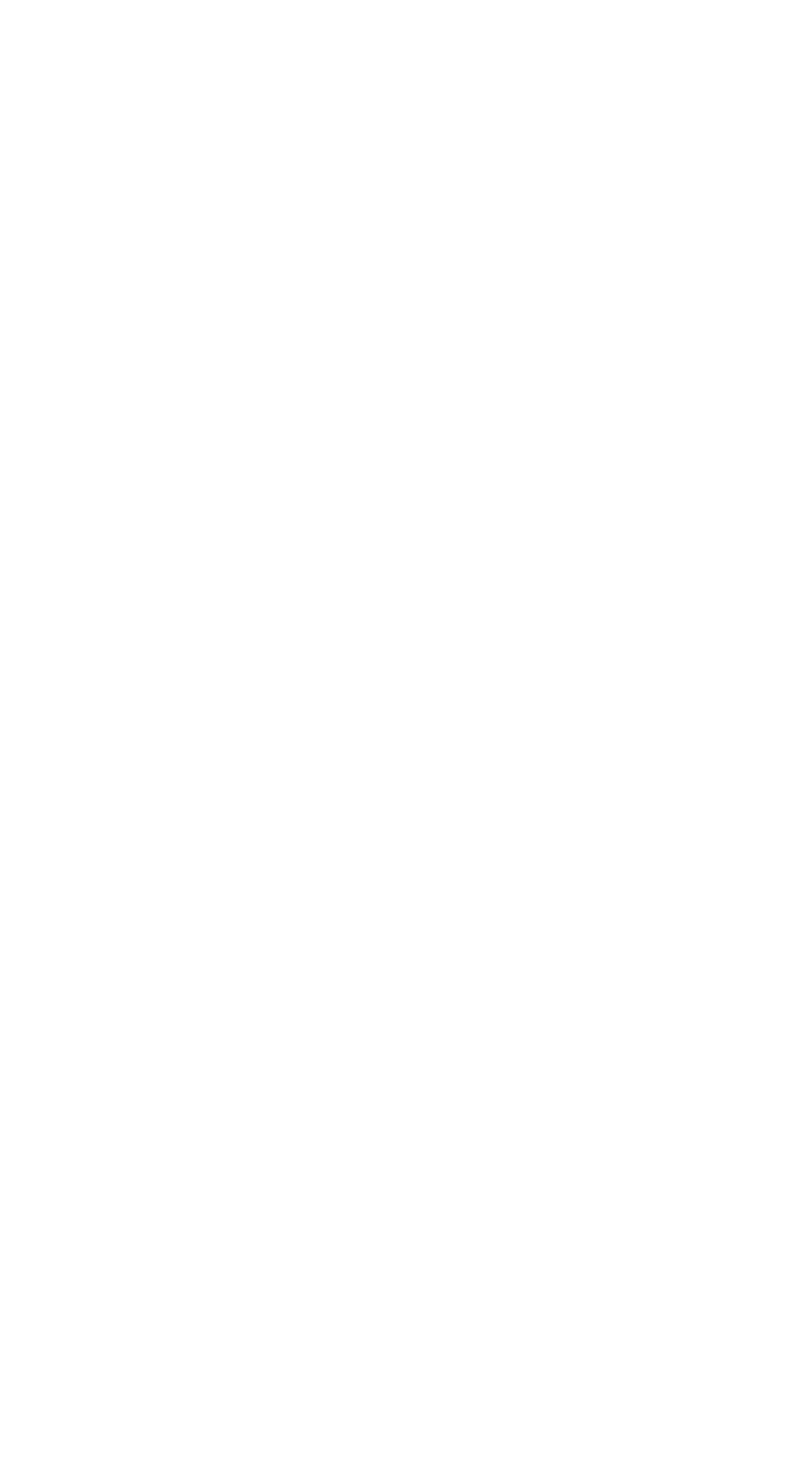




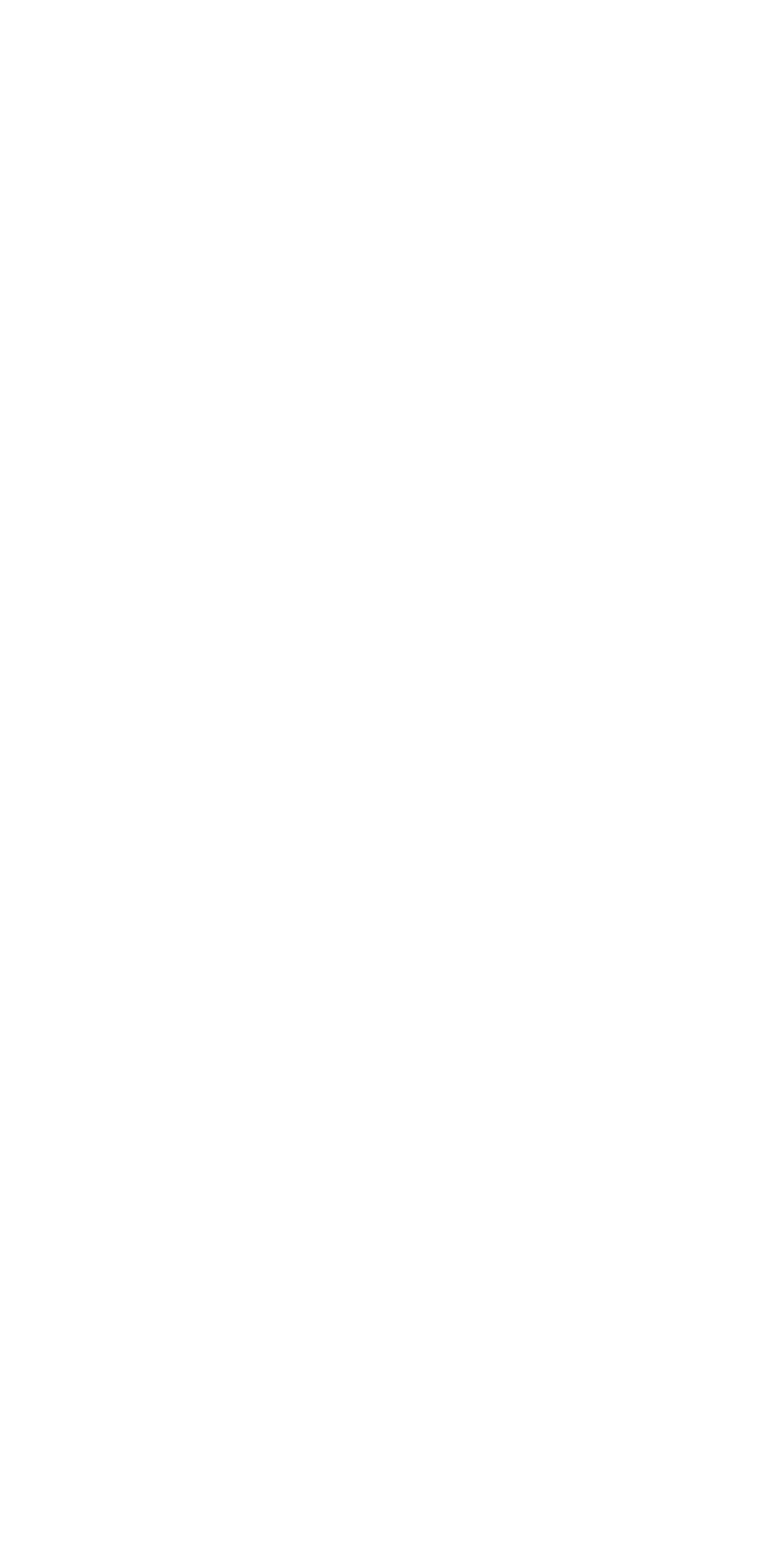




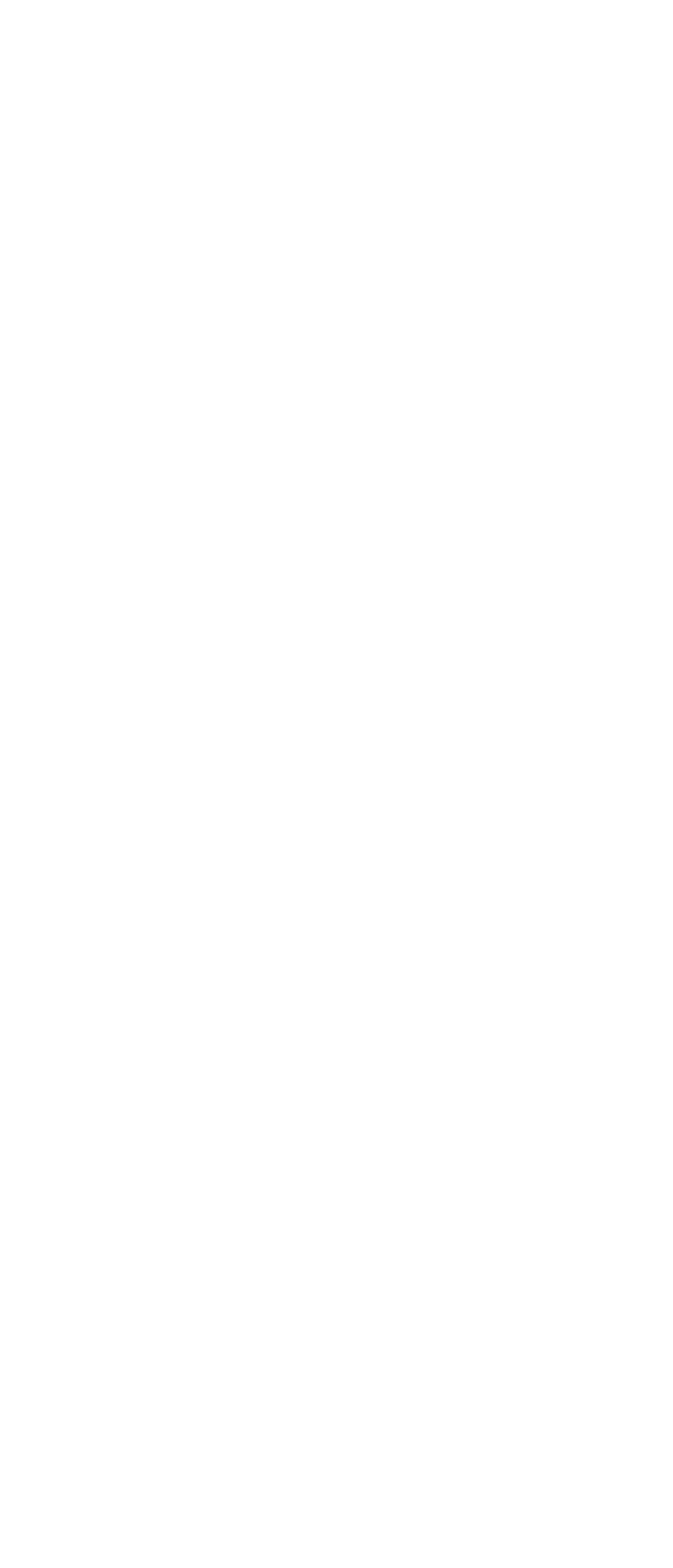




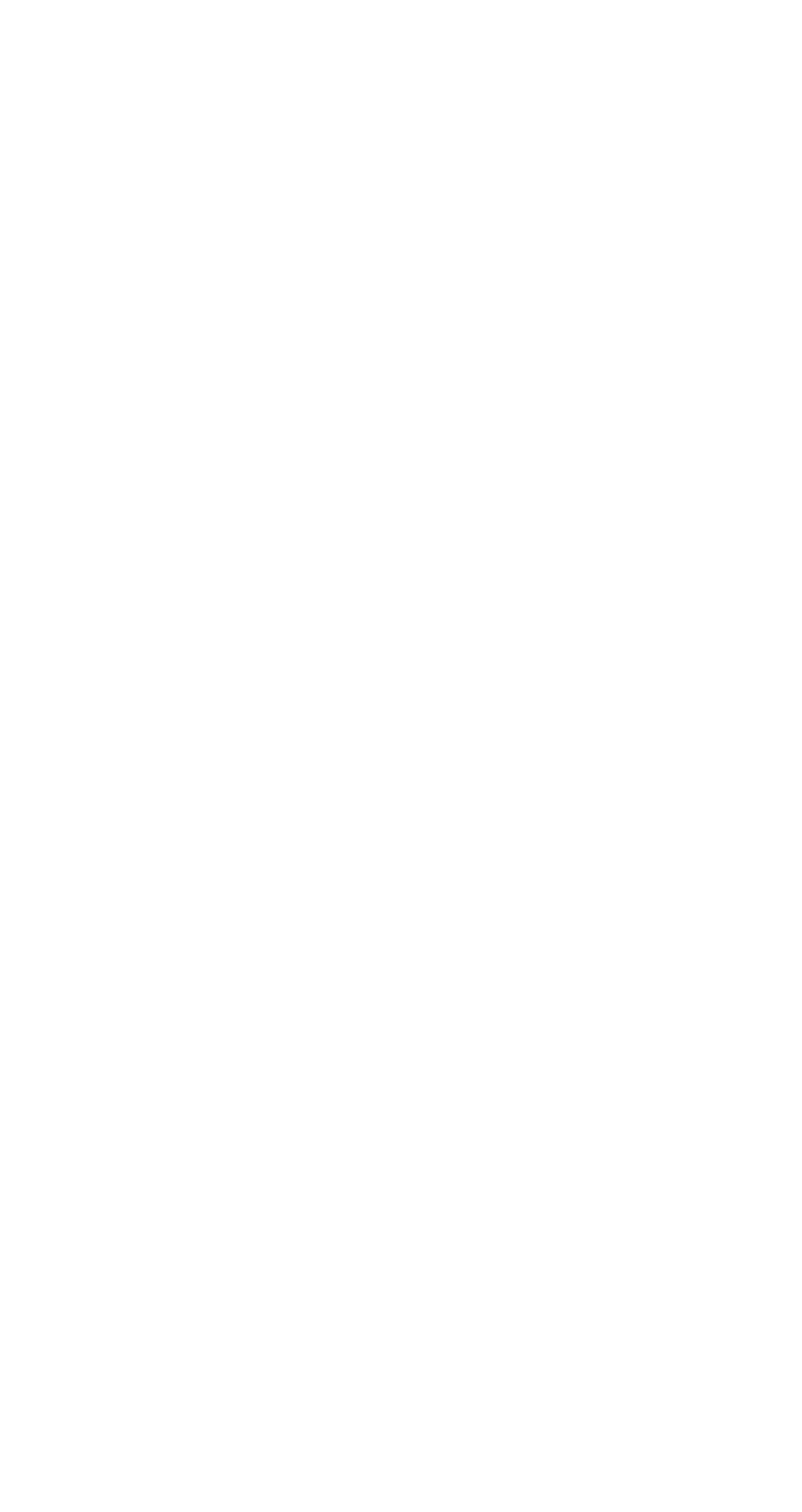




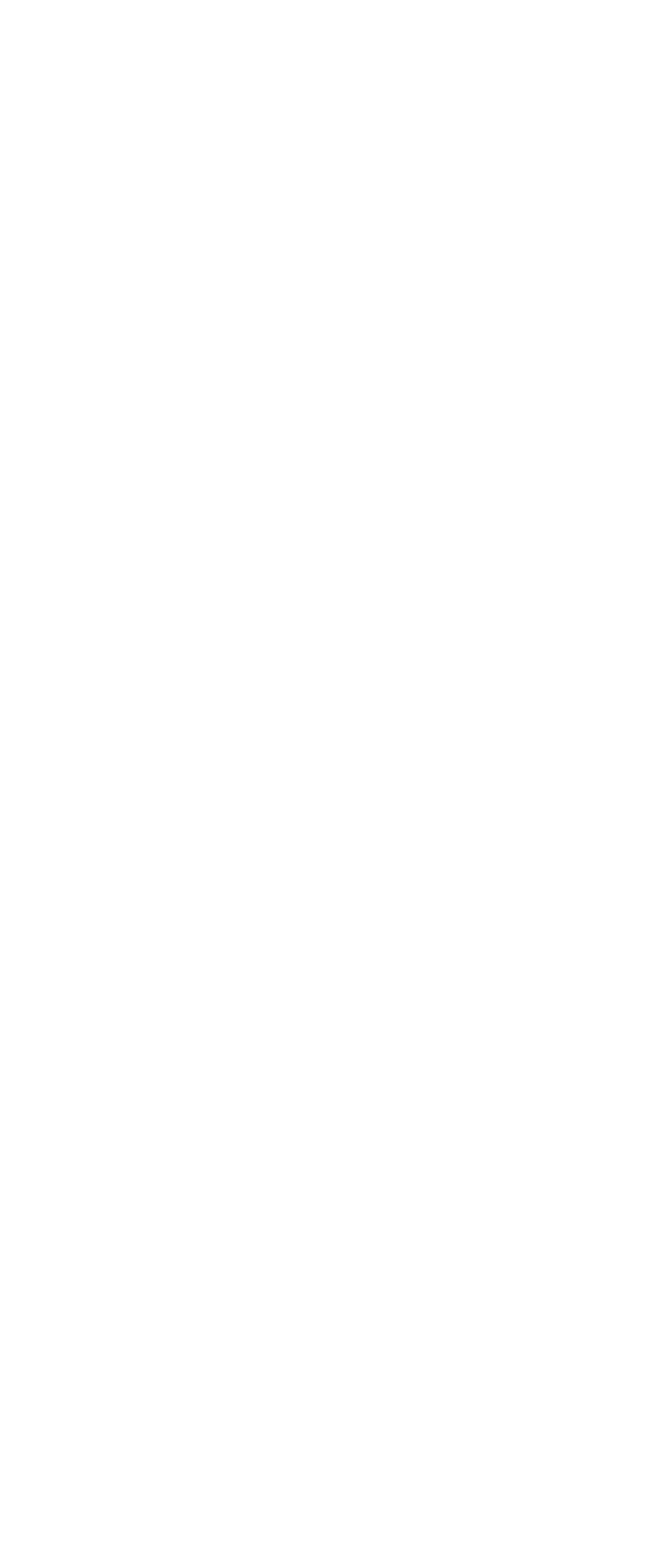




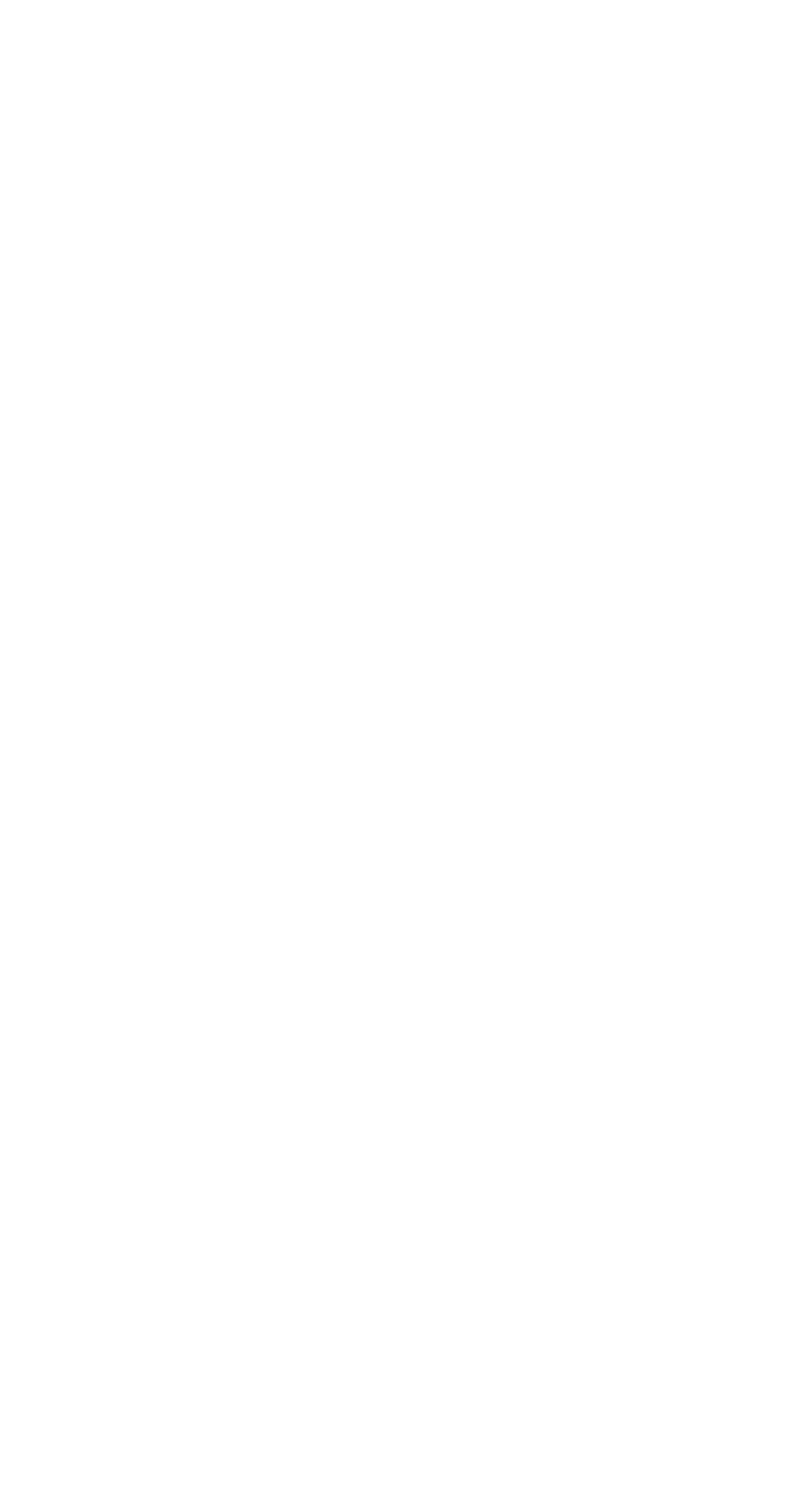




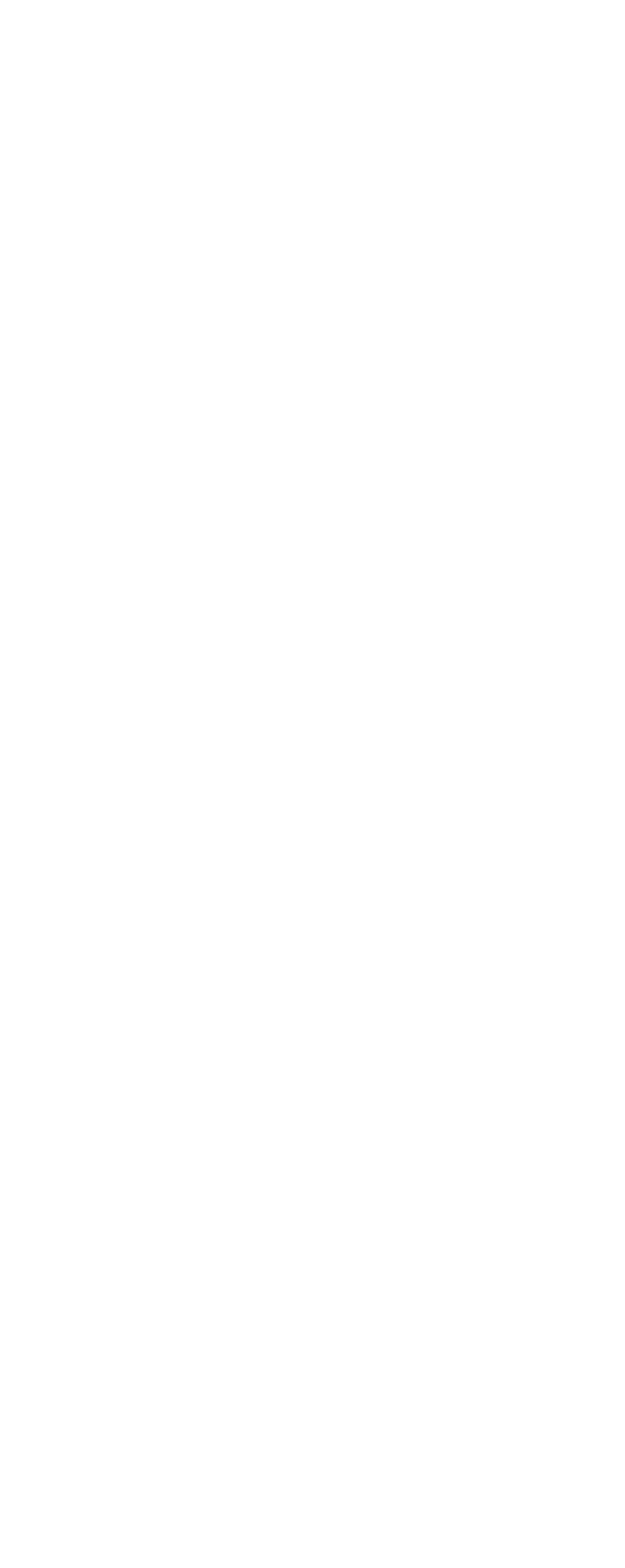




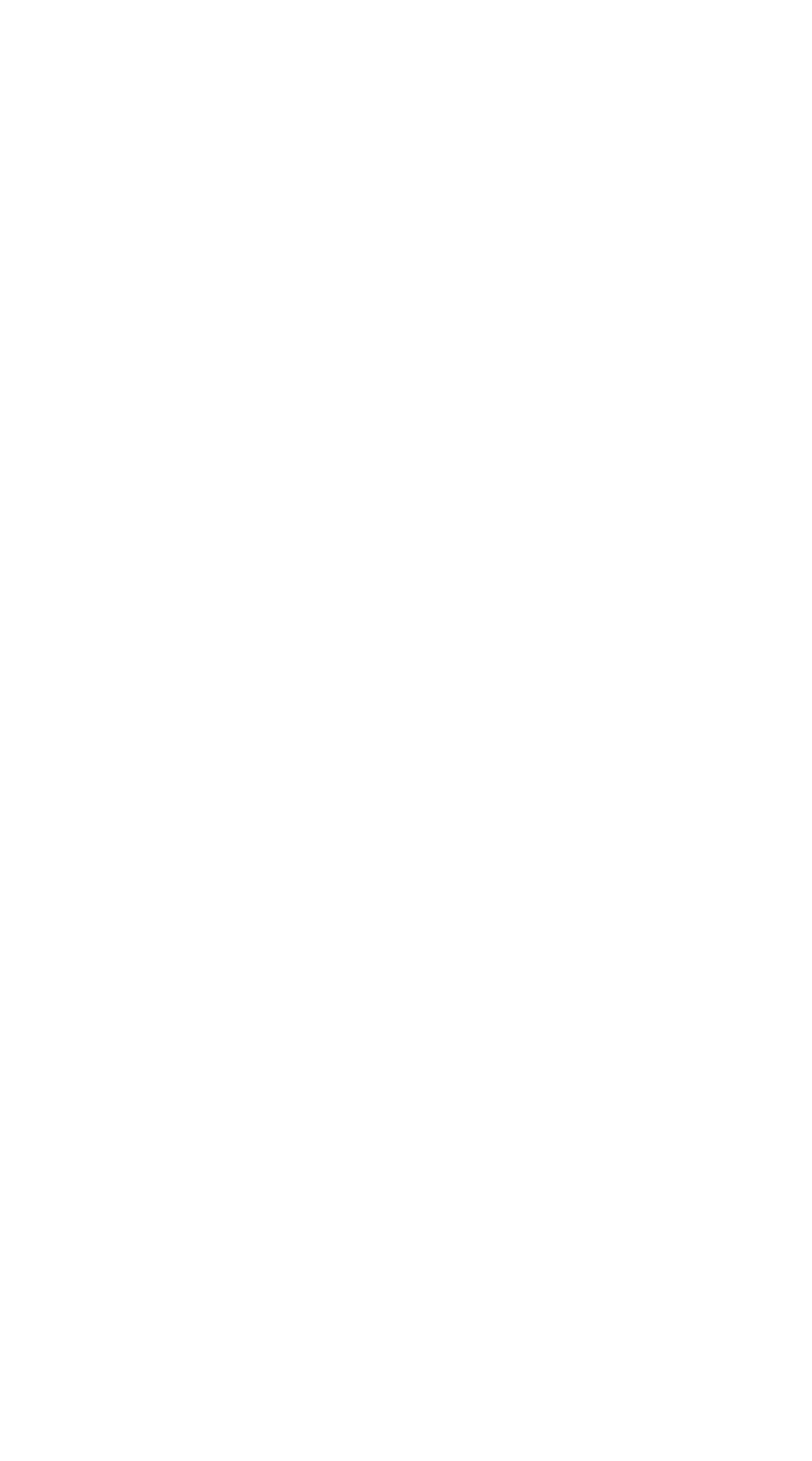




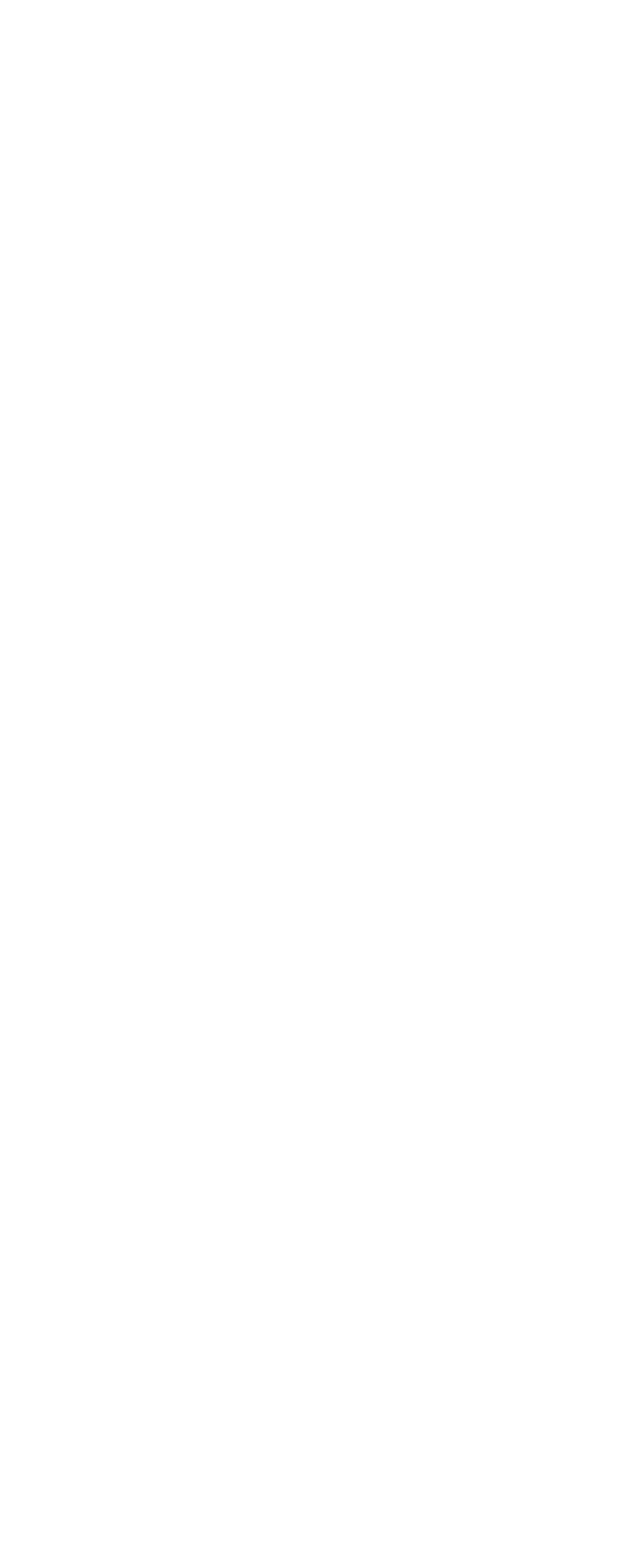




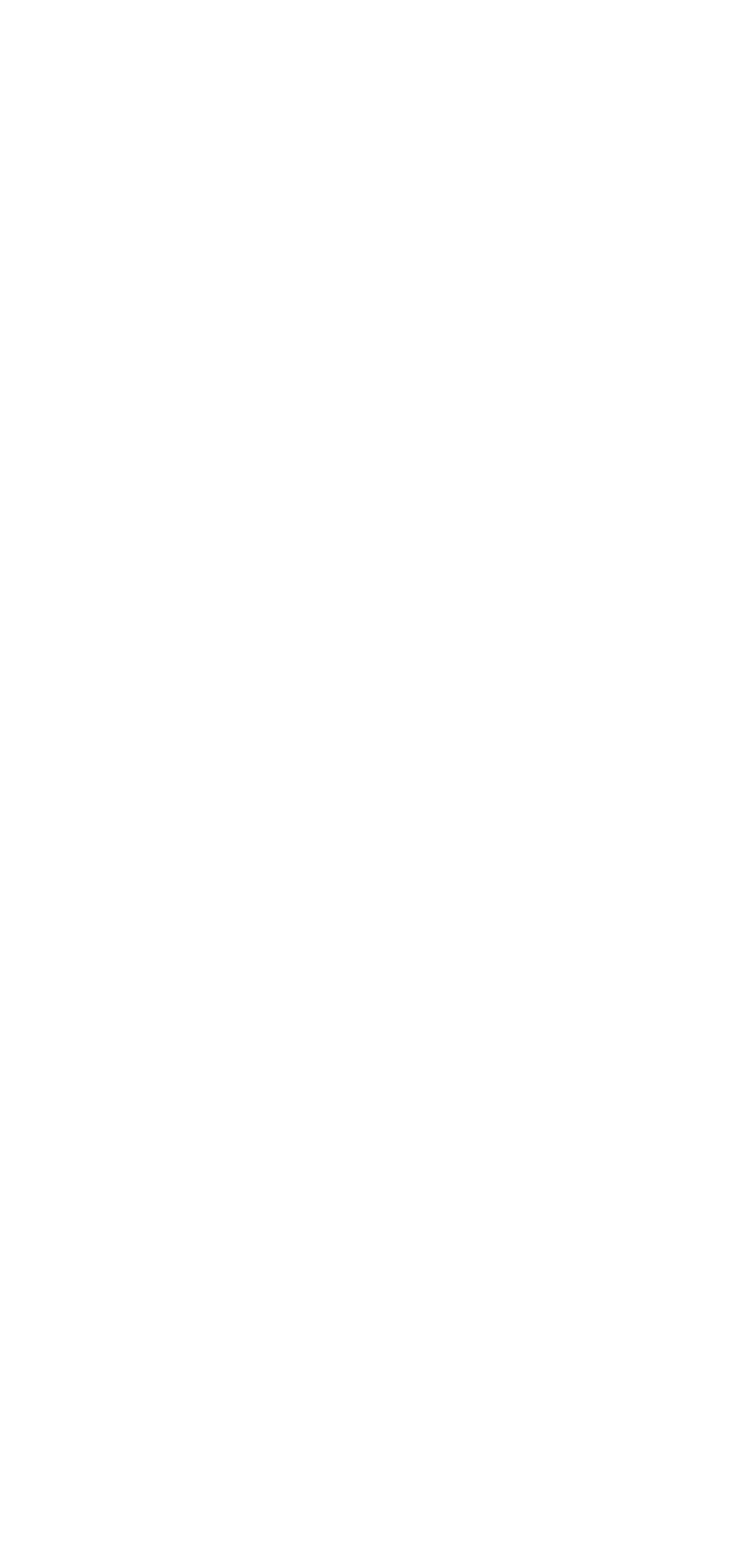




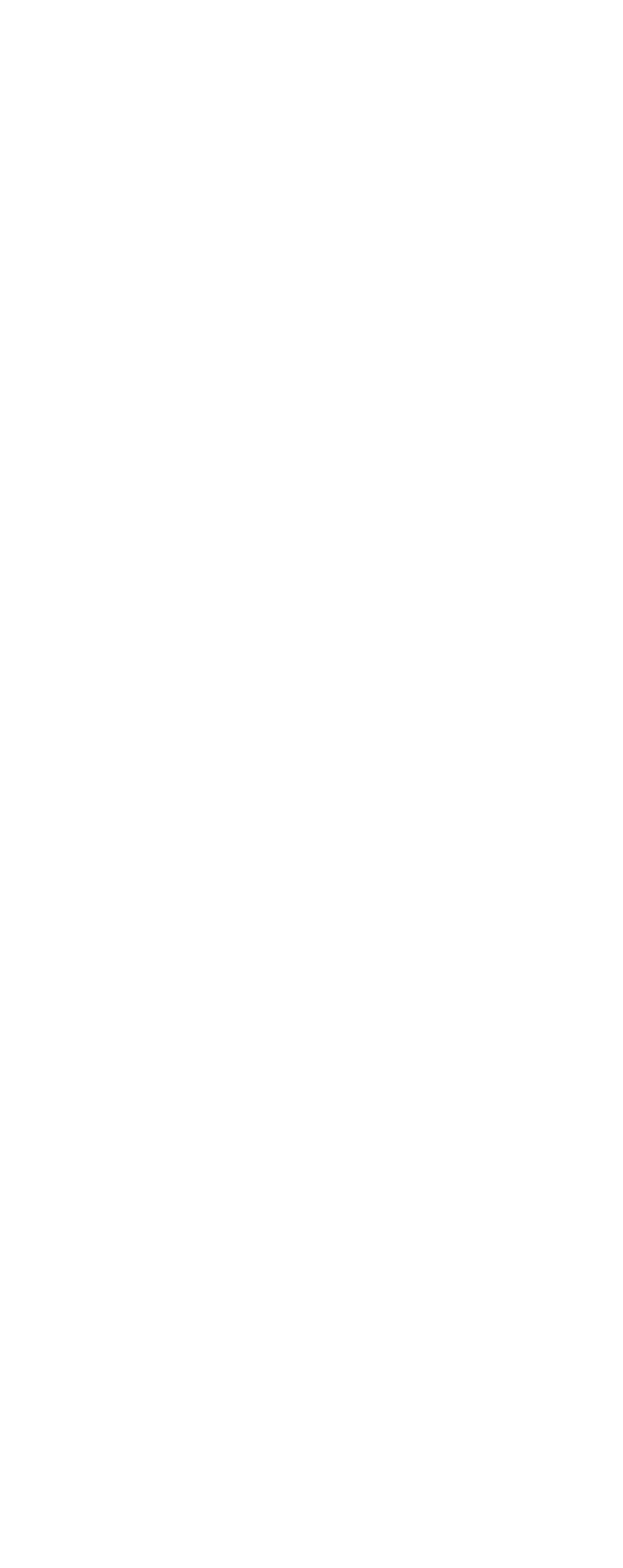




\section{INDEX}

A

Abiquiu Dam, N. Mex., Rio Chama

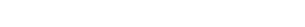

Abiquiu Reservolr, N. Mex., Rio

Chama above.

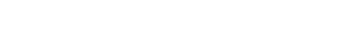

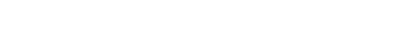$$
\text { below. }
$$

Albany, Tex... Hubbard Creek near.....

North Fork Hubbard Creek near....

Salt Prong Hubbard Creek near.

Snailum Creek near.

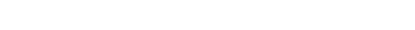

Alto Tex. Neches Biver near ..... 85-89

Aluminum..................... r r 11

Amarillo, Tex, Canadian River near. 194-195

Anahuac, Tex., Trinity Bay near.... 326-328

Trinity River at............ 323-325

Angelina River below Sam Rayburn

Dam, near Jasper, Tex........ 294-295

nea $r$ Lufkin, Tex............ 292-293

Angleton, Tex, Brazos River near.

Anzalduas Dam, Tex., Rio Grande

$$
\text { below. }
$$

Apishapa River near Fowler Colo.

Arkadelphia, Ark., Ouachita River at 239-240

Arkansas River, at Arkansas City

$$
\text { Kans. }
$$

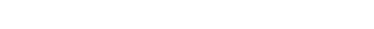

at Dodge City, Kans.

at Great Bend, Kans

at Lamar, Colo.

at Las Animas, Colo.

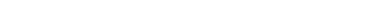

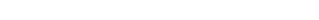

Tulsa, Okla.

Tulsa, Okla ............. 150-153

at Van Buren, Ark........... 210-214

below John Martin Reservoir, Colo.

below Wichita, Kans.

near Coolidge, Kans

near Kinsinson, Kans...........

near La Junta Colo.

near Nepesta, Colo.

near Pueblo, Colo.

rkansas River basin

0-218, 268-272, 278-279, 282

Artesia, N, Mex, Pecos River near. 515-520

Aspermont, Tex., Double Mountain

Haystack Creek near.

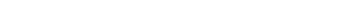

Salt Fork Brazos River near...... 339-341

Whitewater River at ............. 131

Austin, Tex., Colorado River at ...., 411-412

Ballinger, Tex., Colorado River at. 403-405

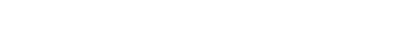
Base-flow investigation, Littie Cypress Creek in Texas...

San Gabriel River in Texas ...... 254-257

Baxter Springs, Kans., Spring River

$$
\text { near }
$$

Bayou Bartholomew near McGehee, Ark

Bayou D'Arbonne at Homer, La.........

Beals Creek near Westbrook, Tex.....

Beals Cre

Belton, Tex., Lampasas River near..

Leon'River' near.

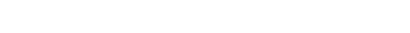

Bernalilio floodwater retarding reservolr No. 1 (Piedra Lisa

Arroyo) near Bernalil

Bernalillo, N. Mex, Rio Grande near 466-469

Bernardo, $N$, Mex., Rio Grande near. 470-475

Rio Puerco near.............4 476-479

Bicarbonate, carbonate and hydroxide

Big Cabin Creek near Big Cabin,

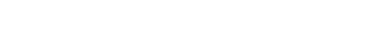

(

Big River near DeSoto, Mo.................

Tex................. 358-360

Biochemicai oxygen demand $\ldots \ldots . . .22$

Black Bear Creek at Pawnee, Okia.... 148

Black River at Harkey Crossing, neàr

Malaga, $\mathbf{N}$, Mex............ 528

Black River at Jonesville, La ...... 251-253

Blackwel1, Olka., Chikaskia River

near................. 145-147

Boron....................... 15

Bourbeuse River above Union, Mo..... 43-44

Brazos River, at Brazoria Reservoir. near Brazorta, Tex........... 389

at Harris Reservoir, near Angleton, Tex............... 388

at Possum Kingdom Dam, near

Graford, Tex.

. $\ldots \ldots \ldots \ldots \ldots, 386-387$

Seymour, Tex............... 342-343

State Highway 21, near Bryan, Brazos Rtver basin. 332-392,57?-586,597 Breckenridge, Tex., Big Sandy Creek

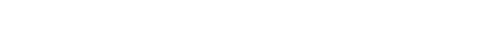
Bromide. . . . . . . . . . . . . . . 18 Bryan Tex., Brazos River near...... 378-379 Navasota River near............ 383-385 Buffalo City, Ark., White River at.. 51 Bull Shoals Dam, Ark., White River

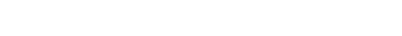

Calctum......... 11-12

Callfornia Creek near stamford, Tex. 344-345 Calista, Kans., South Fork Ninnescah River near.

Cameron, Tex., Littie River at...... 376-377

Canadian River, near Amarillo, Tex. 194-195

near Glenrio, N. Mex........... 193 near whitefield, Okla ........... 207-209 Caney, Okla., Clear Boggy Creek near 232-233 Canon City, Colo., Arkansas River at 60 Carlsbad, N, Mex., Pecos River at ... 526-527

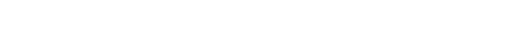
Carnegie, Okla., Washita River at ... 223-225 Cartervilie, Mo., Center Creek near. 178-181 Cedar Creek near Cedar Point, Kans. 170 Center Creek near Carterville, Mo... 178-181 Chambers Creek near Corsicana, Tex. 307-309 Chamita, $N$ Mex. Rio Chama near... 447-452 Chemical oxygen demand........... 22 Chemical quality................. Cheney, Kans. North Fork Ninnescah

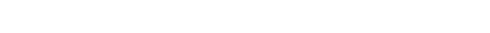
Chlkaskia River near Blackweil; Okla................... 145-147 near Corbin, Kans.............. 140-144 Chloride................... 13-14

Chromi um.

Cimarron River near Guy, N. Mex,... 149

Cla rendon, Ark., White River at .... 58-59

Clear Boggy Creek near Caney, Okla.. 232-233
Clear Fork Brazos River at Eilasville, Tex........................363-365

Clifton, Tex., Nortb Bosque River 369

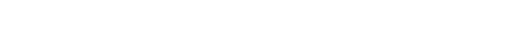

Collection and examination of .... samples ........... 3-6

Colorado City, Colo., Colorado River at . . . . . . . . . . . . . . . Colorado River, at Austin, Tex..... 411-412 at Ballinger, Tex...........4 403-405 at Colorado City, Tex.......... 396-397 at Columbus, Tex............ 413-416 
Colorado River, at San Saba, Tex... 406-407 at Wharton Tat San Saba, Tex.... near San Saba, Tex near Silver, Tex.

Colorado River basin... $393-418,587-590,597$ Columbus, Tex., Colorado River at... 413-416 Commerce, Okla., Neosho River near.

Composition of surface waters...

Conchas Canal below Conchas Dam,

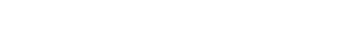
Conroe, Tex., West Fork San Jacinto River near. . . Arkansas River nea Coolidge, Kans, Arkansas River near
Cooper, Tex., South Sulphur River

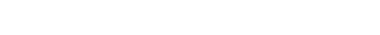

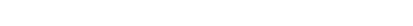
Copper.

Corbin Kans Chikaskia River near 140-144

Corsicana Tex., Chambers Creek near. 307-309 Cottonwood River, at Cottonwood

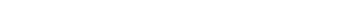

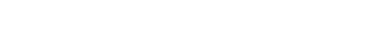
near plymouth, Kans.

Cove, Tex., Old River near.

Covington, La., Tchefuncta River

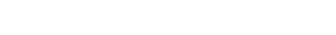

Cow Creek near Lyons, Kans ....
Coyville, Kans., Verdigris River

177

$10-24$

188

$329-331$
$72-73$ 234-235

Crockett Tex Trinity River near.

Croton Creek near Jayton, Tex...... 334-33

Dawson N. Mex., Vermejo River near. . Dayton, $\dot{N}$, Mex., Rio Penasco at.... Deep Creek at Moran,

Deep Fork near Beggs, Okia.

Denison, Tex Red River near....... 204-206

DeSoto, Mo. Big River near.... $45-46$

DeValls Bluff, Ark., White River at. 56-57

Dissolved oxygen. . . . . . . . . . .

Dissolved solids.

Division of work.......................

Dodge City, Kans., Arkansas River at $74-75$

Domingo, N. Mex., Galisteo Creek at, 460-463

Double Mountain Fork Brazos River near Aspermont, Tex.

Douglass, Kans, Walnut River near.

Durwood, Okla., Washita River near. E

East Branch Walnut River near E Dorado, Kans

E1 Dorado, Kans., Wai nut River near West Branch Wainut River near...

Eliasville, Tex., Clear Fork Brazos River at.

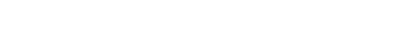

Elm Fork Trinity River near

Muenster, Tex.

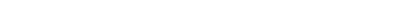

Eureka, Kans, , Fall River near......

Evadale, Tex., Neches River at......

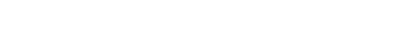

$$
\text { F }
$$

Fairfield, Tex., Richland Creek near 310-311 Falcon Dam, Tex, Rio Grande below.

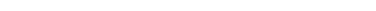

near Eureka, Kans.

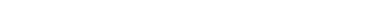

$$
\text { near. A. , Ouachita }
$$

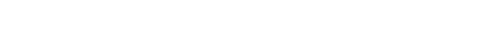

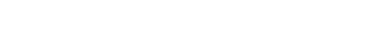

Fort Quitman, Tex., Rio Grande at...

Foss Reservoir near Foss, Okla.

Fountain Creek at Pueblo, Colo...

Fowler, Colo. Apishapa River near.

Fredonia, Kans, Fall River at....

Galisteo Creek at Domingo, N. Mex... 460-463 Gallinas River at Montezuma, N. Mex. near Montezuma, N. Mex.

Ganado, Tex., Navidad River near.... 419-421 Georgetown, Tex., San Gabriel River

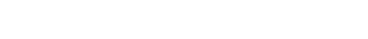

Girvin Tex... Pecos River near .... 540-54

Glenrio, $\mathrm{N}$. Mex., Canadian River

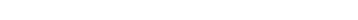
$417-418$ $393-395$ $408-410$ $400-402$

Goliad, Tex, San Antonio River at , 425-427 Gore, Okla.' Illinois River near... 186 Graford, Tex., Brazos River near.... 366 Great Bend, Kans. Arkansas River at 83-84 Guadalupe River at Victoria, Tex..., 422-423 Guadalupe River basin...... 422-427, 591-592 Guy, N. Mex., Cimarron River near... 149 H

Hardness

Haystack Creek near Aspermont, Tex. Heber Springs, Ark., Little Red Henrietta, Tex., Littie Wichita River near.............. 219-221 Hollywood, N Mex., Rio Ruidoso at. 514 Homer, La., Bayou D'Arbonne at ..... 236- 248 Hubbard Creek, near Albany, Tex..... 350-351 near Breckenridge, Tex......... 361-362 near Breckenridge, Tex, ........

Hubbard, Tex., Pin Oak Creek near... 305-306 Hutchinson, Kans., Arkansas River Hydrogen-ion concentration........ 20.21

I

Illinois River near Gore, Okla.

Independence, Kans., Verdigris River at ................... 158-160 Inola, Okia., Verdigris River near. 164-166 Introduction............... 1-3 Ira, Tex.. Colorado River near..... $393-395$ Iron.................

Jasper Tex., Angelina River near. . . 294-295 Jayton, Tex. Croton Creek near.... 334-335 Jemez River below East Fork, near

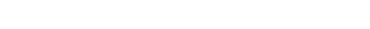

John Hartin Reservoir, Colo. Arkansas River below.

Johnson Ranch, Tex. Rio Grande .... 68-70 Jonesville, La., Black River at .... 251-253 Joplin, Mo., Turkey Creek near..... 182
132 121

\section{Inas}

Kingman, Kans, , South Fork Ninnescah

River near, South Fork Ninnescah 105-106 Kingsley, Kans., Arkansas River near $\begin{array}{r}76-80 \\ 76-106\end{array}$

La Junta, Colo., Arkansas River rear Lake Arthur, La., Mermentau River at 283-285 Lamar, Colo., Arkansas River at..... $\begin{array}{r}71 \\ 372-373\end{array}$ mear Langtry, Tex., Rio Grande at ......... 503 Laredo, Tex., Rio Grande at ......... 543 Larned, Kans., Pawnee River near.... $\begin{array}{lr}\text { Larned, Kans, Pawnee River near. . . } & 81-82 \\ \text { Las Animas, Colo., Arkansas River at } & 66 \\ \text { Purgatolre River near........... } & 67\end{array}$ Las Cruces, N. Mex., Tortugas Arroyo near.................. 498 Lavaca River basin ........... 419-421,590 Lenapah, Okia., verdigris River near................. 161-163 Leon River near Beiton, Tex....... 370-371 Literature cited...............

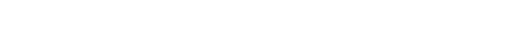
Little Arkansas River at Vailey $\cdots$

Center, Kans....................
Little Cypress Creek base-fiow investigation in Texas.

98

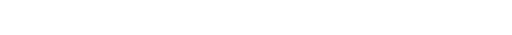
Little River below Hog Creek, near Norman, Okla.

Little River near Poilock, La........ 250 Little River near Pollock, La .....
Little River near Sasakwa, Okia ..... 197-199 Little River near Sasakwa, Okla .... 197-199
Little Rock, Ark., Arkansas River at 215-218 Little Hichita River near Henrietta, Tex. .

Livingston, Tex., Long king Creek near....................... 314-315 Lobatos, Coio. "Rio Grande nea $x \ldots .4433-434$ Logan, N. Mex., Revuelto Creek niear. 190-193 
Long King Creek near Livingston, Tex. Lower Mississippi River basin.

Lufkin. Tex. Angelina Rtver ...... 37-282 Luling Ferry, La, Mississippi River

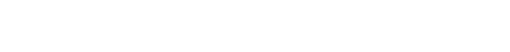

McGehee, Ark., Bayou Bartholomew

McMillan Dam, N. Mex, Pecos River below.

Malaga, $N$. Mex, Black River near

528

Manganese........................ 11

Marion, Kans... Cottonwood River near 168

Mud Creek near.............. 169

Mathis, Tex., Nueces River near.... 431-432

Maysville, Okla., Rush Creek near... 226

Meramec River at Paulina Hills, Mo. near Sullivan, Mo.

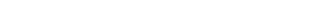

Mermentau River at Lake Arthur. La.. 283-285

Mermentau River basin.......... 283-285

Mineral constituents in solution.

Miscellaneous analyses of lakes and streams in lower Mississippi River basin.

in western Guif of Mexico basins. . $570-597$

Mission River near Refugto, Tex.

Mission River basin.................

Mississippi River, at Luling Ferry.

La . . . . . . . . 260 261

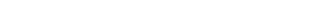

near St. Francisville, La. . 26.26. 258-259

Mississippi River Delta.... 262-266, 279-281

Montezuma, N. Mex., Gallinas River

at Gallinas river near

505

505

Moran, Tex., Deep Creek at

Moss Bluff, Tex., Trinity River

$$
\text { near. }
$$

348-349

Mud Creek i

Muenster, Tex, Elm Fork Trinity

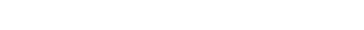

Murdock, Kans., South Fork Ninnescah River near.............. 107-110

Nambe, N, Mex., Rio Nambe near. Navasota River near Bryan, Tex................ Navidad River near Ganado, Tex.

Neches River at Evadale, Tex. near Alto, Tex.

Neches River basin.......... 290-297, 570-571

Neosho River, at Buriington, Kans.

below Fort Gibson Reservoir, near Fort Gibson, Okla............

near Americus, Kans.

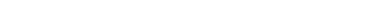

near Parsons, Kans.

Nepesta, Colo, Arkansas River near

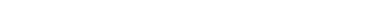

Ninnescah River near Peck, Kans,....

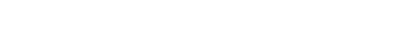

Norfork, Ark., North Fork River near

Norfork, Ark., North

Norphlet, Ark., Smackover Creek near 241-244

North Bosque River near Clifton, Tex...

\section{3}

167

74-176

63

16

North Canadian River near Wetumka.

Okla . . . . . . ............ 200-203

North Fork Hubbard Creek near

Albany, Tex..................

North Fork Ninnescah River near

Cheney, Kans..................

North Fork River at Nor
near Norfork, Ark

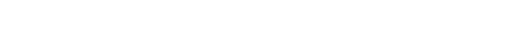

... 354-355

$\ldots . .$.

100

Nueces River basin....... 431-432,594-595

O

Old River near Cove, Tex.....

321-322

Orla, Tex., Pecos River near....... 538-539

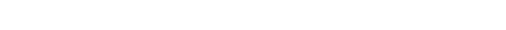

at Monroe, La......... 249

at Monroe, La $\ldots \ldots \ldots \ldots \ldots \ldots \ldots \ldots, \quad 24249$

oxygen consumed................
Park View, N. Mex., Willow Creek near ............. Kasho River near Paulina Hills, Mo., Meramec River at 47-48 Pawnee, Okla., Black Bear Creek at.. 148 Pawnee River near Larned, Kans..... $81-82$ Peck. Kans., Ninnescah River near... 111-113 Peck, Kans., Ninnescah River near... 111-113 at damsite 3 , near Carisbad,

at Pierce Canyon Crossing, near Malaga, $N$. Mex

at Red Bluff, $N$. . . . . . . . . 532-533 at Santa Rosa, $N$ Mex......... $534-537$ below Alamogordo Dam, N. Mex..... 510-511 below McMilian Dam, $N$. Mex...... 523 below Red Bluff Dam, near Oria, Tex...

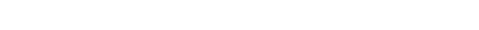

near

near Artesia, $N$. Mex............ 515-520

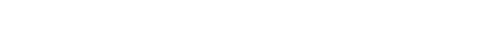

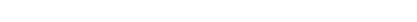

seepage investigations.......... 548-568

water delivery study in Texas.... 547

Pin Oak Creek near Hubbard, Tex..... 305-306

P1 ymouth, Kans., Cottonwood River

near..................... 172

Pollock, La., Littie River near... . 250

Ponchatoula, La., Tangipahoa River near..... South Fork Ninnescah

River near............... 101-102

Presidio, Tex Rio Grande near. 501

Properties and characteristics of

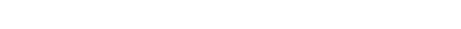

Publications ...............

Pueblo, Colo., Arkansas River near. . 61

Fountain Creek at .............. 62

Purgatoire River near Las Animas, Colo................. 67

$\mathbf{R}$

Rattlesnake Creek near Raymond.

Red Bluff, N. Mex., Pecos River at. . $534-537$ Red River, at Denison Dam, near

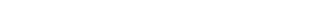

below Zwergle damsite, near Red

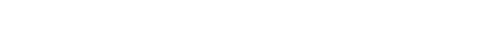
near Red River, $\mathbf{N}$. Mex........ 435 Red River basin........... $219-257,273-277$ Refugto, Tex., Mission River near... 428-430 Revuel to Creek near Logan, N. Mex... 190-193 Richland Creek near Fairfield, Tex. 310-311 R1chmond, Tex., Brazos River at... 386-387 io Chama, above Abiquiu Reservoir. N. Mex.................. 439-442 below Abiquiu Dam, $\mathrm{N}$. Mex....... 443-446 near Chamita, $N$. Mex............. 447-452 R10 Grande, ahove Culebra Creek, near Lobatos, Colo.............. above Rio Conchos near Presidio, 433-434 Tex

at Fort Ringgold, Rio Grande city,

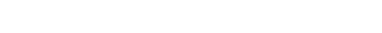

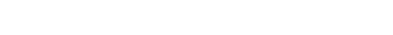
at Fort Quttman, Tex.........

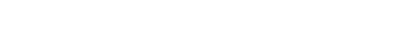
at Langtry, Tex. at Otow1 Bridge, near $\operatorname{San} \ldots \ldots \ldots$ Ildefonso, $N$. Mex. . . . . . . . 454-459 below Anzalduas Dam, Tex....... 546 below Falcon Dam, Tex. San Acacia

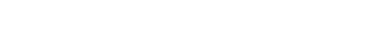
conveyance channel at San Marcial, N. Mex... . . . . . . . . . . 489-494 floodway at San Acacia, $N$. Mex... 485-488 floodway at San Marciai, $\dot{N}$. Mex... 495-497 near Bernali1lo, $N_{\text {. Mex }} \ldots \ldots \ldots \ldots 46-469$ near Bernardo, N. Mex..... Rio Grande City, Tex.., Rio Grande at 545 Rio Grande de Ranchos near Talpa, N. Mex.

Rio Hondo at damsite at vajdez, N. Mex................ 436

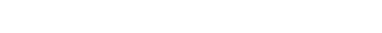


Rio Penasco at Dayton, N. Mex. Rio Puerco near Bernardo, N. Mex. Rio Ruidoso at Hollywood, N. Mex. Rio Salado near San Acacia, N. Mex. Rio Tularosa near Bent, N. Mex. Romayor, Tex., Trinity River at. Romar. Tex. Trintty River at. Rosser, Tex., Trinity River near.... Rush. Ark. Buffalo River near..... Rush, Ark, Buffalo River near .....

Rush Creek near Maysvi

Sabine River near Ruliff, Tex.

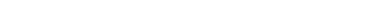
Sabine River basin ...................... Rtver near. Louis, Mo., Mississippi River

\section{at . Mo., Mississippi kiver}

Salt Croton Creek at weir $D$, near Aspermont, Tex

near Aspermont, Tex.

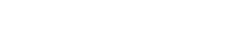
mont, Trazos River near Asper-

Salt Prong Hubbard Creek at Ù. Highway 380 , near Albany, Tex. .

San Acacia, N. Mex., Rio Grande conveyance channel at ..........

Rio Grande floodway at $\ldots \ldots \ldots \ldots \ldots$

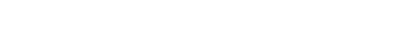

San Antonio River at Goliad, Tex.
San Gabriel River at Georgetown,

San Gabriel River at Georgetown, Tex .... $\ldots \ldots \ldots \ldots \ldots \ldots$

San Ildefonso, N. Mex., Rio Grande

454-459

San Jacinto River basin........ 329-331,577

San Marcial, N. Mex., Rio Grande conveyance channel at. . . . . . 489-494

Rio Grande floodway at . . . . . . 495-497

San Saba River at San Saba, Tex.

San Saba, Tex. Colorado River near.

Santa Rosa, N. Mex., Pecos River at.

Sasakwa, Okla., Little River near.

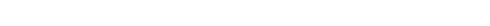

Sedw1ck, Tex., Hubbard Creek near. . 346-347

Seymour, Tex., Brazos River at..... 342-343

Shipps Ferry, Ark. White River at

Shroeder Tex Coleto Creek near...

Shroeder, Tex. Coleto Creek near...

Shumla, Tex., Pecos River near.

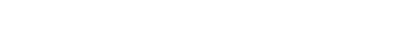

Silver, Tex., Colorado River near...

Snatlum Creek near Albany, Tex.....

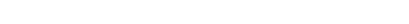

Somerville, Tex., Yegua Creek$$
\text { near. }
$$

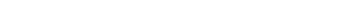

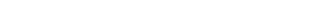
Calista, Kans.

near Kingman, Kans.

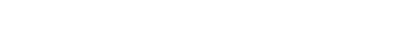

near hudock, Kans.............

South Sulphur River near Cooper.

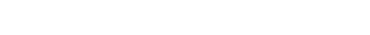

Specific conductance .... s. $\ldots \ldots$

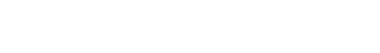

Stamford, Tex., Caiffornia Creek

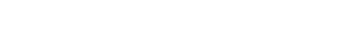

Streamflow.

Strontium . . . . . . . . .

St rontium.

Sulfate..... Meramec River near...

Talpa, N, Mex., Rio Grande de Ranchos near.............................

Tangipahoa River near Ponchatoula,

Latum, Tex.. Sabine River near.......

$7-40$ 336
Tchefuncta River South of Covingto?,

Temperature................ 262-263

Towanda, Kans... Whitewater River at. 126-130 Trinity Bay at mouth of Trinity

River, near Anahuac, Tex....... 326-328

Trintty River, at Anahuac, Tex..... 323-325

at Romayor, Tex................316-318

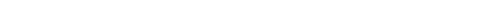
near Moss Bluff, Tex.............. 319-320 near Rosser Tex. ........... 302-304 Trinity River basin..... 298-328,572-576,597 Tulsa, Okla., Arkansas River near... 150-153 Turbidity................ 24 Turkey Creek near Jopiin, Mo....... 182

Unton, Mo., Bourbeuse River above... 43-44

\section{$\mathbf{U}$}

$\mathbf{v}$

Valdez, N. Mex, Rio Hondo damsite

valley Center Kans... Lititie $\ldots \ldots$.

Arkansas River at ........... 98

Van Buren, Ark., Arkansas River at.. 210-214

Verdigris' River, at Independence,

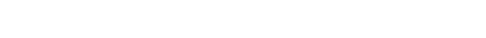
near Coyvilile, Kans $\ldots \ldots \ldots \ldots \ldots \ldots \ldots$, 164-166 near Lenapah, Okla ............. 161-163 Vermejo River, near Dawson, i . Mer.. 187 Victoria, Tex., Guadalupe River at.. 422-423

Walnut Creek at Albert, Kans...... 85-89 Walnut River, at Winfield, Kans..... near Augusta, Kans............. 124-125 near Augusta, Kans.............

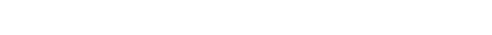
Washita River at Cornegie, Okia..... 223-225 near Durwood, Okla............. 227-229 Water-quality stations in downstream

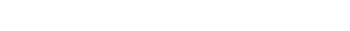

West Branch Wainut River near Ei

542 Dorado, Kans............... 122

10-11 Westbrook, Tex., Beals Creek near. . 398-399

400 West Fork San Jacinto River near

329-331

Western Gulf of Mexico basins....... 283-597

Wetumka, Okla. North Canadian River near.............. 200-203 wharton, Tex, Colorado River at.... 417-418 Whitefield, Okla., Canadian River near.

207-209

White River basin.......... 49-59, 267-268

105-106 Wichita, Kans, Arkansas River be-

07-110 low.......................... 99

101-102 White River, at Buffalo City, Ark... 5 59-50 at Bull Shoals Dam, Ark. . . . . . . .
at Clarendon, Ark. $49-50$

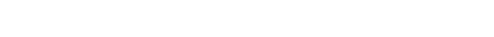
at Shipps Ferry, Ark......... White River basin............ 49-59 Whitewater River at Augusta, Kans... 131 at Towanda, Kans.............. 126-130 Whitney, Tex., Brazos River near... 367-368 Willow Creek near Park View, N. M $3 x, \quad 438$ Winfield, Kans., Walnut River at... 133-139$$
\text { Y }
$$

Yegua Creek near Somerville, Tex... 380-382 Youngsport, Tex., Lampasas River at. 372-373 